\title{
National Health and Nutrition Examination Survey (NHANES)
}

MEC Laboratory Procedures Manual

January 2013 


\section{TABLE OF CONTENTS}

\section{Chapter}

Page

1.1 Overview.

1.2

1.3

1.4

1.5

1.6

1.7

1.8

The Laborat

Tasks.....

Organization of the Laboratory.....

$1-2$

$1-2$

$1-3$

Tasks - Phlebotomy Room

$1-5$

Tasks - Workstation 1

$1-5$

Tasks - Workstation 2

$1-6$

1.9

Tasks - Workstation 3

Cross-training

1-8

$1-9$

2.1 Laboratory-Specific Functionality

2.4.1 File Menu Options......................................................... 2 2-4

2.4.2 Utilities Menu Options ................................................. 2-5

2.4.3 Quality Control Menu ................................................... 2-6

2.4.4 Report Menu Options ..................................................... 2-7

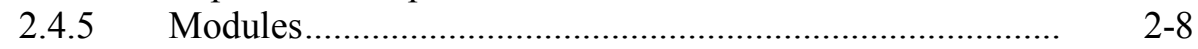

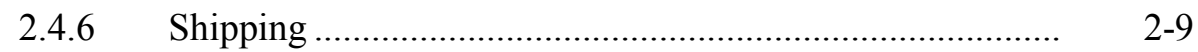

2.5 Informed Consent Exclusions ..................................................... $\quad 2-10$

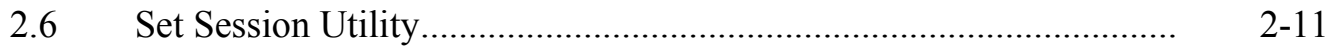

2.7 Container Map ................................................................... 2-13

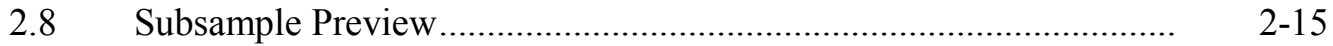

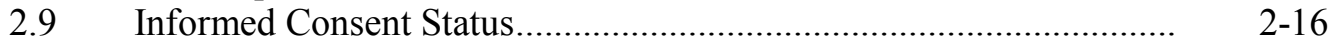

2.10 Access a Processing Module........................................................ 2-18

2.11 Warning and Error Messages.......................................................... 2-19

2.11.1 Not Filled Require a Comment ........................................ 2-20

2.11.2 Labels Must be Printed in Advance .................................... 2-20

2.12 Coulter and Urine Scale Icons ...................................................... 2-21

VENIPUNCTURE ............................................................................. $3-1$

3.1 Introduction....................................................................

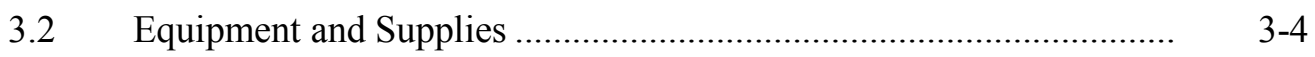

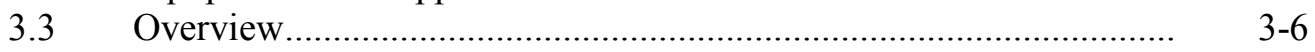




\section{TABLE OF CONTENTS (continued)}

3.4 Gaining Cooperation

\subsubsection{Refusal Conversion ......................................................}

3.5 Performing the Venipuncture on SPs Who Do Not Speak English .... 3-12

3.6 Open the Exam and Log the SP into the Exam................................. 3-13

3.7 The Phlebotomy Interview...........................................................

3.7.1 Pregnancy Shared Exclusion Question ............................. 3-18

3.7.2 Safety Exclusion Questions.............................................. 3-21

3.8 Administering the Fasting Questionnaire ....................................... 3-31

3.9 Venipuncture Procedures ............................................................. $3-41$

3.9.1 Preparation of the Puncture Site....................................... 3-42

3.9.2 Venipuncture Technique for the Nipro (19 Gauge) ............ 3-44

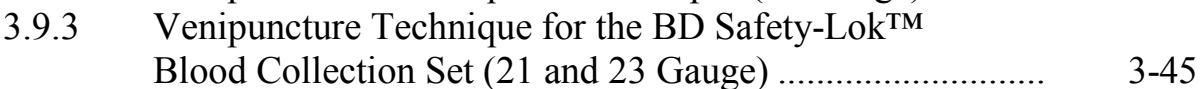

3.9.4 Concluding the Venipuncture............................................. 3-47

3.10 Pediatric Venipuncture ….......................................................... 3-48

3.10.1 Age-Specific Techniques in Phlebotomy Competency....... 3-50

3.11 Hand Venipuncture ........................................................................ 3-63

3.12 Veinlite EMS ….................................................................... 3-63

3.13 Recording the Results of the Venipuncture Procedure ...................... 3-66

3.14 Venipuncture Status ................................................................... $\quad$ 3-70

3.15 Repeat Blood Draw....................................................................

3.16 Phlebotomy Quality Control ........................................................... 3-76

3.16.1 Daily QC …..................................................................

3.16.2 Supply Use Control Log............................................... 3-79

3.17 Red Cross Procedures for Handling Fainting (Syncope) .................. 3- 3-82

3.18 How to Deal With System Failure .................................................... 3-83

$4 \quad$ URINE SPECIMEN COLLECTION AND PROCESSING ..........................

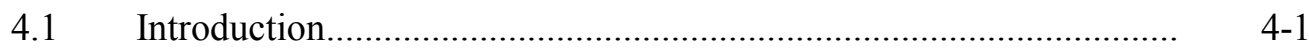

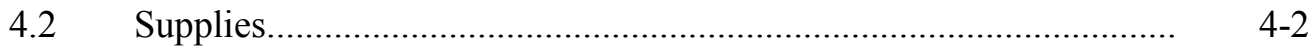

4.3 Urine Flow Rate Background ..................................................... 4- 4

4.4 Using the Scale for Urine Collection ................................................ 4-4 


\section{TABLE OF CONTENTS (continued)}

4.5.1 Quantity Not Sufficient (QNS) Urines.............................. 4-11

4.5.2 Quantity Not Sufficient (QNS) Urines and Enabling Home Urine Collection (HUC)

4.6 Manually Documenting Urine Collection...

4.6.1 Manually Document Urine Collection With

4.6.2 Barcode Scanning.................................................

Barcode Scanning.

4.7 Urine Specimen Assays

4.8 Urine Specimen Protocols

4.9 Labeling Urine Processing Vessels................................................. 4-29

4.10 Urine Specimen Processing .......................................................... 4-30

4.11 Record the Results of Urine Specimen Processing........................... 4-31

4.12 Second and Third Urine Samples .......................................................

4.13 Specimen Storage ..................................................................

4.14 How to Deal With System Failure .....................................................

URINE PREGNANCY TEST ...........................................................

5.1 Purpose and Principle of the Test ..................................................... 5-1

$5.2 \quad$ Special Safety Precautions............................................................. $5-2$

5.3 Computerization: Integrated Survey and Information System (ISIS) ..... $\quad 5-3$

5.4 Specimen Collection and Preparation................................................ 5-3

5.5 Procedure for Microscopic Examination ............................................ 5-3

5.6 Reagents and Supplies ..............................................................

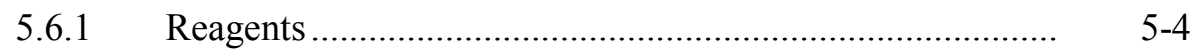

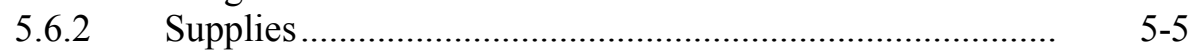

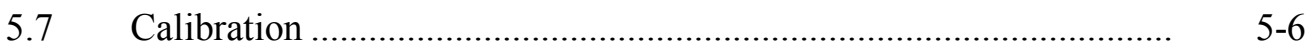

5.8 Assay Procedure …….....................................................

5.9 Reportable Range of Results...........................................................

5.10 Quality Control ..............................................................

5.11 Interpretation of Results and Remedial Action................................. 5-. 5-23

5.12 Record Results ...................................................................... $\quad 5-26$

5.13 Limitations of Method: Specimen Rejection, Interfering

5.14 Reference Ranges .............................................................. $\quad 5-44$

5.15 Action Limits ..............................................................................

5.16 Specimen Storage and Handling During Testing............................ $\quad 5-44$ 


\section{TABLE OF CONTENTS (continued)}

Chapter

$\underline{\text { Page }}$

5.17 Alternative Method for Performing Test or Storing Specimens if Test System Fails ...............................................................................

5.18 Test Results Reporting System Protocol for Reporting Action Limits ... $\quad$ 5-45

5.19 Specimen Accountability and Tracking ......................................... $5-45$

5.20 Quality Control Summary Statistics and Graphs ............................ $\quad 5-45$

$5.21 \quad$ References........................................................................... $5-45$

COMPLETE BLOOD COUNT (CBC).

6.1 Introduction.

6-1

6.2 Purpose and Principle of Test .......................................................

6.2.1 Coulter Method ...............................................................

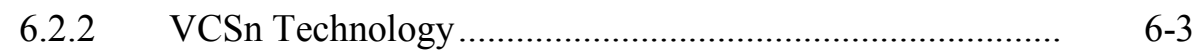

6.2.3 Dataplot Development ................................................. $\quad 6-4$

6.2.4 Parameter Measurement, Derivation, and Calculation........ 6-5

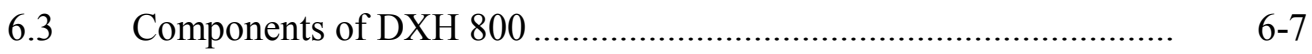

6.3.1 The Specimen Processing Module (SPM) ......................... 6-7

6.3.2 The System Manager (SM) .............................................. 6- 6

6.3.3 Pneumatic Supply Module (PSM) .................................... 6-8

6.4 Special Safety Precautions..................................................................

6.5 Computerization: Integrated Survey and Information System (ISIS) ..... 6- 6

6.6 Specimen Collection and Preparation................................................ 6

6.6.1 Specimen Collection .......................................................

6.6.2 Specimen Preparation.......................................................... 6 6-10

6.7 Procedure for Microscopic Examination ......................................... 6-10

6.7.1 Reagents and Supplies................................................... $\quad 6-10$

6.7.2 DxH 800 Supplies ...................................................... $6-13$

6.8 Shutdown of DxH 800 System ................................................... $6-13$

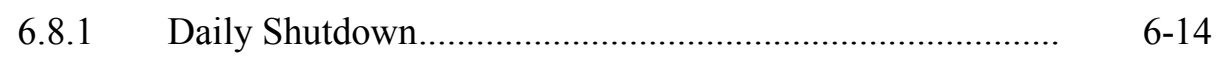

6.8.2 Shutdown at the Mobile Examination Center (MEC) .......... 6-14

6.8.3 Cancel Automatic Shutdown........................................... 6-15

6.8.4 Manual Shutdown of DxH Units...................................... 6-15

6.9 Logon as User .................................................................... $\quad 6-16$ 


\section{TABLE OF CONTENTS (continued)}

Chapter

$\underline{\text { Page }}$

6.10 Run Daily Checks ....................................................................

6.10.1 Daily Checks Screen ...................................................... 6-17

6.10.2 Additional Tasks From the Daily Checks Screen ............... 6-17

6.11 Quality Control .................................................................... $6-18$

6.11.1 Frequency of Performing Quality Control ......................... 6-18

6.11.2 Setting Up a Control File .................................................... 6-19

6.11.3 Setting Up a Latron Control File........................................ 6-19

6.11.4 What To Do When a Control is Out................................... 6-19

6.11.5 Viewing Control Files - Data View ................................. 6-20

6.11.6 Selecting a Control ......................................................... $6-22$

6.11.7 Viewing Control Files Graphically ................................... 6-23

6.11.8 Using and Interpreting the Levey-Jennings Graphs............ $\quad 6-24$

6.11.9 Add, Modify, or Delete Comments to QC Results ............. $\quad 6-25$

6.11.10 View Control Files Under Error Conditions ....................... . 6-26

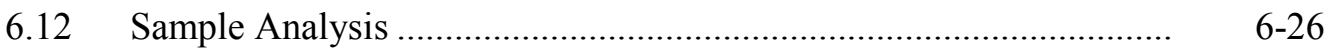

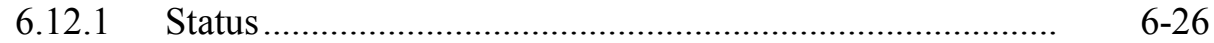

6.12.2 Cassette Presentation....................................................... 6-27

6.12.3 Single-Tube Presentation ................................................... 6-27

6.12.4 Handling Alarms …....................................................... 6-28

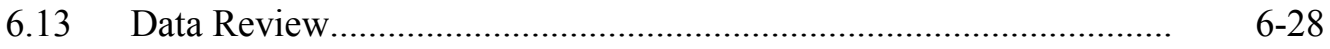

6.13.1 Worklist Screen ...........................................................

6.13.2 Accessing the Worklist Screen........................................... 6-29

6.13.3 Worklist Screen Layout...................................................... 6-29

6.13.4 Parameters For Each Tab ................................................... $\quad 6-30$

6.13.5 Refresh Icon ................................................................. 6-31

6.14 Evaluation of Data .................................................................. $6-31$

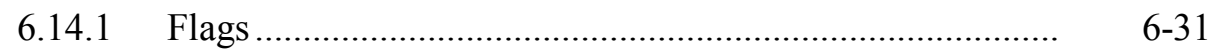

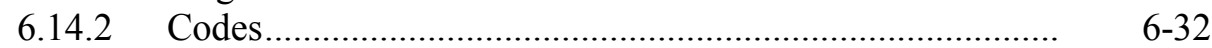

6.14.3 Messages ............................................................. $\quad 6-34$

6.14.4 All Specimens .............................................................. $\quad 6-35$

6.14.5 Capillary Specimen Collection......................................... $\quad 6-35$

6.15 Laser Safety .................................................................. $\quad 6-37$ 


\section{TABLE OF CONTENTS (continued)}

Chapter

$\underline{\text { Page }}$

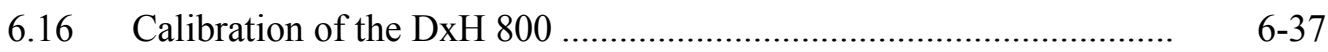

6.16.1 Perform Calibration............................................................ 6-37

6.16.2 Verify the Calibration of Your Instrument................................

6.16.3 Coulter Pre-Start-up Procedures........................................... 6-38

6.16.4 Initial DxH 800 Startup...................................................

6.16.5 Calibration, Repeatability, and Carryover Check ............... 6-39

6.16.6 Carryover ..................................................................

6.16.7 Calibration................................................................. 6

6.17 Assay Procedure ….................................................................

6.17.1 Run Daily Checks at the Beginning of the First

Session Each Operational Day ......................................... $\quad 6-43$

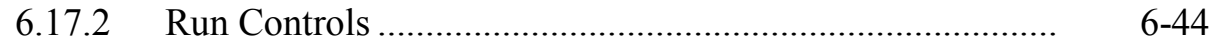

6.17.3 Run Sample Persons....................................................... 6-44

6.17.4 Review Data and Transmit................................................. 6-45

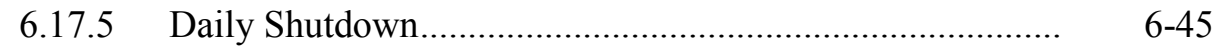

6.17.6 Prolonged Shutdown .......................................................

6.17.7 Beginning and End of Stand Operations ............................ 6-45

6.18 Coulter ${ }^{\circledR}$ Reportable Range of Results............................................

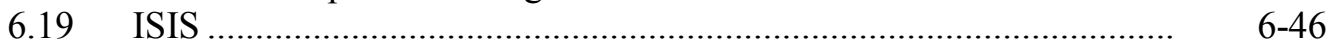

6.19.1 Record 6C Cell and Latron ${ }^{\circledR}$ Lot Numbers in ISIS ............. 6-46

6.19.2 Interlaboratory Quality Assurance Program (IQAP)........... 6-49

6.19.3 Proficiency Testing ............................................................. $\quad 6-50$

6.19.4 Linearity for WBC, RBC, Hgb, and Plt Parameters........... $\quad 6-50$

6.20 Interpretation of Results and Remedial Action.................................

6.20.1 Sample Person Hemoglobin and Hematocrit Review and Remedial Actions ......................................................

6.20.2 Sample Person Parameter Value Review and Remedial Actions .........................................................................

6.20.3 DxH 800 Suspect Messages ............................................

6.20.4 Physician Review ........................................................... $\quad 6-84$

6.21 Limitations of Method: Specimen Rejection, Interfering Substances, and Conditions .......................................................... $\quad 6-87$

6.21.1 Specimen Rejection.......................................................

6.21.2 Interfering Substances and Conditions............................... $\quad 6-88$ 


\section{TABLE OF CONTENTS (continued)}

Chapter

$\underline{\text { Page }}$

6.22

Reference Ranges

6-90

6.23

Action Limits

6-91

6.24

6.25

Specimen Storage and Handling During Testing.

6-92

Alternative Method for Performing Test or Storing Specimens

if Test System Fails.

6-92

6.26 Test Results Reporting System: Protocol for Reporting Action Limits ..

6.27

Quality Control Summary Statistics and Graphs

$6-92$

6-93

6.28

References

BLOOD PROCESSING

7.1

Introduction.

$7-1$

7.2

Equipment and Supplies

7.3

7.4

Protocols

$7-2$

7.5

Blood Processing Age-Specific Protocols

$7-6$

Labeling Vessels

$7-13$

7.6

Equipment.

7.6.1 Benchtop Centrifuges

7.6.2 Laminar Flow Biological Safety Cabinet Operating Sequence

7.7 Blood Processing Procedures.......

7.7.1 Eppendorf Research Pro Pipettes .

$\begin{array}{ll}\text { 7.7.2 } & \text { Process the } 3-\mathrm{mL} \text { and 4-mL EDTA Tubes........................ } \\ \text { 7.7.3 } & \text { Process the 2-mL Gray Tube for Glucose and OGTT. }\end{array}$

7.7.4 Process the 3-mL, 7-mL, 10-mL, and 15-mL Red Top and $6 \mathrm{~mL}$ Royal Blue Tubes for Pooled Sera

7.7.5 Process the 15-mL Red Top Tubes for Environmental Subsamples.....

7.7.6 Reagent Processing and Preparation.

7.8 Record the Results of Specimen Processing ....................................... 7-55

7.9 Blood Specimen Storage .................................................................

7.9.1 Blood Specimen Storage Protocol...................................... 7-69

7.10 How to Deal With System Failure .................................................. 7-72

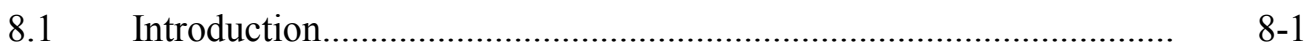

8.2 Equipment and Supplies …..................................................... $8-5$ 


\section{TABLE OF CONTENTS (continued)}

Chapter

Page

8.3 Print Vessel Labels and Label Blood Processing Racks..................... 8-6

8.3.1 Print Vessel Labels Using the Label Print Application....... 8-7

8.3.2 Main Printing Screen............................................................ 8-8

8.3.3 Filter Options .............................................................. 8-9

8.3.4 Print Key ................................................................. $\quad 8-10$

8.3.5 Print Options: Print Labels .............................................. 8 8-11

8.3.6 Print Options: Group By ............................................ $\quad 8-12$

8.3.7 Print Options: Module Filter ................................................ 8-13

8.3.8 Print Order..................................................................... 8.

8.3.9 Print Display Options..................................................... $\quad 8-14$

8.3.10 Printing One SP's Labels ................................................... 8-15

8.3.11 Printing Individual Labels ............................................... $\quad 8-16$

8.3.12 Label Action................................................................. 8-17

8.4 Create New Container Labels ..................................................... $\quad 8-22$

8.5 Trash or Reprint Container Labels................................................... $\quad 8-35$

8.5.1 Reprint Container Labels for Existing Containers .............. $\quad$ 8-38

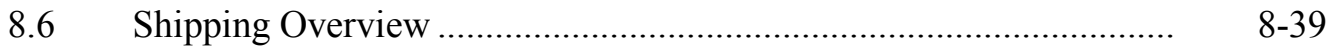

8.7 Assign Containers to Shippers ....................................................... $\quad 8-40$

8.8 Create Shipping Manifests ............................................................. 8

8.9 Informed Consent Exclusions ........................................................ 8-55

8.10 Packing Procedure for the Shippers.............................................. $\quad 8-59$

8.10.1 Pack Boxed Specimens ...................................................... 8-66

8.10.2 Pack Medium Shipping Containers for Frozen

8.10.3 Pack Small Shipping Containers for Frozen Shipment ....... $\quad$ 8-67

8.10.4 Pack Medium Shipping Container for Refrigerated Shipment ............................................................... $\quad 8-68$

8.10.5 Label Shippers..................................................... $\quad 8-69$

8.11 Container Protocol and Weekly Shipping List ............................... 8-69

HPV SWAB PROCESSING ................................................................

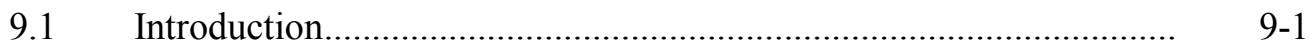

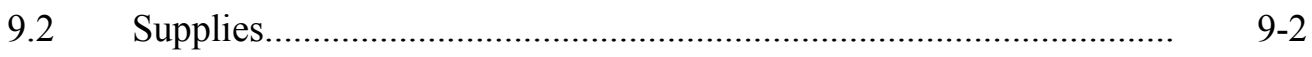

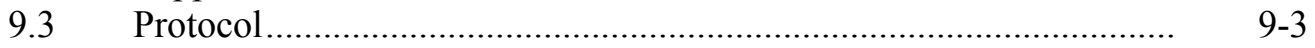

9.4 Labeling Vessels ........................................................................ $9-4$

9.5 HPV Protocol........................................................................ 94

9.6 Record Processing Results............................................................. 9-4

9.7 Specimen Storage …............................................................. $9-15$ 


\section{TABLE OF CONTENTS (continued)}

Chapter

$\underline{\text { Page }}$

9.8 Shipping Procedures

$9-15$

9.8.1 Pack and Ship Vessel 78 at Room Temperature ............... $\quad 9-16$

9.8.2 Label Shippers........................................................ $\quad 9-16$

9.9 How to Deal With System Failure _.................................................... 9-17

$10.1 \quad$ Introduction...................................................................... $10-1$

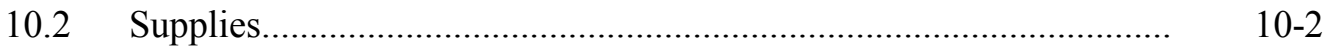

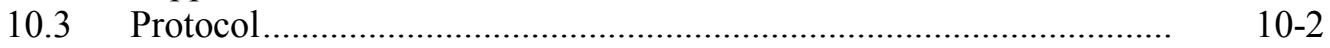

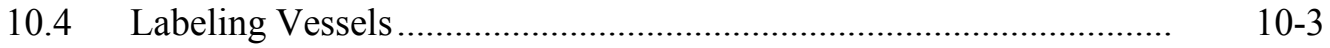

$10.5 \quad$ HPV Rinse Protocol ...................................................................... 10-3

10.6 Record Processing Results.......................................................... 10-4

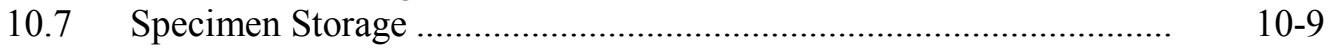

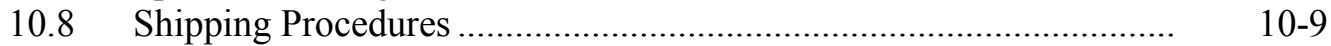

10.9 How to Deal With System Failure ................................................. 10-10

11 ORAL GLUCOSE TOLERANCE TEST (OGTT) APPLICATION............... 11-1

11.1 OGTT Application - Section Two (Blood Draw) ............................ 11-1

11.2 Laboratory Processing ….............................................................. 11-8

11.2.1 Laboratory Overview ..................................................... 11-8

11.2.2 Process the 2-mL Gray Top Tube for OGTT ...................... 11-8

11.2.3 Record the Results of Specimen Processing ........................ 11-9

11.2.4 Vessel 98 Storage Protocol ................................................. 11-19

11.2.5 Vessel 98 Shipping Protocol ............................................... 11-19

11.2.6 Performing the OGTT on SPs Who Do Not Speak English ... 11-20

12 QUALITY CONTROL AND QUALITY ASSURANCE - POLICIES

AND PROCEDURES ...........................................................................

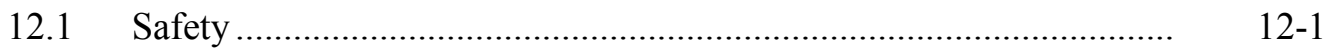

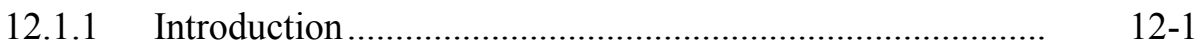

12.2 Specimen Submission and Handling ............................................. 12-1

12.2.1 Introduction ................................................................. $12-1$

12.2.2 Identification of Specimens............................................ $12-2$

12.2.3 Questions on Specimen Submission and Handling............. 12-2 


\section{TABLE OF CONTENTS (continued)}

Chapter

$\underline{\text { Page }}$

12.3 Test Requisition

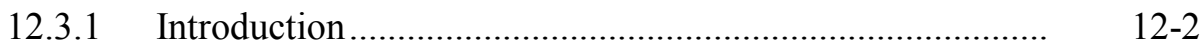

12.3.2 Laboratory Tests....................................................... 12-3

12.3.3 Human Subjects Review ................................................ 12-3

$12.4 \quad$ Test Records ...................................................................

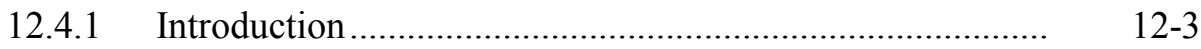

12.4.2 Components of Test Records .......................................... 12-4

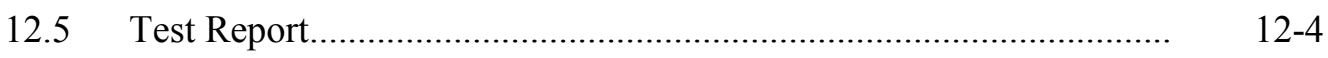

12.5.1 Introduction ................................................................. $12-4$

12.5.2 Review, Approval, and Release of Test Results ................ 12-4

12.5.3 List of Methods and Method Details................................. 12-5

12.5.4 Format of the Test Report ................................................. 12-6

12.5.5 Format for Laboratory Report Cover Letter....................... $\quad 12-6$

12.5.6 Format for Listing of Laboratory Results .......................... 12-6

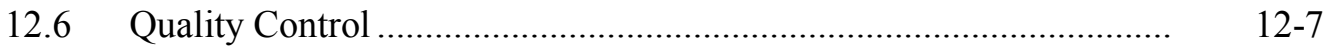

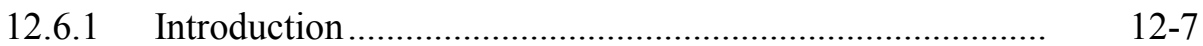

12.6.2 Method-Specific QC Procedures........................................ 12-8

12.6.3 QC Definitions ............................................................ 12-8

12.6.4 Overview of the Relationship Between Internal QC,
Proficiency Testing, and External QC.............................. $12-9$

12.6.5 Internal ("Bench") QC .................................................... 12-10

12.6.6 Coulter Interlaboratory Quality Assurance Program (IQAP).... 12-11

12.6.7 Proficiency Testing ........................................................... 12-11

12.6.8 Calibration Verification ..................................................... 12-13

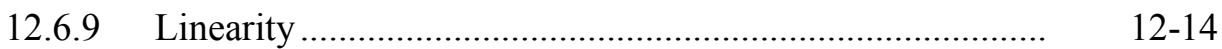

12.6.10 Quality Control Records .............................................. 12-15

12.7 Test Methods, Equipment, Reagents, Supplies, and Facilities ........... 12-15

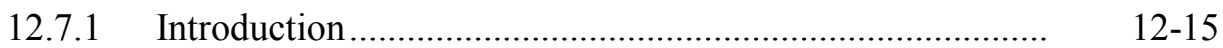

12.7.2 Labeling of Reagents, Solutions, Supplies ........................ 12-15

12.7.3 Facilities ................................................................ 


\section{TABLE OF CONTENTS (continued)}

Chapter

Page

12.8 Analytical Procedure Manual ..................................................... 12-16

12.8.1 Introduction................................................................

12.8.2 Contents of the Analytical Procedure Documentation......... 12-17

12.8.3 Approval and Record Maintenance..................................... 12-18

12.8.4 Method Performance Specifications ................................. 12-18

12.9 Equipment Maintenance and Function Checks............................... 12-19

12.9.1 Introduction ................................................................. 12-19

12.9.2 Laboratory Equipment Calibration................................... $12-19$

12.9.3 Temperature Monitoring ................................................ 12-20

12.10 Calibration and Calibration Verification ......................................... 12-22

12.10.1 Introduction ................................................................... 12-22

$\begin{array}{ll}\text { 12.10.2 Comparison of Methods Performed on Multiple } & \\ \text { Instruments or at Multiple Sites ........................................... 12-22 }\end{array}$

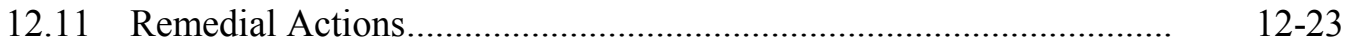

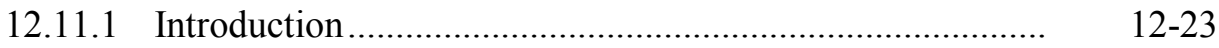

12.12 Integrated Survey Information System (ISIS) ….............................. 12-23

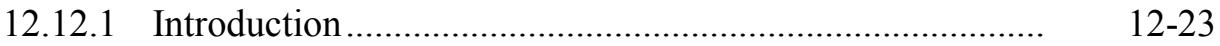

12.12.2 Integrated Survey and Information System Data

Down Times ................................................................ $12-24$

12.12.3 Maintenance of LAN Hardware .......................................... 12-24

12.12.4 Backup Schedule of Integrated Survey and Information System Data ............................................................. $12-24$

12.13 Communications and Complaints ................................................. 12-24

12.13.1 Introduction .......................................................... $12-24$

12.14 Quality Promotion.................................................................. 12-25

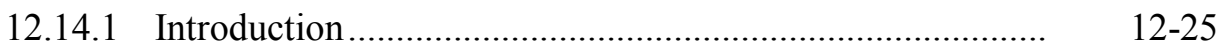

12.15 Quality Assurance Program ..................................................... 12-25

12.15.1 Introduction................................................................... $12-25$

12.15.2 Quality Audit: Survey Participant Test Management ......... 12-26

12.15.3 Quality Audit: Quality Control ........................................ 12-27 


\section{TABLE OF CONTENTS (continued)}

Chapter

$\underline{\text { Page }}$

12.15.4 Quality Audit: Personnel................................................. 12-28

12.15.5 Quality Assurance Review with Staff.............................. 12-28

12.16 Personnel Training and Evaluation................................................... 12-29

12.17 Abbreviations ............................................................................. 12-30

13.1 Start of Stand Activities ................................................................ 13-1

13.2 End of Stand Activities ...................................................................... 13-4

13.3 Inventory Procedures and Supplies.............................................. 13-7

13.3.1 General Inventory Procedures.......................................... 13-8

13.3.2 End of Stand Inventory Procedures..................................... 13-9

13.3.3 Mid-Stand Inventory Procedures ...................................... 13-10

13.3.4 Start of Stand Inventory Procedures.................................... 13-11

13.3.5 Consumables vs. Nonconsumables ................................... 13-12

13.3.6 Shipping Excess Inventory Back to the Warehouse............ 13-12

13.3.7 Tracking of Expired and Broken Inventory ........................ 13-12

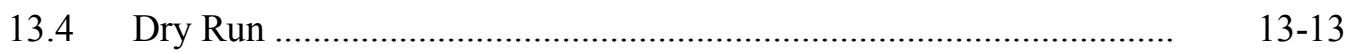

13.4.1 Phlebotomy Protocol and Procedures ................................. 13-13

13.4.2 Urine Processing and Pregnancy Testing............................ 13-16

13.4.3 Urine Processing ............................................................ 13-28

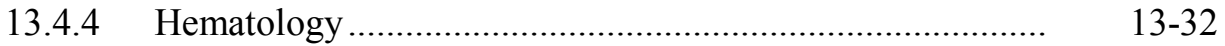

13.4.5 Blood Processing and Storage........................................... 13-32

13.4.6 Shipping Dry Run Specimens ......................................... 13-34

13.5 Centrifuges - Equipment Maintenance............................................ 13-35

13.6 Refrigerators and Freezers - Equipment Maintenance ...................... 13-36

13.7 Use of the Temperature Recording Instrument................................. 13-37

13.8 Use of the Class II Type A Biological Safety Cabinet ...................... 13-39

13.9 Ambient Air Temperature........................................................... 13-52

13.10 Precision Instruments Osmette II................................................. 13-52

13.11 Mettler Toledo Scale............................................................ 13-55

13.12 Laboratory Quality Control Module ................................................. 13-62

13.13 Hard-Copy Supply Use Control Log ............................................ 13-64

13.14 Eppendorf Pipette Care, Sterilization, Maintenance,

13.15 Power Loss and Laboratory Operations......................................... 13-68

13.15.1 Scenario 1 - Power Loss during Exam Session with No Backup Generator............................................. 13-68 


\section{TABLE OF CONTENTS (continued)}

Chapter

Page

13.15.2 Scenario 2 - Power Loss when the MEC is Not Conducting Exams

13.15.3 Scenario 3 - Power Loss with a Backup Generator...

$\underline{\text { Appendixes }}$

List of Appendixes

A INTERNATIONAL AIR TRANSPORT ASSOCIATION (IATA) DANGEROUS GOODS REGULATIONS JANUARY 2012........................ A-1

B

BIOSAFETY

B-1

$\mathrm{C}$

LABORATORY PROTOCOL

C-1

$\mathrm{D}$

LABORATORY AND PHLEBOTOMY INVENTORY

D-1

List of Tables

$\underline{\text { Tables }}$

4-1

NHANES urine processing protocol - primary

NHANES urine processing protocol - VIP guest.

4-3 Storage and shipping protocol for urine.

$4-42$

\section{List of Figures}

$\underline{\text { Figures }}$

$1-1$

Laboratory layout.

$1-4$

$1-2$

Phlebotomy room layout.

$1-4$

$3-1$

Floor plan for phlebotomy room.

$3-4$

$3-2$

UMF 8678 phlebotomy chair

Blood collection cart

$3-8$ 


\section{TABLE OF CONTENTS (continued)}

Exhibits

List of Exhibits

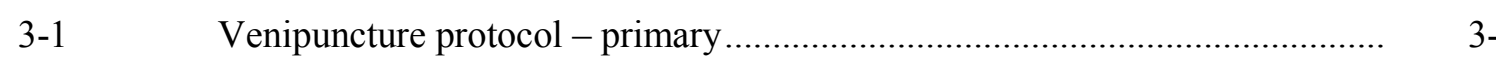

3-2 Venipuncture protocol - VIP guest..................................................

3-3 Venipuncture protocol - guest .............................................................. $3-3$

3-4 Alternative venipuncture protocol - primary.............................................

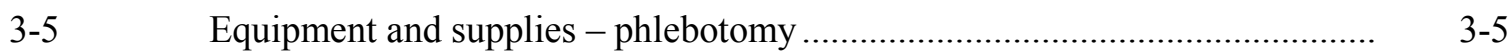

3-6 Phlebotomy worksheet - English Primary .................................................... 3-84

3-7 Phlebotomy worksheet - Spanish Primary ……........................................ $\quad 3-85$

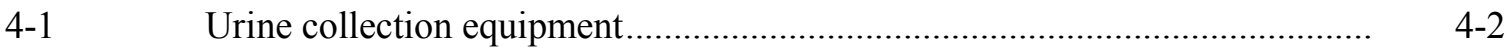

4-2 Urine collection supplies - urine processing and storage ............................... $4-3$

4-3 Comment codes for insufficient urine collection.......................................... 4-18

4-4 Workstation 2 processing worksheet ......................................................

7-1 Nonconsumable supplies for blood processing .........................................

7-2 Consumable supplies for blood processing ............................................ $\quad$ 7-2

7-3 Primary SP's whole blood processing protocol ..........................................

7-4 Primary SP's plasma processing protocol....................................................

7-5 Primary SP's packed cells processing protocol ….......................................

7-6 Primary SP's serum processing protocol ….............................................

7-7 VIP Guest's blood whole blood processing protocol.....................................

7-8 VIP Guest's plasma processing protocol ................................................

7-9 VIP Guest's serum processing protocol.................................................

7-10 Guest serum processing protocol ........................................................

7-11 Blood processing protocol for primary SPs aged 1-2 years............................ 7-6 


\section{TABLE OF CONTENTS (continued)}

Exhibits

List of Exhibits (continued)

7-12 Blood processing protocol for primary SPs aged 3-5 years........................... 7-6

7-13 Blood processing protocol for primary SPs aged 6-11 years......................... 7-7

7-14 Blood processing protocol for primary SPs aged 12-19 years....................... 7-8

7-15 Blood processing protocol for primary SPs aged 20+ years ......................... 7-10

7-16 Blood processing protocol for VIP guest aged 1-2 years............................. 7-11

7-17 Blood processing protocol for VIP guest aged 3-5 years............................. 7-12

7-18 Blood processing protocol for VIP guest aged 6-11 years........................... 7-12

7-19 Blood processing protocol for VIP guest aged 12+ years........................... 7-12

7-20 Blood processing protocol for guest aged $12+$ years ..................................

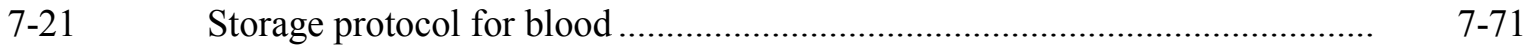

7-22 Workstation 2 processing worksheet …..............................................

8-1 Blood and urine shipping protocol........................................................ $8-2$

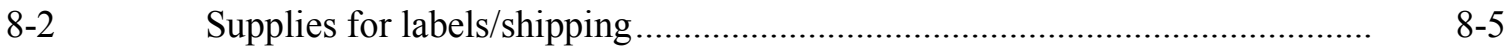

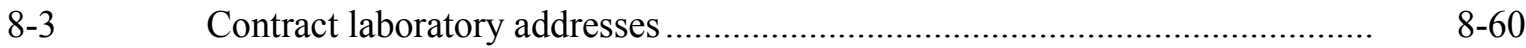

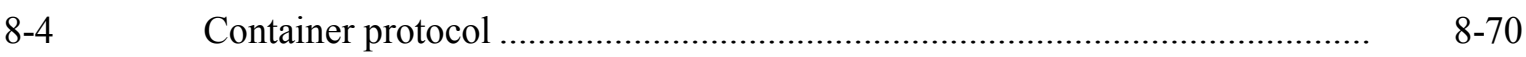

8-5 FedEx shipping list by lab number …..................................................

9-1 Equipment and supplies - HPV processing and storage .............................. 9-3

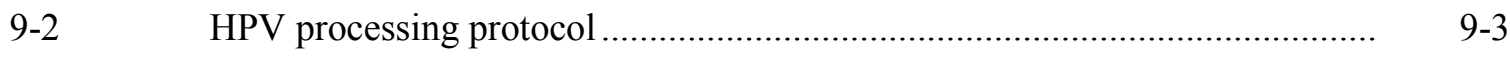

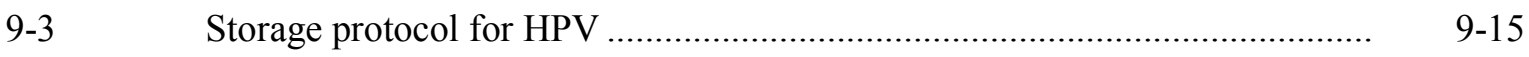

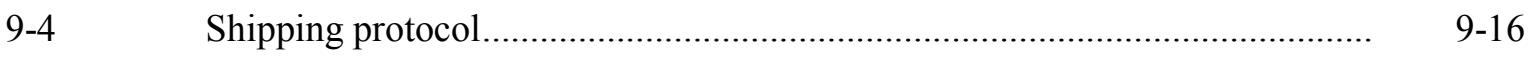

9-5 HPV Processing worksheet............................................................. $9-17$

10-1 Equipment and supplies - HPV processing and storage ........................... $10-2$ 


\section{TABLE OF CONTENTS (continued)}

\begin{tabular}{|c|c|c|}
\hline Exhibits & 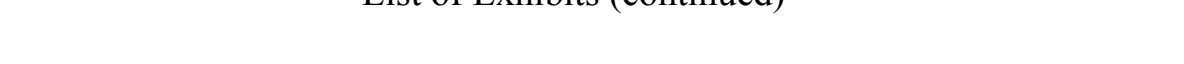 & Page \\
\hline $10-2$ & HPV Rinse processing protocol .......................... & $10-2$ \\
\hline $10-3$ & Storage protocol for HPV ....................... & $10-9$ \\
\hline $10-4$ & 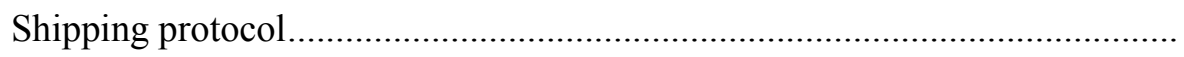 & $10-9$ \\
\hline $10-5$ & HPV Oral Rinse Processing worksheet....... & $10-10$ \\
\hline $11-1$ & Primary SP's OGTT processing protocol.. & $11-8$ \\
\hline $11-2$ & Storage protocol for OGTT & $11-19$ \\
\hline $11-3$ & OGTT shipping protocol.............. & $11-19$ \\
\hline $11-4$ & Contract laboratory addresses... & $11-20$ \\
\hline $13-1$ & Equipment and supplies ....................... & $13-8$ \\
\hline $13-2$ & 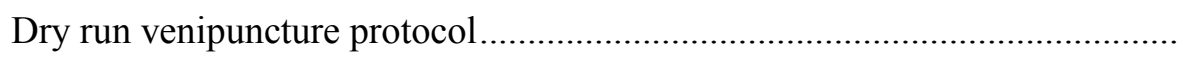 & $13-14$ \\
\hline $13-3$ & 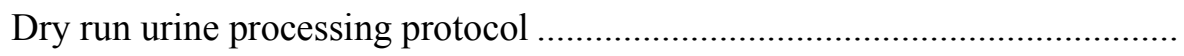 & $13-28$ \\
\hline $13-4$ & 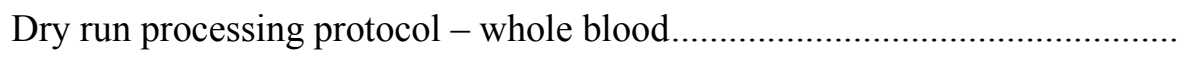 & $13-32$ \\
\hline $13-5$ & Dry run blood processing protocol - serum ..... & $13-33$ \\
\hline $13-6$ & Operating the laminar flow biological safety cabinet ........ & $13-40$ \\
\hline $13-7$ & 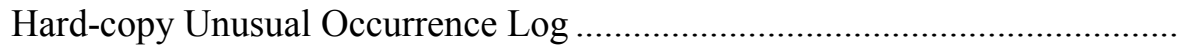 & $13-63$ \\
\hline $13-8$ & Hard-copy Supply Use Control Log ... & $13-65$ \\
\hline
\end{tabular}




\section{OVERVIEW AND LABORATORY TEAM RESPONSIBILITIES}

\subsection{Overview}

The laboratory component of NHANES includes the collection, processing, storage, and shipping of blood, urine, and other biological and environmental specimens. Collectively, these specimens provide data about the health status of the U.S. population.

The blood collection (venipuncture) procedure consists of (1) administering a questionnaire to screen for conditions that exclude the participant from the blood draw; (2) determining fasting status; and (3) performing a blood draw. The phlebotomist also collects one additional blood tube from SPs selected for the VOC (volatile organic compounds) special study on consenting SPs aged 20 years and older. They may administer a dextrose solution $\left(\right.$ Trutol $^{\circledR}$ ) to, and will collect an additional 2-mL gray-top tube from, SPs selected for the oral glucose tolerance (OGTT) component.

The purpose of urine collection and processing is to collect sufficient urine from participants aged 6 years and older to be able to (1) perform a pregnancy test on selected females aged 8 to 17 years and all females aged 18 to 59 years to exclude pregnant participants from other components that could put the participant at risk; (2) allocate urine into vessels for storage and transport to multiple government and contact laboratories for analysis; (3) calculate a urine flow rate; and (4) allocate urine into a vessel for future studies.

The purpose of blood processing is to (1) perform a complete blood count (CBC); (2) allocate blood (whole blood, plasma, and serum) into vessels for transport to multiple government and contract laboratories for analysis; (3) allocate serum into vessels for future studies; and (4) process serum for environmental testing.

The purpose of collecting and processing a vaginal swab and a penile swab is to be able to store and transport the samples to a laboratory for HPV (human papilloma virus) analysis.

The purpose of performing a complete blood count on blood specimens is to provide a study of blood cells and coagulation. 
The purpose of pregnancy testing is to exclude pregnant women aged 8 to 59 years from participating in the dual-energy x-ray absorptiometry (DXA) section of the body composition component of the MEC exam.

The purpose of conducting the special volatile organic compounds (VOCs) study is to collect blood to determine the prevalence of exposures to certain chemicals.

The purpose of collecting and processing an oral rinse for HPV is to be able to store and transport the samples to a laboratory for HPV (human papilloma virus) analysis. This sample is used to determine the prevalence and type of distribution of infection and to investigate the demographic and behavioral factors associated with infection.

\subsection{The Laboratory Team}

Each laboratory team includes three ASCP (American Society of Clinical Pathologists) certified medical technologists or medical laboratory technologists who have experience in all aspects of laboratory practice and a certified phlebotomist who has experience in venipuncture.

The chief medical technologist is the most senior member of the team. He or she is responsible for overseeing all the activities of the medical technologists and phlebotomist in the MEC, as well as quality control, equipment calibration, and maintenance. On a routine basis, the chief medical technologist performs the same duties as the other medical technologists.

\subsection{Tasks}

The phlebotomist's primary tasks include conducting the venipuncture, administering the Trutol $^{\circledR}$ solution, conducting a second venipuncture 2 hours after the Trutol ${ }^{\circledR}$ solution has been consumed, assisting the medical technologists as needed, managing the phlebotomy, OGTT component inventories, performing MEC equipment QC activities and recording the results, printing laboratory labels, labeling blood processing racks, and assisting with the shipping process at the end of each stand. In addition, the 
phlebotomist acts as a backup examiner and recorder for the body measures exam and oral health recorder for the oral health exam.

The medical technologist's tasks include printing labels and labeling vessels; performing CBCs; conducting pregnancy tests; processing blood, urine, vaginal and penile swabs, and oral rinse samples; performing MEC equipment QC activities and recording the results; and shipping specimens to contract laboratories. On a routine basis, the medical technologists assist the phlebotomist as needed and manage the component inventory.

\section{Organization of the Laboratory}

The laboratory consists of two areas - the phlebotomy room and the laboratory (label/ship and environmental sample processing area, biological sample processing, and hematology/pregnancy testing) area. The phlebotomy room and laboratory area are located in trailer 4. Figures 1-1 and 1-2 illustrate the laboratory layout and phlebotomy room layout.

The phlebotomist performs the following activities in the phlebotomy room: collects blood, administers the Trutol ${ }^{\circledR}$ solution, and collects a second blood sample from SPs who consumed the Trutol ${ }^{\circledR}$.

The medical technologist processes, stores, and ships biological and environmental samples; analyzes blood for a complete blood count; and performs pregnancy testing. The technologist also prints labels, labels blood processing racks, and prepares shipping documents. 


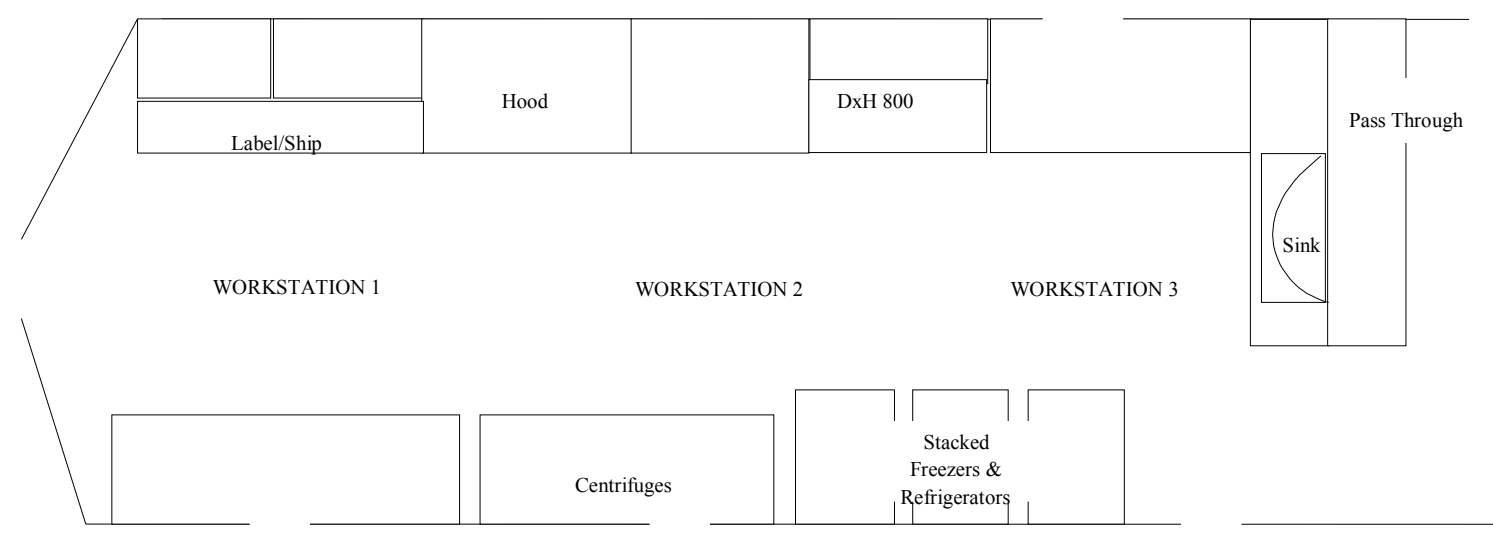

Figure 1-1. Laboratory layout

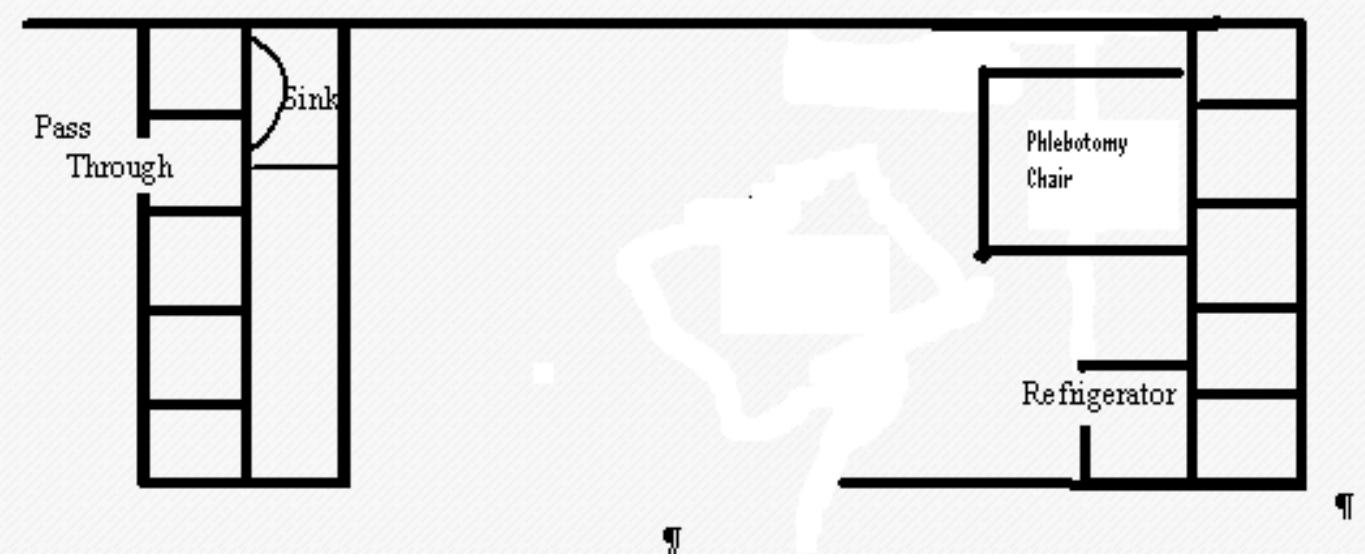

Figure 1-2. Phlebotomy room layout

\section{Laboratory Area Floor Plan}

The laboratory area contains three workstations designated workstation 1, 2, and 3. Printing labels, labeling vials for each SP's blood-processing rack, and shipping activities occur at workstation 1. Blood processing and storage occur at workstation 2. Urine collection, urine processing and storage; pregnancy testing; running CBCs; vaginal and penile swab specimen processing; oral rinse specimen processing; and routine quality control activities occur at workstation 3. 
- Set up supplies and check expiration dates of all items with lot numbers, such as the Vacutainer ${ }^{\mathbb{R}}$ tubes;

- Enter lot numbers and expiration dates into the automated Supply Use Control Log;

- Complete the phlebotomy examination, including conducting the venipuncture interview, administering the fasting questionnaire, and performing the venipuncture;

- Complete the oral glucose tolerance test examination including administering the Trutol $^{\circledR}$ solution to eligible SPs and performing a second blood draw on all GTT SPs who consumed the Trutol ${ }^{\circledR}$;

- Label and transfer all blood tubes to the laboratory area;

- Disinfect all the counters and blood draw chair at the end of each session, or as needed when soiled with blood or other body fluids;

- Take and record the QC readings;

- Maintain the appearance of the venipuncture area and the equipment;

- Monitor the inventory and restock supplies;

- Conduct the start and end of stand inventory counts;

- Clean and stock the phlebotomy room, including the equipment, at the start and end of each stand;

- Assist in printing laboratory labels and label and assemble blood-processing racks;

- Assist the lab staff when they pack the shippers; and

- Serve as the backup examiner and recorder in body measures and recorder in oral health.

\subsection{Tasks - Workstation 1}

- Process water samples for Fluoride, vessel 144.

- Complete blood processing tasks for the first 45 minutes of each session;

- Print blood, urine, pregnancy, and oral rinse testing labels;

- Prelabel the vials for each SP's blood-processing rack; 
- Assist the phlebotomist with venipuncture;

- Generate container IDs at the start of stand;

- Assign a container ID label to one container for each vessel;

- When shipping, close all open containers, create airbills, and assign containers to shippers;

- Print shipping manifests;

- Prepare blood (whole blood, serum, and plasma), urine, vaginal and penile swab, and oral rinse samples for shipping;

- Pack, label, and ship shippers;

- Open all new containers after shipping;

- Maintain appearance of the label/ship area and the equipment;

- Monitor inventory and restock supplies;

- Assist phlebotomist with venipuncture;

- Complete the Trutol ${ }^{\circledR}$ section in the GTT examination as assigned;

- Conduct start and end of stand inventory counts;

- Clean the laboratory at the start and end of each stand; and

- Disinfect counters and work area at the end of each day.

\subsection{Tasks - Workstation 2}

- Stock blood processing and storage supplies;

- Receive blood specimens;

- Select the correct prelabeled rack and age-appropriate template;

- Set the red and royal top blood tubes aside to clot;

- Pipette whole blood into separate 2-mL cryovials from the 3-mL or 4-mL lavender tube for vessel 1 ( $\mathrm{Lead} / \mathrm{Cad} / \mathrm{HG})$, vessel 3 (RBC Folate), vessel 4 (Glycohem), and vessel 5 (Mercury); 
- Pipette the plasma from the 3-mL or 4-mL lavender tube for vessel 156 (Fluoride) and perform cell washing on the remaining cells for vessel 88 (Acrylamide).

- Make the dilution for RBC Folate vessels and serum Folate vessels;

- Transfer the 3-mL or 4-mL lavender tube to the workstation 3 technologists for hematology if needed;

- Centrifuge the 2-mL gray tube;

- Pipette the plasma from the 2-mL gray tube into a 2-mL cryovial for vessel 9 (glucose);

- Centrifuge the red top tubes and royal blue top tube;

- Aliquot serum from the 6-mL royal blue top tube into a 2-mL cryovial for vessel 136 (selenium/copper/zinc);

- Pool the serum from the 3-mL, 7-mL, 15-mL and 10-mL red top tubes, plus the remaining serum from the 6-mL royal blue top tube for each SP and mix well;

- Aliquot serum into separate 2-mL cryovials for the serum vessels according to the age-specific protocols;

- Pool serum from $2-15-\mathrm{mL}$ red top tubes into a $15-\mathrm{mL}$ conical tube and aliquot 1.5$\mathrm{mL}$ into vessel 90 and the remaining serum into vessels 29 and 89. Aliquot equal volumes of serum from a third 15-mL red top tube into vessels 92 and 93 ;

- Enter all blood processing results;

- Centrifuge the second 2-mL gray tube;

- Pipette the plasma from the second 2-mL gray tube into a 2-mL cryovial for vessel 98 (OGTT);

- Enter the blood processing results;

- Refrigerate or freeze the processed vessels;

- Check contents of blood storage boxes (containers) using the container map module at the end of each session;

- Disinfect all the counters and the laminar flow hood at the beginning and end of each session or when the area becomes soiled with blood or other body fluids;

- Maintain appearance of the blood processing area and the equipment;

- Monitor the inventory and restock supplies; 
- Conduct the start and end of stand inventory counts;

- Clean the laboratory at the start and end of each stand; and

- Assist in printing laboratory labels and label and assemble blood-processing racks.

\section{$1.8 \quad$ Tasks - Workstation 3}

- Perform start of stand, start of session, end of session, weekly, and end of stand quality control checks on laboratory equipment and record results;

- Prepare the $1 \%$ and $7 \%$ ascorbic acid solutions daily;

- Enter the ascorbic preparation into Daily QC;

- Stock supplies for urine processing, hematology, and pregnancy;

- Verify and accept the urine volume and record the urine collection results;

- Run the pregnancy test controls;

- Perform startup procedures and run quality control materials on the Coulter ${ }^{\circledR} \mathrm{DxH}$ 800 ;

- Run specimens on the Coulter ${ }^{\circledR}$ DxH 800 and enter results;

- Refrigerate VOC blood (vessel 54) tube;

- Conduct the pregnancy test and enter results;

- Label urine vessels and aliquot into 2- or 5-mL cyrovials, 14-mL Falcon snap cap, or 10-mL VWR screwcap tubes according to the processing protocol;

- Enter the urine processing results;

- Store the urine vessels;

- Process vaginal swab (vessel 78);

- Process penile swab (vessel 140)

- Process oral health rinse (vessel 129);

- Check contents of urine, vaginal swab, penile swab, and oral rinse boxes and bags using the container maps module at the end of each session;

- Disinfect all counters at the beginning of the stand and end of each session; 
- Maintain the appearance of the urine processing/hematology area and the equipment;

- Monitor the inventory and restock supplies;

- Conduct the start and end of stand inventory counts;

- Clean the laboratory at the start and end of each stand;

- Assist in printing laboratory labels and label and assemble blood-processing racks; and

- Disinfect work areas at the end of each day and when they become soiled with blood or other body fluids.

\section{Cross-training}

Cross-training the medical technologists and the phlebotomist increases an individual's skills and provides scheduling flexibility and a safety net during staff absences. The primary phlebotomist helps the lab team set up and tear down. Medical technologists trained in phlebotomy (backup phlebotomists) work in phlebotomy on a routine basis to ensure competency and proficiency. The basic underlying assumption is that the blood draw must never be compromised. The primary phlebotomists must be available to step in and complete the blood draw even when backups are working in phlebotomy.

- When the backup phlebotomist is assigned to workstation 1 (label/ship):

- $\quad$ The primary phlebotomist works at workstation 1, except on Monday when the lab team ships. The backup phlebotomist always works the scheduled workstation 1 assignment on shipping Mondays.

- $\quad$ The backup phlebotomist works in phlebotomy when the primary phlebotomist works at workstation 1.

- The primary phlebotomist always performs the pediatric blood draws while the backup phlebotomist observes and holds the child.

- If the backup phlebotomist encounters a difficult blood, then the primary phlebotomist comes into the room and they work together so that the blood draw is successful. This is intended as a learning experience; the backup phlebotomist can continue to learn from the primary phlebotomist.

- There are no rules for how many days the backup phlebotomist works in phlebotomy during each stand. The objective is at least 2-3 days per stand with emphasis on sessions with 8-10 adult SPs. 
On set-up day, the phlebotomist completes his or her room set-up and assigned tasks before helping lab staff. On set-up day, the phlebotomist should, at a minimum, make the racks for dry run day. On tear-down day, he or she helps pack the shippers and assists the lab staff in other ways as directed by the chief medical technologist. 


\section{LABORATORY ISIS FUNCTIONALITY}

\subsection{Laboratory-Specific Functionality}

While MEC applications are custom built for each specific component, they share a common design. This commonality provides all component applications the same "look and feel" which minimizes reorienting technologists who rotate from component to component. A complete ISIS overview and these features and characteristics are described in the ISIS User Guide. The laboratory application has additional functionality that has been developed to accommodate and facilitate processing specimens and reporting results. This chapter describes the laboratory-specific functionality.

\subsection{Laboratory Heads-up Display}

Use the laboratory heads-up display to view the SPs scheduled into the session, the modules for which they are eligible, and their current process status.

Use the heads-up display to access modules.

\begin{tabular}{|c|c|c|c|c|c|c|c|c|c|c|c|c|c|c|c|c|}
\hline \multicolumn{17}{|c|}{ File Yiew Untilities Quality Control Reports Modules Shipping Wiindow Help } \\
\hline 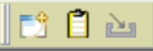 & ? 15 & 军 & (?) & 1 & & & & & & & & & & & & \\
\hline \multirow[t]{2}{*}{ SPID } & \multirow{2}{*}{ Sample ID } & \multirow{2}{*}{ Status } & \multirow[t]{2}{*}{ Type } & \multirow[t]{2}{*}{ Gender } & \multirow[t]{2}{*}{ Age } & \multirow[t]{2}{*}{ Name } & \multirow[b]{2}{*}{ uC } & \multicolumn{5}{|c|}{ Process Status } & \multicolumn{3}{|c|}{ HPV } & \multirow[b]{2}{*}{ OR } \\
\hline & & & & & & & & $\mathrm{u}$ & P & $\mathrm{CB}$ & $\theta$ & GTT & $\mathrm{PC}$ & $\mathrm{F}$ & M & \\
\hline 256339 & 256339 & SC & $P$ & M & 90 & MOORE, LANDON J & 0 & 0 & . & 0 & 0 & 0 & . & . & . & . \\
\hline 329869 & 329869 & $B E$ & $P$ & M & 69 & HOWARD, SOPHIA S & $\bullet$ & $\bullet$ & . & 0 & 0 & 0 & 0 & . & . & 0 \\
\hline 416714 & 416714 & $\mathrm{BE}$ & $P$ & $\mathrm{~F}$ & 20 & BAILEY, ANTHONY J & - & $\bullet$ & 0 & 0 & 0 & 0 & 0 & . & . & 0 \\
\hline 480354 & 480354 & $\mathrm{SC}$ & $P$ & M & 3 & LEE, OWEN S & . & . & . & 0 & 0 & . & . & . & . & . \\
\hline 525757 & 525757 & $\mathrm{Cl}$ & $P$ & $\mathrm{~F}$ & 70 & ADAMS, CALEB L & - & $\bullet$ & . & 0 & 0 & 0 & 0 & . & . & . \\
\hline 545182 & 545182 & $\mathrm{SC}$ & $P$ & $\mathrm{~F}$ & 4 & POWELL, AALIYAH H & . & . & . & 0 & 0 & . & . & . & . & . \\
\hline 553872 & 553872 & SC & $P$ & M & 14 & WRIGHT, LAYLA B & 0 & 0 & . & 0 & 0 & 0 & . & . & 0 & 0 \\
\hline 745052 & 745052 & $\mathrm{SC}$ & $P$ & $\mathrm{~F}$ & 41 & EVANS, SAVANNAH M & 0 & 0 & 0 & 0 & 0 & 0 & . & 0 & . & 0 \\
\hline 767995 & 767995 & $\mathrm{Cl}$ & $P$ & M & 15 & MURPHY, ISAAC N & - & 0 & . & 0 & 0 & 0 & . & . & 0 & 0 \\
\hline 784174 & 784174 & $\mathrm{BE}$ & $P$ & M & 50 & GONZALEZ, SIENNAN & $\odot$ & $\bullet$ & . & 0 & 0 & 0 & . & . & 0 & 0 \\
\hline 813366 & 813366 & $\mathrm{SC}$ & $P$ & $\mathrm{~F}$ & 19 & STEWART, SOPHIE J & 0 & 0 & 0 & 0 & 0 & 0 & . & 0 & . & 0 \\
\hline 836040 & 836040 & SC & $P$ & M & 19 & TORRES, LUCAS S & 0 & 0 & . & 0 & 0 & 0 & . & . & 0 & 0 \\
\hline 8ว76ว? & 837622 & sc. & b & $\mathrm{F}$ & 1 & MARTINF7 ANINA I & & & . & n & $n$ & . & & & & \\
\hline
\end{tabular}


The heads-up display lists all SPs with appointments for the current session. It includes the SP ID, Sample ID, Status ( $\mathrm{SC}=$ scheduled, $\mathrm{LI}=$ Logged-in, $\mathrm{CI}=$ checked-in, $\mathrm{BE}=$ being examined, $\mathrm{EX}=$ exited), Appointment Type $(\mathrm{P}=$ primary, $\mathrm{G}=$ guest, $\mathrm{VIP}=\mathrm{VIP}$ guest, $\mathrm{D}=$ dry run $)$, Gender $(\mathrm{M}=$ male, $\mathrm{F}=$ female), Age, and Name (last, first.)

The heads-up display provides the Process Status for the following modules: UC (urine collection), U (urine processing), $\mathrm{P}$ (pregnancy testing), CBC (complete blood count), B (blood processing), GTT (glucose tolerance test or vessel 98 processing), PC (Packed Cell or vessel 88 processing) HPV (HPV swab processing; F for female and M for Male), and OR (oral rinse processing). The SP is ineligible for a module when the process status is $\bigcirc$. The SP is eligible for a module, but no results have been recorded when the process status is $\mathrm{O}$. The SP is eligible for a module, and some results have been recorded when the process status is $\odot$. The SP is eligible for a module, all results have been recorded, and the module is complete when the process status is

The SP is eligible for a module $(\mathrm{O})$, but the process status for blood processing (B) and the CBC will not enable until at least one blood tube has been recorded as collected in the phlebotomy component. Only SPs who have checked in are eligible for HPV processing. The process status is $\bigcirc$ for urine processing $(\mathrm{U})$, and pregnancy testing $(\mathrm{P})$, until the SP's urine is marked as collected in the UC module. Once these samples are collected, the process status changes from $\bigcirc$ to $\bigcirc$. The GTT and OR modules will enable as soon as one SP has the OGTT blood tube drawn in phlebotomy and one SP has the oral rinse collected in the oral health examination. The process status regularly updates and changes after each result is saved.

The active SP is contained in a rectangular box. Use the heads-up display to select the correct SP. Select a different SP if the active SP is not the SP of choice.

\subsection{Laboratory Task Bar}

Monitor the activity in the laboratory task bar, which is located on the bottom of the screen.

\begin{tabular}{|c|c|c|c|c|}
\hline Ready & |HUP: idle & |MEC Layer: 12/11/2008| & Application: 9.0 .10 & Connected to Coordinator $\quad 04: 24 \mathrm{PM}$ \\
\hline 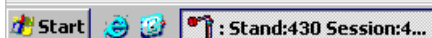 & & & & 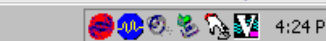 \\
\hline
\end{tabular}


The laboratory task bar displays the following information:

- The status of the refresh routine such as when the labels are printing or the data are updating;

- The status of the heads-up display such as when it is "idle;"

- The current version of the MEC layout;

- The current version of the application;

- The status of the connection to the coordinator; and

- The current time.

\subsection{Menu Bar Options}

Each MEC application has tailored menu options to support the specific examination. The menu options below list all available options. The options marked with an asterisk appear on all MEC examination programs. Options are grayed (inverted) or not present when they are not available for a specific subsystem. 


\subsection{1}

File Menu Options

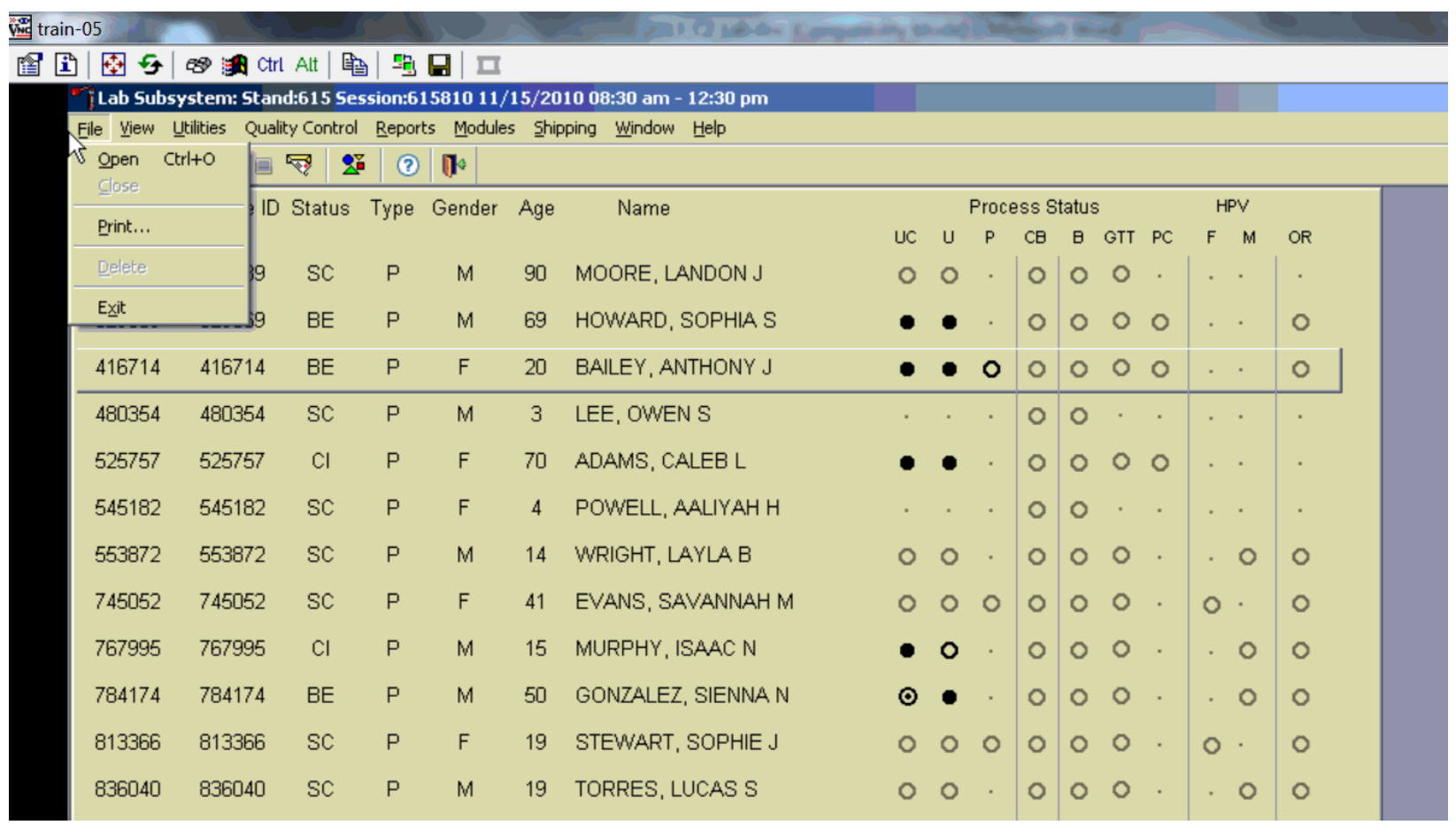

Asterisks (*) denote menu options common to all MEC examination components.

\begin{tabular}{ll}
\hline$*$ File & \\
\hline * Open & $\begin{array}{l}\text { Opens a new, partial, or not done SP exam; the SP must be assigned by the } \\
\text { coordinator if the application is not in standalone mode. }\end{array}$ \\
\hline${ }^{*}$ Close & This option is grayed out and not applicable. \\
\hline$*$ Print & Prints the current examination screen or report. \\
\hline$*$ Delete & This option is grayed out and not applicable. \\
\hline$*$ Exit & Exits the application. \\
\hline
\end{tabular}




\subsubsection{Utilities Menu Options}

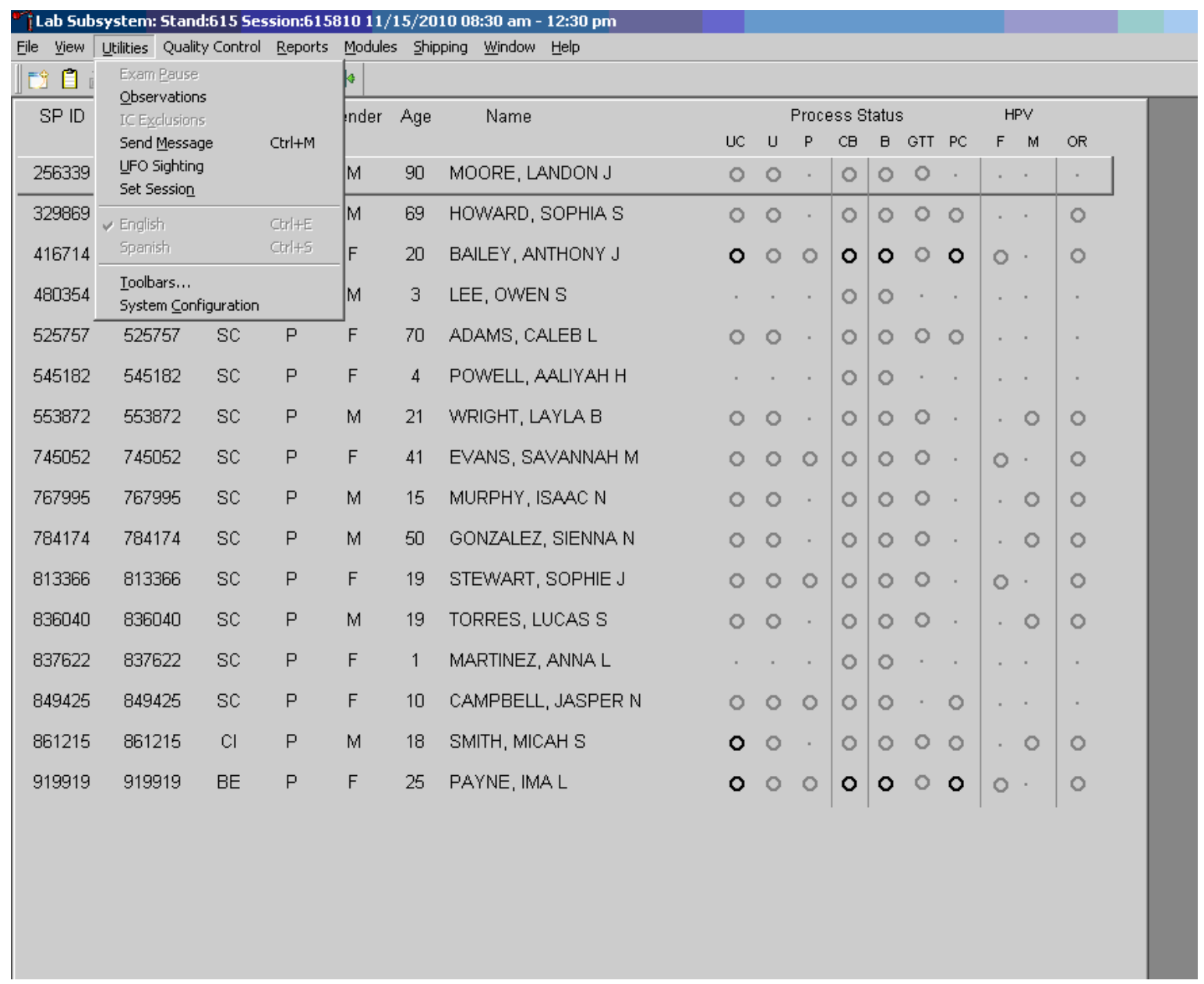

\section{* Utilities}

\begin{tabular}{ll}
\hline${ }^{*}$ Exam $\underline{\text { Pause }}$ & This option is grayed out and not applicable. \\
\hline${ }^{*}$ Observation & Submits an observation to the physician for possible referral. \\
\hline IC Exclusions & $\begin{array}{l}\text { This option is grayed out in the Laboratory application and is not } \\
\text { applicable. } \\
\text { Posts an exclusion record for those SPs who do not want HIV, STD, PSA, } \\
\text { or HPV tests performed, genetic tubes processed, or samples stored for } \\
\text { future research; this utility is available only for coordinator, physician, and } \\
\text { phlebotomy. A complete description of this functionality is described in } \\
\text { Section 3.5. }\end{array}$ \\
\hline $\begin{array}{l}\text { *Send } \underline{\text { Message }} \\
C t r l+M\end{array}$ & Opens a dialog box to send messages to the coordinator. \\
\hline *UFO Sighting & $\begin{array}{l}\text { Opens the Unusual Field Occurrence utility to document unusual } \\
\text { equipment, SP, protocol, trailer, or supply issues. }\end{array}$ \\
\hline
\end{tabular}




\begin{tabular}{ll}
\hline *Utilities & \\
\hline Set Session & $\begin{array}{l}\text { Allows the laboratory medical technologist to change the current session } \\
\text { in the laboratory application. A complete description of this functionality } \\
\text { is described in Section 2.6. }\end{array}$ \\
\hline English & $\begin{array}{l}\text { This option is grayed out in the Laboratory application and is not } \\
\text { applicable. } \\
\text { Ttrl+E }\end{array}$ \\
\hline$\underline{\text { Toggle option to set exam language to English. }}$ \\
Ctrl $+\mathrm{S}$ & $\begin{array}{l}\text { This option is grayed out in the Laboratory application and is not } \\
\text { applicable. }\end{array}$ \\
\hline${ }^{*}$ Toolbars & Toggle option to set exam language to Spanish. \\
\hline${ }^{*}$ System Configuration & Configures placement of toolbars. \\
\hline
\end{tabular}

\subsection{3} Quality Control Menu

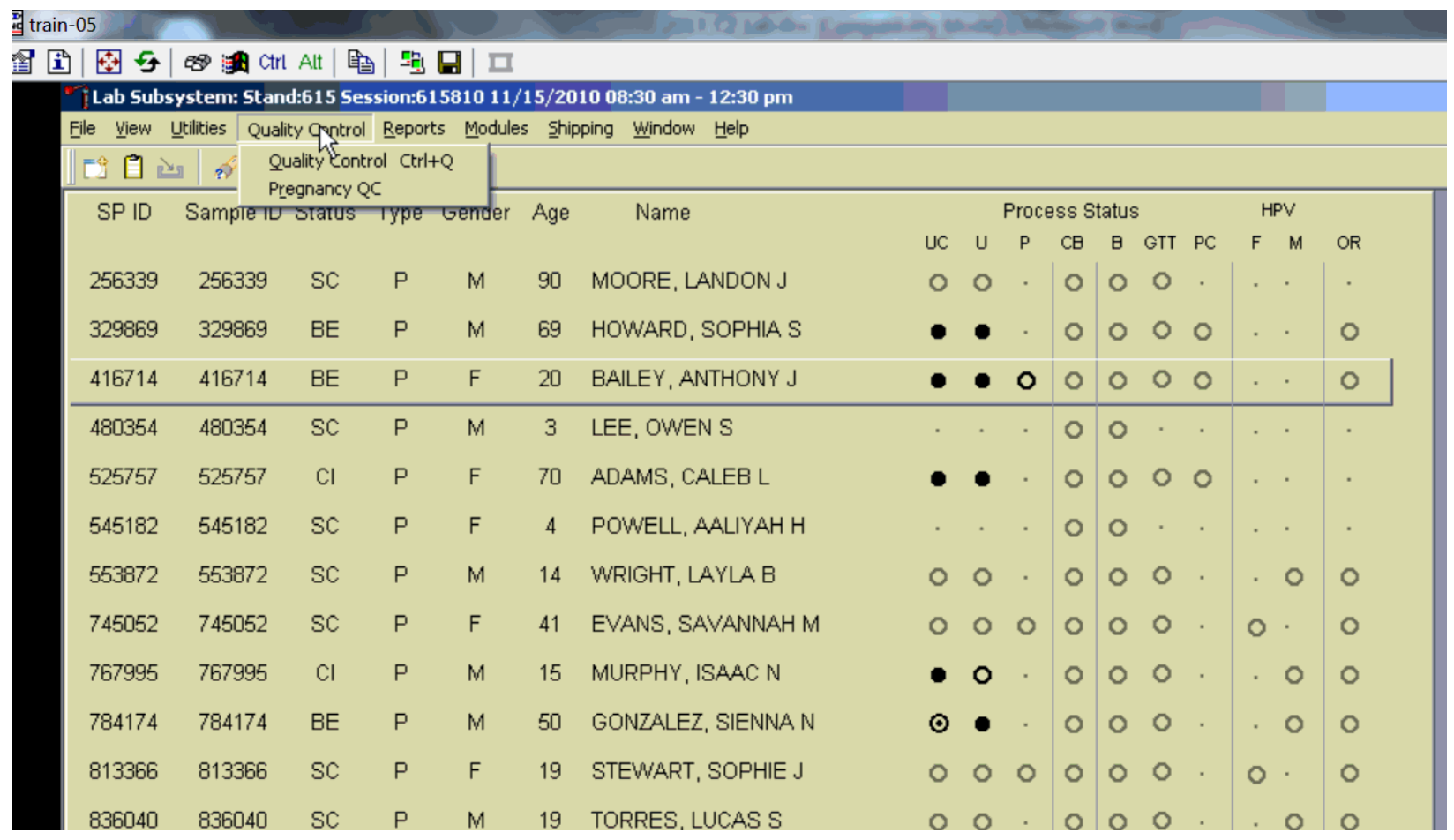

\section{*Quality Control}

Quality Control

Opens Quality Control for Start of Stand, End of Stand, Daily, Weekly, and NCHS QC.

Pregnancy QC Opens pregnancy quality control window. 


\subsection{4}

\section{Report Menu Options}

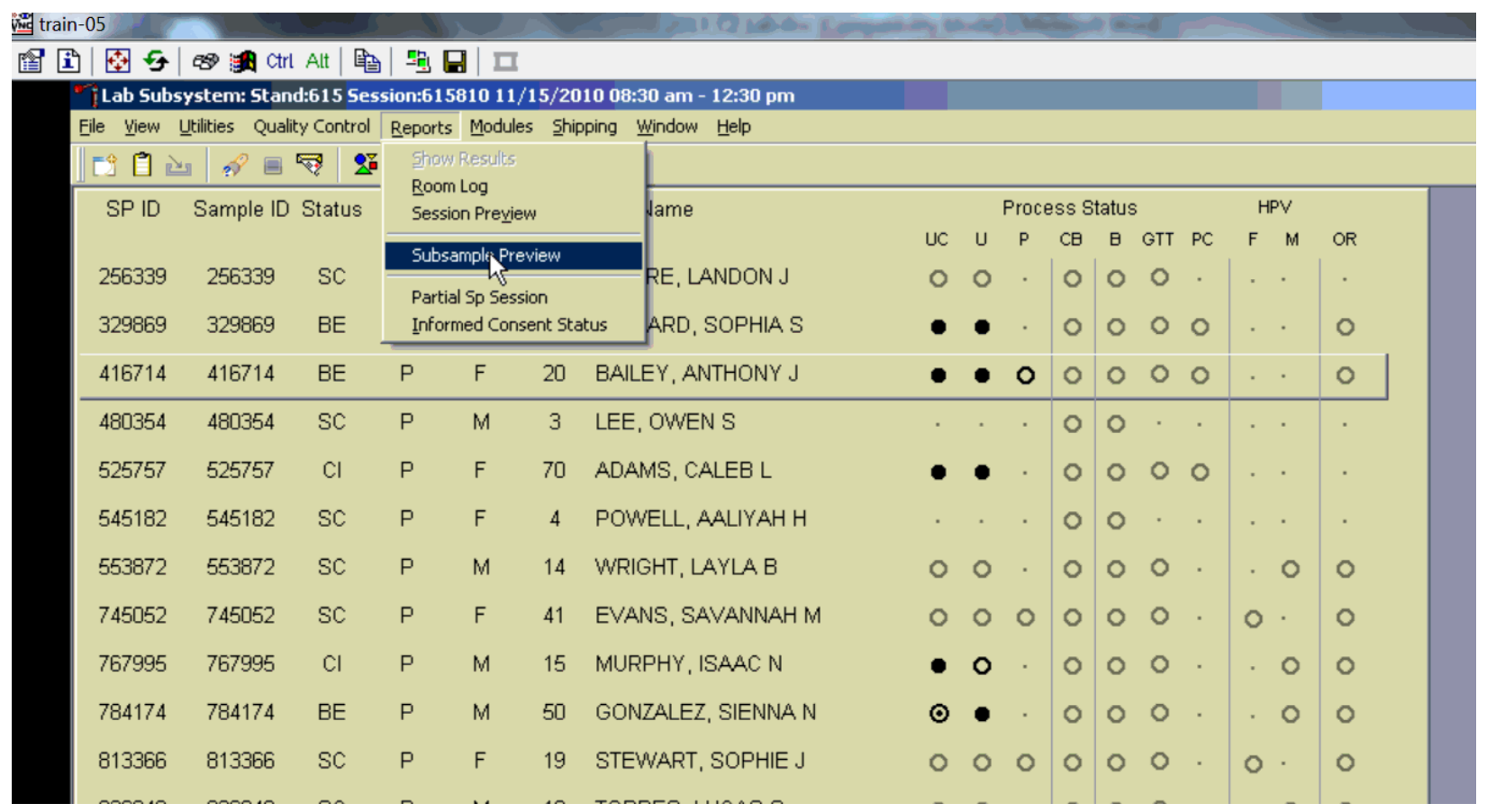

\section{* Reports}

\begin{tabular}{ll}
\hline Show Results & This option is grayed out and not applicable. \\
\hline *Room Log & $\begin{array}{l}\text { Lists the SPs who have completed the component, including appt/exam and } \\
\text { component status. }\end{array}$ \\
\hline${ }^{*}$ Session Preview* & Lists all SPs in current session. \\
\hline Subsample Preview & $\begin{array}{l}\text { List the SPs who are eligible for the various subsamples. A complete } \\
\text { description of this functionality is described in Section 2.8. }\end{array}$ \\
\hline Partial SP Session & Lists the original session that the partial SP was scheduled. \\
\hline Informed Consent Status & $\begin{array}{l}\text { Lists the various modules that require consent and displays the result of the } \\
\text { consent }- \text { excluded or not excluded. A complete description of this } \\
\text { functionality is described in Section 2.9. }\end{array}$ \\
\hline
\end{tabular}




\subsubsection{Modules}

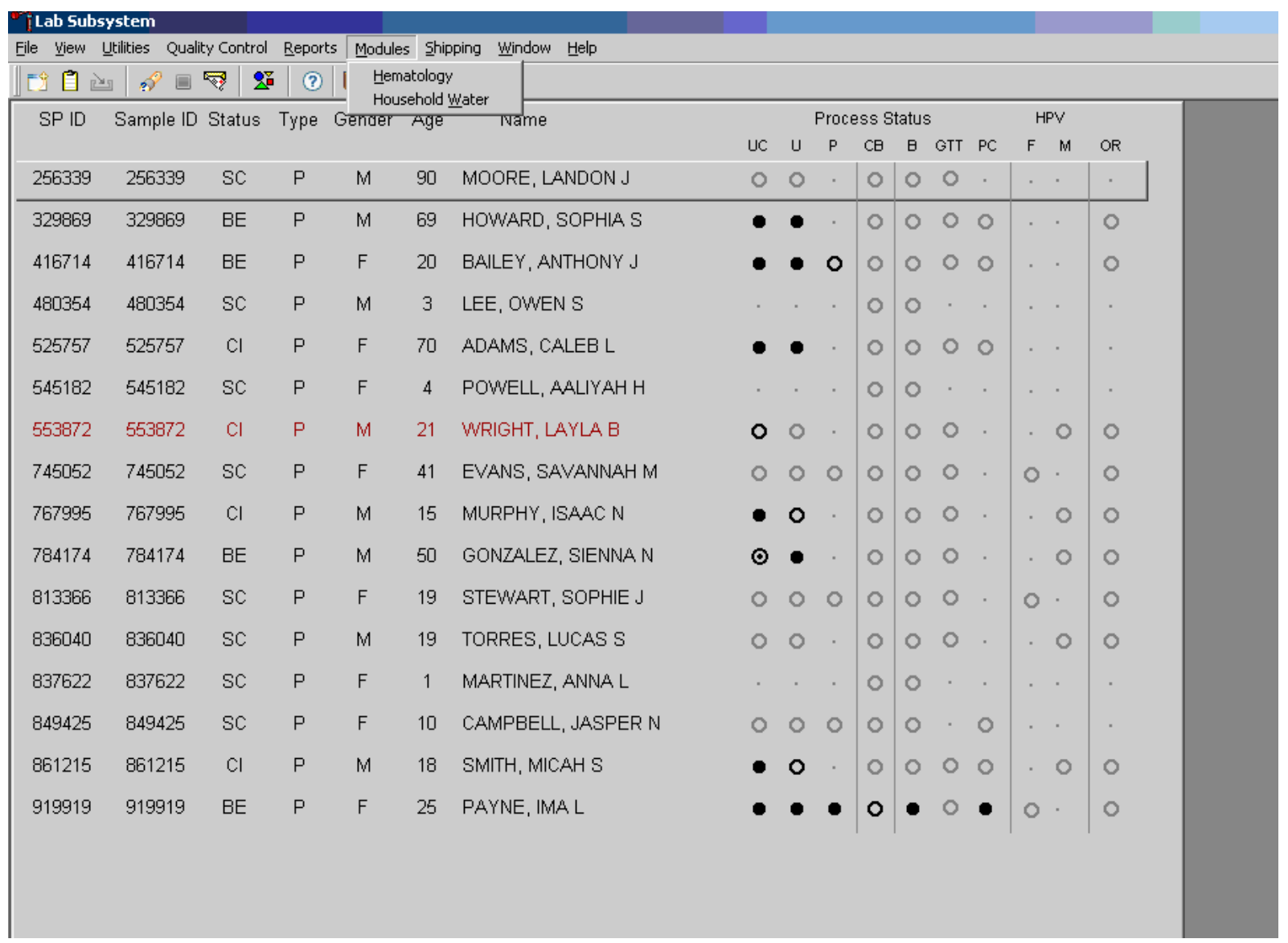

\section{Modules}

Hematology

Opens the laboratory hematology module. A complete description of this functionality is described in Section 7.XI.

Household Water Opens the household water processing module. 


\subsection{6}

Shipping

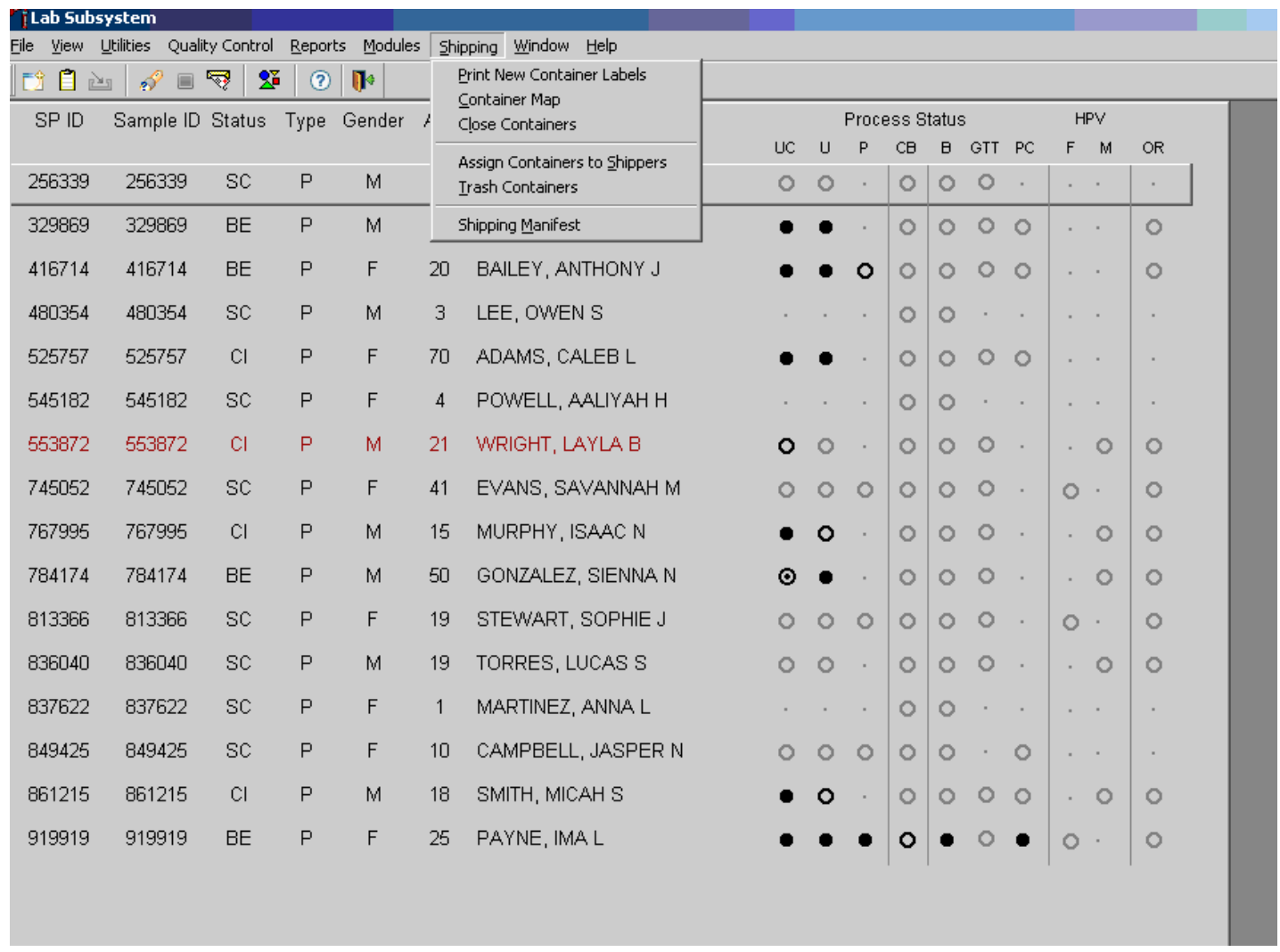

\section{Shipping}

Print New Container

Labels

Container Map
Allows the medical technologist to print labels for containers such as a 5 -inch box with $9 \times 9$ grid. A complete description of this functionality is described in Section 8.5.

Allows the medical technologist to view a list of all containers with a graphic representation of the samples assigned to that container. A complete description of this functionality is described in Section 2.7.

Close Containers Allows the medical technologist to "close" active containers that have been previously opened. A complete description of this functionality is described in Section 8.9.

Assign Containers to Shippers Allows the medical technologist to assign or associate a closed container with a FedEx airbill number. A complete description of this functionality is described in Section 8.10.

\begin{tabular}{ll}
\hline Trash Containers & $\begin{array}{l}\text { Allows the medical technologist to delete container label numbers in the } \\
\text { database for labels that are damaged. A complete description of this } \\
\text { functionality is described in Section 8.6. }\end{array}$ \\
\hline Shipping Manifest & $\begin{array}{l}\text { Allows the medical technologist to print hard-copy manifests and } \\
\text { electronically send email files to the contract laboratories. A complete } \\
\text { description of this functionality is described in Section } 8.11 .\end{array}$ \\
\hline
\end{tabular}


If at any time during the phlebotomy or physician exam, the SP indicates that he or she does not want his or her biological specimens tested for HIV (AIDS), STD (sexually transmitted diseases include chlamydia and trichomonas), HPV, or future studies, then the staff enter this information using the IC Exclusions module.

Access the IC Exclusions module.

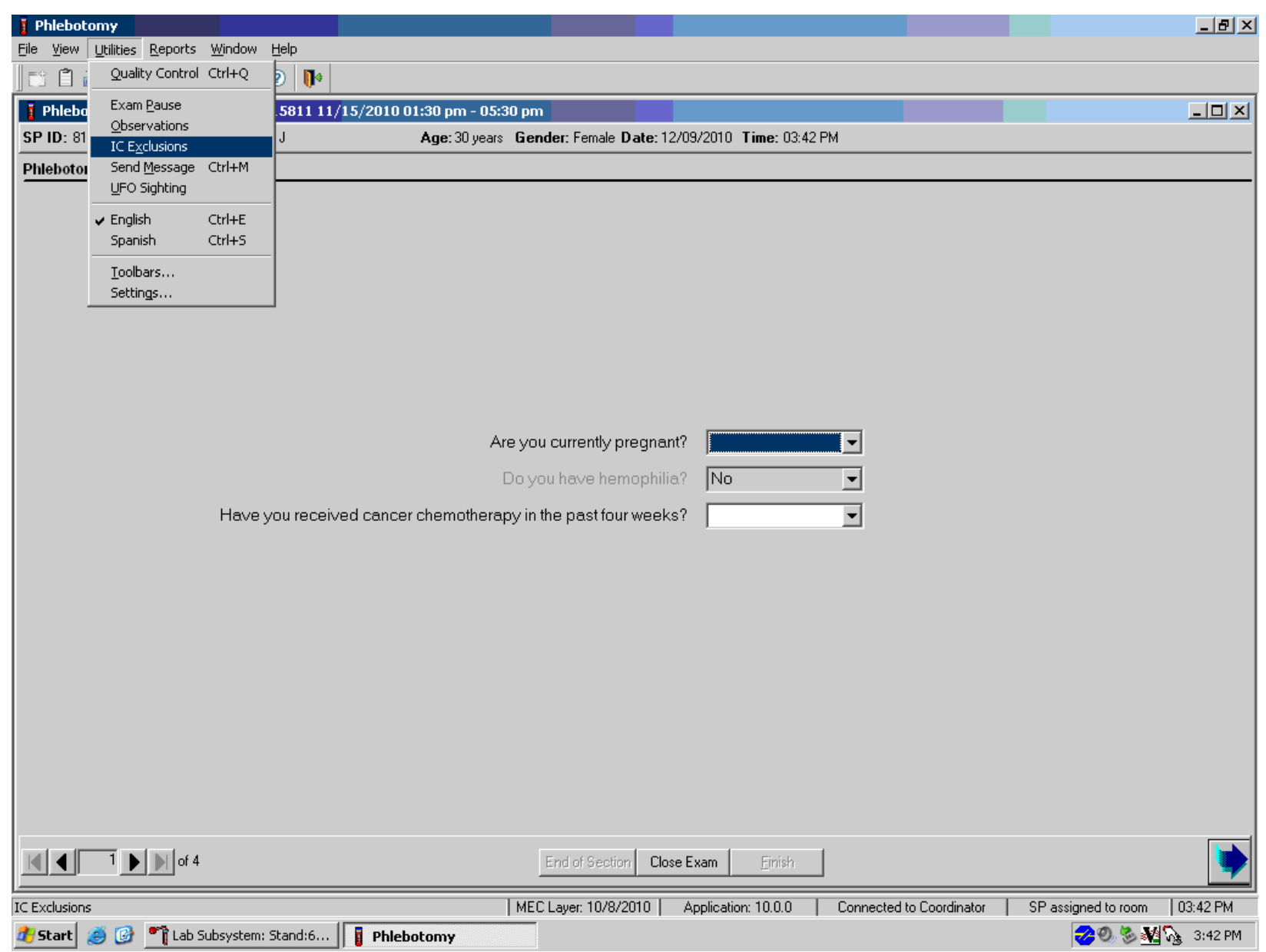

To access the IC (informed consent) Exclusions module, use the mouse to direct the mouse arrow to $\{$ Utilities $\}$ in the menu bar, left click, drag the arrow to $\{\mathrm{IC}$ Exclusions $\}$, and left click, or type [Alt] [U/i], [X/X]. 
The IC Exclusions window displays all the specific exclusion item tests or groups of tests for which the SP is eligible and the status for each exclusion item.

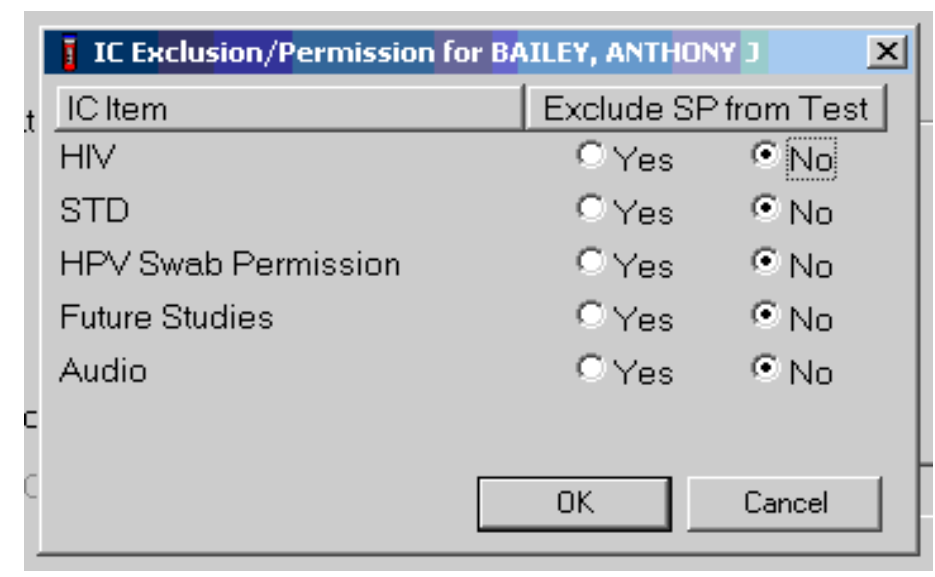

Record each item as directed by the SP. To mark each item, use the mouse to direct the mouse arrow to the "Yes" or "No" radio button and left click. To record these actions and save them to the database, use the mouse to direct the mouse arrow to the OK button and left click, or select [Enter]. To cancel these actions without saving the data to the database, use the mouse to direct the mouse arrow to the Cancel button and left click.

\subsection{Session Utility}

Sign onto the computer terminal. Open the laboratory application. If the laboratory is conducting a MEC session, the heads-up display will default to the current session. Use the Set Session utility to access past or future sessions. 


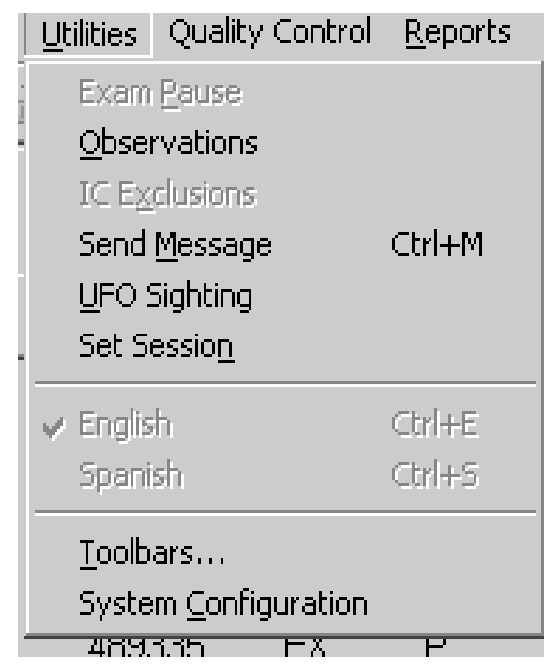

To access the Set Session utility, use the mouse to direct the mouse arrow to Utilities and left click. Drag the arrow to Set Session and left click.

The Session PickUp list displays. Select the correct session from the pick list.

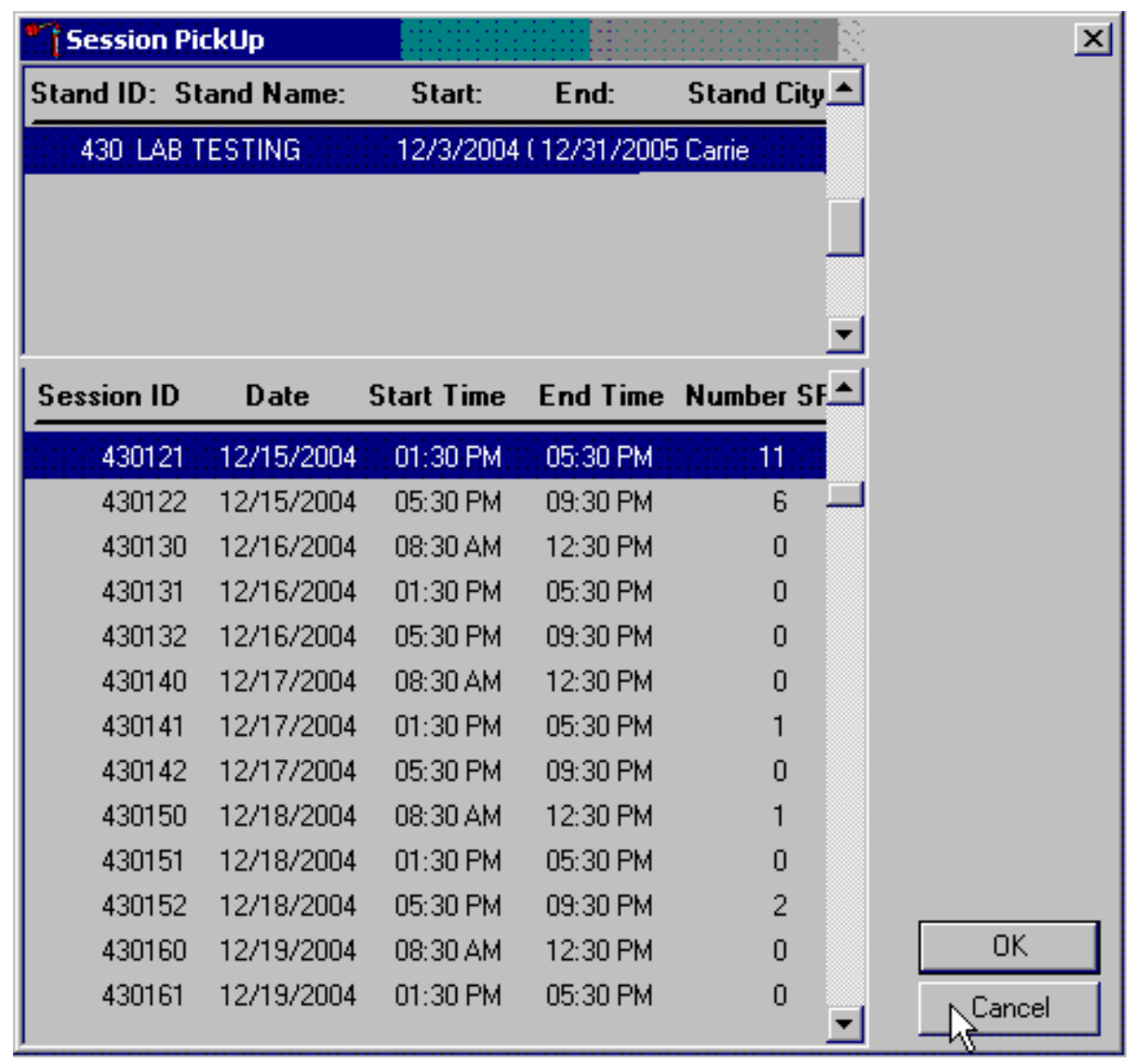


The Session PickUp window displays. The current stand is displayed in the top section of the window. Use the lower vertical scroll bar to view all sessions. Use the mouse to select the desired session. To proceed, hit the OK button or press [Enter]. To cancel and return to the previous session, click on the Cancel button.

After selecting a session from the Session PickUp list and selecting OK, the laboratory heads-up screen displays.

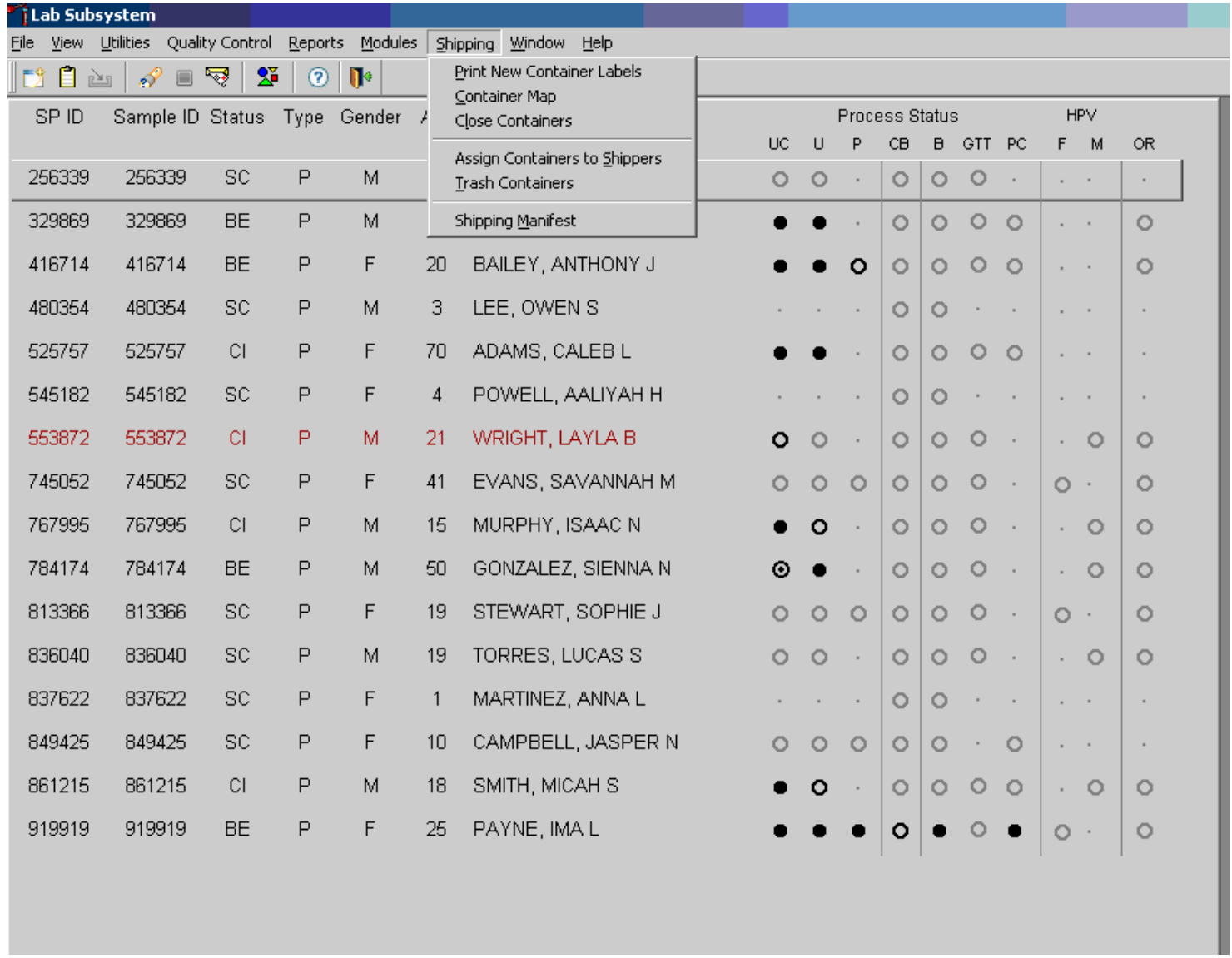

\subsection{Container Map}

Check the slot assignment of all containers at the end of each session. Access the Container Map report under the Reports or Shipping menu, or type [Alt] [R/r] [C/c] or [Alt] [S/s], [C/c]. 


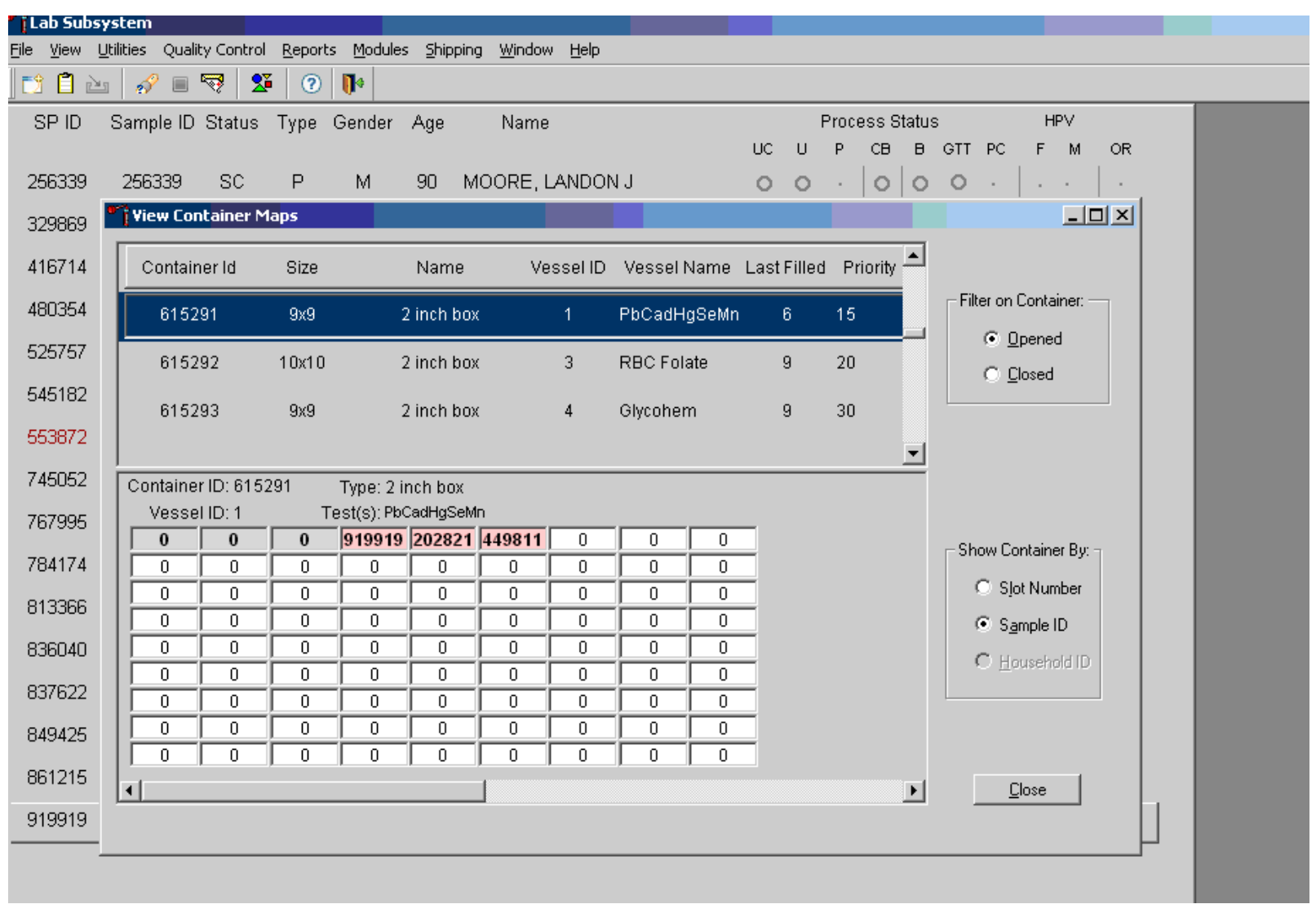

Verify the contents of each container against the container map.

- If "Opened" under "Filter on Container" is selected, then all open containers will display.

- If "Sample ID" under "Show Container By:" is selected, then sample IDs will display in the assigned slot in the graphical representation of the container.

- Container IDs may be selected by mouse or by using the up and down keys.

- Verify the container ID on the box against the container ID listed on the screen and verify each vessel sample ID against its location in the map.

- Mark the specimens that have been checked by placing a black dot in the center of the vial cap. When subsequently checking the Container Map report for this container, begin checking the specimens that are not marked.

Continue checking each Container Map report for the remaining vessels.

To exit the Container Map report, click on the Close button. 
Access the Subsample Preview to view all SPs in the current session and the selected subsamples for which they are eligible.

\begin{tabular}{|c|c|c|c|c|c|c|c|c|c|c|c|c|c|c|c|}
\hline \multicolumn{16}{|c|}{ 尊 train-05 } \\
\hline 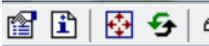 & es $\mathrm{ctrl}$ & Alt | 䠗 & | 骂回 & 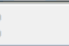 & 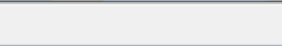 & & & & & & & & & & \\
\hline iLab Subsy: & ystem: Stand & d:615 Sess & sion:615810 & $/ 15 / 20$ & $1008: 30 \mathrm{am}-12: 30 \mathrm{pm}$ & & & & & & & & & & \\
\hline File view ut & Untilities Quality & ity Control & Reports Moc & es Shir & oping Window Help & & & & & & & & & & \\
\hline E9 自 & ? & 布 2 & Show Resu & & & & & & & & & & & & \\
\hline SPID & Sample ID & Status & Session Pre & & lame & & & Proce & ess St & tatus & & & & PV & \\
\hline & & & Subsamplas & eview & & uc & $\mathrm{u}$ & $\mathrm{P}$ & $\mathrm{CB}$ & B & GTT & $\mathrm{PC}$ & $\mathrm{F}$ & M & OR \\
\hline & 256339 & SC & Partial Sp S & & RE, LANDON J & 0 & 0 & . & 0 & 0 & 0 & & . & . & . \\
\hline 329869 & 329869 & $B E$ & Informed C & sent St: & ARD, SOPHIA S & $\bullet$ & $\bullet$ & . & 0 & 0 & 0 & 0 & . & - & 0 \\
\hline 416714 & 416714 & $B E$ & $\mathrm{P}$ & 20 & BAILEY, ANTHONY J & $\bullet$ & $\bullet$ & 0 & 0 & 0 & 0 & 0 & $\cdot$ & - & 0 \\
\hline 480354 & 480354 & SC & $\mathrm{P}$ & 3 & LEE, OWEN S & . & . & . & 0 & 0 & . & . & . & . & . \\
\hline 525757 & 525757 & $\mathrm{Cl}$ & $\mathrm{P}$ & 70 & ADAMS, CALEB L & $\bullet$ & $\bullet$ & . & 0 & 0 & 0 & 0 & $\cdot$ & - & . \\
\hline 545182 & 545182 & SC & $\mathrm{P}$ & 4 & POWELL, AALIYAH H & . & . & . & 0 & 0 & . & . & · & . & . \\
\hline 553872 & 553872 & SC & $\mathrm{P}$ & 14 & WRIGHT, LAYLA B & 0 & 0 & . & 0 & 0 & 0 & . & . & 0 & 0 \\
\hline 745052 & 745052 & SC & $\mathrm{P}$ & 41 & EVANS, SAVANNAH M & 0 & 0 & 0 & 0 & 0 & 0 & . & 0 & - & 0 \\
\hline 767995 & 767995 & $\mathrm{Cl}$ & $\mathrm{P}$ & 15 & MURPHY, ISAAC N & $\bullet$ & 0 & . & 0 & 0 & 0 & . & . & 0 & 0 \\
\hline 784174 & 784174 & $B E$ & $\mathrm{P}$ & 50 & GONZALEZ, SIENNA N & $\odot$ & $\bullet$ & . & 0 & 0 & 0 & . & $\cdot$ & 0 & 0 \\
\hline 813366 & 813366 & SC & $\mathrm{P}$ & 19 & STEWART, SOPHIE J & 0 & 0 & 0 & 0 & 0 & 0 & - & 0 & - & 0 \\
\hline 836040 & 836040 & $\mathrm{SC}$ & $\mathrm{P}$ & 19 & TORRES, LUCAS S & 0 & 0 & . & 0 & 0 & 0 & . & . & 0 & 0 \\
\hline
\end{tabular}

Access the Subsample Preview report under Reports in the menu bar. 
Review the Subsample Preview report.

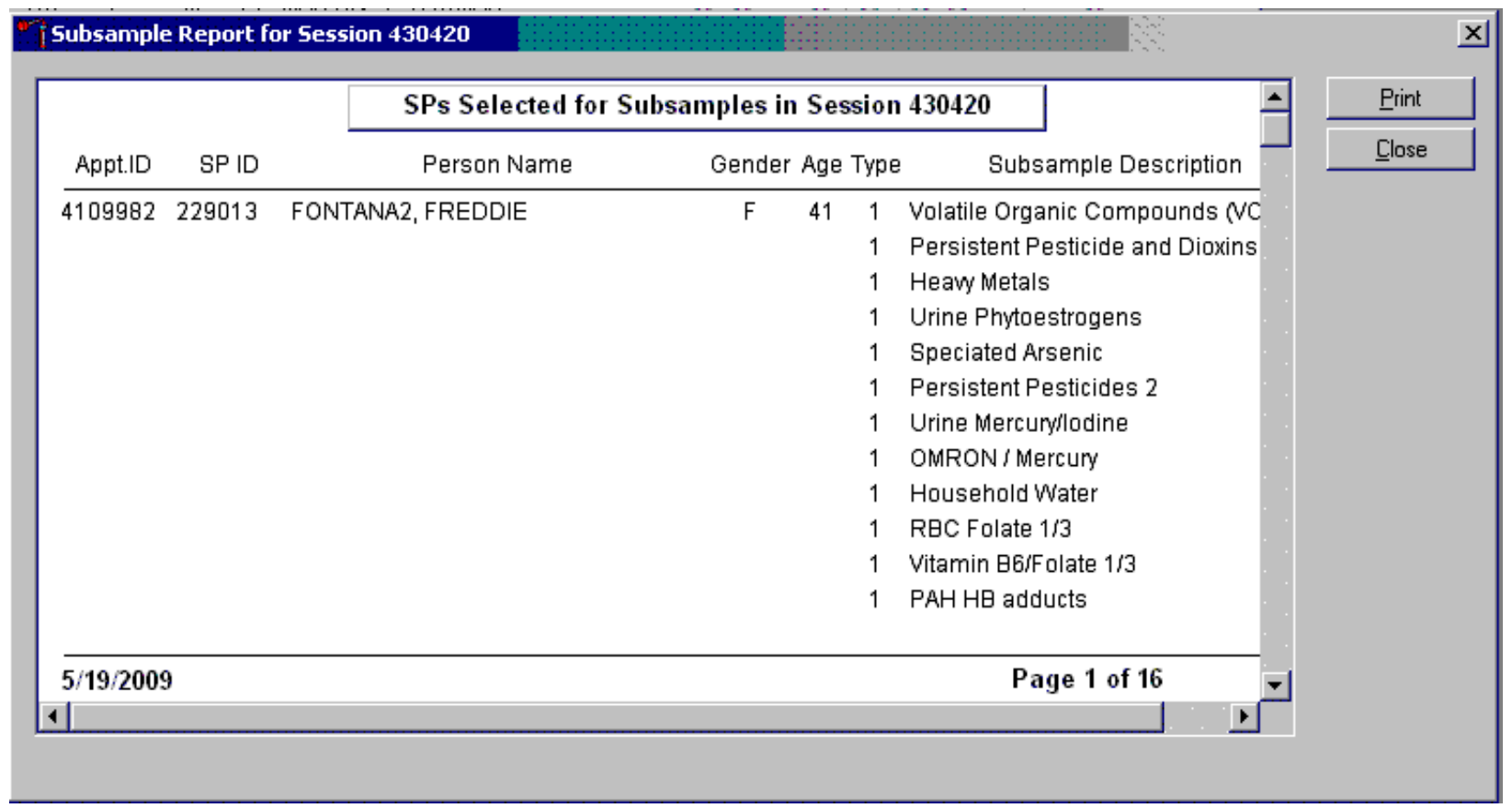

The Subsample Preview report includes all SPs assigned to the current session and the subsamples for which they are eligible. This report contains the appointment ID, SP ID, person name, gender, age, appointment type, and subsample description. Only SPs selected for inclusion in specific subsamples are eligible to have the related vessel filled. To view the entire report, use the scroll bar. To print a copy of the report, use the mouse to direct the mouse arrow to the Print button and left click. To exit and close the report, click on the Close button.

\section{Informed Consent Status}

Access the Informed Consent Status report to view SPs and the status of the informed consent result for various tests and/or groups of tests. 


\begin{tabular}{|c|c|c|c|c|c|c|c|c|c|c|c|c|c|c|c|c|c|}
\hline \multirow{2}{*}{\multicolumn{18}{|c|}{ TLab Subsystem }} \\
\hline File View 나il & \multicolumn{2}{|c|}{ lities Quality Control } & \multirow{3}{*}{\multicolumn{4}{|c|}{$\begin{array}{l}\text { Reports Modules Shipping } \\
\text { Show Results } \\
\text { Room Log } \\
\text { Session Preview }\end{array}$}} & \multicolumn{6}{|l|}{ Window Help } & & & & & \\
\hline$\|=9$ & $? \square$ & 石 & & & & & & & & & & & & & & & \\
\hline \multirow[t]{2}{*}{ SPID } & \multirow{2}{*}{\multicolumn{2}{|c|}{ Sample ID Status }} & & & & & \multirow[t]{2}{*}{ Jame } & \multicolumn{7}{|c|}{ Process Status } & \multicolumn{3}{|c|}{ HPV } \\
\hline & & & \multirow{2}{*}{\multicolumn{4}{|c|}{ Subsample Preview }} & & UC & \multirow{2}{*}{$\begin{array}{l}u \\
0\end{array}$} & \multirow{2}{*}{$\mathrm{P}$} & \multirow{2}{*}{$\mathrm{CB}$} & \multirow{2}{*}{$\begin{array}{l}\mathrm{B} \\
\mathrm{O}\end{array}$} & \multirow{2}{*}{ GTT } & \multirow{2}{*}{$\mathrm{PC}$} & \multirow{2}{*}{$\mathrm{F}$} & \multirow{2}{*}{ M } & \multirow{2}{*}{ OR } \\
\hline 256339 & 256339 & $\mathrm{SC}$ & & & & & RE, LANDON J & 0 & & & & & & & & & \\
\hline 329869 & 329869 & $\mathrm{BE}$ & \multicolumn{4}{|c|}{$\begin{array}{l}\text { Partial Sp Session } \\
\text { Informed Consent Status }\end{array}$} & ARD, SOPHIA S & - & - & . & 0 & 0 & 0 & 0 & . & . & 0 \\
\hline 416714 & 416714 & $\mathrm{BE}$ & $P$ & $\mathrm{~F}$ & 20 & BAIL & EY, ANTHONY J & - & - & 0 & 0 & 0 & 0 & 0 & . & . & 0 \\
\hline 480354 & 480354 & $\mathrm{SC}$ & $\mathrm{P}$ & $M$ & 3 & LEE & OWEN S & . & . & . & 0 & 0 & . & . & . & . & . \\
\hline 525757 & 525757 & $\mathrm{Cl}$ & $P$ & $\mathrm{~F}$ & 70 & $\mathrm{ADA}$ & MS, CALEB L & - & - & . & 0 & 0 & 0 & 0 & . & . & . \\
\hline 545182 & 545182 & $\mathrm{SC}$ & $P$ & $\mathrm{~F}$ & 4 & $\mathrm{POV}$ & WELL, AALIYAH H & . & . & . & 0 & 0 & . & . & . & . & . \\
\hline 553872 & 553872 & $\mathrm{Cl}$ & $P$ & $\mathrm{M}$ & 21 & WRI & GHT, LAYLA B & 0 & 0 & . & 0 & 0 & 0 & . & . & 0 & 0 \\
\hline 745052 & 745052 & $\mathrm{SC}$ & $P$ & $\mathrm{~F}$ & 41 & EVA & NS, SAVANNAH M & 0 & 0 & 0 & 0 & 0 & 0 & . & 0 & . & 0 \\
\hline 767995 & 767995 & $\mathrm{BE}$ & $P$ & $\mathrm{M}$ & 15 & MUF & RPHY, ISAAC N & - & 0 & . & 0 & 0 & 0 & . & . & 0 & 0 \\
\hline 784174 & 784174 & $\mathrm{BE}$ & $P$ & $\mathrm{M}$ & 50 & $\mathrm{GON}$ & AZALEZ, SIENNA N & $\odot$ & - & . & 0 & 0 & 0 & . & . & 0 & 0 \\
\hline 813366 & 813366 & $\mathrm{SC}$ & $P$ & $\mathrm{~F}$ & 19 & STE & WART, SOPHIE J & 0 & 0 & 0 & 0 & 0 & 0 & . & 0 & . & 0 \\
\hline 836040 & 836040 & $\mathrm{SC}$ & $P$ & $M$ & 19 & TOR & RES, LUCAS S & 0 & 0 & . & 0 & 0 & 0 & . & . & 0 & 0 \\
\hline 837622 & 837622 & $\mathrm{SC}$ & $P$ & $\mathrm{~F}$ & 1 & MAF & RTINEZ, ANNA L & . & - & . & 0 & 0 & . & . & . & - & . \\
\hline 849425 & 849425 & $\mathrm{SC}$ & $P$ & $\mathrm{~F}$ & 10 & CAM & APBELL, JASPER N & 0 & 0 & 0 & 0 & 0 & . & 0 & . & . & . \\
\hline 861215 & 861215 & $\mathrm{Cl}$ & $P$ & $\mathrm{M}$ & 18 & SMI & TH, MICAH S & - & 0 & . & 0 & 0 & 0 & 0 & . & 0 & 0 \\
\hline 919919 & 919919 & $\mathrm{BE}$ & $P$ & $\mathrm{~F}$ & 25 & PAY & NE, IMA L & - & 0 & - & 0 & - & 0 & 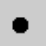 & 0 & . & 0 \\
\hline
\end{tabular}

Access the Informed Consent Status report under the Reports menu in the menu bar.

Review the SPs scheduled into the current session and the status of their informed consent by using the Informed Consent Status report. 


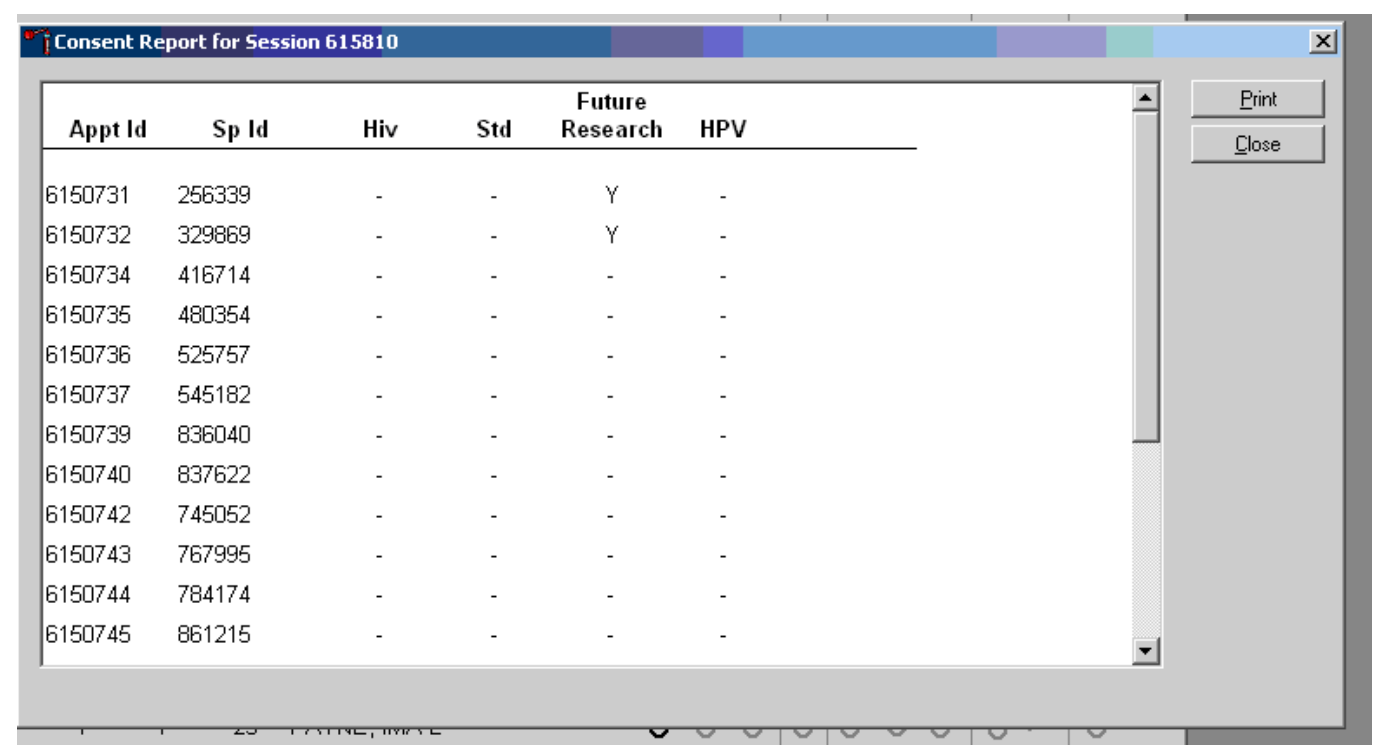

The consent report lists all SPs scheduled in the current session, their appointment and SP IDs, and the exclusion status for the following tests or groups of tests:

- $\quad$ HIV (vessel 22 - serum);

- $\quad$ STD (vessel $24-\mathrm{HSV})$;

- Future research (32-39 - serum, 52 and 139 - urine); and

- HPV Swab (vessel 78 and vessel 140).

The status is "Y" or "Yes" if the SP is excluded and "N" if the SP is not excluded.

\section{Access a Processing Module}

Select the correct SP and access the module of interest. Use the scroll bar to view all SPs scheduled into the session. The active SP is contained in a rectangular box. Use the heads-up display to select the correct SP. Select a different SP if the active SP is not the correct SP. 


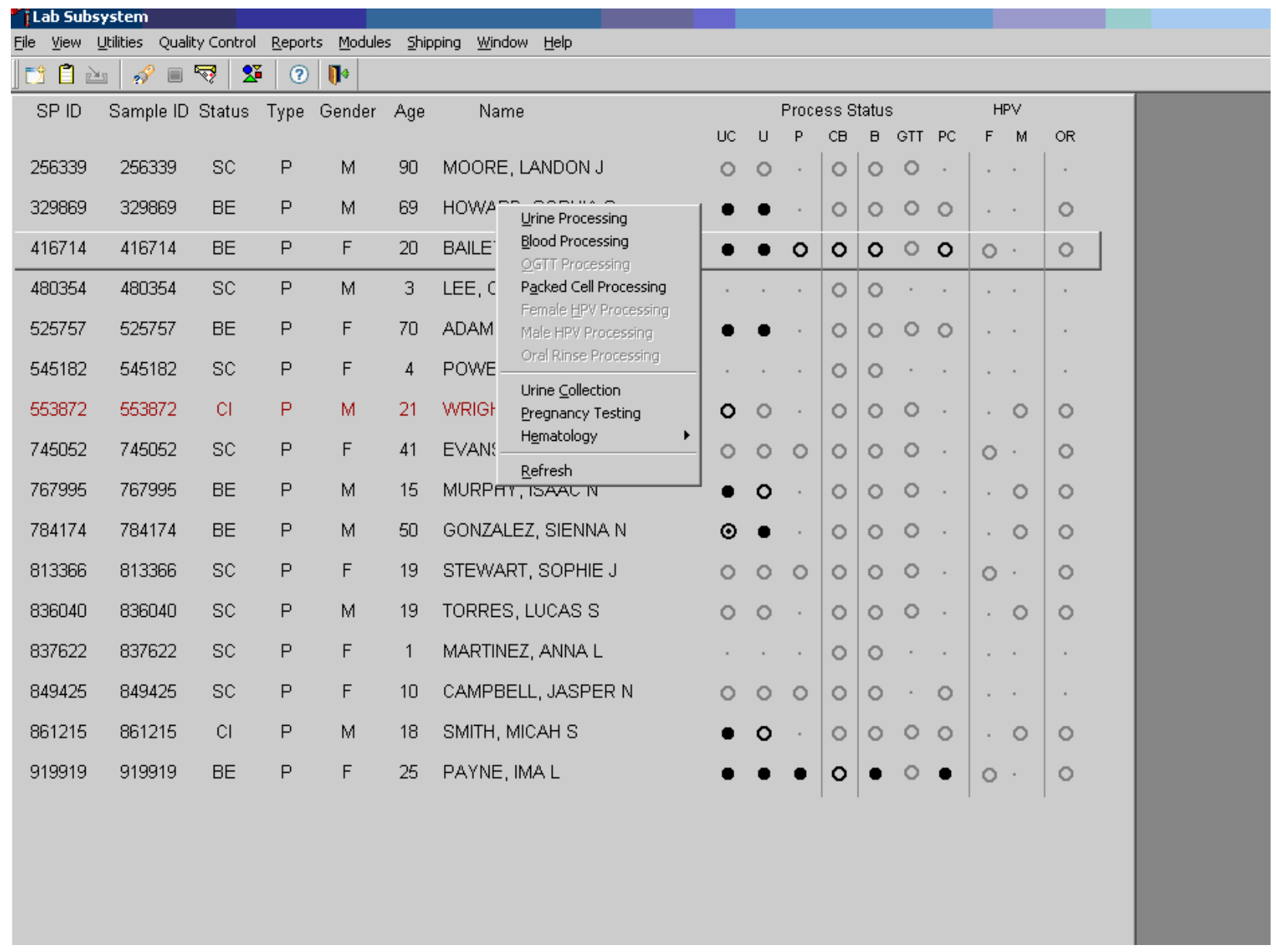

To select an SP, use the up and down keys to move up and down the list until the correct SP is highlighted or click on the SP. To access a specific processing module, click on the module on the heads-up screen: UP\{Urine Processing\}, BP\{Blood Processing\}, GTT\{OGTT Processing), PC \{Packed Cell Processing\}, HPV \{HPV Processing (Male or Female)\}, OR \{Oral Rinse Processing\}, UC\{Urine Collection $\}, \mathrm{P}\{$ Pregnancy Testing\}, or $\mathrm{CB}\{$ Hematology $\}$ and left click. Alternatively, right click on an SP and select the correct module.

\subsection{Warning and Error Messages}

Throughout the course of an examination, warning and error messages may appear when an attempt is made to perform an action the program cannot execute or that requires confirmation to continue the action. The message normally appears with statements explaining the error condition. Complying with 
the error message statement will normally remedy the error. When required to confirm an action, such as deleting records, be sure the action is necessary because, in most cases, it is irreversible.

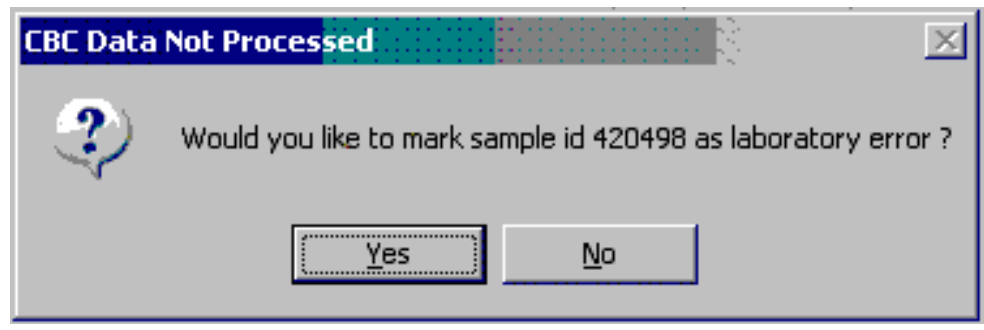

For example, if the SP ID is incorrect in the SP Logon window, a warning message box displays indicating the problem and giving remedial instructions. To remove the warning message box, click on the OK button.

\subsubsection{Not Filled Require a Comment}

Data cannot be saved until all vessels marked as Filled-"No" are assigned a comment.

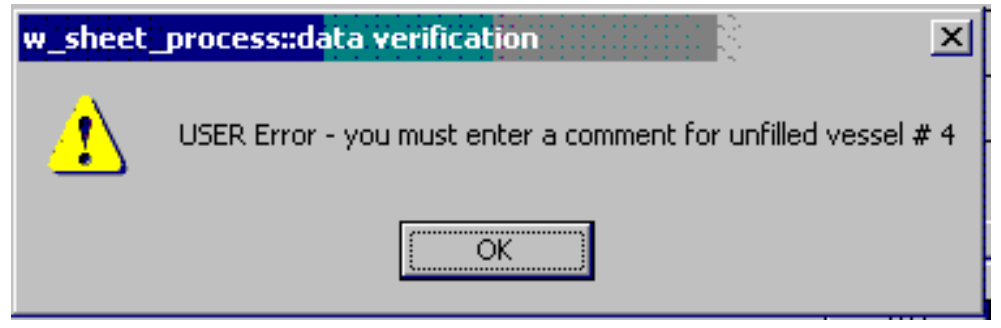

If the Save or the OK button is selected and all vessels are not marked as either Filled-"Yes" or Filled-"No," a warning message box displays requesting a comment for each unfilled vessel. To remove the warning message box, click on the OK button.

\subsubsection{Labels Must be Printed in Advance}

If labels have not been printed in advance, all SPs will be displayed in red. A pop-up box will appear with Label Action Required. 
Specimen processing cannot occur unless labels have been printed in advance. Reprint the labels for all SPs or an individual SP in a session. See Chapter 9 for detailed instructions on the Lab Print Application.

\subsection{Coulter and Urine Scale Icons}

\section{Coulter}

This is the NHANES Coulter ${ }^{\circledR}$ Monitor. It must be open and running at the start of each session. This icon stores all $\mathrm{DxH}^{\mathrm{TM}} 800$ runs in the ISIS database. Open the icon by double-clicking on the Coulter icon on the desktop. The Coulter icon looks like this:

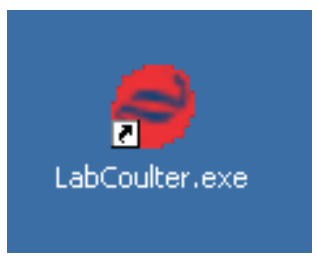

Note the red icon in the lower left hand corner of the system tray.

\section{«at}

NOTE: If there is a problem with the database and the Coulter application, the Hematology module does not need to be open before running SP samples on theDxH 800 . 


\section{Urine Scale}

Ensure that computer workstation 1 (Urine/Sink) is turned on and a technician is logged into the Laboratory application. Open the Urine Scale application by double clicking on the Urine Scale icon on the desktop. The Urine Scale icon looks like this:

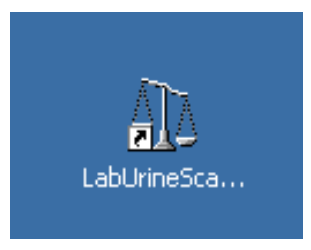

The Urine Scale application icon will appear in the system tray, similar to the Coulter application. This application captures data from the scale and logs it into the database. The Urine Scale application must be opened and in the system tray in order to capture any urine collection readings from the scale.

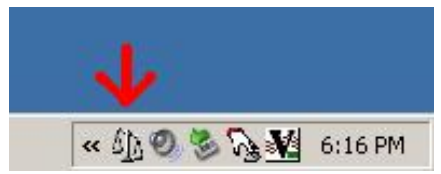




\section{VENIPUNCTURE}

\subsection{Introduction}

The blood collection procedure consists of (1) administering a questionnaire to screen for conditions that exclude the participant from the blood draw; (2) determining fasting status; and (3) performing a blood draw. The phlebotomist also collects one additional blood tube from SPs selected for the VOC (volatile organic compounds) special study, and administers a dextrose solution (Trutol) to and collects an additional 2-mL gray top tube from SPs selected for the Oral Glucose Tolerance (OGTT) component.

The phlebotomist conducts an interview to screen SPs for conditions that exclude them from the blood draw. The phlebotomist also administers a fasting questionnaire to determine fasting compliance. The venipuncture protocol is dependent on the SP's age, appointment type, and session time. Exhibits 3-1 through 3-4 illustrate the various venipuncture protocols. These protocols indicate the types and numbers of tubes in priority order for each age group and person type. A chart located on the wall in the phlebotomy room also illustrates this information. It is extremely important to perform the venipuncture protocol as described for each SP. Use comments to describe differences between the actual tubes drawn and the established protocol. After the results of the blood draw are entered, labels automatically print for the blood tubes. The phlebotomist immediately labels collected tubes, places them through the pass-through window to the laboratory, and escorts the SP to the coordinator area or to his or her next component. The phlebotomist may offer juice and crackers to SPs less than 12 years of age and SPs not selected for the OGTT component. 
Exhibit 3-1. Venipuncture protocol - primary

\begin{tabular}{|c|c|c|c|c|c|}
\hline & \multicolumn{5}{|c|}{ Age in Years } \\
\hline & $1-2$ & $3-5$ & $6-11$ & $12-19$ & $20+$ \\
\hline 3-mL Lavender & 1 & 2 & & & \\
\hline 4-mL Lavender & & & 2 & 2 & 2 \\
\hline $\begin{array}{l}\text { 2-mL Gray } \\
\text { (Morning sessions only) }\end{array}$ & & & & 1 & 1 \\
\hline 6-mL Royal Blue ${ }^{3}$ & & & 1 & 1 & 1 \\
\hline 3-mL Red & 2 & 1 & & & \\
\hline 7-mL Red & & 1 & 1 & 1 & \\
\hline 15-mL Red & & & & 2 & 3 \\
\hline 10-mL Red & & & 1 & & \\
\hline $15-\mathrm{mL} \mathrm{Red}^{\circ}$ & & & & 3 & 3 \\
\hline 7-mL Gray $(12+)^{1}$ & & & & 1 & 1 \\
\hline $\begin{array}{l}\text { 2-mL Gray }{ }^{2} \\
\text { (GTT morning sessions) }\end{array}$ & & & & 1 & 1 \\
\hline
\end{tabular}

${ }^{\circ}$ SPs selected for inclusion in the Persistent Pesticides, Perfluoronated or BFR subsamples

${ }^{1}$ SPs selected for inclusion in the VOC subsample

${ }^{2}$ Glucose tolerance test

${ }^{3}$ Selenium/Copper/Zinc

Exhibit 3-2. Venipuncture protocol - VIP guest

\begin{tabular}{lcccc}
\hline & \multicolumn{4}{c}{ Age in Years } \\
\cline { 2 - 5 } & $1-2$ & $3-5$ & $6-11$ & $12+$ \\
\hline 3-mL Lavender & 1 & 1 & 1 & 1 \\
\hline 4-mL Lavender & & & & 1 \\
\hline 2-mL Gray & & & & \\
(Morning sessions only) & & 1 & & \\
\hline 3-mL Red & 1 & & & 1 \\
\hline 7-mL Red & & & & 1 \\
\hline 15-mL Red & & & & \\
\hline 10-mL Red
\end{tabular}


Exhibit 3-3. Venipuncture protocol - guest

\begin{tabular}{llc} 
& \multicolumn{2}{c}{ Age in Years } \\
\cline { 2 - 3 } & $1-11$ & $12+$ \\
\hline 3-mL Lavender & 1 & 1 \\
\hline 3-mL Red & & 1 \\
\hline
\end{tabular}

If the veins of an SP appear too fragile to accommodate the size of the large red top tubes, substitute smaller red top tubes as an alternative protocol. Exhibit 3-4, Alternative venipuncture protocol - primary, illustrates the alternative size and number of tubes in boldface with the original tube protocol in parentheses. When the alternative protocol is substituted for the original protocol, a comment must be recorded. This protocol constitutes a deviation from the established standard; it should be used only in rare circumstances.

Exhibit 3-4. Alternative venipuncture protocol - primary

\begin{tabular}{lccc}
\hline & \multicolumn{3}{c}{ Age in Years } \\
\cline { 2 - 4 } & $3-5$ & $6-11$ & $12+$ \\
\hline 6-mL Royal Blue & & $\mathbf{1}(1)$ & $\mathbf{1}(1)$ \\
\hline 3-mL Red & $\mathbf{3}$ & $\mathbf{2}$ & $\mathbf{4}$ \\
\hline 7-mL Red & $(1)$ & $\mathbf{2 ( 1 )}$ & $(3)$ \\
\hline 15-mL Red & & & $\mathbf{5}$ \\
\hline 10-mL Red & & $\mathbf{( 1 )}$ & $\mathbf{( 3 )}$ \\
\hline 15-mL Red & & &
\end{tabular}

NOTE: Alternative venipuncture protocol tubes are shown in boldface; regular protocol tubes are shown in parentheses. 


\subsection{Equipment and Supplies}

The phlebotomy room contains a phlebotomy chair, sink, data terminal, Dymo direct thermal label printer, refrigerator, phlebotomy cart, and cabinets. Figure 3-1 illustrates the floor plan for the phlebotomy room.

Figure 3-1. Floor plan for phlebotomy room

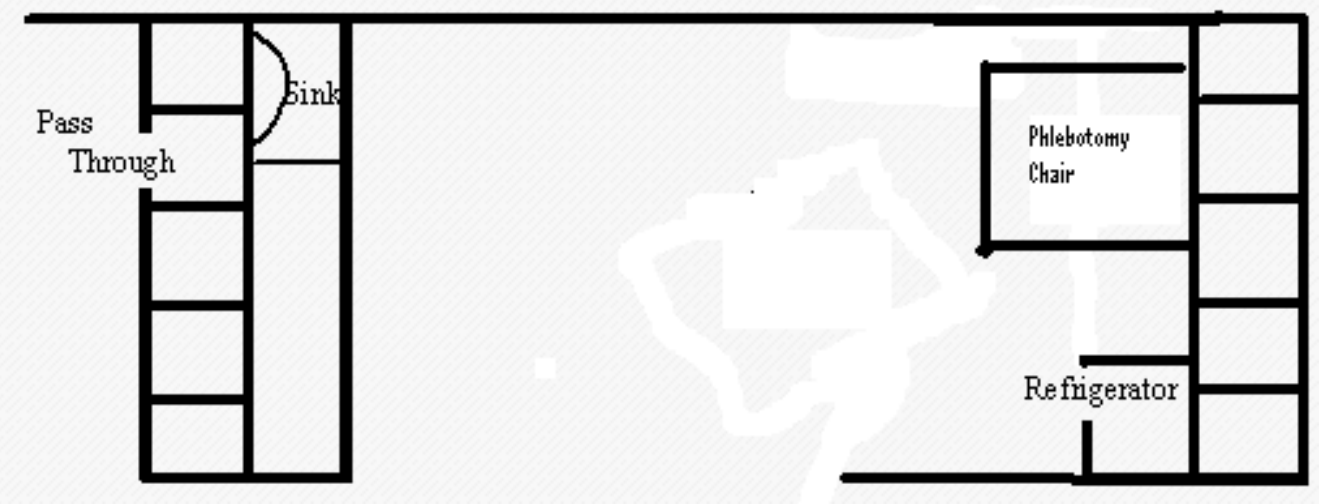

At the start and end of each stand, take a complete inventory using procedures described in Chapter 13, Section 13.3. 
The equipment and supplies used in venipuncture are listed in Exhibit 3-5.

Exhibit 3-5. Equipment and supplies - phlebotomy

\begin{tabular}{|c|c|}
\hline BD Hemogard Vacutainer® 3-mL EDTA & Trutol \\
\hline BD Hemogard Vacutainer ${ }^{\circledR}$ 4-mL EDTA & Alcohol wipe \\
\hline BD Hemogard Vacutainer ${ }^{\circledR}$ 2-mL Gray & $2 \times 2$ gauze square \\
\hline BD Hemogard Vacutainer ${ }^{\circledR}$ 3-mL Red & Co-Flex NL cohesive bandage \\
\hline BD Hemogard Vacutainer ${ }^{\circledR}$ 7-mL Red & Adhesive bandage - Coverlet \\
\hline Kendall Sherwood 15-mL Red & Adhesive bandage - children \\
\hline BD Vacutainer ${ }^{\circledR} 10-\mathrm{mL}$ Red & Transpore tape \\
\hline BD Vacutainer ${ }^{\circledR}$ 7-mL Gray (VOC) & BD Vacutainer $6 \mathrm{~mL}$ Royal Blue \\
\hline BD Hemogard Vacutainer ${ }^{\circledR} 10-m L$ EDTA & Cellestis TB In-Tube \\
\hline Nipro19 gauge & Surgical paper tape \\
\hline BD Luer adapter for use with the 19 gauge Saf- T EZ® set & Cartoon stickers \\
\hline BD Vacutainer Safety-Lok ${ }^{\mathrm{TM}} 21$ gauge with adapter & Ammonia inhalant packet \\
\hline BD Vacutainer Safety-Lok ${ }^{\mathrm{TM}} 23$ gauge with adapter & Pillow and disposable pillow cover \\
\hline BD Vacutainer ${ }^{\circledR}$ needle holder - single use & Squeeze ball \\
\hline Air sickness bag & Juice and crackers \\
\hline Heel warmer & List of reportable tests (handout) \\
\hline Liquid Band-Aid & Labels for Dymo printer \\
\hline Sani-Cloth & Sharps container 2 gallon (8 qt.) \\
\hline Purell hand soap & Biohazard bags 14 x 19 (5 gallon) \\
\hline Medicine cup & Batteries, AA \\
\hline \multicolumn{2}{|l|}{ Stretch disposable tourniquet } \\
\hline \multicolumn{2}{|l|}{ Nonconsumables } \\
\hline Step stool & Tube racks \\
\hline Phlebotomy cart & Floor mat \\
\hline Solar wall thermometer & CPR mask \\
\hline \multicolumn{2}{|l|}{ Emergency blanket } \\
\hline \multicolumn{2}{|l|}{ Obtain From Lab } \\
\hline Hand cream & Kleenex \\
\hline $\begin{array}{l}\text { Nonsterile, powder-free, nonlatex gloves -- small, medium, } \\
\text { large }\end{array}$ & Bleach bottle \\
\hline
\end{tabular}

For each SP, the phlebotomist routinely requires the following materials:

- Alcohol wipes

- 2"x 2" gauze squares

- Vacutainer ${ }^{\circledR}$ tubes of the appropriate size and type

- Disposable tourniquet

- Needle assembly

- Bandage 


\subsection{Overview}

The coordinator tracks each SP through the MEC using the coordinator system. This system tracks the SP throughout the exam, including arrival, location during the session, and exit. The coordinator uses this system to direct the SP to the appropriate workstations in the MEC and to determine if all the appropriate examinations are complete. The MEC coordinator monitors exam component status using responses from examination stations.

It is critical to assign SPs aged 12 years and older to phlebotomy as soon as possible at the start of a session, especially a morning session. It is also important to assess the fasting status of SPs aged 12 years and older assigned to a morning session because they are not eligible for the OGTT examination if they have not met their 9-hour fast requirement. The coordinator gives each SP a hard-copy verification form as part of the check-in process. For SPs aged 12 years and older who are examined during a morning session, the form includes the question, "Did you eat or drink anything, other than plain water, after 11:30 last night?" The SP records "Yes" or "No" in a checkbox and the coordinator enters this information into the coordinator application. The coordinator application then highlights (hot pink) the names of SPs who responded "Yes" since they have not met the 9-hour fast requirement. The coordinator uses the highlighted names to aid in prioritizing SPs for the phlebotomy component; SPs who are not highlighted are assigned to phlebotomy before SPs whose names are highlighted.

Each SP receives a barcoded ID bracelet upon arrival at the MEC. The bracelet remains on the SP throughout the session. This bracelet contains the SP's ID number in barcode and eye-readable format. The phlebotomist "wands" the bracelet barcode with a barcode scanner (wand) to log the SP into the phlebotomy component. If necessary, the ID number can be entered manually by reading it from the bracelet and typing the numbers using the keyboard. 
The phlebotomist is responsible for completing the venipuncture section of the phlebotomy exam.

\section{- Phlebotomy seating options}

For the blood draw, the seating options are dependent on the weight of the SP.

The UMF 8678 phlebotomy chair (Figure 3-2) features an electric motor for up and down patient positioning, three positions - upright, recline, and full recline-and fully adjustable padded armrests that recline as the patient reclines. The chair includes both hand-operated and foot-operated controls to recline the chair with a touch of a button. To recline the chair, push the bottom button on the controller. The foot support will rise and the armrests will recline with the chair.

Figure 3-2. UMF 8678 phlebotomy chair

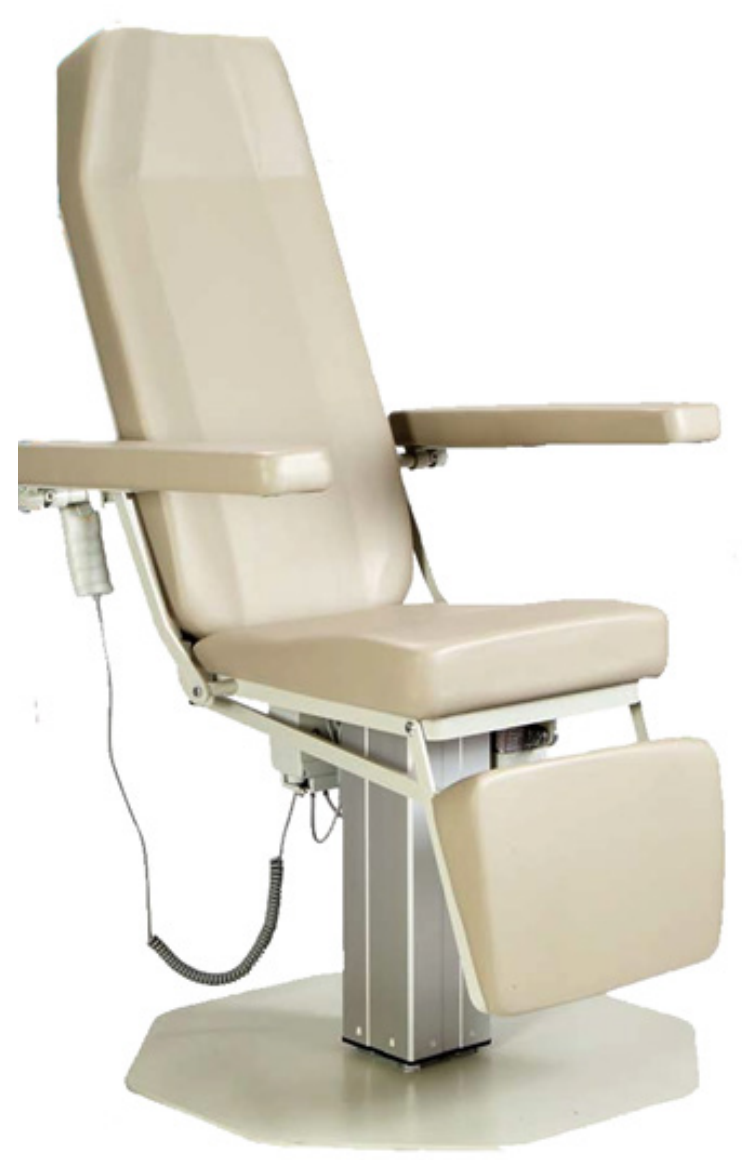


The chair weight restriction is $375 \mathrm{lbs}$. If the phlebotomist estimates that the weight of the SP is less than 375 pounds, and the SP will fit comfortably in the phlebotomy chair, then ask the SP to sit in the chair. Roll wheelchairs completely into the room. If the SP feels faint, then recline the chair either before or during the blood draw as needed.

\section{- Blood collection cart}

Set up the appropriate blood collection cart in advance, making sure there are sufficient supplies for all SPs in the session (see Figure 3-3).

Figure 3-3. Blood collection cart

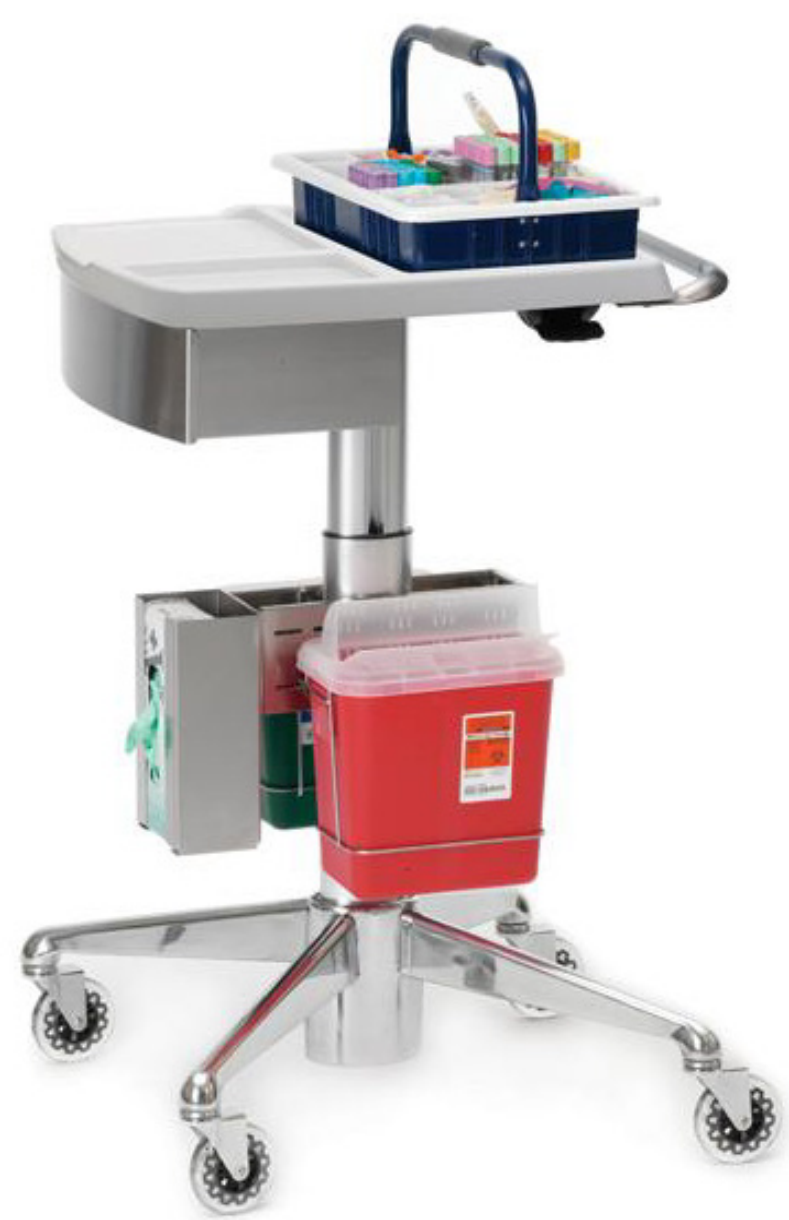

Adjust the height by lifting up on the black lever. Line an empty biohazard waste container with a biohazard bag and use it as a trash receptacle. Discard all Sharps in the 8-quart biohazard container. 
Access the phlebotomy application and open the phlebotomy exam. Log the SP into phlebotomy by scanning the barcode on the SP ID bracelet or manually typing the SP ID when the SP arrives in phlebotomy.

Conduct the phlebotomy interview and administer the fasting questionnaire. The phlebotomy protocol screen displays. Draw the appropriate tubes, and enter the number of tubes collected next to the expected values. Labels generate automatically. Enter the appropriate comment(s) for any tube that was not collected. Label the blood tubes. Verify that the phlebotomy completion status is correct. The phlebotomy section status is "Complete" if all blood tubes are collected, "Partial" if some but not all blood tubes are collected, and "Not Done" if no blood tubes are collected.

\subsection{Gaining Cooperation}

The coordinator will introduce the SP to the examination and briefly explain the examination process. The coordinator can answer any general questions the SP has about venipuncture. However, the phlebotomist must be prepared to answer all the questions the SP poses about the venipuncture procedure. In addition, the phlebotomist must convince the SP of the importance of cooperation in the venipuncture component of the examination.

Prepare to answer questions about the rationale for the venipuncture, the discomfort involved, the amount of blood being drawn, and the possibility of contracting an infectious disease from the process. To address SPs' concerns effectively, know the following information about the procedures used for the study:

\section{- Rationale}

Although the SP has provided much useful information in the household and individual interviews, the successful completion of the venipuncture component of NHANES is critical to the success of the study. Using the various specimens, researchers and laboratories are able to perform more than 250 different biochemical tests (SPs 12+), which provide detailed information about the SP's health and nutritional status. NHANES data produce descriptive statistics that measure and monitor the health of the U.S. population. Much of this information would not be available in any other way. The laboratories that conduct the analysis are considered the "gold standard" for their particular analyte(s). 


\section{- Discomfort}

Venipuncture causes only minimal discomfort. A certified, experienced, phlebotomist performs venipunctures. A variety of blood collection needles are available so that the most appropriate size can be selected for each SP.

\section{- Amount}

Phlebotomists draw the following amount of blood:

1-2 years primary SP, 9-mL ( 0.3 ounces $), 0.6$ tablespoons

3-5 years primary SP, $13-\mathrm{mL}$ ( 0.5 ounces $), 1.0$ tablespoons

6-11 years primary SP, 32-mL (1.0 ounces), 2.0 tablespoons

12-19 years primary SP, 117-mL (3.6 ounces), 7.2 tablespoons

20+ years primary SP, 145-mL (3.9 ounces), 7.8 tablespoons

1-2 years VIP guest, 6-mL (0.2 ounces), 0.4 tablespoons

3-5 years VIP guest, 6-mL (0.2 ounces), 0.4 tablespoons

6-11 years VIP guest, 10-mL (0.3 ounces), 0.7 tablespoons

$12+$ years VIP guest, $15-\mathrm{mL}$ (0.5 ounces), 1.0 tablespoons

$1+$ years guest and surplus, $3-\mathrm{mL}$ ( 0.1 ounces $), 0.2$ tablespoons

$12+$ years guest and surplus, $6-\mathrm{mL}$ ( 0.2 ounces $), 0.4$ tablespoons

An average adult male has 12 pints of blood and an average female has 9 pints. The Red Cross routinely draws $450-\mathrm{mL}$ (or one pint) during a routine donation. Their requirements limit donation to every 8 weeks. The Red Cross does allow autologous donations where an individual can donate his or her own blood before surgery. This consists of one unit per week for up to 6 consecutive weeks. The maximum volume drawn during a MEC exam amounts to slightly more than 25 percent $(128-\mathrm{mL})$ of the amount drawn from regular donors by the Red Cross. The body manufactures blood daily and replaces this volume of blood within 24 hours.

\section{- Infection control}

The supplies used for venipuncture are completely sterile and are used only once. There is absolutely no possibility of the SPs being infected by any blood-borne disease, such as hepatitis or AIDS, because of participation in the venipuncture component of the NHANES exam. 
Gaining the cooperation of an SP is easier if the atmosphere in the phlebotomy room is pleasant and makes the SP feel comfortable. Below is a list of suggestions for creating a pleasant atmosphere in the phlebotomy room.

- Maintain a clean and uncluttered work area. This is especially important because of today's concern with blood-borne infectious diseases, such as hepatitis and AIDS.

- Be aware of body image; a positive body image inspires confidence. Maintain a tidy appearance, erect posture, and a smile.

- Speak face to face with the subject and maintain eye contact. Staring at other areas in the room may cause the SP some uneasiness since it implies that he or she is not important. It also implies disinterest.

- Avoid nervous behaviors, such as squirming and tapping that can be distracting. The SP may begin to feel nervous, hurried, and anxious because of such behaviors.

\subsubsection{Refusal Conversion}

The phlebotomy component maintains the highest priority among MEC examinations. If an SP comes to the MEC and can complete only one exam, it should be the phlebotomy component.

The Field Office and Interviewing teams use "Special Consideration" codes to describe SPs with reservations about the phlebotomy procedure. The codes are part of the special consideration section of the MEC Appointment Schedule in the coordinator application. The phlebotomist prints the Session Preview Report each session and notes CB/NB codes under the Special Considerations column. The codes entered by the interviewers should be viewed as "warning codes" rather than strict edicts.

- When an SP expressed no reservations or concerns about phlebotomy during the home interview, no code will appear, and no special action is needed from MEC staff.

- $\quad \mathbf{C B}$ - represents "Convert Blood"-This means that the SP has expressed concern or fear about the blood draw, but did not indicate that he or she intends to refuse having blood drawn. If an SP is coded $\mathrm{CB}$, phlebotomy remains a priority exam.

- NB - represents "No Blood"-This means the SP indicated during the home interview that he or she intended to refuse the blood draw. When an SP has a NB code, the coordinator may send the SP to phlebotomy either (1) after all other SPs have completed phlebotomy or (2) after the SP has completed the exams in the profile. The coordinator should use the following script to simply explain that "We need to send you to the exam room; we can scan your ID band barcode into the system." 
The MEC manager and coordinator should work in collaboration with the phlebotomist in assigning the NB-coded SPs; the phlebotomist is trained in refusal conversion techniques and is responsible for the overall response rate for this component. At all times, the coordinator and MEC manager must use their judgment in assigning SPs with NB to phlebotomy. If the SP is vehemently adamant about not giving blood, there is no reason to provoke the SP by sending him or her to the phlebotomy room. In this instance, the coordinator will mark the phlebotomy component as a refusal.

\subsection{Performing the Venipuncture on SPs Who Do Not Speak English}

When the phlebotomist must administer the venipuncture procedure to an SP who does not speak English and the phlebotomist does not speak the language of the SP, a translator who does speak the language of the SP assists the phlebotomist.

The translator stays with the phlebotomist and the SP for the entire procedure. It is very important that the phlebotomist be able to communicate with the SP if the SP becomes ill during the venipuncture. Refer to the MEC Operations manual for detailed instructions on the interpreter module. 
Open and log onto the Phlebotomy application.

The message center window identifies the SP assigned to phlebotomy.

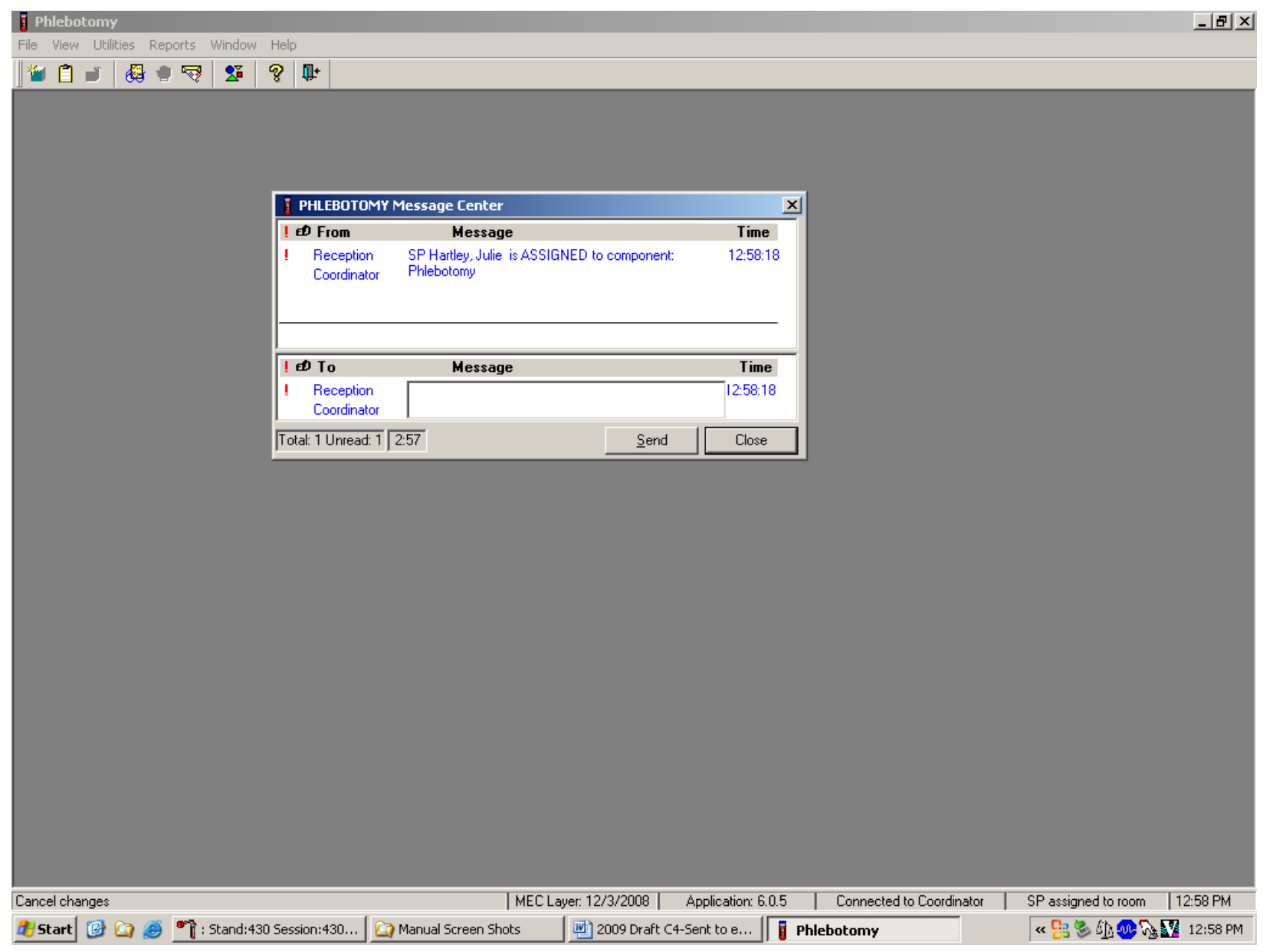

A Message Center message text box displays containing a message from the coordinator indicating the name of the SP who is assigned to the phlebotomy component. Enter an optional text message and, to send the message to the coordinator, use the mouse to direct the mouse arrow to the "Send" button and left click. To exit without sending a message to the coordinator, use the mouse to direct the mouse arrow to the "Close" button and left click, or select [Enter]. 
Open the Phlebotomy exam.

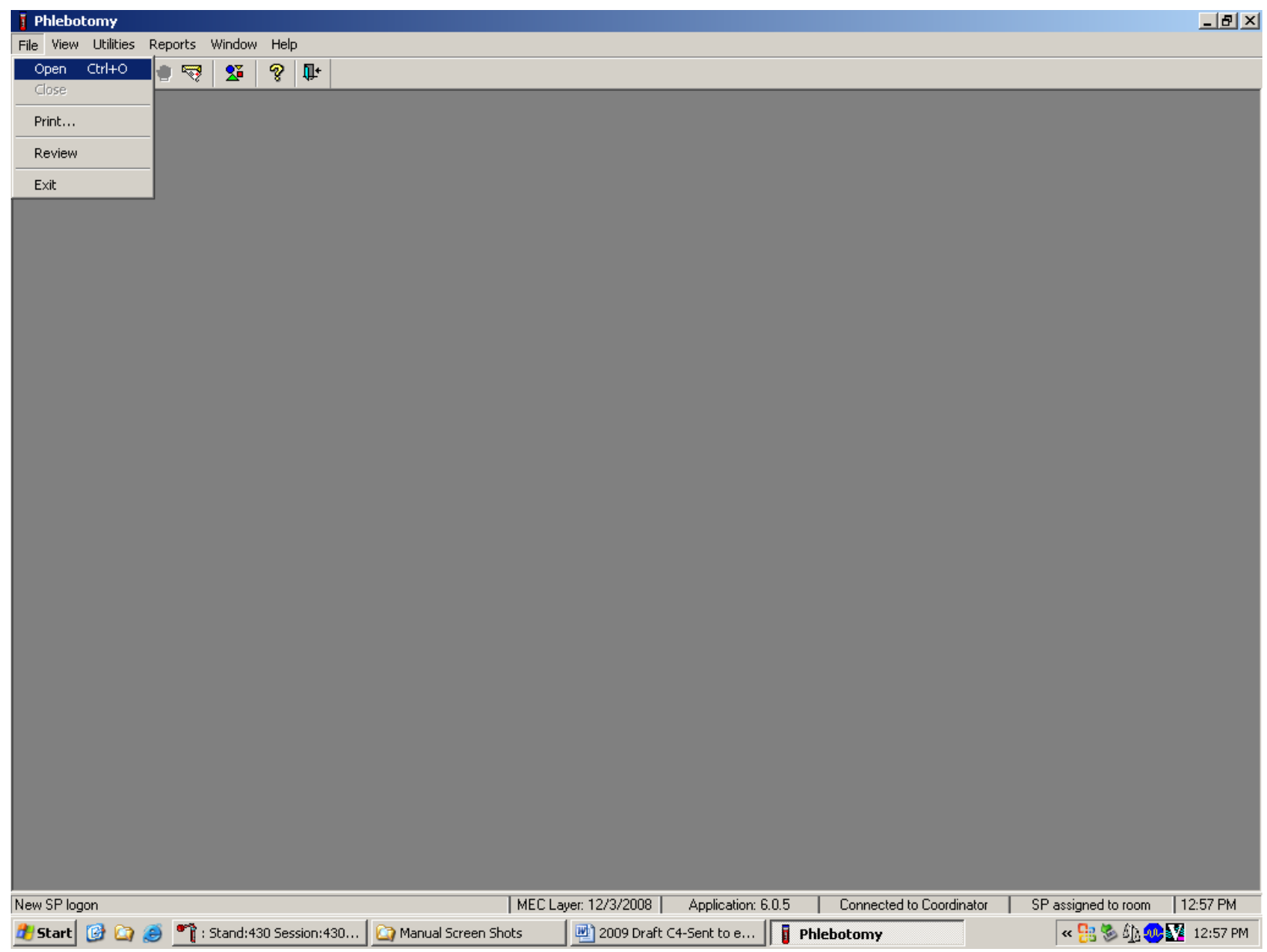

To open an exam, move the mouse to $\{$ File $\}$ in the menu bar, click on $\{$ Open $\}$, or type [Alt] $[\mathrm{F} / \mathrm{f}],[\mathrm{O} / \mathrm{o}]$, or $[\mathrm{Ctrl}][\mathrm{O} / \mathrm{o}]$. 
The SP Logon window displays.

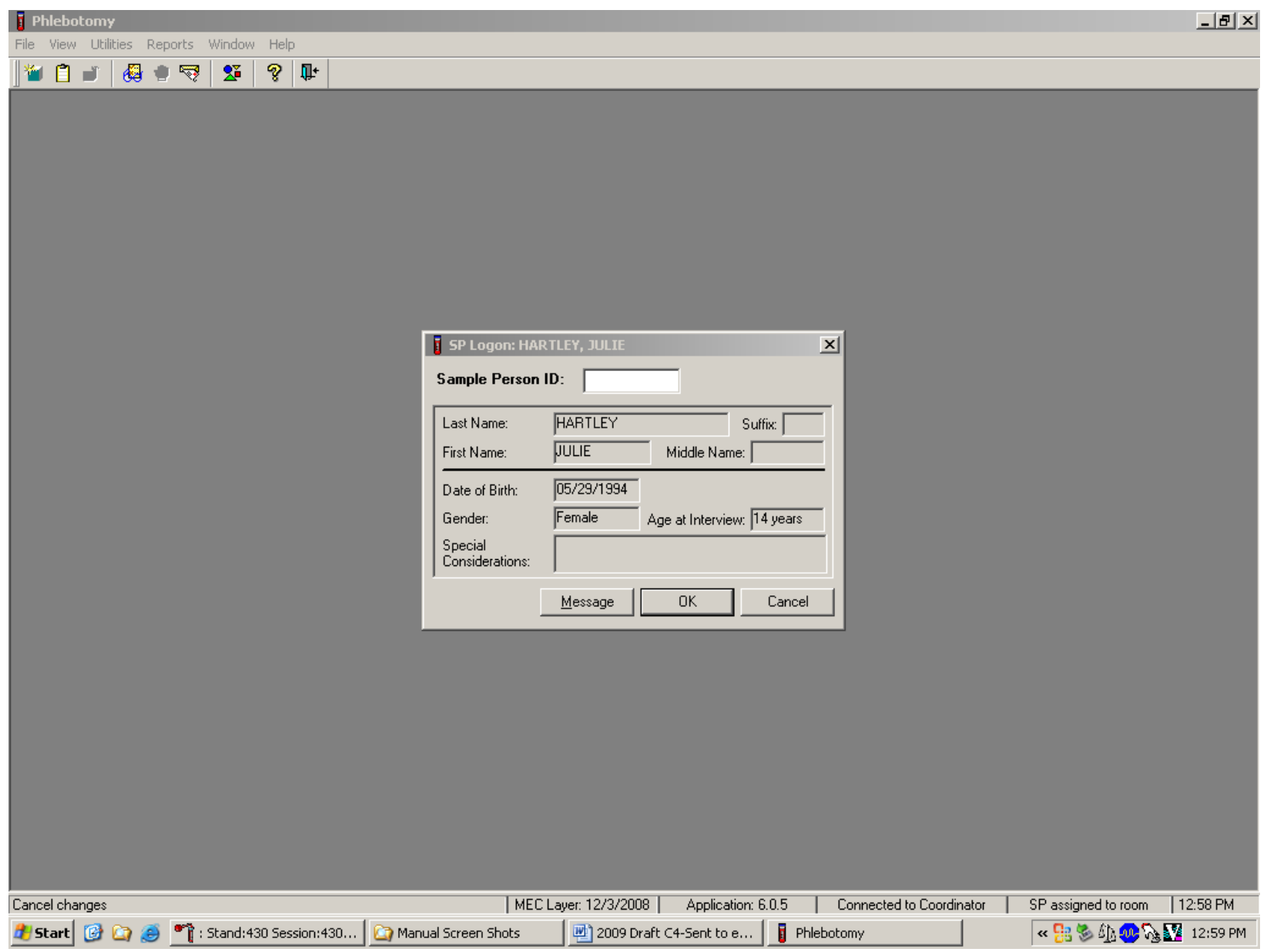

The SP Logon window displays for the SP assigned to the component. 
Log the SP into the component.

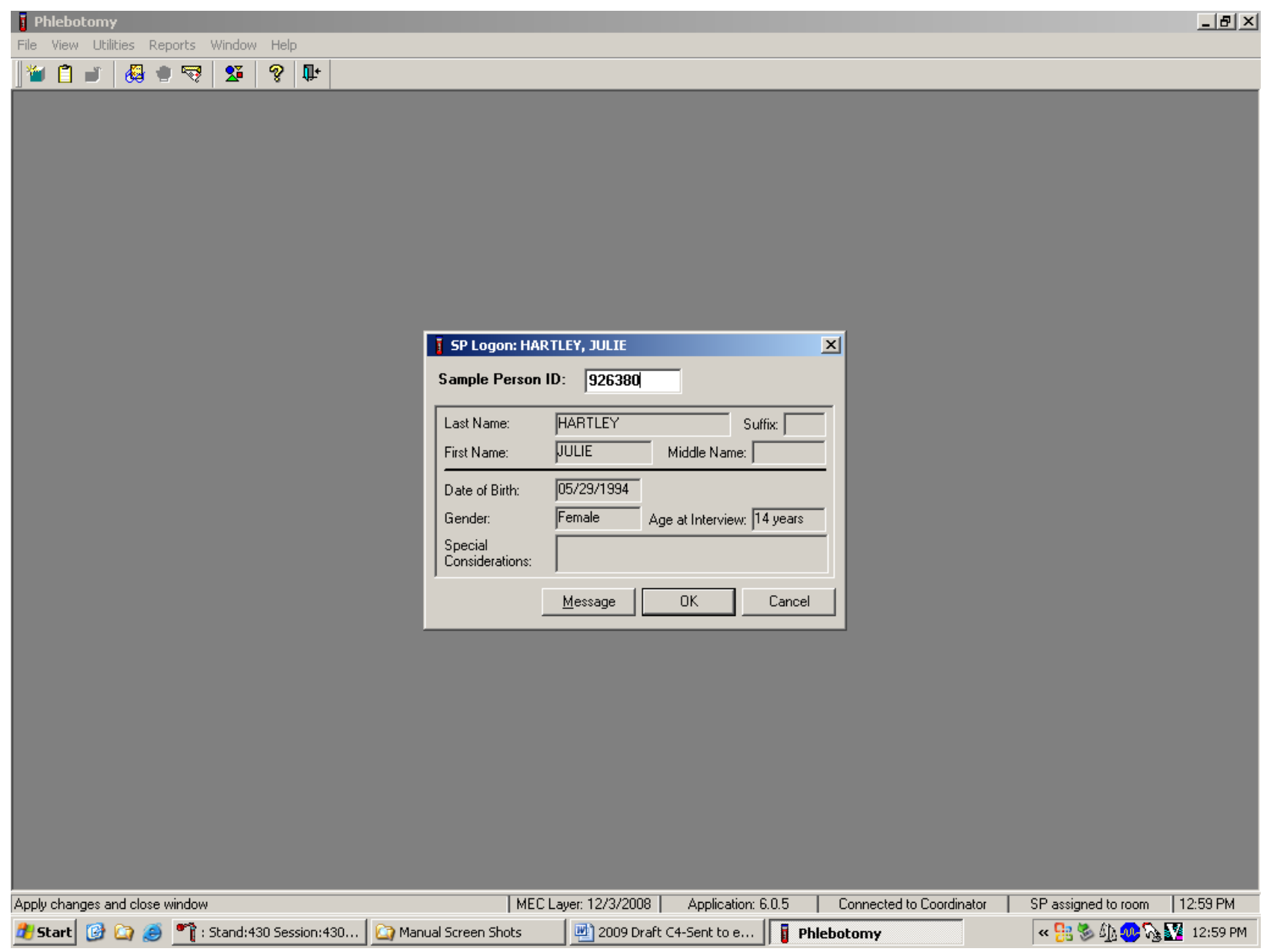

To log the SP into the component, either read the SP ID from the SP's bracelet and manually type this number into the Sample Person ID text box or use the barcode wand to scan the bracelet barcode. To continue, select [Enter] or use the mouse to direct the mouse arrow to the "OK" button and left click. To cancel the Logon process and to remove the window, use the mouse to direct the mouse arrow to the "Cancel" button and left click.

Verify all information that appears in the SP Logon window. If there is an error in any of this information, inform the coordinator immediately. The coordinator will verify and correct the information as necessary. 
The Phlebotomy Interview screen includes a shared exclusion pregnancy question for females aged 16-59 years and two safety exclusion questions. There are only two reasons to exclude an SP from venipuncture-hemophilia, and having received chemotherapy within the past 4 weeks. Administer the interview (and fasting questionnaire) directly to SPs over the age of 16, or administer the interview questions (and fasting questionnaire) to the SP's parent or guardian for SPs under age 16.

Administer the phlebotomy interview immediately before performing the venipuncture. Read the text exactly and record the responses.

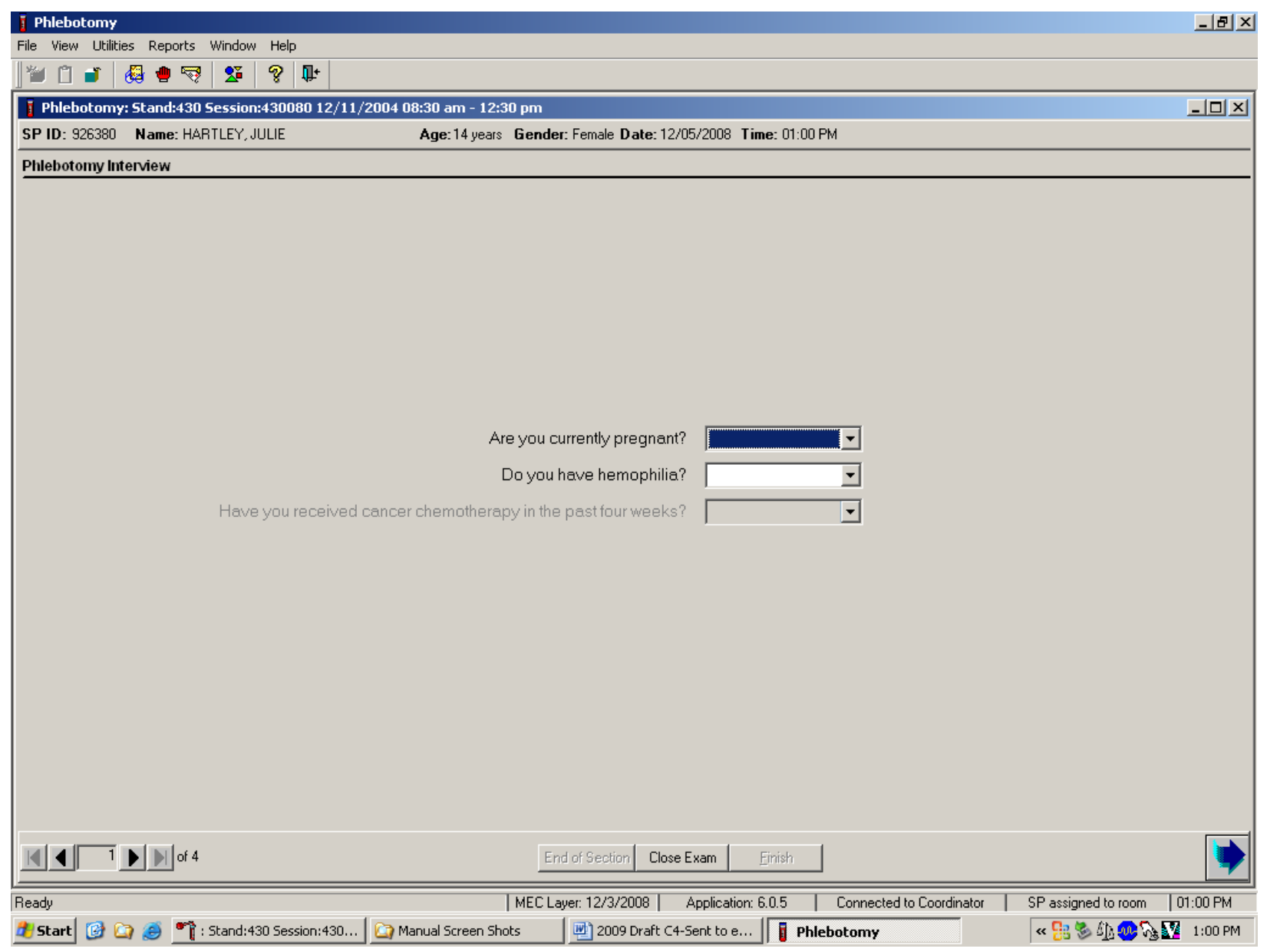




\subsubsection{Pregnancy Shared Exclusion Question}

For female SPs aged 12-59 years, the shared exclusion pregnancy questions are enabled and are asked during the MEC examination in the first examination where shared exclusions are asked. Each question is asked only once, even when the question is relevant for more than one component. Some examinations, like phlebotomy, do not require all the shared exclusions to be asked. In this situation, there may be some questions answered in one examination and then disabled in the remaining examinations. Shared exclusion questions are component specific. Answers provided during previous components appear on the screen for the next examiner to see, but the text box for data entry is disabled. Only shared exclusion questions relevant to the age and component that have not been answered are enabled. Example: If the SP goes to the physician component before phlebotomy, the questions about "pregnancy" and "how many weeks" would be asked by the physician. The "pregnancy" question would be disabled in the phlebotomy component.

- If the SP is a male, a female less than 12 years, or a female older than 60 years, the shared exclusion pregnancy questions will not be displayed.

- If the SP is a female aged 12-59 years, the first shared exclusion pregnancy question is displayed and enabled. 
Record the response to the shared exclusion question.

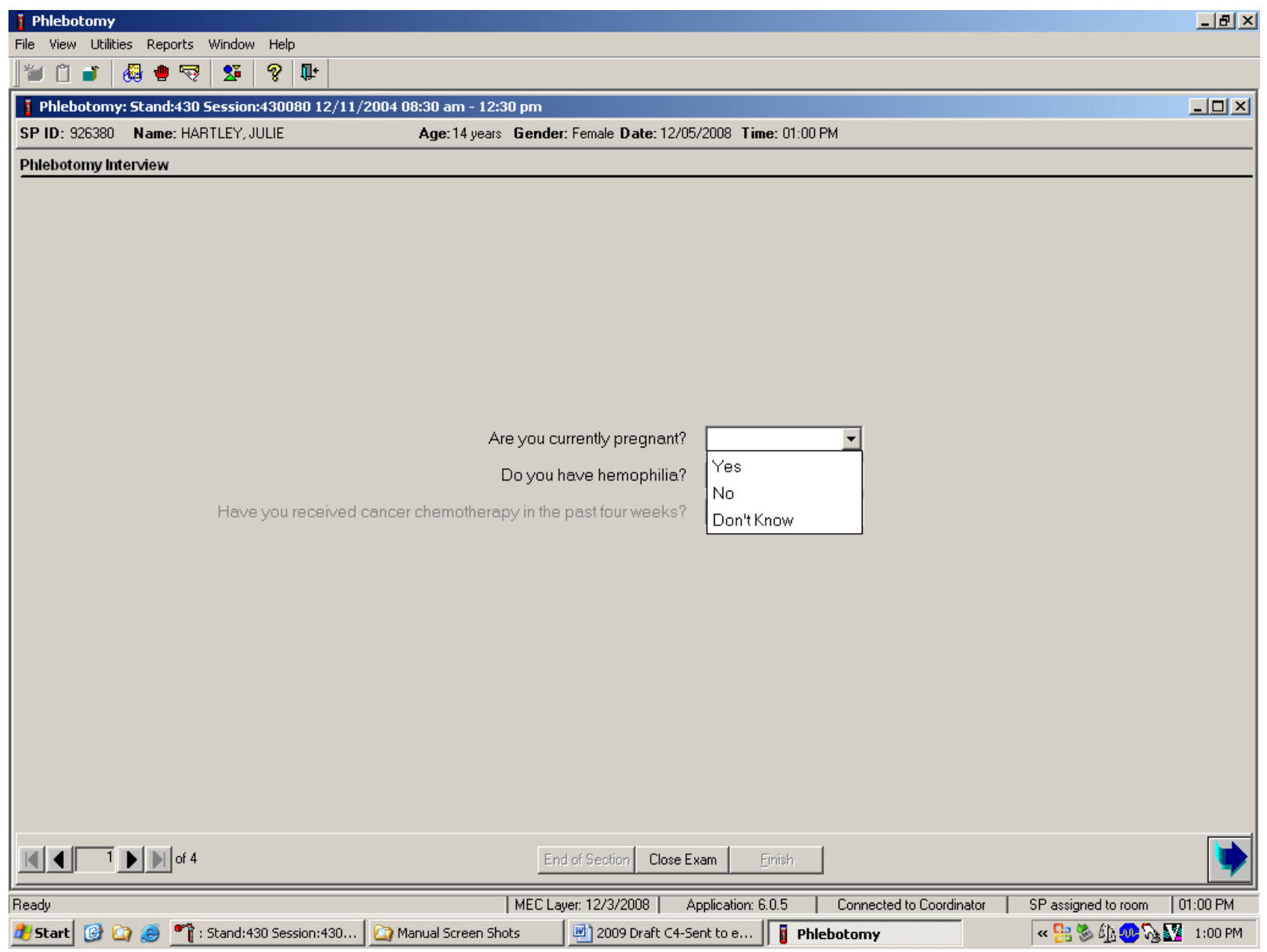

Record the response by typing [Y/y] for "Yes," [N/n] for "No," or [D/d] for "Don't know." Alternatively, move the mouse to the drop-down arrow on the drop-down list, and drag the mouse arrow to "Yes," "No," or "Don't Know" and left click. Select [TAB] to move to the next text box. 
If the response to the question "Are you currently pregnant?" is "Yes," the SP is excluded from GTT due to pregnancy status. At the coordinator application, the component status for GT defaults to "Not Done" with the comment "Blocked: Not Done [SP pregnant]."

If the response to the question "Are you currently pregnant?" is "No" or "Don't Know," the SP is not excluded from the GTT. Ask the next two phlebotomy safety exclusion questions.

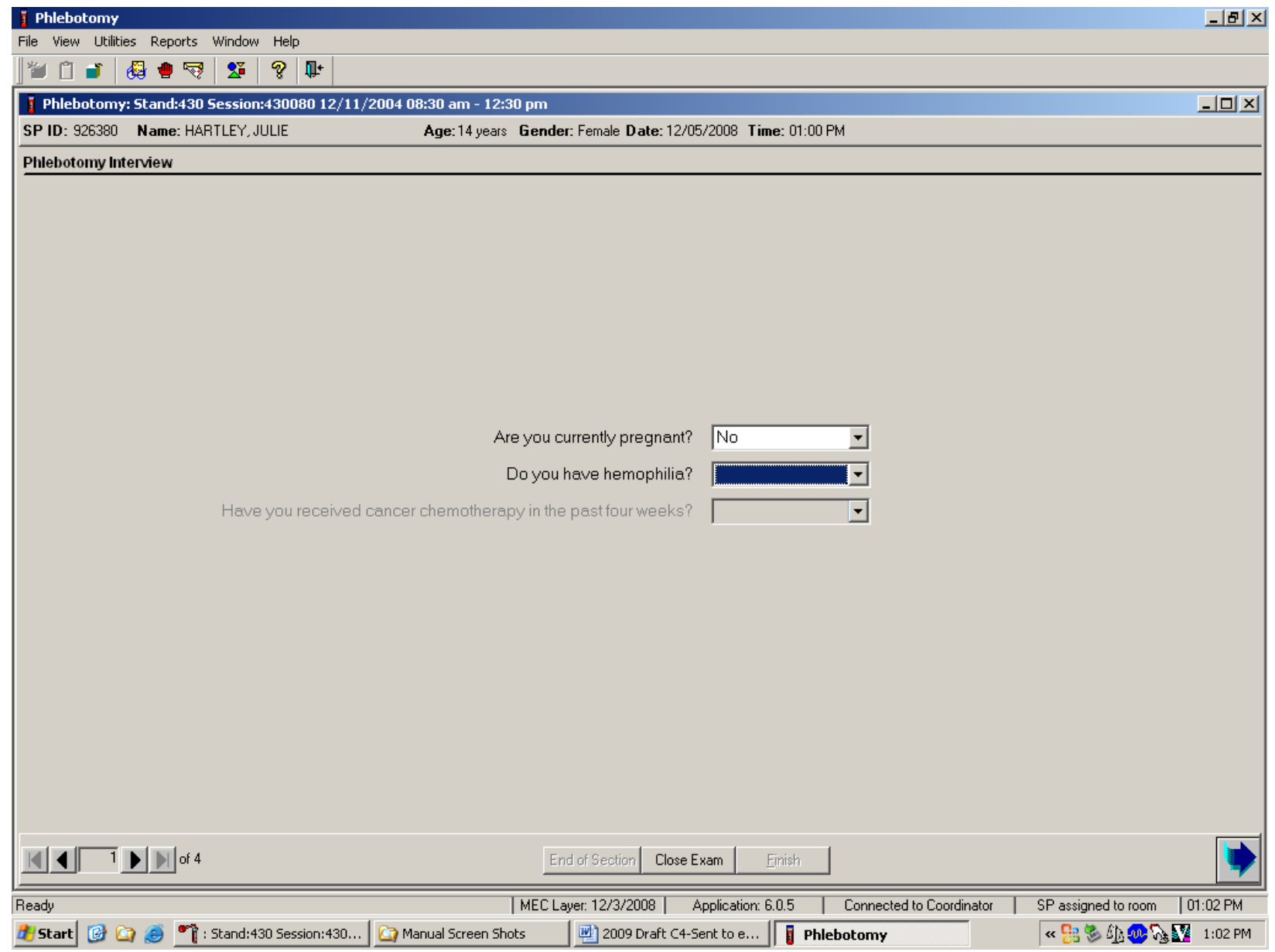

If the answer to the shared exclusion pregnancy question is "No" or "Don't know," then select [TAB] to proceed to the first safety exclusion question, "Do you have hemophilia?" 


\subsubsection{Safety Exclusion Questions}

Continue to conduct the phlebotomy interview by asking the question displayed on the screen, "Do you have hemophilia?"

\section{Q1: Do you have hemophilia?}

Q1: This question asks whether the SP has hemophilia, which is an exclusion criterion for this procedure.
Explain that we cannot perform phlebotomy on participants who have hemophilia. Hemophilia is a rare disease where an individual's blood does not clot. If an SP's relative has hemophilia but the SP does not, the SP is not excluded. If the SP is excluded, the Blood Draw Exclusion box displays. Read the text in the box to the SP and escort the SP back to the coordinator station. 
Record the exact response.

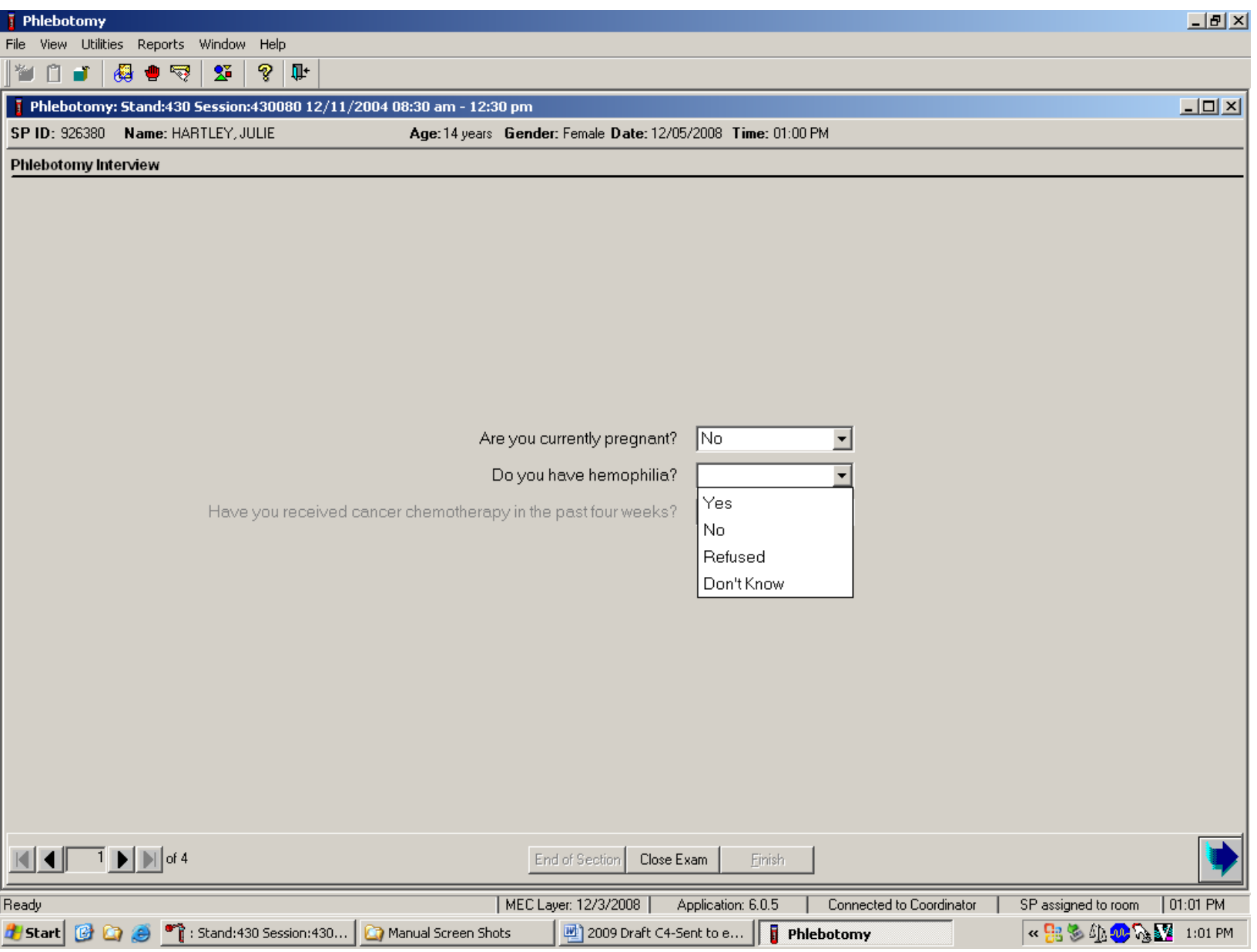

Record the response by typing [Y/y] for "Yes," [N/n] for "No," [R/r] if he or she refuses, or [D/d] for "Don't know." Alternatively, move the mouse to the drop-down arrow on the drop-down list, click to display the responses, and drag the mouse arrow to "Yes," "No," "Refused," or "Don't Know" and left click. If the response is "Yes," "Refused," or "Don't Know," use the mouse to direct the mouse arrow to the bright blue right arrow in the bottom right corner of the screen and left click or select [Enter]. 
If the SP is excluded from the phlebotomy exam due to hemophilia, the Blood Draw Exclusion informational message text box displays.

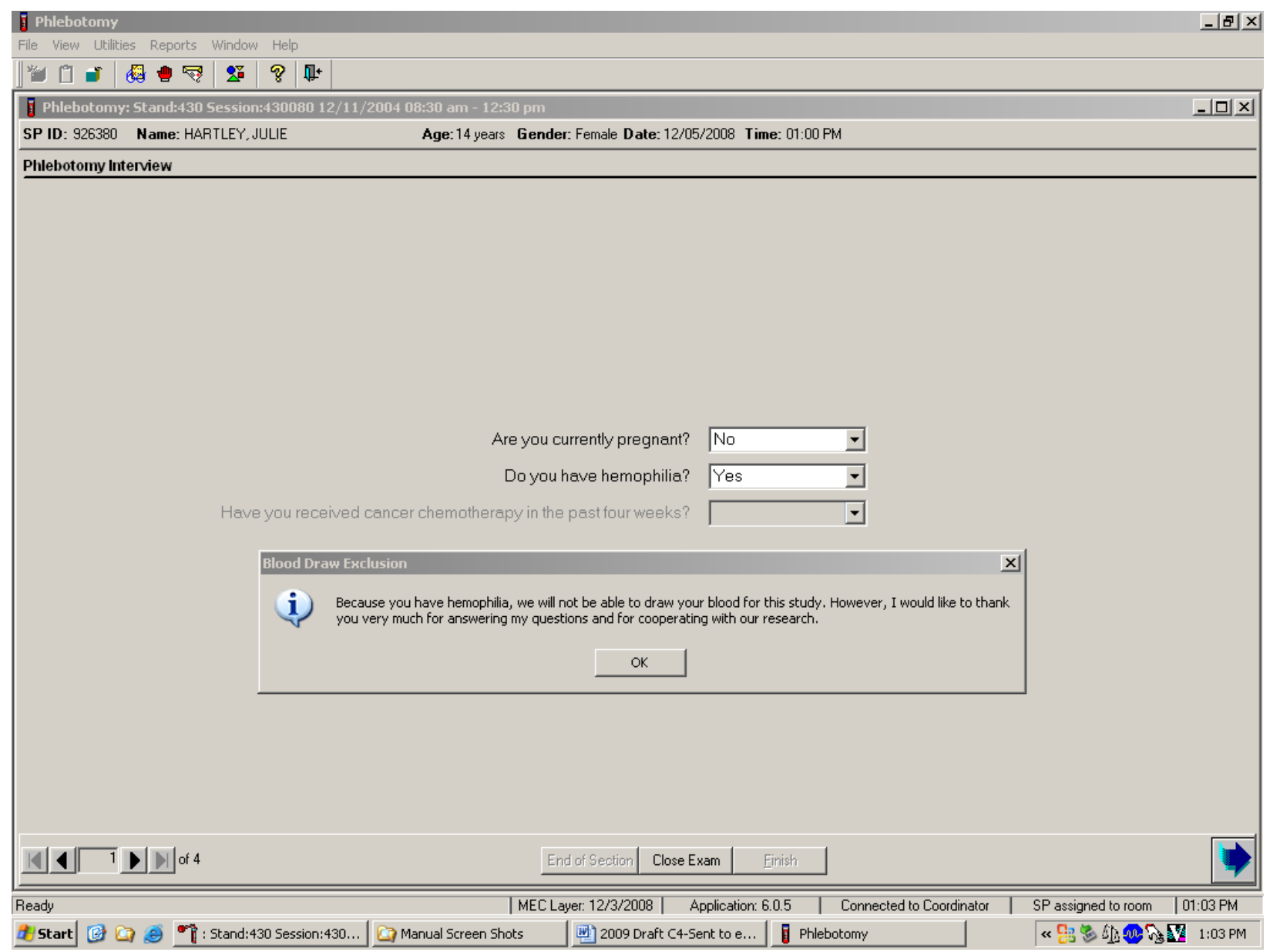

Read the script to the SP. To remove the Blood Draw Exclusion message text box, move the mouse arrow to the "OK" button and left click, or select [Enter]. 
If the SP is excluded from the phlebotomy component due to hemophilia, an informational Exclusion message text box displays. In some cases more than one Exclusion message box will display, one after the other.

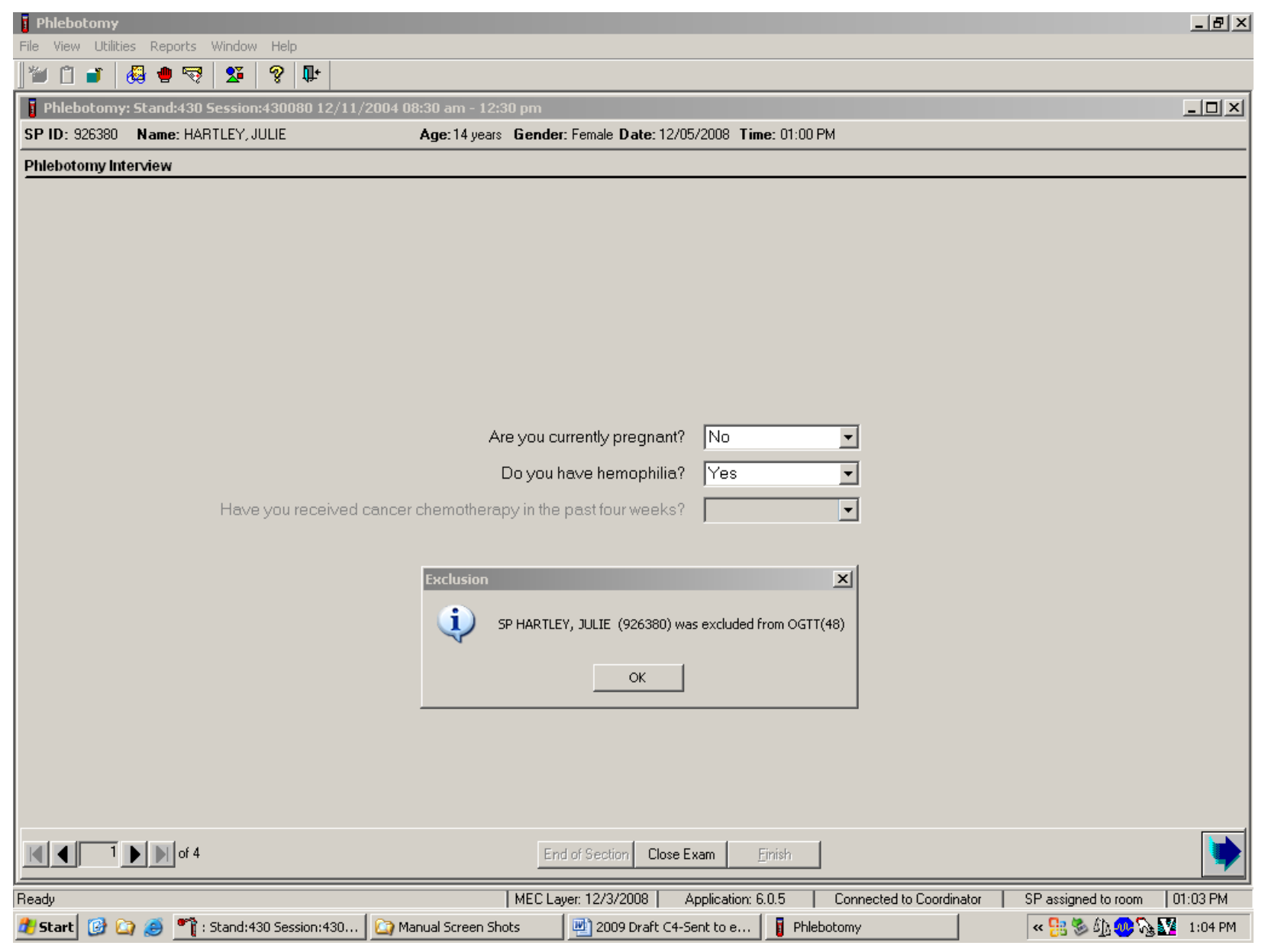

To remove the Exclusion message text box, move the mouse to the "OK" button and left click, or select [Enter]. 
Verify the status.

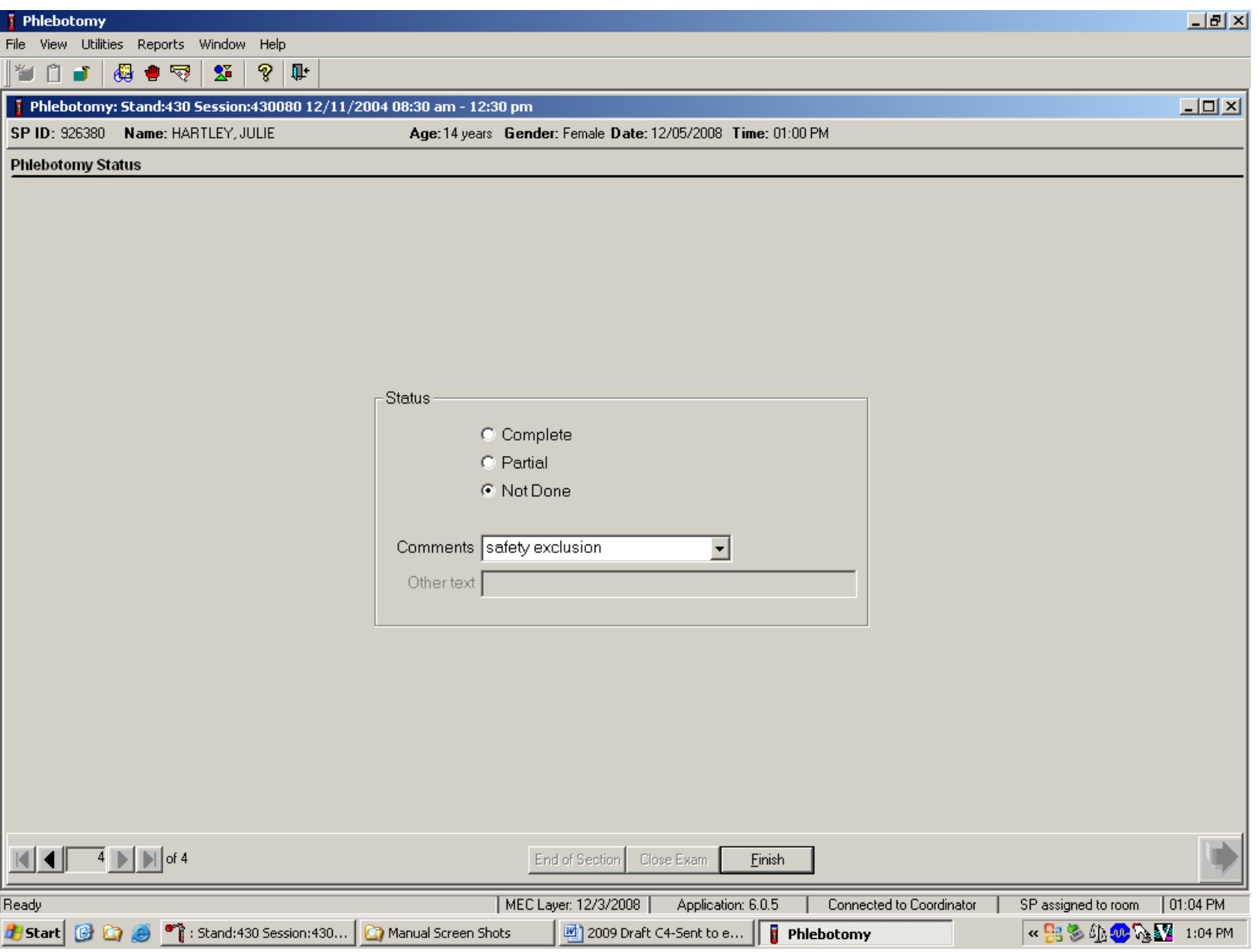

SPs excluded because of hemophilia are automatically coded by the application as "safety exclusion." Escort the SP back to the coordinator or to the next component as directed by the Message Center. 
If the SP has not been excluded because of hemophilia, continue the phlebotomy interview by asking the second question displayed on the screen, "Have you received cancer chemotherapy in the past 4 weeks?"

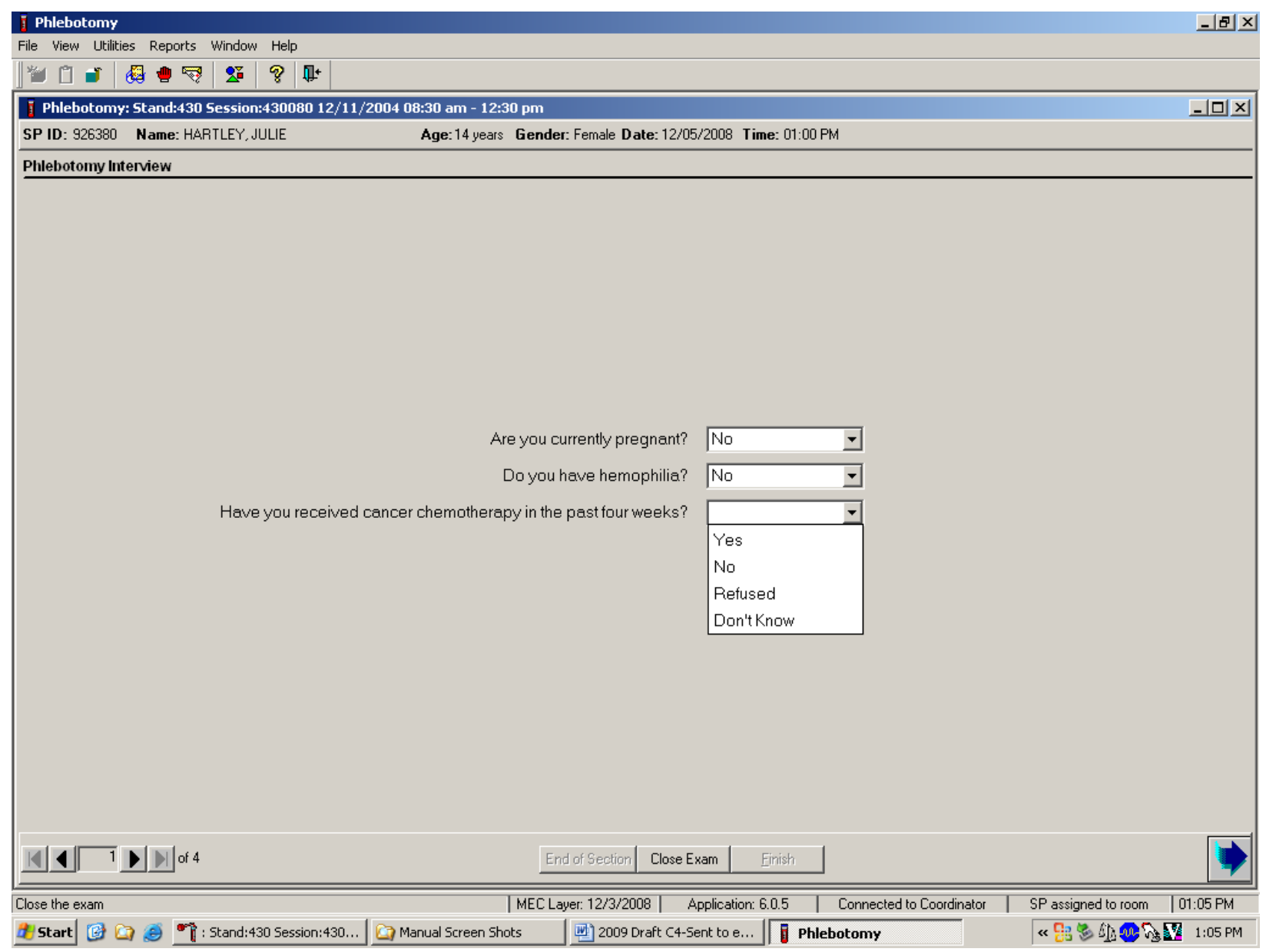

\section{Q2: Have you received cancer chemotherapy in the past 4 weeks?}

Q2: This question asks whether the SP has received chemotherapy in the past 4 weeks. This situation excludes the SP from this procedure.
Explain that we cannot perform phlebotomy on participants who have received cancer chemotherapy within the past 4 weeks. If the SP is excluded, the Blood Draw Exclusion box is displayed. Read the text in the box to the SP and escort him or her back to the coordinator station. 
Record the response by typing $[\mathrm{Y} / \mathrm{y}]$ for "Yes," [N/n] for "No," $[\mathrm{R} / \mathrm{r}]$ if he or she refuses, or [D/d] for "Don't know." Alternatively, move the mouse to the drop-down arrow on the drop-down list, click to display the responses, and drag the mouse arrow to "Yes," "No," "Refused," or "Don't Know" and left click. If the response is "Yes," "Refused," or "Don't Know," use the mouse to direct the mouse arrow to the bright blue right arrow in the bottom right corner of the screen and left click.

If the SP is excluded from the phlebotomy exam because of cancer chemotherapy, the Blood Draw Exclusion informational message text box displays.

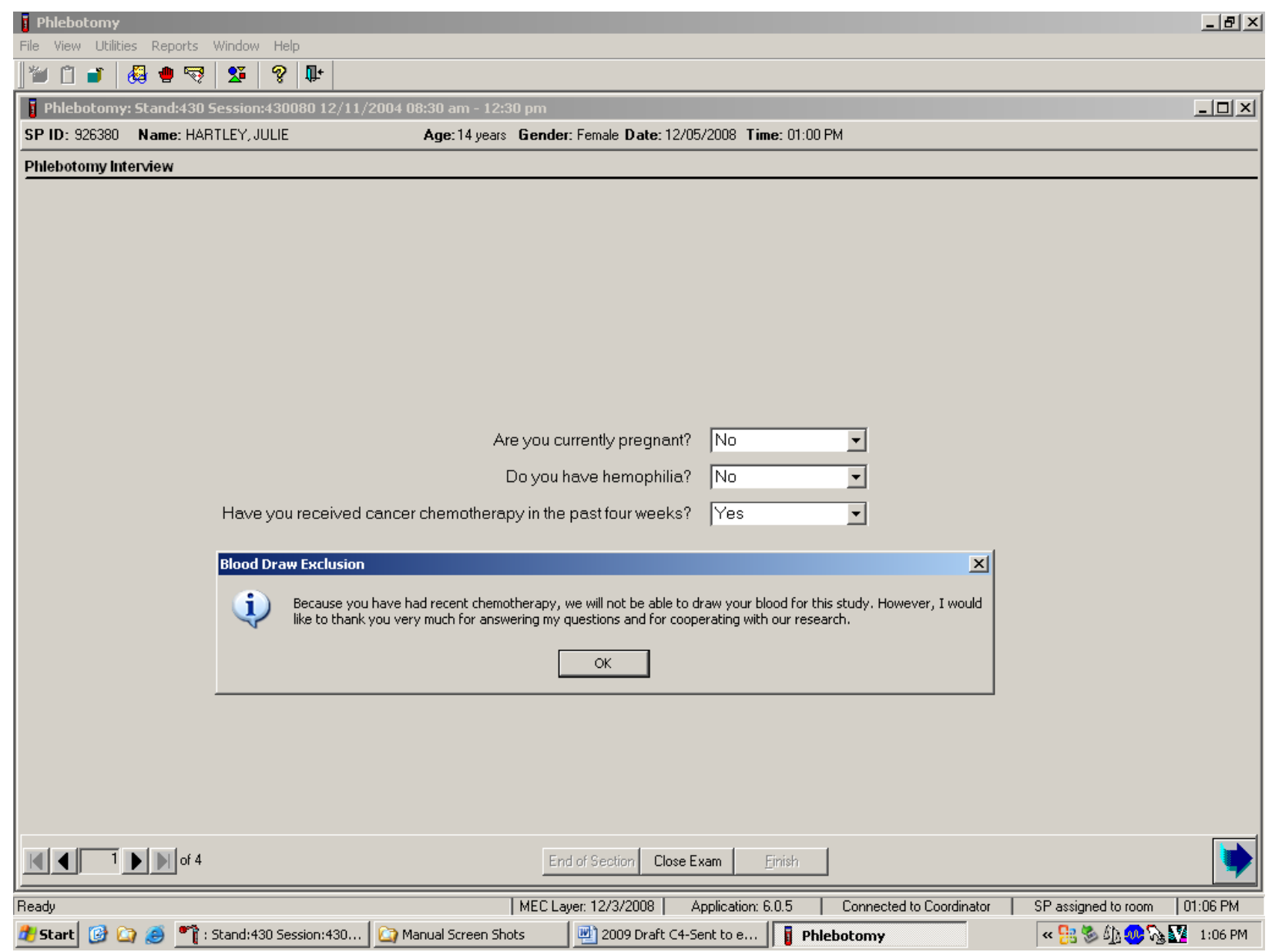

Read the script to the SP. To remove the Blood Draw Exclusion message box, use the mouse to direct the mouse arrow to the "OK" button and left click. 
If the SP is excluded from the phlebotomy component because of chemotherapy, an informational Exclusion message text box displays. In some cases more than one Exclusion message box will display, one after the other.

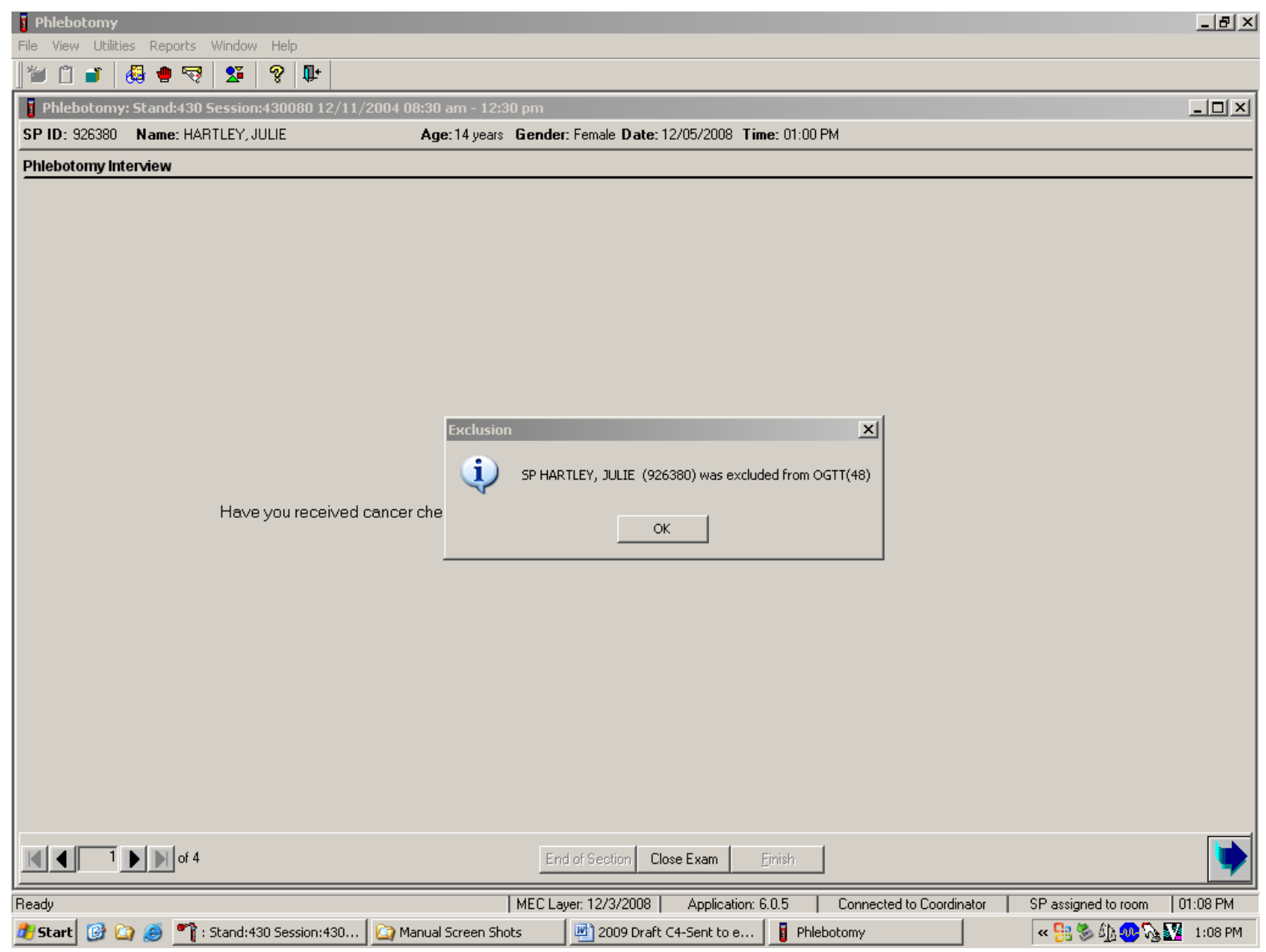

To remove the Exclusion message text box, move the mouse to the "OK" button and click, or select [Enter]. To progress to the component status screen, use the mouse to direct the mouse arrow to the bright blue arrow in the bottom right corner and left click, or select [Enter]. 
Review the Status.

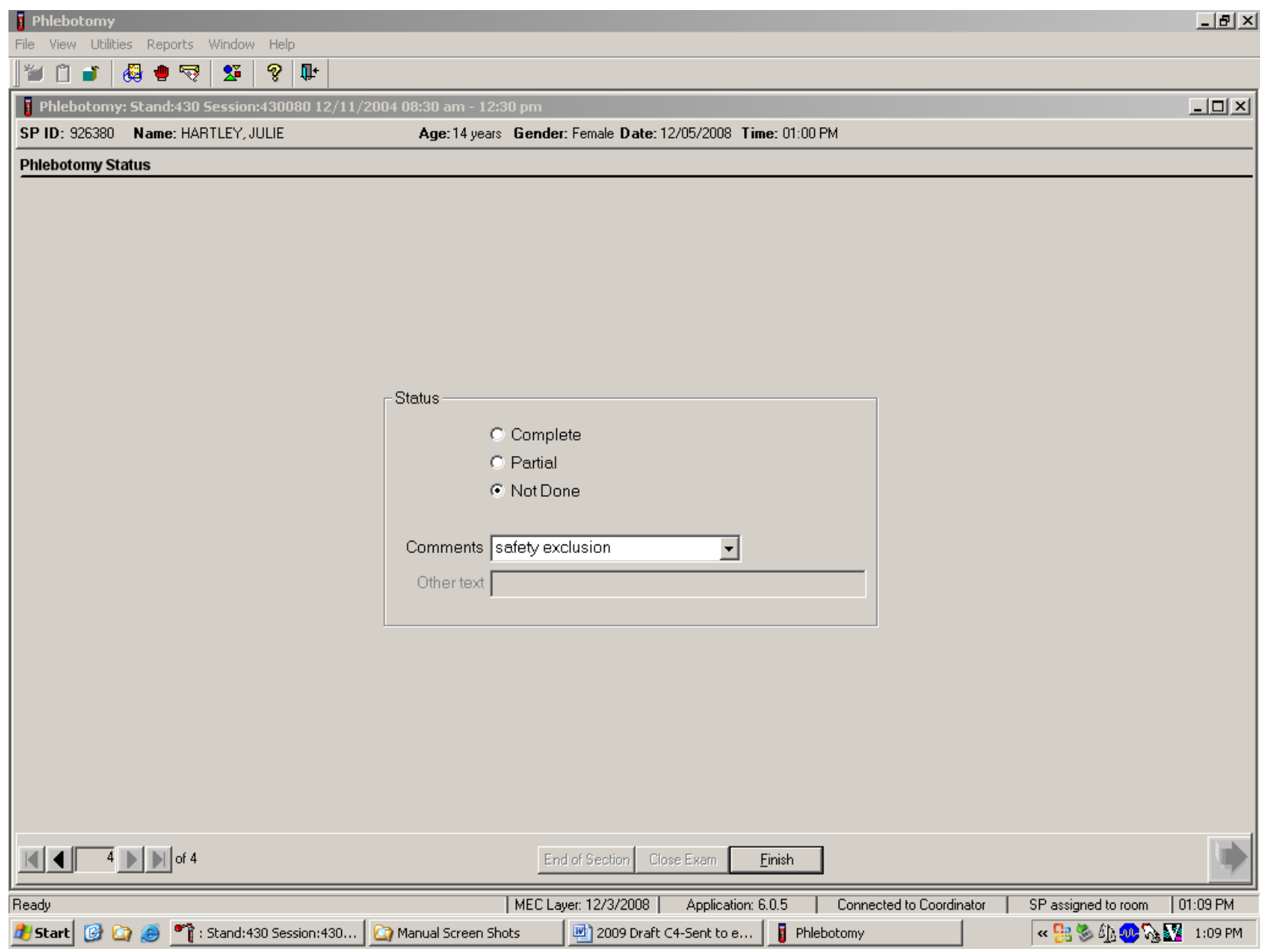

SPs excluded because of chemotherapy are automatically coded by the application as "safety exclusion." Escort the SP back to the coordinator or to the next component as directed by the Message Center. 
If the response to both exclusion questions is "No," continue to the next screen.

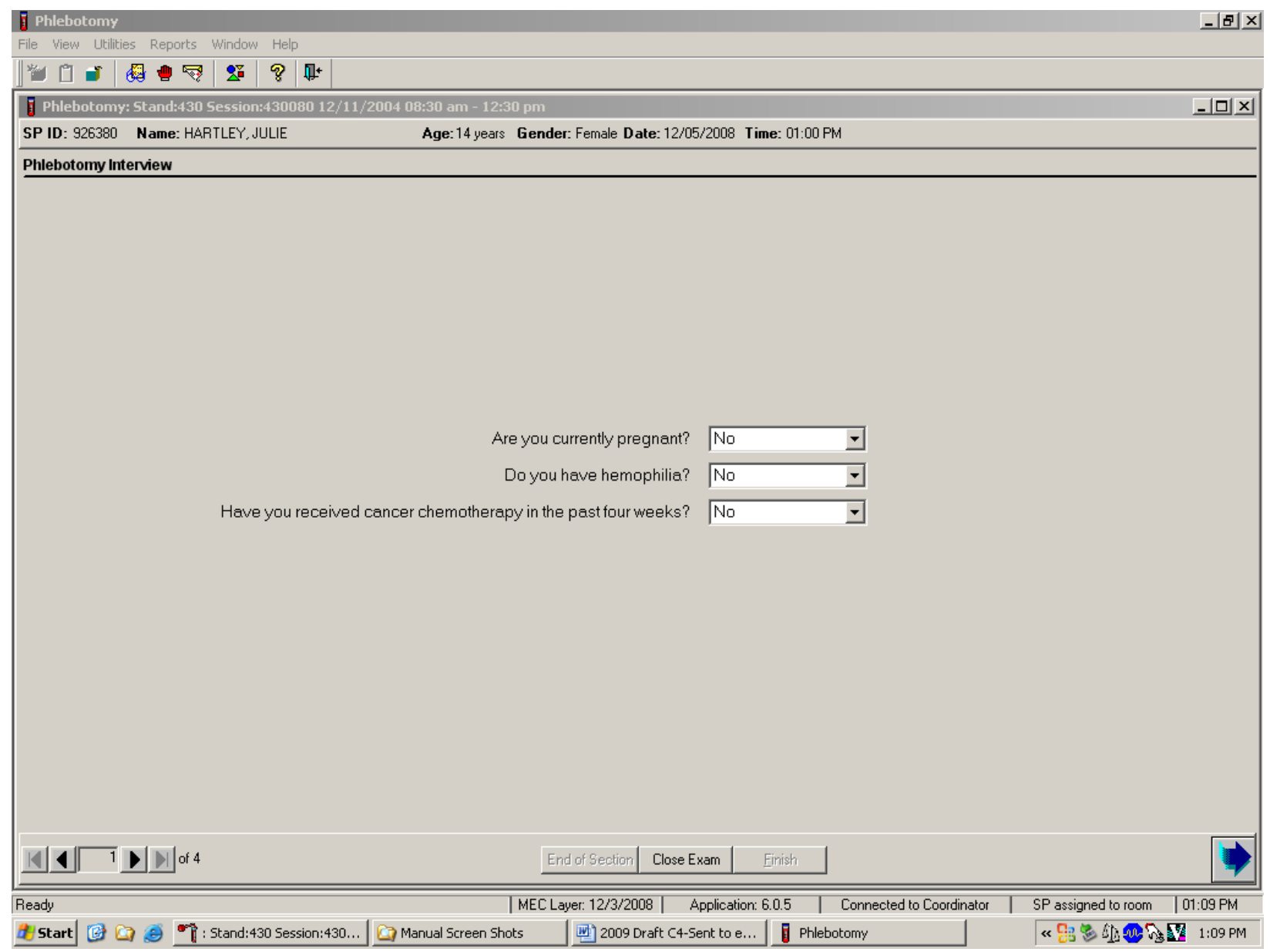

To progress to the fasting questionnaire screen, use the mouse to direct the mouse arrow to the bright blue arrow in the bottom right corner and left click, or select [Enter]. 


\subsection{Administering the Fasting Questionnaire}

Fasting status is critical to many of the laboratory analytes, and fasting data are required for correct interpretation of laboratory results. Fasting is especially critical because only SPs who have met the fast are eligible for the OGTT component. No primary SPs aged 1-11 or SPs assigned to afternoon or evening sessions are asked to fast. All other primary SPs aged 12 years and older appointed to a morning session are asked to fast for 9 hours. This includes diabetics taking insulin, although these SPs are always given the highest priority so the venipuncture can be completed as soon as possible after the SP checks into the MEC.

The phlebotomist administers the fasting questionnaire to all SPs, and the application calculates and displays the fasting time in number of hours and minutes. The phlebotomist then performs the venipuncture.

The overall objective is to perform phlebotomy on all eligible SPs. The coordinator must base the decision to assign an SP to phlebotomy on the greater goal of completing as many components as possible within the time constraints of the session, with phlebotomy as the highest priority component. Sometimes an SP insists on leaving the MEC before he or she has been assigned to phlebotomy. It is always preferable to get blood from this SP before he or she leaves the MEC. The coordinator and MEC manager can provide the best overall assessment of the status of a particular SP.

Guests and VIP guests do not have a fasting requirement, even during morning sessions.

SPs may opt to return to the MEC to complete exams that were missed or to complete exams that were begun but not finished. These SPs are designated as "Partial" SPs. When the SP returns to the phlebotomy component, ask and record the fasting questions a second time so that the data are accurate. Delete any data in the text boxes (previous responses from the initial visit) and enter the new responses. 
Read the text exactly and record the time.

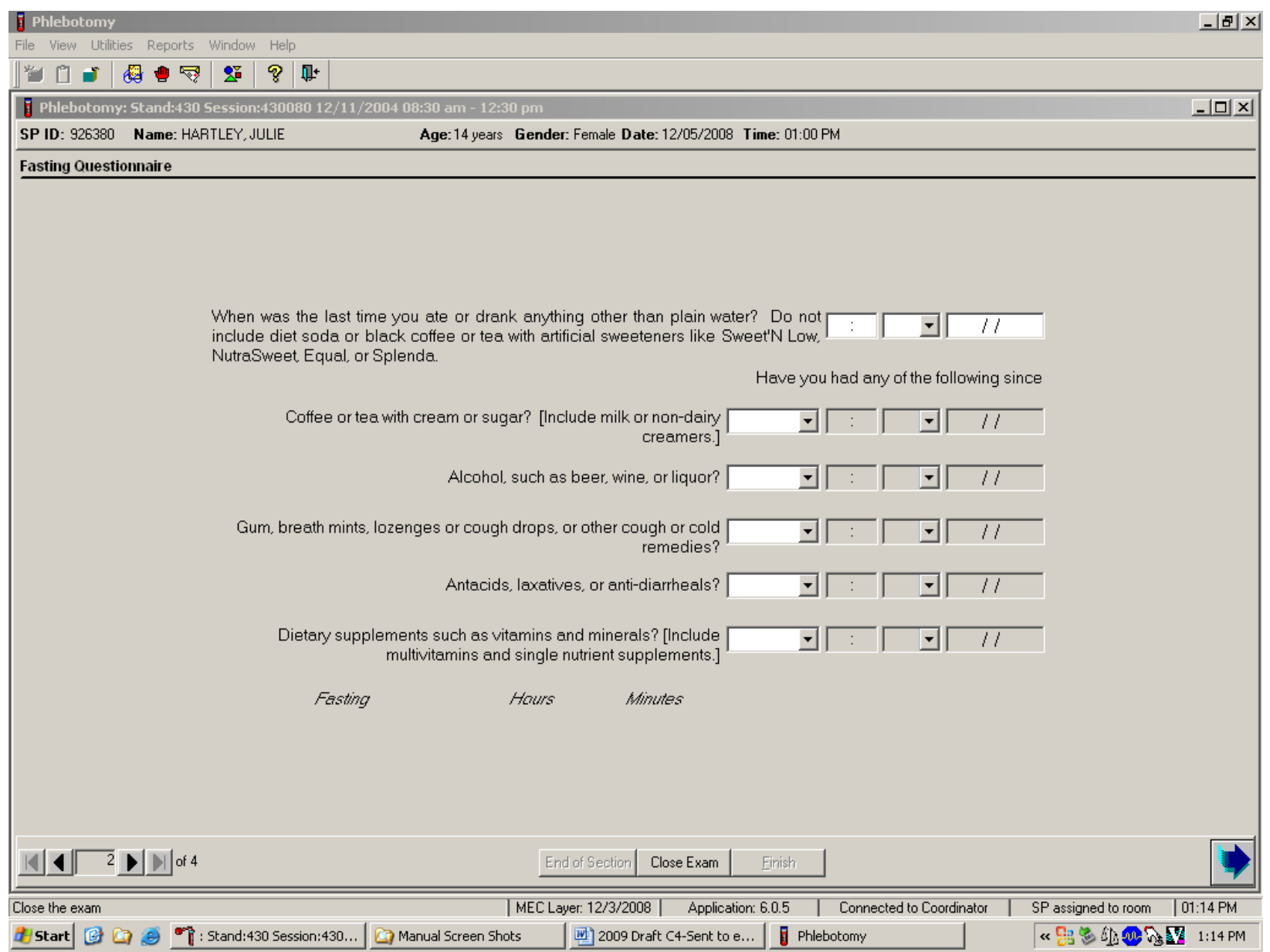

Q1: When was the last time you ate or drank anything other than plain water? Do not include diet soda or black coffee or tea with artificial sweeteners like Sweet' $N$ Low, NutraSweet, Equal, or Splenda.

Q1: This question elicits the last time the SP ate or drank anything and determines fasting time.

SPs are allowed to consume diet soda, black coffee, or tea with artificial sweeteners like Sweet'N Low, NutraSweet, Equal, or Splenda since these have no effect on study analytes. Do not include flavored waters. 
Enter the response.

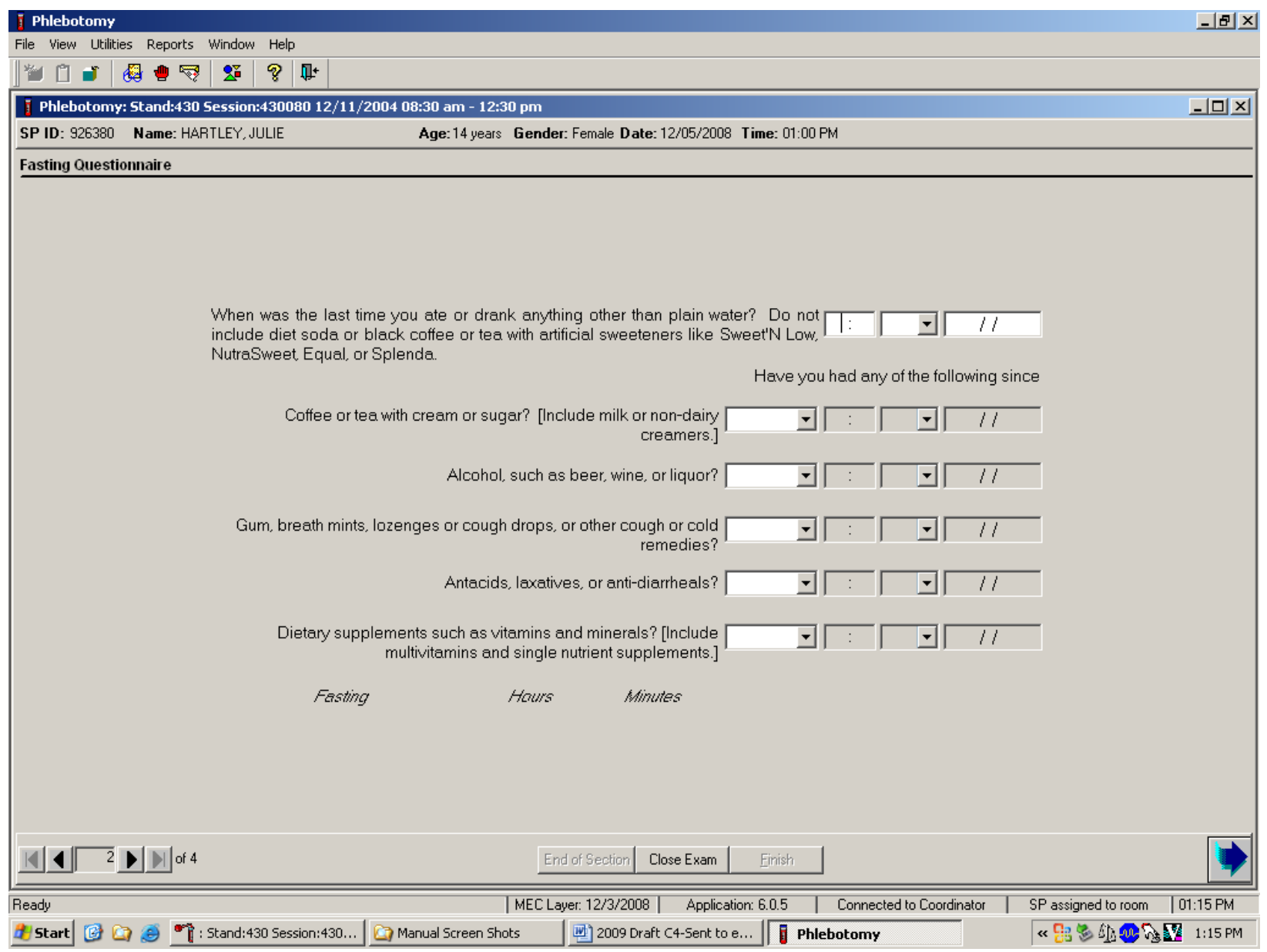

Type in the reported time using the numeric keys and select [Tab] to move to the AM/PM space. Type in $[\mathrm{A} / \mathrm{a}]$ for times between midnight and 11:59 $\mathrm{AM}$ or $[\mathrm{P} / \mathrm{p}]$ for times between 12:00 noon and 11:59 PM and select [Tab]. Alternatively, to select AM or PM, use the mouse to direct the mouse arrow to the drop-down arrow on the right side of the text box, left click, drag the mouse arrow to "AM" or "PM," and left click. 
Enter the date.

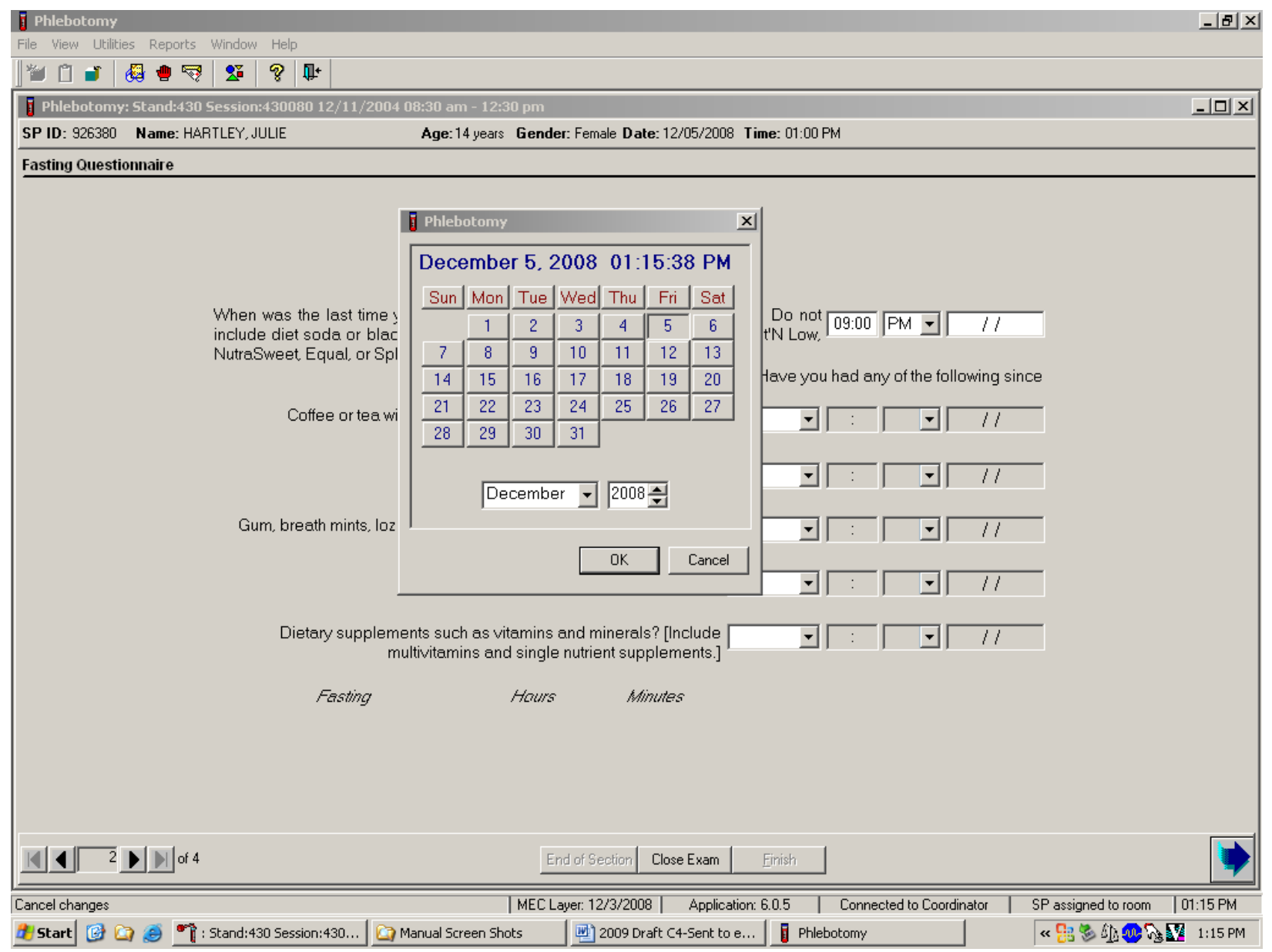

Type in the date using the keyboard's numeric keys and the $\mathrm{mm} / \mathrm{dd} / \mathrm{yyyy}$ format and select [Tab], or use the calendar to enter the date. To access the calendar, select [F2]. To select the correct month, use the mouse to direct the mouse arrow to the drop-down list, drag the arrow to the correct month (use the scroll bar if necessary), and left click. To select the correct day, use the mouse to direct the mouse arrow to the correct day on the displayed month and left click. To correct the year, use the mouse to direct the mouse arrow to the up-down controls on the spin box and toggle the number up and down. To transfer this date into the date space, use the mouse to direct the mouse arrow to the "OK" button and left click, or select [Enter]. To exit the calendar function, use the mouse to direct the mouse arrow to the "Cancel" button and left click. 
Continue administering the questionnaire. Verify the initial time response by asking the remaining questions.

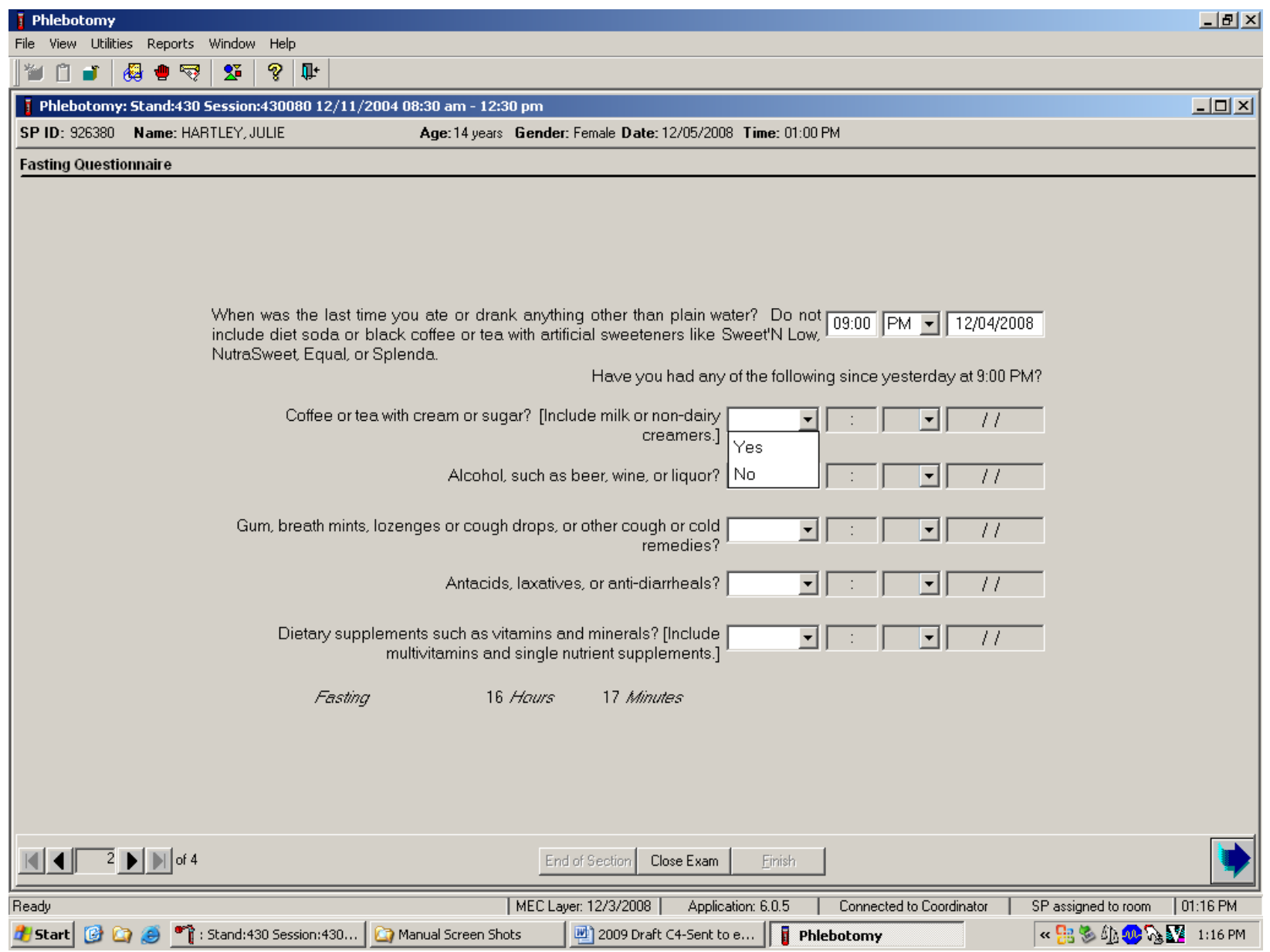


Q2: Have you had any of the following since (time from question 1 inserted here)?

\begin{tabular}{|c|c|}
\hline $\begin{array}{l}\text { Coffee or tea with cream or sugar? } \\
\text { [Include milk or non-dairy } \\
\text { creamers. }]\end{array}$ & $\begin{array}{l}\text { Black coffee is acceptable but coffee with any additives other than } \\
\text { artificial sweeteners is unacceptable. Include all milk products } \\
\text { such as half-and-half, light cream, non-fat milk, and all other non- } \\
\text { dairy creamers like Cremora or Coffee-Mate. }\end{array}$ \\
\hline $\begin{array}{l}\text { Alcohol, such as beer, wine, or } \\
\text { liquor? }\end{array}$ & $\begin{array}{l}\text { Alcohol includes all types of spirits including light beer and wine. } \\
\text { If the answer is "Yes," clarify the response. Ask the SP to describe } \\
\text { the activity, item consumed, and the correct time. }\end{array}$ \\
\hline $\begin{array}{l}\text { Gum, breath mints, lozenges, or } \\
\text { cough drops, or other cough or } \\
\text { cold remedies? }\end{array}$ & $\begin{array}{l}\text { Include sugar-free gum and mints in this category. Give the SP the } \\
\text { laminated card that lists common over-the-counter cough drops } \\
\text { and cough and cold remedies to refresh his or her memory if he or } \\
\text { she appears unsure about these items. If the answer is "Yes," } \\
\text { clarify the response. Ask the SP to describe the activity, item } \\
\text { consumed, and the correct time. }\end{array}$ \\
\hline $\begin{array}{l}\text { Antacids, laxatives, or anti- } \\
\text { diarrheals? }\end{array}$ & $\begin{array}{l}\text { Antacids neutralize stomach acids. Laxatives stimulate evacuation } \\
\text { of the bowels. Anti-diarrheals relieve diarrhea and cramping. } \\
\text { Include all over-the-counter antacids, laxatives, and anti- } \\
\text { diarrheals. Give the SP the laminated card that lists all over-the- } \\
\text { counter antacids, laxatives, and anti-diarrheals to refresh his or her } \\
\text { memory if he or she appears unsure about these items. If the } \\
\text { answer is "Yes," clarify the response. Ask the SP to describe the } \\
\text { activity, item consumed, and the correct time. }\end{array}$ \\
\hline $\begin{array}{l}\text { Dietary supplements such as } \\
\text { vitamins and minerals? [Include } \\
\text { multivitamins and single nutrient } \\
\text { supplements.] }\end{array}$ & $\begin{array}{l}\text { Vitamins refer to various relatively complex organic substances } \\
\text { occurring naturally in plant and animal tissue. They are essential in } \\
\text { small amounts for the control of metabolic processes. Many are } \\
\text { available over the counter as multivitamin-multimineral or single- } \\
\text { nutrient supplements like Vitamin C. Include all of these when } \\
\text { clarifying the response to this question. Give the SP the laminated } \\
\text { card that lists all over-the-counter vitamins, antioxidants, } \\
\text { multivitamins, multivitamins with minerals, and therapeutics to } \\
\text { refresh his or her memory if he or she appears unsure about these } \\
\text { items. If the answer is "Yes," clarify the response. Ask the SP to } \\
\text { describe the activity, item consumed, and the correct time. }\end{array}$ \\
\hline
\end{tabular}


To enter a "Yes" or "No" response, type [Y/y] for "Yes" and [N/n] for "No." Use the up and down arrow keys to toggle between the two choices. Alternatively, use the mouse to direct the mouse arrow to the drop-down arrow on the response text box, select "Yes" or "No" and left click. If "No" is entered, the next response box is highlighted. If "Yes" is entered, the time and date text boxes are highlighted. Type in the reported time using the numeric keys and select [Tab] to move to the AM/PM space. Type in $[\mathrm{A} / \mathrm{a}]$ for times between midnight and 11:59 $\mathrm{AM}$ or $[\mathrm{P} / \mathrm{p}]$ for times between 12:00 noon and 11:59 PM and select [Tab]. Alternatively, to select AM or PM, use the mouse to direct the mouse arrow to the drop-down arrow on the right side of the text box, left click, drag the mouse arrow to "AM," or "PM," and left click.

Type in the date using the keyboard's numeric keys and the $\mathrm{mm} / \mathrm{dd} / \mathrm{yyyy}$ format and select [Tab], or use the calendar to enter the date. To access the calendar, select [F2]. To select the correct month, use the mouse to direct the mouse arrow to the drop-down list, drag the arrow to the correct month (use the scroll bar if necessary), and left click. To select the correct day, use the mouse to direct the mouse arrow to the correct day on the displayed month and left click. To correct the year, use the mouse to direct the mouse arrow to the up-down controls on the spin box and toggle the number up and down. To transfer this date into the date space, use the mouse to direct the mouse arrow to the "OK" button and left click, or select [Enter]. To exit the calendar function, use the mouse to direct the mouse arrow to the "Cancel" button and left click. 
Review the data in the Fasting Questionnaire.

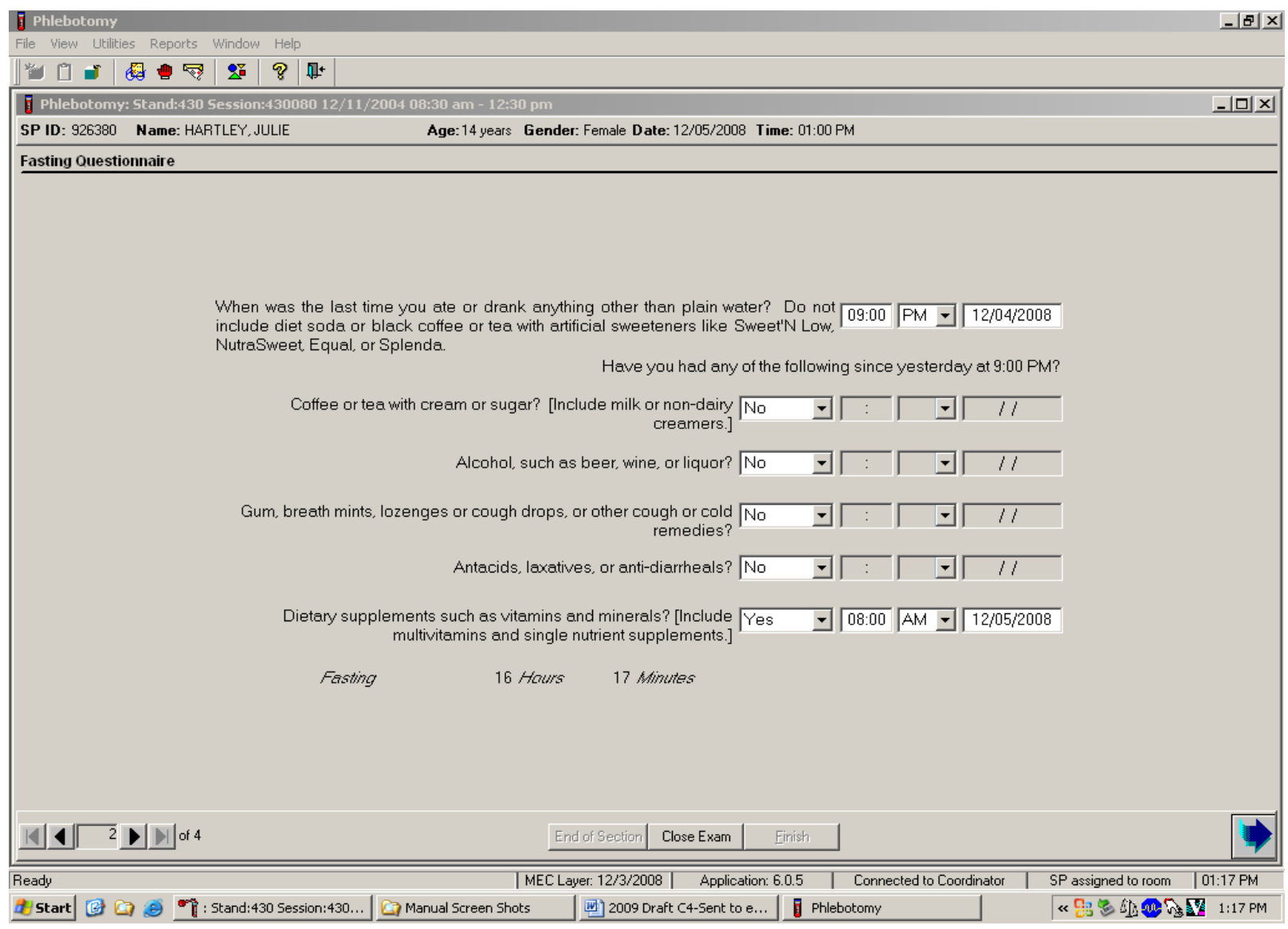

To progress to the next screen, use the mouse to direct the mouse arrow to the bright blue arrow in the bottom right corner and left click, or select [Enter].

\section{SPs Not Meeting 9-Hour Fast - Morning Session Only}

A system was implemented to ensure that SPs who would meet their 9-hour fast and still have enough time to complete the OGTT exam, would wait until they met their fast to have their first venipuncture. The coordinator screen will turn yellow and the phlebotomy application would give a message "I will need to have you wait until approximately (time) before I can collect your blood. I will have the coordinator bring you here at the right time." When the SP has met the 9-hour fast, the coordinator screen will turn green, which indicates the SP has met the fast and can return to phlebotomy. 


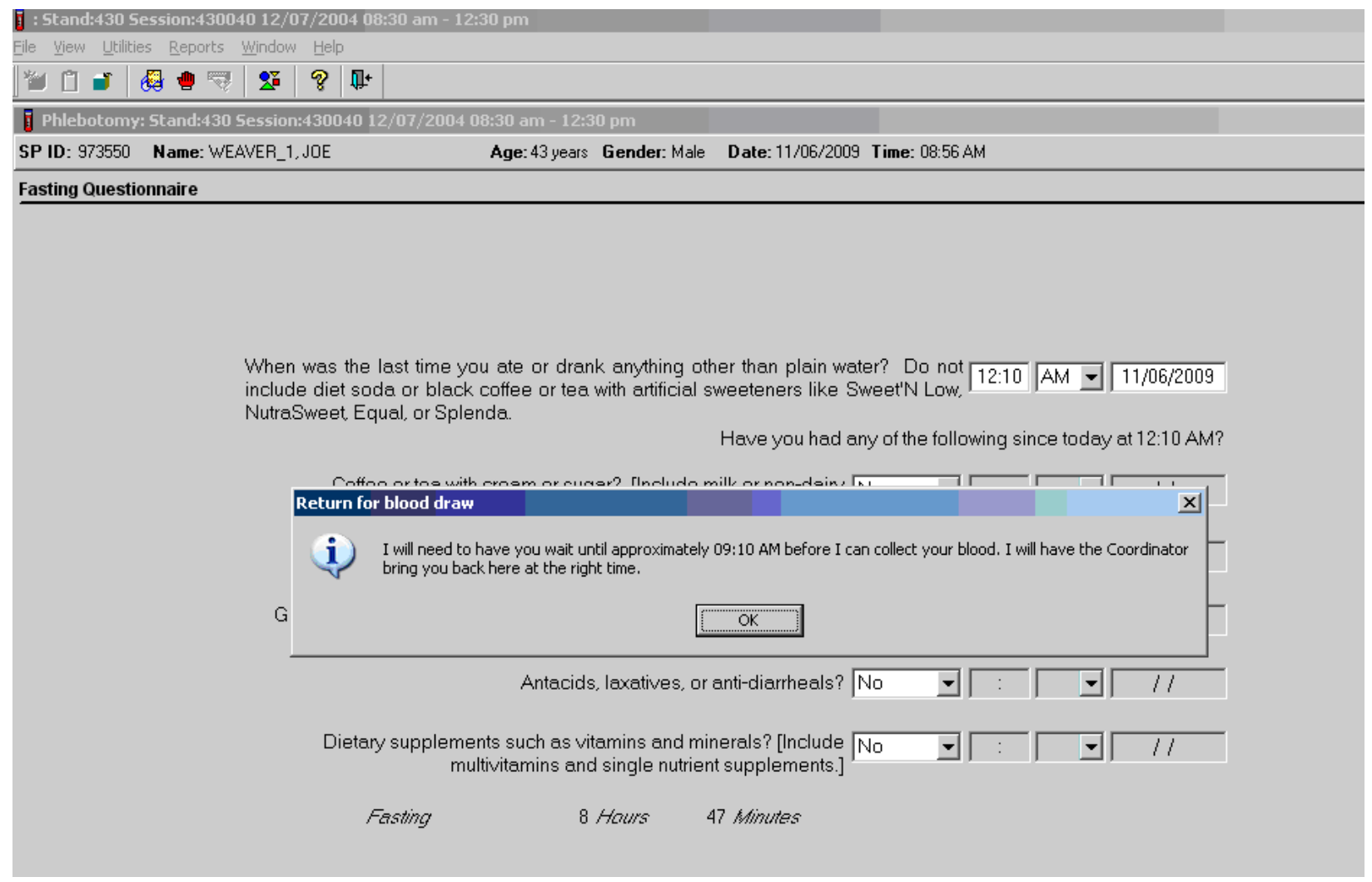

The phlebotomy status screen will automatically default to Not Done with a comment of "Waiting to meet fast." 


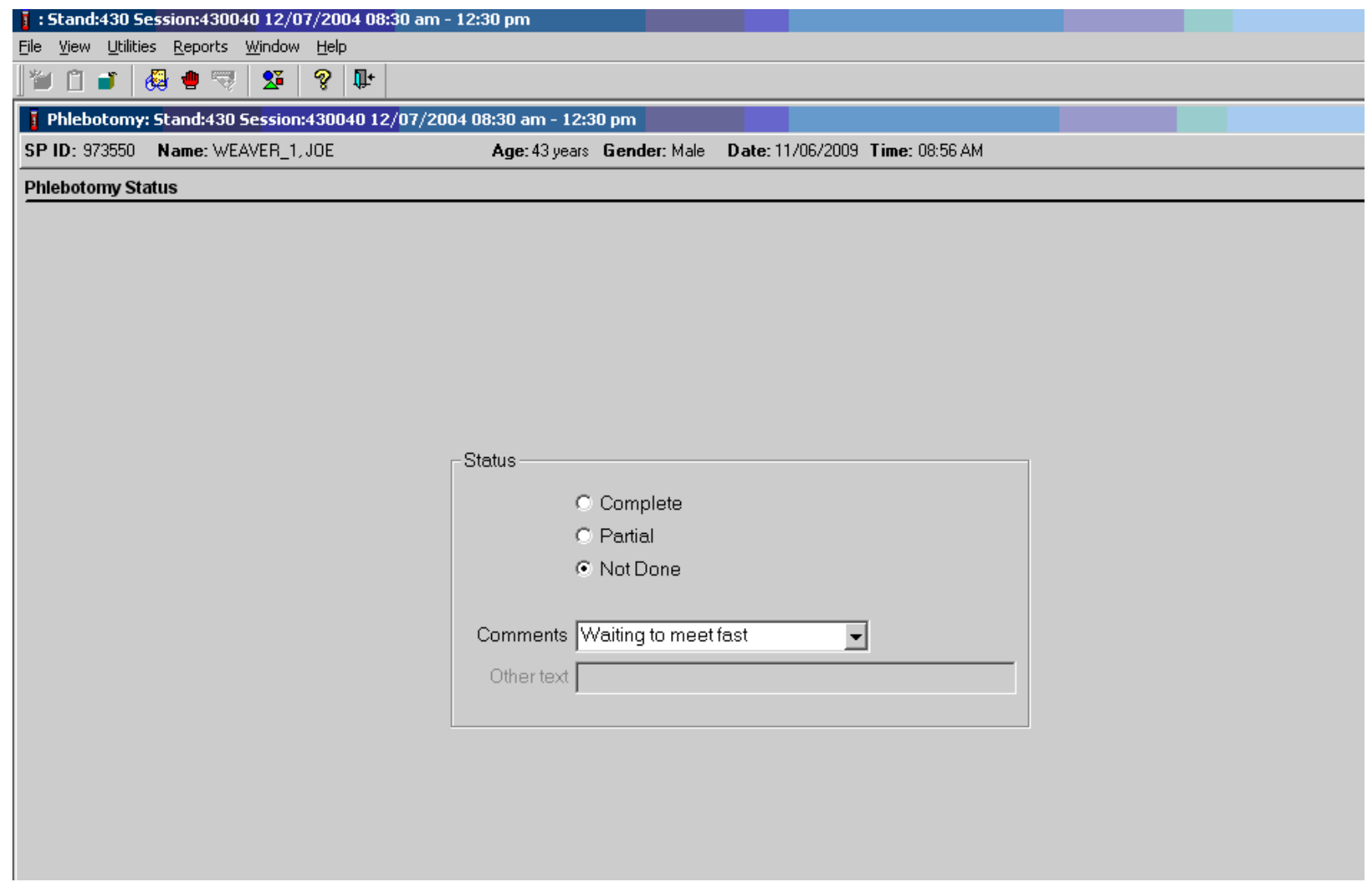

If the SP will never meet the 9-hour fast during the session, he or she is excluded from the OGTT exam and is prompted to have his or her blood drawn at that time without waiting. If the SP will meet the 9-hour fast during the session, but doesn't have enough time to complete the OGTT exam, he or she will be excluded from the GTT exam and will wait until meeting the 9-hour fast for the first venipuncture. 


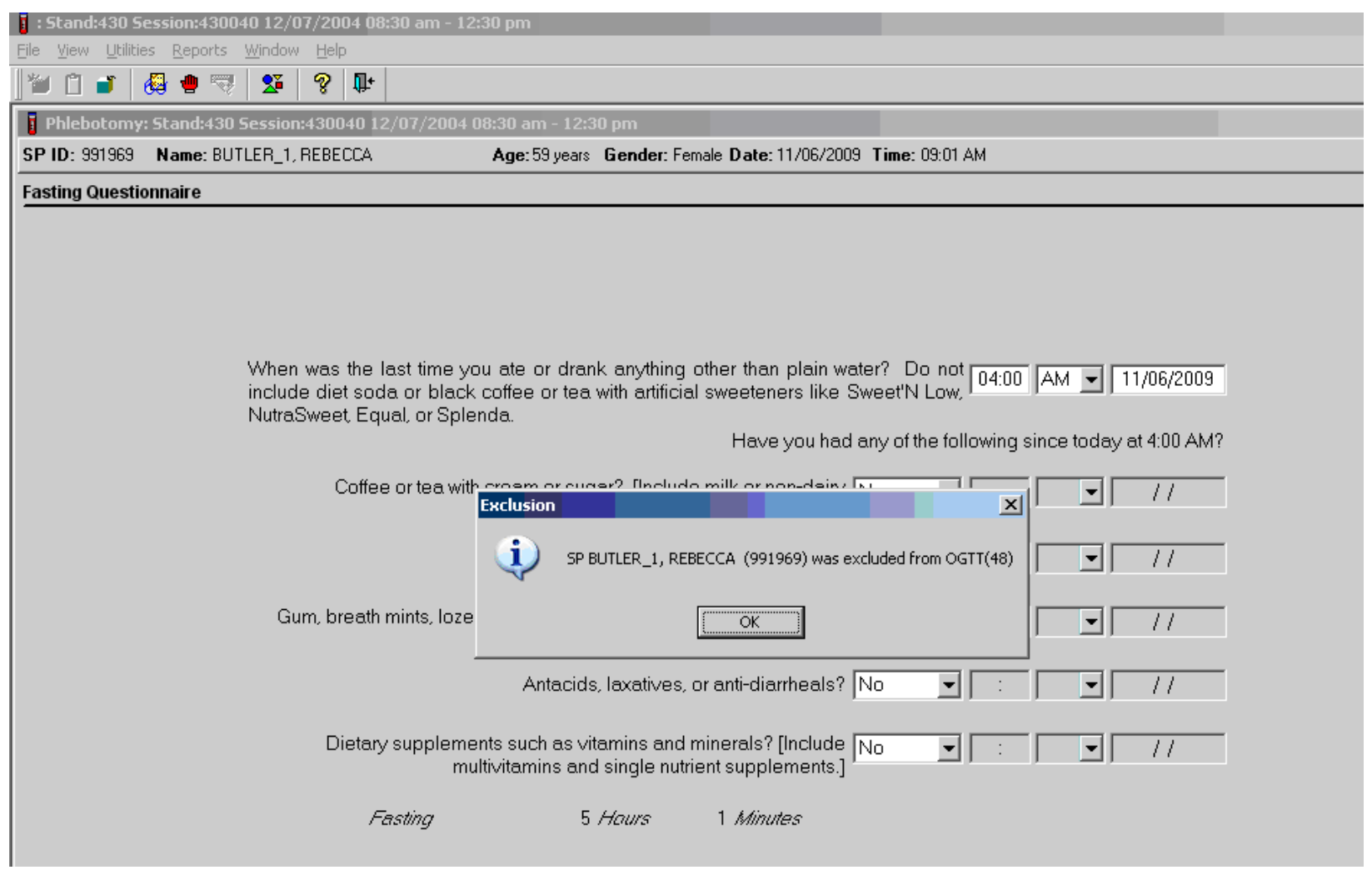

\section{9}

\section{Venipuncture Procedures}

Venipuncture should generally be performed using the median cubital, cephalic, or basilic veins in the left arm unless this arm is unsuitable. If the veins in the left arm are unsuitable, look for suitable veins in the right arm. If the veins in the antecubital space on both arms are not suitable, then look for veins in the forearm or dorsal side of the hand on the left arm/hand and then the right arm/hand.

The Vacutainer® system of blood collection consists of glass or plastic tubes with colorcoded stoppers containing a premeasured vacuum that provides a controlled draw. Some tubes may contain additives to prohibit coagulation or inhibit glycolysis of blood cells. Draw the tubes in the order designated by the venipuncture protocol. 
The exact quantity of blood drawn into each tube varies slightly with altitude, ambient temperature, and venous pressure. Fill tubes with additives completely to ensure proper ratio of blood to additive. Thoroughly mix the lavender tubes, $2-\mathrm{mL}$ and $10-\mathrm{mL}$ gray top tubes immediately after drawing the tubes and place them on the specimen rocker until the tubes are labeled and passed through to the window to the laboratory.

Use Vacutainer® tubes at room temperature. Protect tubes from extreme temperatures and store in a cool place. Note the expiration date printed on Vacutainer ${ }^{\circledR}$ tubes. Do not use expired tubes unless they are the only tubes available and they still contain a vacuum. Indicate if using expired tubes by using the "Other, specify" comment option on the venipuncture status screen.

Document the use of each Vacutainer® type in the automated Supply Use Control Log. When opening a new box, record the date, the lot number of the box, date in use, the expiration date, and tech ID number.

Allow two venipuncture attempts with the SP's verbal consent or with the parent's consent if an SP is a child or an infant.

If an SP faints or becomes ill, causing termination of the procedure without collecting all of the blood, repeat the procedure with the SP's consent after the SP has recovered.

\subsubsection{Preparation of the Puncture Site}

It is extremely important that the anticipated puncture site is thoroughly cleaned and all necessary equipment, including needles and tubes, is kept sterile and free from contamination.

Follow the steps outlined below to prepare the puncture site.

- Place venipuncture equipment where it is readily available but not in danger of being upset. Keep extra equipment within easy reach.

- Thoroughly wash hands.

- Put on gloves. 
- Place appropriate blood collection tubes in a test tube rack in the order dictated by the venipuncture protocol. If an SP exhibits nervousness, keep the tubes covered.

- Instruct the SP to sit on the exam table. Never attempt a venipuncture on a standing subject. Having the subject sit helps guard against any injury that might result if the subject faints. Place the SP in a supine position if it is impossible for him or her to sit upright during the procedure. Instruct the SP to extend the arm palm up and straight at the elbow. If the SP is a child or infant, have a medical technologist hold the SP in the proper position.

- Position the SP's arm on the armrest or a pillow so that the veins are readily accessible and you are able to work in a comfortable position. Be sure that the arm is in a downward position with the elbow lower than the heart to prevent backflow. Dispose of the pillow weekly for hygienic purposes. Change the pillowcase daily.

- Inspect the arm. Use the left arm unless unsuitable. The veins of choice are the cephalic, basilic, and median cubital veins located in the antecubital fossa area.

- If the veins in the antecubital space are not suitable, look for suitable veins on the forearm or on the dorsal side of the hand.

- Apply the tourniquet several inches above the selected site.

- Select a vein that is palpable and well fixed to surrounding tissue. Palpate even when the vein is visible. Use the following techniques if the veins do not distend quickly.

1. Massage the arm from wrist to elbow to force blood into the veins.

2. Tap the area sharply with the index and second finger two or three times to cause the veins to dilate.

3. Allow the arm to hang at the SP's side without a tourniquet to allow the veins to fill to their capacity.

4. Examine the SP's other arm; sometimes the veins in one arm are larger than in the other.

5. Check carefully for scar tissue or tendons near the vein.

Apply the tourniquet for no more than 1 minute while searching for a vein and then release the tourniquet for 2 to 3 minutes. Avoid prolonged obstruction of blood flow by the tourniquet because it is uncomfortable for the SP and may alter certain results (e.g., cholesterol).

- Reapply the tourniquet when ready to perform the venipuncture.

- Cleanse the area with an alcohol wipe. Do not touch the side of the alcohol wipe that is in contact with the puncture site. Cleanse the area using a circular motion beginning 
with a narrow radius and moving outward so as not to cross over the area already cleansed.

- Dry the cleansed area using a $2 \times 2$ gauze pad. The area should be completely dry before performing the venipuncture to reduce the burning sensation caused by alcohol penetrating the skin.

- Determine the correct needle size. Use a $19 \mathrm{~g}, 21 \mathrm{~g}$, or $23 \mathrm{~g}$ butterfly depending on the condition of the SP's veins. The $19 \mathrm{~g}$ butterfly should be suitable for most SPs. If the SP's veins appear fragile or small, use a $21 \mathrm{~g}$ or $23 \mathrm{~g}$ butterfly.

\subsubsection{Venipuncture Technique for the Nipro (19 Gauge)}

1. Prepare the blood collection set. Obtain one blood collection set and one luer adapter. Open the blood collection package and inspect the unit. Open the blue adapter cover by twisting the two ends at the seal. Push the male luer adapter firmly and securely into the female end of the needle assembly. Attach a yellow tube holder onto the adapter.

2. Ask the SP to make a fist. Do not have the SP pump his or her fist since this action may alter certain results.

3. Fix the vein about one inch below the proposed point of entry by pulling the skin taut with the thumb of your less dominant hand.

4. Approach the vein in the same direction that the vein runs, holding the needle with bevel up and at a 15-degree angle to the SP's arm.

5. Remove the needle cover. Grasp the wings and push the needle firmly and deliberately into the vein. Do not grasp the translucent needle shield to insert. If the needle is in the vein, a small amount of blood will appear in the butterfly tubing. Quickly push the first Vacutainer ${ }^{\circledR}$ tube down on the needle. If the needle is in the vein, blood will flow freely into the butterfly tubing. If no blood enters the tube and no bruise is forming, probe the vein until blood begins flowing into the tube. If no blood enters the tube and a bruise is forming, remove the needle. Place gauze squares over the puncture site and apply firm pressure to the puncture site for 3 minutes. Switch to the other arm using a new needle. Wait 10 minutes before beginning the procedure again if using the same arm for a second try.

6. Hold the tube with the tube stopper uppermost and with the tube lower than the needle to prevent backflow through the tube. It is very important to prevent possible backflow because of the possibility of adverse reactions to the SP.

7. As the Vacutainer ${ }^{\circledR}$ tube is filling, transfer the tube holder to the left (less dominant) hand, leaving the dominant hand free to pick up and change the tubes. If left-handed, do the reverse. 
8. Fill all tubes completely. Make sure the tube contents do not touch the stopper or the end of the needle during the procedure.

9. Immediately invert the lavender and gray tubes to ensure proper mixing of blood and anticoagulant or additive. Place the lavender and gray tubes on the rocker. Do not invert or agitate the red top tubes.

10. Insert the next tube and push it down gently onto the adapter.

11. Because prolonged application causes vasoconstriction, remove the tourniquet after 2 minutes to ensure valid test results. If necessary (that is, if the blood flows more slowly), reapply the tourniquet after 2 minutes. If this is the last tube to fill, loosen the tourniquet when the tube begins filling and remove it as the last tube fills.

12. Fill tubes in the proper order, according to the protocol.

13. When the last tube has filled, remove the needle in a smooth quick motion. Avoid heavy pressure as the needle is being withdrawn because it may cause the point of the needle to cut the vein. To activate the shielding mechanism, either grab the tubing and hold the needle guard at the end closest to the tubing and pull the tubing until the needle guard locks in place, or hold the needle guard at the end closest to the tubing, grab the wings and slide the wings back until the needle guard locks in place.

14. Discard the entire blood collection set, including the needle and adapter, in the Sharps needle disposal unit. Do not recap the needle. Discard tourniquet in trash.

\subsubsection{Venipuncture Technique for the BD Safety-Lok ${ }^{\mathrm{TM}}$ Blood Collection Set (21 and 23 Gauge)}

The BD Vacutainer ${ }^{\mathrm{TM}}$ Push Button Blood Collection Set provides immediate protection against needle stick injury when properly activated within the vein, and in accordance with package insert directions.

1. Prepare the blood collection set. Open the package and inspect the unit. Check to ensure that the female luer adapter is firmly and securely attached on the male luer.

2. Ask the SP to make a fist. Do not have the SP pump his or her fist since this action may alter certain results.

3. Fix the vein about one inch below the proposed point of entry by pulling the skin taut with the thumb of your less dominant hand.

4. Approach the vein in the same direction that the vein runs, holding the needle with bevel up and at a 15-degree angle to the SP's arm. 
5. Remove the needle sheath. With thumb and index finger, grasp wings together and access vein using standard needle insertion technique. If the needle is in the vein, a small amount of blood will appear in the butterfly tubing. Quickly push the first Vacutainer ${ }^{\circledR}$ tube down on the needle. If the needle is in the vein, blood will flow freely into the butterfly tubing. If no blood enters the tube and no bruise is forming, probe the vein until blood begins flowing into the tube. If no blood enters the tube and a bruise is forming, remove the needle. Place gauze squares over the puncture site and apply firm pressure to the puncture site for 3 minutes. Switch to the other arm using a new needle. Wait 10 minutes before beginning the procedure again if using the same arm for a second try.

6. Hold the tube with the tube stopper uppermost and with the tube lower than the needle to prevent backflow through the tube. It is very important to prevent possible backflow because of the possibility of adverse reactions to the SP.

7. As the Vacutainer ${ }^{\circledR}$ tube is filling, transfer the tube holder to the left (less dominant) hand, leaving the dominant hand free to pick up and change the tubes. If left-handed, do the reverse.

8. Fill all tubes completely. Make sure the tube contents do not touch the stopper or the end of the needle during the procedure.

9. Immediately invert the lavender and gray tubes to ensure proper mixing of blood and anticoagulant or additive. Place the lavender and gray tubes on the rocker.

10. Insert the next tube and push it down gently onto the adapter.

11. Because prolonged application causes vasoconstriction, remove the tourniquet after 2 minutes to ensure valid test results. If necessary (that is, if the blood flows more slowly), reapply the tourniquet after 2 minutes. If this is the last tube to fill, loosen the tourniquet when the tube begins filling and remove it as the last tube fills.

12. Fill tubes in the proper order, according to the protocol.

13. When the last tube has filled, remove the Safety-Lok set by:

- $\quad$ Grasping the translucent yellow safety shield grip area with thumb and index finger while at the same time grasping the tubing, OR

- $\quad$ Grasping either one wing or both wings and withdrawing.

14. Activate the safety shield:

- $\quad$ One-Hand Technique. Hold tubing in hand and advance translucent yellow safety shield with thumb and index finger until a click is heard indicating that the needle is completely retracted and safety shield is locked in place over needle tip, OR. 
- $\quad$ Modified One-Hand Technique. Apply pressure to site using your fingers. Withdraw blood collection set by grasping the translucent yellow safety shield grip area with the thumb and index finger. With opposite hand, grasp tubing between thumb and index finger while pushing the yellow safety shield forward until a click is heard indicating that the needle is completely retracted and the safety shield is locked in place, OR.

- $\quad$ Two-Hand Technique. Grasp either wing with one hand (A) and grip area of the yellow safety shield base with the other hand (B). Slide the wings back into the rear slot of the safety shield, until a click is heard indicating that the needle is completely retracted and locked into place over needle tip.

\section{Do not grasp safety shield by body; this will interfere with safety shield activation.}

15. Discard the entire blood collection set, including the needle and adapter, in the Sharps needle disposal unit. Do not recap the needle. Discard tourniquet in trash.

\subsubsection{Concluding the Venipuncture}

1. Have the SP place two fingers on the gauze to hold it in place, then ask the SP to raise the arm straight up, elevating the arm above the level of the heart, without bending the elbow. The SP should remain in this position for 2 to 3 minutes to help prevent hematomas.

2. Corresponding computer-generated barcode labels print automatically, after the phlebotomist enters the quantity of tubes drawn. Label the 10-mL gray top tube with the label containing the Vessel ID - 54. Label all other tubes with barcoded labels with the barcode vertical on the tube. The time prints on the label.

3. Place the rack containing the blood collection tubes in the pass-through window located over the sink. Alternatively, deliver the rack to the laboratory, and distribute as described below:

- $\quad$ Place the lavender and gray tubes on the rocker located next to the biological safety hood.

- Place the red top tubes upright in the test tube rack located next to the biological safety hood in the laboratory.

4. Place all used needles in a Sharps container and dispose of all visibly contaminated waste in a biohazard bag.

5. Check the venipuncture site for clotting.

Venipuncture should generally be performed using the median cubital, cephalic, or basilic veins in the left arm unless this arm is unsuitable. If the veins in the left arm are 
unsuitable, look for suitable veins in the right arm. If the veins in the antecubital space on both arms are not suitable, then look for veins in the forearm or dorsal side of the hand on the left arm/hand and then the right arm/hand.

\section{If the venipuncture site is in the right arm:}

For all SPs aged 1-11 and SPs not eligible for OGTT - Apply an adhesive bandage over the gauze pad. Instruct the SP to remove it in no less than 45 minutes if the bleeding has stopped. Also, suggest that the SP sit quietly for a few minutes. If bleeding continues, keep direct pressure on the site for 5 minutes or more.

For all SPs aged 12 years and older who are eligible for the OGTT - Apply CoFlex dressing to the puncture site in place of the regular adhesive bandage.

6. Report any adverse reaction to the venipuncture to the physician immediately and document the event by using the automated Unusual Field Occurrence utility.

7. If the SP has been fasting and is eligible for the OGTT, then do not offer him or her snack and juice. If the SP has not been fasting or is not eligible for the OGTT, then offer him or her juice and crackers.

8. Escort the SP back to the coordinator.

9. If blood has spilled on the phlebotomy chair, wash the area with a 10 percent solution of bleach and water, then prepare the workstation for the next SP. Routinely disinfect the phlebotomy chair with Sani-Wipes to disinfect. Wash hands and change gloves before approaching the next SP.

10. Wash hands and change gloves before approaching the next SP.

\subsection{Pediatric Venipuncture}

Pediatric venipuncture requires special techniques. Because you will be dealing with children of different age ranges and levels of understanding, it is important to be able to recognize at what stage a child is in early in the venipuncture process.

Infants and toddlers, aged 1 to 2 years, experience the world through their senses and do not have much of a language base. Therefore, verbal explanations are virtually meaningless. Expect crying and resistance from the start, even at the first touch. Restrain children of this age by enlisting the assistance of a medical technologist. The best techniques to use with these children are to maintain a reassuring tone, to reinforce that the child is a good boy or girl, and to draw the blood quickly. Be sure to reassure the child after the procedure and to use cartoon adhesive bandages and stickers when finished. 
Preschoolers, aged 2 to 6 years, think concretely and in absolute terms; things are good or bad, right or wrong, painful or not painful. For these children, use simple, concrete terms when describing the procedure and its consequences. Always be honest. Try to avoid the word "take" which implies remove, "test" that implies pass or fail, and "fix" which implies broken. With children of this age, it is still important to emphasize that the child is a good boy or girl and to use colorful adhesive bandages and stickers. Try using distractions, such as holding something, counting things in the room, and breathing deeply. These techniques may work with the older children in this age group. The phlebotomist may have a medical technologist assist with most of these children.

School-aged children, 7 to 12 years, have a better grasp of language and view the world more realistically. They understand past and future, that is, "this will be over soon," and relative terms, such as "this will hurt a little or a lot." Detailed explanations of the procedure are extremely effective. Again, always be honest. Include demonstrations, with a doll for example, whenever possible. Having the child help during the procedure distracts the child as well as letting him or her have some control. Be sure to reinforce how big a help he or she was after the draw. Give the child realistic choices, such as which arm to choose. You may need to consider having a medical technologist assist with some children even at this stage. Adhesive bandages continue to be important.

Although adolescents aged 12 to 18 years are beginning to think abstractly, they often regress when placed in stressful situations. It is best to assume that they will act younger than their chronological age. However, it is still very important to address the adolescent as an adult and not as a child. Provide detailed explanations and inform him or her that the best technique is being used. It is very important that the adolescent maintain control of the situation; therefore, give him or her some choices, as well as allowing him or her to help if offered. Be very clear about rules, such as remaining motionless, but do not restrain the adolescent.

Pediatric venipunctures are most successful when children are immobile, the veins are maximally distended, and all supplies are handy. Many children prefer a cloth or gauze under the tourniquet to prevent pinching of skin.

The usual site for pediatric venipunctures is the antecubital fossa. Most venipunctures in children are successful using a 23-gauge butterfly needle. Follow the general guidelines in Section 4.9 for performing venipunctures. 


\subsubsection{Age-Specific Techniques in Phlebotomy Competency}

Advance for Medical Laboratory Professionals

Vol. $14 \cdot$ Issue $6 \cdot$ Page 11

The Learning Scope:

Understanding and incorporating age-specific competence in evaluating the phlebotomist's performance

By Donna R. Kirven, BA, BPPVE, PBT(ASCP), CLA

\section{Learning Objectives}

At the conclusion of this article the participant will be able to:

State the importance of communicating and incorporating techniques in the performance of phlebotomy procedures based on the physical age and developmental stage of the patient.

Identify at least three major developmental considerations in the performance of phlebotomy procedures for each age-specific group.

Formulate an age-specific competency that can be used as part of the phlebotomy evaluation process.

All health care professions that involve direct patient contact require certain specialized techniques and competencies that are based on characteristics of the patient receiving service. The invasive procedures of phlebotomy are no exception.

Traditionally for some phlebotomists, the majority of their patients could be categorized in one or two age groups, while the experience of others includes all age groups. Age-specific concerns are age, growth, development and safety considerations that must be assessed and incorporated into phlebotomy practices for successful outcomes with all patient age groups.

Simply defined, age-specific competence is the knowledge, skills, ability and behaviors essential for providing care to specific populations of patients and their families. These considerations require expanded knowledge and an increased experience level of physical, psychological, and interpersonal assessment skills. The newly certified (or trained) phlebotomist should begin using that training on older children, and as confidence and technical skills are attained, progress to performing venipunctures on younger children and, subsequently, infants. ${ }^{1}$ Performing a venipuncture on pediatric patients can present a greater technical and emotional challenge to the phlebotomist and, without proper age-specific techniques, can result in an unnecessarily unpleasant experience for the child, the parents, and the phlebotomist.

To make quality health care services available to communities served by acute care facilities, age-specific phlebotomy techniques are now becoming a standard part of the phlebotomy training curriculum and annual competencies. 


\section{Factors in Age-Specific Techniques}

Age-specific techniques in phlebotomy involve much more than simply using a smaller tourniquet and collecting a minimum volume of blood. Considerations for motor skills, communication skills development, social needs, and physical and learning disabilities also are a part of the age-specific assessment. The rationale for age-specific techniques is based on the fact that different age groups of patients have special physical and psychosocial needs. Knowledge of the basic nutritional and social needs such as feeding, sucking, and physical affection in the infant patient can exemplify professionalism and confidence to anxious parents. Recognition of the increased potential for diminished hearing, vision and mobilization can dispel feelings of embarrassment and frustration in the elderly patient.

Safety is also an age-specific criteria issue. While the phlebotomist should exercise safe practices with all patients, it is important to be aware of the special safety precautions that should be used with pediatric, elderly, and disabled patients that are specifically attributable to age. Communication skills on the part of the patient as well as the phlebotomist are also important factors. When performing phlebotomy procedures on an infant, informing the parent or guardian of the details of the procedure in addition to reassurance, can alleviate anxiety and build his or her trust before attempting the procedure. Becoming a highly qualified, experienced phlebotomist skilled in age-specific techniques requires good interpersonal skills such as integrity, effective communication and confidence, as well as demonstrated technical skill. By incorporating awareness growth and development theories into phlebotomy training, the phlebotomist can provide service and perform procedures in a manner that meets the practical (quality specimen collection) and personal (trust, reassurance, respect) needs of patients and their family members. Table 1 shows six stages of development and the corresponding age divisions.

\section{The Neonate/Infant}

Venipunctures should not be performed on children under the age of 6 months unless the test ordered requires the volume and type of specimen that can only be obtained by a needle stick. Nonetheless, heel sticks, the most frequently used method performed for mandatory newborn screens that test for phenylketonuria (PKU), galactosemia, hypothyroidism, primary congenital hypothyroidism and several other hemoglobinopathies, ${ }^{2}$ are performed on a routine basis and require highly specialized skills and agespecific techniques.

The phlebotomist must select the appropriate method of obtaining a blood sample after considering two factors: the patient's age, and sample requirements for the test ordered. The novice phlebotomist must study anatomy and physiology, become familiar with special types of equipment specifically designed for the pediatric patient, observe various techniques as performed by experienced phlebotomists or nurses, and practice the techniques themselves to develop the necessary skills. They must make every attempt to collect the minimum amount of blood required for testing because of the small blood volume of infants. Removal of more than 10 percent of an infant's blood volume at one time can lead to cardiac arrest, ${ }^{1}$ while removal of large amounts of blood over an extended period of time can lead to anemia, possibly requiring the infant to undergo a blood transfusion. Improperly performing a heel incision, or using a safety lancet that is not of appropriate pediatric size on small children, can lead to puncture of the calcaneus bone, infection, and osteomyelitis. 
Specific guidelines for performing a heel stick include:

Refrain from puncturing the anteromedial aspect or the posterior curve of the heel to avoid puncturing the underlying calcaneus.

Hold the infant's foot securely but gently so as not to bruise his/her delicate tissue or restrict blood flow. Avoid milking or squeezing the foot, which can dilute the blood with interstitial (tissue) fluid or cause hemolysis of the specimen.

Refrain from covering the puncture site with adhesive bandages that can macerate the infant's fragile skin underneath.

The emotional challenge of performing phlebotomy procedures on neonates requires that the phlebotomist be prepared to handle not only the infant's reaction to the procedure, but also the parent or guardian who may be present. It is important that the experienced phlebotomist build trust with the parent by communicating in a warm, compassionate manner while displaying a calm and confident approach. These types of interpersonal skills, in addition to requesting the parent's assistance in comforting the infant, can help calm parental anxiety and foster trust in the phlebotomist, allowing her to perform the procedure in a less frenzied environment. Consider the following:

\section{Case Study}

Jordan Smith is a 9-month old boy who has been brought to the outpatient laboratory by his parents for some lab work ordered by Jordan's pediatrician. Aside from the newborn screening performed shortly after his birth, Jordan has never had a blood test. His pediatrician has ordered electrolytes and a complete blood count.

Once Jordan's parents have completed the necessary laboratory registration, Monica, a phlebotomist with 15 years experience, having reviewed the requisition and assembled her phlebotomy supplies beforehand, calls Jordan and his parents into the outpatient drawing room. After introducing herself and completing the patient identification procedure with Jordan's mother, Monica greets Jordan and notices that Jordan's father appears to be frozen with fear, hasn't spoken a word and has large beads of perspiration covering his forehead. Jordan's mother, on the other hand, appears significantly less nervous and is engaged in physically and verbally comforting him. Her years of experience have taught Monica that she should address these observations before beginning the procedure. The dialogue proceeds as follows:

Monica: Mr. and Mrs. Smith, has Jordan ever had a blood test performed?

Mother: No.

Father: (Shakes his head designating "No")

Monica: I'd like to take a minute or two to explain the procedure to you, but first I'd like to reassure you that I will do my best to perform this procedure with as little discomfort as possible for Jordan and for you.

Mother: Thank you. 
Father: (Mumbles “Thank you”)

Monica: Mr. Smith, you appear to be a little nervous, and that's quite understandable. Our phlebotomy staff here have an average of 10 years experience in performing these procedures on patients who are Jordan's age and younger. I understand your hesitation and respect your concern. If you feel that observing the procedure may be a bit too upsetting for you, you're welcome to have a seat in our waiting area while your wife remains here with us during the procedure, but if you prefer to stay that's fine too.

Father: Well, it's just that he's so small—and do you really have to stick him with a needle to get blood, can't you just prick his foot or something like they did when he was born?

Monica: Children who are Jordan's age have usually begun to stand or walk, and in preparation for this, the skin of the foot has grown thicker and more resilient. To incise or cut the heel at his age and size, in the same method as that for newborns, would not allow sufficient blood flow required to complete the tests that his physician has ordered. Our standard practice for a patient Jordan's age is to obtain a blood specimen from a vein in the arm, which is believed to be a more efficient and less traumatic method of performing a blood test.

Father: How can you find a vein in a little arm like this?

Monica: That's a question that I'm often asked by parents. My training for collecting blood specimens from children required that I learn to feel the veins in little arms just as proficiently as I did in older arms, and after proper training and practice, I did. Sometimes, it's easier to find the veins in someone Jordan's size than it is to find them on an adult.

Father: So you've been doing this a long time?

Monica: I personally have more than 10 years experience drawing infants and children.

Father: Well, that's good to know. I feel a little bit better now so I think I'll stay in here.

Monica: That's great. Mrs. Smith do you have any questions?

Mother: No. I just want this done and over with.

Monica: Great! If I can get you to help me by holding Jordan just this way, it will really help us complete this procedure quickly and ensure that we have collected a quality specimen. Dad, you can help too by giving Jordan lots of encouragement and praise during the procedure. 


\section{The Pre-School Child}

Although the pre-school aged patient has begun to verbally communicate in small statements, it is important that the phlebotomist explain the intended procedure in terminology appropriate for the child. As this patient age group is heavily engaged in security-seeking behavior, the presence of the phlebotomist - a stranger clad in a white lab coat - can often exacerbate their fear and increase their anxiety level even before they see a needle. Approach them slowly and gain their trust before handling equipment or touching their arms to look for a vein. ${ }^{2}$

One of the goals of age-specific techniques for this group is to attempt to keep the child calm and reduce crying which, if prolonged, can actually raise the white blood cell count and the blood $\mathrm{pH}$ level. ${ }^{3}$ Several methods can be used to accomplish this, some of which may not work for every child; however, the appropriate choices can be more easily made with the phlebotomist's increasing experience level.

Some of these methods are:

Prepare materials and gather equipment before you encounter the child. Doing this in the child's presence will allow more time for the child's anxiety to build.

Approach the child in a friendly, cheerful, and empathetic manner. Speak before you touch the child, even if the child cannot or will not respond. Introduce yourself and address the child by name.

Speak in a soothing, non-threatening tone of voice. Even if the child does not completely understand what you are saying, a calming tone communicates a non-threatening intention.

Explain the procedure in terms that are appropriate for the child's age. This, coupled with a soothing tone of voice, can help to lessen the child's fear.

Do not say, "It will not hurt." Explain that some pain will be involved in the procedure, and that it is OK to say "ouch" or cry a bit, but that it is important to keep the arm as still as possible.

During the procedure, keep the child informed of how much longer it will be-"Just one more tube"while using appropriate praise for the child, even if he/she did not cooperate as much as possible. Phrases such as "You are doing such a good job of helping me. Thank you, (patient's name)" can provide reinforcing recognition that may encourage the child to approach his/her next phlebotomy experience with less anxiety.

\section{The School-Aged Patient}

Performing phlebotomy procedures on the 8-12 year old patient can be more of a physical challenge than an emotional one. This age group has mastered the meaning and use of the word "No," and is prepared to defend their fear with it. Proper immobilization of the pediatric patient is necessary to ensure the safety of the child as well as the phlebotomist. Instructing the parent/guardian on the correct "hugging" hold method for a child seated on the parent/guardian's lap, or the appropriate restraint for the prone child can allow the phlebotomist to collect the specimen in a manner that will also ensure accurate test results.

The school-aged patient has moved from security-seeking behavior to independence-seeking behavior and, as such, can exhibit unceasing curiosity. It is important that all phlebotomy equipment be kept out of 
reach of the child; however, be sure to respond to the child's inquiries as appropriate. Asking questions is an integral part of the learning process, and responding initiates effective communication skills. These young patients have begun to establish their identities, and the phlebotomist must communicate with them as individuals, in age-appropriate terminology.

Also, most of these children have begun the initial stages of puberty, so the phlebotomist must allow for the privacy of the child in preparation for the phlebotomy procedure. In an outpatient setting, if a urine specimen is ordered, give the child instructions for collection, in age-appropriate terminology, and allow $\mathrm{him} / \mathrm{her}$ to attempt specimen collection assisted by the parent/guardian or alone.

\section{The Adolescent Patient}

In dealing with the adolescent patient, it is essential for the health care provider to remember the importance of demonstrating an understanding of the significance of peer relationships to the adolescent. Embarrassment can be this age group's mascot. The phlebotomist can implement this understanding by recognizing that some reassuring comments or approaches, if not age-appropriate, can actually instill more anxiety for the adolescent patient. Providing age-appropriate praise and positive feedback for this group of patients can lessen their anxiety.

Adolescents in the higher age range are usually engaged in thoughts, goals, and behavior that they believe will eliminate the perception of a child, and establish that of an adult. These behaviors can, however, manifest themselves in a sensitivity-heightened and non-interactive manner that can test the communication skills of the phlebotomist, as well as the parent/guardian. It is important to continue to initiate communication with adolescent patients and welcome their limited inquiries.

The older adolescent male, for some unknown cause, possibly hormonal, can demonstrate a greater potential for fainting during a venipuncture procedure. One of the age-specific safety techniques that can be used with this patient age group is to more closely assess the patient before, during, and after the procedure. Listen for a previous history of syncope or light-headedness when first encountering the adolescent patient.

During the procedure, periodically check for pre-syncope signs such as profuse perspiration, change in skin pallor, and/or the onset of clammy hands. Another indication of pre-syncope can be the adolescent patient who is suddenly silent in the middle of a conversation or extremely talkative after having been previously silent.

It is important that the phlebotomist be familiar with the department's policy/procedure for dealing with patients who experience syncope, light-headedness, or dizziness.

\section{The Adult Patient}

Some of the typical conditions that commonly affect the adult patient are stress and depression, which are usually related to pressures of the workplace, marriage, childbearing, parenting, and social expectations. Additionally, these roles can induce a stronger identification with their parents or primary caregivers, known as generativity, as well as a closer reflection with their own mortality.

For the average adult, the focus of learning patterns and thinking lies mainly on problem solving. Accordingly, the phlebotomist must use effective communication skills by clearly and honestly informing 
the adult patient of the procedure while continually maintaining a professional, confident manner. Adult patients are more than willing to cooperate if they are aware of the benefits of their cooperation.

An adult patient can possess the same fear as that of a pediatric patient; however, the adult patient is usually more willing to allow the procedure as long as it is performed in a professional and concise manner. Conditions that may begin to affect the middle-aged adult patient are the onset of cardiovascular disease and menopause, stroke, and cancers. On the other hand, a significant percentage of adult patients who are directed toward maintaining good mental and physical health, optimal energy, and social activity exists. These patients are usually less stressed about phlebotomy procedures and view them as an integral part of the preventive health care process.

One of the most effective tools of good communication is eye contact. Eye contact promotes a sense of trust and honesty between the patient and phlebotomist; however, if a patient does not return the eye contact when being spoken to, he/she may prefer less direct eye contact due to cultural or modesty reasons. Experienced phlebotomists will take cues from the patient to monitor and satisfy the patient's comfort level needs.

When interacting with adult patients, the phlebotomist should refrain from engaging in negative body language such as crossed arms, wrinkled forehead, or frequently glancing at a clock or watch. These behaviors can detract from the professional image when communicating with adult patients.

\section{The Elderly Patient}

With the expanding life expectancy tables, many phlebotomists' patients are likely to be elderly. ${ }^{3}$ As with infants and younger patients, this can present both physical and psychological challenges for the health care professional. Likewise, working with the older adult can be extremely rewarding for the phlebotomist who continues to perfect her technical expertise and take the time to treat the patient with respect and compassion.

Elderly patients may feel that they are in less control of their medical situation than other patients, may feel apprehensive about having the procedure performed, and embarrassed about feeling this way about it. As with all patients, elderly patients should be treated with consideration for their special needs and with tender, loving care. However, elderly patients are sometimes under the impression that they are a burden and become anxious about the procedure because they don't wish to be a bother. Phlebotomists can help to ease this anxiety by being friendly and cheerful, and taking the time to not only listen to their patients and talk with them, but also to encourage verbalization of their concerns and fears. This can help establish the patient's trust in the phlebotomist and dispel feelings of being a bother.

As people age, they usually undergo a number of physical changes that can have a significant impact on their safety and the effectiveness of normal collection procedures. The elderly patient experiences skin changes, such as the loss of elasticity and moisture, as well as the thinning of skin layers. Loss of supportive connective tissue can lead to "loosened" skin, and loss of muscle tissue and tone may allow for greater movement of veins. ${ }^{3}$ The phlebotomist must treat the skin of the elderly patient in a gentle manner. Sometimes the tourniquet that is applied in a tight or rough fashion can easily tear this delicate skin.

In this age group, patients may experience a decrease in peripheral circulation due to narrowed vessels that are the result of atherosclerosis ${ }^{4}$ and, combined with loss of vessel elasticity, become more fragile. 
Gently massaging the intended site rather than slapping or tapping the arm or hand can effectively increase circulation to the site. As skin layers thin, arteries can lay closer to the skin surface in elderly patients, so the phlebotomist must take care to palpate for the "best" vein, and ensure that it does not have a pulse.

Hearing loss is another disorder that is commonly found in the elderly patient. The phlebotomist should approach a patient who communicates that he/she has a hearing impairment by speaking in a slowed, deliberate and distinct voice to confirm that the patient understands. Some conditions, including Parkinson's disease and stroke, can lead to impaired or unclear speech. This can be a source of frustration for the elderly patient. The phlebotomist must allow the patient to complete his/her statements without being made to feel "rushed," and remember that speech difficulty does not imply difficulty hearing or understanding.

Arthritis also affects a large percentage of the elderly population and may restrict the patient from fully straightening or extending the fingers or arms. The phlebotomist should never force a patient's limb, but should ask the patient whether this action is painful or uncomfortable.

Tremor is also common in advanced aging ${ }^{3}$ and may present some difficulty for the patient to hold his/her arm steady during the venipuncture procedure.

A large number of adults over the age of 50 begin to experience loss of visual acuity and, as such, it is vitally important that the phlebotomist ensure that the entrance and floor areas are free of any debris that may not be visible to the elderly patient. Clear plastic needle covers and small pieces of tape can be falling hazards. Inpatient beds that are raised should be lowered before leaving the patient's room.

Poor nutrition and chronic degenerative disease may lead to emaciation, which can make the elderly patient more susceptible to infection due to loss of immune function, ${ }^{3}$ so these potential falling hazards should be eliminated to avoid cuts, lacerations, and broken limbs.

Forgetfulness, confusion, and dementia are more commonly found in the elderly population. ${ }^{3}$ Reliability on the inpatient's armband to confirm identification is a must. Some confused patients may not fully understand your intentions and may lash out in defense. If the patient does not demonstrate a clear understanding of the procedure, the phlebotomist should check with the nurse and seek assistance if appropriate.

\section{Summary}

Age-specific care techniques are a requirement for all health care professions involving direct patient contact, such as phlebotomy. It is vitally important that these techniques be introduced and incorporated into all phlebotomy training programs, competencies, and continuing education. Learning these techniques requires not only a fundamental knowledge of human growth and development, but also the ability to combine technical expertise with age-specific interpersonal skills.

Incorporating the physical, psychological, and social needs of patients into the formal phlebotomy training curriculum can provide a vital foundation for all those who aspire to successfully perform phlebotomy procedures. Probationary and annual competencies should specifically address age-specific topics such as tone of voice, eye contact, and active listening skills. The implementation of age-specific 
techniques into the phlebotomy training curriculum and competency format will yield more effective phlebotomy practices and, subsequently, quality patient care.

\section{Children Observing Phlebotomy}

No children who are SPs will be allowed in or near the phlebotomy room until they are assigned for a venipuncture. Children that aren't assigned to phlebotomy should remain in the coordinator area and be supervised by the local or other MEC staff. This will reduce the number of SP refusals in children.

\section{REFERENCES}

1. Garza, D., Becan-McBride., K. Phlebotomy Handbook: Blood Collection Essentials, pediatric procedures, $6^{\text {th }}$ Edition. Upper Saddle River, NJ, Prentice Hall, 2002.

2. McCall, R., Tankersley, C. Pediatric venipuncture In: Phlebotomy Essentials, $2^{\text {nd }}$ Edition. Philadelphia, Lippencott-Raven, 1998.

3. Sommer, S., Warekois, R. Blood collection in special populations In: Phlebotomy: Worktext and Procedures Manual. Philadelphia, W.B. Saunders, 2002.

4. Klosinski, D. Collecting specimens from the elderly patient. Laboratory Medicine. 1997;28(8): 515-522.

Donna Kirven is phlebotomy education and training coordinator at John Muir Medical Center in Walnut Creek, $C A$.

http://www.advanceformlp.com/common/editorialsearch/viewer.aspx?FN=03mar10_mtp11.html\& $\mathrm{AD}=3 / 10 / 2003 \& \mathrm{FP}=\mathrm{mt}$

\section{Vol. $\bullet$ Issue $\bullet$ Page LS answers}

\section{Learning Scope Questions}

\section{Which of the following is considered a personal need of the patient?}
a) reassurance
b) respect
c) trust
d) all of the above 
2. Which of the following age groups is heavily engaged in security-seeking behavior?
a) elderly
b) preschool
c) adolescent
d) school-aged

3. Immobilizing the pediatric patient can allow the phlebotomist to:
a) collect a specimen in a manner that will ensure accurate results.
b) build trust.
c) demonstrate confidence.
d) facilitate development and growth.

4. Which of the following age groups includes a population that demonstrates a greater potential for fainting during a venipuncture procedure?
a) elderly
b) preschool
c) adolescent
d) school-aged

5. To monitor and satisfy the adult patient's comfort level needs, the experienced phlebotomist will take his/her cues from the:
a) physician.
b) nurse.
c) family.
d) patient. 
6. To ease anxiety with the elderly patient, the phlebotomist can:
a) be friendly and cheerful.
b) listen and talk with the patient.
c) encourage verbalization of concerns and fears.
d) do all of the above.

7. One of the age-specific goals for the school-aged group is to:
a) facilitate bonding.
b) satisfy practical needs.
c) encourage commitment to task and contribution.
d) assess limiting physical conditions.

\section{Infant cardiac arrest can result from removal of:}
a) 10 percent of infant's blood volume over an extended period of time.
b) small amounts of blood over an extended period of time.
c) 10 percent of infant's blood volume at one time.
d) 2 percent of infant's blood volume at one time.

9. When performing venipuncture on the preschool patient, the phlebotomist should:
a) say nothing.
b) say, "it will not hurt."
c) not say, "it will not hurt."
d) say none of the above.

10. The experienced phlebotomist must demonstrate an understanding of the importance of peer relationships when performing phlebotomy procedures with patients in the:
a) elderly age group.
b) adolescent age group.
c) adult age group.
d) preschool age group. 
11. Some of the typical conditions that commonly affect the adult age group are:
a) diminished hearing.
b) loss of skin elasticity.
c) stress and depression.
d) embarrassment.

12. One of the conditions of advanced aging that can make it difficult for the elderly patient to hold his/her arm steady during the venipuncture procedure is:
a) skin layer thinning.
b) loss of visual acuity.
c) tremor.
d) poor nutrition.

13. Which of the following is considered one of the basic characteristics of the pre-school aged patient?
a) feeding.
b) privacy.
c) imagination.
d) generativity.

14. Which of the following should be specifically addressed in probationary and annual competencies?
a) eye contact.
b) tone of voice.
c) active listening skills.
d) all of the above. 
15. Age, growth and development, and safety considerations must be assessed and incorporated into phlebotomy practices for:
a) increased facility profit.
b) addressing practical patient needs only.
c) successful outcomes with all patient age groups.
d) enhanced interdepartmental communications.

16. In the case study, which of the following is a method that the phlebotomist used to build trust?
a) taking the time to explain the procedure.
b) asking the parents if the patient had a blood test performed before.
c) explaining the venipuncture procedure.
d) all of the above.

17. The phlebotomist in the case study demonstrated professional and timely assessment skills by:
a) explaining the procedure to the parents before beginning the procedure.
b) communicating her years of experience before beginning the procedure.
c) addressing the father's anxiety before beginning the procedure.
d) assembling the phlebotomy equipment before beginning the procedure.

18. By thoroughly answering the parent's questions and communicating her length of experience, the phlebotomist in the case study demonstrated:
a) compassion.
b) proper immobilization technique.
c) satisfaction of practical needs.
d) confidence. 
19. By explaining why the child was too old to have the specimen collected by skin puncture, the phlebotomist in the case study illustrated:
a) age-appropriate terminology.
b) non-threatening tone of voice.
c) knowledge of growth and development.
d) respect for patient privacy.

20. In the case study, which of the following demonstrates that the phlebotomist addressed the personal needs of the patient and his parents?
a) explaining the procedure with compassion.
b) reassuring the parents.
c) addressing the father's anxiety.
d) all of the above.

\subsection{Hand Venipuncture}

It is acceptable to use dorsal hand veins as the venipuncture site. This site should be used only if the antecubital veins are unsuitable, such as when the antecubital area is edematous, burned or scarred, there are casts on both arms, or the veins are thrombosed.

- Select a vein that is palpable, lightly brushing a finger across the back of the hand as it is palpated. This helps select the best vein and determines its direction. Palpate even when the vein is visible.

- Use a $21 \mathrm{~g}$ or $23 \mathrm{~g}$ butterfly blood collection set, depending on the condition of the SP's veins.

- Follow the venipuncture technique described in Section 3.9.3 to draw the blood.

\subsection{Veinlite EMS}

\section{Supplies}

- $\quad 2$ AA Alkaline Batteries

- Disposable Plastic Covers 
The TransLite Veinlite EMS is a hand-held battery powered transillumination device for finding veins and assisting in vein access. It utilizes side-transillumination, which enables the VeinLite EMS to uniformly illuminate a small region of skin and subcutaneous tissue, anywhere on the body, without any areas of shadow.

- Place the Veinlite EMS inside the disposable plastic cover. Check the cover to make sure it is intact.

- Wipe the area of the plastic cover coming in contact with the study participant with an alcohol prep pad.

- Turn off any overhead fluorescent lights as these may interfere with transillumination. Low power, nonfluorescent side lighting may be used.

- Visually locate the region of the skin you wish to examine for veins and then place the Veinlite EMS over it, with the LEDs facing down toward the skin.

- Apply gentle pressure to the Veinlite EMS to ensure it is in contact with the skin.

- Depress the orange button to switch on the LEDs.

- To access a vein while viewing with the Veinlite EMS, place the Veinlite EMS so the opening in the circle of the LEDs faces away from the heart. Apply gentle pressure on the Veinlite EMS to hold the vein. Pull back slightly on the Veinlite EMS to stretch the skin and provide a taut surface for the venipuncture.

- Insert needle in the normal way.

- Discard the disposable plastic cover after each study participant and switch off the Veinlite EMS when it is not in use. 


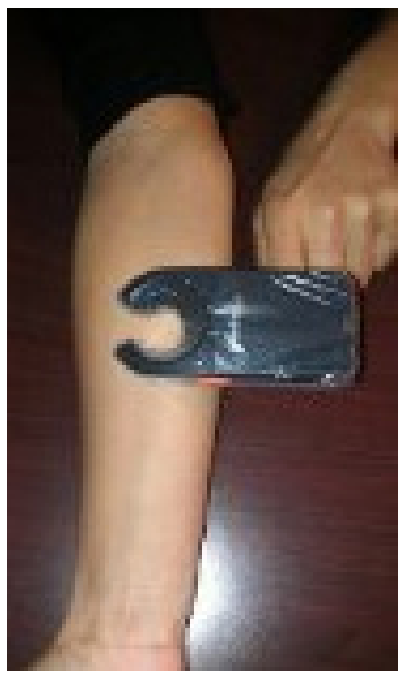

Position of Veinlite EMS while looking for a vein

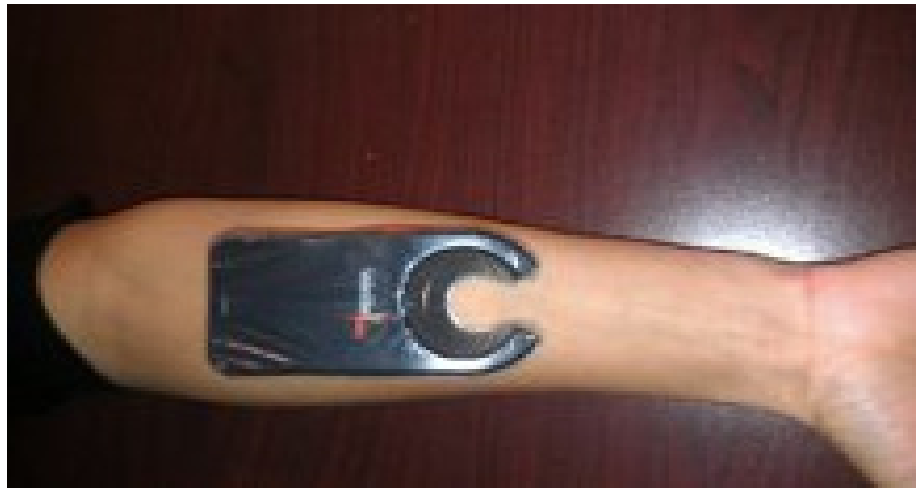

Position of Veinlite EMS while accessing vein

Caution: Do not look at the LEDs when they are switched on or shine the light from the LEDs into anyone's eyes.

\section{Cleaning}

Wipe the device with a Sani-Wipe. Do not immerse the device in liquid. The Veinlite EMS should be wiped daily with an alcohol pre pad to disinfect. 


\subsection{Recording the Results of the Venipuncture Procedure}

The Venipuncture screen displays the tube protocol for the SP.

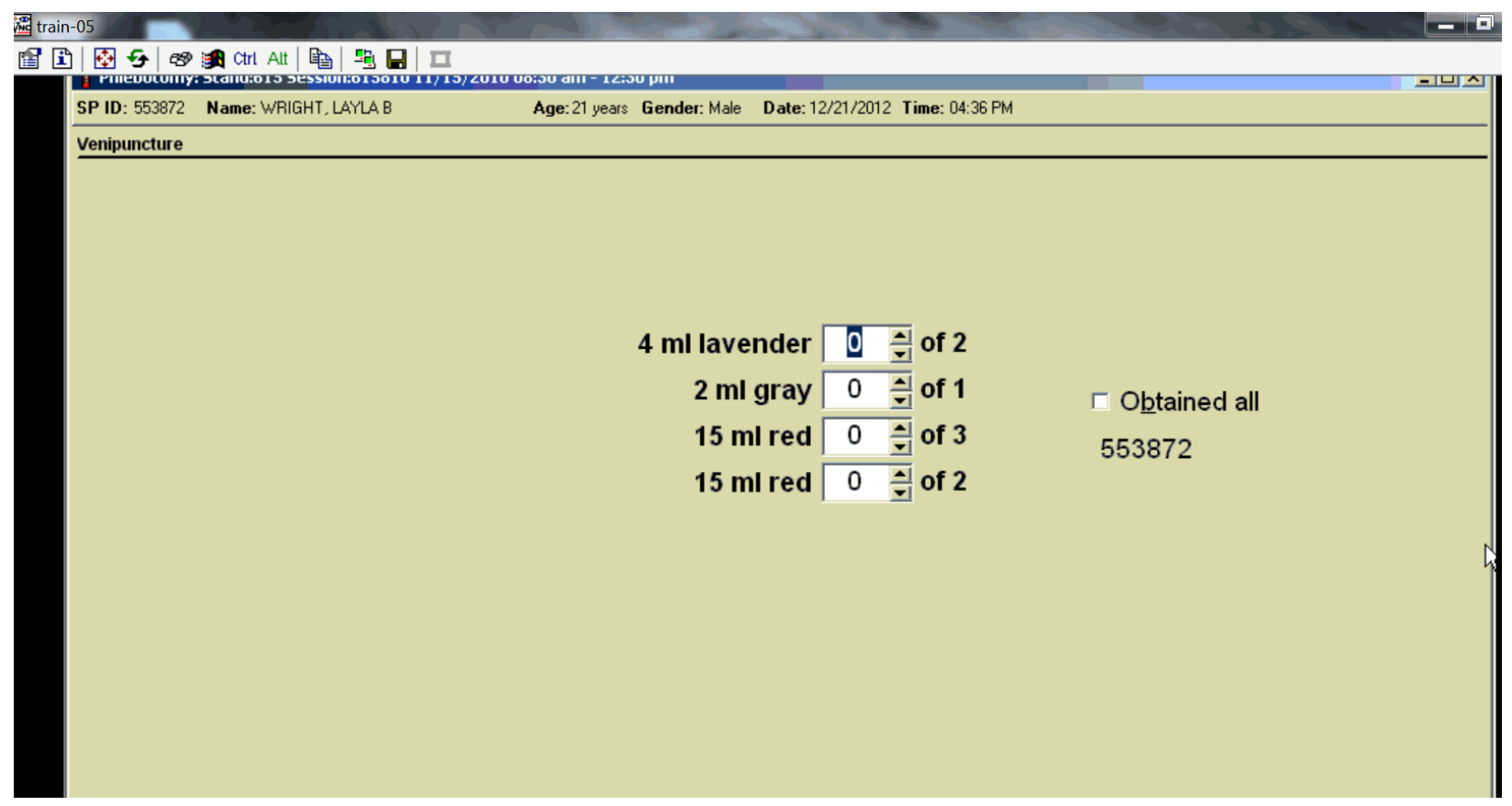

Each SP's profile is dependent on his or her age, appointment type, selection for inclusion into randomly-selected subsamples, and the session time. 
Immediately after completing the venipuncture, enter the results of the blood draw, the reasons for a tube not being drawn according to the protocol, and any comments about the venipuncture.

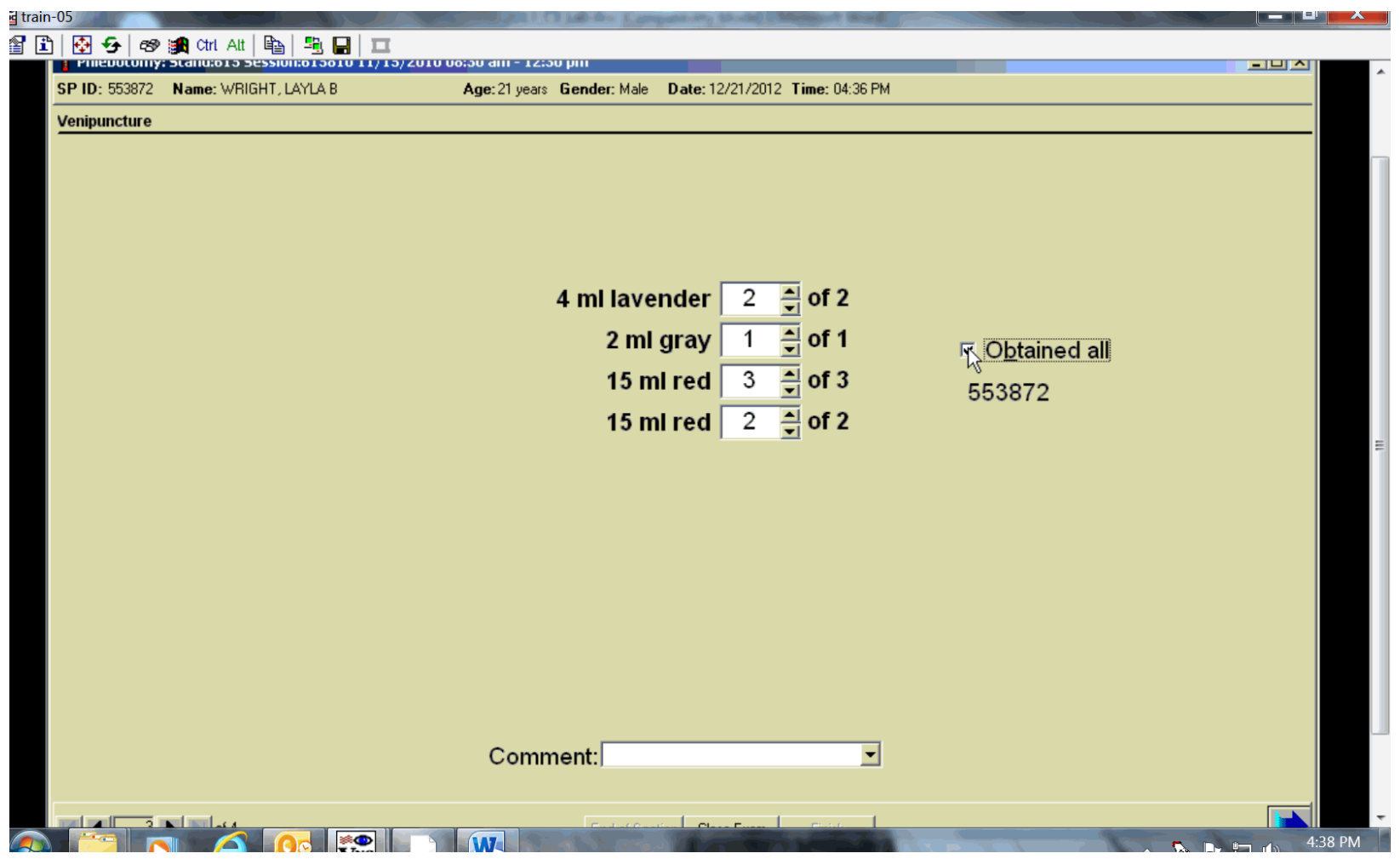

To record all tubes as filled or obtained, move the mouse to the "Obtained all" checkbox and click, or type $[\mathrm{Alt}][\mathrm{B} / \mathrm{b}]$. This records a checkmark in the box and marks all tubes as obtained. To mark individual tubes as filled or obtained, use the mouse to direct the mouse arrow to the up-down controls on the spin box and toggle the number of each tube up or down or type the correct number using the numeric keys.

Document circumstances where the alternative protocol was used or the SP was in the supine position. 


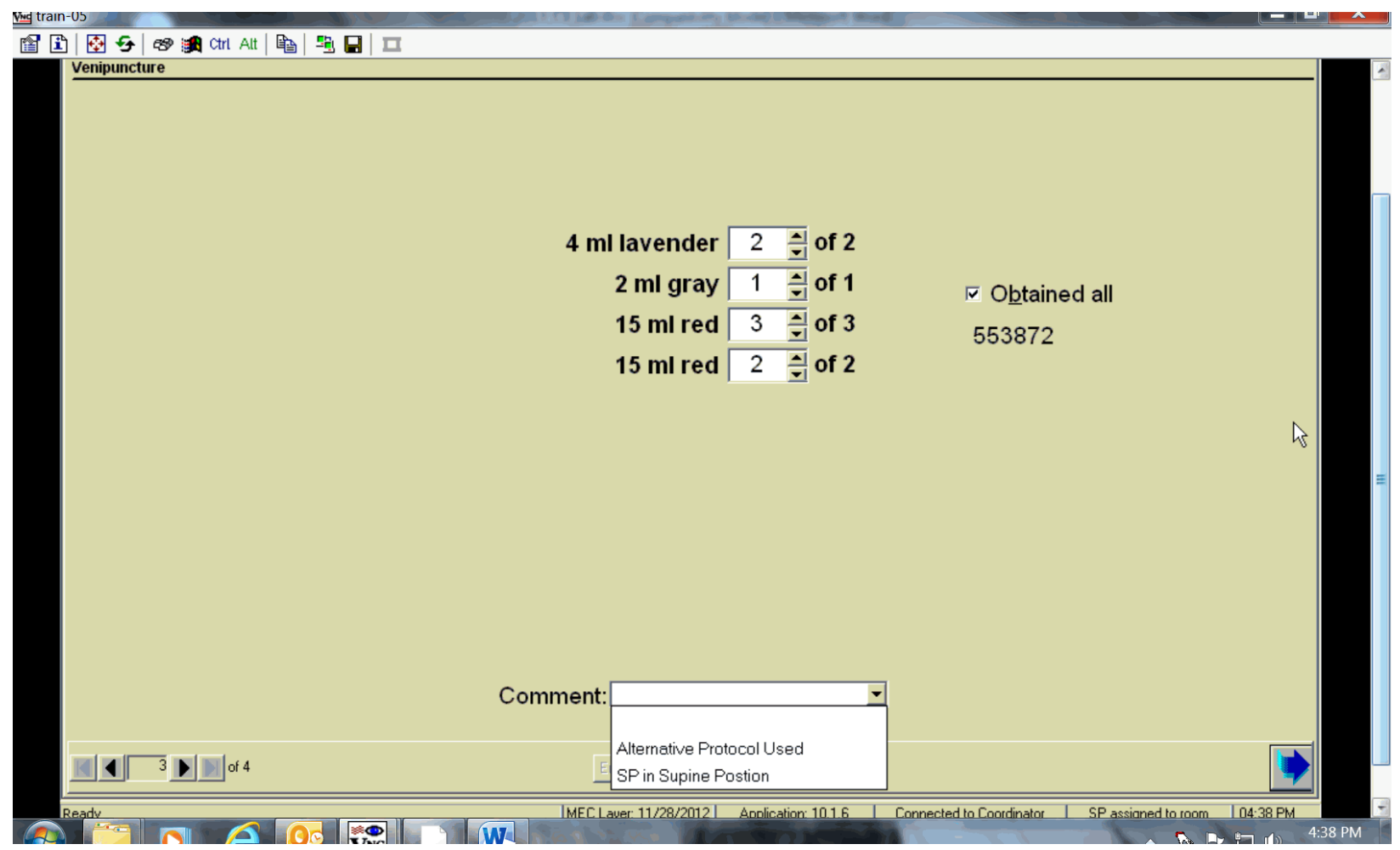

To record either "Alternative Protocol Used" or "SP in Supine Position" comments in the Comment text box, move the mouse to the arrow on the drop-down list, left click, drag the mouse arrow to the desired choice, and left click. Alternatively, type [A/a] for "Alternative Protocol Used" or [S/s] for "SP in Supine Position" and [Enter], or use the up and down arrows to toggle between the two choices and [Tab.]

To progress to the next screen, use the mouse to direct the mouse arrow to the bright blue arrow in the bottom right corner and left click, or select [Enter] when this blue arrow is highlighted.

To reprint the Dymo labels, move forward one screen and back using the navigation arrows. A Print Labels informational text box displays. It is also possible to reprint labels in the review mode. 


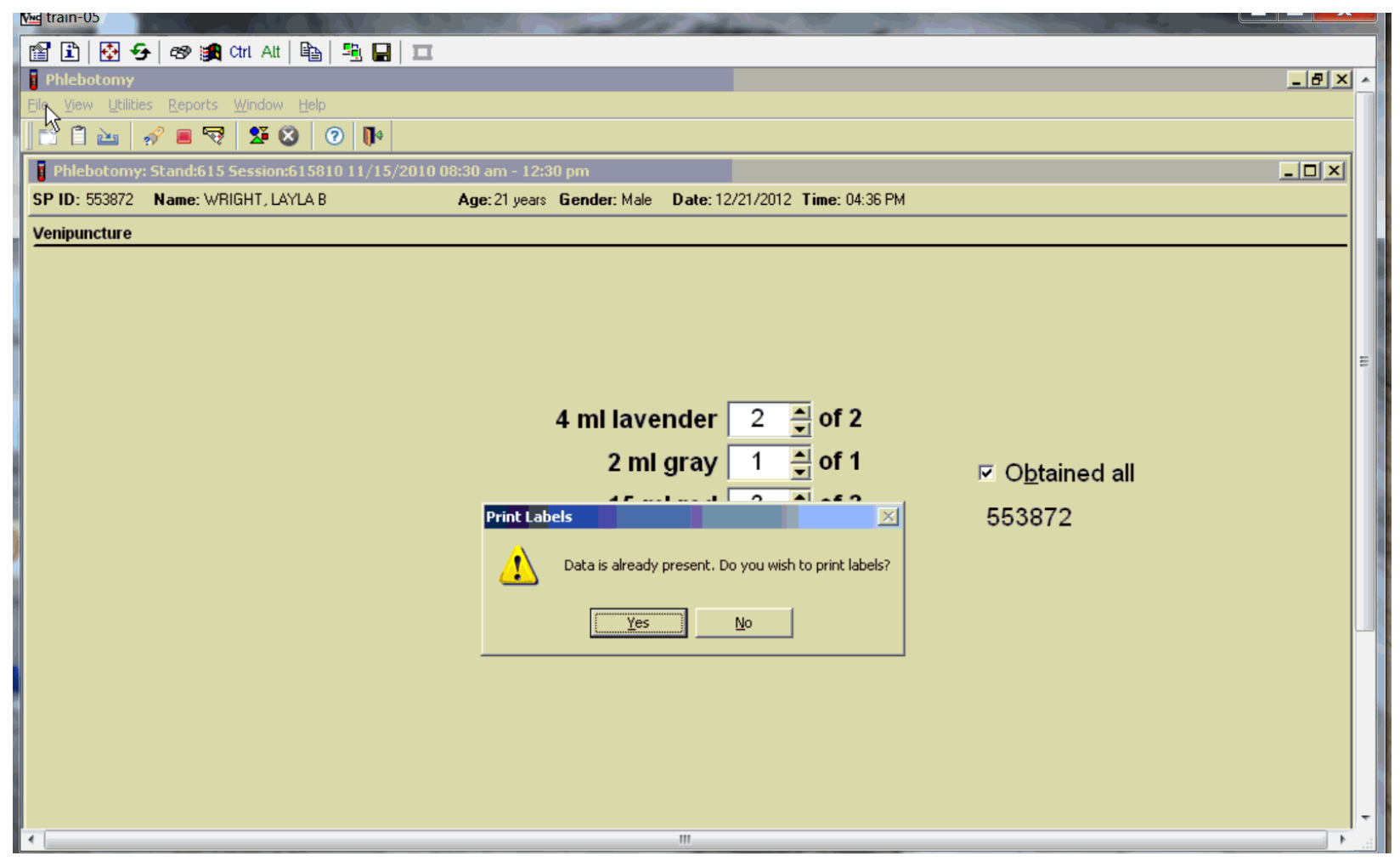

To reprint the labels, use the mouse to direct the mouse arrow to the arrow on the "Yes" button, and left click. To remove the Print Labels informational text box without reprinting the labels, use the mouse to direct the mouse arrow to the "No" button and left click. 


\subsection{Venipuncture Status}

Verify the venipuncture status.

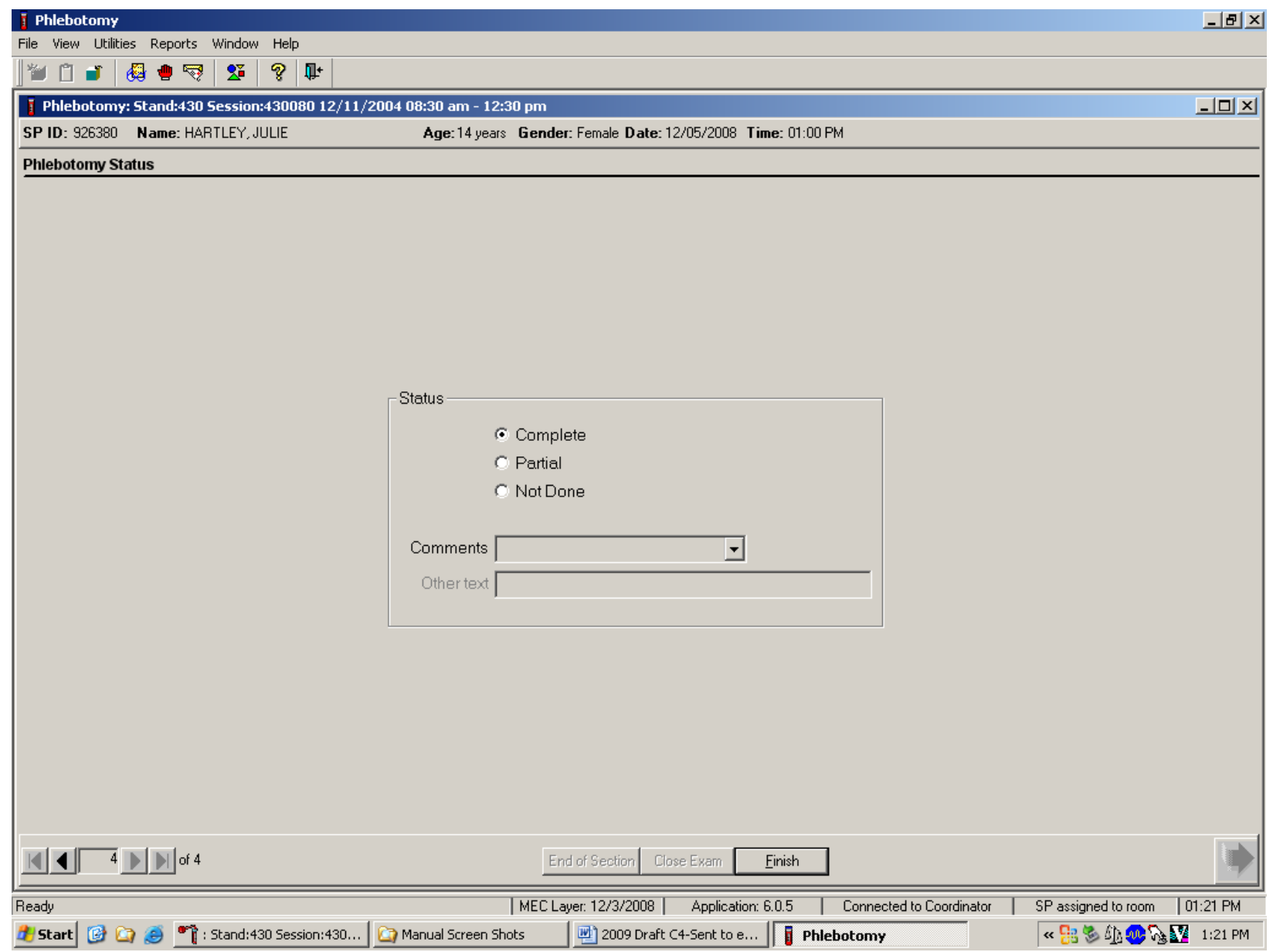

The venipuncture status is complete if all tubes were collected. 
Comment codes are used to explain Partial and Not Done status codes.

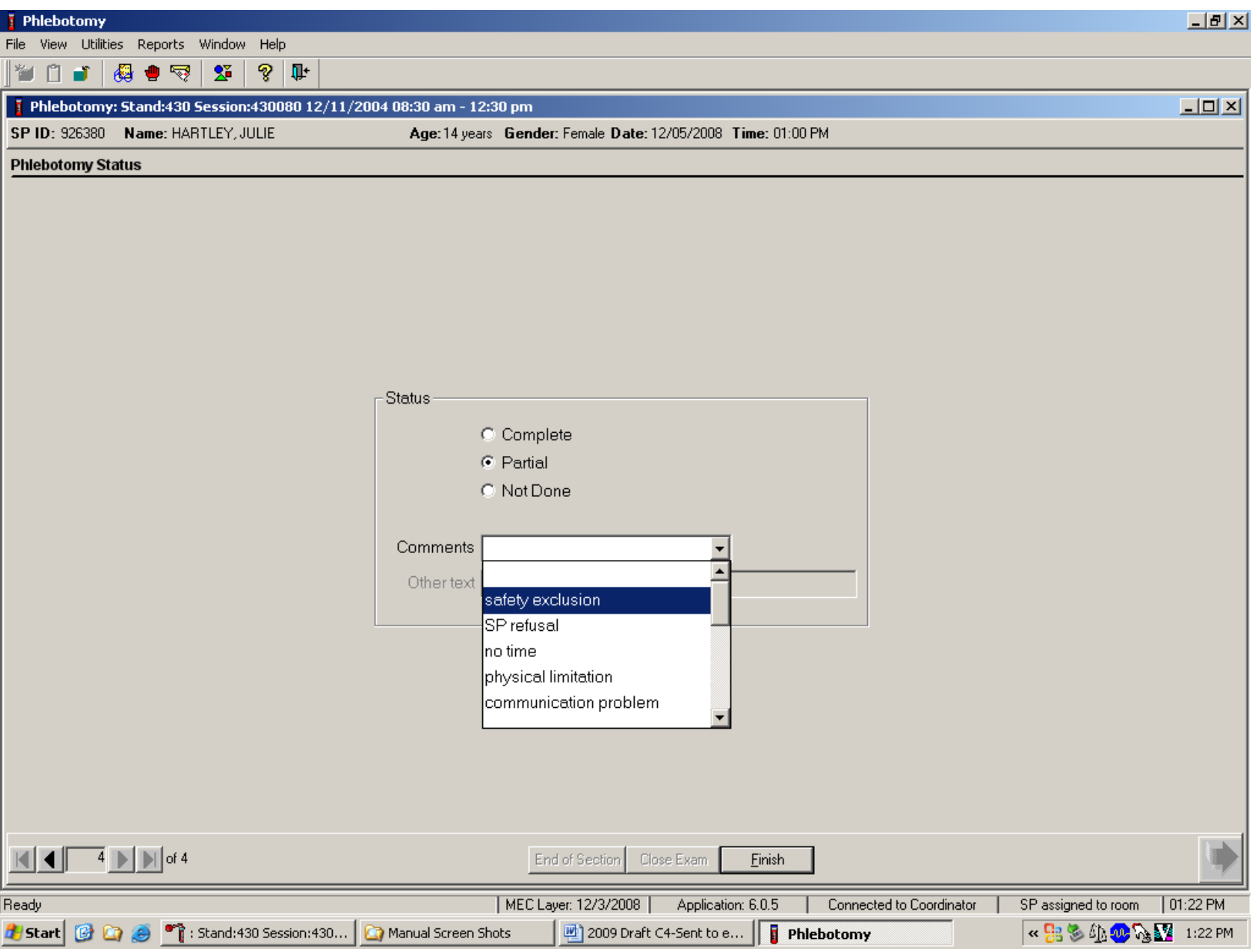

Choose and enter the appropriate comment code when the venipuncture section status is Partial or Not Done. To record a comment in the Comment text box, use the mouse to direct the mouse arrow to the scroll arrow on the drop-down list, left click, drag the mouse arrow to the desired choice, and left click. Use the scroll bar to view all choices. Alternatively, use the up and down keyboard arrows to scroll through the choices or type the first letter of the desired comment code. 


\begin{tabular}{|c|c|}
\hline Comment code & Use when: \\
\hline Safety exclusion & $\begin{array}{l}\text { Not applicable. } \\
\text { This is reserved for positive responses to the hemophilia and chemotherapy } \\
\text { exclusion questions and it is automatically coded by the application. } \\
\text { The coordinator may code exams using this comment. }\end{array}$ \\
\hline SP refusal & $\begin{array}{l}\text { The SP refuses to have his or her blood collected. This is an SP-initiated } \\
\text { nonresponse due to refusal. The SP refuses the component for any reason other } \\
\text { than an illness or emergency. All SPs will be sent to the exam room to have } \\
\text { their arm band barcode scanned and coded. If the SP refuses after starting the } \\
\text { exam, the examiner codes the exam. Use this comment to code partial exams } \\
\text { when the SP refuses after one tube has been drawn, or the blood flow stops } \\
\text { after one tube but before all tubes have been successfully drawn. }\end{array}$ \\
\hline No time & Not applicable. \\
\hline Physical limitation & Not applicable. \\
\hline $\begin{array}{l}\text { Communication } \\
\text { problem }\end{array}$ & Not applicable. \\
\hline Equipment failure & Not applicable. \\
\hline SP ill/emergency & Not applicable. \\
\hline Interrupted & Not applicable. \\
\hline $\begin{array}{l}\text { Error } \\
\text { (technician/software/ } \\
\text { supply) }\end{array}$ & $\begin{array}{l}\text { Use this comment to code Partial and Not Done exams when there are } \\
\text { phlebotomist errors, or software or supply issues. }\end{array}$ \\
\hline Other, specify & $\begin{array}{l}\text { If the above reason for a status code of Not Done is not explained by one of the } \\
\text { comment codes, the examiner must choose Other, specify and record a } \\
\text { comment in the text field. }\end{array}$ \\
\hline No suitable vein & $\begin{array}{l}\text { Use this comment to code exams as Not Done (no tubes drawn) when the SP } \\
\text { does not have an accessible vein, any arms available, casts on both arms, there } \\
\text { is a rash over the entire area, or the blood draw was started but there was no } \\
\text { blood in the tubing. }\end{array}$ \\
\hline Vein collapsed & $\begin{array}{l}\text { Use this comment to code exams as Partial when one or more tubes are } \\
\text { successfully drawn but blood flow stops before all tubes are drawn or when the } \\
\text { vein collapses. }\end{array}$ \\
\hline SP not feeling well & $\begin{array}{l}\text { Use this comment to code Not Done or Partial when the SP is about to faint or } \\
\text { the SP became ill and the test could not be performed on the SP. }\end{array}$ \\
\hline Fainting episode & $\begin{array}{l}\text { Use this comment to code Not Done or Partial when the SP faints and the test } \\
\text { could not be performed on the SP. }\end{array}$ \\
\hline No tubes drawn & Use this comment to code exams as Partial when no tubes are drawn. \\
\hline Language barrier & Not applicable. \\
\hline
\end{tabular}

When finished, use the mouse to direct the mouse arrow to the bright blue arrow in the bottom right corner and left click to exit or select [Enter] when this blue arrow is highlighted. 
Escort the SP to the location indicated in the Message Center text box.

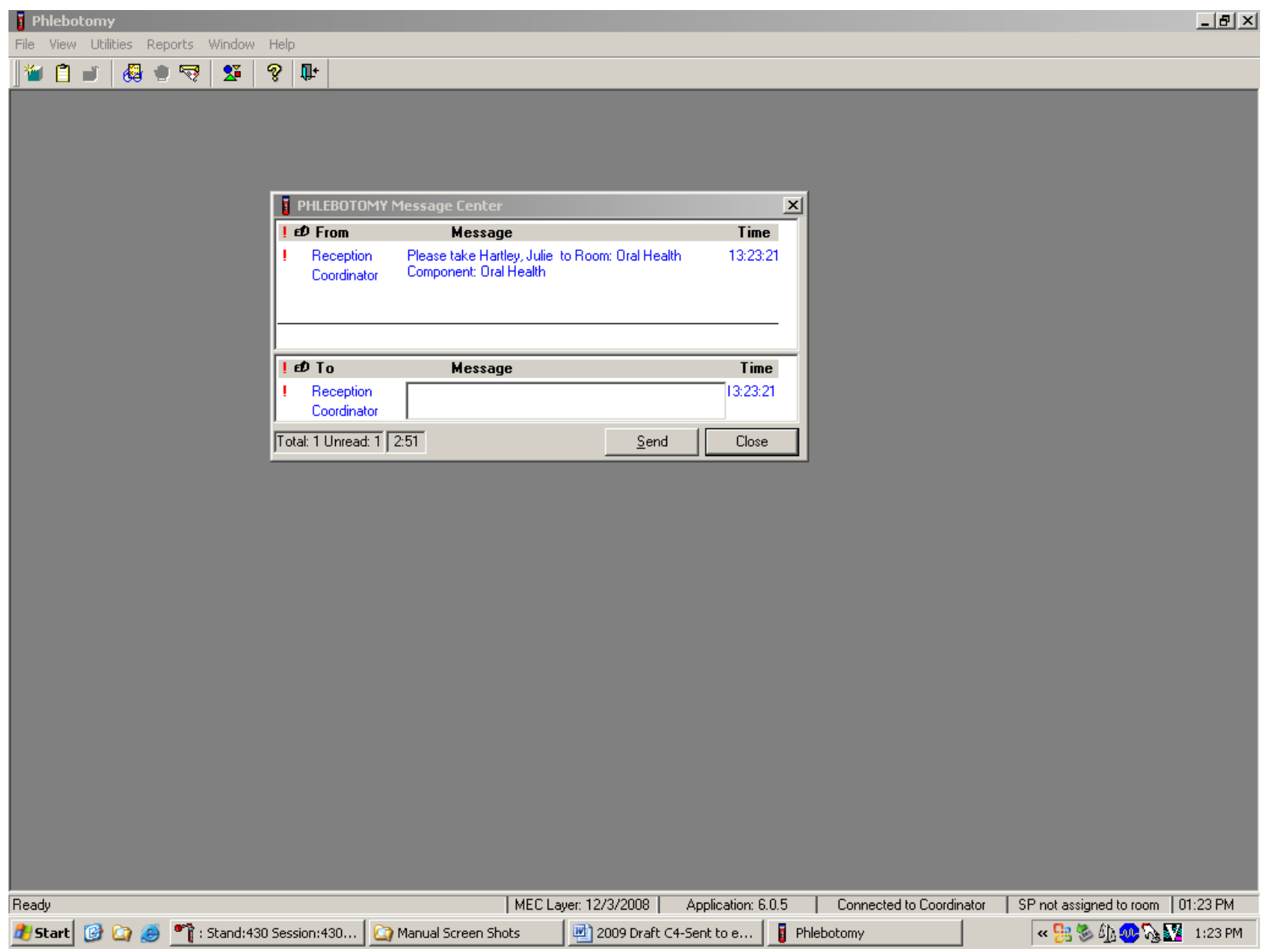

Review the instructions in the Message Center text box and follow the directions. 


\subsection{Repeat Blood Draw}

In rare circumstances, a result from the complete blood count may be an extreme value that would be considered a "panic" value in clinical practice. An example is severe thrombocytopenia. The physician may repeat the complete blood count if the participant reports no history of nosebleeds or prolonged bleeding and the physician verifies that the site of the first venipuncture clotted normally. The second complete blood count will overwrite the first results in ISIS and will be used for reporting and referrals.

\section{Problem Identification}

Rare occurrences of critical CBC results, particularly low platelet counts, have been observed that resulted in an awkward medical referral for the physicians on the MEC. While the med techs and physician may suspect a laboratory instrument or blood collection effect in the reported results, it is not standard practice to repeat the test with a new blood specimen from a second phlebotomy. There is nothing in the phlebotomy, laboratory, and physician protocols that requires a second blood draw on an SP when critical results are obtained.

False low platelet results (low) are most likely the result of micro clots that cannot be observed. The techs do visually look for clots but they will not see micro clots. Many false low platelet results are caused by cold agglutinins. The techs are able to recognize cold agglutinins because the platelet number will decrease each time the blood is run. In this instance they warm the blood and rerun the sample.

The NHANES protocol is unusual because it requires the techs to run all samples in duplicate (whenever possible), whereas a clinical lab would rerun a sample only if the result was abnormal.

\section{Recommended Action}

If the physician feels a repeat blood count is needed, then he or she has the discretion to discuss this with the SP and have the phlebotomist draw a 3-mL EDTA tube for a repeat CBC if the SP 
agrees. Prior to a second phlebotomy, the physician should visually inspect the site of the first venipuncture to rule out prolonged bleeding. If the puncture site looks normal, then a second phlebotomy can be requested. The SP always has the right to refuse the blood draw. The need for this action should be rare as evidenced by the four total low platelet counts in the last 2 years.

After the physician reviews the findings with the SP, and the SP agrees to a repeat CBC phlebotomy:

- The physician should consult with the MEC manager and chief med tech to arrange to have the blood drawn at the next earliest opportunity.

- If the SP is eligible for the second GTT blood draw, we currently require the phlebotomist to draw any tubes that might not have been collected during the first blood draw. The second 3-mL EDTA tube could be drawn at that time but the phlebotomist will need to be alerted because the tube will not display on the GTT blood draw protocol screen.

- If the SP is not eligible for the GTT blood draw, then he or she will need to be walked to phlebotomy to have the blood drawn.

- This additional blood draw will not be supported by the laboratory and phlebotomy applications.

- As long as the SP has not checked out of the MEC or if the Report of Findings has not been printed, the lab application will allow the med techs to overwrite the existing $\mathrm{CBC}$ data with the new data and the new data will become the final result of record.

- The physician will document this event under observations in the physician application. 
Perform phlebotomy quality control at the end of the second session even if there are no SPs scheduled into either session. Record the room temperature reading and the action that the counters were disinfected using the End of Session tab. Record the supply lot numbers and expiration dates by using the Supply Use tab.

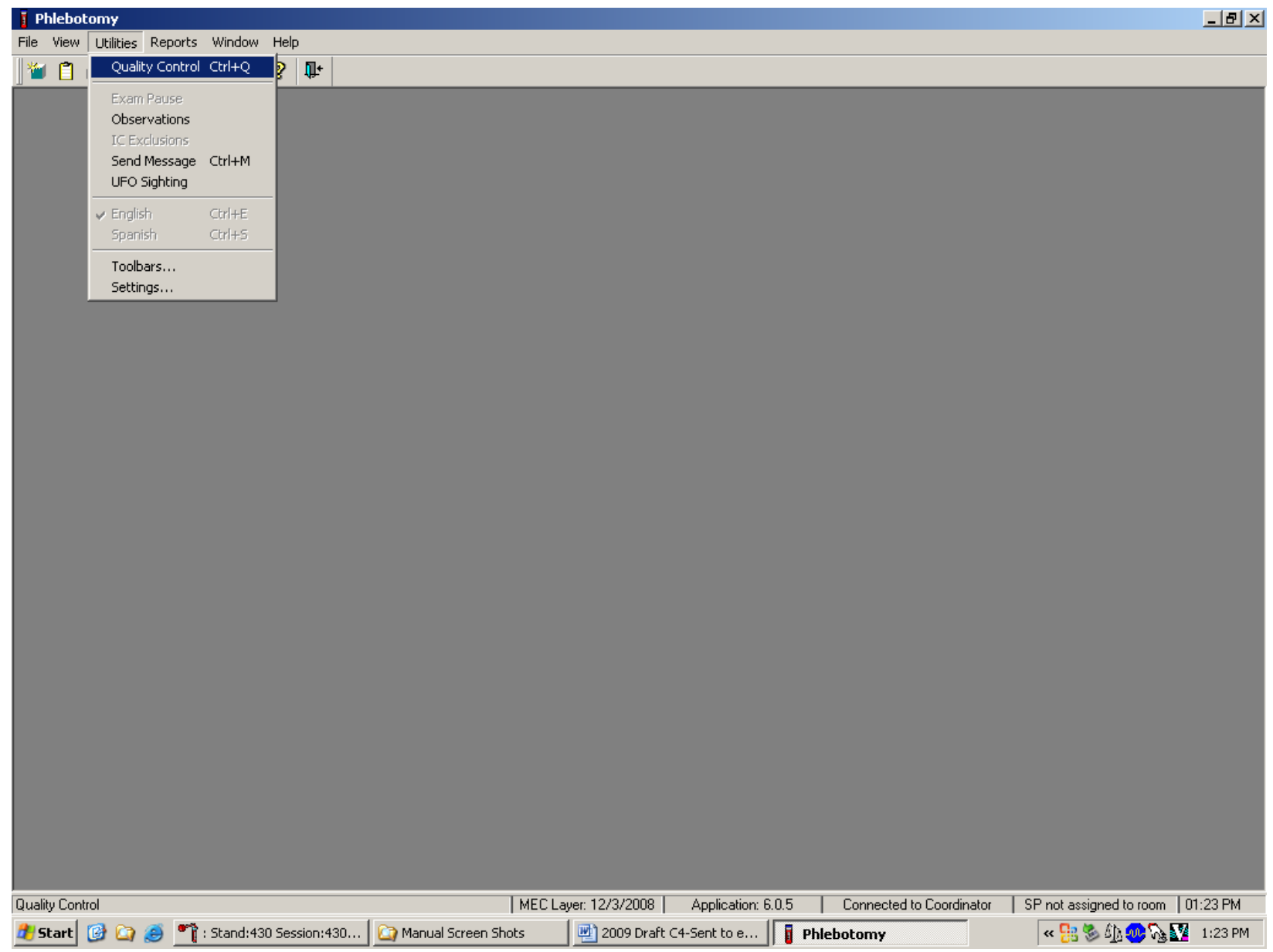

To access the phlebotomy Quality Control module, use the mouse to direct the mouse arrow to $\{$ Utilities\} in the menu bar, left click, drag the arrow to \{Quality Control\} and left click, or select [Alt] $[\mathrm{U} / \mathrm{u}],[\mathrm{Q} / \mathrm{q}]$ or $[\mathrm{Ctrl}][\mathrm{Q} / \mathrm{q}]$. 


\subsubsection{Daily QC}

Record the daily quality control activities at the end of the second session. Mark each Done checkbox with a checkmark.

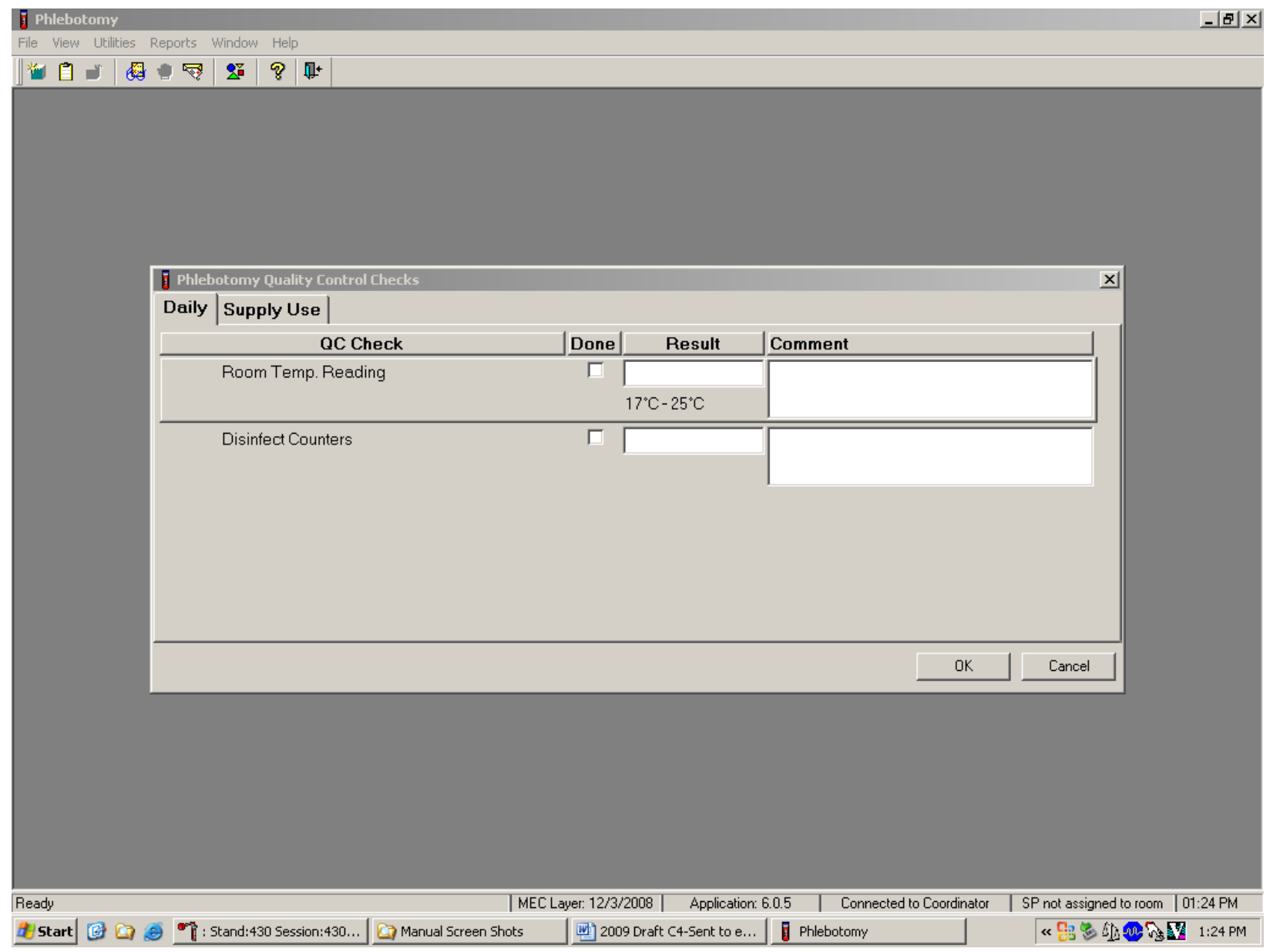

To record a checkmark in the Room Temp. Reading checkbox, use the mouse to direct the mouse arrow to the checkbox in the Done column and left click, then select [Tab] to move to the Result text box. Enter the temperature in the Result text box and select [Tab] to move to the Comment text box. If the reading is outside the established range $\left(17-25^{\circ} \mathrm{C}\right)$, document the actions taken to resolve the situation in the Comment text box. Select [Tab] to move to the next QC item. 
Continue entering the QC.

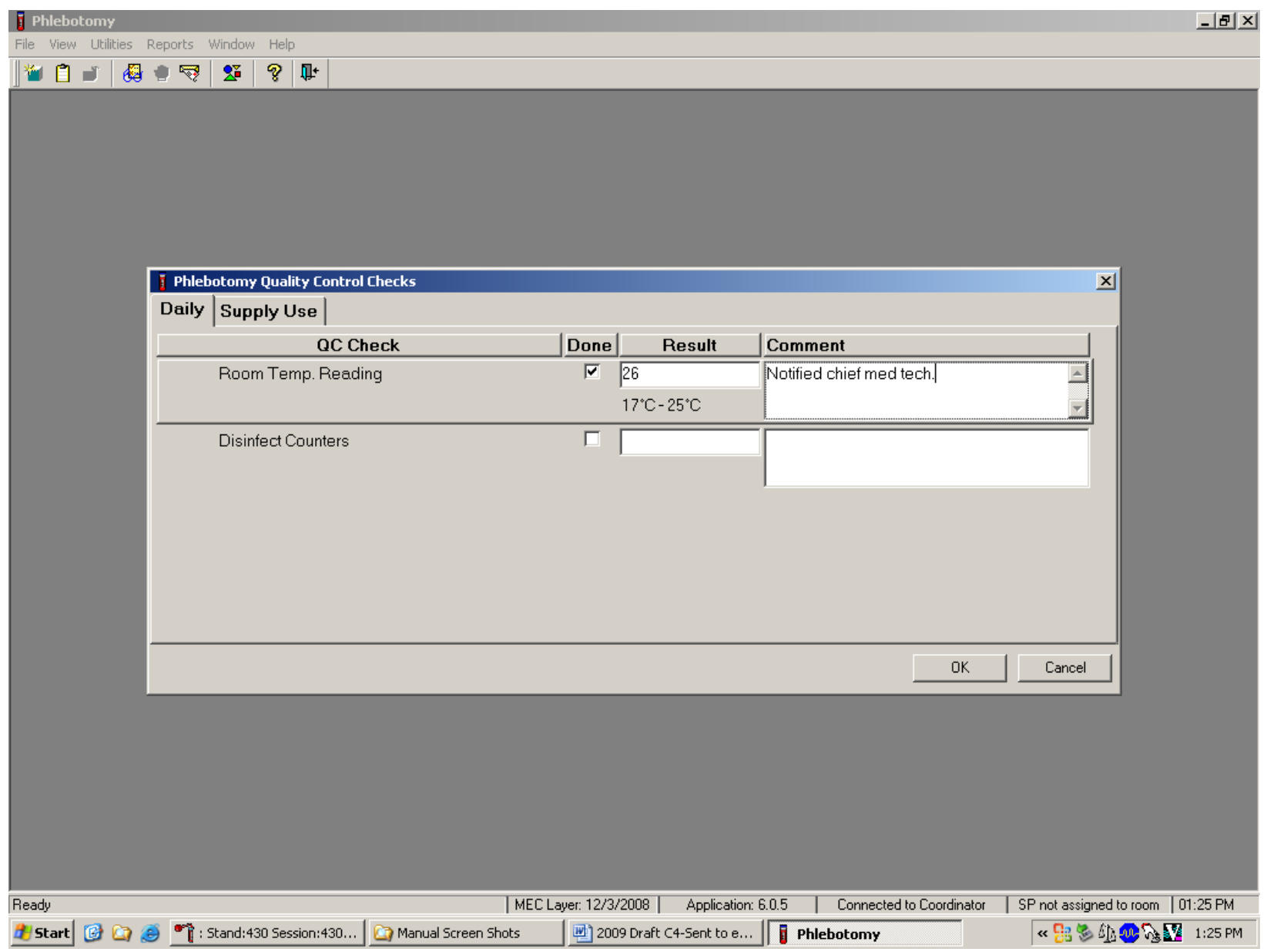

To record a checkmark in the Disinfect Counters checkbox, use the mouse to direct the mouse arrow to the checkbox and left click, then select [Tab] to move to the Result box. Type "Yes" or "Not Done" in the Disinfect Counters Comment text box and then [Tab] to add free form text explaining why the counters were not disinfected for any "Not Done" response. 


\subsubsection{Supply Use Control Log}

Many items have specific lot numbers and expiration dates. Enter the lot numbers and expiration dates in the Supply Use module. This module lists every inventory item associated with the phlebotomy component that includes a lot number and expiration date.

\begin{tabular}{lcc}
\hline \multicolumn{1}{c}{ Item } & Lot Number & Expiration Date \\
\hline 19G Nipro Safe Touch Vein Set & Yes & Yes \\
\hline Luer Adapter & Yes & No \\
\hline Vacutainer Safety-Lok 21G Needle & Yes & Yes \\
\hline Vacutainer Safety-Lok 23G Needle & Yes & Yes \\
\hline BD Vacutainer 6-mL Royal Blue Tube & Yes & Yes \\
\hline BD Vacutainer 3-mL EDTA Blood Tube & Yes & Yes \\
\hline BD Vacutainer 4-mL EDTA Blood Tube & Yes & Yes \\
\hline BD Vacutainer 2-mL Gray Blood Tube & Yes & Yes \\
\hline BD Vacutainer 3-mL Red Blood Tube & Yes & Yes \\
\hline BD Vacutainer 7-mL Red Blood Tube & Yes & Yes \\
\hline BD Vacutainer 10-mL Red Blood Tube & Yes & Yes \\
\hline Sherwood Kendall 15-mL Red Blood Tube & Yes & Yes \\
\hline BD Vacutainer 10-mL EDTA Blood Tube & Yes & Yes \\
\hline VOC 7-mL Vacutainer Blood Tube & Yes & Yes \\
\hline Trutol Glucose Drink - Fruit Punch & Yes & Yes \\
\hline Trutol Glucose Drink - Lemon/Lime & Yes & Yes \\
\hline Trutol Glucose Drink - Orange & Yes & Yes \\
\hline Apple Juice & No & Yes \\
\hline Orange Juice & No & Yes \\
\hline Solar Thermometer & No & Yes \\
\hline Ammonia Ampules & Yes & \\
\hline
\end{tabular}


Use the Supply Use tab to enter lot numbers and expiration dates.

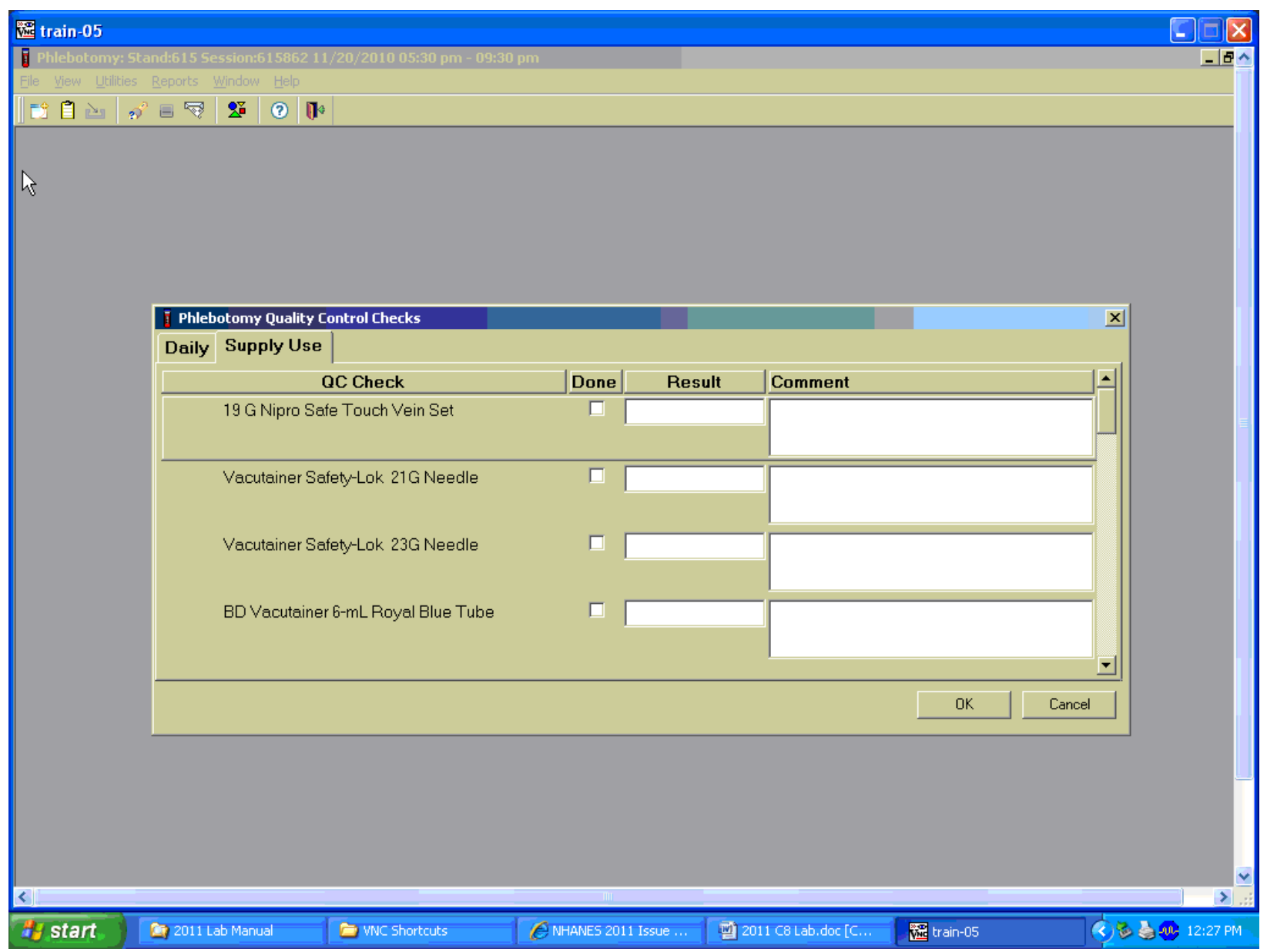

Select the correct activity (Supply Use) by using the mouse to direct the mouse arrow to the correct heading on the top of the file and left click. Mark each checkbox and record the lot and expiration date of each item. Perform this activity at the start of the stand and whenever a new lot is put into use. 
Mark activities as done and enter the lot number and expiration date in the Comment text box.

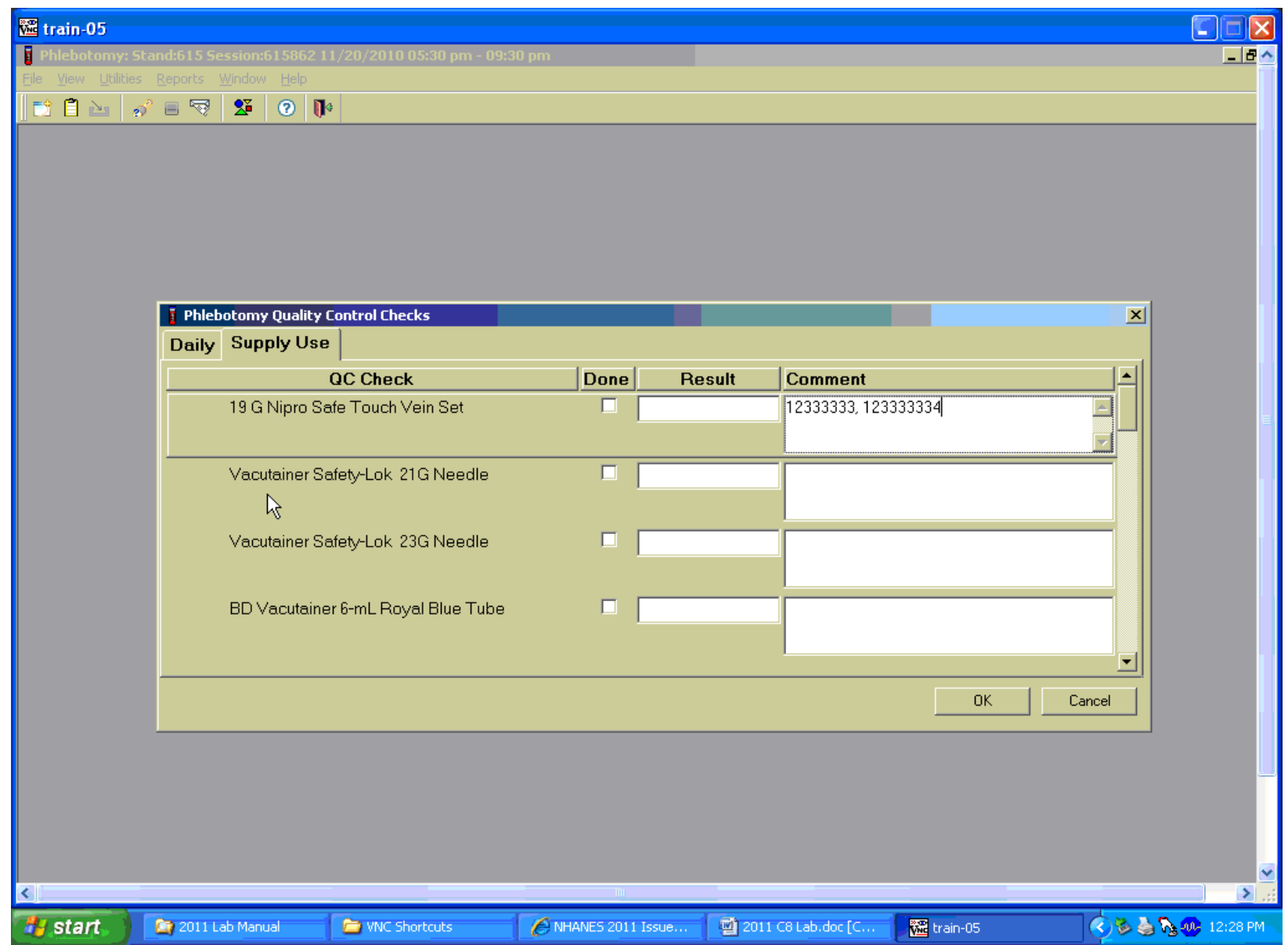

To record an activity as complete or done, use the mouse to direct the mouse arrow to the checkbox in the "Done" column and left click to insert a checkmark. Select [Tab] or [Enter] to move to the Result text box. Do not enter the response or reading in the Result text box. Select [Tab] or [Enter] to move to the Comment text box.

\section{For each item:}

- Record the lot number (if the item does not have a lot number, then record "no lot");

- Add a comma;

- Add a space; and

- Record the expiration date using the MM/DD/YY format. 
There is no frequency requirement for this module within the QC application. Update the information each time a new lot is put into use.

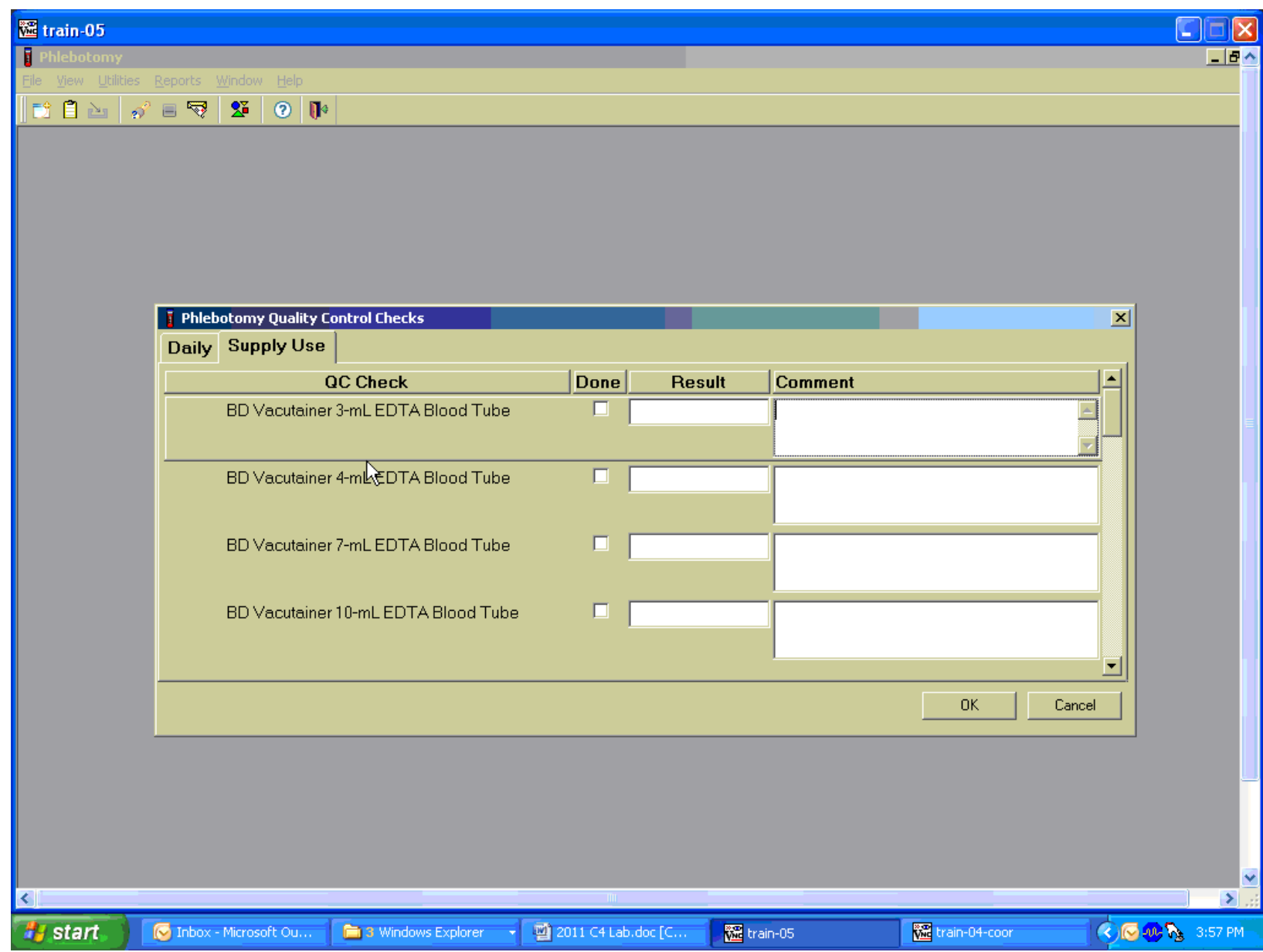

To save these results in the database, use the mouse to direct the mouse arrow to the "OK" button and left click, or select [Enter]. To exit the QC module without saving these actions in the database, use the mouse to direct the mouse arrow to the "Cancel" button and left click.

\subsection{Red Cross Procedures for Handling Fainting (Syncope)}

"Fainting" is a partial or complete loss of consciousness. This is due to a reduced supply of blood to the brain for a short time. Occasionally, a person collapses without warning. Recovery of consciousness usually occurs when the victim falls. Injury may occur from the fall. To prevent a fainting 
attack, a person who feels weak and dizzy should lie down or bend over with his or her head at the level of the knees.

- Signs and symptoms include:

1. Extreme paleness

2. Sweating

3. Coldness of the skin

4. Dizziness

5. Numbness and tingling of the hands and feet

6. Nausea

7. Possible disturbance of vision

If at any time during the venipuncture procedure the SP exhibits any of the manifestations listed above, conclude the venipuncture immediately and perform the first aid procedures listed below.

- Place the SP in a supine position.

- Loosen any tight clothing and keep crowds away.

Provide a disposable airsickness bag if the SP vomits and either roll the SP onto his or her side or turn the SP's head to the side.

- Maintain an open airway.

- Do not pour water over the SP's face because of the danger of aspiration; instead, provide a cool wet cloth to the forehead.

- Do not give any liquid unless the SP has revived.

- Examine the SP to determine if he or she has suffered injury from falling.

- Seek medical assistance. Do not leave the SP. Call for assistance or use the intercom to speak to the coordinator. Observe the SP carefully afterward because fainting might be a brief episode in the development of a serious underlying illness. ${ }^{1}$

\subsection{How to Deal With System Failure}

If the computer system fails, record results on a preprinted Phlebotomy Worksheet (Exhibits 3-6 and 3-7). Complete a Phlebotomy worksheet for each SP while conducting the exam. Enter the results after the system is operational.

\footnotetext{
${ }^{1}$ From Standard First Aid and Personal Safety, Second Edition, the American Red Cross, New York: Doubleday and Company, Inc., 1981, pp. 173-174.
} 
Exhibit 3-6. Phlebotomy worksheet - English Primary

\section{PHLEBOTOMY INTERVIEW, FASTING INTERVIEW, and PROTOCOL PRIMARY SP}

SP ID

\begin{tabular}{|c|c|c|}
\hline Phlebotomy Interview & \multicolumn{2}{|c|}{$\begin{array}{l}\text { Responses } \\
\text { Yes, No, Refused, Don't Know }\end{array}$} \\
\hline \multicolumn{3}{|l|}{ Do you have hemophilia? } \\
\hline \multicolumn{3}{|l|}{ Have you received cancer chemotherapy in the past 4 weeks? } \\
\hline Fasting Questionnaire & \multicolumn{2}{|c|}{ Record time and date } \\
\hline \multicolumn{3}{|l|}{$\begin{array}{l}\text { Q1. When was the last time you ate or drank anything other } \\
\text { than plain water? Do not include diet soda or black coffee } \\
\text { or tea with artificial sweeteners like Sweet'N Low, } \\
\text { NutraSweet, Equal, or Splenda. }\end{array}$} \\
\hline $\begin{array}{l}\text { Confirmation Question: } \\
\text { Q2. Have you had any of the following since \{insert time from } \\
\text { Q1 here\}? }\end{array}$ & $\begin{array}{l}\text { Responses } \\
\underline{\text { Yes, No }}\end{array}$ & $\begin{array}{l}\text { If Yes, record time } \\
\text { and date }\end{array}$ \\
\hline \multicolumn{3}{|l|}{$\begin{array}{l}\text { Coffee or tea with cream or sugar? [Include milk or non- } \\
\text { dairy creamers.] }\end{array}$} \\
\hline \multicolumn{3}{|l|}{ Alcohol, such as beer, wine, or liquor? } \\
\hline \multicolumn{3}{|l|}{$\begin{array}{l}\text { Gum, breath mints, lozenges, or cough drops, or other } \\
\text { cough or cold remedies? }\end{array}$} \\
\hline \multicolumn{3}{|l|}{ Antacids, laxatives, or anti-diarrheals? } \\
\hline \multicolumn{3}{|l|}{$\begin{array}{l}\text { Dietary supplements such as vitamins and minerals? } \\
\text { [Include multivitamins and single nutrient supplements.] }\end{array}$} \\
\hline Primary SP Protocol & \# Filled & Not Filled \\
\hline \multicolumn{3}{|l|}{ 3-mL lavender (1 age 1-2 and2 age 3-5) } \\
\hline \multicolumn{3}{|l|}{ 4-mL lavender $(2$ ages $6+$} \\
\hline \multicolumn{3}{|l|}{ 2-mL gray (morning only 1 age $12+$ ) } \\
\hline \multicolumn{3}{|l|}{$6-\mathrm{mL}$ royal blue tops $(1$ age $6+)$} \\
\hline \multicolumn{3}{|l|}{$3-\mathrm{mL}$ red $(2$ age $1-2,1$ age $3-5)$} \\
\hline \multicolumn{3}{|l|}{ 7-mL red ( 1 age $3-5,1$ age $6-11$ and 1 age $12-19)$} \\
\hline \multicolumn{3}{|l|}{$15-\mathrm{mL}$ red $(2$ age $12-19,3$ age $20+)$} \\
\hline \multicolumn{3}{|l|}{ 10-mL red (1 age 6-11) } \\
\hline \multicolumn{3}{|l|}{$\begin{array}{l}\text { 15-mL red ( } 3 \text { age } 12+\text { if eligible for Persistent Pesticides, } \\
\text { Perfluoronated, and BFR subsamples) }\end{array}$} \\
\hline \multicolumn{3}{|l|}{ 7-mL gray ( 1 age $12+$ if eligible for VOC subsample) } \\
\hline 2-mL gray ( 1 age $12+$ OGTT $)$ & & \\
\hline Comments: & & \\
\hline
\end{tabular}


Exhibit 3-7. Phlebotomy worksheet - Spanish Primary

\section{PHLEBOTOMY INTERVIEW, FASTING INTERVIEW, and PROTOCOL PRIMARY SP}

SP ID

\begin{tabular}{|c|c|c|}
\hline Phlebotomy Interview & \multicolumn{2}{|c|}{$\begin{array}{l}\text { Responses } \\
\text { Yes, No, Refused, Don't Know }\end{array}$} \\
\hline \multicolumn{3}{|l|}{ ¿Tiene hemofilia? } \\
\hline \multicolumn{3}{|l|}{$\begin{array}{l}\text { ¿Ha recibido algún tratamiento para el cáncer en las cuatro } \\
\text { semanas pasadas?? }\end{array}$} \\
\hline Fasting Questionnaire & \multicolumn{2}{|c|}{ Record time and date } \\
\hline \multicolumn{3}{|l|}{$\begin{array}{l}\text { Q1. ¿Cuándo fue la última vez que comió o tomó alguna cosa } \\
\text { aparte de agua sola? No incluya soda de dieta o café o té } \\
\text { solo endulzado con endulzadores artificiales como } \\
\text { Sweet'N Low, NutraSweet, Equal o Splenda. }\end{array}$} \\
\hline $\begin{array}{l}\text { Confirmation Question: } \\
\text { Q2. ¿Ha tomado o comido algo de lo siguiente desde ayer a las } \\
\quad \text { \{insert time from Q1 here }\} \text { ? }\end{array}$ & $\begin{array}{l}\text { Responses } \\
\text { Yes, No }\end{array}$ & $\begin{array}{l}\text { If Yes, record time } \\
\text { and date }\end{array}$ \\
\hline \multicolumn{3}{|l|}{$\begin{array}{l}\text { ¿Café o té con crema o azúcar? } \\
\text { [Incluya leche o cremas que no sean productos lácteos.] }\end{array}$} \\
\hline \multicolumn{3}{|l|}{ ¿Alcohol, tal como cerveza, vino o licor? } \\
\hline \multicolumn{3}{|l|}{$\begin{array}{l}\text { ¿Chicle, mentas para el aliento, tabletas o pastillas para la } \\
\text { tos, u otra medicina para la tos o el resfriado? }\end{array}$} \\
\hline \multicolumn{3}{|l|}{ ¿Antiácidos, laxantes, o antidiarréicos? } \\
\hline \multicolumn{3}{|l|}{$\begin{array}{l}\text { ¿Suplementos para la dieta tales como vitaminas y } \\
\text { minerales? [Incluya multivitaminas y suplementos } \\
\text { nutritivos individuales.] }\end{array}$} \\
\hline Primary SP Protocol & \# Filled & Not Filled \\
\hline \multicolumn{3}{|l|}{ 3-mL lavender (1 age $1-2$ and 2 age $3-5)$} \\
\hline \multicolumn{3}{|l|}{ 4-mL lavender (2 age $6+)$} \\
\hline \multicolumn{3}{|l|}{ 2-mL gray (morning only 1 age $12+$ ) } \\
\hline \multicolumn{3}{|l|}{$6-\mathrm{mL}$ royal blue tops $(1$ age $6+)$} \\
\hline \multicolumn{3}{|l|}{$3-\mathrm{mL}$ red (2 age $1-2,1$ age $3-5)$} \\
\hline \multicolumn{3}{|l|}{ 7-mL red (1 age $3-5,1$ age $6-11$ and 1 age $12-19)$} \\
\hline \multicolumn{3}{|l|}{$15-\mathrm{mL}$ red $(2$ age $12-19,3$ age $20+)$} \\
\hline \multicolumn{3}{|l|}{$10-\mathrm{mL}$ red $(1$ age $6-11)$} \\
\hline \multicolumn{3}{|l|}{$\begin{array}{l}15-\mathrm{mL} \text { red ( } 3 \text { age } 12+\text { if eligible for Persistent Pesticides, } \\
\text { Perfluoronated, and BFR subsamples) }\end{array}$} \\
\hline \multicolumn{3}{|l|}{ 7-mL gray ( 1 age $12+$ if eligible for VOC subsample) } \\
\hline 2 -mL gray $(1$ age $12+$ OGTT $)$ & & \\
\hline
\end{tabular}


The following four screenshots document the English text and official Spanish translations for the phlebotomy component:

- Phlebotomy Interview English,

- Phlebotomy Interview Spanish,

- Fasting Questionnaire English, and

- Fasting Questionnaire Spanish.

Phlebotomy Interview English

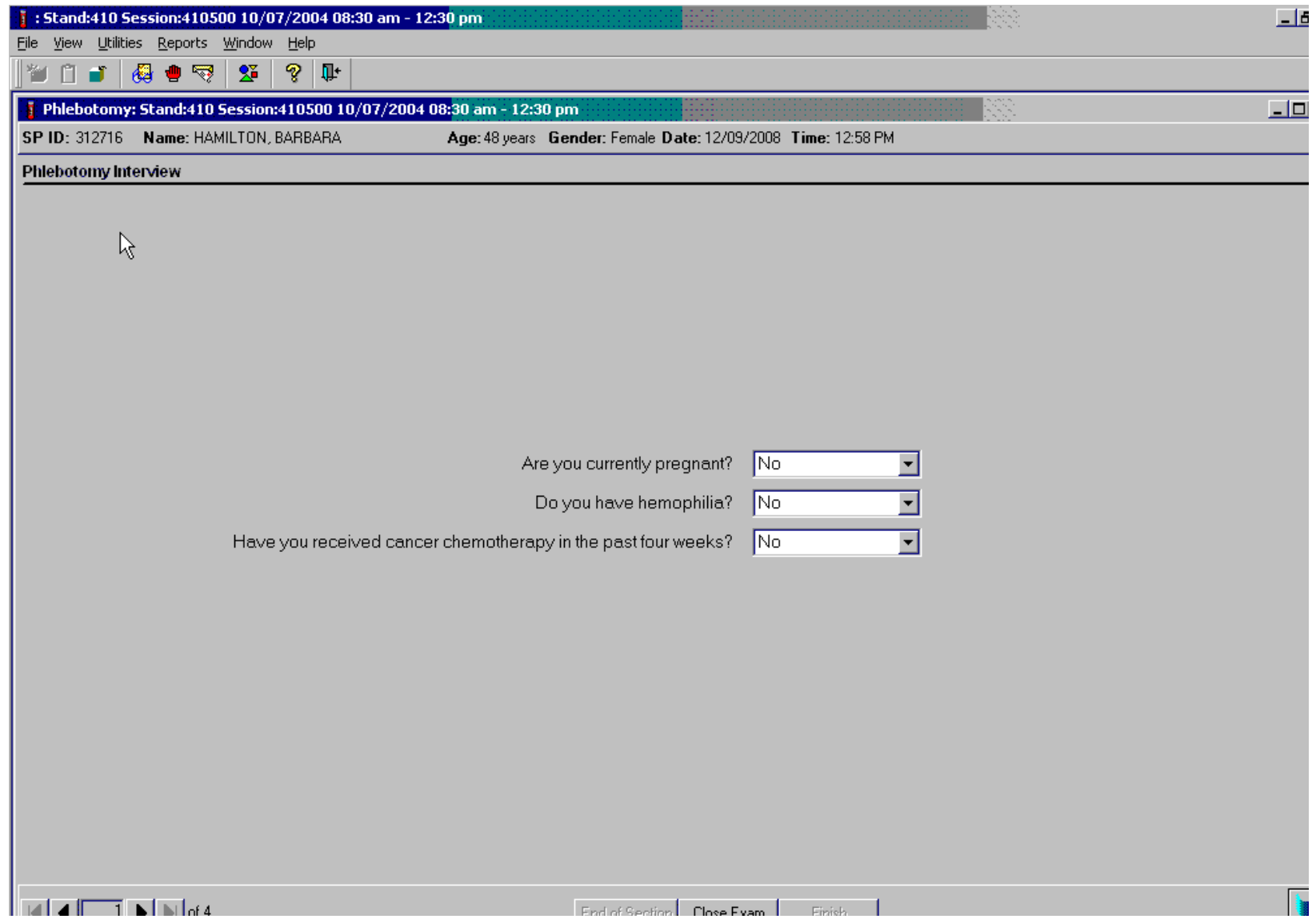


Phlebotomy Interview Spanish

: : Stand:410 Session:410500 10/07/2004 08:30 am - 12:30 pm

File View Uttilities Reports window Help

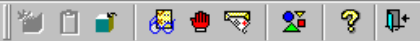

Phlebotomy: Stand:410 Session:410500 10/07/2004 08:30 am - 12:30 pm

Age: 48 years Gender: Female Date: 12/09/2008 Time: 12:58 PM

SP ID: 312716 Name: HAMILTON, BARBARA

Phlebotomy Interview

4

$$
\begin{aligned}
\text { ¿Está usted actualmente embarazada? No } & \text { ¿Tiene hemofilia? No No }
\end{aligned}
$$

¿Ha recibido tratamiento de quimioterapia para el cáncer en las cuatro semanas pasadas?

No

$4 \sqrt{1}+1+1+4$

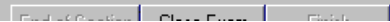




\section{Fasting Questionnaire English}

\section{Phlebotomy}

File View untilities Reports Window Help

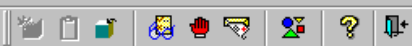

Phlebotomy: Stand:410 Session:410500 10/07/2004 08:30 am -12:30 pm

SP ID: 306325 Name: KAIGA, NEWTON

Age: 13 years Gender: Ma

Date: 12/09/2008 Time: 12:54 PM

Fasting Questionnaire

When was the last time you ate or drank anything other than plain water? Do not $09: 30$ PM 12/08/2008

NutraSweet. Equal, or Splenda

Have you had any of the following since yesterday at 9:30 PM?

$$
\text { Coffee or tea with cream or sugar? [Include milk or non-dairy } \begin{gathered}
\text { creamers.] } \\
\text { No }
\end{gathered}
$$

Alcohol, such as beer, wine, or liquor? No

Gum, breath mints, lozenges or cough drops, or other cough or cold $\begin{gathered}\text { Nomedies? } \\ \text { reme }\end{gathered}$

Antacids, laxatives, or anti-diarrheals? No $\quad$ N

Dietary supplements such as vitamins and minerals? [Include No N multivitamins and single nutrient supplements.]
Fasting
15 Hours
26 Minutes
4 
Fasting Questionnaire Spanish

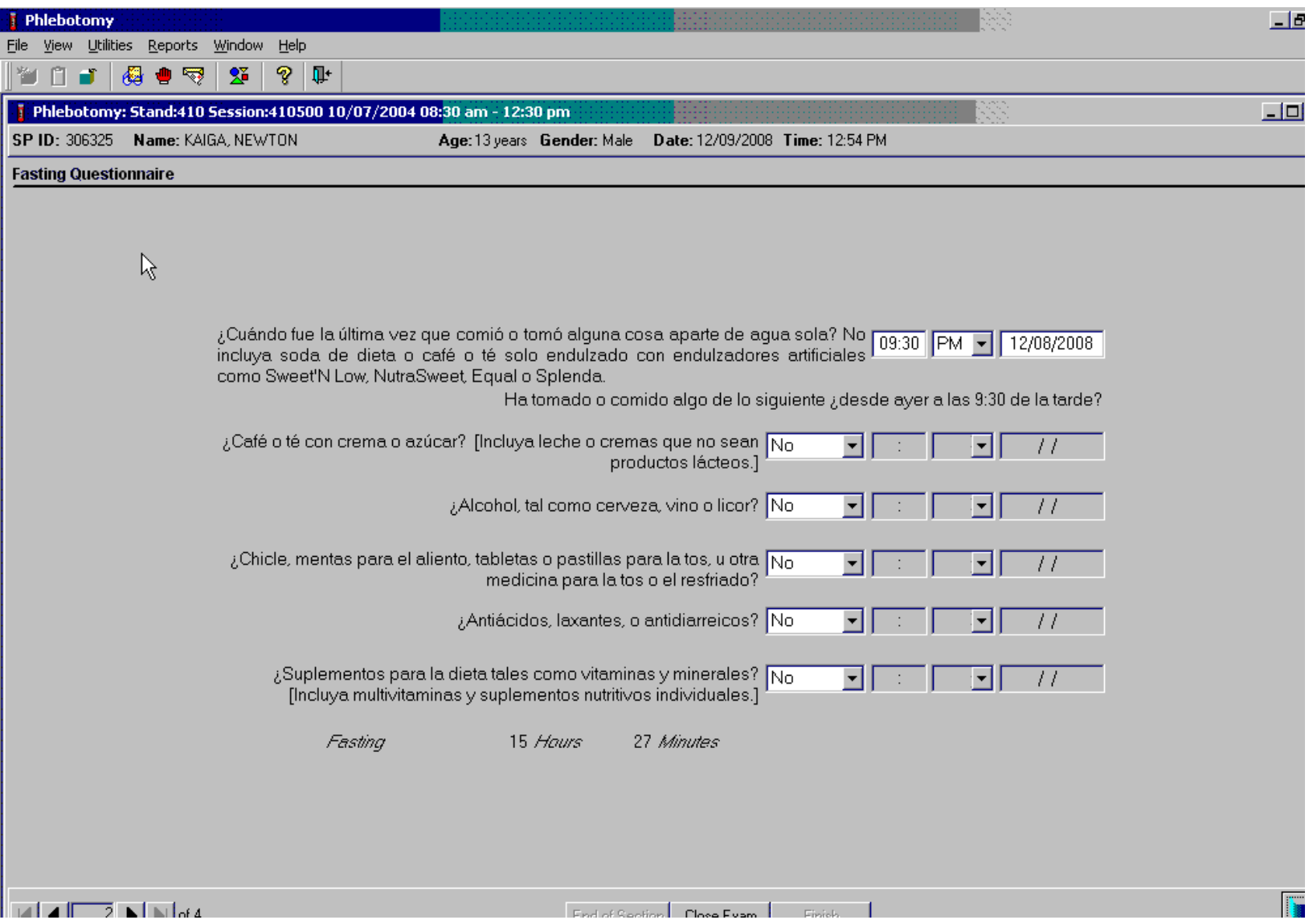




\section{URINE SPECIMEN COLLECTION AND PROCESSING}

\subsection{Introduction}

The purpose of urine collection and processing is to collect sufficient urine from participants aged 6 years and older to be able to (1) collect a date, time, and volume of urine collection for analysis of urine flow rate; (2) perform a pregnancy test on selected females aged 8 to 11 years and all females aged 12 to 59 years to exclude pregnant participants from other components that could put the participant at risk; (3) aliquot urine into vessels for storage and transport to multiple government and contact laboratories for analysis; and (4) aliquot urine into a vessel for future studies.

Collect a urine specimen from all SPs aged 6 years and older. The volume of urine required to complete each SP's protocol is displayed in the urine collection module. Place the urine container on the Mettler Toledo scale and then scan the label on the barcode. The weight is converted to volume and transferred to the urine collection module. Visually compare the volume in the urine container to the volume displayed in the urine collection screen. Accept or correct the weight. Perform the pregnancy test if indicated, perform the osmolality test, process the urine protocol, and store the vessels. At least 5-mL of urine is required on VIP guest and guest appointment types.

The MEC coordinator instructs each SP aged 6 years and older to provide a urine sample as soon as possible when he or she enters the MEC. Ideally, this sample would be collected at the same time that the SP changes into the exam outfit. If the SP cannot provide a urine sample at that time, the coordinator offers the SP a glass of water and asks the SP to provide a sample as soon as possible. The SP is instructed to collect a complete void by emptying the bladder into the urine collection cup. The coordinator, assistant coordinator, or any MEC staff member is responsible for transporting the urine specimen to the laboratory.

The coordinator explains the following instructions to the subject before urine collection:

- It is important to collect ALL urine by COMPLETELY emptying your bladder into the cup.

- Wash your hands with soap and water.

- Take the cap off the cup. 
- Urinate directly into the cup until it is full or until you have finished urinating.

- DO NOT OVERFILL THE CUP.

- The inside of the cup and cap should not touch or come into contact with any part of your body, clothing, or external surfaces.

- Recap the collection cup and seal tightly.

- Please remember to wash your hands.

\subsection{Supplies}

The supplies needed for urine collection and urine processing and storage are listed in Exhibits 4-1 and 4-2.

Exhibit 4-1. Urine collection equipment

\begin{tabular}{|c|}
\hline Blue Scale Carrying Case \\
\hline Mettler Toledo PL601-S Scale \\
Cone protector \\
Weigh pan \\
One Troemner 100g weight (start of stand QC) \\
Two Troemner 300g weights (start of stand QC) \\
\hline Care Pac M \\
\hline Cleaning cloth \\
Two gloves \\
Weight transfer tool for 500g weight \\
Weight transfer forceps for 10g weight \\
Cleaning brush \\
One Ohaus 500g weight (daily QC) \\
One Ohaus 10g weight (daily QC) \\
\hline Clear Acrylic Scale Cover \\
\hline Barcode Scanner \\
\hline
\end{tabular}


Exhibit 4-2. Urine collection supplies - urine processing and storage

\begin{tabular}{|l|l|}
\hline 5-mL cryovial sterile & 14-mL Falcon snap cap test tube \\
\hline 2-mL cryovial & Cube rack -4 way flipper rack \\
\hline $\begin{array}{l}\text { 10-mL VWR screwcap vial with colored dot } \\
\text { (Urn Merc) }\end{array}$ & 10-mL VWR screwcap vial \\
\hline Bench Kote paper & Kimwipes \\
\hline $10 \times 10$ three-inch box & $\begin{array}{l}5.25 \times 5.25 \times 3.0-\text { inch cardboard box with } 9 \times 9 \\
\text { cardboard grid }\end{array}$ \\
\hline $5.25 \times 5.25 \times 3.0$-inch cardboard box without grid & Three-inch 5 x 5 cardboard grid \\
\hline 3.5 -mL-Starstedt Transfer pipette & $\begin{array}{l}\text { VWR 15-mL narrow stem, large bulb disposable } \\
\text { transfer pipette }\end{array}$ \\
\hline $\begin{array}{l}\text { Nonsterile, powder-free, gloves-small, medium, } \\
\text { large }\end{array}$ & Windex for scale cleaning \\
\hline
\end{tabular}

\subsection{Urine Flow Rate Background}

The NHANES protocol includes many measures of environmental toxicants or their metabolites in urine. It is difficult to quantify the level of exposure based on measurements in urine because concentrations will vary by the amount of urine produced. Currently, NHANES urine measurements are adjusted by the urinary creatinine concentration to account for variations in urinary dilution. However, creatinine excretion into the urine varies by many factors other than the urinary dilution (e.g., age, gender, muscle mass, and diet). Also, the amount of toxicant excreted in a 24-hour period is often needed to quantify exposures for risk and exposure assessment purposes. Ideally, this requires 24-hour urine collections to quantify. However, it is possible to estimate the mass of analyte excreted in the urine in a 24-hour period by estimating the urine flow for the spot urine specimen collection (i.e., equivalent to a single urine specimen as collected in the current NHANES protocol).

The date and time of last urine void, along with the volume of the urine specimen and the date and time of urine collection captured during urine collection, will provide NCHS with a measurement of urine flow rate. These items are captured during the SP's visit to the MEC. All SPs aged 6 years and older will be eligible for urine flow rate. Urine specimen volume and date and time of collection will be captured only on SPs who submit a urine specimen.

The MEC appointment reminder letter will include the reminder "Before arriving at the exam center, please make a note of the time you last went to the bathroom and urinated (peed). Please write the time on the appointment card you were given. You will be asked for this time when you get to 
the exam center," and will provide a space for the SP to write a response. When the SP arrives at the MEC, the coordinator will prompt the SP to write down the time of his or her last urine void on the verification form. The coordinator will record this time. If there is any discrepancy between the time on the reminder letter and the time reported at the MEC, the coordinator should use the time reported at the MEC.

\subsection{Using the Scale for Urine Collection}

The Mettler Toledo scale is located in the clean laboratory area near workstation 3. Check to make sure the scale's weighing pan is free of any objects or debris. Turn on the Mettler Toledo scale by pressing the "On" button. After the display briefly lights up all segments, the LED will display "0.0 g." This indicates that the scale is ready to weigh samples. The scale should be used only when set to measure in grams. Cover the scale with the clear acrylic scale cover until a sample is available for weighing.

Ensure that computer workstation 3 (Urine/Sink) is turned on and a technician is logged into the Laboratory application. Open the Urine Scale application by double clicking on the Urine Scale icon on the desktop. The Urine Scale icon looks like this:

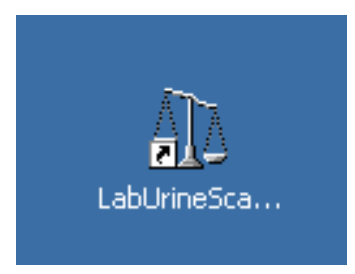

The Urine Scale application icon will appear in the system tray, similar to the Coulter application. This application captures data from the scale and logs it into the database. The Urine Scale application must be opened and in the system tray in order to capture any urine collection readings from the scale.

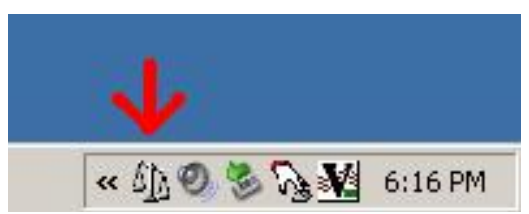


Place the urine collection cup on the Mettler Toledo scale. Cover the scale with the clear box cover to protect the scale from draft. Look at the scale's LED display. In the bottom left corner of the display the stability detector "o" will appear briefly. Once the stability indicator goes away, the weight result is valid. Scan the barcode on the cup using the barcode scanner next to the scale.

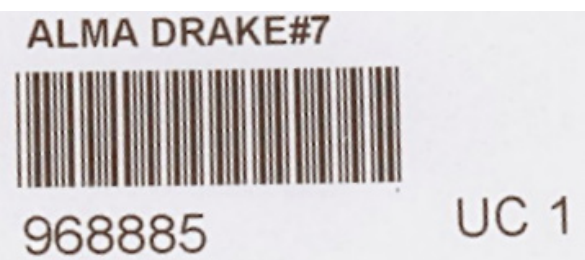

Look at the scale's LED screen to ensure that the SP ID displays. Checking for the SP ID display on the LED screen is verification that the collection was recorded in the database. 


\section{5}

Document Urine Collection

Once a urine sample has been scanned in, the urine collection (UC) circle on the heads-up screen for that SP will turn blue $(\odot$ for quantity not sufficient (QNS) or $\odot$ for Yes). The blue color indicates that there is a urine sample in the lab ready to be validated by the laboratory technician.

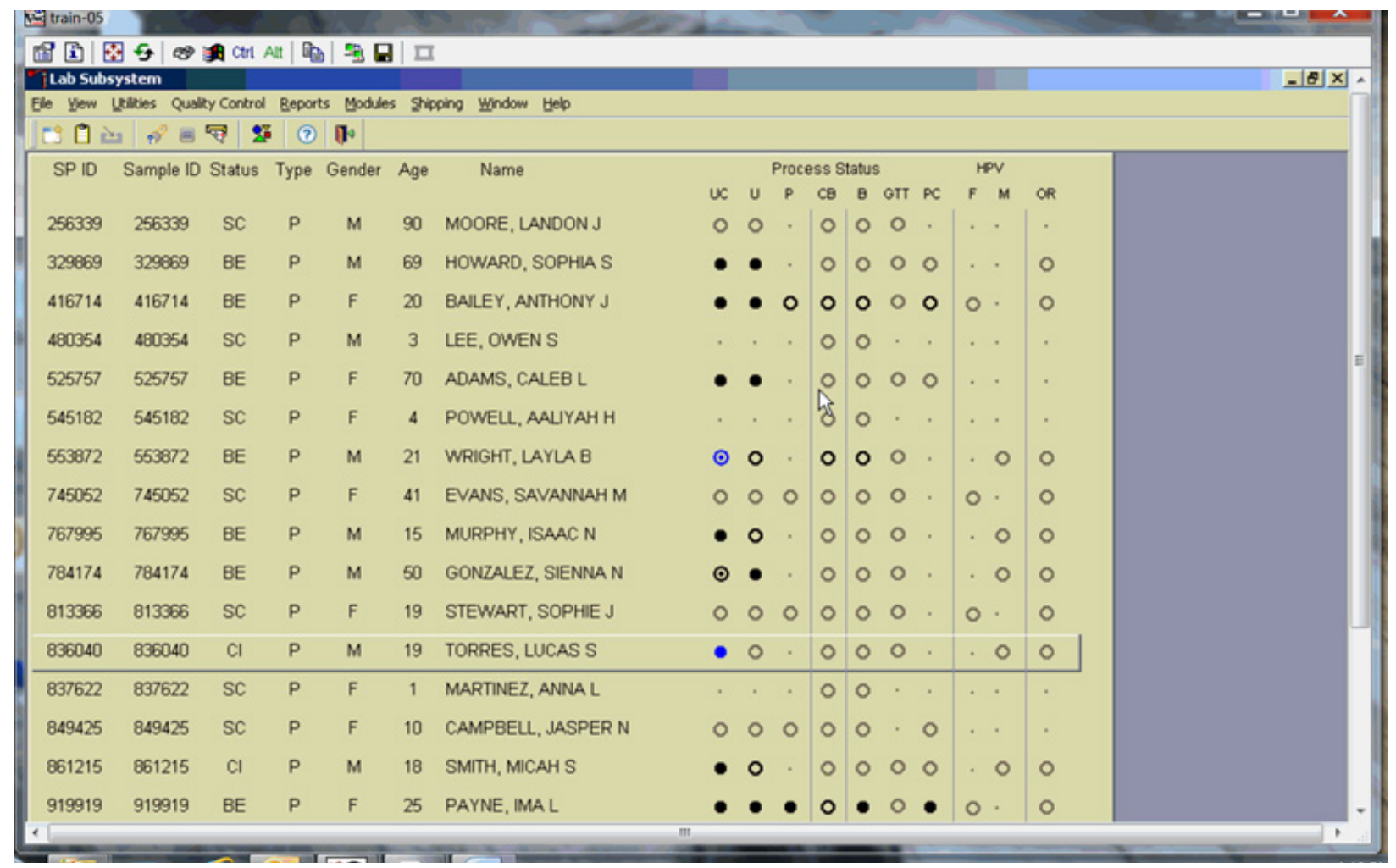


Select an SP with a blue UC circle and access the Urine Collection module.

\begin{tabular}{|c|c|c|c|c|c|c|c|c|c|c|c|c|c|c|c|c|c|}
\hline 4 & 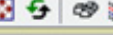 & (f) $\cot A$ & Alt $\mid$ & 5 & 므 & & & & & & & & & & & & \\
\hline eg 0 i & $\Rightarrow=$ & 守 8 & 8 & 10 & & & & & & & & & & & & & \\
\hline SP ID & Sample ID & Status & Type & Gender & Age & Name & & & & Proce & ess 8 & tatus & & PV & & & \\
\hline & & & & & & & & uc & u & $P$ & $c \theta$ & 8 oा & $P C$ & $F \quad M$ & OR & & \\
\hline 256339 & 256339 & $\mathrm{Cl}$ & $P$ & M & 90 & MOORE, L & ANDON J & 0 & 0 & . & 0 & 00 & $\cdot$ & $\cdots$ & $\cdot$ & & \\
\hline 329069 & 329069 & $B E$ & P & M & 69 & HOWARD & SOPHIAS & $\bullet$ & $\bullet$ & . & 0 & 00 & 0 & . . & 0 & & \\
\hline 416714 & 416714 & $B E$ & P & $F$ & 20 & BAILEY, A & NTHONY J & $\bullet$ & $\bullet$ & $\circ$ & 0 & $\circ 0$ & $\circ$ & 0 . & 0 & & \\
\hline 480354 & 480354 & SC & $P$ & M & 3 & LEE, OWE & ENS & . & $\cdot$ & . & 0 & 0. & $\cdot$ & . . & . & & \\
\hline 525757 & 525757 & $\mathrm{BE}$ & P & $F$ & 70 & ADAMS, $C$ & ALEB L & $\bullet$ & $\bullet$ & . & 0 & 00 & 0 & . . & . & & \\
\hline 545182 & 545182 & SC & $P$ & $F$ & 4 & POWELL. & AALUYAH H & . & . & . & 0 & 0. & . & . . & . & & \\
\hline 553872 & 553872 & $B E$ & $P$ & M & 21 & WRIGHT, L & LAYLAB & $\odot$ & 0 & . & 0 & $\circ 0$ & . & . 0 & 0 & & \\
\hline 745052 & 745052 & SC & $P$ & $F$ & 41 & EVANS, S & AVANNAH M & 0 & 0 & 0 & 0 & 00 & . & 0. & 0 & & \\
\hline 767995 & 767995 & $B E$ & $P$ & M & 15 & MURPHY. & ISAAC N & $\bullet$ & 0 & . & 0 & 00 & . & . 0 & $\circ$ & & \\
\hline 784174 & 784174 & $\mathrm{BE}$ & $P$ & M & 50 & GONZALF & 7 CIFNNA N & P & $\bullet$ & . & 0 & 00 & . & . 0 & $\circ$ & & \\
\hline 813366 & 813366 & $\mathrm{Cl}$ & $\mathrm{P}$ & $\mathrm{F}$ & 19 & STEWAR & Elood Processing & $p$ & 0 & 0 & 0 & 00 & . & 0. & 0 & & \\
\hline 836040 & 836040 & Cl & $P$ & M & 19 & TORRES, & Pecked Cell Processing & p & 0 & . & 0 & 00 & . & $\cdot 0$ & 0 & & \\
\hline 837622 & 837622 & SC & $P$ & $\mathrm{~F}$ & 1 & MARTINE & Mole HPV Processong & & . & . & 0 & $\circ$. & . & . . & . & & \\
\hline 849425 & 849425 & Sc & $\mathrm{P}$ & $F$ & 10 & CAMPBE & Oral Runse Processing & & & 0 & 0 & o. & 0 & . $\cdot$ & . & & \\
\hline 861215 & 861215 & Cl & $P$ & M & 18 & SMITH, M & $\begin{array}{l}\text { Urine Sollection } \\
\text { Bregnoncy Testing }\end{array}$ & & $\circ$ & . & 0 & 00 & $\circ$ & . 0 & $\circ$ & & \\
\hline 919919 & 919919 & $\mathrm{BE}$ & $\mathrm{P}$ & $F$ & 25 & PAYNE,I & Hempotology & & & & 0 & & $\bullet$ & & 0 & & \\
\hline & & & & & & & Befresh & & & & & & & & & & \\
\hline
\end{tabular}

To select an SP, use the up and down keys to move up and down the list until the correct SP is highlighted, or drag the mouse arrow to the correct SP and right click. To access the Urine Collection module, drag the mouse arrow to \{Urine Collection\}, and left click or left click and type [C/c]. 
The urine collection window displays.

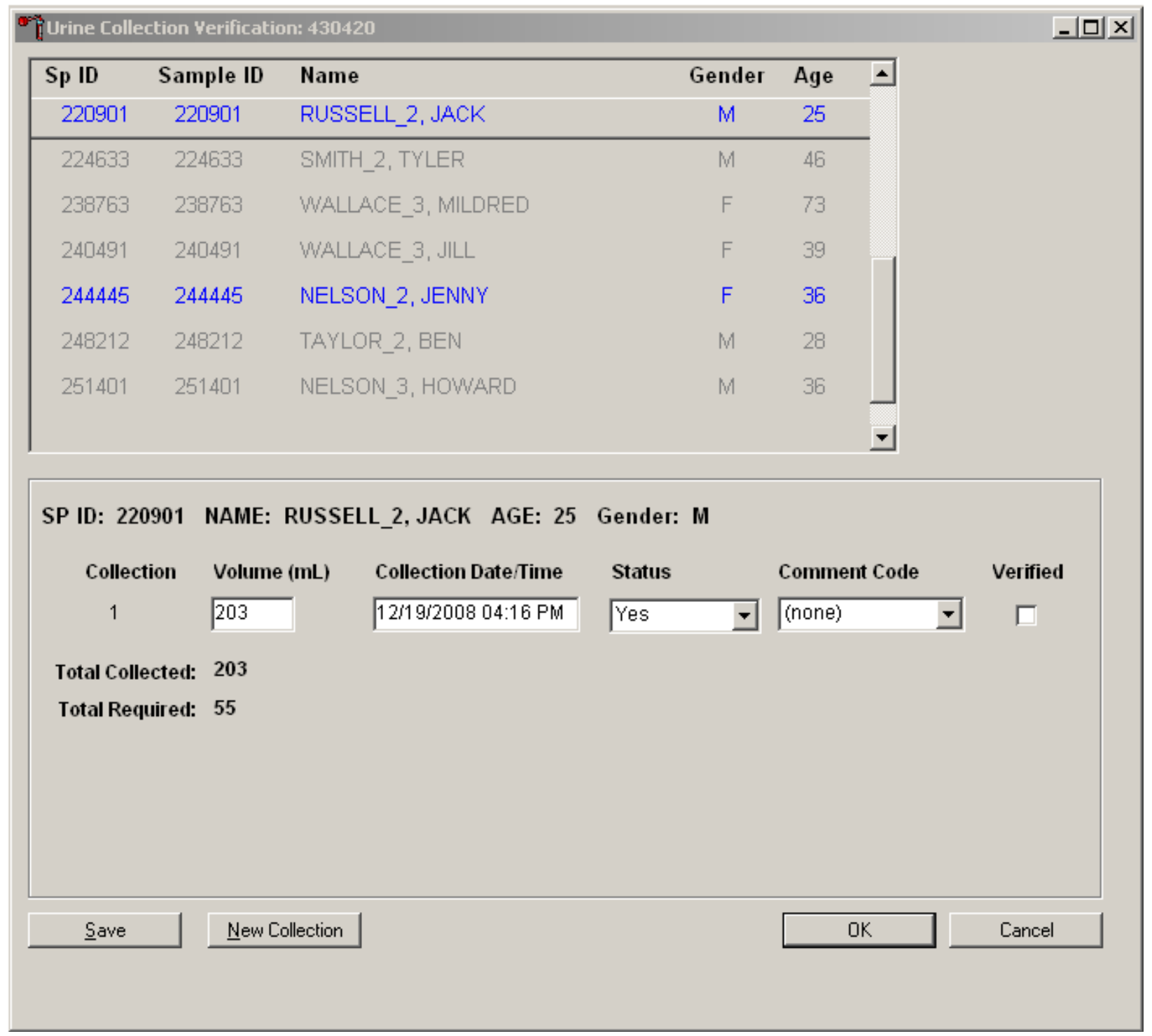

The top of the urine collection window contains columns for the SP ID, Person Name (last, first), Gender, and Age. The row for checked-in SPs is indicated in black, while SPs who have not checked into the MEC session are gray. SPs who have a urine collection ready for verification display in blue. Use the scroll bar to view all SPs scheduled into the session. To select an SP, use the up and down keys to move up and down the list until the correct SP is highlighted, or drag the mouse arrow to the correct SP and right click. 
The lower portion of the urine collection window displays the selected SP ID, Person Name (last, first), Gender, and Age. Below that are columns for Collection instance, Volume (mL) in whole numbers, Collection date/time, Collection status, Comment code, and a checkbox for Verify. The bottom of the screen displays the Total Collected (sum in $\mathrm{mL}$ of all urine collections) and Total Required (total $\mathrm{mL}$ required to meet the SP's urine protocol). The total volume includes 2-mL for pregnancy testing and osmolality for eligible female SPs, and $1-\mathrm{mL}$ for osmolality testing only on pregnancy test ineligible female SPs and all male SPs.

One gram of urine is equal to one $\mathrm{mL}$ of urine. The application records the weight of the urine in whole numbers following standard rounding procedures.

If the SP has submitted a urine collection that has been weighed and scanned into the database, the volume, collection status, and collection date and time will automatically display in the lower portion of the Urine Collection screen. If the collection did not meet the minimum requirement for the protocol, the system will automatically select "QNS" with the comment code "quantity not sufficient." A QNS result immediately notifies the coordinator to prompt for an additional urine collection. A second instance of urine collection for the first QNS sample or third instance of urine collection for the second QNS sample will appear below the current sample. If the quantity of urine collection does meet the requirement of the urine protocol, the system will automatically select "Yes" and no additional collections will appear. 
Evaluate the quantity of urine received in the laboratory.

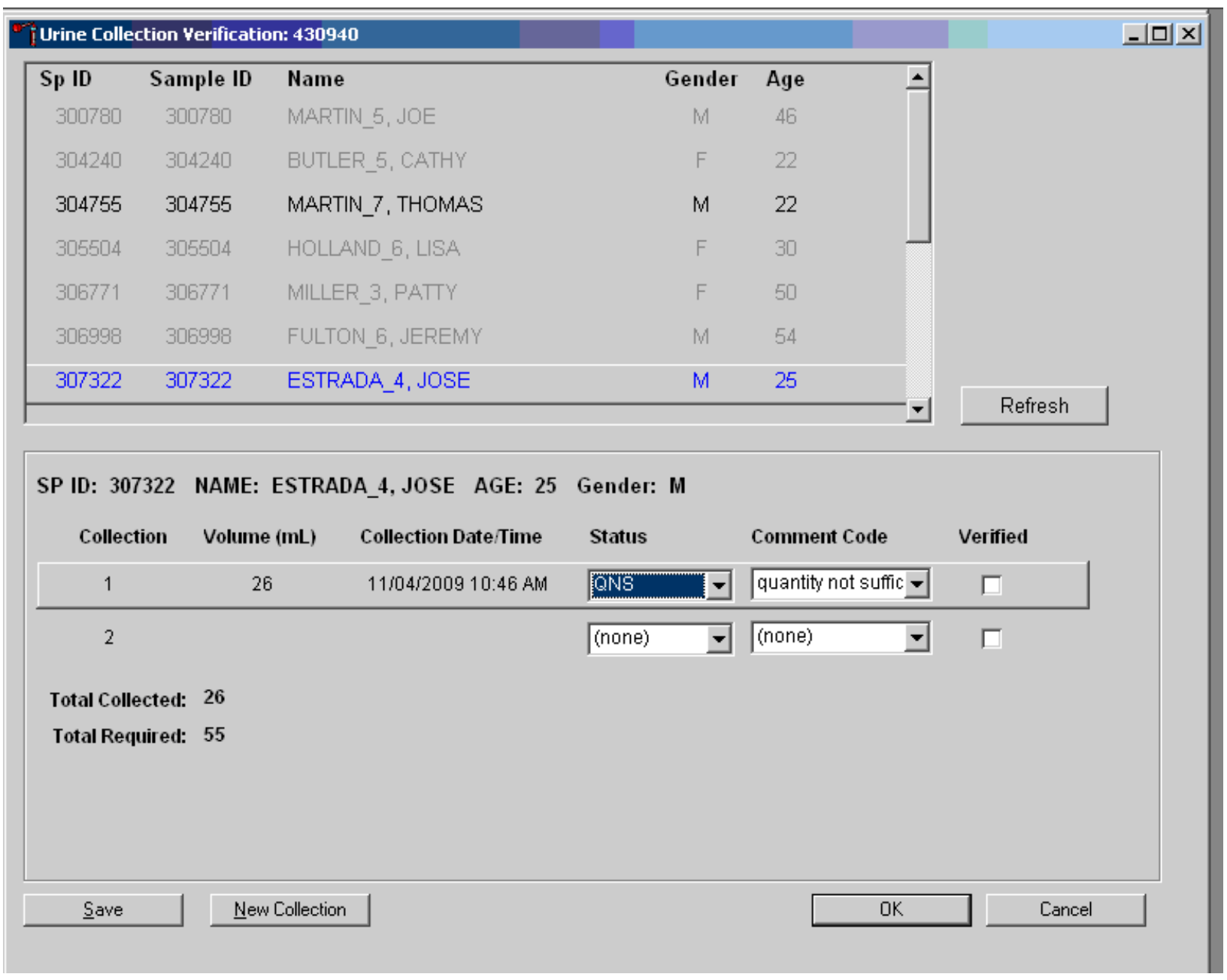

Compare the number displayed in the Total Collected column and the actual volume of urine in the cup. If manual adjustment is needed, follow instructions listed in Section 4.6.

Record the urine collection result for each SP by typing [Y/y] for "Yes," [N/n] for "No," or [Y/y] a second time for "QNS." Alternatively, use the mouse to direct the mouse arrow to the drop-down list, left click to display the responses, drag the mouse arrow to "Yes," "No," or "QNS" and left click. A "QNS" result automatically turns the lab progress bar yellow on the Coordinator screen. This yellow progress bar alerts the coordinator, who then asks the SP to provide an additional urine specimen. 


\subsubsection{Quantity Not Sufficient (QNS) Urines}

Store all QNS urines at room temperature. If the SP has voided $<1.0 \mathrm{~mL}$, the lab will ask the coordinator for a re-collection of Urine Collection (UC) 1. Do not record any results for this urine instance. Discard this sample and wait for a new UC 1.

If the SP has voided $>1 \mathrm{~mL}$, but not enough to fill his or her profile, run the urine pregnancy and osmolality on this sample. When additional urine is obtained or collected and it's enough to fill the SPs profile, pool the urine into the most recently collected cup (the cup with the highest urine collection instance number on the cup label), mix, and process.

Do not enter a result until urine arrives in the laboratory. At the end of each session, review the results for each SP. Enter "No" in the result field for all SPs who have not produced a urine sample. Record a result other than "(none)" for all SPs. The coordinator also has the ability to mark urine as "No" with a comment code. The Laboratory application will not be able to modify any entries made by the coordinator. 


ए Urine Collection Yerification: $\mathbf{4 3 0 4 2 0}$
\begin{tabular}{|cclccc|}
\hline Sp ID & Sample ID & Name & Gender & Age & A \\
220901 & 220901 & RUSSELL_2, JACK & M & 25 & \\
224633 & 224633 & SMITH_2, TYLER & M & 46 & \\
238763 & 238763 & WALLACE_3, MILDRED & F & 73 & \\
240491 & 240491 & WALLACE_3, JILL & F & 39 & \\
244445 & 244445 & NELSON_2, JENNY & F & 36 & \\
\hline 248212 & 248212 & TAYLOR_2, BEN & M & 28 & \\
251401 & 251401 & NELSON_3, HOWARD & M & 36 & \\
& & & & & -1
\end{tabular}

SP ID: 244445 NAME: NELSON_2, JENNY AGE: 36 Gender: F

$\begin{array}{cllll}\text { Collection } & \text { Volume (mL) } & \text { Collection Date/Time } & \text { Status } \\ 1 & 25 & 12 / 19 / 200804: 13 \mathrm{PM} & \text { ONS } & \\ 2 & & & \end{array}$

Total Collected: 25

Total Required: 56

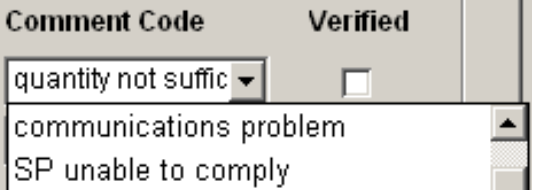

SP unable to comply

quantity not sufficient

SP refusal

SP ill/emergency

no time

Save

New Collection

OK

Cancel

The urine collection cup capacity may contain a sample that weighs more than the scale weight capacity of 610 grams. Look at the scale display for full specimens. If the scale displays dashes when it should display a weight, the reading is over the scale limit: $\ulcorner----\urcorner$. When the barcode for the sample is scanned in, instead of the barcode number displaying on the LCD screen, the words "over Lnt" display.

When the scale is over limit it does not transmit data to the ISIS application. Manually enter the volume $610 \mathrm{~mL}$ in the volume column. 


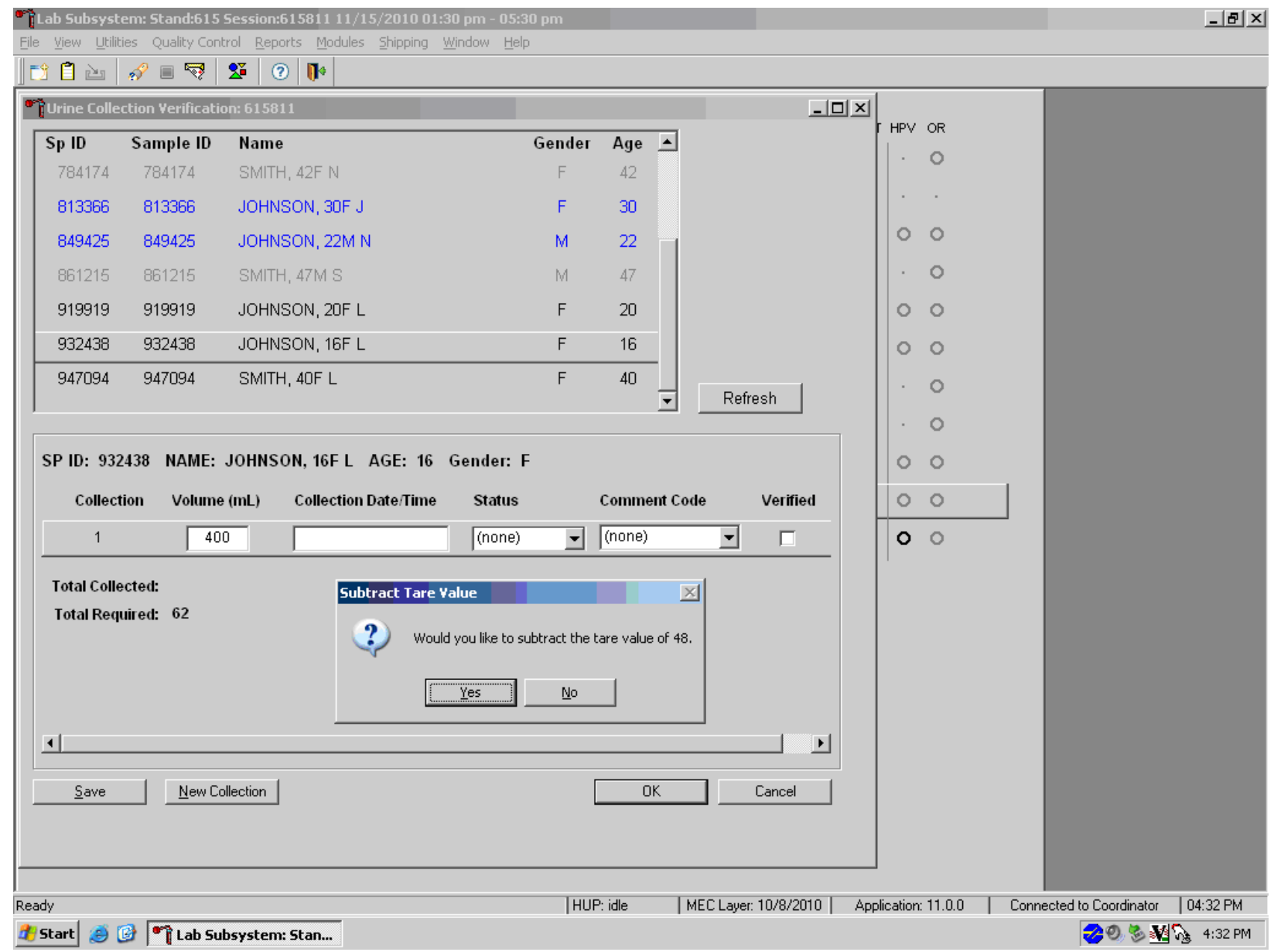

Press "Tab" to move to the Collection Date/Time field. The application will ask "Would you like to subtract the tare value of 48 ?" Select OK. Enter the date and time of specimen collection. Press tab to continue on to the Status field. 


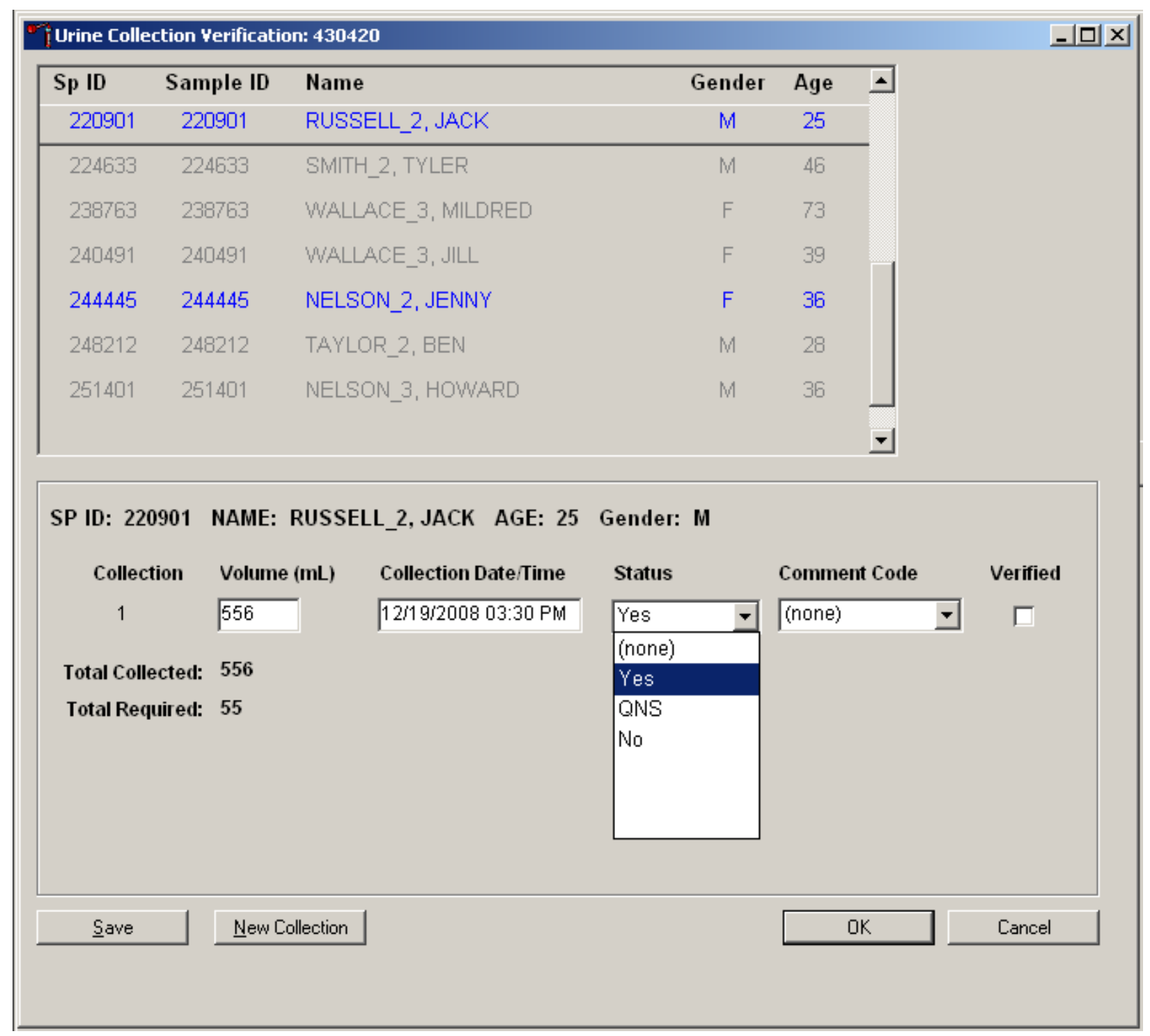

Record urine collections that exceed the scale as "Yes." To mark the urine collection status, direct the mouse arrow to the drop-down list, click to display the different status, and select "Yes" by clicking once on "Yes" with the mouse arrow. Use the Tab key to proceed on to the Comment Code field.

Record a comment for sufficient urine collections if the weight of the urine exceeds the scale weight capacity of 610 grams. 


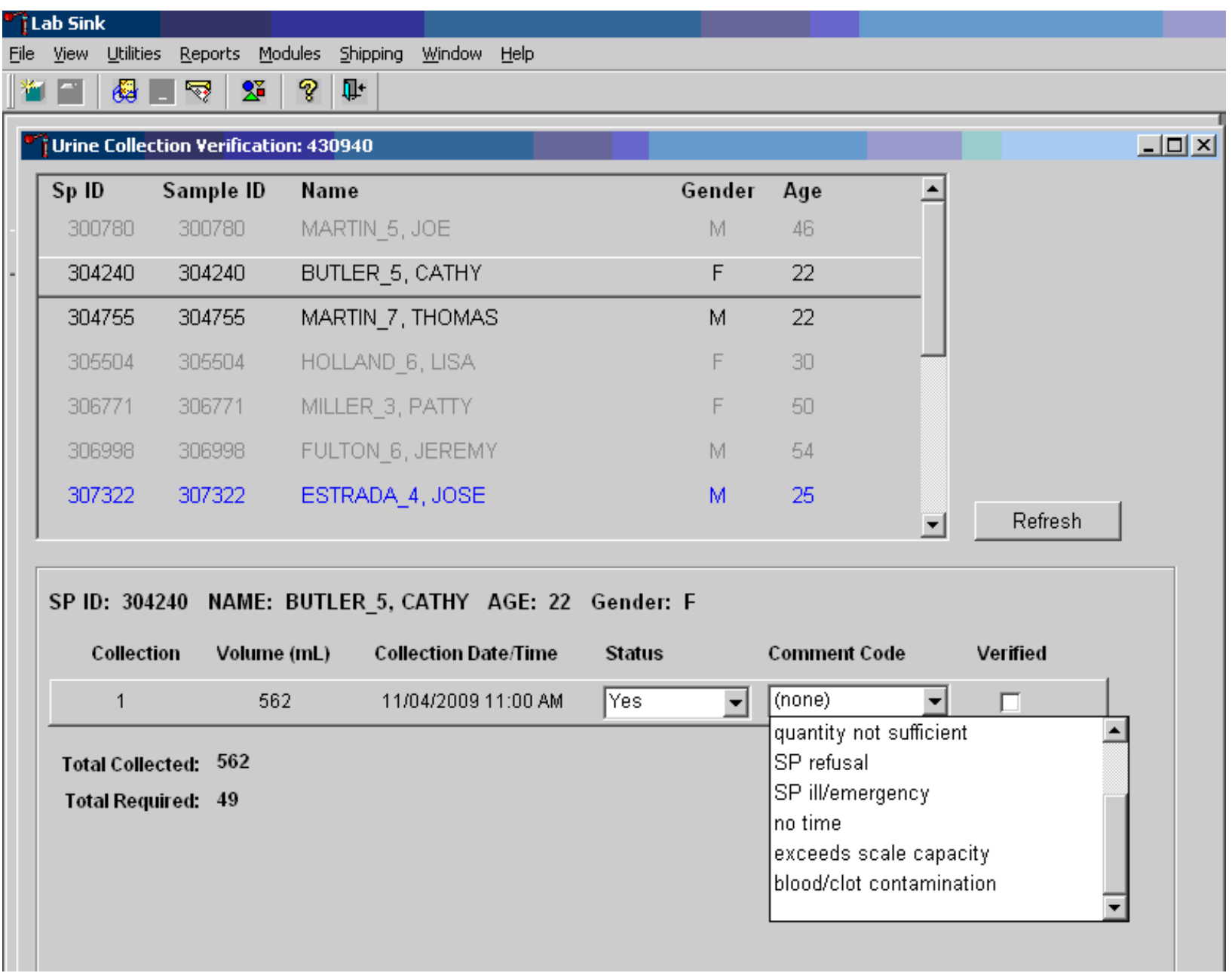

To record a comment, use the mouse to direct the mouse arrow to the drop-down list, click to display the Comment Codes, and select "exceeds scale capacity" from the drop-down list. Alternatively, use the up and down keyboard arrows to scroll through the choices or type the first letter of the desired comment code and, when the correct choice is highlighted, left click. 
Visually evaluate the urine sample for blood contamination. Record a comment for sufficient and QNS urine collections if the sample is contaminated with blood.

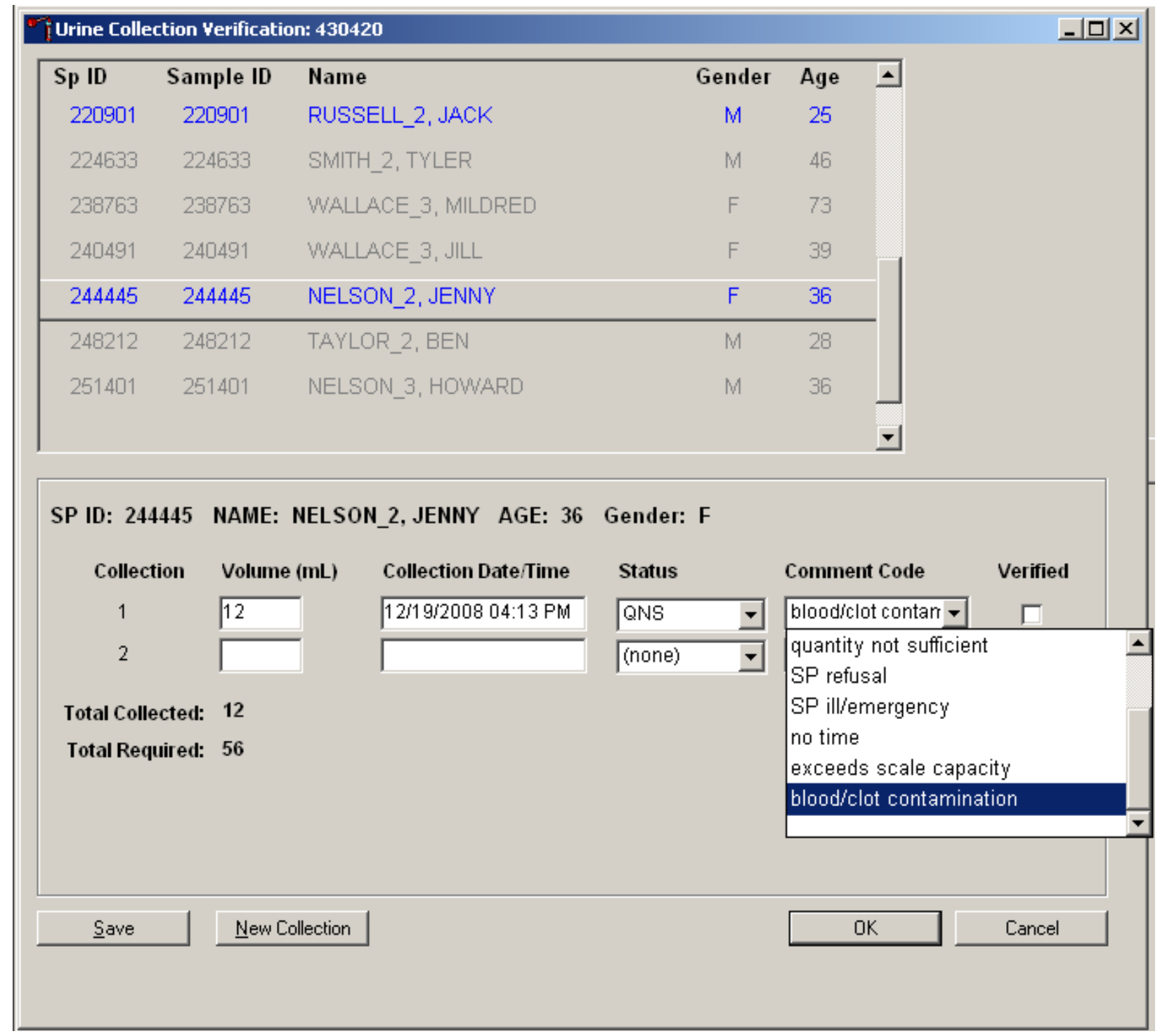

The "blood/clot contamination" comment should supersede the "quantity not sufficient" comment code when blood is present in a QNS specimen. To record a comment, use the mouse to direct the mouse arrow to the drop-down list, click to display the comment codes, and select "blood/clot contamination" from the drop-down list. Alternatively, use the up and down keyboard arrows to scroll through the choices or type the first letter of the desired comment code and, when the correct choice is highlighted, left click. 
Record a comment for all insufficient urine collections. The laboratory application will automatically assign the comment code "quantity not sufficient" for all urine collections that are calculated as QNS by the scale application. The laboratory technician may also assign this comment code to all samples that are changed to QNS during the verification process.

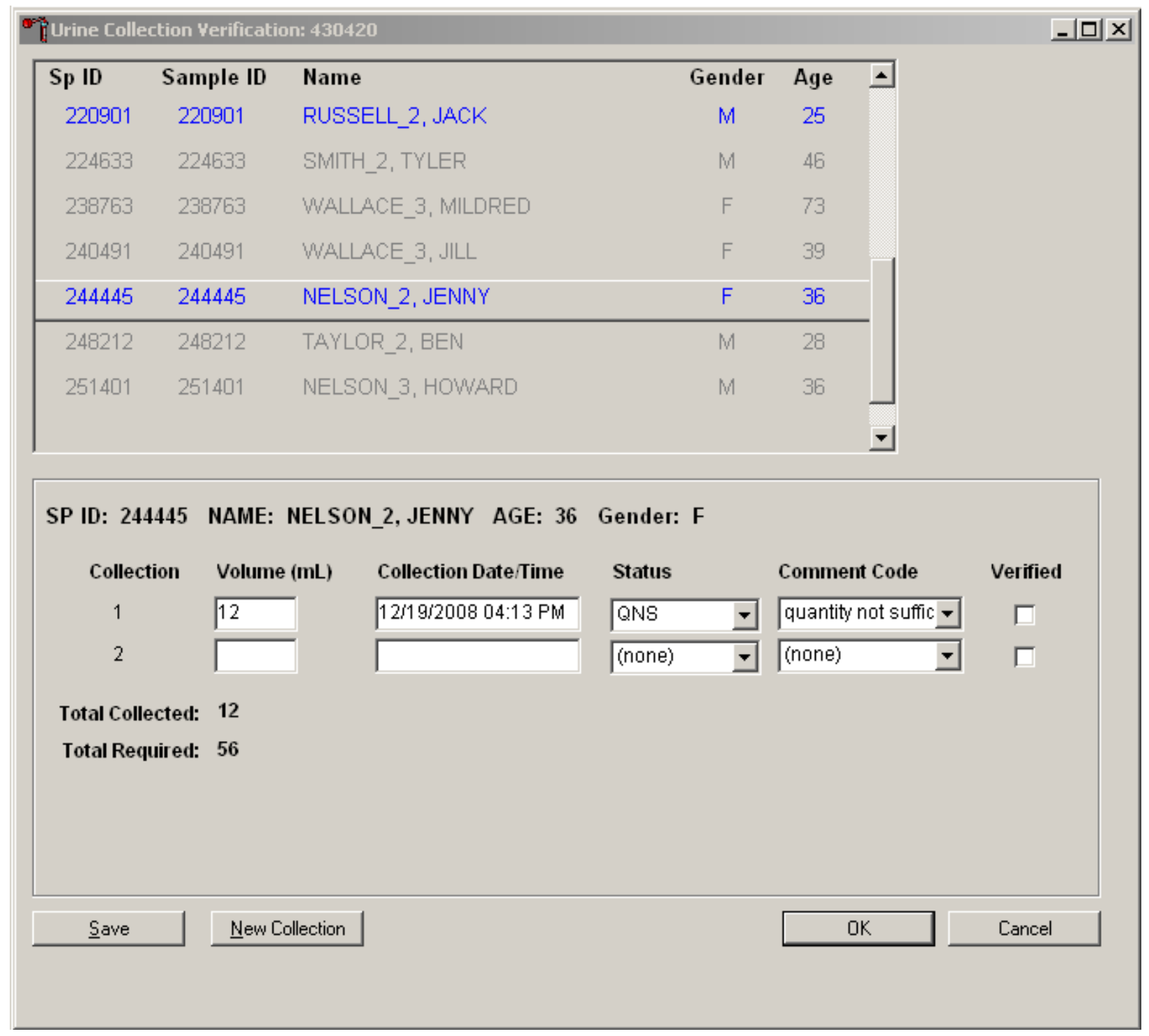


To record a comment, use the mouse to direct the mouse arrow to the drop-down list, click to display the comment codes, and select the most appropriate choice. Alternatively, use the up and down keyboard arrows to scroll through the choices or type the first letter of the desired comment code (Exhibit 4-3) and, when the correct choice is highlighted, left click.

Exhibit 4-3. Comment codes for insufficient urine collection

\begin{tabular}{|l|l|}
\hline Comment Code & Use when: \\
\hline $\begin{array}{l}\text { communication } \\
\text { problem }\end{array}$ & $\begin{array}{l}\text { SP is unable to understand and follow instructions for the component due to } \\
\text { language, cognitive impairment, or other problem, and is unable to complete } \\
\text { the test. }\end{array}$ \\
\hline SP unable to comply & $\begin{array}{l}\text { SP has a physical limitation that prohibits him or her from producing a urine } \\
\text { specimen or the SP is unable to produce urine during the session. }\end{array}$ \\
\hline quantity not sufficient & The quantity of urine is insufficient to process any or all urine vessel(s). \\
\hline SP refusal & SP refuses to provide urine during the session. \\
\hline SP ill/emergency & Not applicable. \\
\hline no time & Not applicable. \\
\hline exceeds scale capacity & The weight of the urine exceeds the scale weight capacity of 610 grams. \\
\hline $\begin{array}{l}\text { blood/clot } \\
\text { contamination }\end{array}$ & Blood or clots are present in the urine and may affect the volume capture. \\
\hline
\end{tabular}


Verify the results of the urine collection. To verify, use the mouse to direct the mouse arrow to the "Verified" checkbox. Left click on the checkbox and a checkmark will appear in the box. Verifying the results will save them to the database.

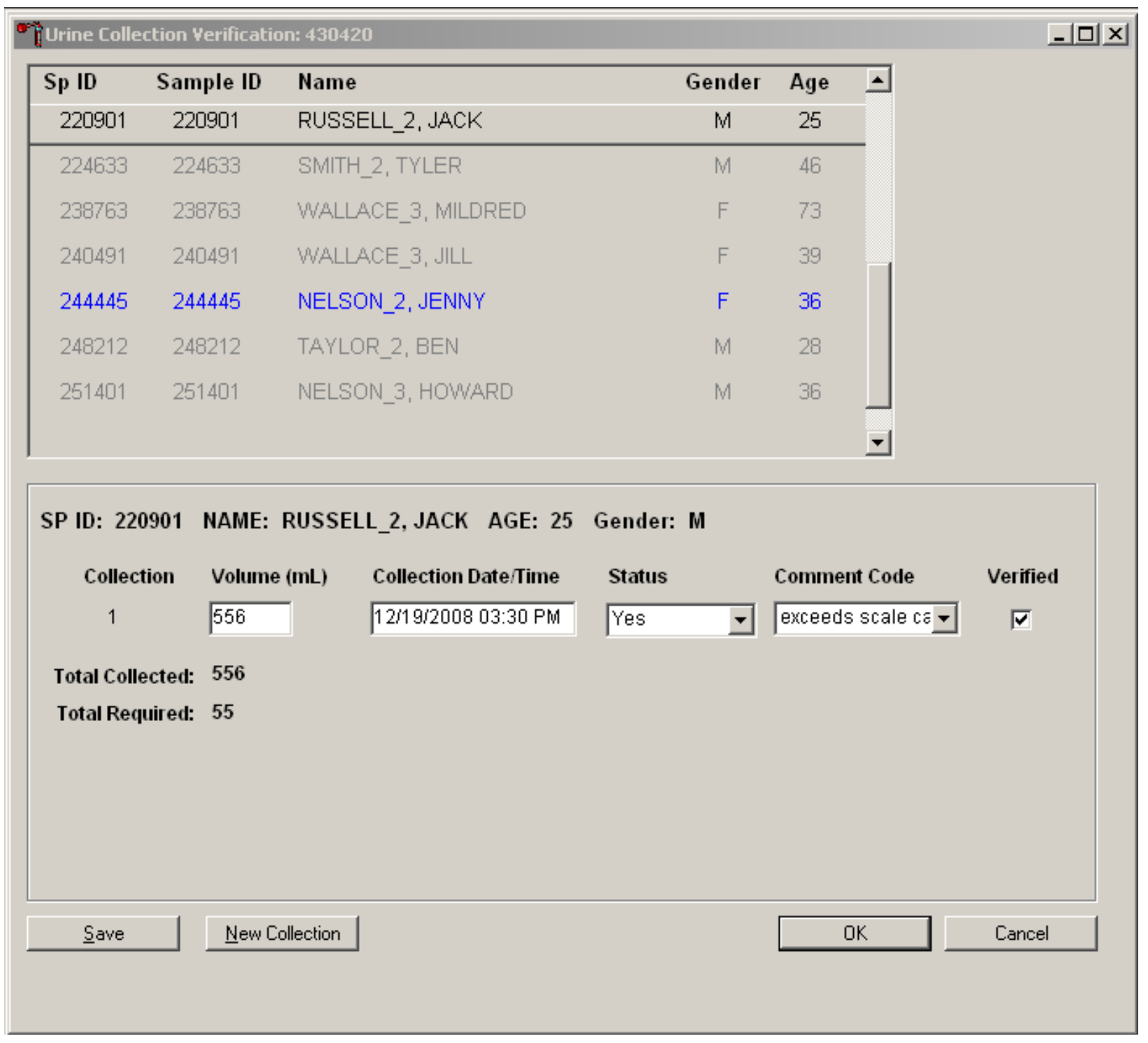


Continue recording and verifying urine collection results as specimens are received in the laboratory. Use the scroll bar to view all SPs in the top part of the Urine Collection screen. SPs who need their urine verified will appear in blue on the top screen.

An SP will be prompted for additional urine only if (1) the total of his or her urine collection was insufficient to meet the SP's protocol requirement and (2) the SP has submitted less than three collection samples. The Urine Collection screen will display up to three instances of urine collection. Urine collection is complete regardless of the amount of urine collected after three attempts. When urine is collected as QNS, a message is sent to the coordinator to prompt the SP for more urine.

\begin{tabular}{|c|c|c|c|c|c|}
\hline Sp ID & Sample ID & Name & Gender & Age & $\Delta$ \\
\hline 220901 & 220901 & RUSSELL_2, JACK & $M$ & 25 & \\
\hline 224633 & 224633 & SMITH_2, TYLER & M & 46 & \\
\hline 238763 & 238763 & WALLACE_3, MILDRED & $\mathrm{F}$ & 73 & \\
\hline 240491 & 240491 & WALLACE_3, JILL & $\mathrm{F}$ & 39 & \\
\hline 244445 & 244445 & NELSON_2, JENNY & $\mathrm{F}$ & 36 & \\
\hline 248212 & 248212 & TAYLOR_2, BEN & M & 28 & \\
\hline 251401 & 251401 & NELSON_3, HOWARD & $M$ & 36 & 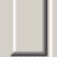 \\
\hline
\end{tabular}

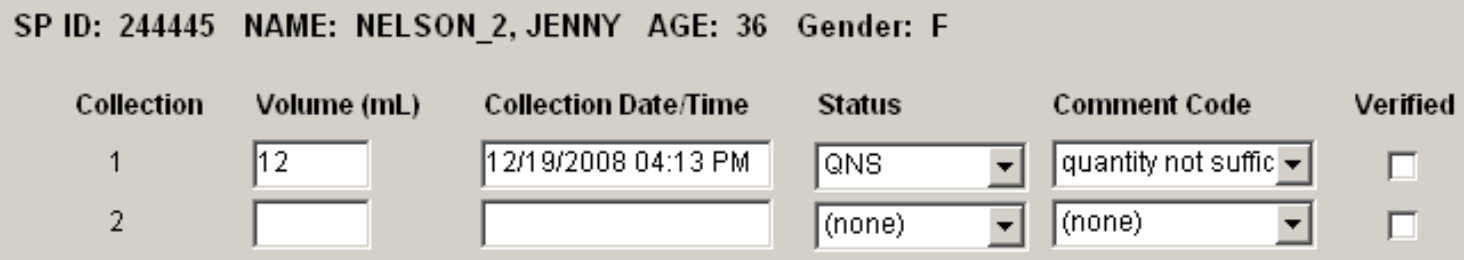

Total Collected: 12

Total Required: 56 
If the urine collection is the first or second collection and the status is QNS, a record for the second and third instances of urine collection will automatically be added by the Laboratory application. Second and third collections will display as empty rows after the last urine collection. The instance number is displayed under the "Collection" column.

Verify the urine collection by checking the "Verified" box. Use "Save" to save changes or select the $\mathrm{X}$ in the upper right corner to exit out of the Urine Collection screen.

Once a urine collection has been recorded for an SP, the circle on the heads-up screen will turn black $(\odot$ for QNS or $\odot$ for Yes). A black urine collection circle indicates that no additional action needs to be taken on that SP for urine collection at that time.

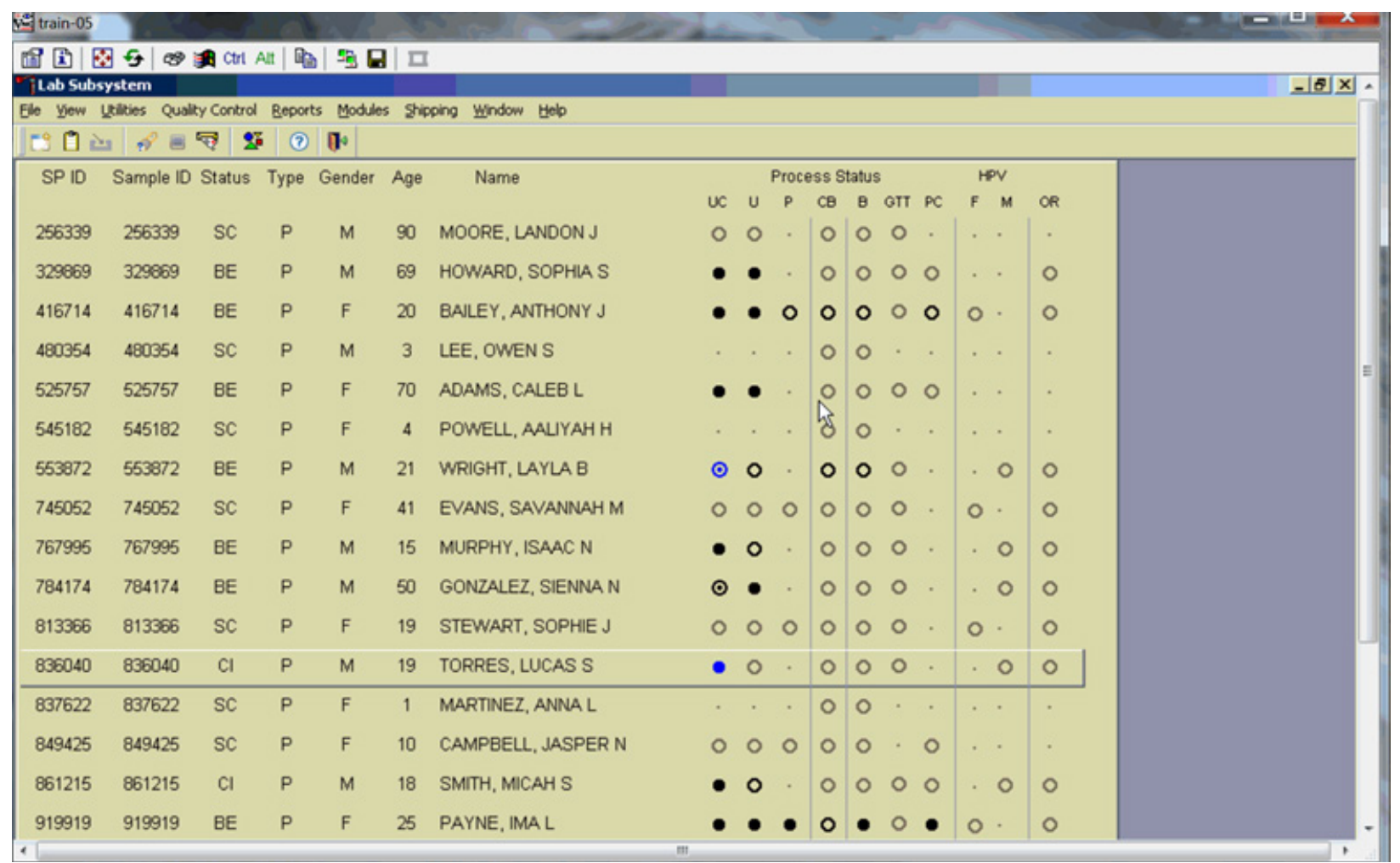




\subsubsection{Quantity Not Sufficient (QNS) Urines and Enabling Home Urine Collection (HUC)}

For QNS urine specimens, HUC will be enabled as soon as the lab verifies the SP has collected greater than or equal to 6-mLs of urine. This verification will turn the HUC box green on the coordinator screen and allow the SP to enter HUC. If UC 1 is QNS, hold until enough urine is available for the entire profile. Pool samples together and proceed filling the vessels.

\subsection{Manually Documenting Urine Collection}

\subsubsection{Manually Document Urine Collection With Barcode Scanning}

The technician can manually record the volume, status, and collection date and time in the urine collection module within the laboratory application. Place the urine collection cup on the scale and cover with the clear acrylic scale cover. Scan the barcode on the urine cup.

Open the Urine Collection screen from the heads-up as described in Section 2.2. The urine collection window displays. At this point, the technician can modify the volume of the urine and the collection date/time.

To manually adjust the Volume $(\mathrm{mL})$, use the mouse to direct the mouse arrow to the text box under the Volume $(\mathrm{mL})$ column. Double click to open boxes for modification and then click on the text box to enable the cursor in the box. Make changes using the number pad on the keyboard. Once any necessary adjustments have been made, use the mouse to direct the mouse arrow to the box under the Collection Date/Time column or press the Tab key. A prompt will pop up asking if you would like to subtract the weight of the cup. If the volume of the sample was transmitted from the scale and you have made a manual adjustment to that reading, select "No." 
In the Collection Date/Time box, right click on the text box to enable the cursor inside the box. Adjust the date and time by entering the date in the format "MMDDYYYY \#\#:\#\#" using the number pad on the keyboard. Adjust "am/pm" by using the up or down arrows. Once any necessary adjustments have been made, use the mouse to direct the mouse arrow to the box under the Status column or press the Tab key.

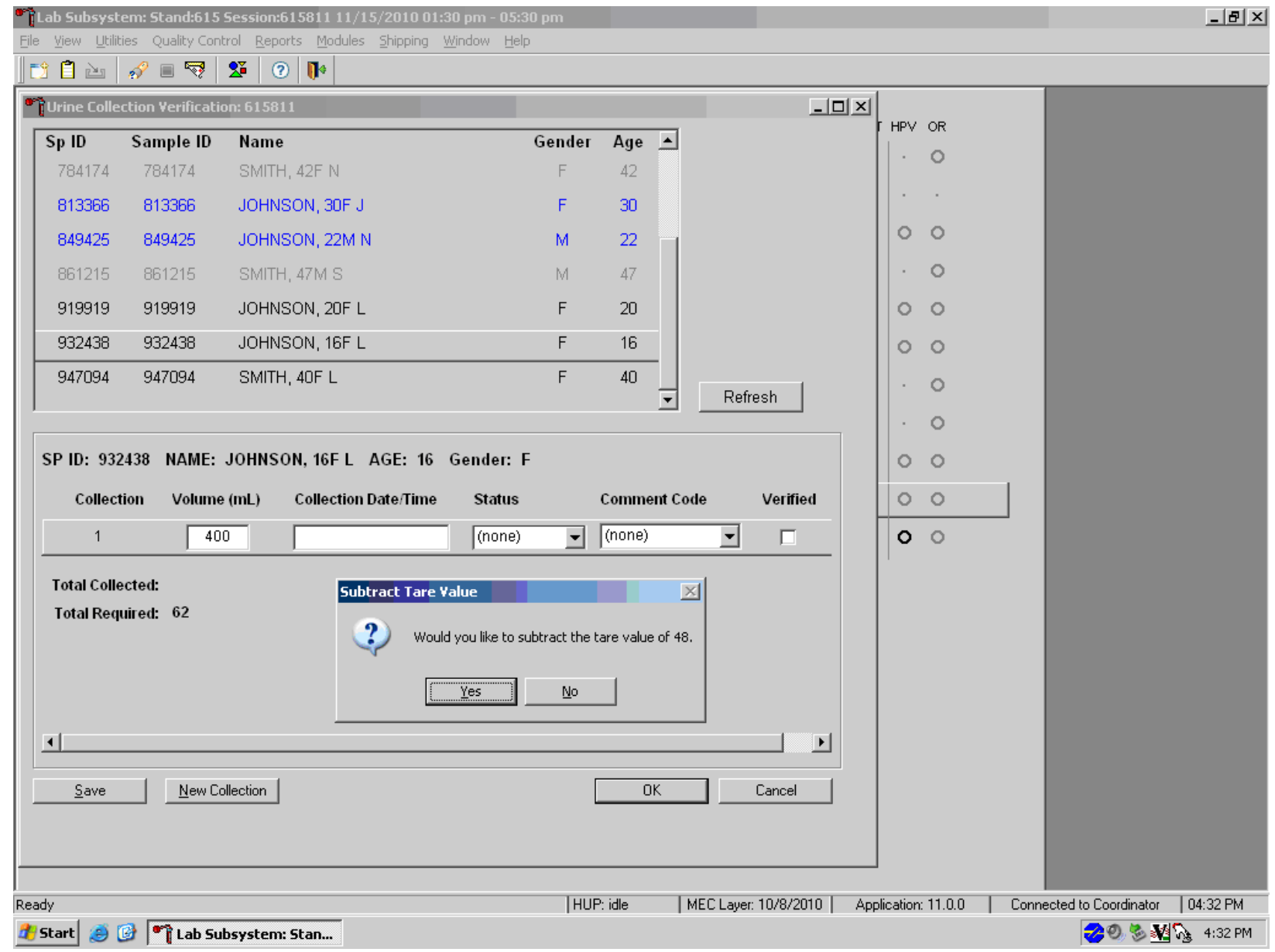

Continue to enter the collection date/time, selecting the appropriate urine collection status, and a comment code if applicable. Verify the urine collection by checking the "Verified" box. 
If the urine collection is the first or second collection and is QNS, second and third instances of urine collection may be added by using the "New Collection" button at the bottom of the screen.

\begin{tabular}{|l|l|l|l|}
\hline Save & New Collection & CK & Cancel \\
\hline
\end{tabular}

Add a second or third collection only if the previous collection was QNS. Enter the second or third urine collection results when the sample is submitted to the MEC laboratory. Do this by typing in the boxes for volume and collection date/time and then selecting the appropriate urine collection status and a comment code if applicable.

Verify the urine collection by checking the "Verified" box. Use "Save" to save changes or select the $\mathrm{X}$ in the upper right corner to exit out of the Urine Collection screen.

\subsubsection{Manually Document Urine Collection Without Barcode Scanning}

If the connection between the scale and workstation 3 is down, the technician can manually record the volume, status, and collection date and time in the urine collection module within the laboratory application. Place the urine collection cup on the scale and cover with the clear acrylic scale cover. Note the weight reading from the LCD. 
Open the Urine Collection screen from the heads-up as described in Section 2.2. The urine collection window displays. Double click in the area underneath Volume or Collection Date/Time to open the boxes where the values can be entered. Enter the urine collection results by typing in the weight reading from the scale's display in the box for volume.

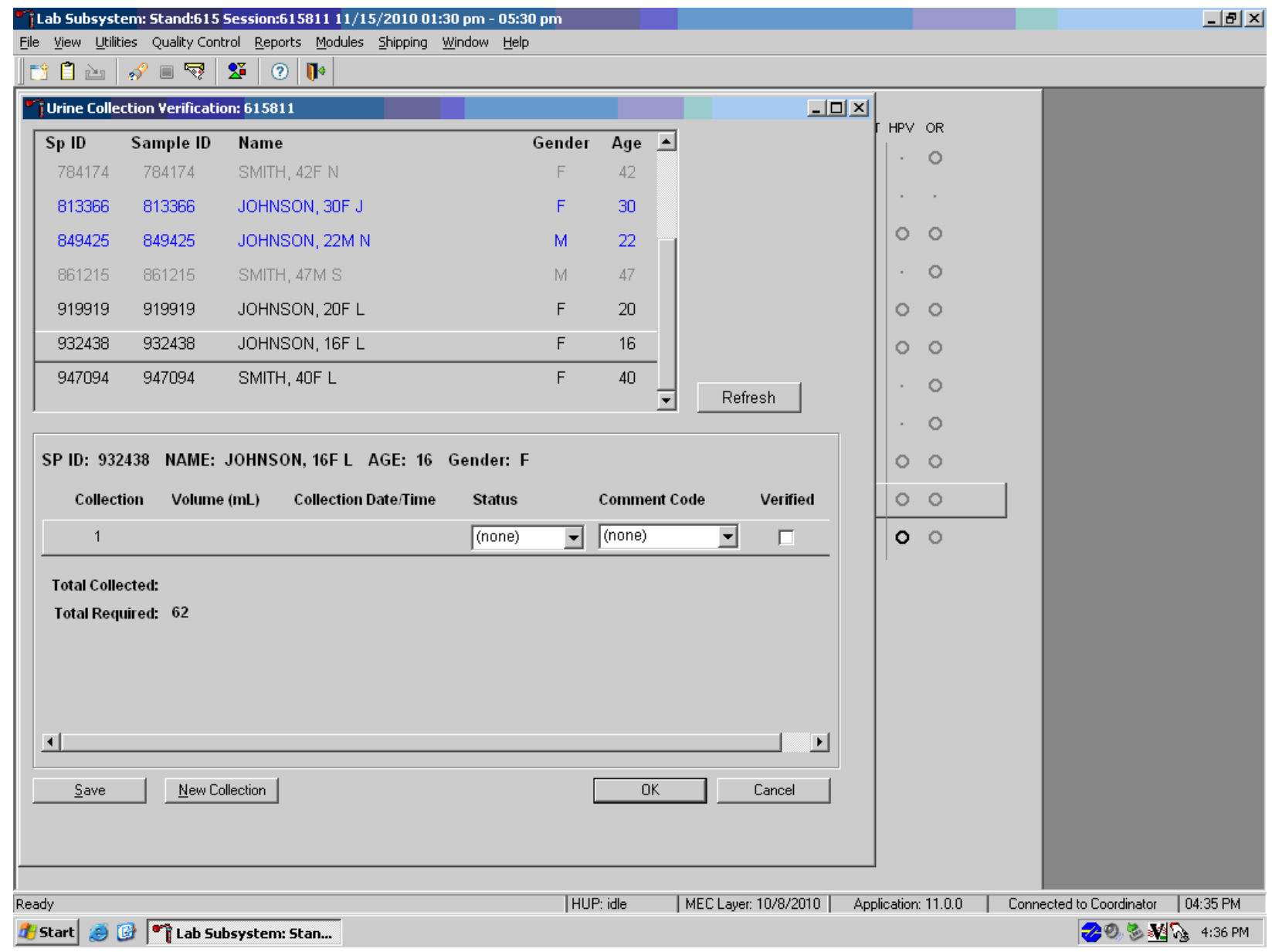


If the next box is selected by pressing the "tab" button or selected by the mouse, a prompt will pop up asking if you would like to subtract the weight of the urine cup from the result entered. Select "Yes."

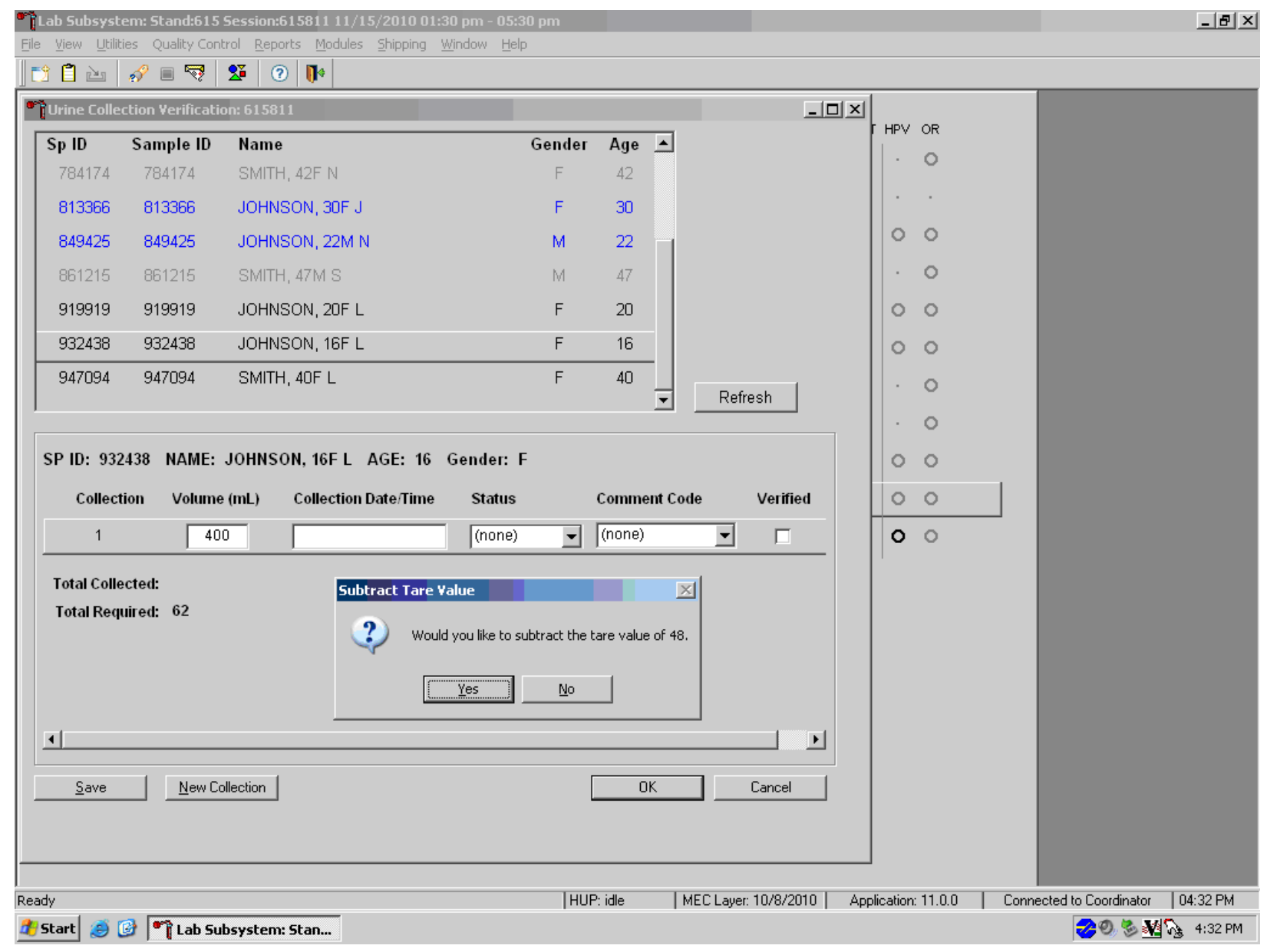

Continue to enter the collection date/time, selecting the appropriate urine collection status, and a comment code if applicable. Verify the urine collection by checking the "Verified" box. 
If the urine collection is the first or second collection and is QNS, second and third instances of urine collection may be added by using the "New Collection" button at the bottom of the screen.

\begin{tabular}{|l|l|l|l|}
\hline Save & New Collection & CK & Cancel \\
\hline
\end{tabular}

Add a second or third collection only if the previous collection was QNS. Enter the second or third urine collection results when the sample is submitted to the MEC laboratory. Do this by typing in the boxes for volume and collection date/time and then selecting the appropriate urine collection status and a comment code if applicable.

Verify the urine collection by checking the "Verify" box. Use "Save" to save changes or select the $\mathrm{X}$ in the upper right corner to exit out of the Urine Collection screen.

\subsection{Urine Specimen Assays}

Process urine specimens for the following tests:

- Pregnancy test for selected females aged 8-11 and all females aged 12 to 59 years;

- Albumin/Creatinine (Alb/Creat) on primary SPs and VIP guests aged 6 years and older;

- Chlamydia (Chlam) and Trichomonas (Trich) on primary SPs 14-39 years old;

- Trichomonas (Trich) on SPs 40-59 years old;

- Speciated Arsenic (Spc Arsenic) on selected SPs aged 6 years and older;

- Priority Pesticides (Prior Pest) on selected SPs aged 6 years and older;

- Organophosphates (Organophos) on selected SPs aged 6 years and older;

- Urine Mercury/Iodine (Urin Merc) on selected SPs aged 6 years and older;

- Heavy Metal on selected SPs aged 6 years and older;

- Polyaeromatic Hydrocarbons (PAH) on selected SPs aged 6 years and older;

- Phthalates on selected SPs aged 6 years and older; 
- Perchlorate on selected SPs aged 6 years and older;

- Caffeine on selected SPs aged 6 years and older;

- NNAL/TSNA(a tobacco-specific nitrosamine) on SPs aged 6 years and older;

- Aromatic Amines on SPs aged 6 years and older;

- Volitile Nitrosamines on SPs aged 6 years and older;

- HAA/Nicotine Analogs on SPs aged 6 years and older; and

- Extra urine (Xtra Urine) on SPs aged 6 years and older.

\subsection{Urine Specimen Protocols}

Table 4-1 summarizes the urine processing protocol for primary SPs. Process urine in priority order with vessel 45 filled first, 46 second, etc.

Table 4-1. NHANES urine processing protocol - primary

\begin{tabular}{|c|c|c|c|c|}
\hline $\begin{array}{c}\text { Vessel } \\
\text { no. }\end{array}$ & Assay & $\begin{array}{c}\text { Age in } \\
\text { years }\end{array}$ & $\begin{array}{l}\text { Sample } \\
\text { size } \mathrm{mL}\end{array}$ & Vessel \\
\hline 45 & Alb/Creat & $6+$ & 3 & 5-mL cryovial \\
\hline \multirow[t]{2}{*}{46} & Chlam/Trichomonas & 14-39 & 10 & 14-mL Falcon \\
\hline & Trichomonas & $40-59$ & 10 & 14-mL Falcon \\
\hline 86 & Spc Arsenic & $6+$ & 3 & 5-mL cryovial \\
\hline 48 & Prior Pest & $6+$ & 7 & 10-mL VWR screwcap \\
\hline 49 & Organophos & $6+$ & 7 & 10-mL VWR screwcap \\
\hline 62 & Urin Merc/Iodine & $6+$ & 5 & 10-mL VWR screwcap \\
\hline 50 & Heavy Metal & $6+$ & 8 & 14-mL Falcon \\
\hline 66 & PAH & $6+$ & 3 & 5-mL cryovial \\
\hline 67 & Phthalates & $6+$ & 3 & 5-mL cryovial \\
\hline 102 & Perchlorate/VOC/Thiocyante & $6+$ & 5 & 5-mL cryovial \\
\hline 121 & NNAL/TNSA & $6+$ & 10 & 10-mL VWR screwcap \\
\hline \multirow[t]{4}{*}{128} & Caffeine & $6+$ & 1 & 2-mL cryovial \\
\hline & Aromatic Amines & $6+$ & 5 & 5-mL cryovial \\
\hline & Volatile Nitrosamines & $6+$ & 5 & 5-mL cryovial \\
\hline & HAA/Nicotine Analogs & $6+$ & 5 & 5-mL cryovial \\
\hline 52 & Xtra Urine 1 & $6+$ & 5 & 5-mL cryovial \\
\hline 139 & Xtra Urine 2 & $6+$ & 5 & 5-mL cryovial \\
\hline
\end{tabular}


Table 4-2 summarizes the urine processing protocol for the VIP guest appointment type.

Table 4-2. NHANES urine processing protocol - VIP guest

\begin{tabular}{|c|c|c|c|c|}
\hline $\begin{array}{c}\text { Vessel } \\
\text { No. }\end{array}$ & Assay & $\begin{array}{c}\text { Age in } \\
\text { years }\end{array}$ & $\begin{array}{c}\text { Sample } \\
\text { size } \mathrm{mL}\end{array}$ & Vessel \\
\hline 45 & Alb/Creat & $6+$ & 3 & $5-\mathrm{mL}$ Cryovial \\
\hline
\end{tabular}

\subsection{Labeling Urine Processing Vessels}

The appropriate barcode labels automatically print for each SP based on the SP's age, sex, appointment type, and subsample selection. The label/ship technologist at workstation 1 prints the labels at least one session in advance. The printing procedure is described in Chapter 8. Place labels at workstation 3 .

Process urine on one SP at a time. Set up each urine processing rack using the preprinted labels. Label each vessel with the appropriate vessel label, according to the protocol. Place the label on the vessel, wrapping it around the vessel horizontally, making sure the label wraps onto itself. Place the label so the first digit of the vessel number is at the top of the vessel. Set the vessels in the cube racks in priority order.

One hour into the session, if an SP has not checked in, separate the labels for SPs who have checked in from SPs who have not checked in. Set the labels aside for SPs who have not checked in. If the SP does not show up for the session, then discard the labels at the end of the session. 


\section{Urine Specimen Processing}

- Perform pregnancy test and record results before aliquoting the urine specimen.

- Label all urine aliquot vessels for the SP and place in priority order in the rack.

Using the large $15-\mathrm{mL}$ plastic transfer pipette for all but vessel 62 , aliquot the urine sample for each of these tests using the following procedure:

45. Aliquot $3 \mathrm{~mL}$ of urine for Alb/Creat into a $5-\mathrm{mL}$ vessel.

46. Aliquot $10 \mathrm{~mL}$ of urine for Chlam/Trich (14-39 years old) into 14-mL vessel.

154. Aliquot $10 \mathrm{~mL}$ of urine for Trich (40-59 years old) into a 14-mL vessel.

86. Aliquot $3 \mathrm{~mL}$ of urine for Spc Arsenic into a 5-mL vessel.

48. Aliquot $7 \mathrm{~mL}$ of urine for Prior Pest into a $10-\mathrm{mL}$ vessel.

49. Aliquot $7 \mathrm{~mL}$ of urine for Organophos into a $10-\mathrm{mL}$ vessel.

62. Urin Merc. Transfer exactly $5 \mathrm{~mL}$ of urine into the $10-\mathrm{mL}$ VWR screwcap vessel, close the cap, and mix gently. Keep vessel 62 upright at all times; do not invert the tube because the preservative could drain into the cap and spill when opened. If the vessel does become inverted, stand it upright for at least several hours or overnight, then open the cap, and pipette the urine into the $10-\mathrm{mL}$ vessel. Tap the bottom of the capped vessel against a hard surface before opening. Do not discard the prepared tubes even if the preservative crystallizes. If the preservative crystallizes, add 5-ml of urine with the transfer pipette and mix extremely well. Do not use these tubes for any other purpose since they contain a preservative.

50. Aliquot $8 \mathrm{~mL}$ of urine for Heavy Metal into a 14-mL Falcon.

66. Aliquot $3 \mathrm{~mL}$ of urine for PAH into a 5-mL vessel.

67. Aliquot $3 \mathrm{~mL}$ of urine for Phthalates into a 5-mL vessel.

102. Aliquot $5 \mathrm{~mL}$ of urine for Perchlorate/VOC/Thiocyanate into a 5-mL vessel.

121. Aliquot $1 \mathrm{~mL}$ of urine for NNAL/TSNA into a $10-\mathrm{mL}$ vessel. 
128. Aliquot $1 \mathrm{~mL}$ of urine for caffeine into a $2-\mathrm{mL}$ vessel.

152. Aliqout $5 \mathrm{~mL}$ of urine for Aromatic Amines into a 5-mL vessel.

151. Aliqout $5 \mathrm{~mL}$ of urine for Volatile Nitrosamines into a 5-mL vessel.

153. Aliqout $5 \mathrm{~mL}$ of urine for $\mathrm{HAA} /$ Nicotine Analogs into a 5-mL vessel.

52. Aliquot $5 \mathrm{~mL}$ of urine for Xtra Urine 1 into a 5-mL vessel.

139. Aliquot $5 \mathrm{~mL}$ of urine for Xtra Urine 2 into a $5-\mathrm{mL}$ vessel.

If the SP has opted out of Future Studies, the SP won't have vessels 52 or 139 in his or her profile. Inform the blood processing tech that there are not any extras on that SP.

Discard any remaining urine specimen at the end of each session. Pour urine down the drain in the laboratory sink that is connected to the sewer. Flush the sink with water. Discard urine cup in the regular trash, after peeling off the label or marking out the name.

\section{Do not process urine on more than one SP at a time.}

\subsection{Record the Results of Urine Specimen Processing}

After filling the urine specimen vessels for each SP, use the Urine Processing module to enter the results of urine processing. 
Access the Urine Processing module.

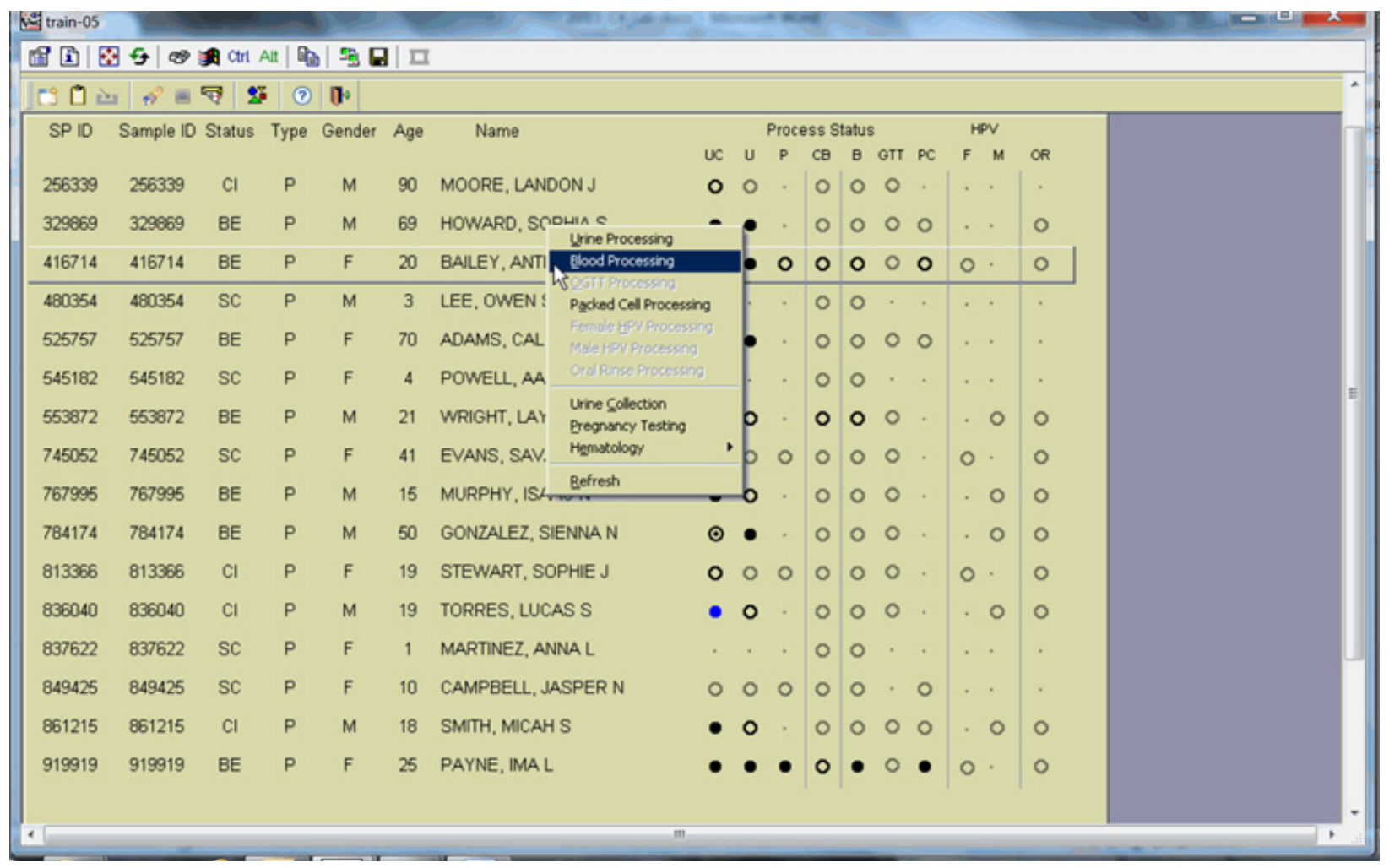

To access the Urine Processing module, use the mouse to direct the mouse arrow to the correct SP, right click, drag the mouse arrow to \{Urine Processing\}, and right click or right click and type $[\mathrm{U} / \mathrm{u}]$. Alternatively, use the up and down keys to move up and down the list until the correct SP is highlighted, right click, drag the mouse arrow to \{Urine Processing\}, and right click or right click and type $[\mathrm{U} / \mathrm{u}]$. 
The SP's urine processing window displays.

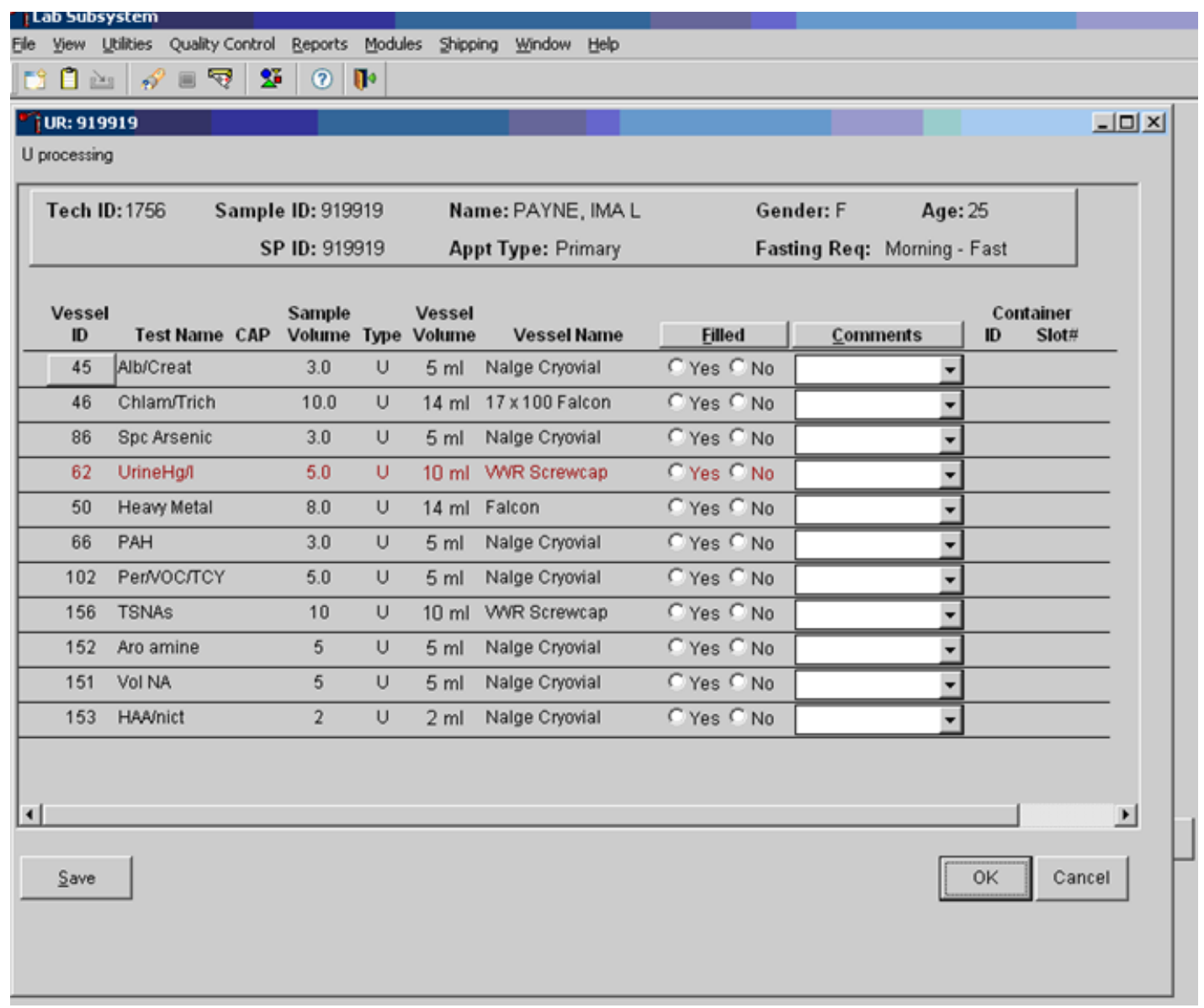

The urine processing window contains the following information for SPs: Tech ID, Sample ID, SP ID, Name (last, first), Gender, Age, Appt (appointment) Type, and Fasting Req. There are columns that list the Vessel ID, Test Name, Sample Volume, Sample Type (U = urine), Vessel Volume, Vessel Name, Filled (Yes or No) radio buttons, Comment drop-down box, and Container ID and Container Slot\# column. 
Individually mark each vessel as Filled - "Yes."

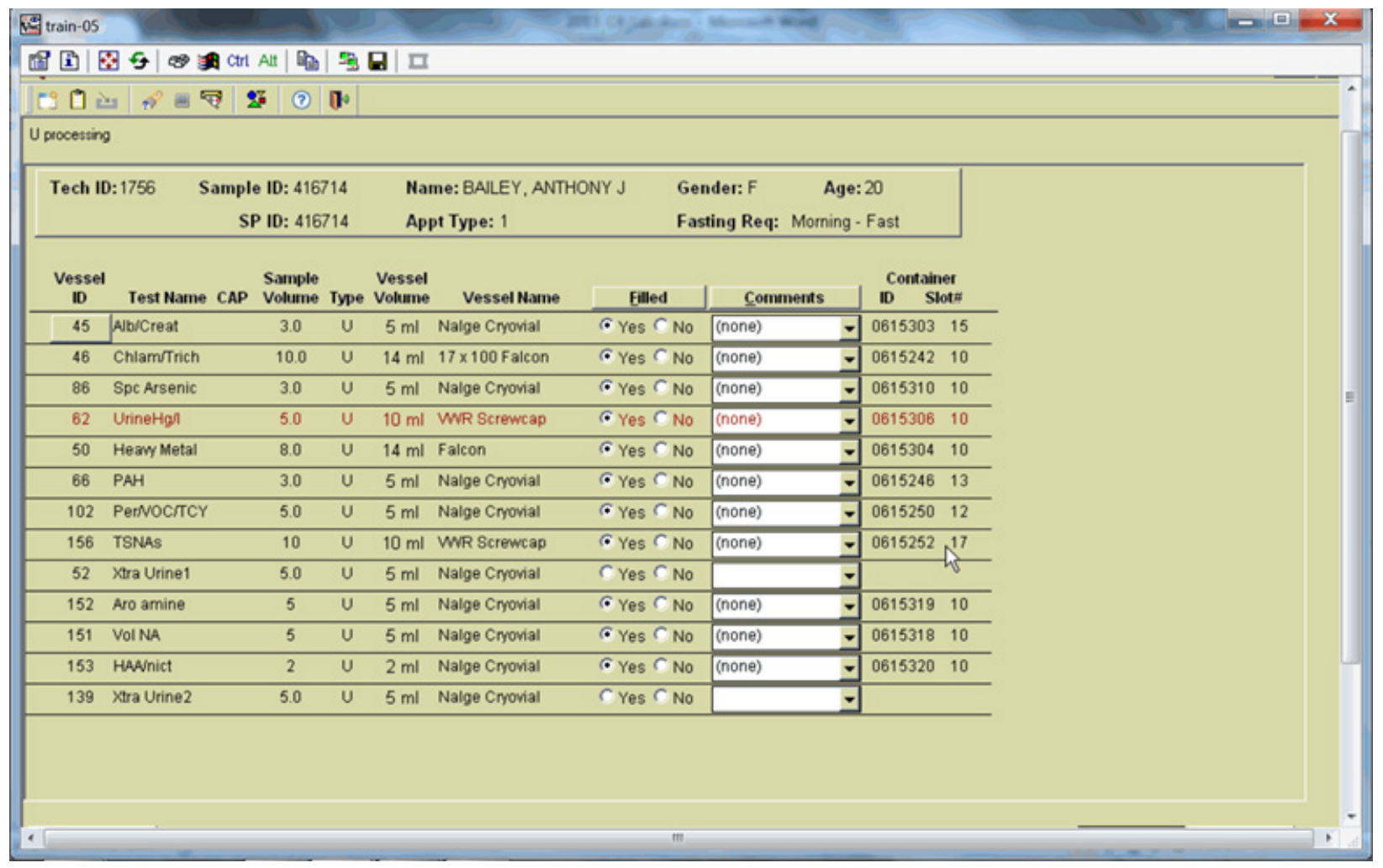

To mark an individual urine vessel as collected or Filled - "Yes," use the mouse to direct the mouse arrow to the center of the "Yes" radio button and left click. As each vessel is marked as Filled -"Yes," it is automatically assigned to a slot in an existing (open) container and the next open slot number. 
Individually mark each vessel as Filled - "No."

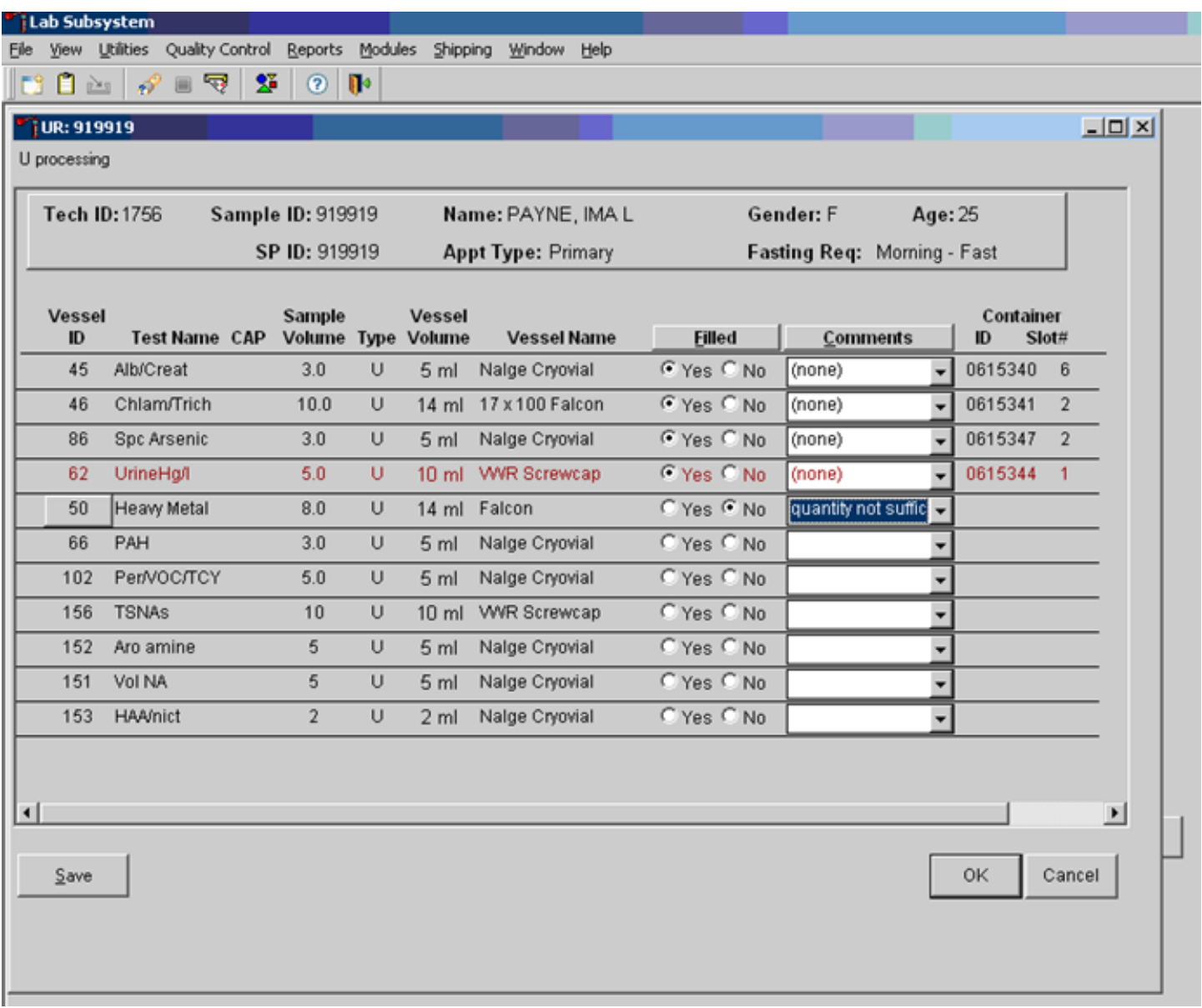

To mark an individual urine vessel as not collected or Filled - "No," use the mouse to direct the mouse arrow to the center of the "No" radio button and left click. As each vessel is marked as Filled "No," the comment "quantity not sufficient" is automatically entered in the Comments column. 
Collectively mark all vessels as Filled - "Yes."

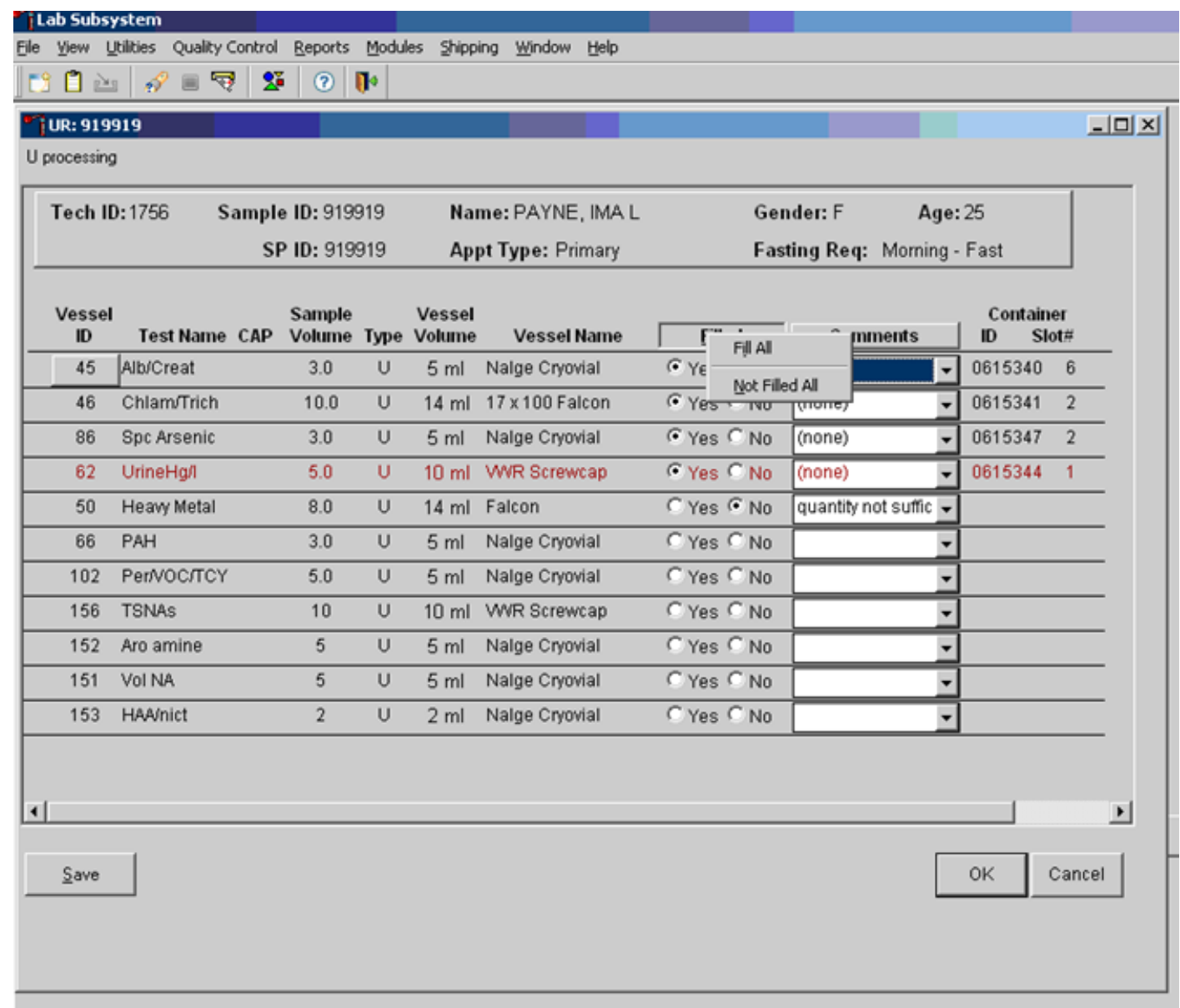

To collectively mark all vessels as Filled - "Yes," use the mouse to direct the mouse arrow to the "Filled" button on the top of the radio buttons, left click, and drag the arrow to \{Fill All $\}$ and left click or type [Shift] [F/f], [I/i]. 
Collectively mark all vessels as Filled - "No."

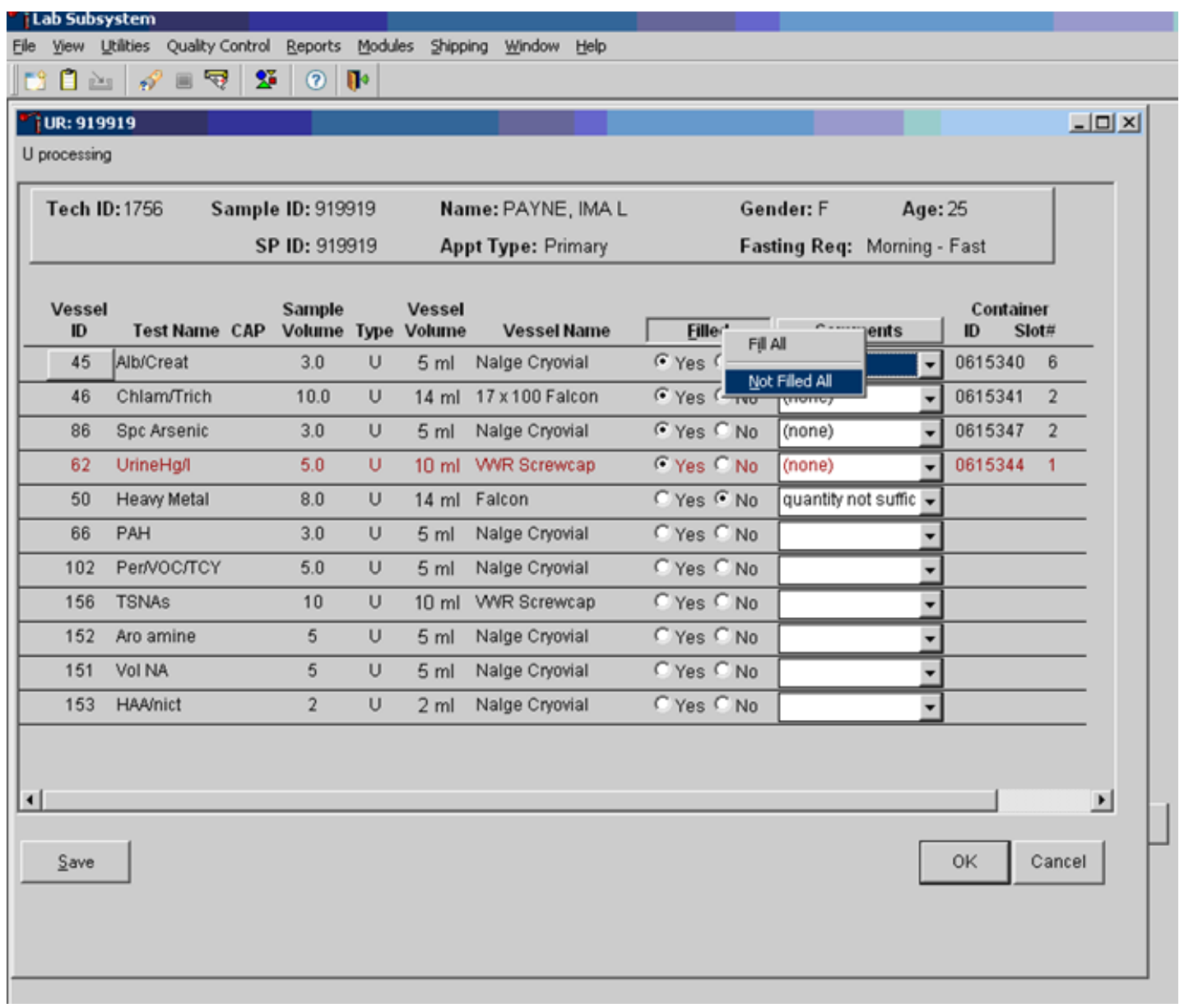

To mark all vessels as Filled - "No," use the mouse to direct the mouse arrow to the Filled box, left click, drag the mouse arrow to \{Not Filled All\}, and left click or type [Shift] [F/f], [N/n.] 
Use the quality comment code to indicate if blood is present.

\begin{tabular}{|l|l|}
\hline Comment Code & Use when: \\
\hline \hline Blood present & Blood is visible in the specimen. \\
\hline
\end{tabular}

Attach the quality comment code to indicate if blood is present.

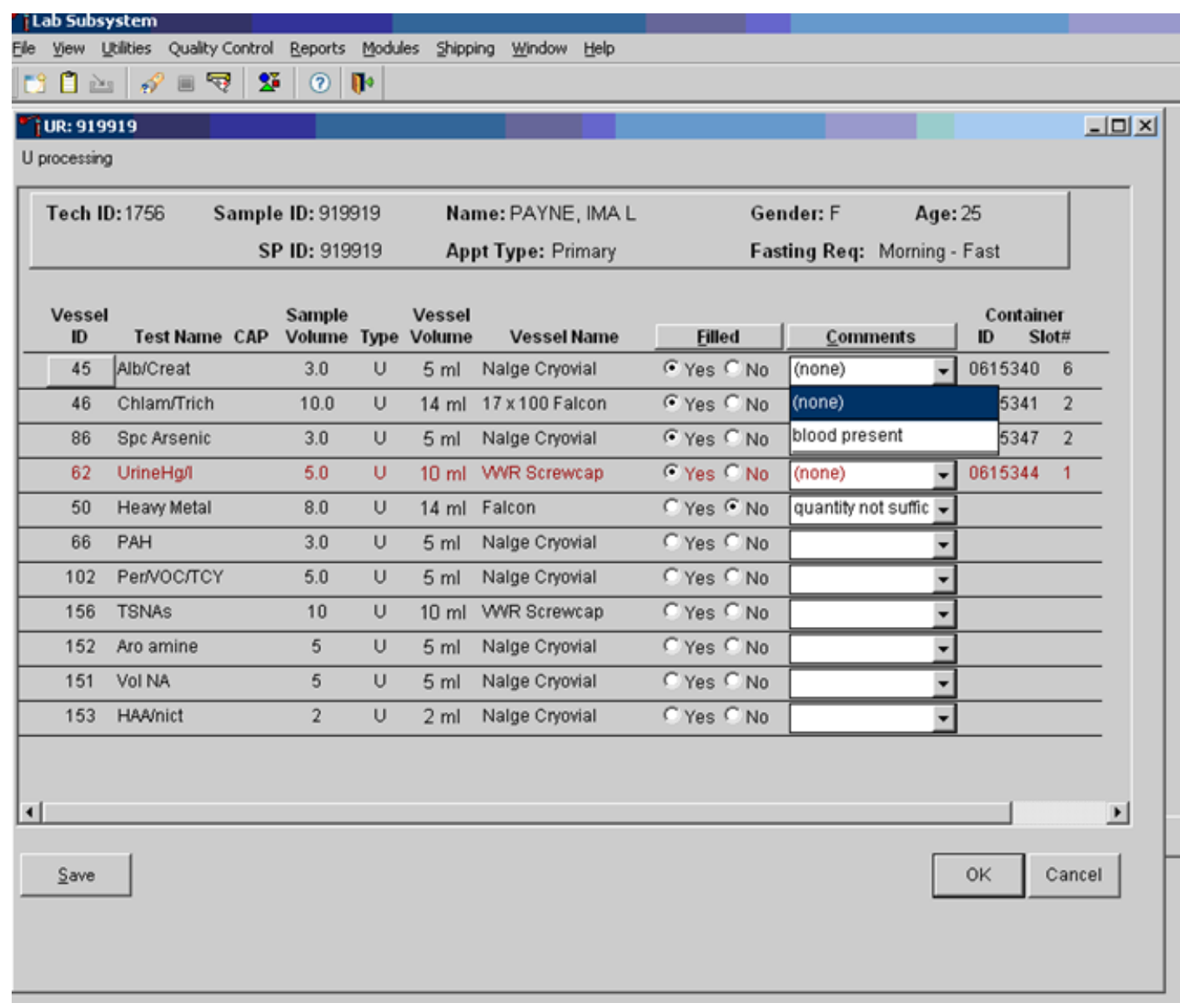

To enter the quality control comment "blood present" for a filled vessel, use the mouse to direct the mouse arrow to the drop-down list in the Comments column, left click to display the choices, drag the mouse arrow to \{blood present\}, and left click. 
If a vessel is marked as Filled-"No," the comment "quantity not sufficient" is automatically entered. Review the comment for all insufficient urine collections and correct the comment, when necessary.

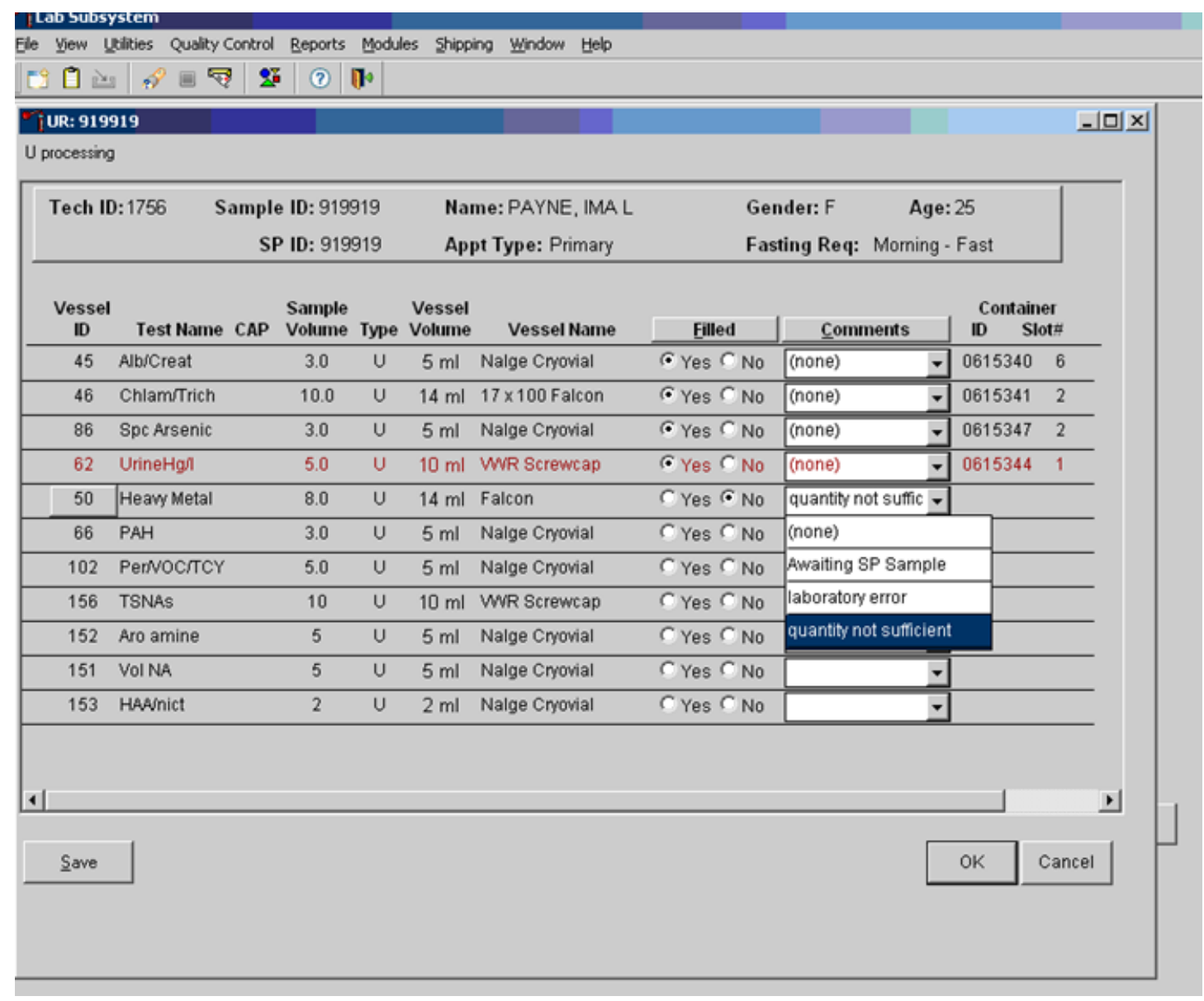

\begin{tabular}{|l|l|}
\hline Comment Code & Use when: \\
\hline \hline laboratory error & A technologist makes an inadvertent error when processing. \\
\hline quantity not sufficient & The amount of urine received in the lab is insufficient to fill this vessel. \\
\hline
\end{tabular}

Continue recording and updating urine collection results as specimens are delivered to the laboratory. 
Review the information in the urine-processing window and save the data to the database.

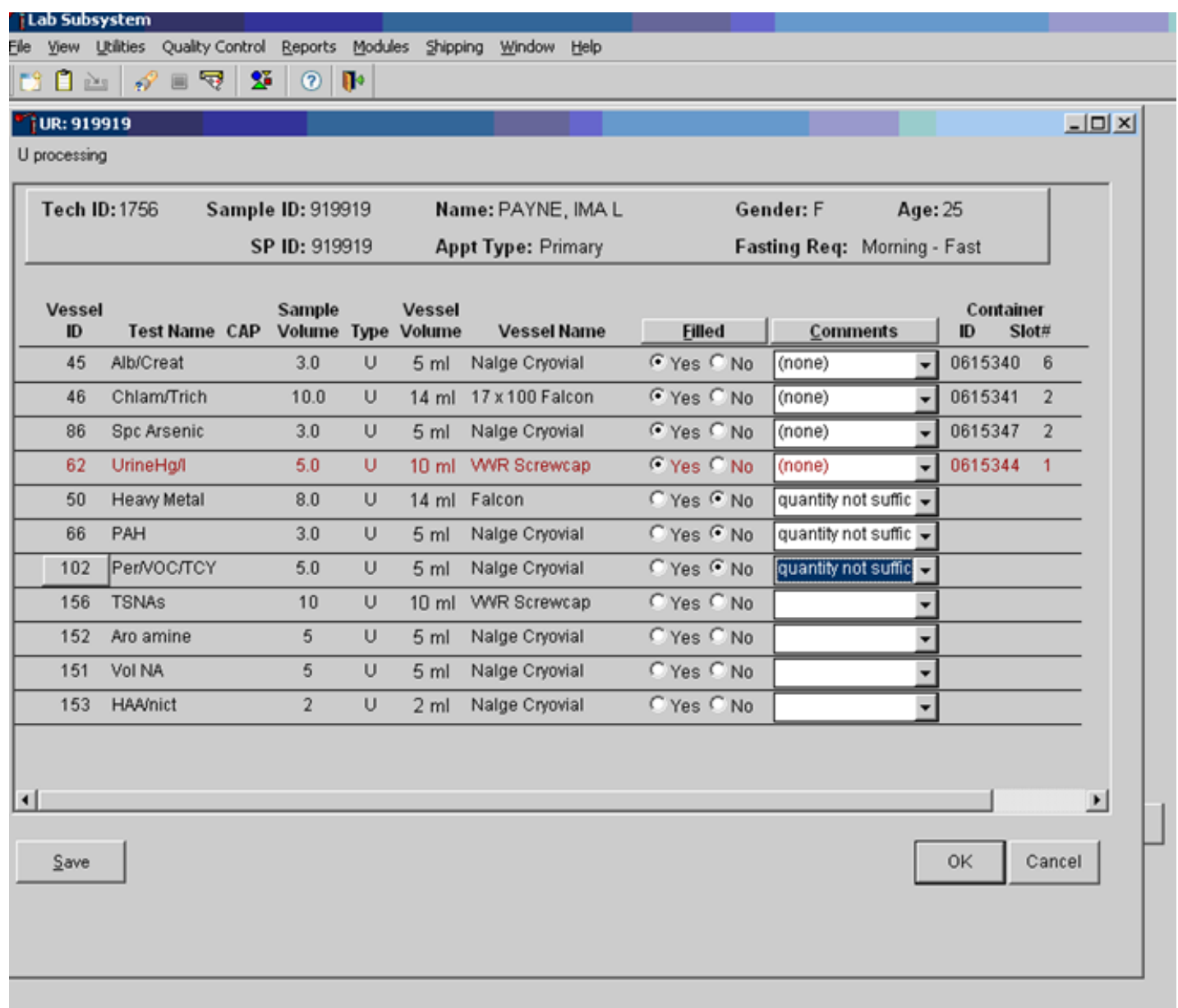

Store each filled vessel in the assigned slot in the assigned container. To record this action or to save the data to the database, use the mouse to direct the mouse arrow to the "Save" button and left click. To record this action or to save the data to the database and exit the module, use the mouse to direct the mouse arrow to the "OK" button and left click. To close the window without saving any data in the database, use the mouse to direct the mouse arrow to the "Cancel" button and left click. 


\subsection{Second and Third Urine Samples}

If the initial urine sample does not meet the minimum volume requirement, refrigerate the initial urine until enough additional urine is received in the second or third urine collection to meet the minimum requirements to complete the protocol or the session ends. If a subsequent sample collection fulfills the protocol requirement, pool the initial and subsequent urine samples into the most recently collected specimen urine cup, update the Urine Collection module, access the Urine Processing module, fill the vessels, and record the results. If the session ends and no additional urine has been collected, access the Urine Processing module, fill as many vessels as possible in priority order, mark the remaining vessels as filled "No," with the comment code "quantity not sufficient," and save the results.

\subsection{Specimen Storage}

Fill the urine vessels, enter the urine processing results, and prepare to store the vessels. Store vessels in numbered storage boxes according to test as indicated in Table 4-3. Check the slot assignment of each urine processing container at the end of each processing session by accessing the Container Map Report. Verify the contents of each container against the container map.

At the beginning of each stand, generate and print a series of barcoded, numbered labels for storage containers as described in Chapter 8. Use the shipping module to assign a barcode label to a specific storage container (test). This process "opens" a storage box. Each vessel is assigned to a specific slot in a specific container as processing results are entered. Slots in containers are assigned according to a standard left to right, top to bottom procedure. Store each vessel in the appropriate slot in the correct container immediately after processing. 
Table 4-3. Storage and shipping protocol for urine

\begin{tabular}{|c|c|c|c|c|c|}
\hline Lab \# & Shipping location & Vessels & Conditions & Frequency & Vessel storage \\
\hline 16 & $\begin{array}{l}\text { University of } \\
\text { Minnesota }\end{array}$ & $45 \mathrm{Alb} /$ Creat & Frozen & Weekly & 9x9 three inch box \\
\hline 5 & $\begin{array}{l}\text { CDC/NCID } \\
\text { NCHSTD }\end{array}$ & 46 Chlam/Trich & Frozen & Weekly & $5 \times 5$ three inch box \\
\hline 5 & $\begin{array}{l}\text { CDC/NCID } \\
\text { NCHSTD }\end{array}$ & Trich & Frozen & Weekly & $5 \times 5$ three inch box \\
\hline 61 & CASPIR & $86 \mathrm{Spc}$ Arsenic & Frozen & Weekly & $9 \times 9$ three inch box \\
\hline 26 & $\begin{array}{l}\text { CDC/NCEH Pesticide } \\
\text { Laboratory }\end{array}$ & 48 Prior Pest & Frozen & $\begin{array}{l}\text { End of } \\
\text { stand }\end{array}$ & $5 \times 5$ three inch box \\
\hline 26 & $\begin{array}{l}\text { CDC/NCEH Pesticide } \\
\text { Laboratory }\end{array}$ & 49 Organophos & Frozen & $\begin{array}{c}\text { End of } \\
\text { stand }\end{array}$ & $5 \times 5$ three inch box \\
\hline 61 & CASPIR & 62 Urin Merc & Frozen & Weekly & $5 \times 5$ three inch box \\
\hline 61 & CASPIR & 50 Heavy Metal & Frozen & Weekly & $5 \times 5$ three inch box \\
\hline 31 & CASPIR & $66 \mathrm{PAH}$ & Frozen & $\begin{array}{c}\text { End of } \\
\text { stand }\end{array}$ & $9 \times 9$ three inch box \\
\hline 24 & $\begin{array}{l}\text { CDC/NCEH } \\
\text { Environmental Health } \\
\text { Laboratory }\end{array}$ & 67 Phthalates & Frozen & $\begin{array}{c}\text { End of } \\
\text { stand }\end{array}$ & 9x9 three inch box \\
\hline 62 & CASPIR & $\begin{array}{l}102 \\
\text { Perchlorate/VOC/Thioc } \\
\text { yanate }\end{array}$ & Frozen & $\begin{array}{l}\text { End of } \\
\text { stand }\end{array}$ & $5 \times 5$ three inch box \\
\hline 59 & CASPIR & 121 NNAL/TSNA & Frozen & $\begin{array}{c}\text { End of } \\
\text { stand }\end{array}$ & $5 \times 5$ three inch box \\
\hline 54 & CASPIR & 128 Caffeine & Frozen & Weekly & 10x10 two inch box \\
\hline 6 & CASPIR & Aromatic Amines & Frozen & Weekly & $9 \times 9$ three inch box \\
\hline 6 & CASPIR & Volatile Nitrosamines & Frozen & Weekly & 9x9 three inch box \\
\hline 6 & CASPIR & HAA/Nicotine Analogs & Frozen & Weekly & 9x9 three inch box \\
\hline 6 & CASPIR & 52 Xtra Urine 1 & Frozen & $\begin{array}{c}\text { End of } \\
\text { stand }\end{array}$ & $9 \times 9$ three inch box \\
\hline 6 & CASPIR & 139 Xtra Urine 2 & Frozen & $\begin{array}{c}\text { End of } \\
\text { stand }\end{array}$ & 9x9 three inch box \\
\hline
\end{tabular}

\subsection{How to Deal With System Failure}

If the computer system fails, record results on a preprinted workstation 2 processing worksheet (Exhibit 4-4). Complete a workstation 2 worksheet for each SP after performing the pregnancy test, and while processing the urine specimens. Enter the results after the system is operational. Send the worksheets to the home office at the end of the stand. 
Exhibit 4-4. Workstation 2 processing worksheet

URINE COLLECTION, PREGNANCY TEST, URINE PROCESSING

SP ID

\begin{tabular}{|c|c|c|c|c|c|c|c|c|}
\hline & & \multicolumn{2}{|l|}{$\begin{array}{c}\text { Urine Collected } \\
\text { Age } 6+\end{array}$} & Yes & $\underset{\downarrow}{\mathrm{Yes} / \mathrm{QNS}}$ & No & \multicolumn{2}{|c|}{$\begin{array}{l}\text { Comments: } \\
\text { Communication problem } \\
\text { No time } \\
\text { Quantity not sufficient } \\
\text { SP } \text { ill/emergency } \\
\text { SP refusal } \\
\text { SP unable to comply }\end{array}$} \\
\hline \multicolumn{9}{|c|}{ Result } \\
\hline & & \multicolumn{2}{|l|}{$\begin{array}{l}\text { Pregnancy Test } \\
\text { Age } 8-17\end{array}$} & $\underset{\checkmark}{\text { Negative }}$ & $\begin{array}{c}\text { Invalid } \\
\checkmark \\
\end{array}$ & $\begin{array}{c}\text { Positive } \\
\checkmark\end{array}$ & \multicolumn{2}{|c|}{$\begin{array}{l}\text { Serum Confirmation Result } \\
\text { (Send observation to physician) }\end{array}$} \\
\hline \multicolumn{9}{|c|}{ Result } \\
\hline & & \multicolumn{2}{|l|}{$\begin{array}{l}\text { Pregnancy Test } \\
\text { Age } 18-59\end{array}$} & $\underset{\checkmark}{\text { Negative }}$ & $\underset{\vee}{\text { Invalid }}$ & $\begin{array}{l}\text { Positive } \\
\checkmark\end{array}$ & & \\
\hline \multicolumn{9}{|c|}{ Result } \\
\hline ID & Name & & Ages & $\begin{array}{l}\text { Sample } \\
\mathrm{mL}\end{array}$ & $\begin{array}{l}\text { Sample } \\
\text { Type }\end{array}$ & $\underset{\checkmark}{\text { Filled }}$ & $\begin{array}{l}\text { Comments } \\
\text { QNS } \\
\text { Blood Present }\end{array}$ & Slot \# \\
\hline 45 & \multicolumn{2}{|c|}{ Alb/Creat } & $6+$ & 3 & Urine & & & \\
\hline 46 & \multicolumn{2}{|c|}{ Chlam/Trich } & $14-39$ & 10 & Urine & & & \\
\hline 154 & \multicolumn{2}{|l|}{ Trich } & $40-59$ & 10 & Urine & & & \\
\hline 86 & \multicolumn{2}{|c|}{ Spc Arsenic } & $6+$ & 3 & Urine & & & \\
\hline 48 & \multicolumn{2}{|c|}{ Prior Pest } & $6+$ & 7 & Urine & & & \\
\hline 49 & \multicolumn{2}{|c|}{ Organophos } & $6+$ & 7 & Urine & & & \\
\hline 62 & \multicolumn{2}{|c|}{ Urn Merc/Iodine } & $6+$ & 5 & Urine & & & \\
\hline 50 & \multicolumn{2}{|c|}{ Heavy Metal } & $6+$ & 8 & Urine & & & \\
\hline 66 & \multicolumn{2}{|l|}{ PAH } & $6+$ & 3 & Urine & & & \\
\hline 67 & \multicolumn{2}{|c|}{ Phthalates } & $6+$ & 3 & Urine & & & \\
\hline 102 & \multicolumn{2}{|c|}{ Perchlorate/VOC/Thiocyanate } & $6+$ & 5 & Urine & & & \\
\hline 121 & \multicolumn{2}{|c|}{ NNAL/TSNA } & $6+$ & 10 & Urine & & & \\
\hline 128 & \multicolumn{2}{|c|}{ Caffeine } & $6+$ & 1 & Urine & & & \\
\hline 152 & \multicolumn{2}{|c|}{ Aromatic Amines } & $6+$ & 5 & Urine & & & \\
\hline 151 & \multicolumn{2}{|c|}{ Volitile Nitrosamines } & $6+$ & 5 & Urine & & & \\
\hline 153 & \multicolumn{2}{|c|}{ HAA/Nicotine Analogs } & $6+$ & 5 & Urine & & & \\
\hline 52 & \multicolumn{2}{|c|}{ Xtra Urine 1} & $6+$ & 5 & Urine & & & \\
\hline 139 & \multicolumn{2}{|c|}{ Xtra Urine 2} & $6+$ & 5 & Urine & & & \\
\hline
\end{tabular}




\section{URINE PREGNANCY TEST}

\subsection{Purpose and Principle of the Test}

Perform a pregnancy test on females of all appointment types who are aged 12-59 years, and girls 8-11 who report that they are menstruating when asked during the home interview. A positive test result excludes pregnant women aged 8-59 from participating in the dual-energy x-ray absorptiometry (DXA), OGTT, and Bronchodilator sections of the MEC exam. Report test results immediately since these components are not assigned until the results of the pregnancy test are documented. If a urine pregnancy test is positive on any female SP aged 8-17 years, the result is confirmed using a serum test. If no blood is drawn, repeat the urine test. Notify the physician of the second positive or negative confirmatory test or the inability to perform a confirmation test using the observation function.

Tests for confirming pregnancy are based on detecting elevated levels of human chorionic gonadotropin (hCG), a hormone that the placenta begins to produce in increasing amounts shortly after fertilization. The ICON ${ }^{\circledR} 25$ hCG (Urine/Serum) test kit is a rapid chromatographic immunoassay for the qualitative detection of human chorionic gonadotropin (hCG) in urine or serum to aid in the early detection of pregnancy.

In normal pregnancy, hCG can be detected in both urine and serum as early as 7 to 10 days after conception. The hCG levels continue to rise very rapidly, frequently exceeding $100 \mathrm{mIU} / \mathrm{mL}$ by the first missed menstrual period, and peaking in the 100,000 to $200,000 \mathrm{mIU} / \mathrm{mL}$ range about $10-12$ weeks into pregnancy. The appearance of hCG in both urine and serum soon after conception, and its subsequent rapid raise in concentration during early gestational growth, make an excellent marker for the detection of pregnancy.

The ICON ${ }^{\circ} 25 \mathrm{hCG}$ test is a rapid test that qualitatively detects the presence of hCG in urine or serum sample at the sensitivity of $25 \mathrm{mIU} / \mathrm{mL}$. The test utilizes a combination of monoclonal and polyclonal antibodies to selectively detect elevated levels of hCG in urine or serum. At the level of claimed sensitivity, the ICON ${ }^{2} 25$ hCG test shows no cross-reactivity interference from the structurally related glycoprotein hormones $\mathrm{hFSH}, \mathrm{hLH}$, and hTSH at high physiological levels. 
The assay is conducted by adding urine or serum sample to the sample well of the test device. The sample migrates via capillary action along the membrane to react with colored conjugate. Positive samples react with the specific antibody-hCG-colored conjugate to form a colored line at the test region of the membrane. Absence of this colored line suggests a negative result. To serve as a procedural control, a colored line will always appear at the control line region if the test has been performed properly.

\subsection{Special Safety Precautions}

ICON® 25 hCG Test (Urine or Serum)

- This test is for professional in vitro diagnostic use only.

- Do not use the kit beyond the expiration date.

- The test device should remain in the sealed pouch until use.

- Observe standard guidelines for handling biological hazards.

- Wear gloves while handling specimens. After use, dispose of gloves and other contaminated materials appropriately in a proper biohazard container and wash hands.

Sure-Vue ${ }^{\mathrm{TM}}$ hCG Urine and Serum Control Sets

- Sure-Vue ${ }^{\mathrm{TM}}$ hCG urine and serum controls are intended to be used to monitor the performance and accuracy of pregnancy test kits. These controls are for professional use only.

- The use of known controls in the laboratory is invaluable. It is important to verify testing procedures to confirm the validity of the results reported. Testing Sure-Vue ${ }^{\mathrm{TM}}$ hCG Urine and Serum Controls will provide assurance that the pregnancy test kit is performing properly.

- Reagents in these kits contain 0.2 percent of sodium azide, which may react with lead or copper plumbing to form potentially explosive metal azides. When disposing of such reagents, always flush with large volumes of water to prevent azide buildup. This reagent is harmful if swallowed. Contact with acids liberates very toxic gas. After contact with skin, wash immediately with plenty of water.

- Do not use the controls beyond the expiration date. 


\subsection{Computerization: Integrated Survey and Information System (ISIS)}

The MEC-automated ISIS system captures all data and stores it electronically at Westat's home office in Rockville, Maryland. Access the Pregnancy QC module to enter the quality control results. The session number, technologist ID, and run number are automatically captured; enter the kit lot number and expiration date, and the urine and serum control lot and expiration dates. Record control results and save them to the database. All pregnancy quality control results are immediately available electronically at the home office. All data are backed up and stored at Westat's home office.

\subsection{Specimen Collection and Preparation}

Collect a urine specimen on all SPs 6 years and older into a clean and dry plastic container and document the collection in the urine collection module as described in Chapter 4. Perform a urine pregnancy test on females of all person types who are aged 12-59 years and girls aged 8-11 who report that they are menstruating when asked during the home interview. If a urine pregnancy test is positive on any female SP aged 8-17, confirm the result using serum. If no blood is drawn, repeat the urine test. Notify the physician of the second positive or negative confirmatory test or the inability to perform a confirmation test using the observation function. Assay all specimens immediately and record results in the Laboratory Pregnancy Testing module.

\subsection{Procedure for Microscopic Examination}

Not applicable. 


\subsubsection{Reagents}

ICON® 25 hCG Urine/Serum test kit

Product Number 43025 (25)

Beckman Coulter

4300 N. Harbor Blvd

Fullerton, CA 92834-3100

$1-800-877-6242$ or $650-845-3526$

- Components

- $\quad$ One test device. (25) - Contains anti-hCG particles and anti-hCG coating on the membrane.

- $\quad$ Disposable sample droppers - Plastic pipettes for measuring and dispensing patient samples. (Packaged together with the test device in a white foil pouch.)

- $\quad$ Zip closable bag with two extra sample droppers

- $\quad$ Product instructions

- Storage and Stability

- $\quad$ Store the ICON 25 hCG test kit at $2-30^{\circ} \mathrm{C},\left(59-83^{\circ} \mathrm{F}\right)$. The test device is stable up to the expiration date printed on the sealed pouch. Keep the test device in the sealed pouch until use. DO NOT FREEZE. Do not use beyond expiration date. Date and initial the kit when putting it into use.

Sure-Vue ${ }^{\mathrm{TM}}$ hCG Control Sets - Urine and Serum

Sure-Vue ${ }^{\mathrm{TM}}$ hCG Control Sets - Urine

Product Number SA087413-F

Fisher Scientific Company

1-888-727-3315 (Technical assistance)

- Components - Sure-Vue ${ }^{\mathrm{TM}}$ hCG Control Set

- Urine is a stable human urine-based material for use as a quality control material. Use controls in the same manner as SP specimens in accordance with the protocol provided in the Sure-Vue ${ }^{\mathrm{TM}}$ hCG Control Sets directional insert. 
- $\quad$ Low Positive Control: Human urine containing approximately $25 \mathrm{mIU}$ hCG/mL and $0.2 \%$ sodium azide as a preservative. $(1 \times 5 \mathrm{~mL})$

- High Positive Control: Human urine containing approximately $250 \mathrm{mIU}$ $\mathrm{hCG} / \mathrm{mL}$ and $0.2 \%$ sodium azide as a preservative. ( $1 \times 5 \mathrm{~mL}$ )

- $\quad$ Negative Control: Contains $0.2 \%$ sodium azide as a preservative. ( 1 x $5 \mathrm{~mL}$ )

- Storage and Stability - Sure-Vue ${ }^{\mathrm{TM}}$ hCG Control Set

- $\quad$ Store the urine control set between 2 to $8^{\circ} \mathrm{C}\left(35\right.$ to $\left.46^{\circ} \mathrm{F}\right)$ at which temperatures these controls are stable until the expiration date printed on the label.

Sure-Vue ${ }^{\mathrm{TM}}$ hCG Serum Control Set

Product Number 087712-F

Fisher Scientific Company

1-888-727-3315 (Technical assistance)

- Components - Sure-Vue ${ }^{\mathrm{TM}}$ hCG Serum Control

- $\quad$ The Sure-Vue ${ }^{\mathrm{TM}}$ hCG Serum Control is a stable human serum-based material for use as a quality control material. Use controls in the same manner as SP specimens in accordance with the protocol provided in the Sure-Vue ${ }^{\mathrm{TM}}$ hCG Control Set directional insert.

- Low Positive Control: Human serum containing approximately $25 \mathrm{mIU}$ $\mathrm{hCG} / \mathrm{mL}$ and $0.2 \%$ sodium azide as a preservative. $(1 \times 5 \mathrm{~mL})$

- High Positive Control: Human serum containing approximately $250 \mathrm{mIU}$ $\mathrm{hCG} / \mathrm{mL}$ and $0.2 \%$ sodium azide as a preservative. $(1 \times 5 \mathrm{~mL})$

- $\quad$ Negative Control: Contains $0.2 \%$ sodium azide as a preservative. ( 1 x $5 \mathrm{~mL}$ )

- Storage and Stability - Sure-Vue ${ }^{\mathrm{TM}}$ hCG Serum Control

- $\quad$ Store the Sure-Vue ${ }^{\mathrm{TM}}$ hCG Serum Control Set between 2 to $8^{\circ} \mathrm{C}\left(35\right.$ to $\left.46^{\circ} \mathrm{F}\right)$ at which temperatures these controls are stable until the expiration date printed on the label.

\subsubsection{Supplies}

- Timer 
Not applicable

\section{Assay Procedure}

\section{ICON® 25 hCG Test kit}

- Label each test device with the preprinted label or write the SP ID on a clear section of the test device using a felt tip pen.

- Use a new transfer dropper for each specimen.

Sure-Vue ${ }^{\mathrm{TM}}$ hCG Urine and Serum Control Sets

- The Sure-Vue ${ }^{\mathrm{TM}}$ urine and serum controls have their own droppers. The use of the control droppers to add three drops of sample has been validated; do not use the ICON $25 \mathrm{hCG}$ plastic pipette to add controls.

- Allow controls to reach room temperature before testing. The controls are ready to use. No dilution is required.

- The controls are used in place of the specimen and should be tested according to the $\mathrm{ICON} \circledast 25 \mathrm{hCG}$ test procedure.

- Discard the high positive urine and serum vials $(250 \mathrm{mIU} / \mathrm{mL})$.

Urine or Serum Testing Procedure

Perform the test procedure for ICON® $25 \mathrm{hCG}$ Urine/Serum test at room temperature $\left(15-30^{\circ} \mathrm{C}, 59-86^{\circ} \mathrm{F}\right)$. Before proceeding, carefully read Section XII titled "Limitations of Method: Specimen Rejection, Interfering Substances, and Conditions,” found on pages 6-26 through 6-47.

1. Remove the test device from the sealed pouch and use it as soon as possible.

2. Place the test device on a clean and level surface. Hold the dropper vertically and transfer three full drops of urine or serum (approximately $100 \mu \mathrm{L}$ ) to the sample well (S) of the test device, and then start the timer. Avoid trapping air bubbles in the sample well. 
3. Wait for the red line(s) to appear. Read the results at 3 minutes when testing a urine sample or 5 minutes when testing a serum sample. It is important that the background is clear before the result is read.

NOTE: A low hCG concentration might result in a weak line appearing in the test region (T) after an extended period; therefore, do not interpret the result after 3 minutes when testing a urine sample or after 5 minutes when testing a serum sample.

\subsection{Reportable Range of Results}

Report test results as Positive, Negative, or Invalid.

\subsection{Quality Control}

Run negative and low positive urine controls containing hCG at concentrations of 0 and 25 $\mathrm{mIU} / \mathrm{mL}$, respectively, just prior to or concurrently with the first examinee pregnancy test each exam day, and when putting a new lot into use. Run negative and low positive serum controls with each serum pregnancy test. The pregnancy QC module contains the following information:

- The session ID number links to the stand number, stand location, date, including day, month, and year. The technologist ID is automatically captured.

- Kit lot number and expiration date fill in automatically from the prior QC report. Update or manually enter this information if necessary.

- The control lot number and expiration date for both the urine and serum control fills in automatically from the prior QC report. Update or manually enter this information if necessary.

- The Control QC Type is either urine or serum. The default type is set as urine.

- Negative Control result.

- Positive Control result. 


\section{CAP Proficiency Testing}

For purposes of this document, proficiency testing is one method of external quality control in which the analytical performance of a method is evaluated using specimens provided on a periodic basis (usually every 3 months).

Participation in the College of American Pathologist (CAP) EXCEL proficiency-testing program is part of the comprehensive quality control program. Each MEC submits results for CAP specimens for evaluation. CAP compares the results to established values and issues a report.

CAP sends samples three times a year for qualitative serum hCG and urine hCG. Each serum survey includes five lyophilized sera specimens and each urine survey includes five liquid specimens. Handle and analyze these samples in a manner identical to SP samples. Record results on the correct CAP form, located on the CAP web site, and submit results. Send a copy of the CAP result form to the home office at the end of the stand. 
Open the Pregnancy QC module.

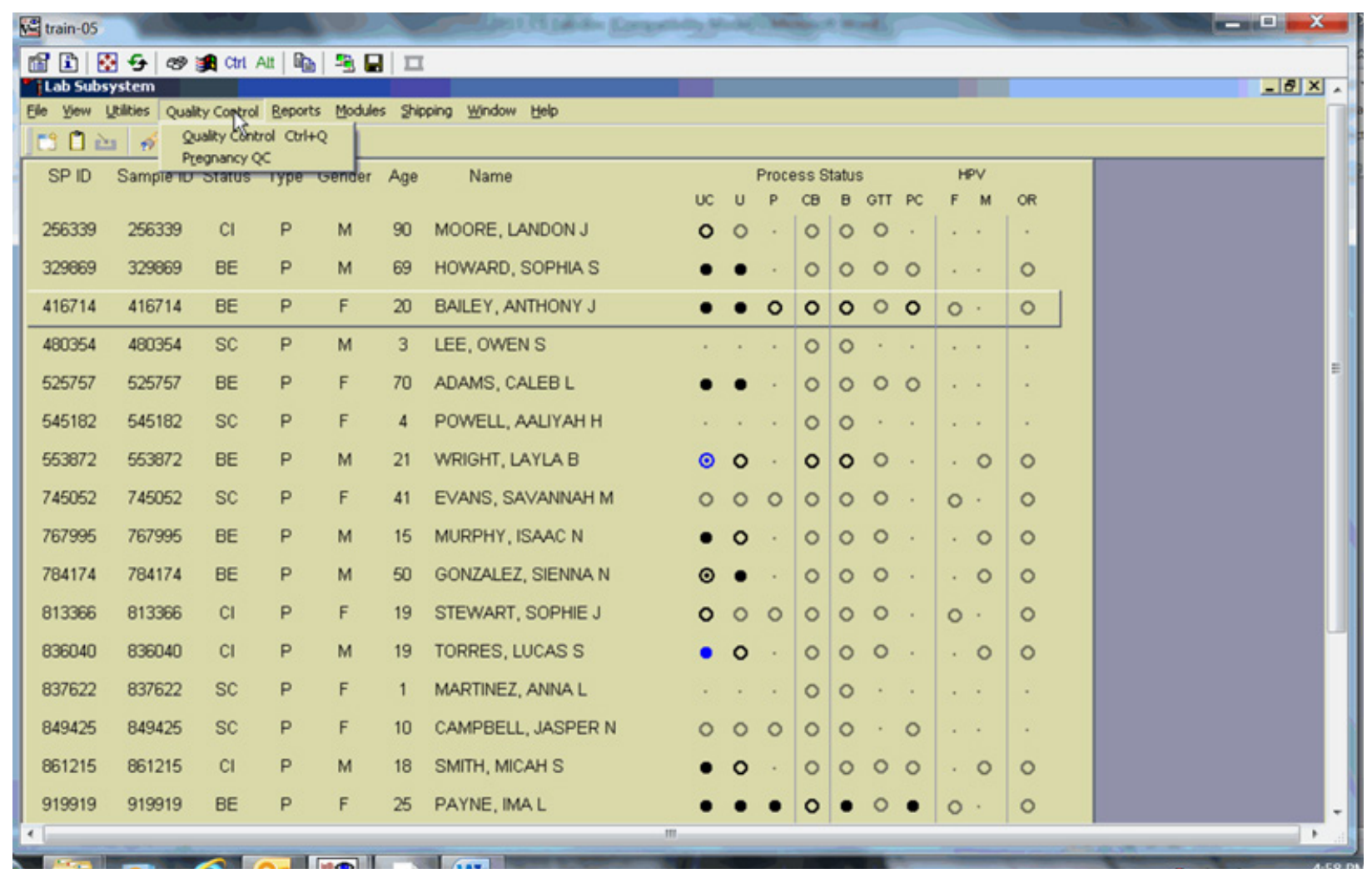

To open the pregnancy QC module, use the mouse to direct the mouse arrow to \{Utilities\} in the top menu bar, drag the arrow to \{Pregnancy QC\}, and left click or type [Alt] [U/u], $[\mathrm{R} / \mathrm{r}]$. 
Alternatively, open the Pregnancy QC module from the heads-up screen.

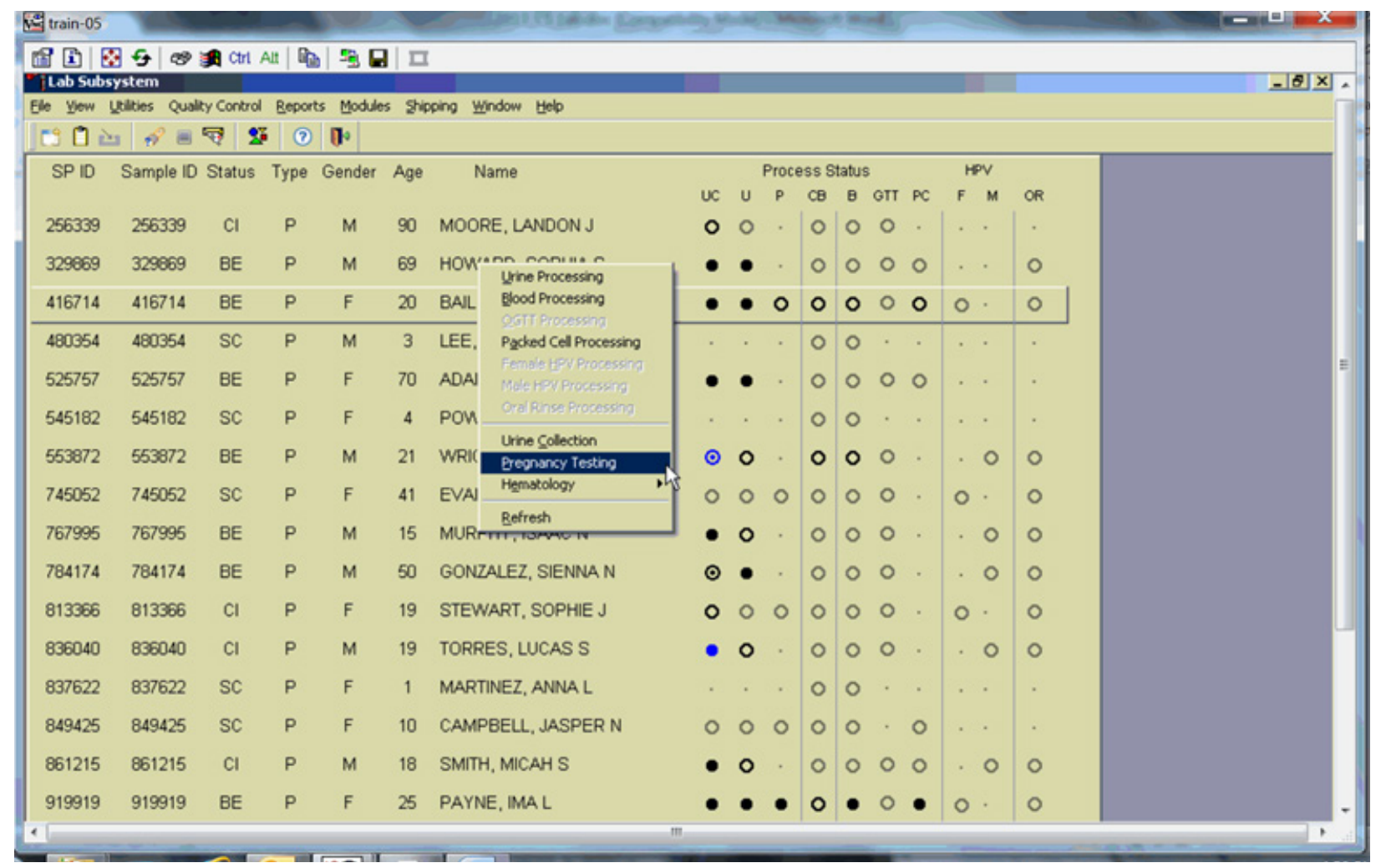

To access the Pregnancy QC module from the heads-up screen, use the up and down keys to move up and down the list until the correct SP is highlighted, or use the mouse to direct the mouse arrow to the correct SP, right click, drag the mouse arrow to \{Pregnancy Testing\}, and left click or left click and type $[\mathrm{P} / \mathrm{p}]$. 
The pregnancy QC window displays.

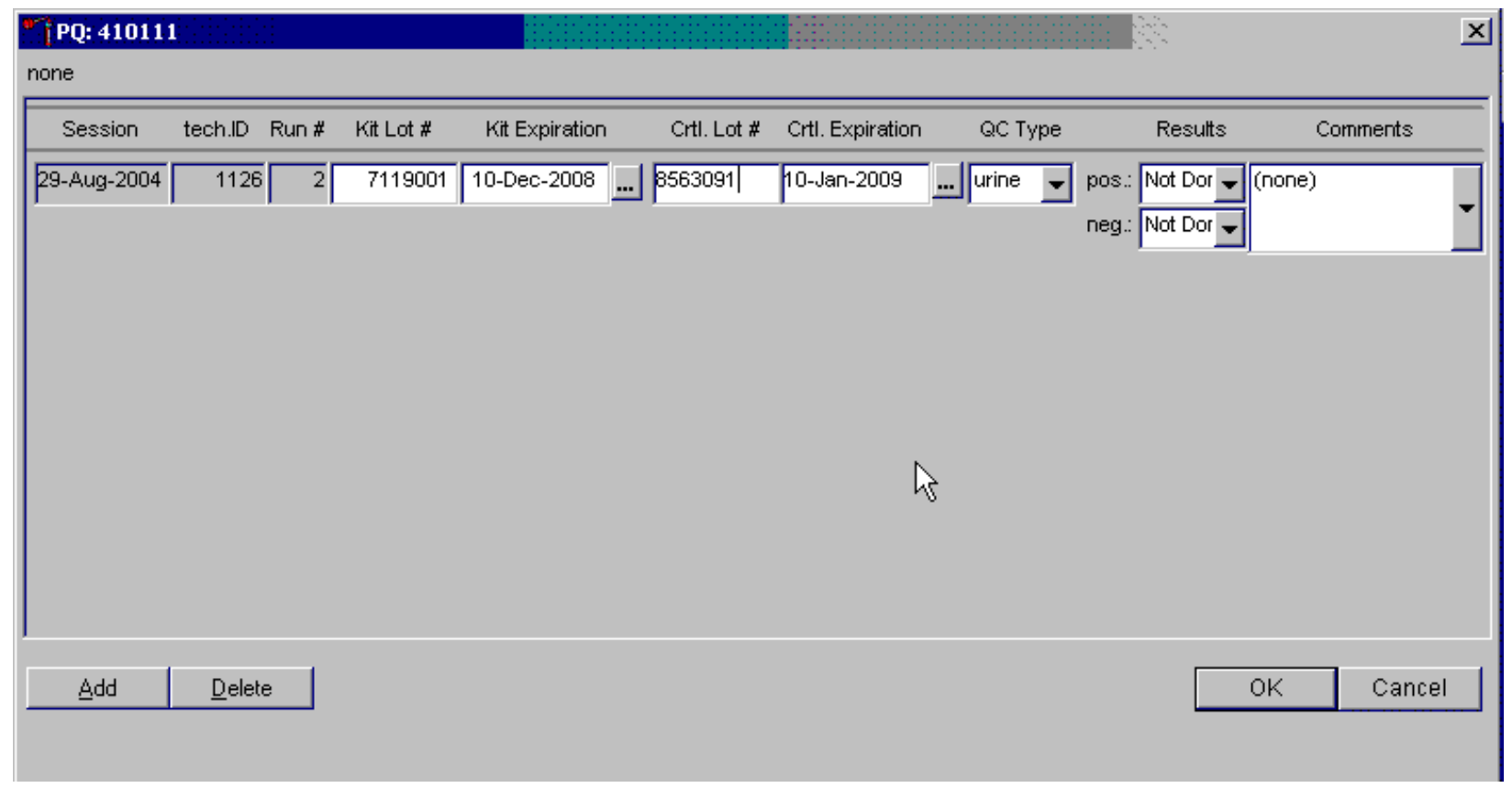

The pregnancy QC module automatically captures the session date and technologist ID and assigns the run number. The module defaults to the last kit lot number, kit expiration date, Ctrl (control) lot number, Ctrl expiration date, and the QC type - urine. Verify the existing kit lot number and expiration date. If the lot number is incorrect, type in the new kit lot number using the keyboard's numeric keys and select [Tab] to progress to the Kit Expiration blank. Use the calendar to correct the Kit Expiration date. If the screen defaults to the current $\mathrm{Ctrl}$ lot number, verify the existing lot number and expiration date. If the lot number is incorrect, type in the new control lot number using the keyboard numeric keys and select [Tab] to progress to the Ctrl Expiration blank. Use the calendar to correct either the Kit or the Ctrl Expiration date. 
Update the Kit or Ctrl Expiration date using the calendar function.

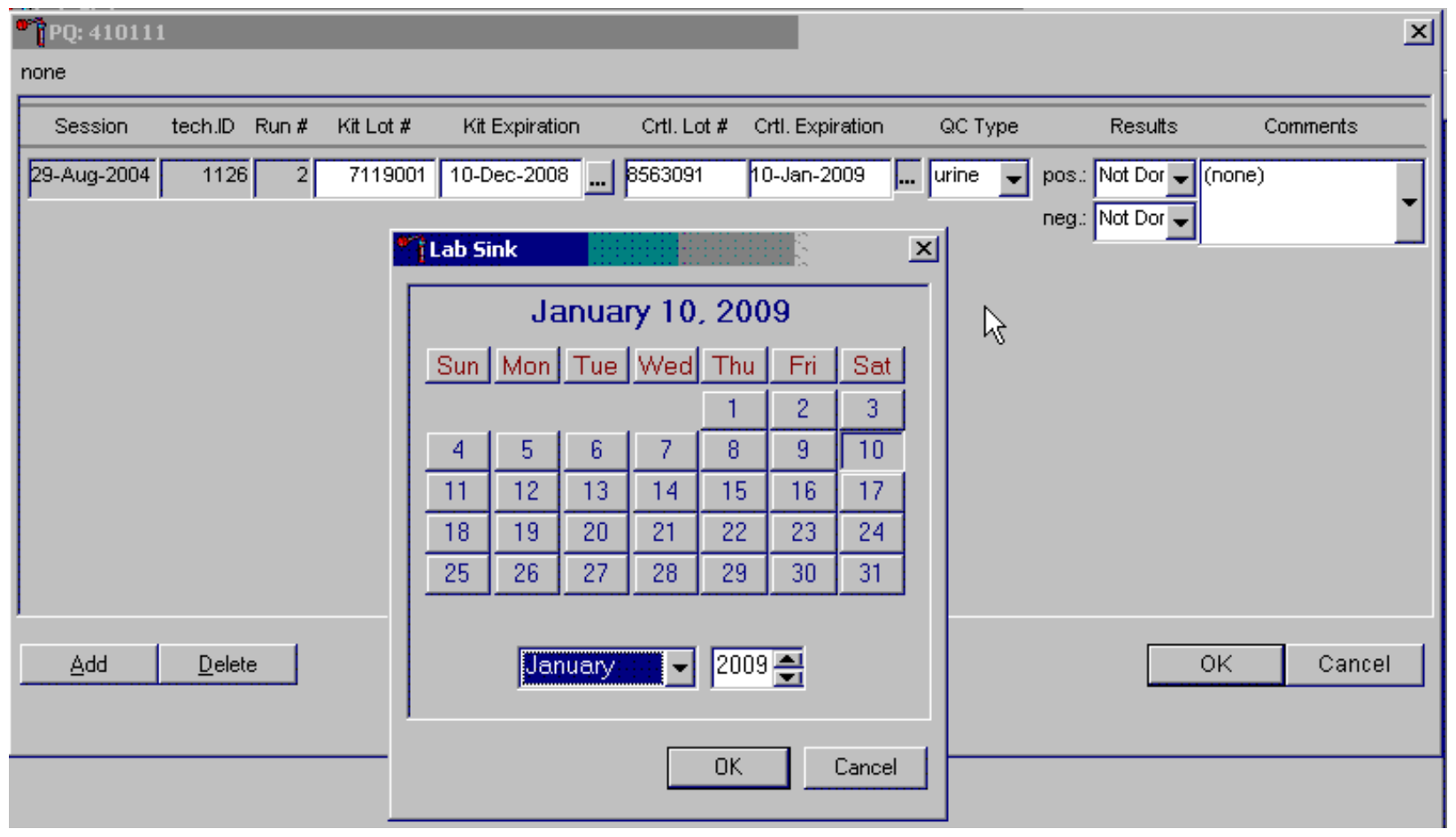

Type in the date using the keyboard's numeric keys and the $\mathrm{dd} / \mathrm{mm} / \mathrm{yyyy}$ format and select [Tab] or use the calendar to enter the date. To access the calendar, use the mouse to direct the mouse arrow to the ellipsis and left click. To select the correct month, use the mouse to direct the mouse arrow to the drop-down list, drag the arrow to the correct month (use the scroll bar if necessary) and left click. To select the correct day, use the mouse to direct the mouse arrow to the correct day on the displayed month and left click. To correct the year, use the mouse to direct the mouse arrow to up/down controls on the spin box and toggle the year up and down. To transfer this date into the text box, use the mouse to direct the mouse arrow to the OK button and left click; to exit the calendar function, use the mouse to direct the mouse arrow to the Cancel button and left click. 
Select the control QC type.

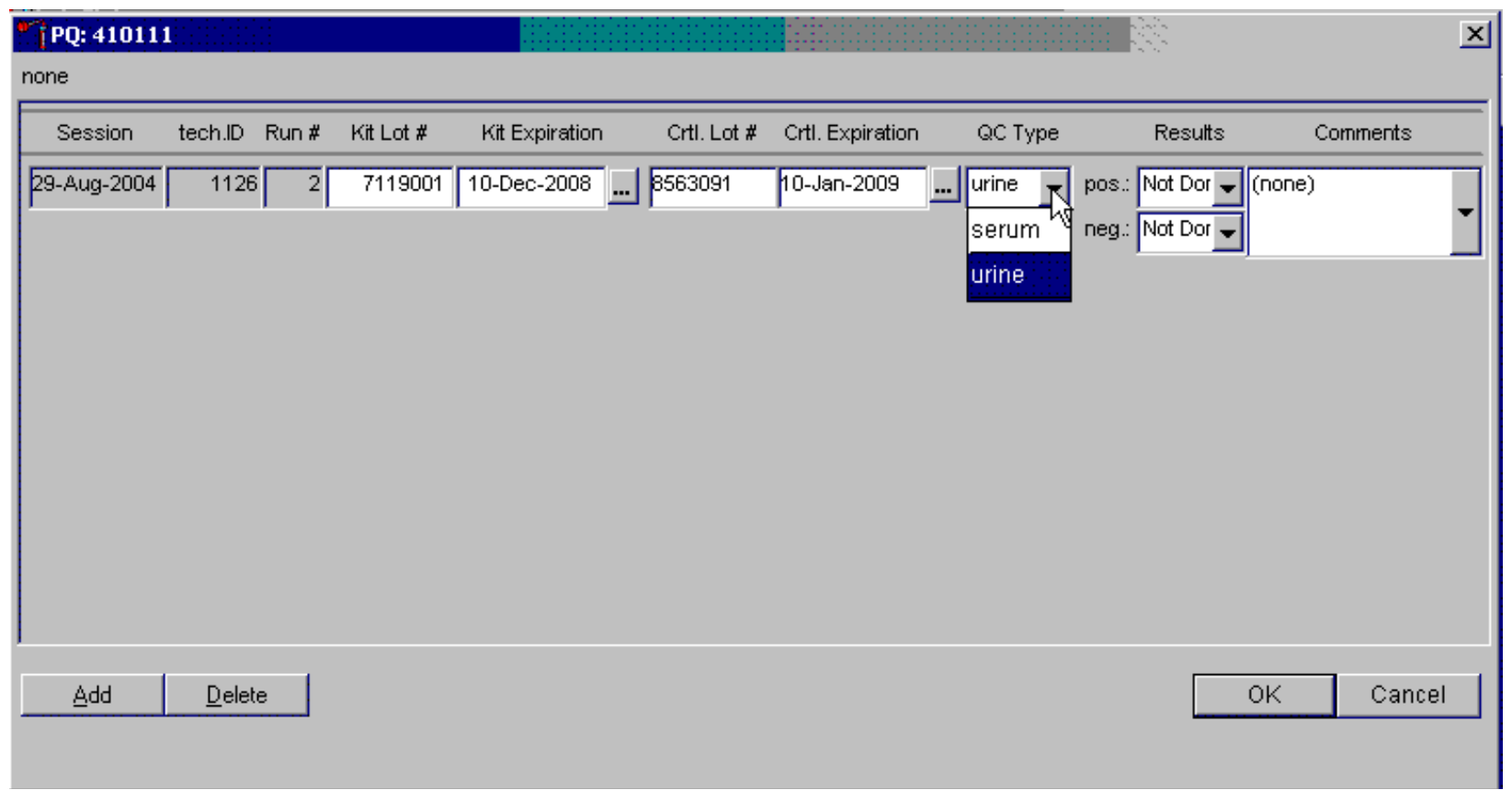

The QC control type default is "Urine." To change the QC type to serum or to select urine, use the mouse to direct the mouse arrow to the drop-down list, drag the mouse arrow to either serum or urine, and left click. Alternatively, highlight the QC type text box and type [S/s] for serum or [U/u] for urine and $[\mathrm{Tab}]$ to move to the result test boxes. 
Record the positive and negative control results.

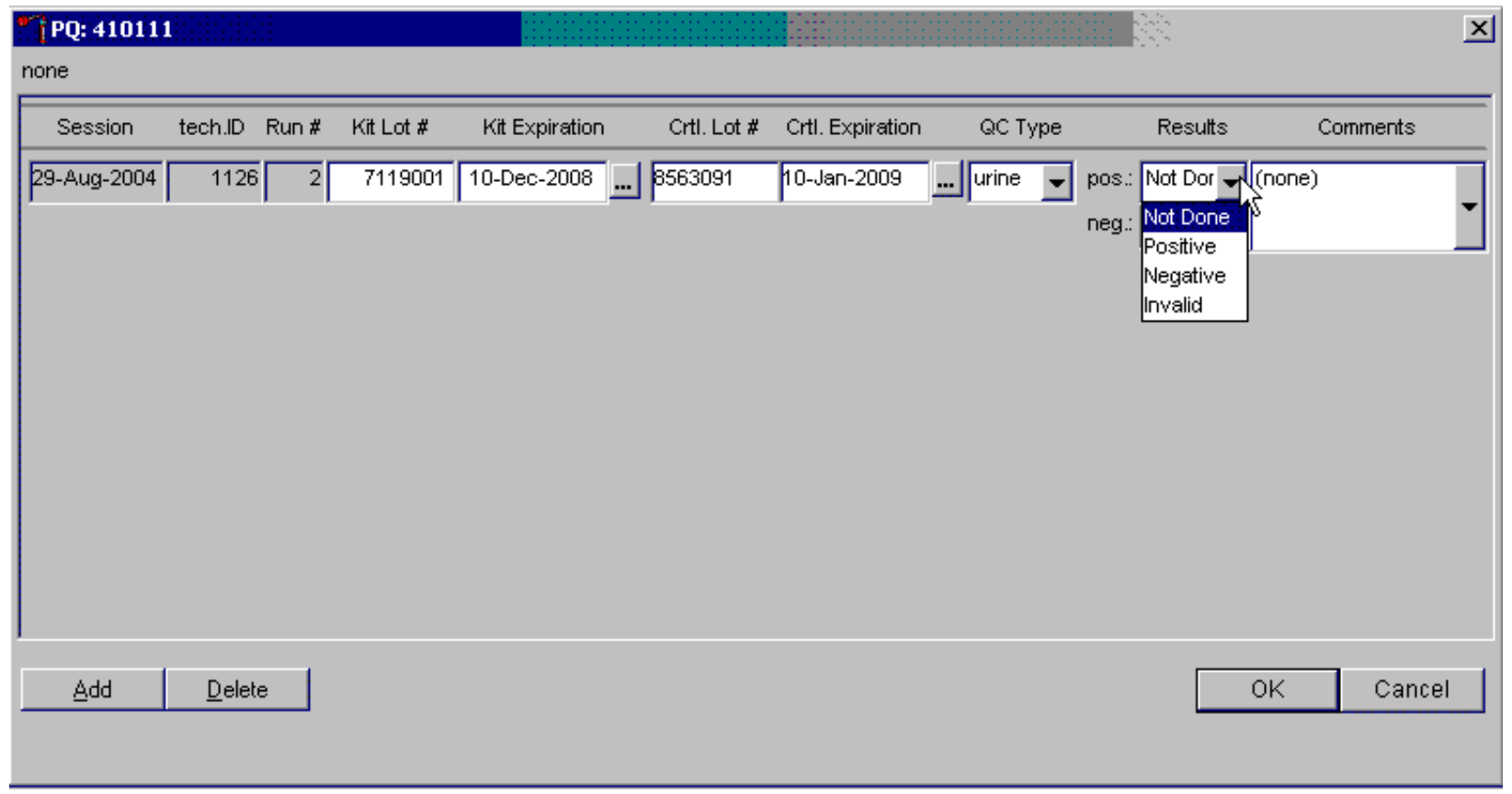

To enter a result in the positive result text box, type $[\mathrm{P} / \mathrm{p}]$ for "Positive," $[\mathrm{N} / \mathrm{n}]$ for "Negative," or [I/i] for "Invalid" or use the up and down arrow keys to toggle among the three choices: Positive, Negative, and Invalid and select [Tab]. Alternatively, use the mouse to direct the mouse arrow to the drop-down list, drag the mouse arrow to "Positive," "Negative," or "Invalid" and left click. If "Positive" is entered, the Negative response box is highlighted. Type [Tab] to move to the negative result text box. 
Enter a result in the negative result text box.

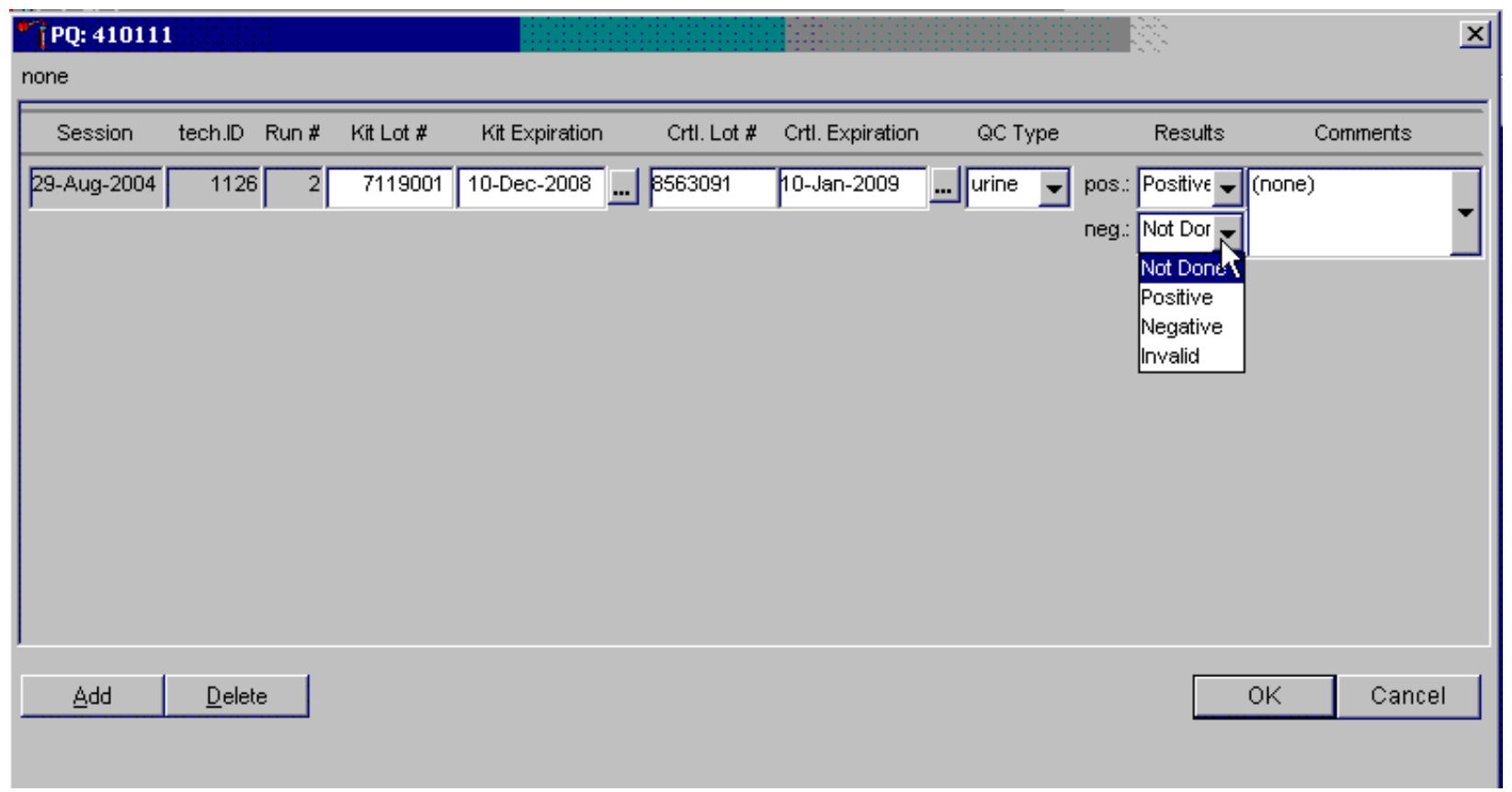

To enter a result in the negative result text box, type $[\mathrm{P} / \mathrm{p}]$ for "Positive," $[\mathrm{N} / \mathrm{n}]$ for "Negative," or [I/i] for "Invalid" or use the up and down arrow keys to toggle among the three choices: Positive, Negative, and Invalid and [Tab]. Alternatively, use the mouse to direct the mouse arrow to the drop-down list, drag the mouse arrow to "Positive," "Negative," or "Invalid," and left click. 
Save results when positive and negative controls demonstrate the expected result.

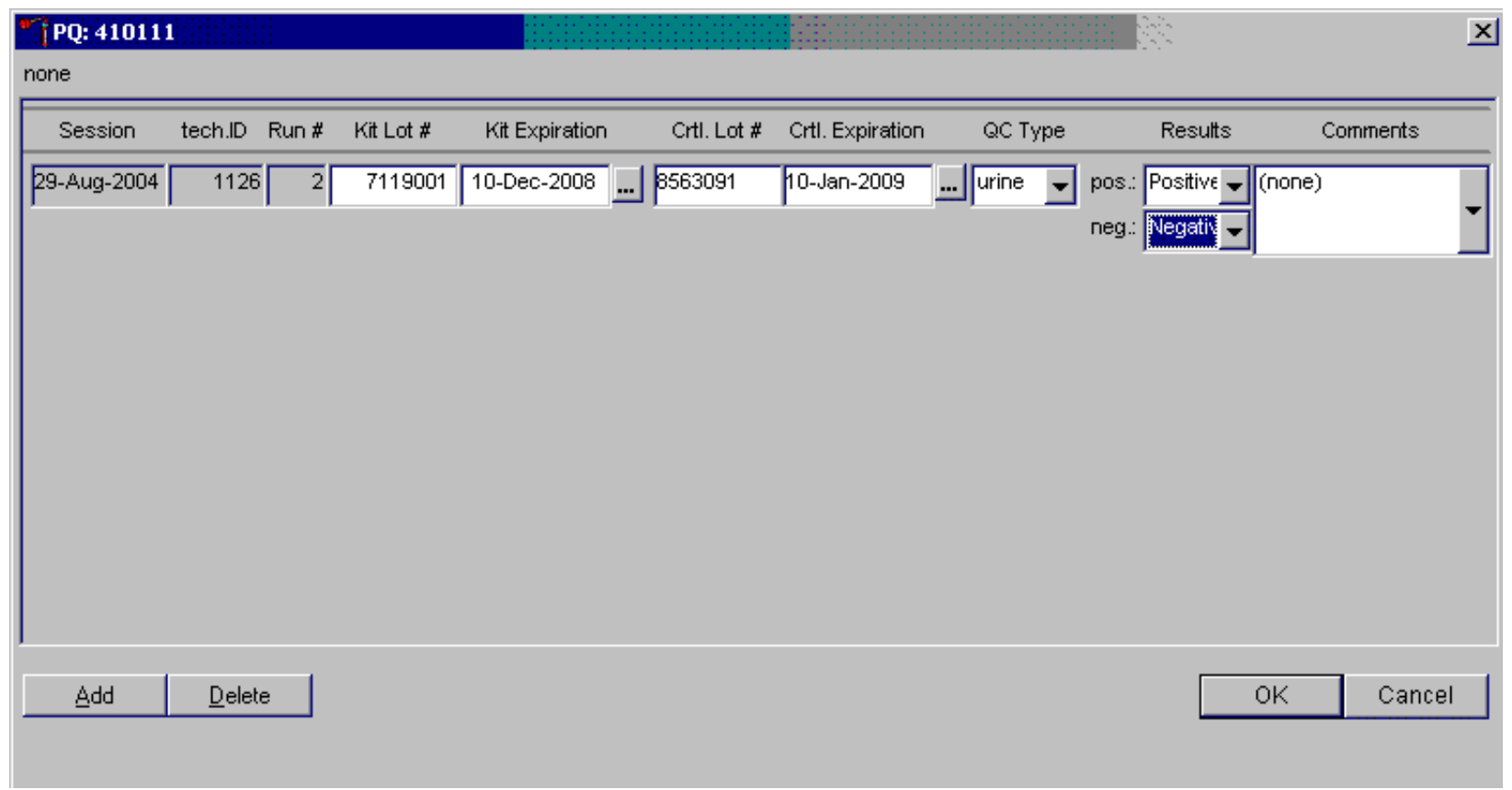

If the positive and negative controls demonstrate the expected result, use the mouse to direct the mouse arrow to the OK button and left click to save these results and to proceed with pregnancy testing. To cancel and exit the module without recording or saving any QC results to the database, use the mouse to direct the mouse arrow to the Cancel button and left click. To delete the information in the window and exit the module without recording or saving any QC results to the database, use the mouse to direct the mouse arrow to the Delete button and left click or type [Shift] [D/d]. 
Enter a comment for any result that is inconsistent with expected results. If the OK button is selected before a comment is entered, the ERROR informational message box displays. Review the text in this box.

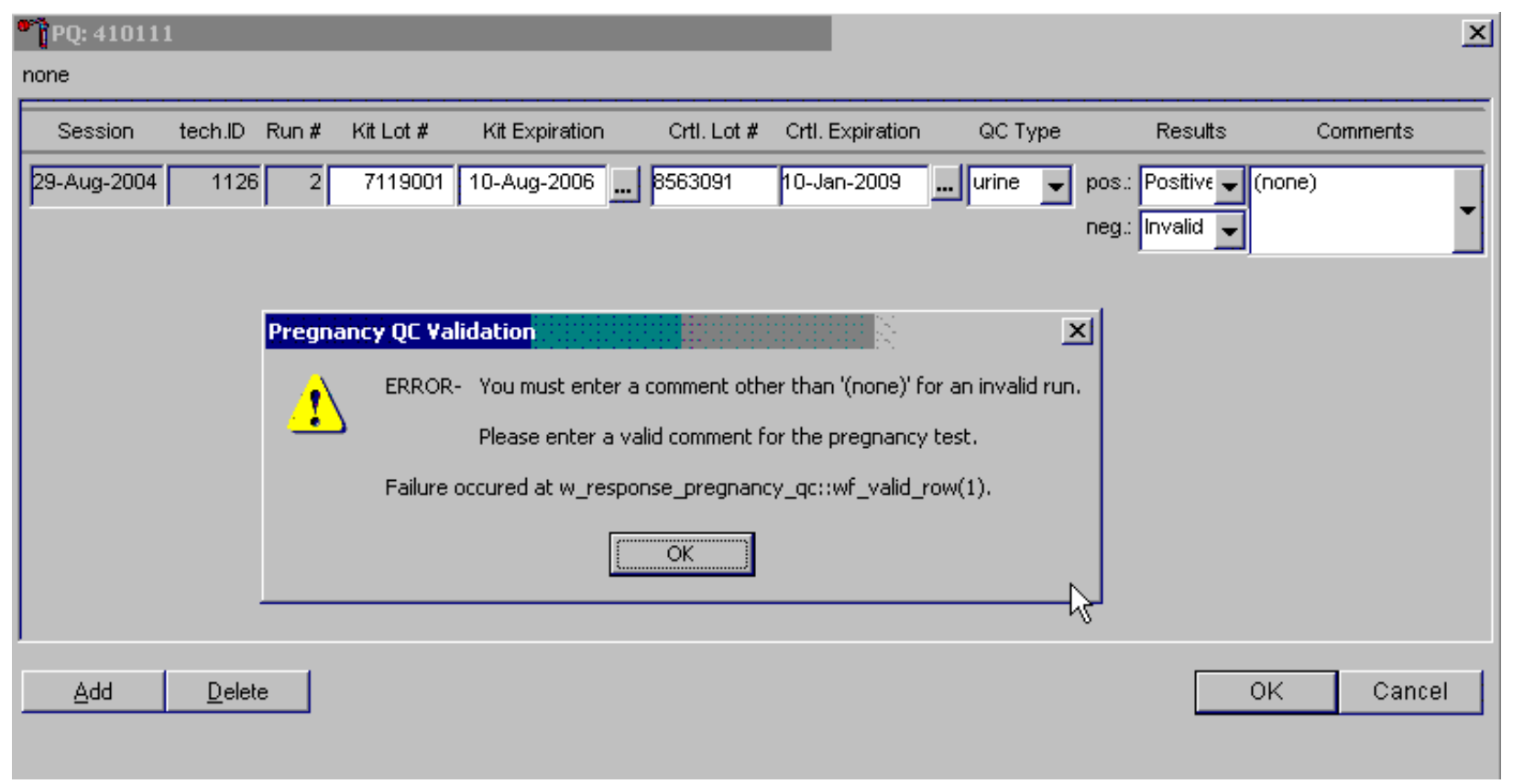

To close the box, use the mouse to direct the mouse arrow to the OK button and left click or select [Enter]. 
Enter a comment for any result that is inconsistent with expected results.

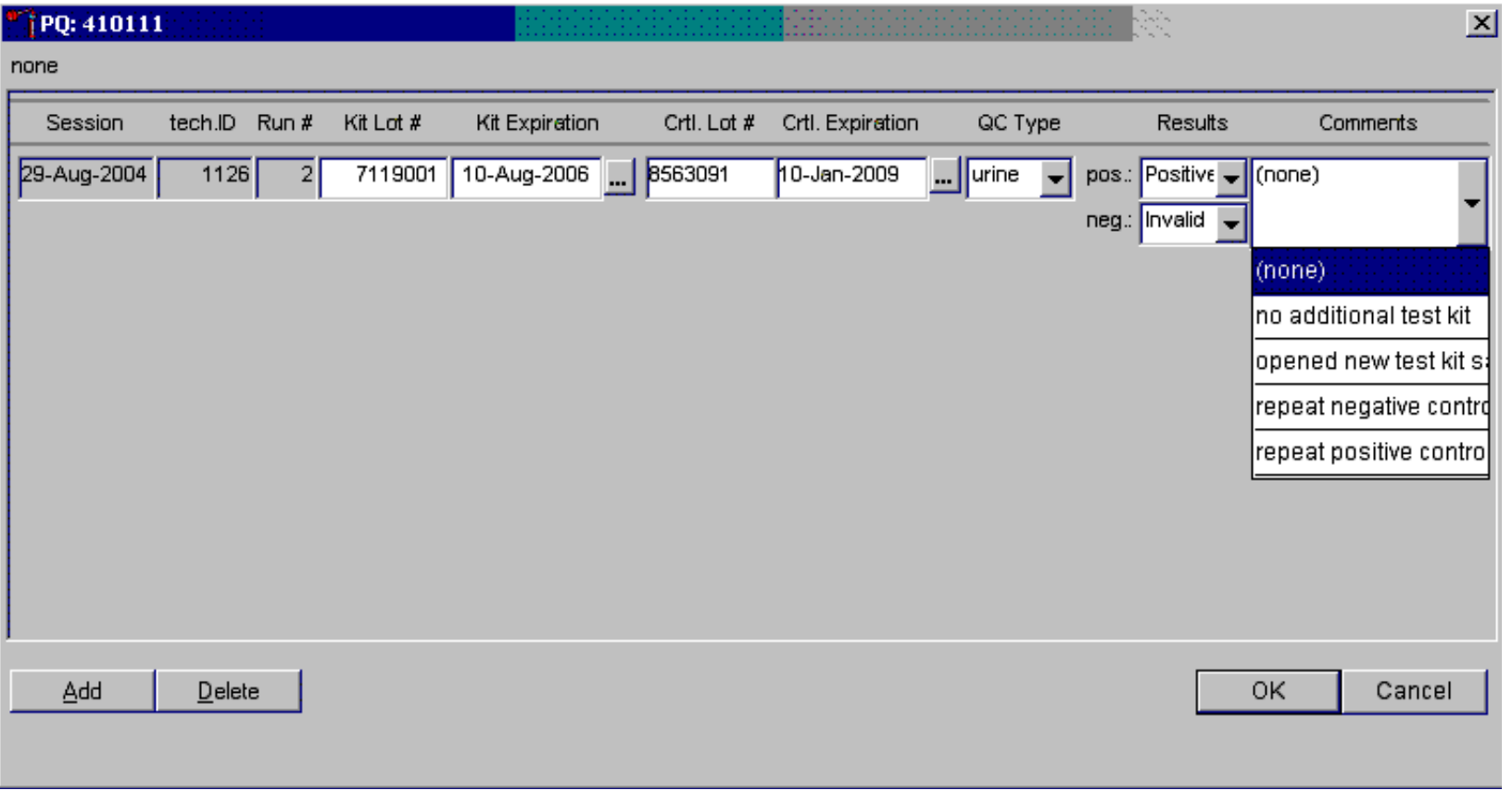

Enter the appropriate comment in the "Comments" column by using the mouse to direct the mouse arrow to the drop-down list, left click to display the choices, drag the mouse arrow to the most appropriate choice, and left click when the correct choice is highlighted. Alternatively, use the up and down keyboard arrows to scroll through the choices or type the first letter of the desired comment code. 
Review the comment.

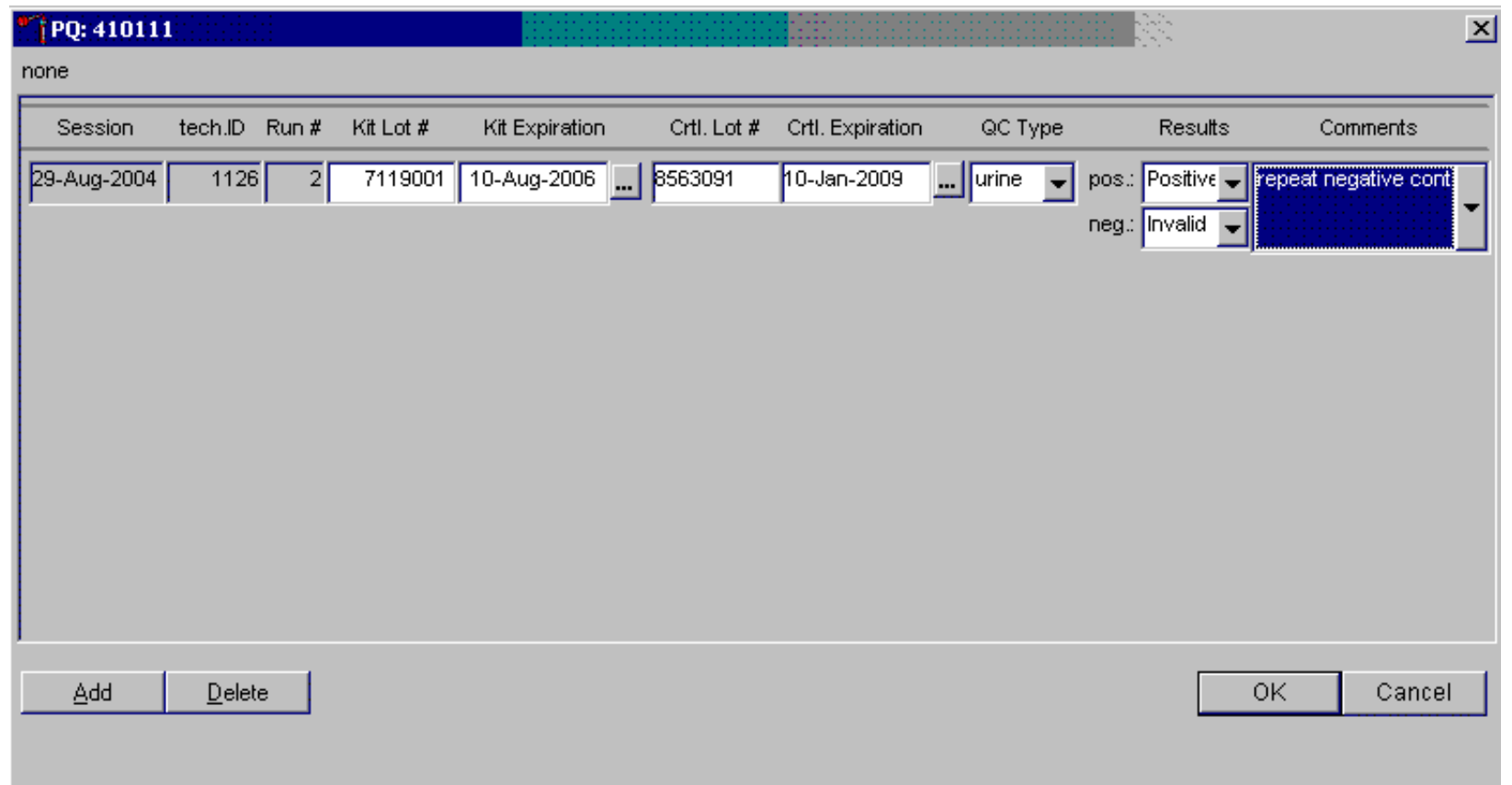

Review all the information in the QC window to verify its accuracy. 
Add a new QC record and repeat the control for invalid or unexpected results.

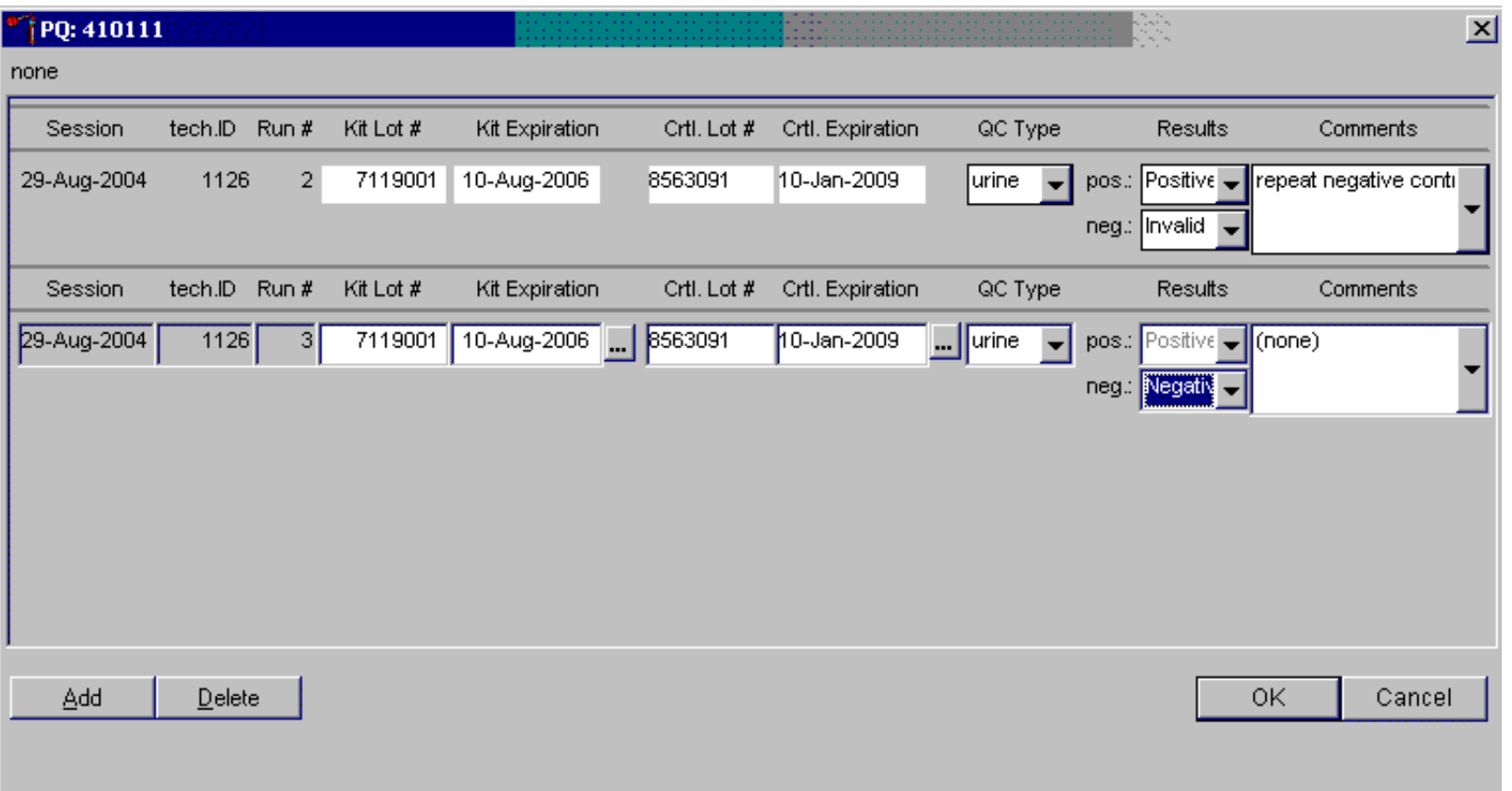

Rerun or repeat the control that demonstrated the unexpected result. Add a new test line to record the new control results. To add a new QC test line, use the mouse to direct the mouse arrow to the Add button and left click or type [Shift] [A/a]. Record the new results. If this resolves the inconsistency, use the mouse to direct the mouse arrow to the OK button and left click to save these results and to proceed with pregnancy testing.

If this does not resolve the situation, repeat the procedure with a different lot of controls. If this fails to resolve the problem, discard the entire lot of pregnancy test kits and test a new lot. 
Add a new test line each time a control is run and remember to record the serum QC each time it is performed.

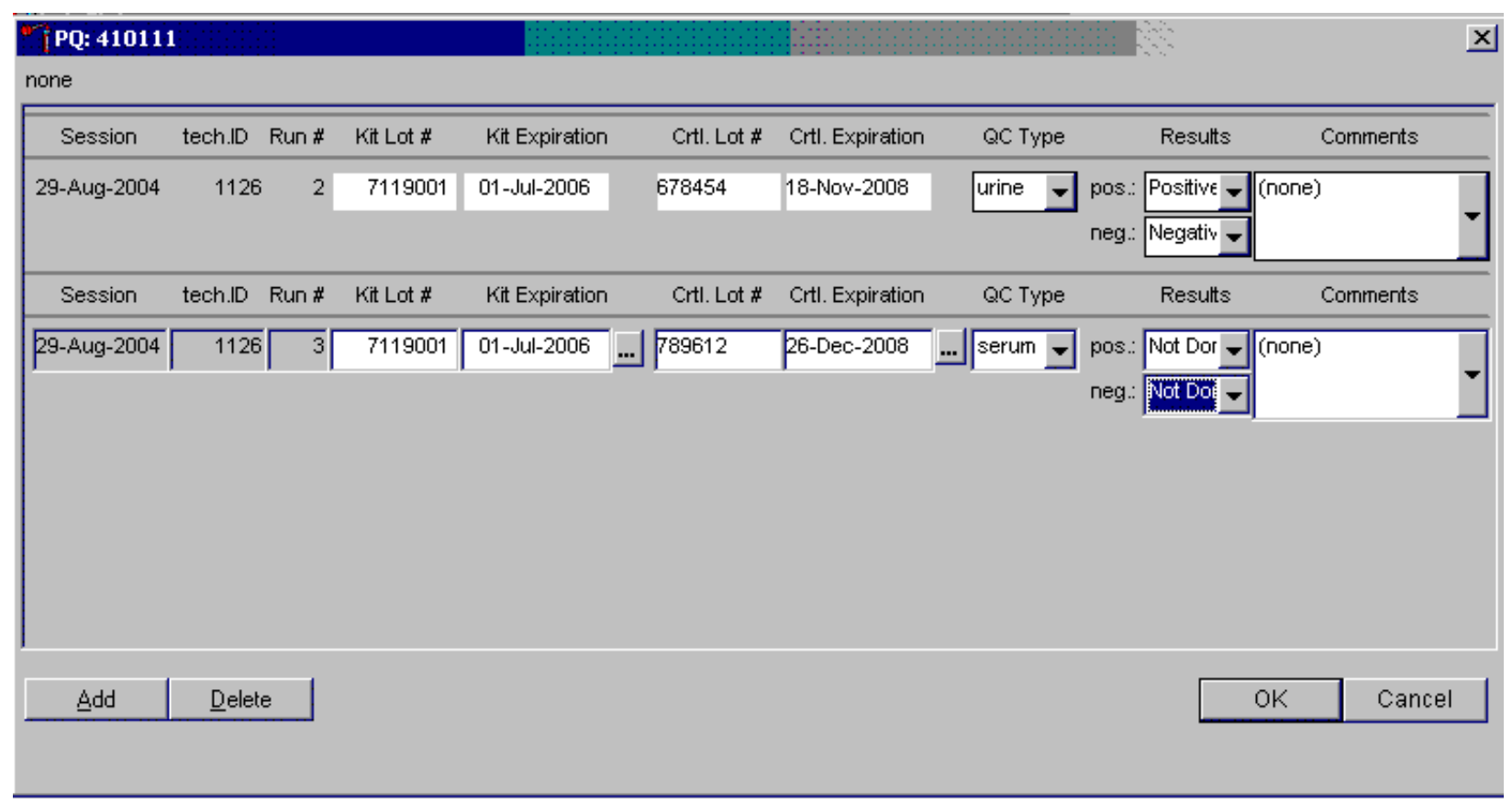

To add a new QC test line, use the mouse to direct the mouse arrow to the Add button and left click or type [Shift] [A/a]. The QC Type defaults to "Urine." Correct the control lot number and expiration date. To change the QC type to serum, use the mouse to direct the mouse arrow to the dropdown list, drag the mouse arrow to serum, and left click. 
Record the serum control results.

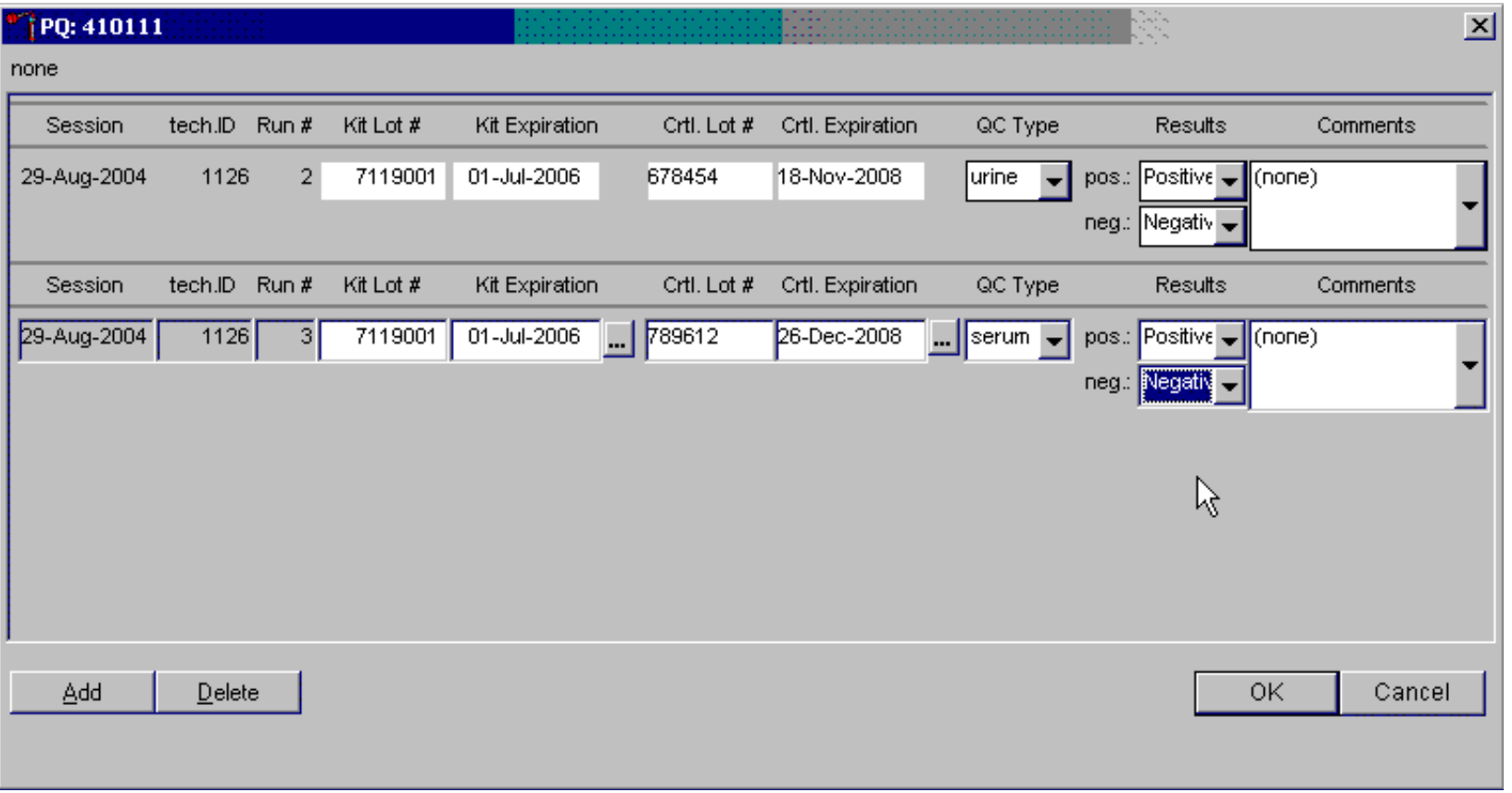

If the positive and negative serum controls demonstrate the expected result, use the mouse to direct the mouse arrow to the OK button and left click to save these results and to proceed with pregnancy testing. If the positive and/or the negative serum controls fail to demonstrate the expected result(s), add a new QC record, and repeat the control. 


\subsection{Interpretation of Results and Remedial Action}

ICON® 25 hCG Test

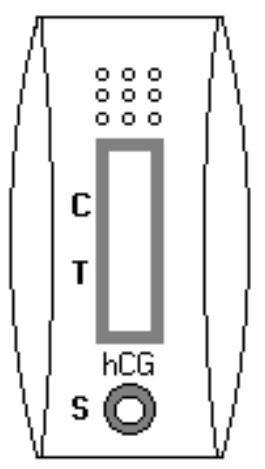

Figure 1.

ICON 25 test device

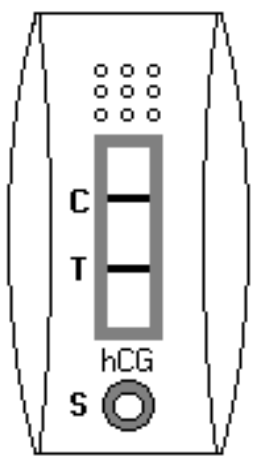

Figure 2.

Positive Result

Two distinct red lines appear. One line should be in the control region (C) and another line should be in the test region (T). 


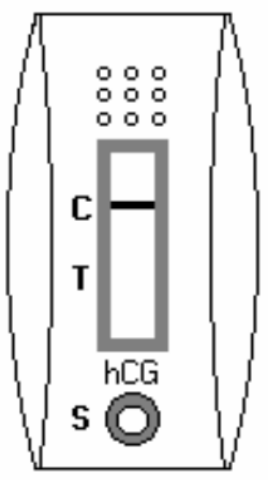

Figure 3.

Negative Result

One red line appears in the control region $(\mathrm{C})$. No apparent red or pink line appears in the test region.
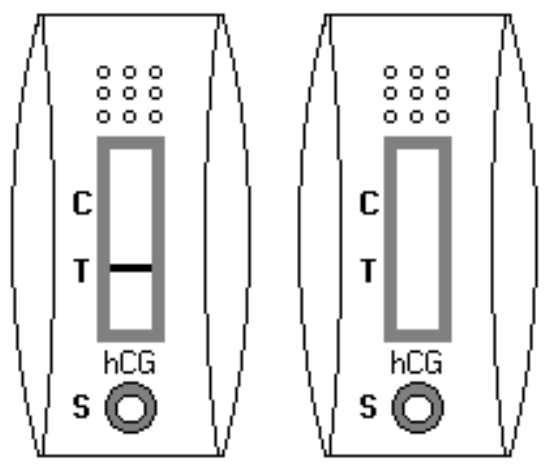

Figure 4. Invalid Result

Control line fails to appear.

\section{Positive Result:}

A specimen that produces two distinct red lines is positive for the presence of hCG. One line should be in the control region (C) and another line should be in the test region (T).

\section{Negative Result:}

A specimen that does produce a red line appears in the control region (C) or no apparent red or pink line in the test region is negative for the presence of hCG. 


\section{Invalid Result (any of the following):}

For the test to be valid, a red line must appear in the control region (C). Insufficient sample volume or incorrect procedural techniques are the most likely reasons for control line failure. Review the procedure and repeat the test with a new test device. If the problem persists, discontinue using the test kit and immediately contact the technical supervisor.

If an invalid test result occurs repeatedly, or if you need technical assistance, contact the Technical Support Department, 1-800-877-6242.

\section{Sure-Vue ${ }^{\mathrm{TM}}$ hCG Urine and Serum Control Sets}

\section{Positive Result:}

The $25 \mathrm{mIU} / \mathrm{mL}$ hCG positive urine and serum controls should produce positive results.

\section{Negative Result:}

The negative urine and serum control should produce a negative result. 
Use the heads-up display and the Pregnancy Testing module to enter the results of the ICON® 25 hCG test kit pregnancy test result for each SP.

Use the heads-up display to access the Pregnancy Testing module. Note that the first urine collection must be marked as verified for the pregnancy testing module to enable on the heads-up display and on the right click menu.

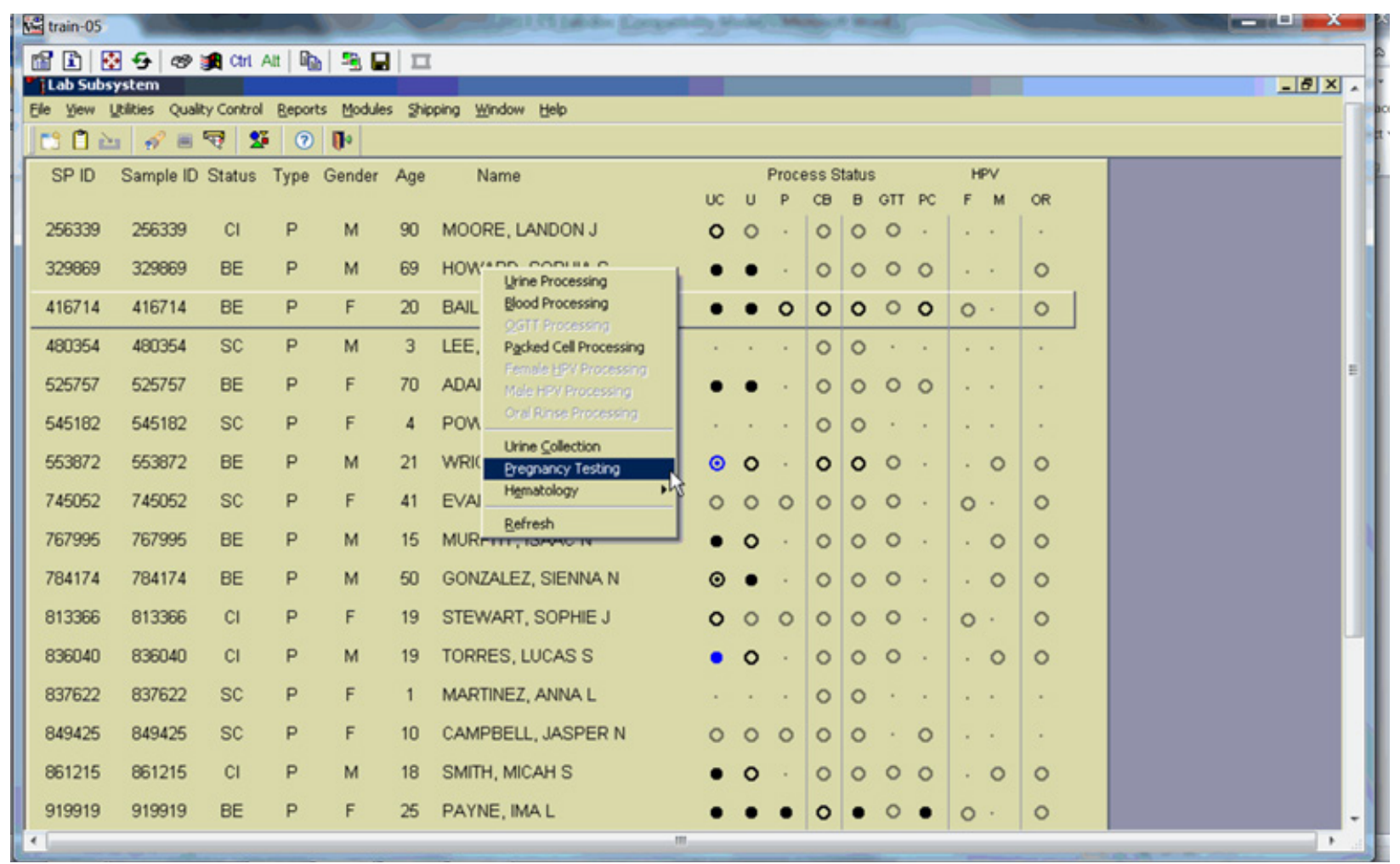


The Pregnancy Testing window displays.

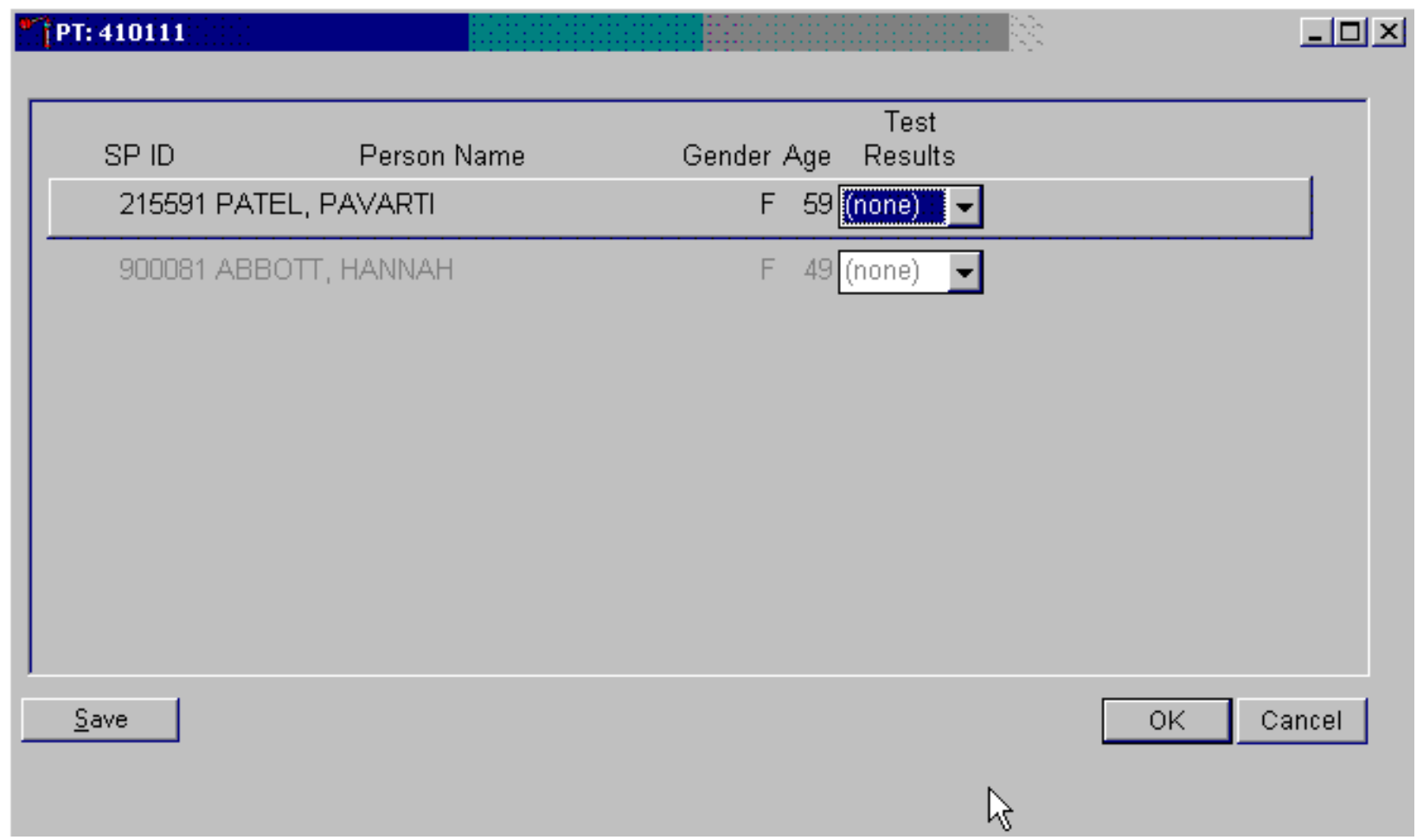

The text is black for SPs who have urine collection results recorded as "Yes" or "Yes/QNS." The text is gray for SPs who do not have urine collection results recorded. The window lists the SP ID, Person Name, Gender, and Age and includes a Test Results column (text box). 
Report the results for each SP as positive, negative, not done, or invalid.

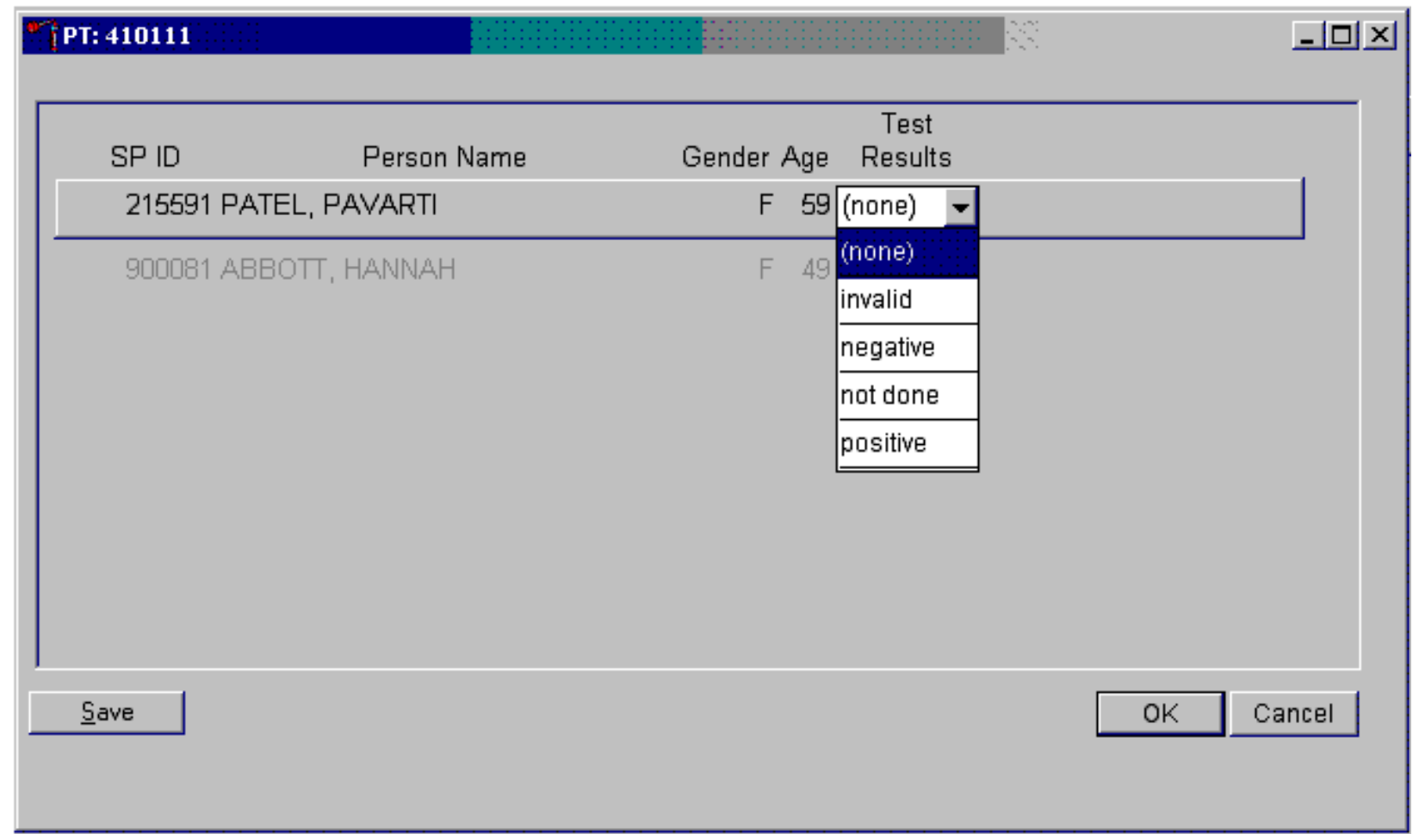

If the Test Result field is black or highlighted, record the result by typing $[\mathrm{P} / \mathrm{p}]$ for positive, $[\mathrm{N} / \mathrm{n}]$ for negative, or $[\mathrm{I} / \mathrm{i}]$ for an invalid result. Enter $[\mathrm{N} / \mathrm{n}]$ not done when the test is not performed. Alternatively, to record a result, use the mouse to direct the mouse arrow to the drop-down list, drag the arrow to the correct choice, and left click. To update the results without exiting the window, use the mouse to direct the mouse arrow to the Save button and left click or type [ALT] [S/s]. To update the results and exit the window, use the mouse to direct the mouse arrow to the OK button and left click or select [Enter]. To exit the module without saving any result, use the mouse to direct the mouse arrow to the Cancel button and left click. 
Continue performing pregnancy tests and recording the results. Save the test results after entering each positive, negative, or invalid result. To update the results without exiting the window, use the mouse to direct the mouse arrow to the Save button and left click or type [Shift] [S/s]. To update the results and exit the window, use the mouse to direct the mouse arrow to the OK button and left click or select [Enter]. To exit the module without saving any result, use the mouse to direct the mouse arrow to the Cancel button and left click.

A series of message boxes displays to inform that an SP has been excluded (blocked) or is eligible (unblocked.) After each negative result is saved to the database, an unblocking message box displays.

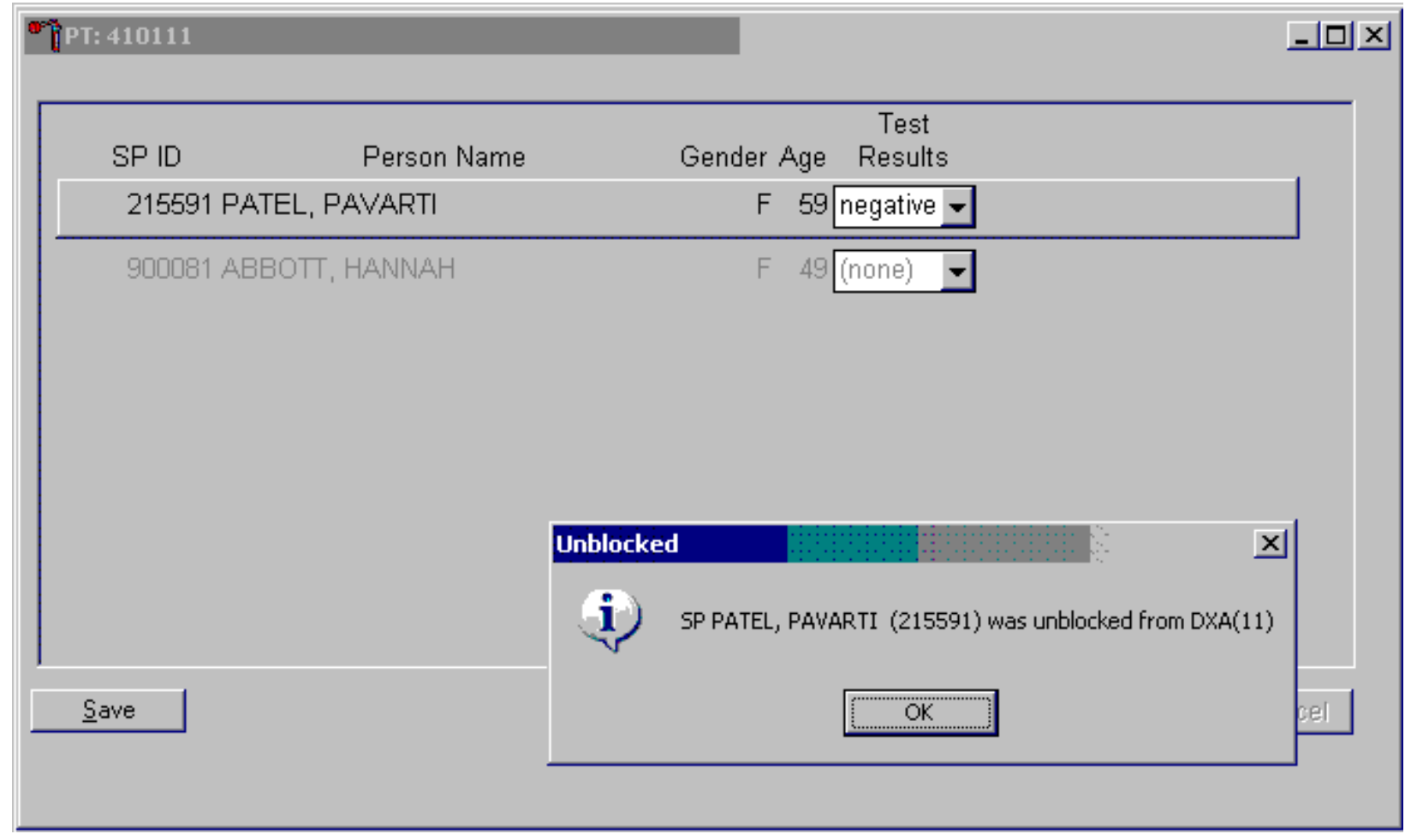

To remove the informational message box, use the mouse to direct the mouse arrow to the OK button and left click, or select [Enter]. 
As each positive result is saved to the database, a blocking message displays.

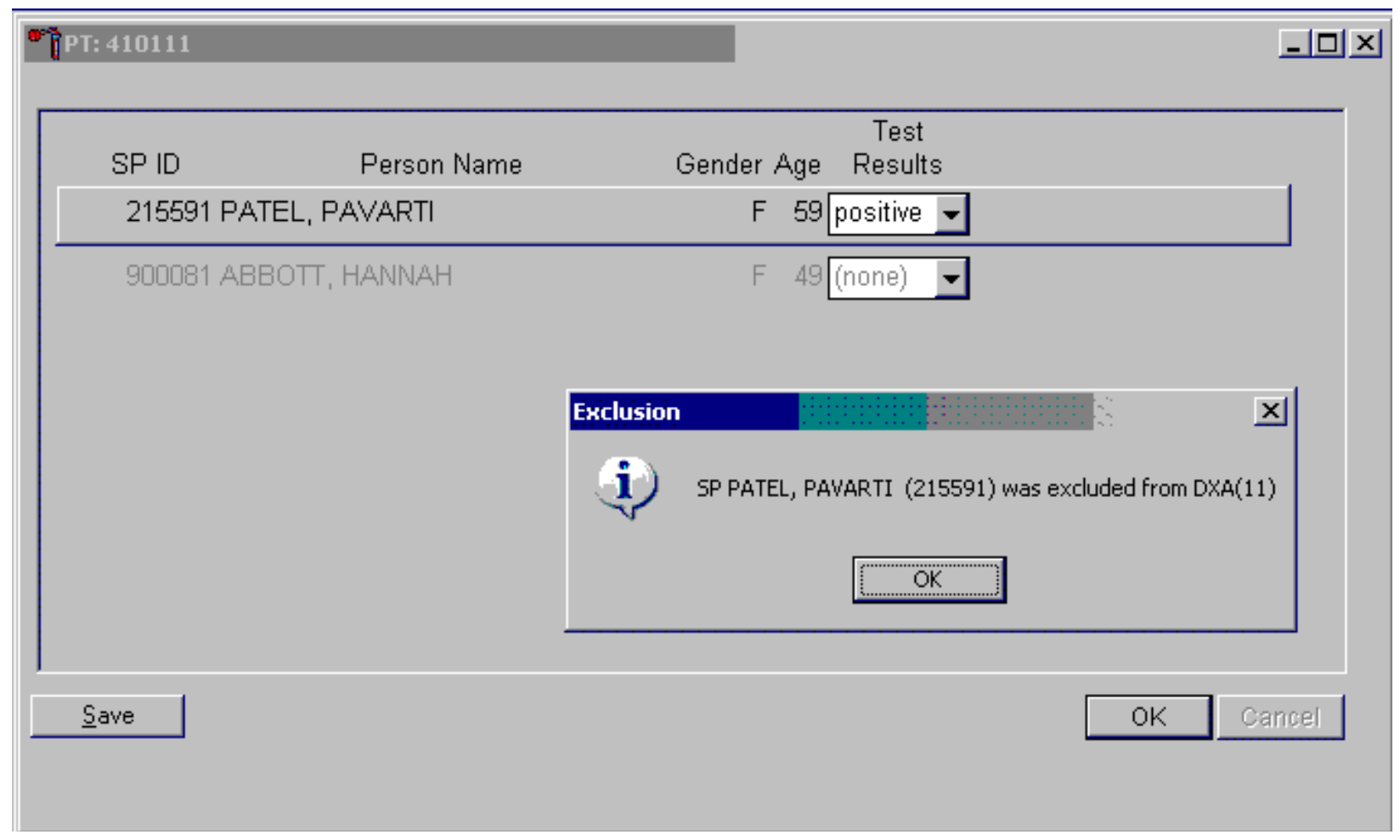

To remove the informational message box, use the mouse to direct the mouse arrow to the OK button and left click, or select [Enter]. 
If a urine pregnancy test is positive on any female SPs aged 8-17 years, confirm the result using serum. An informational pop-up box displays as a reminder to perform the confirmation test.

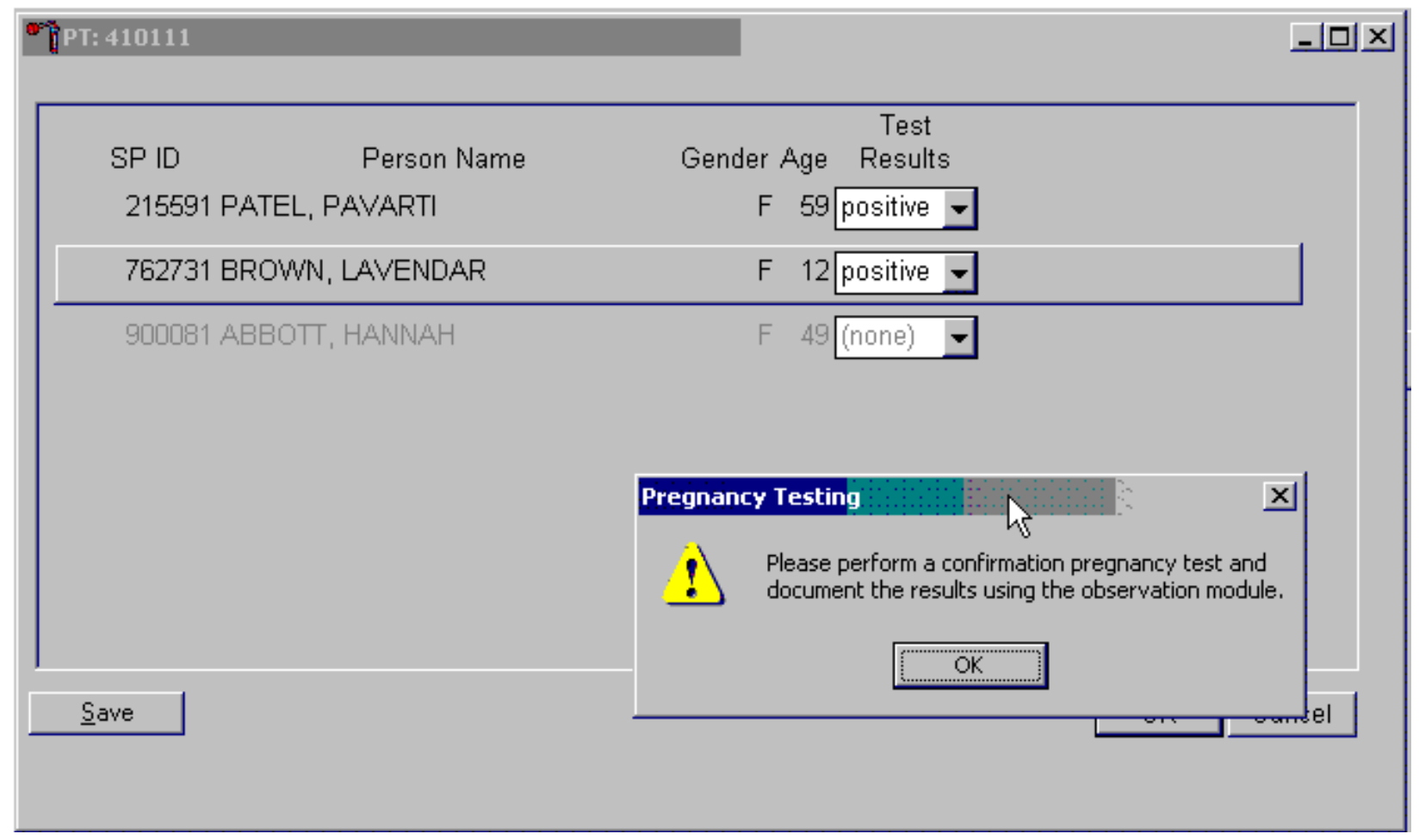

To remove the informational message box, use the mouse to direct the mouse arrow to the OK button and left click, or select [Enter].

If no blood is drawn, repeat the urine test. Notify the physician of the second positive or negative confirmatory test or the inability to perform a confirmation test using the observation function. 
Access the observation function to send an observation to the physician.

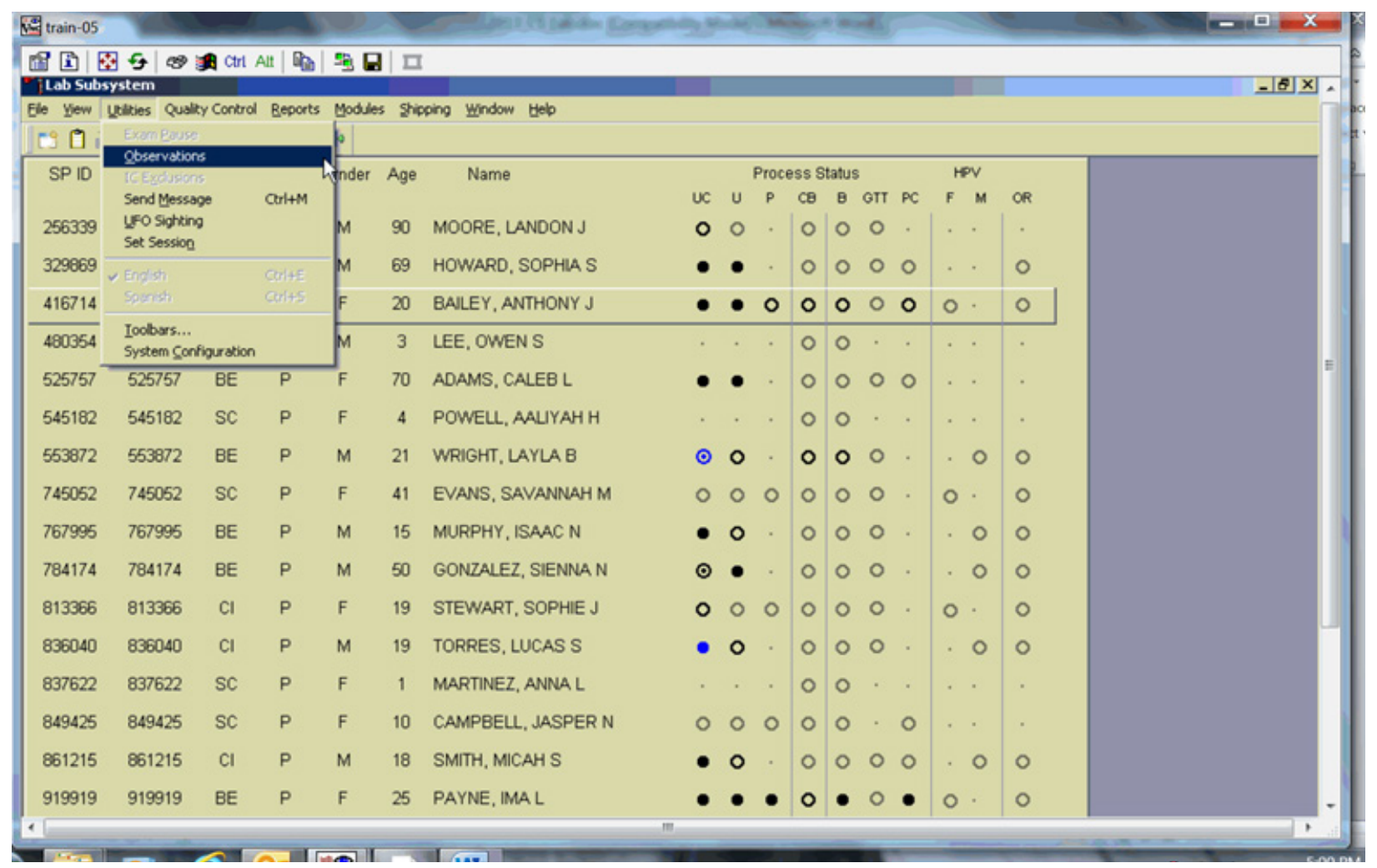

To send an observation to the physician, use the mouse to direct the mouse arrow to \{Utilities\} in the top menu bar, drag the mouse arrow to \{Observations\}, and left click or type [Alt] $[\mathrm{U} / \mathrm{u}],[\mathrm{O} / \mathrm{o}]$. 
Select or highlight the correct SP.

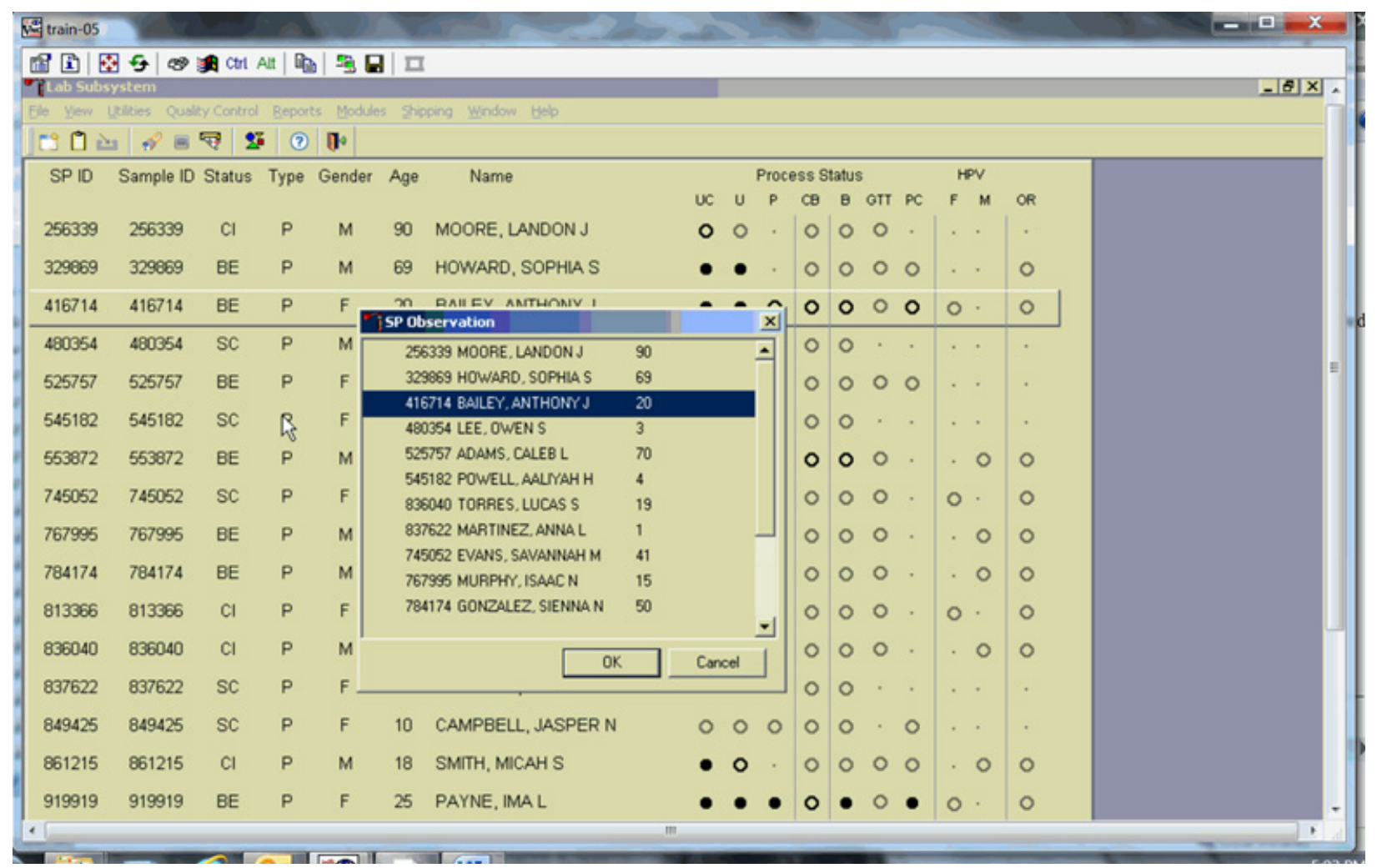

To select or highlight an SP, drag the mouse arrow to the correct SP and left click. Verify that the SP ID, name, and age are correct. Use the vertical scroll bar to view the complete list of SPs. To continue, use the mouse to direct the mouse arrow to the OK button and left click or select [Enter]. To cancel these actions and exit the observation function, use the mouse to direct the mouse arrow to the Cancel button and left click. 
The observation text window displays.

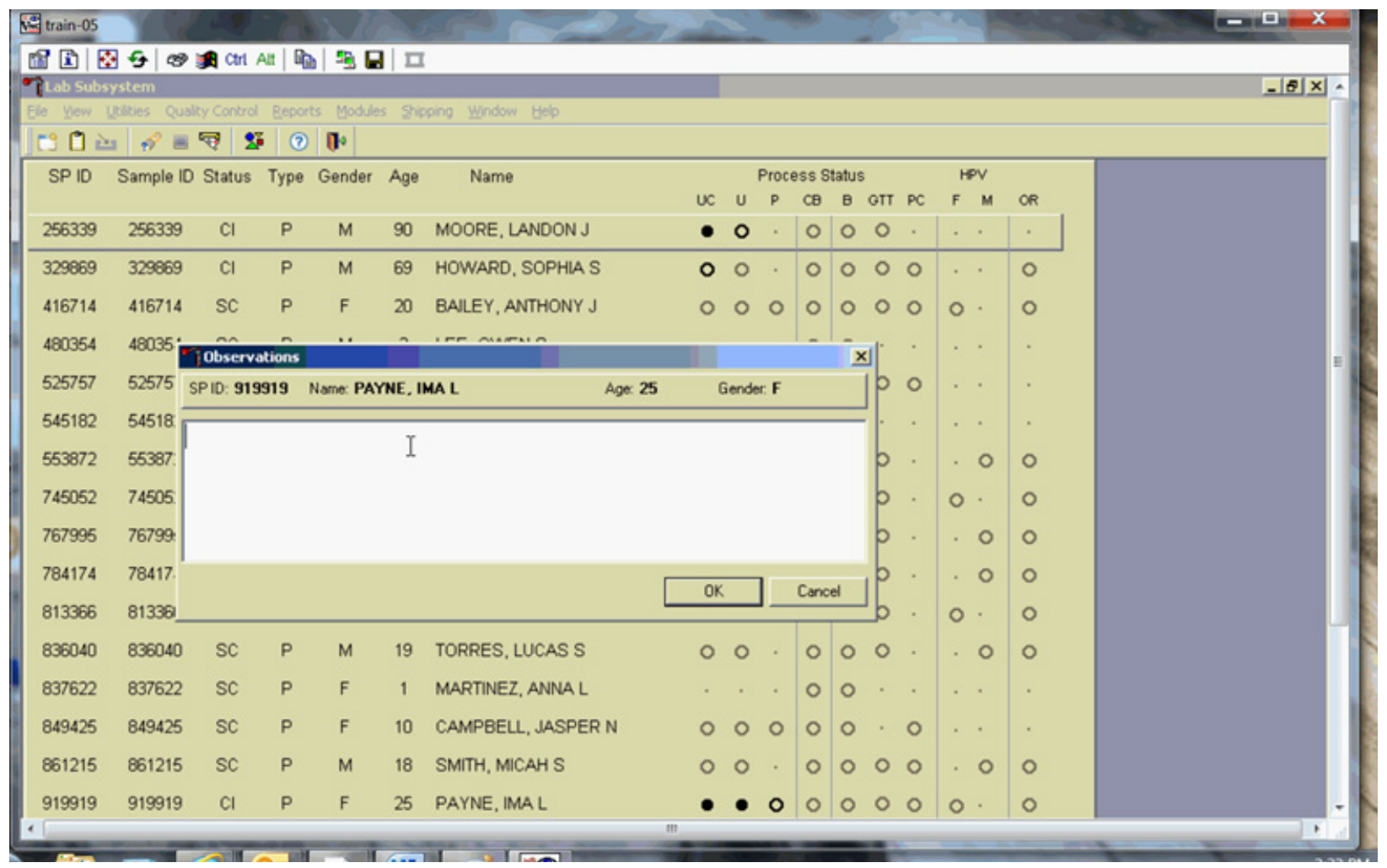

The observation text window contains the SP ID, name, age, and gender. Type the observation using the keyboard's keys. To send the observation to the physician, use the mouse to direct the mouse arrow to the OK button and left click or select [Enter]. To cancel these actions or to exit the observation window without entering an observation, use the mouse to direct the mouse arrow to the Cancel button and left click. 
Send an observation to report a negative serum test result on SPs aged 8-17 years.

\begin{tabular}{|c|c|c|c|c|c|c|c|c|c|c|c|c|c|c|c|c|c|c|}
\hline AS train -05 & 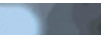 & & & & & & & & & & & & & & & & 프 $x$ & \\
\hline 圆国| E & 89 6 & 周 ctrl & Alt $\mid$ QD & D. 5 & | 므 & & & & & & & & & & & & & \\
\hline plab Dabi & system & & & & & & & & & & & & & & & & $-\theta \mid x$ & - \\
\hline Ele yed & Uallies Qu & wilky Contro & Bepor & its Moduh & ss shiph & oping Window Heb & & & & & & & & & & & & \\
\hline $\operatorname{cg} 9$ iे & $\Rightarrow+8=$ & 军 & 6 (3) & 0 & & & & & & & & & & & & & & \\
\hline SP 10 & Sample IC & ID Status & Type & Gender & Age & Name & & & Proce & ess St & tatus & & & HP & & & & \\
\hline & & & & & & & uc & $u$ & $P$ & $C \theta$ & $\theta$ & बाT & PC & $F$ & M & OR & & \\
\hline 256339 & 256339 & $\mathrm{Cl}$ & P & M & 90 & MOORE, LANDON J & - & 0 & - & 0 & 0 & 0 & . & . & . & · & & \\
\hline 329869 & 329869 & $\mathrm{Cl}$ & $\mathbf{P}$ & M & 69 & HOWARD, SOPHIA S & 0 & 0 & . & 0 & 0 & 0 & 0 & . & . & 0 & & \\
\hline 416714 & 416714 & $\mathrm{SC}$ & P & $F$ & 20 & BAILEY, ANTHONY J & 0 & 0 & 0 & 0 & 0 & 0 & 0 & 0 & . & 0 & & \\
\hline 480354 & 48035 & Tobserv & ations & .. & $=$ & .rr numan & & & & - & - & $x \mid$ & . & . & . & . & & \\
\hline 525757 & 52575 & SPID: 915 & 9919 & Name: PAI & YNE, IN & Age: 25 & & Gende & & & & p & 0 & . & . & . & & \\
\hline 545182 & 54518 & The sen & um hor & $\mathrm{G}$ is ned & gative & & & & & & & 1. & . & . & . & . & & \\
\hline 553872 & 55387 & & & & & & & & & & & $p$ & . & . & 0 & 0 & & \\
\hline 745052 & 74505 & & & & & & & & & & & $p$ & . & 0 & . & 0 & & \\
\hline 767995 & 76799 & & & & & & & & & & & p & . & . & 0 & 0 & & \\
\hline 784174 & 78417 & & & & & & $O K$ & & & Cance & & b & . & . & 0 & 0 & & \\
\hline 813366 & 81338 & & & & & & & & $1-$ & & & b & . & 0 & . & 0 & & \\
\hline 836040 & 836040 & SC & P & M & 19 & TORRES, LUCAS S & 0 & 0 & . & 0 & 0 & 0 & . & . & 0 & 0 & & \\
\hline 837622 & 837622 & SC & $P$ & $\mathrm{~F}$ & 1 & MARTINEZ, ANNA L & . & . & . & 0 & 0 & . & . & . & . & . & & \\
\hline 849425 & 849425 & SC & P & $F$ & 10 & CAMPBELL, JASPER N & 0 & 0 & 0 & 0 & 0 & . & 0 & . & . & . & & \\
\hline 861215 & 861215 & SC & P & M & 18 & SMITH, MICAHS & 0 & 0 & . & 0 & 0 & 0 & ० & . & 0 & 0 & & \\
\hline 919919 & 919919 & $\mathrm{Cl}$ & $P$ & $\mathrm{~F}$ & 25 & PAYNE, IMA L & $\bullet$ & $\bullet$ & 0 & 0 & 0 & 0 & 0 & 0 & . & 0 & & - \\
\hline
\end{tabular}

The observation window contains the SP ID, name, age, and gender. Type the observation, "The serum hCG test is negative," using the keyboard keys. To send the observation to the physician, use the mouse to direct the mouse arrow to the OK button and left click or select [Enter]. To cancel these actions or to exit the observation window without entering an observation, use the mouse to direct the mouse arrow to the Cancel button and left click. 
Send an observation to report a positive serum test result on SPs aged 8-17 years.

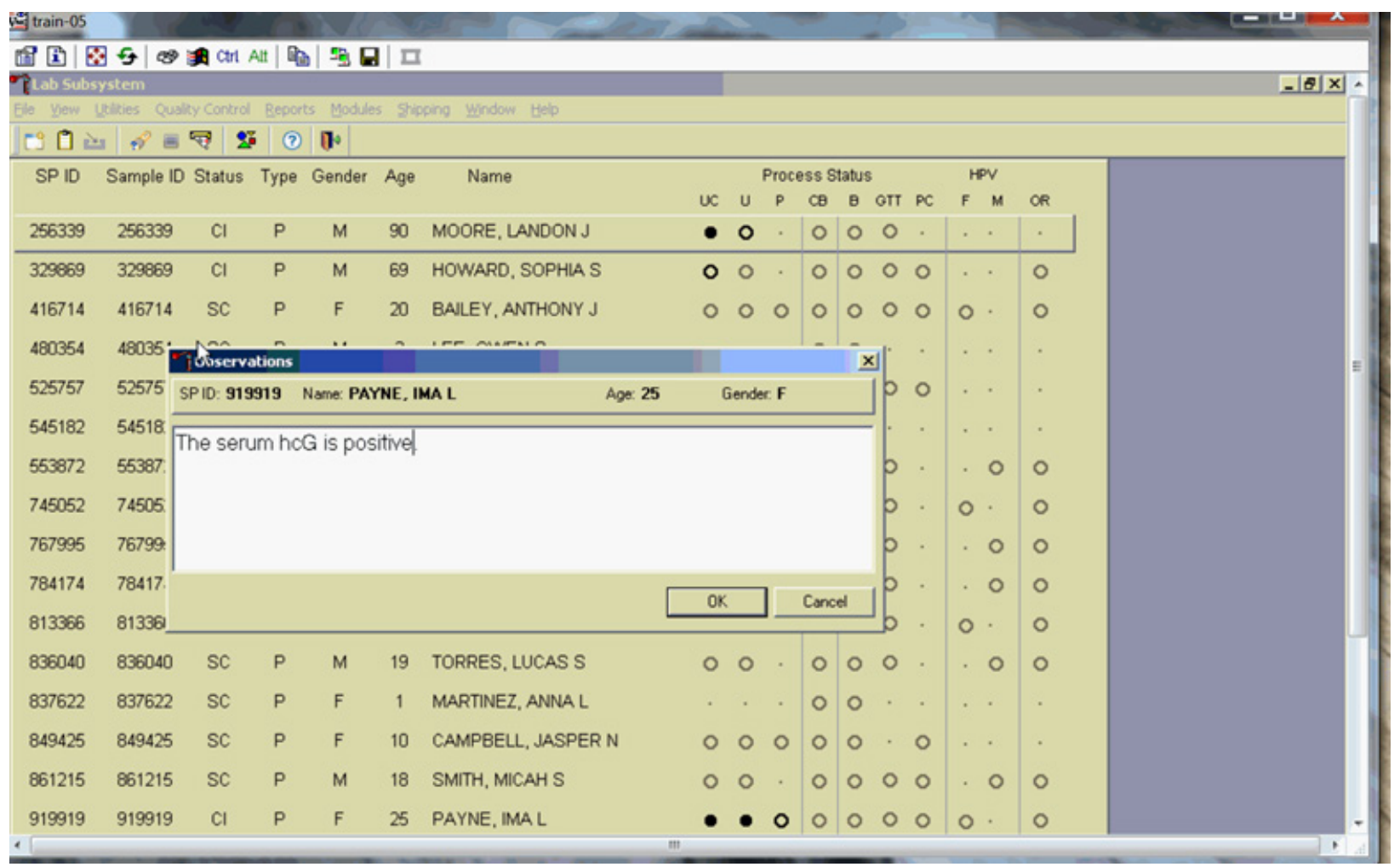

The observation window contains the SP ID, name, age, and gender. Type the observation, "The serum hCG test is positive," using the keyboard keys. To send the observation to the physician, use the mouse to direct the mouse arrow to the OK button and left click or select [Enter]. To cancel these actions, or to exit the observation window without entering an observation, use the mouse to direct the mouse arrow to the Cancel button and left click. 
Send an observation to report the inability to perform the serum confirmation test and to report the results of a repeat negative urine test on female SPs aged 8-17 years.

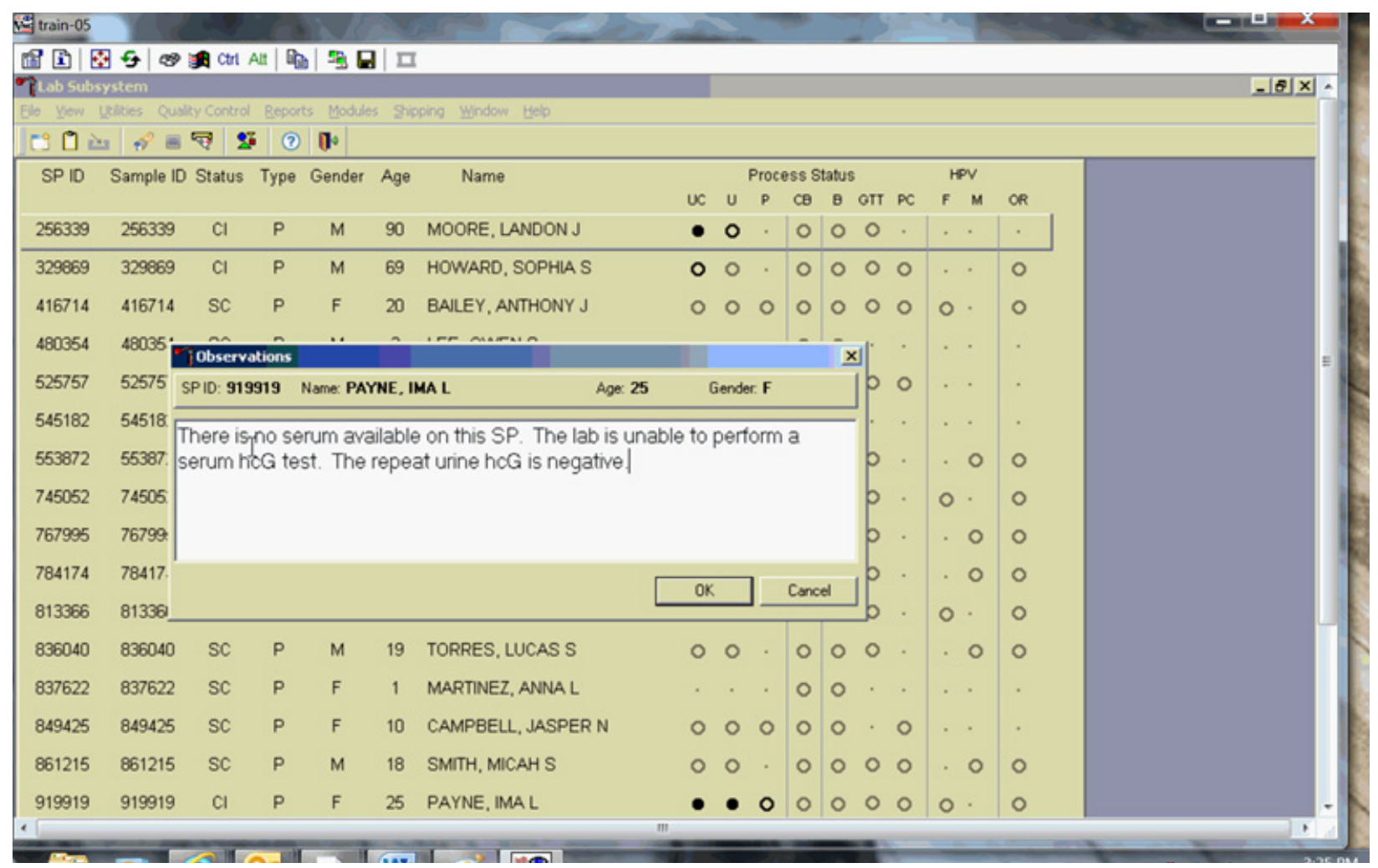

The observation window contains the SP ID, name, age, and gender. Type the observation, "There is no serum available on this SP. The lab is unable to perform a serum hCG test. The repeat urine hCG test is negative," using the keyboard keys. To send the observation to the physician, use the mouse to direct the mouse arrow to the OK button and left click or select [Enter]. To cancel these actions or to exit the observation window without entering an observation, use the mouse to direct the mouse arrow to the Cancel button and left click. 
Send an observation to report the inability to perform the serum confirmation test and to report the results of a repeat positive urine test on female SPs aged 8-17 years.

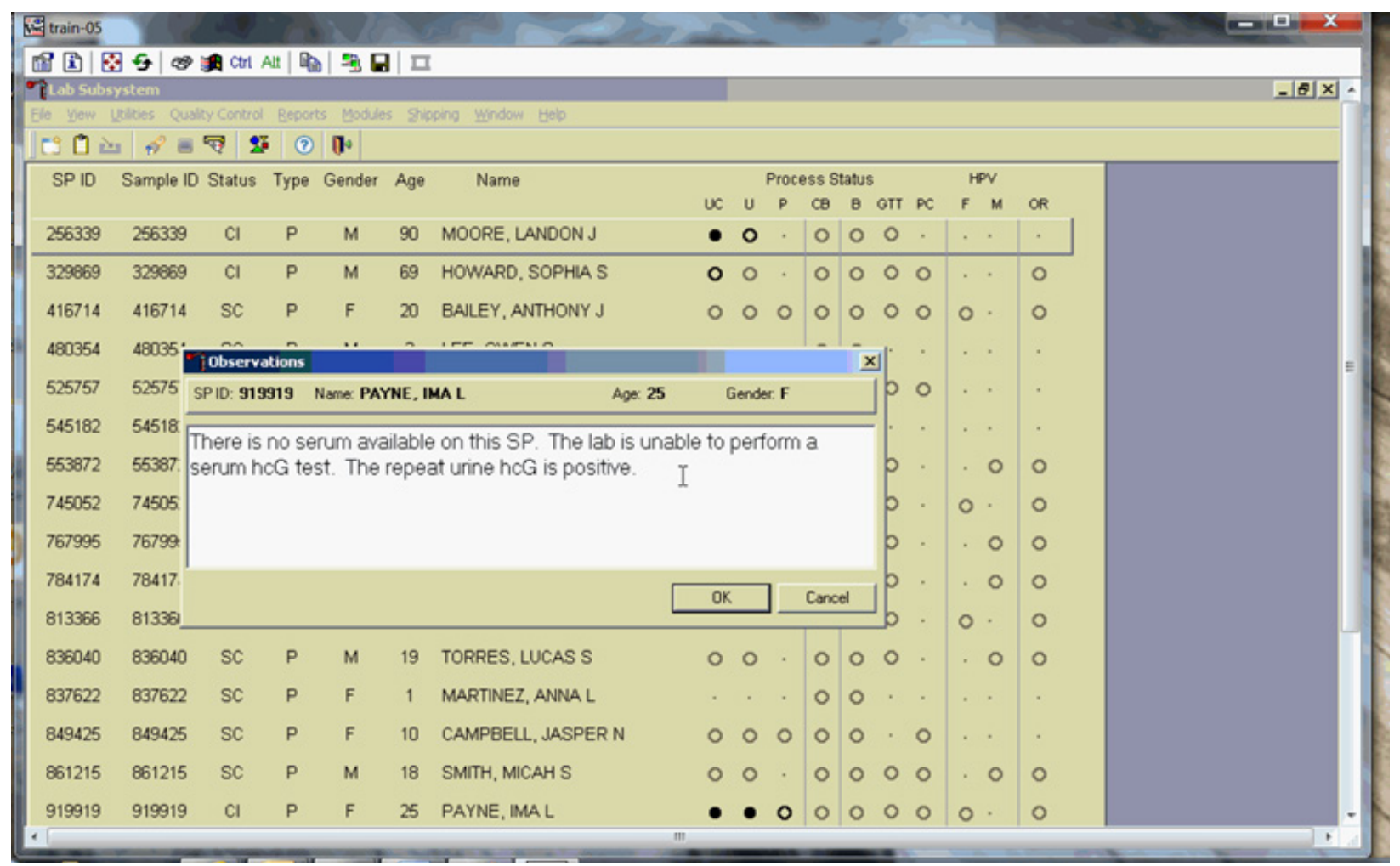

The observation window contains the SP ID, name, age, and gender. Type the observation, "There is no serum available on this SP. The lab is unable to perform a serum hCG test. The repeat urine test is positive." using the keyboard keys. To send the observation to the physician, use the mouse to direct the mouse arrow to the OK button and left click or select [Enter]. To cancel these actions or to exit the observation window without entering an observation, use the mouse to direct the mouse arrow to the Cancel button and left click. 
Send an observation to report the inability either to perform the serum confirmation test or to repeat the urine test on female SPs aged 8-17 years.

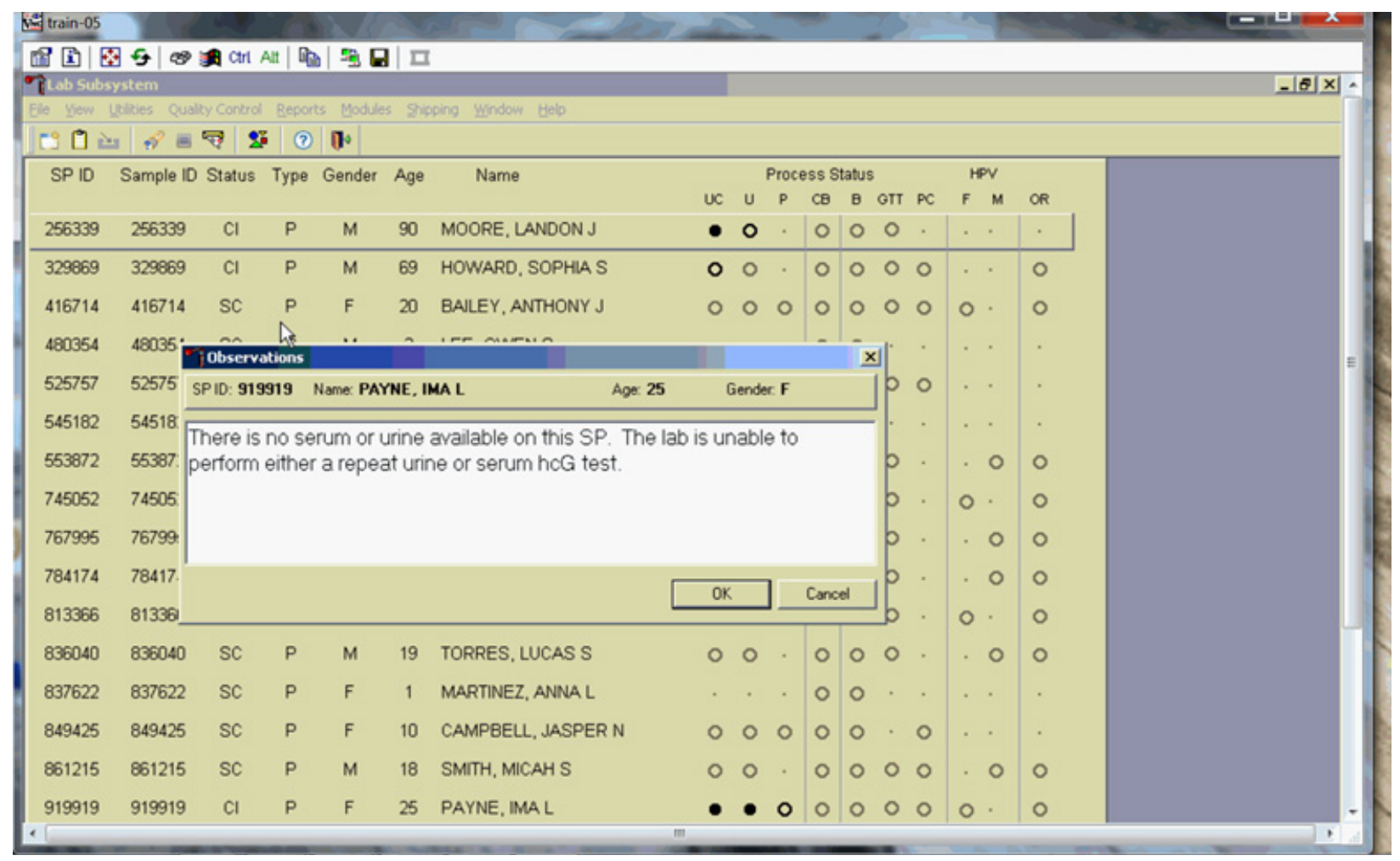

The observation window contains the SP ID, name, age, and gender. Type the observation, "There is no serum or urine on this SP. The lab is unable to perform either a repeat urine or serum hCG test." using the keyboard keys. To send the observation to the physician, use the mouse to direct the mouse arrow to the OK button and left click or select [Enter]. To cancel these actions or to exit the observation window without entering an observation, use the mouse to direct the mouse arrow to the Cancel button and left click. 
The physician reviews all observations.

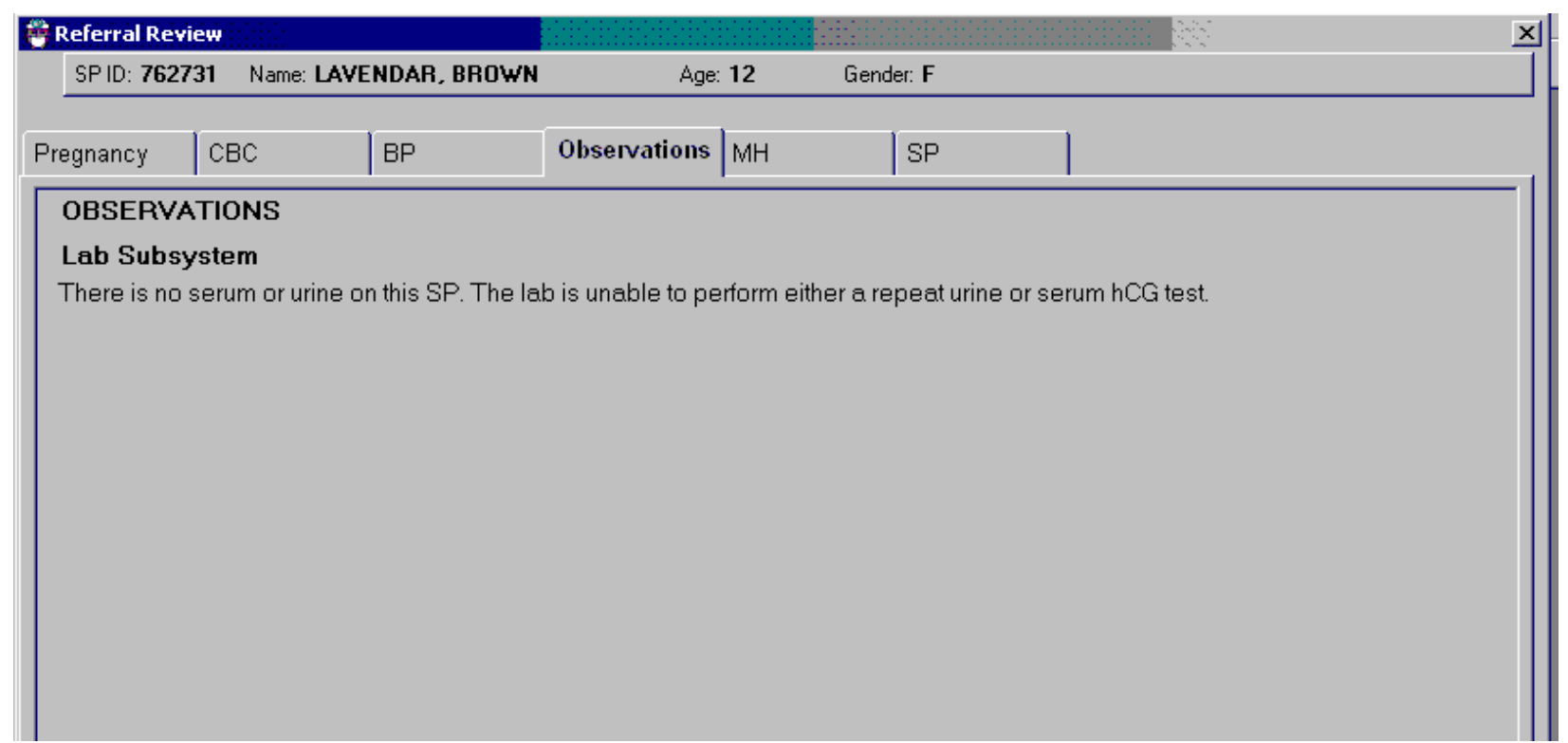

The physician accesses the observations using the Review in Box Details.

\subsection{Limitations of Method: Specimen Rejection, Interfering Substances, and Conditions}

Read the following procedural notes to ensure the best results with the ICON 25 hCG test kit. 


\section{ICON® 25 hCG Test}

- Dispensing Specimen - Do not reuse a test device or use a test device for multiple patient samples or controls, as this will produce inaccurate results.

- Incubation Times - The intensity of the red color in the test line region (T) will vary depending on the concentration of hCG present in the sample. However, neither the qualitative value nor the rate of increase in hCG can be determined by this qualitative test.

- Batch Processing - If several specimens are to be tested during one run, perform each test step for all specimens and controls at timed intervals before proceeding to the next test step. It is recommended that no more than ten (10) test devices be run in one batch.

- Specimens Containing Particulate Matter - Urine samples exhibiting visible precipitates should be centrifuged or allowed to settle to obtain a clear sample for testing.

- Very dilute urine samples, as indicated by a low specific gravity, may not contain representative levels of hCG.

- False negative results may occur when the levels of hCG are below the sensitivity level of the test.

- Very low levels of hCG (less than $50 \mathrm{mIL} / \mathrm{mL}$ ) are present in urine and serum samples shortly after implantation. However, because a significant number of first trimester pregnancies terminate for natural reasons, a test result that is weakly positive should be confirmed.

- A number of conditions other than pregnancy, including trophoblastic disease and certain nontrophoblastic neoplasms including testicular tumors, prostate cancer, breast cancer, and lung cancer, cause elevated levels of hCG. Therefore, the presence of hCG in urine or serum should not be used to diagnose pregnancy unless these conditions have been ruled out.

- This test provides a presumptive diagnosis for pregnancy. A physician should make a confirmed pregnancy diagnosis only after all clinical and laboratory findings have been evaluated.

- Negative results are expected in healthy nonpregnant women and healthy men. Healthy pregnant women have hCG in their urine and serum samples. The amount of hCG will vary greatly with gestational age and between individuals. Concentrations of hCG in pregnant women are generally between 10 and $30 \mathrm{mIU} \mathrm{hCG} / \mathrm{mL}$ in the $7-10$ days following implantation or 3 weeks after the last menstrual period (LMP). During the latter part of the first trimester of pregnancy, the hCG concentration reaches a maximum level of greater than $100,000 \mathrm{mIL} / \mathrm{mL}$. 
- The ICON $25 \mathrm{hCG}$ test has a stated sensitivity of $25 \mathrm{mIU} / \mathrm{mL}$ and is capable of detecting pregnancy as early as 1 day after the first missed menses.

- Do not use urine samples containing grossly hemolyzed blood since they may give inaccurate or erratic results.

\section{Sure-Vue ${ }^{\mathrm{TM}}$ hCG Urine and Serum Control Sets}

- These controls are formulated for use as quality control specimens in the reagent verification of pregnancy test kits.

\section{Accuracy, Sensitivity, and Specificity}

ICON® 25 hCG Test

\section{Accuracy}

A multicenter clinical evaluation was conducted comparing the results obtained using the ICON ${ }^{\circledR} 25$ hCG test and another commercially available urine/serum membrane hCG test. The urine study included 159 samples and both tests identified 88 negative and 71 positive results. The serum study included 73 samples and both assays identified 51 negative and 21 positive and 1 inconclusive result. The results demonstrated a 100 percent overall agreement (for an accuracy of $>99 \%$ ) of the ICON® 25 hCG test when compared to the other urine/serum membrane hCG test.

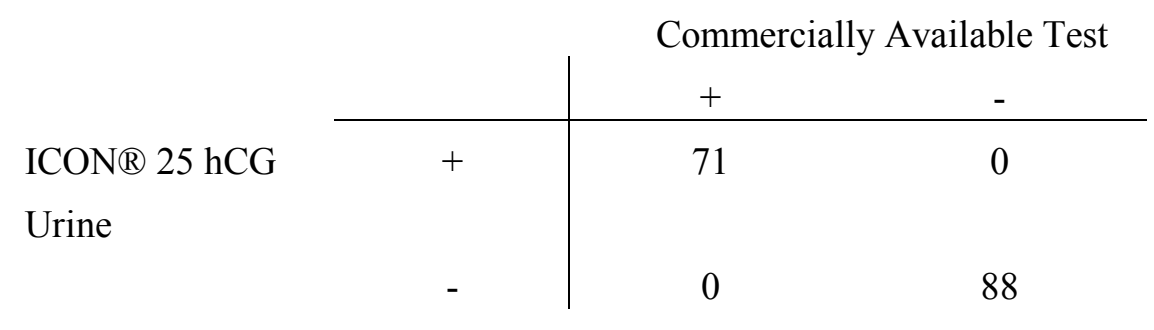

\begin{tabular}{|c|c|c|c|}
\hline & & Com & able Test \\
\hline & & + & - \\
\hline $\mathrm{ICON} ® 25 \mathrm{hCG}$ & + & 21 & 0 \\
\hline Serum & & & \\
\hline & - & 0 & 51 \\
\hline
\end{tabular}




\section{Sensitivity}

- The ICON $® 25 \mathrm{hCG}$ test detects $\mathrm{hCG}$ at concentrations of $25 \mathrm{mIU} \mathrm{hCG} / \mathrm{mL}$ or greater. The test has been standardized to the World Health Organization (W.H.O.) Third International Standard. The addition of $300 \mathrm{mIU} / \mathrm{mL}$ of luteinizing hormone, (LH), $1,000 \mathrm{mIU} / \mathrm{mL}$ of follicle stimulating hormone $(\mathrm{FSH})$, and $1,000 \mathrm{mIU} / \mathrm{mL}$ of thyroid stimulating hormone $(\mathrm{TSH})$ to negative $(0 \mathrm{mIU} / \mathrm{mL} \mathrm{hCG})$ urine/serum specimens and to positive $(25 \mathrm{mIU} / \mathrm{mL}$ hCG) urine/serum specimens did not exhibit cross-reactivity in the assay.

\section{Specificity}

- The following potentially interfering substances were added to hCG negative and positive samples.

- None of the substances at the concentrations tested interfered in the test.

\section{Interfering Substances}

\begin{tabular}{lc}
\hline Substance Added & Concentration \\
\hline Acetaminophen & $20 \mathrm{mg} / \mathrm{dL}$ \\
Ascorbic Acid & $20 \mathrm{mg} / \mathrm{dL}$ \\
Caffeine & $20 \mathrm{mg} / \mathrm{dL}$ \\
Gentisic Acid & $20 \mathrm{mg} / \mathrm{dL}$ \\
Acetylsalicylic Acid & $20 \mathrm{mg} / \mathrm{dL}$ \\
Atropine & $20 \mathrm{mg} / \mathrm{dL}$ \\
Bilirubin (serum) & $40 \mathrm{mg} / \mathrm{dL}$ \\
Glucose & $2 \mathrm{~g} / \mathrm{dL}$ \\
Hemoglobin & $1 \mathrm{mg} / \mathrm{dL}$ \\
Bilirubin (Urine) & $2 \mathrm{mg} / \mathrm{dL}$ \\
Triglycerides & $1,200 \mathrm{mg} / \mathrm{dL}$ \\
\hline
\end{tabular}

\section{Sure-Vue ${ }^{\mathrm{TM}}$ hCG Urine and Serum Controls}

These controls have been designed to produce correct results in pregnancy test kits. These controls have been tested with the ICON ${ }^{2} 25$ hCG test and were found to produce satisfactory results. 


\section{$5.14 \quad$ Reference Ranges}

Not applicable.

\subsection{Action Limits}

Not applicable.

\subsection{Specimen Storage and Handling During Testing}

\section{Serum}

Serum is required; do not use plasma. Specimens may be stored for 48 hours at $2{ }^{\circ} \mathrm{C}-8^{\circ} \mathrm{C}$ $\left(36^{\circ} \mathrm{F}-46^{\circ} \mathrm{F}\right)$ before testing. Specimens held for longer times should be frozen at $-20^{\circ} \mathrm{C}\left(-4^{\circ} \mathrm{F}\right)$ before testing. Bring all specimens to room temperature before beginning the assay procedure and mix before testing. Use clear nonhemolyzed samples when possible.

\section{Urine}

A urine sample must be collected in a clean and dry container. Centrifuge very turbid urine specimens at 2,500 RPM for 5 minutes before use. Do not shake or disturb specimens containing particulate matter, such as salts that have settled out of solution; pipette samples from the clear supernatant of such specimens.

\subsection{Alternative Method for Performing Test or Storing Specimens if Test System Fails}

There is no alternative method for testing. 


\subsection{Test Results Reporting System Protocol for Reporting Action Limits}

Not applicable.

\subsection{Specimen Accountability and Tracking}

All records, including $\mathrm{QA} / \mathrm{QC}$ data are maintained for 6 years. Use only numerical identifiers for SP results.

\subsection{Quality Control Summary Statistics and Graphs}

Quality control reports are monitored by stand and maintained for 6 years.

\section{$5.21 \quad$ References}

Batzer, F.R. (1980). Hormonal evaluation of early pregnancy, Fertility \& Sterility, 34(1), 1-13.

Catt, K.J., Dafau, M.L., and Vaitukaitis, J.L. (1975). Appearance of hCG in pregnancy plasma following the initiation of implantation of the blastocyte, J. Clin. Endocrinol. Metab, 40(3), 537-540.

Braunstein, G.D., Rasor, J., Adler, J., and Danzer, H. (1976). ME Wade Serum human chorionic gonadotropin levels throughout normal pregnancy, AM. J. Obstet. Gynecol., 126(6), 678-681.

Lenton, E.A., Neal, L.M., and Sulaiman, R. (1982). Plasma concentrations of human chorionic gonadotropin from the time of implantation until the second week of pregnancy, Fertil. Steril., 37(6), 773-778.

Steier, J.A., Bergsjo, P., and Myking, O.L. (1984). Human chorionic gonadotropin in maternal plasma after induced abortion, spontaneous abortion and removed ectopic pregnancy, Obstet. Gynecol., 64(3), 391-394.

Dawood, M.Y., Saxeba, B.B., and Landersman, R. (1977). Human chorionic gonadotropin and its subunits in hydatiform mole and choriocarcinoma, Obstet. Gynecol., 5(2), 172-181.

Braunstein, G.D., Vaitukatitis, J.L., Carbone, P.P., and Ross, G.T. (1973). Ectopic production of human chorionic gonadotropin by neoplasms, Ann. Inern. Med., 78(1), 39-45. 


\section{COMPLETE BLOOD COUNT (CBC)}

\subsection{Introduction}

Perform a complete blood count (CBC) in duplicate on all Sample Persons (SPs) aged 1 year and older. Perform the CBC on the Coulter ${ }^{\circledR}$ DxH 800. Run a CBC on the SP's EDTA blood tubes.

The three Coulter ${ }^{\circledR}$ DxH 800 serial numbers and system IDs are:

\begin{tabular}{|c|c|c|c|}
\hline DxH 800 Location & Serial Number & System ID & IQAP ID \\
\hline MEC 1 & AU36520 & 3770768 & $32979-1-B 3$ \\
\hline MEC 2 & AU32459 & 3702621 & $32979-1-B 2$ \\
\hline MEC 3 & AU23263 & 3348667 & $32979-1-B 1$ \\
\hline
\end{tabular}

\subsection{Purpose and Principle of Test}

The UniCel DxH 800 Analyzer is a quantitative, automated hematology analyzer for in-vitro diagnostic use in screening patient populations found in clinical laboratories. The UniCel DxH 800 Analyzer provides a:

Complete Blood Count (CBC), Leukocyte 5-Part Differential (Diff), Reticulocyte (Retic) and Nucleated Red Blood Cell (NRBC) on whole blood. 
The UniCel DxH 800 Analyzer measures the following parameters:

\begin{tabular}{|c|c|}
\hline WBC & White Blood Cell count \\
\hline UWBC & Uncorrected White Blood Cell count \\
\hline $\mathrm{RBC}$ & Red Blood Cell count (for Whole Blood and Body Fluids) \\
\hline HGB & Hemoglobin \\
\hline HCT & Hematocrit \\
\hline $\mathrm{MCV}$ & Mean Corpuscular Volume \\
\hline $\mathrm{MCH}$ & Mean Corpuscular Hemoglobin \\
\hline $\mathrm{MCHC}$ & Mean Corpuscular Hemoglobin Concentration \\
\hline RDW & Red Cell Distribution Width \\
\hline RDW-SD & Red Cell Distribution Width Standard Deviation (SD) \\
\hline PLT & Platelet count \\
\hline MPV & Mean Platelet Volume \\
\hline $\mathrm{NE}$ & Neutrophil percent \\
\hline LY & Lymphocyte percent \\
\hline MO & Monocyte percent \\
\hline EO & Eosinophil percent \\
\hline $\mathrm{BA}$ & Basophil percent \\
\hline NE\# & Neutrophil absolute number \\
\hline LY\# & Lymphocyte absolute number \\
\hline MO\# & Monocyte absolute number \\
\hline EO\# & Eosinophil absolute number \\
\hline BA\# & Basophil absolute number \\
\hline NRBC & Nucleated Red Blood Cell percent \\
\hline NRBC\# & Nucleated Red Blood Cell absolute number \\
\hline RET & Reticulocyte percent \\
\hline RET\# & Reticulocyte absolute number \\
\hline MRV & Mean Reticulocyte Volume \\
\hline IRF & Immature Reticulocyte Fraction \\
\hline $\mathrm{TNC}$ & Total Nucleated Cell (Body Fluids) \\
\hline
\end{tabular}




\subsubsection{Coulter Method}

In 1956, Wallace Coulter described the Coulter Principle as follows: A suspension of blood cells is passed through a small orifice simultaneously with an electric current. The individual blood cells passing through the orifice introduce an impedance change in the orifice determined by the size of the cell.

Each cell suspended in a conductive liquid (diluent) acts as an insulator. As each cell goes through the aperture, it momentarily increases the resistance of the electrical path between the submerged electrodes on either side of the aperture. This causes a measurable electronic pulse. For counting, the vacuum used to pull the diluted suspension of cells through the aperture must be at a regulated volume. While the number of pulses indicates particle count, the size of the electrical pulse is proportional to the cell volume.

The system counts the individual cells and provides cell size distribution. The number of cells counted per sample is approximately 100 times greater than the usual microscope count reducing statistical error approximately ten times.

\subsubsection{VCSn Technology}

Historically, Beckman Coulter analyzers housed a flow cell in a Triple Transducer Module (TTM), first introduced commercially in the 1980's. The TTM flow cell was the location for detection of the processed samples. The TTM produced three measurement signals - volume, conductivity, and light scatter.

The DxH 800 system replaces the TTM with the Multi-Transducer Module (MTM), which measures additional multiple angles of light scatter, a major improvement over the single light scatter measured by the TTM.

All Diff, NRBC, and Retic analysis occurs in the VCSn module. The VCSn module is responsible for controlled sample preparation and delivery of the prepared sample to the flow cell for analysis of the WBC differential, reticulocyte, and NRBC. The VCSn module includes the Air Mix and Temperature Control (AMTC) and the Multi-Transducer Module (MTM). 
In the flow cell, low-frequency, direct current measures volume, while high-frequency (RF) current senses cellular internal content through measuring changes in conductivity.

The MTM utilizes a flow cell to pass particles through a sensing zone one particle at a time and a diode laser to illuminate the particles. The illuminated particles both scatter and absorb a portion of the incident light. Sensors strategically placed around the flow cell collect the scattered light of interest. An additional sensor placed in the laser path measures the amount of light removed due to light scatter and absorption. This measurement is called Axial Light Loss.

\subsubsection{Dataplot Development}

The System Manager (SM) performs a series of operations on the stored digital raw values received from the flow cell to identify populations and calculate the frequency of cells within each population. The system produces the Dataplot displays for visual representation of the Differential, NRBC, and Reticulocyte membership and density.

The DxH 800 System algorithm uses tools designed for finding optimal separation between overlapping clusters of data.

The algorithm can:

- Adapt to unusual population shifts and overlaps.

- Define highly irregular separation.

- Make subsequent analysis of the identified regions.

- Correct deficiencies in separation.

In the Dataplots, different colors represent different memberships (types of cells). Shades of colors represent density (concentration): dark colors for low density and bright colors for high density. 


\begin{tabular}{llllll}
\multicolumn{2}{l}{ NRBC Analysis } & Diff Analysis & & \multicolumn{2}{l}{ Retic Analysis } \\
NRBC & Red & Lymphocyte & Blue & WBC & Blue \\
Other* & Green & Monocyte & Green & RBC & Red \\
WBC & Blue & Neutrophil & Purple & PLT & Green \\
& & Eosinophil & Orange & RETIC & Purple \\
& & Basophil & White & & \\
& Non-White Cell & Red & & \\
* Others include RBC debris, and PLT debris, etc. & & &
\end{tabular}

\subsubsection{Parameter Measurement, Derivation, and Calculation}

The following table lists each reportable parameter and the method used to obtain the results. A description of the method is also included.

\begin{tabular}{|c|c|c|}
\hline $\begin{array}{l}\text { Parameter } \\
\text { (Reporting } \\
\text { Units in US-1 } \\
\text { Format) }\end{array}$ & Method & Description \\
\hline WBC & $\begin{array}{l}\text { Coulter } \\
\text { Principle }\end{array}$ & $\begin{array}{l}\text { White Blood Cell Count or Leukocyte Count } \\
\text { - Measure directly, multiplied by the calibration factor. } \\
\text { - Corrected for interference if necessary. If no } \\
\text { correction is required, then } \mathrm{WBC}=\mathrm{UWBC} \text {. } \\
\text { - } \mathrm{WBC}=\mathrm{N} \times 10^{3} \text { cells } / \mu \mathrm{L} \text {. }\end{array}$ \\
\hline UWBC & $\begin{array}{l}\text { Coulter } \\
\text { Principle }\end{array}$ & $\begin{array}{l}\text { Uncorrected White Blood Cell } \\
\text { - Measure directly, multiplied by the calibration factor. } \\
\text { - } \mathrm{UWBC}=\mathrm{N} \times 10^{3} \text { cells } / \mu \mathrm{L} \text {. }\end{array}$ \\
\hline RBC & $\begin{array}{l}\text { Coulter } \\
\text { Principle }\end{array}$ & $\begin{array}{l}\text { Red Blood Cell Count or Erythrocyte Count } \\
\text { - Measure directly, multiplied by the calibration factor. } \\
\text { - Corrected for very high white count if necessary. } \\
\text { - } \mathrm{RBC}=\mathrm{N} X 10^{6} \text { cells } / \mu \mathrm{L} \text {. }\end{array}$ \\
\hline
\end{tabular}




\begin{tabular}{|c|c|c|}
\hline $\begin{array}{c}\text { Parameter } \\
\text { (Reporting } \\
\text { Units in US-1 } \\
\text { Format) }\end{array}$ & Method & Description \\
\hline $\mathrm{Hgb}$ & $\begin{array}{l}\text { Photometric } \\
\text { Measurement }\end{array}$ & $\begin{array}{l}\text { Hemoglobin or Hemoglobin Concentration } \\
\text { - Transmittance of light at } 525 \mathrm{~nm} \text { through a lysed } \\
\text { WBC solution in the Hgb cuvette, compared to the } \\
\text { transmittance of the same light through a reagent } \\
\text { blank. The system converts this ratio to the Hgb value } \\
\text { using a calibration factor. } \\
\text { - Weight (mass) of Hgb determined from the degree of } \\
\text { absorbance found through photo current transmittance } \\
\text { expressed in } \mathrm{g} / \mathrm{dL} \text {. } \\
\text { - Corrected for WBC interference. } \\
\text { - } \mathrm{HgB}(\mathrm{g} / \mathrm{dL})=\left[\text { constant } \mathrm{X} \log ^{10} \text { (Reference }\right. \\
\% \mathrm{~T} / \mathrm{Sample} \% \mathrm{~T})] \text {. }\end{array}$ \\
\hline Hct & Calculated & $\begin{array}{l}\text { Hematocrit } \\
\text { - The relative volume of packed erythrocytes to whole } \\
\text { blood. } \\
\left.\text { - Hct }(\%)=\mathrm{RBC} \mathrm{X} \mathrm{MCVV}^{10}\right) \text {. }\end{array}$ \\
\hline $\mathrm{MCV}$ & $\begin{array}{l}\text { Derived from } \\
\text { RBC } \\
\text { Histogram }\end{array}$ & $\begin{array}{l}\text { Mean Corpuscular Volume } \\
\text { - The average volume of individual erythrocytes derived } \\
\text { from the RBC histogram. } \\
\text { - The system multiples the number of RBC in each } \\
\text { channel by the size of the RBC in that channel. The } \\
\text { products of each channel between } 36 \text { and } 360 \\
\text { femtoliters (fL) are added. This sum is divided by the } \\
\text { total number of RBC between } 36 \text { and } 360 \mathrm{fL} \text {. The } \\
\text { Analyzer then multiplies by a calibration factor. } \\
\text { - Corrected for WBC interference. } \\
\text { - Expressed in } \mathrm{fL} \text {. }\end{array}$ \\
\hline
\end{tabular}

The DxH 800 System includes a Specimen Processing Module (SPM), a Pneumatic Supply Module (PSM), and a System Manager (SM), precisely integrated to provide process control, data consolidation, and cassette or single-tube delivery of specimens. 


\subsection{Components of DXH 800}

\subsubsection{The Specimen Processing Module (SPM)}

The SP is made up of the following functional modules:

- $\quad$ Specimen Transport Module (STM). The STM automatically transports specimens. It delivers closed-vial and open-vial specimens accommodating a wide range of specimen tubes. The STM supports the following:

- $\quad$ Operator loading and unloading of specimens.

- $\quad$ Transport and queuing of specimens.

- $\quad$ Mixing and presentation of specimens.

- $\quad$ A Magnetic Transport System to move cassettes containing specimens.

- Sample Aspiration Module (SAM). The SAM aspirates from capped specimen tubes (presented via Cassette or Single-tube presentation) or open vials (Single-tube presentation). Body fluids are presented via Single-tube, while whole bloods can be presented in either presentation mode. The identification and integrity of samples are verified prior to delivery and transfer to the analytical modules within the system. The SAM design provides for two methods of sample delivery: sample segmentation and sample dispensing. In sample segmentation, aspirated sample is transferred through the BSV where it is segmented for whole blood CBC and body fluid. In sample dispensing, whole blood sample segments are then dispensed via the aspiration probe into the mixing chambers on the Air Mix Temperature Control Module (AMTC) for the WBC differential, NRBC, and reticulocyte analyses.

- VCSn Module provides the physical processing elements necessary for the Differential, NRBC, and Retic sample preparation and subsequent measurement. The VCSn Module includes the Distribution Valve (DV), Air Mix Temperature Control (AMTC) module, Multi-transducer Module (MTM) and their associated electronic and fluidics. Samples prepared at the AMTC are delivered to the MTM where the sample detection occurs.

- $\quad \mathrm{CBC}$ Assembly provides the physical processing elements necessary for CBC sample conditioning (combining of reagents and the sample segment, mixing, and incubation) and measurement via the aperture bath assemblies, the HGB assembly, and the electrical signal conditioning circuits. 
- Common Services consist of the Electronic Supply Module, Pneumatic Services, and Reagent Services (supply and distribution). Common Services provides and monitors electronic power and supplies and monitors the reagent and waste levels as well as the pressure and vacuum.

\subsubsection{The System Manager (SM)}

The SM is responsible for:

- Controlling processes, such as analysis and diagnostic procedures.

- Producing test results.

- Managing data, such as test ordering, results review, results release, quality control, LIS interface, logging, and report generation.

- The SM includes an LCD flat panel monitor with touch screen capability, a computer with CD/DVD RW drive running Microsoft Windows XP Professional operating system, the DxH 800 software, a standard keyboard, and an optical mouse.

\subsubsection{Pneumatic Supply Module (PSM)}

The Pneumatic Supply Module supplies vacuum and pressure to the SPM.

\subsection{Special Safety Precautions}

All specimens may be potentially positive for infectious agents including HIV and the hepatitis $\mathrm{B}$ and $\mathrm{C}$ viruses. Observe universal precautions. It is mandatory to wear gloves and lab coat when handling all human blood products and Coulter ${ }^{\circledR}$ controls. Wear safety glasses whenever operating the instrument in SECONDARY mode. Dispose of all biological samples in a biohazard container and wipe down all work surfaces with $10 \%$ bleach solution at the end of each session.

The mobile examination center (MEC) Working Safely with Hazardous Chemicals manual contains all Coulter ${ }^{\circledR}$ material safety data sheets (MSDS). 


\subsection{Computerization: Integrated Survey and Information System (ISIS)}

The DxH 800 System Manager (SM) transmits individual SP results to the MEC automated ISIS system. Review all SP results at the DxH 800 SM monitor.

The SM is responsible for the following:

- Controlling processes, such as analysis and diagnostic procedures.

- Producing test results.

- Manages data, such as test ordering, results review, results release, quality control, LIS interface, logging and report generation.

- The SM includes an LCD flat panel monitor with touch screen capability, a computer with CD/DVD RW drive running Microsoft Windows XP Professional operating system, the DxH 800 software, a standard keyboard, and an optical mouse.

The ISIS hematology module in the laboratory application automatically receives the results or transmits them manually to the hematology module. The laboratory application evaluates the data for completeness and accuracy. The final decision to accept or reject a result is the responsibility of the medical technologist.

All data are backed up and stored at Westat's home office.

\subsection{Specimen Collection and Preparation}

\subsubsection{Specimen Collection}

- The phlebotomist collects a 3 or 4-mL K3 EDTA tube, used for a CBC, on all SPs aged 1 year and older, following established venipuncture protocol and procedures. (A $1-2 \%$ dilution effect occurs in this liquid EDTA tube.)

- Sample volume is $165 \mu \mathrm{L}$ of whole blood in the closed-vial mode or the single tube presentation mode. The minimum sample volume per tube in the closed-vial mode is $1-\mathrm{mL}$ with the proper proportion of blood to anticoagulant. 


\subsubsection{Specimen Preparation}

- For SPs 1-5 years old, the blood specimen-processing technologist initially processes the tube by taking off whole blood for various tests. The blood specimen-processing technologist places the specimen on a rocker until the hematology technologist can perform the $\mathrm{CBC}$. Run the $\mathrm{CBC}$ as soon as possible; there is no requirement to wait any length of time between drawing the blood and running the $\mathrm{CBC}$.

- For SPs aged 6 years and older, there is a dedicated tube drawn for the CBC.

\subsection{Procedure for Microscopic Examination}

Not Applicable - Do not prepare differential microscopic slides.

\subsubsection{Reagents and Supplies}

DxH Reagents, including storage and handling requirements, are listed below:

1. Coulter DxH Diluent (store at $2-40^{\circ} \mathrm{C}$ ).

- Coulter DxH diluent is shipped in ready-to-use containers.

- Opened containers are stable for 60 days.

- Do not use this product beyond the expiration date.

- Coulter DxH Diluent is a cyanide-free, isotonic buffered saline solution.

- Coulter DxH Diluent dilutes the specimen, is used for rinsing SPM components between sample analyses, and provides a sheath stream to transport the specimen through the flow cell.

2. Coulter DxH Lyse Reagent (store at $2-40^{\circ} \mathrm{C}$ ).

- Coulter DxH Lyse reagent is shipped in ready-to-use containers.

- Opened containers are stable for 60 days.

- Do not use this product beyond the expiration date. 
- Coulter DxH Lyse is a cyanide-free, CBC lytic reagent that lyses red blood cells for the white blood cell count and works in conjunction with Coulter DxH Diluent to generate a stable hemoglobin measurement.

- Coulter DxH Cell Lyse is also used to lyse the red blood cells and discriminates nucleated red blood cells from white blood cells.

3. Coulter DxH Diff Pack (store at $2-25^{\circ} \mathrm{C}$ ).

- Coulter DxH Diff Pack is shipped in ready-to-use containers.

- Opened containers are stable for 60 days.

- Do not use this product beyond the expiration date.

- The Coulter DxH Diff Pack consists of the Erythrolyse Lytic Reagent and StabliLyse Preservative Reagent.

- The Erythrolyse Lytic Reagent is a cyanide-free lytic reagent that dilutes the blood sample and lyses red blood cells in preparation for white blood cell measurement in the flow cell.

- The StabiLyse Preservative Reagent neutralizes the Diff lytic reagent and preserves the white blood cells for measurement in the flow cell. Together, Erythrolyse and StabiLyse provide the five-part differential.

4. Coulter DxH Cleaner (store at $2-25^{\circ} \mathrm{C}$ ).

- Coulter DxH Cleaner is shipped in ready-to-use containers.

- Opened containers are stable for 90 days.

- Do not use this product beyond the expiration date.

- DxH Cleaner is a cyanide-free, aldehyde-free cleaning agent that degrades residual materials so that they may be flushed from the system with diluent.

NOTE: If any Coulter DxH reagent has been partially or completely frozen, allow product to warm to room temperature. Mix product by gentle inversion prior to placement on the instrument.

DxH Controls including storage and handling requirements are listed below:

1. Coulter $6 \mathrm{C} \mathrm{Cell} \mathrm{Control} \mathrm{(store} \mathrm{at} 2-8^{\circ} \mathrm{C}$ ).

- The Coulter 6C Cell Control is an integrated control that enables monitoring of system performance and calibration status for all directly measured and calculated CBC, Diff, and NRBC parameters. Instructions for use are as follows: 
- Remove $6 \mathrm{C}$ Cell Control vials from refrigerator and warm at Ambient Temperature for 10 to 15 minutes.

- Roll the tube slowly between the palms of the hand 8 times in an upright position. Invert the tube and slowly roll between the palms of the hand 8 times. Then gently invert the tube 8 times. Repeat.

- Run the controls in the cassette presentation on the instrument

- Return the control tubes to the refrigerator with 30 minutes.

2. The Coulter LIN-X Linearity Control is recommended for verification of the reportable range and linearity, and calibration assessment of the $\mathrm{WBC}, \mathrm{RBC}, \mathrm{HGB}$, and PLT parameters.

- Remove Coulter Lin-X Cell Control vials from refrigerator and warm at Ambient Temperature for 15 minutes.

- Roll the tube slowly between the palms of the hand 8 times in an upright position. Invert the tube and slowly roll between the palms of the hand 8 times. Then gently invert the tube 8 times. Repeat mixing procedure 3 times.

- Run the Lin-X Cell Controls in consecutive order beginning with Level 0 and ending with Level 11. Run controls in cassette presentation on the instrument

- Level 11 is used to clean the system. The values for this level are not recorded.

3. Coulter Latron CP-X Control (store at 2 to $30^{\circ} \mathrm{C}$ ) is recommended for the verification of the system calibration status of the VCSn parameters. Coulter Latron CP-X control checks the alignment of the laser and the flow cell on the DxH 800.

- Perform Daily Checks prior to running Latron Control.

- Gently mix Latron CP-X control by inversion 5 to 8 times. Avoid foaming.

- Run the controls in the cassette presentation on the instrument.

4. Coulter S-Cal Calibrator is traceable to reference methods and recommended for determining adjustment factors to ensure accurate SPM measurements of directly measured CBC parameters. Calibrator results should be monitored with Coulter 6C Cell Control.

- Remove S-Cal from the refrigerator and warm at Ambient Temperature for 15 minutes.

- Roll the tube slowly between the palms of the hand 8 times in an upright position. 
- Invert the tube and slowly roll between the palms of the hand 8 times. Then gently invert the tube 8 times. Repeat.

- Inspect the tube contents to determine if all cells have been uniformly distributed. Repeat the mixing procedure if contents have not been uniformly distributed.

- Complete entire procedure within 1 hour.

\subsubsection{DxH 800 Supplies}

1. 3-mL $\mathrm{K}_{2}$ EDTA BD Hemogard Vacutainer ${ }^{\circledR}$ tube (367856)

2. $\quad$ 4-mL $\mathrm{K}_{2}$ EDTA BD Hemogard Vacutainer ${ }^{\circledR}$ tube (367861)

3. Tube rocker

4. Bleach, 5.25\% sodium hypochlorite

5. Bottled Dionized Water

6. Plastic squirt bottle

7. Cotton gauze pads

8. Three-hole paper punch

9. Notebook

10. Flashlight

11. Precision screwdriver set

12. CD-RW

\subsection{Shutdown of DxH 800 System}

The DxH 800 SPM, System Manager, and Monitor are connected to an Uninterruptible Power Supply (UPS). In the event of a power outage at your facility, the components will continue to 
operate for a short time so that you can shut down the system. The printer is connected to the hospital power. Shutdown the System Manager as follows:

- Logoff. Select the Logoff utility icon

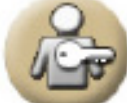
, then the Exit Workstation button.

- Shutdown the operating system by using the routine Windows application.

- Power Off the computer. Powering off the computer also powers off the monitor. You do not need to power off the monitor separately. The computer does not need to be shut down on a routine basis. Power Off the computer only when circumstances are indicated.

\subsubsection{Daily Shutdown}

Beckman Coulter recommends that the SPM remain in cleaner for at least 30 minutes every 24 hours. If the SPM has power, and is in Shutdown, but the pneumatics is off, an automatic cycling occurs every 24 hours to prevent flow cell and sample line clogging.

- Shutdown can be initiated manually or automatically.

- Shutdown removes diluent from the Unicell DxH 800 and replaces it with cleaner.

- At the end of the time with cleaner, the cleaner is replaced with diluent. Then, the compressor automatically shuts off.

\subsubsection{Shutdown at the Mobile Examination Center (MEC)}

- Shutdown will be set automatically.

- Daily Checks is programmed to immediately follow the Start-Up.

- Latron CP-X control should be run immediately following Daily Checks. 


\subsubsection{Cancel Automatic Shutdown}

At certain times Automatic Shutdown will have to be cancelled. Shutdown might have to be scheduled earlier or later depending on volume. To cancel Automatic Shutdown:

- From the Daily Checks screen, select Auto Configuration.

- Select Configure Shutdown.

- Remove the Checkmark from the Enable Automatic Shutdown box.

- To perform a manual shutdown when Auto Configuration is disabled, please see the information that follows.

\subsubsection{Manual Shutdown of DxH Units}

1. From the Daily Checks screen, select the Shutdown button to display the Manual Shutdown dialog box.

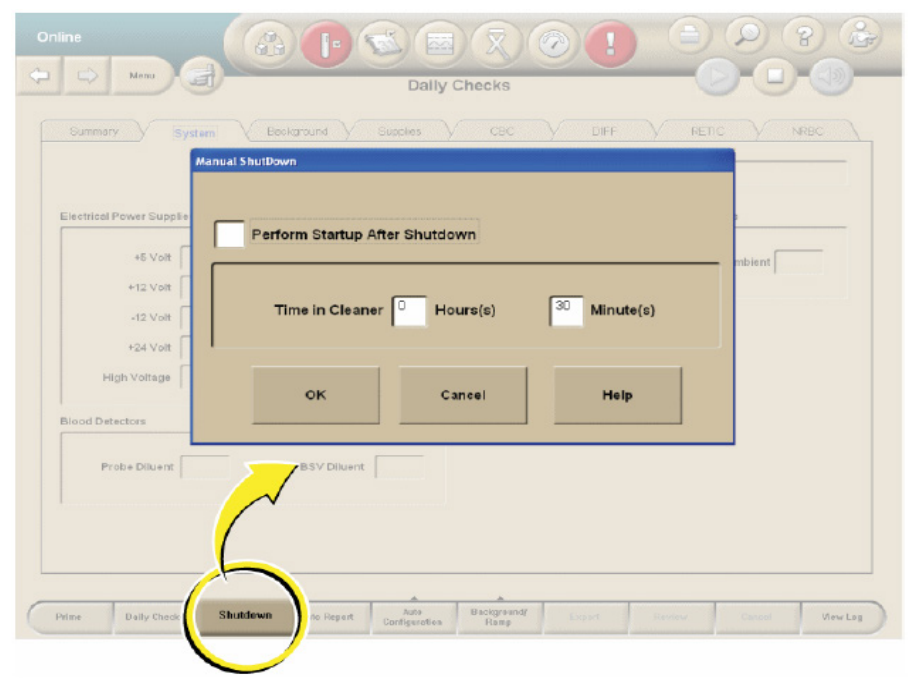

2. Select the Perform Startup after Shutdown checkbox to automatically begin Daily Checks after Shutdown.

3. Type a number in the Time in Cleaner Hours and Minutes checkboxes to determine the time in cleaner.

4. Select OK to begin Shutdown or Cancel to exit the pop-up window without starting Shutdown. 
Logging on to the System Manager:

- Click on the

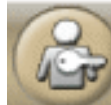
icon (Person with key).

- Type your User Name.

- Type your Password. If you forget your password, ask one of the senior techs to reset it for you.

6.10 Run Daily Checks (formerly known as StartUP) (Menu $>$ QA $>$ Daily Checks)

Running Daily Checks:

- Select the Daily Checks button (Calendar Icon) from the top of any screen to display the Daily Checks Summary screen with results of the most recent Daily Checks.

- Select the Daily Checks button at the bottom of the screen. A DxH 800 dialog box displays the following:

Are you sure you want to start a Daily Checks procedure? Press OK to continue.

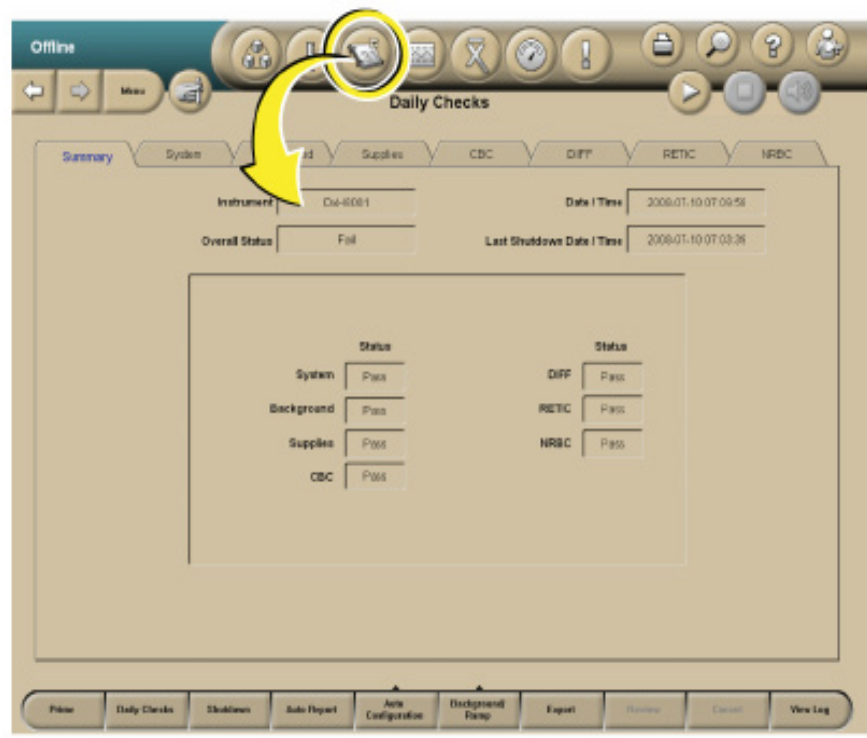


Select OK to run Daily Checks or Select Cancel.

\subsubsection{Daily Checks Screen}

- The results of Daily Checks display on eight tabbed views: Summary (default view), System, Background, Supplies, CBC, Diff, Retic, and NRBC.

- When Daily Checks pass, the Daily Checks Icon is neutral. Select the individual tabs to view those results.

If any of the Daily Checks do not pass, the following occurs:

- The Daily Checks icon is red.

- The problematic result is backlit in red on the Summary tab.

- The tab that contains the problematic result has a red indicator.

- If there are any failures, you must Review Daily Checks (Event Logs $>$ General Tab) in order to proceed with further analysis.

- The Daily Checks must pass or be reviewed in order to run specimens.

- From any tab on the Daily Checks screen, select the Review button on the Local Navigation bar to indicate that the Daily Checks results have been reviewed. Note: If Daily Checks pass, the Review button is disabled.

- You only have to review results that are questionable.

\subsubsection{Additional Tasks From the Daily Checks Screen}

- Select the Histogram button at the right of the screen on the DIFF, RETIC or NRBC tabs to display the VCSn Ramp Tests Histogram screen.

- NOTE: The VCSn Ramp Tests Histogram screen is not for routine use; however, you may at some time be asked to access this screen when on the phone with a Beckman Coulter Representative.

- Prime the SPM.

- Prime provides a method to remove cleaner in case an error occurred during a Shutdown cycle. 
- Select the Prime button at the bottom of the Daily Checks screen to prime the SPM.

- A DxH 800 dialog box will display the following message; "You have requested to perform a Prime Cycle."

- Select OK to continue or Cancel.

\subsection{Quality Control}

- Quality Control is the routine monitoring of performance and service using commercial or patient controls.

- Controls have known characteristics when run on a given system and are analyzed periodically in the same manner that patient specimens are analyzed.

- The results of analyzed controls are then compared to the know characteristics using statistical methods.

- This comparison allows changes in the SPM performance to be detected.

- You can then take some action if the changes detected are significant.

\subsubsection{Frequency of Performing Quality Control}

1. Beckman Coulter 6C Cell Control will be run each shift, at the start of the session. The time of QC performance is set to follow the preprogrammed Start-Up of the analyzers.

2. Beckman Coulter 6C Cell Control is run in the cassette mode on the DxH analyzers.

3. Beckman Coulter Latron CP-X Control is run once each 24 hours following Daily Checks on the instrument and is run in the cassette mode on the DxH analyzers.

4. Beckman Coulter Lin-X will be run once per year or at high elevation stands.

5. Calibration with S-Cal will be performed at the start of every stand or when recommended to troubleshoot a problem. 


\subsubsection{Setting Up a Control File}

1. Entering 6C Cell Control lot Numbers and Expiration Dates

2. Menu $>$ QA $>$ QC $>$ More Options $>$ QC Setup $>$ New Control from Barcode $>$ Scan the $6 C$ Cell Insert Barcode $>$ Select ALL \& Auto Transmit $>$ Select [OK].

\subsubsection{Setting Up a Latron Control File}

1. Entering Latron Control Lot Numbers and Expiration Dates

2. Menu $>$ QA $>$ QC $>$ More Options $>$ QC Setup $>$ New Control from Barcode $>$ Scan the Latron Insert Barcode $>$ Select ALL \& Auto Transmit $>$ Select [OK].

\subsubsection{What To Do When a Control is Out}

1. When a commercial control is out:

- $\quad$ Ensure the control material was mixed properly. If not, mix it according to the package insert.

- $\quad$ Verify that the control was identified properly. If using a barcode reader, ensure the barcode labels are clean and positioned correctly. If entering the ID manually, ensure that you typed the correct information.

- Verify that the assigned setup information (assigned values and expected ranges) matches either the control package insert, or your labs established values. If they do not, contact a senior technologist to change the control's information to match.

- $\quad$ If the problems above are ruled out then rerun the control to ensure the problem was not a statistical outlier.

- $\quad$ Ensure the control material was not contaminated by running another vial or level of control.

- $\quad$ Ensure there are no errors during the cycle. If necessary, call Beckman Coulter for help in resolution. 
2. When Latron QC is out:

- $\quad$ Ensure that the control setup information (assigned values and expected ranges) matches the package insert. If it does not, change the control information to match the package insert, then rerun the control.

- $\quad$ Ensure that the Latron control is not contaminated, properly mixed, not expired, and you have a sufficient volume of sample.

- If necessary, use a new vial of control. Be sure to mix it according to the directions listed above.

- $\quad$ Ensure the flow cell is clear by performing the Flush Flow Cell procedure.

- Rerun the control. If the control is still outside the expected ranges, call Beckman Coulter. The SPM can be set to the CBC mode and continue to process samples.

\subsubsection{Viewing Control Files - Data View (Menu $>$ QA $>Q C)$}

- To view control files on the Quality Control (Data View) screen select the QC Status icon at the top of any screen.

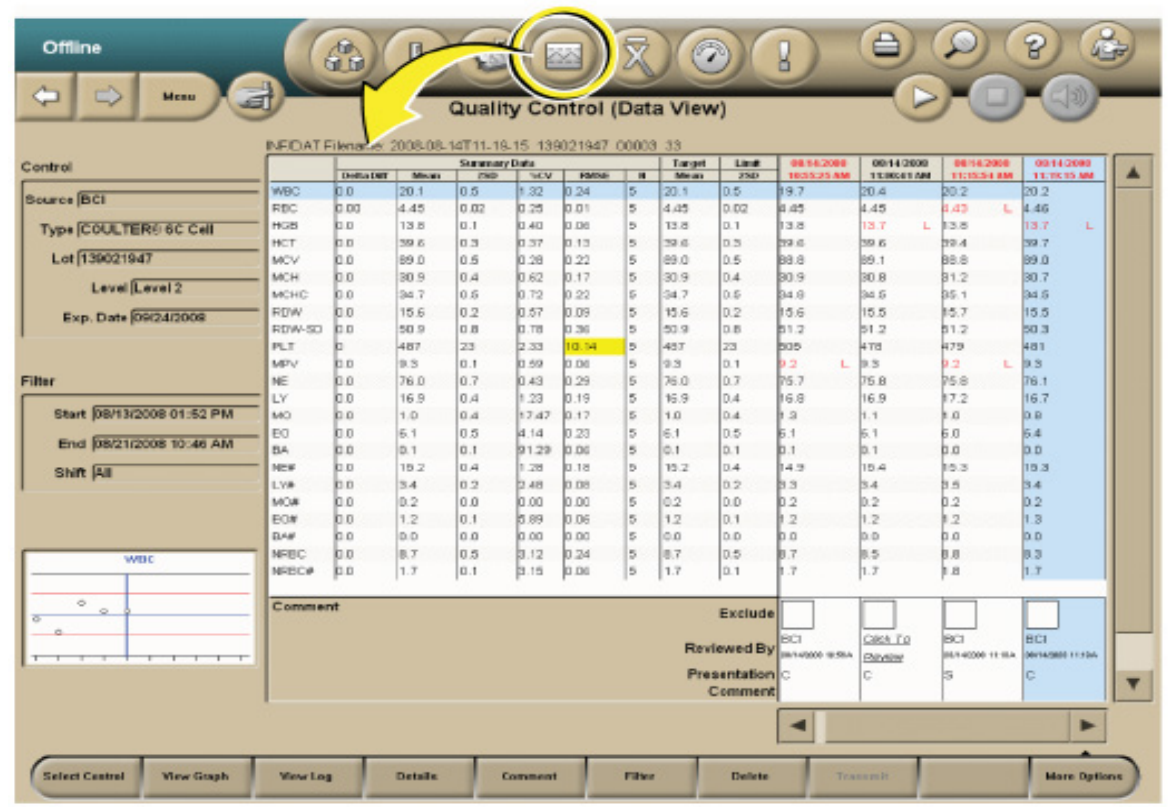

1. The Quality Control -Data View screen displays the most recently analyzed control lot with the most recently analyzed run selected by default. 
2. If controls have not been reviewed, a dialog box displays a list of those controls that have not been reviewed.

3. Out of control data is displayed in red text as well as the date and time.

4. The Quality Control (Data View) screen includes summary data, configuration data, a filter section, QC run data, run status and one thumbnail Levy-Jennings Graph for the selected parameter (row).

The following table lists the components seen on the QC Data View screen.

\begin{tabular}{|c|c|}
\hline Component & Description \\
\hline \multirow[t]{3}{*}{ Delta Diff } & $\begin{array}{l}\text { The difference between the calculated mean and the assigned } \\
\text { target of the parameter within the specified filter. }\end{array}$ \\
\hline & $\begin{array}{l}\text { NOTE: If the Extended QC is enabled and Extended QC limits } \\
\text { have been configured and the equation below is greater than } \\
\text { (absolute Delta Diff/BCI Target Value) } x 100 \text { is greater than the } \\
\text { Systematic Error limit, the Delta Diff will be highlighted in } \\
\text { yellow for that parameter if } N>=2 \text { and } N<15 \text { or in red if } N>=15 \text {. }\end{array}$ \\
\hline & $\begin{array}{l}\text { If the parameter's target is not applicable, N/A displays in this } \\
\text { field. }\end{array}$ \\
\hline Mean & $\begin{array}{l}\text { The calculated mean of the included points within the specified } \\
\text { filter. }\end{array}$ \\
\hline $25 \mathrm{D}$ & $\begin{array}{l}\text { The calculated SD of the included points within the specified } \\
\text { filter. }\end{array}$ \\
\hline \multirow[t]{2}{*}{$\% \mathrm{CV}$} & $\begin{array}{l}\text { The calculated Coefficient of Variation of the included points } \\
\text { within the specified filter. }\end{array}$ \\
\hline & $\begin{array}{l}\text { NOTE: If the Extended QC is enabled and Extended QC limits } \\
\text { have been configured and the CV value is greater than the } \\
\text { Random Error limit, the } \% C V \text { will be highlighted in yellow for } \\
\text { that parameter if } N>=2 \text { and } N<15 \text {, or in red if } N>=15 \text {. }\end{array}$ \\
\hline \multirow[t]{2}{*}{ RMSE } & $\begin{array}{l}\text { Root Mean Square Error displays when Extended QC is enabled. } \\
\text { See Figure 4.2. The RMSE is a Single Measurement Error. If the } \\
\text { value exceeds the Single Measurement Error limit, the RMSE } \\
\text { value will be highlighted in yellow for that parameter if } \mathrm{N}>=2 \\
\text { and } \mathrm{N}<15 \text {. The value is highlighted in red if } \mathrm{N}>=15 \text {. }\end{array}$ \\
\hline & $\begin{array}{l}\text { If the parameter's target is not applicable, NA displays in this } \\
\text { field. }\end{array}$ \\
\hline $\mathrm{N}$ & The number of included points within the specified filter. \\
\hline
\end{tabular}




\begin{tabular}{|c|c|}
\hline Component & Description \\
\hline Target & $\begin{array}{l}\text { The assigned target of the parameter being used in your lab at the } \\
\text { time of the control analysis. The target used by Extended QC } \\
\text { will be based on what has been configured for Traditional QC } \\
\text { (either assigned or Mean to Target values). }\end{array}$ \\
\hline (Assigned or Mean) & $\begin{array}{l}\text { If Beckman Coulter or manually-entered targets are used, then } \\
\text { the label below this heading reads "Assigned:" If means are } \\
\text { used, then the label below the heading reads "Means." If the } \\
\text { parameter's target is not applicable, N/A displays in this field. }\end{array}$ \\
\hline Limit & $\begin{array}{l}\text { The traditional expected limit of the parameter in use in your lab } \\
\text { at the time of the control analysis. }\end{array}$ \\
\hline (Manual, 2SD, or Lab) & $\begin{array}{l}\text { If assigned limits are used, then the label below this heading } \\
\text { reads "Manual." If SDs or Lab Limits are used, then the label } \\
\text { below this heading reads " } 2 \text { SD" or "Lab," respectively. }\end{array}$ \\
\hline Date/Time & The date and time of the control analysis. \\
\hline Exclude & $\begin{array}{l}\text { Allows you to exclude the results of that run from the control } \\
\text { statistics calculations. }\end{array}$ \\
\hline Reviewed By & $\begin{array}{l}\text { Displays one of the following: } \\
\text { - IF the run has not yet been reviewed: A Click to Review link } \\
\text { that allows you to review the run. } \\
\text { - If the run has been reviewed: The reviewer's username and } \\
\text { the review date and time. }\end{array}$ \\
\hline Presentation & $\begin{array}{l}\text { Displays the method of presentation for each run in the control } \\
\text { file. } \\
\text { - } \mathrm{C}=\text { Cassette Presentation } \\
\text { - } \mathrm{S}=\text { Single-tube Presentation }\end{array}$ \\
\hline Comment & $\begin{array}{l}\text { A comment icon } \Xi \text { displays in this column if any comments } \\
\text { have been added. }\end{array}$ \\
\hline Ref. RBC & $\begin{array}{l}\text { If the control type is a RETIC only, a REF, RBC heading and } \\
\text { numeric value display on the left side of the screen under the } \\
\text { Summary Data columns. If the control is RETIC only and the } \\
\text { Reference RBC Target and Limit are not sec, No Value will } \\
\text { display in the Ref. RBC field. }\end{array}$ \\
\hline
\end{tabular}

\subsubsection{Selecting a Control}

- From the Quality Control (Data View) screen or the Quality Control (Graph View) screen, select the Select Control button on the Local Navigation Bar to display the QC Select Controls dialog box. 


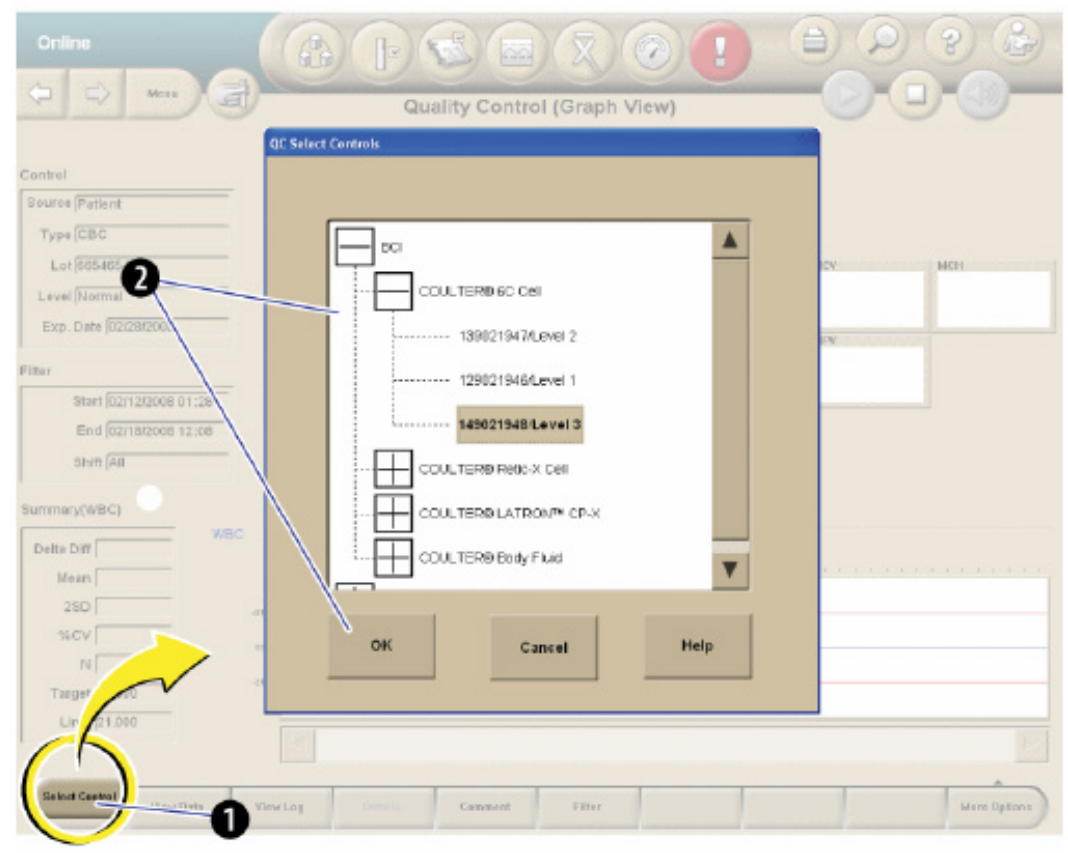

- Highlight the desired control and select OK.

\subsubsection{Viewing Control Files Graphically (Menu $>\mathrm{QA}>\mathrm{QC}>$ View Graph)}

From the Quality Control - Data View screen, select the View Graph button OR Select a parameter (row) and double tap the Thumbnail Levey-Jennings graph.

The Quality Control (Graph View) screen displays the following:

- Up to 31 thumbnail Levey-Jennings graphs, one graph per parameter.

- An expanded Levey-Jennings graph for the selected parameter data.

- Summary data for the selected parameter. 


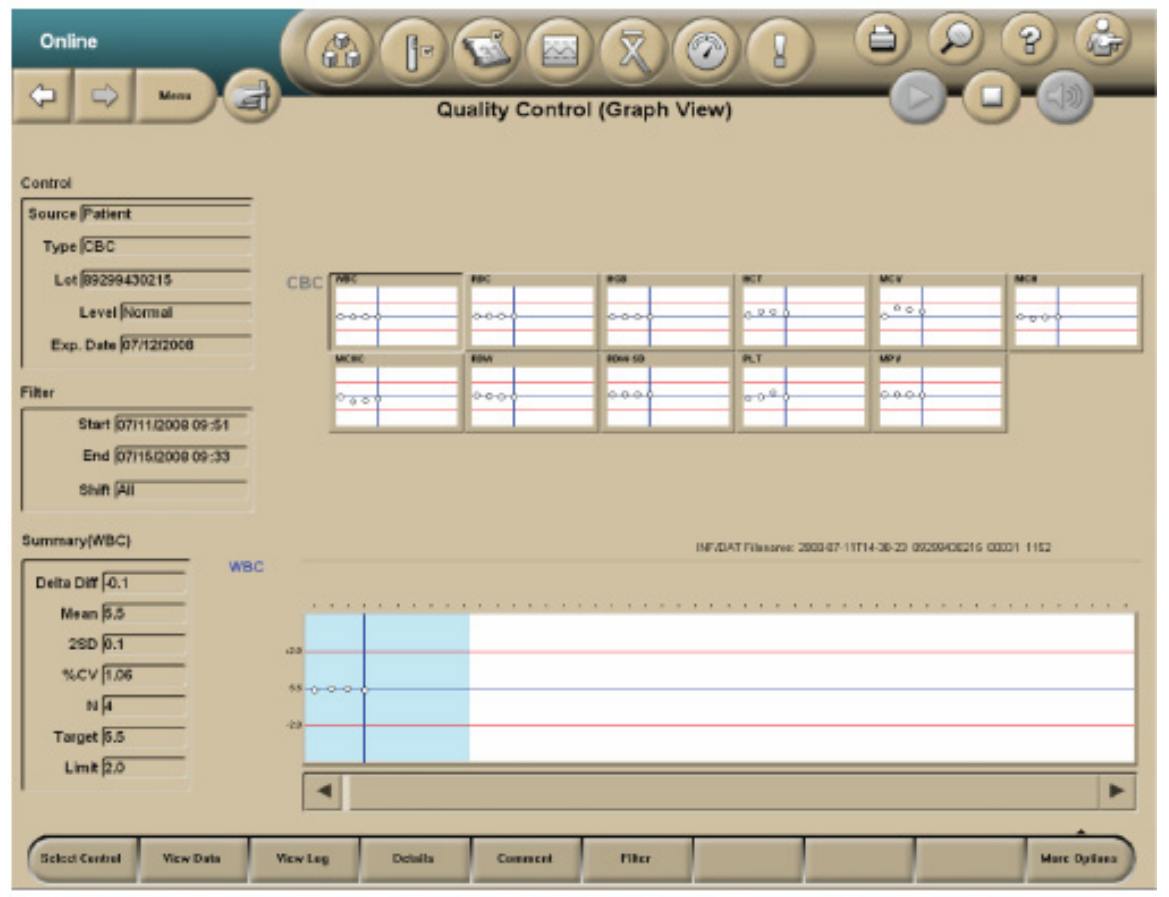

\subsubsection{Using and Interpreting the Levey-Jennings Graphs}

1. The top half of the Quality Control (Graph View) screen displays all parameters associated with controls.

- $\quad$ Levey-Jennings graphs display the latest run points for all parameters.

- $\quad$ Each graph displays up to 10 points and these points change to reflect the scrolling of the expanded graph.

- The points shown in the blue shaded window of the expanded graph reflect those shown in the thumbnail graphs.

2. The point cursor on the thumbnail graphs is synchronized with the selected run within the data grid and the point cursor in the expanded graph.

3. If you select a thumbnail graph that graph will be displayed in the expanded graph section on the lower half of the screen.

4. Any thumbnail graph's border displays red if it contains a QC Out point.

a. Once all runs in a graph are reviewed, its border will return to normal color, but the QC Out point will remain red in the graph. 
b. If the results violate the extended QC Single Measurement Error, the point will be yellow.

5. The expanded Levey-Jennings graph at the bottom of the Quality Control (Graph View) screen displays all of the results for a selected parameter in the control file.

a. The blue-shaded window on the expanded graph, which contains up to 10 points and the point cursor, determines the points and cursor displayed in the thumbnail graphs.

6. The point cursor is a blue vertical line on the graph that reflects the date selected in the Data View.

a. The cursor can be moved left or right by either using the scroll bar or by selecting either the data points on the graph or the control run analysis Date/Times in the Data View table.

b. If the cursor moves to a point outside the window, the window shifts so that the new point displays in the window as the furthest right or left point.

\subsubsection{Add, Modify, or Delete Comments to QC Results}

- From the Quality Control (Data View) or the Quality Control (Graph View) screen, select the Comment button to display the QC Comment Dialog Box.

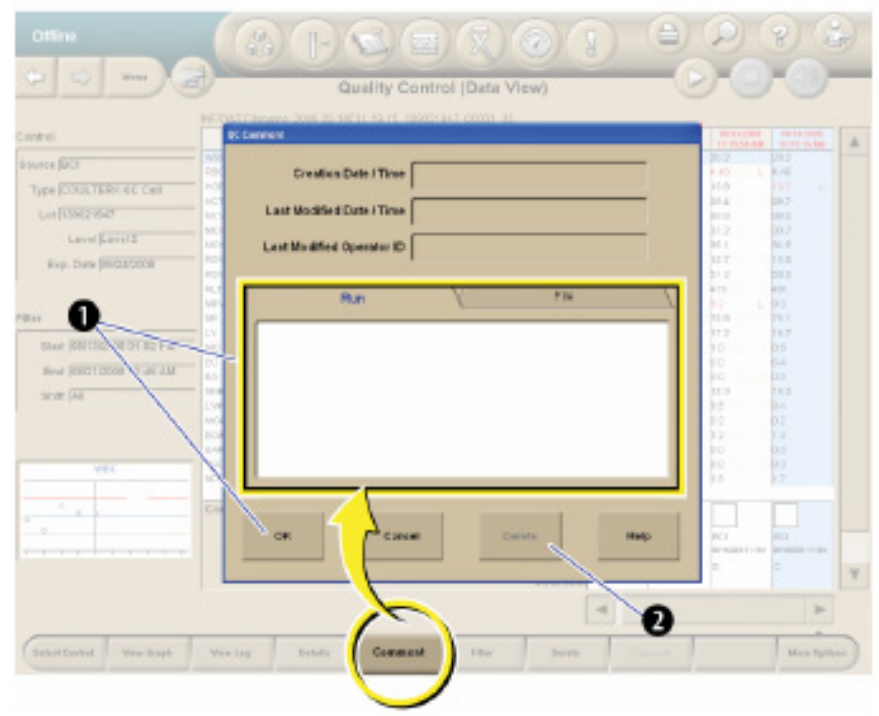

- The creation date/time, modified date/time, and last modified Operator ID will not be populated until the entry of the comment has been completed.

- To add or modify a comment on a control run, select the Run tab, type in the text box and select $\mathrm{OK}$. 
- To add or modify a comment on a control file, select the File tab, type in the text box and select $\mathrm{OK}$.

- To delete a comment, select the Delete button.

\subsubsection{View Control Files Under Error Conditions}

- Under Error conditions the Quality Control Status icon at the top of the screen is red.

- If more than one lot triggered an error condition, the Select Control File to Review dialog box displays.

- All lots that are OUT are displayed on the dialog box.

- To review a specific log, select the lot and select the OK button.

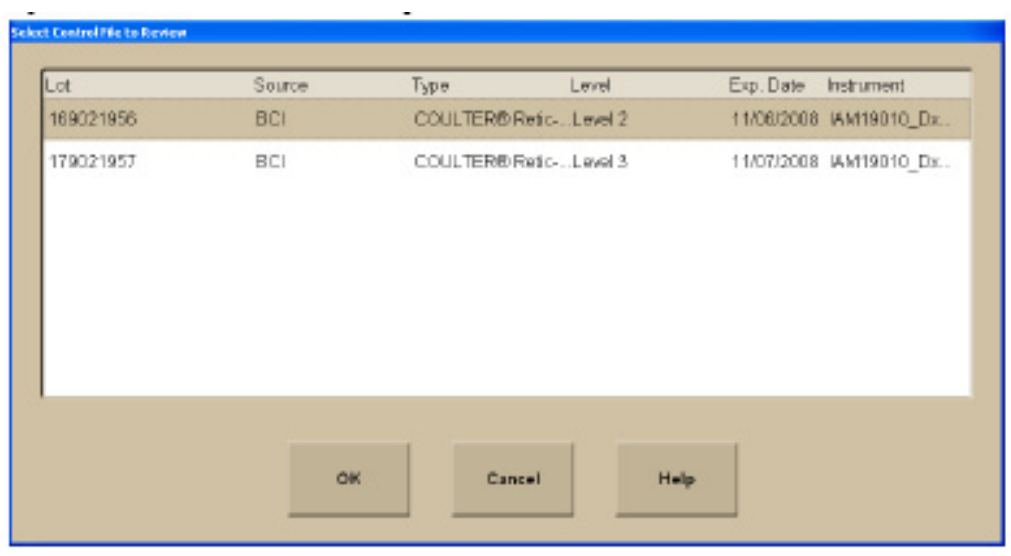

\subsection{Sample Analysis}

\subsubsection{Status}

- The SPM must be online to run samples.

- You can view the status of the SPM in the Status Mode area at the upper left hand corner of any screen.

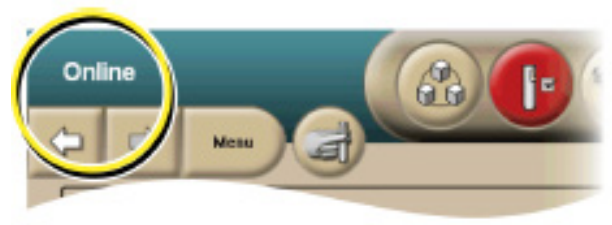


- Ensure the SPM is set up for the appropriate test for your workflow.

- Ensure your specimens have been collected and stored properly.

- Load the specimens into the cassettes. Narrow tubes with small internal diameters will require manual premixing prior to analysis to ensure proper cell and plasma distribution and to avoid possible erroneous results. Premix these tubes before placing them in the cassette and then analyze the cassette by placing it in the Stat position of the Input Buffer.

- Place the cassettes into the input buffer to the right of the SPM. The SPM automatically begins cycling the cassettes.

- After the SPM cycles the samples, review the sample results at the System Manager. To avoid serious injury, do not place your hand through the cassette presentation opening on the SPM.

\subsubsection{Single-Tube Presentation}

- Ensure your specimens have been collected and stored properly.

- Select the Single-Tube Presentation icon

at the top of any screen to display the Single-Tube Presentation dialog box.

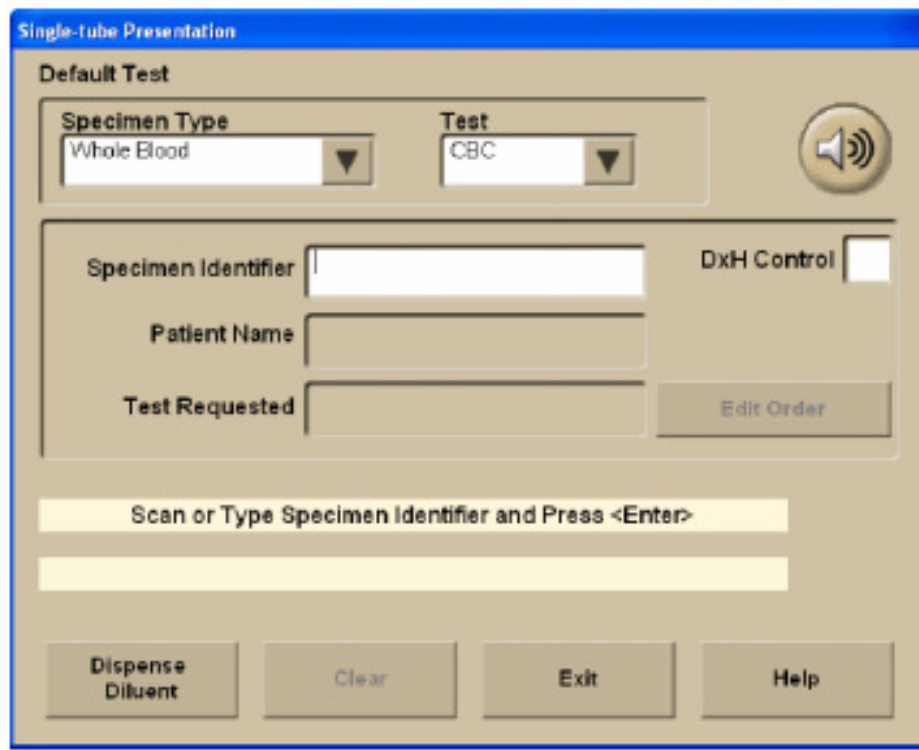


- Place the specimen on the barcode reader platform of the Single-Tube Presentation Station with the barcode facing the SPM to allow the Single-Tube Presentation BarCode Reader to scan the specimen label. The barcode may also be scanned with the handheld scanner or the Specimen Accession Number may be typed into the Specimen Identifier field.

- Verify the Specimen Accession number and test request. Acknowledging the ID that displays on the System Manager screen indicates that you accept the barcode label read or manual entry.

- Thoroughly mix the specimen.

- Place the specimen into the correct Single-tube position.

- Do not place a closed tube or a $16 \mathrm{~mm}$ diameter tube in the right position of the Singletube Presentation Station. Doing so could result in an incomplete aspiration and an erroneous result.

\subsubsection{Handling Alarms}

- An audible or visible alarm on the DxH 800 System should be addressed by reviewing the Even Logs on the History Log screen.

\subsection{Data Review}

\subsubsection{Worklist Screen}

The worklist screen manages test orders and results within the database. The worklist screen allows you to:

- Use predefined filters for display and monitoring of patient test orders and results.

- Specify sort/filter criteria for display and monitoring of patient test orders and results.

- Add, delete, and modify patient test orders.

- Print, transmit, and export patient result.

- Clear notification for specimens that were not processed. 


\subsubsection{Accessing the Worklist Screen}

- Select the Worklist Icon $\sqrt{ }$ from the top of any screen OR

- $\quad$ Select Menu $>$ Worklist.

\subsubsection{Worklist Screen Layout}

- Each tab displays a particular filter view of the database.

- The Review, Released, and Custom tabs organize information according to the currently selected filter.

- Only one tab can be viewed at a time.

The five tabs on the Worklist screen are:

1. Pending Tab.

- $\quad$ The pending tab displays all patient test orders with a pending or partially complete result status.

2. Not Processed Tab.

- The Not Processed tab displays exceptions for specimens that have been skipped.

- You need to address the problem and reload the skipped specimens for processing. If exceptions are posted to the Not Processed tab, the Worklist alert

status icon is red.

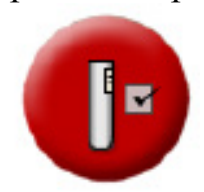

- $\quad$ The tab (view) automatically displays when you click the red Worklist icon.

- $\quad$ Specimens skipped because of a No Read or No Match are posted to the Not Processed Tab.

- $\quad$ A corresponding Event is posted in the Event Log for each. 


\section{Review Tab.}

- $\quad$ The Worklist-Review tab displays specimens that have been held (not released) and requires attention.

- $\quad$ Use the scroll bar to view all the components and data on this screen.

- $\quad$ The filter at the top right allows you to search by the following categories; all held, held with exception status, and held with slide review action.

- $\quad$ To release a result, you must be at the Patient Results screen.

4. Released Tab.

- $\quad$ The Worklist-Released tab displays the released results according to the filter that you select.

- $\quad$ The filter name drop-down list at the top right of the Released Tab allows you to filter by selecting All or All (Last 30 days).

5. Custom Tab.

- $\quad$ The Worklist-Custom tab allows one to select from predefined or user-defined filters.

- $\quad$ The following filter options may be chosen from the Filter Name drop-down list; Chartable Report Not Printed, Lab Report Not Printed, Not Transmitted, Rejected, Removed, Studies, and site specific Custom filters.

\subsubsection{Parameters For Each Tab}

- Disabled parameters (via Setup $>$ System $>$ Analysis) are disabled (grayed out) on any tab.

- Disabled parameters (via Setup $>$ Reporting $>$ Tests) are not displayed on any tab.

- For unreleased results with multiple runs, the last run (most recent) displays.

- Rejected results display with \#\#\#\#\# signs.

- Unreleased values display in brackets.

- Unordered but received parameters display in a grayed out color.

- A "lock" icon displays in the first column for any result that is saved, meaning it cannot be removed, pruned, or deleted. 
- Data that does not match the filter criteria is not displayed.

- If a row no longer matches the filter criteria (for example, Pending Tab is selected and all tests are now completed), all fields display in a disabled color.

\subsubsection{Refresh Icon}

The green Refresh icon at the top of the screen indicates that you need to select the Refresh button on the Local Navigation bar at the bottom of the screen.

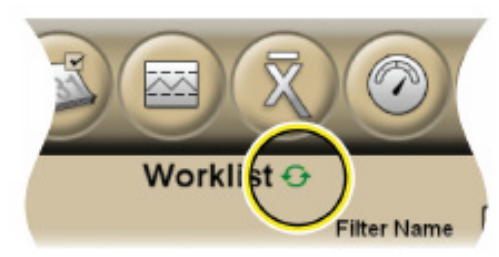

\subsection{Evaluation of Data}

\subsubsection{Flags}

Flags appear to the right of the result:

- For some parameters, flagging occurs as a result of the flagging or editing of other parameters.

- Flags in the following table are shown in order of placement on screens and printouts, with the highest priority flags at the top within each space.

- A yellow background on the screen indicates results were above or below a reference range.

- A red background on the screen indicates results exceeded an action or critical limit, or another flag was present.

- Flags appear in one of four positions to the right of the result.

- The flags are listed in order of priority within each space. It is possible to have flags in each of the four positions. 
- For some parameters, flagging occurs as a result of the flagging or editing of other parameters.

- Refer to the chart below to interpret DxH Flags.

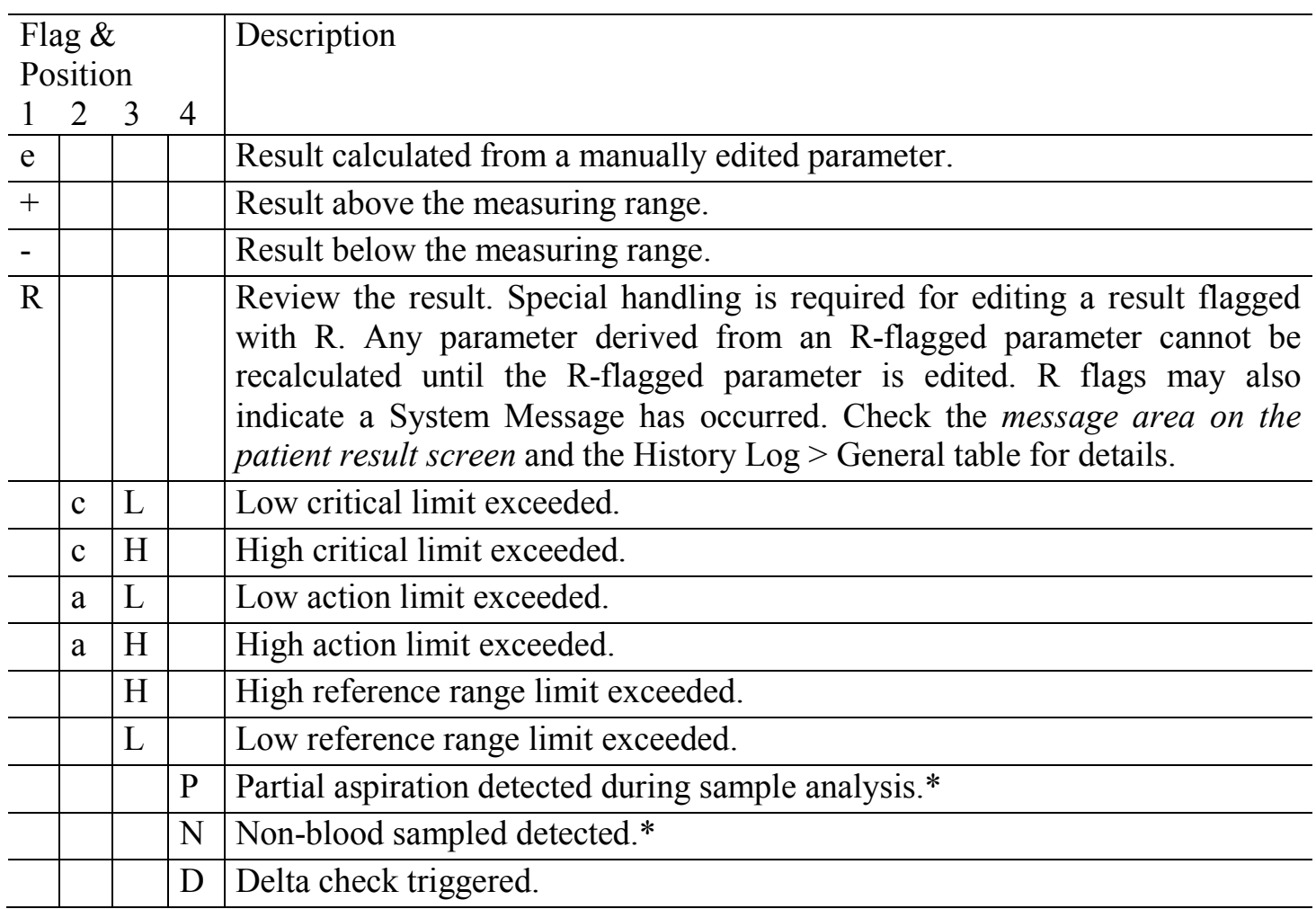

\subsubsection{Codes}

Codes appear in place of results when the system cannot generate results:

- Codes are also called nonnumeric results.

- Codes in the following table are shown in order of placement on screens and printouts, with the highest priority flags at the top within each space. 
- Refer to the chart below to interpret DxH Codes.

$====\quad$ Analysis was disabled at the configuration level of the System Manager (Menu $>$ Setup $>$ System $>$ Analysis).

For example:

IF NRBC is temporarily disabled, any results with an NRBC enumeration in the panel will display $====$ in place of the NRBC vlaues. The $====$ persists in the database after the analysis has been re-enabled.*

$\operatorname{xxxxx}$

Although available on a panel, this parameter was not enabled as a Test (Menu $>$ Setup $>$ Reporting $>$ Tests) at the time of analysis; however, after this analysis, the parameter was enabled as a Test.

For example:

Sample $A$ was analyzed for a CR panel, but the IRF and MRV were not enabled as Tests.

Later, IRF and MRV were enabled as Tests; therefore, the system will not display the IRF and MRV parameters for CR panel results, but, for Sample $A$, xxxxx displays in place of a value for those parameters.

:::: $\quad$ Flow cell clog was detected.*

Total voteout occurred. No average histogram will appear for the affected parameter.

Incomplete computation.

May occur in place of calculated parameters because a voteout or overranged occurred for a primary parameter used in the calculation. Occurs when the instrument cover is opened.*

$++++\quad$ Rresult exceeds the operating range.

???? Result is outside of the range of values that can be formatted for display.

\#\# \# \# $\quad$ Results were rejected. 


\subsubsection{Messages}

Messages can be results-related or demographic- or status-related:

- Results-Related Messages:

- Suspect messages (appear in red) are generated by internal algorithms to convey that a clinical condition may exist with a specimen based on an abnormal cell population or distribution.

-- $\quad$ Refer to the Unicel DxH 800 Help Manual on board the analyzer for a detailed list of all Suspect messages.

- $\quad$ System messages (appear in green) indicate an event occurrence that may affect the operation of a system, requires operator notification, or entry into a History Log.

-- $\quad$ The majority of System messages are accompanied by an R (review) flag. Some are accompanied by $\mathrm{N}$ or P flags.

-- $\quad$ Refer to the Unicel DxH 800 Help Manual on board the analyzer for a detailed list of all System messages.

- $\quad$ Definitive messages (appear in blue) appear for results based on exceed limits configured as part of an individual flagging set.

-- $\quad$ Definitive messages can be created by copying reference ranges, or by manual entry of your own message definition.

-- $\quad$ Refer to Definitive Messages in the Setup chapter of the on board Unicel DxH System Help Manual.

- Demographic/Status-related Messages.

- $\quad$ Exception Messages.

-- If there are any exceptions for a specimen, a comments indicator displays in the System Status Message area below the patient demographics.

-- $\quad$ Select the Additional Data button to view the Exceptions.

- System Status Messages indicate that the instrument was operating in some nonstandard state when a specimen was analyzed.

- $\quad$ These states are usually the result of some user action. 
- They do not indicate that any problem was seen when the specimen was analyzed; instead, they indicate that the system was being operated in a manner in which some problems might not be detected.

- Lab Action Messages are triggered by Decision Rules programmed in the analyzer.

These messages are listed in the Lab Actions area of the Patient Results screen.

\subsubsection{All Specimens}

- Misleading results can occur if the specimen is not properly collected, stored or transported. Beckman Coulter, Inc. recommends that you follow CLSI or equivalent procedures to ensure proper specimen collection, storage and transport. Always follow manufacturer's recommendations when using micro-collection devices for capillary specimen collection.

- Misleading results can occur if specimens contain clots. Always use good laboratory practices for inspecting specimens for clots and verifying results.

- Misleading results can occur if the specimen is not properly mixed. Always use good laboratory practices to ensure specimens are appropriately mixed. Do not bypass or circumvent the automated mixing process used on the $\mathrm{DxH} 800$.

- When running a test panel, with NRBC analysis enabled, the information from the $\mathrm{NRBC}$ analysis is used to supplement interference detection, flagging and correction.

\subsubsection{Capillary Specimen Collection}

\section{- WBC and TNC}

- NRBCs, giant platelets, platelet clumps, malarial parasites, precipitated elevated proteins, cryoglobulin, microlymphoblasts, very small lymphocytes, fragmented white cells, agglutinated white cells, lyse resistant red cells, unlysed particles $>35 \mathrm{fl}$ in size.

- $\quad$ Elevated WBC counts may have a carryover effect on subsequent leukopenic specimens, within the limits specified by Beckman Coulter. (See the Carryover section in the Unicel DxH Help Manual on board the analyzer).

\section{- $\quad \mathbf{R B C}$}

- Very high WBC count, high concentration of very large platelets, autoagglutination. 
- If hemolysis is occurring in vivo, the instrument RBC may be flagged as low, reflecting the true circulating cells. If however, the hemolysis is in vitro, the specimen may give falsely low RBC results. Cell counts due to in vitro hemolysis do not represent the number of circulating red blood cells.

- HGB - Severe lipemia, heparin, certain unusual RBC abnormalities that resist lysing.

- MCV - Very high WBC count, high concentration of very large platelets, autoagglutination.

- RDW - Very high WBC count, high concentration of very large platelets, autoagglutination.

- $\quad$ PLT - Giant Platelets, platelet clumps, white cell fragments, electronic noise very small red cells, red cell fragments.

- HCT - Known interferences related to RBC and MCV.

- $\mathbf{M C H}$ - Known interferences related to HGB and RBC.

- MCHC - Known interferences related to HGB, RBC, and MCV.

- NRBC - Known interferences may be related to the following:

- $\quad$ Lyse resistant red cells.

- $\quad$ Malarial parasites.

- $\quad$ Very small or multi-population lymphocytes.

- $\quad$ Precipitated elevated proteins.

\section{- Differential}

- Hypogranular granulocytes, agranular granulocytes, lyse resistant red cells, very small or multi-population lymphocytes, elevated triglycerides, precipitated elevated proteins.

- A transient basophilia may be observed in samples that have been exposed to high temperatures $\left(90^{\circ} \mathrm{F}\right.$ or $\left.32^{\circ} \mathrm{C}\right)$. The temporary basophilia should resolve after stabilization at room temperature $\left(72^{\circ} \mathrm{F}\right.$ or $\left.22^{\circ} \mathrm{C}\right)$.

- Highly viscous synovial fluids may trap cells leading to misleading or erroneous results. 
6.15 Laser Safety

- The Multi-Transducer Module contains a laser.

- A laser is a unique light source that exhibits characteristics different from conventional light sources.

- The safe use of the laser depends upon familiarity with the instrument and the properties of coherent, intense beams of light.

- The beam can cause eye damage and instrument damage. There is enough power from the laser to ignite substances placed in the beam path, even at some distance. The beam might also cause damage if contacted indirectly from reflective surfaces (specular reflection).

- The laser on the DxH 800 is covered by a protective housing.

\subsection{Calibration of the DxH 800}

(The S-CAL ${ }^{\circledR}$ calibrator kit determines the adjustment factors for the calibration of the Coulter ${ }^{\circledR}$ DxH 800 . Calibration is a procedure to standardize the instrument by determining its deviation from calibration references and to apply any necessary correction factors. Perform calibration in the closed-vial mode at ambient room temperature range $\left(16-32^{\circ} \mathrm{C}, 60-90^{\circ} \mathrm{F}\right)$, using $\mathrm{S}-\mathrm{CAL} \AA$ as an alternative to whole blood.

\subsubsection{Perform Calibration}

- At the start of each stand, before you begin analyzing samples;

- After you replace any component dealing with dilution preparation, such as the BSV primary measurement, such as an aperture; and

- If your Beckman Coulter ${ }^{\circledR}$ representative suggests that you calibrate.

\subsubsection{Verify the Calibration of Your Instrument}

- If controls show unusual trends or are outside limits; and

- When room temperature varies more than $10^{\circ} \mathrm{F}\left(5.5^{\circ} \mathrm{C}\right)$ from the room temperature during the last calibration. 


\subsubsection{Coulter Pre-Start-up Procedures}

Arrangements for a Beckman Coulter ${ }^{\circledR}$ Representative to perform the necessary Pre-Startup procedures on the DxH 800, prior to the arrival of the team to the stand, will be arranged by the chief field medical technologist in advance. These Pre-Start Up procedures for the representative will include:

- Removal of the travel brackets

- Placement of all reagent lines in the reagents: Diluent, Cell Lyse, Diff Pack, and Cleaner

- Power up the analyzer

- "Wetting" the analyzer (Full Prime of the System)

- Adjustment of the flow cell

*A placard will be available for the representative (placed on the side of the analyzer), with detailed instructions of the tasks that will be needed to be performed prior to the arrival of the team. As usual, the representative will be given the Chief Technologist's telephone number and email address, in case any questions concerning the instructions should arise.

\subsubsection{Initial DxH 800 Startup}

Perform a Daily Check after the instrument has been "Shutdown" for at least 30 minutes. Select Menu $>$ QA $>$ Daily Checks $>$ Daily Checks $>$ Select [OK].

Inventory all Coulter Reagents - marking them with receive dates. (Once reagents have been opened, mark with an open date and tech initials). *

- Log into the DxH (System Manager) SM

- Delete previously used 6C Cell Control Files - Menu $>$ QA $>$ QC $>$ More Options $>$ QC Setup $>$ Select Previous (Older) Control File (will be highlighted) $>$ Delete Control $>$ Select $[\mathrm{OK}]$.

- $\quad$ *You can only delete control files one at a time.

- Enter Reagent Lot Numbers and Expiration Dates - Menu $>$ Supplies $>$ Setup $>$ Scan the Reagent Barcodes $>$ Select [OK]. 
- Enter 6C Cell Control Lot Numbers and Expiration Dates - Menu $>$ QA $>$ QC $>$ More Options $>$ QC Setup $>$ New Control from Barcode $>$ Scan the 6 C Cell Insert Barcode $>$ Select ALL \& Auto Transmit $>$ Select [OK].

- Enter Latron Control Lot Numbers and Expiration Dates - Menu $>$ QA $>$ QC $>$ More Options $>$ QC Setup $>$ New Control from Barcode $>$ Scan the Latron Insert Barcode $>$ Select ALL \& Auto Transmit $>$ Select [OK].

- Open Coulter Icon in ISIS.

- Enter 6C Cell and Latron Control Lot Information into ISIS - Right click on the Lab Coulter Icon in the Task Bar > Select Expected Values $>$ Scan the Insert Barcode $>$ Tab $>$ Verify Results $>$ Select $[\mathrm{OK}]$. Perform for both $6 \mathrm{C}$ Cell and Latron Control File Inserts.

- Place the 6C Cell and Latron package inserts in the Coulter binder.

*NOTE: If the barcode on a reagent doesn't scan properly when trying to add reagents to the DxH 800, manually change the third or fourth character from the end of the first (long) barcode. Then proceed as normal.

Storage, handling and stability: Sealed vials are stable through the expiration date when stored at $2-8^{\circ}\left(35-46^{\circ} \mathrm{F}\right)$. Opened and/or pooled vials are stable for 1 hour.

\subsubsection{Calibration, Repeatability, and Carryover Check}

*Draw three 4-mL EDTA tubes from a person with a normal CBC*

\section{Repeatability}

Supply List: (2) 4-mL EDTA tubes filled with normal blood.

*Ensure you have enough normal whole blood from a single donor for a minimum of 10 cycles, and delete any data on the Repeatability screen prior to starting a new study.

- Use $2-4 \mathrm{~mL}$ EDTA tubes of well mixed normal specimen (Aspiration probe damage can occur if you pierce a specimen tube more than five times). 
- $\quad$ Select Menu >QA > Repeatability > Repeatability Setup:

- $\quad$ Presentation: Cassette

- $\quad$ Test Panel: CD

- $\quad$ Number of Aspirations: 5

- $\quad$ Select $[\mathrm{OK}]$ and follow the screen prompts.

- Place the two tubes into consecutive positions in a cassette and place the cassette in the input buffer and Select [OK] on the DxH dialog box to start the cycle.

- When the cycle has completed, review the results on the Repeatability screen. Use the scroll bar to review all the results. Exclude the first result from the run.

- Verify that the Coefficient of Variation (CV) does not exceed the established Repeatability Limits:

- $\quad$ WBC $2.5 \%$

- $\quad$ RBC $2.0 \%$

- $\quad$ HGB $1.5 \%$

- $\quad$ MCV $2.0 \%$

- $\quad$ PLT $5.0 \%$

- $\quad$ MPV 3.0\%

- Check the low to high difference (bottom line) for the Diff parameters with these Limits:

$-\quad \mathrm{LY} \% \leq 4.8$

$-\quad \mathrm{MO} \% \leq 3.2$

$-\quad \mathrm{NE} \% \leq 4.8$

$-\quad \mathrm{EO} \% \leq 1.6$

$-\quad \mathrm{BA} \% \leq 1.6$

If any of the results are outside the limits, call Beckman Coulter ${ }^{\circledR}$ Customer Service.

- If all the results are within these parameters: Print $>$ Select [Finish], and proceed with Carryover Check. 
*Use the \%CV line for CBC parameters only and the Diff line for the differential parameters only. Please see Chapter 1: System Overview - Performance Specifications and Characteristics section located in the Help Menu for additional information regarding Repeatability limits for acceptability.

\subsubsection{Carryover}

Supply List: (1) 4-mL EDTA tube filled with normal blood \& (3) empty 4-mL EDTA tubes.

*Before you can start or restart the Carryover process, the SPM must be offline.

- $\quad$ Fill three empty 4-mL Vacutainer ${ }^{\circledR}$ EDTA tubes with diluent. Select Single-Tube Presentation Icon $>$ Dispense Diluent $>$ Select $[$ OK]. Place one empty capped EDTA tube in the purple tube holder on the left-hand side of the cradle. $1 \mathrm{~mL}$ of diluent is dispensed during this process. Once completed "Do you want to dispense more diluent in the same tube?" box will appear > Select [No]. Remove the filled EDTA tube, and repeat the above steps two more times to fill the 2 remaining empty EDTA tubes with diluent. Select [Exit] > "Are you sure you want to exit Single-tube Presentation?" > Select [Yes].

- $\quad$ Select Menu $>$ QA $>$ Carryover $>$ Carryover Setup:

- $\quad$ Presentation: Cassette

- $\quad$ Test Panel: CD

- $\quad$ Select $[\mathrm{OK}]$ and follow the screen prompts.

- Select $[\mathbf{O K}]$ to start the Carryover procedure

- Place a cassette in the input buffer with one blood tube followed by three diluent tubes consecutively and Select $[\mathbf{O K}]$ to start the Carryover procedure.

- When Carryover is complete, review the results on the Carryover screen. If any of the Carryover results "FAIL," repeat the procedure.

If the Carryover results "FAIL" after the second attempt, call Beckman Coulter ${ }^{\circledR}$ Customer Service.

- If the status of all parameters is "PASS:" Print > Select [Finish], and proceed with Calibration. 
*When performing a Carryover procedure, the calculated \% carryover and/or background for each parameter is compared to the Carryover and Background limits for acceptability. Please see Chapter 1: System Overview - Performance Specifications and Characteristics section located in the Help Menu for additional information regarding carryover and background limits for acceptability.

Place all printed charts and control inserts into the DxH Logbook, and have all results verified by a Beckman Coulter ${ }^{\circledR}$ Representative via a VIP Service Call.

\subsubsection{Calibration}

Supply List: (1) S-CAL® Calibrator.

*Before you can start or restart the Calibration procedure, the SPM must be offline.

The Calibration Procedure:

- Allow S-CAL ${ }^{\circledR}$ to warm to ambient (room) temperature for approximately 15 minutes.

- $\quad$ Select Menu $>$ QA $>$ CBC Calibration $>$ Calibration Setup. You will be prompted by a dialog box which states: Existing Data will be deleted. Select [OK] to continue.

- $\quad$ Number of Aspirations: 12

- $\quad$ Presentation: Cassette

- $\quad$ Calibrator Type: BCI

- $\quad$ Select Upload $>$ Scan the S-Cal Insert Barcode > Verify All Values > Select [OK].

- Mix the S-CAL ${ }^{\circledR}$ by hand by rolling the tube slowly between the palms of the hands eight times in an upright position. Invert the tube and slowly roll it again eight times. Gently invert the tube eight times. Repeat these actions again. When all the cells are uniformly distributed, place the Calibrator in the cassette.

- Place the cassette in the input buffer and Select [OK] to start Calibration Procedure.

- Exclude the first and second run by checking the boxes beside those runs. (Note: first run is located at the bottom of list of runs).

- Review the calibration results. 
- If all the results are within the specified parameters: Print $>$ Select [Finish].

- If results recommend calibration (the boxes at the bottom of the calibration screen are check marked), Select [OK].

*The background color of the Factor \%Diff, \%CV, and Difference cells change color when the presented value is out of the normal range as follows:

- Yellow for Difference indicates that the value is out to range, which means that calibration is recommended.

- Red only applies to the \%CV and indicates that the statistical value is NOT within range and the system does NOT allow calibration.

- When all results are acceptable, the Edit System Recommendations button at the bottom right hand corner of the screen is enabled. This button allows the modification of the calibration recommended by the system by selecting or deselecting checkboxes.

- Please see Chapter 1: System Overview - Performance Specifications and Characteristics section located in the Help Menu for additional information regarding Calibration limits for acceptability.

- Print out a copy of the calibration, initial and date.

- Verify the calibration by running controls. Menu $>$ QA $>$ QC $>$ Select Control $>$ Select the Control that you want to view $>$ Select $[$ OK] $>$ Select "Reviewed By" to review controls $>$ Print.

6.17 Assay Procedure

6.17.1 Run Daily Checks at the Beginning of the First Session Each Operational Day

- Turn the DxH 800 to online and operate with all panels closed.

- Change tech initials.

- To begin Daily Checks, select the Calendar Icon, select Daily Checks at the bottom of screen and press $\mathrm{OK}$.

- After Daily Checks is complete, evaluate the display. Results automatically print. Results outside limits turn red and must be reviewed in order to proceed with further analysis. File in the logbook. 


\subsubsection{Run Controls}

Run Coulter ${ }^{\circledR}$ Latron CP-X control, and all three levels, of Coulter ${ }^{\circledR} 6 \mathrm{C} \circledast$ cell control daily at the beginning of the first session. Run all three levels of Coulter ${ }^{\circledR} 6 \mathrm{C} \circledast$ cell control at the beginning of the second session.

\subsubsection{Run Sample Persons}

- Place tube in the cassette and place in the SPM.
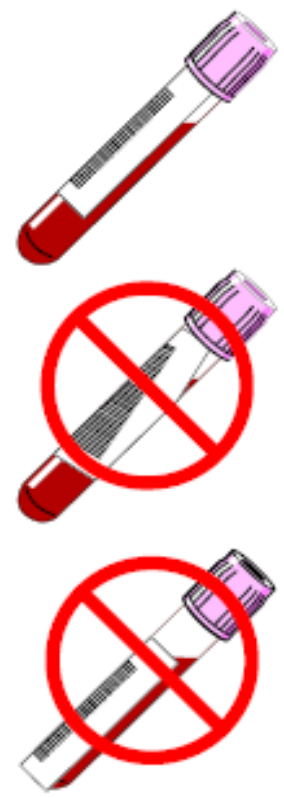

- Run all samples in duplicate.

- Run samples in the Primary mode.

Check to make sure the printer is working properly. Operate the analyzer with the doors and panels closed. Monitor reagent levels. 


\subsubsection{Review Data and Transmit}

- Review the data.

- Set host computer to transmit. Data from the Coulter ${ }^{\circledR}$ DxH 800 are automatically transmitted to the ISIS system.

- Use the Worklist screen to sort, retrieve, transmit, and archive data to CD . Transmit data from the Coulter ${ }^{\circledR}$ DxH 800 to ISIS as necessary. Save all data at the end of each stand to $\mathrm{CD}$

\subsubsection{Daily Shutdown}

1. Shut down the instrument for at least 30 minutes every 24 hours.

2. Perform Daily Checks before running samples or controls.

\subsubsection{Prolonged Shutdown}

1. If the instrument is going to be idle for $>72$ hours, call Coulter Support for a service call.

\subsubsection{Beginning and End of Stand Operations}

1. Beginning of stand:

a. Arrange for a Coulter Service Representative to remove all travel brackets and re-wet the system.

b. Follow procedure for Calibration, Repeatability, and Carryover Check.

2. End of stand

a. Call Coulter Support to have an engineer install the travel brackets and perform the prolonged shutdown procedure for the DxH800.+ 


\begin{tabular}{lll}
\hline Parameter & Linearity & Limits: The greater of \\
\hline $\mathrm{WBC} \times 10^{3}$ cells $/ \mu \mathrm{L}$ & 0 to 99.9 & 0.2 or $3.0 \%$ \\
$\mathrm{RBC} \times 10^{6}$ cells $/ \mu \mathrm{L}$ & 0 to 7.00 & 0.05 or $2.0 \%$ \\
$\mathrm{Hgb} \mathrm{g} / \mathrm{dL}$ & 0 to 25.0 & 0.2 or $3.0 \%$ \\
$\mathrm{MCV} \mathrm{fL}$ & 50.0 to 150.0 & 2.0 or $3.0 \%$ \\
$\mathrm{Plt} \times 10^{3}$ cells $/ \mu \mathrm{L}$ & 0 to 999 & 10.0 or $7.0 \%$ \\
$\mathrm{MPV} \mathrm{fL}$ & 5.0 to 20.0 & $5.0 \%$ \\
\hline
\end{tabular}

\subsection{ISIS}

\subsubsection{Record 6C Cell and Latron ${ }^{\circledR}$ Lot Numbers in ISIS}

The ISIS maintains the capability to download all 6C cell and Latron ${ }^{\circledR}$ control data. The data are used to monitor quality control results. Upload or enter data for each lot number each time a new lot number is put into use. The ISIS uses the same $6 \mathrm{C}$ cell control package insert and Latron package insert as the DxH800.

- $\quad$ Access the Coulter® QC Lot Info module.

- Right click on Coulter icon in the bottom right hand corner of window.

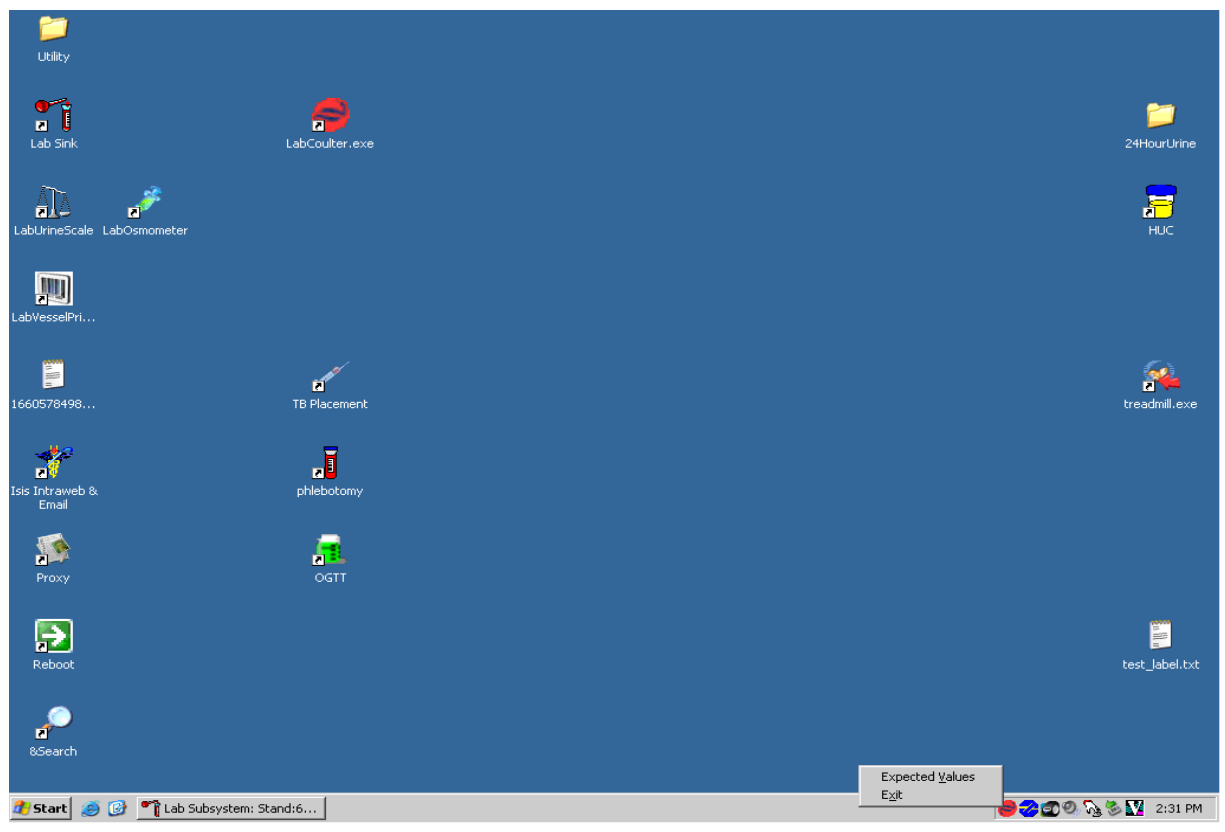


- Click on Expected Values to bring up the Coulter QC Input Screen for 6C Cell Controls and Latron QC.

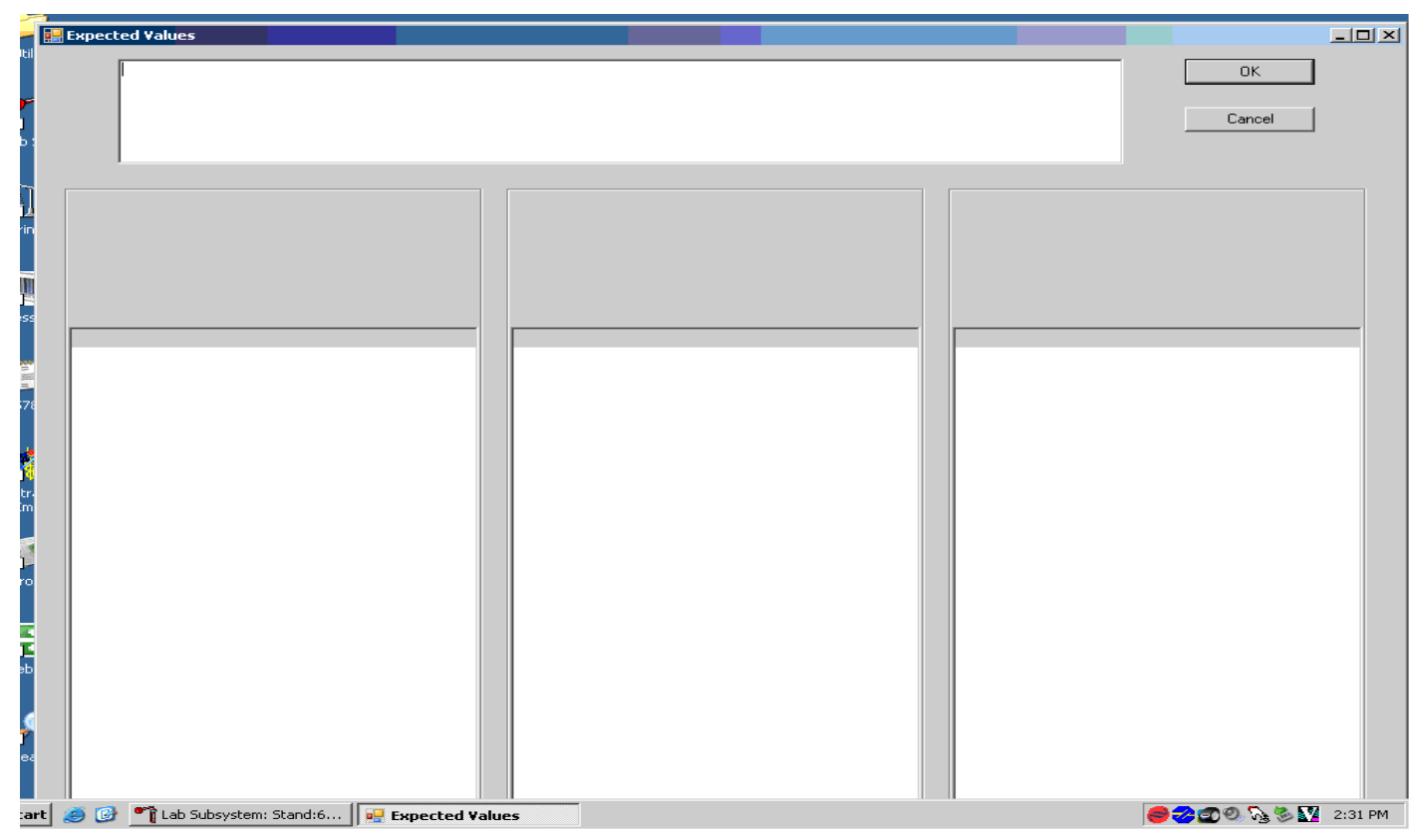

- Make sure cursor is in the top window, scan the barcode on the Coulter Package insert, and wait until data fill in the top window.

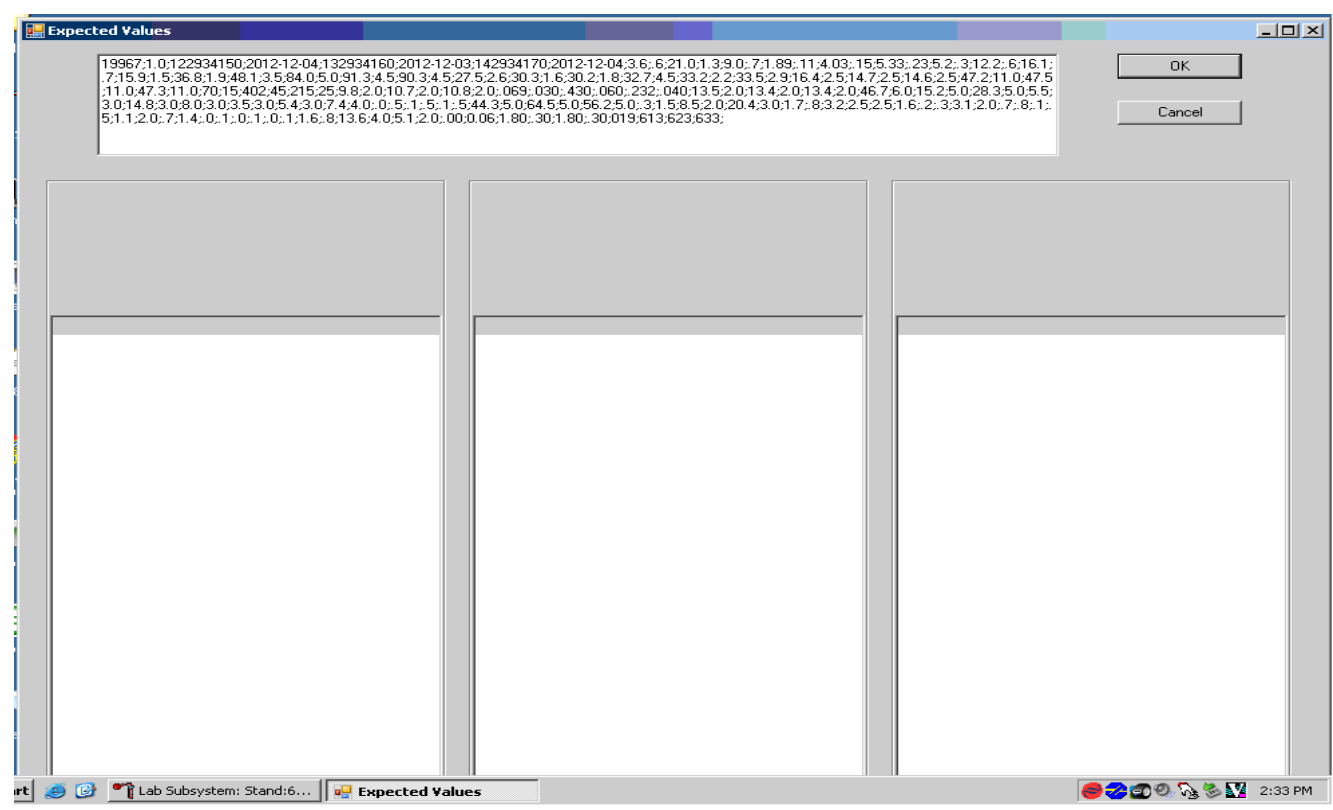

- Tab off to populate the bottom windows for each level of 6C Cell Control. 


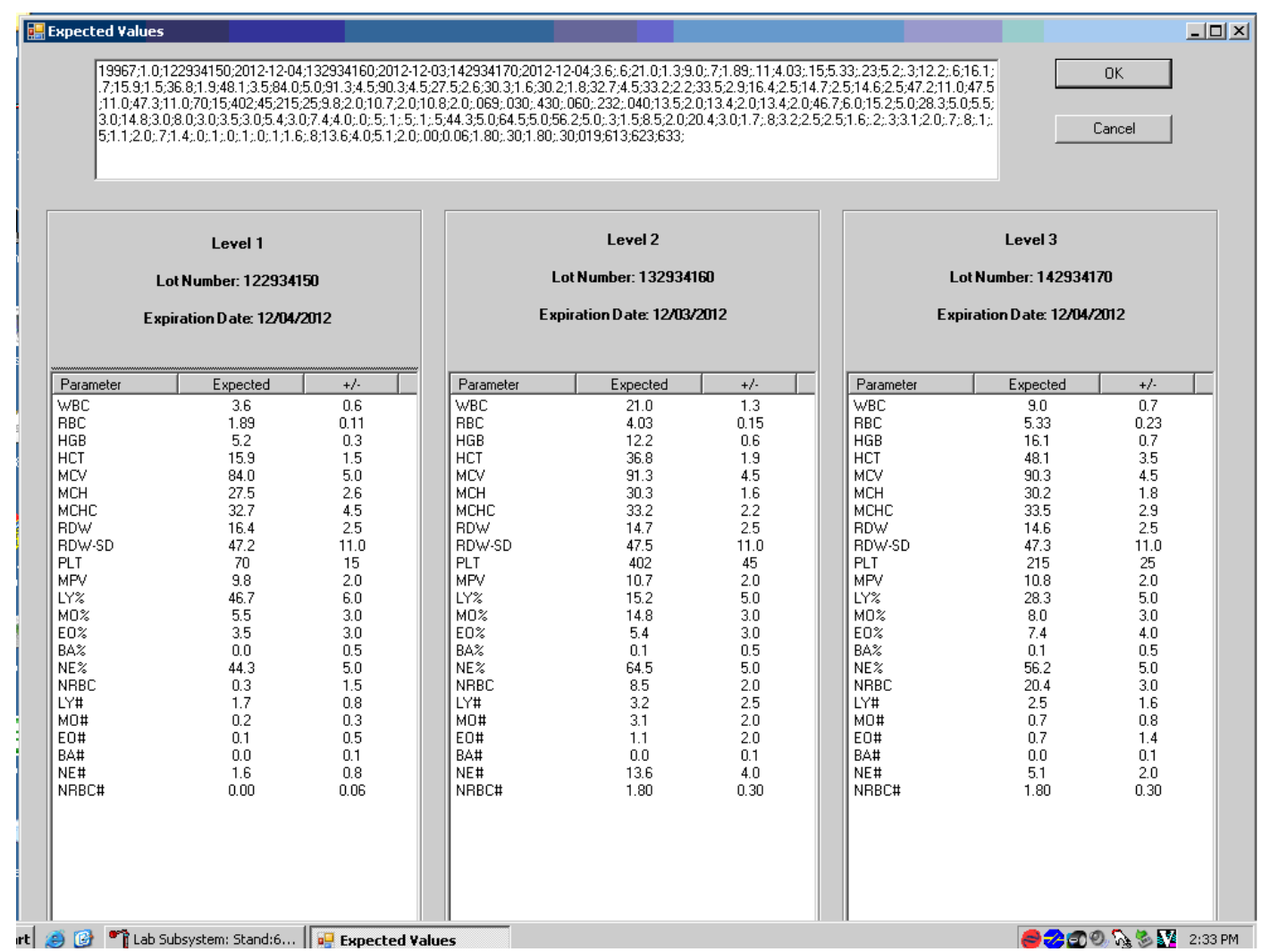

- Screenshot depicting Latron QC expected values for Diff, Retic, and nRBC. 


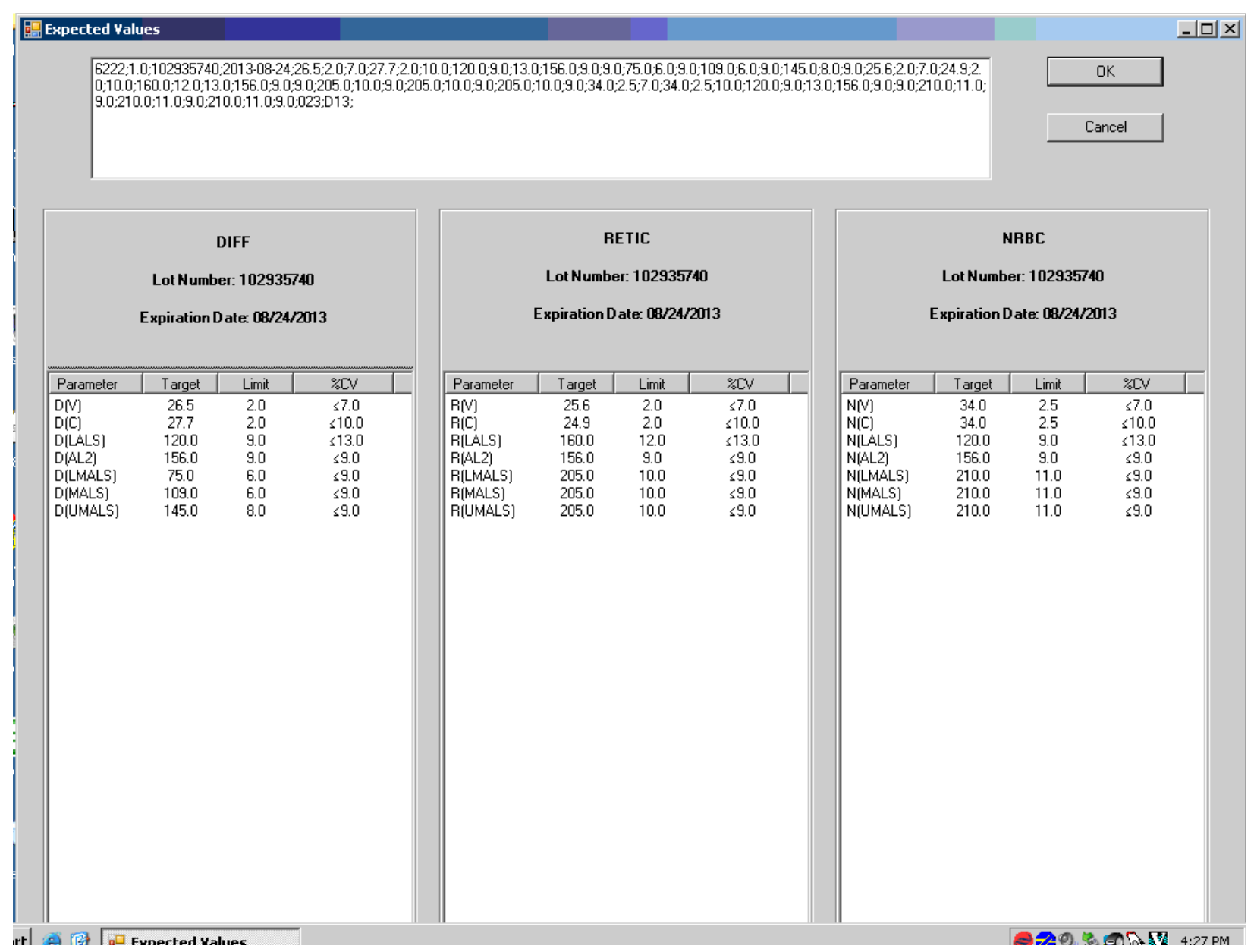

- Check the expected values against the package insert and click OK to save and exit.

\subsubsection{Interlaboratory Quality Assurance Program (IQAP)}

All instruments participate in Coulter's IQAP program. The IQAP program includes saving results of $6 \mathrm{C}$ cell controls, transferring them to a $\mathrm{CD}$, and sending them to Coulter ${ }^{\circledR}$ who compares the results to other laboratories. Coulter ${ }^{\circledR}$ issues a report that contains a statistical analysis to evaluate performance. Perform this procedure at the end of each stand, or when a lot expires.

1. Procedure to download IQAP files to CD

- $\quad$ Slide the CD into the drive and gently close CD holder

- $\quad$ Menu $>$ QA $>$ QC $>$ More options (bottom of screen) $>$ Export 


$$
\begin{array}{ll}
- & \text { Type: CVS } \\
- & \text { Choose options } \\
- & \text { Selected Run } \\
- & \text { All runs in current filter } \\
- & \text { All runs in control file } \\
- & \text { Folder: CD Recorder }
\end{array}
$$

*Must reinsert disc after level of QC is copied. Repeat for each of the three levels of QC.

\subsubsection{Proficiency Testing}

Evaluation and participation in the College of American Pathologist (CAP) proficiencytesting program is part of the comprehensive quality control program. These survey materials are shipped three times per year and consist of $53-\mathrm{mL}$ whole blood specimens. Follow all CAP instruction in preparing the materials before performing the test. Run specimens in a manner identical to routine specimens. Fill out the CAP result form, make a copy for the logbook, and send results to CAP.

\subsubsection{Linearity for WBC, RBC, Hgb, and PIt Parameters}

Lin-X ${ }^{\mathrm{TM}}$ (PN 628029 ) - The Lin-X ${ }^{\mathrm{TM}}$ linearity controls verifies the reportable range of Coulter ${ }^{\circledR}$ hematology system parameters for WBC, RBC, Hgb and Plt.

\section{OR}

CAP Hematology Calibration Verification/Linearity Survey (LN9) - These materials are shipped twice per year and consist of 18 3-mL liquid specimens. Follow all CAP instruction in preparing and running the materials before performing the test. Fill out the CAP result form, make a copy for the logbook, and send results to CAP. 
Run either the Lin-X ${ }^{\mathrm{TM}}$ or CAP LN9 survey material:

- At installation

- At least yearly

- Whenever experiencing an altitude change of one mile or more between stands

Principle - Lin-X ${ }^{\mathrm{TM}}$ linearity controls are human blood components from which repeated measurements verify the reportable range of Coulter ${ }^{\circledR}$ hematology systems. Controls contain 12 vials, ranging from 0-12. . Lin- $\mathrm{X}^{\mathrm{TM}}$ verifies ranges for the following parameters: WBC, RBC, Hgb, and Plt.

To ensure the accuracy of linearity control ranges, Coulter ${ }^{\circledR}$ system calibrates with S-CAL ${ }^{\circledR}$ calibrator.

\subsection{Interpretation of Results and Remedial Action}

\subsubsection{Sample Person Hemoglobin and Hematocrit Review and Remedial Actions}

Review all results to make sure the hemoglobin and hematocrit are acceptable. The hematocrit should be approximately three times the hemoglobin.

\subsubsection{Sample Person Parameter Value Review and Remedial Actions}

1. Access the Hematology module or reject a clotted blood tube.

Open the Hematology module.

The Hematology module does not need to be open before running SP samples on the DxH800. Note the red icon in the lower right corner of the system tray.

$$
\text { «อ) }
$$


This is the NHANES Coulter ${ }^{\circledR}$ Monitor icon. It must be open and running at the start of each session. This icon stores all DxH800runs in the ISIS database. Open the icon by double-clicking on the Coulter ${ }^{\circledR}$ icon on the desktop. The Coulter ${ }^{\circledR}$ icon looks like this:

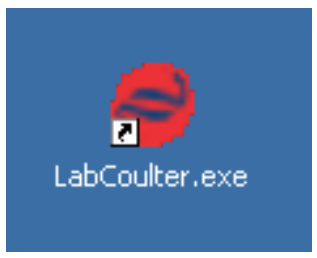

Open the Hematology module.

\begin{tabular}{|c|c|c|c|c|c|c|c|c|c|c|c|c|c|c|c|c|}
\hline \multicolumn{17}{|c|}{ ¡ Lab Subsystem } \\
\hline Ele vew ut & ptitities Quality & ty Control & Beport & ts Modules & 5 Ship & ping Window Help & & & & & & & & & & \\
\hline \multicolumn{17}{|c|}{ 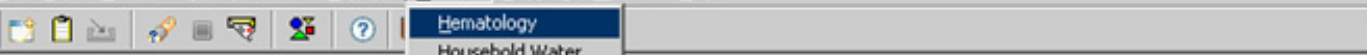 } \\
\hline \multirow{2}{*}{ SP ID } & \multirow[t]{2}{*}{ Sample ID } & \multirow[t]{2}{*}{ Status } & \multirow{2}{*}{ Type } & \multirow{2}{*}{\multicolumn{3}{|c|}{ Gencer Age Name }} & \multicolumn{7}{|c|}{ Process Status } & \multicolumn{3}{|c|}{ HPV } \\
\hline & & & & & & & uc & u & $\mathrm{P}$ & $C \theta$ & B & GIT & PC & $\mathrm{F}$ & M & OR \\
\hline 256339 & 256339 & $\mathrm{sC}$ & $P$ & M & 90 & MOORE, LANDON J & 0 & 0 & $\cdot$ & 0 & 0 & 0 & · & . & · & · \\
\hline 329669 & 329869 & $\mathrm{BE}$ & $P$ & M & 69 & HOWARD, SOPHIA S & 0 & 0 & $\cdot$ & 0 & 0 & 0 & 0 & . & . & 0 \\
\hline 416714 & 416714 & BE & $\mathrm{P}$ & $\mathrm{F}$ & 20 & BAILEY, ANTHONY J & 0 & 0 & 0 & 0 & 0 & 0 & 0 & 0 & . & 0 \\
\hline 480354 & 480354 & $\mathrm{SC}$ & $P$ & M & 3 & LEE, OWEN S & . & . & · & 0 & 0 & . & . & . & . & . \\
\hline 525757 & 525757 & $\mathrm{BE}$ & $P$ & $\mathrm{~F}$ & 70 & ADAMS, CALEB L & 0 & 0 & . & 0 & 0 & 0 & 0 & . & . & . \\
\hline 545182 & 545182 & $\mathrm{SC}$ & $P$ & $\mathrm{~F}$ & 4 & POWELL, AALIYAH H & . & . & . & 0 & 0 & . & . & . & $\cdot$ & . \\
\hline 553872 & 553872 & $\mathrm{Cl}$ & $P$ & M & 21 & WRIGHT, LAYLA B & 0 & ० & · & 0 & 0 & 0 & · & . & 0 & 0 \\
\hline 745052 & 745052 & $\mathrm{Cl}$ & $P$ & $\mathrm{~F}$ & 41 & EVANS, SAVANNAH M & 0 & 0 & o & 0 & 0 & 0 & · & 0 & . & 0 \\
\hline 767995 & 767995 & $\mathrm{BE}$ & $P$ & M & 15 & MURPHY, ISAAC N & 0 & 0 & · & 0 & 0 & 0 & . & . & 0 & 0 \\
\hline 784174 & 784174 & $\mathrm{BE}$ & $P$ & M & 50 & GONZALEZ, SIENNA N & 0 & $\circ$ & $\cdot$ & 0 & 0 & 0 & · & . & 0 & 0 \\
\hline 813366 & 813366 & $\mathrm{BE}$ & $P$ & $\mathrm{~F}$ & 19 & STEWART, SOPHIE J & 0 & 0 & 0 & 0 & 0 & 0 & · & 0 & - & 0 \\
\hline 836040 & 836040 & $\mathrm{SC}$ & $P$ & M & 19 & TORRES, LUCAS S & 0 & 0 & . & 0 & 0 & 0 & . & . & 0 & 0 \\
\hline 837622 & 837622 & $\mathrm{SC}$ & P & $\mathrm{F}$ & 1 & MARTINEZ, ANNA L & · & · & · & 0 & 0 & · & · & . & · & . \\
\hline 849425 & 849425 & $\mathrm{sc}$ & $\mathrm{P}$ & $\mathrm{F}$ & 10 & CAMPBELL, JASPER N & 0 & 0 & 0 & 0 & 0 & . & 0 & . & . & . \\
\hline 861215 & 861215 & $B E$ & P & M & 18 & SMITH, MICAHS & 0 & 0 & . & 0 & 0 & 0 & 0 & . & $\circ$ & 0 \\
\hline 919919 & 919919 & $\mathrm{Cl}$ & $P$ & $\mathrm{~F}$ & 25 & PAYNE, IMA L & 0 & 0 & 0 & 0 & 0 & 0 & 0 & 0 & . & 0 \\
\hline
\end{tabular}


To open the Hematology module, use the mouse to direct the mouse arrow to \{Modules\}, in the top menu bar, left click, drag the mouse arrow to $\{$ Hematology $\}$, and right click, or type $[\mathrm{Alt}][\mathrm{M} / \mathrm{m}]$, $[\mathrm{H} / \mathrm{h}]$.

Alternatively, open the Hematology module from the heads-up display.

\begin{tabular}{|c|c|c|c|c|c|c|c|c|c|c|c|c|c|c|c|c|}
\hline \multicolumn{17}{|c|}{ Tab Subsystem } \\
\hline File view $\underline{\underline{u}}$ & Ittilities Qualit: & ity Control & Reports & ts Module & ss Shipt & pping Window & Help & & & & & & & & & \\
\hline \multicolumn{17}{|c|}{ 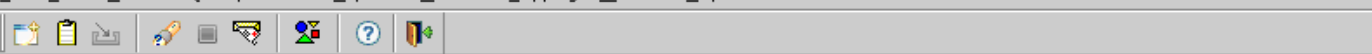 } \\
\hline \multirow[t]{2}{*}{ SP ID } & \multirow[t]{2}{*}{ Sample ID } & \multirow[t]{2}{*}{ Status } & \multirow[t]{2}{*}{ Type } & \multirow[t]{2}{*}{ Gender } & \multirow[t]{2}{*}{ Age } & \multirow{2}{*}{\multicolumn{2}{|c|}{ Name }} & \multicolumn{6}{|c|}{ Process Status } & \multicolumn{3}{|c|}{ HPV } \\
\hline & & & & & & & & uc & $u$ & $P$ & $\mathrm{CB}$ & B GTT & $\mathrm{PC}$ & $\mathrm{F}$ & M & $\mathrm{OR}$ \\
\hline 256339 & 256339 & SC & $P$ & M & 90 & MOORE, LAI & ANDON J & 0 & 0 & . & 0 & 00 & . & . & . & . \\
\hline 329869 & 329869 & $B E$ & $P$ & M & 69 & HOWARD, & Upine Processing & & 0 & . & 0 & 00 & 0 & . & . & 0 \\
\hline 416714 & 416714 & $B E$ & $P$ & $\mathrm{~F}$ & 20 & BAILEY, AI & $\begin{array}{l}\text { Elood Processing } \\
\text { OGTT Processing }\end{array}$ & & 0 & 0 & 0 & 00 & 0 & 0 & . & 0 \\
\hline 480354 & 480354 & SC & $P$ & M & 3 & LEE, OWE & $\begin{array}{l}\text { Pạcked Cell Processing } \\
\text { Fernale HPy Processing }\end{array}$ & & . & . & 0 & 0. & . & . & . & . \\
\hline 525757 & 525757 & $\mathrm{BE}$ & $P$ & $\mathrm{~F}$ & 70 & ADAMS, $C$, & $\begin{array}{l}\text { Male HPY Processing } \\
\text { Oral Rinse Processing }\end{array}$ & & 0 & . & 0 & 00 & 0 & . & . & . \\
\hline 545182 & 545182 & SC & $P$ & $\mathrm{~F}$ & 4 & POWELL, , & Urine Collection & & . & . & 0 & 0. & . & . & . & . \\
\hline 553872 & 553872 & $\mathrm{Cl}$ & $P$ & M & 21 & WRIGHT, L & Bregnancy Testing & & $n$ &. & $\ln$ & $\ln 0$ & & . & 0 & 0 \\
\hline 745052 & 745052 & $\mathrm{Cl}$ & $P$ & $\mathrm{~F}$ & 41 & EVANS, St & $\begin{array}{l}\text { Hematology } \\
\text { Refresh }\end{array}$ & 中 & & cess $C$ & EBC Da & ata & & 0 & . & 0 \\
\hline 767995 & 767995 & $\mathrm{BE}$ & $P$ & M & 15 & MURPHY, IS & SAACN & $c$ & & view $C$ & BC Re: & sults & & & 0 & 0 \\
\hline 784174 & 784174 & $B E$ & $P$ & M & 50 & GONZALEZ, & SIENNA N & 0 & 0 & . & 0 & 00 & . & . & 0 & 0 \\
\hline 813366 & 813366 & $\mathrm{BE}$ & $P$ & $\mathrm{~F}$ & 19 & STEWART, S & SOPHIE J & 0 & 0 & 0 & 0 & 00 & . & 0 & . & 0 \\
\hline 836040 & 836040 & SC & $P$ & M & 19 & TORRES, LU & UCAS S & 0 & 0 & . & 0 & 00 & . & . & 0 & 0 \\
\hline 837622 & 837622 & SC & $P$ & $\mathrm{~F}$ & 1 & MARTINEZ, & ANNA L & . & . & . & 0 & 0. & . & . & . & . \\
\hline 849425 & 849425 & $S C$ & $P$ & $\mathrm{~F}$ & 10 & CAMPBELL, & JASPER N & 0 & 0 & 0 & 0 & 0. & 0 & . & . & . \\
\hline 861215 & 861215 & $B E$ & $P$ & M & 18 & SMITH, MICA & AH S & 0 & 0 & . & 0 & 00 & 0 & . & 0 & 0 \\
\hline 919919 & 919919 & $\mathrm{Cl}$ & $P$ & $\mathrm{~F}$ & 25 & PAYNE, IMA & & 0 & 0 & 0 & 0 & 00 & 0 & 0 & . & 0 \\
\hline
\end{tabular}

To access the Hematology module, use the mouse to direct the mouse arrow to any SP, right click, drag the mouse arrow to $\{$ Hematology then to Process CBC Data\}, and right click or right click and type [Shift] [E/e] [P/p]. Alternatively, use the up and down keys to move up and down the list until a particular SP is highlighted, right click, drag the mouse arrow to \{Hematology then to Process CBC Data $\}$, and right click or right click and type [Shift $[\mathrm{E} / \mathrm{e}][\mathrm{P} / \mathrm{p}]$. 
Either open the module or reject the specimen and add a reason or comment for every CB record where blood was drawn in phlebotomy AND there are no $\mathrm{CBC}$ results.

\begin{tabular}{|c|c|c|c|c|c|c|c|c|c|c|c|c|c|c|}
\hline \multicolumn{15}{|c|}{ ¡Lab Subsystem } \\
\hline \multicolumn{15}{|c|}{ File Wiew untilities Quality Control Reports Modules shhipping Window Help } \\
\hline & $\because \square$ & 惯 & & & (2) & 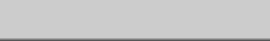 & & & & & & & & \\
\hline \multirow{2}{*}{ SP ID } & \multirow{2}{*}{ Sample ID } & \multirow{2}{*}{ Status } & \multirow{2}{*}{ Type } & \multirow{2}{*}{ Gender } & \multirow[t]{2}{*}{ Age } & \multirow{2}{*}{ Name } & \multicolumn{6}{|c|}{ Process Status } & $\mathrm{HPV}$ & \multirow[b]{2}{*}{ OR } \\
\hline & & & & & & & uc & $u \quad F$ & P & $\mathrm{CB} \mathrm{E}-2-C$ & $B \quad$ GTT & $\mathrm{PC}$ & F M & \\
\hline 256339 & 256339 & Sc & $P$ & M & 90 & MOORE, LANDON J & 0 & 0. & . & $0 \mid c$ & 00 & . & . . & - \\
\hline 329869 & 329869 & $\mathrm{BE}$ & $P$ & M & 69 & HOWARD, SOPHIAS & $\hat{\text { Urine Processing }}$ & & & $p c$ & 00 & 0 & . . & 0 \\
\hline 416714 & 416714 & $\mathrm{BE}$ & $P$ & $\mathrm{~F}$ & 20 & BAILEY, ANTHONY J & \multirow{4}{*}{\multicolumn{3}{|c|}{$\begin{array}{l}\text { Elood Processing } \\
\text { OGgTT Processing } \\
\text { Packed Cell Processing } \\
\text { Fernale HPY Processing } \\
\text { Male HPY Processing } \\
\text { Oral Rinse Processing }\end{array}$}} & $p c$ & 00 & 0 & 0. & 0 \\
\hline 480354 & 480354 & SC & $P$ & M & 3 & LEE, OWEN S & & & & $p c$ & 0. & - & . . & . \\
\hline 525757 & 525757 & $\mathrm{BE}$ & $P$ & $\mathrm{~F}$ & 70 & ADAMS, CALEB L & & & & $p c$ & 00 & 0 & $\cdot \cdot$ & $\cdot$ \\
\hline 545182 & 545182 & SC & $P$ & $\mathrm{~F}$ & 4 & POWELL, AALIYAH H & & & & $p c$ & 0. & . & . $\cdot$ & . \\
\hline 553872 & 553872 & $\mathrm{Cl}$ & $P$ & M & 21 & WRIGHT, LAYLA B & \multicolumn{3}{|c|}{$\begin{array}{l}\text { Urine Collection } \\
\text { Pregnancy Testing }\end{array}$} & $p<c$ & 00 & & . 0 & 0 \\
\hline 745052 & 745052 & $\mathrm{Cl}$ & $\mathrm{P}$ & $\mathrm{F}$ & 41 & EVANS, SAVANNAH M & Hematology & & & \multicolumn{4}{|c|}{ Process CBC Data } & \\
\hline & & & & & & & \multirow{2}{*}{\multicolumn{3}{|c|}{ Refresh }} & \multirow{2}{*}{\multicolumn{4}{|c|}{$\begin{array}{l}\text { Not Processed CBC Data } \\
\text { Review CBC Results } \\
\end{array}$}} & \multirow{3}{*}{$\begin{array}{l}\text { Glotted } \\
\text { Not Enough Bloc } \\
\text { Equipment Failur } \\
\text { Lab Error }\end{array}$} \\
\hline $76 / 995$ & $/ 6 / 995$ & BE & $P$ & $\mathrm{M}$ & 15 & MURPHY, ISAAC N & & & & & & & & \\
\hline 784174 & 784174 & BE & $P$ & M & 50 & GONZALEZ, SIENNA N & 0 & 0. & . & $0 c$ & 00 & - & $\cdot 0$ & \\
\hline 813366 & 813366 & $\mathrm{BE}$ & $P$ & $\mathrm{~F}$ & 19 & STEWART, SOPHIE J & 0 & $0 c$ & 0 & $0 c$ & 00 & . & 0 & 0 \\
\hline 836040 & 836040 & $\mathrm{SC}$ & $P$ & M & 19 & TORRES, LUCAS S & 0 & 0. & . & $0 c$ & 00 & . & .0 & 0 \\
\hline 837622 & 837622 & $\mathrm{SC}$ & $P$ & $\mathrm{~F}$ & 1 & MARTINEZ, ANNA L & . & . & . & $0 c$ & 0. & . & .. & . \\
\hline 849425 & 849425 & SC & $P$ & $\mathrm{~F}$ & 10 & CAMPBELL, JASPER N & 0 & $0 c$ & 0 & $0 c$ & 0. & 0 & $\cdot \cdot$ & - \\
\hline 861215 & 861215 & $B E$ & $P$ & M & 18 & SMITH, MICAH S & 0 & 0. & . & $0 c$ & 00 & 0 & . 0 & 0 \\
\hline 919919 & 919919 & $\mathrm{Cl}$ & $P$ & $\mathrm{~F}$ & 25 & PAYNE, IMA L & 0 & $0 c$ & 0 & $0 c$ & 00 & 0 & 0. & 0 \\
\hline
\end{tabular}

Open the module \{Process CBC Data\} or record a reason why the $\mathrm{CBC}$ is not being run $\{$ Not Processed CBC Data\}. Select or record a comment for every CBC that is not run. To complete opening or accessing the Hematology module, use the mouse to direct the mouse arrow to \{Process CBC Data $\}$ and right click or type $[\mathrm{P} / \mathrm{p}$.] To record a reason why a $\mathrm{CBC}$ is not being run, use the mouse to direct the mouse arrow to the exact SP's line on the heads-up display, right click, drag the mouse arrow to \{Not Processed CBC Data\}, and drag the mouse arrow to the correct reason and right click. Alternatively, type [Shift] [E/e] [N/n] and [C/c] for Clotted, [N/n] for Not Enough Blood, [E/e] for Equipment Failure, or $[\mathrm{L} / \mathrm{l}]$ for Lab Error. 
A pop-up window will display. Confirm the selection.

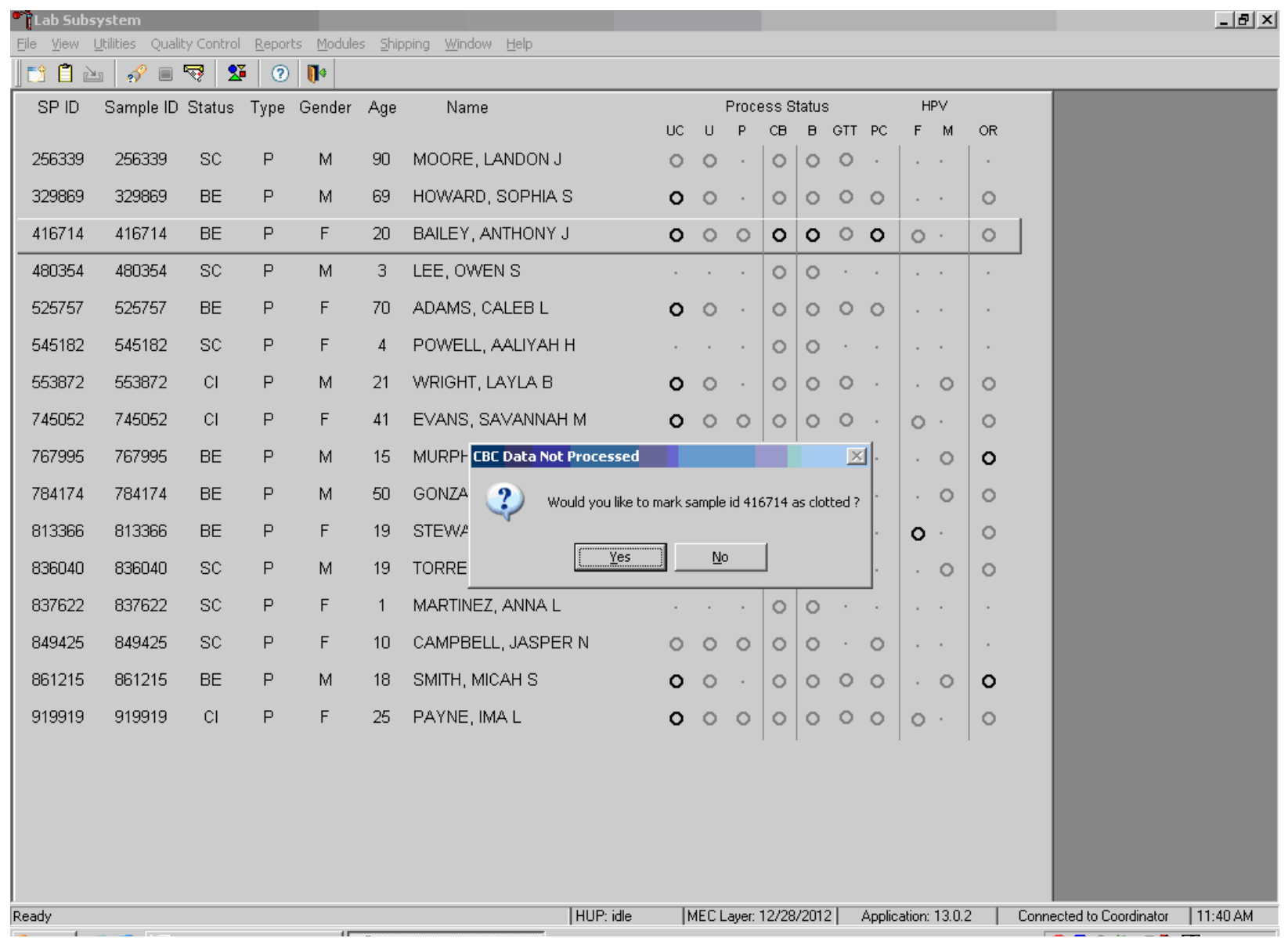

A CBC Data Not Processed message text box displays that asks, "Would you like to mark sample id XXXXXX as Clotted/Not Enough Blood/Equipment Failure/Lab Error?" To record a "Yes" response, use the mouse to direct the mouse arrow to the Yes button and left click, type [Y/y], or press [Enter]. To record a "No" response, use the mouse to direct the mouse arrow to the No button and left click, or type [N/n]. If a Yes response is recorded, the comment is saved to the database. If a No response is recorded, no comment is saved to the database. If the record is marked with the selection in the database, then the heads-up display updates to complete (the CB circle fills in black).

\section{Hematology module overview}

Coulter ${ }^{\circledR}$ does not automatically transmit results to the Laboratory application. Use the Retrieve button to send the results from the DMS to the Hematology module. Select after each run. Make 
sure the Coulter ${ }^{\circledR}$ DMS host computer icon (HC) is displaying an up arrow ( $\uparrow$ ) in the DMS bottom tile bar.

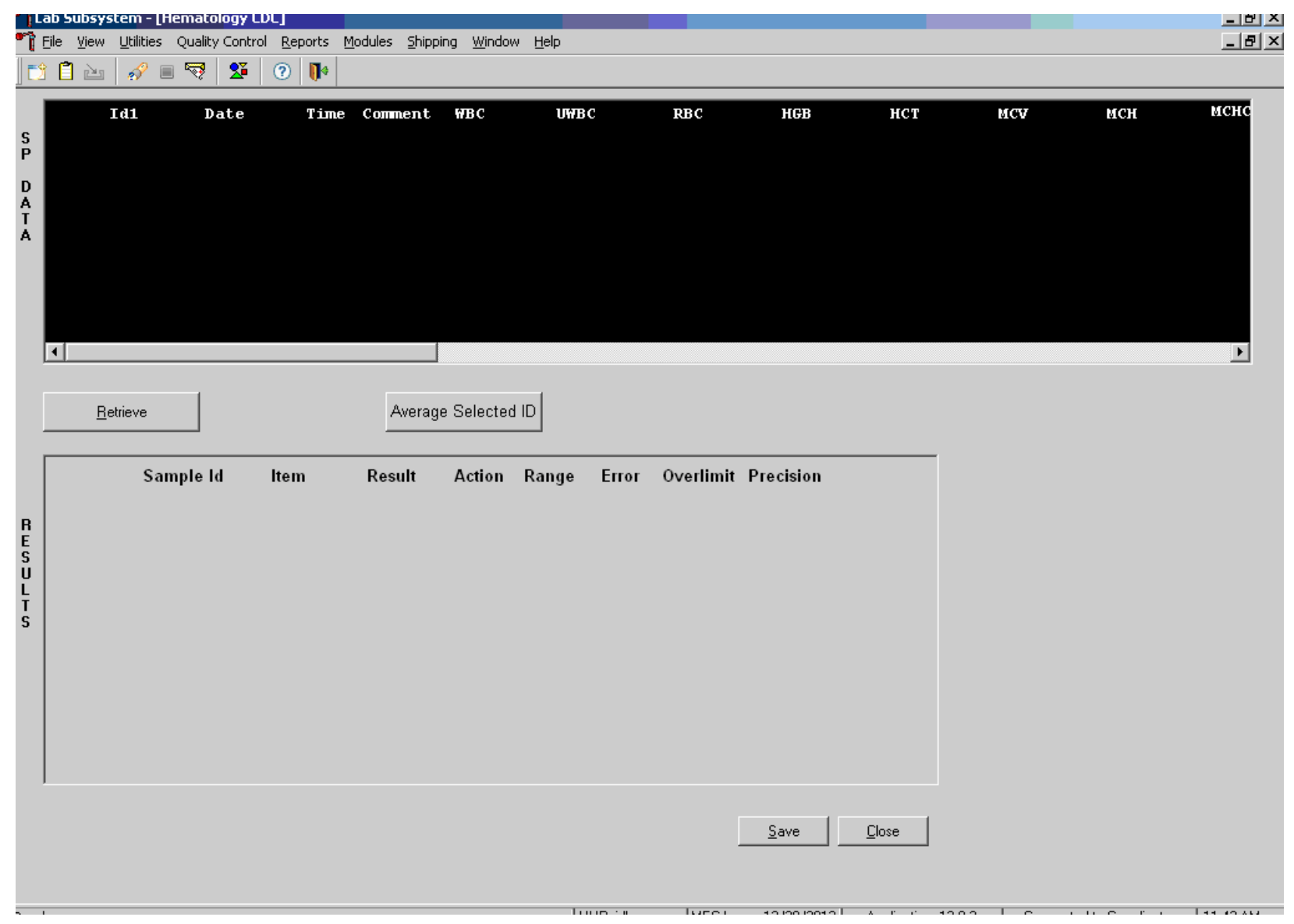

After data are retrieved, they display in the top portion of the window, the SP Data section. This section includes the SP ID, the date and time the CBC was run, and columns for each individual parameter. The bottom portion of the window is the Results section. Results display after the Average Selected ID button is selected. 
Review all Coulter ${ }^{\circledR}$ data in the SP Data section after they are retrieved.

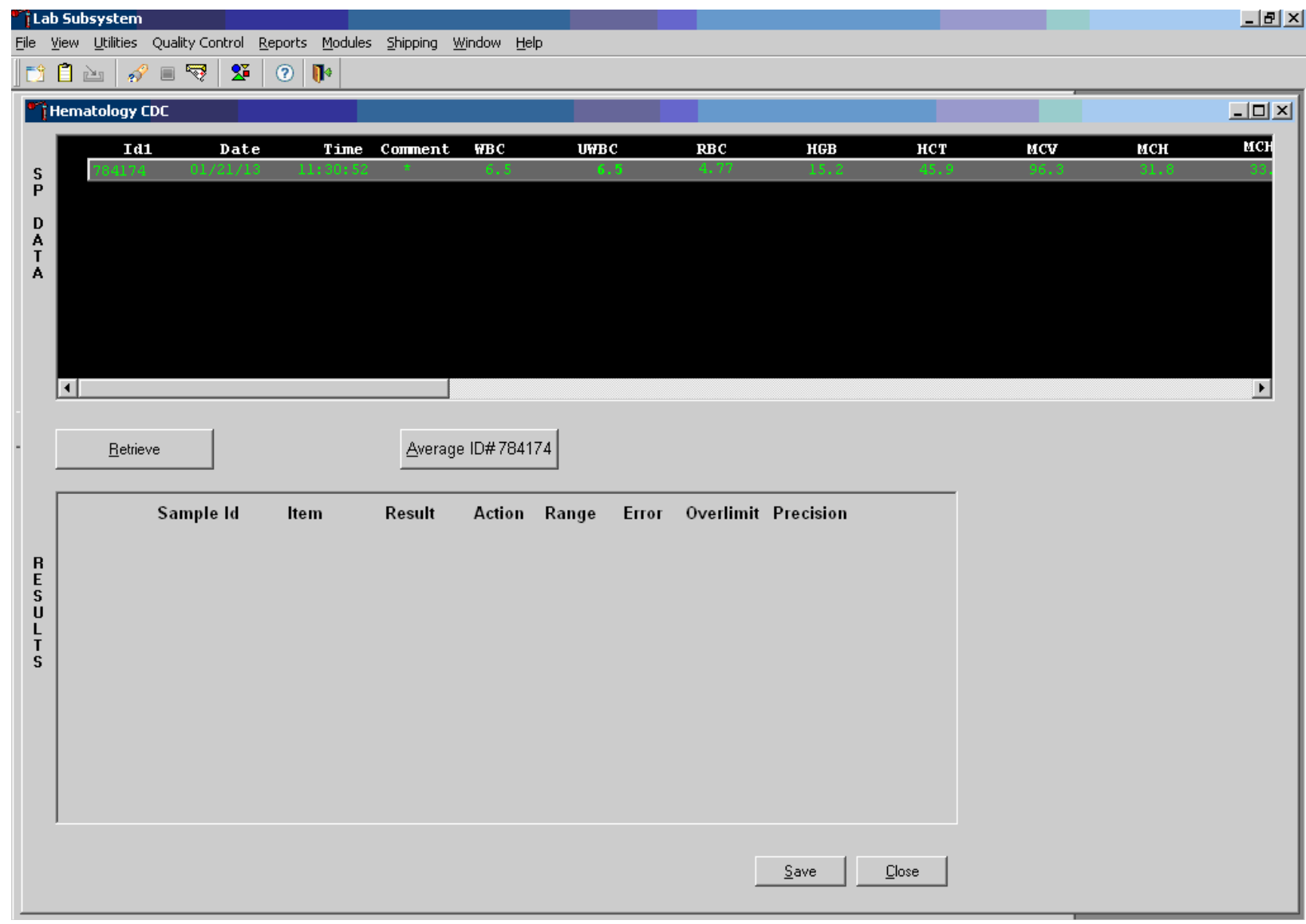

To view SP data for all parameters, use the mouse to direct the mouse arrow to the horizontal scroll bar at the bottom of the SP Data section and drag the scroll bar to the left or right. Alternatively, to view the SP data for all parameters, use the mouse to direct the mouse arrow to the small scroll arrows on the left and right edges of the horizontal scroll bar, and left click.

The Hematology Results section overview.

The Result columns include: Sample ID, Item (CBC parameter), Result (Coulter® data for a single run or the ISIS averaged result for multiple runs), Action (CDC established critical limits), Range (CDC established reference ranges for both genders and four age groups), Error (Coulter ${ }^{\circledR}$ transmitted instrument errors), Overlimit (Coulter ${ }^{\circledR}$ transmitted result that exceeds the instrument's linearity limit), and Precision (CDC established values for the difference between any two runs.) Checkmarks display in 
boxes for parameters that have errors, are overlimit, or for those that exceed precision limits. An " $\mathrm{H}$ " for "high" and "L" for "low" display for parameters that exceed action limits or reference ranges.

Average, evaluate, and save results for all parameters. The SP's results display in the bottom portion of the window after the Average Selected ID button is selected. To view results for all parameters, use the mouse to direct the mouse arrow to the vertical scroll bar at the right side of the Results section and drag the bar up or down. Alternatively, to view all the results, use the mouse to direct the mouse arrow to the small scroll arrows on the top and bottom edges of the vertical scroll bar, and left click. Evaluate each parameter for error, overlimit, and precision checkmarks, and " $H$ " or "L" action limit and reference range flags. To save the result to the database, use the mouse to direct the mouse arrow to the Save button and left click or type [Shift] [S/s]. After results are saved, they are erased from the screen. To exit without saving the result to the database, use the mouse to direct the mouse arrow to the Cancel button and left click or type [Shift] [C/c].

A warning text box displays if the Close button is selected before the results have been saved. 


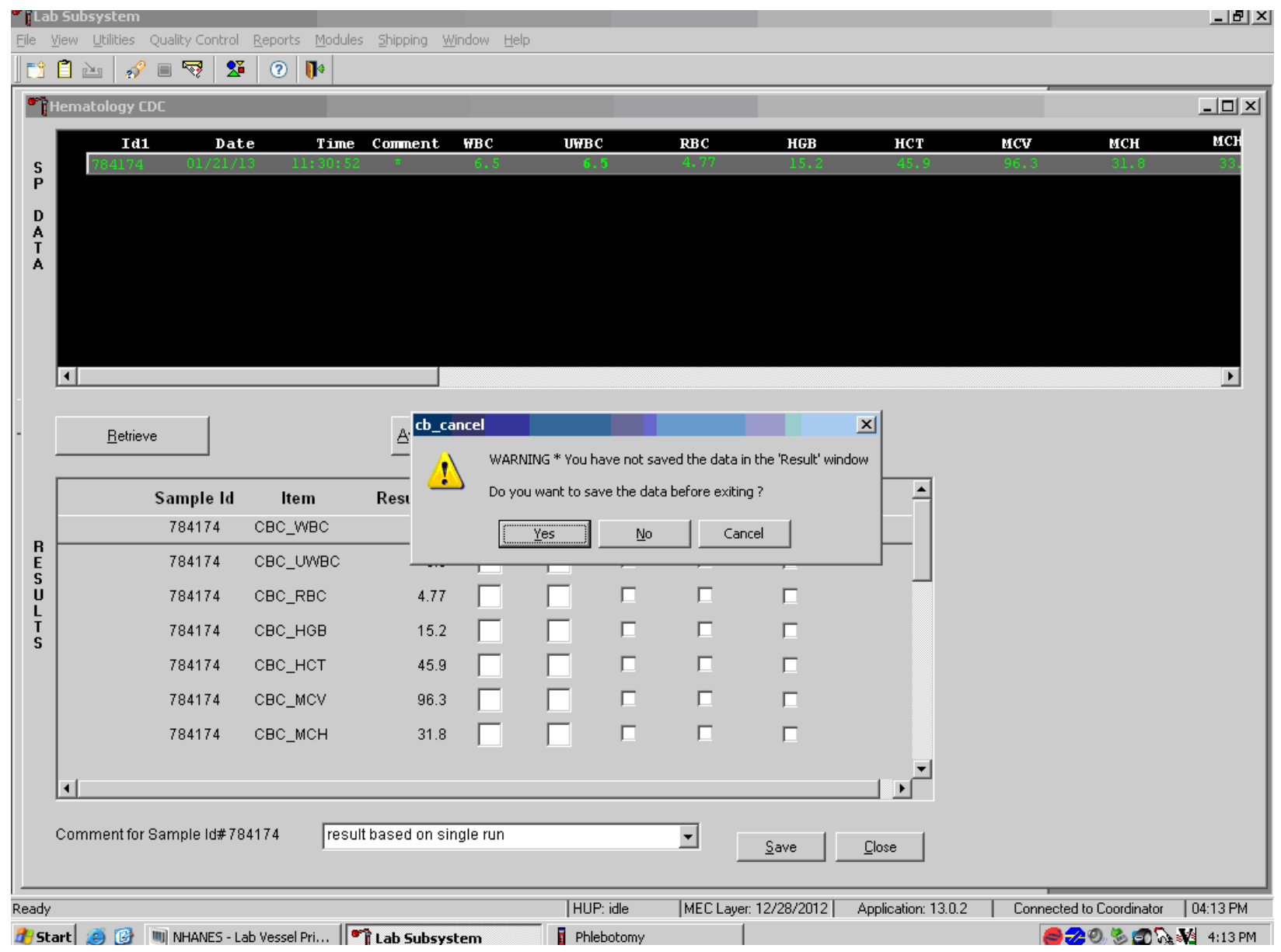

If the Close button is selected before the results are saved to the database, a warning message text box displays that states, "WARNING *You have not saved the data in the 'Result' window" and asks, "Do you want to save the data before exiting?" To record a "Yes" response, use the mouse to direct the mouse arrow to the Yes button and left click, type [Y/y], or press [Enter]. To record a "No" response, use the mouse to direct the mouse arrow to the No button and left click, or type [N/n]. To cancel the action, use the mouse to direct the mouse arrow to the Cancel button and left click. If a Yes response is recorded, the $\mathrm{CBC}$ results are saved to the database and erased. If a No response is recorded, no results are saved to the database and the data in the Results section is erased. A Cancel response returns the screen to its previous state. 
3. Running samples when only one run is possible

Save results where only one run was possible.

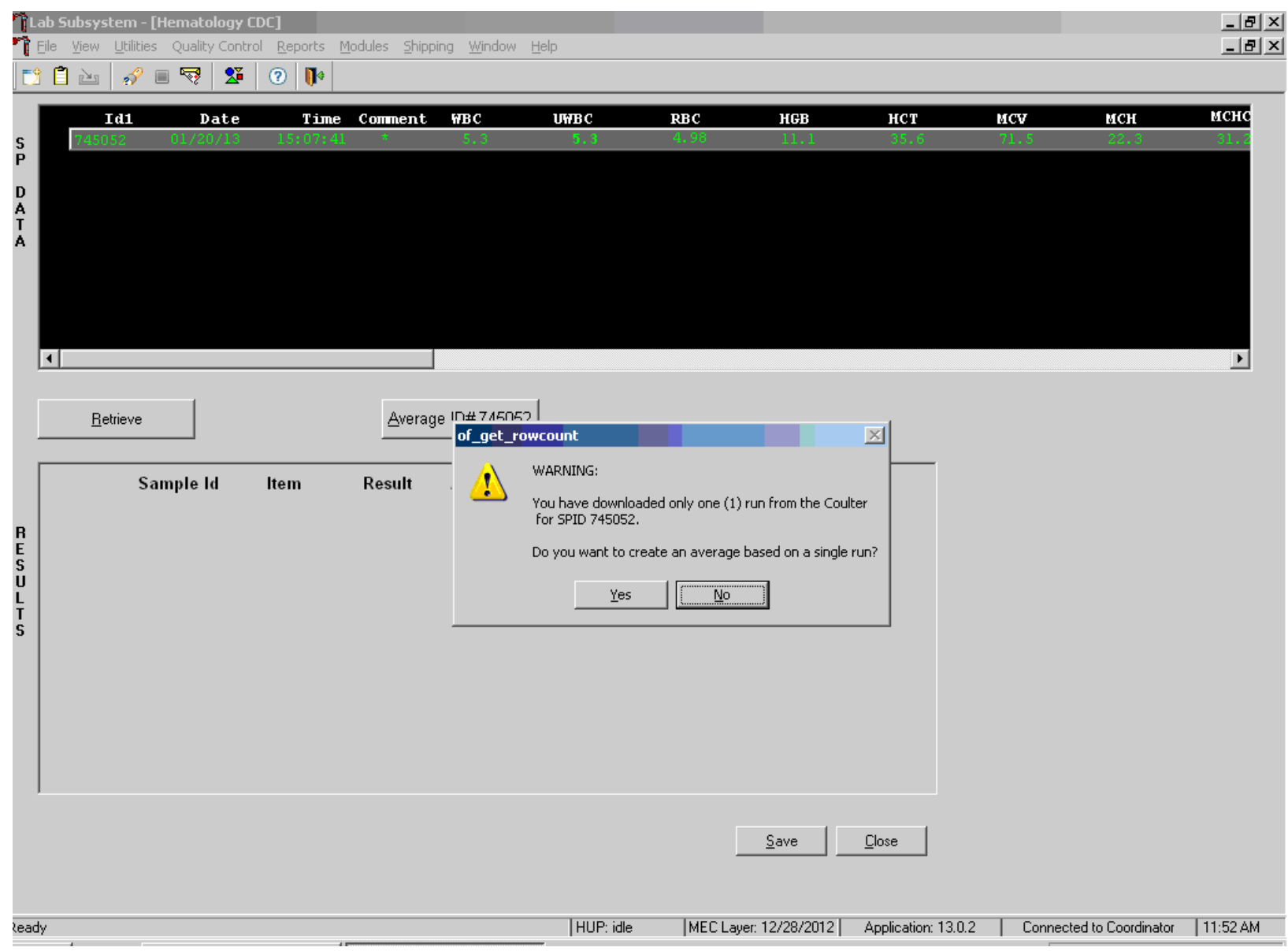

Run samples in duplicate whenever possible. If the whole blood is insufficient, it is acceptable to average and save only one run. To save results where only one run is possible, use the mouse to direct the mouse arrow to the Average Selected ID box and left click. A warning message text box displays that states, "You have downloaded only one (1) run from the Coulter ${ }^{\circledR}$ for SPID $X X X X X X$ " and asks, "Do you want to create an average based on a single run?" To record a "Yes" response, use the mouse to direct the mouse arrow to the Yes button and left click or type [Y/y]. To record a "No" response, use the mouse to direct the mouse arrow to the No button and left click, or type [N/n], or press [Enter]. If a Yes response is recorded, the CBC results display in the bottom Results section of the window. A "No" response cancels the action and returns the window to its previous state. 
For results where only one run was possible, the Comment box at the bottom of the window defaults to "result based on single run."

\section{Running samples in duplicate}

Run all samples in duplicate and average the data.

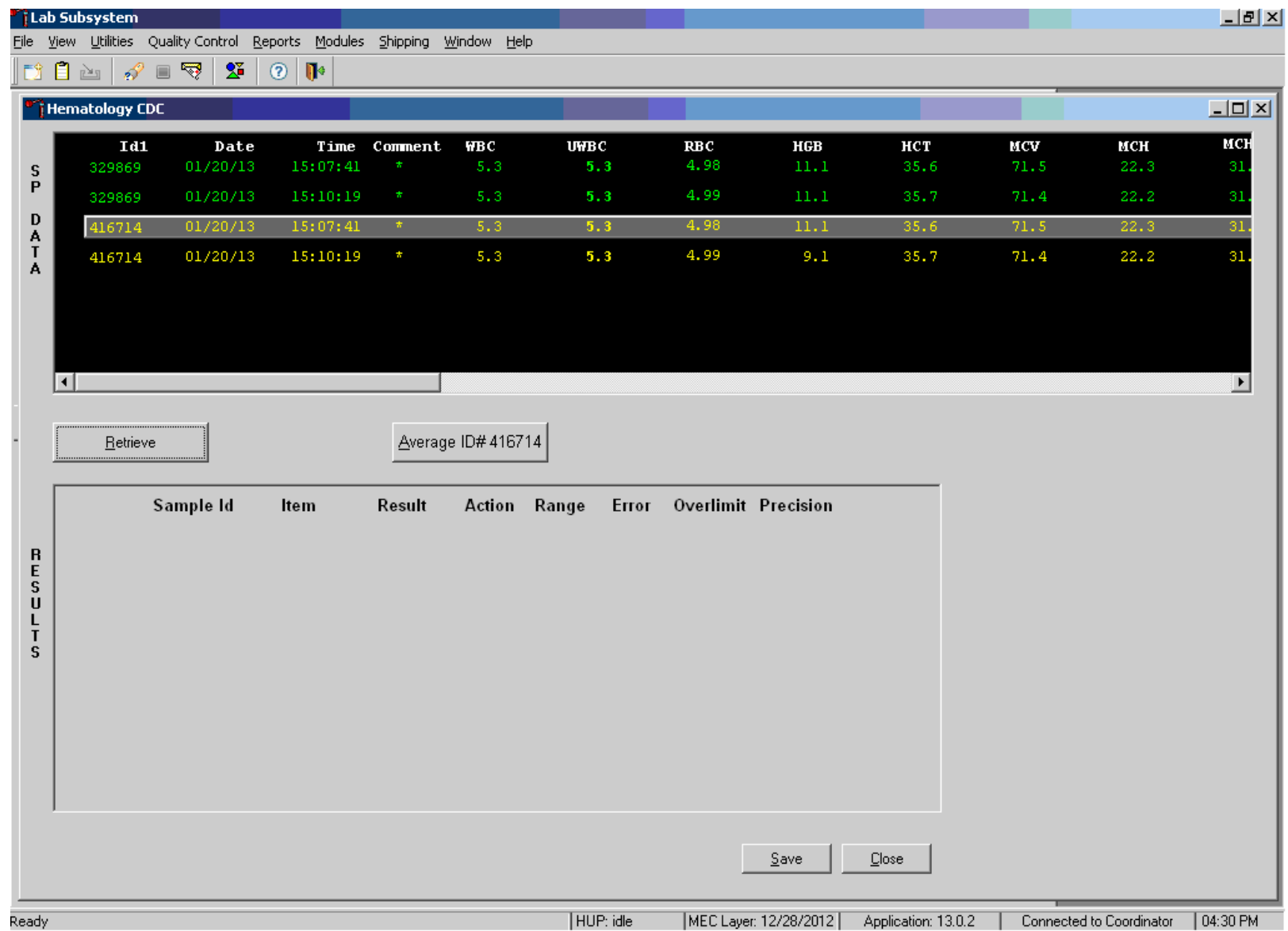

Whenever a sample is run in duplicate, both results display in the SP Data section. To average these two results, use the mouse to direct the mouse arrow to Average ID $\# X X X X X X$ button and left click.

Results display in the lower section of the window. 


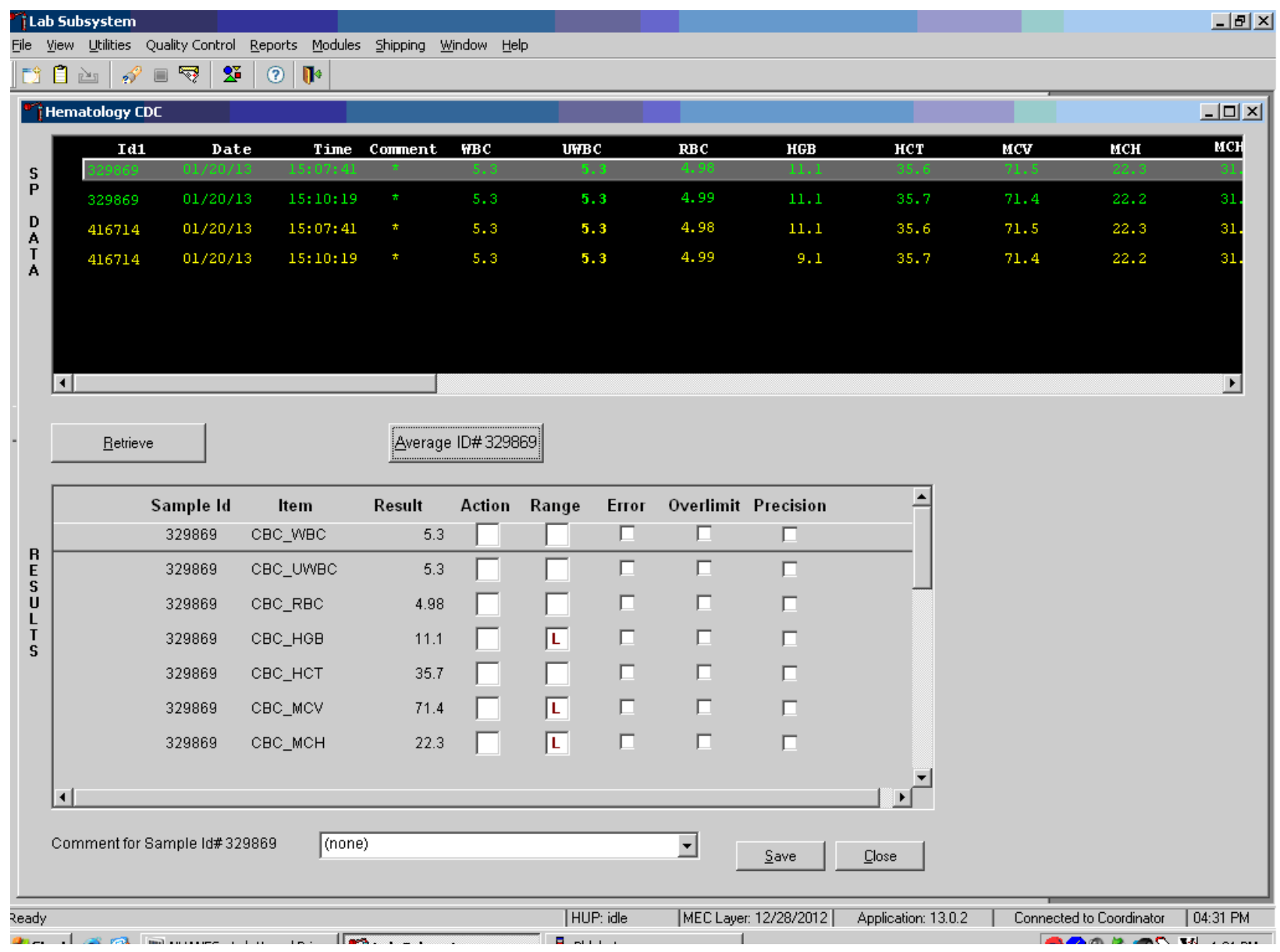

The differences between duplicate values for WBC, RBC, Hgb, MCV, Plt, NE\#, LY\#, MO\#, EO\# and BA\# are calculated and evaluated to determine if the differences are within the following CDC established precision limits.

CDC established precision limits:

$\begin{array}{ll}\underline{\text { Test }} & \underline{\text { Precision Limits }} \\ \text { WBC } & 0.4 \times 10^{3} \\ \text { RBC } & 0.1 \times 10^{6} \\ \text { Hgb } & 0.4 \mathrm{~g} / \mathrm{dL} \\ \text { MCV } & 2.2 \mathrm{fL} \\ \text { Plt } & 23 \times 10^{3} \\ \text { NE\# } & 0.4 \times 10^{3} \\ \text { LY\# } & 0.2 \times 10^{3} \\ \text { MO\# } & 0.2 \times 10^{3} \\ \text { EO\# } & 0.2 \times 10^{3} \\ \text { BA\# } & 0.2 \times 10^{3}\end{array}$


If $\mathrm{RBC}$ parameters are out of range, evaluate the data for drift. If drift is evident, evaluate the possibility of an instrument malfunction. If any WBC differential absolute number is out of range, check the WBC scattergram for abnormal cell population(s).

Evaluate and save results when precision limits are not exceeded.

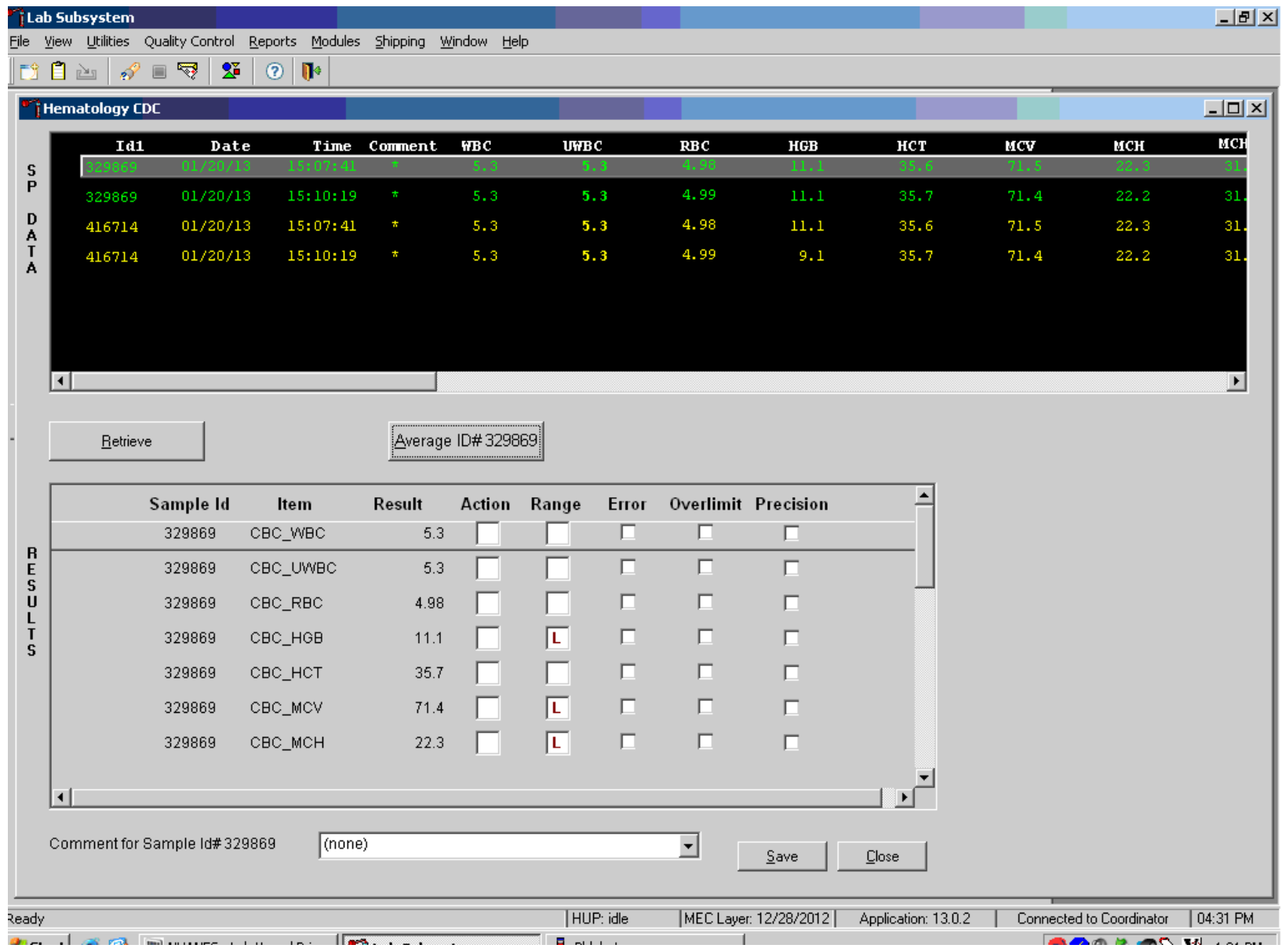

Evaluate results for all parameters. Evaluate each parameter for error, overlimit, and precision checkmarks, and " $\mathrm{H}$ " or "L" action limit and reference range flags. If there are no checkmarks in the Precision column (no precision limit was exceeded), save the results to the database. To save the results to the database, use the mouse to direct the mouse arrow to the Save button and left click or type [Shift] [S/s]. After results are saved, they are erased from the screen. To exit without saving the result to the database, use the mouse to direct the mouse arrow to the Close button and left click or type [Shift] $[\mathrm{C} / \mathrm{c}]$. 
Average, evaluate, and rerun specimens that exceed precision limits.

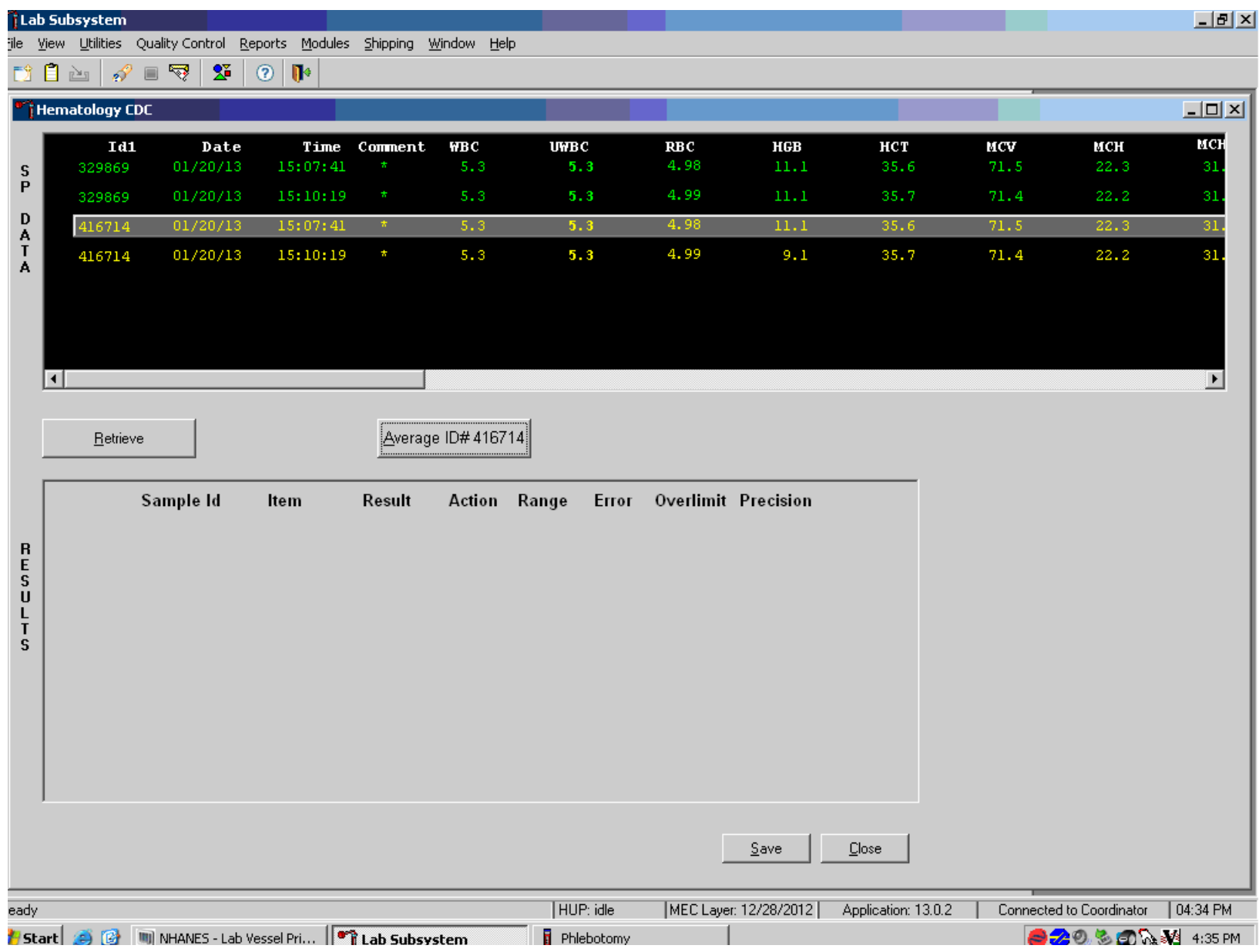

Average the two results by using the mouse to direct the mouse arrow to the Average ID\#XXXXXX button and left click. If at this point, precision limits are exceeded for any individual parameter, the technologist is prompted to run a control and evaluate the control to determine if all control values fall within the control's established range. 
If any parameter exceeds its precision limit, the Hematology Control Run window displays.

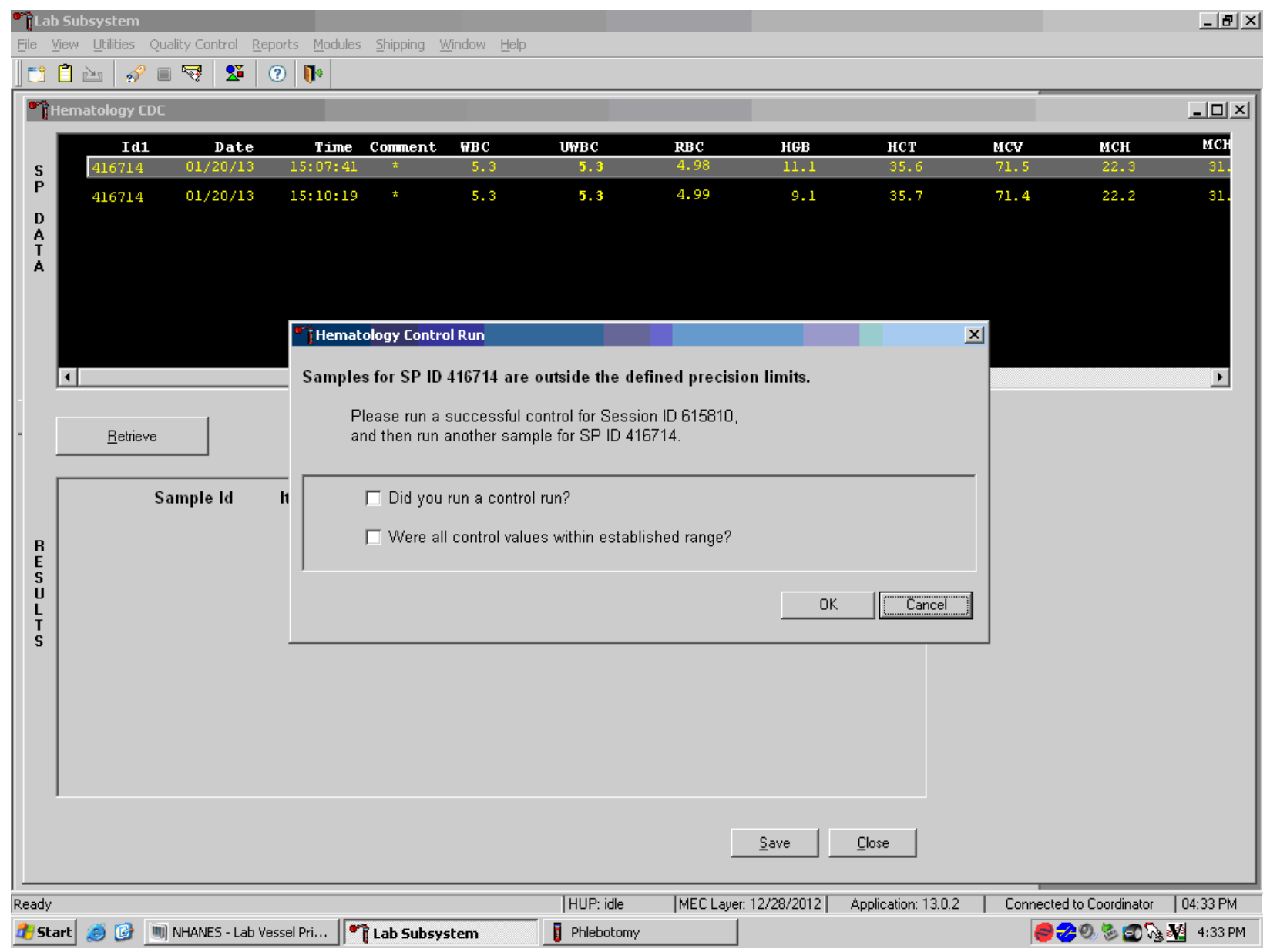

The Hematology Control Run window indicates that the "Samples for SP ID $X X X X X X$ are outside the defined precision limits." It instructs the technologist to "Please run a successful control for Session ID $X X X X X X$, and then run another sample for SP ID $X X X X X X$." Run any one level of 5C cell control and evaluate the results. Respond to the two questions, "Did you run a control run?" and "Were all control values within established range?" To confirm these actions, use the mouse to direct the mouse arrow to each of the two checkboxes and left click to record the checkmarks. 
Save the responses to the checkbox questions.

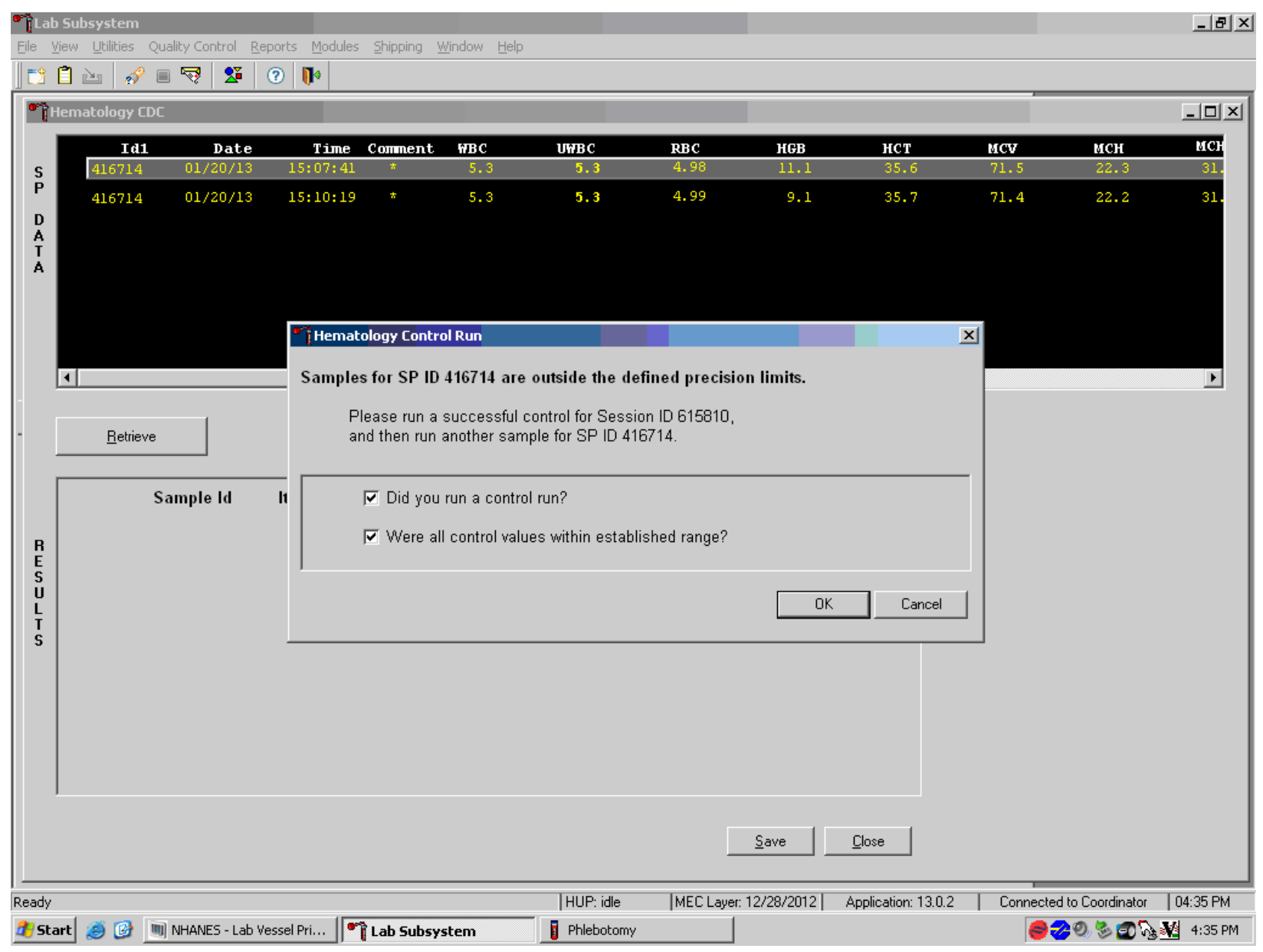

To save these responses to the database, use the mouse to direct the mouse arrow to the OK button and left click or type [Enter], or to exit the Hematology Control Run window without saving the responses, use the mouse to direct the mouse arrow to the Cancel button and left click. As soon as the OK button is selected, the averages display in the Results section of the window. 
Evaluate the results displayed in the Results section of the window.

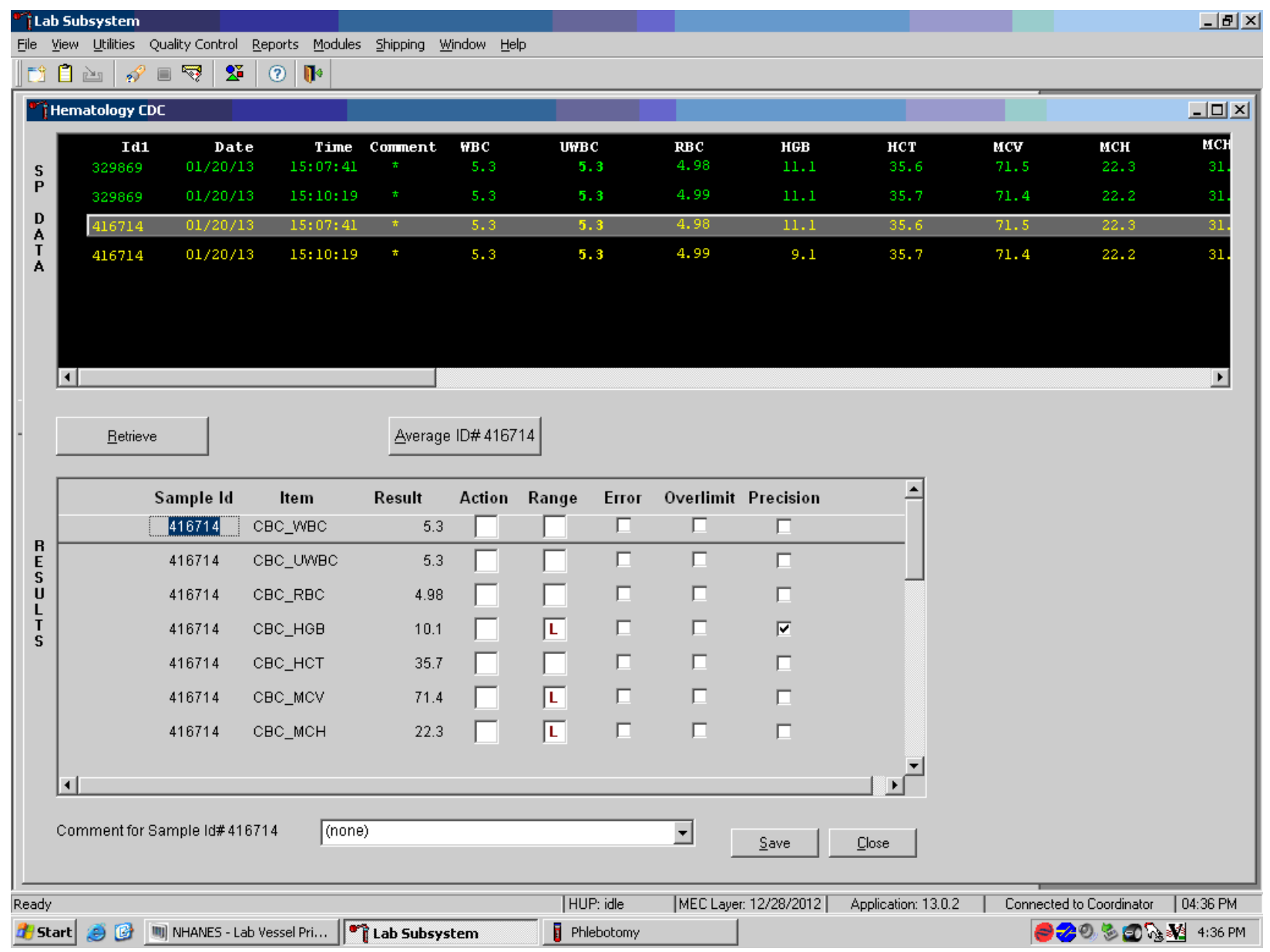

Evaluate each parameter for error, overlimit, and precision checkmarks, and " $H$ " or " $L$ " action limit, reference range flags and comment in the above screen. Use the scroll bar to view all results. When there are checkmarks in any of the Precision columns, meaning that the precision limit was exceeded, rerun the blood sample a third time. If there is insufficient blood to run the CBC a third time, save the result to the database. To save the results to the database, use the mouse to direct the mouse arrow to the Save button and left click or type [Shift] [S/s]. After results are saved, they are erased from the screen. To exit without saving the result to the database, use the mouse to direct the mouse arrow to the Close button and left click or type [Shift] [C/c]. 
Run the specimen a third time when any precision limit is exceeded or if one run has a comment and the second run doesn't.

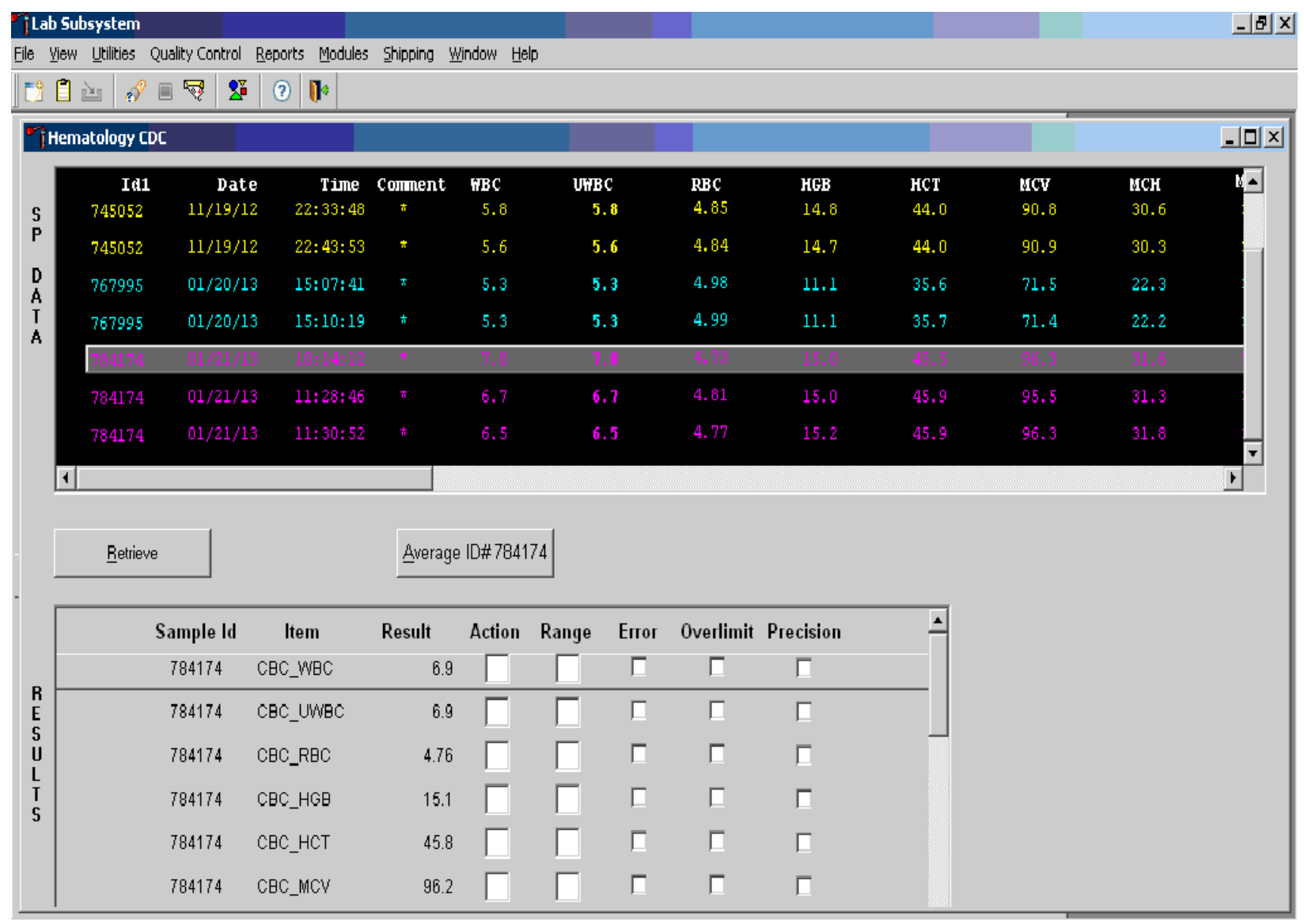

Run the blood sample a third time. Whenever a sample is run more than once, all results in the SP Data section are displayed. Scroll through the top of the SP data window and determine which of the two runs match the best. Delete the outlier run, which could be the run that caused the precision error or the run that doesn't/does have a comment and re-average the two runs. 


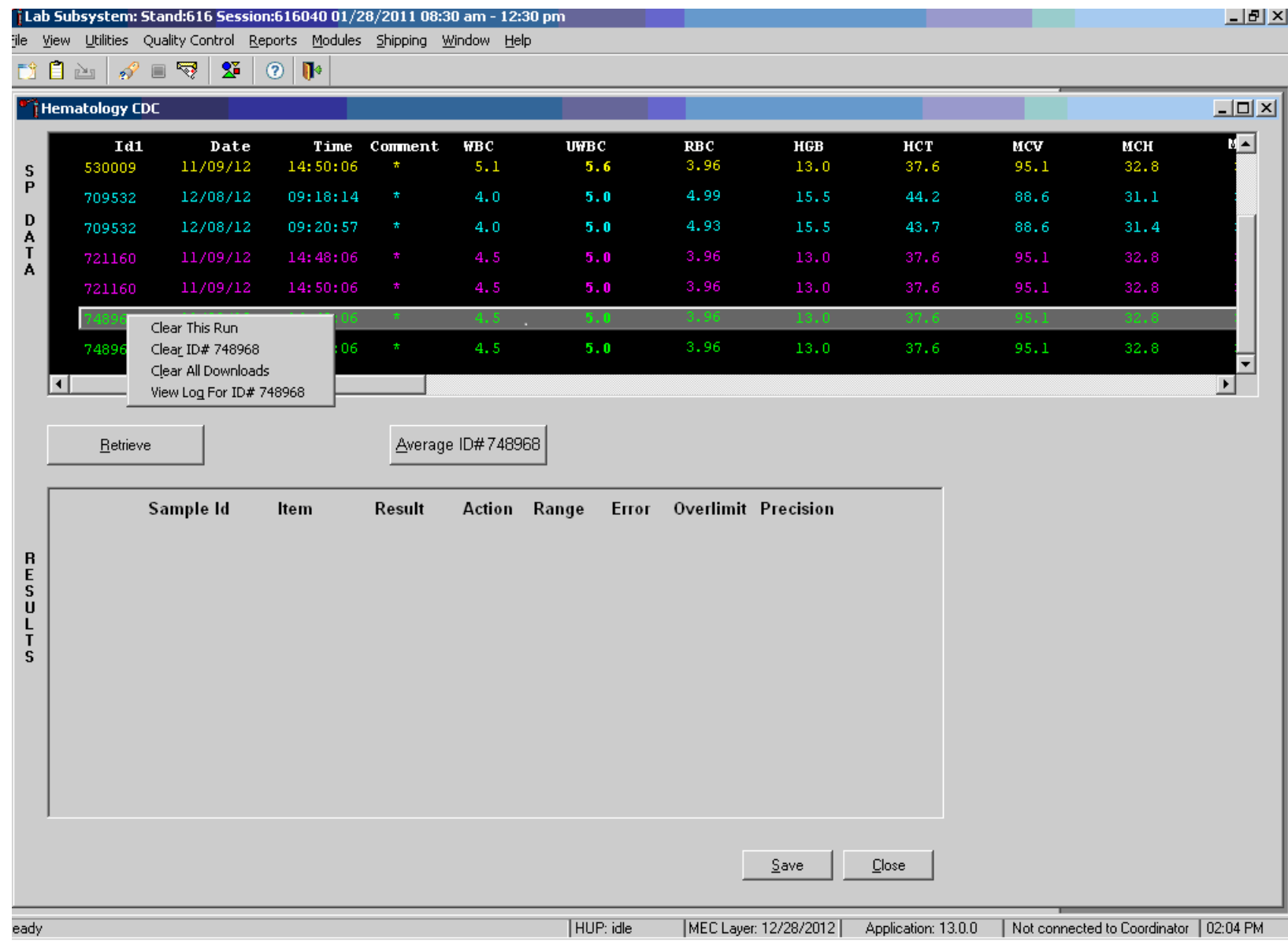

After clearing the outlier run, re-average the two runs and analyze the results. 
Override the current averaged results for parameters that exceeded the precision limit with a new average.

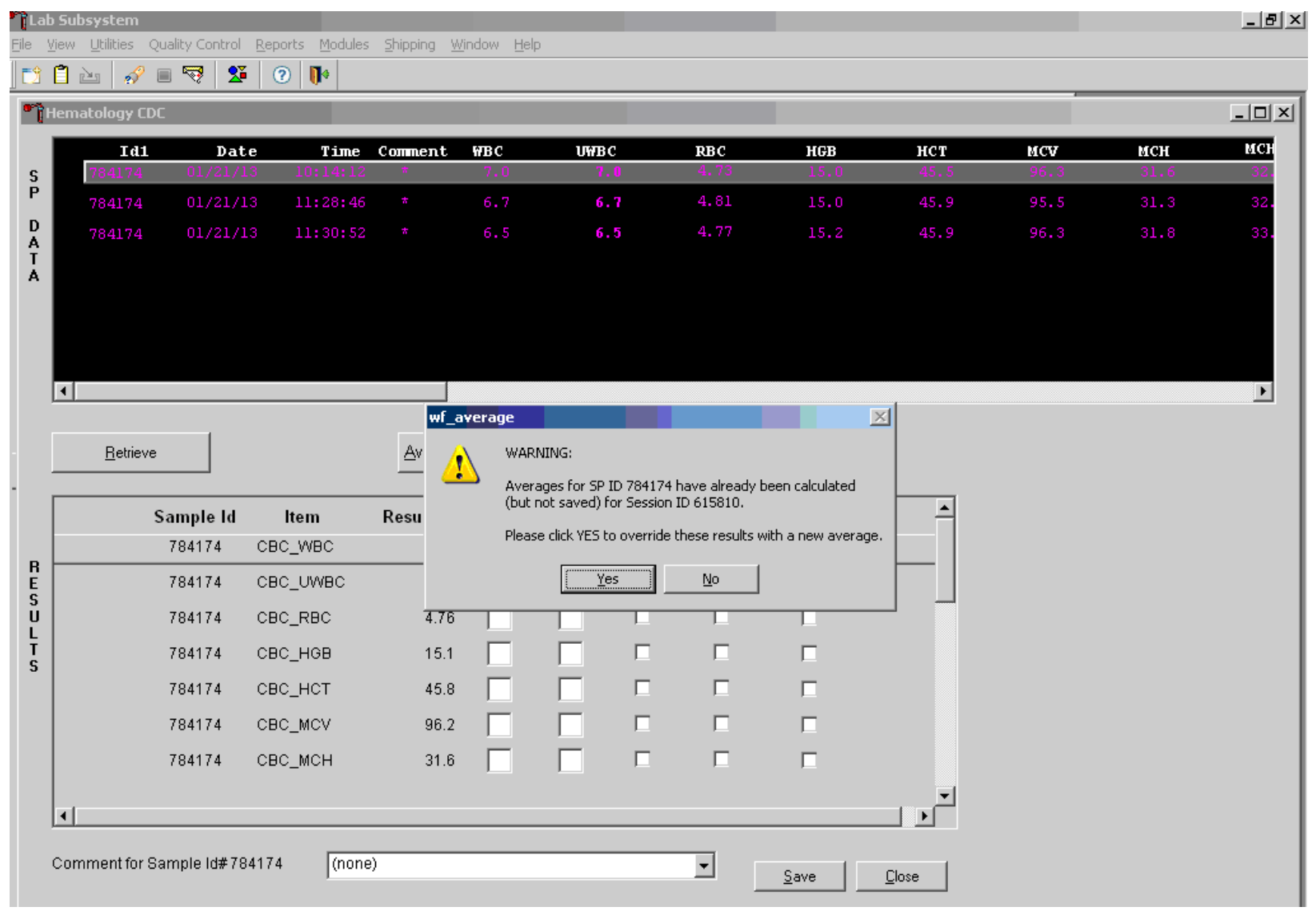

Whenever a sample has previously been averaged, and a new average is calculated, a warning message text box displays stating, "Averages for SP ID $X X X X X X$ have already been calculated (but not saved) for session ID $X X X X X X$." The text box instructs, "Please click YES to override these results with a new average." To override the current average with a new average (but for only those parameters that exceeded precision limits), use the mouse to direct the mouse arrow to the Yes button and left click or type [Shift] [Y/y], or press [Enter]. To exit or return to the previous screen containing the previous averages, use the mouse to direct the mouse arrow to the No button and left click, or type [Shift] $[\mathrm{N} / \mathrm{n}]$. 
Evaluate the new results.

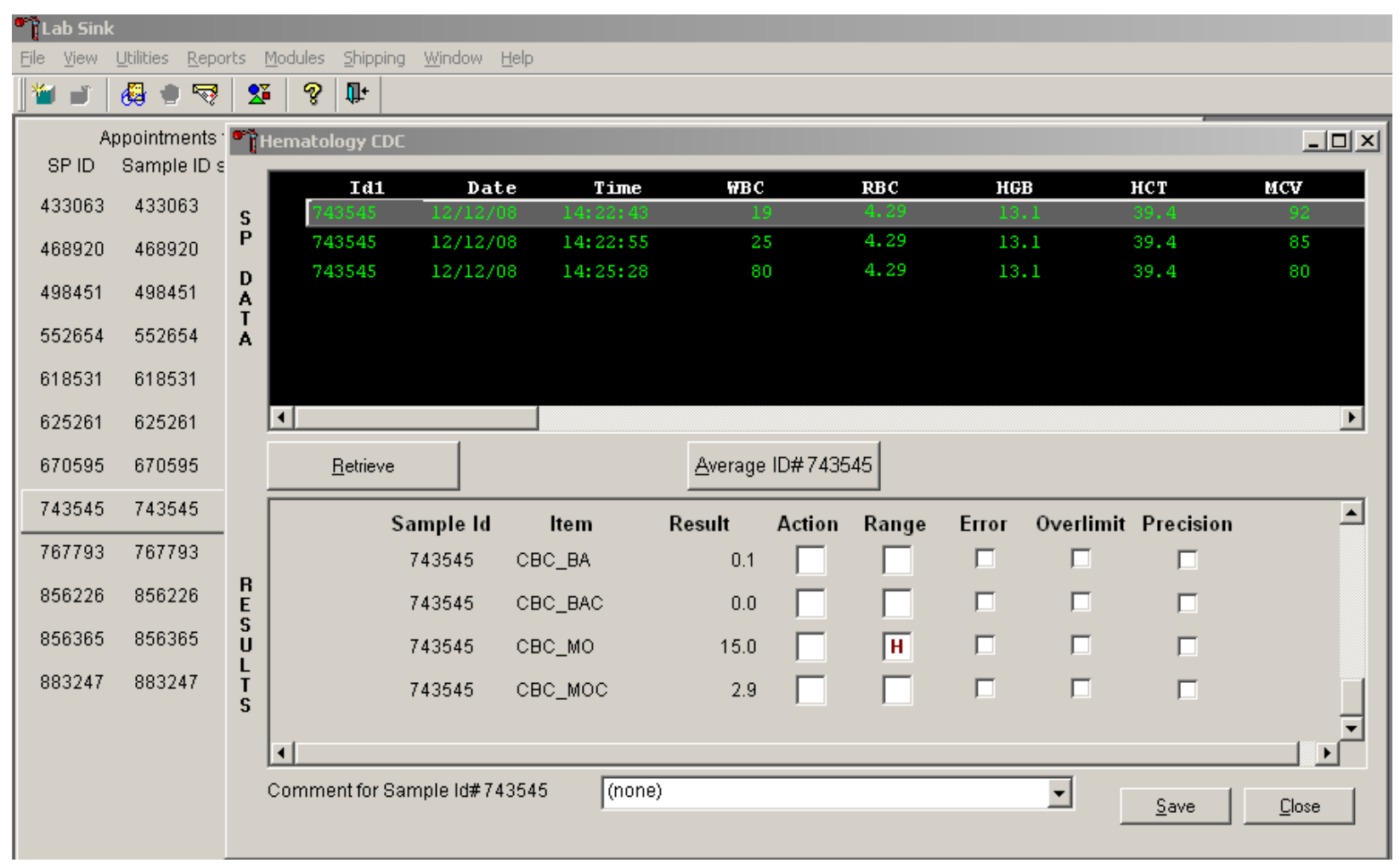




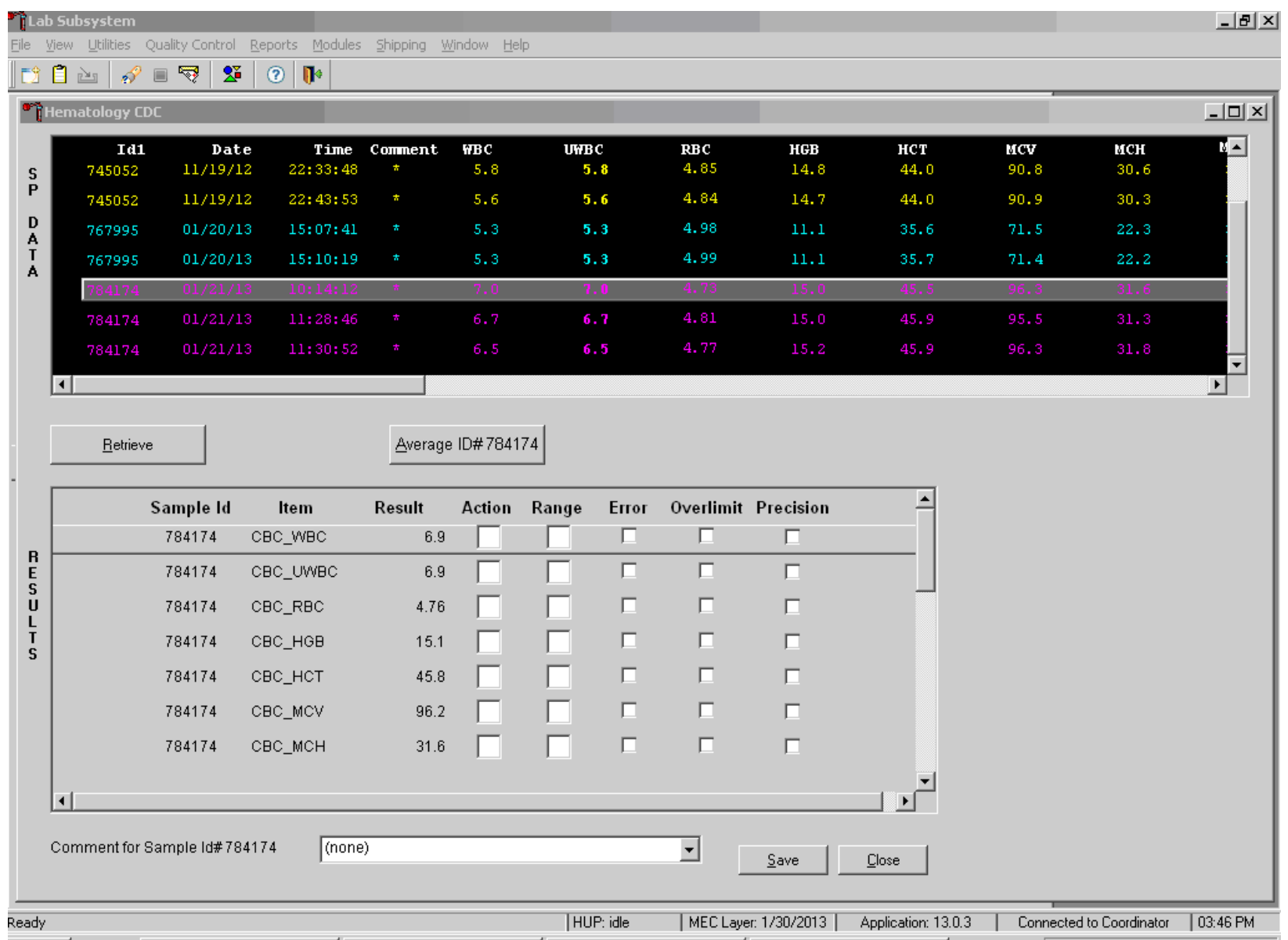

If all parameters of the two runs now meet precision limits (there are no checkmarks in the Precision column), save the results to the database. To save the result to the database, use the mouse to direct the mouse arrow to the Save button and left click or type [Shift] [S/s]. After results are saved, they are erased from the screen. To exit without saving the result to the database, use the mouse to direct the mouse arrow to the Close button and left click or type [Shift] [C/c].

If necessary, run the specimen a fourth time and evaluate.

If precision limits are still not met for any parameter, run the blood tube a fourth time. Delete the outlier run, re-average, evaluate, and save the results. If after four runs precision limits are still not met for any parameter, a comment is automatically attached to the results. Do not run a specimen more than four times. 


\section{Attaching comments to the results}

Enter a comment for any run where results were repeated and confirmed, the equipment failed, or there was a laboratory error.

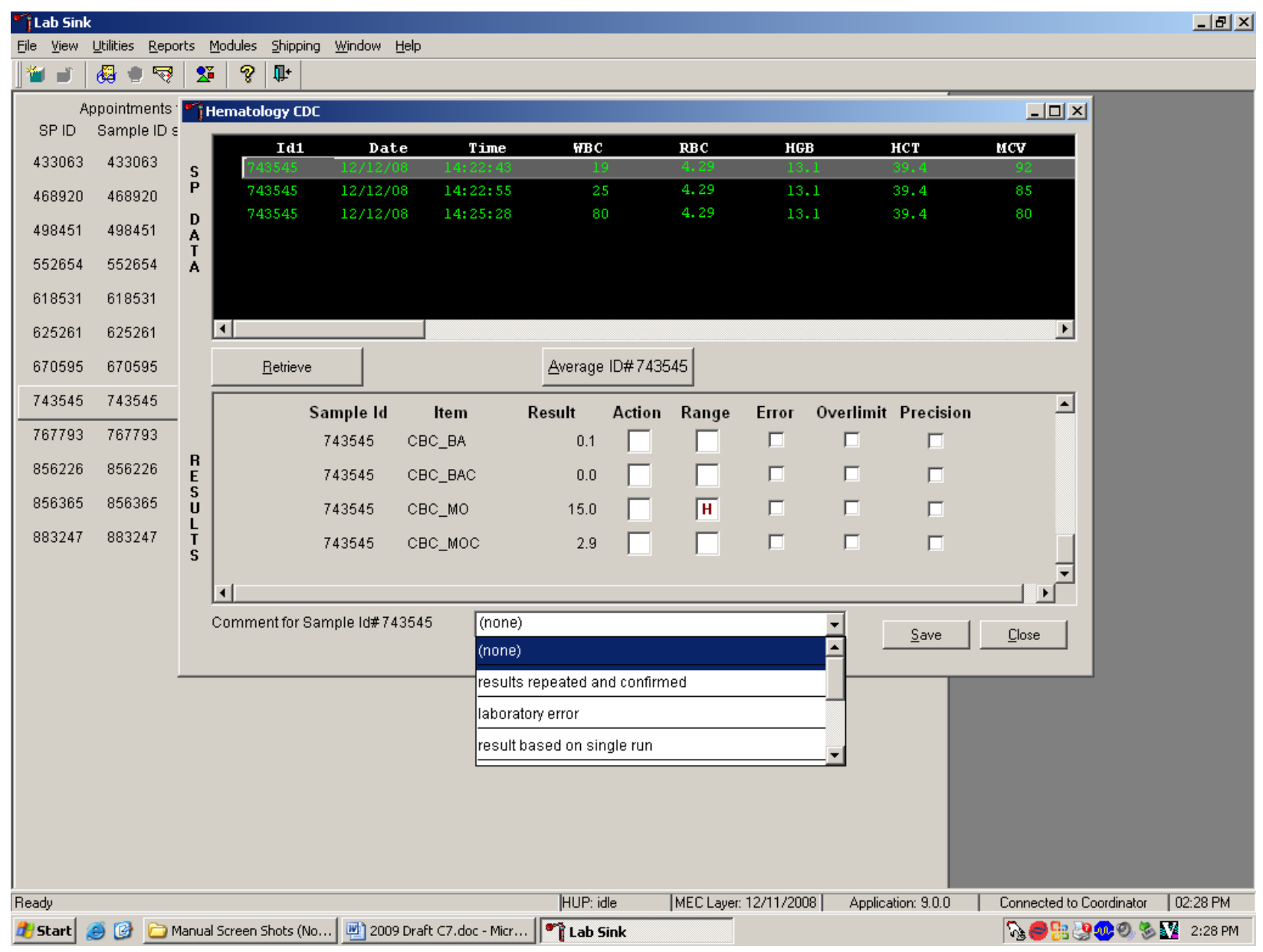




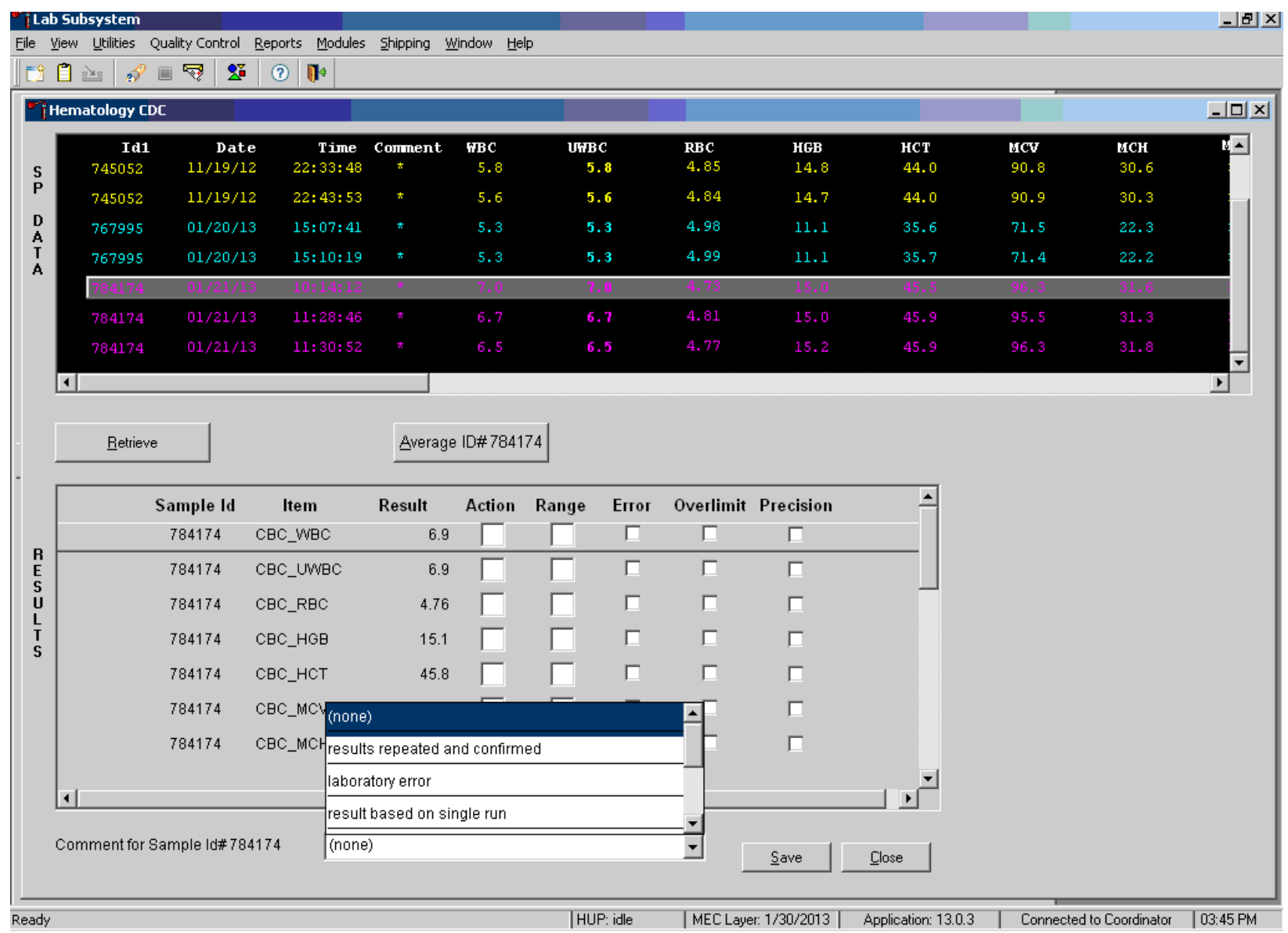

To attach a comment to the results in the database, use the mouse to direct the mouse arrow to the drop-down list on the Comment box, left click to display the codes, drag the mouse arrow to the most appropriate choice, and left click. Alternatively, to record a comment, use the mouse to direct the mouse arrow to the drop-down list on the Comment box, click to display the codes, use the up and down keyboard arrows to scroll through the choices or type the first letter of the desired comment code, and when the correct choice is highlighted, left click. 


\begin{tabular}{|l|l|}
\hline \multicolumn{1}{|c|}{ Comment } & \multicolumn{1}{c|}{ Use when: } \\
\hline Results repeated and confirmed & $\begin{array}{l}\text { Any parameter exceeded an action, range, or } \\
\text { precision limit and the specimen (blood tube) } \\
\text { was run more than once. }\end{array}$ \\
\hline Laboratory error & There was a technologist error. \\
\hline Result based on single run & $\begin{array}{l}\text { The quantity of specimen is insufficient to run it } \\
\text { more than once. }\end{array}$ \\
\hline Equipment failure & The Coulter ${ }^{\circledR}$ malfunctioned. \\
\hline No diff results/diff voteout & $\begin{array}{l}\text { The Coulter }{ }^{\circledR} \text { does not report parameter results } \\
\text { for any part of the differential or the instrument } \\
\text { reported a voteout for any of the differential } \\
\text { parameters. }\end{array}$ \\
\hline Clotted & The blood is clotted. \\
\hline Not enough blood & $\begin{array}{l}\text { There is insufficient blood to perform at least one } \\
\text { complete aspiration. }\end{array}$ \\
\hline
\end{tabular}

Save the result after attaching a comment.

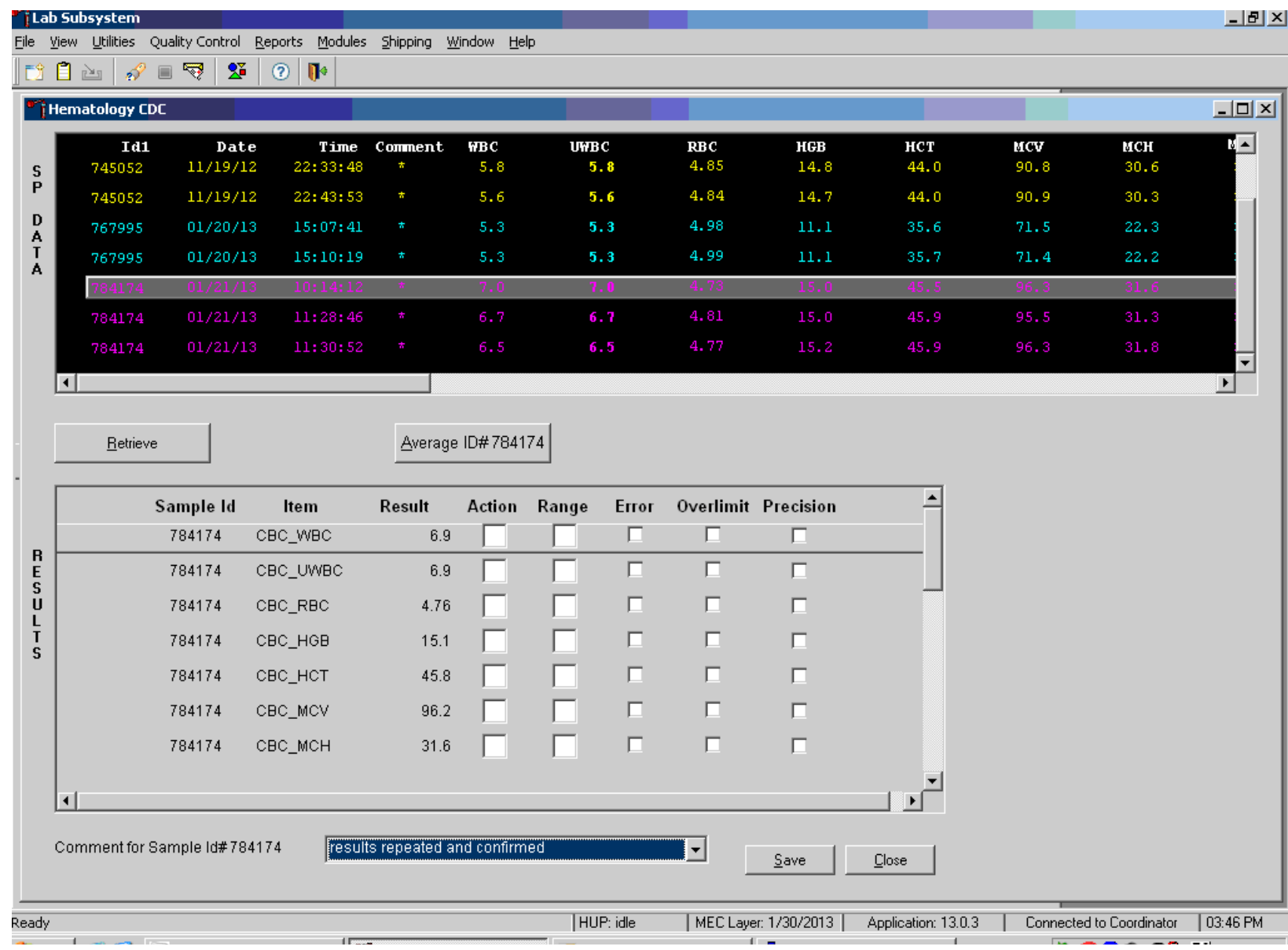


Save the averaged result and update the comment, by using the mouse to direct the mouse arrow to the Save button and left click or type [Shift] $[\mathrm{S} / \mathrm{s}]$. To exit without saving any data to the database, use the mouse to direct the mouse arrow to the Close button and left click or type [Shift] [C/c].

6. Using alternative data manipulation choices

Retain data for multiple SPs in the SP Data section of the window.

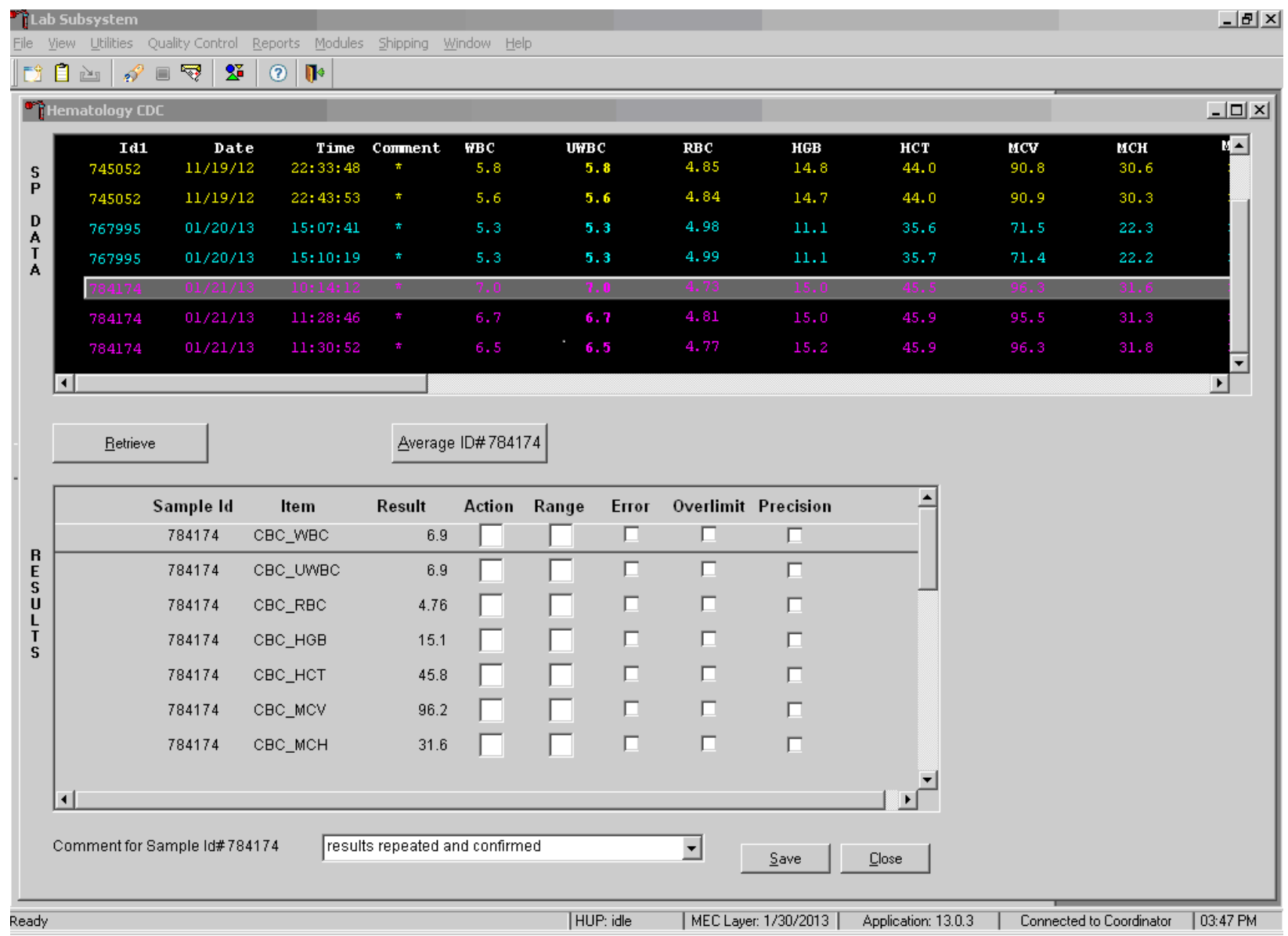

In general, run one SP in duplicate through the Coulter, retrieve the data, average the result, and save the data to the database. The Hematology module will display data for multiple SPs in the SP Data section. Each individual SP's results or pairs of results are displayed in a different color. To select or highlight any one SP, use the mouse to direct the mouse arrow to the row containing the correct SP ID and left click. 
Average the results for one SP at a time.

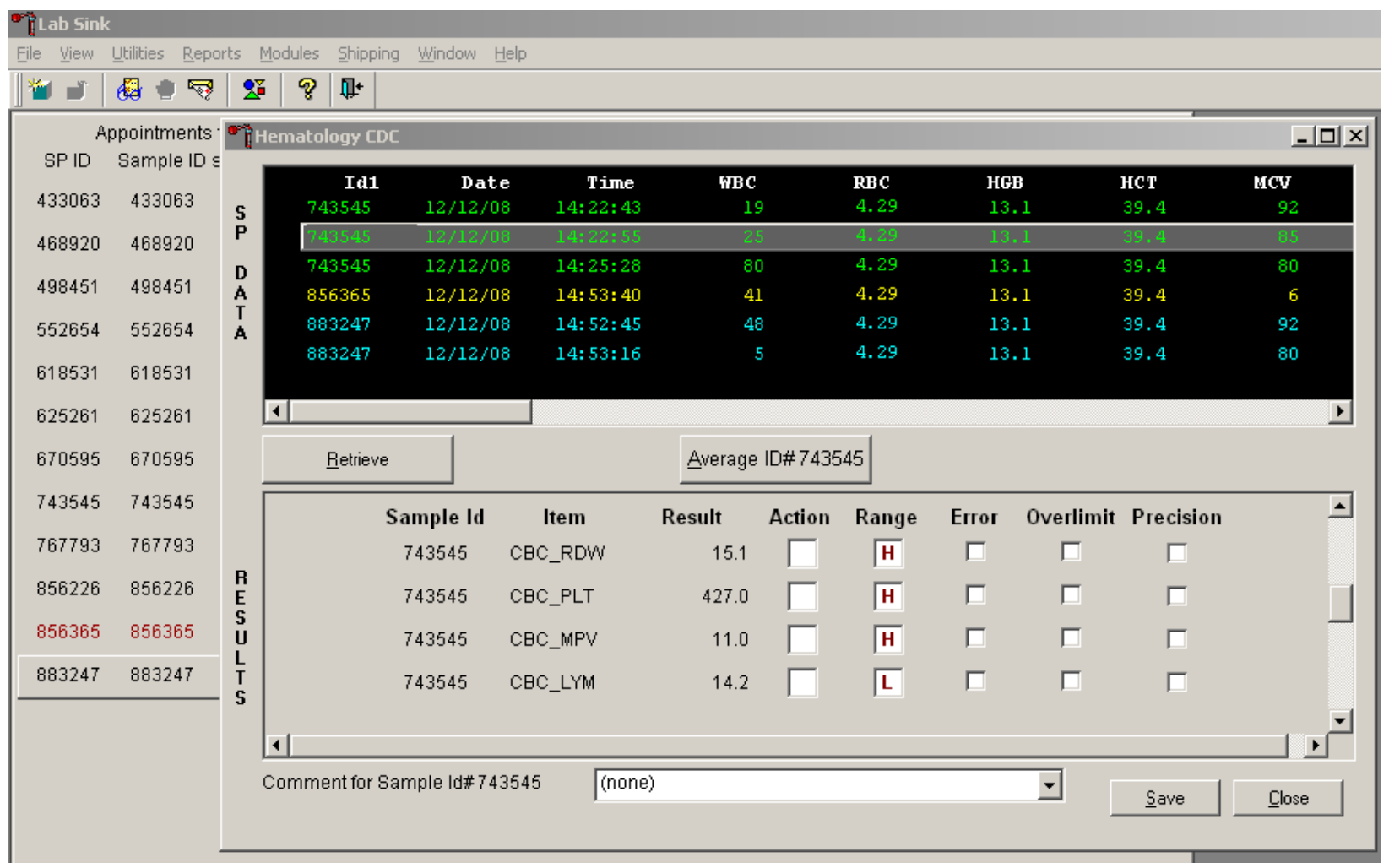

Evaluate each parameter for error, overlimit, and precision checkmarks, and " $\mathrm{H}$ " or "L" action limit and reference range flags. If there are no checkmarks in the Precision column (no precision limit was exceeded), save the result to the database. To save the result to the database, use the mouse to direct the mouse arrow to the Save button and left click or type [Shift] [S/s]. To exit without saving the result to the database, use the mouse to direct the mouse arrow to the Close button and left click or type [Shift] [C/c]. If there are any Precision checkmarks, run a 5C cell control, evaluate the control results, rerun the blood tube a third time, re-average, evaluate, and save the results. 
Use alternative data manipulation choices as desired.

Use the "Clear All Downloads" function to delete all data for all SPs in the SP Data section.

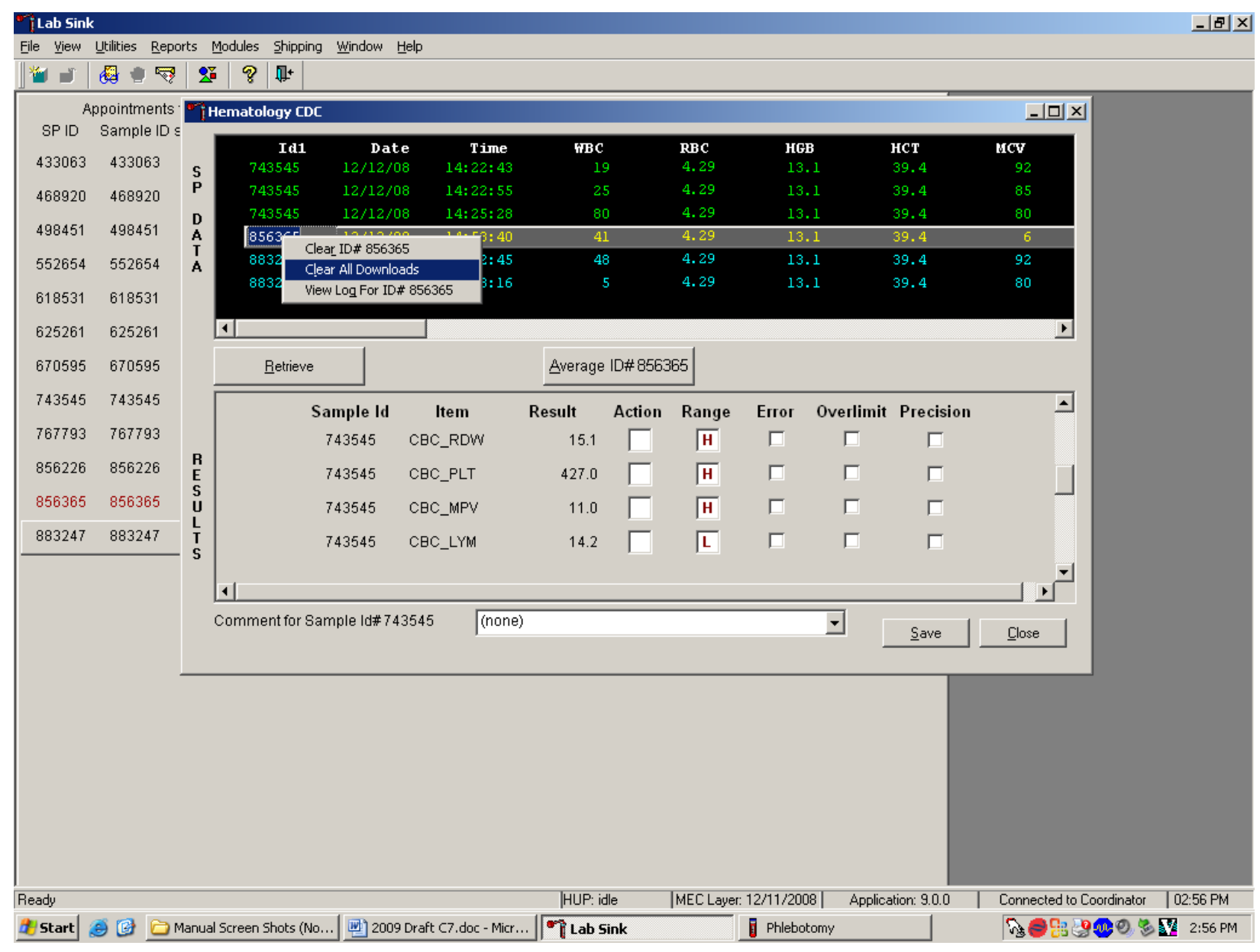




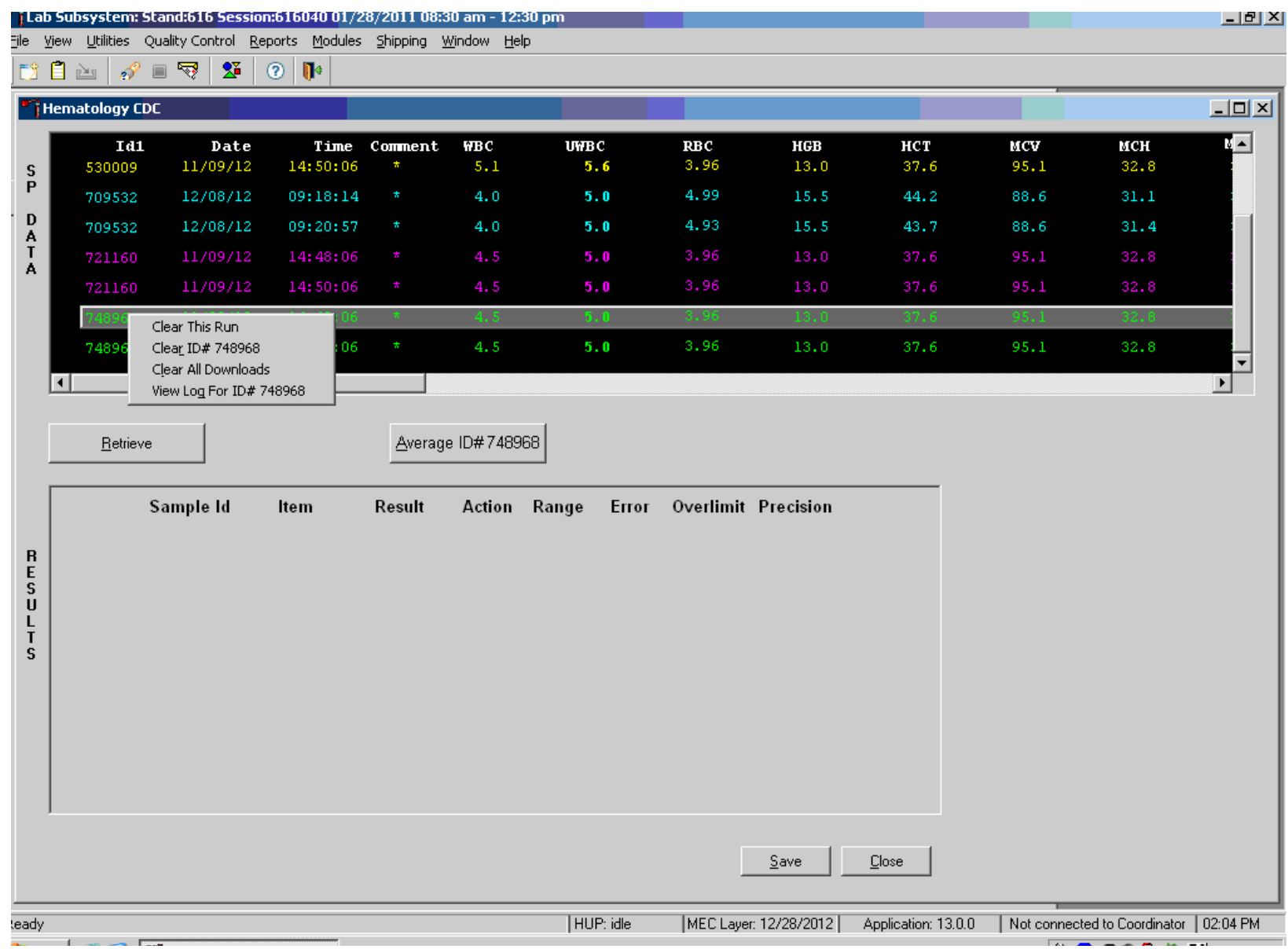

To clear all downloads from the SP Data section, use the mouse to direct the mouse arrow to any individual row in the SP Data section and right click to display the alternative data manipulation choices. To select "Clear All Downloads," drag the mouse arrow to \{Clear All Downloads\} and left click or type [Shift] [L/1]. 
Release the mouse button to clear all downloads.

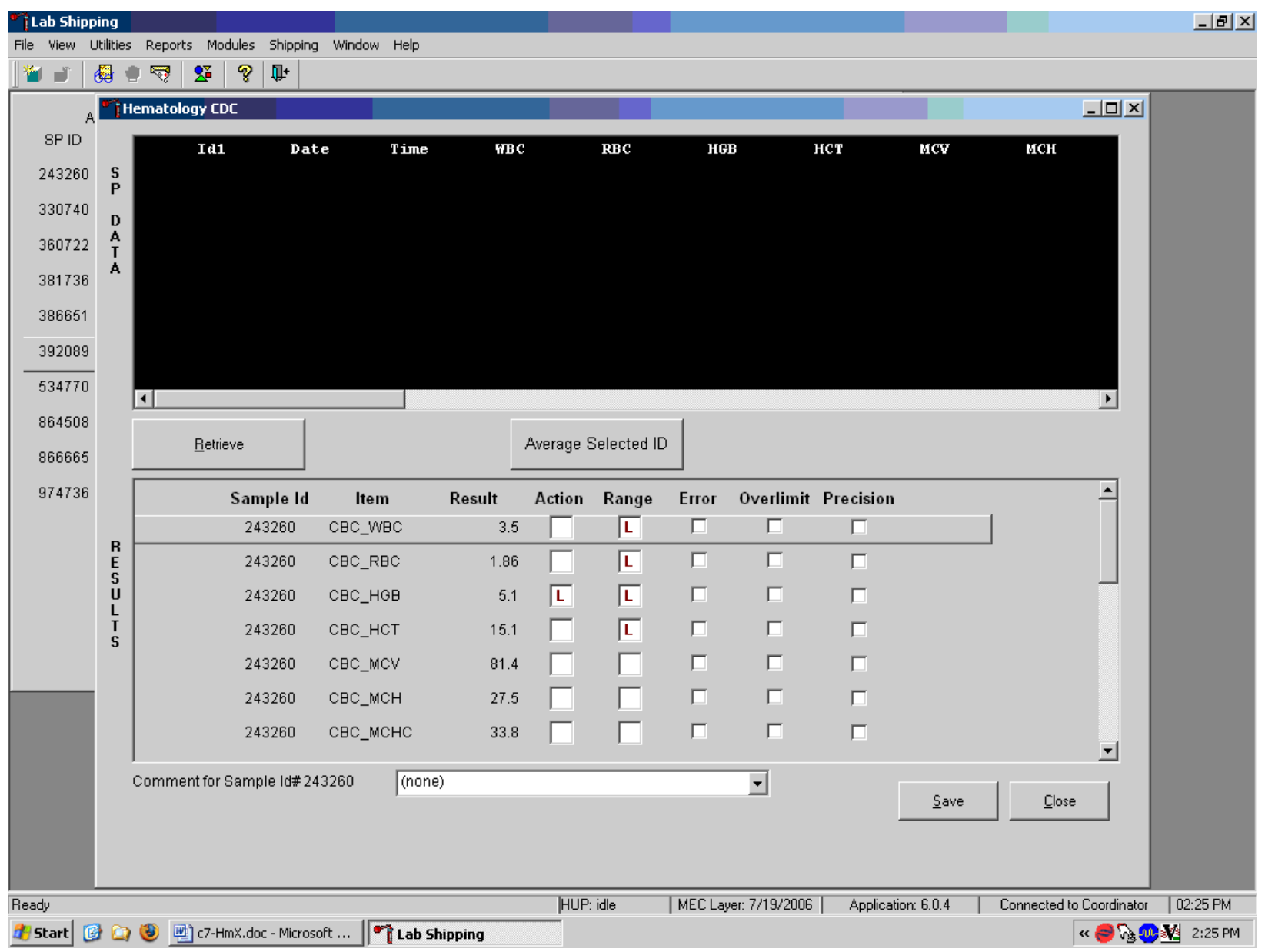

All data in the SP Data section are erased but any averaged result remains in the Results section of the window. To clear the result section, close the Hematology module. To close the hematology module, use the mouse to direct the mouse arrow to the Close button and left click or type [Shift] [C/c]. 
Use the data manipulation functions to erase or delete one SP's data in the SP Data section.

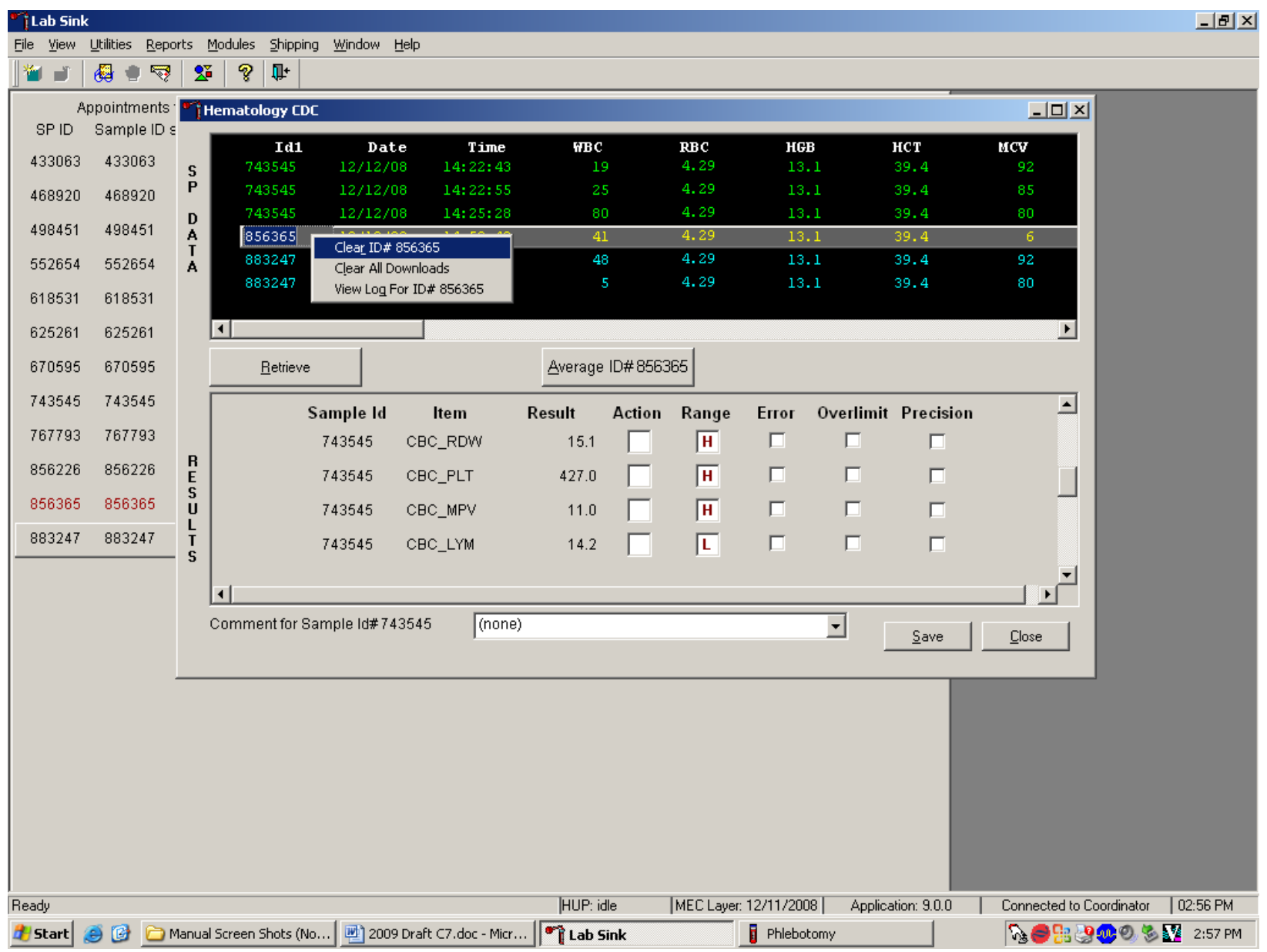

To clear the Coulter ${ }^{\circledR}$ downloads for any one SP, use the mouse to direct the mouse arrow to the individual row in the SP Data section, right click to display the alternative data manipulation choices, drag the mouse arrow to $\{$ Clear ID $\# X X X X X X\}$, and right or left click or type [Shift] $[\mathrm{R} / \mathrm{r}]$. Release the mouse button to clear this download. Retransmit, retrieve, average, and save the data. 
Use the "View Log for ID\# $X X X X X X$ " choice to view all Coulter ${ }^{\circledR}$ data, averages, and precision limit values for any SP.

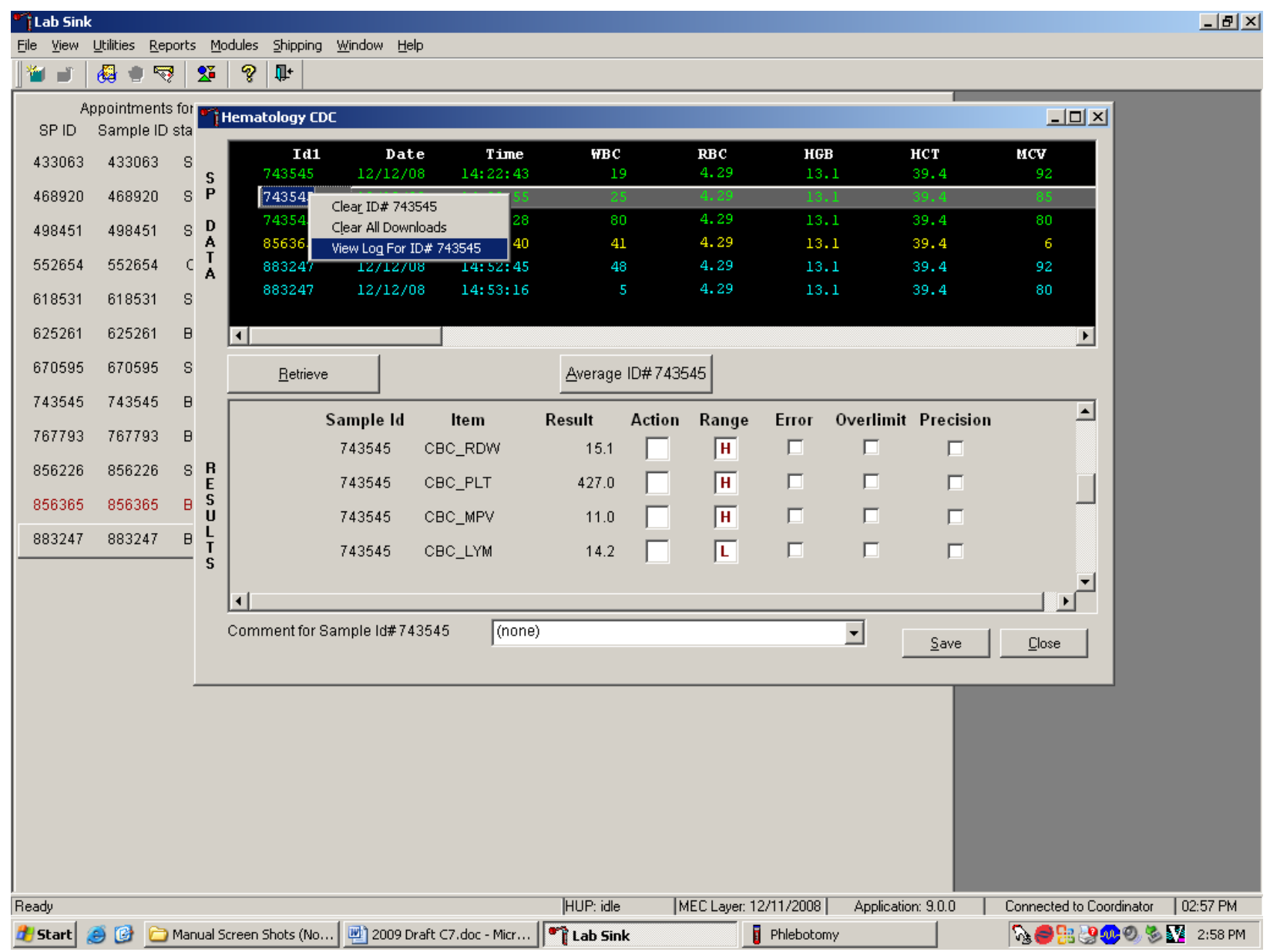

To view the detailed log for any one SP's result, use the mouse to direct the mouse arrow to the individual row in the SP Data section and right click to display the alternative data manipulation choices. Drag the mouse arrow to $\{$ View Log for ID $X X X X X X\}$ and release the mouse button to view the detailed log for this SP's results. 
Review the SP's log.

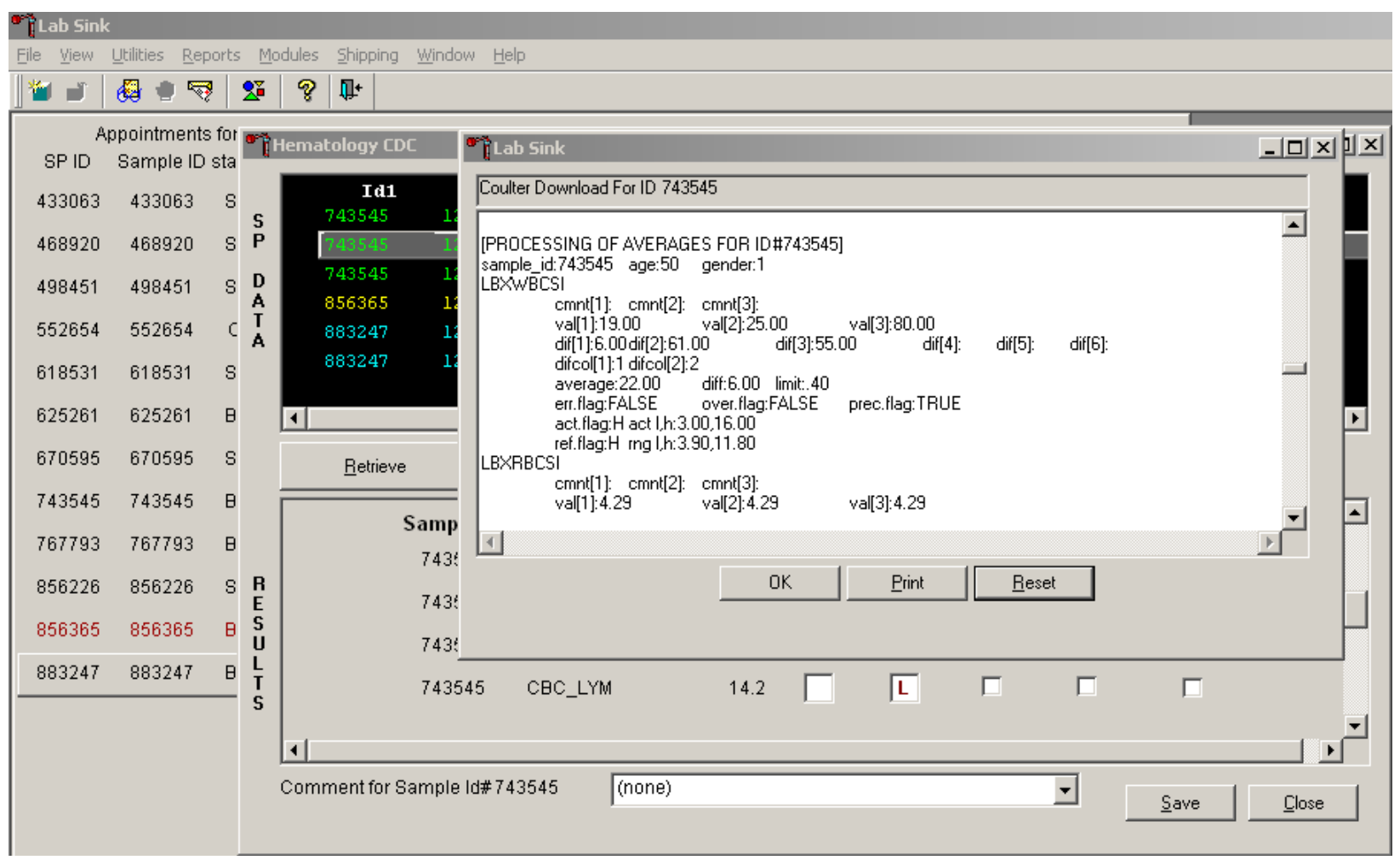

The SP's log contains detailed information for each parameter and each run. When finished reviewing the information, use the mouse to direct the mouse arrow to the OK button to close the window. To print the log, drag the mouse arrow to the Print button and left click or type [Shift] [P/p]. To clear the log and close the screen, use the mouse to direct the mouse arrow to the Reset button and left click, or type [Shift] [R/r], or select [Enter].

\subsubsection{DxH 800 Suspect Messages}

1. Suspect messages flag an abnormal cell distribution or population. The system generates these messages according to an internal algorithm. Specific suspect messages indicate some abnormalities that exhibit characteristic cluster patterns. Suspect messages indicate the possibility of a particular abnormality. Not every atypical scatterplot has a corresponding suspect message.

2. Remedial action - Rerun the specimen. 
1. The MEC physician reviews and interprets all $\mathrm{CBC}$ results. Results outside action limits flash and transmit to the physician immediately. The physician determines if referral for the SP for treatment is necessary.

2. The medical technologist sends an observation to the physician whenever a critical or action limit is detected for any $\mathrm{CBC}$ parameter. This observation includes the date, time, responsible laboratory individual, person notified, and test results.

Send an observation on any SP scheduled into the MEC session. Access the observation function.

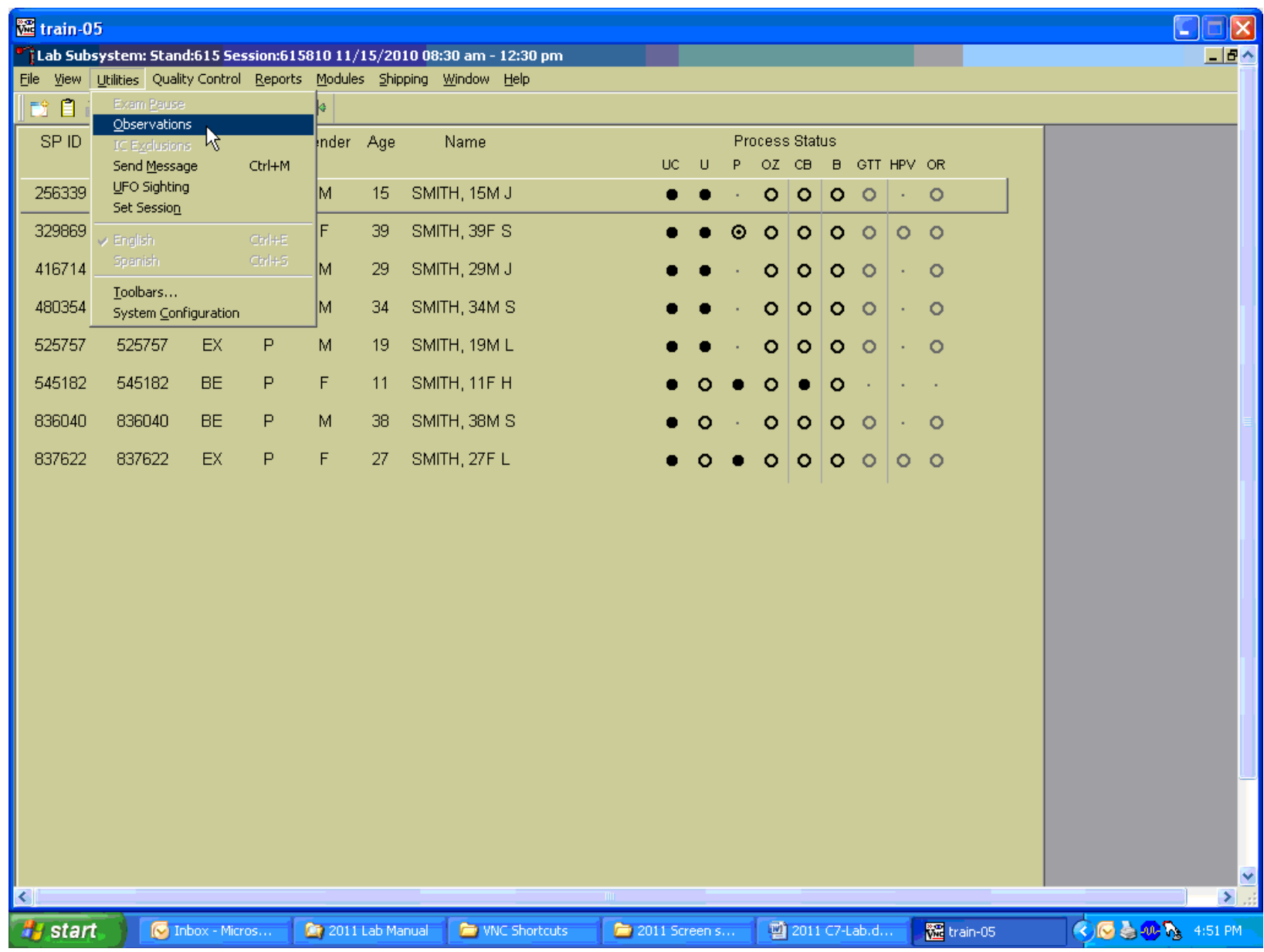

To access the observation function, use the mouse to direct the mouse arrow to \{Utilities\} in the menu bar, left click, drag the mouse arrow to \{Observations\}, and left click, or type [Alt] [U/u], [O/o]. 
Select or highlight the correct SP.

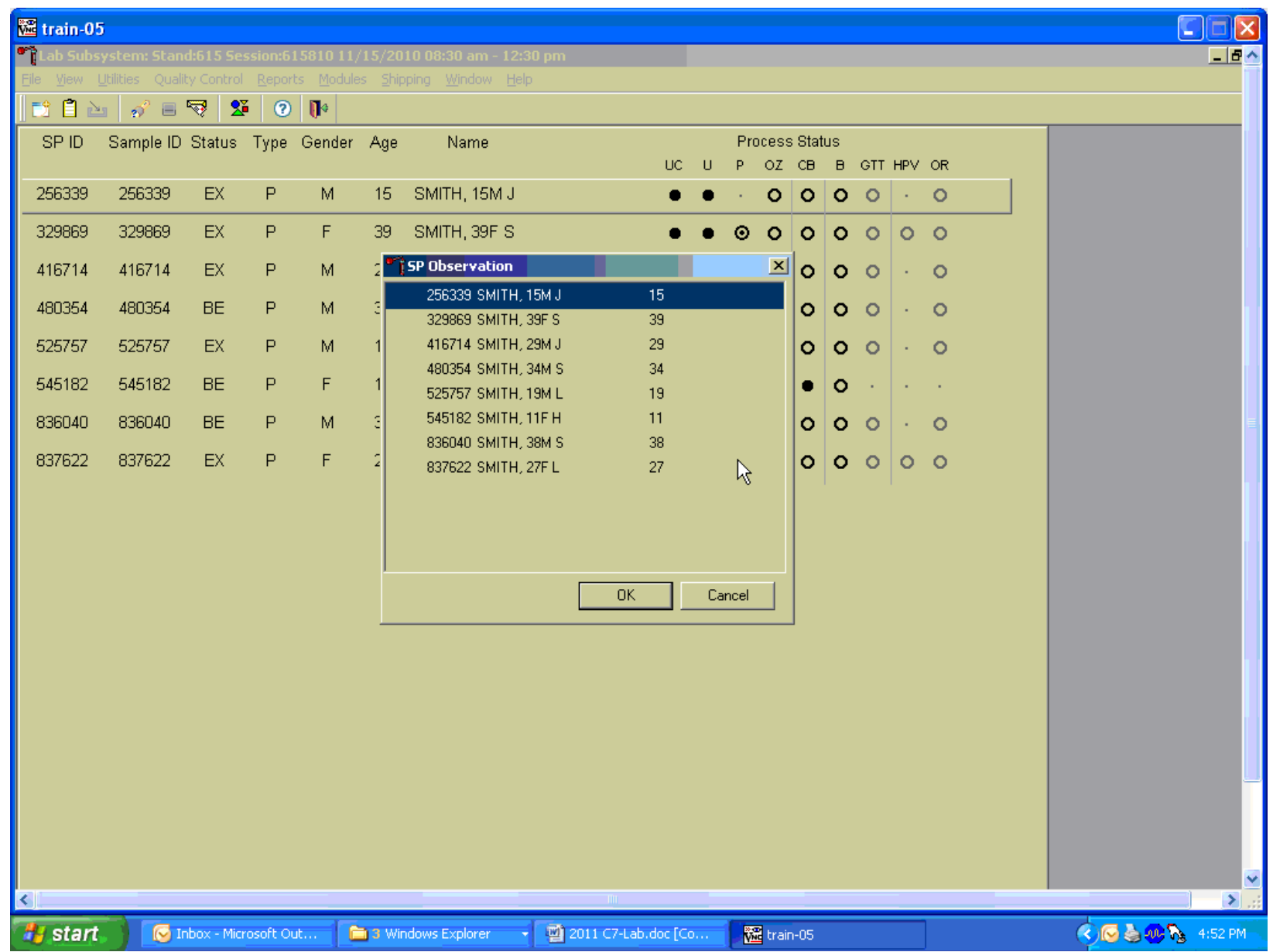

To select or highlight an SP, drag the mouse arrow to the correct SP and left click or use the up and down arrows to move up and down the list. Verify that the SP ID, name, and age are correct. Use the scroll bar to view the complete list of SPs. To continue, use the mouse to direct the mouse arrow to the OK button and left click, or select [Enter]. To cancel these actions and exit the observation function, use the mouse to direct the mouse arrow to the Cancel button and left click. 
The observation window displays.

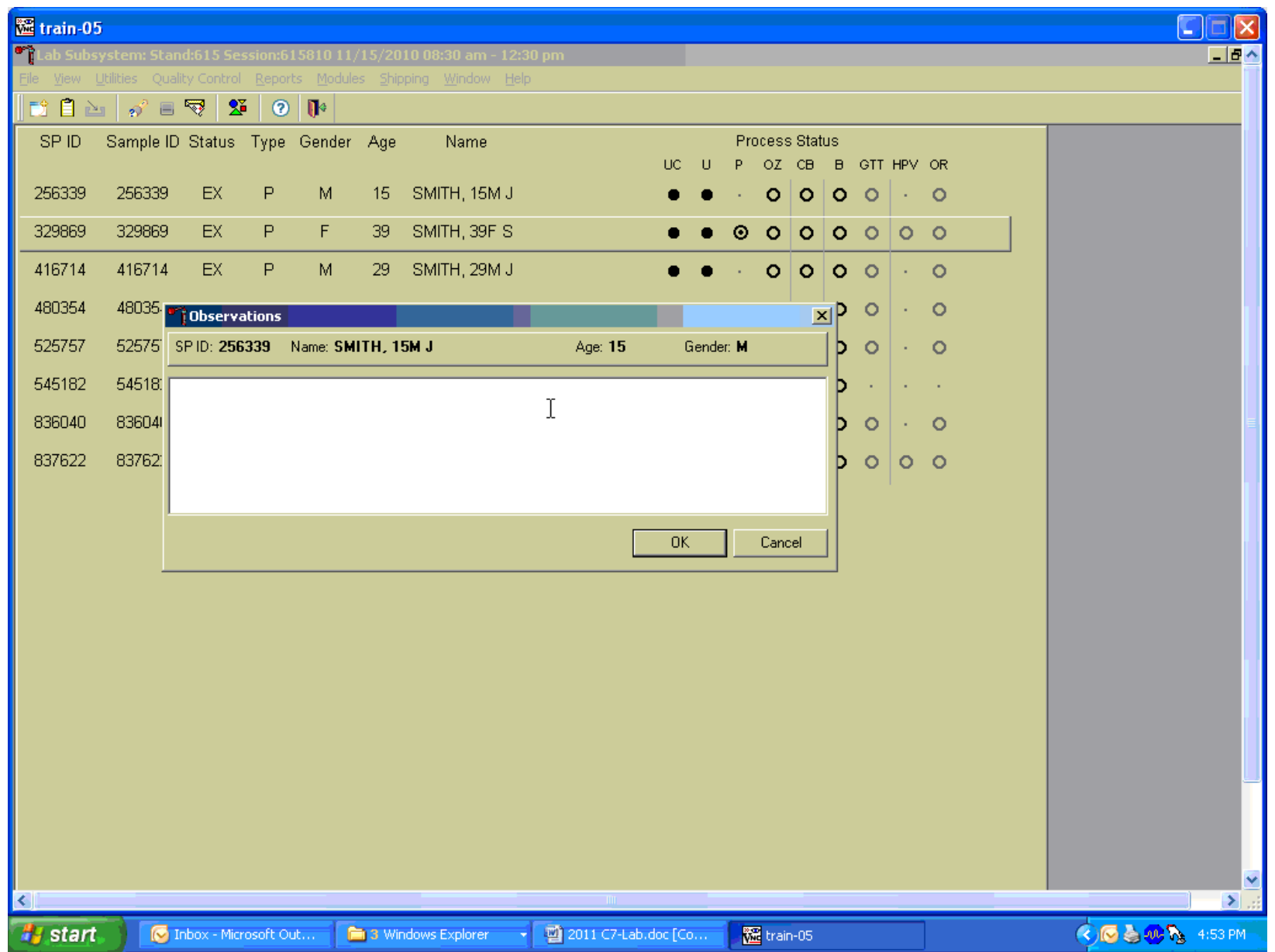

The observation window contains the SP ID, name, age, and gender. Type the observation using the keyboard. To send the observation to the physician, use the mouse to direct the mouse arrow to the OK button and left click, or select [Enter]. To cancel these actions or to exit the observation window without entering an observation, use the mouse to direct the mouse arrow to the Cancel button and left click. 
Enter the observation on a SP.

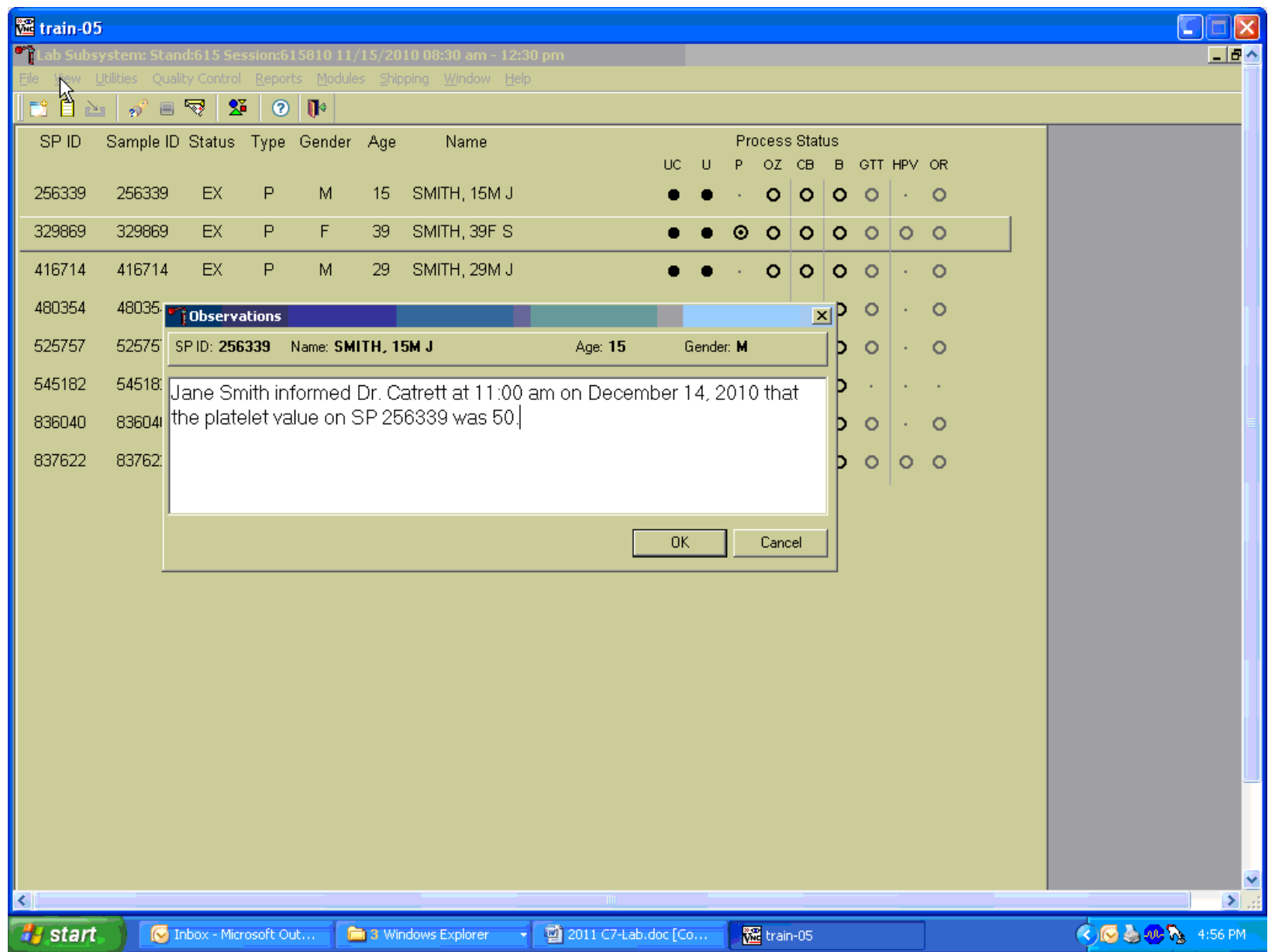

Document the date, time, responsible laboratory individual, person notified, and test results, including the parameter.

6.21 Limitations of Method: Specimen Rejection, Interfering Substances, and Conditions

This method limits samples to human whole blood.

\subsubsection{Specimen Rejection}

1. Reject clotted specimens and recollect. 


\subsubsection{Interfering Substances and Conditions}

Because the Coulter ${ }^{\circledR}$ directly measures RBC, WBC, Hgb, and Diff \%, it is most important to concentrate on analytes and substances that interfere with these parameters. The Coulter ${ }^{\circledR}$ calculates HCT, MCH, MCHC, and DIFF \# parameters. The Coulter ${ }^{\circledR}$ derives MCV, RDW, PLT, and MPV from $\mathrm{RBC}$ or platelet histograms. The following are possible interfering substances or conditions:

- Abnormal BUN, glucose, or sodium levels could affect the MCV.

- Abnormal WBCs could affect lymphocytes, monocytes, and granulocytes.

- Abnormally small WBCs could affect white count, lymphocytes, monocytes, and granulocytes.

- Clumped platelets could affect white count, lymphocytes, monocytes, granulocytes, $\mathrm{RBC}, \mathrm{MCV}, \mathrm{RDW}$, platelet count, and MPV.

- Cryofibrinogen and cryoglobulin crystals could affect white count, lymphocytes, monocytes, granulocytes, RBC, hemoglobin, platelet count, and MPV.

- An elevated WBC count could affect RBC, hemoglobin, MCV, RDW, platelet count, and MPV parameters.

- Fragile WBCs could affect white count, lymphocytes, monocytes, granulocytes, platelet count, and MPV.

- Giant platelets could affect white count, lymphocytes, monocytes, granulocytes, RBC, $\mathrm{MCV}, \mathrm{RDW}$, platelet count, and MPV.

- Hemolyzed specimens could affect RBC, hemoglobin, platelet count, and MPV.

- Lipemic specimens could affect MCV.

- Severely icteric plasma causes increased hemoglobin. Evaluate CBC result carefully and report all parameters except the hemoglobin result.

- Nucleated RBCs could affect the white count, lymphocytes, monocytes, granulocytes, and hemoglobin values.

- WBC - Certain unusual RBC abnormalities that resist lysing, nucleated RBCs, fragmented WBCs, agglutinated WBCs, any unlysed particles greater than $35 \mathrm{fL}$, very large or aggregated platelets as when anticoagulated with oxalate or heparin, specimens containing fibrin, cell fragments, or other debris such as pediatric and oncology specimens. 
- $\quad \mathrm{RBC}-$ Very high WBC count, high concentration of very large platelets, agglutinated RBCs, RBCs smaller than $36 \mathrm{fL}$, specimens containing fibrin, cell fragments, or other debris such as pediatric and oncology specimens.

- Hgb - Very high WBC count, severe lipemia, heparin, certain unusual RBC abnormalities that resist lysing, or anything that increases the turbidity of the sample such as elevated levels of triglycerides.

- $\mathrm{MCV}-$ Very high WBC count, high concentration of very large platelets, agglutinated $\mathrm{RBCs}, \mathrm{RBC}$ fragments that fall below the 36-fL threshold, or rigid RBCs.

- $\quad$ RDW - Very high WBC count, high concentration of very large or clumped platelets as in blood anticoagulated with oxalate or heparin, RBCs below the 36-fL threshold, two distinct populations of RBCs, RBC agglutinates, or rigid RBCs.

- Plt - Very small red blood cells near the upper threshold, cell fragments, clumped platelets as with oxalate or heparin, platelet fragments, or cellular debris near the lower platelet threshold.

- MPV - Known factors that interfere with the Plt count and shape of the histogram or known effects of EDTA.

- Hct - Known factors that interfere with the parameters used for computation: RBC and MCV.

- $\mathrm{MCH}-\mathrm{Known}$ factors that interfere with the parameters used for computation: Hgb and $\mathrm{RBC}$.

- $\mathrm{MCHC}-\mathrm{Known}$ factors that interfere with the parameters used for computation: Hgb, $\mathrm{RBC}$, and $\mathrm{MCV}$.

- Diff Parameters - Known factors that affect the WBC count as listed above or high triglycerides that affect lysing. 


\subsection{Reference Ranges}

\section{Males}

\begin{tabular}{|l|c|c|c|c|c|c|c|c|}
\hline \multirow{2}{*}{\multicolumn{1}{|c}{ Age in years }} & \multicolumn{2}{c|}{$1-5$} & \multicolumn{2}{c|}{$6-18$} & \multicolumn{2}{c|}{$19-65$} & \multicolumn{2}{c|}{$66+$} \\
\cline { 2 - 9 } & 2.5 & 97.5 & 2.5 & 97.5 & 2.5 & 97.5 & 2.5 & 97.5 \\
\hline White blood cell count (SI) & 4.3 & 14.6 & 3.6 & 11.5 & 3.9 & 11.8 & 3.8 & 12.1 \\
\hline Red cell count (SI) & 3.98 & 5.3 & 4.14 & 5.78 & 4.18 & 5.86 & 3.57 & 5.67 \\
\hline Hemoglobin (g/dL) & 10.7 & 14.2 & 11.9 & 16.9 & 13.1 & 17.5 & 11.4 & 17.1 \\
\hline Hematocrit (\%) & 32.1 & 41.7 & 35.3 & 49.9 & 38.7 & 51.4 & 33.9 & 50.9 \\
\hline Mean cell volume (fL) & 68.2 & 88.8 & 75.6 & 94.6 & 79.8 & 99.1 & 81.4 & 102.7 \\
\hline Mean cell hemoglobin (pg) & 22.3 & 30.6 & 25.0 & 32.3 & 26.3 & 34.0 & 26.3 & 35.0 \\
\hline MCHC (g/dL) & 32.3 & 35.6 & 32.3 & 35.3 & 32.3 & 35.3 & 32.1 & 35.1 \\
\hline Red cell distribution width (\%) & 11.4 & 15.8 & 11.4 & 14.0 & 11.4 & 14.5 & 11.8 & 16.2 \\
\hline Platelet count (\%) SI & 212 & 546 & 179 & 439 & 152 & 386 & 124 & 384 \\
\hline Mean platelet volume (fL) & 6.1 & 8.9 & 6.6 & 10 & 6.8 & 10.1 & 6.6 & 10.2 \\
\hline Lymphocyte percent (\%) & 22.8 & 68.4 & 17.5 & 54.3 & 16.1 & 47.9 & 12.3 & 46.4 \\
\hline Monocyte percent (\%) & 4.6 & 15.2 & 4.8 & 13.7 & 4.4 & 13.5 & 4.6 & 14.0 \\
\hline Segmented neutrophils percent (\%) & 17.6 & 67.1 & 30.3 & 72.8 & 37.8 & 74.6 & 39.5 & 78.1 \\
\hline Eosinophils percent (\%) & 0.7 & 11.3 & 0.7 & 11.5 & 0.7 & 8.5 & 0.6 & 8.8 \\
\hline Basophils percent (\%) & 0.1 & 2.5 & 0.1 & 1.6 & 0.1 & 1.6 & 0.1 & 1.6 \\
\hline
\end{tabular}




\section{Females}

\begin{tabular}{|c|c|c|c|c|c|c|c|c|}
\hline \multirow[b]{2}{*}{ Age in years } & \multicolumn{2}{|c|}{$1-5$} & \multicolumn{2}{|c|}{$6-18$} & \multicolumn{2}{|c|}{$19-65$} & \multicolumn{2}{|c|}{$66+$} \\
\hline & 2.5 & 97.5 & 2.5 & 97.5 & 2.5 & 97.5 & 2.5 & 97.5 \\
\hline White blood cell count (SI) & 4.3 & 14.0 & 3.9 & 12.2 & 4.1 & 12.9 & 4.0 & 11.6 \\
\hline Red cell count (SI) & 3.96 & 5.28 & 3.84 & 5.24 & 3.64 & 5.2 & 3.51 & 5.34 \\
\hline Hemoglobin $(\mathrm{g} / \mathrm{dL})$ & 11.0 & 14.2 & 11.2 & 15.1 & 10.6 & 15.6 & 10.9 & 15.9 \\
\hline Hematocrit (\%) & 32.5 & 41.9 & 33.5 & 44.6 & 32.0 & 45.9 & 32.8 & 47.0 \\
\hline Mean cell volume (fL) & 70.2 & 89.1 & 74.7 & 94.9 & 74.6 & 98.2 & 80.3 & 100.6 \\
\hline Mean cell hemoglobin (pg) & 23.3 & 30.8 & 24.5 & 32.6 & 24.3 & 33.8 & 26.4 & 34.5 \\
\hline $\mathrm{MCHC}(\mathrm{g} / \mathrm{dL})$ & 32.4 & 35.5 & 32.3 & 35.3 & 32.1 & 35.3 & 32.3 & 35.1 \\
\hline Red cell distribution width (\%) & 11.3 & 15.4 & 11.3 & 14.8 & 11.4 & 16.3 & 11.6 & 16.3 \\
\hline Platelet count (\%) SI & 215 & 547 & 190 & 446 & 168 & 441 & 155 & 428 \\
\hline Mean platelet volume (fL) & 6.1 & 8.9 & 6.6 & 10.0 & 6.8 & 10.2 & 6.7 & 10.5 \\
\hline Lymphocyte percent (\%) & 21.6 & 68.8 & 17.2 & 54.7 & 14.1 & 47.6 & 13.7 & 46.9 \\
\hline Monocyte percent $(\%)$ & 4.2 & 14.4 & 4.3 & 12.7 & 3.8 & 11.6 & 4.4 & 12.8 \\
\hline Segmented neutrophils percent (\%) & 19.4 & 69.5 & 31.9 & 74.3 & 39.8 & 78.1 & 40.9 & 78.1 \\
\hline Eosinophils percent (\%) & 0.6 & 9.9 & 0.6 & 9.9 & 0.6 & 7.3 & 0.6 & 7.5 \\
\hline Basophils percent $(\%)$ & 0.1 & 2.5 & 0.1 & 1.6 & 0.1 & 1.7 & 0.1 & 1.7 \\
\hline
\end{tabular}

3. Reference ranges for normal values were calculated from the NHANES data set (1999-2004) using 95 percent reference interval(s) determined nonparametrically, through ranking the observations and determining the lower (2.5th percentile) and the upper (97.5th percentile) reference limits. The nonparametric (ranking) method was used because most measured hematology parameters have a skewed, non-Gaussian distribution.

\subsection{Action Limits}

Action limits are a guide to inform the physician that a $\mathrm{CBC}$ result(s) is/are abnormal. Since all specimens are run in duplicate, there is no reason to retest the sample.

- WBC male and female (all ages) $<$ or $=$ to $3 \times 10^{3} \mu \mathrm{L}$ or $>$ or $=$ to $16.0 \times 10^{3} \mu \mathrm{L}$

- Hgb male and female $\quad(<6$ years $) \quad<6.5 \mathrm{~g} / \mathrm{dL}$ or $>14.5 \mathrm{~g} / \mathrm{dL}$

- Hgb female ( $>6$ years) $\quad<6.5 \mathrm{~g} / \mathrm{dL}$ or $>16.0 \mathrm{~g} / \mathrm{dL}$

- Hgb male ( $>6$ years) $<6.5 \mathrm{~g} / \mathrm{dL}$ or $>18.0 \mathrm{~g} / \mathrm{dL}$

- PLT male and female (all ages) $\quad<50 \times 10^{3} \mu \mathrm{L}$ or $>800 \times 10^{3} \mu \mathrm{L}$ 
Possible causes of abnormal parameters:

- High RBC, Hgb, or HCT - dehydration, polycythemia, shock, chronic hypoxia

- Low RBC, Hgb, or HCT - anemia, thalassemia, and other hemoglobinopathies

- Low MCV - microcytic anemia

- High MCV - macrocytic anemia, liver disease

- Low WBC - sepsis, marrow hypoplasia

- High WBC - acute stress, infection, malignancies

- Low platelets - risk of bleeding

- High platelets - risk of thrombosis

\subsection{Specimen Storage and Handling During Testing}

Specimen Storage:

- Store specimens capped and place on a rocker at room temperature until processed.

- Run within 24 hours of drawing.

\subsection{Alternative Method for Performing Test or Storing Specimens if Test System Fails}

There is no alternative method for this test. Store EDTA tube at room temperature for no more than 24 hours. Restore the instrument to functionality and then run the specimen.

\subsection{Test Results Reporting System: Protocol for Reporting Action Limits}

Results outside the action limits are automatically brought to the physician's attention for a decision as to "course-to-follow." 
All records, including QA/QC data will be maintained for 6 years. Use only numerical identifiers for SP results.

\subsection{Quality Control Summary Statistics and Graphs}

Chapter 13 includes a separate detailed description of the comprehensive quality control

plan. Monitor $6 \mathrm{C} \AA$ cell control results for bias and maintain results for the entire study period. Compare all three instruments using the CAP proficiency results.

\subsection{References}

UniCel DxH 800 Coulter Cellular Analysis System Instructions for Use. Beckman Coulter, Inc. Fullerton, CA 92835. www.BeckmanCoulter.com. 


\section{BLOOD PROCESSING}

\subsection{Introduction}

The purpose of blood processing is to (1) perform a complete blood count; (2) allocate blood into vessels for storage and transport to laboratories across the United States for analysis; and (3) allocate serum into vessels for future studies and storage. The purpose of packed cell processing is to allocate washed packed cells for storage and transport to two separate analysis laboratories Processing includes centrifuging and storing blood collection tubes; aliquoting whole blood into storage vessels; centrifuging, separating, and aliquoting plasma and serum into storage vessels; performing dilutions; washing and lysing whole blood cells; and storing the specimens.

\subsection{Equipment and Supplies}

The blood processing area includes two laboratory-grade refrigerators, four laboratory-grade freezers, two refrigerated centrifuges, two data terminals, a laminar flow hood, and two specimen rockers. This area also includes counter space for processing blood. Store extra processing supplies in cabinets and/or the trailer compartments. Exhibits 7-1 and 7-2 list nonconsumable and consumable supplies for blood processing.

Exhibit 7-1. Nonconsumable supplies for blood processing

\begin{tabular}{|ll|}
\hline Blood processing template $(6-11 / 12+$ and $1-2 / 3-5)$ & Nalge cryovial racks \\
\hline One Eppendorf pipette $100-5,000 \mu \mathrm{L}$ & Two Eppendorf pipettes $50-1,000 \mu \mathrm{L}$ \\
\hline One Eppendorf pipette $5-100 \mu \mathrm{L}$ & Pipette charging stand \\
\hline One pipette battery & 50 -mL graduated cylinder \\
\hline $13 \mathrm{~mm}$ half rack & Cube rack - 4-way flipper rack \\
\hline $16 \mathrm{~mm}$ full rack & Floor mat \\
\hline $16 \mathrm{~mm}$ half rack & Protective eyewear \\
\hline Deionized water bottle & Saline squeeze bottle \\
\hline
\end{tabular}


Exhibit 7-2. Consumable supplies for blood processing

\begin{tabular}{|c|c|}
\hline Cotton swabs & Eight gallon biohazard sharps container \\
\hline \multicolumn{2}{|l|}{ Biohazard bags $25 \times 35$} \\
\hline Kimwipes & Bench Kote paper \\
\hline Pipette tips $1,000 \mu \mathrm{L}$ & Pipette tips $200 \mu \mathrm{L}$ \\
\hline Pipette tips 5-mL & Wooden applicator sticks \\
\hline Zip closable bags & 3.5-mL transfer pipette nonsterile \\
\hline \multicolumn{2}{|l|}{$15.0 \mathrm{~mL}$ transfer pipette nonsterile } \\
\hline 5-mL cryovial sterile & 14-mL snap cap test tube \\
\hline Conical tube $50-\mathrm{mL}$ & 2-mL cryovial sterile \\
\hline 2-mL cryovial non-sterile & Caps for 2-mL cryovial \\
\hline Serum filters $13 \mathrm{~mm} \times 4$ inches & Serum filters $16 \mathrm{~mm} \times 6$ inches \\
\hline $\begin{array}{l}5.25 \times 5.25 \times 2.0 \text { inch cardboard box with } \\
9 \times 9 \text { cardboard grid }\end{array}$ & $\begin{array}{l}5.25 \times 5.25 \times 3.0 \text { inch cardboard box with } \\
9 \times 9 \text { cardboard grid }\end{array}$ \\
\hline $5.25 \times 5.25 \times 3.0$ inch cardboard box with $5 \times 5$ grid & 10x10 three inch box \\
\hline $\begin{array}{l}5.25 \times 5.25 \times 5.0 \text { inch cardboard box with } \\
5 \times 5 \text { foam insert }\end{array}$ & $7 \times 7$ two inch cardboard box \\
\hline $10 \times 10$ two-inch box & $5.25 \times 5.25 \times$ 3inch box with $7 \times 7$ grid \\
\hline Ascorbic acid 0.3 gm per vessel & $\begin{array}{l}\text { Nonsterile, powder-free, nonlatex gloves-small, } \\
\text { medium, large }\end{array}$ \\
\hline Ascorbic Acid 0.14 gm per vessel & Red cap insert \\
\hline Sterile saline & Deionized water \\
\hline Sharpie pens & Lab marker - fine \\
\hline
\end{tabular}

\subsection{Protocols}

Exhibits 7-3 through 7-6 illustrate the primary SP's protocol for blood processing. It is extremely important to follow the processing procedures outlined in these protocols and in this manual. Specimen loss or biased results will occur if the exact procedures are not followed. 
Exhibit 7-3. Primary SP's whole blood processing protocol

\begin{tabular}{|l|l|c|c|l|c|c|l|}
\hline ID & \multicolumn{1}{|c|}{ Test name } & Ages & $\begin{array}{c}\text { Sample } \\
(\mathrm{mL})\end{array}$ & Sample type & $\begin{array}{c}\text { Collection } \\
\text { type }\end{array}$ & $\begin{array}{c}\text { Vessel } \\
\text { type }\end{array}$ & \multicolumn{1}{|c|}{ Remarks } \\
\hline 54 & VOC Blood & $12+$ & 10.0 & Whole blood & $\begin{array}{c}7-\mathrm{mL} \\
\text { Gray }\end{array}$ & $10-\mathrm{mL}$ & $\begin{array}{l}1 / 2 \text { subsample } \\
1,2,4,5,10,11\end{array}$ \\
\hline 1 & $\begin{array}{l}\text { Lead/Cad/HG/ } \\
\text { Se/Mn }\end{array}$ & $1+$ & 0.4 & Whole blood & EDTA & $\begin{array}{l}2-\mathrm{mL} \\
\text { sterile }\end{array}$ & $\begin{array}{l}1 / 2 \text { subsample } \\
(1,2,4,5,10,11)\end{array}$ \\
\hline 3 & RBC Folate & $1+$ & 0.1 & Whole blood & EDTA & $2-\mathrm{mL}$ & $\begin{array}{l}\text { Dilute with 1-mL AA } \\
1 \%\end{array}$ \\
\hline 4 & Glycohem & $12+$ & 0.4 & Whole blood & EDTA & 2-mL & Refrigerate \\
\hline 5 & $\begin{array}{l}\text { Mercury, Ethyl } \\
\text { \& Methyl }\end{array}$ & $1+$ & 0.5 & Whole blood & EDTA & $\begin{array}{l}2-\mathrm{mL} \\
\text { sterile }\end{array}$ & $\begin{array}{l}1 / 2 \text { subsample } \\
(1,2,4,5,10,11)\end{array}$ \\
\hline
\end{tabular}

Exhibit 7-4. Primary SP's plasma processing protocol

\begin{tabular}{|l|l|c|c|c|c|c|c|}
\hline \multicolumn{1}{|c|}{ ID } & \multicolumn{1}{|c|}{ Test name } & Ages & $\begin{array}{c}\text { Sample } \\
(\mathrm{mL})\end{array}$ & Sample type & $\begin{array}{c}\text { Collection } \\
\text { type }\end{array}$ & $\begin{array}{c}\text { Vessel } \\
\text { type }\end{array}$ & Remarks \\
\hline 150 & Fluoride & $3-19$ & 1.0 & Plasma & EDTA & $2 \mathrm{~mL}$ & \\
\hline 9 & Glucose & $12+$ & 0.5 & Plasma & Gray & $2-\mathrm{mL}$ & Morning SPs only \\
\hline 98 & OGTT & $12+$ & 0.5 & Plasma & Gray & $2-\mathrm{mL}$ & $\begin{array}{l}\text { Morning SPs only, if } \\
\text { eligible }\end{array}$ \\
\hline
\end{tabular}

Exhibit 7-5. Primary SP's packed cells processing protocol

\begin{tabular}{|c|c|c|c|c|c|c|c|}
\hline ID & Test name & Ages & $\begin{array}{c}\text { Sample } \\
(\mathrm{mL})\end{array}$ & Sample type & $\begin{array}{c}\text { Collection } \\
\text { type }\end{array}$ & $\begin{array}{c}\text { Vessel } \\
\text { type }\end{array}$ & \multicolumn{1}{c|}{ Remarks } \\
\hline 88 & Acrylamide & $6+$ & 1.0 & Packed cells & EDTA & $2 \mathrm{~mL}$ & $\begin{array}{l}\text { Wash 3 times, lyse } \\
1 / 3 \text { subsample } \\
(1,2,5,11) \text { plus adult } \\
\text { smokers }\end{array}$ \\
\hline
\end{tabular}


Exhibit 7-6. Primary SP's serum processing protocol

\begin{tabular}{|c|c|c|c|c|c|c|c|}
\hline ID & Test name & Ages & $\begin{array}{c}\text { Sample } \\
(\mathrm{mL})\end{array}$ & $\begin{array}{c}\text { Sample } \\
\text { type }\end{array}$ & \begin{tabular}{|c|}
$\begin{array}{c}\text { Collection } \\
\text { type }\end{array}$ \\
\end{tabular} & $\begin{array}{c}\text { Vessel } \\
\text { type }\end{array}$ & Remarks \\
\hline 136 & $\mathrm{Se} / \mathrm{Cu} / \mathrm{Zn}$ & $6+$ & 1.0 & Serum & $\begin{array}{l}\text { Royal } \\
\text { Blue }\end{array}$ & $\begin{array}{c}2 \mathrm{~mL} \\
\text { sterile }\end{array}$ & 1/3 Subsample $1,2,5,11$ \\
\hline 110 & Folate & $1+$ & 0.7 & Serum & Red & $2-\mathrm{mL}$ & Add $0.05 \mathrm{~mL} \mathrm{AA} 7 \%$ \\
\hline 70 & Vitamin D/PUFA & $\begin{array}{l}1-5 \\
6+ \\
\end{array}$ & $\begin{array}{l}0.5 \\
0.7\end{array}$ & Serum & Red & $2-\mathrm{mL}$ & \\
\hline 122 & Hep Profile & $6+$ & 1.0 & Serum & Red & 2-mL & \\
\hline 87 & Hepatit ab & $\begin{array}{c}2 \\
3-5 \\
\end{array}$ & $\begin{array}{l}0.3 \\
0.5 \\
\end{array}$ & Serum & Red & $2-\mathrm{mL}$ & Age $2-5$ only \\
\hline 17 & Cotinine & $\begin{array}{c}3-5 \\
6-11 \\
12+\end{array}$ & $\begin{array}{l}0.8 \\
1.0 \\
1.8\end{array}$ & Serum & Red & $2-\mathrm{mL}$ & \\
\hline 21 & Lipids & $\begin{array}{l}6-11 \\
12+\end{array}$ & $\begin{array}{l}0.5 \\
1.0\end{array}$ & Serum & Red & $2-\mathrm{mL}$ & \\
\hline 77 & HPV Serum & $14-59$ & 0.5 & Serum & Red & $2-\mathrm{mL}$ & $14-59$ only \\
\hline 18 & Biochem & $12+$ & 0.8 & Serum & Red & 2-mL & \\
\hline 23 & Insulin & $12+$ & 0.5 & Serum & Red & $2-\mathrm{mL}$ & Morning SPs only \\
\hline 12 & Vit B12/MMA & $19+$ & 0.7 & Serum & Red & $2 \mathrm{~mL}$ & \\
\hline 22 & HIV & $18-59$ & 0.8 & Serum & Red & $2-\mathrm{mL}$ & $18-59$ only \\
\hline 24 & HSV & $14-49$ & 0.2 & Serum & Red & $2-\mathrm{mL}$ & 14-49 only \\
\hline 127 & IgA-TTG/EMA & $6+$ & 0.5 & Serum & Red & $2-\mathrm{mL}$ & \\
\hline 137 & Testosterone/Estradiol/SHBG & $6+$ & 0.7 & Serum & Red & $2 \mathrm{~mL}$ & \\
\hline 150 & Aldehyde & $12+$ & 0.6 & Serum & Red & $2 \mathrm{~mL}$ & $\begin{array}{l}1 / 3 \text { subsample } \\
1,2,5,11 \text { plus adult } \\
\text { smokers }\end{array}$ \\
\hline 32 & Xtra Sera1 & $3-11$ & 0.5 & Serum & Red & $2-\mathrm{mL}$ & \\
\hline 33 & Xtra Sera2 & $3-11$ & 0.5 & Serum & Red & $2-\mathrm{mL}$ & \\
\hline 34 & Xtra Sera3 & $12+$ & 1.0 & Serum & Red & 2-mL & \\
\hline 35 & Xtra Sera4 & $12+$ & 1.0 & Serum & Red & $2-\mathrm{mL}$ & \\
\hline 36 & Xtra Sera5 & $12+$ & 1.0 & Serum & Red & 2-mL & \\
\hline 37 & Xtra Sera6 & $12+$ & 1.0 & Serum & Red & $2-\mathrm{mL}$ & \\
\hline 29 & Persis Pest1 & $12+$ & 4.0 & Serum & 10-mL Red & $5-\mathrm{mL}$ & $\begin{array}{l}1 / 3 \text { Subsample } \\
0,3,7,10\end{array}$ \\
\hline 89 & Persis Pest2 & $12+$ & 4.0 & Serum & 10-mL Red & $\begin{array}{c}5-\mathrm{mL} \\
\text { sterile }\end{array}$ & $\begin{array}{l}1 / 3 \text { Subsample } \\
0,3,7,10\end{array}$ \\
\hline 90 & Perfluor & $12+$ & 1.5 & Serum & 10-mL Red & $\begin{array}{l}5-\mathrm{mL} \\
\text { sterile }\end{array}$ & $\begin{array}{l}1 / 3 \text { subsample } \\
0,3,7,10\end{array}$ \\
\hline 92 & BFR1 & $12+$ & 4.0 & Serum & 10-mL Red & $\begin{array}{l}5 \text {-mL } \\
\text { sterile }\end{array}$ & $\begin{array}{l}1 / 3 \text { Subsample } \\
4,6,8,9\end{array}$ \\
\hline 93 & BFR2 & $12+$ & 4.0 & Serum & 10-mL Red & $\begin{array}{l}5 \text {-mL } \\
\text { sterile }\end{array}$ & $\begin{array}{l}\text { 1/3 Subsample } \\
4,6,8,9\end{array}$ \\
\hline
\end{tabular}


Exhibits 7-7 through 7-9 illustrate the VIP guest's protocol for blood processing. It is extremely important to follow the processing procedures outlined in these protocols and in this manual. Specimen loss or biased results will occur if the exact procedures are not followed.

Exhibit 7-7. VIP Guest's blood whole blood processing protocol

\begin{tabular}{|l|l|c|c|c|c|c|c|}
\hline ID & \multicolumn{1}{|c|}{ Test name } & Ages & $\begin{array}{c}\text { Sample } \\
(\mathrm{mL})\end{array}$ & Sample type & $\begin{array}{c}\text { Collection } \\
\text { type }\end{array}$ & $\begin{array}{c}\text { Vessel } \\
\text { type }\end{array}$ & Remarks \\
\hline 1 & $\begin{array}{l}\text { Lead/Cad/HG/ } \\
\text { Se/Mn }\end{array}$ & $1+$ & 0.4 & Whole blood & EDTA & $\begin{array}{c}2-\mathrm{mL} \\
\text { sterile }\end{array}$ & \\
\hline 3 & RBC Folate & $1+$ & 0.1 & Whole blood & EDTA & $2-\mathrm{mL}$ & $\begin{array}{l}\text { Dilute with 1 mL of the } \\
1 \% \text { AA solution }\end{array}$ \\
\hline 5 & $\begin{array}{l}\text { Mercury, } \\
\text { Ethyl \& } \\
\text { Methyl }\end{array}$ & $1+$ & 0.5 & Whole blood & EDTA & $\begin{array}{c}2-\mathrm{mL} \\
\text { sterile }\end{array}$ & \\
\hline
\end{tabular}

Exhibit 7-8. VIP Guest's plasma processing protocol

\begin{tabular}{|l|l|l|c|c|c|c|c|}
\hline ID & Test name & Ages & $\begin{array}{c}\text { Sample } \\
(\mathrm{mL})\end{array}$ & Sample type & $\begin{array}{c}\text { Collection } \\
\text { type }\end{array}$ & $\begin{array}{c}\text { Vessel } \\
\text { type }\end{array}$ & Remarks \\
\hline 9 & Glucose & $12+$ & 0.5 & Plasma & Grey & 2-mL & Morning SPs only \\
\hline
\end{tabular}

Exhibit 7-9. VIP Guest's serum processing protocol

\begin{tabular}{|c|l|c|c|c|c|c|c|}
\hline ID & Test name & Ages & $\begin{array}{c}\text { Sample } \\
(\mathrm{mL})\end{array}$ & Sample type & $\begin{array}{c}\text { Collection } \\
\text { type }\end{array}$ & $\begin{array}{c}\text { Vessel } \\
\text { type }\end{array}$ & Remarks \\
\hline 110 & Folate & $1+$ & 0.7 & Serum & Red & 2-mL & $\begin{array}{l}\text { Dilute with 0.5 mL of } \\
7 \% \text { AA solution }\end{array}$ \\
\hline 21 & Lipids & $6+$ & 1.0 & Serum & Red & 2-mL & \\
\hline 23 & Biochem & $12+$ & 0.8 & Serum & Red & 2-mL & \\
\hline
\end{tabular}

Exhibit 7-10 illustrates the guest and surplus protocol for blood processing. It is extremely important to follow the processing procedures outlined in these protocols and in this manual. Specimen loss or biased results will occur if the exact procedures are not followed.

Exhibit 7-10. Guest serum processing protocol

\begin{tabular}{|c|c|c|c|c|c|c|c|}
\hline ID & Test name & Ages & $\begin{array}{c}\text { Sample } \\
(\mathrm{mL})\end{array}$ & Sample type & $\begin{array}{c}\text { Collection } \\
\text { type }\end{array}$ & $\begin{array}{c}\text { Vessel } \\
\text { type }\end{array}$ & Remarks \\
\hline 18 & Biochem & $12+$ & 0.8 & Serum & Red & $2-\mathrm{mL}$ & \\
\hline
\end{tabular}


Exhibits 7-11 through 7-15 illustrate the blood processing vessels for each primary SP by age group.

Exhibit 7-11. Blood processing protocol for primary SPs aged 1-2 years

\begin{tabular}{|c|l|c|c|c|c|c|}
\hline ID & \multicolumn{1}{|c|}{ Test name } & Ages & $\begin{array}{c}\text { Sample } \\
(\mathrm{mL})\end{array}$ & Sample type & Collection type & $\begin{array}{c}\text { Vessel } \\
\text { type }\end{array}$ \\
\hline 1 & $\begin{array}{l}\text { Lead/Cad/HG/Se/ } \\
\text { Mn }\end{array}$ & $1-2$ & 0.4 & Whole blood & 3-mL EDTA & $\begin{array}{c}2-\mathrm{mL} \\
\text { sterile }\end{array}$ \\
\hline 3 & RBC Folate & $1-2$ & 0.1 & Whole blood & 3-mL EDTA & $2-\mathrm{mL}$ \\
\hline 5 & $\begin{array}{l}\text { Mercury, Ethyl \& } \\
\text { Methyl }\end{array}$ & $1-2$ & 0.5 & Whole blood & $3-\mathrm{mL}$ EDTA & $\begin{array}{c}2-\mathrm{mL} \\
\text { sterile }\end{array}$ \\
\hline 110 & Folate & $1-2$ & 0.7 & Serum & 3-mL Red & 2-mL \\
\hline 70 & Vitamin D/PUFA & $1-2$ & 0.5 & Serum & 3-mL Red & 2-mL \\
\hline 87 & Hepatitis ab & 2 & 0.3 & Serum & 3-mL Red & 2-mL \\
\hline
\end{tabular}

Exhibit 7-12. Blood processing protocol for primary SPs aged 3-5 years

\begin{tabular}{|c|l|c|c|c|l|c|}
\hline ID & \multicolumn{1}{|c|}{ Test name } & Ages & $\begin{array}{c}\text { Sample } \\
(\mathrm{mL})\end{array}$ & Sample type & \multicolumn{1}{c|}{ Collection type } & $\begin{array}{c}\text { Vessel } \\
\text { type }\end{array}$ \\
\hline 1 & Lead/Cad/HG/Se/Mn & $3-5$ & 0.4 & Whole blood & 3-mL EDTA & $\begin{array}{c}2-\mathrm{mL} \\
\text { sterile }\end{array}$ \\
\hline 3 & RBC Folate & $3-5$ & 0.1 & Whole blood & 3 -mL EDTA & $2-\mathrm{mL}$ \\
\hline 5 & $\begin{array}{l}\text { Mercury, Ethyl \& } \\
\text { Methyl }\end{array}$ & $3-5$ & 0.5 & Whole blood & $3-\mathrm{mL}$ EDTA & $\begin{array}{c}2-\mathrm{mL} \\
\text { sterile }\end{array}$ \\
\hline 155 & Fluoride & $3-5$ & 1.0 & Plasma & 3-mL EDTA & $2 \mathrm{~mL}$ \\
\hline 110 & Folate & $3-5$ & 0.7 & Serum & 7 and 3-mL Red & $2-\mathrm{mL}$ \\
\hline 70 & Vitamin D/PUFA & $3-5$ & 0.5 & Serum & 7 and 3-mL Red & $2-\mathrm{mL}$ \\
\hline 87 & Hepatitis ab & $3-5$ & 0.5 & Serum & 7 and 3-mL Red & $2-\mathrm{mL}$ \\
\hline 17 & Cotinine & $3-5$ & 0.8 & Serum & 7 and 3-mL Red & $2-\mathrm{mL}$ \\
\hline 32 & Extra Sera1 & $3-5$ & 0.5 & Serum & 7 and 3-mL Red & $2-\mathrm{mL}$ \\
\hline 33 & Extra Sera2 & $3-5$ & 0.5 & Serum & 7 and 3-mL Red & $2-\mathrm{mL}$ \\
\hline
\end{tabular}


Exhibit 7-13. Blood processing protocol for primary SPs aged 6-11 years

\begin{tabular}{|c|c|c|c|c|c|c|}
\hline ID & Test name & Ages & $\begin{array}{l}\text { Sample } \\
(\mathrm{mL})\end{array}$ & Sample type & Collection type & $\begin{array}{l}\text { Vessel } \\
\text { type }\end{array}$ \\
\hline 1 & $\begin{array}{l}\text { Lead/Cad/HG/Se/ } \\
\text { Mn }\end{array}$ & $6-11$ & 0.4 & Whole blood & 4-mL EDTA & $\begin{array}{l}2-\mathrm{mL} \\
\text { sterile }\end{array}$ \\
\hline 3 & RBC Folate & $6-11$ & 0.1 & Whole blood & 4-mL EDTA & $2-\mathrm{mL}$ \\
\hline 5 & $\begin{array}{l}\text { Mercury, Ethyl \& } \\
\text { Methyl }\end{array}$ & $6-11$ & 0.5 & Whole blood & 4-mL EDTA & $\begin{array}{l}2-\mathrm{mL} \\
\text { sterile }\end{array}$ \\
\hline 88 & Acrylamide & $6-11$ & 1.0 & Packed Cells & $4 \mathrm{~mL}$ EDTA & $2 \mathrm{~mL}$ \\
\hline 155 & Fluoride & $6-11$ & 1.0 & Plasma & $4 \mathrm{~mL}$ EDTA & $2 \mathrm{~mL}$ \\
\hline 136 & $\mathrm{Se} / \mathrm{Cu} / \mathrm{Zn}$ & $6-11$ & 1.0 & Serum & $6 \mathrm{~mL}$ Royal blue & $\begin{array}{c}2 \mathrm{~mL} \\
\text { sterile }\end{array}$ \\
\hline 110 & Folate & $6-11$ & 0.7 & Serum & $\begin{array}{l}10 \text { and } 7-\mathrm{mL} \text { Red, } \\
6 \mathrm{~mL} \text { Royal blue }\end{array}$ & $2-\mathrm{mL}$ \\
\hline 70 & Vitamin D/PUFA & $6-11$ & 0.7 & Serum & $\begin{array}{l}10 \text { and } 7-\mathrm{mL} \text { Red, } \\
6 \mathrm{~mL} \text { Royal blue }\end{array}$ & $2-\mathrm{mL}$ \\
\hline 122 & Hep Profile & $6-19$ & 1.0 & Serum & $\begin{array}{l}10 \text { and } 7-\mathrm{mL} \text { Red, } \\
6 \mathrm{~mL} \text { Royal blue }\end{array}$ & $2-\mathrm{mL}$ \\
\hline 17 & Cotinine & $6-11$ & 0.5 & Serum & $\begin{array}{l}10 \text { and } 7-\mathrm{mL} \text { Red, } \\
6 \mathrm{~mL} \text { Royal blue }\end{array}$ & $2-\mathrm{mL}$ \\
\hline 21 & Lipids & $6-11$ & 1.0 & Serum & $\begin{array}{l}10 \text { and } 7-\mathrm{mL} \text { Red, } \\
6 \mathrm{~mL} \text { Royal blue }\end{array}$ & $2-\mathrm{mL}$ \\
\hline 127 & IGA-TTG/EMA & $6-11$ & 0.5 & Serum & $\begin{array}{l}10 \text { and } 7-\mathrm{mL} \text { Red, } \\
6 \mathrm{~mL} \text { Royal blue }\end{array}$ & $2-\mathrm{mL}$ \\
\hline 137 & $\begin{array}{l}\text { Testosterone/ } \\
\text { Estradiol/SHBG }\end{array}$ & $6-11$ & 0.7 & Serum & $\begin{array}{l}10 \text { and } 7 \mathrm{~mL} \text { Red, } \\
6 \mathrm{~mL} \text { Royal blue }\end{array}$ & $2 \mathrm{~mL}$ \\
\hline 32 & Xtra Sera1 & $6-11$ & 0.5 & Serum & $\begin{array}{l}10 \text { and } 7-\mathrm{mL} \text { Red, } \\
6 \mathrm{~mL} \text { Royal blue }\end{array}$ & $2-\mathrm{mL}$ \\
\hline 33 & Xtra Sera2 & $6-11$ & 0.5 & Serum & $\begin{array}{l}10 \text { and } 7-\mathrm{mL} \text { Red, } \\
6 \mathrm{~mL} \text { Royal blue }\end{array}$ & $2-\mathrm{mL}$ \\
\hline
\end{tabular}


Exhibit 7-14. Blood processing protocol for primary SPs aged 12-19 years

\begin{tabular}{|c|c|c|c|c|c|c|}
\hline ID & Test name & Ages & $\begin{array}{l}\text { Sample } \\
(\mathrm{mL})\end{array}$ & Sample type & Collection type & $\begin{array}{l}\text { Vessel } \\
\text { type }\end{array}$ \\
\hline 54 & VOC Blood & $12-19$ & 7.0 & Whole blood & 7-mL Gray & $7-\mathrm{mL}$ \\
\hline 1 & $\begin{array}{l}\mathrm{Lead} / \mathrm{Cad} / \mathrm{HG} / \mathrm{S} \\
\text { e/Mn }\end{array}$ & $12-19$ & 0.4 & Whole blood & 4 -mL EDTA & $\begin{array}{l}\text { 2-mL } \\
\text { sterile }\end{array}$ \\
\hline 3 & RBC Folate & $12-19$ & 0.1 & Whole blood & 4-mL EDTA & $2-\mathrm{mL}$ \\
\hline 4 & Glycohem & $12-19$ & 0.4 & Whole blood & 4-mL EDTA & $2-\mathrm{mL}$ \\
\hline 5 & $\begin{array}{l}\text { Mercury, } \\
\text { Methyl \& Ethyl }\end{array}$ & $12-19$ & 0.5 & Whole blood & 4-mL EDTA & $\begin{array}{l}\text { 2-mL } \\
\text { sterile }\end{array}$ \\
\hline 88 & Acrylamide & $12-19$ & 1.0 & Packed Cells & $4 \mathrm{~mL}$ EDTA & $2 \mathrm{~mL}$ \\
\hline 155 & Fluoride & $12-19$ & 1.0 & Plasma & $4 \mathrm{~mL}$ EDTA & $2 \mathrm{~mL}$ \\
\hline 9 & Glucose & $12-19$ & 0.5 & Plasma & 2-mL Gray & 2-mL \\
\hline 98 & OGTT & $12-19$ & 0.5 & Plasma & 2-mL Gray & $2-m L$ \\
\hline 136 & $\mathrm{Se} / \mathrm{Cu} / \mathrm{Zn}$ & $12-19$ & 1.0 & Serum & $6 \mathrm{~mL}$ Royal Blue & $2 \mathrm{~mL}$ \\
\hline 110 & Folate1 & $12-19$ & 0.7 & Serum & 15 and $10-\mathrm{mL}$ Red & $2-\mathrm{mL}$ \\
\hline 70 & $\begin{array}{l}\text { Vitamin } \\
\text { D/PUFA }\end{array}$ & $12-19$ & 0.7 & Serum & $\begin{array}{l}15 \text { and } 7-\mathrm{mL} \text { Red, } \\
6 \mathrm{~mL} \text { Royal blue }\end{array}$ & $2-\mathrm{mL}$ \\
\hline 122 & Hep Profile & $12-19$ & 1.0 & Serum & 15 and $7-\mathrm{mL}$ Red & $2-\mathrm{mL}$ \\
\hline 17 & Cotinine & $12-19$ & 1.8 & Serum & $\begin{array}{l}15 \text { and } 7-\mathrm{mL} \text { Red, } \\
6 \mathrm{~mL} \text { Royal blue }\end{array}$ & 2-mL \\
\hline 21 & Lipids & $12-19$ & 1.0 & Serum & $\begin{array}{l}15 \text { and } 7-\mathrm{mL} \text { Red, } \\
6 \mathrm{~mL} \text { Royal blue }\end{array}$ & $2-\mathrm{mL}$ \\
\hline 77 & HPV Serum & $14-19$ & 0.5 & Serum & $\begin{array}{l}15 \text { and } 7-\mathrm{mL} \text { Red, } \\
6 \mathrm{~mL} \text { Royal blue }\end{array}$ & $2-\mathrm{mL}$ \\
\hline 18 & Biochem & $12-19$ & 0.8 & Serum & 15 and 7-mL Red & $2-m L$ \\
\hline 23 & Insulin & $12-19$ & 0.5 & Serum & $\begin{array}{l}15 \text { and } 7-m L \text { Red, } \\
6 \mathrm{~mL} \text { Royal blue }\end{array}$ & $2-\mathrm{mL}$ \\
\hline 12 & Vit B12/MMA & 19 & 0.7 & Serum & $\begin{array}{l}15 \text { and } 7-\mathrm{mL} \text { Red, } \\
6 \mathrm{~mL} \text { Royal blue }\end{array}$ & $2 \mathrm{~mL}$ \\
\hline 22 & HIV & $18-19$ & 0.8 & Serum & $\begin{array}{l}15 \text { and } 7-\mathrm{mL} \text { Red, } \\
6 \mathrm{~mL} \text { Royal blue }\end{array}$ & $2-\mathrm{mL}$ \\
\hline 24 & HSV & $14-49$ & 0.2 & Serum & $\begin{array}{l}15 \text { and } 7-\mathrm{mL} \text { Red, } \\
6 \mathrm{~mL} \text { Royal blue }\end{array}$ & $2-\mathrm{mL}$ \\
\hline 127 & IgA-TTG/EMA & $12-19$ & 0.5 & Serum & $\begin{array}{l}15 \text { and } 7-\mathrm{mL} \text { Red, } \\
6 \mathrm{~mL} \text { Royal blue }\end{array}$ & $2-\mathrm{mL}$ \\
\hline 137 & $\begin{array}{l}\text { Testosterone/ } \\
\text { Estradiol/SHBG }\end{array}$ & $12-19$ & 0.7 & Serum & $\begin{array}{l}15 \text { and } 7 \mathrm{~mL} \text { Red, } \\
6 \mathrm{~mL} \text { Royal blue }\end{array}$ & $2 \mathrm{~mL}$ \\
\hline 150 & Aldehyde & $12-19$ & 0.6 & Serum & $\begin{array}{l}15 \text { and } 7 \mathrm{~mL} \text { Red, } \\
6 \mathrm{~mL} \text { Royal blue }\end{array}$ & $2 \mathrm{~mL}$ \\
\hline 34 & Xtra Sera3 & $12-19$ & 1.0 & Serum & $\begin{array}{l}15 \text { and } 7-m L \text { Red, } \\
6 \mathrm{~mL} \text { Royal blue }\end{array}$ & 2-mL \\
\hline 35 & Xtra Sera4 & $12-19$ & 1.0 & Serum & $\begin{array}{l}15 \text { and } 7-\mathrm{mL} \text { Red, } \\
6 \mathrm{~mL} \text { Royal blue }\end{array}$ & 2-mL \\
\hline
\end{tabular}


Exhibit 7-14. Blood processing protocol for primary SPs aged 12-19 years (continued)

\begin{tabular}{|c|l|c|c|c|c|c|}
\hline ID & \multicolumn{1}{|c|}{ Test name } & Ages & $\begin{array}{c}\text { Sample } \\
(\mathrm{mL})\end{array}$ & Sample type & \multicolumn{1}{|c|}{ Collection type } & $\begin{array}{c}\text { Vessel } \\
\text { type }\end{array}$ \\
\hline 36 & Xtra Sera5 & $12-19$ & 1.0 & Serum & $\begin{array}{l}\text { 15 and 7-mL Red, } \\
\text { 6mL Royal blue }\end{array}$ & $2-\mathrm{mL}$ \\
\hline 37 & Xtra Sera6 & $12-19$ & 1.0 & Serum & $\begin{array}{l}\text { 15 and 7-mL Red, } \\
\text { 6mL Royal blue }\end{array}$ & $2-\mathrm{mL}$ \\
\hline 29 & Persis Pest1 & $12-19$ & 4.0 & Serum & $15-\mathrm{mL}$ Red & $\begin{array}{c}5-\mathrm{mL} \\
\text { sterile }\end{array}$ \\
\hline 89 & Persis Pest2 & $12-19$ & 4.0 & Serum & $15-\mathrm{mL}$ Red & $\begin{array}{c}5-\mathrm{mL} \\
\text { sterile }\end{array}$ \\
\hline 90 & Perflour1 & $12-19$ & 1.5 & Serum & $15-\mathrm{mL} \mathrm{Red}$ & $\begin{array}{c}5-\mathrm{mL} \\
\text { sterile }\end{array}$ \\
\hline 92 & BFR1 & $12-19$ & 4.0 & Serum & $15-\mathrm{mL} \mathrm{Red}$ & $\begin{array}{c}5-\mathrm{mL} \\
\text { sterile }\end{array}$ \\
\hline 93 & BFR2 & $12-19$ & 4.0 & Serum & $15-\mathrm{mL} \mathrm{Red}$ & $\begin{array}{c}5-\mathrm{mL} \\
\text { sterile }\end{array}$ \\
\hline
\end{tabular}


Exhibit 7-15. Blood processing protocol for primary SPs aged 20+ years

\begin{tabular}{|c|c|c|c|c|c|c|}
\hline ID & Test name & Ages & $\begin{array}{l}\text { Sample } \\
(\mathrm{mL})\end{array}$ & Sample type & Collection type & $\begin{array}{c}\text { Vessel } \\
\text { type }\end{array}$ \\
\hline 54 & VOC Blood & $20+$ & 10.0 & Whole blood & $10-\mathrm{mL}$ gray & 10-mL Gray \\
\hline 1 & $\begin{array}{l}\mathrm{Lead} / \mathrm{Cad} / \mathrm{HG} / \mathrm{Se} \\
/ \mathrm{Mn}\end{array}$ & $20+$ & 0.4 & Whole blood & 4-mL EDTA & $\begin{array}{l}2-\mathrm{mL} \\
\text { sterile }\end{array}$ \\
\hline 3 & RBC Folate & $20+$ & 0.1 & Whole blood & 4-mL EDTA & $2-\mathrm{mL}$ \\
\hline 4 & Glycohem & $20+$ & 0.4 & Whole blood & 4-mL EDTA & 2-mL \\
\hline 5 & $\begin{array}{l}\text { Mercury, Methyl } \\
\text { \& Ethyl }\end{array}$ & $20+$ & 0.5 & Whole blood & 4-mL EDTA & $\begin{array}{l}2-\mathrm{mL} \\
\text { sterile }\end{array}$ \\
\hline 88 & Acrylamide & $20+$ & 1.0 & Packed Cells & $4 \mathrm{~mL}$ EDTA & $2 \mathrm{~mL}$ \\
\hline 155 & Fluoride & $20+$ & 1.0 & Plasma & $4 \mathrm{~mL}$ EDTA & $2 \mathrm{~mL}$ \\
\hline 9 & Glucose & $20+$ & 0.5 & Plasma & 2-mL Gray & 2-mL \\
\hline 98 & OGTT & $20+$ & 0.5 & Plasma & 2-mL Gray & 2-mL \\
\hline 136 & $\mathrm{Se} / \mathrm{Cu} / \mathrm{Zn}$ & $20+$ & 1.0 & Serum & $6 \mathrm{~mL}$ Royal blue & 2-mL sterile \\
\hline 110 & Folate & $20+$ & 0.7 & Serum & $\begin{array}{l}15 \text { Red, } 6 \mathrm{~mL} \text { Royal } \\
\text { blue }\end{array}$ & $2-\mathrm{mL}$ \\
\hline 70 & $\begin{array}{l}\text { Vitamin } \\
\text { D/PUFA }\end{array}$ & $20+$ & 0.7 & Serum & $\begin{array}{l}15 \text { and } 10-\mathrm{mL} \text { Red, } \\
6 \mathrm{~mL} \text { Royal blue }\end{array}$ & 2-mL \\
\hline 122 & Hep Profile & $20+$ & 1.0 & Serum & $\begin{array}{l}15 \text { and } 7-\mathrm{mL} \text { Red, } \\
6 \mathrm{~mL} \text { Royal blue }\end{array}$ & 2-mL \\
\hline 17 & Cotinine & $20+$ & 1.8 & Serum & $\begin{array}{l}15 \text { Red, } 6 \mathrm{~mL} \text { Royal } \\
\text { blue }\end{array}$ & 2-mL \\
\hline 21 & Lipids & $20+$ & 1.0 & Serum & $\begin{array}{l}15 \text { Red, } 6 \mathrm{~mL} \text { Royal } \\
\text { blue }\end{array}$ & 2-mL \\
\hline 77 & HPV Serum & $20-59$ & 0.5 & Serum & $\begin{array}{l}15 \text { Red, } 6 \mathrm{~mL} \text { Royal } \\
\text { blue }\end{array}$ & 2-mL \\
\hline 18 & Biochem & $20+$ & 0.8 & Serum & $\begin{array}{l}15 \text { Red, } 6 \mathrm{~mL} \text { Royal } \\
\text { blue }\end{array}$ & 2-mL \\
\hline 23 & Insulin & $20+$ & 0.5 & Serum & $\begin{array}{l}15 \text { Red, } 6 \mathrm{~mL} \text { Royal } \\
\text { blue }\end{array}$ & $2-\mathrm{mL}$ \\
\hline 12 & Vit B12/MMA & $20+$ & 0.7 & Serum & $\begin{array}{l}15 \mathrm{~mL} \text { Red, } 6 \mathrm{~mL} \\
\text { Royal blue }\end{array}$ & 2-mL \\
\hline 22 & HIV & $20-59$ & 0.8 & Serum & $\begin{array}{l}15 \text { Red, } 6 \mathrm{~mL} \text { Royal } \\
\text { blue }\end{array}$ & 2-mL \\
\hline 24 & HSV & $20-49$ & 0.2 & Serum & $\begin{array}{l}15 \text { Red, } 6 \mathrm{~mL} \text { Royal } \\
\text { blue }\end{array}$ & $2-m L$ \\
\hline
\end{tabular}


Exhibit 7-15. Blood processing protocol for primary SPs aged 20+ years (continued)

\begin{tabular}{|c|c|c|c|c|c|c|}
\hline ID & Test name & Ages & $\begin{array}{l}\text { Sample } \\
(\mathrm{mL})\end{array}$ & Sample type & Collection type & $\begin{array}{l}\text { Vessel } \\
\text { type }\end{array}$ \\
\hline 127 & IgA-TTG/EMA & $20+$ & 0.5 & Serum & $\begin{array}{l}15 \mathrm{Red}, 6 \mathrm{~mL} \\
\text { Royal blue }\end{array}$ & $2-\mathrm{mL}$ \\
\hline 137 & $\begin{array}{l}\text { Testosterone/ } \\
\text { Estradiol/SHBG }\end{array}$ & $20+$ & 0.7 & Serum & $\begin{array}{l}15 \mathrm{~mL} \text { Red, } 6 \mathrm{~mL} \\
\text { Royal blue }\end{array}$ & $2 \mathrm{~mL}$ \\
\hline 150 & Aldehyde & $20+$ & 0.6 & & & \\
\hline 34 & Xtra Sera3 & $20+$ & 1.0 & Serum & $\begin{array}{l}15 \text { Red, } 6 \mathrm{~mL} \\
\text { Royal blue }\end{array}$ & 2-mL \\
\hline 35 & Xtra Sera4 & $20+$ & 1.0 & Serum & $\begin{array}{l}15 \text { Red, } 6 \mathrm{~mL} \\
\text { Royal blue }\end{array}$ & 2-mL \\
\hline 36 & Xtra Sera5 & $20+$ & 1.0 & Serum & $\begin{array}{l}15 \text { Red, } 6 \mathrm{~mL} \\
\text { Royal blue }\end{array}$ & $2-\mathrm{mL}$ \\
\hline 37 & Xtra Sera6 & $20+$ & 1.0 & Serum & $\begin{array}{l}15 \text { Red, } 6 \mathrm{~mL} \\
\text { Royal blue }\end{array}$ & $2-\mathrm{mL}$ \\
\hline 29 & Persis Pest & $20+$ & 4.0 & Serum & 15-mL Red & $\begin{array}{l}\text { 5-mL } \\
\text { sterile }\end{array}$ \\
\hline 89 & Persis Pest2 & $20+$ & 4.0 & Serum & 105-mL Red & $\begin{array}{l}5-\mathrm{mL} \\
\text { sterile }\end{array}$ \\
\hline 90 & Perflour1 & $20+$ & 1.5 & Serum & 15-mL Red & $\begin{array}{l}5-\mathrm{mL} \\
\text { sterile }\end{array}$ \\
\hline 92 & BFR1 & $20+$ & 4.0 & Serum & 15-mL Red & $\begin{array}{l}5-\mathrm{mL} \\
\text { sterile }\end{array}$ \\
\hline 93 & BFR2 & $20+$ & 4.0 & Serum & 15-mL Red & $\begin{array}{l}\text { 5-mL } \\
\text { sterile }\end{array}$ \\
\hline
\end{tabular}

Exhibits 7-16 through 7-19 illustrate the blood processing vessels for each VIP guest.

Exhibit 7-16. Blood processing protocol for VIP guest aged 1-2 years

\begin{tabular}{|c|l|c|c|c|c|c|}
\hline ID & \multicolumn{1}{|c|}{ Test name } & Ages & $\begin{array}{c}\text { Sample } \\
(\mathrm{mL})\end{array}$ & Sample type & Collection type & $\begin{array}{c}\text { Vessel } \\
\text { type }\end{array}$ \\
\hline 1 & $\begin{array}{l}\mathrm{Lead} / \mathrm{Cad} / \mathrm{HG} / \mathrm{Se} / \\
\mathrm{Mn}\end{array}$ & $1-2$ & 0.4 & Whole blood & 3-mL EDTA & $\begin{array}{c}\text { 2-mL } \\
\text { sterile }\end{array}$ \\
\hline 3 & RBC Folate & $1-2$ & 0.1 & Whole blood & 3-mL EDTA & 2-mL \\
\hline 5 & $\begin{array}{l}\text { Mercury, Methyl \& } \\
\text { Ethyl }\end{array}$ & $1-2$ & 0.5 & Whole blood & 3-mL EDTA & $\begin{array}{c}2-\mathrm{mL} \\
\text { sterile }\end{array}$ \\
\hline 110 & Folate & $1-2$ & 0.7 & Serum & 3-mL Red & 2-mL \\
\hline
\end{tabular}


Exhibit 7-17. Blood processing protocol for VIP guest aged 3-5 years

\begin{tabular}{|c|l|c|c|c|c|c|}
\hline ID & \multicolumn{1}{|c|}{ Test name } & Ages & $\begin{array}{c}\text { Sample } \\
(\mathrm{mL})\end{array}$ & Sample type & \multicolumn{1}{c|}{ Collection type } & $\begin{array}{c}\text { Vessel } \\
\text { type }\end{array}$ \\
\hline 1 & Lead/Cad/HG/Se/Mn & $3-5$ & 0.4 & Whole blood & 3-mL EDTA & $\begin{array}{c}2-\mathrm{mL} \\
\text { sterile }\end{array}$ \\
\hline 3 & RBC Folate & $3-5$ & 0.1 & Whole blood & 3-mL EDTA & 2-mL \\
\hline 5 & $\begin{array}{l}\text { Mercury, Methyl \& } \\
\text { Ethyl }\end{array}$ & $3-5$ & 0.5 & Whole blood & $3-\mathrm{mL}$ EDTA & $\begin{array}{c}2-\mathrm{mL} \\
\text { sterile }\end{array}$ \\
\hline 110 & Folate & $3-5$ & 0.7 & Serum & 3-mL Red & 2-mL \\
\hline
\end{tabular}

Exhibit 7-18. Blood processing protocol for VIP guest aged 6-11 years

\begin{tabular}{|c|l|c|c|c|c|c|}
\hline ID & \multicolumn{1}{|c|}{ Test name } & Ages & $\begin{array}{c}\text { Sample } \\
(\mathrm{mL})\end{array}$ & Sample type & Collection type & $\begin{array}{c}\text { Vessel } \\
\text { type }\end{array}$ \\
\hline 1 & Lead/Cad/HG, Se/Mn & $6-11$ & 0.4 & Whole blood & 4-mL EDTA & $\begin{array}{c}2-\mathrm{mL} \\
\text { sterile }\end{array}$ \\
\hline 3 & RBC Folate & $6-11$ & 0.1 & Whole blood & 4-mL EDTA & 2-mL \\
\hline 5 & $\begin{array}{l}\text { Mercury, Methyl \& } \\
\text { Ethyl }\end{array}$ & $6-11$ & 0.5 & Whole blood & 4-mL EDTA & $\begin{array}{c}\text { 2-mL } \\
\text { sterile }\end{array}$ \\
\hline 110 & Folate & $6-11$ & 0.7 & Serum & 7-mL Red & 2-mL \\
\hline 21 & Lipids & $6-11$ & 1.0 & Serum & 7-mL Red & 2-mL \\
\hline
\end{tabular}

Exhibit 7-19. Blood processing protocol for VIP guest aged 12+ years

\begin{tabular}{|c|l|c|c|c|c|c|}
\hline ID & \multicolumn{1}{|c|}{ Test name } & Ages & $\begin{array}{c}\text { Sample } \\
(\mathrm{mL})\end{array}$ & Sample type & Collection type & $\begin{array}{c}\text { Vessel } \\
\text { Type }\end{array}$ \\
\hline 1 & Lead/Cad/HG/Se/Mn & $12+$ & 0.4 & Whole blood & 3 -mL EDTA & $\begin{array}{c}2-\mathrm{mL} \\
\text { sterile }\end{array}$ \\
\hline 3 & RBC Folate & $12+$ & 0.1 & Whole blood & 3-mL EDTA & $2-\mathrm{mL}$ \\
\hline 5 & $\begin{array}{l}\text { Mercury, Methyl \& } \\
\text { Ethyl }\end{array}$ & $12+$ & 0.5 & Whole blood & $3-\mathrm{mL}$ EDTA & $\begin{array}{c}2-\mathrm{mL} \\
\text { sterile }\end{array}$ \\
\hline 9 & Glucose & $12+$ & 0.5 & Plasma & 2-mL Gray & 2-mL \\
\hline 110 & Folate & $12+$ & 0.7 & Serum & $10-\mathrm{mL}$ Red & $2-\mathrm{mL}$ \\
\hline 21 & Lipids & $12+$ & 1.0 & Serum & $10-\mathrm{mL}$ Red & 2-mL \\
\hline 18 & Biochem & $12+$ & 0.8 & Serum & $10-\mathrm{mL}$ Red & $2-\mathrm{mL}$ \\
\hline
\end{tabular}

Exhibit 7-20 illustrates the blood processing vessel for each guest.

Exhibit 7-20. Blood processing protocol for guest aged $12+$ years

\begin{tabular}{|c|c|c|c|c|c|c|}
\hline ID & Test name & Ages & $\begin{array}{c}\text { Sample } \\
(\mathrm{mL})\end{array}$ & Sample type & Collection type & $\begin{array}{c}\text { Vessel } \\
\text { type }\end{array}$ \\
\hline 18 & Biochem & $12+$ & 0.8 & Serum & 3-mL Red & 2-mL \\
\hline
\end{tabular}




\section{Labeling Vessels}

The phlebotomist and the technologist assigned to shipping are responsible for labeling vessels and assembling the blood processing racks. Use extreme care in setting up the blood processing racks and in labeling the vessels with the appropriate barcode label.

Use the following materials to set up and label blood racks:

- Rack;

- Age-appropriate processing template;

- Storage vessels of the following sizes:

- $\quad$ 2.0-mL sterile vessel;

- $\quad$ 2.0-mL non-sterile vessel;

- $\quad$ 5.0-mL sterile vessel;

- $\quad 14 \mathrm{~mL}$ screwcap

- $\quad$ 50-mL conical tube; and

- Barcoded SP ID labels, each containing the unique 6-digit SP ID number concatenated with the vessel ID.

Prepare one blood processing rack for each SP. The tubes for which each SP is eligible are determined by their sex, appointment type, and inclusion into certain subsamples so each SP's blood processing rack may be unique. When possible, prepare the racks at least 1 day before the SP arrives for the MEC examination.

Before each session, the technologist assigned to workstation 1 prints all labels for all SPs, distributes the labels, and labels the blood processing racks. The correct barcode labels automatically print for each SP based on the SP's unique profile.

While the technologist assigned to shipping is responsible for labeling the vessels and setting up the blood processing racks, it is the blood processing technologist's responsibility to verify that each vessel has the correct label before filling. Do not rely only upon memory or the positioning of the vessels in a rack to indicate which vessels to fill. 
Always double check:

- The SP's ID number on the barcode label;

- The vessel number on the label;

- The adhesion quality of the labels; and

- To ensure that specimens are not missing labels or incorrectly labeled.

Save and recycle unused vessels:

- If the SP does not show up for the MEC exam at the appointed date and time, then it is possible that he or she will reschedule;

- Store the SP's labeled vials in the clear bin or cupboard in workstation 1 until the end of the stand;

- If the SP has not shown up by the end of the stand, discard the unused vessels; and

- If the SP refuses phlebotomy during a session, discard vessels from the rack.

\subsection{Equipment}

\subsubsection{Benchtop Centrifuges}

Each laboratory contains two benchtop refrigerated centrifuges.

\section{Allegra X15R}

The centrifuge has the following safety features:

- An imbalance detector triggers an automatic shutdown if rotor loads are severely out of balance.

- A safety-lock mechanism and secondary manual latch prohibit opening the centrifuge door during a run until the rotor is virtually stopped.

- A lockout system requires the door to be closed and latched before the centrifuge will start. 
- An anchoring system secures the centrifuge in place. Anchoring systems reduce the possibility of injury or damage that could result from centrifuge movement in case of a major rotor mishap.

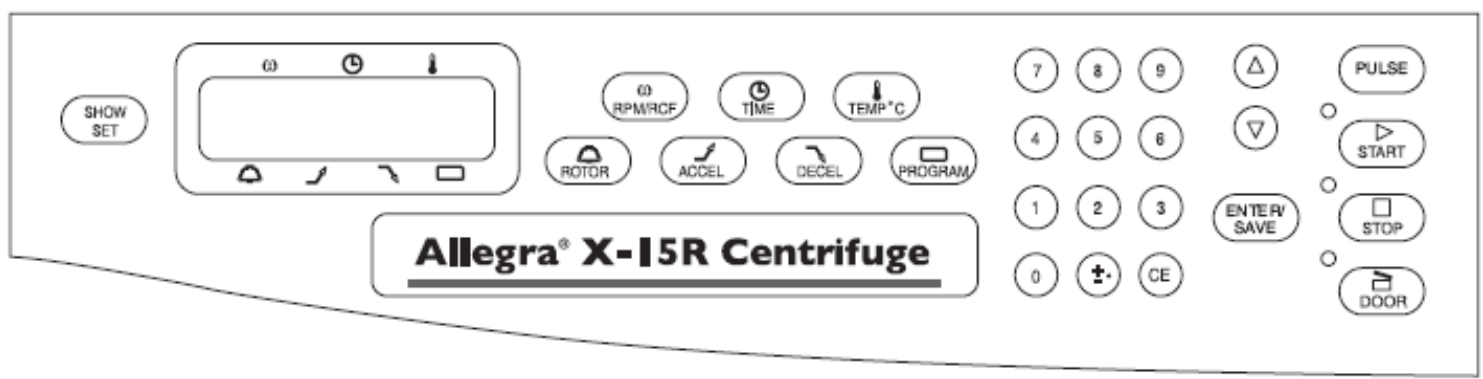

\section{Control Panel}

The control panel is mounted at an angle on the centrifuge front for easy visibility and access. The control panel has the following features:

$\omega$

\section{Digital Displays}

(9The displays provide run information, user messages, and diagnostics. During a run, they show the actual (real-time) operating conditions:

(SPEED)

(TIME)

(TEMPERATURE)

(ROTOR)

$\boldsymbol{\rho}(\mathrm{ACCEL})$

(DECEL)

$\square($ PROGRAM)
Indicates rotor speed in revolutions per minute (rpm)

In a timed run, indicates the remaining run time in hours and minutes.

Indicates rotor temperature in degrees $\mathrm{C}$ at thermoequilibrium.

Displays the name of the selected rotor.

When the ACCED key is pressed, one of ten available acceleration rates can be entered. Displays the selected acceleration rate throughout the run.

When the (DECEL) key is pressed, one of eleven available deceleration rates can be entered. Displays the selected deceleration rate throughout the run.

During setup-when the PROGRAM key is pressed, a program number (one of ten possible) can be selected and program parameters entered. 
During centrifugation - the number of the program being run is displayed.

Pressing ENTER/SAVE, then START begins the run. (START) must be pressed within 5 seconds of pressing ENTER/SAVE.) This key combination can also be used to abort a deceleration process and restart the centrifuge.

Can be pressed at any time while the rotor is spinning to terminate a run. The centrifuge decelerates to a complete stop according to the deceleration setting. The green light next to the key flashes while the rotor decelerates.

Unlocks and opens the door. (The instrument will not accept the unlock command if the rotor is spinning.)

\section{Time}

For plasma and serum - set appropriate time and temperature for each tube as follows:

- $\quad 3-\mathrm{mL}$ or4-mL EDTA

- $\quad 2-m L$ gray

- $\quad 3-\mathrm{mL}, 7-\mathrm{mL}, 10-\mathrm{mL}$ or $15-\mathrm{mL}$ red

- $6 \mathrm{~mL}$ royal blue
10 minutes $\quad 17-25^{\circ} \mathrm{C}$

10 minutes $\quad 17-25^{\circ} \mathrm{C}$

15 minutes $\quad 17-25^{\circ} \mathrm{C}$

15 minutes $\quad 17-25^{\circ} \mathrm{C}$

For washed packed cells - set appropriate time and temperature for each tube as follows:

- Vessel $88 \quad 3$ or 4 mL EDTA 5 minutes $\quad 4-8^{\circ} \mathrm{C}$

\section{Speed}

For plasma and serum - set the speed at 2,900 rpm.

For washed packed cells vessel 88 - set speed at 1,985 rpm. 


\section{Run Procedure}

\section{MANUAL RUN}

\begin{tabular}{|c|c|c|}
\hline 1 & POWER & Turn the power switch on (I). \\
\hline 2 & (DOOR & Press (DOOR) to open the chamber door; lift the door open. \\
\hline 3 & & $\begin{array}{l}\text { Install the rotor according to the applicable rotor manual, then close } \\
\text { the chamber door. }\end{array}$ \\
\hline 4 & ROTOR & $\begin{array}{l}\text { Press ROTOR, then press ROTOR again or use the arrow keys to } \\
\text { select the rotor. }\end{array}$ \\
\hline 5 & RPM/RCF & $\begin{array}{l}\text { Press RPM/RCF, then use the keypad to enter the run speed. Press } \\
\text { RPM/RCF a second time to toggle between RPM and RCF modes, } \\
\text { RPM/RCF RPM/RCF. }\end{array}$ \\
\hline 6 & (TIME) & $\begin{array}{l}\text { Press (TIME), then use the keypad to enter the run time (to } 99 \mathrm{hrs,} \\
59 \text { minutes); or press (TIME) twice to toggle between hold } \\
\text { (continuous) run and set time mode. }\end{array}$ \\
\hline 7 & TEMP ${ }^{\circ} \mathrm{C}$ & $\begin{array}{l}\text { Press } \text { TEMP }^{\circ} \mathrm{C} \text {, then use the keypad to enter the required run } \\
\text { temperature }-10 \text { to }+40^{\circ} \mathrm{C} \text {. TEMP }{ }^{\circ} \mathrm{C}\end{array}$ \\
\hline 8 & ACCEL & $\begin{array}{l}\text { Press ACCEL, then use the keypad or arrow keys to enter the } \\
\text { selected acceleration rate number, } 1 \text { (SLOW) through } 10 \text { (MAX). }\end{array}$ \\
\hline 9 & (DECEL) & $\begin{array}{l}\text { Press (DECEL), then use the keypad or arrow keys to enter the } \\
\text { selected deceleration rate number, } 0 \text { (OFF) through } 10 \text { (MAX). }\end{array}$ \\
\hline 10 & $\begin{array}{l}\text { ENTER/SAVE } \\
\text { START }\end{array}$ & $\begin{array}{l}\text { Check that all parameters are correct and that the door is closed } \\
\text { (door LED will be lit). Press ENTER/SAVE, then press (START) } \\
\text { (within } 5 \text { seconds). ENTER/SAVE }\end{array}$ \\
\hline 11 & STOP & $\begin{array}{l}\text { Wait for the set time to count down to zero, or end the run by } \\
\text { pressing STOP). }\end{array}$ \\
\hline 12 & (DOOR) & $\begin{array}{l}\text { When the rotor stops (a tone sounds and the (DOOR) LED lights), } \\
\text { press (DOOR) to open the chamber door; lift the door. }\end{array}$ \\
\hline
\end{tabular}




\section{PROGRAMMED RUN}

\begin{tabular}{|c|c|c|}
\hline 1 & POWER & Turn the power switch on (I). \\
\hline 2 & (DOOR & Press (DOOR) to open the chamber door. \\
\hline 3 & & $\begin{array}{l}\text { Install the rotor according to the applicable rotor manual, then } \\
\text { close the chamber door. }\end{array}$ \\
\hline 4 & PROGRAM & $\begin{array}{l}\text { Press (PROGRAM), use the keypad or arrow keys to enter the } \\
\text { required program number, then press ENTER/SAVE. }\end{array}$ \\
\hline 5 & $\begin{array}{l}\text { ENTER/SAVE } \\
\text { START }\end{array}$ & $\begin{array}{l}\text { Check that all parameters are correct and that the door is closed } \\
\text { (door LED will be lit). Press ENTER/SAVE, then press (START } \\
\text { (within } 5 \text { seconds). }\end{array}$ \\
\hline 6 & STOP & Wait for the run to end, or end the run by pressing (STOP). \\
\hline 7 & (DOOR) & $\begin{array}{l}\text { When the rotor stops (a tone sounds and the (DOOR) LED } \\
\text { lights), press (DOOR) to open the chamber door; lift the door. }\end{array}$ \\
\hline
\end{tabular}

Maintenance: Use the Beckman Coulter Guide

Instead of using the paint-on graphite which is described in the maintenance section, use the triflow lubricant and use the same maintenance procedure as the old Beckman GS 6R centrifuges. 
End of Stand Centrifuge Procedure:

Remove rotors from the Allegra $\mathrm{x}-15 \mathrm{R}$ centrifuges and pack in the black totes with the green foam for safe traveling. The balance detection mechanism is very sensitive during travel and needs to be protected. See photos for proper packing.

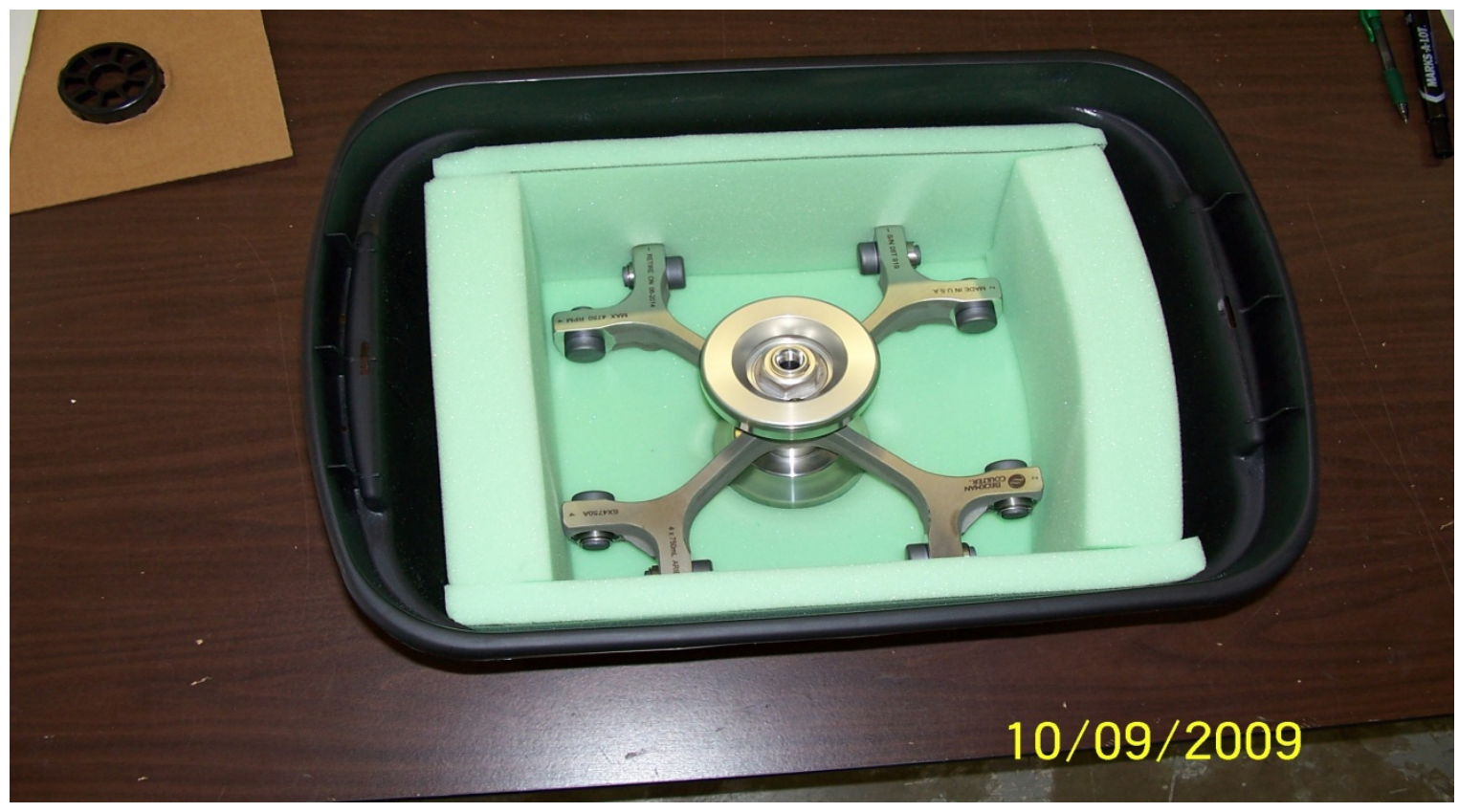




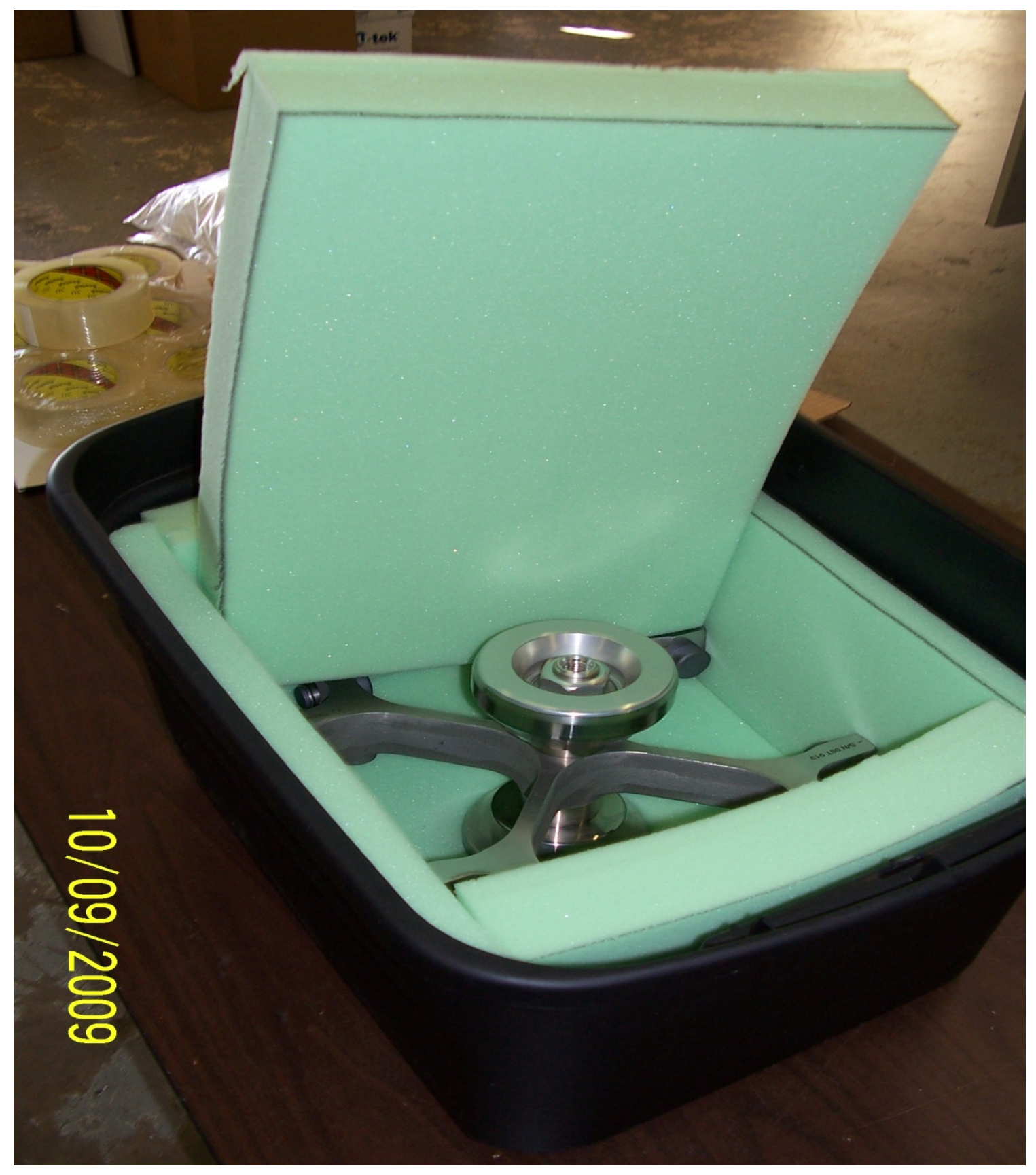




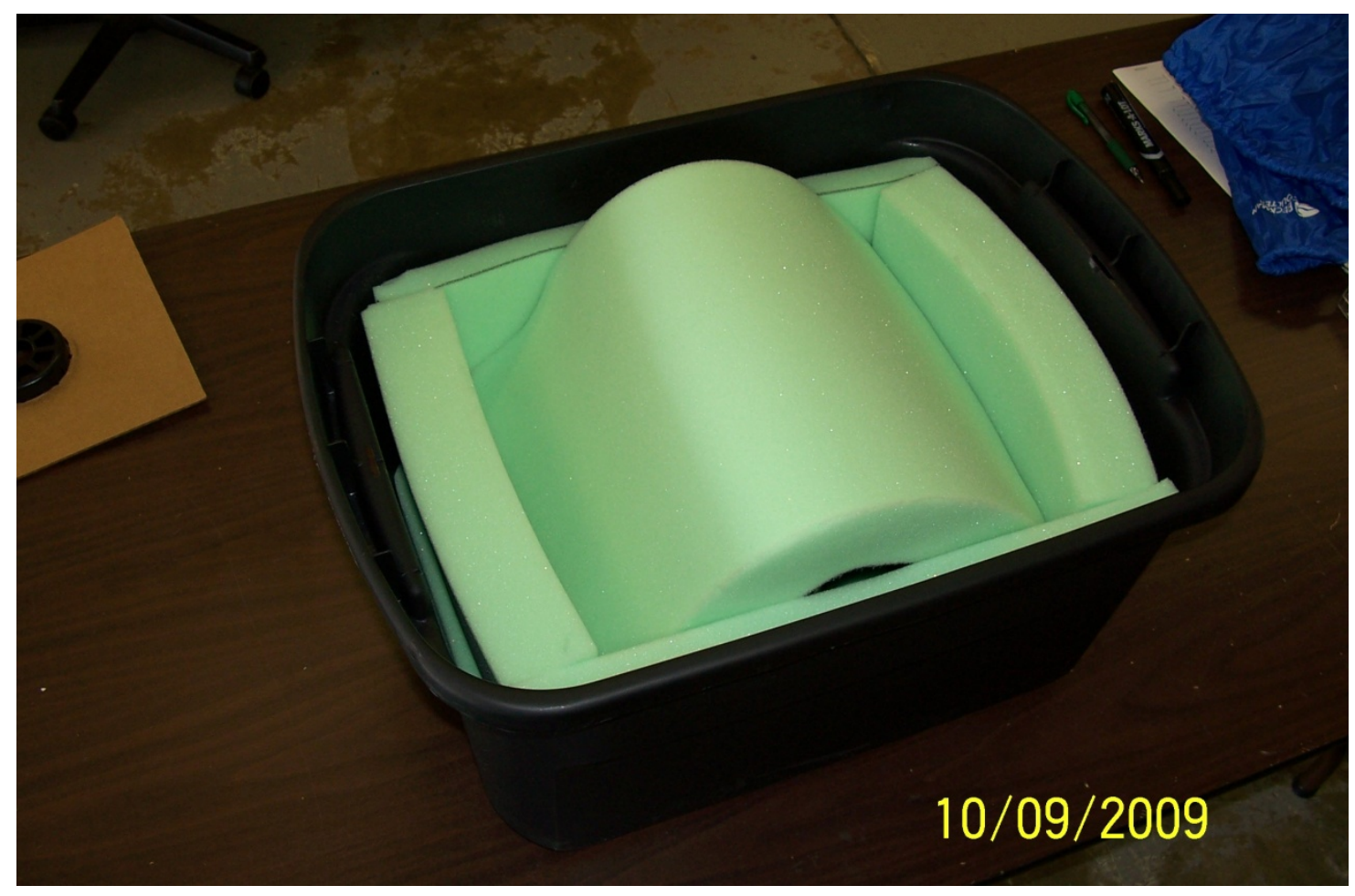

\subsubsection{Laminar Flow Biological Safety Cabinet Operating Sequence}

- Turn on the cabinet blower and lights, and check the air intake and exhaust portals of the cabinet to make sure they are unobstructed.

- Check the pressure on the magnehelic gauge. If the pressure has increased more than 0.1 w.g. since the previous reading, notify the biomedical engineer. Record the pressure reading and any comments in the Laboratory QC module.

- Allow the blowers to operate for a minimum of 15 minutes before beginning work in the cabinet.

- Disinfect the interior of the workspace by wiping it down with a disinfectant Saniwipe.).

- Place a disposable paper blotter on the work surface. Set up the equipment for processing. Do not place any equipment or supplies over the front intake grills.

- Arrange and separate clean and contaminated materials.

- Wait 2 to 3 minutes after placing the materials and supplies in the cabinet before beginning work. This will rid the area of all "loose" contamination. 
- Wear a long-sleeved laboratory coat and gloves. This will minimize the shedding of skin flora into the work area and concurrently protect hands and arms from viable agent contamination. Wash hands with soap and water before and after working in the cabinet.

- After completing work, leave the blower on for 2 to 3 minutes to purge the unit.

- Decontaminate interior surfaces after removal of all specimens and biohazard waste by wiping them with a 10 percent solution of bleach and water (1:10).

- Check the grills and diffuser vents for spills or splashes and clean using appropriate techniques and materials.

\section{Blood Processing Procedures}

Centrifuge all blood specimens unopened, then transport to, and uncap and process in the laminar flow hood. The laminar flow hood provides a HEPA-filtered, recirculated mass airflow within the workspace, and protects from any spattering that may occur when opening the Vacutainer ${ }^{\circledR}$ tubes or when pouring or pipetting the specimens. It also prevents drawing contaminated air or dust over the specimens. It is mandatory to use the blower.

Always wear gloves when handling specimens. Check gloves for small holes or tears. Change gloves if they become visibly contaminated with blood. Immediately soak visibly contaminated racks in 1:10 bleach solution. Decontaminate workstation, including the biological safety hood using Saniwipes or a $10 \%$ bleach solution at the end of the day or immediately when spills occur.

Decontaminate racks and templates by soaking them in $10 \%$ bleach solution at the end of each session. Place on blotter to air dry.

\subsubsection{Eppendorf Research Pro Pipettes}

Pipette whole blood and pooled serum and make dilutions using the Eppendorf Research pro pipettes. Pipette plasma using plastic transfer pipettes. 
The MEC laboratory has four Eppendorf Research pro electronic pipettes: one 5-100 $\mu \mathrm{L}$, two $50-1,000 \mu \mathrm{L}$, and one $100-5,000 \mu \mathrm{L}$. The lab also uses $100 \mu \mathrm{L}$ and 1,000 $\mu \mathrm{L}$ Biopur pipette tips and 5-mL bulk pipette tips. Charge the pipettes in the 4 -stand charger.

- Use the one 5-100 $\mu \mathrm{L}$ pipette in the standard (PIP) mode and set the volume to 100 $\mu \mathrm{L}$. Use this dedicated pipette to aliquot the whole blood for RBC Folate - vessel 3. Use this pipette to aliquot $50 \mu 1$ the ascorbic acid (7\% solution) for Folate1 vessel 110.

- Use one of the two $50-1,000 \mu \mathrm{L}$ pipettes in the standard (PIP) mode and set the volume to $1,000 \mu \mathrm{L}$. Use this dedicated pipette to aliquot the $1 \%$ ascorbic acid solution for RBC Folate - vessel 3.

- Use the second 50-1,000 $\mu \mathrm{L}$ pipette in the FIX mode. Set the five fixed volumes to $300 \mu \mathrm{L}, 400 \mu \mathrm{L}, 500 \mu \mathrm{L}$, and $700 \mu \mathrm{L}$. Use this pipette to aliquot all whole blood tests, except RBC Folate - vessel 3 and pooled serum volumes that match the five volume settings.

- Use the second $50-1,000 \mu \mathrm{L}$ pipette and the $100-5,000 \mu \mathrm{L}$ pipette in the DIS mode. Set the DIS volume on the $50-1,000 \mu \mathrm{L}$ to $100 \mu \mathrm{L}$. Set the DIS volume on the $100-$ $5,000 \mu \mathrm{L}$ to $500 \mu \mathrm{L}$. Use these pipettes to aliquot pooled serum.

- Use the 100-5,000 $\mu \mathrm{L}$ pipette in the FIX mode. Set the five fixed volumes to 1,000 $\mu \mathrm{L}, 2,000 \mu \mathrm{L}, 3,000 \mu \mathrm{L}, 4,000 \mu \mathrm{L}$, and $5,000 \mu \mathrm{L}$. Use this pipette to aliquot pooled serum volumes that match the five volume settings.

- Use the 100-5,000 $\mu \mathrm{L}$ pipette in the ADS mode. Program this mode in slot 2. Set the volume to $500 \mu \mathrm{L}$ and the interval to 1 second. Use this pipette to aliquot pooled serum.

- Use the $100-5,000 \mu \mathrm{L}$ pipette in the SD mode. Set the volumes to match the agespecific processing protocols. Set program 1 to match the adult profile for vessels 7024. Set program 3 to match the child's profile ages 1-2. Set program 4 to match the child's profile ages 3-5. Set program 5 to match the child's profile ages $6-11$. Use this pipette to aliquot pooled serum. 
Eppendorf Research Pro Electronic Pipettes

\begin{tabular}{|c|c|c|c|c|}
\hline Pipette & Tip & Mode & Volume & Matrix \\
\hline$* 5-100 \mu L$ & $200 \mu L$ & $F I X$ & $100 \mu L$ & Whole blood \& 7\% Ascorbic Acid \\
\hline$* 50-1,000 \mu L$ & $1,000 \mu L$ & $P I P$ & $1,000 \mu L$ & $1 \%$ Ascorbic acid \\
\hline $50-1,000 \mu L$ & $1,000 \mu L$ & $F I X$ & $\begin{array}{l}100 \mu L \\
400 \mu L \\
500 \mu L \\
700 \mu L \\
1,000 \mu L\end{array}$ & $\begin{array}{l}\text { Whole blood } \\
\text { Pooled serum }\end{array}$ \\
\hline $100-5,000 \mu L$ & $5,000 \mu L$ & $S D$ & $\begin{array}{l}\text { Program } 1-12+ \\
\text { Program } 3-\text { Child } 1-2 \\
\text { Program } 4-\text { Child } 3-5 \\
\text { Program } 5-\text { Child } 6-11\end{array}$ & Pooled serum \\
\hline
\end{tabular}

Italic $=$ Pipette's primary program

* Dedicated pipette for RBC Folate and folate dilutions

\subsubsection{Safety Precautions, Handling, Care, Maintenance, and Battery for the Research Pro}

Before using the Research pro for the first time, please read the entire Eppendorf Research Pro Instruction Manual. Observe the following points:

\section{Handling}

- Use the Research pro only when a pipette tip is attached.

- Do not lay down the Research pro when a filled pipette tip is attached.

- After liquid has been aspirated, press the Actuate key only when you are sure the liquid will be dispensed correctly.

- When the pipette is switched on and when the Reset key is pressed, the piston may move. Please ensure beforehand that the liquid in the pipette tip will be dispensed correctly. 
- Emergency stop: pressing the Reset key can stop a moving piston.

- Operate the Research pro at temperatures between $15^{\circ} \mathrm{C}$ and $40^{\circ} \mathrm{C}$. Operate at a maximum humidity of $80 \%$ at a temperature up to $31^{\circ} \mathrm{C}$.

\section{Care and Maintenance}

- Do not clean the display or any of the labeling using acetone or aggressive solutions.

- Do not allow any liquid to enter the device.

- Only authorized service personnel may carry out repairs.

- Use original spare parts and accessories (battery, pipette tips) only.

\section{Battery}

- Charge the battery before operating the device for the first time.

- Charge the battery in the pipette only, using the charging stand and the original power unit.

- If a flashing battery symbol appears in the display, stop dispensing and charge the battery.

- When charging the battery, do not allow the battery to be exposed to any metallic surfaces.

- Dispose of used batteries as special waste.

- Never charge the Research pro without a battery.

- Before storing the Research pro for a long period, remove the battery. 


\subsubsection{Technical Data for the Research Pro}

Single Channel Pipette

\begin{tabular}{|l|c|l|l|l|c|c|}
\hline $\begin{array}{c}\text { Volume } \\
\text { Range } \mu \mathrm{L}\end{array}$ & $\begin{array}{c}\text { Step } \\
\text { size } \mu \mathrm{L}\end{array}$ & \multicolumn{1}{c|}{ Color code } & \multicolumn{1}{|c|}{ Pipette tip } & \multicolumn{1}{|c|}{ Test volume } & $\begin{array}{c}\text { Inaccuracy } \\
\%\end{array}$ & $\begin{array}{c}\text { Imprecision } \\
\%\end{array}$ \\
\hline $5-100$ & 0.1 & Yellow & $200 \mu \mathrm{L}$ & $100 \mu \mathrm{L}$ & \pm 0.8 & $\leq 0.2$ \\
\hline $50-1,000$ & 1 & Blue & $1,000 \mu \mathrm{L}$ & $1,000 \mu \mathrm{L}$ & \pm 0.6 & $\leq 0.2$ \\
\hline $100-5,000$ & 10 & Violet & $5-\mathrm{mL}$ & $\begin{array}{l}500 \mu \mathrm{L} \\
5,000 \mu \mathrm{L}\end{array}$ & $\begin{array}{l} \pm 3.0 \\
\pm 0.6\end{array}$ & $\leq 0.6$ \\
& & & & & \\
\hline
\end{tabular}

The technical data are valid only when the Research pro is used with Eppendorf pipette tips.

- Test conditions in accordance with DIN 12650 for piston-stroke pipettes with an air cushion by means of a standardized fine balance with a moisture trap;

- Number of determination: 10 ;

- Degassed distilled water, $20^{\circ} \mathrm{C}-25^{\circ} \mathrm{C} \pm 0.5^{\circ} \mathrm{C}$;

- Maximum speed;

- Standard operation;

- Prewetted pipette tip, dispensing onto inside of tube due to possible evaporation; and

- Check volumes $\leq 10 \mu \mathrm{L}$ immediately after aspiration.

\section{Battery}

- Nickel-metal hydride battery pack, 1,200 mAh/2.4 V with reversible over-current and over-temperature protection

- Charging time: approximately 9.5 hours for a fully discharged battery; overcharging protection with the pipette 


\section{Power Unit}

- Input voltage, country-specific: $230 \mathrm{~V} \pm 10 \%, 50 / 60 \mathrm{~Hz} ; 115 \mathrm{~V} \pm 10 \%, 60 \mathrm{~Hz} ; 100 \mathrm{~V}$ $\pm 10 \%, 50 / 60 \mathrm{~Hz}$.

- $\quad$ Output voltage: 9 V DC; 200 mA (1.8 VA)

\section{Delivery Package}

The Research pro is supplied as a single-channel pipette for different volume ranges. The pipette is delivered without a charging adapter, but each lab is supplied with a charging stand. Each package contains the following:

- Charging stand with separate power unit;

- Special tool(s) according to pipette type;

- Operating manual with measuring protocol and CE conformity assurance document; and

- Ni-MH battery pack.

\section{Inserting the Battery}

Charge the battery in the pipette. Charging may only be carried out using the charging stand with the original power unit. Do not try to recharge a pipette if the battery is not installed. A new battery does not attain its full capacity until two or three complete charging/discharging cycles have been completed.

For charging purposes, place the pipette in the charging stand.

During the start and termination of the charging process, "CON" appears briefly on the left side of the display. The display is switched on during the charging process. The battery symbol appears in a rolling form. During charging, the dispensing function is frozen. When the charging process has finished, the battery symbol appears in the display without moving. When the pipette has been fully charged, it may remain in the charging stand. To increase the charging capacity, the battery should be 
discharged until the flashing battery symbol appears. To avoid accidental discharge, remove the battery from the Research pro during extended periods of nonuse. In this case, all data remain stored.

\subsubsection{Operating Principle}

\section{Display and Keypad}

- To switch on: Press any key firmly.

- To switch off: Unit does this automatically after 10 minutes of nonuse.
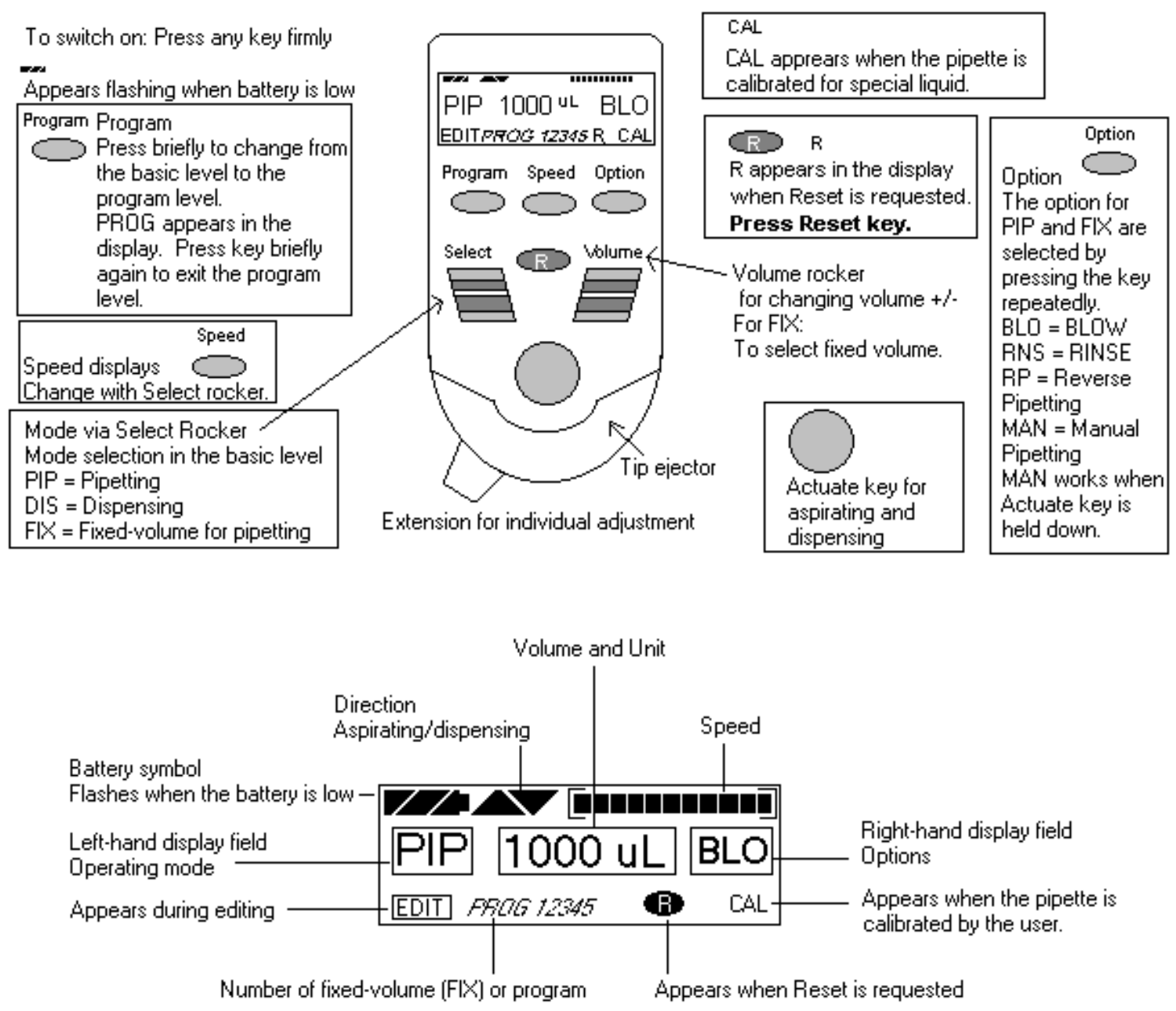


\subsubsection{Functions of the Unit}

The Research pro is a microprocessor-controlled pipette, which executes the piston movement with the aid of a stepper motor. The power supply is a rechargeable nickel-metal hydride battery.

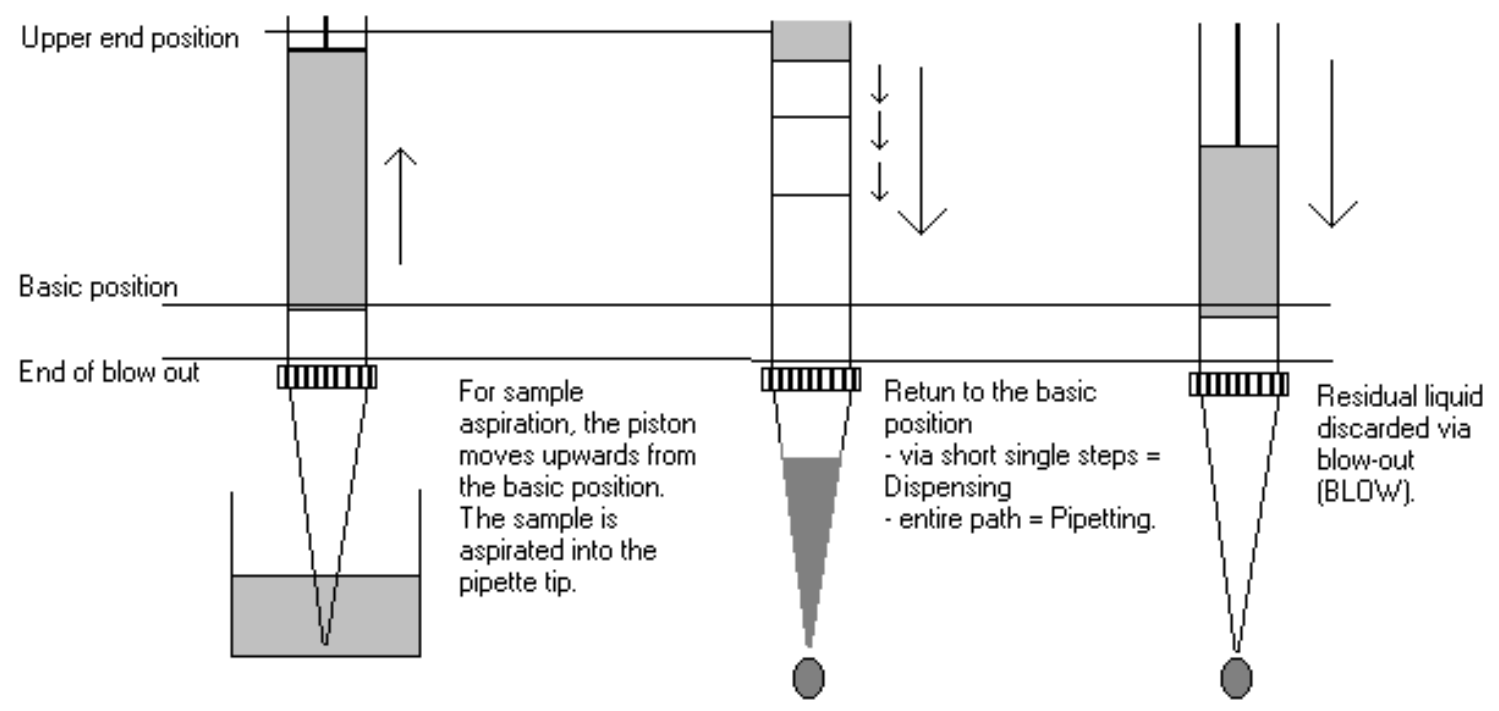

Dispensing and reverse pipetting require a different basic position than that for pipetting. The request to change the basic position is accomplished by selecting the Reset button.

\section{Ejector with Extension}

The force translation of the ejector for pipette tips ensures that the tips are ejected very easily. The extension can be individually adjusted to accommodate left- and right-handed users as well as different hand sizes. Select the desired setting by simply sliding the extension into the position as required.

\section{Hook on the Hand Rest}

Loosening the screw enables the hook on the rear of the pipette to be adjusted to suit all hand sizes. This can be done easily with a small Phillips screwdriver. 


\section{Contact Surface on the Head of the Pipette}

Both upper surfaces are required for charging the nickel-metal hydride battery.

The lower surfaces are the data interfaces for the service department.

The contact to the charging adapter or the charging stand cannot be confused with any other contacts.

\section{Acoustic Signals}

Certain signals assist the user for different operations:

- Short signal to acknowledge that the keypad has been pressed;

- Slightly higher tone to indicate that the function for which the key has been pressed cannot be executed;

- Louder tone to indicate that the piston movement has ended;

- Louder tone to indicate that a specific sequence of steps (e.g., dispensing) has ended; and

- Louder tone to indicate an error message.

The acoustic signals are a great help when you are familiarizing yourself with the operating procedure of the Research pro. They can be switched off if required.

\subsubsection{Mode of Operation}

If the display of the Research pro is dark, activate the pipette by pressing any key firmly. If the pipette has not been used for a long period, a motor reset is executed after it has been activated.

- Attach an Eppendorf pipette tip to the Research pro. The color coding of the Actuate key corresponds to the color code of the racks for the pipette tips. The dispensing liquid is aspirated into the tip; 
- When aspirating liquid, immerse the pipette tip vertically - and as little as possible into the liquid. Following aspiration, remove the pipette tip from the liquid after the acoustic signal has been emitted. If the air bubbles have been aspirated, repeat this process;

- Never lay the pipette down when the tip is filled;

- To dispense the liquid, position the pipette tip against the side of the aspirating vessel; and

- Discard the pipette tip by pressing the Eject key, attach a new tip, and reaspirate liquid.

\section{Essential Operating Information}

The following sections contain systematic explanations of the operating procedure. It is essential to work through these sections with the pipette in your hand.

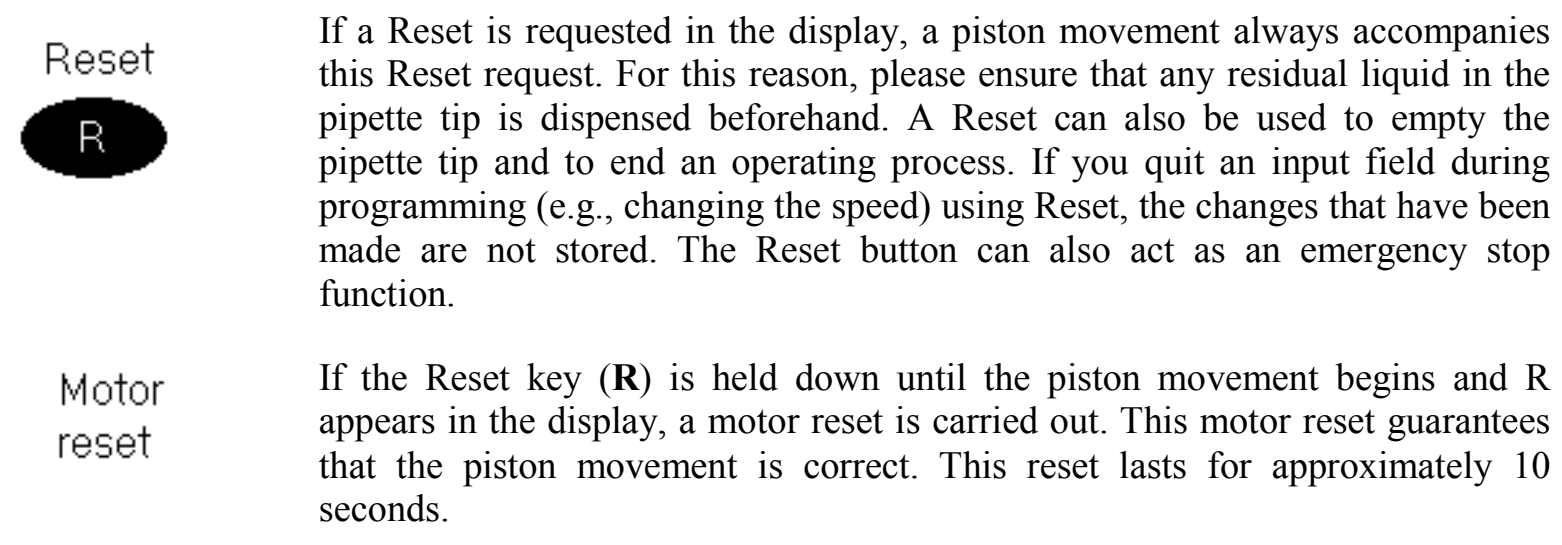

Please refer to the following illustrations when going through each operation.

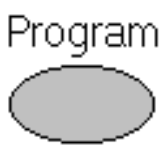

The Research pro has two operating levels. The basic modes for pipetting (PIP and FIX) and (DIS) are accessed in the first level by pressing the Select rocker. PROG appears in the display by pressing the "Program" key. You are now in the second level. Using the Select rocker, select from the five available program slots. To quit this level, press the "Program" key briefly and PROG will disappear from the display. 


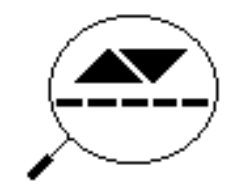

If the piston movements appear in the display without any volumes, this indicates a process that is not connected to liquid aspiration or dispensing.

\section{Aspirating and Dispensing Speeds}

The selected speed can be viewed by pressing the Speed key several times. The speed is changed as follows:
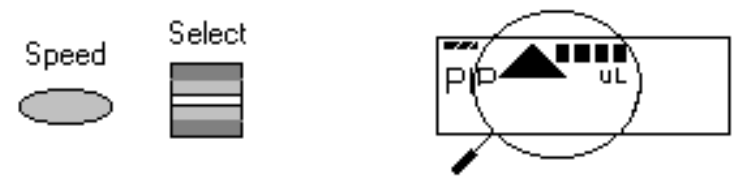

Display and selection of aspirating speed.

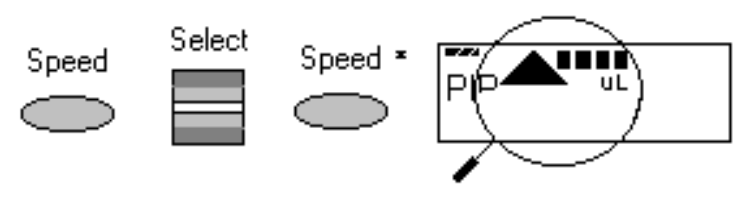

Display and selection of dispensing speed.

- This procedure does not have to be completed using the Speed key. Any key, with the exception of Reset, may be used. At the same time, pressing the key executes the respective key function;

- The direction of the arrow for speed flashes in this input field;

- The speed can be altered before every dispensing or aspiration operation; and

- Important - high speeds have a much lower current consumption than low speeds. 
7.7.1.6 Pipetting in the Standard Mode (PIP) (Use this mode for whole blood RBC folate and Vitamin C [5-100 $\mu$ L pipette] and ascorbic acid [50-1,000 $\mu$ L pipette].)

In the standard mode, blowout is executed automatically when liquid is dispensed. The standard mode is recommended for rapid series pipetting with aqueous solutions.

\begin{tabular}{|l|l|}
\hline \multicolumn{2}{|c|}{ Programming - PIP } \\
\hline Step 1 & $\begin{array}{l}\text { Select the pipetting mode "PIP." Use the Select rocker switch to move up } \\
\text { and down through the three modes (PIP, DIS, and FIX) until the "PIP" } \\
\text { choice appears in the left side of the display. } \\
\text { (If PROG appears in the display: Press the Program key briefly until PROG } \\
\text { disappears.) }\end{array}$ \\
\hline Step 2 & $\begin{array}{l}\text { Select the volume. Use the Volume rocker to move the number up or down } \\
\text { until the number (volume) 100 for the 5-100- } \mu \text { L pipette and 1,000 } \mu \text { L for the } \\
\text { 50-1,000- } \mu \text { L pipette is reached. If necessary, press the Option key several } \\
\text { times until the right side of the display is blank. (Do not use the BLO, RNS, } \\
\text { RP, or MAN options.) }\end{array}$ \\
\hline Step 1 & $\begin{array}{l}\text { Aspirate the liquid. Make sure the Aspirating arrow in the display points up. } \\
\text { Immerse the tip of the pipette into the liquid and press the Actuate key to } \\
\text { draw the liquid into the pipette tip. }\end{array}$ \\
\hline Step 2 & $\begin{array}{l}\text { Place the pipette tip against the side of the receiving vessel. Make sure the } \\
\text { Dispensing arrow in the display points down. To dispense the liquid with a } \\
\text { blowout, press the Aspirate button. }\end{array}$ \\
\hline Step 3 & $\begin{array}{l}\text { The pipette is ready to aspirate again if the Aspirating arrow in the display } \\
\text { points up. }\end{array}$ \\
\hline
\end{tabular}




\subsubsection{Pipetting with a Preselected Fixed (FIX) Volume}

(Use this mode for aliquoting whole blood volumes 300, 400, 500, 600, and 1,000 $\mu \mathrm{L}$ and for $400 \mu L M P A-50-1,000 \mu L$ pipette, and for pipetting pooled serum volumes 1,000, 2,000, 3,000, 4000, and 5,000 $\mu \mathrm{L}-100-5,000 \mu \mathrm{L}$.)

FIX is used to rapidly set and recall five frequently used pipette volumes. Five FIX volumes are predefined in the pipette. FIX volumes can be changed via programming.

\begin{tabular}{|c|c|}
\hline \multicolumn{2}{|r|}{ Programming FIX fixed volumes } \\
\hline Step 1 & $\begin{array}{l}\text { Select the pipetting mode "FIX." Use the Select rocker switch to move up } \\
\text { and down through the three modes (PIP, DIS, FIX) until the "FIX" choice } \\
\text { appears in the display. } \\
\text { (If PROG appears in the display: Press the Program key briefly until PROG } \\
\text { disappears.) }\end{array}$ \\
\hline Step 2 & $\begin{array}{l}\text { Hold down the Program key until EDIT appears in the lower left corner of } \\
\text { the display. The volume flashes. }\end{array}$ \\
\hline Step 3 & $\begin{array}{l}\text { Select the first memory slot using the Select rocker. Use the Select rocker to } \\
\text { move the memory slot number up and down until the } \# 1 \text { is displayed. }\end{array}$ \\
\hline Step 4 & $\begin{array}{l}\text { Select the volume for memory slot } \# 1 \text {. Use the Volume rocker to move the } \\
\text { number (volume) up and down until the number (volume) } 300 \mu \mathrm{L} \text { for the } 5 \text { - } \\
1,000-\mu \mathrm{L} \text { pipette or } 1,000 \mu \mathrm{L} \text { for the } 100-5,000 \mu \mathrm{L} \text { pipette is reached. }\end{array}$ \\
\hline Step 5 & $\begin{array}{l}\text { Select the next (second) memory slot for purposes of changing the volume. } \\
\text { Select the second memory slot using the Select rocker. Use the Select rocker } \\
\text { to move the memory slot number up and down until the } \# 2 \text { is displayed. }\end{array}$ \\
\hline Step 6 & $\begin{array}{l}\text { Select the volume for memory slot } \# 2 \text {. Use the Volume rocker to move the } \\
\text { number (volume) up and down until the number (volume) } 400 \mu \mathrm{L} \text { for the } 5 \text { - } \\
1,000-\mu \mathrm{L} \text { pipette or } 2,000 \mu \mathrm{L} \text { for the } 100-5,000 \mu \mathrm{L} \text { pipette is reached. }\end{array}$ \\
\hline Step 7 & $\begin{array}{l}\text { Select the next (third) memory slot for purposes of changing the volume. } \\
\text { Select the third memory slot using the Select rocker. Use the Select rocker to } \\
\text { move the memory slot number up and down until the } \# 3 \text { is displayed. }\end{array}$ \\
\hline Step 8 & $\begin{array}{l}\text { Select the volume for memory slot \#3. Use the Volume rocker to move the } \\
\text { number (volume) up and down until the number (volume) } 500 \mu \mathrm{L} \text { for the } 5 \text { - } \\
1,000-\mu \mathrm{L} \text { pipette or } 3,000 \mu \mathrm{L} \text { for the } 100-5,000 \mu \mathrm{L} \text { pipette is reached. }\end{array}$ \\
\hline Step 9 & $\begin{array}{l}\text { Select the next (fourth) memory slot for purposes of changing the volume. } \\
\text { Select the fourth memory slot using the Select rocker. Use the Select rocker } \\
\text { to move the memory slot number up and down until the } \# 4 \text { is displayed. }\end{array}$ \\
\hline Step 10 & $\begin{array}{l}\text { Select the volume for memory slot } \# 4 \text {. Use the Volume rocker to move the } \\
\text { number (volume) up and down until the number (volume) } 600 \mu \mathrm{L} \text { for the } 5 \text { - } \\
1,000-\mu \mathrm{L} \text { pipette or } 4000 \mu \mathrm{L} \text { for the } 100-5,000 \mu \mathrm{L} \text { pipette is reached. }\end{array}$ \\
\hline Step 11 & $\begin{array}{l}\text { Select the next (fifth) memory slot for purposes of changing the volume. } \\
\text { Select the fifth memory slot using the Select rocker. Use the Select rocker to } \\
\text { move the memory slot number up and down until the } \# 5 \text { is displayed. }\end{array}$ \\
\hline
\end{tabular}




\begin{tabular}{|l|l|}
\hline \multicolumn{2}{|c|}{ Programming FIX fixed volumes (continued) } \\
\hline Step 12 & $\begin{array}{l}\text { Select the volume for memory slot } \# 5 \text {. Use the Volume rocker to move the } \\
\text { number (volume) up and down until the number (volume) } 1,000 \mu \mathrm{L} \text { for the 5- } \\
1,000-\mu \mathrm{L} \text { pipette or } 5,000 \mu \mathrm{L} \text { for the } 100-5,000 \mu \mathrm{L} \text { pipette is reached. }\end{array}$ \\
\hline Step 13 & $\begin{array}{l}\text { End the programming. Press the Program key to stop programming. } \\
\text { Alternatively, to stop programming and aspirate the volume of liquid in the } \\
\text { display, press the Aspirate button. EDIT and the flashing volume disappear } \\
\text { from the display. }\end{array}$ \\
\hline Step 1 & $\begin{array}{l}\text { Select the correct mode. Use the Select rocker to move up and down through } \\
\text { the three mode choices (PIP, DIS, FIX) until FIX displays. }\end{array}$ \\
\hline Step 2 & $\begin{array}{l}\text { Select one of the five programmed fixed volumes. Select the correct memory } \\
\text { slot. Use the Volume rocker to move the volume number up and down until } \\
\text { the correct choice displays. }\end{array}$ \\
\hline Step 3 & $\begin{array}{l}\text { Aspirate the liquid. Make sure the Aspirating arrow in the display points up. } \\
\text { Immerse the tip of the pipette into the liquid and press the Actuate key to } \\
\text { draw the liquid into the pipette tip. }\end{array}$ \\
\hline Step 4 & $\begin{array}{l}\text { Place the pipette tip against the side of the receiving vessel. Make sure the } \\
\text { Dispensing arrow in the display points down. To dispense the liquid with a } \\
\text { blowout, press the Aspirate button. }\end{array}$ \\
\hline Step 5 & $\begin{array}{l}\text { The pipette is ready to aspirate again if the Aspirating arrow in the display } \\
\text { points up. }\end{array}$ \\
\hline
\end{tabular}

\subsubsection{Dispensing (DIS)}

(Use this mode to pipette $100 \mu \mathrm{L}$ whole blood and/or pooled serum - 50-1,000 $\mu \mathrm{L}$ pipette and $500 \mu \mathrm{L}$ pooled serum 100-5,000 $\mu \mathrm{L}$ pipette.)

During this procedure, the aspirating liquid is dispensed in partial steps. As with "Reverse Pipetting," slightly more liquid is aspirated than is necessary for the sum of the dispensing steps. The maximum number of dispensing steps per filling of the pipette tip is displayed on the right side of the display. The maximum number of dispensing steps is calculated by the pipette and is based on the dispensing volume and the pipette tip. For example, if the dispensing volume is set at $100 \mu \mathrm{L}$ and the pipette tip is $1,000 \mu \mathrm{L}$ then there are 10 dispensing steps. If the dispensing volume is set at $500 \mu \mathrm{L}$ and the pipette tip is $5,000 \mu \mathrm{L}$, then there are also 10 dispensing steps. 


\begin{tabular}{|c|c|}
\hline \multicolumn{2}{|r|}{ Programming DIS } \\
\hline Step 1 & $\begin{array}{l}\text { Select the pipetting mode "DIS." Use the Select rocker switch to move up } \\
\text { and down through the three modes (PIP, DIS, FIX) until the "DIS" choice } \\
\text { appears in the display. } \\
\text { (If PROG appears in the display: Press the Program key briefly until PROG } \\
\text { disappears.) }\end{array}$ \\
\hline Step 2 & $\begin{array}{l}\text { Select the volume per dispensing step. Use the Volume rocker to move the } \\
\text { number up or down until the number (volume) } 100 \text { for the } 50-1,000-\mu \mathrm{L} \\
\text { pipette or } 500 \mu \mathrm{L} \text { for the } 100-5,000 \mu \mathrm{L} \text { pipette is reached. }\end{array}$ \\
\hline Step 3 & $\begin{array}{l}\text { Select the aspirating speed using the Speed key and the Select rocker. Press } \\
\text { the Speed key, then use the Select rocker to move the speed indicator all the } \\
\text { way to the right. When finished, press the Speed key. The pipette is ready for } \\
\text { entry of the dispensing speed. }\end{array}$ \\
\hline Step 4 & $\begin{array}{l}\text { Select the dispensing speed. Use the Select rocker to move the speed } \\
\text { indicator all the way to the right. Press the Speed key to exit. }\end{array}$ \\
\hline \multicolumn{2}{|r|}{ Pipetting Procedure } \\
\hline Step 1 & $\begin{array}{l}\text { Select the correct mode. Use the Select rocker to move up and down through } \\
\text { the three mode choices (PIP, DIS, FIX) until DIS displays. }\end{array}$ \\
\hline Step 2 & $\begin{array}{l}\text { Aspirate the liquid. Make sure the Aspirating arrow in the display points up. } \\
\text { Immerse the tip of the pipette into the liquid and press the Actuate key to } \\
\text { draw the liquid into the pipette tip. (If } \mathbf{R} \text { appears in the display: Press the R } \\
\text { key briefly.) }\end{array}$ \\
\hline Step 3 & $\begin{array}{l}\text { Place the pipette tip against the side of the first receiving vessel. Make sure } \\
\text { the Dispensing arrow in the display points down. To dispense the first } \\
\text { aliquot, press the Aspirate button. The dispensing step counter (number) on } \\
\text { the right-hand side of the display counts down one number. }\end{array}$ \\
\hline Step 4 & $\begin{array}{l}\text { Place the pipette tip against the side of the second receiving vessel. Make } \\
\text { sure the Dispensing arrow in the display points down. To dispense the second } \\
\text { aliquot, press the Aspirate button. The dispensing step counter (number) on } \\
\text { the right side of the display counts down one more number. }\end{array}$ \\
\hline Step 5 & $\begin{array}{l}\text { Continue dispensing until the dispensing step number reaches } 0 \text {. A special } \\
\text { acoustic signal is emitted. }\end{array}$ \\
\hline Step 6 & $\begin{array}{l}\text { Complete the "blank" step. Press the Actuate key to perform the blank step. } \\
\text { This is not a dispensing step. A special acoustic signal is emitted. }\end{array}$ \\
\hline Step 7 & $\begin{array}{l}\text { Discard the last drop. Press the Actuate key to blow out the last drop. This is } \\
\text { not a dispensing step. Dashes replace the volume number in the display. If } \\
\text { the Actuate key is held down during the final blowout, the device does not } \\
\text { return the Aspiration arrow to the up position until the key is released. }\end{array}$ \\
\hline Step 8 & $\begin{array}{l}\text { The pipette is ready to aspirate again if the Aspirating arrow in the display } \\
\text { points up and the volume number and dispensing step display. }\end{array}$ \\
\hline
\end{tabular}




\subsubsection{Notes on the Programs}

- To reach the programming level, press the Program key briefly;

- PROG appears in the display together with a memory slot number from 1 to 5;

- With the aid of the Select rocker, five programs can be shown on the left side of the display; and

- To program the five memory slots, it is possible to select from seven different program sequences.

\section{1. $\quad \mathrm{PIP}=$ Pipetting}

As per PIP outside program level. The complete sequence with volume, option, and speeds is programmed in one memory slot.

2. $\mathrm{SP}=$ Sequential Pipetting

Up to five different pipette steps can be linked to each other. Volume, option, and speeds are defined for each pipetting sequence.

3. $\quad$ DIS $=$ Dispensing

As per DIS outside the program level. The complete procedure is programmed in one memory slot with dispensing volume, dispensing steps, and speeds.

4. $\quad \mathrm{SD}=$ Sequential Dispensing

Up to 20 dispensing steps can be defined. Each dispensing step may have a different partial volume. The total volume may exceed the filling level of a pipette tip. This means that new liquid for the pipette tip must be aspirated during the dispensing procedure. Only one aspirating and dispensing speed is defined for all dispensing steps.

5. $\quad \mathrm{ADS}=$ Automatic Dispensing

With the Actuate key held down, all dispensing strokes are executed automatically with the same volume and in a user-defined rhythm (.01-10.0 seconds). Automatic dispensing may be interrupted by releasing the Actuate key. Apart from this, the procedure is identical to the DIS function.

6. $\quad$ DIL $=$ Diluting

During the diluting process, a diluent, then an air bubble, and finally a sample are aspirated into the pipette tip. The user defines the diluent volume and the sample volume. The air bubble is dependent on the sample volume and is always specified by the program. Selecting a pipetting option can dispense the entire 
contents of the pipette tip. Depending on the pipette used, the dilution ratio is between approximately 1:2 and approximately 1:20.

7. $\quad \mathrm{SDI}=$ Serial Diluting

For serial diluting, a defined volume of liquid is aspirated into the pipette tip. This liquid is dispensed and then mixed with a specific liquid volume by means of a programmable mixing process (volume, mixing cycles). Pressing the Actuate key separately starts the mixing process (MIX).

\section{EDIT}

1. If PROG is in the display, holding down the Program key can open the programming level. The Program key must be held down until the word EDIT appears in the display in front of PROG 1-5. Systematically programming is explained in the following subsection.

2. If the programming of a program sequence is quit by pressing Reset, the entries for the program sequence are not stored.

3. The same program sequence - with, for example, different volumes or options - can be stored in several memory slots. It would, therefore, be possible for different pipetting sequences to be stored permanently in the program level.

4. Programming sequences can be overwritten at any time under EDIT (hold down the Program key). During the programming process, the entry that is to be defined flashes in the display. Selections are made using the Select rocker. Volumes are selected using the Volume rocker.

5. Data are stored by pressing the Actuate key (ENTER function).

6. Options and speeds can be selected in the programming level by using the Select rocker or by using the Option and Speed key. We recommend that you read these sections before you start programming. The following sections on programming for pipetting contain only a description of operations using the rockers. Programming and execution of the other programs are contained in Part B of the Research pro manual.

\subsubsection{Introduction - Additional Program Sequences}

During programming (EDIT and PROG in display), the Speed and Option keys and the Volume rocker can also be used. This enables users to skip backward and forward during the program sequence. 
When the programs are executed (only PROG is in the display), the Speed and Option keys and the Volume rocker are blocked.

If only parts of the program have been changed, programming can be ended prematurely by pressing the Program key briefly.

If a memory slot is quit using Reset before programming has been completed, the changes that have not been carried out for this memory slot are not saved. EDIT disappears from the display.

\subsubsection{SD $=$ Sequential Dispensing}

(Use this mode to pipette the 4 age-specific (1-2, 3-5, 6-11 and 12+) serum protocols - 100$5,000 \mu$ L pipette.)

The sequential dispensing program can be used when different volumes of a solution have to be dispensed consecutively. Up to 20 steps can be defined. If the sum of the dispensing steps is larger than the volume of the pipette tip, the procedure for normal dispensing is applicable when a liquid is aspirated repeatedly.

\begin{tabular}{|c|c|}
\hline \multicolumn{2}{|r|}{ Programming SD } \\
\hline Step 1 & $\begin{array}{l}\text { Select the programming mode. Press the Program key. PROG appears in the } \\
\text { display. }\end{array}$ \\
\hline Step 2 & $\begin{array}{l}\text { Select the memory slot number } 1 \text {. Use the Select rocker to move up and } \\
\text { down through the } 5 \text { program numbers until " } 1 \text { " is selected. }\end{array}$ \\
\hline Step 3 & Hold down the Program key until EDIT appears in the display. \\
\hline Step 4 & $\begin{array}{l}\text { Select the SD program. Use the Select rocker to move through the } \\
\text { programming choices until "SD" appears in the display. Press the Actuate } \\
\text { key. }\end{array}$ \\
\hline Step 5 & $\begin{array}{l}\text { Determine the dispensing steps } 1-20 \text {. Use the Select rocker to move the } \\
\text { number up or down until the number } 19 \text { is displayed. Press the Actuate key } \\
\text { to store the number of steps in the program. }\end{array}$ \\
\hline Step 6 & $\begin{array}{l}\text { Select and store the volume for the first dispensing step. Use the Select or } \\
\text { Volume rocker to move the number up or down until the number } 1,000 \text { is } \\
\text { displayed. Press the Actuate key to store the volume for step } 1 \text {. }\end{array}$ \\
\hline Step 7 & $\begin{array}{l}\text { Continue programming the remaining } 18 \text { steps and volumes for the } 12+ \\
\text { protocol. }\end{array}$ \\
\hline
\end{tabular}




\begin{tabular}{|c|c|}
\hline \multicolumn{2}{|r|}{ Programming SD (continued) } \\
\hline Step 8 & $\begin{array}{l}\text { Select the aspirating and dispensing speed using the Speed key and the Select } \\
\text { rocker. Press the Speed key, then use the Select rocker to move the speed } \\
\text { indicator all the way to the right. Press the Actuate key to store the speed in } \\
\text { the program. }\end{array}$ \\
\hline Step 9 & $\begin{array}{l}\text { Programming is complete. The display should show: } \\
\text { "SD } 500 \mu \mathrm{L} 19 \text { " }\end{array}$ \\
\hline \multicolumn{2}{|r|}{ Pipetting Procedure } \\
\hline Step 1 & $\begin{array}{l}\text { The necessary or largest possible amount of serum is aspirated. Aspirate the } \\
\text { serum. Make sure the direction of the aspirating arrow is pointed up. Press } \\
\text { the Actuate key once. The pipette will aspirate } 1,000 \mu \mathrm{L} \text {. }\end{array}$ \\
\hline Step 2 & Dispense the serum. Make sure the aspirating arrow is pointed downward. \\
\hline Step 3 & $\begin{array}{l}\text { Place the pipette tip against the side of the first receiving vessel. To dispense } \\
\text { the first aliquot, press the Actuate key once to dispense one } 1,000 \mu \mathrm{L} \text { aliquot. } \\
\text { The dispensing step counter (number) on the right side of the display counts } \\
\text { down one number. }\end{array}$ \\
\hline Step 4 & Place the pipette tip against the side of the second receiving vessel. \\
\hline Step 5 & $\begin{array}{l}\text { Continue dispensing the remaining aliquots until the (number) on the right } \\
\text { side of the display reaches } 0 \text {. If a slot in the processing rack is empty, } \\
\text { dispense the aliquot back into the polled serum vessel. }\end{array}$ \\
\hline Step 6 & $\begin{array}{l}\text { Complete the "blank" step. Press the Actuate key to perform the blank step. } \\
\text { This is not a dispensing step. A special acoustic signal is emitted. }\end{array}$ \\
\hline Step 7 & $\begin{array}{l}\text { Discard the last drop. Press the Actuate key to blow out the last drop. This is } \\
\text { not a dispensing step. Dashes replace the volume number in the display. If } \\
\text { the Actuate key is held down during the final blowout, the device does not } \\
\text { return the Aspiration arrow to the up position until the key is released. }\end{array}$ \\
\hline Step 8 & $\begin{array}{l}\text { The pipette is ready to aspirate again if the Aspirating arrow in the display } \\
\text { points up and the volume number and dispensing step display. }\end{array}$ \\
\hline
\end{tabular}

Continue programming the remaining program slots, age-specific protocols, and volumes.

\subsubsection{ADS $=$ Automatic Dispensing}

(Use this mode to repetitively pipette $500 \mu \mathrm{L}$ pooled serum - 100-5,000 $\mu$ L pipette.)

The automatic dispensing function allows a single volume to be dispensed repetitively with a predetermined time interval of between 0 and 10 seconds. 


\begin{tabular}{|c|c|}
\hline \multicolumn{2}{|r|}{ Programming ADS } \\
\hline Step 1 & $\begin{array}{l}\text { Select the programming mode. Press the Program key. PROG appears in the } \\
\text { display. }\end{array}$ \\
\hline Step 2 & $\begin{array}{l}\text { Select the memory slot number } 2 \text {. Use the Select rocker to move up and } \\
\text { down through the } 5 \text { program numbers until " } 2 \text { " is selected. }\end{array}$ \\
\hline Step 3 & Hold down the Program key until EDIT appears in the display. \\
\hline Step 4 & $\begin{array}{l}\text { Select the ADS program. Use the Select rocker to move through the } \\
\text { programming choices until "ADS" appears in the display. Press the Actuate } \\
\text { key. }\end{array}$ \\
\hline Step 5 & $\begin{array}{l}\text { Select and store the dispensing volume. Use the Select or Volume rocker to } \\
\text { move the number until } 500 \text { is displayed. Press the Actuate key to store the } \\
\text { volume in the program. }\end{array}$ \\
\hline Step 6 & $\begin{array}{l}\text { Select the aspirating and dispensing speed using the Speed key and the Select } \\
\text { rocker. Press the Speed key, and then use the Select rocker to move the speed } \\
\text { indicator all the way to the right. Press the Actuate key to store the speed in } \\
\text { the program. }\end{array}$ \\
\hline Step 7 & $\begin{array}{l}\text { Reduction of the dispensing steps. The total number of dispensing steps } \\
\text { displays on the right side of the display. Do not use the Select rocker to } \\
\text { change this number. The pipette has calculated the number of dispensing } \\
\text { steps based on the aspirating volume and the size of the pipette tip. Press the } \\
\text { Actuate key to store the number of calculated dispensing steps and to move } \\
\text { to the final programming step. }\end{array}$ \\
\hline Step 8 & $\begin{array}{l}\text { Set the dispensing frequency. Use the Select rocker to move the number of } \\
\text { seconds (SEC) up or down until } 1 \text { is displayed before SEC in the display. } \\
\text { Press the Actuate key to store this frequency in the program. }\end{array}$ \\
\hline Step 9 & $\begin{array}{l}\text { Programming is complete. The display should show: } \\
\text { "ADS } 500 \mu \mathrm{L} 10 "\end{array}$ \\
\hline
\end{tabular}




\begin{tabular}{|l|l|}
\hline \multicolumn{2}{|c|}{ Pipetting Procedure } \\
\hline Step 1 & $\begin{array}{l}\text { Aspirate the serum. Make sure the aspirating arrow is pointed upward. Press } \\
\text { the Actuate key once. The pipette will aspirate 5,000 } \mu \mathrm{L} .\end{array}$ \\
\hline Step 2 & $\begin{array}{l}\text { Dispense the serum. Make sure the aspirating arrow is pointed downward. } \\
\text { Press the Actuate key once. Dispensing is carried out while the Actuate key } \\
\text { is held down. An acoustic signal is emitted after the dispensing step. To } \\
\text { interrupt the action, release the Actuate key. }\end{array}$ \\
\hline Step 3 & $\begin{array}{l}\text { Place the pipette tip against the side of the first receiving vessel. To dispense } \\
\text { the first aliquot, press the Actuate key once to dispense one 500 } \mu \text { L aliquot, } \\
\text { or to automatically pipette sequential 500 } \mu \text { L aliquots, hold the actuate key } \\
\text { down. The dispensing step counter (number) on the right side of the display } \\
\text { counts down one number. As soon as you hear the acoustic signal, move the } \\
\text { pipette tip to the next vessel. }\end{array}$ \\
\hline Step 4 & $\begin{array}{l}\text { Place the pipette tip against the side of the second receiving vessel. } \\
\text { Continue dispensing 500 } \mu \text { L aliquots until the (number) on the right side of } \\
\text { the display reaches 0. }\end{array}$ \\
\hline Step 5 6 & $\begin{array}{l}\text { Complete the "blank" step. Press the Actuate key to perform the blank step. } \\
\text { This is not a dispensing step. A special acoustic signal is emitted. }\end{array}$ \\
\hline Step 7 & $\begin{array}{l}\text { Discard the last drop. Press the Actuate key to blow out the last drop. This is } \\
\text { not a dispensing step. Dashes replace the volume number in the display. If } \\
\text { the Actuate key is held down during the final blowout, the device does not } \\
\text { return the Aspiration arrow to the up position until the key is released. }\end{array}$ \\
\hline Step 8 & $\begin{array}{l}\text { The pipette is ready to aspirate again if the Aspirating arrow in the display } \\
\text { points up and the volume number and dispensing step display. }\end{array}$ \\
\hline
\end{tabular}




\subsubsection{Device Parameters}

Trained personnel may change device parameters only after a thorough inspection.

Device parameters are made up of the following:

BE1 To switch on/off the acoustic signal for the keypad (short beep).

BE2 To switch on/off the second acoustic signal for the end position of the dispensing piston, for warning, and for confirmation (different beeps).

CAL To alter the calibration data $\mu \mathrm{L}$, axle section (b) and gradient (m).

OPT Option for volume restriction:

When the $200 \mu \mathrm{L}$ tips are used with the $300 \mu \mathrm{L}$ pipettes (limit: $200 \mu \mathrm{L}$ ).

With "RP" and "DIS" when filter tips are used.

INI To initialize the original pipette data upon delivery.

The device parameters can all be found on one list. The Actuate key functions as an Enter key. After Enter has been pressed, the next line appears in the display.

\begin{tabular}{|l|l|}
\hline $\begin{array}{l}\text { To access the device } \\
\text { parameters }\end{array}$ & $\begin{array}{l}\text { With the pipette switched on, press both the Speed and Option keys } \\
\text { briefly at the same time. }\end{array}$ \\
\hline $\begin{array}{l}\text { To switch off/on acoustic } \\
\text { signal for the keypad }\end{array}$ & $\begin{array}{l}\text { Use the Select rocker to move through the selections until "BE 1" is } \\
\text { displayed in the left side of the display window. Press the Actuate key. }\end{array}$ \\
\hline $\begin{array}{l}\text { To switch on/off (on), second } \\
\text { acoustic signal }\end{array}$ & $\begin{array}{l}\text { Use the Select rocker to move through the selections until "BE 2" is } \\
\text { displayed in the left side of the display window. Press the Actuate key. }\end{array}$ \\
\hline To exit the device parameters & $\begin{array}{l}\text { Press the Actuate key when "INI" is displayed in the left side of the } \\
\text { display window. }\end{array}$ \\
\hline $\begin{array}{l}\text { To initialize the pipette and to } \\
\text { erase all settings }\end{array}$ & $\begin{array}{l}\text { Using "ENTER," access "INI" in device parameters. Hold down the } \\
\text { Program key. Initialization is completed when the device parameter } \\
\text { "INI" disappears from the display. }\end{array}$ \\
\hline
\end{tabular}




\subsubsection{Care, Sterilization, Maintenance, and Ordering Information}

The outside of the pipette can be wiped with a moist cloth. The use of water with a wetting agent is also permitted.

DO NOT ALLOW ANY LIQUID TO ENTER THE PIPETTE.

Only the lower part of the pipette should be rinsed with distilled water and dried. The pipette may be wiped clean with 60 percent isopropanol.

If the pipette is very contaminated, or if very aggressive chemicals are dispensed, the lower half of the Research pro should be disassembled. The individual parts are rinsed in distilled water and then dried. The piston is then lightly lubricated using silicone grease.

After opening the pipette, check that the piston is correctly positioned. A motor reset must also be executed. The motor reset is triggered by holding down the Reset key until $\mathbf{R}$ appears in the display.

\section{Sterilization}

Only the lower half of the pipette can be disassembled. The individual parts of the lower part must be disassembled as separate pieces. Please review the diagrams on page 27 of the Eppendorf Research Pro Instruction Manual before attempting to disassemble any pipette.

To loosen the lower part of the pipette for all pipettes except the $5,000 \mu \mathrm{L}$ :

1. Hold down the ejector and pull off the ejector sleeve. Force may be required.

2. Unscrew the lower part using the key. During disassembly, do not damage the piston!

3. Unscrew the piston. If necessary, first loosen the piston at the upper end using flat nose pliers. The piston is moved to the lowest position. 
To loosen the lower part of the $5,000 \mu \mathrm{L}$ pipette:

1. Hold down the ejector and pull off the ejector sleeve. Force may be required.

2. Unscrew the cylinder. The piston is held in place magnetically. During reassembly, do not damage the piston!

3. Pull the piston out of the upper part.

Assemble in reverse order. Engage the magnetic coupling of the 5,000 $\mu \mathrm{L}$ piston as shown in the diagram on page 27 of the instruction manual. Check that the dispensing function of the pipette is working correctly.

\section{Maintenance}

Apart from general care and occasional discharging (when the battery symbol starts flashing) and recharging of the pipette, no special maintenance is necessary if the pipette is used correctly. The lower part of the pipette may be replaced completely. The process for replacing the seals in the lower part is described in Part B of the Research pro manual: "Ordering information/service parts." The Service Department may carry out maintenance on the Research pro. Contact Brinkmann Instruments at 800-645-3050 Ext. 404.

A complete list of troubleshooting information is included on pages 28 and 29 of the Eppendorf Research Pro Instruction Manual. 


\section{Ordering Information}

\begin{tabular}{|l|c|c|}
\hline \multicolumn{1}{|c|}{ Item } & Size & Part number \\
\hline Eppendorf Research pro & $5-100 \mu \mathrm{L}$ & $2246-136-2$ \\
\hline Eppendorf Research pro & $50-1,000 \mu \mathrm{L}$ & $2246-138-9$ \\
\hline Eppendorf Research pro & $100-5,000 \mu \mathrm{L}$ & $2246-139-7$ \\
\hline Charging stand & Four pipettes & $2246-155-9$ \\
\hline Pipette Tips & $5-\mathrm{mL}$ & $2235-081-1$ \\
\hline Bulk-packaged, in bags & $200 \mu \mathrm{L}$ & \\
\hline $\begin{array}{l}\text { Eppendorf Biopur, colorless, sterile, pyrogen-free, } \\
\text { DNA-free, Rnase-free } \\
\text { In Boxes 5 x 96 = 480 tips }\end{array}$ & $2249-002-8$ \\
\hline $\begin{array}{l}\text { Eppendorf Biopur, colorless, sterile, pyrogen-free, } \\
\text { DNA-free, Rnase-free } \\
\text { In Boxes 5 x 96=480 tips }\end{array}$ & $1,000 \mu \mathrm{L}$ \\
\hline Additional accessories & & $2246-701-8$ \\
\hline Spare Ni-MH battery, 1,200 mAh
\end{tabular}

Page 102 in the instruction manual contains a complete list of additional parts and accessories.

- Use the dedicated 5-100 $\mu \mathrm{L}$ and the dedicated 50-1,000 $\mu \mathrm{L}$ Eppendorf Research pro pipettes and trace metal-free pipette tips to aliquot whole blood and perform the dilution for RBC Folate - vessel 3 and RBC Foll - vessel 106 using 1.0-uL ascorbic acid.

Pipetting Procedure

1. Using the pipette tip dispenser tray, press the pipette nozzle firmly into a fresh tip.

2. Aspirate and dispense the whole blood, serum, and ascorbic acid using procedures described in Section 7.7.

3. Place the tip against the inner wall of the cryovial as close to the bottom as possible.

4. Discard the tip in the Sharps container.

5. Cover the pipette tips at the end of each session to prevent contamination. 
The EDTA (tripotassium salts of ethylene diamine-tetracetic acid) acts as a chelating agent to combine with calcium in the blood to prevent coagulation. Use the well-mixed lavender 3-mL and 4-mL EDTA tubes for the hematology profile complete blood count (CBC) and certain whole blood tests. Use the 3- and/or 4-mL (age 12+) EDTA tubes for washed packed cells.

\subsubsection{Process the 3-mL or 4-mL EDTA Tube for Lead/Cad/HG/Se/Mn, RBC Folate, Glycohem, and Mercury}

The hematology technologist runs the $\mathrm{CBC}$ on the room temperature 3-mL (age 1-11 years) or $4 \mathrm{~mL}$ (age 6+ years) EDTA tube after the blood-processing technologist verifies proper identification and labeling, and processes the tube for the Lead/Cad/HG/Se/Mn, RBC Folate, Glycohem, and Mercury. If the EDTA tube is clotted, do not process vessel 1 (Lead/Cad/HG/Se/Mn), vessel 3 (RBC Folate), vessel 4 (Glycohem), or vessel 5 (Mercury). If the CBC was accidentally run before the whole blood for vessels $1(\mathrm{Lead} / \mathrm{Cad} / \mathrm{HG} / \mathrm{Se} / \mathrm{Mn})$ and 5 (Mercury) was pipetted, do not process these two vessels and add the comment "laboratory error" to vessel 1 and vessel 5.

Complete the following process for all SPs. Use the following tubes for the CBC and whole blood protocol:

1. Ages 1-2 years

a. Process the 3-mL EDTA tube for CBC and all whole blood tests.

2. Ages 3-5 years

a. Process the first 3-mL EDTA tube for CBC and all whole blood tests.

b. Process the $3 \mathrm{~mL}$ EDTA tube for plasma test (vessel 155).

3. Ages 6-11 years

a. Process the first $4 \mathrm{~mL}$ EDTA tube all whole blood tests and for $\mathrm{CBC}$.

b. Process the $4 \mathrm{~mL}$ EDTA tube for plasma test (vessel 155) and the washed packed cells (vessel 88). 
4. Ages 12-19 years

a. Process the first 4-mL EDTA tube for all whole blood tests and the CBC.

b. Process the second $4 \mathrm{~mL}$ EDTA tube for plasma (vessel 155) and washed packed cells (vessel 88).

5. Ages $20+$ years

a. Process the 4-mL EDTA tube for all whole blood tests and the CBC .

b. Process the second $4 \mathrm{~mL}$ EDTA tube for washed packed cells (vessel 88).

- The blood-processing technologist initially processes the 3- or 4-mL EDTA tube for the whole blood tests.

- Invert the appropriate EDTA tube several times to remix contents thoroughly.

- $\quad$ Pre-wet pipette tip.

- $\quad$ Refrigerate L-ascorbic acid diluent when not in use. Discard unused diluent at end of each session.

\section{Vessel 1}

- $\quad$ Use trace metal-free pipette tips and the $50-1,000 \mu \mathrm{L}$ Eppendorf pipette to aliquot $0.4-\mathrm{mL}$ whole blood for vessel $1(\mathrm{Lead} / \mathrm{Cad} / \mathrm{HG} / \mathrm{Mn} / \mathrm{Se})$ on primary SPs and VIP guests aged 1+ into a 2-mL sterile vessel.

\section{Vessel 3}

- $\quad$ Use trace metal-free pipette tips and the dedicated 5-100 $\mu \mathrm{L}$ Eppendorf pipette to pipette $0.1-\mathrm{mL}$ whole blood for vessel 3 (RBC Folate) on primary SPs and VIP guests aged 1+ into a 2-mL vessel.

- $\quad$ Pre-wet the pipette tips. Dilute the whole blood with ascorbic acid. Use trace metal free pipette tips and the dedicated $50-1,000 \mu \mathrm{L}$ Eppendorf pipette to immediately pipette $1.0-\mathrm{mL}$ into the $1 \%$ ascorbic acid into the $2-\mathrm{mL}$ vessel. Mix well.

\section{Vessel 4}

- Use trace metal-free pipette tips and the $50-1,000 \mu \mathrm{L}$ Eppendorf pipette to aliquot $0.4-\mathrm{mL}$ whole blood for vessel 4 (Glycohem) on primary SPs aged 12+ into a $2-\mathrm{mL}$ vessel. 


\section{Vessel 5}

- Use trace metal-free pipette tips and the 50-1,000 $\mu \mathrm{L}$ Eppendorf pipette to aliquot $0.5-\mathrm{mL}$ whole blood for vessel 5 (Mercury) on primary SPs and VIP guests aged $1+$ into a $2-\mathrm{mL}$ sterile vessel.

- Securely close all vessels to prevent leakage and evaporation.

- Record results using the blood-processing module as described in Section 7.8.

- Store all specimens immediately as outlined in Section 7.9.

- Place the EDTA tube on a hematology rocker until the hematology technologist performs the $\mathrm{CBC}$.

Run the $\mathrm{CBC}$ within 2 hours. If unable to run the $\mathrm{CBC}$ within 2 hours, store the 3- or 4-mL EDTA blood tube at room temperature for no more than 24 hours. After 24 hours, the blood will undergo changes that will render the $\mathrm{WBC}, \mathrm{RBC}, \mathrm{HgB}$, and $\mathrm{HCT}$ results inaccurate.

\subsubsection{Process the 3 or $4 \mathrm{~mL}$ EDTA for Plasma Fluoride (vessel 155)}

Place the tube in the centrifuge carrier of the refrigerated tabletop centrifuge. Balance the tubes in the centrifuge by using water-filled tubes if necessary. Immediately centrifuge the whole blood from the 3 - or $4-\mathrm{mL}$ EDTA tube in the $17-25^{\circ} \mathrm{C}$ or $4-8^{\circ} \mathrm{C}$ centrifuge at $2,900 \mathrm{rpm}$ for 10 minutes to yield plasma for vessel 155 (Fluoride).

\section{Vessel 155}

- Use a calibrated plastic transfer pipette to aliquot $1.0 \mathrm{~mL}$ of plasma for vessel 155.

- Determine if the plasma is hemolyzed, turbid, lipemic, or icteric. If so, enter a comment to describe the plasma when entering the processing results.

- Securely close all vessels to prevent leakage and evaporation.

- $\quad$ Record results using the blood-processing module as described in Section 7.8.

- Store all specimens immediately as outlined in Section 7.9. 


\subsubsection{Process the 3 or $4 \mathrm{~mL}$ EDTA for Washed Packed Cells for Acrylamide (vessel 88)}

Centrifuge the EDTA tube and remove the plasma as described in Section 7.7.2.2. Leave the cells in the original blood collection tube. Wash the remaining red blood cells (erythrocytes) with sterile saline three times and then lyse the cells with distilled water. Store the saline in the refrigerator and use it cold. Pour saline and deionized water into squeeze bottles. It is acceptable to batch the samples as long as the samples and reagents are refrigerated.

Use the following tubes for the washed packed cells protocol for vessel 88:

- Process the cells from the 4-mL EDTA tube on SPs aged 6+ years.

- $\quad$ Place the tube(s) in the centrifuge carrier of the refrigerated tabletop centrifuge. Balance the tube(s) in the centrifuge by using water-filled tubes if necessary. Take care not to disturb the pellet of red blood cells when removing the plasma.

\section{Vessel 88}

1. Centrifuge the $4-\mathrm{mL}$ EDTA tube at $17-25^{\circ} \mathrm{C}$ or $4-8^{\circ} \mathrm{C}$. Centrifuge at $1,985 \mathrm{rpm}$ for 5 minutes to separate the plasma from the cells.

2. Use a disposable transfer pipette to remove the plasma, which is the upper clear layer, and add it to vessel 155.

3. Use the refrigerated squeeze bottle of saline to add an equal amount (to the packed cells) of 0.9 percent cold saline to the packed cells.

4. Close vial and mix by gently shaking.

5. Centrifuge at $1,985 \mathrm{rpm}$ and $4^{\circ} \mathrm{C}$ for $5 \mathrm{~min}$.

6. Remove and discard the clear upper layer.

7. Repeat steps 3 to 6 two more times (total of 3 wash cycles).

8. After the last wash cycle, use the squeeze bottle of deionized water to add an equal amount of deionized water to the packed cells. (The final volume should be at least $1.0-\mathrm{mL}$ of washed lysed packed cells.)

9. Close vial and mix by gently shaking.

10. Transfer the lysed cells to a 2-mL cryovial.

- Close all vessels securely to prevent leakage and evaporation. 
- Record results using the blood-processing module as described in Section 7.8.

- Store all specimens immediately as outlined in Section 7.9.

7.7.3

Process the 2-mL Gray Tube for Glucose and OGTT

Centrifuge and separate the plasma from the 2-mL gray top tube within 30 minutes from the time the blood was collected. Process the specimen even if the contents of the gray tube are clotted.

- Place the tube in the centrifuge carrier of either tabletop centrifuge. Balance the tube in the centrifuge by using water-filled tubes if necessary. Centrifuge the 2-mL gray tube at $17-25^{\circ} \mathrm{C}$ or $4-8^{\circ} \mathrm{C}$ and $2,900 \mathrm{rpm}$ for 10 minutes to yield plasma for vessel 9 (Glucose) or vessel 98 (OGTT.)

\section{Vessel 9 or 98}

- $\quad$ Pre-wet pipette tip.

- $\quad$ Use a calibrated plastic transfer pipette to transfer all plasma from this tube but at least 0.5 -mL plasma for vessel 9 (Glucose) or vessel 98 (OGTT) on primary SPs aged $12+$ into a $2-\mathrm{mL}$ vessel.

- Determine if the plasma is hemolyzed, turbid, lipemic, or icteric. If so, enter a comment to describe the plasma when entering the processing results.

- Close all vessels securely to prevent leakage and evaporation.

- Record results using the blood-processing module as described in Section 7.8.

- $\quad$ Store all specimens immediately as outlined in Section 7.9.

7.7.4 Process the 3-mL, 7-mL, 10-mL, and 15-mL Red Top and $6 \mathrm{~mL}$ Royal Blue Tubes for Pooled Sera (10-mL Reds are pooled by themselves for environmental testing)

- Gently invert the tubes 5-6 times as soon as possible after receiving them in the laboratory.

- Place 3-mL, 7-mL, 10-mL and 15-mL on the rocker for 10 minutes and then place in the rack to clot for the remaining time indicated on timer.

- Allow the blood in each 3-mL, 7-mL, 10-mL, and 15-mL Red top and 6mL Royal Blue top tubes to clot for 30-45 minutes at room temperature. Do not let clot for more than 1 hour. 
- Place the tubes in the room temperature centrifuge carrier of either tabletop centrifuge. Balance the tubes in the centrifuge by using water-filled tubes if necessary. Centrifuge the Red top and Royal Blue top tubes at $17-25^{\circ} \mathrm{C}$ and $2,900 \mathrm{rpm}$ for 15 minutes to yield serum.

- $\quad$ Determine if the serum is hemolyzed, turbid, lipemic, or icteric. If so, enter a comment to describe the serum when entering the processing results.

If the serum in any one Red top tube from an SP is grossly hemolyzed, do not pool it with serum from the remaining tubes. Reserve it for the storage vessels 32-37.

If the serum in all Red top tubes for a SP is turbid, lipemic, or icteric, pool it and allocate as usual.

\section{- Vessel 136}

- Use serum separator to remove serum from the $6 \mathrm{~mL}$ Royal Blue top tube by pushing the serum separator to the clot barrier gently to ensure cell-free separation. Do not push the separator too hard.

- $\quad$ Use trace metal-free pipette tips to aliquot $1.0 \mathrm{~mL}$ from the Royal Blue top tube, into a $2 \mathrm{~mL}$ sterile cryovial, prior to pooling the serum with the Red top tubes. Do not substitute any other tube for this vessel. The $6 \mathrm{~mL}$ Royal Blue top is prescreened by the CDC for these tests.

- Vessels 70, 122, 87, 17, 21, 77, 18, 23, 12, 22, 24, 127, 137, 150 and 32-37.

- $\quad$ Use a serum separator to remove serum from all the Red top tubes by pushing the serum separator to the clot barrier gently to ensure cell-free separation. Do not push the separator too hard. Do not touch the serum separator to the clot barrier.

- $\quad$ Label the 50-mL conical tube with SP ID \# label. Pool all clear sera. For each SP pool the serum from all Red top tubes by decanting the separated serum into the $50-\mathrm{mL}$ polypropylene tube. Hold the tube and the separator level while decanting the serum. Be careful to avoid introducing any cellular debris. Discard all tubes and separators in a sharps container.

- Securely close the 50-mL polypropylene tube and mix its contents by gentle inversion.

- Confirm that all vessels have the correct information with the appropriate SP's barcode ID label before filling them.

- $\quad$ Use the 100-5,000 Eppendorf ${ }^{\circledR}$ pipette and 5-mL pipette tips to aliquot the serum for all vessels. 
- Using clear serum only, fill as many vessels as possible in the priority order shown in Section 7.3. Exhibits 7.10 - 7.18 describe the order of allocation and specific procedures to follow for each person type and vessel.

- $\quad$ If excess serum remains after completing the entire protocol, add an additional $1.5-\mathrm{mL}$ serum beginning with vessel 32 and continuing through vessel 37 until the serum is exhausted. If extra serum remains, continue to top off the other serum vessels, beginning with vessel 138, until the serum is exhausted.

\section{Vessel 110}

- $\quad$ Use trace metal-free pipette tips and the dedicated $100-5,000 \mu \mathrm{L}$ Eppendorf pipette to pipette $0.7-\mathrm{mL}$ serum for vessel 110 (Folate1) on primary SPs and VIP guests aged 1+ into a 2-mL vessel. Pre-wet the pipette tips. Dilute the serum with $50 \mu \mathrm{L}$ of the $7 \%$ ascorbic acid, using trace metal free pipette tips and the dedicated 50-1,000 $\mathrm{LL}$ Eppendorf pipette. Mix well.

- Securely close all vessels to prevent leakage and evaporation.

- Record results using the blood processing module as described in Section 7.8.

- Store all specimens immediately as outlined in Section 7.9.

- If the serum cannot be aliquoted immediately, refrigerate it at $4^{\circ} \mathrm{C}$ for no more than 4 hours.

\subsubsection{Process the 15-mL Red Top Tubes for Environmental Subsamples}

The environmental subsamples represent a 1/3 subsample of SPs aged 12+. SPs in the 0, 3, 7, 10 subsample group are eligible for vessels 29, 89 and 90. SPs in the 4, 6, 8, 9 subsample group are eligible for vessels 92 and 93.

- Gently invert the tubes 5-6 times as soon as possible after receiving them in the laboratory.

- Allow the blood in the 15-mL Red top tubes to clot for 30-45 minutes at room temperature. Do not let clot for more than 1 hour.

- Place the tubes in the room temperature centrifuge carrier of either tabletop centrifuge. Balance the tubes in the centrifuge by using water-filled tubes if necessary. Centrifuge the red top tubes at $17-25^{\circ} \mathrm{C}$ and $2,900 \mathrm{rpm}$ for 15 minutes to yield serum. 


\section{Vessels 29, 89 and 90}

- Determine if the serum is hemolyzed, turbid, lipemic, or icteric. If so, enter a comment to describe the serum when entering the processing results.

- $\quad$ Use a serum separator to carefully remove serum from 2 -15-mL Red top tubes by pushing the serum separator to the clot barrier gently to ensure cell-free separation. Do not push the separator too hard or some flowthrough may occur. Do not touch the serum separator to the clot barrier.

- $\quad$ Pool serum from the $2-15 \mathrm{~mL}$ red tops into a $14 \mathrm{~mL}$ falcon and aliquot the serum immediately, $4.0 \mathrm{~mL}$ for vessel 29 (Persis Pest1) into a sterile $5 \mathrm{~mL}$ cryovial, $4.0 \mathrm{~mL}$ for vessel 89 ( Persis Pest2) into a $5 \mathrm{~mL}$ sterile cryovial, and $1.5 \mathrm{~mL}$ into vessel 90 (Perfluor) into a sterile $2 \mathrm{~mL}$ cryovial on primary SPs aged $12+$.

\section{Vessels 92 and 93}

- Determine if the serum is hemolyzed, turbid, lipemic, or icteric. If so, enter a comment to describe the serum when entering the processing results.

- $\quad$ Use a serum separator to carefully remove serum from one 15-mL Red top tube by pushing the serum separator to the clot barrier gently to ensure cell-free separation. Do not push the separator too hard or some flowthrough may occur. Do not touch the serum separator to the clot barrier.

- $\quad$ Aliquot the serum immediately by pouring the serum for vessel 92 (BFR1) and vessel 93 (BRF2) on primary SPs aged $12+$ into a $5 \mathrm{~mL}$ sterile cryovial.

- Securely close all vessels to prevent leakage and evaporation.

- Record results using the blood-processing module described in Section 7.8.

- Store all specimens immediately as outlined in Section 7.9.

\subsubsection{Reagent Processing and Preparation}

The Centers for Disease Control and Prevention supplies premeasured 1 percent ascorbic acid (AA) for vessel 3 (RBC Folate), and a premeasured 7 percent AA solution for vessel 110 (Folate1). Prepare both reagents before processing the blood samples. Allow the reagent to equilibrate to room temperature before pipetting and performing the dilution. Use the Eppendorf Research pro pipettes to 
make the dilution. Add 1.0-mL of $1 \%$ ascorbic acid to $0.1-\mathrm{mL}$ whole blood for vessel 3 (RBC Folate) and $0.05 \mathrm{~mL}$ of the $7 \%$ AA to vessel 110 . Mix by gentle inversion.

- The $1 \%$ ascorbic acid powder is in a $50-\mathrm{mL}$ Falcon tube. Dissolve the powder in 30 $\mathrm{mL}$ of deionized water using a graduated cylinder to measure the water and mix gently. Prepare this reagent daily. Date and label the Falcon tube. If the powder appears discolored or the solution appears cloudy, discard and prepare fresh reagent. If no bottled distilled water is available, use tap water. Record the reagent in the Daily QC Log each time it is prepared. Record Lot \# in the Supply Use Control Log.

- The 7\% AA powder is in a 2-mL Nalgene cryovial. Dissolve the powder in $2 \mathrm{~mL}$ of deionized water using a graduated cylinder to measure the water and mix gently. Prepare this reagent daily. Date and label the Nalgene tube. If the powder appears discolored or the solution appears cloudy, discard and prepare fresh reagent. If no bottled distilled water is available, use tap water. Record the reagent in the Daily QC Log each time it is prepared. Record Lot \# in the Supply Use Control Log.

- Discard both the 1\% and 7\% AA prepared reagents at the end of each day and make fresh vials at the start of the next session.

\subsection{Record the Results of Specimen Processing}

After filling or processing the whole blood, plasma, serum, and/or the EDTA or Gray top tubes for each SP, use the Blood Processing module to enter the blood processing results.

Open and log onto the Laboratory application. Use the heads-up display to view the SPs, the modules for which they are eligible, and their current process status. 
Select the correct SP and access the Blood Processing module.

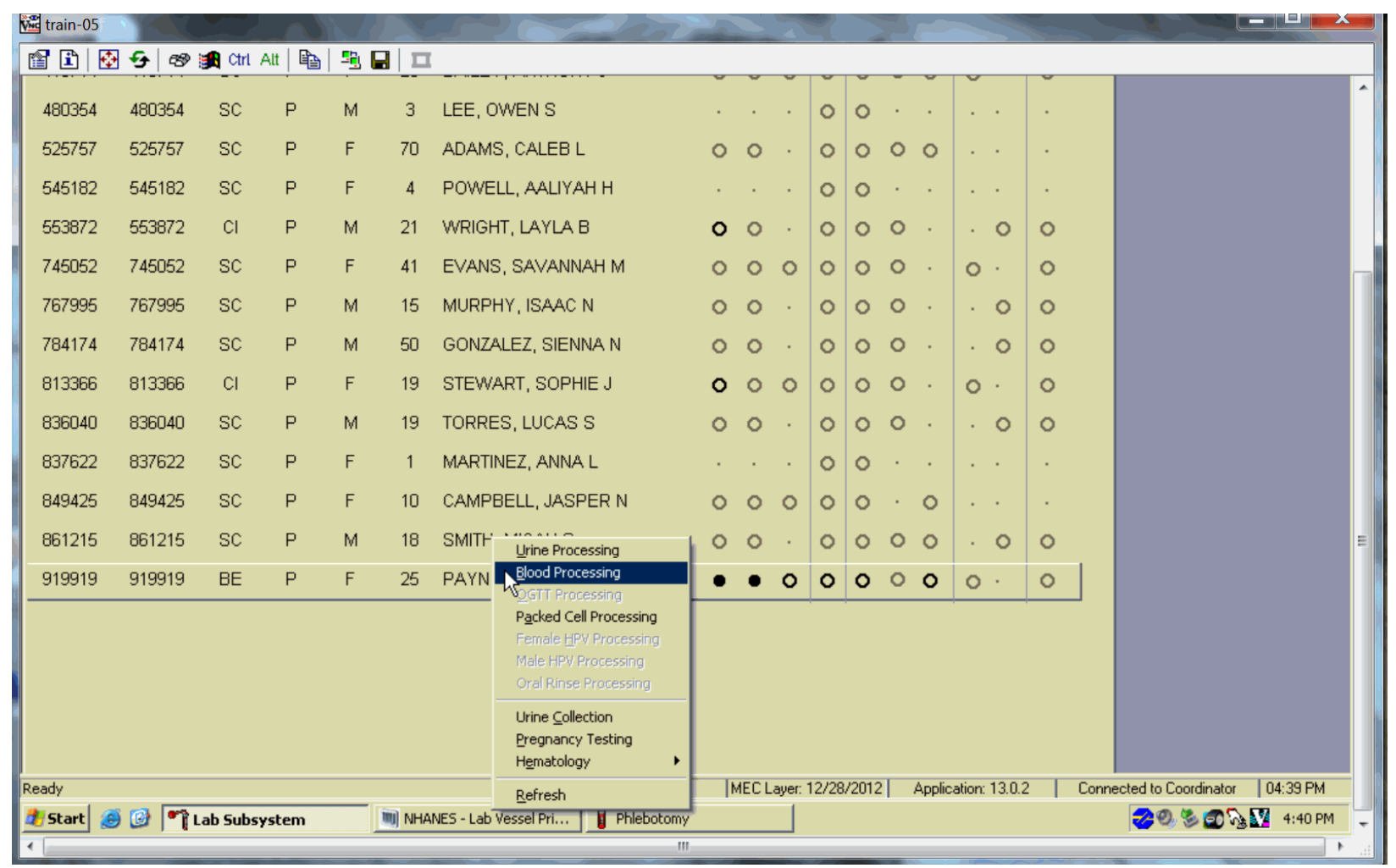

If labels have not been printed in advance the SPs will show up in a red font on the heads-up display. To access the blood processing module, highlight the SP, right click, drag the mouse arrow to $\{$ Blood Processing , and left click or type [Alt] [B/b]. 
The SP's blood processing screen displays.

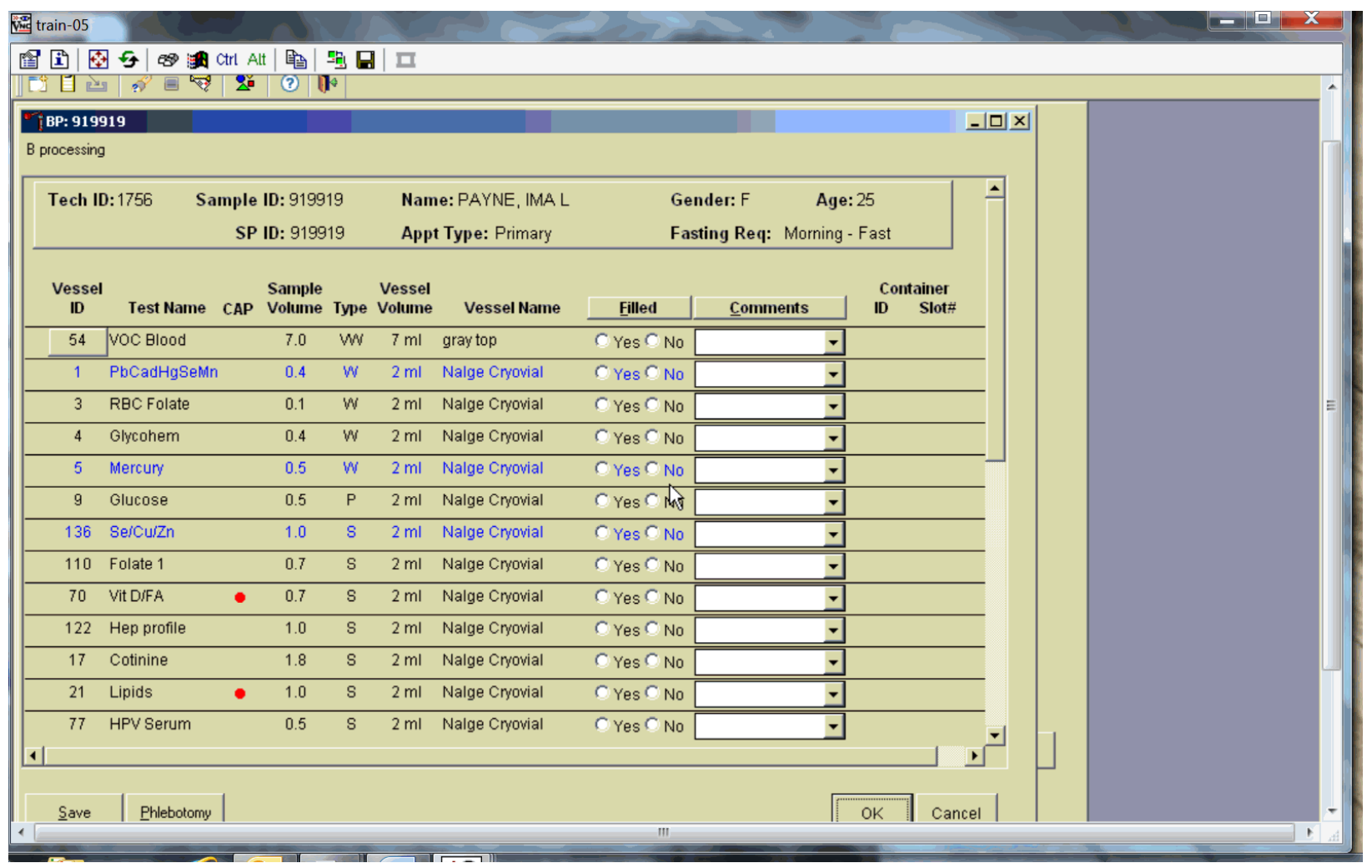

The blood processing window for an SP contains the following information: Tech ID, Sample ID, SP ID, Name (last, first), Gender, Age, Appt (appointment) Type, Fasting Req, columns for Vessel ID, Test Name, Sample Volume, Type (whole blood, plasma, serum), Vessel Volume, Vessel Name, Filled radio buttons, Comments text box, and a Container ID/Slot\# column. Vessels that require sterile 2-mL cryovials are displayed in blue.

Inspect the SPs Vacutainer ${ }^{\circledR}$ tubes. Compare the Sample ID on the tubes to the barcode label on the vessels. Verify that the number of tubes and the recorded amount of blood collected are correct. 


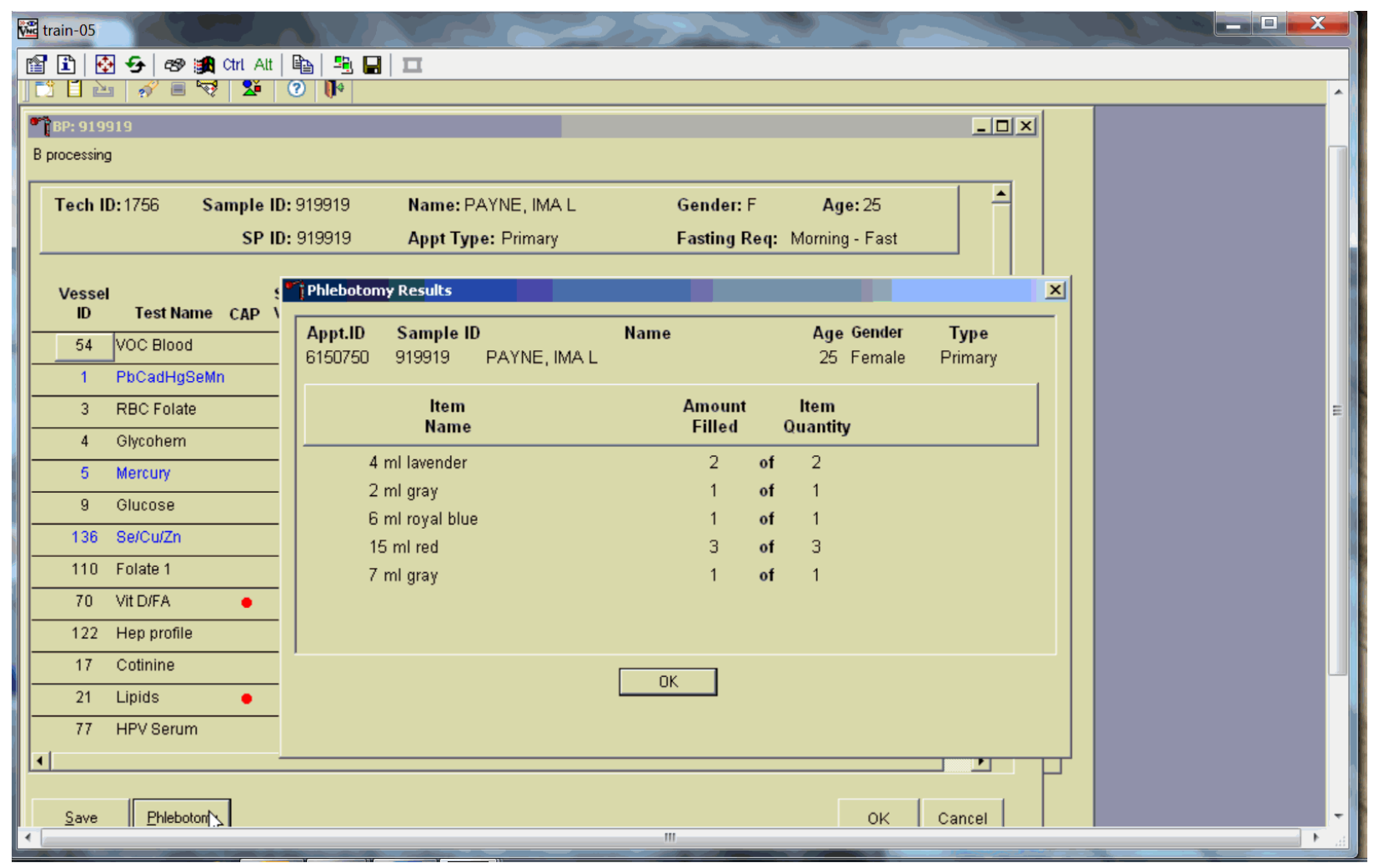

To review the phlebotomy results, use the mouse to direct the mouse arrow to the "Phlebotomy" button on the blood processing screen and left click or type [Shift] [P/p]. The phlebotomy protocol and number of tubes successfully collected displays. To close this screen, use the mouse to direct the mouse arrow to the "OK" button and left click.

Record blood processing results for each SP. Individually mark each vessel as Filled-"Yes." 


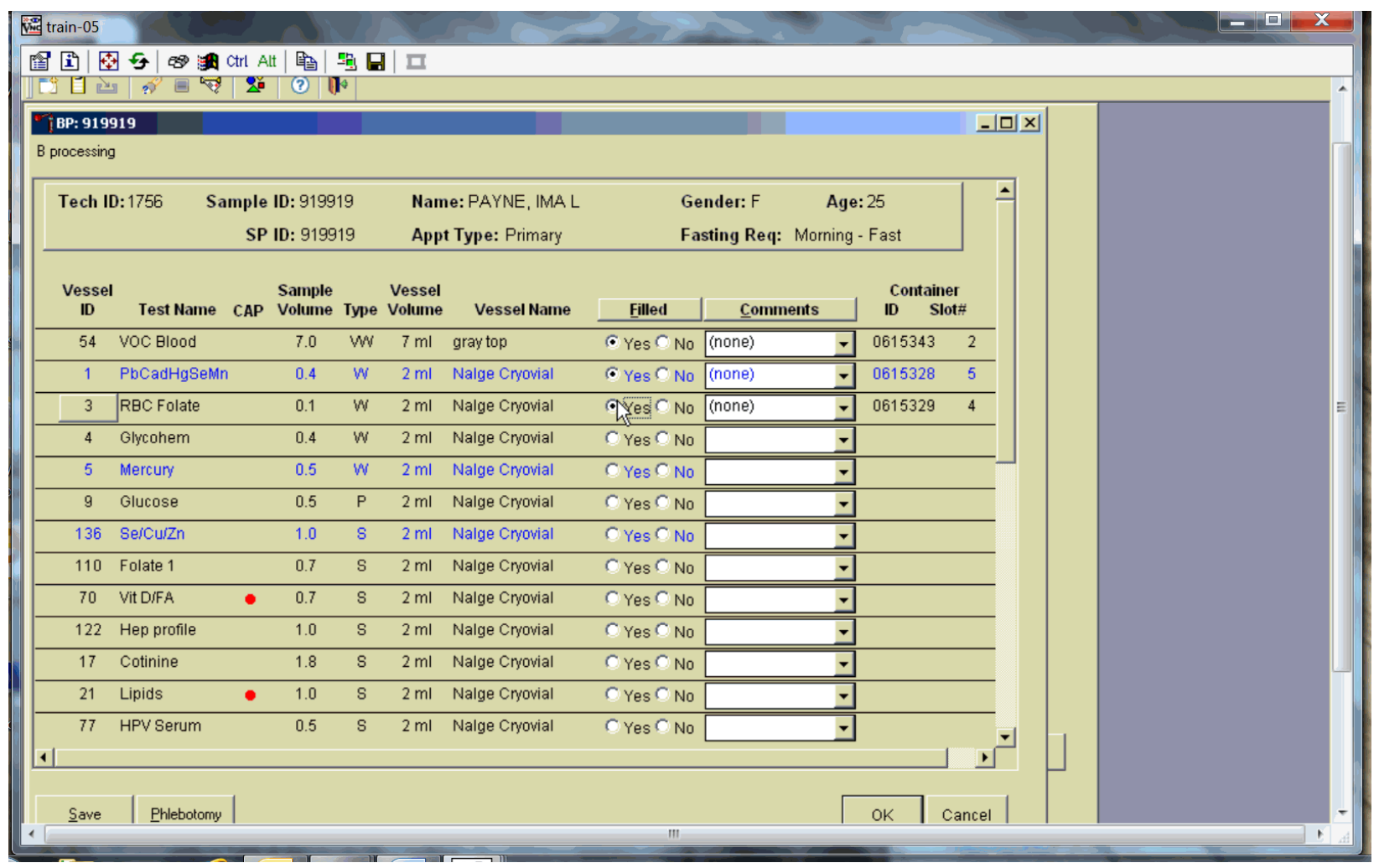

To mark an individual blood vessel as collected or Filled-"Yes" use the mouse to direct the mouse arrow to the center of the "Yes" radio button and left click. As each vessel is marked as Filled"Yes," it is automatically assigned to a slot in an existing (open) container.

Individually mark each vessel as Filled-"No." 


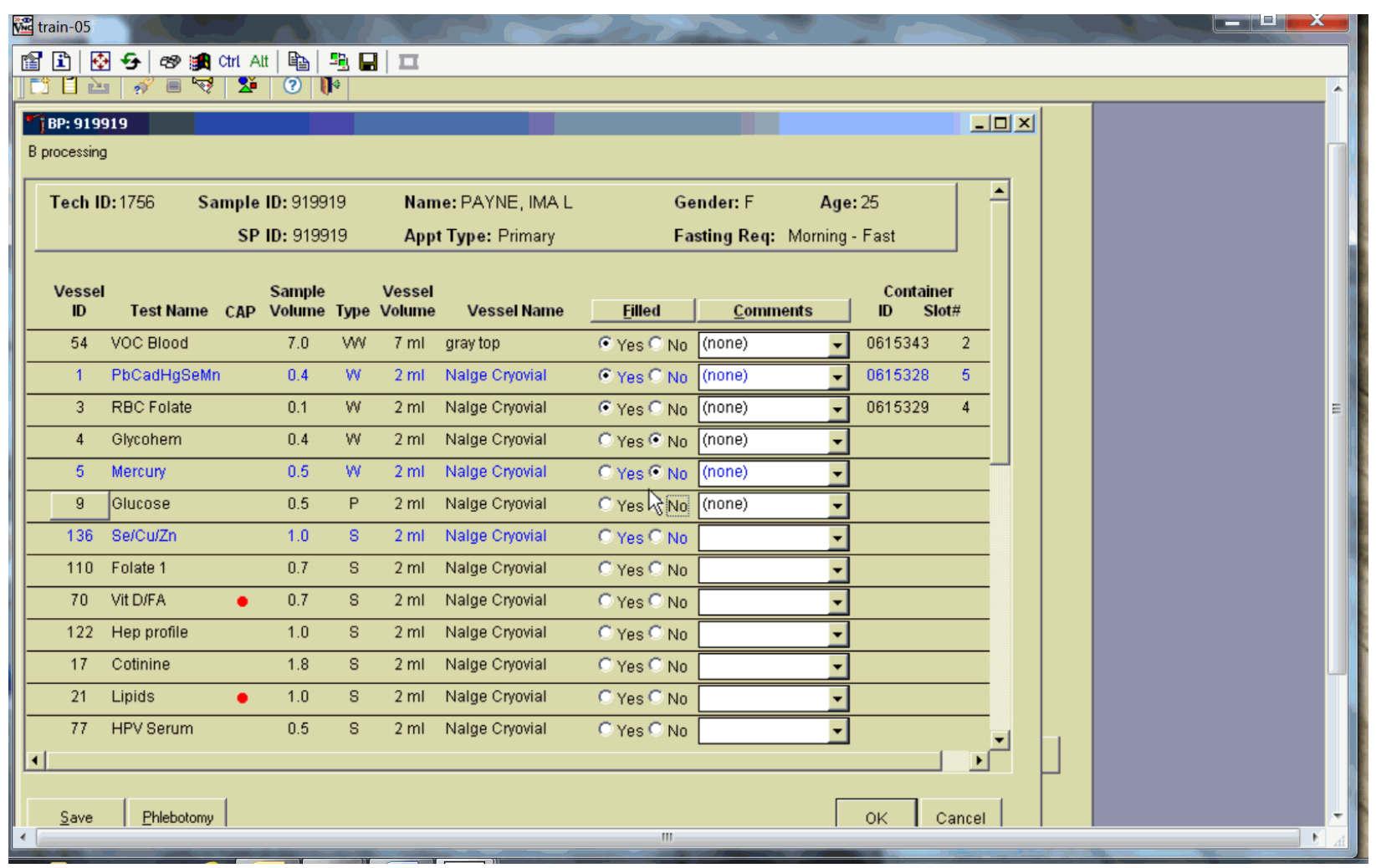

To mark an individual blood vessel as not collected or Filled-"No," use the mouse to direct the mouse arrow to the center of the "No" radio button and left click. Vessels marked as "No" require a comment.

Collectively mark all vessels as Filled-"Yes." 


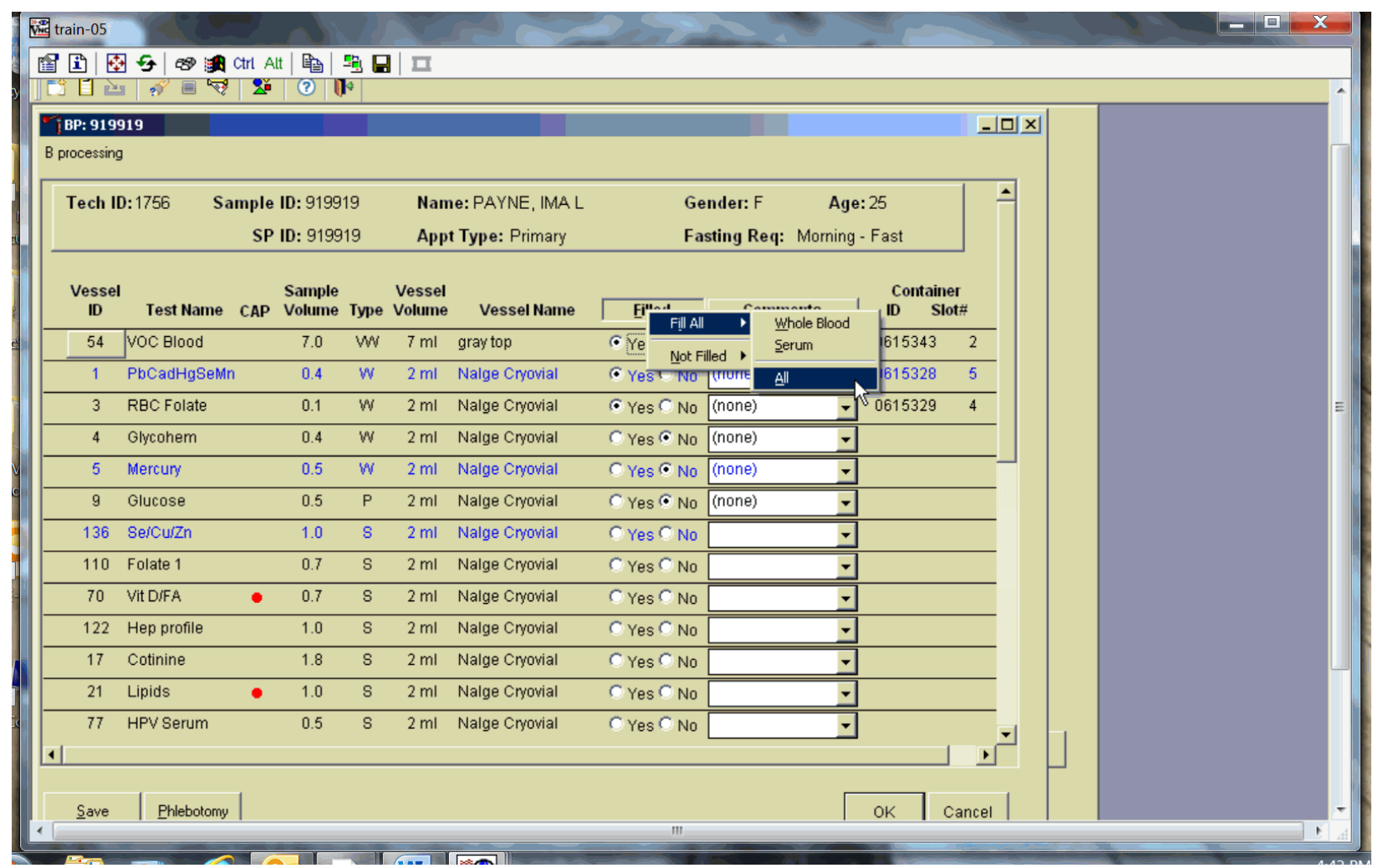

To collectively mark all vessels as Filled-"Yes," use the mouse to direct the mouse arrow to the "Filled" button on the top of the radio buttons, left click, drag the arrow to \{Fill All\}, drag the mouse arrow to $\{$ All $\}$, and left click. Alternatively, to collectively mark all vessels as Filled-"Yes," type [Shift] $[\mathrm{F} / \mathrm{f}],[\mathrm{I} / \mathrm{i}]$, and $[\mathrm{A} / \mathrm{a}]$.

Selectively mark all whole blood or serum vessels as Filled-“'Yes.” 


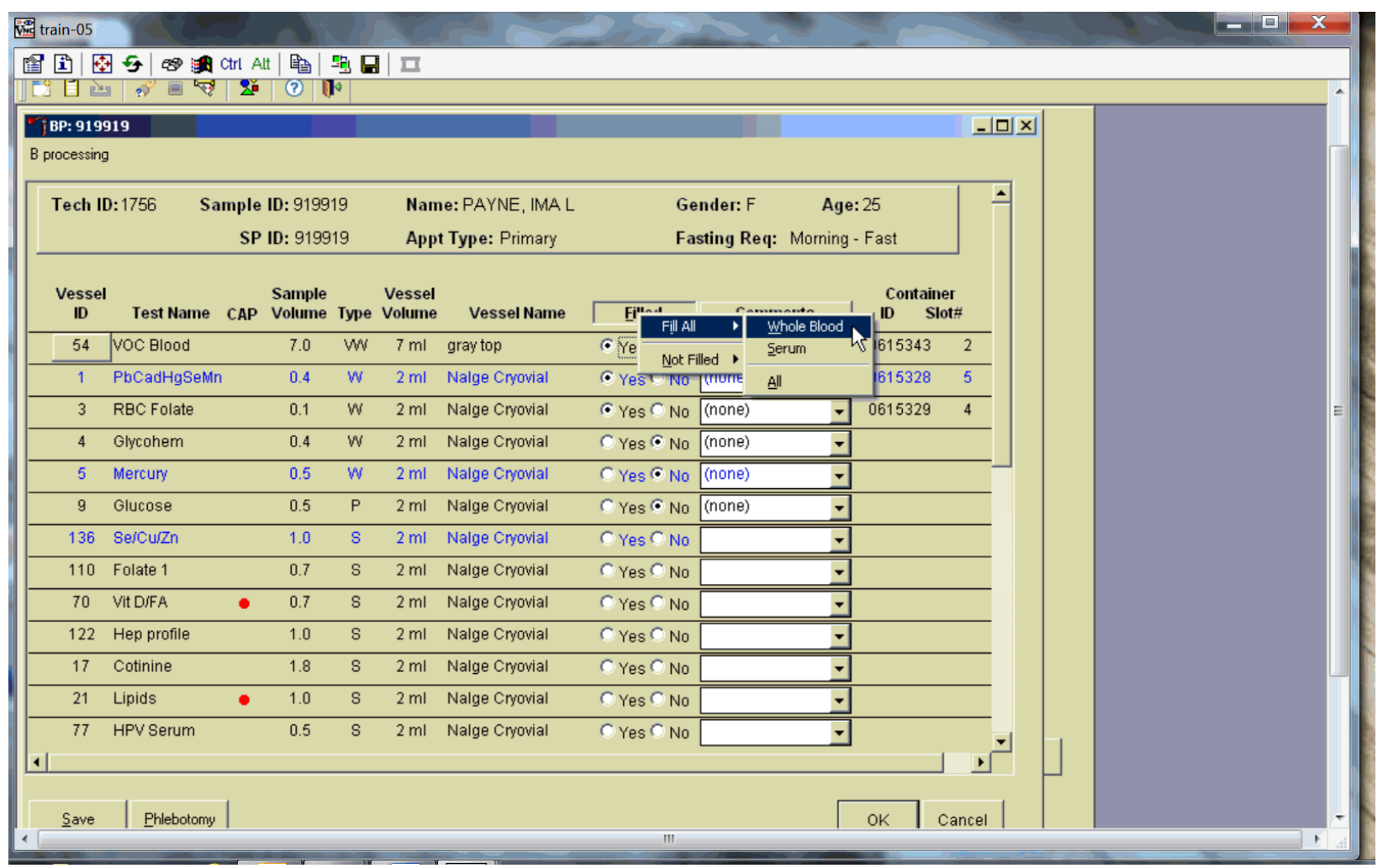

To collectively mark all whole blood vessels as Filled-"Yes," use the mouse to direct the mouse arrow to the "Filled" button on the top of the radio buttons, left click, drag the arrow to \{Fill All\}, drag the mouse arrow to \{Whole Blood\}, and left click. To collectively mark all serum vessels as Filled"Yes," use the mouse to direct the mouse arrow to the "Filled" button on the top of the radio buttons, left click, drag the arrow to $\{$ Fill All\}, drag the mouse arrow to $\{$ Serum $\}$, and left click. As each vessel is marked as "Filled," it is automatically assigned to a slot in an existing (open) container. Alternatively, to collectively mark all whole blood vessels as Filled-"Yes," type [Shift] [F/f], [I/i] and [W/w] and to collectively mark all serum vessels as Filled-“Yes," type [Shift] [F/f], [I/i], and [S/s]. 
Quality comment codes are available to describe the quality of the specimen.

\begin{tabular}{|l|l|}
\hline Comment for filled vessels & \multicolumn{1}{|c|}{ Use when: } \\
\hline Clotted & $\begin{array}{l}\text { The whole blood sample contains a fibrin formation. This is due to } \\
\text { inadequate mixing or inadequate clotting. }\end{array}$ \\
\hline Equipment failure & Pipettes are malfunctioning. \\
\hline Hemolyzed & $\begin{array}{l}\text { There is a red appearance to the serum or plasma. Hemolysis refers to the } \\
\text { abnormal lysis of erythrocytes. Hemolysis can occur before venipuncture } \\
\text { (in vivo hemolysis) or during the analytic procedure (in vitro hemolysis). If } \\
\text { in vivo, hemolysis will give plasma a red color. Use when describing visible } \\
\text { hemolysis, which occurs when the concentration of hemoglobin exceeds } \\
\text { 200 mg/L. }\end{array}$ \\
\hline Icteric & $\begin{array}{l}\text { There is visible orange color to the serum or plasma. Icteric refers to orange } \\
\text { color imparted to a sample because of the presence of bilirubin. Bilirubin } \\
\text { present in serum results in visible "jaundice" color when the concentration } \\
\text { of bilirubin is above 430 umol/L. }\end{array}$ \\
\hline Lipemic & $\begin{array}{l}\text { There is turbid appearance to the serum or plasma. Lipemia refers to the } \\
\text { presence of lipid particles (usually very low-density lipoprotein) in a } \\
\text { sample, which gives the sample a turbid appearance. Plasma appears visibly } \\
\text { lactescent or lipemic when the concentration of triglycerides exceeds } 4.6 \\
\text { mmol/L. }\end{array}$ \\
\hline Post OGTT draw & $\begin{array}{l}\text { During the OGTT blood draw (2 hours after the initial venipuncture and } \\
\text { after the SP has consumed the Trutol), the phlebotomist will draw any tubes } \\
\text { that were missed during the first venipuncture. If any vessel is filled from } \\
\text { one of the tubes drawn during the OGTT blood draw, then add this } \\
\text { comment. }\end{array}$ \\
\hline
\end{tabular}

Attach a comment to an individual filled vessel. 


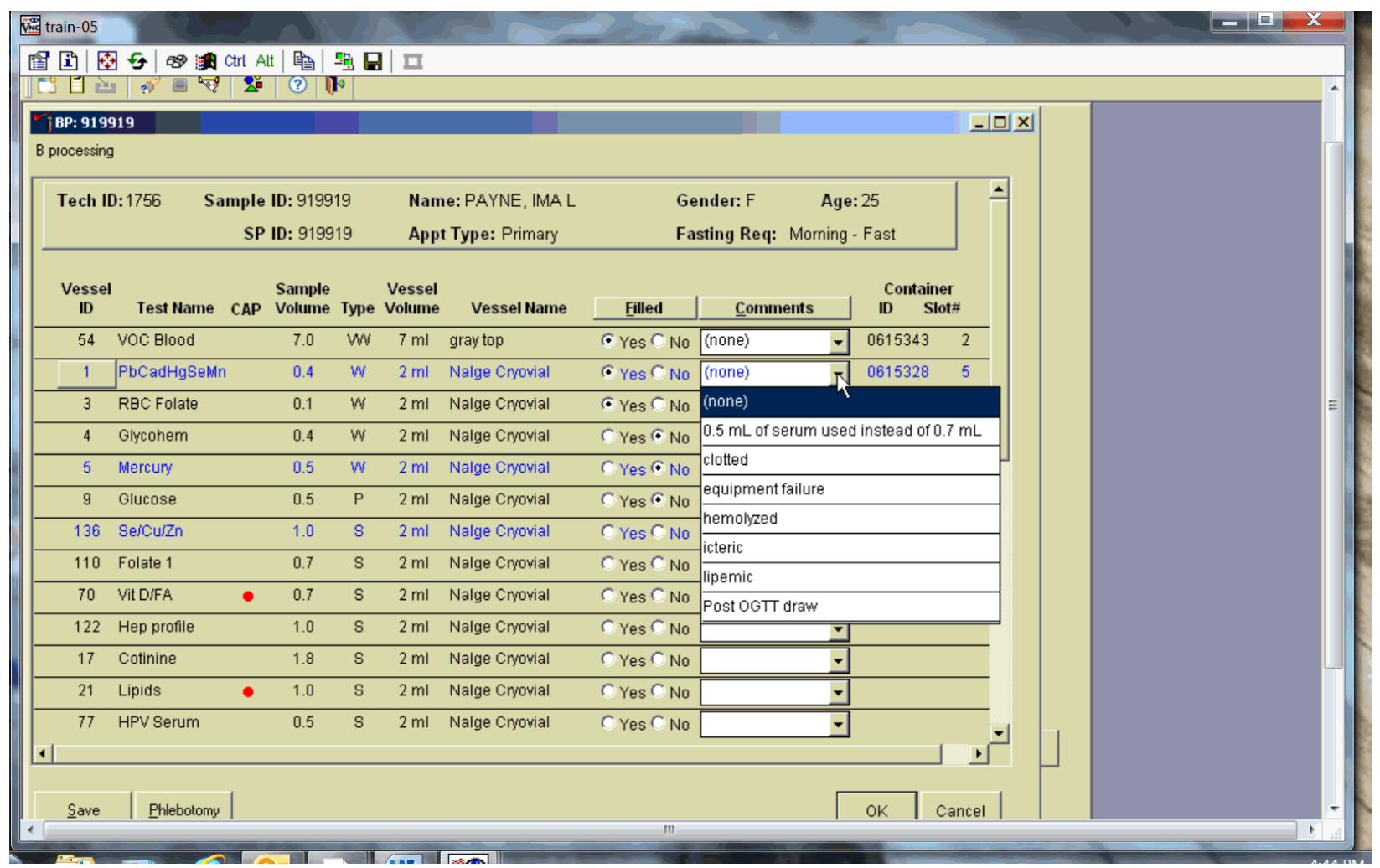

To attach a quality control comment to an individual filled vessel, use the mouse to direct the mouse arrow to the drop-down list under the "Comments" button to display the choices, drag the arrow to the correct choice, left click or highlight the Comment text box, and type the first letter of the desired choice (c, e, h, i, l, p.)

Use the following procedure when all filled vessels require the same comment. 


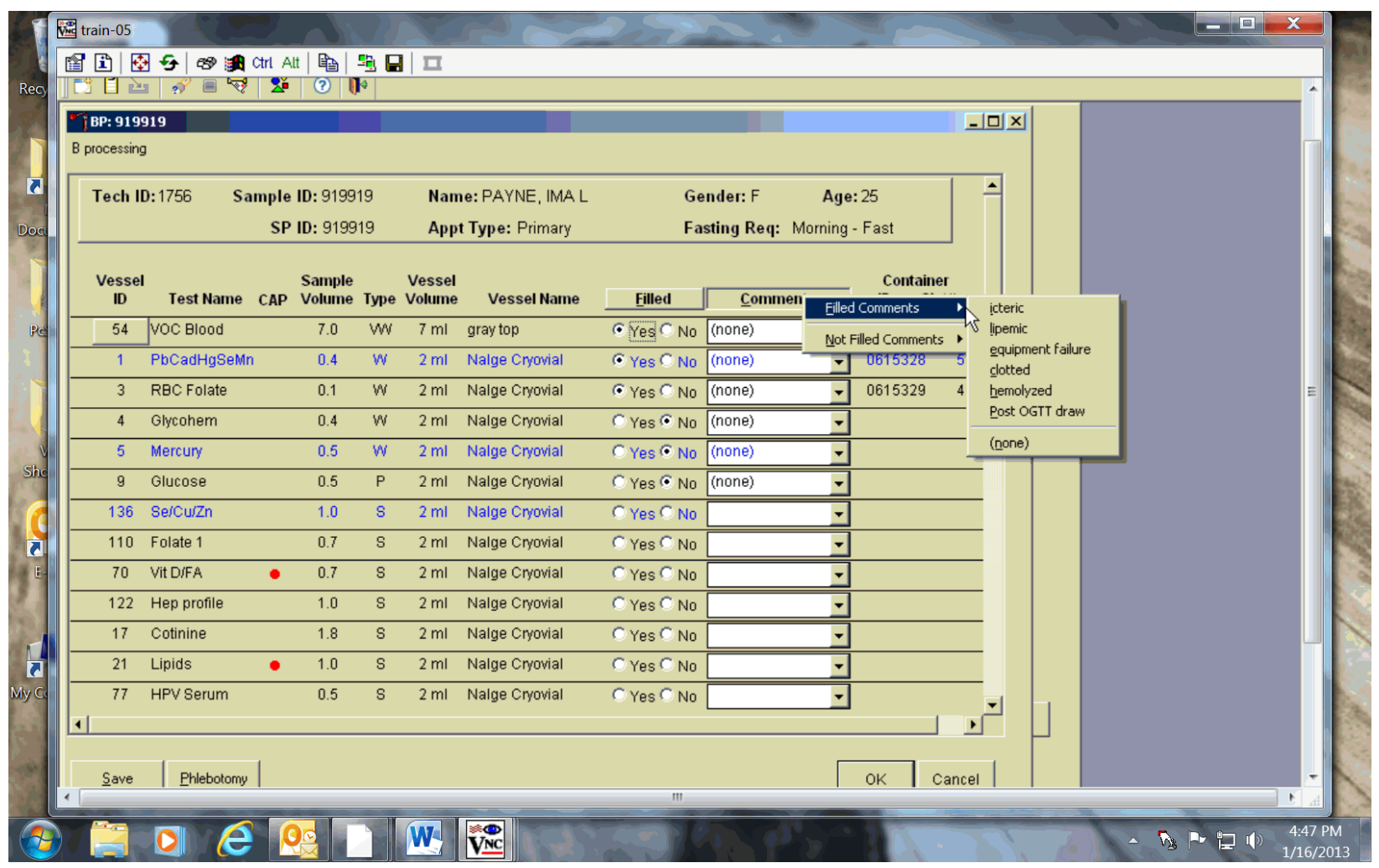

To collectively assign the same comment to all vessels, use the mouse to direct the mouse arrow to the "Comments" button on the top of the radio buttons, left click, drag the arrow to \{Filled Comments $\}$, drag the mouse arrow to the correct choice, and left click. Alternatively, to assign the same comment to all filled vessels type [Shift] [C/c], and the first letter of the correct choice (i, l, e, c, h, p.)

All blood tubes (vessels 54) must be marked individually.

All vessels marked as Filled-"No" require a comment or explanation. 
Enter comment codes to describe the reasons why a vessel is marked as Filled-"No," or why the protocol is incomplete.

\begin{tabular}{|l|l|}
\hline Comment for not filled vessels & Use when: \\
\hline Laboratory error & $\begin{array}{l}\text { A tube breaks in the centrifuge, contents spill, or other technologist } \\
\text { error. }\end{array}$ \\
\hline Quantity not sufficient & Quantity of blood is not sufficient to complete the protocol. \\
\hline SP refusal & The SP does not provide consent and consequently, excluded from HIV. \\
\hline
\end{tabular}

Attach a comment to an individual Filled-"No" vessel.

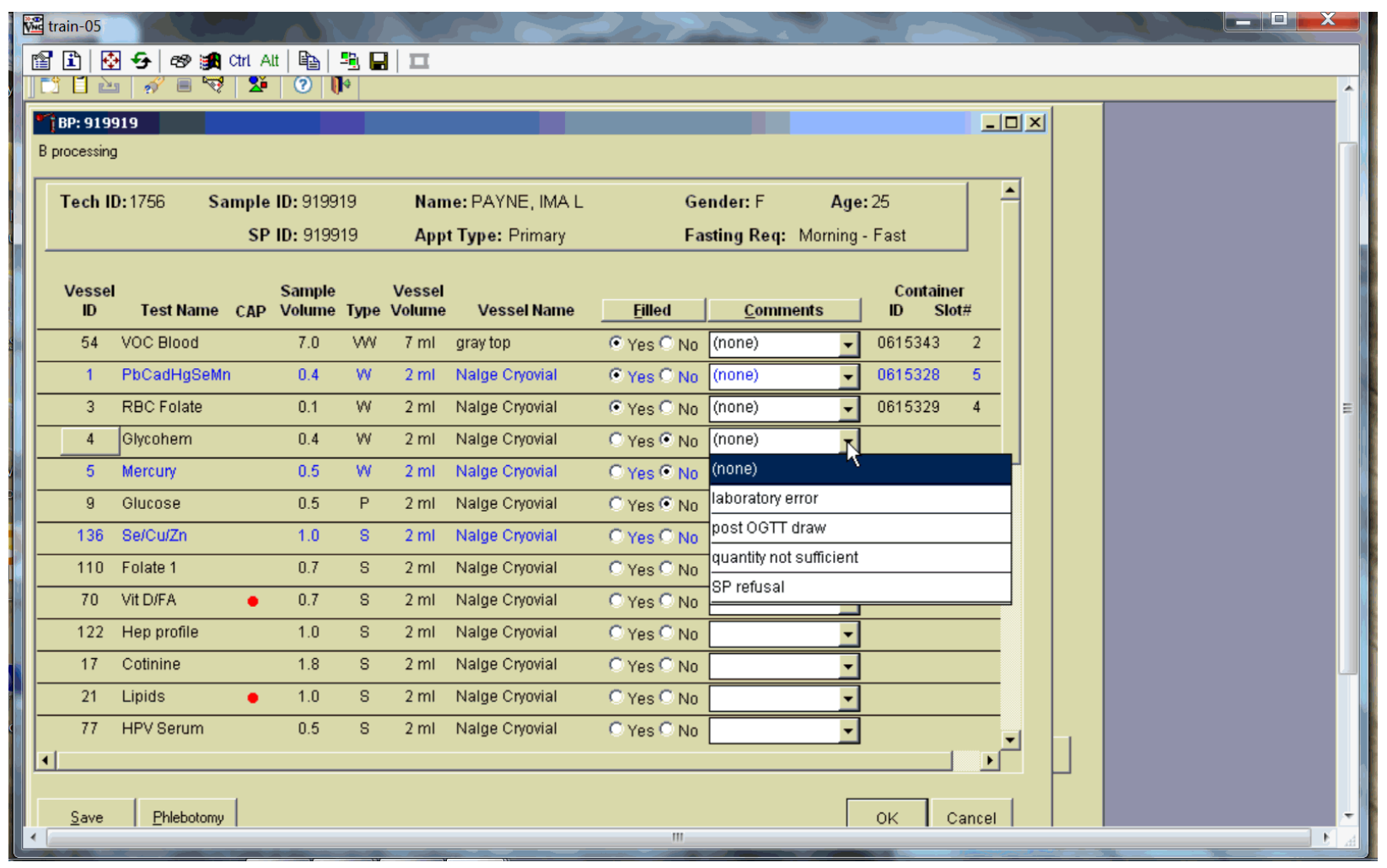

To attach a Filled-"No" comment to an individual vessel, use the mouse to direct the mouse arrow to the drop-down list under the "Comments" button to display the choices, drag the arrow to the correct choice, and left click. Alternatively, highlight the Comment text box and type the first letter of the desired choice.

Use the following procedure when all Filled - "No" vessels require the same comment. 


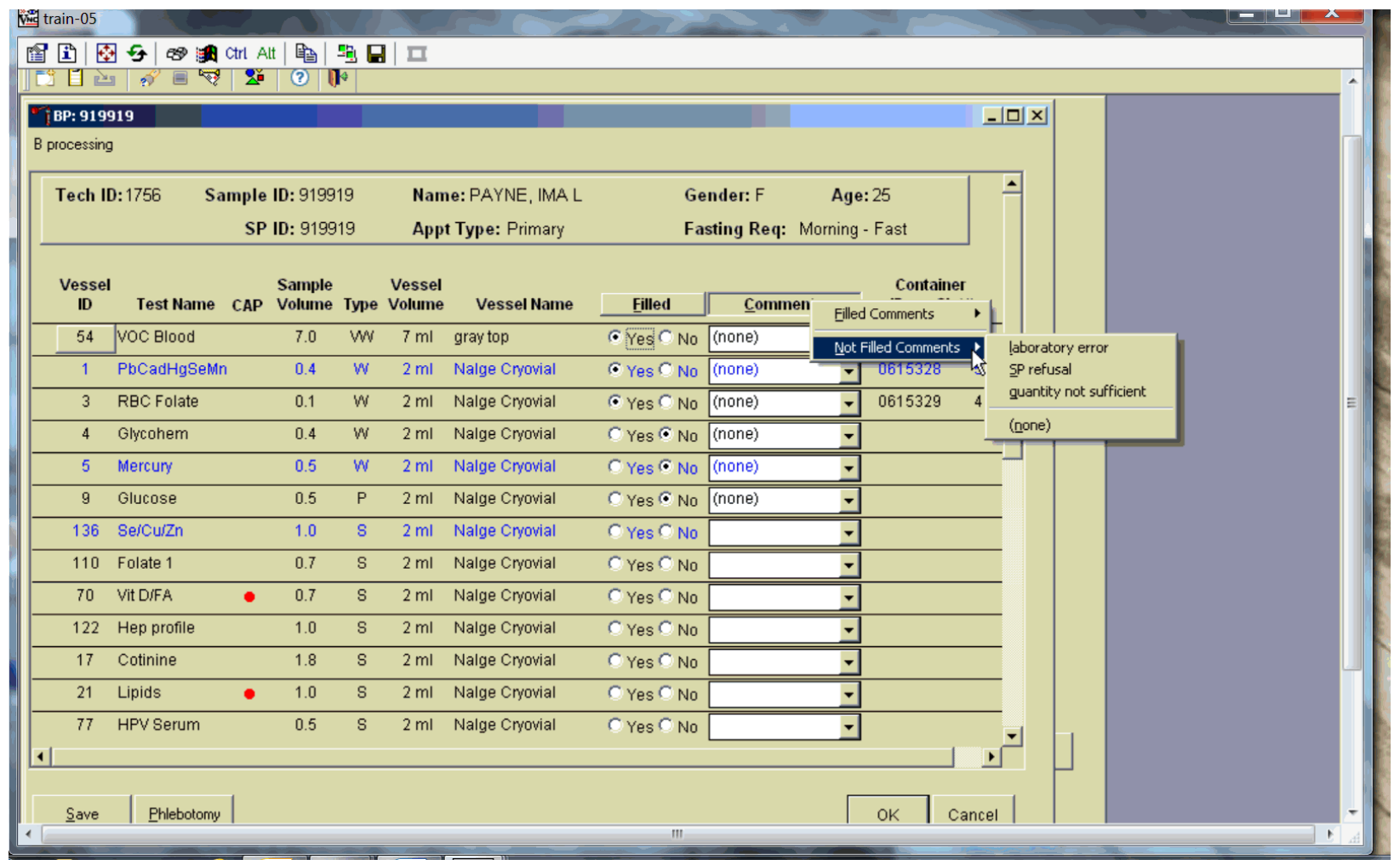

To collectively attach the same comment to all Filled-"No" vessels, use the mouse to direct the mouse arrow to the "Filled" button on the top of the radio buttons, left click, drag the arrow to \{Not Filled All $\}$, drag the mouse arrow to the desired choice, and left click. Alternatively, to collectively attach the same comment to all vessels marked as Filled - "No" type [Shift] [L/l], [N/n], and the first letter of the desired choice (l=laboratory error, $\mathrm{s}=\mathrm{SP}$ refusal, $\mathrm{q}=$ quantity not sufficient.).

If the EDTA tube is clotted, do not process vessel 1 ( $\mathrm{Lead} / \mathrm{Cad}$ ), vessel 3 (RBC Folate), vessel 4 (Glycohem), or vessel 5 (Mercury).

Attach the "Laboratory error" comment to a Filled-No result for vessels 1 and 5, if the $\mathrm{CBC}$ was run before the vessels were processed. 


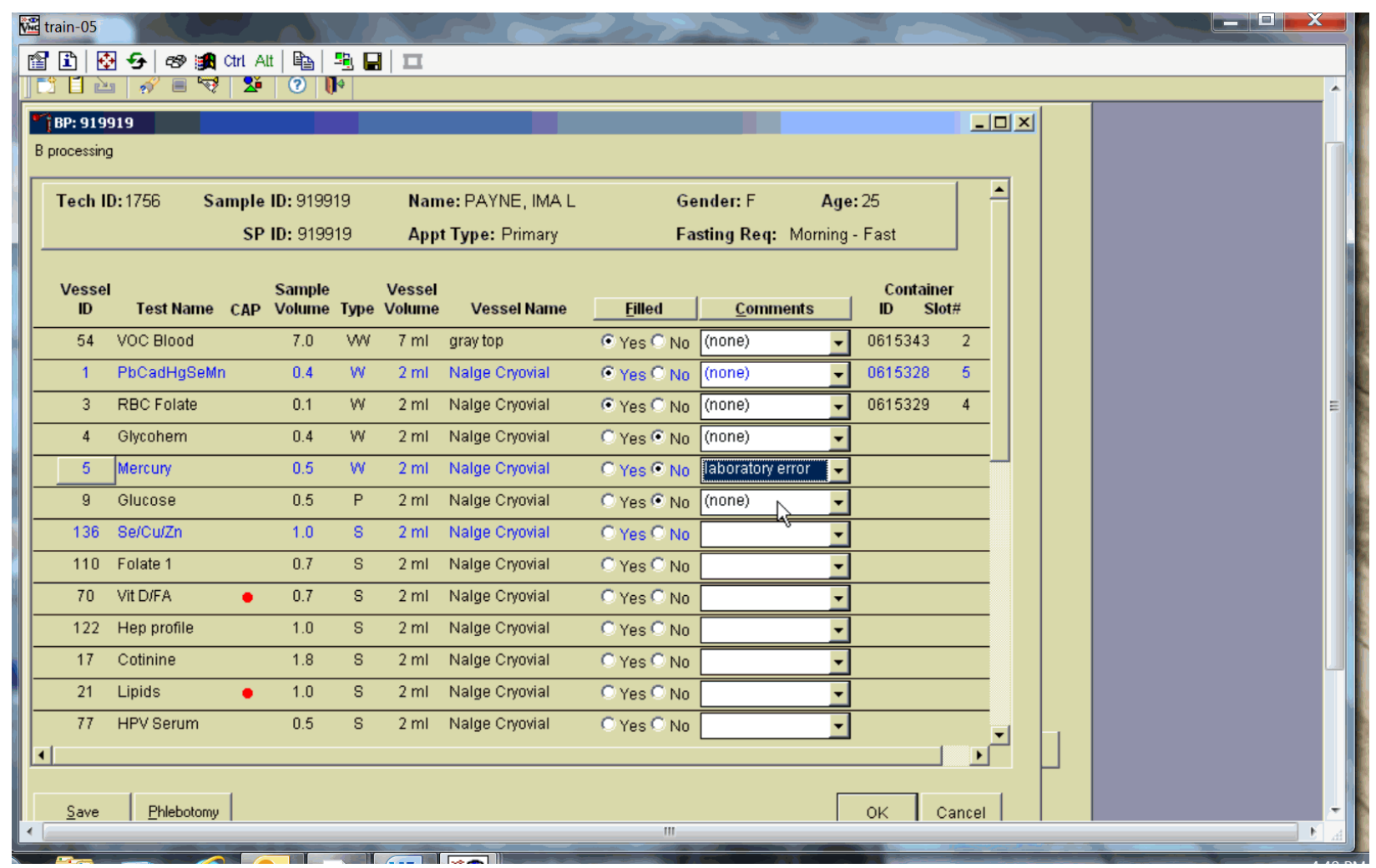

If the $\mathrm{CBC}$ was accidentally run before the whole blood for vessels $1(\mathrm{Lead} / \mathrm{Cad})$ and 5 (Mercury) was pipetted, then do not process these two vessels. Mark vessel 1 and 5 as Filled-"No" and attach the comment "laboratory error." Continue to pipette the whole blood for vessels 3 and 4 even if the $\mathrm{CBC}$ was run before the whole blood was pipetted.

Continue to enter results as vessels are processed. Review the information in the bloodprocessing window and save the data to the database. 


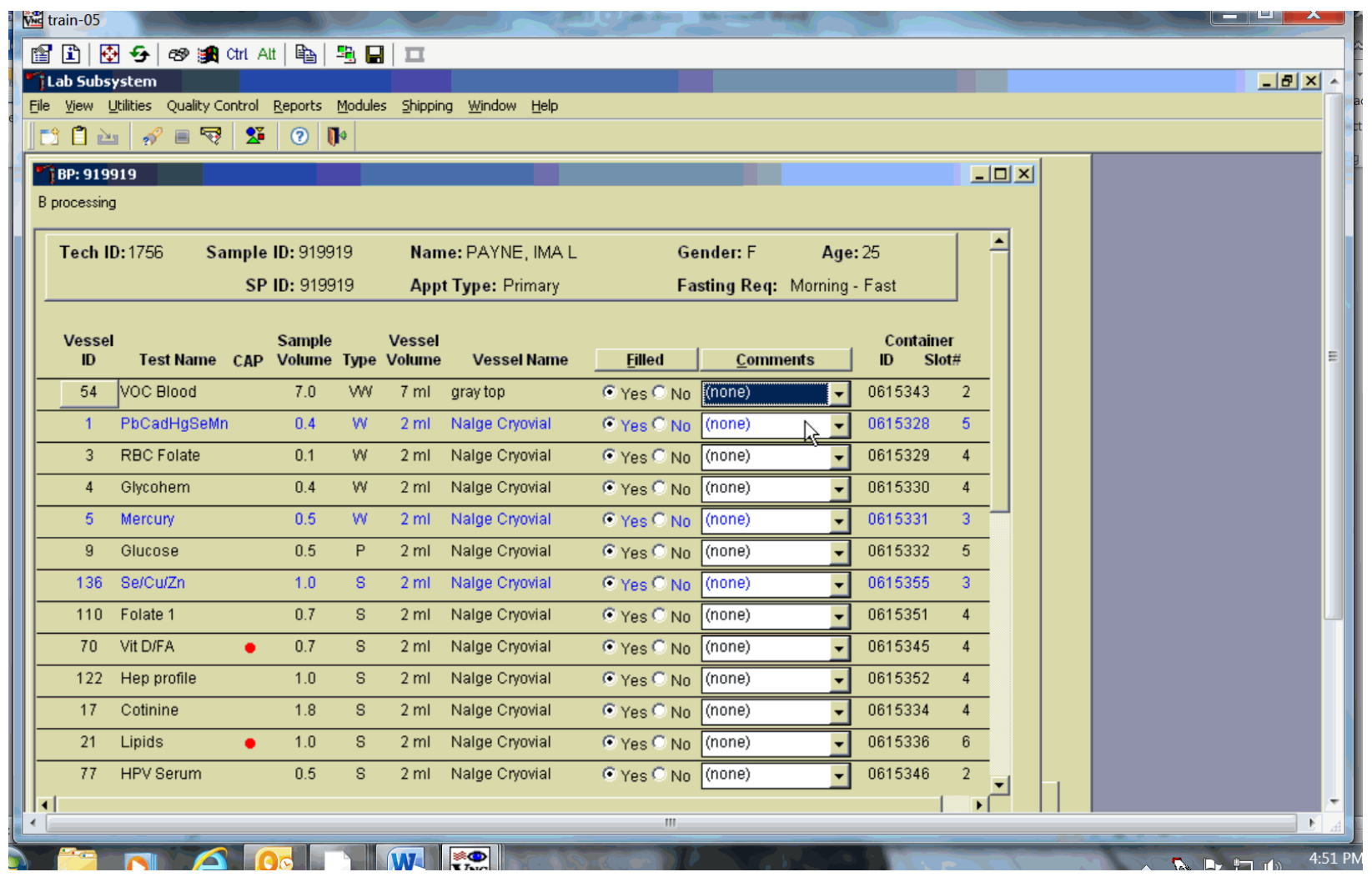

Store each filled vessel in the assigned slot in the assigned container. To record this action or to save this data to the database, use the mouse to direct the mouse arrow to the "Save" button and left click. To record this action or to save this data to the database and to exit the module, use the mouse to direct the mouse arrow to the "OK" button and left click. To close the window without saving any data in the database, use the mouse to direct the mouse arrow to the "Cancel" button and left click.

\subsection{Blood Specimen Storage}

\subsubsection{Blood Specimen Storage Protocol}

Fill the blood vessels, enter the blood processing results, and prepare to store the vessels. Store vessels in numbered storage boxes according to test as indicated in Exhibit 7-21. 
At the beginning of each stand, generate and print a series of barcoded, numbered labels for storage containers as described in Chapter 8. Use the shipping module to assign a barcode label to a specific storage container (test). This process "opens" a storage box. Each vessel is assigned to a specific slot in a specific container as processing results are entered. Slots in containers are assigned according to a standard left to right, top to bottom procedure. Store each vessel in the appropriate slot in the correct container immediately after processing. 
Exhibit 7-21. Storage protocol for blood

\begin{tabular}{|c|c|c|c|c|}
\hline Shipping Location & Vessels & Conditions & Vessel Storage & $\begin{array}{c}\text { Ship } \\
\text { Frequency }\end{array}$ \\
\hline $\begin{array}{l}\text { CDC/NCEH Air Toxicant } \\
\text { Branch }\end{array}$ & 54 VOC Blood & Refrigerated & $\begin{array}{l}5 \times 5 \text { five inch foam } \\
\text { box }\end{array}$ & Weekly \\
\hline CASPIR & $\begin{array}{l}1 \mathrm{Lead} / \mathrm{Cad} / \mathrm{HG} / \\
\mathrm{Se} / \mathrm{Mn}\end{array}$ & Frozen & $9 \times 9$ two inch box & Weekly \\
\hline CASPIR & 3 RBC Folate & Frozen & 10x10 two inch box & Weekly \\
\hline NHANES Diabetes Laboratory & 4 Glycohem & Refrigerated & $9 \times 9$ two inch box & Weekly \\
\hline CASPIR & $\begin{array}{l}5 \text { Mercury, Ethyl } \\
\text { \& Methyl }\end{array}$ & Frozen & $9 \times 9$ two inch box & Weekly \\
\hline CASPIR & 88 Acrylamide & Frozen & $9 \times 9$ two inch box & Weekly \\
\hline Georgia Health Systems & 155 Fluoride & Frozen & $9 \times 9$ two inch box & Weekly \\
\hline NHANES Diabetes Laboratory & 9 Glucose & Frozen & $9 \times 9$ two inch box & Weekly \\
\hline NHANES Diabetes Laboratory & 98 OGTT & Frozen & $9 \times 9$ two inch box & Weekly \\
\hline CASPIR & $136 \mathrm{Se} / \mathrm{Cu} / \mathrm{Zn}$ & Frozen & 9x9 two inch box & Weekly \\
\hline CASPIR & 110 Folate & Frozen & $\begin{array}{l}10 \times 10 \text { two inch } \\
\text { box }\end{array}$ & Weekly \\
\hline CASPIR & $\begin{array}{l}\text { 70 Vitamin } \\
\text { D/Fatty Acids }\end{array}$ & Frozen & 10x10 two inch box & Weekly \\
\hline CDC/NCID Hepatitis Ref Lab & 122 Hep Profile & Frozen & $9 \times 9$ two inch box & Weekly \\
\hline CDC/NCID Hepatitis Ref Lab & 87 Hepatitis ab & Frozen & $9 \times 9$ two inch box & Weekly \\
\hline CASPIR & 17 Cotinine & Frozen & $9 \times 9$ two inch box & Weekly \\
\hline NHANES Diabetes Laboratory & 21 Lipids & Frozen & $9 \times 9$ two inch box & Weekly \\
\hline CDC /DVRD HPV & 77 HPV Serum & Frozen & $9 \times 9$ two inch box & Weekly \\
\hline $\begin{array}{l}\text { Collaborative Laboratory } \\
\text { Services }\end{array}$ & 18 Biochem & Refrigerated & $9 \times 9$ two inch box & Weekly \\
\hline NHANES Diabetes Laboratory & 23 Insulin & Frozen & $9 \times 9$ two inch box & Weekly \\
\hline CASPIR & 12 Vit B12/MMA & Frozen & 9x9 two inch box & Weekly \\
\hline CDC NCID AIDS Division & $22 \mathrm{HIV}$ & Frozen & $9 \times 9$ two inch box & Weekly \\
\hline Emory University & $24 \mathrm{HSV}$ & Frozen & $9 \times 9$ two inch box & Weekly \\
\hline Mayo Clinic & $\begin{array}{l}\text { 127 IgA- } \\
\text { TTG/EMA }\end{array}$ & Frozen & $9 \times 9$ two inch box & Weekly \\
\hline CASPIR & $\begin{array}{l}137 \\
\text { Testosterone/Estra } \\
\text { diol/SHBG }\end{array}$ & Frozen & 9x9 two inch box & Weekly \\
\hline CASPIIR & 150 Aldehyde & Refrigerated & 9x9 two inch box & Weekly \\
\hline CASPIR & 32-37 Xtra Sera & Frozen & $9 \times 9$ two inch box & End of stand \\
\hline CASPIR & $\begin{array}{l}29 \text { Persis Pest1 } \\
89 \text { Persis Pest2 }\end{array}$ & Frozen & $9 \times 9$ three inch box & End of stand \\
\hline $\begin{array}{l}\text { CDC/NCEH Environmental } \\
\text { Health Laboratory }\end{array}$ & 90 Perfluor & Frozen & $9 \times 9$ two inch box & End of stand \\
\hline CASPIR & $\begin{array}{l}92 \text { BFR } 1 \\
93 \text { BFR } 2\end{array}$ & Frozen & $9 \times 9$ three inch box & End of stand \\
\hline
\end{tabular}


If the computer system fails, record results on a preprinted workstation 1 processing worksheet (Exhibit 7-22). Complete a Blood Processing worksheet for each SP while processing the blood specimens. Enter the results after the system is operational.

Exhibit 7-22. Workstation 2 processing worksheet

SP ID

\section{BLOOD PROCESSING}

\begin{tabular}{|c|c|c|c|c|c|c|c|c|}
\hline ID & Name & Ages & $\begin{array}{c}\text { Sample } \\
\text { mL } \\
\end{array}$ & $\begin{array}{c}\text { Sample } \\
\text { Type }\end{array}$ & $\underset{\checkmark}{\text { Filled }}$ & $\begin{array}{c}\text { Filled } \\
\text { Comments } \\
\text { IctericLipemic } \\
\text { Equip Failure } \\
\text { Clotted } \\
\text { Hemolyzed } \\
\end{array}$ & $\begin{array}{c}\text { Not Filled } \\
\text { Comments } \\
\text { ONSSP } \\
\text { Refusal } \\
\text { Lab Error }\end{array}$ & Slot \# \\
\hline 54 & VOC Blood & $12+$ & 10.0 & WB & & & & \\
\hline 1 & $\begin{array}{l}\mathrm{Lead} / \mathrm{Cad} / \\
\mathrm{HG} / \mathrm{Se} / \mathrm{Mn}\end{array}$ & $1+$ & 0.4 & WB & & & & \\
\hline 3 & RBC Folate & $1+$ & 0.1 & WB & & & & \\
\hline 4 & Glycohem & $12+$ & 0.4 & WB & & & & \\
\hline 5 & $\begin{array}{l}\text { Mercury, Ethyl } \\
\text { \& Methyl }\end{array}$ & $1+$ & 0.5 & WB & & & & \\
\hline 88 & Acrylamide & $6+$ & 1.0 & $\mathrm{PC}$ & & & & \\
\hline 155 & Fluoride & $3-19$ & 1.0 & $\mathrm{P}$ & & & & \\
\hline 9 & Glucose & $12+$ & 0.5 & $\mathrm{P}$ & & & & \\
\hline 98 & OGTT & $12+$ & 0.5 & $\mathrm{P}$ & & & & \\
\hline 136 & $\mathrm{Se} / \mathrm{Cu} / \mathrm{Zn}$ & $6+$ & 1.0 & $\mathrm{~S}$ & & & & \\
\hline 110 & Folate & $1+$ & 0.7 & $S$ & & & & \\
\hline 70 & $\begin{array}{l}\text { Vitamin D/ } \\
\text { Fatty Acids }\end{array}$ & $\begin{array}{l}1-5 \\
6+\end{array}$ & $\begin{array}{l}0.5 \\
0.7\end{array}$ & $\mathrm{~S}$ & & & & \\
\hline 122 & Hep Profile & $6+$ & 1.0 & $\mathrm{~S}$ & & & & \\
\hline 87 & $\begin{array}{l}\text { Hepatitis } \mathrm{B}_{\mathrm{s}} \\
\mathrm{Ab}\end{array}$ & $\begin{array}{c}2 \\
3-5 \\
\end{array}$ & $\begin{array}{l}0.3 \\
0.5 \\
\end{array}$ & $\mathrm{~S}$ & & & & \\
\hline 17 & Cotinine & $\begin{array}{c}3-5 \\
6-11 \\
12+ \\
\end{array}$ & $\begin{array}{l}0.8 \\
1.0 \\
1.8 \\
\end{array}$ & $\mathrm{~S}$ & & & & \\
\hline 21 & Lipids & $\begin{array}{c}6-11 \\
12+ \\
\end{array}$ & $\begin{array}{l}0.5 \\
1.0 \\
\end{array}$ & $\mathrm{~S}$ & & & & \\
\hline
\end{tabular}


Exhibit 7-22. Workstation 2 processing worksheet (continued)

SP ID

\section{BLOOD PROCESSING}

\begin{tabular}{|c|c|c|c|c|c|c|c|c|}
\hline ID & Name & Ages & $\begin{array}{c}\text { Sample } \\
\mathrm{mL}\end{array}$ & $\begin{array}{c}\text { Sample } \\
\text { Type }\end{array}$ & $\underset{\checkmark}{\text { Filled }}$ & $\begin{array}{c}\text { Filled } \\
\text { Comments } \\
\text { IctericLipemic } \\
\text { Equip Failure } \\
\text { Clotted } \\
\text { Hemolyzed } \\
\end{array}$ & $\begin{array}{c}\text { Not Filled } \\
\text { Comments } \\
\text { QNSSP } \\
\text { Refusal } \\
\text { Lab Error }\end{array}$ & Slot \# \\
\hline 77 & HPV Serum & $14-59$ & 0.5 & $S$ & & & & \\
\hline 18 & Biochem & $12+$ & 0.8 & $\mathrm{~S}$ & & & & \\
\hline 23 & Insulin & $12+$ & 0.5 & $\mathrm{~S}$ & & & & \\
\hline 12 & $\begin{array}{l}\text { Vit } \\
\text { B12/MMA }\end{array}$ & $20+$ & 0.7 & $\mathrm{~S}$ & & & & \\
\hline 22 & HIV & $18-59$ & 0.8 & $S$ & & & & \\
\hline 24 & $\mathrm{HSV}$ & $14-49$ & 0.2 & $\mathrm{~S}$ & & & & \\
\hline 127 & $\begin{array}{l}\text { IgA-TTG/ } \\
\text { EMA }\end{array}$ & $6+$ & 1.0 & $\mathrm{~S}$ & & & & \\
\hline 137 & $\begin{array}{l}\text { Testosterone/ } \\
\text { Estradiol/ } \\
\text { SHBG }\end{array}$ & $6+$ & 0.7 & $S$ & & & & \\
\hline 150 & Aldehyde & $6+$ & 0.6 & $\mathrm{~S}$ & & & & \\
\hline 32 & Xtra Sera1 & $3+$ & 0.5 & $\mathrm{~S}$ & & & & \\
\hline 33 & Xtra Sera2 & $3+$ & 0.5 & $S$ & & & & \\
\hline 34 & Xtra Sera3 & $12+$ & 1.0 & $\mathrm{~S}$ & & & & \\
\hline 35 & Xtra Sera4 & $12+$ & 1.0 & $\mathrm{~S}$ & & & & \\
\hline 36 & Xtra Sera5 & $12+$ & 1.0 & $S$ & & & & \\
\hline 37 & Xtra Sera6 & $12+$ & 1.0 & $\mathrm{~S}$ & & & & \\
\hline 29 & Persis Pest1 & $12+$ & 4.0 & $\mathrm{~S}$ & & & & \\
\hline 89 & Persis Pest2 & $12+$ & 4.0 & $\mathrm{~S}$ & & & & \\
\hline 90 & Perfluor1 & $12+$ & 1.5 & $\mathrm{~S}$ & & & & \\
\hline 92 & BFR1 & $12+$ & 4.0 & $\mathrm{~S}$ & & & & \\
\hline 93 & BFR2 & $12+$ & 4.0 & $\mathrm{~S}$ & & & & \\
\hline
\end{tabular}

$\mathrm{WB}=$ Whole Blood $\quad \mathrm{P}=$ Plasma $\quad \mathrm{PC}=$ Packed cells $\quad \mathrm{S}=$ Serum 


\section{PRINTING LABELS AND SPECIMEN SHIPMENT}

\subsection{Introduction}

Labels are essential to the laboratory component. Labels support each sample person's (SP's) unique processing protocol. The laboratory record is created when the medical technologist accesses a session for the first time. This record forms the basis for each SP's data in the database. Container labels are generated and assigned to vessel storage containers and allow a processed sample to be tracked from mobile examination center (MEC) processing through contract laboratory receipt.

Biological specimens are shipped to a variety of contract laboratories in double-sided Styrofoam and corrugated cardboard shippers or hard-sided shippers with Styrofoam inserts using dry ice or wet ice (refrigerant packs). A copy of the shipping manifest is included with each shipment that contains a list of the items contained in the shipment and certain demographic information. Exhibit 8-1, the Blood and urine shipping protocol, illustrates the specifications for the shipment of blood and urine specimens to each contract laboratory.

Packages are shipped using FedEx. The chief medical technologist is responsible for contacting FedEx at the start of each stand to determine availability. The facility and equipment specialist contacts the dry ice company at the start of each stand to establish a contact but the chief medical technologist arranges for the delivery of dry ice on shipping days. It is the chief medical technologist's responsibility to confirm the arrangements for shipping and dry ice delivery with the facility and the equipment specialist at the beginning of each stand.

The chief medical technologist works with the MEC manager and the home office to determine exactly which days to ship specimens to the contract laboratories. As a rule, pack and ship most specimens once a week during the MEC split examination sessions. Hold the containers for vessels 32-37, 29/89, 90, 92/93, 48, 49, 66, 67, 102, 52, and 139 and ship once they are full, or hold and ship at the end of the stand. All specimens should arrive at the destination the day after shipping. The next-day limitation may conflict with off days, holidays, and vacations. If a conflict with the shipping schedule, working schedule, or the contract laboratory schedule occurs, consult the MEC manager and the home office technical supervisor. 
Exhibit 8-1. Blood and urine shipping protocol

\begin{tabular}{|c|c|c|c|c|}
\hline Location & Vessels & Conditions & Vessel storage & $\begin{array}{l}\text { Shipping } \\
\text { frequency }\end{array}$ \\
\hline \multicolumn{5}{|l|}{ Whole Blood } \\
\hline $\begin{array}{l}\text { CDC/NCEH Air Toxicant } \\
\text { Branch } \\
\text { Vessel } 54\end{array}$ & VOC Blood & Refrigerated & $\begin{array}{l}5 \times 5 \text { five-inch box } \\
\text { with foam insert }\end{array}$ & Weekly \\
\hline $\begin{array}{l}\text { CASPIR } \\
\text { Vessel } 1\end{array}$ & Lead/Cad/HG & Frozen & $9 \times 9$ two-inch box & Weekly \\
\hline $\begin{array}{l}\text { CASPIR } \\
\text { Vessel } 3\end{array}$ & RBC Folate & Frozen & $\begin{array}{l}10 \times 10 \text { two-inch } \\
\text { box }\end{array}$ & Weekly \\
\hline $\begin{array}{l}\text { University of Missouri } \\
\text { Vessel } 4\end{array}$ & Glycohem & Refrigerated & $9 \times 9$ two-inch box & Weekly \\
\hline $\begin{array}{l}\text { CASPIR } \\
\text { Vessel } 5\end{array}$ & $\begin{array}{l}\text { Mercury, Ethyl } \\
\text { and Methyl }\end{array}$ & Frozen & $9 \times 9$ two-inch box & Weekly \\
\hline \multicolumn{5}{|l|}{ Plasma } \\
\hline $\begin{array}{l}\text { Georgia Health Systems } \\
\text { Vessel } 155\end{array}$ & Fluoride & Frozen & 9 x9 two-inch box & Weekly \\
\hline $\begin{array}{l}\text { University of Missouri } \\
\text { Vessel } 9\end{array}$ & Glucose & Frozen & $9 \times 9$ two-inch box & Weekly \\
\hline $\begin{array}{l}\text { University of Missouri } \\
\text { Vessel } 98\end{array}$ & OGTT & Frozen & $9 \times 9$ two-inch box & Weekly \\
\hline \multicolumn{5}{|c|}{ EDTA Washed Packed Cells } \\
\hline $\begin{array}{l}\text { CASPIR } \\
\text { Vessel } 88\end{array}$ & Acrylamide & Frozen & $9 \times 9$ two-inch box & Weekly \\
\hline \multicolumn{5}{|l|}{ Serum } \\
\hline $\begin{array}{l}\text { CASPIR } \\
\text { Vessel } 136\end{array}$ & $\mathrm{Se} / \mathrm{Cu} / \mathrm{Zn}$ & Frozen & $9 \times 9$ two-inch box & Weekly \\
\hline $\begin{array}{l}\text { CASPIR } \\
\text { Vessel } 110\end{array}$ & Folate & Frozen & 10x10 two-inch box & Weekly \\
\hline $\begin{array}{l}\text { CASPIR } \\
\text { Vessel } 70\end{array}$ & $\begin{array}{l}\text { Vitamin D/Fatty } \\
\text { Acids }\end{array}$ & Frozen & $\begin{array}{l}10 \times 10 \text { two-inch } \\
\text { box }\end{array}$ & Weekly \\
\hline
\end{tabular}


Exhibit 8-1. Blood and urine shipping protocol (continued)

\begin{tabular}{|c|c|c|c|c|}
\hline Location & Vessels & Conditions & Vessel storage & $\begin{array}{l}\text { Shipping } \\
\text { frequency }\end{array}$ \\
\hline \multicolumn{5}{|l|}{ Serum } \\
\hline $\begin{array}{l}\text { CDC/NCID Hepatitis Ref Lab } \\
\text { Vessel } 122\end{array}$ & Hep Profile & Frozen & $9 \times 9$ two-inch box & Weekly \\
\hline $\begin{array}{l}\text { CDC NCID Hepatitis Ref Lab } \\
\text { Vessel } 87\end{array}$ & Hepatitis ab & Frozen & $9 \times 9$ two-inch box & Weekly \\
\hline $\begin{array}{l}\text { CASPIR } \\
\text { Vessel } 17\end{array}$ & Cotinine & Frozen & $9 \times 9$ two-inch box & Weekly \\
\hline $\begin{array}{l}\text { NHANES Diabetes Laboratory } \\
\text { Vessel } 21\end{array}$ & Lipids & Frozen & $9 \times 9$ two-inch box & Weekly \\
\hline $\begin{array}{l}\text { CDC /DVRD/Molecular } \\
\text { Epidemiology } \\
\text { Vessel } 77\end{array}$ & HPV Serum & Frozen & $9 \times 9$ two-inch box & Weekly \\
\hline $\begin{array}{l}\text { Collaborative Laboratory } \\
\text { Services } \\
\text { Vessel } 18\end{array}$ & Biochem & Refrigerated & $9 \times 9$ two-inch box & Weekly \\
\hline $\begin{array}{l}\text { University of MissouriVessel } \\
23\end{array}$ & Insulin & Frozen & $9 \times 9$ two-inch box & Weekly \\
\hline $\begin{array}{l}\text { CASPIR } \\
\text { Vessel } 12\end{array}$ & Vit B12/MMA & Frozen & 9x9 two-inch box & Weekly \\
\hline $\begin{array}{l}\text { CDC/NCID AIDS Division } \\
\text { Vessel } 22\end{array}$ & HIV & Frozen & $9 \times 9$ two-inch box & Weekly \\
\hline $\begin{array}{l}\text { Emory University } \\
\text { Vessel } 24\end{array}$ & HSV & Frozen & $9 \times 9$ two-inch box & Weekly \\
\hline $\begin{array}{l}\text { Mayo Clinic } \\
\text { Vessel } 127\end{array}$ & IgA-TTG/EMA & Frozen & $9 \times 9$ two-inch box & Weekly \\
\hline $\begin{array}{l}\text { CASPIR } \\
\text { Vessel } 137\end{array}$ & Testosterone & Frozen & 9x9 two-inch box & Weekly \\
\hline CDC NCEH Air Toxicant & Aldehyde & Refrigerated & $9 \times 9$ two-inch box & Weekly \\
\hline $\begin{array}{l}\text { CASPIR } \\
\text { Vessels 32-37 }\end{array}$ & Xtra Sera & Frozen & $9 \times 9$ two-inch box & $\begin{array}{l}\text { Full or end } \\
\text { of stand }\end{array}$ \\
\hline $\begin{array}{l}\text { CASPIR } \\
\text { Vessels } 29 \text { and } 89\end{array}$ & $\begin{array}{l}\text { Persis Pest1 } \\
\text { Persis Pest } 2\end{array}$ & Frozen & $9 \times 9$ three-inch box & $\begin{array}{l}\text { Full or end } \\
\text { of stand }\end{array}$ \\
\hline $\begin{array}{l}\text { CDC/NCEH Environmental } \\
\text { Health Laboratory } \\
\text { Vessel } 90\end{array}$ & Perfluor1 & Frozen & $9 \times 9$ three-inch box & $\begin{array}{l}\text { Full or end } \\
\text { of stand }\end{array}$ \\
\hline
\end{tabular}


Exhibit 8-1. Blood and urine shipping protocol (continued)

\begin{tabular}{|c|c|c|c|c|}
\hline Location & Vessels & $\begin{array}{c}\text { Condition } \\
\text { s }\end{array}$ & Vessel storage & $\begin{array}{l}\text { Shipping } \\
\text { frequency }\end{array}$ \\
\hline $\begin{array}{l}\text { CASPIR } \\
\text { Vessels } 92 \text { and } 93\end{array}$ & $\begin{array}{l}\text { BFR1 } \\
\text { BFR2 } \\
\end{array}$ & Frozen & $\begin{array}{c}9 \times 9 \text { three-inch } \\
\text { box }\end{array}$ & $\begin{array}{l}\text { Full or end } \\
\text { of stand }\end{array}$ \\
\hline \multicolumn{5}{|l|}{ Urine } \\
\hline $\begin{array}{l}\text { University of Minnesota } \\
\text { Vessel } 45\end{array}$ & Alb/Creat & Frozen & $\begin{array}{c}9 \times 9 \text { three-inch } \\
\text { box }\end{array}$ & Weekly \\
\hline $\begin{array}{l}\text { CDC/NCID NCHSTD } \\
\text { Vessel } 46\end{array}$ & Chlam/Trichomonas & Frozen & $\begin{array}{c}5 \times 5 \text { three-inch } \\
\text { box }\end{array}$ & Weekly \\
\hline $\begin{array}{l}\text { CDC/NCID NCHSTD } \\
\text { Vessel } 154\end{array}$ & Trichomonas & Frozen & $\begin{array}{c}5 \times 5 \text { three-inch } \\
\text { box }\end{array}$ & Weekly \\
\hline $\begin{array}{l}\text { CASPIR } \\
\text { Vessel } 86 \\
\end{array}$ & Spc Arsenic & Frozen & $\begin{array}{c}9 \times 9 \text { three-inch } \\
\text { box }\end{array}$ & Weekly \\
\hline $\begin{array}{l}\text { CASPIR } \\
\text { Vessel } 48\end{array}$ & Prior Pest & Frozen & $\begin{array}{c}5 \times 5 \text { three-inch } \\
\text { box }\end{array}$ & $\begin{array}{l}\text { Full or end } \\
\text { of stand }\end{array}$ \\
\hline $\begin{array}{l}\text { CASPIR } \\
\text { Vessel } 49\end{array}$ & Organophos & Frozen & $\begin{array}{c}5 \times 5 \text { three-inch } \\
\text { box }\end{array}$ & $\begin{array}{l}\text { Full or end } \\
\text { of stand }\end{array}$ \\
\hline $\begin{array}{l}\text { CASPIR } \\
\text { Vessel } 62\end{array}$ & Urn Merc & Frozen & $\begin{array}{c}5 \times 5 \text { three-inch } \\
\text { box }\end{array}$ & Weekly \\
\hline $\begin{array}{l}\text { CASPIR } \\
\text { Vessel } 50\end{array}$ & Heavy Metal & Frozen & $\begin{array}{c}5 \times 5 \text { three-inch } \\
\text { box }\end{array}$ & Weekly \\
\hline $\begin{array}{l}\text { CASPIR } \\
\text { Vessel } 66\end{array}$ & PAH & Frozen & $\begin{array}{c}9 \times 9 \text { three-inch } \\
\text { box }\end{array}$ & $\begin{array}{l}\text { Full or end } \\
\text { of stand }\end{array}$ \\
\hline $\begin{array}{l}\text { CDC/NCEH Environmental } \\
\text { Health Laboratory } \\
\text { Vessel } 67\end{array}$ & Phthalates & Frozen & $\begin{array}{c}9 \times 9 \text { three-inch } \\
\text { box }\end{array}$ & $\begin{array}{l}\text { Full or end } \\
\text { of stand }\end{array}$ \\
\hline $\begin{array}{l}\text { CDC/NCEH Air Toxicant } \\
\text { Branch } \\
\text { Vessel } 102\end{array}$ & $\begin{array}{l}\text { Perchlorate/VOC/ } \\
\text { Thiocyante }\end{array}$ & Frozen & $\begin{array}{c}9 \times 9 \text { three-inch } \\
\text { box }\end{array}$ & End of stand \\
\hline $\begin{array}{l}\text { CASPIR } \\
\text { Vessel } 121\end{array}$ & NNAL & Frozen & $\begin{array}{c}5 \times 5 \text { three-inch } \\
\text { box }\end{array}$ & End of stand \\
\hline $\begin{array}{l}\text { CASPIR } \\
\text { Vessel } 128\end{array}$ & Caffeine & Frozen & $\begin{array}{c}10 \times 10 \text { two-inch } \\
\text { box }\end{array}$ & Weekly \\
\hline $\begin{array}{l}\text { CASPIR } \\
\text { Vessel } 152\end{array}$ & Aromatic Amines & Frozen & $\begin{array}{c}9 \times 9 \text { three-inch } \\
\text { box }\end{array}$ & Weekly \\
\hline $\begin{array}{l}\text { CASPIR } \\
\text { Vessel } 151\end{array}$ & Volitile Nitrosamines & Frozen & $\begin{array}{c}9 \times 9 \text { three-inch } \\
\text { box }\end{array}$ & Weekly \\
\hline $\begin{array}{l}\text { CASPIR } \\
\text { Vessel } 153\end{array}$ & HAA/Nicotine analogs & Frozen & $\begin{array}{c}9 \times 9 \text { three-inch } \\
\text { box }\end{array}$ & Weekly \\
\hline $\begin{array}{l}\text { CASPIR } \\
\text { Vessel } 52 \\
\end{array}$ & Xtra Urine1 & Frozen & $\begin{array}{c}9 \times 9 \text { three-inch } \\
\text { box }\end{array}$ & $\begin{array}{l}\text { Full or end } \\
\text { of stand }\end{array}$ \\
\hline $\begin{array}{l}\text { CASPIR } \\
\text { Vessel } 139\end{array}$ & Xtra Urine2 & Frozen & $\begin{array}{c}9 \times 9 \text { three-inch } \\
\text { box }\end{array}$ & $\begin{array}{l}\text { Full or end } \\
\text { of stand }\end{array}$ \\
\hline
\end{tabular}


Exhibit 8-2 contains a list of label/shipping supplies.

Exhibit 8-2. Supplies for labels/shipping

\begin{tabular}{|c|c|}
\hline Small Styrofoam shipper (14 x 14 x 12) & Medium Styrofoam shippers $(16$ x16 x 15.5) \\
\hline 5-mL cryovial sterile & 2-mL cryovial sterile \\
\hline 14-mL snap cap test tube & 2-mL cryovial nonsterile \\
\hline Conical tube $50-\mathrm{mL}$ & Caps for 2-mL cryovial \\
\hline \multirow[t]{2}{*}{$\begin{array}{l}5.25 \times 5.25 \times 2.0 \text { inch cardboard box with } \\
9 \times 9 \text { cardboard grid }\end{array}$} & $\begin{array}{l}5.25 \times 5.25 \times 3.0 \text { inch cardboard box with } 9 \times 9 \\
\text { cardboard grid }\end{array}$ \\
\hline & $5.25 \times 5.25 \times 3.0$ inch box with $7 \times 7$ grid \\
\hline $\begin{array}{l}5.25 \times 5.25 \times 3.0 \text { inch cardboard box with } \\
5 \times 5 \text { grid }\end{array}$ & Scale \\
\hline $10 \times 10$ two-inch box & $10 \times 10$ three-inch box \\
\hline STP-710 Pressure vessel & STP-310 Pressure vessel \\
\hline STP-740 Pressure vessel & U-TEK Reusable Refrigerant Packs Large \\
\hline Plastic bag $9 \times 12$ & Zip closable bags - quart size \\
\hline Absorbent sheets $(2 \times 2)$ & Rubber bands \\
\hline Large padded envelope & Newsprint paper \\
\hline Cryo gloves & Full face shield \\
\hline Hammer & Dry ice scoop \\
\hline Tape gun & Packaging tape 2" \\
\hline Overpack label & Address labels for FedEx class 9 sticker \\
\hline UN 3373 label & Refrigerate Do Not Freeze label \\
\hline Keep Frozen label & Avery 5160 labels \\
\hline Label Zebra (1.125”) & Printer ribbon, Zebra (thermal transfer) \\
\hline Sheet-fed laser labels & Wipes, Terri 13" x 18" \\
\hline FedEx large boxes & FedEx Class 9 label \\
\hline Clear FedEx label pouches & Dymo Labels \\
\hline FedEx form 0215 & FedEx preprinted return airbill - stand \\
\hline Toner cartridge (HP Laser Jet 4000) & Lab jackets (Petite, Lg., X-Lg.) \\
\hline
\end{tabular}




\subsection{Print Vessel Labels and Label Blood Processing Racks}

Before each session, the technologist assigned to workstation 1 prints all labels for all SPs assigned to a session. The correct bar-coded labels automatically print for each SP based on the SP's age, sex, appointment type, and selection into one or more subsamples. Print pregnancy testing, urine processing, blood processing, packed cells processing, GTT processing labels, and HPV oral rinse for all SPs scheduled into a MEC session. The phlebotomist and the technologist assigned to shipping are responsible for labeling vessels and assembling the blood processing racks. The MEC physician prints the HPV swab labels.

\section{Distribute labels}

- Place urine processing, pregnancy testing, and HPV oral rinse labels at workstation 3 in the laboratory.

- If the SP does not show up for the appointment, it is possible he or she will reschedule.

- Store the SP's vessels in a zip-closable bag until the end of the stand.

- If the SP has not shown up by the end of the stand, place racks in clear plastic bins or in cupboard above workstation 3.

\section{Assemble blood-processing racks}

The phlebotomist and the medical technologist assigned to the shipping workstation are responsible for labeling vessels and assembling the blood processing racks. Use extreme care in setting up the blood vessel racks and in labeling the vessels with the appropriate bar-code label.

Label each vessel with the appropriate vessel label, according to the protocol. Place the label on the vessel, wrapping it around the vessel horizontally and making sure the label wraps onto itself. Place the label so the first digit of the vessel number is at the top of the vessel. Set the vessels in the cube racks in priority order. 
Use the following materials to set up and label blood racks:

- Plastic rack

- Template for processing

- Vessels stored of the following sizes:

- $\quad$ 2.0-mL sterile vessel

- $\quad$ 2.0-mL non-sterile vessel

- $\quad$ 5.0-mL sterile vessel

- $\quad$ 14.0-mL tube

- $\quad 50-\mathrm{mL}$ conical tube

- Barcoded SP ID labels each containing the unique 6-digit SP ID number concatenated with the vessel ID

Prepare one blood processing rack for each SP. The set up of the rack depends on the subject's age, sex, appointment type, and inclusion in selected subsamples. When possible, prepare the racks at least 1 day before the SP arrives for the MEC examination.

- Label all GTT tubes (vessel 98) and place in one rack.

- Label all Acrylamide tubes (vessel 88) and place in one rack.

Place the prelabeled blood processing racks and the GTT rack on the counter to the right of the centrifuges at the start of each session.

\subsubsection{Print Vessel Labels Using the Label Print Application}

Open the Laboratory application. Log onto the computer terminal and select the Label Print Application. This application is found on the desktop of all laboratory computers and has an icon that looks like a barcode label.

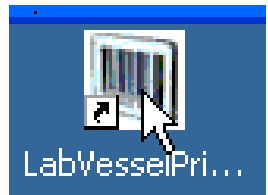




\subsubsection{Main Printing Screen}

From the main printing screen, the current day and next 2 days the MEC is in session will be displayed in a tree view on the left side of the screen. The sessions will be color coded to match the legend at the bottom left hand side of the screen. Sessions in green indicate that a particular session or SPs within that session need to be printed. Sessions in black have already been printed and there are no SP profile changes. Sessions in blue indicate that an SP was added to that session or that a SP or SPs had a profile change and need to be reprinted. If an SP is removed from a session, that session will be displayed in red, which indicates label action is needed.

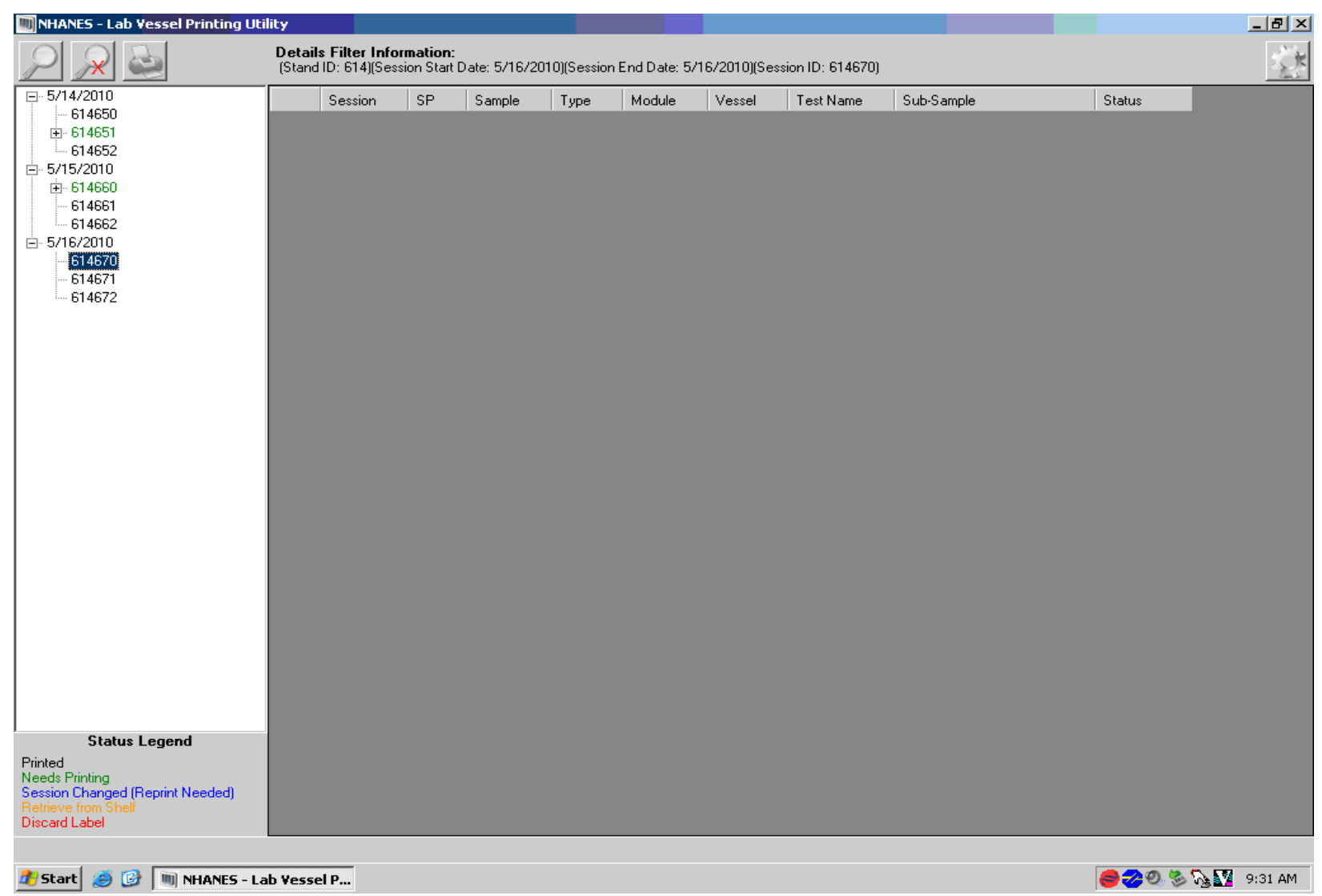




\subsubsection{Filter Options}

The looking glass icon is used for the filtering options, where you can filter labels by stand start and end date, session ID, SP ID, or Sample ID. After picking the criteria you would like to filter with, press the filter button. Clearing the filter takes you back to the current date, plus 2 days and the cancel button takes you back to the main printing screen.

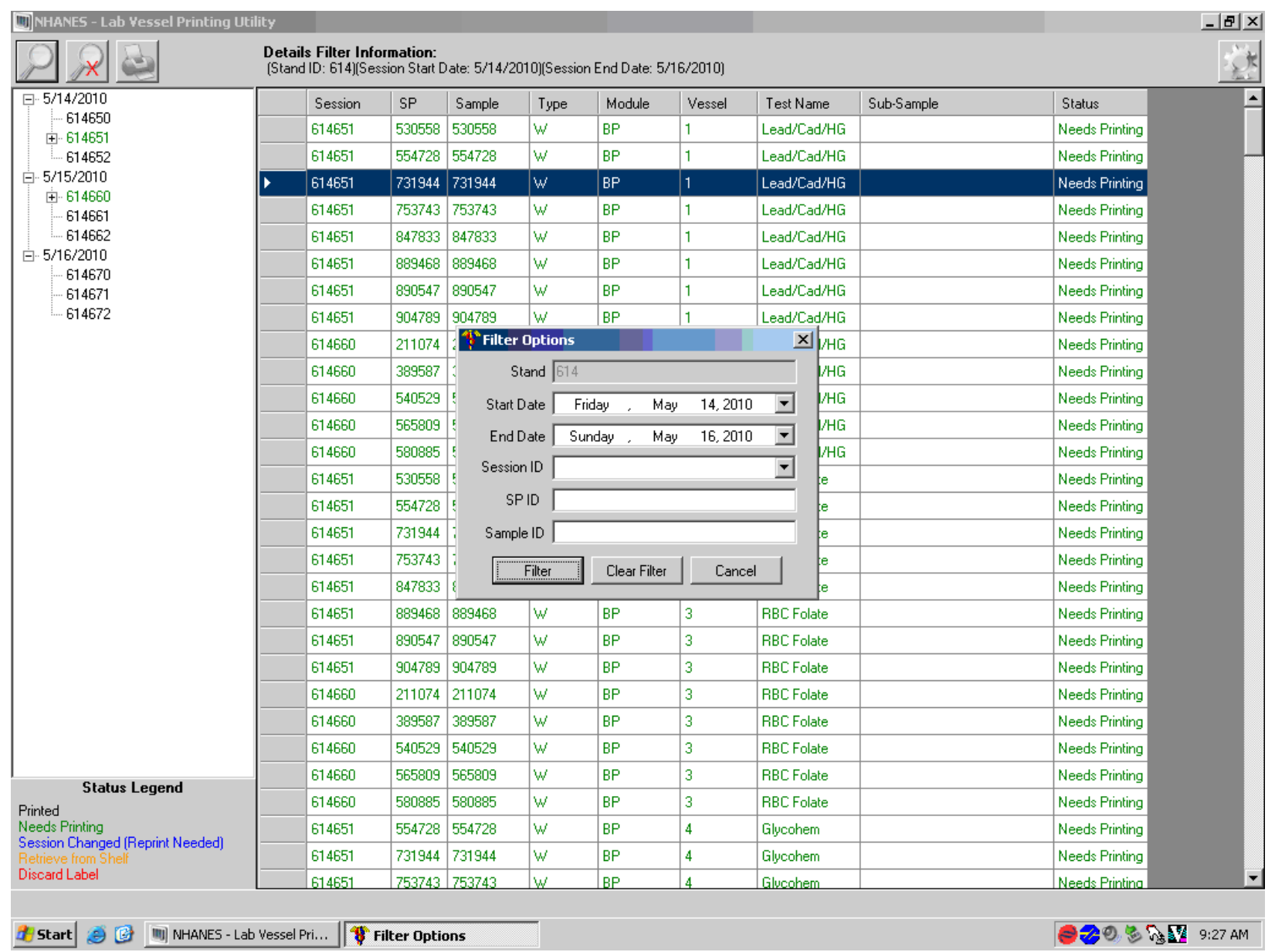




\subsubsection{Print Key}

The print key (represented by the printer) brings up another menu that allows the user to select different print options and view print details.

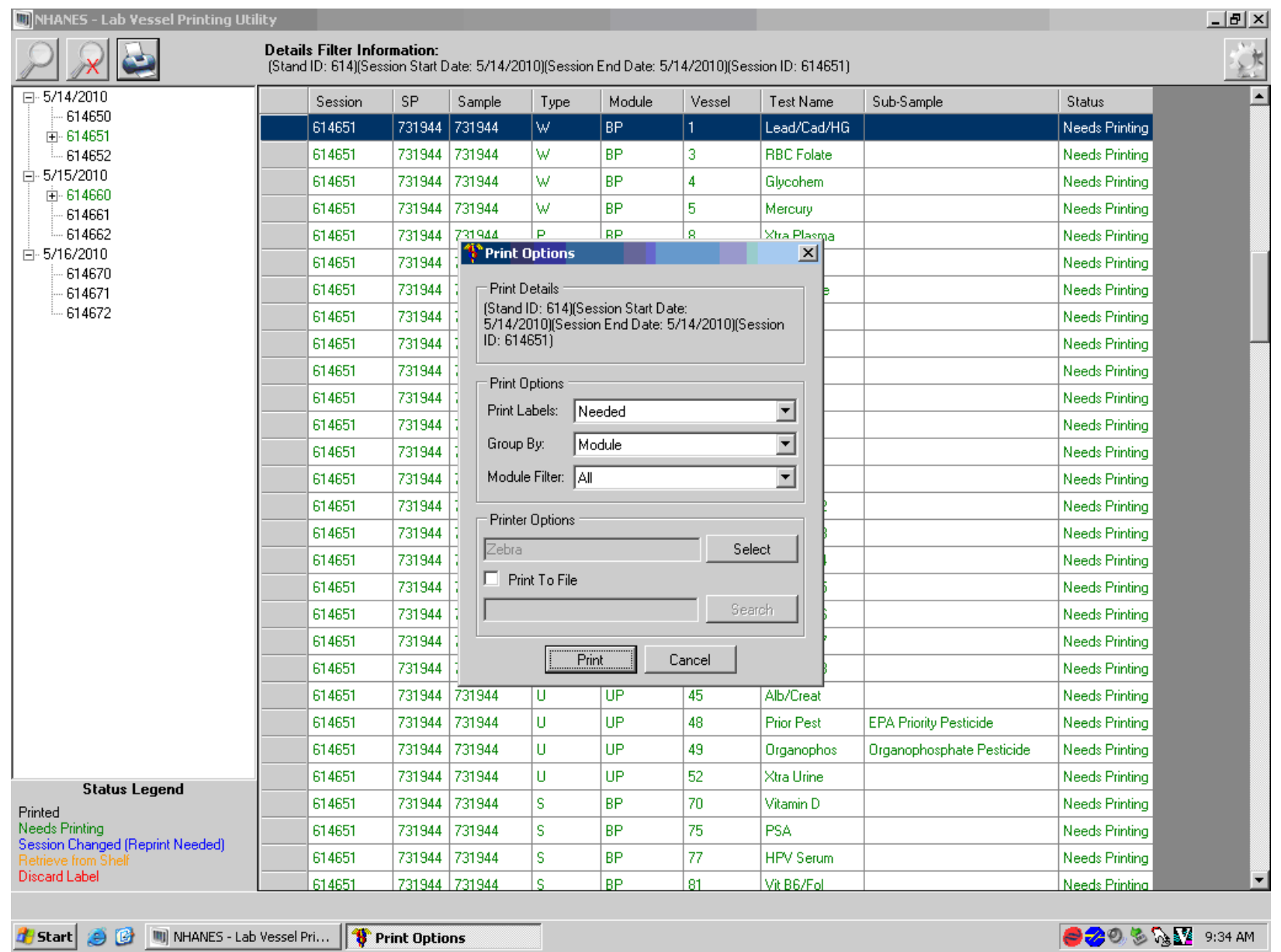




\title{
8.3.5 Print Options: Print Labels
}

Labels can be printed using two options: All or Needed.

\author{
All Vessel Labels:
}

- Print laboratory vessel labels for all modules for an SP or for all SPs in a session.

Needed Vessel Labels:

- Labels that are displayed in green are needed and will print if either option is selected. All should be selected if nothing is in green for that session, but all the labels need to be printed or reprinted.

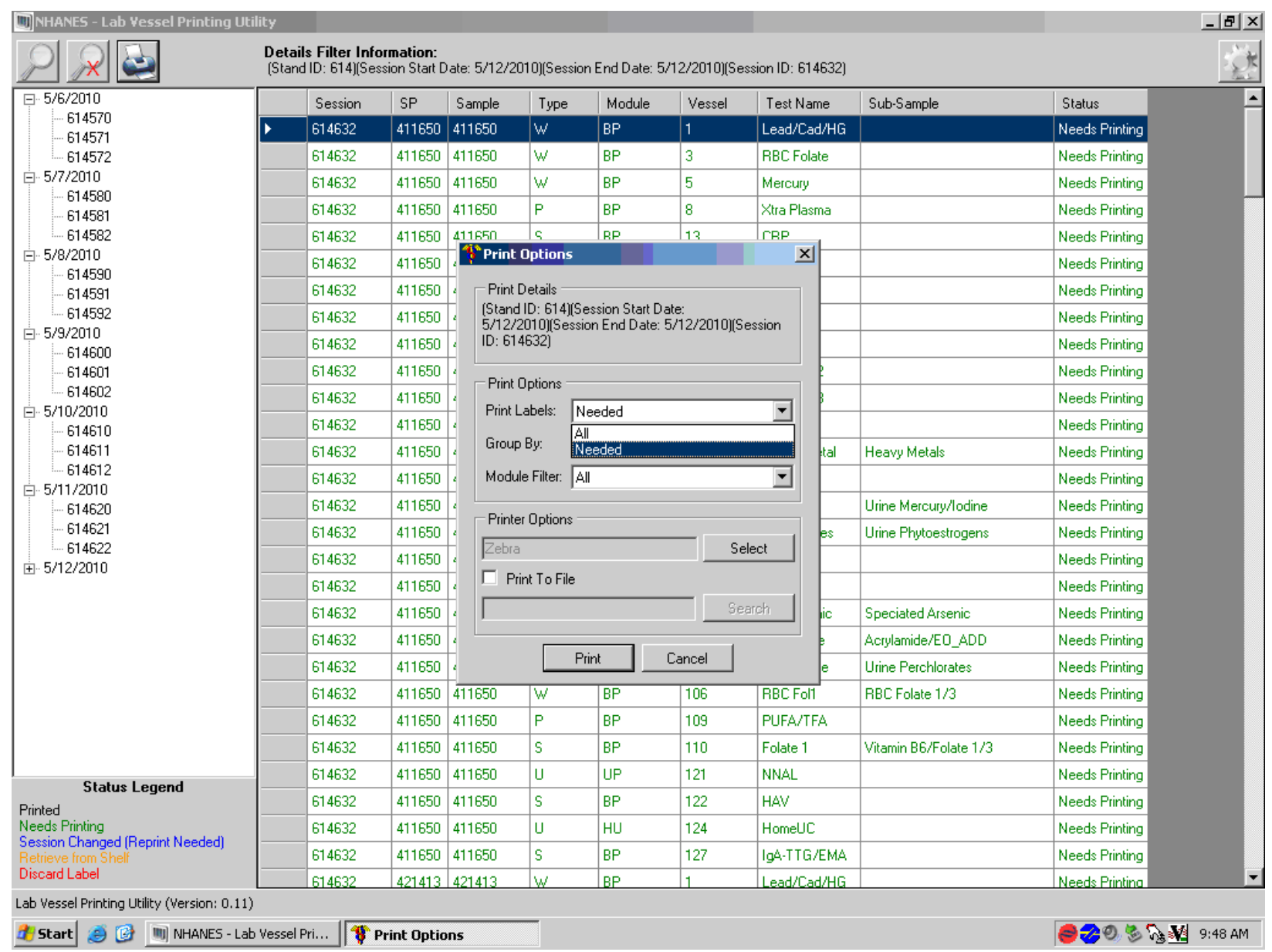




\subsection{6}

\section{Print Options: Group By}

Have the option to print labels grouped by SP, Vessel, Module, or Sample. The Group By will automatically default to module.

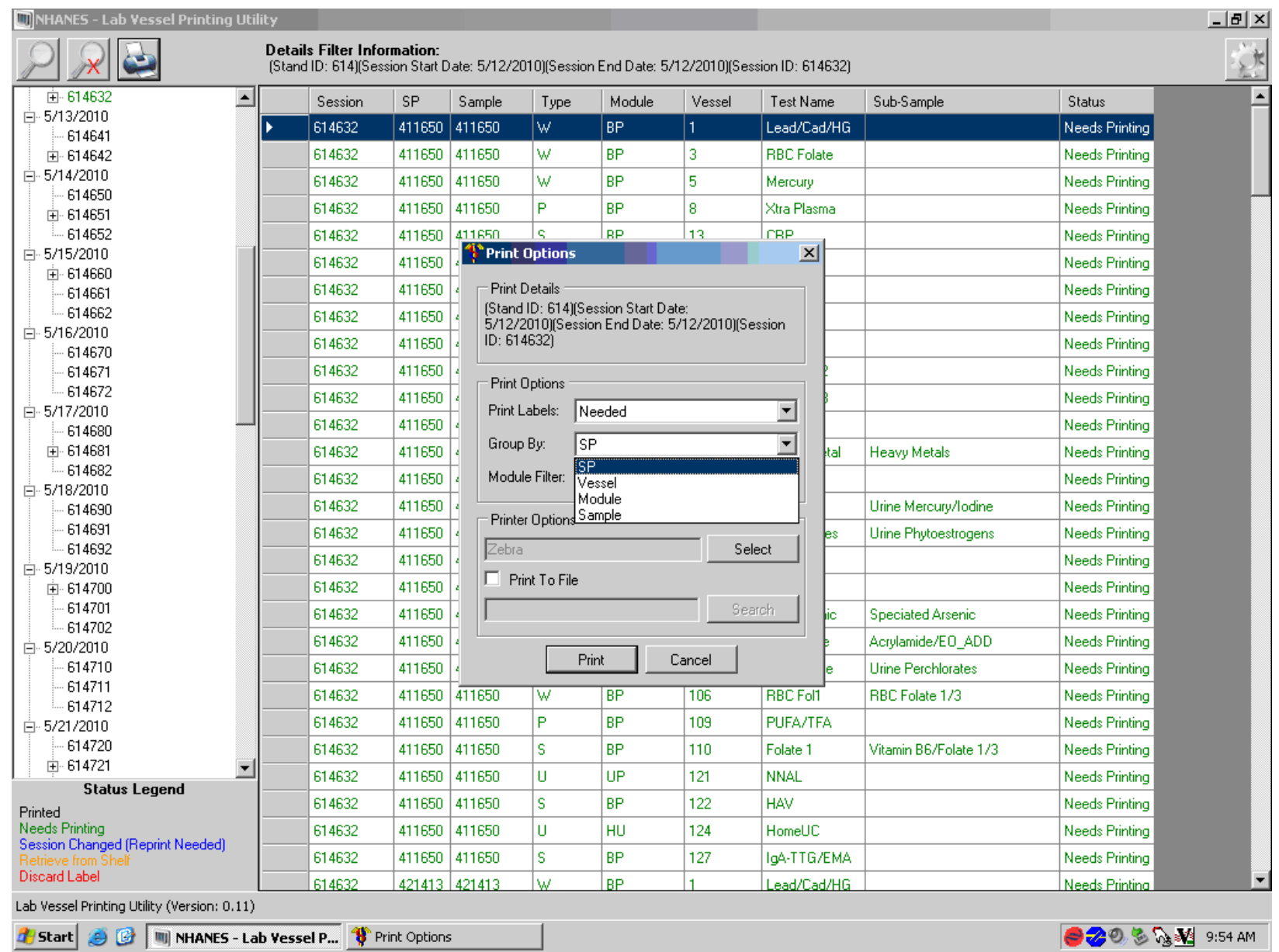




\subsubsection{Print Options: Module Filter}

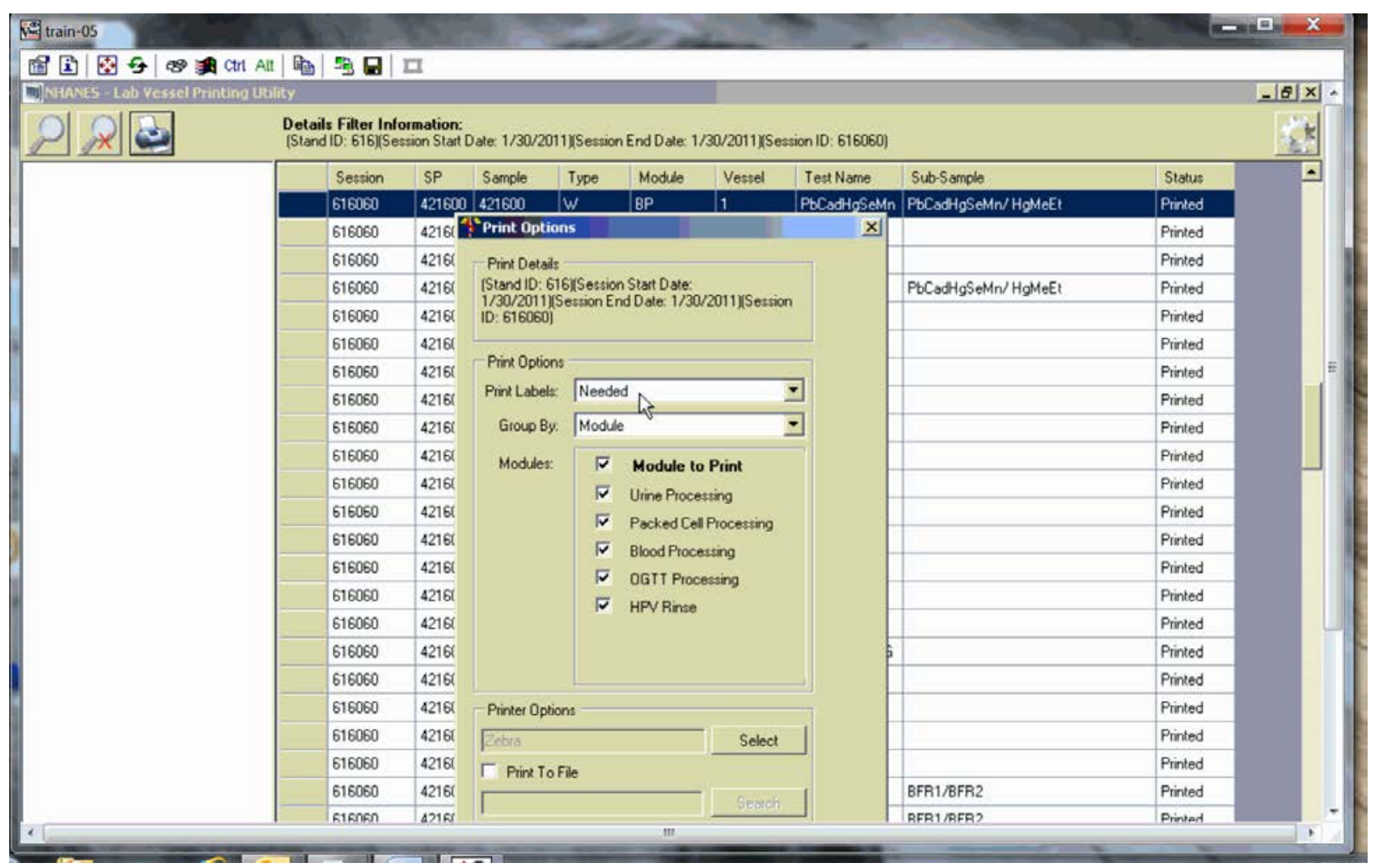

Option to select all modules, a single module, or a combination of modules using the checkbox in front of the desired module.

\subsubsection{Print Order}

The print order matches the order the vessels are displayed on the print screen for the entire session. All urine vessels are printed first, which are grouped by SP ID. Do not label urine vessels; save urine labels together, which will be distributed to workstation 3 the day of the session being labeled. 


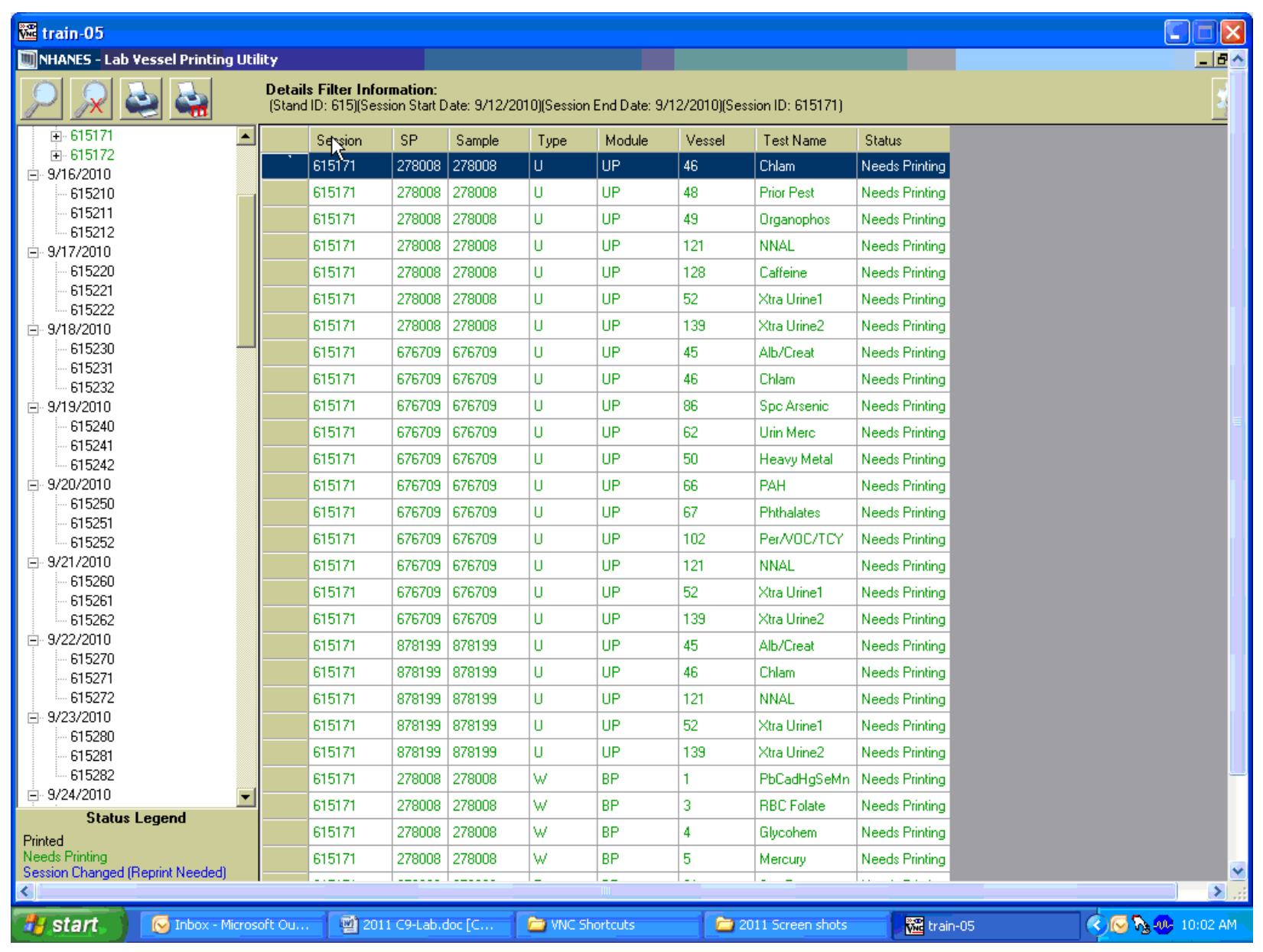

If printing a morning session, the OGTT labels are printed after the blood processing labels. Label the OGTT tubes and put these SPs into a separate rack. The last module to be printed is the HPV rinse labels and they should be kept with the urine labels and distributed to workstation 3 the day of the session you are labeling.

\subsubsection{Print Display Options}

To change the display on the screen to display in numerical order for all SPs, click on "Vessel" tab and the display will change to numerical order for the entire session. 


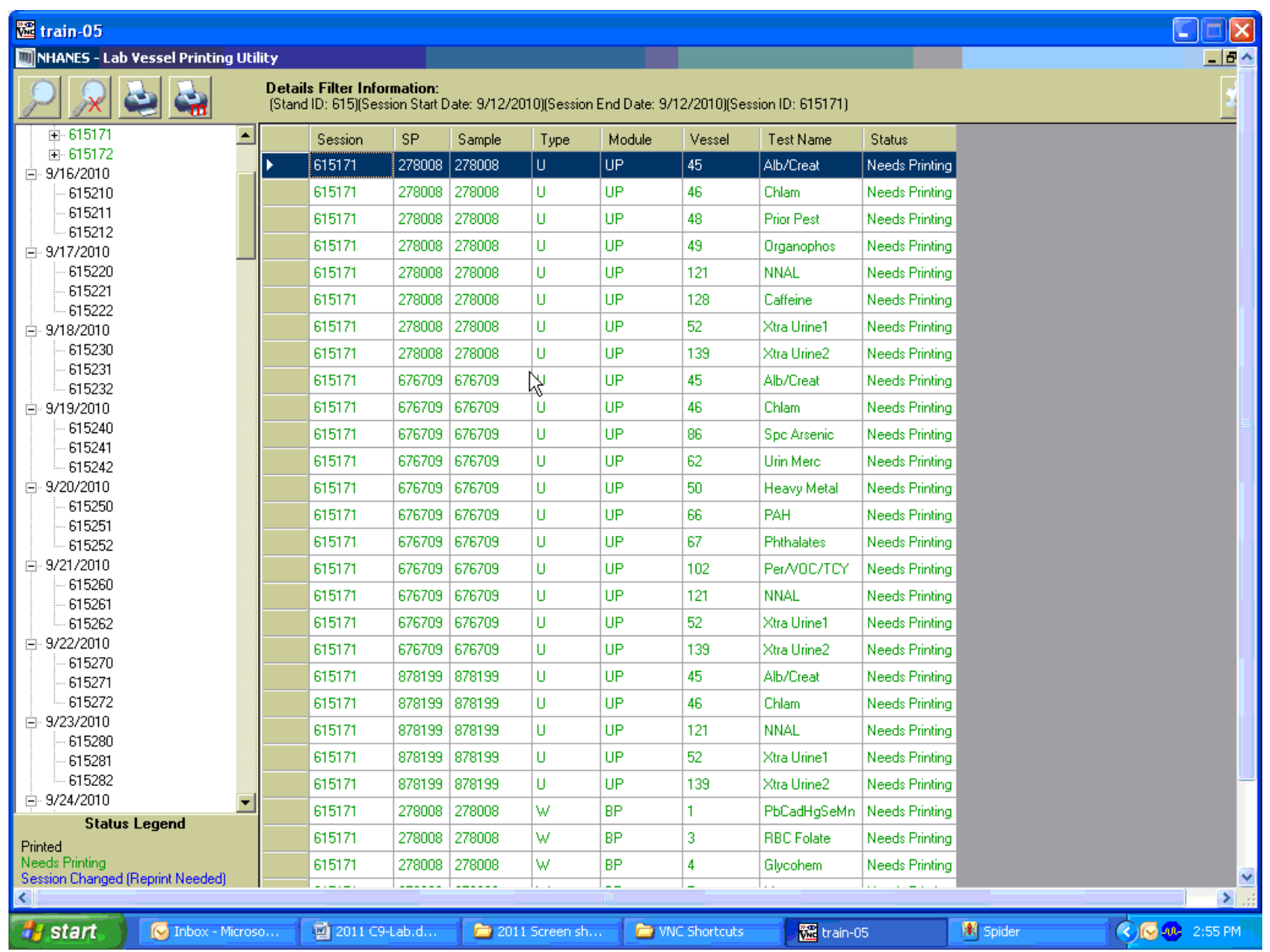

\subsubsection{Printing One SP's Labels}

Using the tree on the left hand side of the screen, expand the session by clicking on the + sign to the left of the session number. This will display all the SPs scheduled for that session. Click on the SP you want displayed. Use the printer icon to print using the print options described in 8.3.5 to 8.3.7. 


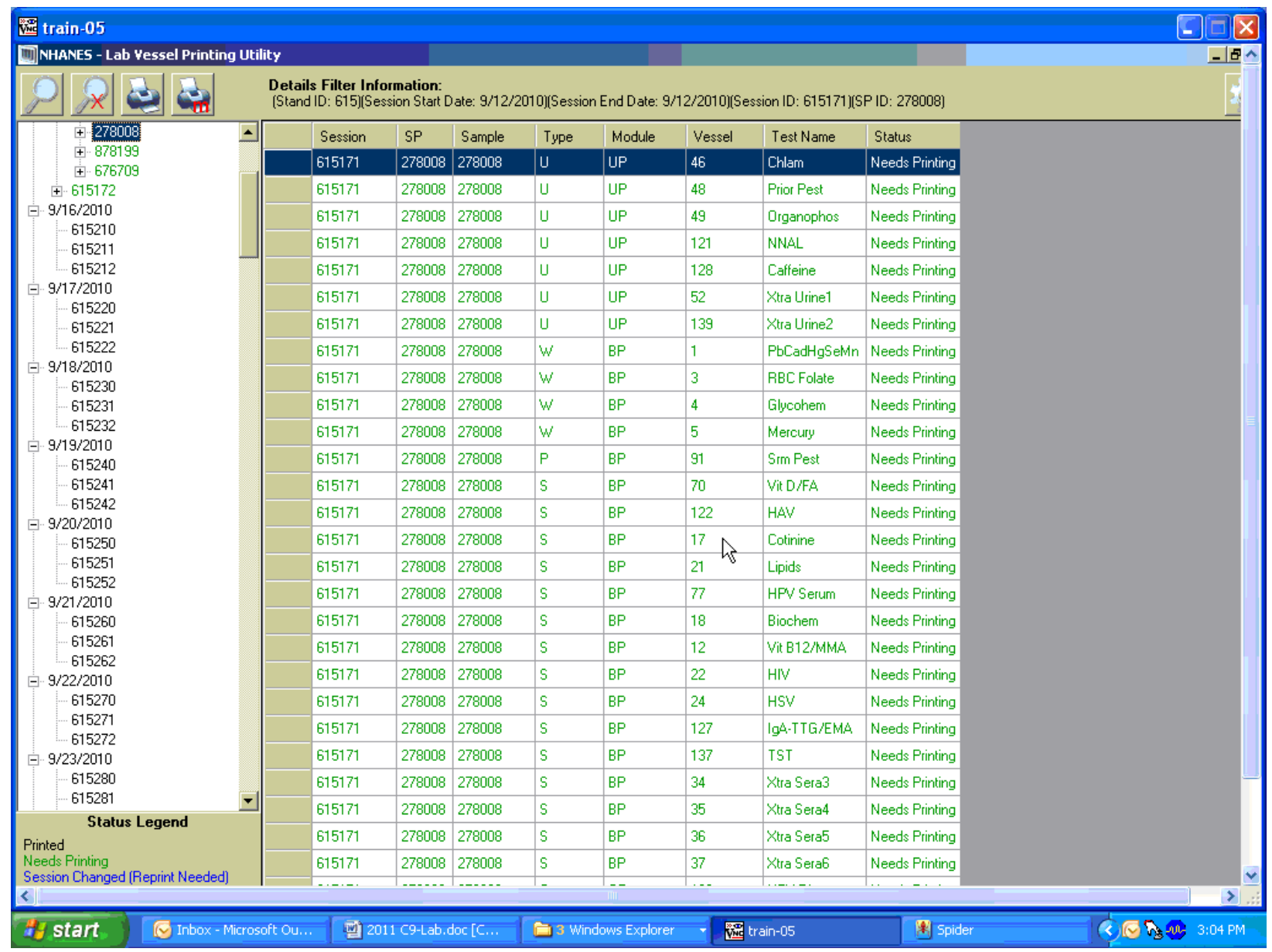

\subsubsection{Printing Individual Labels}

To print a single or individual label, highlight the line you wish to be printed. Use the printer icon to print using the print options described in Sections 8.3.5 to 8.3.7. 


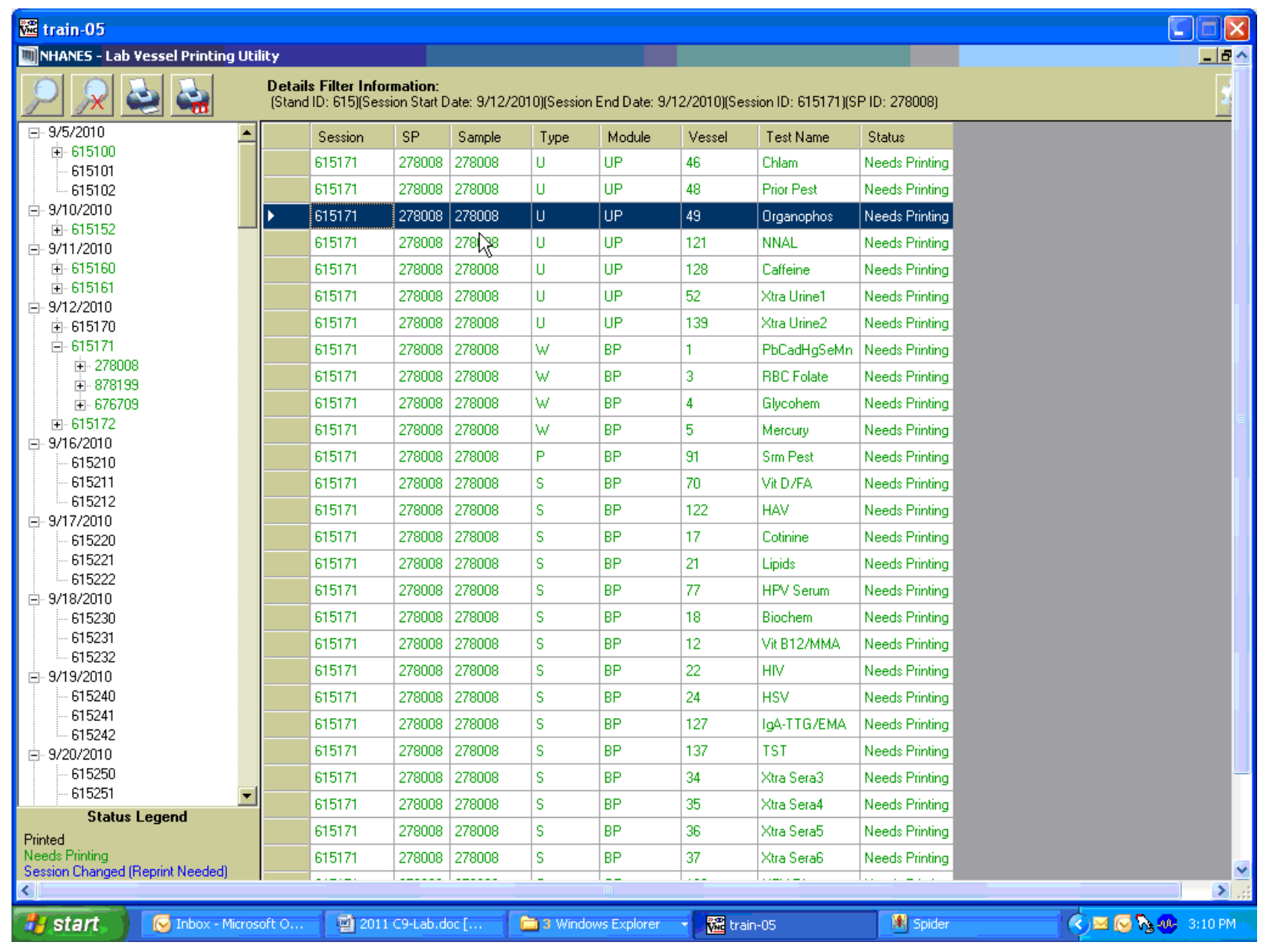

\subsubsection{Label Action}

If an SP's profile has changed and the labels have already been printed, the SP line will turn red on the heads-up display and show "Label Action Required." Blood processing won't be enabled until the label action is performed. 


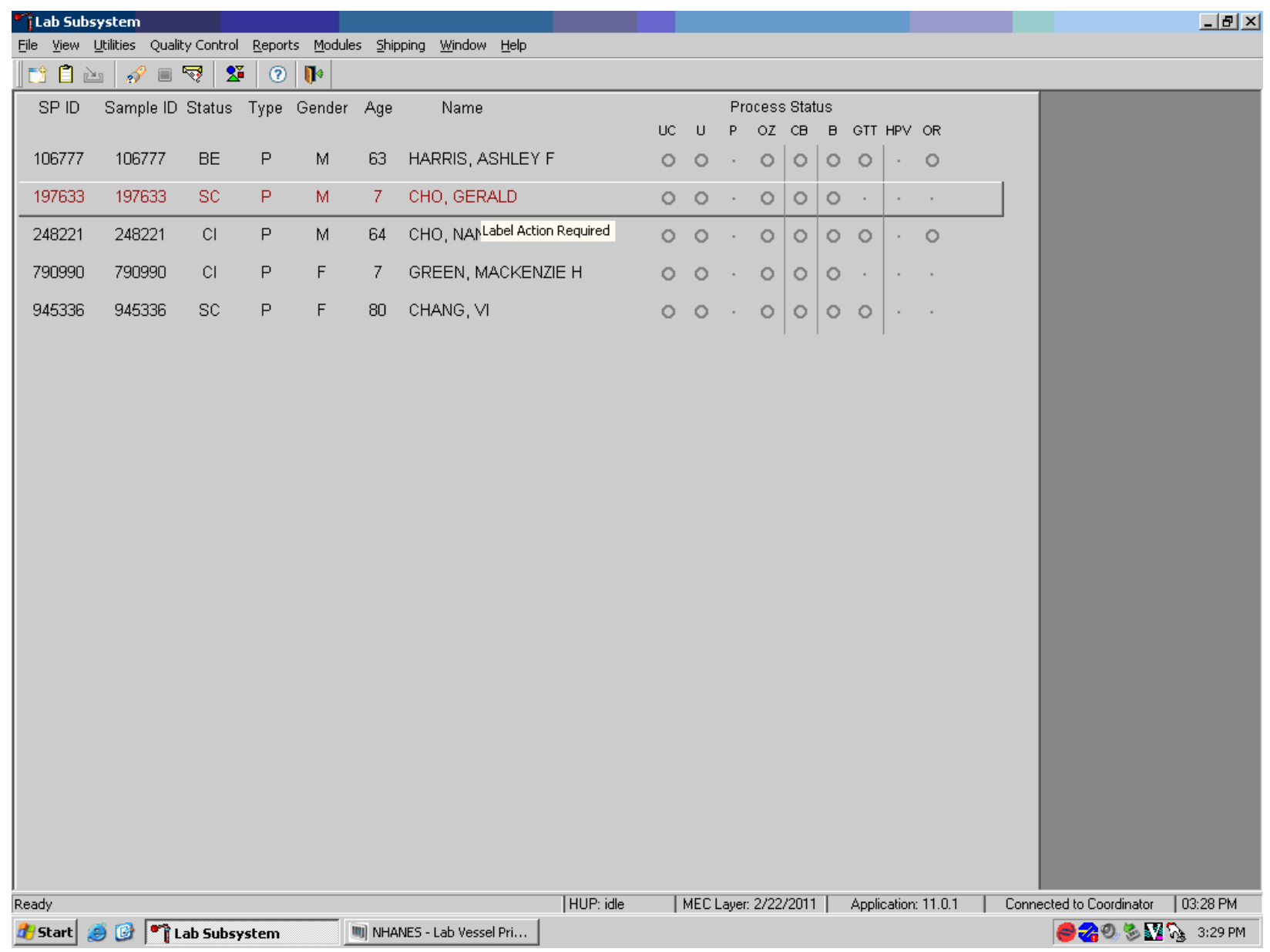

Open up the Lab printing application.

The session will be displayed in the tree in green, which indicates something needs to be printed. Click on the SP ID in the tree; the SP will also be displayed in green because labels will need to be printed and some will need to be discarded. Labels that need to be discarded are displayed in red. 


\begin{tabular}{|c|c|c|c|c|c|c|c|c|c|}
\hline \multicolumn{6}{|l|}{ 四|NHANES - Lab Yessel Printing Utility } & \multirow{2}{*}{\multicolumn{3}{|c|}{ /9/2010)(Session ID: 615440)(SP ID: 197633) }} & \multirow{2}{*}{ 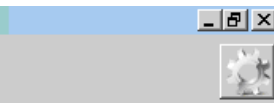 } \\
\hline 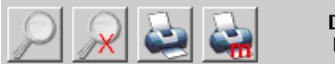 & $\begin{array}{l}\text { ils Filter In } \\
\text { dID: } 615)(S\end{array}$ & $\begin{array}{l}\text { Imation: } \\
\text { ion Start } \mathrm{C}\end{array}$ & ate: $10 / 9$ & 10)(Sessi & End Date: & & & & \\
\hline \multirow{26}{*}{$\begin{array}{c}10 / 9 / 2010 \\
+615440 \\
+945336 \\
+197633 \\
+248221 \\
+790990 \\
+106777 \\
615442 \\
10 / 10 / 2010 \\
615451 \\
615452\end{array}$} & Session & SP & Sample & Type & Module & Vessel & Test Name & Status & $\Delta$ \\
\hline & 615440 & 197633 & 197633 & W & $\mathrm{BP}$ & 3 & RBC Folate & Printed & \\
\hline & 615440 & 197633 & 197633 & W & $\mathrm{BP}$ & 4 & Glycohem & Discard Label & \\
\hline & 615440 & 197633 & 197633 & w & $\mathrm{BP}$ & 5 & Mercury & Printed & \\
\hline & 615440 & 197633 & 197633 & P & BP & 9 & Glucose & Discard Label & \\
\hline & 615440 & 197633 & 197633 & s & $\mathrm{BP}$ & 136 & $\mathrm{Se} / \mathrm{Cu} / \mathrm{Zn}$ & Printed & \\
\hline & 615440 & 197633 & 197633 & S & $\mathrm{BP}$ & 70 & Vit D/FA & Printed & \\
\hline & 615440 & 197633 & 197633 & s & $\mathrm{BP}$ & 122 & HAV & Printed & \\
\hline & 615440 & 197633 & 197633 & s & $\mathrm{BP}$ & 17 & Cotinine & Printed & \\
\hline & 615440 & 197633 & 197633 & S & $\mathrm{BP}$ & 21 & Lipids & Printed & \\
\hline & 615440 & 197633 & 197633 & s & $B P$ & 18 & Biochem & Discard Label & \\
\hline & 615440 & 197633 & 197633 & S & $\mathrm{BP}$ & 23 & Insulin & Discard Label & \\
\hline & 615440 & 197633 & 197633 & S & $B P$ & 12 & Vit B12/MMA & Discard Label & \\
\hline & 615440 & 197633 & 197633 & s & $\mathrm{BP}$ & 118 & Thyroid & Discard Label & \\
\hline & 615440 & 197633 & 197633 & S & $\mathrm{BP}$ & 127 & IgA-TTG/EMA & Printed & \\
\hline & 615440 & 197633 & 197633 & S & $\mathrm{BP}$ & 137 & TST & Printed & \\
\hline & 615440 & 197633 & 197633 & S & $B P$ & 110 & Folate 1 & Printed & \\
\hline & 615440 & 197633 & 197633 & s & $\mathrm{BP}$ & 32 & Xtra Sera1 & Needs Printing & \\
\hline & 615440 & 197633 & 197633 & S & $\mathrm{BP}$ & 33 & Xtra Sera2 & Needs Printing & \\
\hline & 615440 & 197633 & 197633 & s & $\mathrm{BP}$ & 34 & Xtra Sera3 & Discard Label & \\
\hline & 615440 & 197633 & 197633 & s & $\mathrm{BP}$ & 35 & Xtra Sera4 & Discard Label & \\
\hline & 615440 & 197633 & 197633 & S & $\mathrm{BP}$ & 36 & Xtra Sera5 & Discard Label & \\
\hline & 615440 & 197633 & 197633 & s & $\mathrm{BP}$ & 37 & Xtra Sera6 & Discard Label & \\
\hline & 615440 & 197633 & 197633 & s & $\mathrm{BP}$ & 29 & Pers Pest1 & Discard Label & \\
\hline & 615440 & 197633 & 197633 & s & $\mathrm{BP}$ & 89 & Pers Pest2 & Discard Label & \\
\hline & 615440 & 197633 & 197633 & 5 & $\mathrm{BP}$ & 90 & Perfluor & Discard Label & \\
\hline \multirow{4}{*}{$\begin{array}{l}\text { Printed } \\
\text { Needs Printing } \\
\text { Session Changed (Reprint Needed) } \\
\text { Retrieve from Shef } \\
\text { Discard Label }\end{array}$} & 615440 & 197633 & 197633 & s & $\mathrm{BP}$ & 92 & BFR1 & Discard Label & \\
\hline & 615440 & 197633 & 197633 & s & $\mathrm{BP}$ & 93 & BFR2 & Discard Label & \\
\hline & 615440 & 197633 & 197633 & $\mathrm{P}$ & TT & 98 & OGTT & Discard Label & \\
\hline & 615440 & 197633 & 197633 & c & $a B$ & 129 & HPV Rinse & DiscardLabel & $\nabla$ \\
\hline \multicolumn{10}{|l|}{ Lab Vessel Printing Utility (Version: 1.5 ) } \\
\hline d) Start $\&$ [at Lab Subsystem & 间 & HANES - 1 & Lab Vesse & & & & & & - \\
\hline
\end{tabular}

Click on the printer icon and print what is needed. Right click on a line that is displayed in red to mark label as discarded. Two options are available for discarding, either individually or all. 


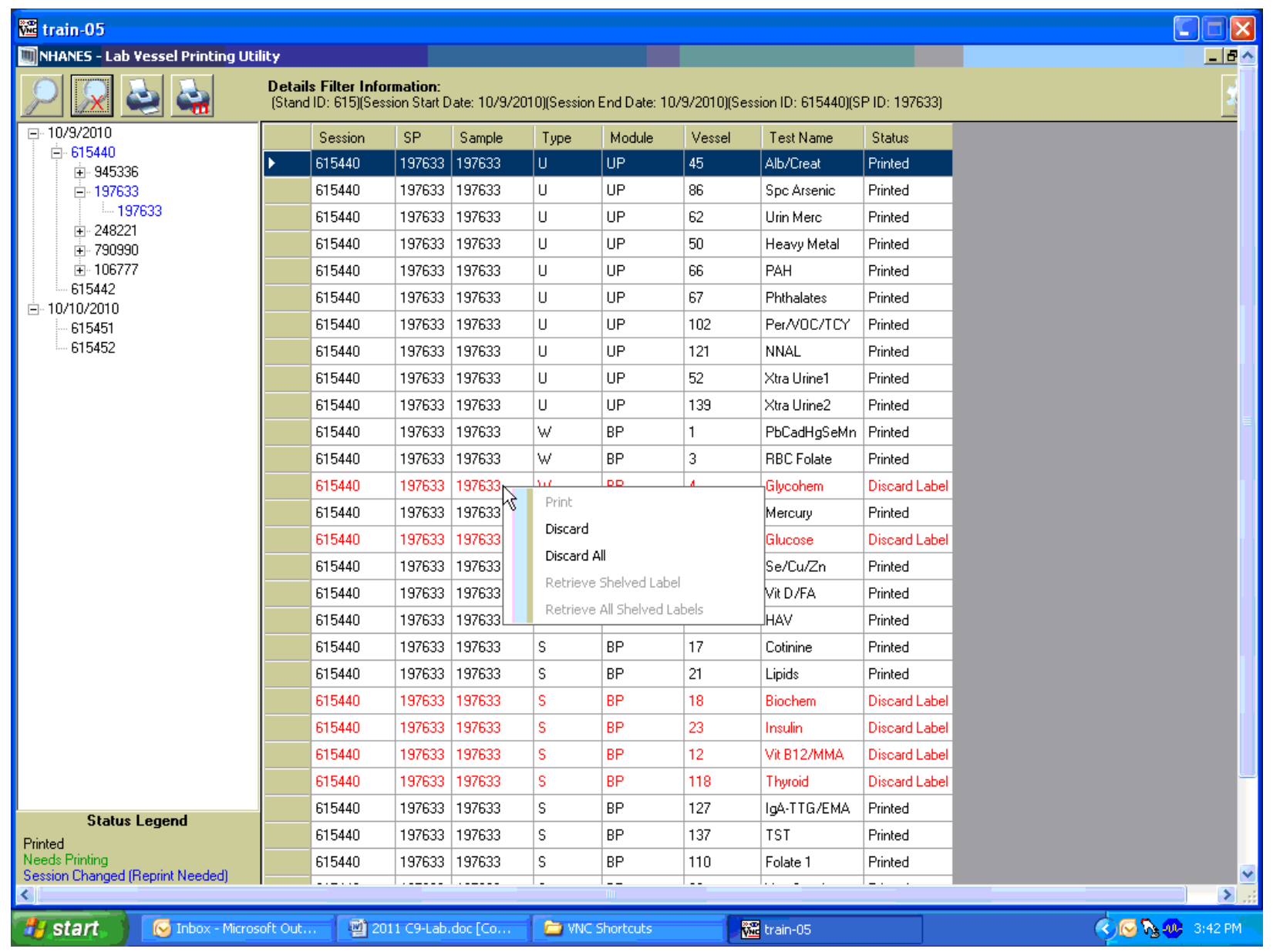

After completing the Label Action, return to heads-up display and allow the display to update before proceeding. After update, the SP will change from red to black and blood processing can be accessed. 


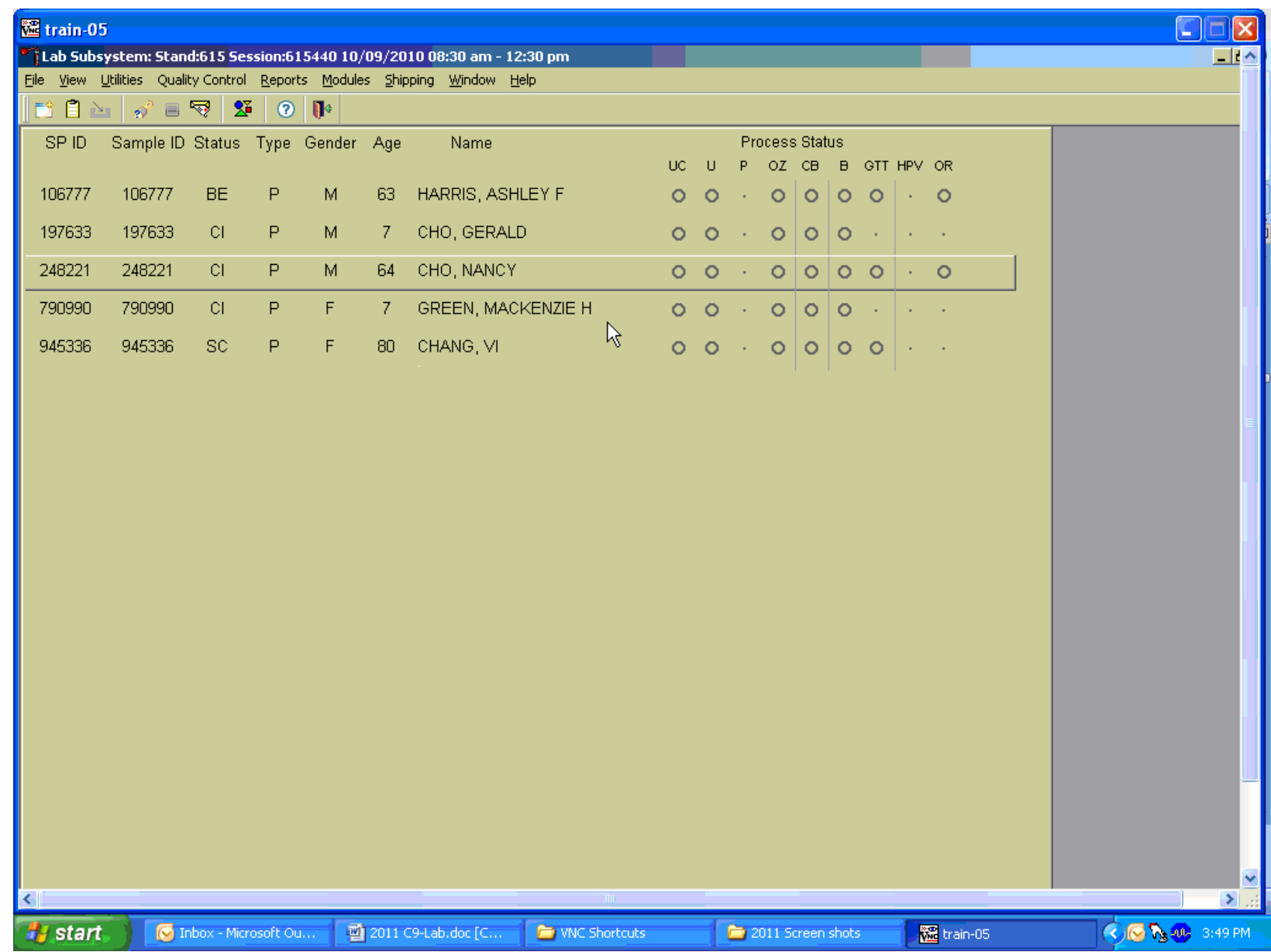




\subsection{Create New Container Labels}

Store processed vessels and biological samples in boxes and bags (containers). Print and assign container labels using the \{Create New Container Labels\} module. Once a label has been created, the container is designated as "open" in the database; it is available for slot assignments as vessels are marked as filled.

At the beginning of each stand, print and assign one new container ID for each vessel using either the \{Print\} module or the \{Shipping\} module. Print additional labels as needed. Each stand is allocated a maximum of 999 labels. The actual container label will include the vessel name, vessel ID, the size of the container ( $9 \times 9$ 2-inch box, etc.), and the container number.

Chlam/GC (46)

$5 \times 53$ inch box

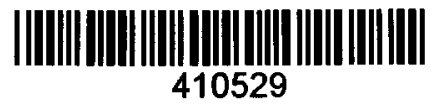

Prior Pest (48)

$5 \times 53$ inch box

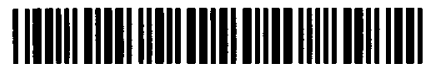

410530 
Use the shipping module within the laboratory application to print and assign new container ID numbers or labels.

Print and assign new container ID numbers using the \{Shipping\} module.

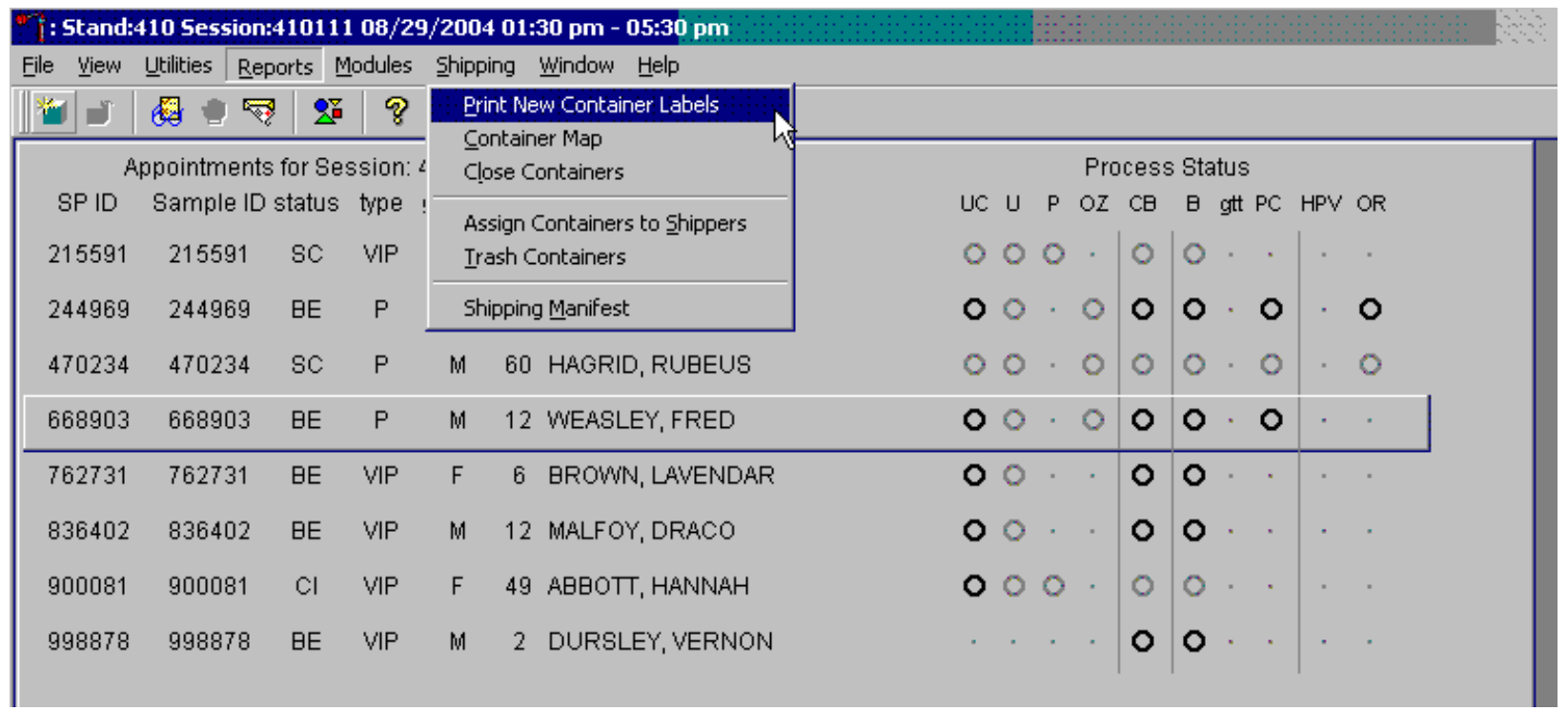

To access the \{Print New Container Labels\} module, use the mouse to direct the mouse arrow to \{Shipping\} in the menu bar and left click, drag the mouse arrow to \{Print New Container Labels $\}$, and left click or type [Alt] [S/s], [P/p]. 
There are three instances when it is appropriate to use this module. At the start of each stand, create one new container label for each vessel ID or "group." Use the same module to create a new label for a vessel after the last slot in an open container is filled. Use this module to print additional labels at any time.

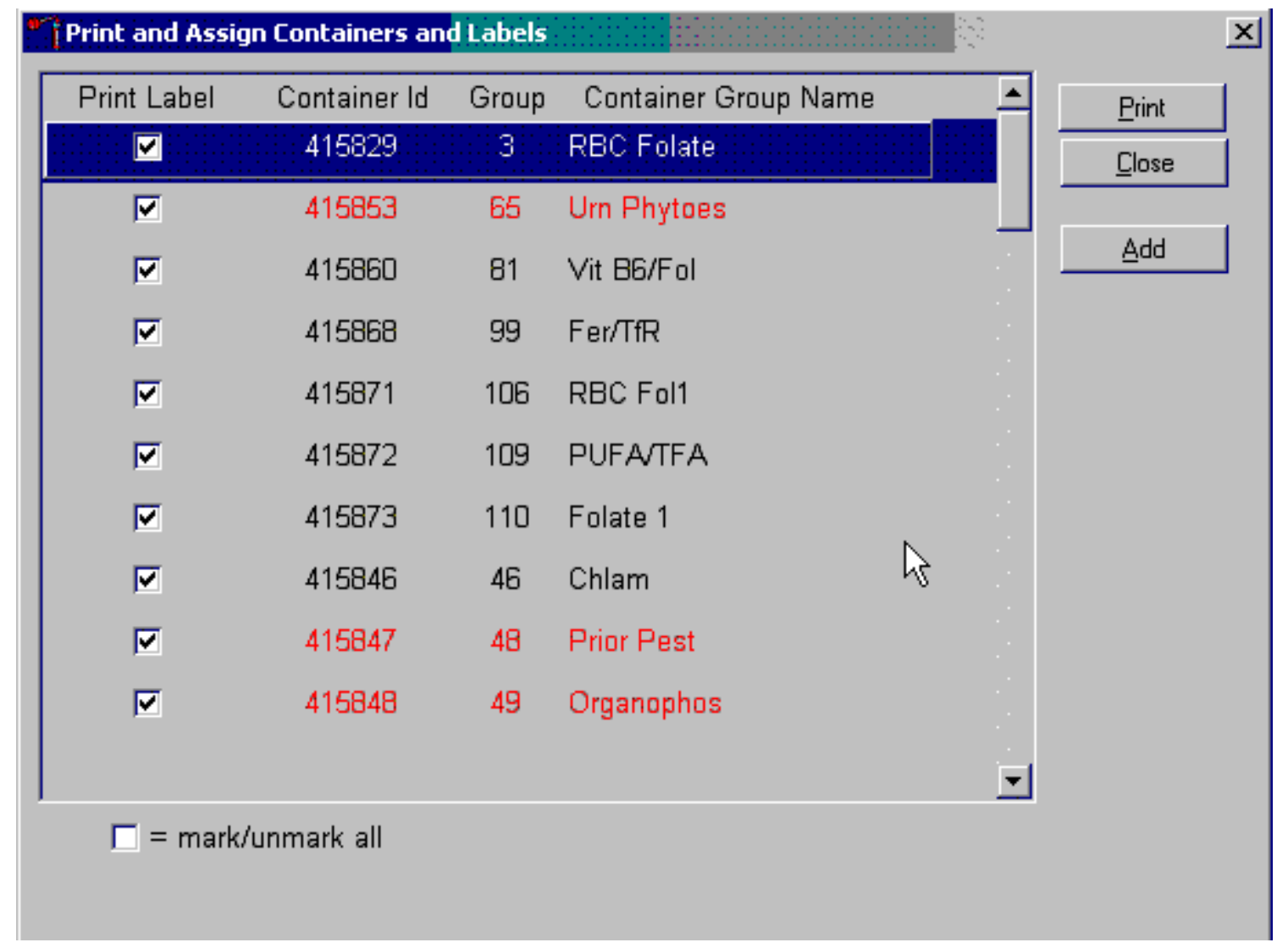

The Print and Assign Containers and Labels window displays once the \{Print New Container module is accessed. The window includes checkboxes to mark or identify specific groups, new Container Ids that are assigned to the Groups, the number of the Group, and the Container Group Name or vessel name. The Group and corresponding Container Group Name listed in this screen represent the Group where the container has been "closed" and a new label has not been created. This screen also includes a "mark/unmark all" checkbox and three buttons: Print, Close, and Add. Use the "Print" button to print the container labels, use the "Close" button to exit the module without printing any labels or assigning Container IDs to the Groups, and use the "Add" button to open another window that allows the user to add additional or multiple labels for any vessel. 
Individually mark the Print Label checkbox to identify vessels that require labels.

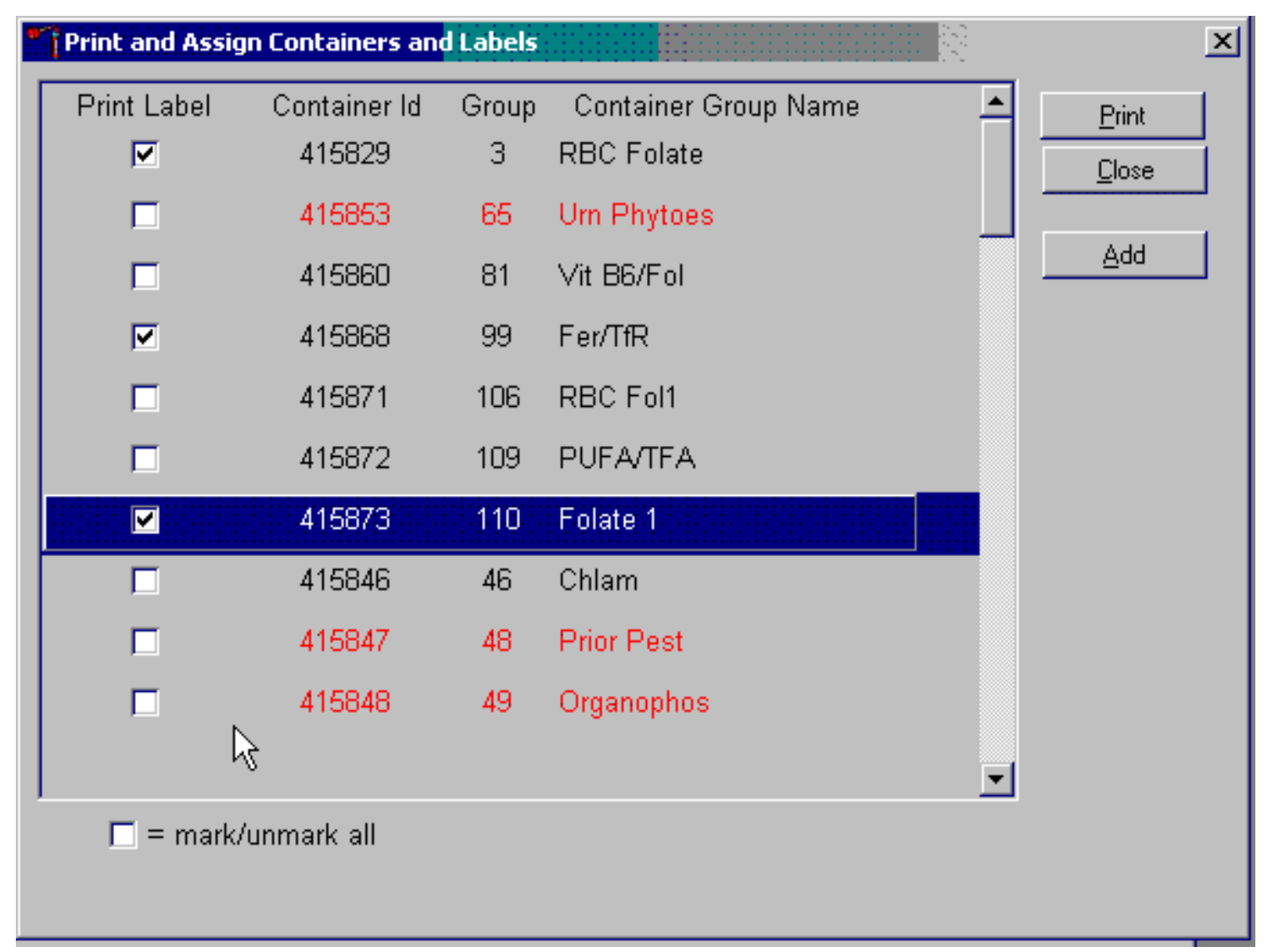

To begin the process of printing and assigning new Container IDs, mark the individual check boxes that correspond to the Group (vessel) of interest. To add a checkmark to the Print Label checkbox, use the mouse to direct the mouse arrow to the Print Label checkbox and left click. 
Collectively mark all the Print Label checkboxes for all the Groups listed in the window.

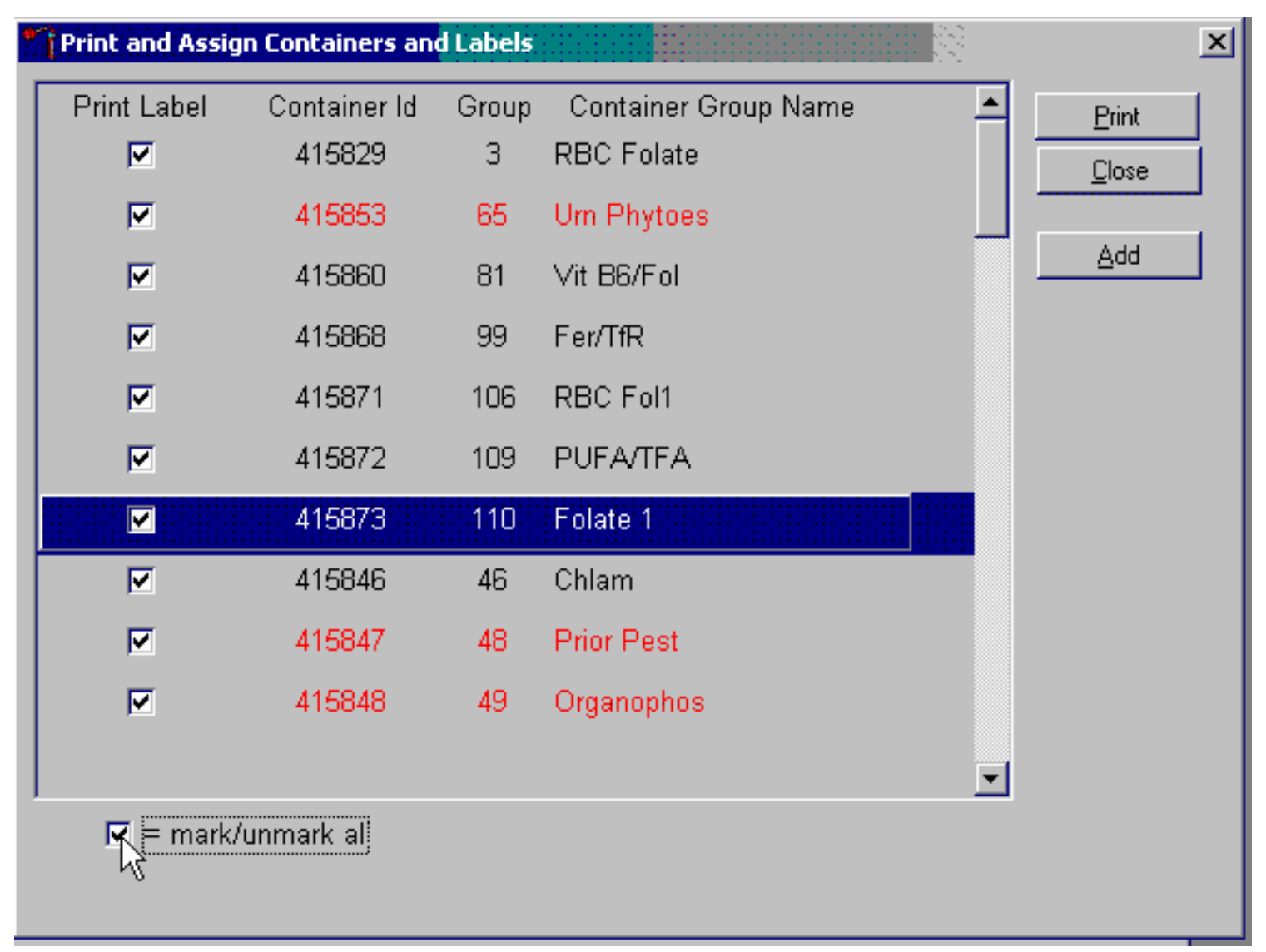

To collectively mark all the checkboxes, use the mouse to direct the mouse arrow to the mark/unmark all checkbox and left click. To collectively unmark all the checkboxes, use the mouse to direct the mouse arrow to the mark/unmark all checkboxes and left click. 
Access the Generate Additional Containers window to print additional labels for any Group at any time.

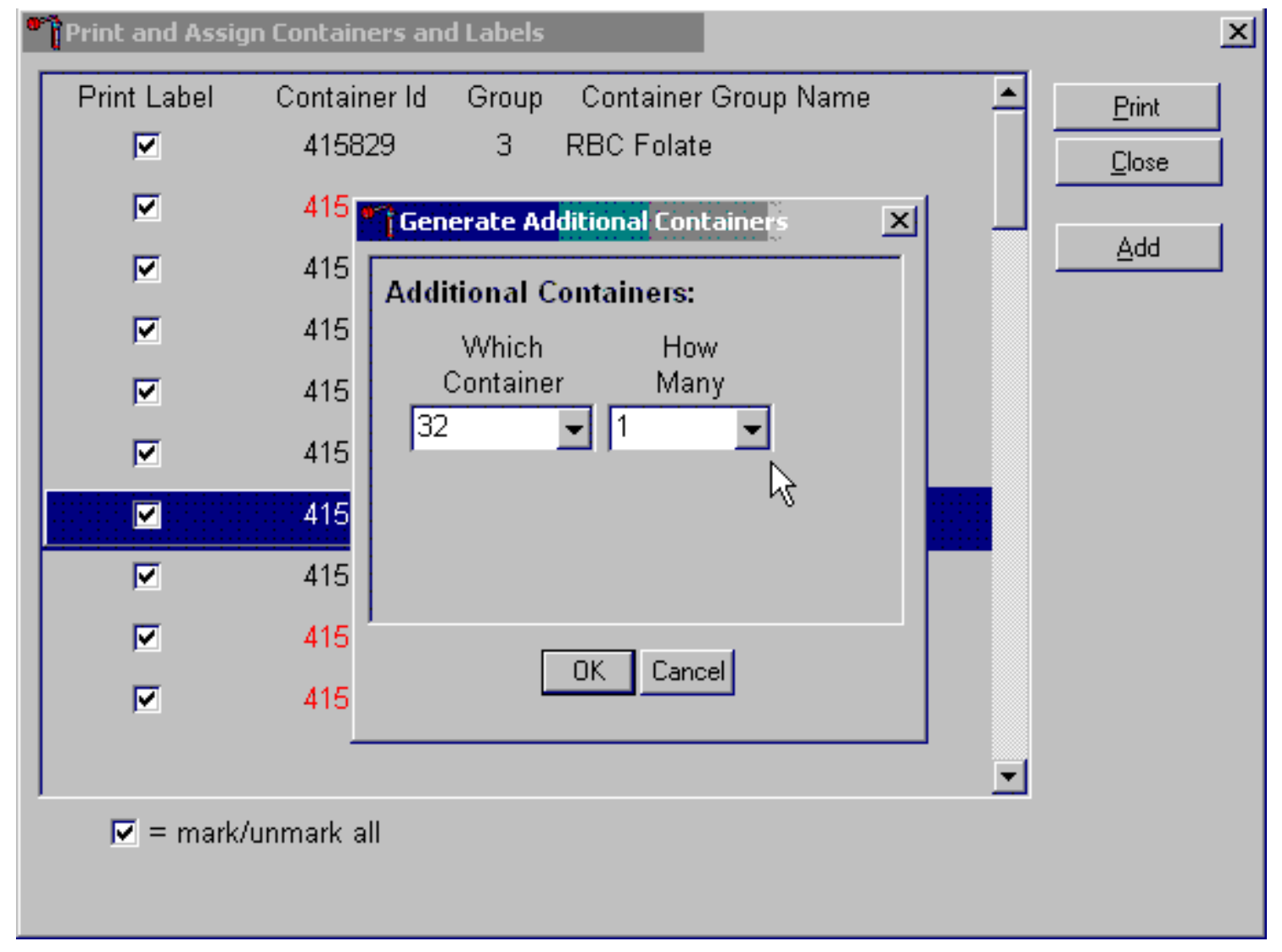

To assess the Generate Additional Containers window, use the mouse to direct the mouse arrow to the "Add" button and left click, or type [Alt] [A]. 
The Generate Additional Containers window displays.

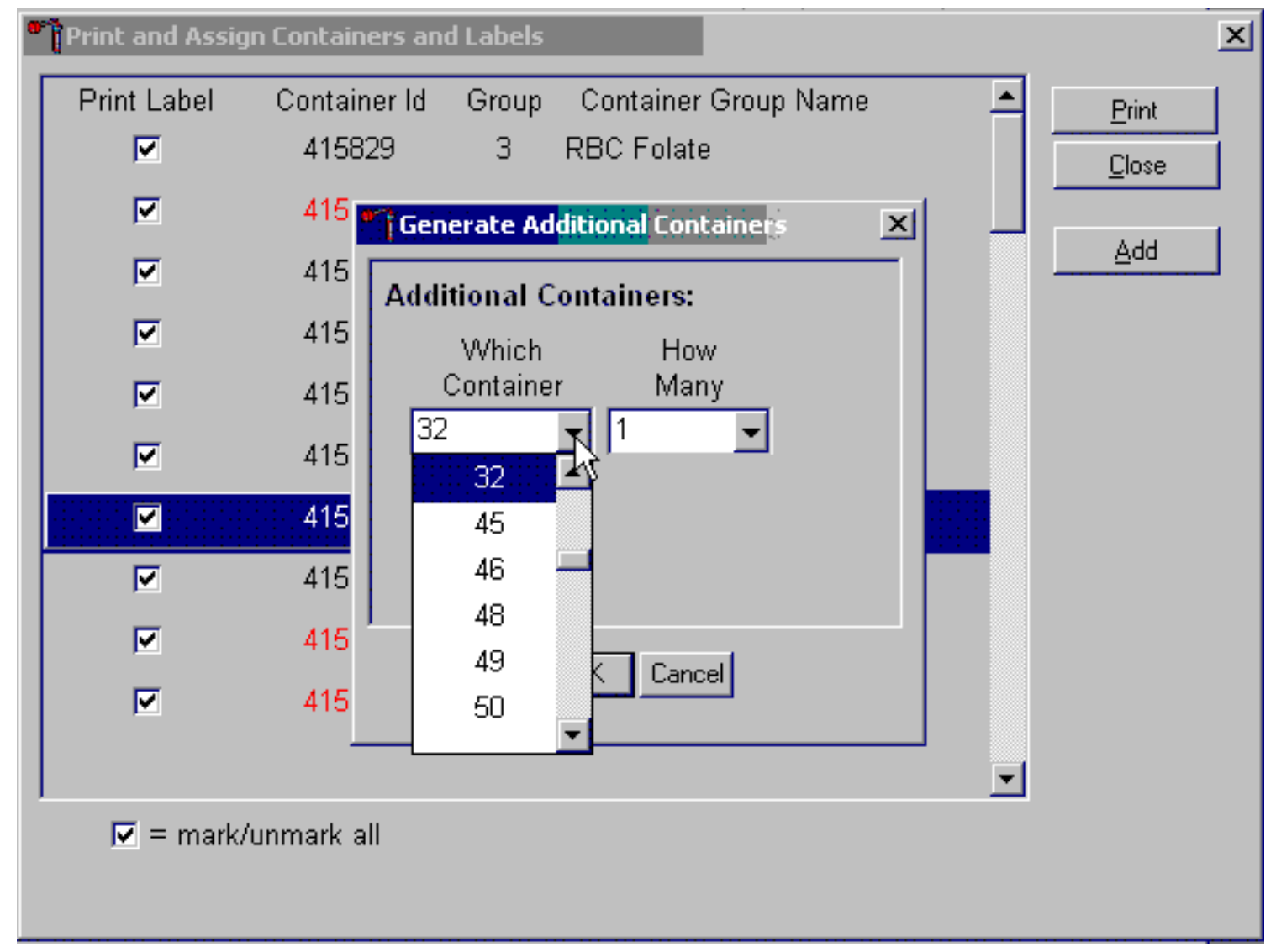

The Generate Additional Containers window includes two text boxes with drop-down lists: Which Container and How Many. The Which Container text box defaults to vessel 32. To select a vessel other than 32 , use the mouse to direct the mouse arrow to the drop-down arrow and left click, drag the arrow to the desired choice and left click. Use the scroll bar to view all the choices. 
Select the number (1-5) of additional container labels.

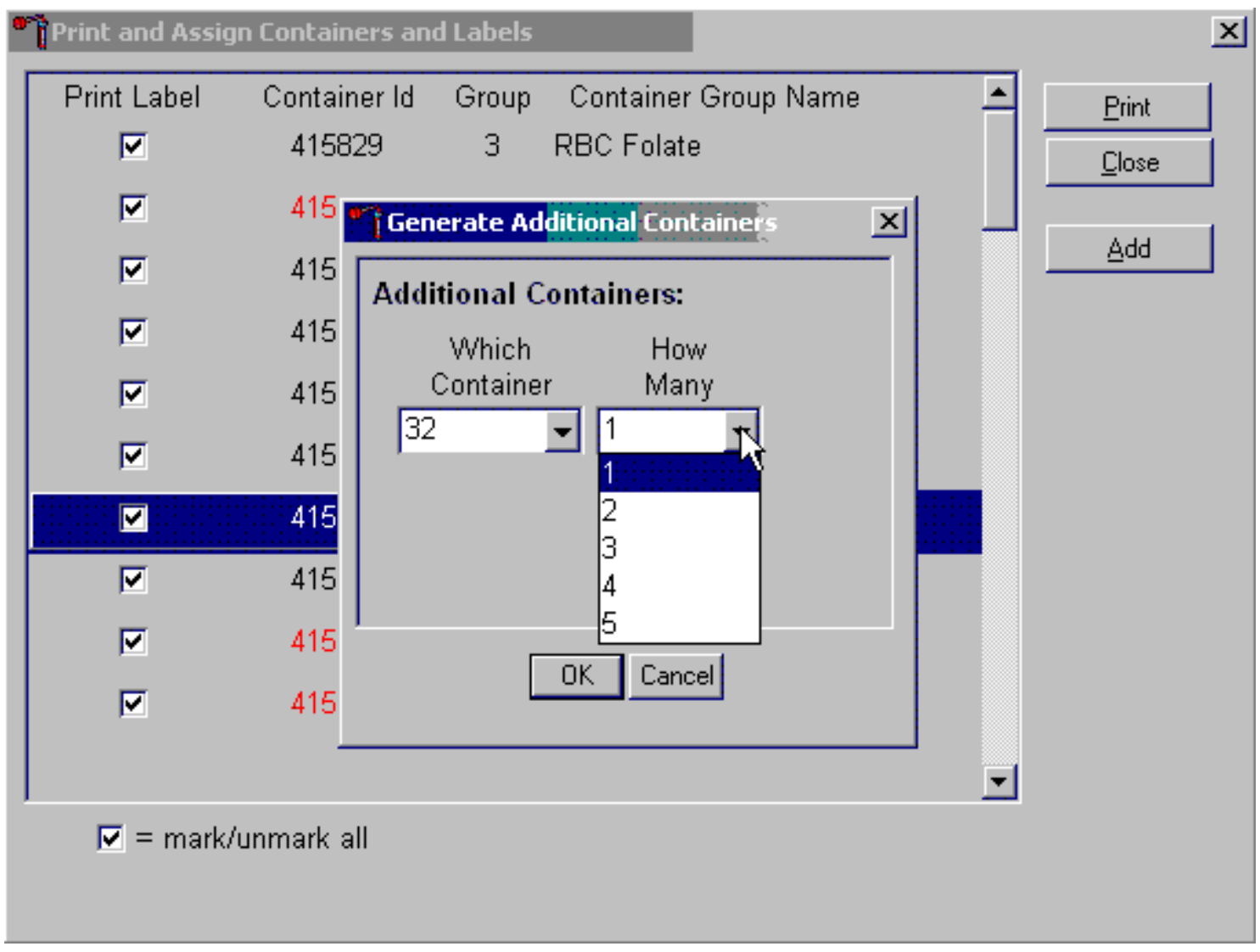

The How Many text box defaults to vessel 1. To select 2, 3, 4, or 5, use the mouse to direct the mouse arrow to the drop-down arrow and left click, then drag the arrow to the desired choice and left click. Use the scroll bar to view all the choices. 
Add these vessels to the Print and Assign Containers and Labels window or cancel the action.

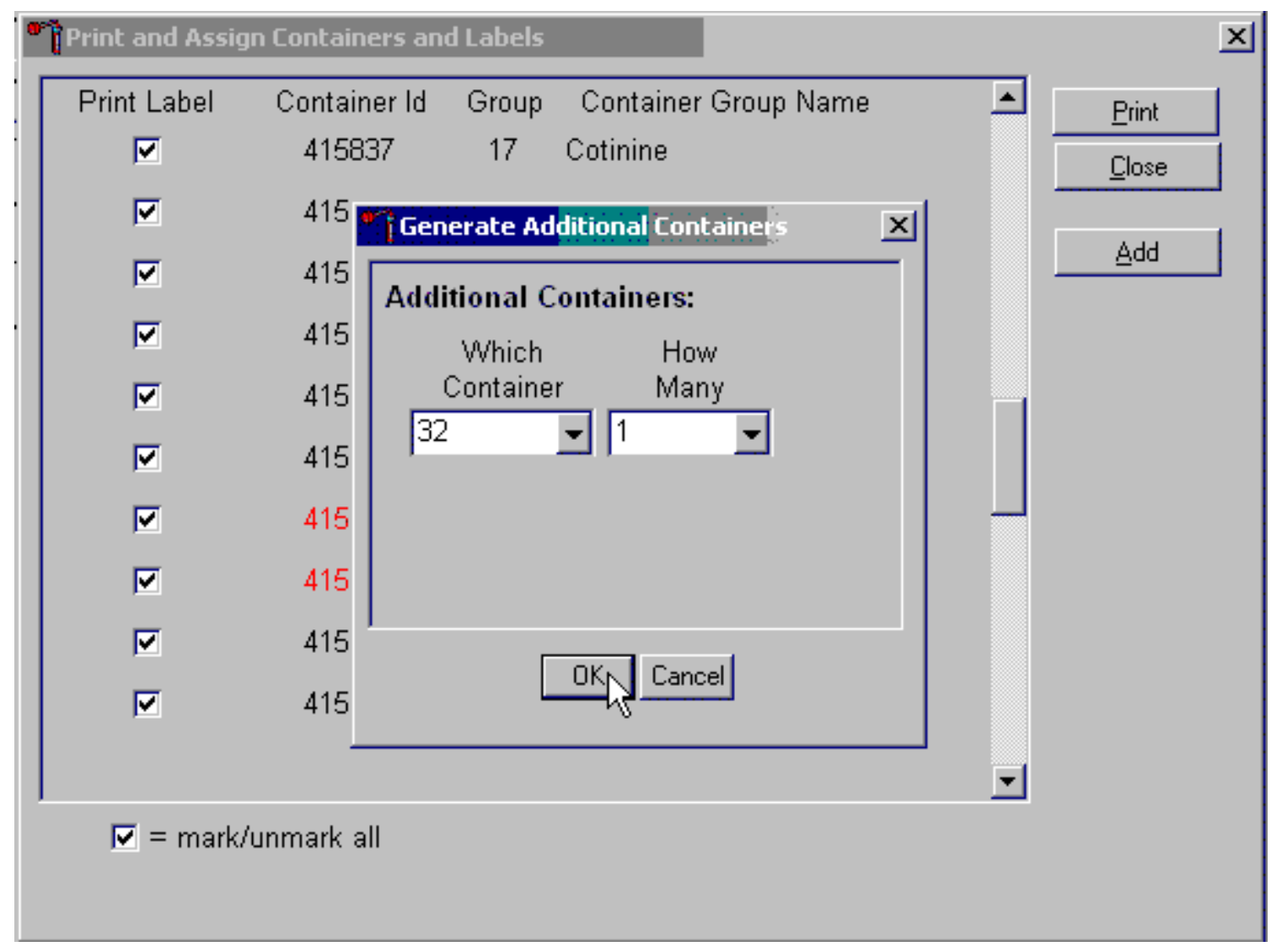

To accept the choices displayed in the Generate Additional Containers window, use the mouse to direct the mouse arrow to the "OK" button and left click or type [Enter]. To close the window without creating a new container, use the mouse to direct the mouse arrow to the "Cancel" button and left click. 
If the "OK" button is selected, then the containers are added to the Print and Assign Containers and Labels window.

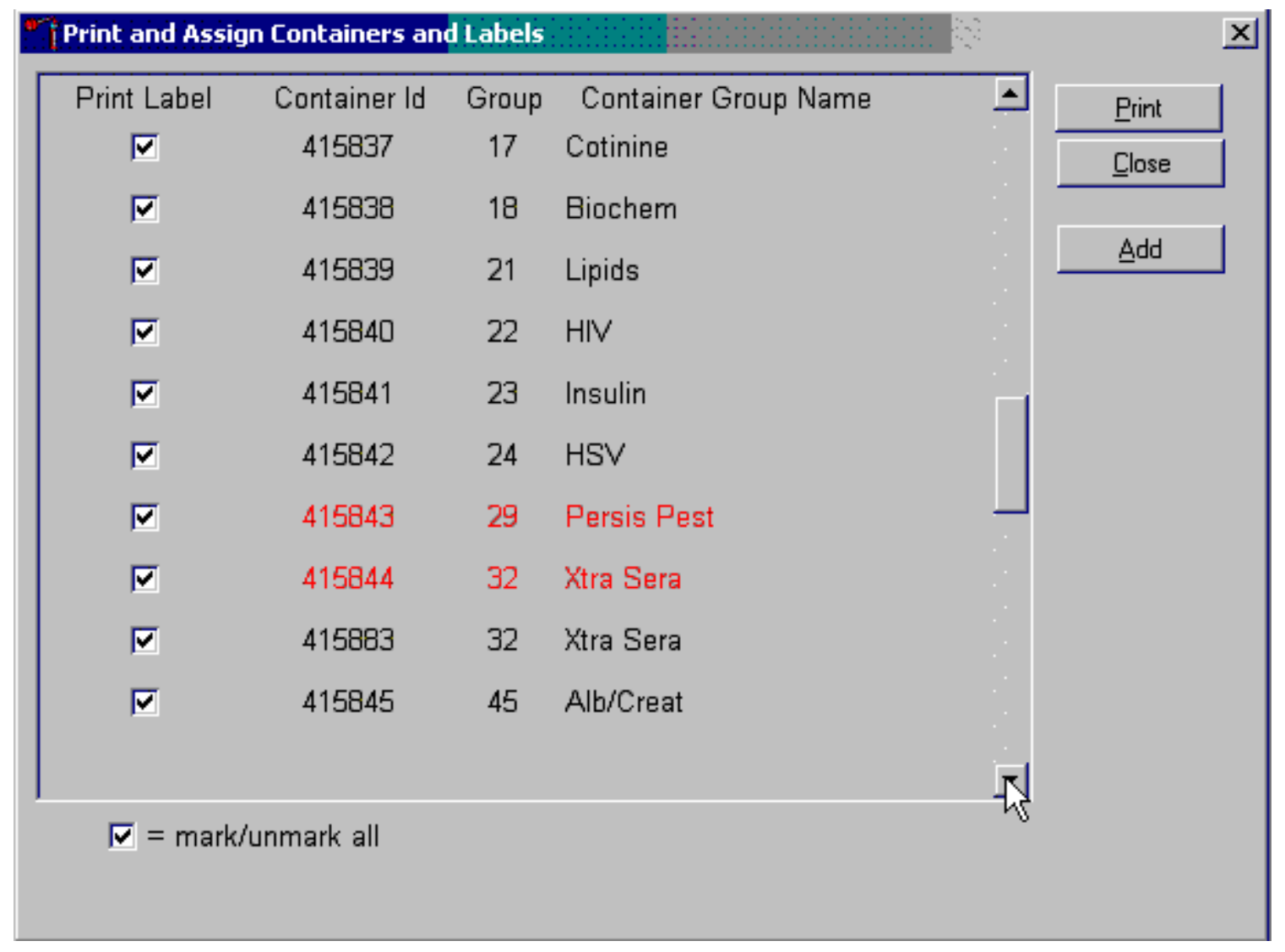

Review the information on the screen. 
Complete the assignment process and print the labels.

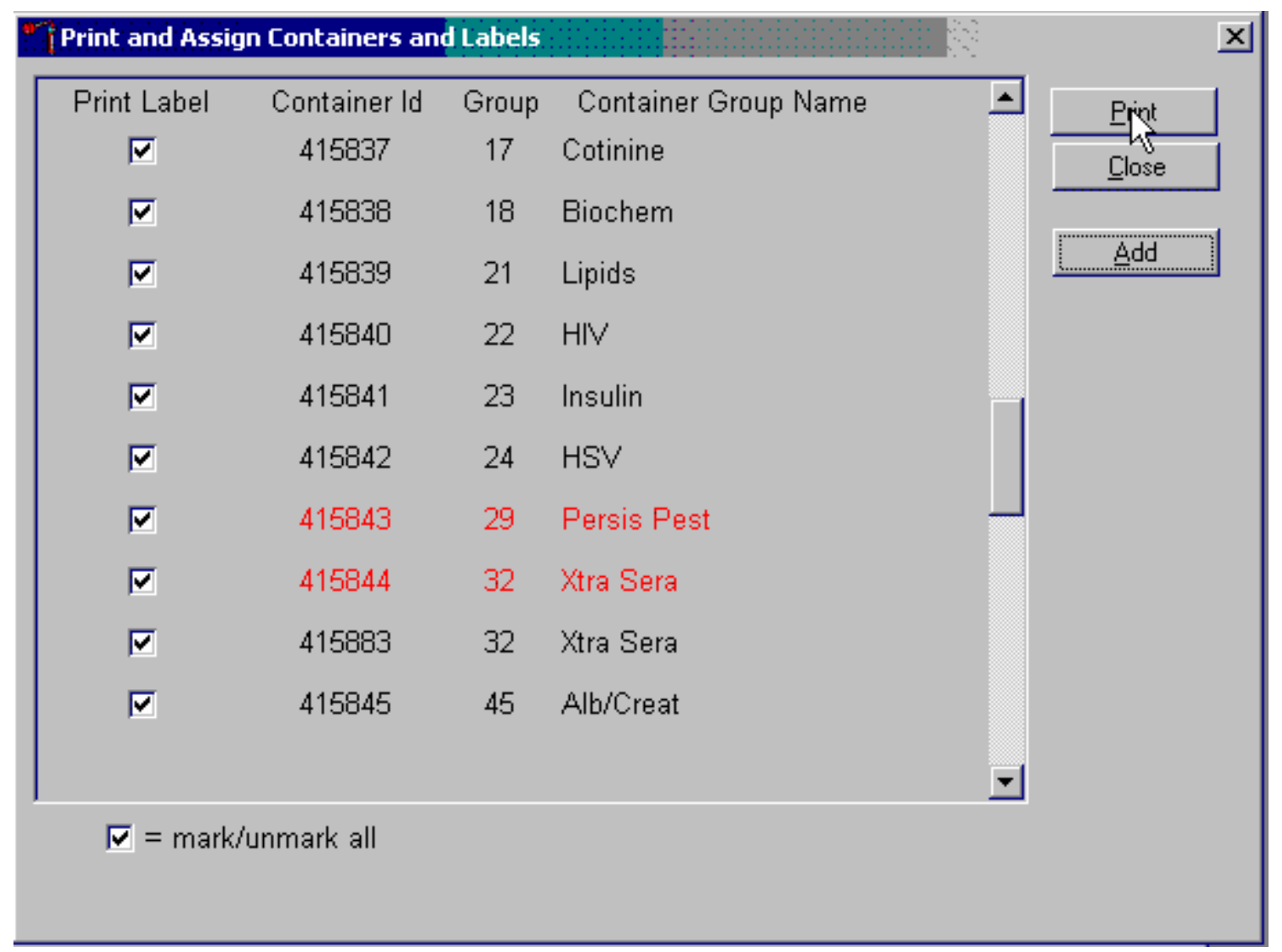

After reviewing the screen, print the labels. To print the labels, use the mouse to direct the mouse arrow to the "Print" button and left click, or type $[\mathrm{Alt}][\mathrm{P} / \mathrm{p}]$. To exit the window without printing any labels, use the mouse to direct the mouse arrow to the "Close" button and left click, or type [Alt] $[\mathrm{C} / \mathrm{c}]$. Affix the container ID label(s) to the container(s) of the correct type. 
If the "Close" button is selected before the labels have been printed, then a Print and Assign Containers and Labels window displays.

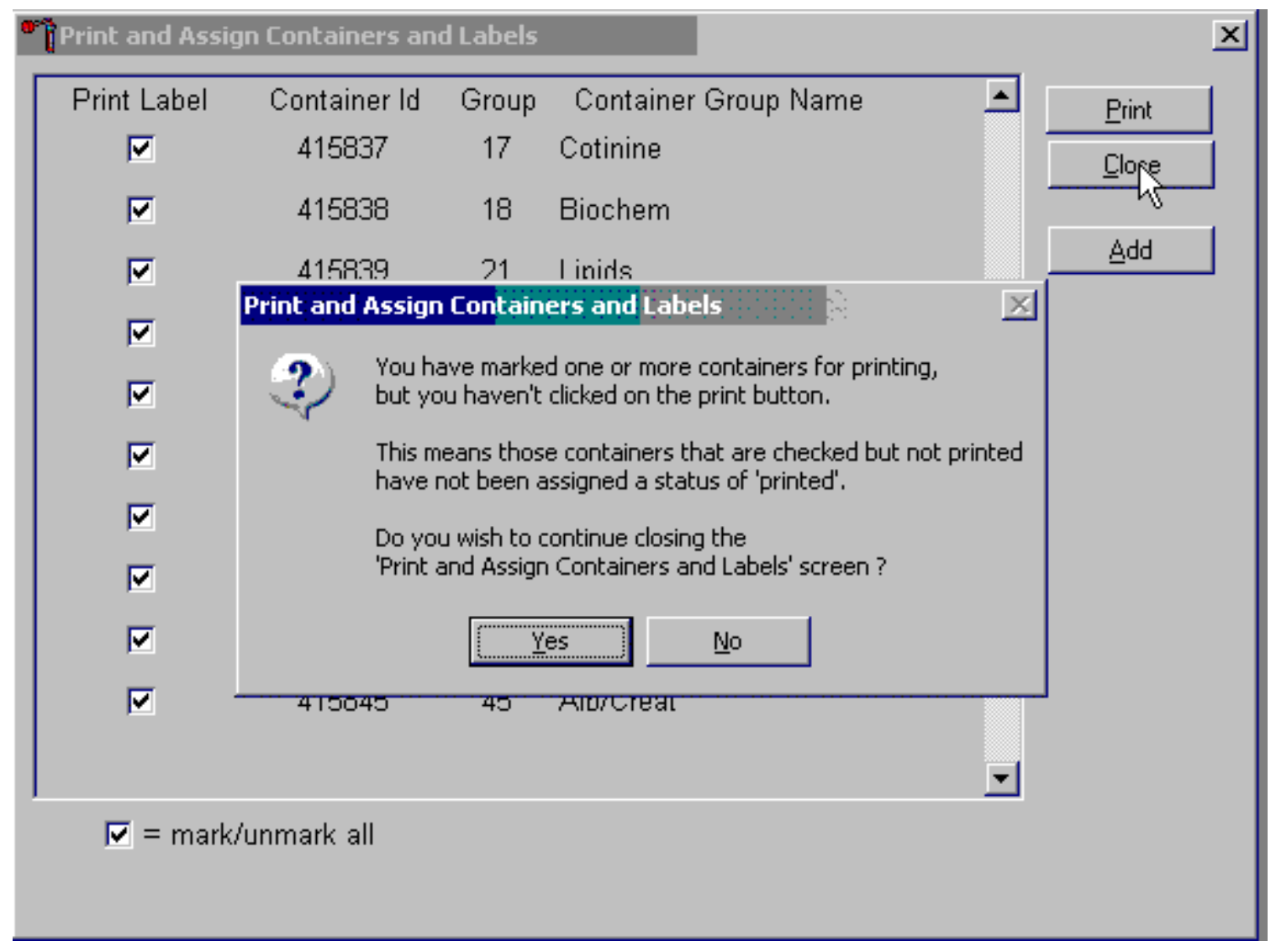

Review the text. To close the Print and Assign Containers and Labels screen, select the "Yes" button. To return to the Print and Assign Containers and Labels screen, select the "No" button. 
When a container is full, the container will automatically "close" and the Print and Assign Containers and Labels window opens with that vessel displayed and the checkmark prefilled. Alternatively, open a new container to replace the closed container by using the Print and Assign Containers and Labels window.

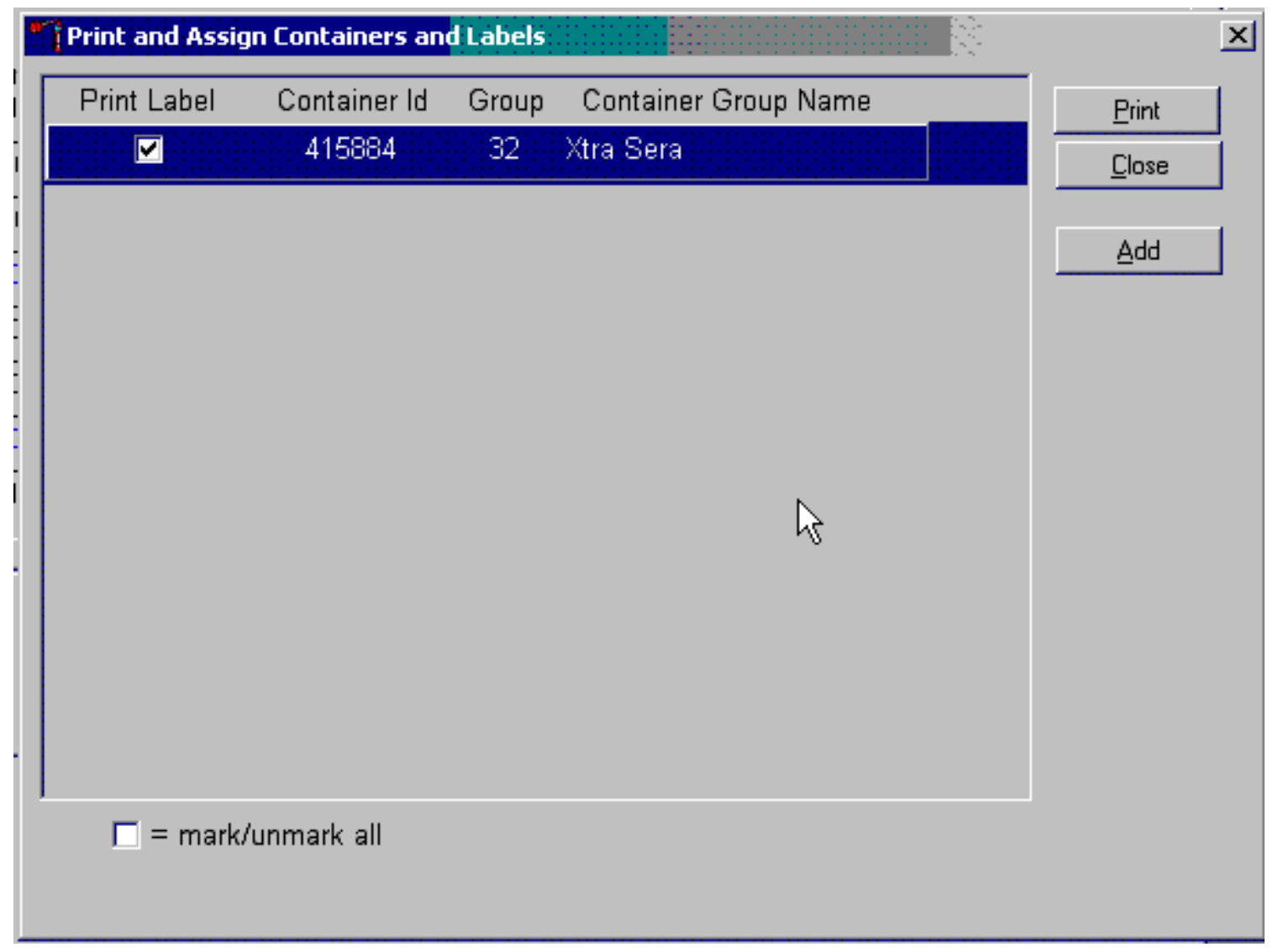

When a container is full, an opportunity to open a new container is automatically displayed on the "Print and Assign Container and Labels" window. Repeat the process of marking the checkbox and selecting the "Print" button. 
Each stand is allocated a maximum of 999 container IDs.

If unused container labels become lost or damaged, use the \{Trash Containers\} module to delete the container IDs. Access the \{Trash Containers\} module.

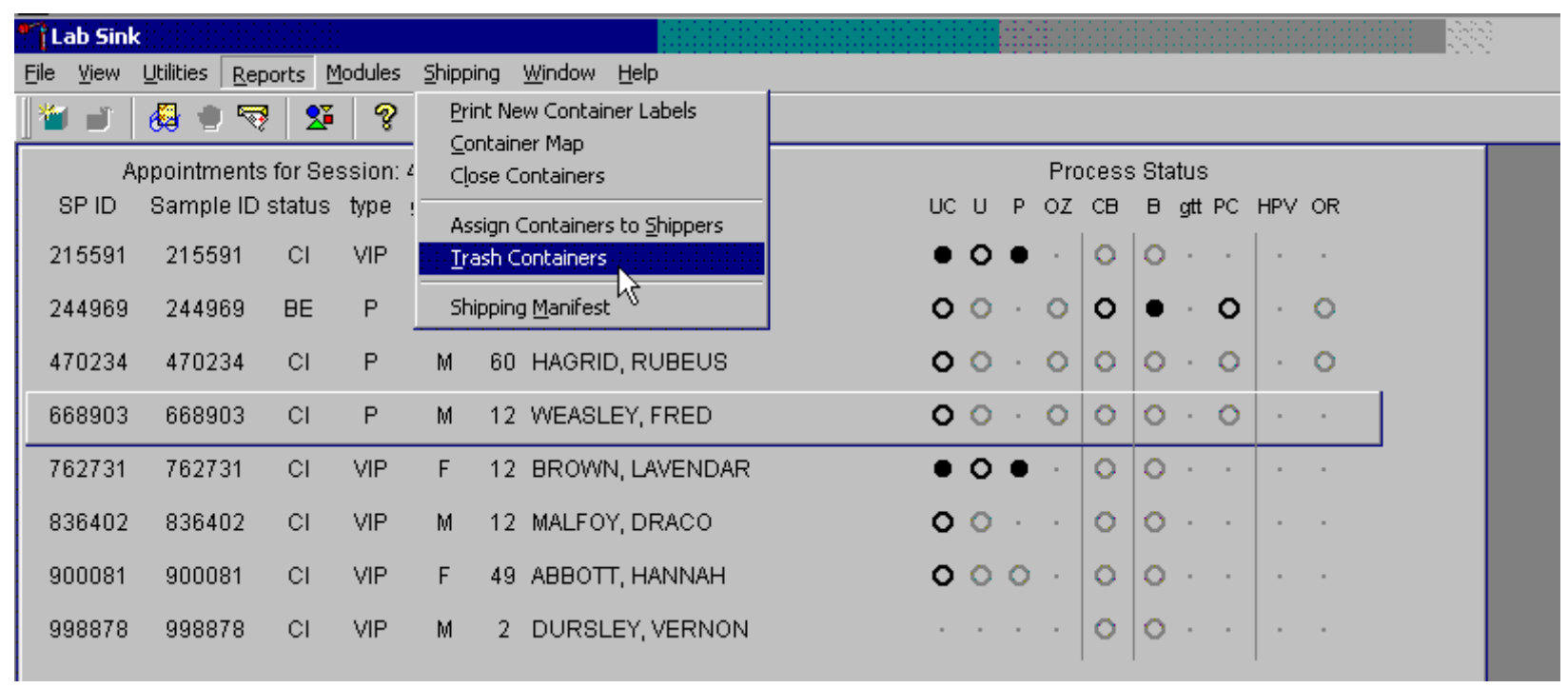

To access the $\{$ Trash Containers $\}$ module, use the mouse to direct the mouse arrow to \{Shipping\}, left click, drag the arrow to \{Trash Containers\}, and left click, or type [Alt] [S/s], [T/t]. 
The Trash Unused Containers window displays.

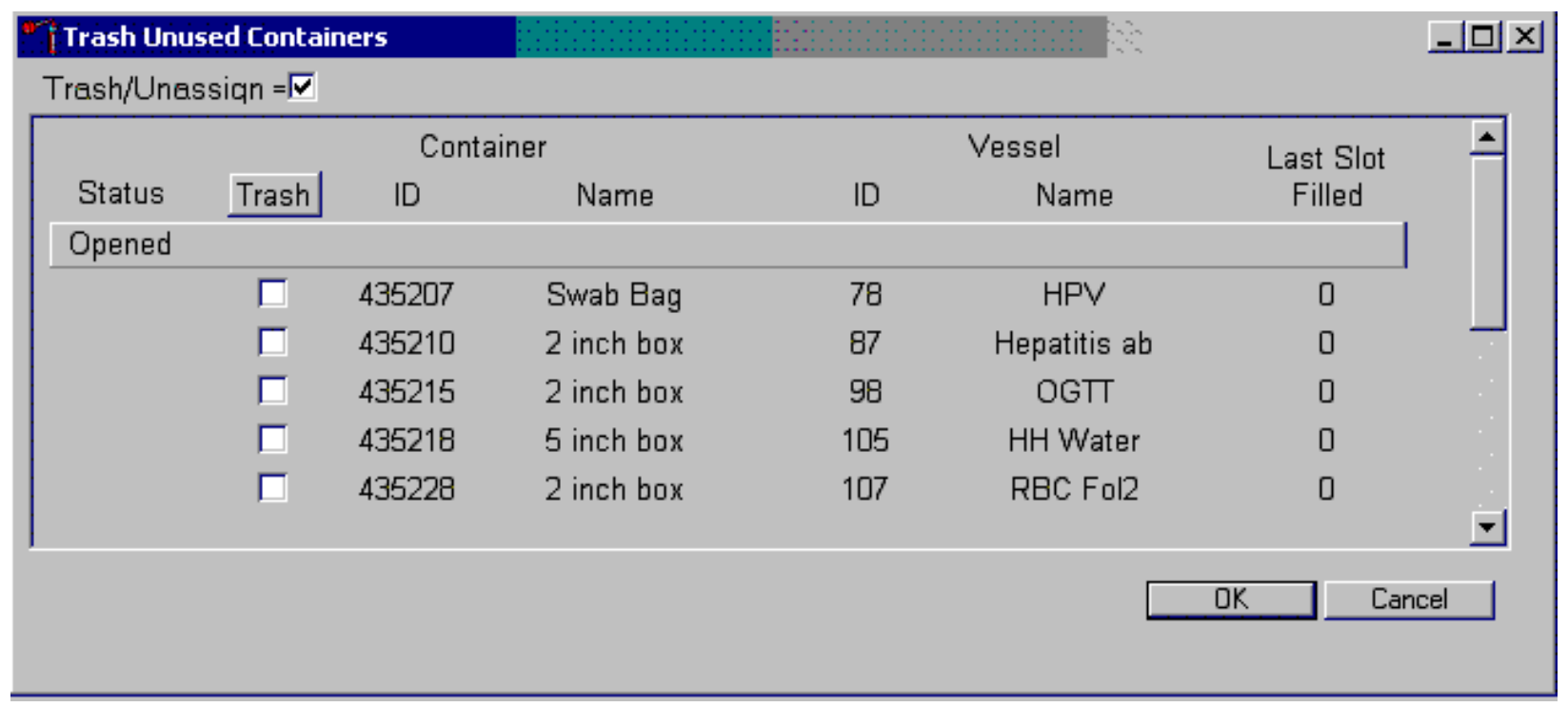

The status of each container ID is "Opened." To individually mark the container IDs for deletion, use the mouse to direct the mouse arrow to the checkboxes and individually left click on specific boxes or, to mark all outstanding container IDs for deletion, use the mouse to direct the mouse arrow to the "Trash" button and left click. To collectively mark ALL containers, use the mouse to direct the mouse arrow to the Trash header and left click. 
As the container IDs are marked for deletion, the status changes from "Opened" to "Trashed."

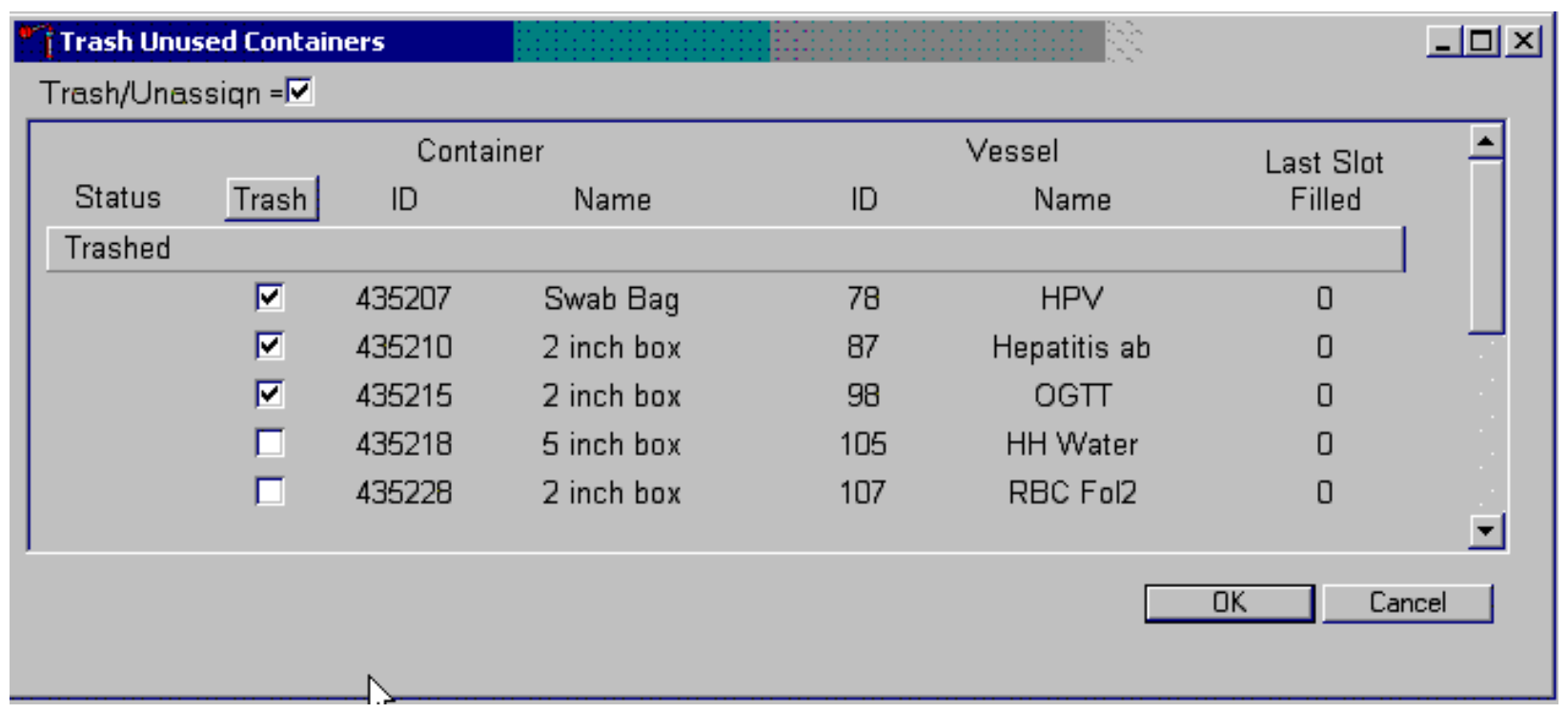

To record these actions and execute the Trash Unused Containers function, use the mouse to direct the mouse arrow to the "OK" button and left click, or select [Enter]. To exit the \{Trash Unused Containers $\}$ module without recording or executing the Trash Containers function, use the mouse to direct the mouse arrow to the "Cancel" button and left click. 


\subsection{1}

\section{Reprint Container Labels for Existing Containers}

Reprint labels for existing, not shipped containers.

\section{Reprinting Container Labels from the Container Map Screen}

In the Container map screen, an option to reprint the container label has been added. Right click anywhere in the line of the container you wish to reprint the label, so it's highlighted, Click on the task "Reprint Label" and remove the label from the Dymo Printer.

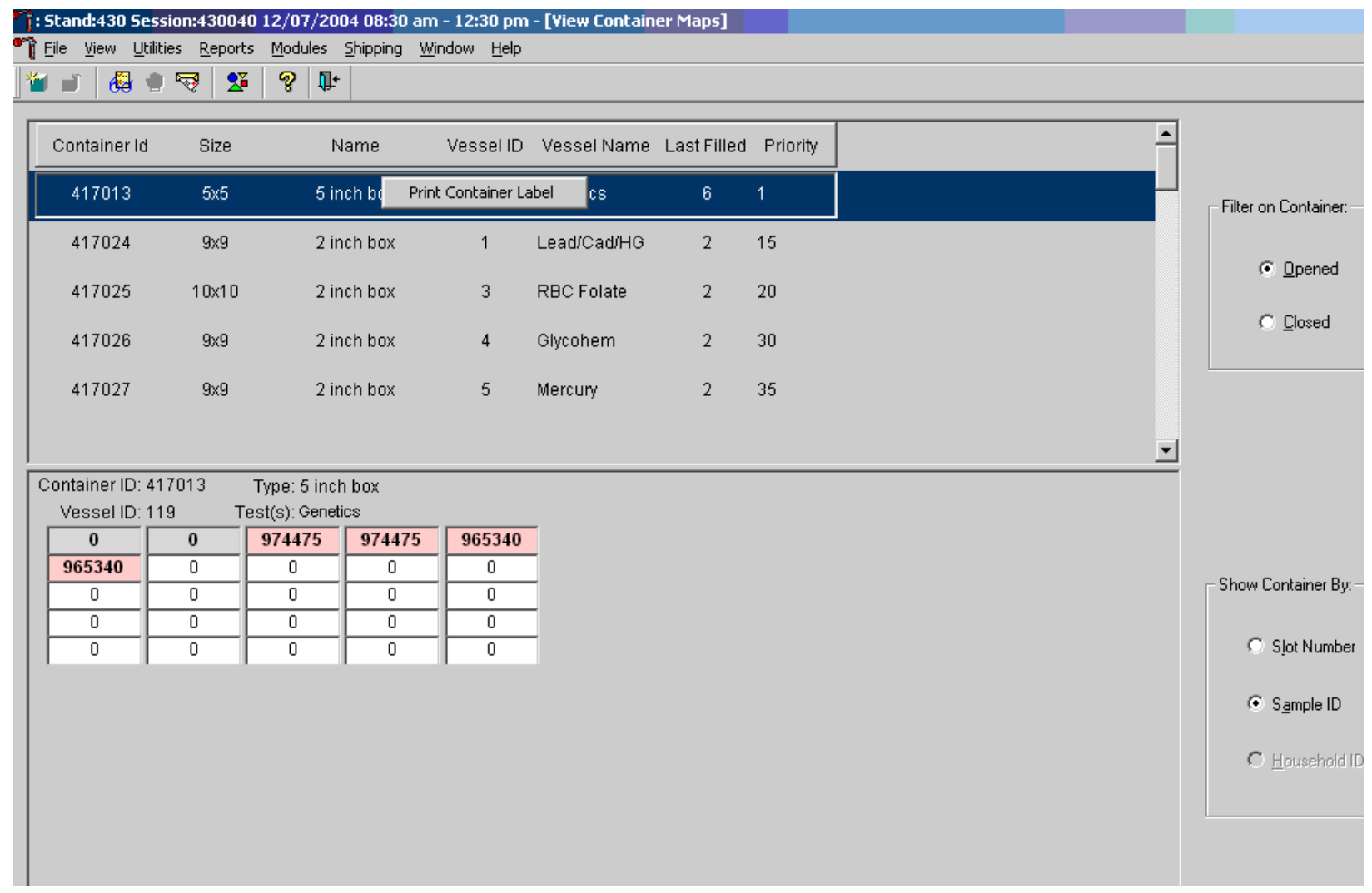




\subsection{Shipping Overview}

The integrity of laboratory specimens is critical to NHANES. Storage temperatures have been established for each analyte in consultation with the National Center for Health Statistics (NCHS) and contract laboratories. It is critical to maintain these established conditions throughout specimen storage and shipping and until their ultimate receipt by the contract laboratories. Maintain these conditions by using documented procedures, which include using sufficient dry ice, refrigerant packs, and sending specimens by FedEx priority overnight.

FedEx Priority Overnight guarantees next-business-day delivery by 10:30 AM to most U.S addresses and by noon, 4:30 PM, or 5 PM in remote areas. Delivery by FedEx can be delayed due to a weekend or holiday. This extraordinary circumstance usually occurs at the end of a stand or just before vacation. In any case, ship all specimens before vacations. The following actions are intended to mitigate unforeseen delay:

- Consider using medium shippers instead of small shippers for all laboratories east of the Mississippi when shipping from areas west of the Mississippi, and for all laboratories west of the Mississippi when shipping from areas east of the Mississippi. Substitute a larger shipper when shipping specimens in extreme summer heat or whenever professional judgment dictates added precautions.

- Use a medium shipper for all international shipments.

- Use dry ice in blocks instead of pellets since blocks sublimate at a slower rate.

- Increase the number of refrigerant packs from four to at least six per shipper.

The shipping technologist is primarily responsible for packing and shipping the specimens to various contract laboratories on a designated day. As necessary, other medical technologists assist in performing each of the steps listed below to complete the shipping procedures.

Prior to the next stand, the laboratory staff creates address labels with the current MEC address to use on the Class 9 labels. Place the current MEC address label in the lower left of the Class 9 label and place the appropriate Lab label in the lower right of the Class 9 label. Trim both labels to fit in the space, so they don't cover up any information. Prepare Class 9 labels with the MEC address and Lab address for each shipping week. 
Shipping procedures:

- On the day before or the morning of a designated shipping day, create and print shipper labels (airbills) using FedEx Ship Manager.

- Prepare the Class 9 labels for shipments that use dry ice.

- On the morning of a shipping day, close all containers, assign containers to a shipper, print the paper shipping manifest for each shipper, and open all new containers.

- Assemble the paper shipping manifest, prepared FedEx label, FedEx preprinted return airbills, and assorted "warning" labels for each shipper. Clip all materials together until ready to pack the shippers.

- Collect the shippers from the compartments under the MEC.

- Pack the shippers with the containers, absorbent material, newsprint (padding), plastic bags, and a sufficient supply of dry ice or refrigerant packs as appropriate.

- Place the appropriate paper manifest and the preprinted FedEx return airbill in a $9 \times 12$ plastic bag and place the plastic bag on top of the Styrofoam shipper lid.

- Label each shipper with the appropriate FedEx label and warning labels.

- When the contract laboratory personnel receive the shippers, they unpack the specimens and return the shippers and refrigerant packs to the warehouse.

\subsection{Assign Containers to Shippers}

Assign closed containers to or associate closed containers with a specific airbill. To associate a specific closed container to the airbill for that laboratory, use the \{Assign Container to Shippers\} module. 
Access the $\{$ Assign Containers to Shippers $\}$ module.

\begin{tabular}{|c|c|c|c|c|c|c|c|c|c|c|c|c|c|c|c|}
\hline \multicolumn{16}{|c|}{ Lab Sink } \\
\hline \multicolumn{4}{|c|}{ File Yiew ㄴtilities Reports Modules } & \multicolumn{12}{|c|}{ Shipping Wwindow Help } \\
\hline 㭗 & 国 & 造 & 8 & \multirow{2}{*}{\multicolumn{2}{|c|}{$\begin{array}{l}\text { Print New Container Labels } \\
\text { Container Map } \\
\text { Close Containers }\end{array}$}} & & & & & & & & & & \\
\hline \multirow{2}{*}{\multicolumn{4}{|c|}{$\begin{array}{l}\text { Appointments for Session: } 2 \\
\text { SP ID Sample ID status type }\end{array}$}} & & & \multirow{5}{*}{\multicolumn{3}{|c|}{$\begin{array}{cc}U C & U \\
0 & 0 \\
0 & 0 \\
0 & 0\end{array}$}} & \multicolumn{4}{|c|}{ Process Status } & \multirow{2}{*}{\multicolumn{2}{|c|}{$\mathrm{HPV}$ OF }} & \\
\hline & & & & & kign Containers to Shippers & & & & $O Z$ & $\mathrm{CB}$ & $\mathrm{B}$ & gtt $P C$ & & & \\
\hline 215591 & 215591 & $\mathrm{Cl}$ & VIP & & S5 Containers & & & & $\cdot$ & 0 & 0 & · $\cdot$ & & $\cdot \cdot$ & \\
\hline 244969 & 244969 & $\mathrm{BE}$ & $P$ & & pping Manifest & & & & & 0 & - & $\cdot \mathrm{C}$ & & $\cdot \mathrm{c}$ & \\
\hline 470234 & 470234 & $\mathrm{Cl}$ & $\mathrm{P}$ & & 60 HAGRID, RUBEUS & & & & 0 & 0 & 0 & $\cdot c$ & 0 & $\cdot 0$ & \\
\hline 668903 & 668903 & $\mathrm{Cl}$ & $P$ & m & 12 WEASLEY, FRED & 0 & 0 & $\cdot$ & 0 & 0 & 0 & $\cdot c$ & 0 & & \\
\hline 762731 & 762731 & $\mathrm{Cl}$ & VIP & $\mathrm{F}$ & 12 BROWN, LAVENDAR & & 0 & $\bullet$ & . & 0 & 0 & $\cdot$. & & & \\
\hline 836402 & 836402 & $\mathrm{Cl}$ & VIP & $M$ & 12 MALFOY, DRACO & 0 & 0 & . & . & 0 & 0 & . . & . & & \\
\hline 900081 & 900081 & $\mathrm{Cl}$ & VIP & $\mathrm{F}$ & 49 ABEOTT, HANNAH & 0 & 0 & 0 & . & 0 & 0 & $\cdot \cdot$ & - & & \\
\hline 998878 & 998878 & $\mathrm{Cl}$ & VIP & $M$ & 2 DURSLEY, VERNON & . & . & . & . & 0 & 0 & .. & . & . & • \\
\hline
\end{tabular}

To access the \{Assign Containers to Shippers\} module, use the mouse to direct the mouse arrow to $\{$ Shipping\} in the top menu bar, left click, drag the mouse arrow to Assign Container to Shippers $\}$, and left click or type [Alt] [S/s], [S/s]. 
The Assign Containers to Shippers window displays.

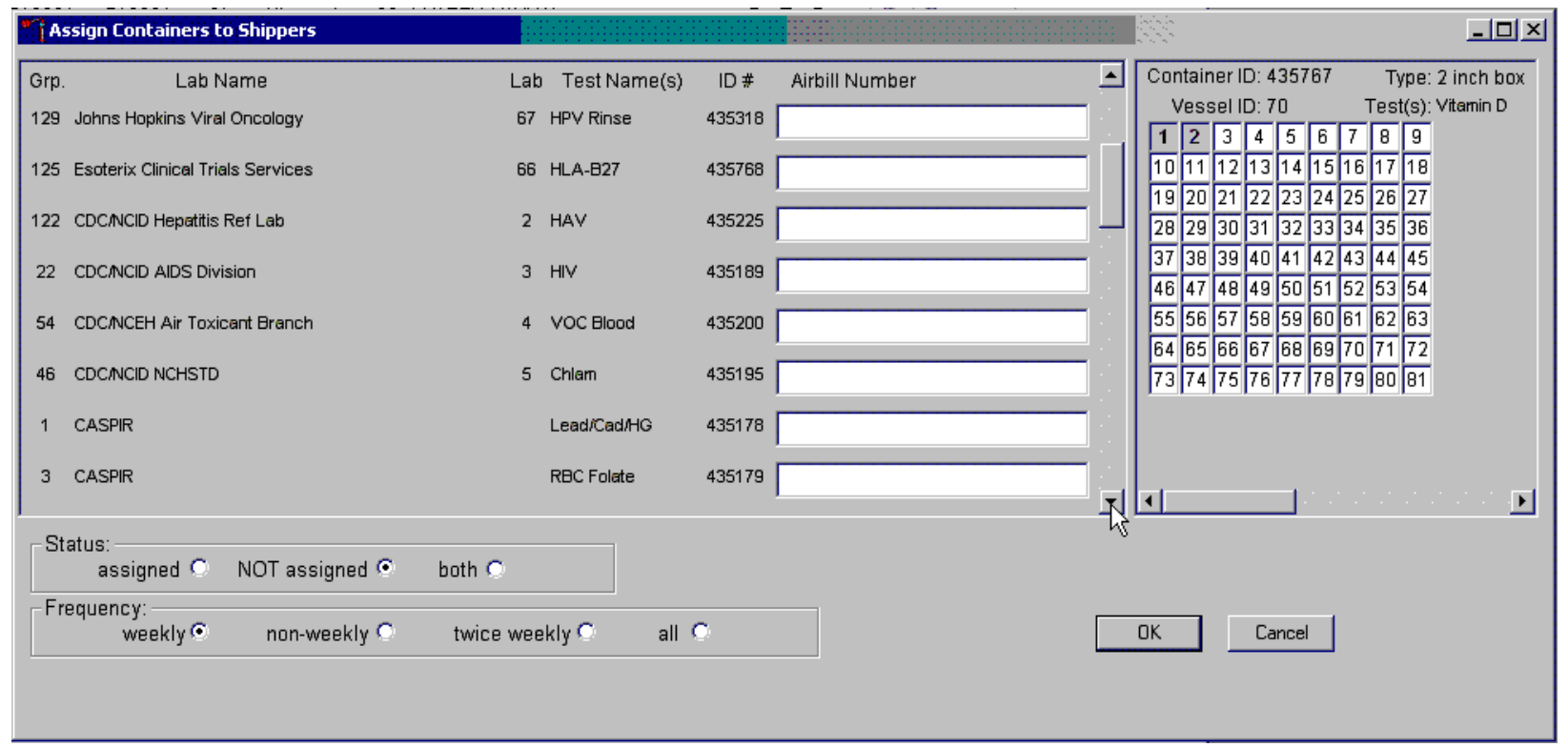

This left side of the screen includes columns for a group designation (which represents a number), Type of container, Lab designation, Test Name(s), Container ID number, and Airbill Number. The container line within the rectangular box is the active container. The right side of the screen displays the corresponding container map for the active container. The left side of the bottom of the window contains three "Status" filters (radio buttons): "assigned," "NOT assigned," and "both." The right side of the bottom of the window contains three "Frequency" filters (radio buttons): "weekly," "non-weekly," "twice weekly," and "all." Use the column titles to sort the rows. To sort the rows, use the mouse to direct the mouse arrow to the desired column title and left click. The window updates based on the selection. 
Enter an airbill number into each Airbill Number text box. There are three ways to enter the airbill number.

- Use the barcode gun to scan the barcode from the FedEx Ship Manager airbill. The barcode is located in the bottom of the top half of the folded airbill. It is the tallest on the airbill. Hold the gun in the right hand, squeeze the trigger, and move the gun up and down to adjust the laser light so that it covers the width of the barcode. Once the gun successfully scans the barcode, an audible beep is emitted.

- Use the barcode gun to scan the barcode on the second page (Recipient's Copy) of the two-part Form ID 0215. This barcode is located on the lower left-hand side toward the bottom of the form. Hold the gun in the right hand, squeeze the trigger, and move the gun up and down to adjust the laser light so that it covers the width of the barcode. Once the gun successfully scans the barcode, an audible beep is emitted.

- Use the keyboard keys to type the airbill number from either the FedEx Ship Manager airbill or the FedEx airbill form 0215.

To assign a container to the corresponding airbill:

- Set the Status filter to: "NOT assigned";

- Set the Frequency to: weekly, non-weekly, or both, depending on the shipping situation; and

- Sort the laboratories by Lab number. 
Set the Status and Frequency filters.

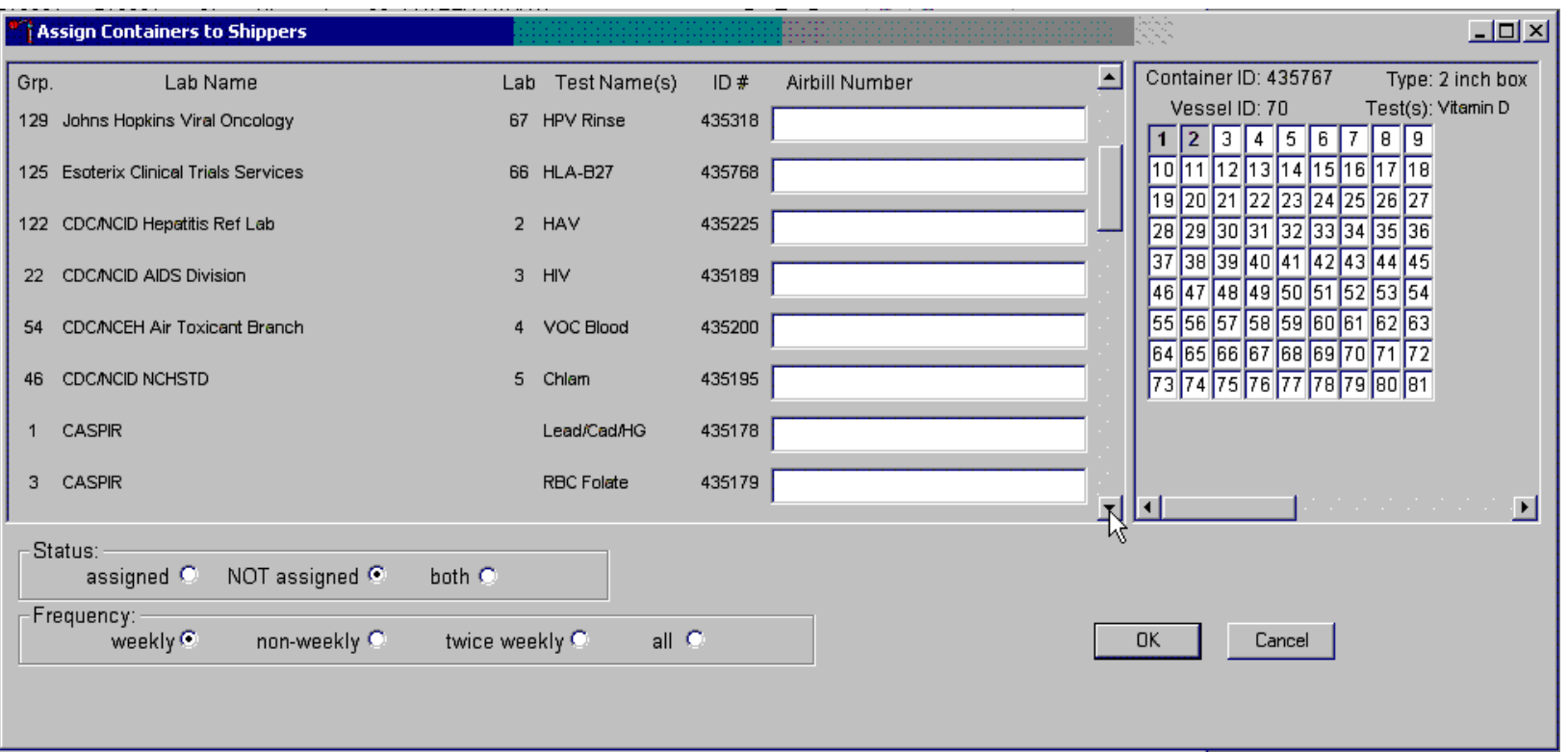

To select the Status filter, use the mouse to direct the mouse arrow to the "assigned," "NOT assigned," or "both," radio button and left click. To select the shipping Frequency, use the mouse to direct the mouse arrow to weekly, non-weekly, twice weekly, or all, and left click. 
Sort the rows by Lab Name.

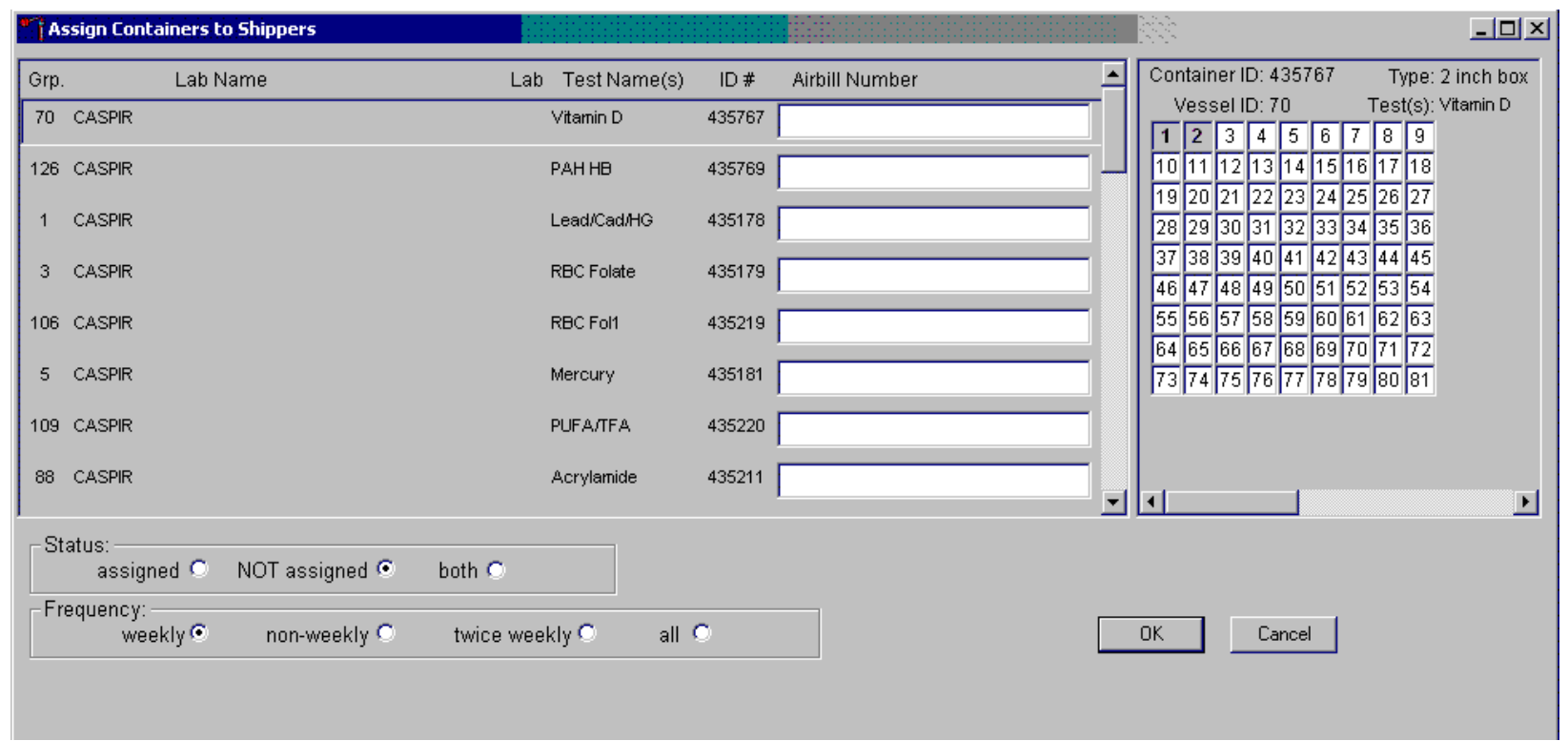

To sort the rows by lab Name, use the mouse to direct the mouse arrow to the top header "Lab Name" and left click. All containers associated with a specific lab will be grouped together in ascending order. 
Enter the airbill number into the Airbill Number text box.

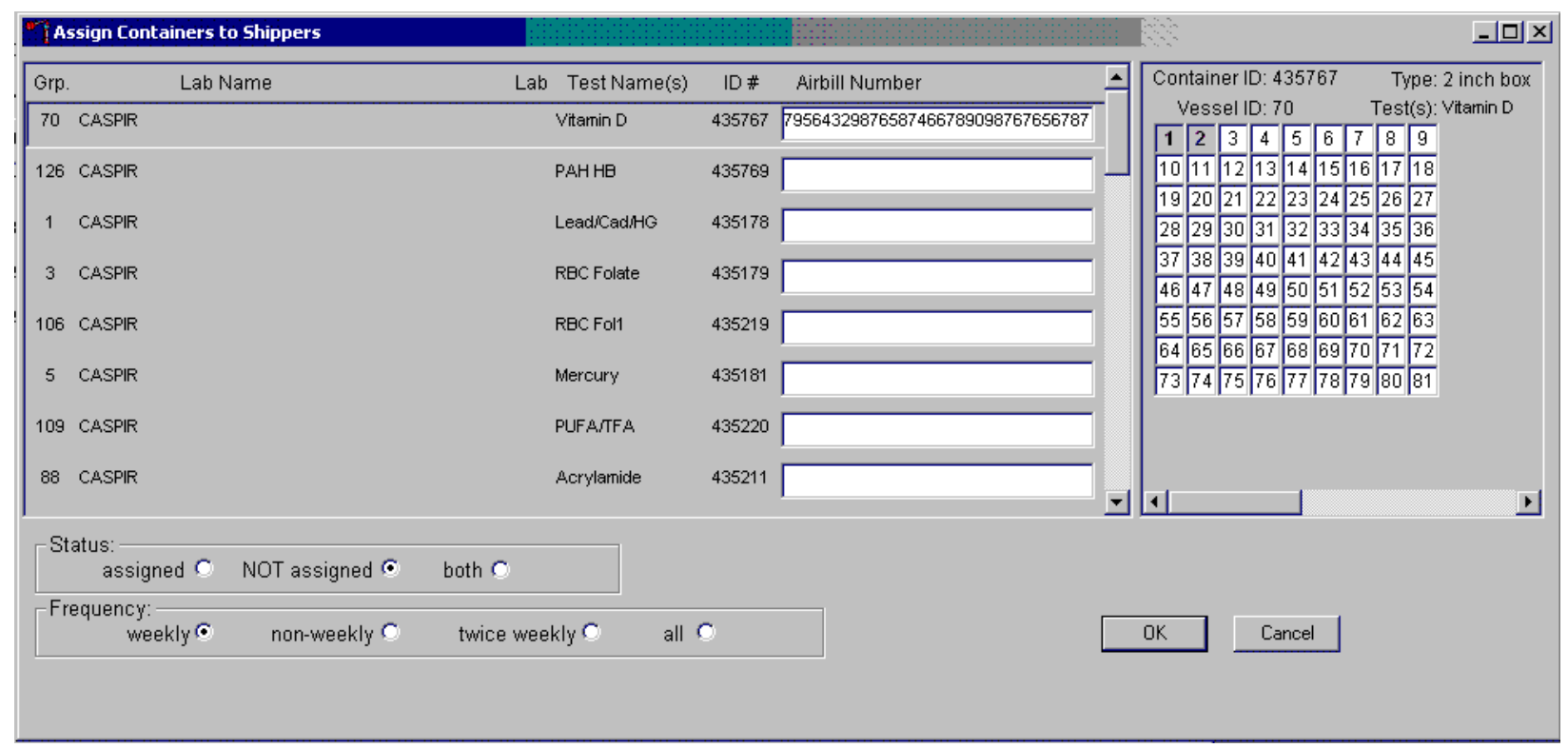

Place the cursor in the first Airbill Number text box. Use the barcode gun to scan the airbill number. The scanned number contains 20 extra digits. The actual 12-digit airbill number is embedded in the 32-digit scanned number. Select the [Tab] key to move to the next text box. Do not select the [Tab] key until the application has been allowed to enter the entire number into the text box. Once the [Tab] key is selected, the program edits the first airbill number to delete the extra numbers. 
Continue the assignment process.

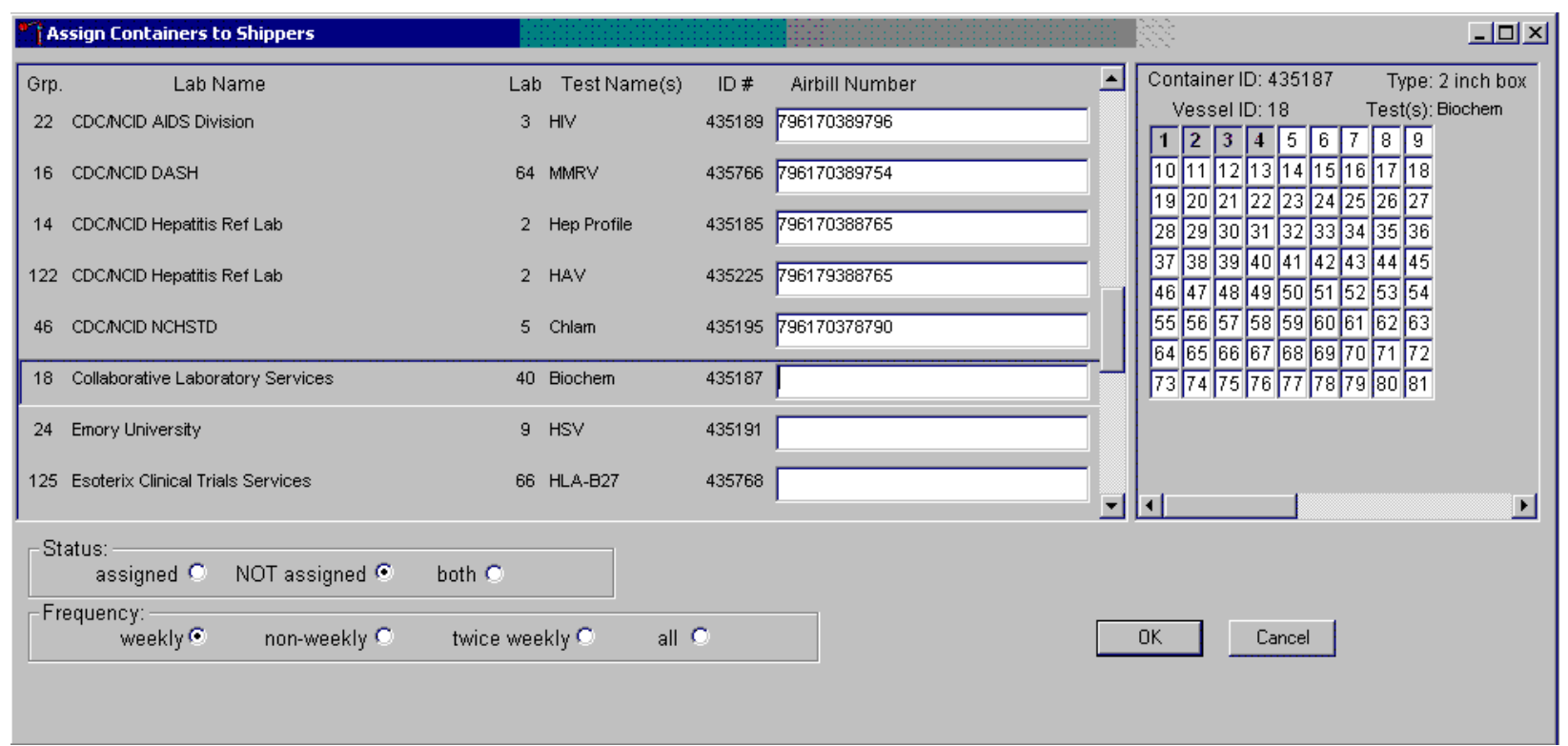

Continue scanning the barcodes and selecting the [Tab] key until all closed containers are assigned to airbills. 
Complete the assignment process.

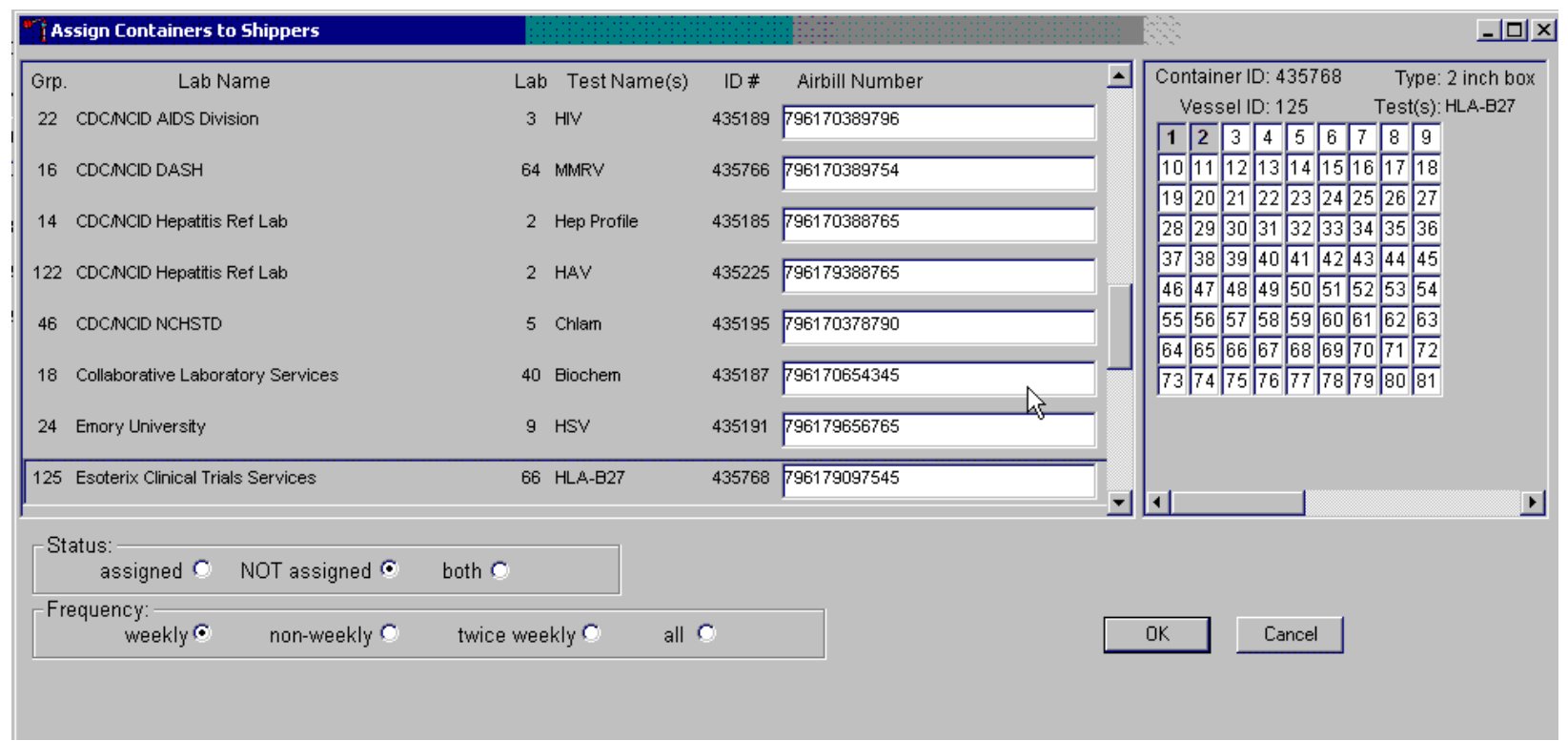

To record the assignment of containers to shippers, use the mouse to direct the mouse arrow to the "OK" button and left click. This step is required to save the information. To exit the Assign Containers to Shippers $\}$ module without recording any assignments, use the mouse to direct the mouse arrow to the "Cancel" button and left click. 


\subsection{Create Shipping Manifests}

Each shipper must include a paper manifest, which lists the inventory of the specimens in the shipping boxes. In addition, each laboratory receives an identical electronic file. Follow the steps listed below to prepare the paper manifest and to send the electronic file.

Access the $\{$ Shipping Manifest $\}$ module.

\begin{tabular}{|c|c|c|c|c|c|c|c|c|c|c|c|c|c|c|}
\hline \multicolumn{15}{|c|}{ Lab Sink } \\
\hline \multicolumn{4}{|c|}{ File View utilities Reports Modules } & \multicolumn{11}{|c|}{ Shipping Window Help } \\
\hline v & 圈 0 咨 & 绍 & $\vartheta$ & \multirow{2}{*}{\multicolumn{2}{|c|}{$\begin{array}{l}\text { Print New Container Labels } \\
\text { Container Map } \\
\text { Close Containers }\end{array}$}} & & & & & & & & & \\
\hline \multicolumn{4}{|c|}{ Appointments for Session: $\angle$} & & & \multirow{5}{*}{$\begin{array}{c}\text { UC } \\
- \\
0 \\
0\end{array}$} & \multicolumn{6}{|c|}{ Process Status } & \multirow{2}{*}{\multicolumn{2}{|c|}{$\mathrm{HPV}$ OF }} \\
\hline SPID & Sample ID s & status & type & \multirow{2}{*}{\multicolumn{2}{|c|}{$\begin{array}{l}\text { Assign Containers to Shippers } \\
\text { Irash Containers }\end{array}$}} & & & $\mathrm{P}$ & $O Z$ & $\mathrm{CB}$ & $\mathrm{Bg}$ & gtt PC & & \\
\hline 215591 & 215591 & $\mathrm{Cl}$ & VIP & & & & 0 & - & . & 0 & 0 & - & - & \\
\hline 244969 & 244969 & $\mathrm{BE}$ & $\mathrm{P}$ & \multicolumn{2}{|c|}{ Shipping Manifest } & & 0 & . & 0 & 0 & e & 0 & & \\
\hline 470234 & 470234 & $\mathrm{Cl}$ & $\mathrm{P}$ & & 60 HAGRID, RUBEUS & & & . & 0 & 0 & 0. & . 0 & & 0 \\
\hline 668903 & 668903 & $\mathrm{Cl}$ & $\mathrm{P}$ & & 12 WEASLEY, FRED & 0 & 0 & . & 0 & 0 & 0. & .0 & . & - \\
\hline 762731 & 762731 & $\mathrm{Cl}$ & VIP & & 12 BROWN, LAVENDAR & - & 0 & - & . & 0 & 0. & $\cdot \cdot$ & - & - \\
\hline 836402 & 836402 & $\mathrm{Cl}$ & VIP & & 12 MALFOY, DRACO & 0 & 0 & - & - & 0 & 0. & $\cdot \cdot$ & - & . \\
\hline 900081 & 900081 & $\mathrm{Cl}$ & VIP & $\mathrm{F}$ & 49 ABBOTT, HANNAH & 0 & 0 & 0 & - & 0 & 0. & $\cdot \cdot$ & . & - \\
\hline 998878 & 998878 & $\mathrm{Cl}$ & VIP & $M$ & 2 DURSLEY, VERNON & . & . & . & . & 0 & 0. & . & . & . \\
\hline
\end{tabular}

To access the \{Shipping Manifest\} module, use the mouse to direct the mouse arrow to \{Shipping\} in the top menu bar, left click, drag the mouse arrow to \{Shipping Manifest\}, and left click or type $[$ Alt] $[\mathrm{R} / \mathrm{r}],[\mathrm{M} / \mathrm{m}]$. 
A Shipping Manifest window displays either after the \{Shipping Manifest\} module is accessed or after a positive response is recorded to the IC Informed Consent Exclusion window.

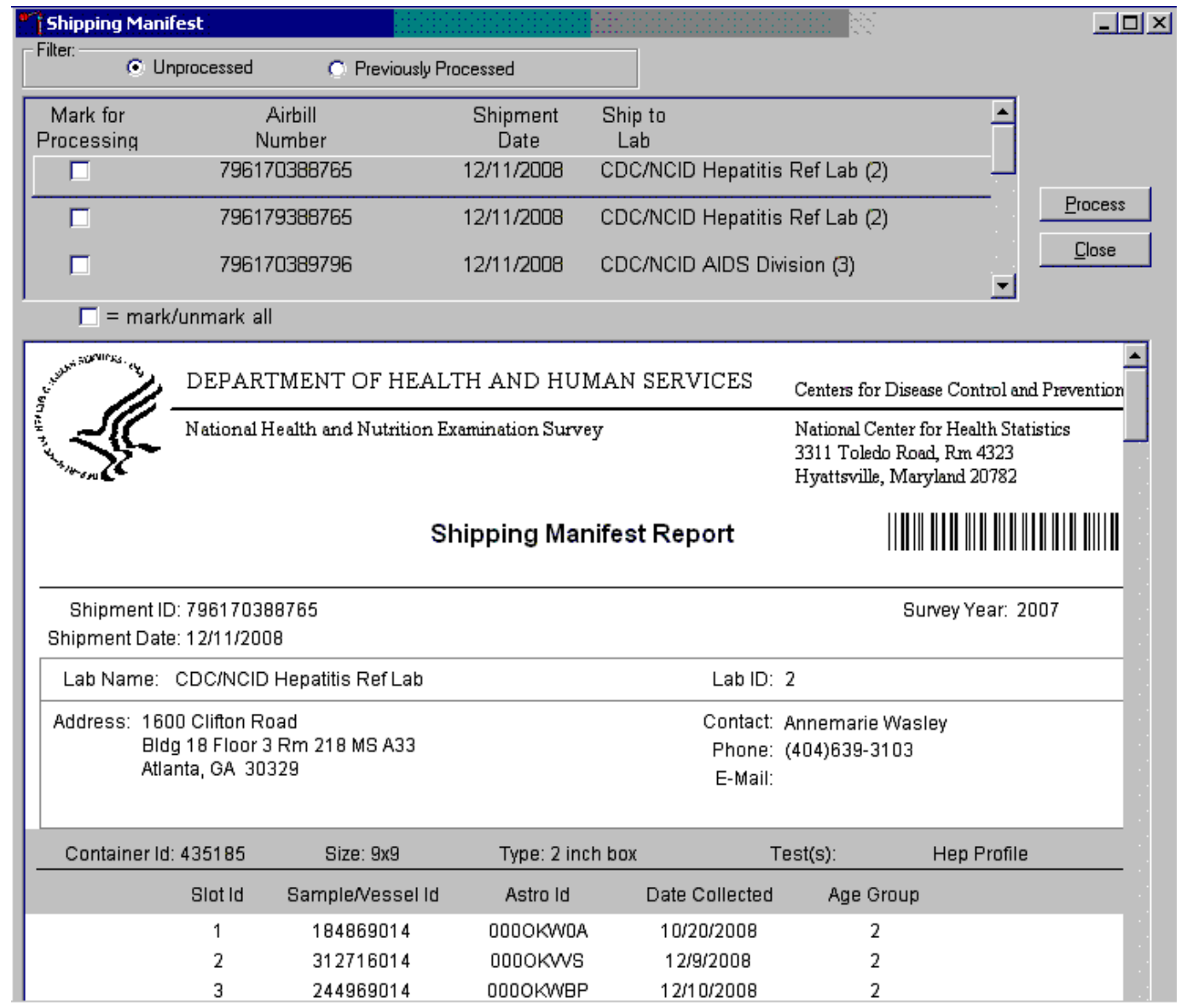

The upper portion of the Shipping Manifest Report contains two filters (Unprocessed and Previously Processed) and one row for each airbill assigned in the (Assign Container to Shipper\} module. The row includes a Mark for Processing checkbox, the Airbill Number, Shipment Date, and Ship to Lab ID, and one checkbox to mark or unmark all airbills.

The lower portion of the window includes the Shipment ID or airbill number, Shipment Date, Lab Name, Lab ID number, Address, Contact personnel, Survey Year, Container ID associated with the shipment ID, container Size, container Type, Test(s) associated with the container, and a list by Slot ID of the Sample/Vessel ID, Astro ID, and Date Collected. 
Select one of the filters.

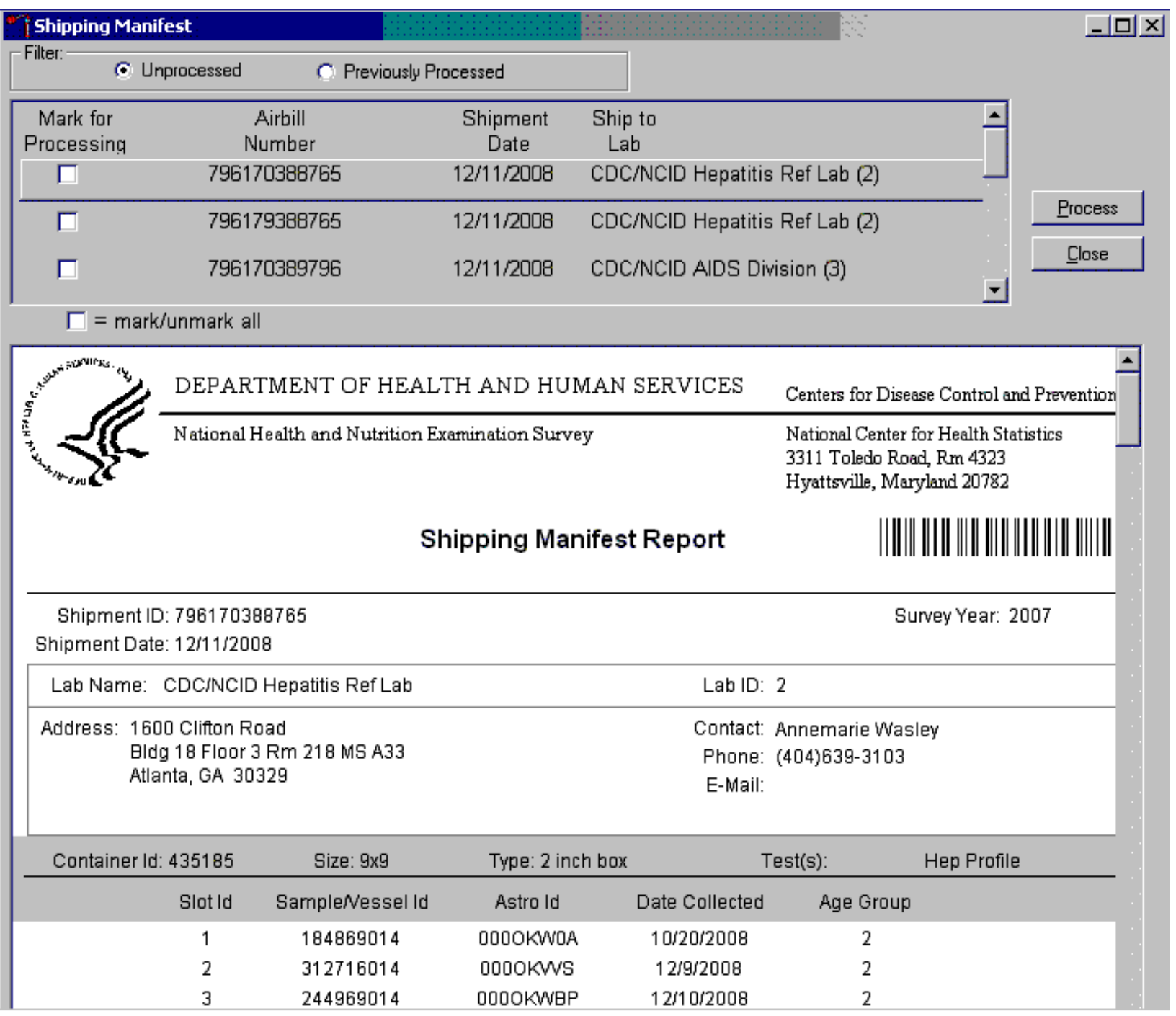

Set the filter to "Unprocessed" to print manifests and generate email files for shipments created but not previously processed using this module. Set the filter to "Previously Processed" to reprint an airbill or to recreate an electronic file for shipments previously processed using this module. To filter on unprocessed airbills, use the mouse to direct the mouse arrow to the "Unprocessed" radio button and left click. To filter on previously processed airbills, use the mouse to direct the mouse arrow to the "Previously Processed" radio button and left click. 
Select one or more manifests by placing a checkmark in the Mark for Processing checkboxes.

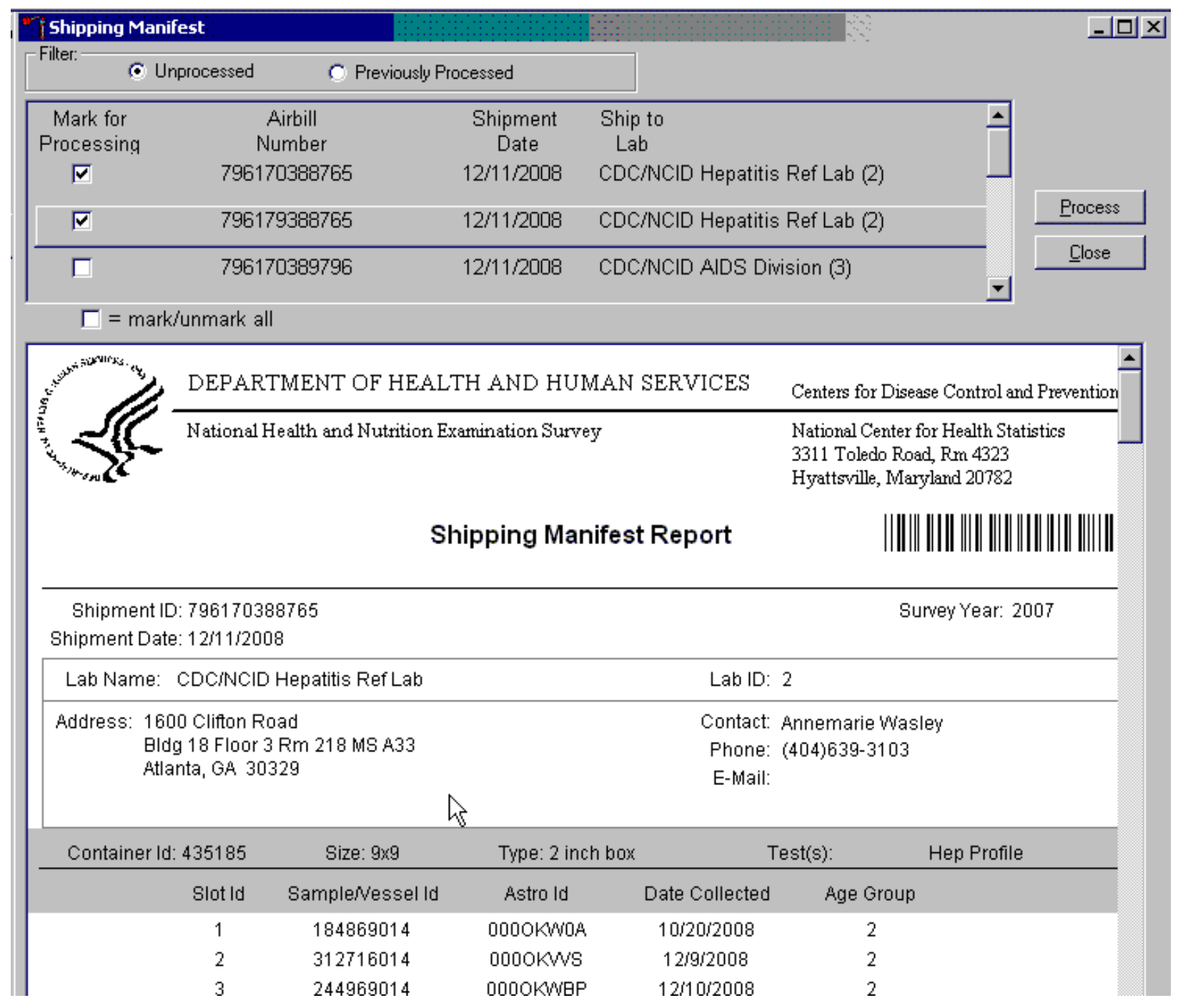

To select or mark one manifest for printing and email, use the mouse to direct the mouse arrow to the checkbox and left click. Repeat this process to include other airbills. Use the scroll bar to display all existing airbills. The lower portion of the window updates to display only marked airbills. 
Collectively mark or unmark all the airbills.

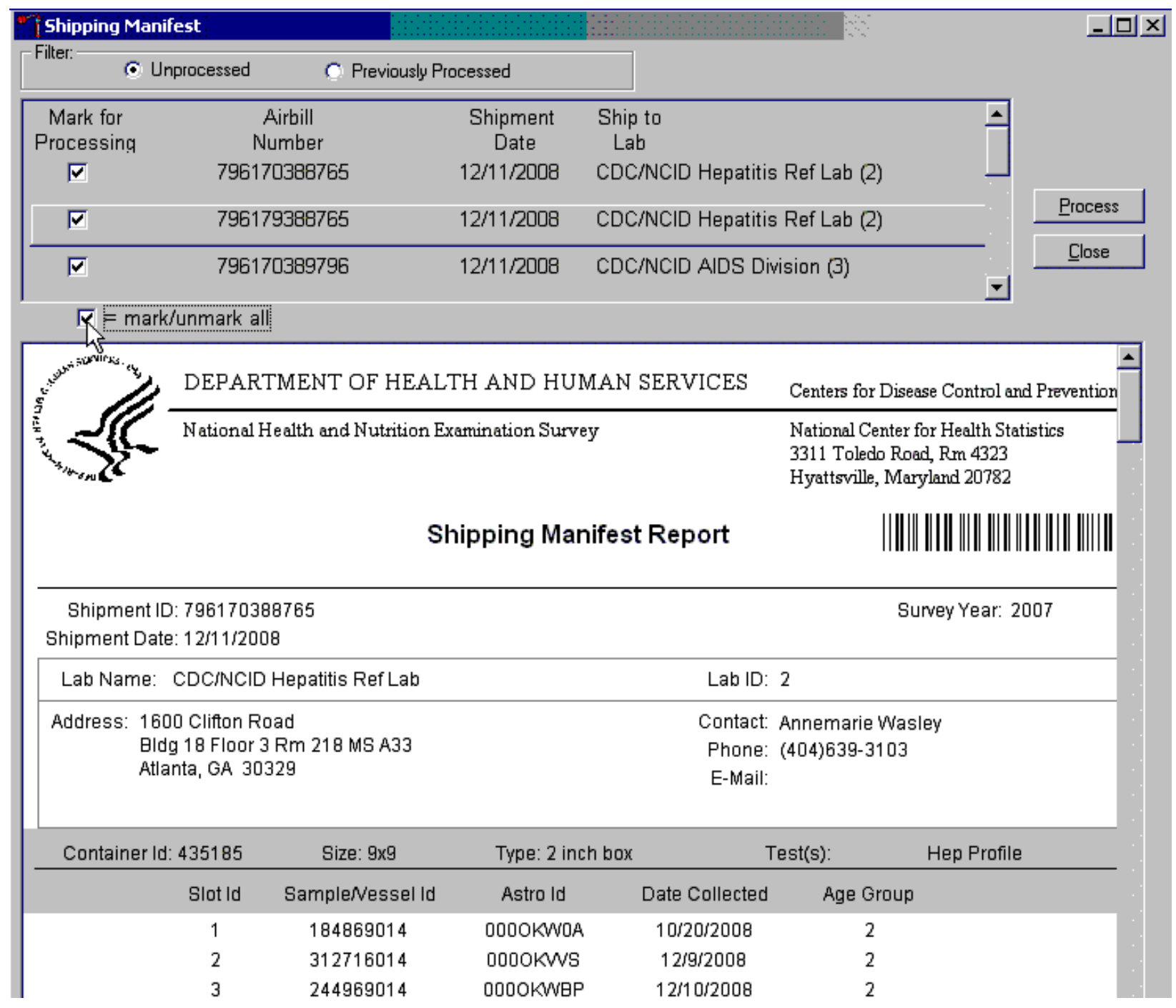

To collectively mark all the airbills, use the mouse to direct the mouse arrow to the mark/unmark all checkbox and left click. To collectively unmark all the airbills, use the mouse to direct the mouse arrow to the mark/unmark all checkbox and left click a second time. The lower portion of the screen will update to display the marked airbills. 
The right side of the window includes two buttons: Process and Close. Process the shipping manifests using the "Process" button.

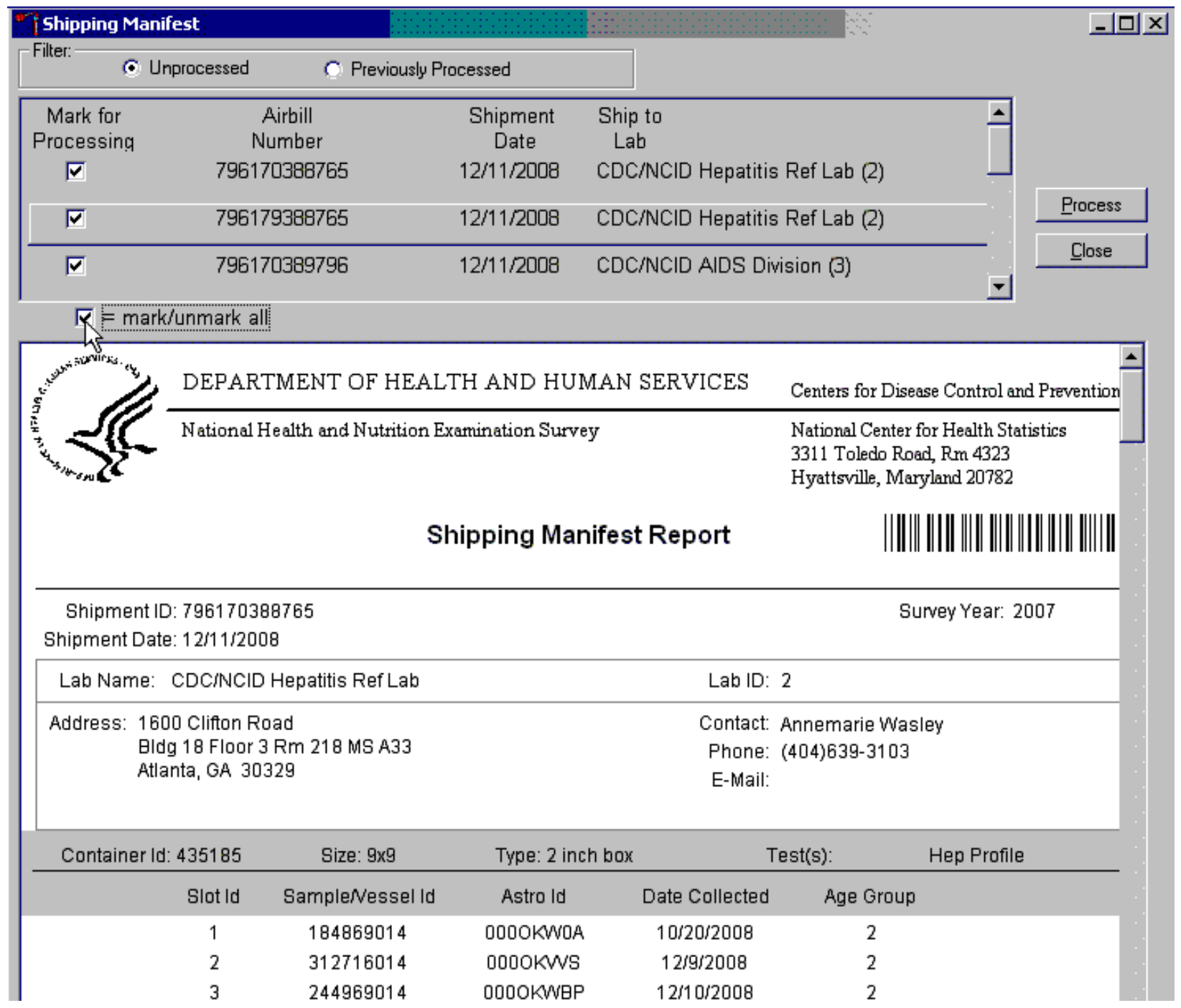

Selecting the "Process" button will generate and print the Shipping Manifest Report(s). To print the manifests, use the mouse to direct the mouse arrow to the Process button and left click, or type [Shift] $[\mathrm{P} / \mathrm{p}]$. To exit the module without printing the manifest, use the mouse to direct the mouse arrow to the "Close" button and left click, or type [Shift] [C/c.] 
The Informed Consent Exclusions window displays either when the \{Close Container\} or \{Shipping Manifest\} module is accessed. It displays when the following conditions have been met:

1. SPs initially signed their consent form thereby agreeing to have their samples tested for HIV, HPV, PSA, STD testing, and/or genetics;

2. They consent to have their vessels stored for future research; and

3. They subsequently revoke either consent; and

4. The associated vessels have already been processed.

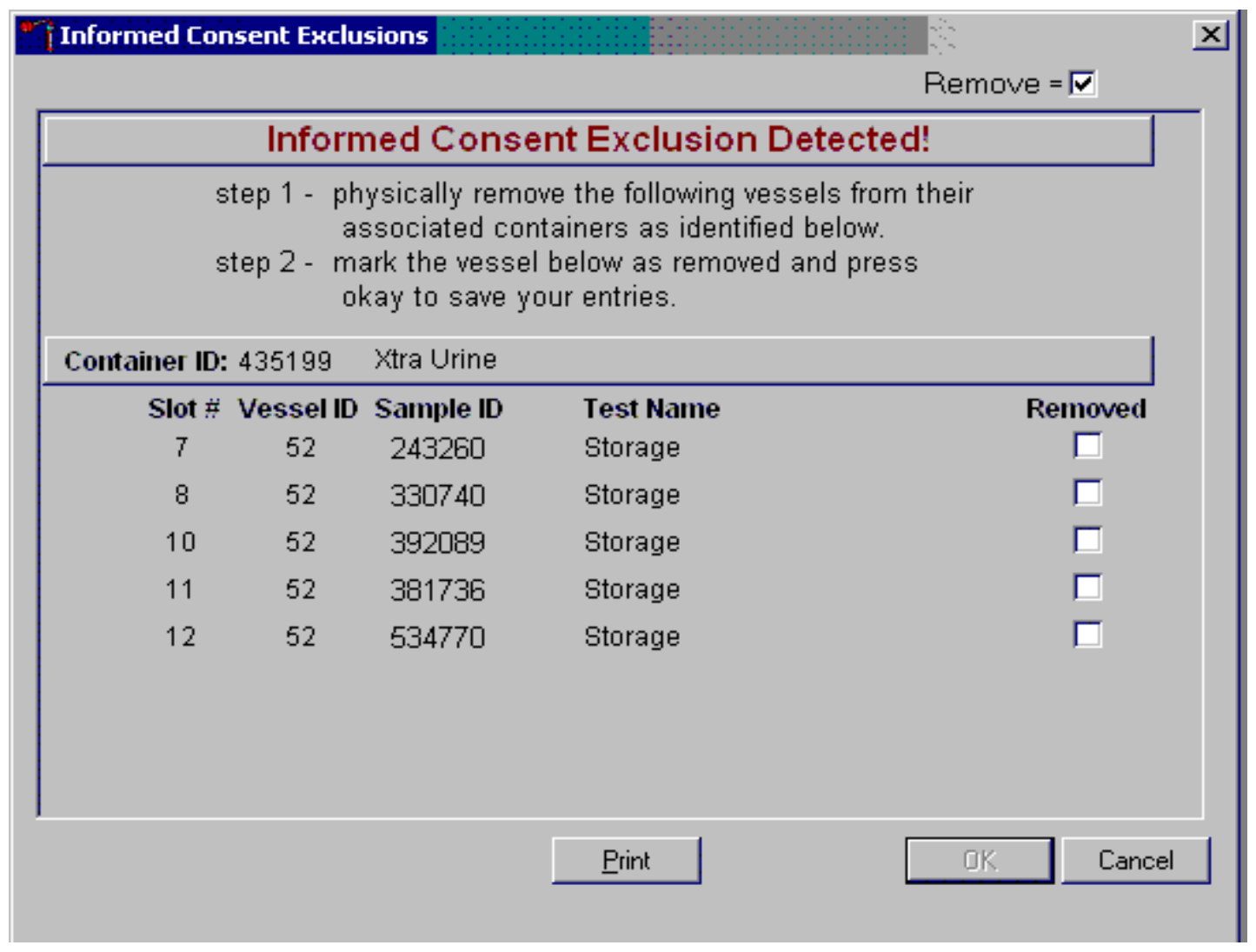

The window contains two steps that must be taken in order to resolve the inconsistencies. It includes the specific container ID and slot number within the container where the vessels are located, the sample ID, and the test name. Each row represents a vessel that must be marked as removed by placing a checkmark in the checkbox. A "Print" button on the bottom of the screen allows the list to be printed. 
These vessels must be removed from the container and destroyed before the container is shipped. Follow the exact instructions indicated in the window-"Physically remove the following vessels from their associated containers as identified below."

Affirm the actions taken to resolve these exclusions.

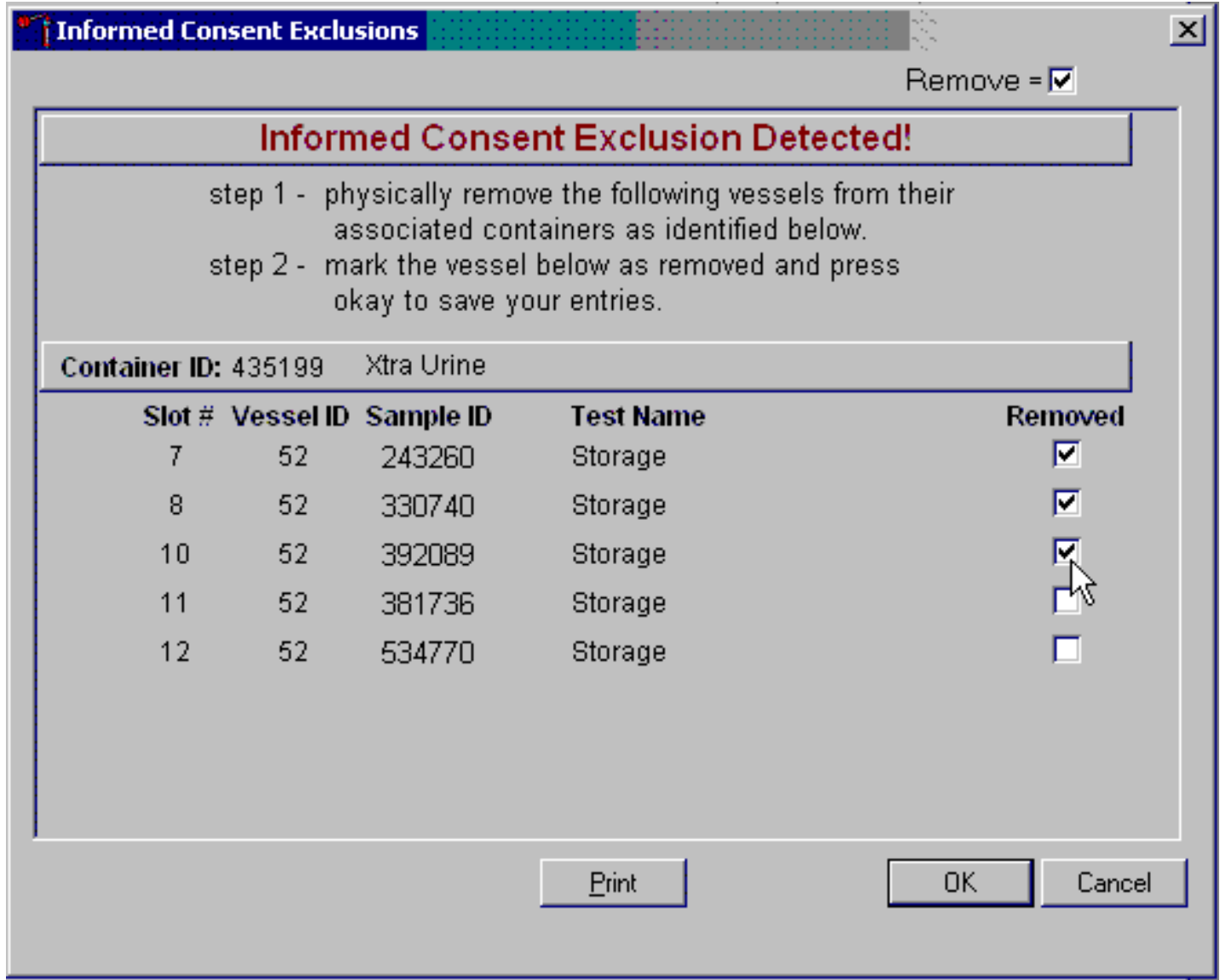

After following the exact instructions displayed in the window, record the actions taken to resolve the exclusions. To record these actions, use the mouse to direct the mouse arrow to the Removed checkboxes and left click to insert a checkmark. To record these actions in the database and to close the Informed Consent Exclusion window, use the mouse to direct the mouse arrow to the "OK" button and left click, or select [Enter]. To remove the window and exit without saving the actions to the database, use the mouse to direct the mouse arrow to the "Cancel" button and left click. 
If all the vessels are not marked, then an Informed Consent Exclusions for Stand XXX warning pop-up box displays.

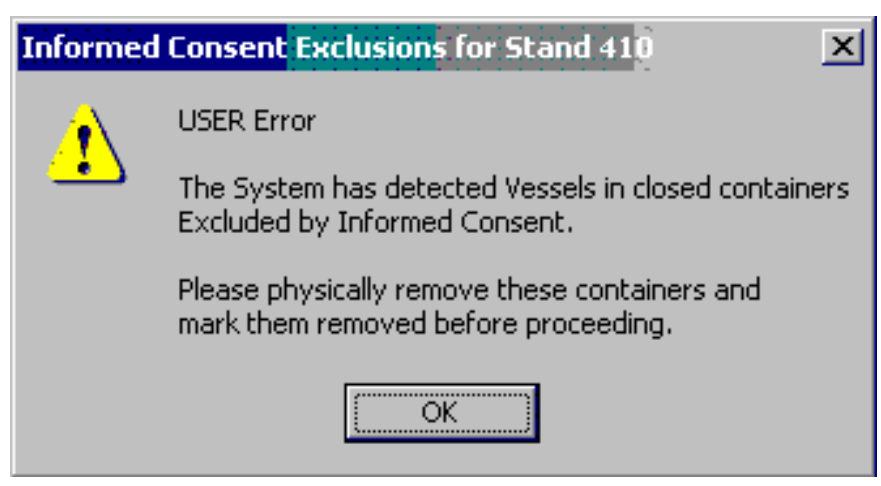

The system tracks each vessel in the \{Informed Consent Exclusion\} module to ensure that every vessel is marked as removed. Follow the instructions in the pop-up box, "Please physically remove these containers and mark them removed before proceeding." To acknowledge the message, use the mouse to direct the mouse arrow to the "OK" button and left click, or type [Enter]. 
If all the vessels were not marked, then access the $\{$ Informed Consent Exclusion $\}$ module a second time.

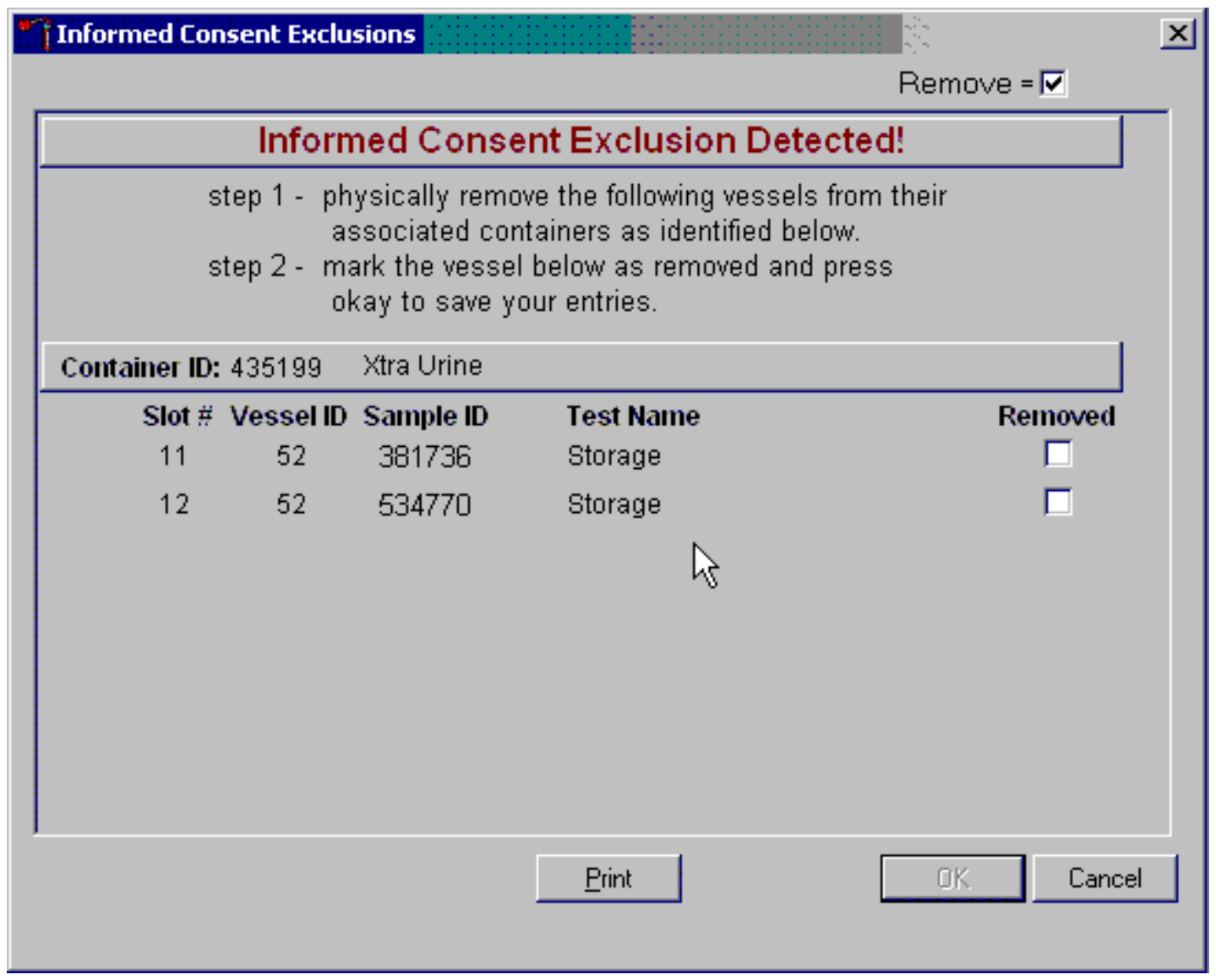

Follow the exact instructions displayed in the window, and then record the actions taken to resolve the exclusions. To record these actions, use the mouse to direct the mouse arrow to the Removed checkboxes and left click to insert a checkmark. To record these actions in the database and to close the Informed Consent Exclusions window, use the mouse to direct the mouse arrow to the "OK" button and left click, or select [Enter]. Once all vessels have been marked correctly, the close container window will automatically display. 
Most of the shipments require dry ice to keep the specimens frozen during shipment. Some shipments require reusable refrigerant packs to keep specimens cold but not frozen. Store reusable refrigerant packs in the freezers. (Distribute the refrigerant packs across all freezers.) It is the chief medical technologist's responsibility to arrange for delivery of dry ice pellets or blocks for shipping specimens. Use sliced blocks whenever possible since these sublimate less than pellets. Well in advance of a scheduled shipment, the shipping technologist estimates how much dry ice to order for the shipment and informs the MEC manager. Store the dry ice in extra shipping containers. When handling dry ice, wear cryo gloves to avoid burning hands. Also, wear an eye shield when filling bags with ice chips or breaking blocks into chunks. Use the scale to ensure that each shipper has the required amount of dry ice. Exhibit 8-3 is a list of all contract laboratory addresses.

Federal regulations require packaging all blood, urine, and other liquid diagnostic specimens according to certain regulations and packaging procedures. A complete description of these regulations is contained in Appendix A.

Diagnostic specimens must be packed in good quality packaging, which must be strong enough to withstand the shocks and loading normally encountered during transport, including transshipment between transport units and warehouses as well as removal from a pallet or overpack for subsequent manual or mechanical handling. Packaging must be constructed and closed to prevent any loss of contents when prepared for transport, which might be caused under normal conditions of transport, by vibration, or by changes in temperature, humidity, or pressure.

The packaging must consist of three components:

1. A primary receptacle(s);

2. A secondary packaging; and

3. A rigid outer packaging. 
Exhibit 8-3. Contract laboratory addresses

\begin{tabular}{|c|c|c|c|}
\hline $\begin{array}{c}\text { Laboratory/destination } \\
\text { specimen shipment address }\end{array}$ & Vessels/assays & Conditions & $\begin{array}{c}\text { Contact name } \\
\text { Telephone number }\end{array}$ \\
\hline $\begin{array}{l}\text { Lab } 2 \\
\text { CDC/NCID Hepatitis Ref Lab } \\
\text { Attn: Annemarie Wasley } \\
\text { Code NH4 } \\
\text { 1600 CLIFTON RD NE } \\
\text { Bldg 18 Floor } 3 \text { Rm } 218 \text { MS A33 } \\
\text { Atlanta, GA 30329-4018 } \\
\text { 404-639-3103 }\end{array}$ & $\begin{array}{l}122-\text { HIV } \\
87-\text { Hepatitis ab }\end{array}$ & Frozen & $\begin{array}{l}\text { Annemarie Wasley } \\
404-639-3103\end{array}$ \\
\hline $\begin{array}{l}\text { Lab } 3 \\
\text { CDC/NCID AIDS Division } \\
\text { Attn: Tim Granade/Jane Feldman } \\
1600 \text { Clifton Road NE } \\
\text { Bldg 17, Room 3127, MS A-25 } \\
\text { Atlanta, GA 30329-4018 } \\
\text { 404-639-3850 }\end{array}$ & $22-\mathrm{HIV}$ & Frozen & $\begin{array}{l}\text { Tim Granade/ } \\
\text { Jane Feldman } \\
404-639-3850\end{array}$ \\
\hline $\begin{array}{l}\text { Lab } 4 \\
\text { CDC/NCEH Air Toxicant Branch } \\
\text { Attn: Dr. Ben Blount } \\
\text { 4770 Buford Highway } \\
\text { Bldg 103 Loading Dock } \\
\text { Atlanta, GA 30341-3717 } \\
\text { 770-488-4312 }\end{array}$ & $54-$ VOC Blood & Refrigerated & $\begin{array}{l}\text { Dr. Ben Blount } \\
770-488-4312\end{array}$ \\
\hline $\begin{array}{l}\text { Lab 4a } \\
\text { CDC/NCEH Air Toxicant Branch } \\
\text { Attn: Dr. Lalith Silva } \\
\text { 4770 Buford Highway } \\
\text { Bldg 103 Loading Dock } \\
\text { Atlanta, GA 30341-3717 } \\
\text { 770-488-3559 }\end{array}$ & 150 - Aldehyde & Refrigerated & $\begin{array}{l}\text { Dr. Lalith Silva } \\
770-488-3559\end{array}$ \\
\hline $\begin{array}{l}\text { Lab 5 } \\
\text { CDC/NCID NCHSTD } \\
\text { Attn: Carol Farshy } \\
\text { 1600 Clifton Road NE } \\
\text { MS A13 } \\
\text { Atlanta, GA 30329-4019 } \\
\text { 404-639-2870 }\end{array}$ & 46 - Chlam/Trichomonas & Frozen & $\begin{array}{l}\text { Carol Farshy } \\
404-639-2870\end{array}$ \\
\hline
\end{tabular}


Exhibit 8-3. Contract laboratory addresses (continued)

\begin{tabular}{|c|c|c|c|}
\hline $\begin{array}{c}\text { Laboratory/destination } \\
\text { specimen shipment address }\end{array}$ & Vessels/assays & Conditions & $\begin{array}{c}\text { Contact name } \\
\text { Telephone number }\end{array}$ \\
\hline $\begin{array}{l}\text { Lab } 6 \\
\text { CASPIR } \\
\text { Attn: Robert White } \\
602 \text { Webb Gin House Road } \\
\text { Building C } \\
\text { Lawrenceville, GA } 30045-5427 \\
770-339-5942\end{array}$ & 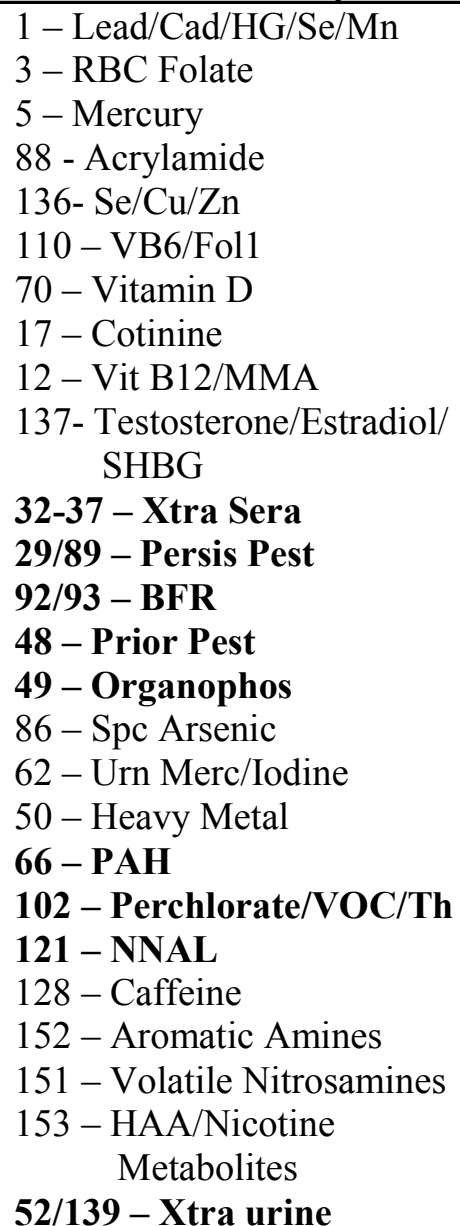 & Frozen & $\begin{array}{l}\text { Robert White } \\
770-339-5942\end{array}$ \\
\hline $\begin{array}{l}\text { Lab } 9 \\
\text { Emory University } \\
\text { Theda Gibson } \\
\text { 2015 UPPERGATE DR. } \\
\text { ROOM 560 } \\
\text { Atlanta, GA 30322-0001 } \\
\text { 404-727-5678 }\end{array}$ & $24-\mathrm{HSV}$ & Frozen & $\begin{array}{l}\text { Theda Gibson } \\
404-727-5678\end{array}$ \\
\hline
\end{tabular}


Exhibit 8-3. Contract laboratory addresses (continued)

\begin{tabular}{|c|c|c|c|}
\hline $\begin{array}{c}\text { Laboratory/destination } \\
\text { specimen shipment address }\end{array}$ & Vessels/assays & Conditions & $\begin{array}{c}\text { Contact name } \\
\text { Telephone number }\end{array}$ \\
\hline $\begin{array}{l}\text { Lab } 10 \\
\text { Diabetes Diagnostic Laboratory } \\
\text { University Of MO - Columbia } \\
1 \text { Hospital Dr. Rm. M772 } \\
\text { Columbia, MO } 65212\end{array}$ & $\begin{array}{l}9-\text { Glucose } \\
98-\text { OGTT } \\
23-\text { Insulin }\end{array}$ & Frozen & $\begin{array}{l}\text { Alethea Tennill } \\
(573) 882-2125\end{array}$ \\
\hline $\begin{array}{l}\text { Lab } 16 \\
\text { University of Minnesota } \\
\text { Attn: Susan Kupcho } \\
\text { 515 Delaware Street SE } \\
\text { Room 13-219 - MOOS Tower } \\
\text { Minneapolis, MN 55455-0357 } \\
\text { 612-624-6153 }\end{array}$ & $45-\mathrm{Alb} /$ Creat & Frozen & $\begin{array}{l}\text { Susan Kupcho } \\
612-624-6153\end{array}$ \\
\hline $\begin{array}{l}\text { Lab } 24 \\
\text { CDC/Environmental Health } \\
\text { Laboratory } \\
\text { Attn: Antonio Calafat } \\
4770 \text { Buford Highway } \\
\text { Building 110, Loading Dock } \\
\text { Atlanta, GA 30341-3717 } \\
\text { 770-488-7886 }\end{array}$ & $\begin{array}{l}90 \text { - Perfluor1 } \\
67 \text { - Phthalates }\end{array}$ & Frozen & $\begin{array}{l}\text { Dr. Antonia Calafat } \\
770-488-7891\end{array}$ \\
\hline $\begin{array}{l}\text { Lab } 37 \\
\text { CDC/Human Papillomavirus } \\
\text { Laboratory } \\
\text { Attn: Sonya Patel } \\
\text { 1600 Clifton Road NE } \\
\text { MS G41 Bldg/Rm 1/1355 } \\
\text { Atlanta, GA 30329-4018 } \\
\text { 404-639-2187 }\end{array}$ & $\begin{array}{l}78 \text { - HPV Female } \\
\text { Swab } \\
140 \text { - HPV Male Swab }\end{array}$ & $\begin{array}{l}\text { Room Temperature } \\
\text { Refrigerated }\end{array}$ & $\begin{array}{l}\text { Sonya Patel } \\
404-639-2187\end{array}$ \\
\hline $\begin{array}{l}\text { Lab } 40 \\
\text { Collaborative Laboratory } \\
\text { Services } \\
\text { Attn: Sue Vanness } \\
1005 \text { Pennsylvania Ave. } \\
\text { Suite } 102 \\
\text { Ottumwa, Iowa 52501-6408 } \\
641684-4621\end{array}$ & $18-$ Biochem & $\begin{array}{l}\text { Refrigerated } \\
\text { Frozen }\end{array}$ & $\begin{array}{l}\text { Sue Vanness } \\
641684-4621\end{array}$ \\
\hline
\end{tabular}


Exhibit 8-3. Contract laboratory addresses (continued)

\begin{tabular}{|c|c|c|c|}
\hline $\begin{array}{c}\text { Laboratory/destination specimen } \\
\text { shipment address }\end{array}$ & Vessels/assays & Conditions & $\begin{array}{c}\text { Contact name } \\
\text { Telephone number }\end{array}$ \\
\hline $\begin{array}{l}\text { Lab 51 } \\
\text { NHANES Diabetes Laboratory } \\
\text { Attn: Jean Bucksa } \\
420 \text { Delaware St SE } \\
\text { Mayo L275 } \\
\text { Minneapolis, MN 55455-0341 } \\
\text { 612-273-3645 }\end{array}$ & 21 - Lipids & Frozen & $\begin{array}{l}\text { Jean Bucksa } \\
612-273-3645\end{array}$ \\
\hline $\begin{array}{l}\text { Lab 52 } \\
\text { CDC/DVRD HPV } \\
\text { Attn: Gitika Panicker } \\
1600 \text { Clifton Rd NE } \\
\text { MS G-41 Bldg 1-South, Rm } 1250 \\
\text { Atlanta, GA 30329-4018 } \\
\text { 404-639-2269 }\end{array}$ & 77 - HPV Serum & Frozen & $\begin{array}{l}\text { Gitika } \\
\text { PanickerMeadows } \\
\text { 404-639-2269 }\end{array}$ \\
\hline $\begin{array}{l}\text { Lab } 65 \\
\text { Mayo Clinic } \\
\text { Attn: Tricia Brantner } \\
2214^{\text {th }} \text { Avenue SW } \\
\text { Guggenheim 10-02 } \\
\text { ROCHESTER, MN } 55902 \\
\text { 507-538-4128 }\end{array}$ & $127-$ IgA-TTG/EMA & Frozen & $\begin{array}{l}\text { Tricia Brantner } \\
507-538-4128\end{array}$ \\
\hline $\begin{array}{l}\text { Lab } 67 \\
\text { Gillison Laboratory } \\
\text { 2001 Polaris Pkwy \# } 1320 \\
\text { OSU - Pathology Core Facility } \\
\text { Columbus, OH43240-2000 } \\
\text { 614-293-5942 }\end{array}$ & 129 - HPV Rinse & Frozen & $\begin{array}{l}\text { Dr. Maura Gillison } \\
614-293-5942\end{array}$ \\
\hline $\begin{array}{l}\text { Lab } 120 \\
\text { Dr Gary Whitford } \\
\text { Department of Oral Biology } \\
\text { Georgia Health Sciences } \\
\text { University } \\
1120 \text { 15th St, Rm AD1435 } \\
\text { Augusta, GA, } 30912 \\
\text { 706-721-0388 }\end{array}$ & $\begin{array}{l}155 \text { - Fluoride, plasma } \\
144 \text { - Fluoride, water }\end{array}$ & $\begin{array}{l}\text { Frozen } \\
\text { Frozen }\end{array}$ & $\begin{array}{l}\text { Gary Whitford } \\
706-721-0388\end{array}$ \\
\hline
\end{tabular}


Primary receptacles must be packed in secondary packaging in such a way that, under normal conditions of transport, they cannot break, be punctured, or leak their contents into secondary packaging. Secondary packaging must be secured in outer packaging with suitable cushioning material. Any leakage of the contents must not substantially impair the protection properties of the cushioning material or of the outer packaging.

\section{Special Packing Instructions for VOC Blood (Vessel 54) and Aldehyde (Vessel 150)}

Place four ice packs in the bottom of a medium shipper, place a barrier of newsprint over the ice packs, then cover with a Blue "chuck." Place the Tyvek wrapped container/s on top of the blue chuck. Place newsprint paper around the sides and top of the container to secure for shipping. Replace the Styrofoam top, add the shipping manifest, close shipper, and add FedEx labeling.

Packages must be prepared as follows:

- 650(a) For Liquids;

- The primary receptacle(s) must be leakproof and must not contain more than $1 \mathrm{~L}$;

- The secondary packaging must be leakproof;

- If multiple fragile primary receptacles are placed in a single secondary packaging, they must be individually wrapped or separated to prevent contact between them;

- Absorbent material must be placed between the primary receptacle and the secondary packaging. The absorbent material, such as cotton wool, must be in sufficient quantity to absorb the entire contents of the primary receptacle (so that any release of the liquid substance will not compromise the integrity of the cushioning material or of the outer packaging;

- The primary receptacle or the secondary packaging must be capable of withstanding, without leakage, an internal pressure producing a pressure differential of not less than $95 \mathrm{kPa}$ in the range of $-40^{\circ} \mathrm{C}$ to $+55^{\circ} \mathrm{C}\left(-40^{\circ} \mathrm{F}\right.$ to $\left.130^{\circ} \mathrm{F}\right)$; and

- The outer packaging must not contain more than $4 \mathrm{~L}$. 
Specific Requirements - Refrigerated or Frozen Specimens: Ice, Dry Ice, and

\section{Liquid Nitrogen}

- When dry ice or liquid nitrogen is used to keep specimens cold, all applicable requirements of these Regulations must be met. When used, ice or dry ice must be placed outside the secondary packaging or in the outer packaging or an overpack. Interior supports must be provided to secure the secondary packaging in the original position after the ice or dry ice (solid carbon dioxide) has been dissipated. If ice is used, the packaging or overpack must be leakproof. If dry ice (solid carbon dioxide) is used, the outside packaging or overpack must be designed and constructed to permit the release of carbon dioxide gas to prevent a buildup of pressure that could rupture the packaging.

- The primary receptacle and the secondary packaging must maintain their integrity at the temperature of the refrigerant used as well as the temperatures and the pressures, which could result if refrigeration were to be lost.

Infectious substances assigned to UN 3373 which are packed and marked in accordance with this packing instruction are not subject to any other requirement of these regulations except for the following:

a. The name, address, and telephone number of a responsible person must be provided on the waybill or on the package.

\section{Packing Instruction 904 - Dry Ice}

Dry ice (solid carbon dioxide), when offered for transport by air, must be in packaging designed and constructed to permit the release of carbon dioxide gas and to prevent a buildup of pressure that could rupture the packaging.

The net weight of dry ice must be marked on the outside of the package.

Arrangements between the shipper and operator(s) must be made for each shipment to ensure that ventilation and safety procedures are followed. 
When a Shipper's Declaration is not required, the following information as required by 8.2.3 for dry ice (solid carbon dioxide) must be contained in the "Nature and Quantity of Dangerous Goods" box on the air waybill:

- Proper shipping name (Dry Ice or Carbon Dioxide, solid);

- UN 1845;

- The number of packages; and

- The net weight of dry ice in each package.

The net weight of the dry ice must be marked on the outside of the package.

\subsubsection{Pack Boxed Specimens}

Store the blood and urine vessels in 2-, 3-, and 5-inch storage boxes. Separate the vessels with cardboard grids or foam inserts. Secure the top of the box to the bottom with a rubber band. Place the box in a pressure vessel, add one absorbent paper, and seal the pressure vessel.

Ship the vessels to the addresses listed in Exhibit 8-3, Contract Laboratory addresses, shown earlier.

\subsubsection{Pack Medium Shipping Containers for Frozen Shipment}

Pack the specimen storage boxes tightly in the Styrofoam container to prevent movement.

- Place the shipper on the scale and tare.

- Place a layer 3-inches thick or at least 15 pounds of chipped dry ice or dry ice slabs in the bottom of a medium Styrofoam shipper.

- Place a single layer of newsprint between the box and the dry ice.

- Place the sealed pressure vessels containing the boxes on top of the dry ice.

- Pack the sides with crumbled newsprint. 
- Place enough chipped dry ice or dry ice slabs on top so that the Styrofoam container is filled with dry ice to $1 / 4$ inch below the rim or add a minimum of another 15 pounds of dry ice.

- Place the Styrofoam lid on top of the Styrofoam bottom.

- Place a plastic envelope containing the appropriate shipping manifest and return preprinted label on top of the Styrofoam lid.

- Weigh the shipper on the scale or assume the medium shipper weighs approximately 35 pounds.

- Record the weight of the dry ice (30 pounds for a medium shipper) on the FedEx Class 9 label.

- Secure the shipper with tape.

- Attach the airbill.

\subsubsection{Pack Small Shipping Containers for Frozen Shipment}

Pack the specimen storage boxes tightly in the Styrofoam container to prevent movement.

- Place the shipper on the scale and tare.

- Place a layer 3-inches thick or at least 10 pounds of chipped dry ice or dry ice slabs in the bottom of a medium Styrofoam shipper.

- Place a single layer of newsprint between the box and the dry ice.

- Place the sealed pressure vessels containing the boxes on top of the dry ice.

- Pack the sides with crumbled newsprint.

- Place enough chipped dry ice or dry ice slabs on top so that the Styrofoam container is filled with dry ice to $1 / 4$ inch below the rim or add a minimum of another 10 pounds of dry ice.

- Place the Styrofoam lid on top of the Styrofoam bottom.

- Place a plastic envelope containing the appropriate shipping manifest and return preprinted label on top of the Styrofoam lid.

- Weigh the shipper on the scale or assume the small shipper weighs approximately 25 pounds.

- Record the weight of the dry ice (20 pounds for a small shipper) on the FedEx Class 9 label. 
- Secure the shipper with tape.

- Attach the airbill.

\subsubsection{Pack Medium Shipping Container for Refrigerated Shipment}

Some shipments require a coolant to keep the specimens cold but not frozen during shipment. For these shipments, use reusable refrigerant packs. Add enough frozen refrigerant packs to the shipper to keep specimens cold for at least 48-72 hours. Keep approximately 30 refrigerant packs in the freezers at all times, replacing as necessary. Pack all refrigerated containers in Styrofoam shippers.

Pack the specimen storage boxes tightly in the Styrofoam container to prevent movement.

- Seal the $9 \times 12$ zip closable plastic bag or the pressure vessel containing the boxes.

- Place at least two large frozen refrigerant packs in the bottom of a shipper.

- Place the sealed plastic bag containing the swabs or the pressure vessel containing the boxes on top of the refrigerant pack.

- Pack the sides with crumbled newsprint.

- Place two additional refrigerant packs on top of the container(s).

- Place the Styrofoam lid on top of the Styrofoam bottom.

- Place the appropriate shipping manifest and one return FedEx preprinted airbill into a $9 \times 12$ plastic bag and seal.

- Place the plastic envelope containing the appropriate shipping manifest and one FedEx preprinted airbill on top of the Styrofoam lid.

- Weigh the shipper on the scale or assume that the package weighs 10 pounds.

- Record the total weight of the shipper (10 pounds) on the appropriate FedEx label. 


\subsubsection{Label Shippers}

For all destinations, include a preprinted FedEx return airbill containing the warehouse address.

All shippers must contain a FedEx barcode label and appropriate orientation and warning labels. Label all shippers with the appropriate warning labels as follows:

1. A "Keep Frozen" label and a FedEx Class 9 - Miscellaneous Dangerous Goods label on shippers containing dry ice.

2. A "Refrigerate" label on shippers containing refrigerant packs.

3. A UN 3373: - "BIOLOGICAL SUBSTANCE, CATEGORY B label on all shippers.

4. An "overpack" label on all shippers

5. Complete the section of the FedEx label that requests the weight of the contents of the shipper, and if applicable, the section that requests the weight of the dry ice contained in the shipper.

6. Place the FedEx label with the appropriate contract laboratory address in a plastic FedEx pouch and attach the window to the cardboard lid of the shipper.

7. Add one additional strip of tape across the FedEx pouch.

\subsection{Container Protocol and Weekly Shipping List}

Exhibit 8-4 illustrates the Container protocol; Exhibit 8-5 illustrates the FedEx shipping list by lab name; and Exhibit 8-6 illustrates the shipping list by lab number. Use the container protocol as an aid at the start of each stand when opening new containers. Use the FedEx shipping lists as an aid when creating airbills in FedEx Ship Manager. 
Exhibit 8-4. Container protocol

2013 Final Container Protocol

2013 Final Container Protocol

\begin{tabular}{|c|c|c|c|c|c|}
\hline $\mathrm{Lab}$ & Vessel & Test & $\mathrm{Lab}$ & Vessel & Container \\
\hline 61 & 1 & $\mathrm{Lead} / \mathrm{Cad} / \mathrm{HG} / \mathrm{Se} / \mathrm{Mn}$ & CASPIR & $2 \mathrm{~mL}$ sterile cryovial & $9 \times 9$ two-inch box \\
\hline 54 & 3 & RBC Folate & CASPIR & $2 \mathrm{~mL}$ cryovial & $\begin{array}{l}10 \times 10 \text { two-inch } \\
\text { box }\end{array}$ \\
\hline 10 & 4 & Glycohem & University of Missouri & $2 \mathrm{~mL}$ sterile cryovial & $9 \times 9$ two-inch box \\
\hline 61 & 5 & $\begin{array}{l}\text { Mercury, Ethyl \& } \\
\text { Methyl }\end{array}$ & CASPIR & $2 \mathrm{~mL}$ cryovial & 9x9 two-inch box \\
\hline 60 & 88 & Acrylamide & CASPIR & $2 \mathrm{~mL}$ cryovial & 9x9 two-inch box \\
\hline 10 & 9 & Glucose & University of Missouri & $2 \mathrm{~mL}$ cryovial & $9 \times 9$ two-inch box \\
\hline 10 & 98 & OGTT & University of Missouri & $2 \mathrm{~mL}$ cryovial & $9 \times 9$ two-inch box \\
\hline 61 & 136 & $\mathrm{Se} / \mathrm{Cu} / \mathrm{Zn}$ & CASPIR & $2 \mathrm{~mL}$ sterile cryovial & 9x9 two-inch box \\
\hline 54 & 110 & Fol & CASPIR & $2 \mathrm{~mL}$ cryovial & $\begin{array}{l}10 \times 10 \text { two-inch } \\
\text { box }\end{array}$ \\
\hline 54 & 70 & Vitamin D/Fatty Acids & CASPIR & $2 \mathrm{~mL}$ cryovial & $\begin{array}{l}10 \times 10 \text { two-inch } \\
\text { box }\end{array}$ \\
\hline 2 & 122 & HAV & CDC/NCID Hepatitis Ref Lab & $2 \mathrm{~mL}$ cryovial & 9x9 two-inch box \\
\hline 2 & 87 & Hepatit ab & CDC/NCID Hepatitis Ref Lab & $2 \mathrm{~mL}$ cryovial & $9 \times 9$ two-inch box \\
\hline 59 & 17 & Cotinine & CASPIR & $2 \mathrm{~mL}$ cryovial & 9x9 two-inch box \\
\hline 51 & 21 & Lipids & NHANES Diabetes Laboratory & $2 \mathrm{~mL}$ cryovial & $9 \times 9$ two-inch box \\
\hline 52 & 77 & HPV Serum & CDC/DVRD HPV & $2 \mathrm{~mL}$ cryovial & $9 \times 9$ two-inch box \\
\hline 40 & 18 & Biochem & $\begin{array}{l}\text { Collaborative Laboratory } \\
\text { Services }\end{array}$ & $2 m L$ cryovial & $9 \times 9$ two-inch box \\
\hline 10 & 23 & Insulin & University of Missouri & $2 \mathrm{~mL}$ cryovial & 9x9 two-inch box \\
\hline 54 & 12 & Vit B12/MMA & CASPIR & $2 \mathrm{~mL}$ cryovial & 9x9 two-inch box \\
\hline 3 & 22 & HIV & CDC/NCID AIDS Division & $2 \mathrm{~mL}$ cryovial & 9x9 two-inch box \\
\hline 9 & 24 & HSV & Emory University & $2 \mathrm{~mL}$ cryovial & 9x9 two-inch box \\
\hline 65 & 127 & IgA-TTG/EMA & Mayo Clinic & $2 \mathrm{~mL}$ cryovial & $9 \times 9$ two-inch box \\
\hline
\end{tabular}




\section{Exhibit 8-4. Container protocol (continued)}

\section{Final Container Protocol}

\section{Final Container Protocol}

\begin{tabular}{|c|c|c|c|c|c|}
\hline Lab & Vessel & Test & $\mathrm{Lab}$ & Vessel & Container \\
\hline 60 & 137 & $\begin{array}{l}\text { Testosterone/Estradiol/ } \\
\text { SHBG }\end{array}$ & CASPIR & $2 \mathrm{~mL}$ cryovial & $9 \times 9$ two-inch box \\
\hline $4 \mathrm{a}$ & 150 & Aldehyde & $\begin{array}{l}\text { CDC NCEH } \\
\text { Air Toxicant }\end{array}$ & $2 \mathrm{~mL}$ cryovial & 9x9 two-inch box \\
\hline 6 & $32-37$ & Xtra sera1 & CASPIR & $2 \mathrm{~mL}$ cryovial & 9x9 two-inch box \\
\hline 28 & $29 / 89$ & Persis Pest1 & CASPIR & $5 \mathrm{~mL}$ cryovial & 9x9 three-inch box \\
\hline 24 & 90 & Perfluor & $\begin{array}{l}\mathrm{CDC} / \mathrm{NCEH} \\
\text { Environmental } \\
\text { Health Lab }\end{array}$ & $2 \mathrm{~mL}$ cryovial & 9x9 two-inch box \\
\hline 28 & $92 / 93$ & BFR1 & CASPIR & $5 \mathrm{~mL}$ cryovial & $9 \times 9$ three-inch box \\
\hline 16 & 45 & Alb/Creat & $\begin{array}{l}\text { University of } \\
\text { Minnesota }\end{array}$ & $5 \mathrm{~mL}$ cryovial & 9x9 three-inch box \\
\hline 5 & 46 & Chlam/Trichomonas & $\begin{array}{l}\text { CDC/NCID } \\
\text { NCHSTD } \\
\end{array}$ & 14 mL Falcon & $5 \times 5$ three-inch box \\
\hline 5 & 154 & Trichomonas & $\begin{array}{l}\text { CDC/NCID } \\
\text { NCHSTD } \\
\end{array}$ & 14 mL Falcon & $5 \times 5$ three-inch box \\
\hline 61 & 86 & Spc Arsenic & CASPIR & $5 \mathrm{~mL}$ cryovial & $9 \times 9$ three-inch box \\
\hline 26 & 48 & Prior Pest & $\begin{array}{l}\text { CDC/NCEH } \\
\text { Pesticide } \\
\text { Laboratory }\end{array}$ & $10 \mathrm{~mL}$ VWR screwcap & $5 \times 5$ three-inch box \\
\hline 26 & 49 & Organophos & $\begin{array}{l}\text { CDC/NCEH } \\
\text { Pesticide } \\
\text { Laboratory }\end{array}$ & $10 \mathrm{~mL}$ VWR screwcap & $5 \times 5$ three-inch box \\
\hline 61 & 62 & Urn Merc/Iodine & CASPIR & $\begin{array}{l}10 \mathrm{~mL} \text { VWR screwcap } \\
\text { with green dot }\end{array}$ & $5 \times 5$ three-inch box \\
\hline 61 & 50 & Heavy Metal & CASPIR & 14 mL Falcon & $5 \times 5$ three-inch box \\
\hline 31 & 66 & PAH & CASPIR & $5 \mathrm{~mL}$ cryovial & 9x9 three-inch box \\
\hline 24 & 67 & Phthalates & $\begin{array}{l}\text { CDC/NCEH } \\
\text { Environmental } \\
\text { Health Lab }\end{array}$ & $5 \mathrm{~mL}$ cryovial & 9x9 three-inch box \\
\hline 4 & 102 & $\begin{array}{l}\text { Perchlorate/VOC/ } \\
\text { Thiocyanate }\end{array}$ & CASPIR & $5 \mathrm{~mL}$ cryoviall & $9 \times 9$ three-inch box \\
\hline 59 & 156 & TSNAs & CASPIR & $10 \mathrm{~mL}$ VWR screwcap & $5 \times 5$ three-inch box \\
\hline 54 & 128 & Caffeine & CASPIR & $2 \mathrm{~mL}$ cryovial & 10x10 two-inch box \\
\hline 4 & 152 & Aromatic Amines & CASPIR & $5 \mathrm{~mL}$ cryovial & 9x9 three-inch box \\
\hline 4 & 151 & Volatile Nitrosamines & CASPIR & $5 \mathrm{~mL}$ cryovial & 9x9 three-inch box \\
\hline
\end{tabular}


Exhibit 8-4. Container protocol (continued)

2013 Final Container Protocol

2013 Final Container Protocol

\begin{tabular}{|c|c|c|c|c|c|}
\hline $\mathrm{Lab}$ & Vessel & Test & $\mathrm{Lab}$ & Vessel & Container \\
\hline 4 & 153 & HAA/Nicotine analogs & CASPIR & $2 \mathrm{~mL}$ cryovial & $9 \times 9$ two-inch box \\
\hline 6 & 52 & Xtra Urine 1 & CASPIR & $5 \mathrm{~mL}$ cryovial & 9x9 three-inch box \\
\hline 6 & & Xtra Urine 2 & CASPIR & $5 \mathrm{~mL}$ cryovial & 9x9 three-inch box \\
\hline 4 & 54 & VOC Blood & $\begin{array}{l}\text { CDC/NCEH Air } \\
\text { Toxicant Branch }\end{array}$ & 7 mL Gray tube & $5 \times 5$ five-inch foam box \\
\hline 37 & 78 & HPV Swab - Female & $\begin{array}{l}\text { CDC/Human } \\
\text { Papillomavirus } \\
\text { Lab }\end{array}$ & Swab & 9x12 Ziplock bag \\
\hline 37 & 140 & HPV Swab - Male & $\begin{array}{l}\text { CDC/Human } \\
\text { Papillomavirus } \\
\text { Lab }\end{array}$ & Swab & $7 x 7$ three-inch box \\
\hline 67 & 129 & HPV Rinse & $\begin{array}{l}\text { Johns Hopkins } \\
\text { Viral Oncology }\end{array}$ & 14 mL Falcon tube & $5 \times 5$ three-inch box \\
\hline 120 & 144 & Water, Fluoride & $\begin{array}{l}\text { Georgia Health } \\
\text { Systems }\end{array}$ & $10 \mathrm{~mL}$ cryovial & $5 \times 5$ three-inch box \\
\hline $\begin{array}{l}\text { Bold } \\
\text { end o }\end{array}$ & $\begin{array}{l}\text { - ship at } \\
\text { f stand. }\end{array}$ & & & $\begin{array}{l}\text { Italics }=\text { ship } \\
\text { refrigerated }\end{array}$ & \\
\hline
\end{tabular}


Exhibit 8-5. FedEx shipping list by lab number

\begin{tabular}{|c|c|c|c|c|c|c|c|}
\hline \multicolumn{8}{|c|}{2013 FedEx by Laboratory Number } \\
\hline LAB & VESSEL & TEST & $\mathbf{L A B}$ & CONDITIONS & $\begin{array}{c}\text { Dry } \\
\text { Ice } \\
\text { (Lbs.) } \\
\end{array}$ & $\begin{array}{c}\text { Dry } \\
\text { Ice } \\
\text { (Kg.) }\end{array}$ & Weight \\
\hline \multirow[b]{2}{*}{2} & 122 & Hep Profile & \multirow{2}{*}{$\begin{array}{l}\text { CDC/NCID } \\
\text { Hepatitis Ref } \\
\text { Lab }\end{array}$} & \multirow[b]{2}{*}{ Frozen } & \multirow[b]{2}{*}{20} & \multirow[b]{2}{*}{9} & \multirow[b]{2}{*}{25} \\
\hline & 87 & Hepatitis $\mathrm{B}_{\mathrm{s}} \mathrm{Ab}$ & & & & & \\
\hline 3 & 22 & HIV & $\begin{array}{l}\text { CDC/NCID } \\
\text { AIDS Division }\end{array}$ & Frozen & 20 & 9 & 25 \\
\hline 4 & 54 & VOC Blood & $\begin{array}{l}\text { CDC/NCEH } \\
\text { Air Toxicant } \\
\text { Branch }\end{array}$ & Refrigerated & & & 10 \\
\hline $4 a$ & 150 & Aldehyde & $\begin{array}{l}\text { CDC/NCEH } \\
\text { Air Toxicant } \\
\text { Branch } \\
\end{array}$ & Refrigerated & & & 10 \\
\hline \multirow{2}{*}{5} & 46 & Chlam/Trich & \multirow{2}{*}{$\begin{array}{l}\text { CDC/NCID } \\
\text { NCHSTD }\end{array}$} & \multirow{2}{*}{ Frozen } & \multirow{2}{*}{20} & \multirow{2}{*}{9} & \multirow[t]{2}{*}{25} \\
\hline & 154 & Trichomonas & & & & & \\
\hline \multirow{25}{*}{6} & 1 & $\mathrm{Lead} / \mathrm{Cad} / \mathrm{HG} / \mathrm{Se} / \mathrm{Mn}$ & \multirow{25}{*}{ CASPIR } & \multirow{25}{*}{ Frozen } & \multirow{25}{*}{30} & \multirow{25}{*}{14} & \multirow{25}{*}{35} \\
\hline & 3 & RBC Folate & & & & & \\
\hline & 5 & Mercury & & & & & \\
\hline & 88 & Acrylamide & & & & & \\
\hline & 136 & $\mathrm{Se} / \mathrm{Cu} / \mathrm{Zn}$ & & & & & \\
\hline & 110 & Folate1 & & & & & \\
\hline & 70 & Vit D/FA & & & & & \\
\hline & 17 & Cotinine & & & & & \\
\hline & 12 & Vit $\mathrm{B}_{12} / \mathrm{MMA}$ & & & & & \\
\hline & 137 & Testosterone/Estradiol/SHBG & & & & & \\
\hline & 32-37 & Xtra Sera & & & & & \\
\hline & $29 / 89$ & Persis Pest1 & & & & & \\
\hline & $92 / 93$ & BFR1 & & & & & \\
\hline & 48 & Prior Pest & & & & & \\
\hline & 49 & Organophos & & & & & \\
\hline & 86 & Spc Arsenic & & & & & \\
\hline & 62 & Urn Merc/Iodine & & & & & \\
\hline & 50 & Heavy Metal & & & & & \\
\hline & 66 & PAH & & & & & \\
\hline & 102 & Perchlorate & & & & & \\
\hline & 156 & TSNA & & & & & \\
\hline & 128 & Caffeine & & & & & \\
\hline & 152 & Aromatic Amines & & & & & \\
\hline & 151 & Volatile Nitrosamines & & & & & \\
\hline & 153 & HAA/Nicotine & & & & & \\
\hline
\end{tabular}


Exhibit 8-5. FedEx shipping list by lab number (continued)

\begin{tabular}{|c|c|c|c|c|c|c|c|}
\hline \multicolumn{8}{|c|}{2013 FedEx by Laboratory Number } \\
\hline LAB & VESSEL & TEST & \multirow[t]{2}{*}{ LAB } & \multirow[t]{2}{*}{ CONDITIONS } & \multirow[t]{2}{*}{$\begin{array}{c}\text { Dry } \\
\text { Ice } \\
\text { (Lbs.) } \\
\end{array}$} & \multirow[t]{2}{*}{$\begin{array}{c}\text { Dry } \\
\text { Ice } \\
\text { (Kg.) } \\
\end{array}$} & \multirow[t]{2}{*}{ Weight } \\
\hline & $52 / 139$ & Xtra Urine & & & & & \\
\hline 9 & 24 & $\mathrm{HSV}$ & $\begin{array}{l}\text { Emory } \\
\text { University }\end{array}$ & Frozen & 20 & 9 & 25 \\
\hline 10 & 4 & Glycohem & $\begin{array}{l}\text { University of } \\
\text { Missouri }\end{array}$ & Refrigerated & & & 10 \\
\hline \multirow{3}{*}{10} & 23 & Insulin & \multirow{3}{*}{$\begin{array}{l}\text { NHANES } \\
\text { Diabetes } \\
\text { Laboratory }\end{array}$} & \multirow{3}{*}{ Frozen } & \multirow{3}{*}{30} & \multirow{3}{*}{14} & \multirow{3}{*}{35} \\
\hline & 9 & Glucose & & & & & \\
\hline & 98 & OGTT & & & & & \\
\hline 16 & 45 & Alb/Creat & $\begin{array}{l}\text { University of } \\
\text { Minnesota }\end{array}$ & Frozen & 20 & 9 & 25 \\
\hline \multirow[b]{2}{*}{24} & 90 & Perfluor & \multirow{2}{*}{$\begin{array}{l}\mathrm{CDC} / \mathrm{NCEH} \\
\text { Environmental } \\
\text { Health Lab }\end{array}$} & \multirow[b]{2}{*}{ Frozen } & \multirow[b]{2}{*}{30} & \multirow[b]{2}{*}{14} & \multirow[b]{2}{*}{35} \\
\hline & 67 & Phthalates & & & & & \\
\hline \multirow{2}{*}{37} & 78 & HPV, Female & \multirow{2}{*}{$\begin{array}{l}\text { CDC/Human } \\
\text { Papillomavirus } \\
\text { Lab }\end{array}$} & Room Temp & & & 1 \\
\hline & 140 & HPV, Male & & Refrigerated & & & 10 \\
\hline 40 & 18 & Biochem & $\begin{array}{l}\text { Collaborative } \\
\text { Laboratory } \\
\text { Services }\end{array}$ & Refrigerated & & & 10 \\
\hline 51 & 21 & Lipids & $\begin{array}{l}\text { NHANES } \\
\text { Lipid Lab }\end{array}$ & Frozen & 20 & 9 & 25 \\
\hline 52 & 77 & HPV Serum & $\begin{array}{l}\text { CDC/DVRD } \\
\mathrm{HPV}\end{array}$ & Frozen & 20 & 9 & 25 \\
\hline 65 & 127 & IgA-TTG/EMA & Mayo Clinic & Frozen & 20 & 9 & 25 \\
\hline 67 & 129 & HPV Rinse & $\begin{array}{l}\text { Gillison } \\
\text { Laboratory, } \\
\text { OSU }\end{array}$ & Refrigerated & & & 10 \\
\hline \multirow[b]{2}{*}{120} & 155 & Fluoride & \multirow{2}{*}{$\begin{array}{l}\text { Georgia } \\
\text { Health } \\
\text { Systems } \\
\end{array}$} & \multirow[t]{2}{*}{ Frozen } & \multirow[t]{2}{*}{30} & \multirow[t]{2}{*}{14} & \multirow[t]{2}{*}{35} \\
\hline & 144 & Fluoride & & & & & \\
\hline
\end{tabular}

Gray fill - Ship Refrigerated

Bold-Ship at end of stand or when full.

Blood

Urine

Other 


\section{HPV SWAB PROCESSING}

\subsection{Introduction}

The purpose of collecting vaginal and penile swabs is to obtain suitable biological specimens to test for human papillomavirus (HPV). Human papillomavirus infection is one of the most common sexually transmitted infections in the United States. Cervical infection with certain types of HPV is a major risk factor for cervical cancer in women. The "high-risk" types of HPV (e.g., HPV 16, 18, 31, 33, $35,39,45,51,52,56,58,59,68)$ are associated with cervical cancer, and the "low-risk" types (e.g., HPV $6,11,42.43,44)$ with genital warts. No national surveillance system exists to measure the full burden of HPV infection, and no reliable national population estimate of HPV exists. Therefore, NHANES offers a unique opportunity to assess the prevalence of HPV infection in the general population.

Reducing the prevalence of HPV infection is a Developmental Healthy People 2010 objective: "Reducing the number of new HPV cases can help minimize the overall number of cases of high-risk subtypes associated with cervical cancer in females." Detection and typing of HPV DNA in vaginal and penile swabs (in conjunction with testing of NHANES sera for HPV 16 antibody) will allow evaluation of trends in prevalence of type-specific HPV infection by age, sexual behavior, and race/ethnicity. Studies have demonstrated that recovery of HPV from self-collected swabs is comparable to that from physician-collected cervical and penile samples.

HPV vaccine is available, and knowledge of the national prevalence of HPV infection will be critical for planning vaccination strategies in the United States. HPV vaccine is recommended for females from 11 to 26 years of age to prevent four common HPV types (two that cause most genital warts and two that cause cancers, including vaginal, penile, and anal cancers). It protects against new HPV infections; however, it does not cure existing HPV infections or disease.

The prevalence of current HPV infection among males in the U.S. is unknown. Data on DNA prevalence will be increasingly important as vaccine is implemented. DNA prevalence data are needed to improve baseline assumptions for models evaluating the impact of a prophylactic HPV vaccine on herd immunity and transmission of HPV 6,11, 16, and 18, and to monitor the impact of HPV vaccine on prevalence of vaccine as well as nonvaccine types to identify trends indicative of type-replacement or vaccine failures. The objectives of this component are to estimate current HPV infection in the U.S. male population 14-59 years old; to describe characteristics of men with HPV infection; and provide baseline estimates to measure impact of prophylactic vaccines. 
For females, the HPV Hybrid Capture ${ }^{\circledR}$ II DNA results will be reported to survey participants via the same reporting system as for STD/HIV testing. This system is a respondent-initiated toll-free phone call to NCHS. Results are given to persons tested after a password is provided to verify identity. The HPV-16 serology and the HPV DNA typology will not be reported.

For males, the Roche Linear Array HPV Genotyping results will not be reported to the survey participant via the same reporting system as for STD/HIV testing. If the participant explicitly requests the results for the HPV testing when calling for other STD test results by phone, the health educator will explain that HPV tests take several months to process. If the participant is willing to wait, results will be mailed at a later date.

Primary female and male SPs aged 14-59 years collect the HPV swab. The physician prints the appropriate label. Each label includes the test name, 6-digit SP ID concatenated with the vessel ID, and the corresponding barcode. The physician counsels each eligible SP and introduces her to the vaginal collection technique and him to the penile collection technique using a prepared collection kit.

The female collection kit is designed for SPs aged 14-59 years and includes illustrated collection instructions and one Epicentre ${ }^{\circledR}$ Dacron swab with foam tip. The physician removes the appropriate swab from the collection kit and labels the Epicentre ${ }^{\circledR}$ Dacron swab with the foam tip with the label displaying the vessel ID \#78. The physician then places the swab(s) back in the collection kit and gives the collection kit to the eligible SP.

The male collection kit is designed for SPs aged 14-59 years and includes illustrated collection instructions. This kit also includes the Diagene specimen transport media (STM) and Dacron swab. The physician opens the package and places a label on the STM vial, which includes the SP ID and vessel ID 140. The physician then places the swab(s) back in the collection kit and gives the collection kit to the eligible SP.

The SP self-collects the appropriate swab in the mobile examination center (MEC) bathroom, places the swab back in its original container, and places the swab back in the plastic bag. The SP hands the plastic bag containing the swab(s) to the assistant coordinator. The assistant coordinator delivers the plastic bag to the laboratory.

The laboratory staff verifies the SP ID and the vessel ID (\#78) on the label of the Epicentre ${ }^{\circledR}$ Dacron swab with the foam tip, records the collection in the HPV module, and stores the swab at room temperature. For vessel 140, the laboratory staff verifies the SP ID and correct vessel number on the 
Digene STM vial and stores it in a 2 -inch box with a $7 \times 7$ grid, in the refrigerator. The laboratory staff ships the specimens to the CDC laboratory weekly.

The laboratory staff also processes serum for vessel 77 (HPV Serum) on SPs aged 14-59 as part of an SP's blood processing profile. The vessels are stored at $-20^{\circ} \mathrm{C}$ and shipped to the $\mathrm{CDC} / \mathrm{Human}$ Papillomavirus Laboratory weekly.

\subsection{Supplies}

The supplies needed for HPV processing and storage are shown in Exhibit 9-1.

Exhibit 9-1. Equipment and supplies - HPV processing and storage

\begin{tabular}{|l|l|}
\hline Large padded envelope & Zip closable bag (9” x 12" clear) \\
\hline $7 \mathrm{x} 7$ three inch box & \\
\hline
\end{tabular}

\subsection{Protocol}

Exhibit 9-2 illustrates the SP's protocol for HPV processing. It is extremely important to follow the processing procedures outlined in these protocols and in this manual. Specimen loss or bias results will occur if the exact procedures are not followed.

Exhibit 9-2. HPV processing protocol

\begin{tabular}{|c|c|c|c|c|l|c|}
\hline ID & Test name & Ages & Sample & Sample type & Vessel type & Appointment type \\
\hline 78 & HPV & $14-59$ females & 1 swab & Vaginal swab & $\begin{array}{l}\text { Epicentre }{ }^{\circledR} \\
\text { Dacron swab }\end{array}$ & Primary only \\
\hline 140 & HPV & $14-59$ males & 1 swab & Penile swab & $\begin{array}{l}\text { Digene transport } \\
\text { media }\end{array}$ & Primary only \\
\hline
\end{tabular}




\subsection{Labeling Vessels}

The physician prints the vessel label. It is possible to reprint the label in the lab using the Ad Hoc functionality.

\subsection{HPV Protocol}

Process male and female HPV swabs and verify that the swabs are labeled correctly and that the label is legible.

Access the HPV Processing module for all SPs.

- Vessel 78 - HPV (female)

- $\quad$ Verify that the tip of the Dacron swab in the clear plastic tube is foam. Verify that the SP ID on the label matches the SP ID on the processing screen. Record the processing results in the HPV module and store the swab at room temperature.

- Vessel 140 - HPV (male)

- $\quad$ Record the processing results in the HPV module and store the swab in the refrigerator.

\subsection{Record Processing Results}

The protocol includes a serum vessel (HPV Serum \#77) for primary SPs aged 14-59. The volume requirement is $0.5-\mathrm{mL}$ of serum; the storage vessel is a 2-mL cryovial, and it is stored in a 2-inch box with a 9 x 9 grid. The vessel is processed after the Lipids vessel (\#21) and before the Biochem vessel (\#18.) The serum vessel is displayed on the blood-processing screen in the correct priority and is recorded in the same manner as the other vessels. This vessel is frozen and shipped to CDC/DVRD HPV weekly. 
Select the correct SP and access the Blood Processing module.

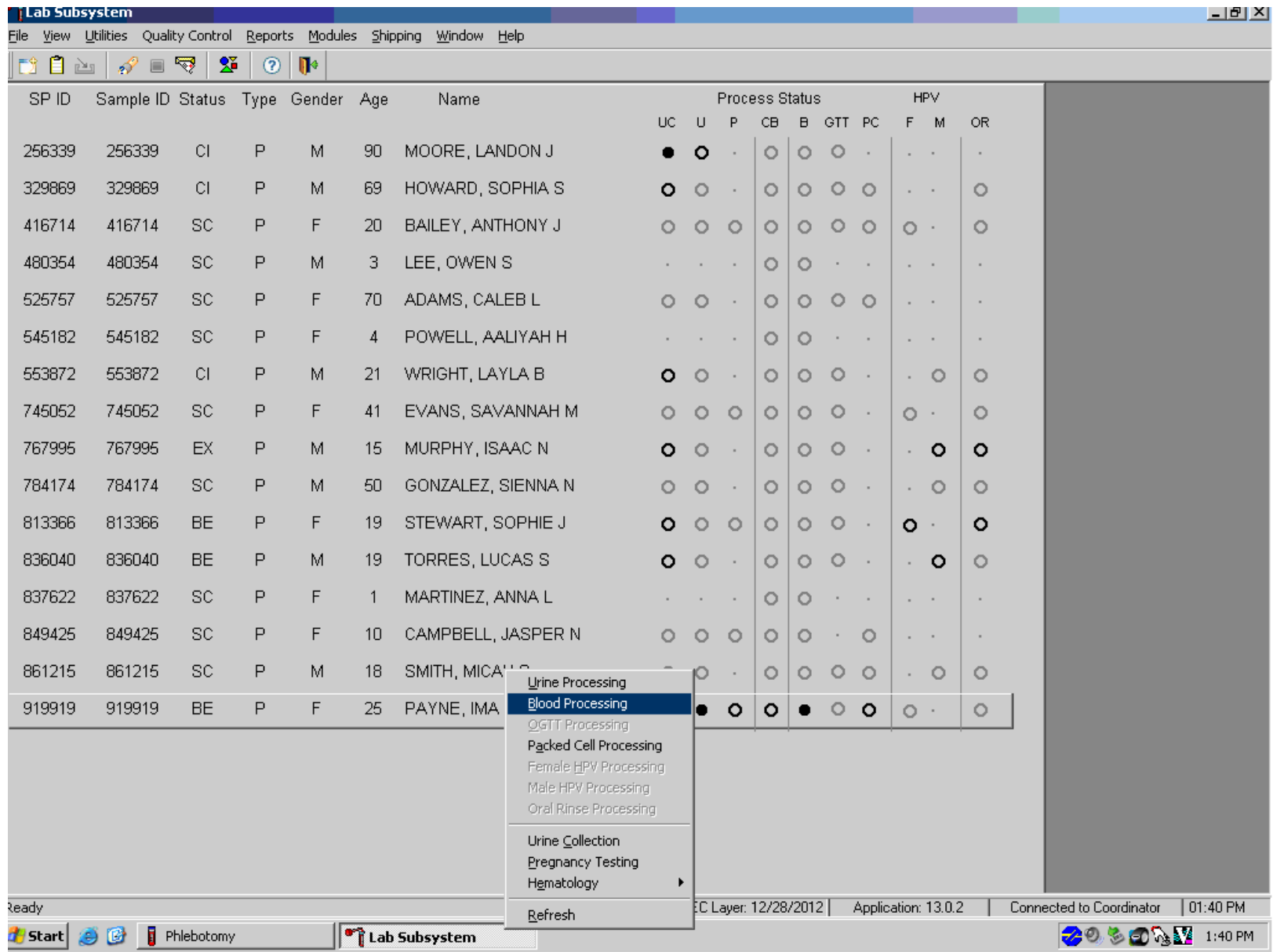

To select an SP, use the up and down keys to move up and down the list until the correct SP is highlighted, or drag the mouse arrow to the correct SP and right click. To access the Blood Processing module, drag the mouse arrow to \{Blood Processing\}, and left click or left click and type [B/b]. 
Record processing results for vessel 77 (HPV Serum) in the Blood Processing module.

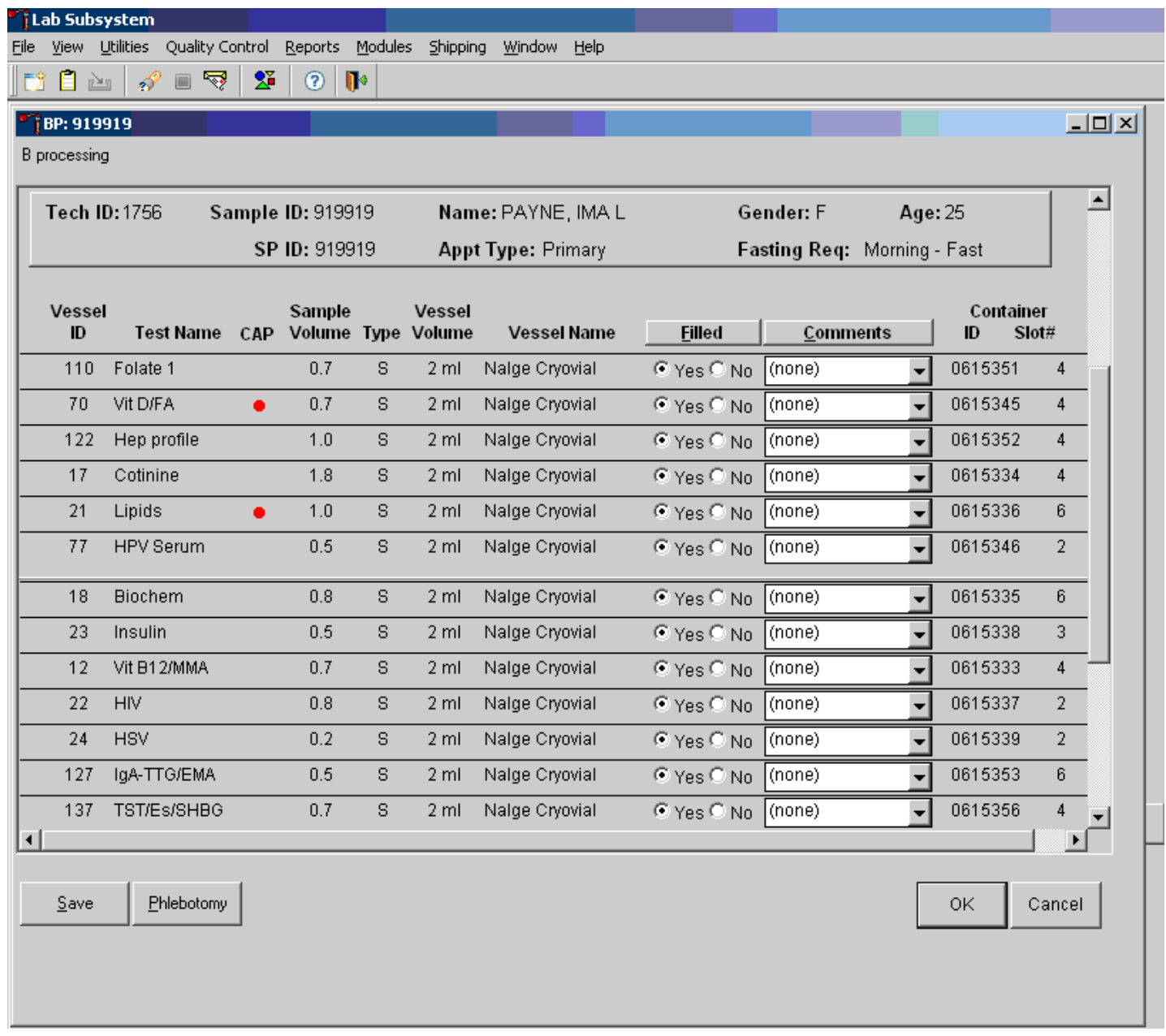

Primary SPs aged 14-59 are eligible for the HPV serum test. Pool the serum and aliquot 0.5-mL into a prelabeled 2.0-mL cryovial. Mark the vessel as Filled-"Yes" or Filled-"No" using procedures described in Chapter 8. Store the vessels in a 2-inch tall container with a $9 \times 9$ grid. Pack the container in a small shipper, add sufficient dry ice, and ship weekly to CDC/DVRD HPV. Use existing procedures described in Chapter 9 to pack, label, and ship the shipper. 
Select the correct SP and access either the Female or Male HPV Processing module.

\section{Female HPV Module}

Tab Subsystem

تile View utilities Quality Control Reports Modules shipping Window Help

\begin{tabular}{|c|c|c|c|c|c|c|c|c|c|c|c|c|c|c|c|c|c|}
\hline 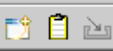 & ? $\square$ & प्र & (2) & 1 & & & & & & & & & & & & & \\
\hline \multirow[t]{2}{*}{ SP ID } & \multirow[t]{2}{*}{ Sample ID } & \multirow[t]{2}{*}{ Status } & \multirow[t]{2}{*}{ Type } & \multirow[t]{2}{*}{ Gender } & \multirow[t]{2}{*}{ Age } & \multirow{2}{*}{\multicolumn{2}{|c|}{ Name }} & \multicolumn{6}{|c|}{ Process Status } & \multicolumn{3}{|c|}{ HPV } & \multirow[b]{2}{*}{ OR } \\
\hline & & & & & & & & UC & $u$ & $P$ & $\mathrm{CB}$ & B & GTT & $\mathrm{PC}$ & $\mathrm{F}$ & M & \\
\hline 256339 & 256339 & $\mathrm{Cl}$ & $P$ & $M$ & 90 & \multicolumn{2}{|c|}{ MOORE, LANDON J } & - & 0 & . & 0 & 0 & 0 & . & . & . & . \\
\hline 329869 & 329869 & $\mathrm{Cl}$ & $\mathrm{P}$ & M & 69 & \multicolumn{2}{|c|}{ HOWARD, SOPHIA S } & 0 & 0 & . & 0 & 0 & 0 & 0 & . & . & 0 \\
\hline 416714 & 416714 & $\mathrm{SC}$ & $\mathrm{P}$ & $\mathrm{F}$ & 20 & \multicolumn{2}{|c|}{ BAILEY, ANTHONY J } & 0 & 0 & 0 & 0 & 0 & 0 & 0 & 0 & . & 0 \\
\hline 480354 & 480354 & $\mathrm{SC}$ & $\mathrm{P}$ & M & 3 & \multicolumn{2}{|l|}{ LEE, OWEN S } & . & . & . & 0 & 0 & . & . & . & . & . \\
\hline 525757 & 525757 & $\mathrm{SC}$ & $\mathrm{P}$ & $\mathrm{F}$ & 70 & \multicolumn{2}{|c|}{ ADAMS, CALEB L } & 0 & 0 & . & 0 & 0 & 0 & 0 & . & . & . \\
\hline 545182 & 545182 & $\mathrm{SC}$ & $\mathrm{P}$ & $\mathrm{F}$ & 4 & \multicolumn{2}{|c|}{ POWELL, AALIYAH H } & . & . & . & 0 & 0 & . & . & . & . & . \\
\hline 553872 & 553872 & $\mathrm{Cl}$ & $P$ & M & 21 & \multicolumn{2}{|c|}{ WRIGHT, LAYLA B } & 0 & 0 & . & 0 & 0 & 0 & . & . & 0 & 0 \\
\hline 745052 & 745052 & $\mathrm{SC}$ & $\mathrm{P}$ & $\mathrm{F}$ & 41 & \multicolumn{2}{|c|}{ EVANS, SAVANNAH M } & 0 & 0 & 0 & 0 & 0 & 0 & . & 0 & . & 0 \\
\hline 767995 & 767995 & EX & $\mathrm{P}$ & M & 15 & \multicolumn{2}{|c|}{ MURPHY, ISAAC N } & 0 & 0 & . & 0 & 0 & 0 & . & . & 0 & 0 \\
\hline 784174 & 784174 & $\mathrm{SC}$ & $\mathrm{P}$ & M & 50 & \multicolumn{2}{|c|}{ GONZALEZ, pirsin in si } & - & 0 & . & 0 & 0 & 0 & . & . & 0 & 0 \\
\hline 813366 & 813366 & $\mathrm{BE}$ & $P$ & $\mathrm{~F}$ & 19 & STEWART, $\subseteq$ & \multirow{2}{*}{\multicolumn{2}{|c|}{$\begin{array}{l}\text { Blood Processing } \\
\text { ogTT Processing } \\
\text { Packed Cell Processing }\end{array}$}} & 0 & 0 & 0 & 0 & 0 & . & 0 & . & 0 \\
\hline 836040 & 836040 & $\mathrm{BE}$ & $\mathrm{P}$ & M & 19 & $\overline{\text { TORRES, LU }}$ & & & 0 & . & 0 & 0 & 0 & . & . & 0 & 0 \\
\hline 837622 & 837622 & $\mathrm{SC}$ & $\mathrm{P}$ & $\mathrm{F}$ & 1 & MARTINEZ, $A$ & \multicolumn{2}{|l|}{ Female HPV Processing } & . & . & 0 & 0 & · & . & . & . & . \\
\hline 849425 & 849425 & $\mathrm{SC}$ & $P$ & $\mathrm{~F}$ & 10 & CAMPBELL, & $\begin{array}{l}\text { Male HPY Processing } \\
\text { Oral Rinse Processing }\end{array}$ & & 0 & 0 & 0 & 0 & . & 0 & . & . & . \\
\hline 861215 & 861215 & $\mathrm{SC}$ & $\mathrm{P}$ & M & 18 & SMITH, MICA & Pregnancy Testing & & 0 & . & 0 & 0 & 0 & 0 & . & 0 & 0 \\
\hline \multirow[t]{2}{*}{919919} & \multirow[t]{2}{*}{919919} & \multirow[t]{2}{*}{$\mathrm{BE}$} & \multirow[t]{2}{*}{$\mathrm{P}$} & \multirow[t]{2}{*}{$\mathrm{F}$} & 25 & PAYNE, IMA & Hematology & > & - & 0 & 0 & e & 0 & 0 & 0 & . & 0 \\
\hline & & & & & & & Refresh & & & & & & & & & & \\
\hline
\end{tabular}




\section{Male HPV Module}

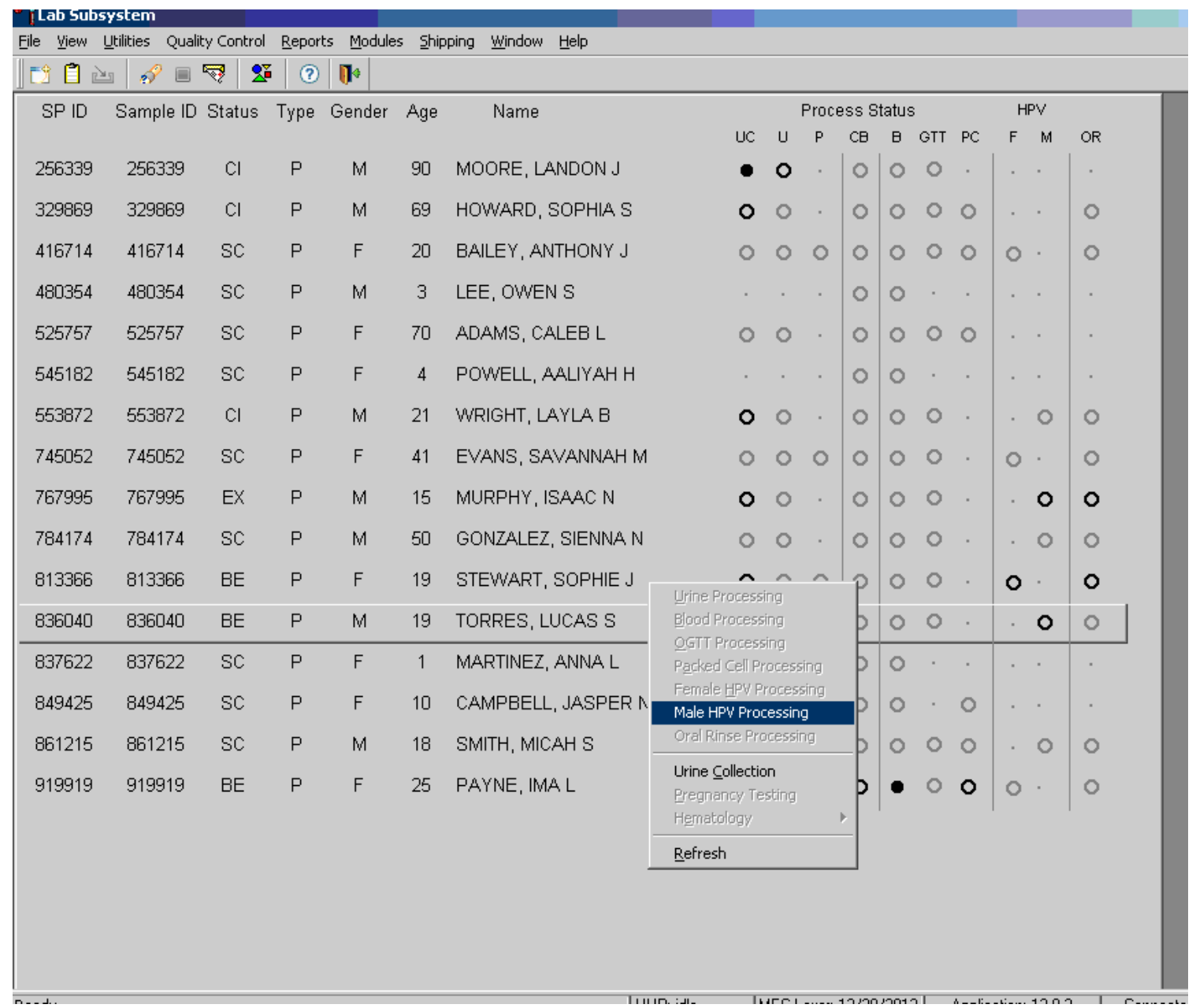

Use the scroll bar to view all SPs scheduled into the session. The active SP is contained in a rectangular box. Use the heads-up display to select the correct SP. Select a different SP if the active SP is not the correct SP.

To select an SP, use the up and down keys to move up and down the list until the correct SP is highlighted or drag the mouse arrow to the correct SP and right click. To access the HPV Processing module, drag the mouse arrow to \{HPV Processing $\}$ and left click or right click and type [S/s]. Alternatively, use the up and down keys to move up and down the list until the correct SP is highlighted, right click, drag the mouse arrow to the correct module, and left click or type $[\mathrm{S} / \mathrm{s}]$. 
Document processing results.

Female HPV Swab Processing

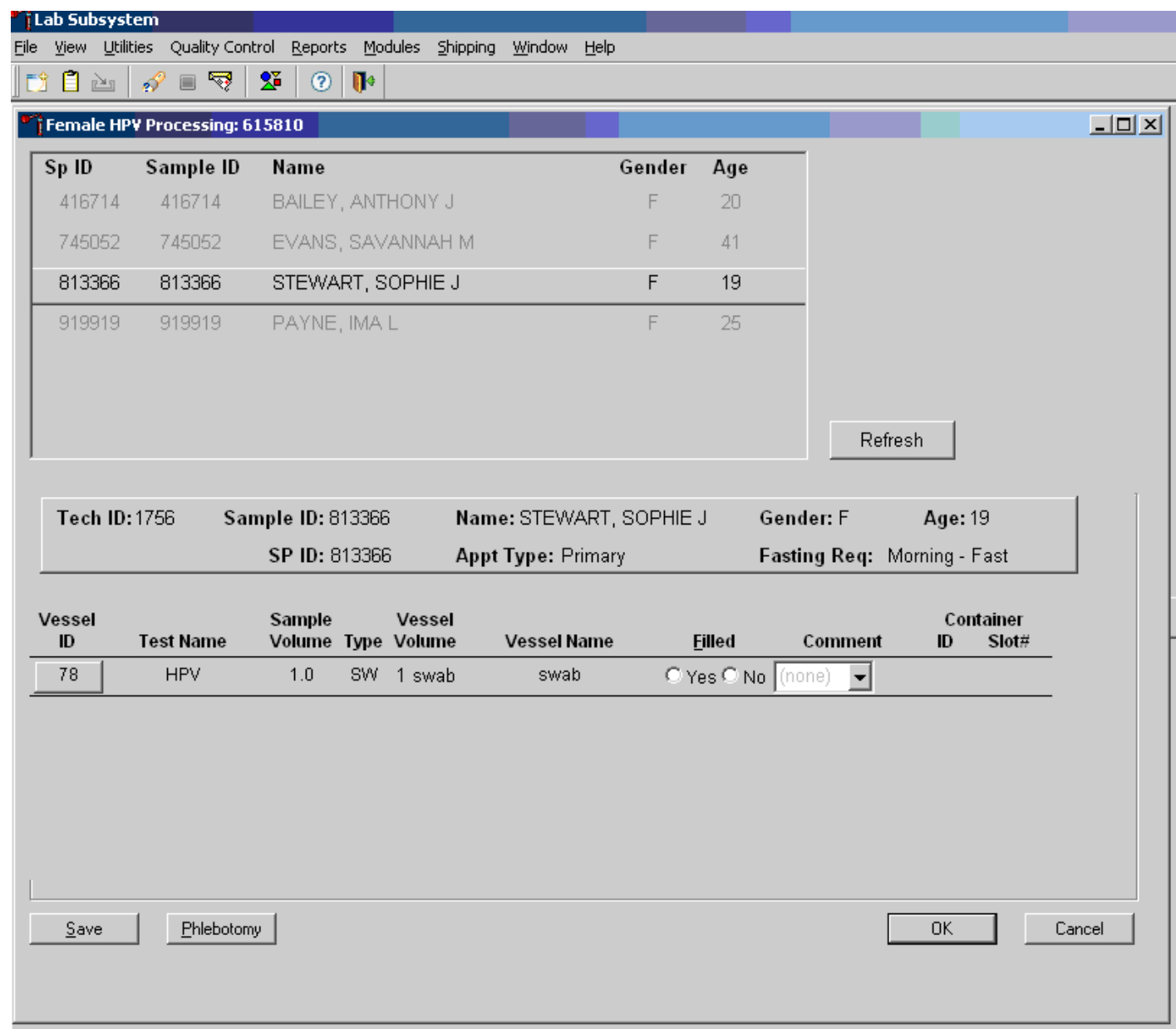


Male HPV Swab Processing

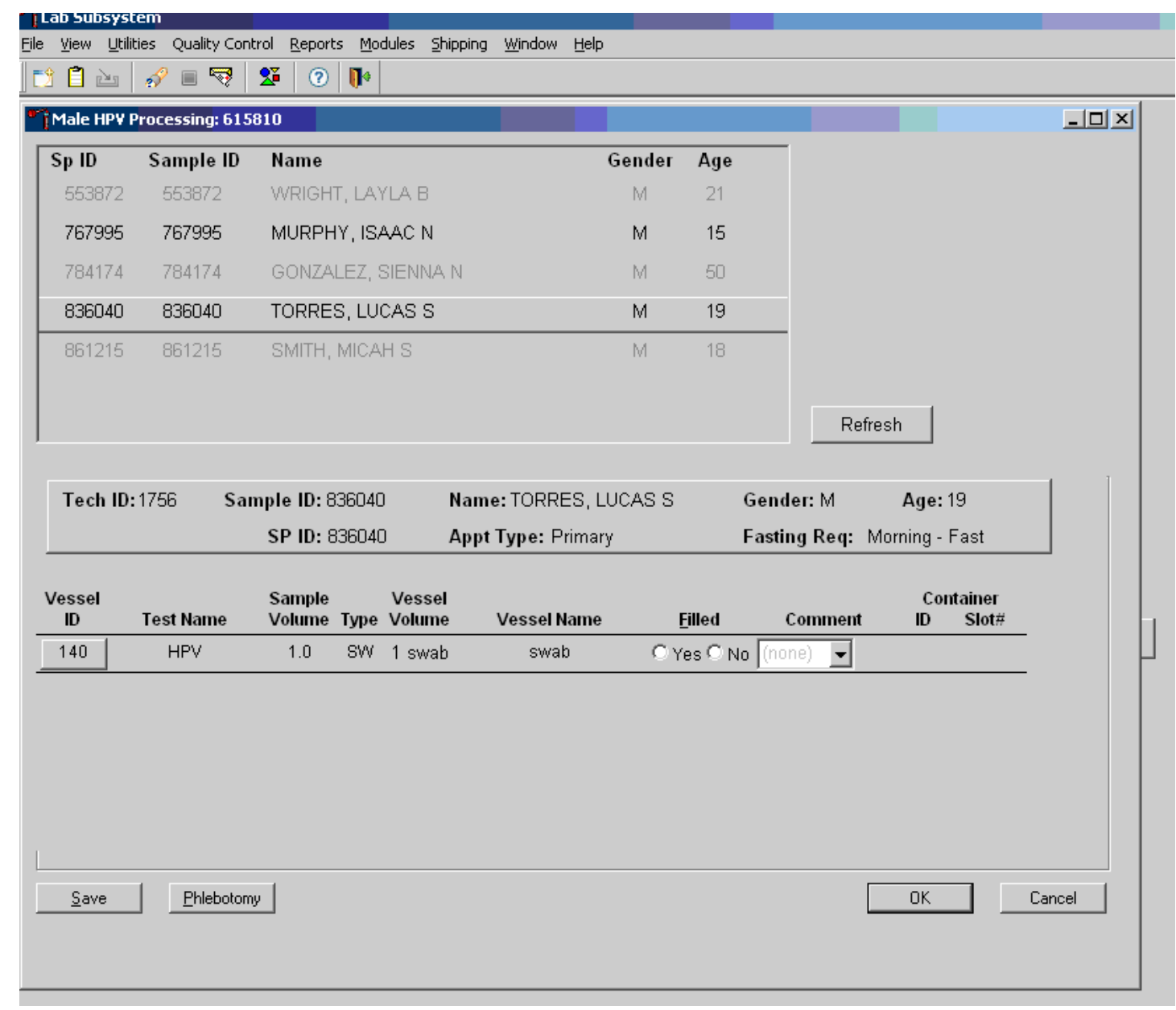

Both the Female and Male HPV Processing windows contains the following information for SPs: Tech ID, Sample ID, SP ID, Name (last, first), Gender, Age, App (appointment) type, and Fasting_Req. There are columns that list the Vessel ID, Test Name, Sample Volume, Sample Type (SW = swab), Vessel Volume, Vessel Name, Filled (Yes or No) radio buttons, Comment drop-down box, and a Container ID and Container Slot\# column. There is one row associated with vessel 78. Use radio buttons to record the processing results, Filled-"Yes" or Filled-"No." If necessary, attach a comment using the Comment drop-down box, and store the vessel in the assigned Container ID and Slot\#. 
Mark vessel 78 as Filled-"Yes" if the swab was collected.

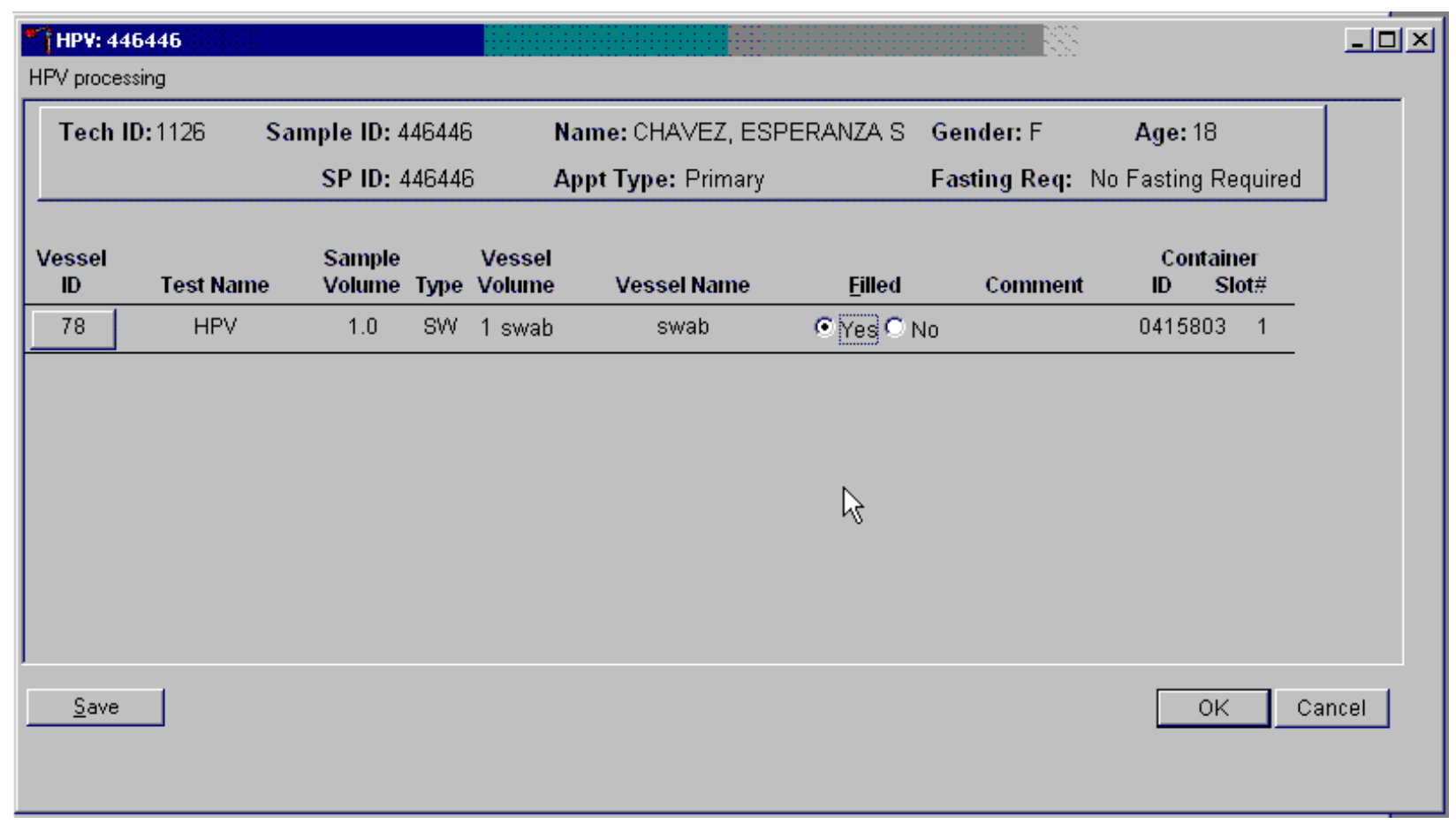

To mark vessel 78 as collected or Filled-"Yes," use the mouse to direct the mouse arrow to the "Yes" radio button and left click. As a vessel is marked as Filled-"Yes," it is automatically assigned to a slot in an existing (open) container. 
Mark vessel 78 as Filled-"No" if the swab was not collected.

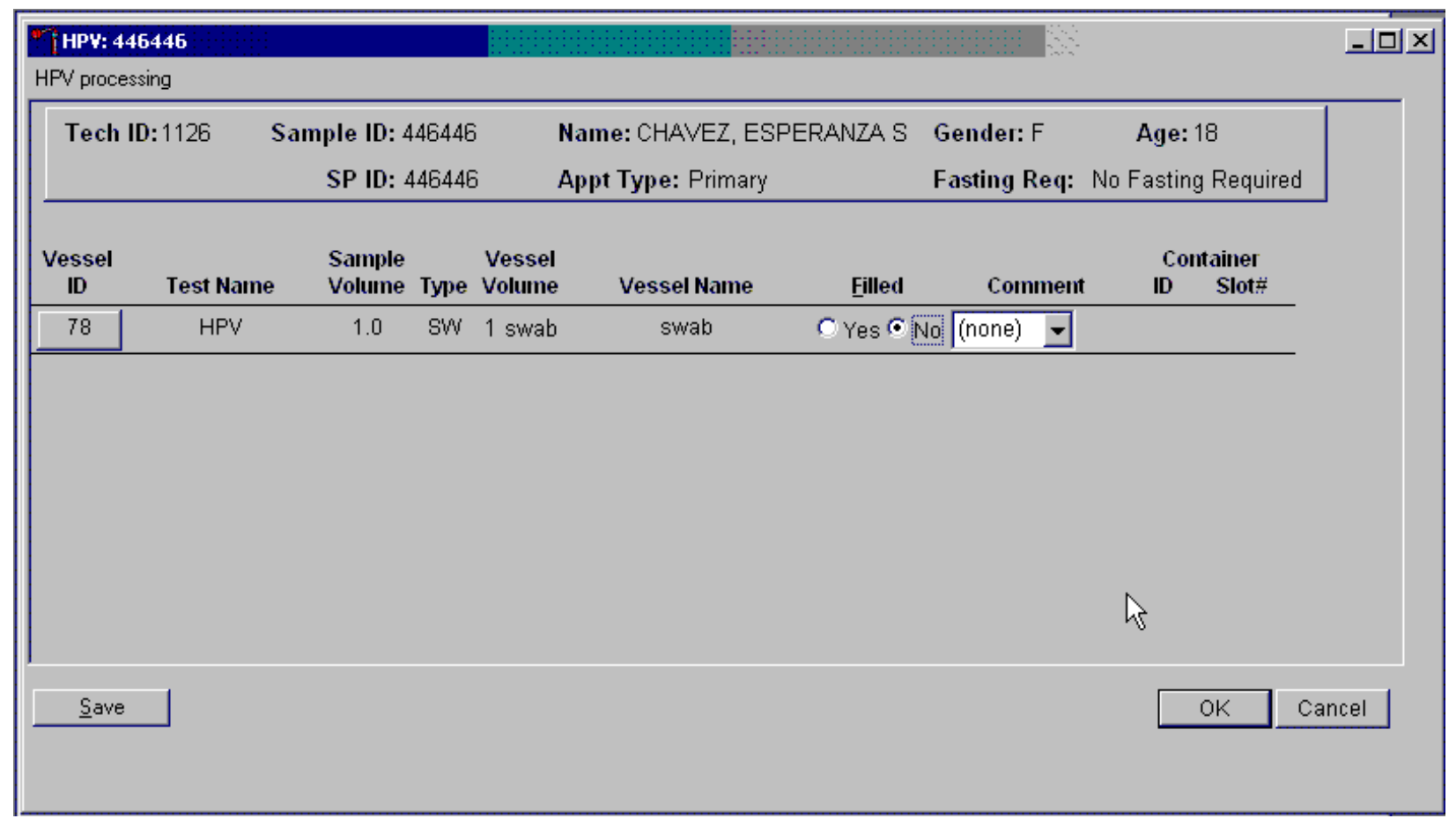

To mark vessel 78 as not collected or Filled-"No," use the mouse to direct the mouse arrow to the "No" radio button and left click. 
Enter a quality control comment for vessel 78 when the swab is dry and the judgment is that the SP did not attempt to collect the sample(s).

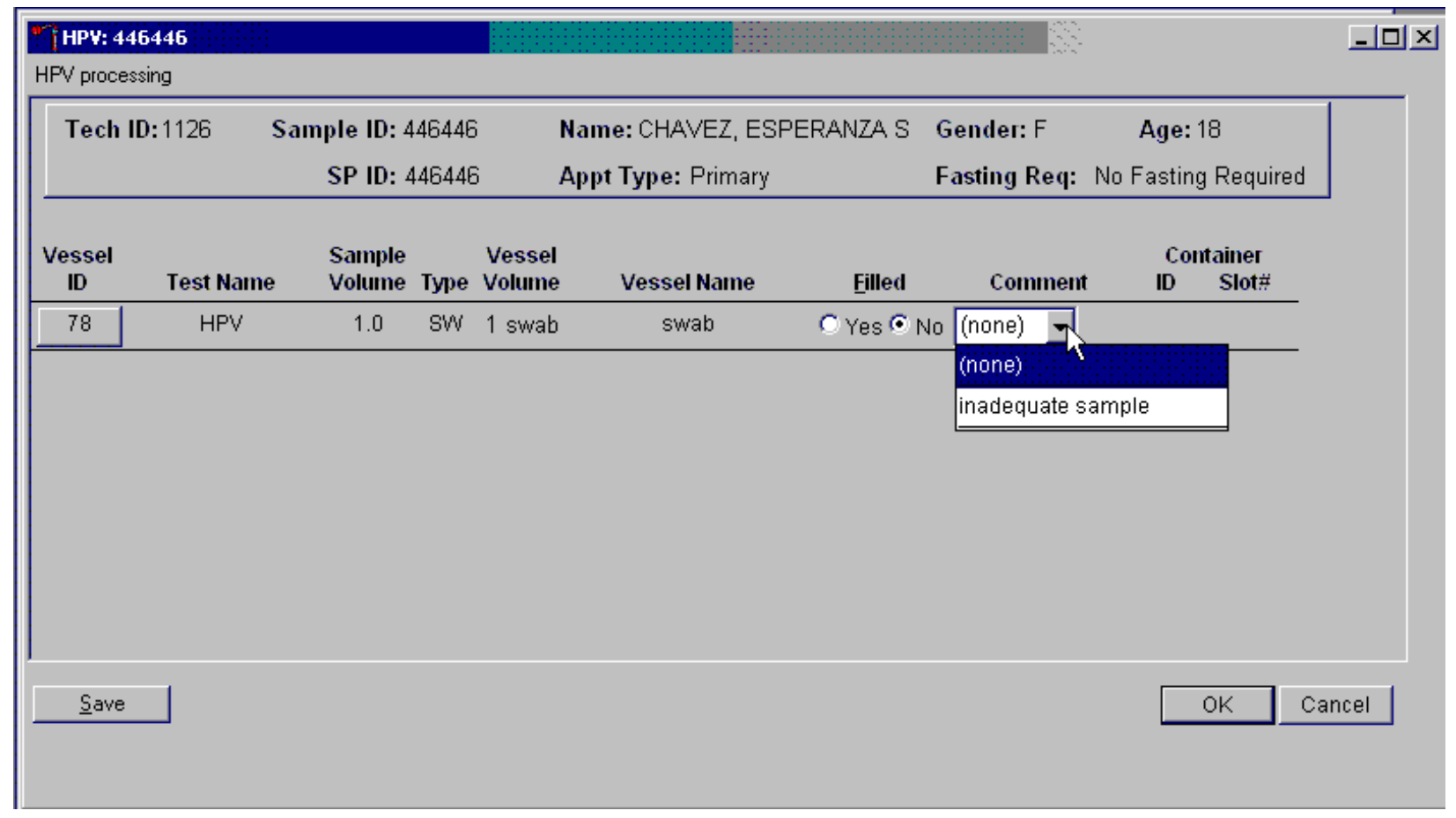

To record an "inadequate sample" comment, use the mouse to direct the mouse arrow to the drop-down list, click to display the comment codes, drag the mouse arrow to "inadequate sample," and left click. Alternatively, to record the comment "inadequate sample," [Tab] to advance to the Comment text box and type $[\mathrm{I} / \mathrm{I}]$ and [Enter]. 
Store the Epicentre ${ }^{\circledR}$ Dacron swab in the assigned 9" x 12" plastic bag at room temperature; review the information in the HPV-Processing window, and save the data to the database.

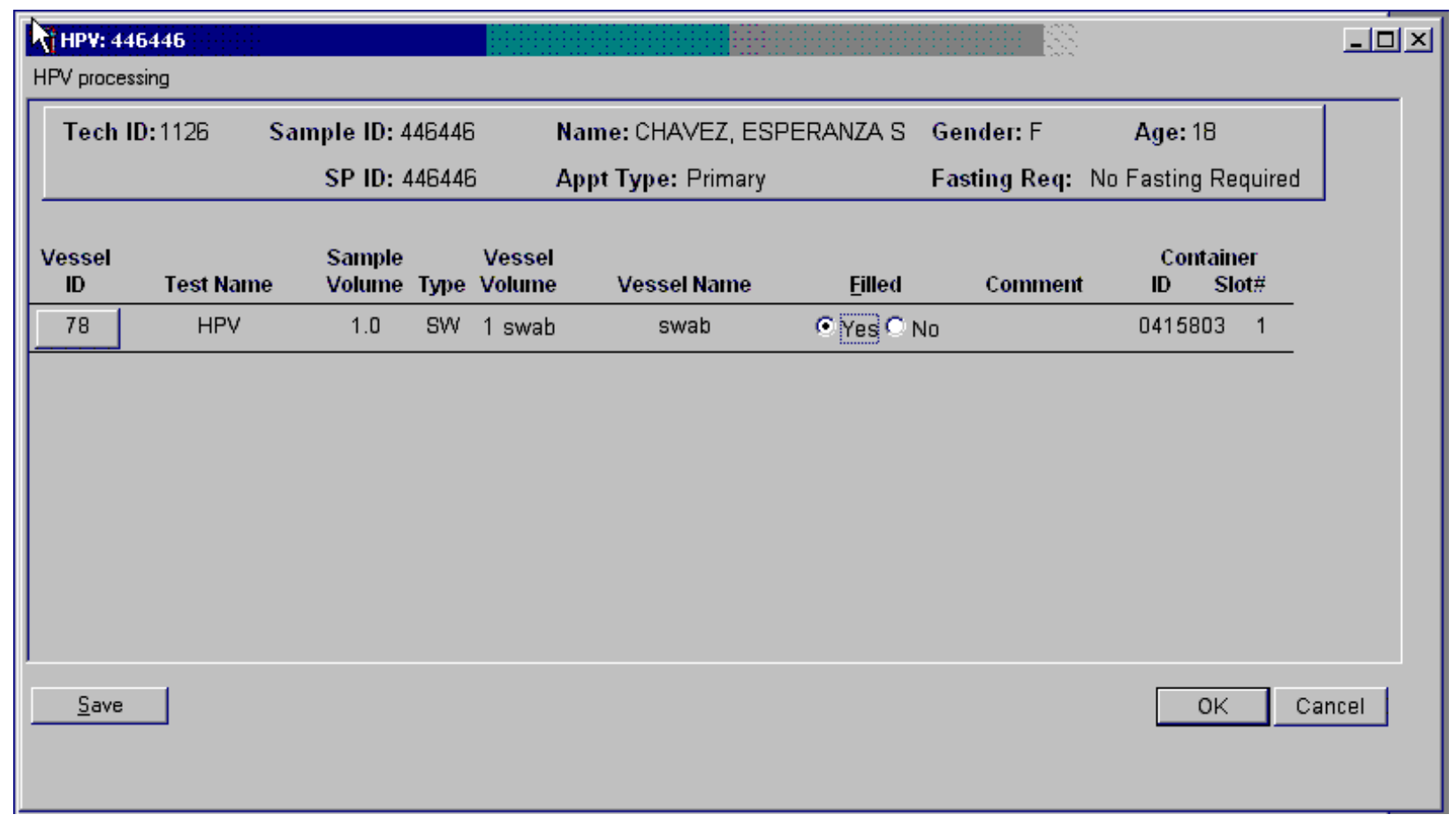

To record this action or to save the data to the database, use the mouse to direct the mouse arrow to the "Save" button and left click or type $[\mathrm{S} / \mathrm{s}]$. To record this action or to save the data to the database and to exit the module, use the mouse to direct the mouse arrow to the "OK" button and left click. To close the window without saving any data in the database, use the mouse to direct the mouse arrow to the "Cancel" button and left click. 
The process status circle in the heads-up display fills in completely for SPs whose vessel 78 is recorded as collected or Filled-"Yes." Do not enter a result until the swab arrives in the laboratory. At the end of each session, review the results for each SP. Access each record and enter the Filled-"No" result for all vessels for SPs for whom no swab was received.

\subsection{Specimen Storage}

Record the processing result for the Epicentre ${ }^{\circledR}$ Dacron swab and store the swab in a 9 " x 12 " plastic bag at room temperature. Store vessels in numbered storage containers according to each vessel as indicated in Exhibit 9-3.

Store each vessel in the appropriate slot in the correct container immediately after processing. Check the slot assignment of containers associated with vessel 78 at the end of each session by accessing the Container Map report. Verify the contents of each container against the container map.

Exhibit 9-3. Storage protocol for HPV

\begin{tabular}{|l|l|l|c|}
\hline \multicolumn{1}{|c|}{ Shipping Location } & \multicolumn{1}{c|}{ Vessel } & \multicolumn{1}{c|}{ Condition } & Vessel Storage \\
\hline CDC/DVRD HPV & 77 HPV Serum & Frozen & $9 \times 9$ two-inch box \\
\hline $\begin{array}{l}\text { CDC/Human Papillomavirus } \\
\text { Laboratory }\end{array}$ & 78 HPV Swab, female & Room temperature & 9 " 12 " plastic bag \\
\hline $\begin{array}{l}\text { CDC/Human Papillomavirus } \\
\text { Laboratory }\end{array}$ & 144 HPV Swab, male & Refrigerated & $7 \times 7$ three-inch box \\
\hline
\end{tabular}

\subsection{Shipping Procedures}

Ship vessel 77 and 144 according to procedures described in Section 8.13. Ship vessel 78 in a padded envelope at room temperature. Include a copy of a shipping manifest, which contains a list of the items contained in the shipment and certain demographic information with each shipment. Exhibit 9-4, the Shipping protocol, illustrates the specifications for the shipment of biological specimens to each contract laboratory. 
Exhibit 9-4. Shipping protocol

\begin{tabular}{|l|c|l|l|l|}
\hline \multicolumn{1}{|c|}{ Laboratory } & Vessel & \multicolumn{1}{|c|}{ Test Name } & \multicolumn{1}{|c|}{ Vessel Storage } & Shipping Frequency \\
\hline CDC/DVRD HPV & 77 & HPV Serum & Frozen & Weekly \\
\hline $\begin{array}{l}\text { CDC/Human } \\
\text { Papillomavirus Laboratory }\end{array}$ & 78 & HPV Swab, female & Room Temperature & Weekly \\
\hline $\begin{array}{l}\text { CDC/Human } \\
\text { Papillomavirus Laboratory }\end{array}$ & 144 & HPV Swab, male & Refrigerated & Weekly \\
\hline
\end{tabular}

\subsubsection{Pack and Ship Vessel 78 at Room Temperature}

Ship the container (9" x 12" plastic bag) for vessel 78 (HPV swab) at room temperature.

- Place the plastic bag containing the specimens in a large padded envelope.

- Place the appropriate shipping manifest in a 9" x 12" plastic bag and seal.

- Place a plastic envelope containing the appropriate shipping manifest in the padded envelope and seal.

- Assume that the package weighs 1 pound.

- Record the total weight of the envelope (1 pound) on the appropriate FedEx label.

\subsubsection{Label Shippers}

All shippers must contain a FedEx barcoded label and appropriate orientation and warning labels. Label all shippers with the appropriate warning labels as follows:

- Complete the section of the FedEx label that requests the weight of the contents of the shipper.

- Place the FedEx label with the appropriate contract laboratory address in a plastic FedEx pouch and attach the window to the cardboard lid of the shipper.

- Add one additional strip of tape across the FedEx pouch. 
If the computer system fails, record results on a preprinted HPV Processing worksheet (Exhibit 9-5). Complete a workstation 2 worksheet for each SP while processing the HPV specimens. Enter the results after the system is operational. Send the worksheets to the home office at the end of the stand.

Exhibit 9-5. HPV Processing worksheet

HPV PROCESSING

SP ID

\begin{tabular}{|c|c|c|c|c|c|c|c|}
\hline ID & Name & $\begin{array}{c}\text { Ages } \\
\text { Gender }\end{array}$ & $\begin{array}{c}\text { Sample } \\
\text { mL }\end{array}$ & Sample Type & $\mathbf{p H}$ & $\begin{array}{c}\text { Filled } \\
\checkmark\end{array}$ & Slot \# \\
\hline 78 & HPV Swab, female & $\begin{array}{c}14-59 \\
\text { females }\end{array}$ & 1 swab & $\begin{array}{l}\text { Epicentre } \AA \text { Dacron } \\
\text { Swab }\end{array}$ & & & \\
\hline 144 & HPV Swab, male & $\begin{array}{l}14-59 \\
\text { males }\end{array}$ & $1 \mathrm{swab}$ & $\begin{array}{l}\text { Diagene Swab } \\
\text { w/ Transport Media }\end{array}$ & & & \\
\hline
\end{tabular}




\section{HPV ORAL RINSE PROCESSING}

\subsection{Introduction}

The overall goal of collecting an oral rinse is to perform the first population-based study of oral HPV infection to determine the prevalence and type of distribution of infection and to investigate the demographic and behavioral factors associated with infection.

Testing for 37 types of HPV will be accomplished by means of a multiple polymerase-chain reaction (PCR) assay targeted to the L1 region of the viral genome, using PGMY09/11 L1 primer pools and primers for Beta globulin followed by hybridization to a linear probe array (Roche Molecular.)

The HPV oral rinse is collected by the dental hygienist during the oral health exam on primary SPs aged 14-69 years. The SP is asked to swish a $10 \mathrm{~mL}$ sample of Scope mouthwash (or a sterile saline solution if preferred by the SP) in their mouth and then expectorate the sample into a sterile collection cup. The cap is secured on the cup, a label is attached, and the sample is then taken to the MEC laboratory for processing.

The hygienist places the cup in the MEC laboratory in the same area as the urine collection samples. The HPV oral rinse samples are easily distinguished from the urine collection samples by the color of the sample (blue-green unless the clear sterile saline solution is used) and the collection cup (clear and cylindrical with a screw-on metal cap) which is much smaller than the urine collection cup.

The MEC laboratory technologists process the sample by transferring the HPV oral rinse from the collection cup to a $14 \mathrm{~mL}$ Falcon snap cap tube. It is critical to transfer the entire sample from the collection cup to the processing vessel. The technologists use the OR (oral rinse) module to document collection status. All SPs are listed on one screen so the samples can be batch processed. They make sure that the snap cap is pressed down fully to prevent leakage during shipment.

The sample is stored frozen at -20 degrees Celsius and shipped weekly to Lab 67, Gillison Laboratory, 2001 Polaris PKWY \# 1320 OSU - Pathology Core Facility Columbus, OH 43240-2000 (614)293-5942. 


\subsection{Supplies}

The supplies needed for HPV Rinse processing and storage are listed in Exhibit 10-1.

Exhibit 10-1. Equipment and supplies - HPV processing and storage

\begin{tabular}{|l|l|}
\hline 14-mL snap cap test tube & Small Styrofoam shipper (14 x 14 x 12) \\
\hline Three-inch 5 x 5 cardboard grid & STP-310 Pressure vessel \\
\hline Absorbent sheets $(2 \times 2)$ & \\
\hline Packaging tape 2" & Plastic bag 9" x 12" \\
\hline Overpack label & \\
\hline Rubber bands & Dry ice \\
\hline
\end{tabular}

\subsection{Protocol}

Exhibit 10-2 illustrates the SP's protocol for HPV Rinse processing. It is extremely important to follow the processing procedures outlined in these protocols and in this manual. Specimen loss or bias results will occur if the exact procedures are not followed.

Exhibit 10-2. HPV Rinse processing protocol

\begin{tabular}{|c|c|c|c|c|c|c|}
\hline ID & Test name & Ages & Sample & Sample type & Vessel type & Appointment type \\
\hline 129 & HPV Rinse & $14-69$ & 1 & Oral rinse & 14-mL snap cap tube & Primary only \\
\hline
\end{tabular}




\section{$10.4 \quad$ Labeling Vessels}

The dental hygienist prints the label for the specimen cup. The medical technologist assigned to workstation 3 prints the vessel labels for all eligible SPs and places these labels at workstation 2. It is possible to reprint the vessel label in the lab using the ad hoc functionality.

\subsection{HPV Rinse Protocol}

Process the oral rinse for HPV Rinse. Verify that the specimen container is labeled correctly and that the label is legible.

Access the OR Processing module for all SPs.

- Vessel 129 - HPV Rinse

- $\quad$ Verify that the SP ID on the label matches the SP ID on the processing screen.

- $\quad$ Label the 14-mL Falcon snap cap tube.

- $\quad$ Pour the oral rinse from the specimen container into the Falcon tube.

- $\quad$ Snap the cap firmly on the Falcon tube.

- $\quad$ Record the processing results in the OR module.

- $\quad$ Store the vessel in a 3-inch tall box with a 5 x 5 grid.

- $\quad$ Store the container in the refrigerator.

- $\quad$ Ship weekly refrigerated. 
Open and log onto the Laboratory application. Use the heads-up display to view the SPs, the modules for which they are eligible, and their current process status. Record oral rinse processing results for vessel 129 using the "OR" module.

\begin{tabular}{|c|c|c|c|c|c|c|c|c|c|c|c|c|c|c|c|}
\hline \multicolumn{16}{|c|}{ Lab Subsystem } \\
\hline File view ut: & tilities Qualit: & ity Control & Report & ts Module: & $s$ Ship & ping Window Help & & & & & & & & & \\
\hline \multicolumn{16}{|c|}{$=\theta \circlearrowright$ ? } \\
\hline \multirow[t]{2}{*}{ SP ID } & \multirow[t]{2}{*}{ Sample ID } & \multirow[t]{2}{*}{ Status } & \multirow[t]{2}{*}{ Type } & \multirow[t]{2}{*}{ Gender } & \multirow[t]{2}{*}{ Age } & \multirow[t]{2}{*}{ Name } & \multicolumn{8}{|c|}{ Process Status } & \multirow[b]{2}{*}{$\mathrm{OR}$} \\
\hline & & & & & & & UC & $u$ & $\mathrm{P}$ & $O Z$ & $\mathrm{CB}$ & $B$ & GTT & HPV & \\
\hline 136701 & 136701 & SC & $P$ & $\mathrm{~F}$ & 60 & OBENDORFER, ED & 0 & 0 & . & 0 & 0 & 0 & 0 & . & 0 \\
\hline 224123 & 224123 & $\mathrm{SC}$ & $P$ & $\mathrm{~F}$ & 60 & HESSELS, JOEL & 0 & 0 & . & 0 & 0 & 0 & 0 & . & 0 \\
\hline 333280 & 333280 & SC & $\mathrm{P}$ & M & 91 & HESSELS, MARK & 0 & 0 & . & 0 & 0 & 0 & 0 & . & . \\
\hline 343133 & 343133 & $\mathrm{Cl}$ & $\mathrm{P}$ & $\mathrm{F}$ & 60 & OBENDORFER, KELLEY & 0 & 0 & . & 0 & 0 & 0 & 0 & . & 0 \\
\hline 588451 & 588451 & $B E$ & $\mathrm{P}$ & $\mathrm{F}$ & 20 & OBENDORFER, ANNMARIE & - & 0 & 0 & 0 & 0 & 0 & 0 & 0 & 0 \\
\hline 635859 & 635859 & $\mathrm{BE}$ & $P$ & $\mathrm{~F}$ & 60 & HESSELS, BENJAMMIN & $\bullet$ & 0 & . & 0 & 0 & 0 & 0 & . & 0 \\
\hline 737421 & 737421 & $\mathrm{BE}$ & $P$ & $\mathrm{~F}$ & 12 & OBENDORFER, JAMIE & 0 & 0 & 0 & 0 & 0 & 0 & 0 & - & $\cdot$ \\
\hline 743644 & 743644 & $B E$ & $\mathrm{P}$ & $\mathrm{F}$ & 60 & OBENDORFER, KIM & 0 & 0 & . & 0 & 0 & 0 & 0 & . & 0 \\
\hline 876427 & 876427 & $S C$ & $P$ & M & 70 & HUNT, JOE & 0 & 0 & . & 0 & 0 & 0 & 0 & . & . \\
\hline 932761 & 932761 & SC & $P$ & $\mathrm{~F}$ & 60 & HESSELS, JONAH & 0 & 0 & $\cdot$ & 0 & 0 & & 0 & . & 0 \\
\hline
\end{tabular}

The "OR" module is grayed out until at least one SP has the oral rinse collected in the oral health component. Once one SP has the oral rinse marked as collected, the processing circle for that SP turns black. 
Select the correct SP and access the Oral Rinse Processing module.

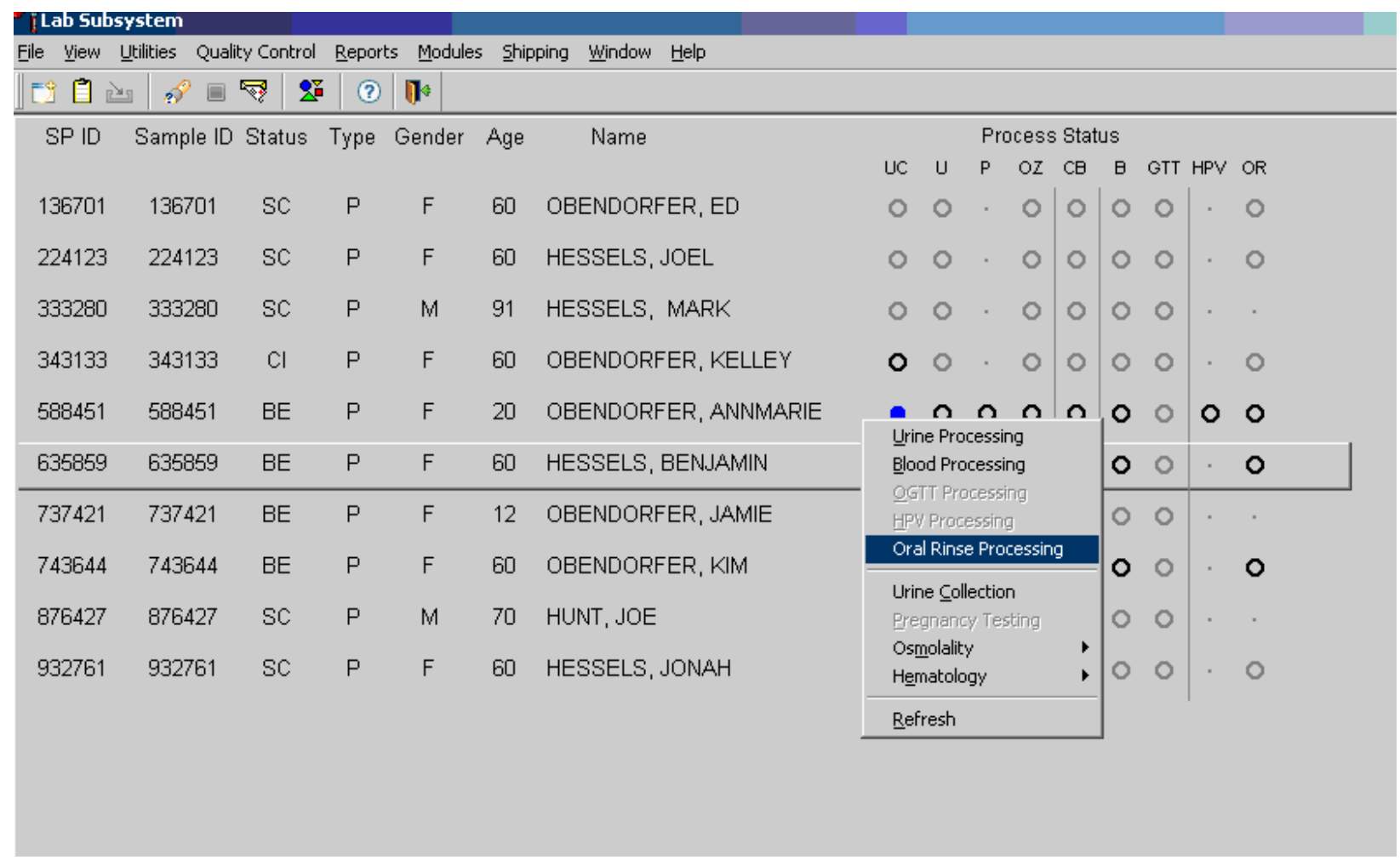

To access the OR module, use the mouse to direct the mouse arrow to the SP on the headsup display, left click, drag the mouse arrow to Oral Rinse Processing, and right click. 
Record the vessel 129 processing results in the Oral Rinse processing window. All SPs are included in the same window but are listed separately by row.

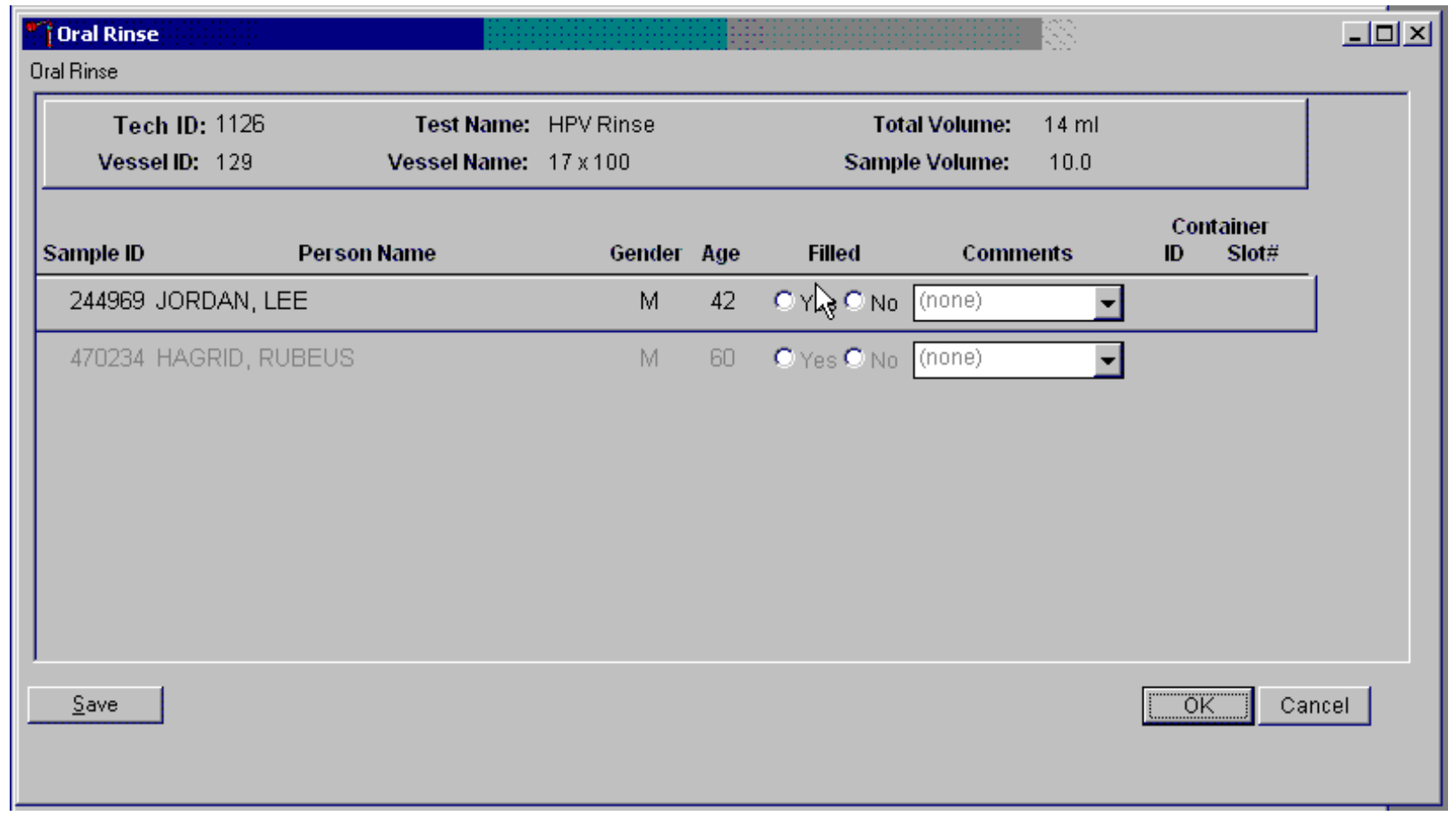

The Oral Rinse processing window lists the vessel requirements across the top. SPs are listed separately in rows. The upper section of the Oral Rinse window contains the following information: Tech ID, Vessel ID (129), Test Name (HPV Rinse), Total Volume (14 ml), Vessel Name (17 x 100), and Sample Volume (10.0.) The lower section of the window contains columns for Sample ID, Person Name (Last, First), Gender, Age, Filled Yes and No radio buttons, Comments text box, and a Container ID/Slot \# column N. Use the Save button to save the results to the database. Use the OK button to save the results to the database and close the module. Use the Cancel button to close the window without saving any data to the database. 
Record oral rinse processing results for each SP. Individually mark each vessel as Filled"Yes."

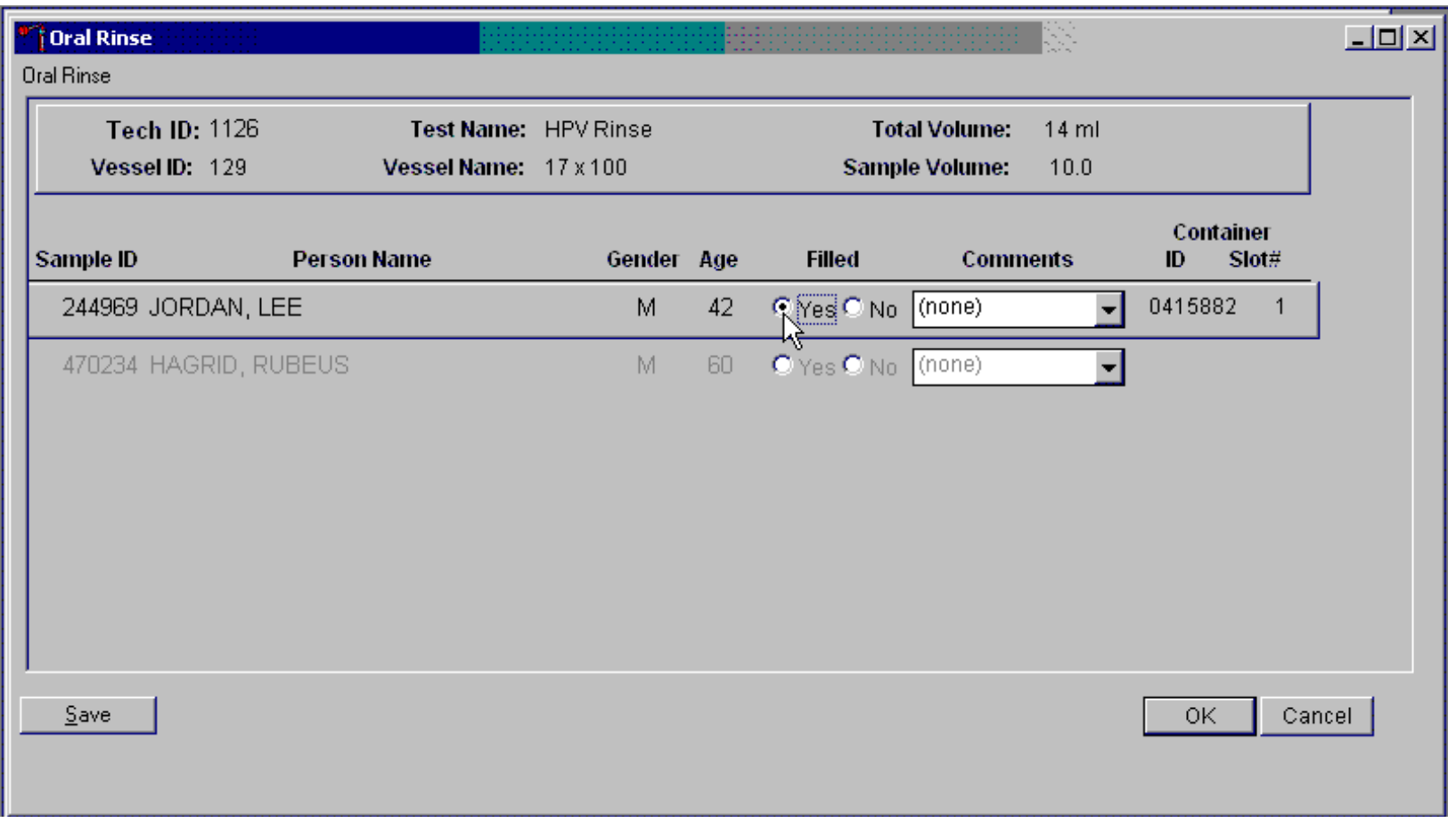

Only SPs who have had the oral rinse marked as collected in the Oral Health application are eligible to be processed. Once the sample is collected, the row changes from gray to black, indicating that the SP is ready to be marked as processed. To mark an individual vessel as collected or Filled-"Yes," use the mouse to direct the mouse arrow to the center of the "Yes" radio button and left click. As each vessel is marked as Filled-"Yes," it is automatically assigned to a slot in an existing (open) container. 
There are no quality comment codes available to describe the quality of the specimen or to explain why a vessel is marked as not filled.

Record oral rinse processing results for each SP. Individually mark each vessel as Filled"No."

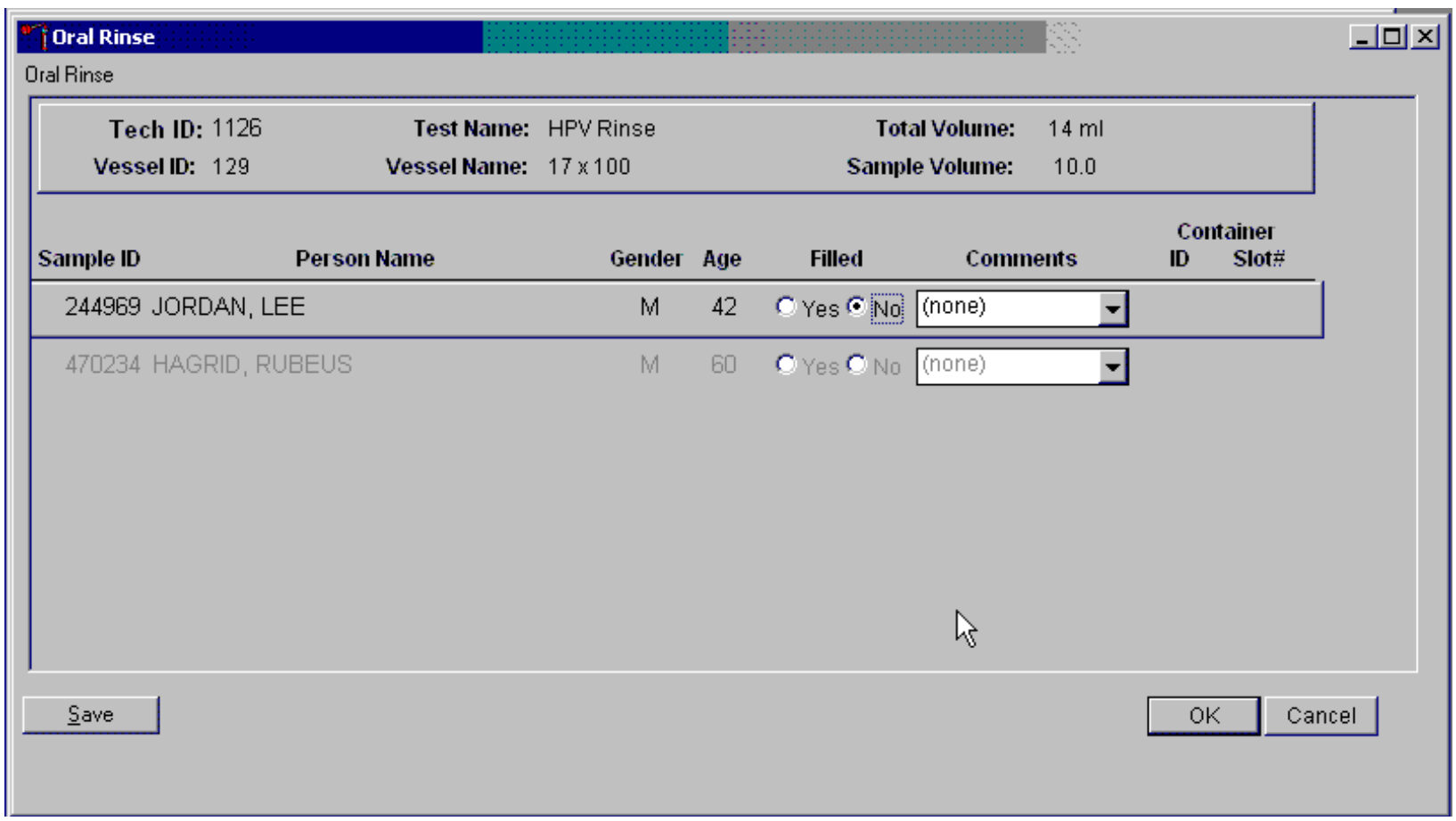

To mark vessel 129 as not collected or Filled-"No," use the mouse to direct the mouse arrow to the center of the "No" radio button and left click. Vessels marked as "No" do not require a comment.

Continue to enter results as vessels are processed. Review the information in the Oral Rinse processing window and save the data to the database.

Store each filled vessel in the assigned slot in the assigned container. To record this action or to save this data to the database, use the mouse to direct the mouse arrow to the Save button and left click. To record this action or to save this data to the database and to exit the module, use the mouse to direct the mouse arrow to the OK button, and left click. To close the window without saving any data in the database, use the mouse to direct the mouse arrow to the Cancel button, and left click. 


\subsection{Specimen Storage}

Record the processing result for the HPV Rinse and store the vessel in a 3-inch tall container with a 5 x 5 grid. Store the container in the refrigerator.

Check the slot assignment of containers associated with vessel 129 at the end of each session by accessing the Container Map report. Verify the contents of each container against the container map.

Exhibit 10-3. Storage protocol for HPV

\begin{tabular}{l|l|l|l}
\hline Shipping Location & Vessel & Condition & Vessel Storage \\
\hline Lab 67 & 129 HPV Rinse & Refrigerated & $5 \times 5$ three-inch box \\
Gillison Laboratory & & & \\
2001 Polaris PKWY \# 1320 & & & \\
OSU - Pathology Core Facility & & & \\
Columbus, OH 43240-2000 & & & \\
$(614) 293-5942$ & & & \\
\hline
\end{tabular}

\subsection{Shipping Procedures}

Ship vessel 129 according to procedures described in Section 8.11. Exhibit 8-4, the Shipping protocol, illustrates the specifications for the shipment of biological specimens to each contract laboratory.

Exhibit 10-4. Shipping protocol

\begin{tabular}{l|l|l|l|l}
\hline Laboratory & Vessel & Test Name & Vessel Storage & Shipping Frequency \\
\hline Gillison Laboratory & 129 & HPV Rinse & Refrigerated & Weekly \\
2001 Polaris PKWY \# 1320 & & & & \\
OSU - Pathology Core Facility & & & & \\
Columbus, OH 43240-2000 & & & & \\
$(614) 293-5942$ & & & & \\
\hline
\end{tabular}

Pack shipper according to the procedure described in Section 8.10. 
If the computer system fails, record results on a preprinted HPV Oral Rinse Processing worksheet (Exhibit 10-5). Complete a workstation 2 worksheet for each SP while processing the HPV specimens. Enter the results after the system is operational. Send the worksheets to the home office at the end of the stand.

Exhibit 10-5. HPV Oral Rinse Processing worksheet

HPV ORAL RINSE PROCESSING

SP ID

\begin{tabular}{|c|c|c|c|c|c|c|}
\hline ID & Name & Ages Gender & Sample mL & Sample Type & $\begin{array}{l}\text { Filled } \\
\checkmark\end{array}$ & Slot \# \\
\hline 129 & HPV Rinse & $14-69$ & 1 oral rinse & 10-ml Scope & & \\
\hline
\end{tabular}




\section{ORAL GLUCOSE TOLERANCE TEST (OGTT) APPLICATION}

\subsection{OGTT Application - Section Two (Blood Draw)}

If an SP consumed the entire calibrated dose of the Trutol solution in 10 minutes, and 1 hour and 45 minutes have elapsed, then he or she is eligible to return to phlebotomy to have (1) one additional 2-mL gray top drawn and (2) any tubes that were not drawn during the initial venipuncture examination drawn.

A pop-up message window will alert the coordinator and the phlebotomist when an SP is eligible to return to phlebotomy for the second blood draw. To close or remove the Message Center box, use the mouse to direct the mouse arrow to the Close button and left click, or use the mouse to direct the mouse arrow to the upper right corner to the $\mathrm{X}$ and left click.

Logon to the gtt application and log the SP into the component. 
The blood draw screen is divided into two sections.

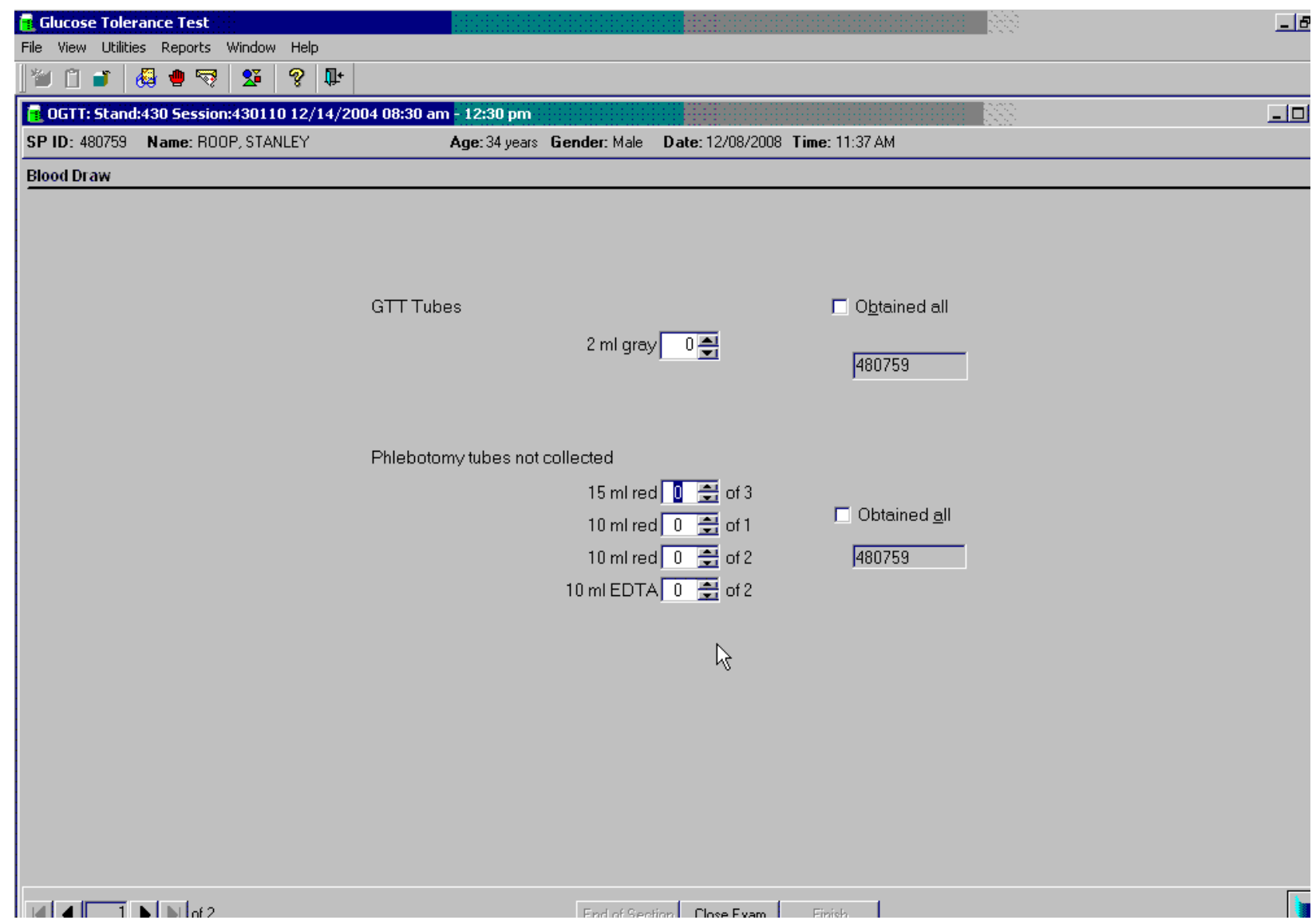

The upper section is identical to the venipuncture screen in the phlebotomy examination except that it contains only one $2-\mathrm{mL}$ gray top tube. The bottom half of the screen lists any tubes that were not drawn during the initial venipuncture examination. The sections function exactly like the venipuncture screen in the phlebotomy examination. 
If all of the blood tubes were drawn during the phlebotomy examination, then only the first half of the blood draw screen will contain the 2-mL gray top tube. The lower section of the blood draw screen will indicate that there are "No phlebotomy tubes to collect."

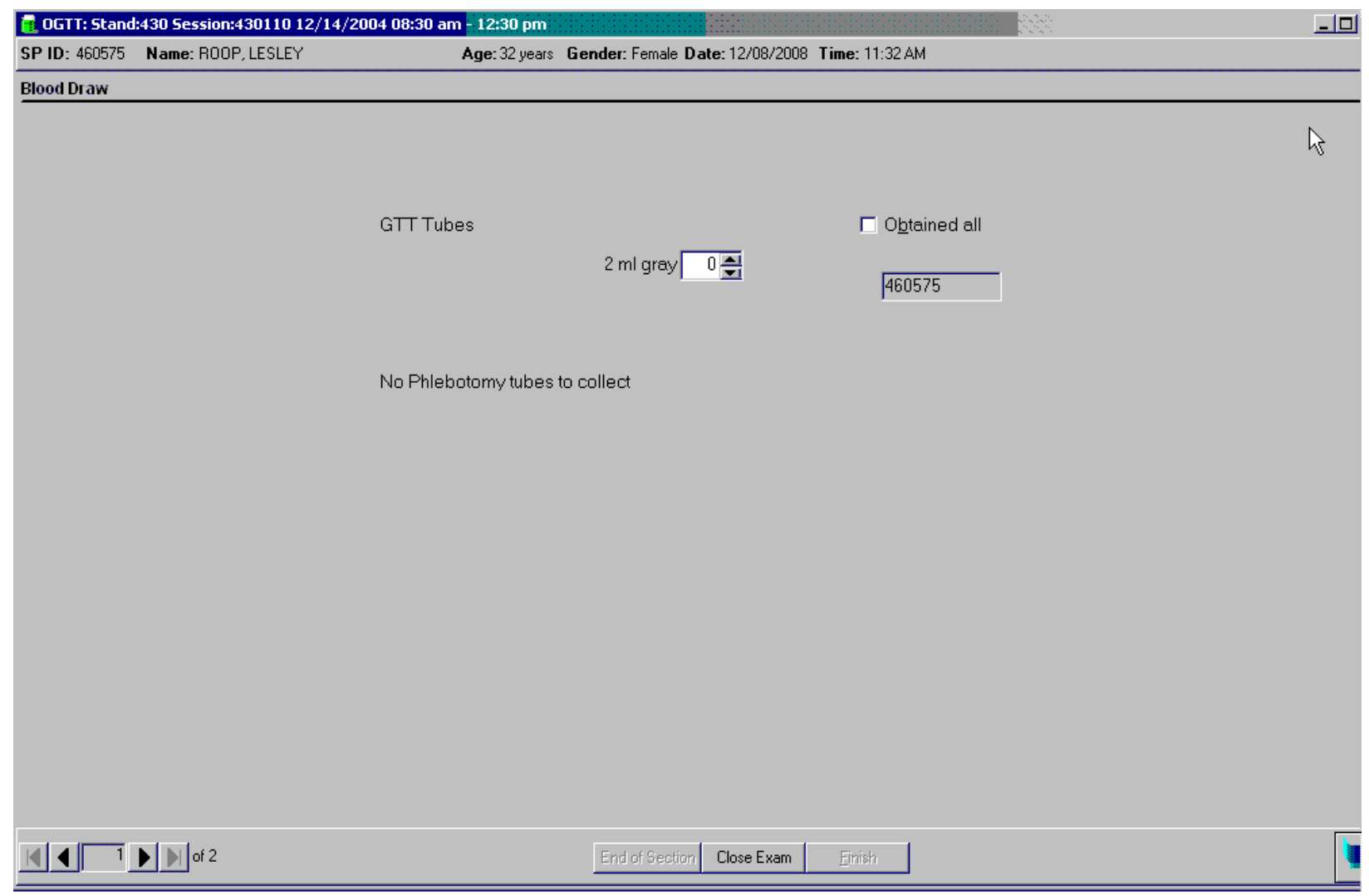

Draw the additional 2-mL gray top tube and then any tubes that were missed during the phlebotomy examination first. Draw all tubes according to the protocol described in Chapter 4 .

Labels will print for all the tubes marked as collected, including the second 2-mL gray top tube. The label for the second 2-mL gray top tube contains a barcode with the SP ID concatenated with the vessel ID (\# 98) and includes the test name "OGTT." 
Immediately after completing the venipuncture, enter the results of the blood draw and any comments about the venipuncture.

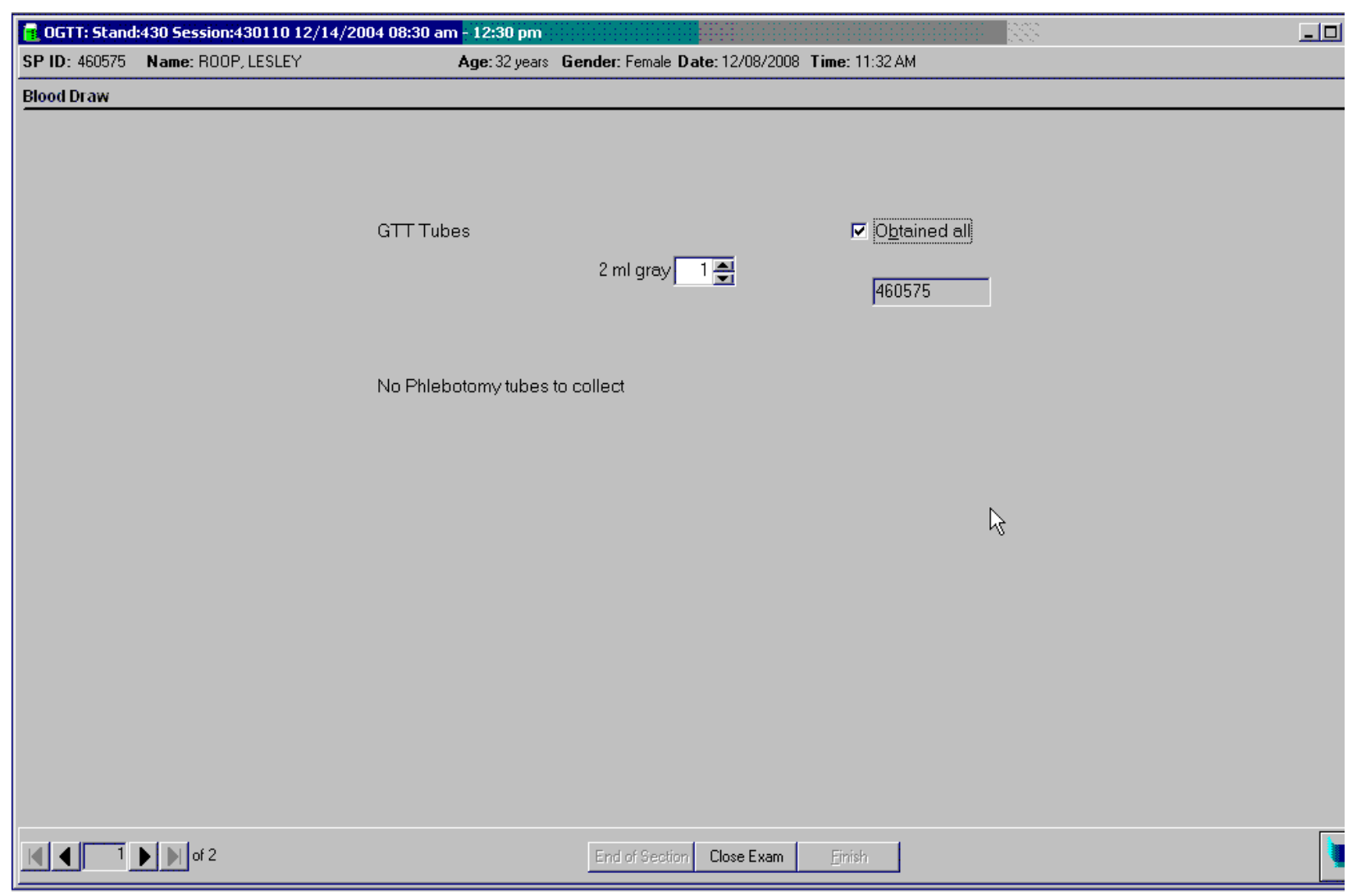

To record all tubes as filled or obtained, use the mouse to direct the mouse arrow to the "Obtained all" check box and left click, or type [Alt] [B/b]. This records a checkmark in the box and marks all tubes as obtained. To mark individual tubes as filled or obtained, use the mouse to direct the mouse arrow to the up-down controls on the spin box and toggle the number of each tube up or down or type the correct number using the numeric keys. 
Verify the Blood Draw Section Status.

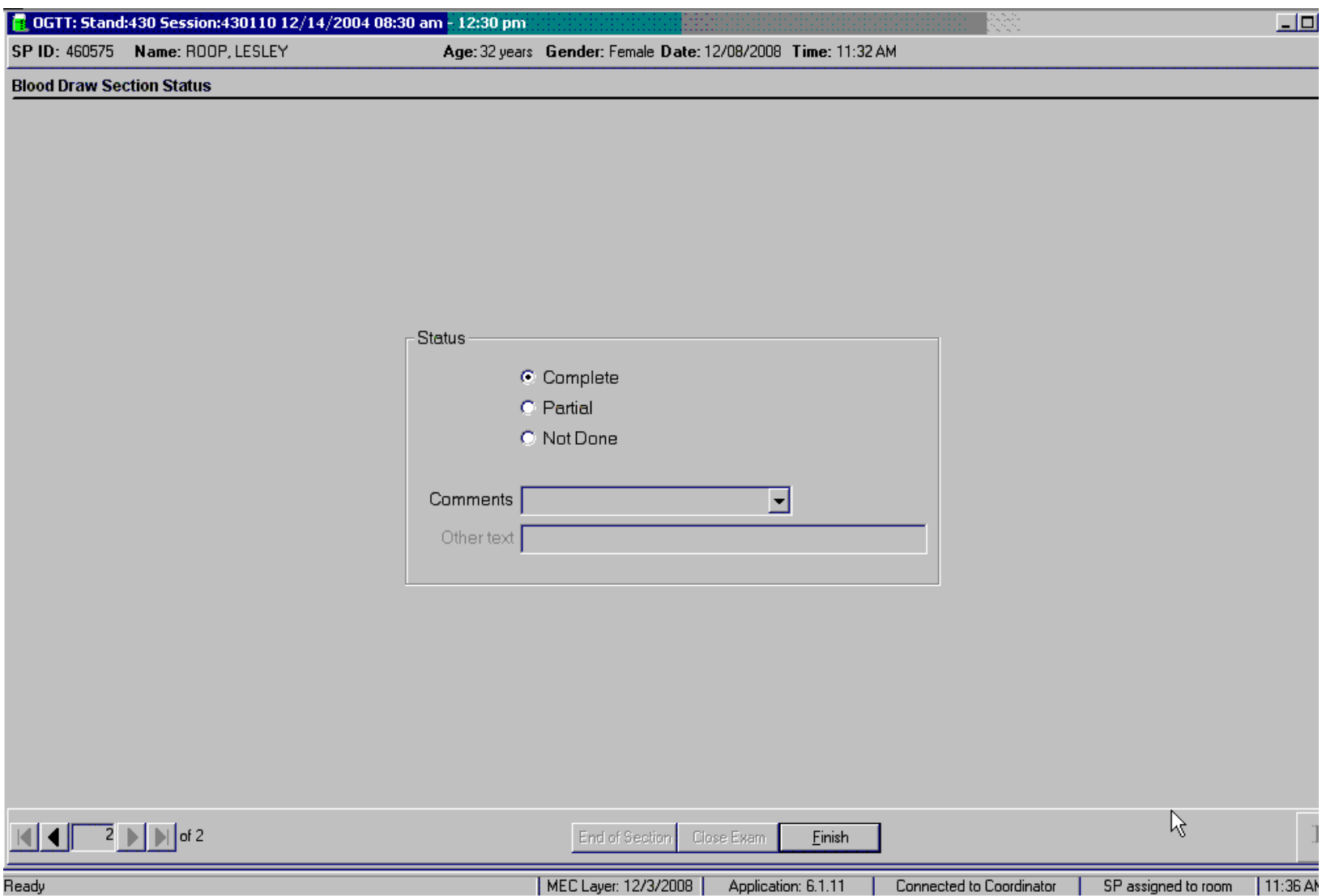

The Blood Draw Section Status is complete if the second 2-mL gray top tube is collected.

If any blood tubes were not drawn during the initial phlebotomy examination and they were drawn during the blood draw section of the OGTT examination, then the phlebotomy section status will automatically update to reflect the additional tubes drawn during the OGTT examination.

Remove the Dymo label from the SP's gown and ask the SP if he or she is allergic to peanuts. If not, then offer the SP peanut butter crackers and juice. If the SP is allergic to peanuts or does not know, then offer the standard crackers and juice. 
Use comment codes to explain Not Done status codes.

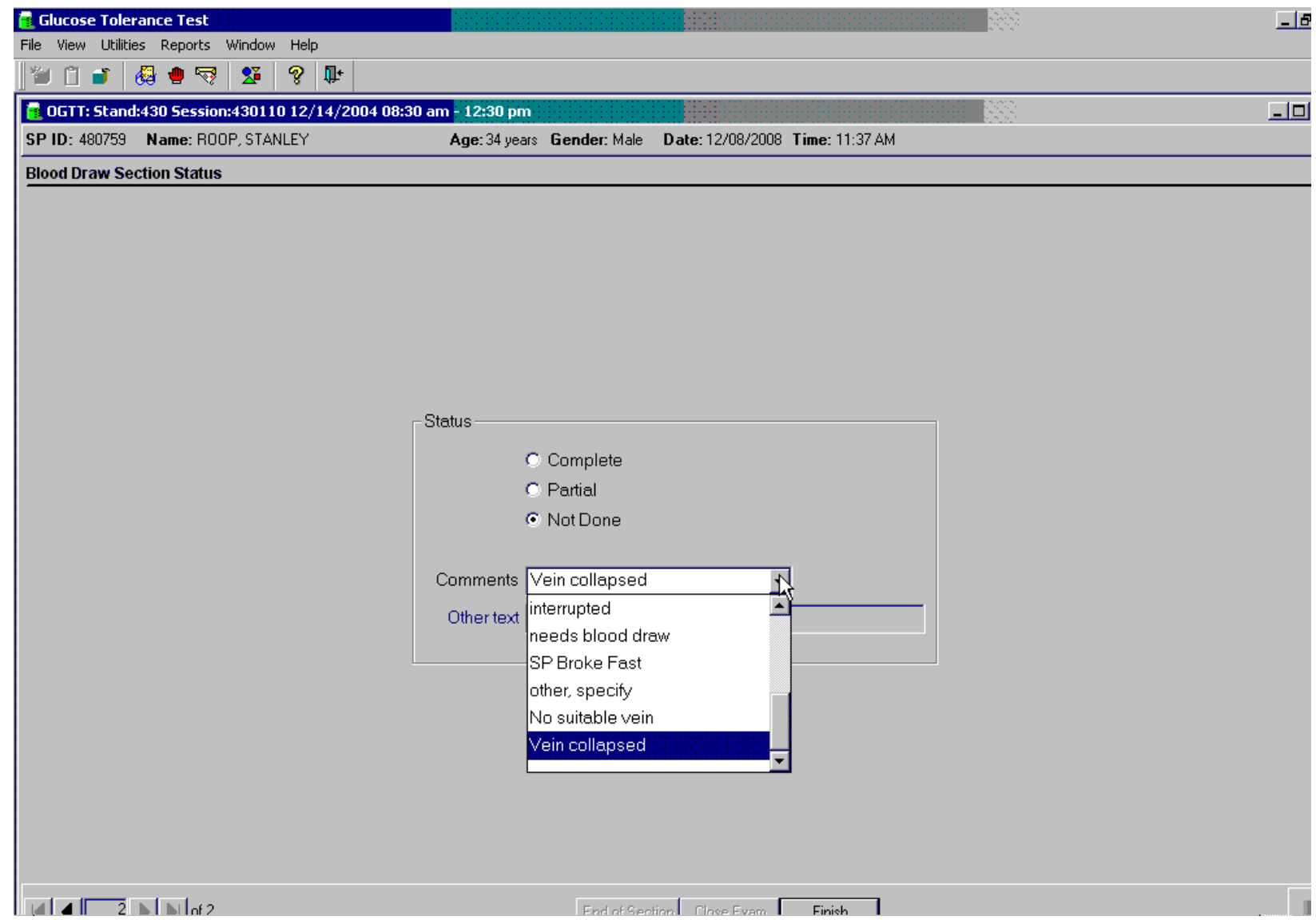

The blood draw section status is Not Done if the second 2-mL gray top tube is not collected.

Once the tube is marked as not obtained, the comment will default to "needs blood draw." Correct the comment by selecting the most appropriate comment code. To record a comment in the Comment text box, use the mouse to direct the mouse arrow to the scroll arrow on the drop-down list, left click, drag the mouse arrow to the desired choice, and left click. Use the scroll bar to view all choices. Alternatively, use the up and down keyboard arrows to scroll through the choices or type the first letter of the desired comment code. 


\begin{tabular}{|c|c|}
\hline Comment Code & Use when: \\
\hline Safety exclusion & $\begin{array}{l}\text { Not applicable. } \\
\text { This is reserved for positive responses to the hemophilia and chemotherapy } \\
\text { exclusion questions and it is automatically coded by the application. The } \\
\text { coordinator may code exams using this comment. }\end{array}$ \\
\hline SP refusal & $\begin{array}{l}\text { The SP refuses to have his or her blood collected. This is an SP-initiated } \\
\text { nonresponse due to refusal. The SP refuses the component for any reason other } \\
\text { than an illness or emergency. If the SP refuses in the reception area, the } \\
\text { coordinator codes the exam. If the SP refuses after starting the exam, the } \\
\text { examiner codes the exam. Use this comment to code partial exams when the } \\
\text { SP refuses after one tube has been drawn, or the blood flow stops after one } \\
\text { tube but before all tubes have been successfully drawn. }\end{array}$ \\
\hline No time & Not applicable. \\
\hline Physical limitation & Not applicable. \\
\hline $\begin{array}{l}\text { Communication } \\
\text { problem }\end{array}$ & Not applicable. \\
\hline Equipment failure & Not applicable. \\
\hline SP ill/emergency & $\begin{array}{l}\text { Use this comment to code not done or partial when the SP faints or is about to } \\
\text { faint, or the SP becomes ill or an emergency occurs and the test cannot be } \\
\text { performed on the SP. }\end{array}$ \\
\hline Interrupted & Not applicable. \\
\hline Needs blood draw & $\begin{array}{l}\text { The system uses this code as a default after the SP consumed the Trutol. } \\
\text { Correct this code depending on the situation. }\end{array}$ \\
\hline SP broke fast & $\begin{array}{l}\text { Use this code if the SP ate or drank anything other than plain water after he or } \\
\text { she drank the Trutol and before the blood draw. }\end{array}$ \\
\hline $\begin{array}{l}\text { Error (technician/ } \\
\text { software/supply) }\end{array}$ & $\begin{array}{l}\text { Use this comment to code partial and not done exams when there are } \\
\text { phlebotomist errors, or software or supply issues. }\end{array}$ \\
\hline Other, specify & $\begin{array}{l}\text { If the above reason for a status Code of Not Done is not explained by one of } \\
\text { the Comment Codes, the examiner must choose Other, specify and record a } \\
\text { comment in the text field. }\end{array}$ \\
\hline No suitable vein & $\begin{array}{l}\text { Use this comment to code exams as not done (no tubes drawn) when the SP } \\
\text { does not have an accessible vein, any arms available, casts on both arms, there } \\
\text { is a rash over the entire area, or the blood draw was started but there was no } \\
\text { blood in the tubing. }\end{array}$ \\
\hline Vein collapsed & $\begin{array}{l}\text { Use this comment to code exams as partial when one or more tubes are } \\
\text { successfully drawn but blood flow stops before all tubes are drawn or when the } \\
\text { veins collapse. }\end{array}$ \\
\hline
\end{tabular}

When finished, use the mouse to direct the mouse arrow to the bright blue arrow in the bottom right-hand corner and left click to exit or select [Enter] when this blue arrow is highlighted. 


\subsection{Laboratory Processing}

\subsubsection{Laboratory Overview}

The vessel for OGTT is \#98 (Exhibit 11-1). Use the gtt processing module to record the blood processing results. Labels for the OGTT vessel print for all SPs after the last blood processing set of labels. Label all OGTT tubes and place all in one rack. Keep with the blood processing racks.

Exhibit 11-1. Primary SP's OGTT processing protocol

\begin{tabular}{|c|c|c|c|c|c|c|c|}
\hline ID & Test name & Ages & $\begin{array}{c}\text { Sample } \\
(\mathrm{mL})\end{array}$ & Sample type & $\begin{array}{c}\text { Collection } \\
\text { type }\end{array}$ & $\begin{array}{c}\text { Vessel } \\
\text { type }\end{array}$ & Remarks \\
\hline 98 & OGTT & $12+$ & 0.5 & Plasma & Gray & 2-mL & Morning SPs only \\
\hline
\end{tabular}

\subsubsection{Process the 2-mL Gray Top Tube for OGTT}

Centrifuge and separate the plasma from the 2-mL gray top tube within 30 minutes from the time the blood was collected. Process the specimen even if the contents of the gray tube are clotted.

- Place the tube in the centrifuge carrier of either tabletop centrifuge. Balance the tube in the centrifuge by using water-filled tubes if necessary. Centrifuge the 2-mL gray tube at $17-25^{\circ} \mathrm{C}$ or $11-8^{\circ} \mathrm{C}$ and $2,900 \mathrm{rpm}$ for 10 minutes to yield plasma for vessel 98 (OGTT.)

\section{Vessel 98}

- $\quad$ Use a calibrated plastic transfer pipette to transfer all plasma from this tube but at least $0.5-\mathrm{mL}$ plasma for vessel 98 (OGTT) on primary SPs into a 2-mL vessel.

- Determine if the plasma is hemolyzed, turbid, lipemic, or icteric. If so, enter a comment to describe the plasma when entering the processing results.

- Close all vessels secured to prevent leakage and evaporation.

- Record results using the GTT module as described in Section 8.3 of the Laboratory Procedures Manual.

- Store all specimens immediately as outlined in Section 8.10 of the Laboratory Procedures Manual. 


\subsubsection{Record the Results of Specimen Processing}

Open and log onto the Laboratory application. Use the heads-up display to view the SPs, the modules for which they are eligible, and their current process status. Record blood processing results for vessel 98 using the "OGTT" module.

The "OGTT" module is grayed out until at least one SP has the second 2-mL gray top tube drawn. Once one SP has the second 2-mL gray top tube drawn, the processing circle for that SP turns black.

Select the correct SP and access the OGTT Processing module.

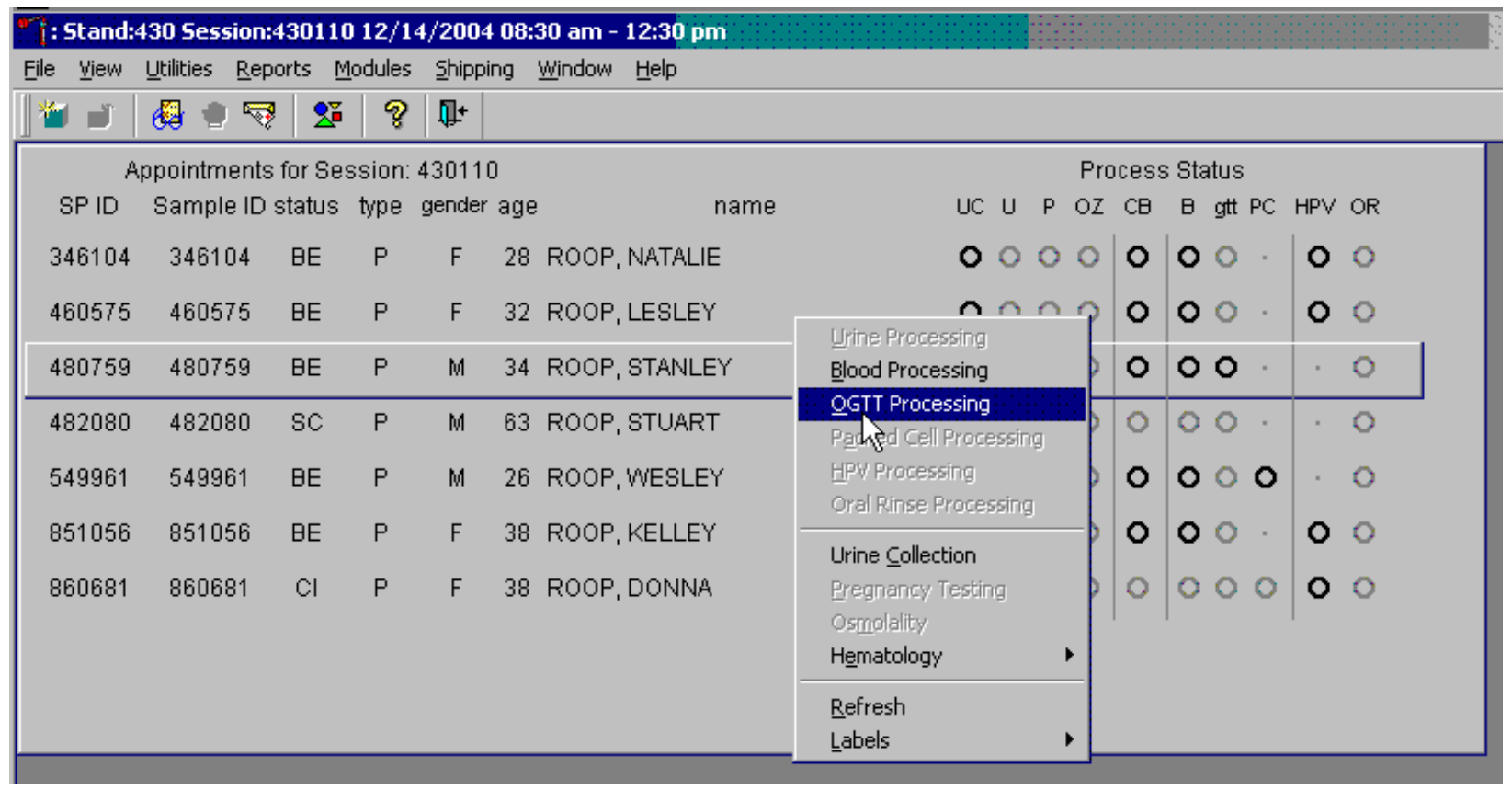

To access the OGTT Processing module, use the mouse to direct the mouse arrow to the SP on the heads-up display, left click, drag the mouse arrow to OGTT Processing, and right click. 
Record the vessel 98 processing results in the OGTT processing window. All SPs are included in the same window but are listed separately by rows.

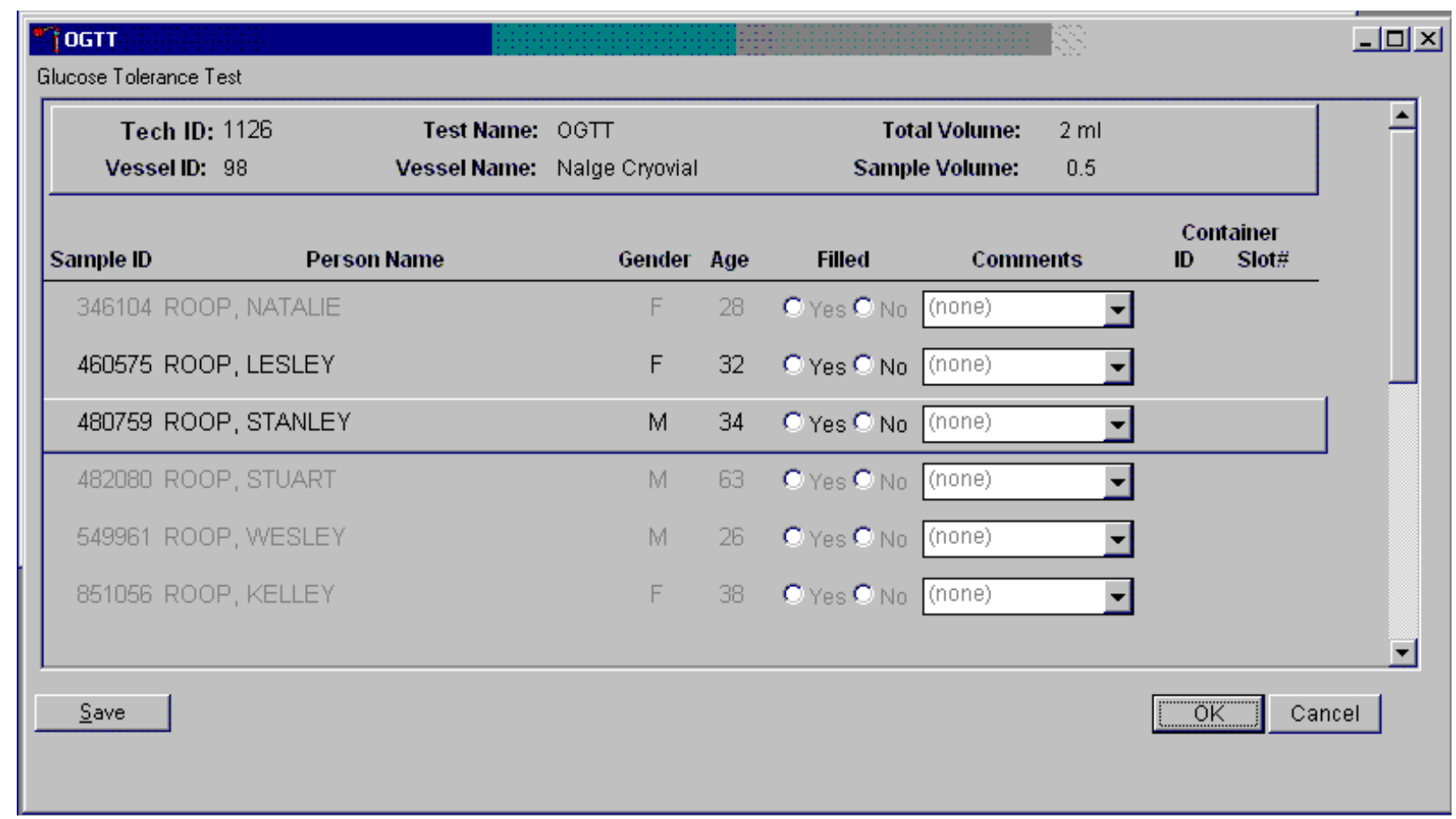

The OGTT processing window lists the vessel requirements across the top. SPs are listed separately in rows. The upper section of the Glucose Tolerance Test window contains the following information: Tech ID, Vessel ID (98), Test Name (OGTT), Total Volume (2 ml), Vessel Name (Nalge cryovial), and Sample Volume (0.5.) The lower section of the window contains columns for Sample ID, Person Name (Last, First), Gender, Age, Filled Yes and No radio buttons, Comments text box, and a Container ID/Slot \# column N. Use the Save button to save the results to the database. Use the OK button to save the results to the database and close the module. Use the Cancel button to close the window without saving any data to the database. 
Record blood processing results for each SP. Individually mark each vessel as Filled-"Yes."

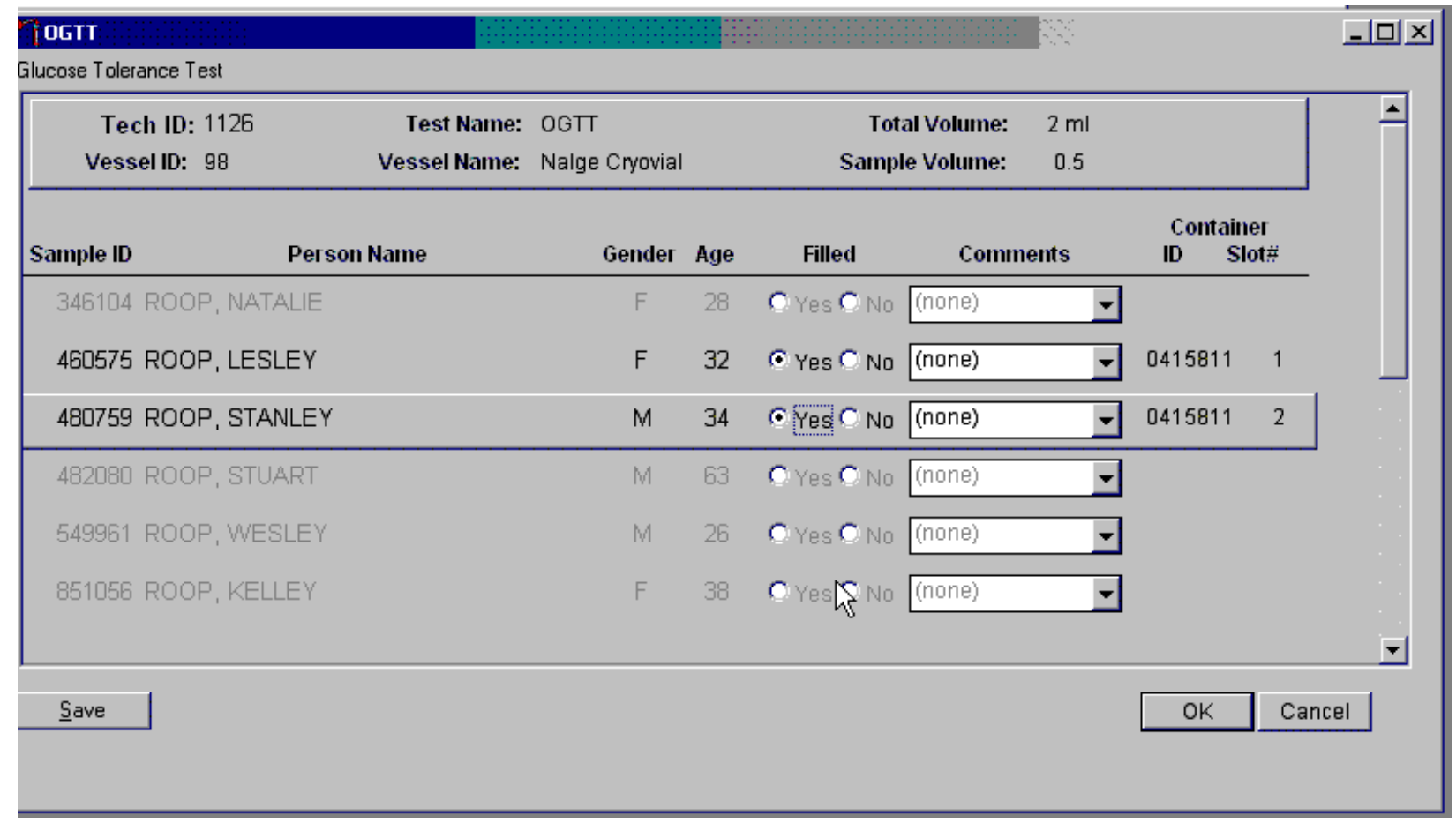

Only SPs who have had the second 2-mL gray top tubes marked as collected in the gtt application are eligible to be processed. Once the tube is collected, the row changes from gray to black, indicating that the SP is ready to be marked as processed. To mark an individual vessel as collected or Filled-"Yes" use the mouse to direct the mouse arrow to the center of the "Yes" radio button and left click. As each vessel is marked as Filled-"Yes," it is automatically assigned to a slot in an existing (open) container. 
Quality comment codes are available to describe the quality of the specimen.

\begin{tabular}{|l|l|}
\hline Comment for filled vessels & Use when: \\
\hline Clotted & $\begin{array}{l}\text { The whole blood sample contains a fibrin formation. This is due to } \\
\text { inadequate mixing or inadequate clotting. }\end{array}$ \\
\hline Equipment failure & Pipettes are malfunctioning. \\
\hline Hemolyzed & $\begin{array}{l}\text { There is a red appearance to the serum or plasma. Hemolysis refers to the } \\
\text { abnormal lysis of erythrocytes. Hemolysis can occur before venipuncture } \\
\text { (in vivo hemolysis) or during the analytic procedure (in vitro hemolysis). If } \\
\text { in vivo, hemolysis will give plasma a red color. Use when describing visible } \\
\text { hemolysis, which occurs when the concentration of hemoglobin exceeds } \\
\text { 200 mg/L. }\end{array}$ \\
\hline Icteric & $\begin{array}{l}\text { There is a visible orange color to the serum or plasma. Icteric refers to } \\
\text { orange color imparted to a sample because of the presence of bilirubin. } \\
\text { Bilirubin present in serum results in visible "jaundice" color when the } \\
\text { concentration of bilirubin is above 1130 umol/L (micromoles per liter). }\end{array}$ \\
\hline Lipemic & $\begin{array}{l}\text { There is a turbid appearance to the serum or plasma. Lipemia refers to the } \\
\text { presence of lipid particles (usually very low-density lipoprotein) in a } \\
\text { sample, which gives the sample a turbid appearance. Plasma appears visibly } \\
\text { lactescent or lipemic when the concentration of triglycerides exceeds 11.6 } \\
\text { mmol/L (millimoles per liter). }\end{array}$ \\
\hline
\end{tabular}


Attach a comment to an individual filled vessel.

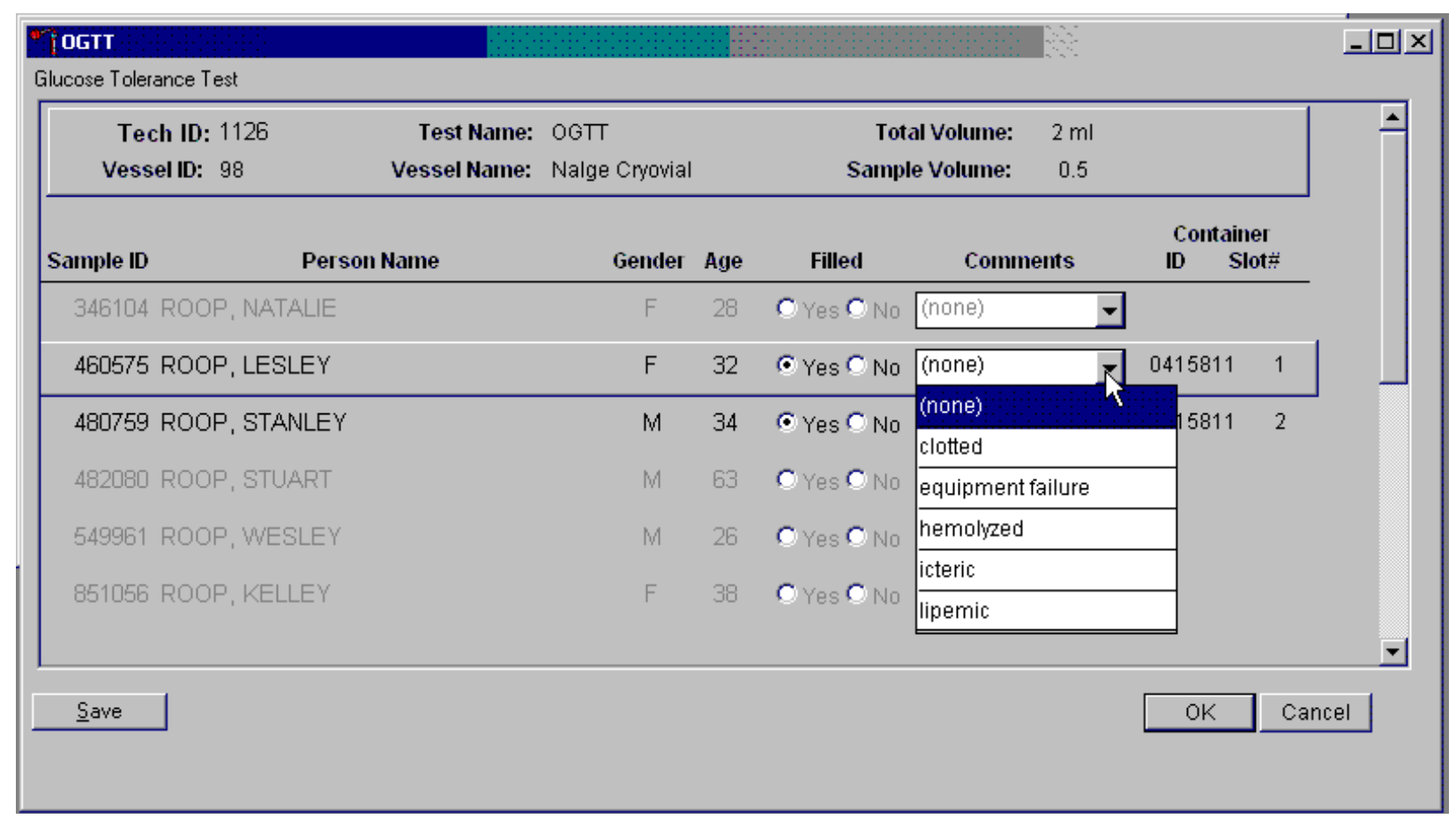

To attach a quality control comment to a filled vessel, use the mouse to direct the mouse arrow to the drop-down list under the Comments button to display the choices, drag the arrow to the correct choice, and left click or highlight the Comment text box and type the first letter of the desired choice (c, e, h, i, l). 
Record blood processing results for each SP. Individually mark each vessel as Filled-"No."

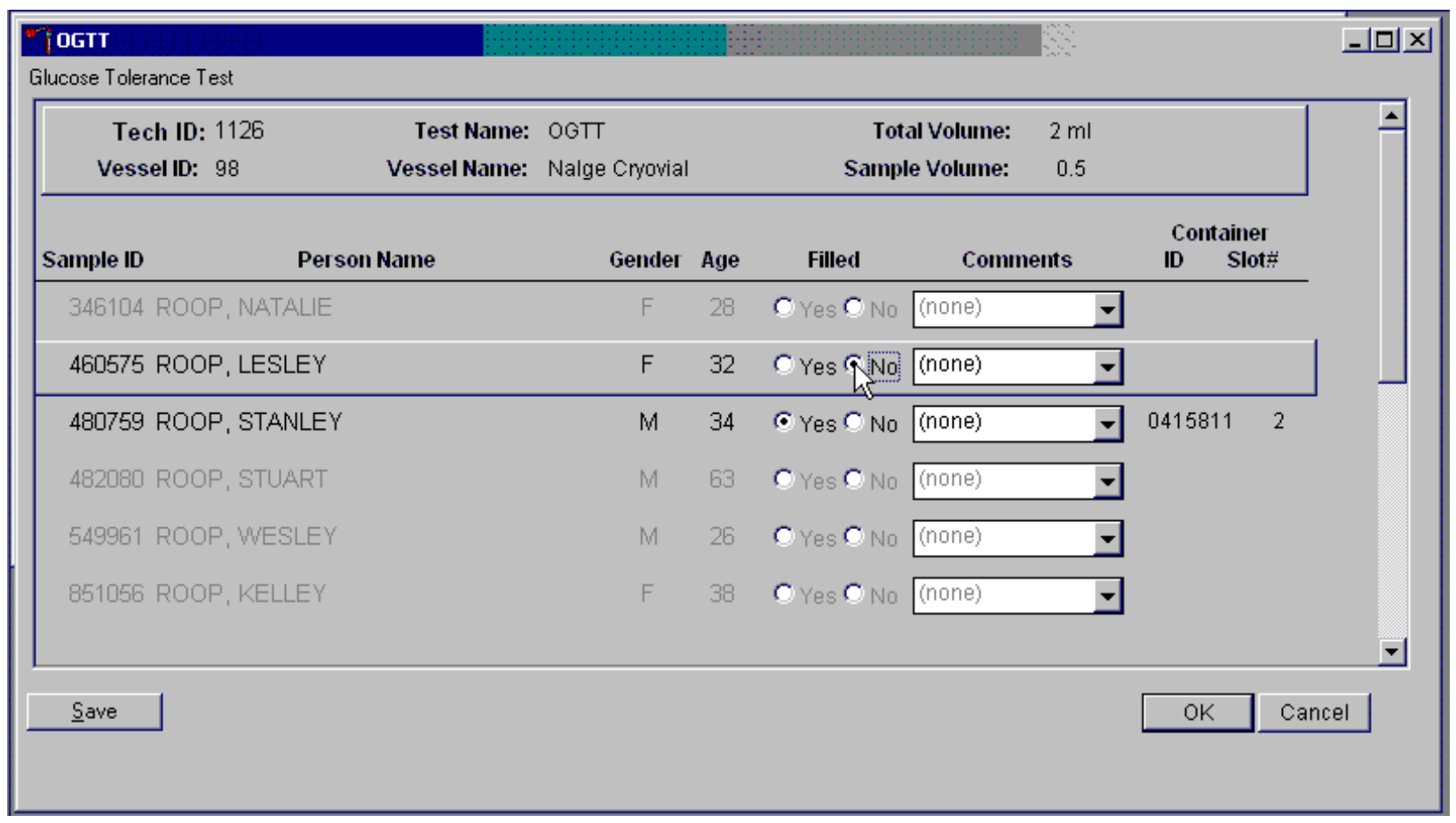

To mark vessel 98 as not collected or Filled-"No," use the mouse to direct the mouse arrow to the center of the "No" radio button and left click. Vessels marked as "No" require a comment.

Enter comment codes to describe the reasons why a vessel is marked as Filled-"No," or why the protocol is incomplete.

\begin{tabular}{|l|l|}
\hline Comment for not filled vessels & Use when: \\
\hline Laboratory error & $\begin{array}{l}\text { A tube breaks in the centrifuge, contents spill, or other technologist } \\
\text { error. }\end{array}$ \\
\hline Quantity not sufficient & Quantity of blood is not sufficient to complete the protocol. \\
\hline SP refusal & The SP refused the blood draw. \\
\hline
\end{tabular}


Attach a comment to an individual Filled-"No" vessel.

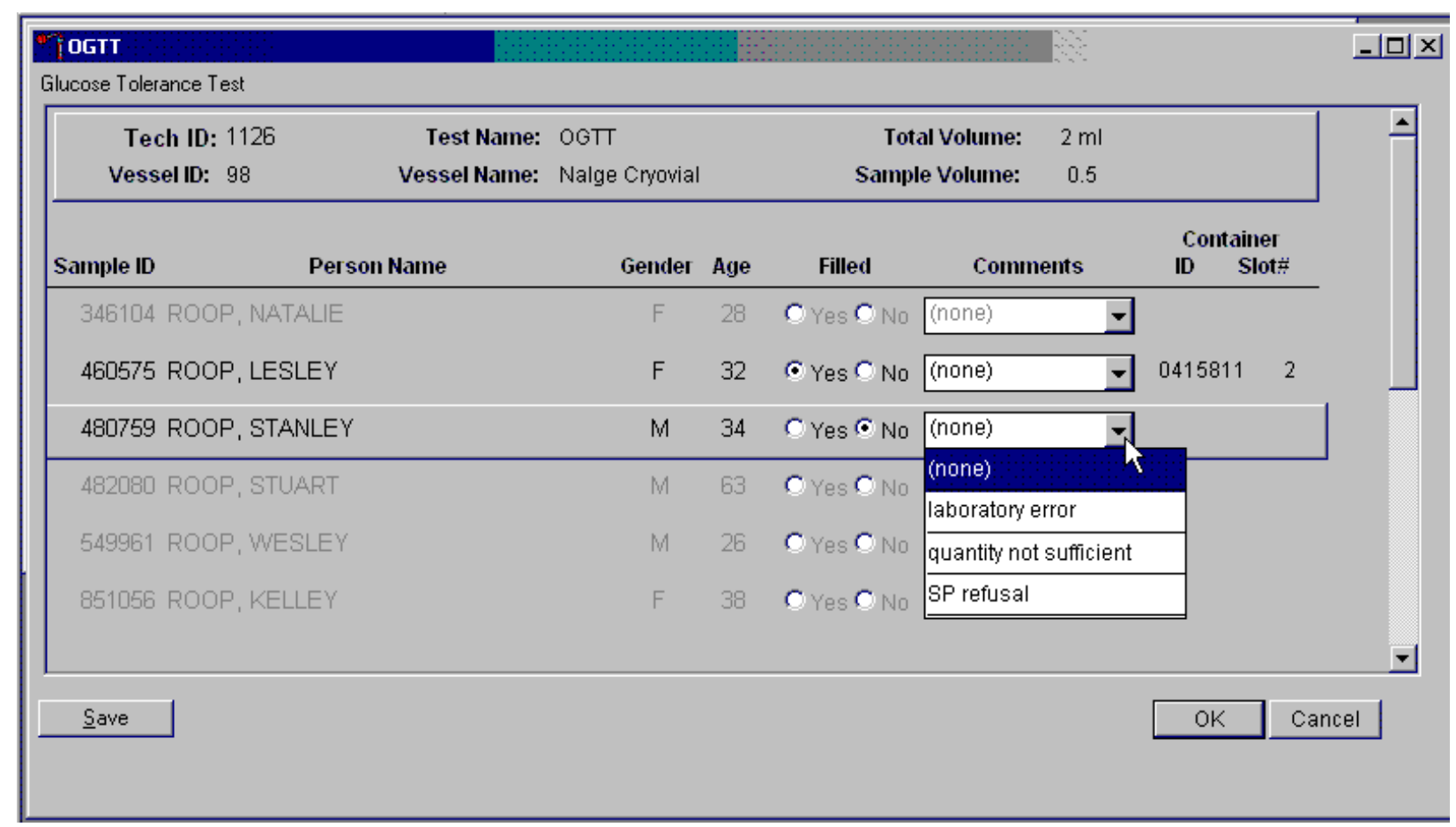

To attach a Filled-"No" comment to a vessel, use the mouse to direct the mouse arrow to the drop-down list under the Comments button to display the choices, drag the arrow to the correct choice, and left click. Alternatively, highlight the Comment text box and type the first letter of the desired choice. 
Continue to enter results as vessels are processed. Review the information in the OGTT processing window and save the data to the database.

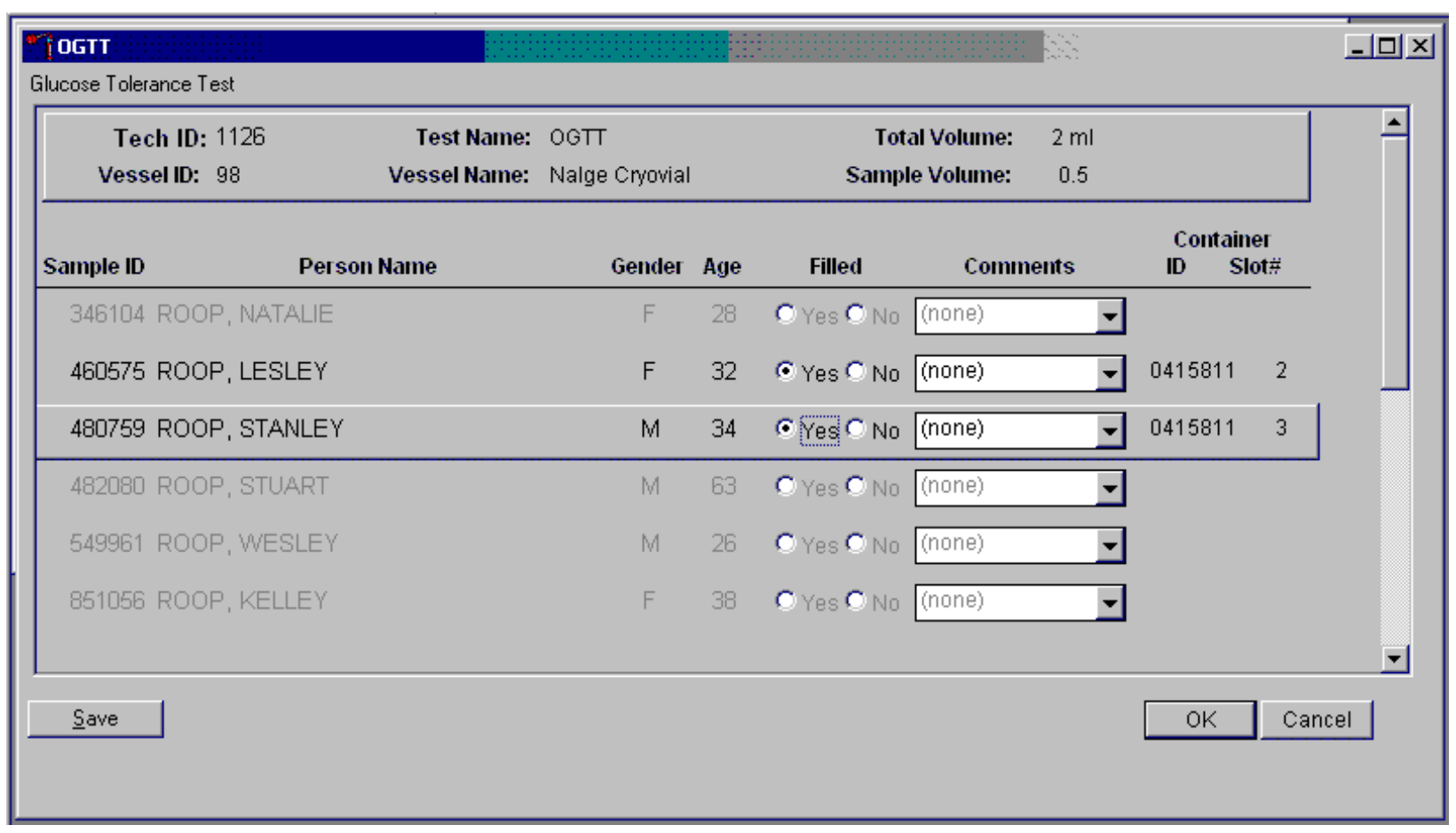

Store each filled vessel in the assigned slot in the assigned container. To record this action or to save this data to the database, use the mouse to direct the mouse arrow to the Save button and left click. To record this action or to save this data to the database and to exit the module, use the mouse to direct the mouse arrow to the OK button, and left click. To close the window without saving any data in the database, use the mouse to direct the mouse arrow to the Cancel button, and left click. 
It is not possible to mark vessels 9 (glucose) and 23 (insulin) in the blood processing module after the second 2-mL gray top tube has been marked as collected in phlebotomy. They are grayed out on the blood processing window.

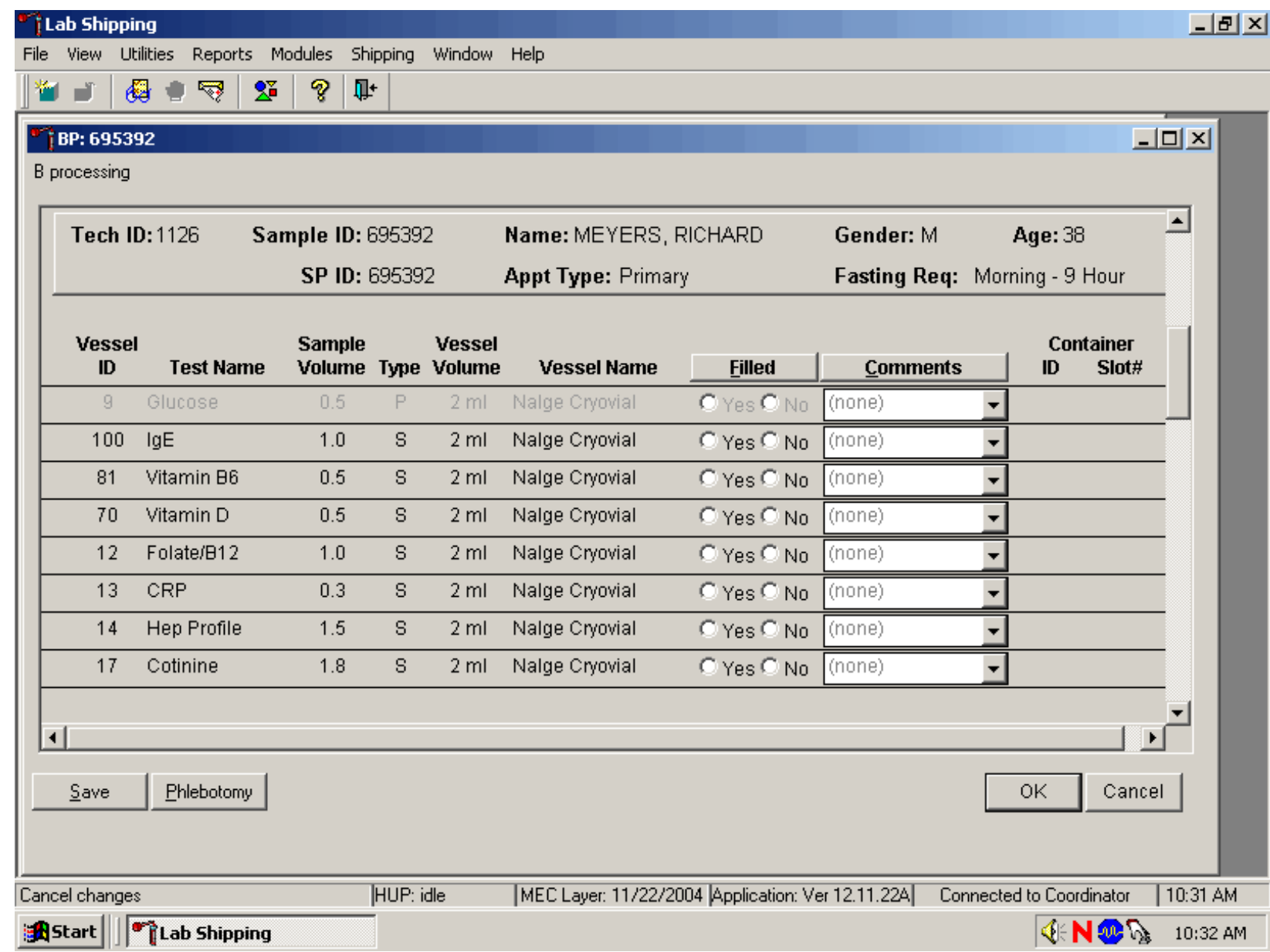

Process the blood from the first venipuncture as soon as possible. Contact the system programmer if the blood tubes for vessels 9 and 23 were drawn before the second 2-mL gray top tube was marked as collected in phlebotomy. 
During the OGTT blood draw (2 hours after the initial venipuncture and after the SP has consumed the Trutol), the phlebotomist will draw any tubes that were missed during the first venipuncture. Add the "Post OGTT draw" comment to any blood vessel processed or filled from blood collected during the OGTT blood draw.

\begin{tabular}{|c|c|c|c|c|c|c|c|c|c|c|c|c|c|}
\hline \multicolumn{14}{|l|}{ B processing } \\
\hline \multicolumn{2}{|c|}{ Tech ID: 1126} & \multicolumn{3}{|c|}{$\begin{array}{r}\text { Sample ID: } 480759 \\
\text { SP ID: } 480759\end{array}$} & \multicolumn{2}{|c|}{$\begin{array}{l}\text { Name: ROOP, STANLEY } \\
\text { Appt Type: Primary }\end{array}$} & \multicolumn{3}{|c|}{$\begin{array}{l}\text { Gender: M Age: } 34 \\
\text { Fasting Req: Morning - Fast }\end{array}$} & & & & $\Delta$ \\
\hline \multirow{2}{*}{$\begin{array}{l}\text { Vessel } \\
\text { ID }\end{array}$} & \multirow{2}{*}{ Test Name } & \multirow[b]{2}{*}{ CAP } & \multirow{2}{*}{$\begin{array}{l}\text { Sample } \\
\text { Volume }\end{array}$} & \multirow[b]{2}{*}{ Type } & \multirow{2}{*}{$\begin{array}{l}\text { Vessel } \\
\text { Volume }\end{array}$} & \multirow[b]{2}{*}{ Vessel Name } & \multirow{2}{*}{\multicolumn{4}{|c|}{$\begin{array}{l}\text { Container } \\
\text { ID Slot }\end{array}$}} & & & \\
\hline & & & & & & & Filled & & & & & & \\
\hline 22 & HIV & & 0.8 & $\mathrm{~s}$ & $2 \mathrm{ml}$ & Nalge Cryovial & C Yes CNo & (none) & 0435743 & 1 & & & \\
\hline 24 & HSV & & 0.2 & $\mathrm{~s}$ & $2 \mathrm{ml}$ & Nalge Cryovial & C Yes $\bigcirc$ No & (none) & 0435745 & 1 & & & \\
\hline 127 & IgA-TTG/EMA & & 0.5 & $\mathrm{~s}$ & $2 \mathrm{ml}$ & Nalge Cryovial & C Yes C No & (none) & 0435764 & 1 & & & \\
\hline 161 & MMRV & & 1.5 & $\mathrm{~s}$ & $2 \mathrm{ml}$ & Nalge Cryovial & C Yes C No & Post OGTT draw $=$ & 0435739 & 1 & & & \\
\hline $32 \gamma$ & Xtra Sera1 & & 0.5 & $\mathrm{~s}$ & $2 \mathrm{ml}$ & Nalge Cryovial & C Yes C No & Post OGTT draw $=$ & 0435675 & 17 & & & \\
\hline $33 \gamma$ & Xtra Sera2 & & 0.5 & $\mathrm{~s}$ & $2 \mathrm{ml}$ & Nalge Cryovial & C Yes C No & Post OGTT draw $=$ & 0435675 & 18 & & & \\
\hline $34>$ & Xtra Sera3 & & 0.5 & $\mathrm{~s}$ & $2 \mathrm{ml}$ & Nalge Cryovial & C Yes C No & Post OGTT draw $=$ & 0435675 & 19 & & & \\
\hline $35>$ & Xtra Sera 4 & & 0.5 & $\mathrm{~s}$ & $2 \mathrm{ml}$ & Nalge Cryovial & $\odot$ Yes CNo & Post OGTT draw $=$ & 0435675 & 20 & & & \\
\hline $36 \gamma$ & Xtra Sera5 & & 1.0 & $\mathrm{~s}$ & $2 \mathrm{ml}$ & Nalge Cryovial & C Yes C No & Post OGTT draw $=$ & 0435675 & 21 & & & \\
\hline $37>$ & Xtra Sera6 & & 1.0 & $\mathrm{~s}$ & $2 \mathrm{ml}$ & Nalge Cryovial & - Yes C No & Post OGTT draw $=$ & 0435675 & 22 & & & \\
\hline $38>$ & Xtra Sera7 & & 1.0 & $\mathrm{~s}$ & $2 \mathrm{ml}$ & Nalge Cryovial & C Yes C No & Post OGTT draw $\nabla$ & 0435675 & 23 & & & \\
\hline $39>$ & Xtra Sera8 & & 1.0 & $\mathrm{~s}$ & $2 \mathrm{ml}$ & Nalge Cryovial & C Yes CNo & Post OGTT draw - & 0435675 & 24 & & & \\
\hline 92 & BFR1 & & 4.0 & $\mathrm{~s}$ & $5 \mathrm{ml}$ & Nalge Cryovial & - Yes CNo & Post OGTT draw & 0435697 & 3 & & & \\
\hline 93 & BFR2 & & 4.0 & 5 & $5 \mathrm{ml}$ & Nalge Cryovial & C Yes CNo & PostogT" draw" & 0435697 & 4 & & & \\
\hline Save & Phleb & otomy & & & & & & s & & & OK & Cancel & \\
\hline
\end{tabular}


To attach a quality control comment to an individual filled vessel, use the mouse to direct the mouse arrow to the drop-down list under the Comments button to display the choices, drag the arrow to the correct choice, and left click or highlight the Comment text box and type the first letter of the desired choice (c, e, h, I, 1, p).

To collectively assign the same comment to all vessels, use the mouse to direct the mouse arrow to the Comments button on the top of the radio buttons, left click, drag the arrow to \{Filled Comments\}, drag the mouse arrow to the correct choice, and left click. Alternatively, to assign the same comment to all filled vessels, type [Shift] [C/c], and the first letter of the correct choice (i, l, e, c, h, p).

\subsubsection{Vessel 98 Storage Protocol}

Fill the vessels, enter the OGTT processing results, and prepare to store the vessels. Store vessels in numbered storage boxes according to test as indicated in Exhibit 11-2.

Exhibit 11-2. Storage protocol for OGTT

\begin{tabular}{|c|c|c|c|}
\hline Shipping Location & Vessels & Conditions & Vessel Storage \\
\hline University of Missouri & 98 OGTT & Frozen & $9 \times 9$ two-inch box \\
\hline
\end{tabular}

\subsubsection{Vessel 98 Shipping Protocol}

Ship the OGTT container weekly on dry ice to the NHANES Diabetes Laboratory. Exhibit 11-3 describes the shipping protocol.

Exhibit 11-3. OGTT shipping protocol

\begin{tabular}{|l|c|c|c|c|}
\hline \multicolumn{1}{|c|}{ Location } & Vessels & Conditions & Vessel storage & $\begin{array}{c}\text { Shipping } \\
\text { frequency }\end{array}$ \\
\hline Blood & OGTT & Frozen & $9 \times 9$ two-inch box & Weekly \\
\hline $\begin{array}{l}\text { University of Missouri } \\
\text { Vessel 98 }\end{array}$ & & & \\
\hline
\end{tabular}


Pack the container in the same shipper as vessels 9, 23, and 21. Close the container using procedures described in Section 8.10 of the Laboratory Procedures Manual. Assign the closed container(s) to, or associate closed containers with, the same air bill that was created for the frozen shipper being sent to the NHANES Diabetes Lab. Exhibit 11-4 includes the contract laboratory address. Use procedures described in Section 8.10 of the Laboratory Procedures Manual. Follow the steps listed in Section 8.8 to create the shipping manifest and to send the electronic file. Pack and label the shipper using procedures described in Section 8.10 of the Laboratory Procedures Manual.

Exhibit 11-4. Contract laboratory address

\begin{tabular}{|c|c|c|c|}
\hline $\begin{array}{c}\text { Laboratory/destination } \\
\text { specimen shipment address }\end{array}$ & Vessels/assays & Conditions & $\begin{array}{l}\text { Contact name } \\
\text { telephone number }\end{array}$ \\
\hline $\begin{array}{l}\text { University of Missouri } \\
1 \text { Hospital Drive, Room M772 } \\
\text { Columbia, Missouri } 65201\end{array}$ & $\begin{array}{l}\text { 9/Glucose } \\
\text { 23/Insulin } \\
\text { 98/OGTT }\end{array}$ & Frozen & $\begin{array}{l}\text { Alethea Tennill } \\
(573) 882-2121\end{array}$ \\
\hline
\end{tabular}

\subsubsection{Performing the OGTT on SPs Who Do Not Speak English}

When the MEC staff must conduct the OGTT procedure with an SP who does not speak English and the staff member does not speak the language of the SP, a translator who does speak the language of the SP assists the phlebotomist.

The translator stays with the phlebotomist and the SP for the entire procedure. It is very important that the phlebotomist be able to communicate with the SP if the SP becomes ill during the examination.

\subsubsection{Convert Screen Text to English or Spanish}

Convert exam screen text from English to Spanish or from Spanish to English at any time before, during, or after an exam.

To view the exam screen text in Spanish, use the mouse to direct the mouse arrow to $\{$ Utilities $\}$ in the menu bar, left click, drag the arrow to $\{$ Spanish $\}$, and left click, or type $[\mathrm{Ctrl}][\mathrm{S} / \mathrm{s}]$. To 
return exam screen text to English, use the mouse to direct the mouse arrow to \{Utilities\} in the menu bar, left click, drag the arrow to $\{$ English $\}$, and left click, or type [Ctrl] [E/e].

\begin{tabular}{ll}
\hline English & Toggle option to set exam language to English. \\
$C t r l+E$ & \\
\hline$\underline{S}$ panish & Toggle option to set exam language to Spanish. \\
$C t r l+S$ & \\
\hline
\end{tabular}




\section{QUALITY CONTROL AND QUALITY ASSURANCE - POLICIES AND PROCEDURES}

This policies and procedures quality control and quality assurance chapter is an adjunct to the Westat Policies Manual.

\subsection{Safety}

\subsubsection{Introduction}

NCHS and Westat maintain an ongoing Health and Safety Committee that acts in cooperation with the CDC Office of Health and Safety to assure safe working conditions for technologists in the NHANES mobile examination center (MEC). Safety precautions for all methods are documented in the Biosafety appendix in the NHANES Laboratory Procedures Manual. This manual is readily available to all laboratory staff. In addition to annual safety training, the NCHS Biomedical Engineer performs safety inspections at the beginning of each location. The technical supervisor assures that the primary laboratory analyst reads and is familiar with safety precautions.

\subsection{Specimen Submission and Handling}

\subsubsection{Introduction}

The goal of specimen submission and handling is to optimize the accurate and reliable measurement of analytes of interest. Each analytical procedure used in NHANES is documented in the NHANES Laboratory Procedures Manual (LPM). Each method in the LPM specifies the procedure to be used for preparation of the survey participant, specimen collection, labeling, processing, and preservation, and conditions for specimen transport that are appropriate for that method. The methods may refer to the NHANES blood collection protocol, or urine collection protocol, noting any special requirements specific to the particular method. These protocols are a starting point due to the special contamination requirements needed for collection of specimens analyzed for heavy metals. 


\subsubsection{Identification of Specimens}

Personal identifiers (e.g., names) are not included on test specimens. NHANES uses a random internal specimen ID number to identify and track individual samples. The Integrated Survey and Information System (ISIS) system at Westat and the National Center for Health Statistics (NCHS) maintain information that links the specimen ID number to the survey participant's name.

\subsubsection{Questions on Specimen Submission and Handling}

The chief medical technologist addresses questions concerning specimen collection including handling and testing.

\subsection{Test Requisition}

\subsubsection{Introduction}

Under a congressional mandate (Section 306 of the Public Health Service Act 42 U.S.C.242k) since 1960, NCHS has collected data on the health of the people of the United States through interviews and extensive physical examinations. Seven surveys using health examination procedures have been completed since 1960. As in previous NHANES programs, the survey's primary purpose is to produce descriptive statistics that can be used to measure and monitor the health and nutritional status of the civilian, noninstitutional U.S. population. Data collection for this survey involves about 7,000 survey participants per year, of whom about 5,000 per year are expected to be examined. Data collection began in January 2005 and continues for about 8 years at 15 different locations (stands) per year.

NHANES performs a complete blood count on survey participants 1 year and older, and urine and/or serum pregnancy tests on female SPs aged 12-59 years, and girls 8-13 who report that they are menstruating when asked during the home interview. If a urine pregnancy test is positive on any female SP aged 8-17 years, the result is confirmed using a serum test. If no blood is drawn, urine test is repeated. The physician is notified of the second positive or negative confirmatory test or the inability to perform a confirmation test using the observation function. 
A record of the test result report is maintained for a period of 7 years by Westat, the primary NHANES contractor, and by NCHS for an indefinite period.

\subsubsection{Laboratory Tests}

"Panic" or abnormally high $\mathrm{CBC}$ results are reported to the survey participant. The report includes laboratory tests that will be performed. The NCHS medical officer or MEC physician involved in the measurement may also confer with the survey participant to obtain any other pertinent information which might facilitate the laboratory measurement process.

\subsubsection{Human Subjects Review}

The NCHS ERB, as defined by the CDC institutional review board (IRB) criteria, shall perform all laboratory analyses conducted on human specimens, which are part of a "survey," only after approval of the survey.

\subsection{Test Records}

\subsubsection{Introduction}

The purpose of test records is to ensure correct and reliable identification of survey participant specimens and to report accurate test results. The laboratory test record system is distinguished from the test report that is provided to the survey participant. 


\subsubsection{Components of Test Records}

Test records include:

- Person performing the test (may be identified by initials);

- Printed or electronically stored output from instrumentation that the technical supervisor judges as necessary to document the analytical result;

- Date of specimen analysis;

- Date and time that specimen was collected by the laboratory; and

- Condition of specimens that did not meet criteria for acceptable specimens (e.g., hemolyzed or insufficient quantity for test).

Test records are maintained for a minimum of 7 years. Records may be bound in laboratory notebooks, in loose-leaf form, or stored electronically.

\subsection{Test Report}

\subsubsection{Introduction}

The laboratory report is provided promptly to the survey participant. This occurs as soon as the quality control has been reviewed and approved. The laboratory maintains confidentiality of the survey participant results since it identifies samples only by a specimen ID number. Westat and NCHS maintain the link between the specimen ID number and the identity of the survey participant.

\subsubsection{Review, Approval, and Release of Test Results}

Upon completion of laboratory testing, results are reviewed by laboratory staff responsible for review of the quality control results to assure that the measurement process was in control for each of the survey participant's analyses.

For NHANES surveys, the initial evaluation of quality control (QC) results is accomplished

electronically by comparing the QC results to QC limits stored in a relational database. The laboratory 
staff performing the complete blood count $(\mathrm{CBC})$ reviews these QC performance results and ensures that only acceptable survey participants' (SPs') data are reported.

Review and approval of the $\mathrm{CBC}$ and pregnancy test results by the MEC physician are documented. Review by the MEC physician includes an evaluation of the consistency of survey participant results with information that may be available on the survey participant (e.g., age, sex). All SPs' CBC samples are run in duplicate whenever possible. If inconsistent results are noted, and an adequate sample is available, the test is repeated a third time. If inconsistent results are again noted, the sample is run a fourth time. If the amount of sample is inadequate for repeat, then the survey participant is informed verbally and in writing of the inconsistent result.

The paper hard copy of the results with the NCHS medical officer's signature is filed by Westat and kept with the test record for 7 years. The NCHS medical officer signs all laboratory reports before release.

Laboratory reports are released only to the authorized survey participant. Each method includes a description of actions taken, such as when results are markedly elevated or are "panic values." The survey participant is contacted immediately by the MEC physician with such results.

In addition to the written laboratory report, the data may be transmitted electronically in a suitable format; for example, a SAS data set or an ASCII file is sent by email to the Westat home office and to NCHS.

\subsubsection{List of Methods and Method Details}

NHANES maintains a Laboratory Procedures Manual (LPM) which includes detailed descriptions of each analytical procedure including analytical sensitivity, specificity, and accuracy, interfering substances, and other information. 


\subsubsection{Format of the Test Report}

The test report includes:

- Name and address of NCHS;

- Test result;

- Units of measurement;

- Reference range for each analyte, if available;

- Condition of specimens not meeting the laboratory's criteria for acceptability; and

- Signature of the NHANES medical officer.

The laboratory report consists of:

- 1st page: a cover letter; and

- 2nd page: an analysis summary table.

A corrected laboratory report has the same format, except the words "corrected laboratory results" replace the words "laboratory results" in the letter and title of the pages. In addition, in the text of the cover letter, the date of the previous laboratory report is noted.

\subsubsection{Format for Laboratory Report Cover Letter}

An example of the Laboratory Report cover letter (report of findings) is available upon request.

\subsubsection{Format for Listing of Laboratory Results}

An example of the laboratory results is available upon request. 


\subsection{Quality Control}

\subsubsection{Introduction}

Most quantitative analytical procedures involve several operations, or steps, and each operation is subject to some inaccuracy or imprecision or to the possibility of a mistake. The immediate aim of quality control is to ensure that the analytical values produced by a clinical laboratory are sufficiently reliable for their intended purpose. A broader objective is to ensure that all laboratories produce analytical values that meet acceptable standards of precision and accuracy.

Quality control in laboratory medicine is the study of those errors that are the responsibility of the laboratory, and the procedures used to recognize and minimize them. An alternate term, "quality assurance," represents the techniques available to ensure with a specified degree of confidence that the result reported by the laboratory is correct. Both "precision control" (the agreement between replicate measurements) and "accuracy control" (the agreement between the mean estimate of a quantity and its true value) are evaluated to establish this degree of confidence.

A quality control program also monitors laboratory performance to detect excessive random or systematic error. Factors contributing to random error are those that affect the reproducibility of the measurement. These include instability of the instrumentation; variations in the temperature; variations in reagents and calibrators; handling techniques such as pipetting, mixing, and timing; and operators. Terms used to describe random error include precision, imprecision, reproducibility, and repeatability. Systematic error describes the error that is consistently low or high. Factors that contribute to systematic error are independent of the analyte concentration. Examples of these factors include interfering substances or incorrect assignment of the amount of substance in the calibrator.

A good quality control program includes both internal (intralaboratory) and external (interlaboratory) surveillance and monitors the following five parameters:

1. Clerical Error: This includes properly documented acknowledgment of transmittal and receipt of specimens (for example, "logging in"), proper labeling of all specimens, correct assignment of laboratory values to the proper SP ID number, and maintenance of proper records for all specimens for future reference.

2. Techniques: This includes continued assurance that all personnel performing an assay understand the principles underlying a particular assay and are cognizant of the proper technique for that assay; that all personnel use the same technique for a particular assay; that there is ready access to a current technique manual; and that periodic review is undertaken to ensure use of the most current and reliable techniques. 
3. Reagents and Materials: This includes confirmation of commercial standards and controls before they reach the bench; proper labeling of reagents, particularly those prepared in the laboratory; ensuring all reagents in use are not outdated; having an adequate supply of current reliable reagents; proper calibration of equipment, such as pipettes; and proper washing of glassware.

4. Bench Performance: This includes the use of controls and standards for each assay performed, a technique based on sound statistical principles which allows the technologist performing the assay to detect error outside of previously determined limits before reporting data; documentation of daily bench performance for detection of less obvious error (particularly those which tend to accumulate over time, so-called "drift"); and established procedures to be followed wherever error is found to exceed previously determined limits.

5. Instrumentation: This includes periodic preventive maintenance of all instruments in use in the laboratory and documentation that each instrument is maintaining a previously determined level of each performance at each check.

In this context, QC procedures monitor and evaluate the quality of the analytical testing process of each method to ensure the accuracy and reliability of test results. Thus, QC procedures referenced here refer to the analytical phase of testing and not to the preanalytic or postanalytic phases. Quality assurance (QA) addresses all three phases of measurement: preanalytic, analytic, and postanalytic. QC is therefore viewed here as one part of the overall QA process.

\subsubsection{Method-Specific QC Procedures}

Specific QC procedures exist for each analytical laboratory procedure in the Laboratory Procedures Manual. The following are some general aspects of QC procedures.

\subsubsection{QC Definitions}

\section{Internal ("Bench") Quality Control}

For purposes of this document, internal quality control is the evaluation of analytical performance that includes QC bench samples for which the analyst knows the expected measurement result. Internal commercial quality control materials contain predetermined amounts of analyte in the matrix, qualify under Clinical Laboratory Improvement Amendments (CLIA) as "calibration materials," and may be used as a "calibration verification" as defined by CLIA. 
- External ("Blind") Quality Control - For purposes of this document, external quality control is the evaluation of analytical performance that includes QC samples for which the analyst does not know the expected measurement result. The analyst is "blind" to the expected measurement result. Examples include proficiency testing samples.

- Interlaboratory Quality Assurance Program (IQAP) - For purposes of this document, NHANES participates in Beckman Coulter's ${ }^{\circledR}$ IQAP program. Beckman Coulter ${ }^{\circledR}$ issues a report that contains a statistical analysis to evaluate performance.

- Proficiency Testing - For purposes of this document, proficiency testing is one method of external quality control in which the analytical performance of a method is evaluated using specimens provided on a periodic basis (usually every 3 months).

- Analytical Run (sometimes referred to just as a run) - An analytical run is a set of samples analyzed in a time within which the measurement system is considered to have stable accuracy and precision. The time for a run may not exceed 24 hours. An analytical run usually consists of both quality control specimens and survey participant specimens.

- Calibration - Defined by CLIA as "the process of testing and adjusting an instrument, kit, or test system to provide a known relationship between the measurement response and the value of the substance that is being measured by the test procedure."

- Calibration Material - Defined by CLIA as "a solution which has a known amount of analyte weighed in or has a value determined by repetitive testing using a reference or definitive test method." Many standards are therefore calibration materials.

- Calibration Verification - Defined by CLIA as "the assaying of calibration materials in the same manner as survey participant samples to confirm that the calibration of the instrument, kit, or test system has remained stable throughout the laboratory's reportable range for survey participant test results."

- QC Requirements of Each Analytical Run - Analysis of survey participant samples is organized into analytical runs. The minimum QC requirement for an analytical run, which is analyzing survey participant specimens, is that the run includes four internal ("bench") QC specimens.

\subsubsection{Overview of the Relationship Between Internal QC, Proficiency Testing, and External QC}

A Beckman Coulter® technical representative performs DxH 800instrument setup and verification and performs and verifies QC checks according to their procedure at the beginning of each stand. Medical technologists perform instrument calibration. 
Most laboratory quality control programs include running internal ("bench") QC with each analytical run to ensure each analytical run is in control. The laboratory participates in proficiency testing programs administered by an outside scientific group to be sure on a periodic basis that accuracy and precision are within acceptable limits. The IQAP compares NHANES 6C cell control results to other laboratories. These QC systems are used within NHANES. The linearity of the instrument is evaluated at least once a year.

In addition to these systems, NHANES uses calibration materials at the beginning of each stand to standardize the three Beckman Coulter ${ }^{\circledR}$ DxH 800instruments and to provide additional objective evaluation of the quality of specimen analysis. S-CAL ${ }^{\circledR}$ is a calibration material as defined by CLIA (i.e., "a solution, which has a known amount of analyte or has a value determined by repetitive testing using a reference or definitive test method") that is useful for monitoring method performance.

\subsubsection{Internal ("Bench") QC}

\section{Complete Blood Count}

The goal of internal QC is to provide a rapid feedback to the analyst on the performance of the measurement process to be sure analytical results and factors affecting analytical results are within acceptable limits. At least three internal QC specimens $(6 \mathrm{C} \cap$ Cell Control) are included in each analytical run. The QC material used as internal QC specimen has levels of the analyte, which are useful for monitoring method performance.

The $6 C \circledR$ Cell Control is a hematology reference control used to monitor the performance of instruments with complete CBC and VCS differential technology. The 6C® Cell Control consists of human blood so repeated measurements to monitor daily performance of the instrument system are possible. The $6 \mathrm{C} \circledast$ Cell Control Tri-Pack contains three levels-normal, abnormal I, and abnormal II. When used with Beckman Coulter's ${ }^{\circledR}$ DxH diluent, DxH Lyse Reagent, DxH Diff Pack, they serve as a check on the accuracy of dilution, WBC counts, RBC counts, platelet counts, hemoglobin determinations, and differential counts.

The technologists run all three levels at the beginning of each session to monitor the precision of the instrument following procedures described in Chapter 6. 
Standard Levy-Jennings QC charts are maintained for this internal QC specimen. A separate QC chart is to be maintained for each QC material used for this internal QC specimen. Standard criteria for run rejection based on statistical probabilities are used to declare a run either in-control or out-ofcontrol. No results for a given analyte are reported from an analytical run that has been declared out-ofcontrol for that analyte as assessed by internal ("bench") QC. Rejection criteria are described in each method procedure in the LPM. Quality control results are captured by and stored in the ISIS. Any remedial actions for out-of-control conditions are documented in a $\mathrm{QC}$ logbook.

In addition, a latex control (Latron ${ }^{\circledR} \mathrm{CP}-\mathrm{X}$ control) is used to monitor the volume, conductivity, and light scatter (VCS) of the diff mode only. This control is run at the beginning of each day.

The Latron ${ }^{\circledR}$ CP-X control is a ready-to-use suspension of latex particles used to monitor differential parameters on the Beckman Coulter ${ }^{\circledR}$ DxH 800. These particles pass through the flow cell and produce characteristic electrical signals. It measures these signals as volume, conductivity, and light scatter. The technologist runs the Latron ${ }^{\circledR}$ CP-X control immediately following the Latron ${ }^{\circledR}$ primer.

\subsubsection{Coulter Interlaboratory Quality Assurance Program (IQAP)}

All DxH 800 instruments participate in Beckman Coulter's ${ }^{\circledR}$ Interlaboratory Quality Assurance Program (IQAP.) The Beckman Coulter $\AA$ Data Management System saves results of 6C® cell controls in files. Technologists transfer the files to a CD-RW and then send the disk to Westat laboratory study manager, who uploads the data in the Beckman Coulter ${ }^{\circledR}$ IQAP system and compares the results to other laboratories. Beckman Coulter ${ }^{\circledR}$ issues a report that contains a statistical analysis to evaluate performance. The technologists perform this procedure on the last day of each month, at the end of each stand, or when a control lot expires, using procedures described in Chapter 6.

\subsubsection{Proficiency Testing}

Participation in the College of American Pathologist (CAP) proficiency-testing (PT) program is part of the comprehensive quality control program. Each MEC submits results for CAP specimens for evaluation. CAP compares the results to established values and issues a report. 
The laboratory will satisfactorily participate in the HCFA-approved PT program for each method used to analyze human samples. Performance in the PT program, along with documentation of remedial action taken for unacceptable performance, is documented in a QC logbook that is available for review.

CAP samples are sent three times a year for hematology. Each shipment includes five 2.5-mL whole blood specimens. Duplicate Beckman Coulter ${ }^{\circledR}$ CBC determinations are performed on each whole blood sample using procedures described in Chapter 6. PT samples are handled and analyzed in a manner identical to SP samples. Results are recorded on the CAP forms and sent to the address specified by CAP Surveys Program Support with a copy to NCHS.

CAP samples are sent three times a year for qualitative serum and urine hCG. Each serum shipment includes five lyophilized sera specimens and each urine shipment contains five liquid specimens. PT samples are handled and analyzed in a manner identical to SP samples.

\section{CAP Proficiency Testing}

For purposes of this document, proficiency testing is one method of external quality control in which the analytical performance of a method is evaluated using specimens provided on a periodic basis (usually every 3 months).

Participation in the College of American Pathologist EXCEL proficiency-testing program is part of the comprehensive quality control program. Each MEC submits results for CAP specimens for evaluation. CAP compares the results to established values and issues a report.

These samples should be handled and analyzed in a manner identical to SP samples. Results are recorded on the CAP forms and are entered directly on the CAP website. A copy of the CAP form is sent to the home office at the end of the stand.

Results are recorded on the CAP forms and sent to the address specified by CAP Surveys Program Support with a copy to NCHS at the following address:

Brenda Lewis, MPH

National Center for Health Statistics

3313 Toledo Road

Hyattsville, MD 20782 


\subsubsection{Calibration Verification}

Calibration verification is defined by CLIA as "the assaying of calibration materials in the same manner as survey participant specimens to confirm that the calibration of the instrument, kit, or test system has remained stable throughout the laboratory's reportable range for survey participant test results." Thus, calibration verification assures that the accuracy of the measurement process across the reportable range is maintained over time. For methods approved by the FDA, calibration verification is performed according to the manufacturer's specification and at the frequency recommended by the manufacturer.

Note that the calibration materials must bracket the reportable range; that is; results should not be reported as a numerical value unless they are within the reportable range. If a result is higher than the upper end of the reportable range and the method permits sample dilution, the sample may be diluted to bring it within the reportable range. If dilution has been necessary, the reported value will exceed the upper end of the reportable range. Each analytical procedure specifies acceptable limits for results of analyses of the calibration materials based on the method precision of these materials.

The reliability of analytical values obtained when using a procedure often depends on the quality of the standards and the calibration procedure. The College of American Pathologists suggests that automated instruments be calibrated using multiple analytical whole blood specimens or a certified, stabilized whole-blood type preparation. The International Committee for Standardization of Hematology (ICSH) by its definition requires that a calibrator "be based on or traceable to a reference preparation or material."

Beckman Coulter ${ }^{\circledR}$ provides the S-CAL ${ }^{\circledR}$ calibrator to meet these requirements. The DxH 800 uses the $\mathrm{S}-\mathrm{CAL}{ }^{\circledR}$ kit that requires a calibrator to convert electronic measurements of each sample into accurate results expressed in clinical terms. The S-CAL $\AA$ calibrates the WBC, RBC, Hgb, MCV, Plt, and MPV parameters. It is a stabilized human-blood preparation. The S-CAL ${ }^{\circledR}$ is an acceptable alternative to whole blood calibration. Hct, $\mathrm{MCH}, \mathrm{MCHC}, \mathrm{RDW}$, and the DIFF parameters do not require calibration.

Calibration is a procedure to standardize the instrument by determining its deviation from calibration references and to apply any necessary correction factors. The calibration procedure uses replicate measurements of S-CAL $\AA$ calibrator. The S-CAL $\AA$ divides the average result into the calibrator Assigned Value to give an Adjustment Factor. Then it obtains and adjusts an instrument reading according to the Adjustment Factor. 
The S-CAL ${ }^{\circledR}$ is used to calibrate the Beckman Coulter ${ }^{\circledR}$ DxH 800 at the beginning of each stand (once every 6-8 weeks), after replacing any component dealing with dilution preparation, if the Beckman Coulter ${ }^{\circledR}$ representative suggests it, if controls demonstrate unusual trends or are outside limits, or when the temperature varies by more than $10^{\circ} \mathrm{F}\left(5.5^{\circ} \mathrm{C}\right)$ from the room temperature during the last calibration.

Instructions for the performance of calibration procedures using S-CAL® calibration are in Chapter 6.

\subsubsection{Linearity}

The linearity controls verify the reportable range of Beckman Coulter ${ }^{\circledR}$ hematology systems. Linearity is performed at installation, at least yearly, and whenever experiencing an altitude change of one mile or more between locations using either Beckman Coulter ${ }^{\circledR}$ Lin-X ${ }^{\mathrm{TM}}$ or CAP LN9 survey material.

Beckman Coulter® Lin- ${ }^{\mathrm{TM}}$ linearity controls are human blood components from which repeated measurements verify the reportable range of Beckman Coulter ${ }^{\circledR}$ hematology systems that use both Beckman Coulter ${ }^{\circledR}$ III diluent and Lyse S ${ }^{\circledR}$ III diff lytic reagent. Controls contain one each of Ultra Low Range, Low Range, Mid Range, High Range, and Ultra High Range. Lin-C ${ }^{\text {TM }}$ verifies ranges for the following parameters: WBC, RBC, Hgb, and Plt. Beckman Coulter ${ }^{\circledR}$ Lin-C ${ }^{\text {TM }}$.

CAP Hematology Calibration Verification/Linearity Survey (LN9) materials are shipped twice per year and consist of 18 3.0-mL liquid specimens. The medical technologist follows all CAP instructions in preparing and running the materials before performing the test. They fill out the CAP result form, make a copy for the logbook, send results to CAP, and send the copy back to the home office at the end of the stand.

The medical technologist follows all CAP instructions in preparing and running the materials before performing the test. They fill out the CAP result form, make a copy for the logbook, send results to $\mathrm{CAP}$, and send the copy back to the home office at the end of the stand. 


\subsubsection{Quality Control Records}

Records of all quality control results are maintained for at least 7 years. Quality control results are captured and stored in the ISIS and are available via the Intraweb. A QC logbook is maintained which documents out-of-control conditions and remedial actions taken to correct out-of-control conditions. The QC logbook also includes copies of results for Proficiency Testing and calibration.

New lot number crossover studies are performed on Coulter $6 \mathrm{C} \circledast$ Cell Controls and Osmolality Human Urine Controls. The new lot is run against the old lot 20 times, before the new lot is put into use.

\subsection{Test Methods, Equipment, Reagents, Supplies, and Facilities}

\subsubsection{Introduction}

Analytical procedures use equipment, reagents, materials, and supplies that are appropriate for achieving acceptable accuracy, precision, analytical sensitivity, and analytical specificity from the method. The documentation of individual analytical procedures included in the Laboratory Procedures Manual (LPM) specifies acceptable equipment, reagents, materials, and supplies. If special requirements concerning water quality, temperature, humidity, electrical power, or other conditions are required for acceptable method performance, then these are described for each procedure in the LPM. Special procedures to monitor these requirements are also included in the documentation of the individual procedure. A lack of requirements in the documentation of the analytical procedure means that the expected day-to-day variation in any of these parameters is acceptable for proper method performance.

Documentation of problems with conditions required (e.g., water quality) for acceptable method performance is maintained in the logbook of the primary instrument used in the analysis. This documentation includes remedial action.

\subsubsection{Labeling of Reagents, Solutions, Supplies}

Reagents, solutions, and other supplies are labeled to indicate the identity of contents, the concentration (if significant), the recommended storage requirements, the preparation and expiration date, 
technologist's initials, and any other pertinent information required for proper use. Reagent, solutions, and other supplies are not used when they have exceeded their expiration date.

If a method uses a manufacturer's kit, components of reagent kits of different lot numbers are not interchanged unless otherwise specified by the manufacturer.

Beckman Coulter ${ }^{\circledR}$ reagents are monitored and documented in the Beckman Coulter ${ }^{\circledR}$ Data Management System and are additionally located on the daily instrument startup result sheet. A copy of all startup results is filed in the quality control notebook and sent back to the home office at the end of each stand. The technologist documents any unusual occurrence in the automated Unusual Occurrence Log or repair of the Beckman Coulter ${ }^{\circledR}$ DxH 800 in the automated Equipment Tracking System.

Logs are maintained for reagents other than Beckman Coulter ${ }^{\circledR}$. The technologist documents reagent use by entering the reagent's name, date made, date put into use, expiration date, and tech ID in the automated Supply Use Control Log. The technical supervisor reviews the Supply Use Control Log at the end of each stand.

\subsubsection{Facilities}

Laboratories are arranged to ensure that adequate space, ventilation, and utilities are available for all phases of testing: preanalytic, analytic, and postanalytic.

\subsection{Analytical Procedure Manual}

\subsubsection{Introduction}

All procedures performed in the laboratory on human specimens are documented in the LPM. A copy of the LPM is maintained at the Westat home office and at NCHS. The individual procedure is also available at or nearby the bench site where the procedure is performed. 


\subsubsection{Contents of the Analytical Procedure Documentation}

Each analytical procedure includes the following, when applicable:

- Requirements for specimen collection and processing including criteria for specimen rejection;

- Step-by-step performance of the procedure including test calculations and interpretation of results;

- Preparation of reagents, calibrators, controls, solutions, and other materials used in testing;

- Calibration and calibration verification procedures;

- The reportable range for survey participant test results;

- Quality control procedures;

- Remedial action to be taken when calibration or control results are outside acceptable limits;

- Limitation in methods, including interfering substances;

- Reference range (normal values);

- Life-threatening or "panic values;"

- Pertinent literature references;

- Specimen storage criteria;

- Protocol for reporting panic values;

- Course of action if test system becomes inoperable; and

- Safety considerations for performing the method. 


\subsubsection{Approval and Record Maintenance}

Each procedure is reviewed, approved, signed, and dated by the clinical consultant and technical supervisor and laboratory director of NHANES. Procedures are reapproved, signed, and dated if the director changes. Each change in a procedure is approved, signed, and dated by the current director. The procedure includes the dates of initial use and discontinuance, if discontinued. The procedure documentation is maintained for 7 years after discontinuance of the method.

\subsubsection{Method Performance Specifications}

Method performance specifications for each analytical method are established or verified:

- Accuracy - accuracy of each analytical method will be determined by analysis of calibrators and yearly linearity checks as described in the section of the LPM on quality control.

- Precision - precision of each analytical method is determined by analysis of QC reference materials as described in the section of this manual on quality control.

- Analytical specificity - analytical specificity is determined for each method including effects of potential interfering substances. This may be verified by testing the effect of potential interfering substances in method development, by analyzing reference materials, by comparing results on split samples with a method considered more definitive, and/or by analyzing a sample of persons $(n>20)$ and examining the measurement output searching for interference. The appropriate procedures for verifying analytical specificity will vary by analytical method. Substances that interfere with the analysis in the reportable range are listed in the method procedure.

- Reportable range of test results - the reportable range of test results is described in the documentation of the analytical procedure.

- Reference range (normal range) - if available, the reference range of test results is described in the documentation of the analytical procedure and on the test report.

- Other pertinent performance specifications - other performance specifications that are required for adequate method performance are specified in the documentation of the analytical procedure. 


\subsection{Equipment Maintenance and Function Checks}

\subsubsection{Introduction}

Laboratory equipment is checked regularly to ensure acceptable performance. Maintenance (including preventive maintenance) and function checks are documented in the Laboratory and Phlebotomy Quality Control modules in the Integrated Survey and Information System (ISIS). The frequency of maintenance and function checks follows manufacturers' recommendations, when available. Manufacturers' recommendations are included in the manuals that are located in notebooks in the laboratory.

Each analytical procedure outlines the equipment maintenance and function checks for proper method performance and acceptable results from the checks. These checks must be made at the interval specified in the documentation of the procedure. Failure of a function check is documented in the automated Unusual Occurrence Log.

\subsubsection{Laboratory Equipment Calibration}

- Eppendorf Pipettes

- $\quad$ The Eppendorf Research pro electronic pipettes are calibrated once per year. Pipettes are sent to the manufacturer and calibration certificates are kept on file at the home office.

- Troemner Scale Weights

- $\quad$ The Troemner Scale Weights are calibrated once per year. Scale weights are sent to the manufacturer and calibration certificates are kept on file at the home office. 


\subsubsection{Temperature Monitoring}

Temperature recordings are required for storage and preservation of reagents and sample material (analytical specimens), as well as quality control and reference materials.

Beckman Coulter ${ }^{\circledR}$, Vacutainer, Precision Instruments, and Quantimetix have recommended the following storage conditions for reagents and tubes. Quality control temperature ranges have been established based on these recommendations.

\begin{tabular}{lcc}
\multicolumn{1}{c}{ Reagent } & $\begin{array}{c}\text { Manufacturer's recommended } \\
\text { storage conditions }\end{array}$ & Established range \\
\hline $\begin{array}{l}\text { Coulter }{ }^{\circledR} \text { reagents } \\
\text { DxH Diluent } \\
\text { CoulterDxH Lyse }\end{array}$ & $2-25^{\circ} \mathrm{C}\left(36-77^{\circ} \mathrm{F}\right)$ & $17-25^{\circ} \mathrm{C}\left(63-77^{\circ} \mathrm{F}\right)$ \\
DxH Diff Pack & & \\
DxH Cleaner & & \\
\hline S-CAL at room temperature & $16-32^{\circ} \mathrm{C}\left(60-90^{\circ} \mathrm{F}\right)$ & $17-25^{\circ} \mathrm{C}\left(63\right.$ to $\left.77^{\circ} \mathrm{F}\right)$ \\
\hline S-CAL at refrigerated temperature & $2-8^{\circ} \mathrm{C}\left(35-46^{\circ} \mathrm{F}\right)$ & $2-8^{\circ} \mathrm{C}\left(36\right.$ to $\left.46^{\circ} \mathrm{F}\right)$ \\
\hline Latron & $2-30^{\circ} \mathrm{C}\left(36-86^{\circ} \mathrm{F}\right)$ & $17-25^{\circ} \mathrm{C}\left(63\right.$ to $\left.77^{\circ} \mathrm{F}\right)$ \\
\hline $6 \mathrm{C} \mathrm{Cell} \mathrm{Control}$ & $2-8^{\circ} \mathrm{C}\left(36-46^{\circ} \mathrm{F}\right)$ & $2-8^{\circ} \mathrm{C}\left(36\right.$ to $\left.46^{\circ} \mathrm{F}\right)$ \\
\hline Vacutainers & Not greater than $77^{\circ} \mathrm{F}$ & $17-25^{\circ} \mathrm{C}\left(63\right.$ to $\left.77^{\circ} \mathrm{F}\right)$ \\
\hline
\end{tabular}


The following ranges have been established for monitoring instrument quality control.

\begin{tabular}{lcc}
\hline \multicolumn{1}{c}{ Instrument } & Manufacturer's instrument range or limit & Established range \\
\hline $\begin{array}{l}\text { Beckman centrifuge } \\
\text { (refrigerated) }\end{array}$ & $<-5$ to $+25^{\circ} \mathrm{C}\left(23-77^{\circ} \mathrm{F}\right)$ & $2-8^{\circ} \mathrm{C}\left(35\right.$ to $\left.46^{\circ} \mathrm{F}\right)$ \\
\hline $\begin{array}{l}\text { Beckman centrifuge } \\
\text { (room temperature })\end{array}$ & -5 to $+25^{\circ} \mathrm{C}\left(23-77^{\circ} \mathrm{F}\right)$ & $17-25^{\circ} \mathrm{C}\left(62\right.$ to $\left.77^{\circ} \mathrm{F}\right)$ \\
\hline Revco freezer & $-30^{\circ} \mathrm{C}\left(-22^{\circ} \mathrm{F}\right)$ Limit & -26 to $-34^{\circ} \mathrm{C}\left(-15\right.$ to $\left.-29^{\circ} \mathrm{F}\right)$ \\
\hline Jewett freezer & $-20^{\circ} \mathrm{C}\left(-4^{\circ} \mathrm{F}\right)$ Limit & -18 to $-23^{\circ} \mathrm{C}\left(0\right.$ to $\left.-10^{\circ} \mathrm{F}\right)$ \\
\hline Jewett refrigerator & $2-5^{\circ} \mathrm{C}\left(35\right.$ to $\left.41^{\circ} \mathrm{F}\right)$ & $2-8^{\circ} \mathrm{C}\left(35\right.$ to $\left.46^{\circ} \mathrm{F}\right)$ \\
\hline LFBSC & 0.450 & 0.350 to 0.550 \\
\hline Phlebotomy refrigerator & None & $2-8^{\circ} \mathrm{C}\left(35\right.$ to $\left.46^{\circ} \mathrm{F}\right)$ \\
\hline Mettler Toledo Scale & One $100 \mathrm{~g}$ weight (start of stand QC) & $+/-1.0$ grams \\
\hline Mettler Toledo Scale & Two $300 \mathrm{~g}$ weights (start of stand QC) & $+/-6.0$ grams \\
\hline Mettler Toledo Scale & One $500 \mathrm{~g}$ weight (daily QC) & $+/-5.0$ grams \\
\hline Mettler Toledo Scale & One $20 \mathrm{~g}$ weight (daily QC) & $+/-0.2$ grams \\
\hline
\end{tabular}

Two thermometers monitor ambient temperature in the laboratory and one thermometer monitors ambient temperature in the phlebotomy room. The allowable temperature range for these rooms is $17-25^{\circ} \mathrm{C}\left(63\right.$ to $\left.77^{\circ} \mathrm{F}\right)$.

The acceptable temperature range for refrigerators used for storage of CLIA-regulated specimens and reagents is $2-8^{\circ} \mathrm{C}$ and is checked and recorded daily.

Freezer temperatures are monitored daily by an automated TempGuard system. The range of allowable temperature for the $-20^{\circ} \mathrm{C}$ Jewett model is -18 to $-23^{\circ} \mathrm{C}$. The range of allowable temperature for the $-30^{\circ} \mathrm{C}$ Revco model is -26 to $-34^{\circ} \mathrm{C}$.

A separate automated continuous TempGuard system monitors the room temperature and the internal temperature of the laboratory refrigerators and freezers. When the system detects a significant increase in the temperature, it begins calling designated staff. The MEC managers and Facilities Equipment Specialists (FES) have been identified as those individuals responsible for responding to the call by typing in a response code. The temperature increase could be confined to one freezer or refrigerator, to multiple units, or all units. The temperature increase could be due to any number of circumstances such as a loss of power because a circuit breaker tripped, a compressor malfunctioned, or a 
system failed. The MEC manager or data manager implements corrective actions that are warranted to prevent loss of biological specimens.

Freezers and refrigerators are regularly monitored for excessive ice deposit, inoperative cooling fans, and frayed or worn electrical power connections. Problems are reported to the MEC manager.

SPs are given a urine collection cup and instructed to void completely into the urine container when they check into the MEC and change into their exam clothing. The container is brought back to the MEC laboratory and placed on the counter near the Mettler Toledo scale at workstation 2.

No later than 15 minutes after the specimen arrives in the laboratory the medical technologist places the cup on the scale and scans in the specimen. The lab application calculates the volume of urine and compares the volume collected to the amount needed for the protocol. The volume and date and time of urine collection are also captured.

\subsection{Calibration and Calibration Verification}

\subsubsection{Introduction}

Calibration and calibration verification are specified in the documentation of the CBC analytical procedure.

\subsubsection{Comparison of Methods Performed on Multiple Instruments or at Multiple Sites}

At least once every 6 to 8 weeks, each of the three DxH 800 instruments are calibrated with S-Cal. CAP data are analyzed by regression analysis and at least two of the three instruments are compared to each other three times per year. 


\subsection{Remedial Actions}

\subsubsection{Introduction}

Remedial actions are taken and documented when:

- Test systems perform outside acceptable performance specifications. Remedial action is documented in the appropriate QC manual.

- Results of control materials and calibration materials fail to meet quality control criteria. Remedial action is noted in the appropriate QC logbook.

- The laboratory cannot report survey participant test results within its established period. If the reporting of data will exceed the agreed upon time frame, the MEC and final Report of Findings indicate that test results are not available.

- Errors in the reported SP test results are detected. When errors are detected in the reporting of survey participant results, the MEC physician notifies the survey participant by phone, followed by the issuance of a corrected report within a time period suitable to the survey participant, but not to exceed 1 week. The corrected report clearly shows the corrected results in the title. Exact duplicates of the original as well as the corrected report are maintained for 7 years.

\subsection{Integrated Survey Information System (ISIS)}

\subsubsection{Introduction}

One of the purposes of the ISIS is to store survey participant results for retrieval in a period acceptable for the intended use of the data. Survey participant test results are stored in a database. The ISIS consists of the PC databases used for storing survey participant results. This form for the ISIS facilitates the interfacing of varied and complex instrumentation used in many of the analytical procedures. The MEC LAN hardware serves as the hardware for the ISIS. The identification of the survey participant result is by specimen ID number, and the result is accessible by this number.

The minimum amount of data stored for each analytical result is the specimen ID number, the analyte name, and the measured result. If the specimen ID does not provide sufficient information to relate the measured result to the QC data for the run, then the run number and run date must also be stored. The specimen ID, run date, and run number should be sufficient information to relate the measured result to the quality control information for that run. Access to the ISIS database is password protected so that only the persons authorized by the Westat and NCHS may access the data. 


\subsubsection{Integrated Survey and Information System Data Down Times}

Each laboratory method is able to run and temporarily store results even if the MEC LAN is inoperable.

\subsubsection{Maintenance of LAN Hardware}

After failure of a LAN component, ISIS should be able to be repaired or replaced within a day by a functioning component. LAN hardware is readily available. PC components and the infrequent problems with this hardware are readily correctable in this period. Westat participates in the standard service contract arranged for NHANES for service of failing or failed computer equipment.

\subsubsection{Backup Schedule of Integrated Survey and Information System Data}

ISIS data stored on LAN hard disk drives are backed up to tape once each weekday (Monday through Friday). If ISIS data are stored on local PC or minicomputer hard drives, they are archived to a second hard disk, tape, optical diskette, or CD-ROM at least once a week.

\subsection{Communications and Complaints}

\subsubsection{Introduction}

Good communication between the laboratory and persons reviewing laboratory analyses is essential for proper use of laboratory results. NHANES encourages collaborative relationships between technologists and physicians utilizing laboratory data. This collaborative interaction is the primary mechanism by which NHANES evaluates the effectiveness of laboratory protocols and other laboratory communications. If the laboratory has a persistent problem with meeting laboratory criteria (e.g., inadequate amount of specimen), the MEC manager, technical supervisor, or laboratory director will discuss the problem with the person in a collaborative spirit.

Similarly, complaints of laboratory performance are carefully considered. Usually, these complaints are fixed with minor adjustment or explanation. Each member of the laboratory staff is responsive to complaints that have merit and relate to her or his responsibilities. Nontrivial complaints are reported to supervisors who will decide appropriate actions, including investigation and remedial action. 
The technical supervisor maintains documentation of nontrivial complaints concerning laboratory performance that are reported to her or him.

\subsection{Quality Promotion}

\subsubsection{Introduction}

High-quality laboratory results are generated when all phases of the measurement process (i.e., preanalytic, analytic, and postanalytic) are conducted properly. Factors that promote high quality results include:

- Competent, well-trained, and motivated laboratory staff;

- Quality laboratory facilities;

- Well-maintained, high-quality laboratory equipment;

- High-quality laboratory analytical methods;

- Clear commitment of management to quality laboratory results; and

NHANES is committed to supporting each one of these factors in order to enhance quality.

\subsection{Quality Assurance Program}

\subsubsection{Introduction}

The goal of the NHANES Quality Assurance Program (QAP) is to monitor and evaluate the overall quality of the testing process including preanalytic, analytic, and postanalytic phases.

The initial database and reporting system design included the following requirements:

- All data are released in a timely manner relative to its intended use. All CBC results are provided to the SP in a preliminary Report of Findings when the SP checks out of the MEC. If the results are not available by the time the SP checks out of the MEC, they are reviewed by the MEC physician as soon as they are available, and the SP is notified by telephone of any abnormal results. A hard copy of the CBC results is included in the final Report of Findings sent to the SP 6-12 weeks after the MEC examination. In the case where no blood was drawn, the preliminary or final Report of Findings states, "Not done." In the case where blood was drawn but no results were 
transmitted, the preliminary or final Report of Findings states, "Data Not available at this time."

- The preliminary and final Report of Findings are ISIS-generated and are based on results in the database; they are not predefined reports stored in the system. The format for the English and Spanish preliminary and final Report of Findings is tested annually to determine if the generic format is legible.

- The preliminary and final Report of Findings format includes reference ranges, units of measure, or notation for inadequate specimen. Sample preliminary and final English and Spanish Report of Findings are printed and the format is tested annually.

- NCHS is responsible for sending the final Report of Findings to each survey participant. Mailing labels are printed, the mailing label is matched to the final Report of Findings, and the final Report of Findings is inserted into the envelope. NCHS monitors communications from SPs who report that they have received the incorrect Report of Findings.

- All results are generated based on results stored in the database. The ISIS system prohibits the MEC technologist from changing data in the database after the SP has checked out of the MEC or if the MEC Report of Findings has been printed. All subsequent copies of results should match the original results. If any result is ever changed in the database, a back-end editing request system form is submitted, tracked, and maintained by ISIS.

- A comprehensive security system guards against unauthorized access. A complete description of this system is maintained by and is available through NCHS.

The basic mechanism for the QAP is the quality audit. The quality audit is conducted by the NHANES technical supervisor or laboratory director, who maintains documentation of the results of the quality audits, including remedial actions taken to address conditions that merited corrective action.

\subsubsection{Quality Audit: Survey Participant Test Management}

The quality audit for survey participant test management is conducted at least yearly and consists of:

- A survey of the laboratory staff, and of the analytical procedures used in NHANES. The survey includes requests for information on:

Any changes which need to be made in survey participant preparation, specimen collection, labeling, preservation, and transportation; 
- $\quad$ Any changes which need to be made in completeness and relevance of survey participant information obtained at the time the specimen is acquired, and the use and appropriateness of specimen rejection criteria based on their experience and interactions with persons requesting the tests;

- $\quad$ Laboratory-reporting errors encountered since the last survey;

- Whether the performance of the Integrated Survey and Information System (ISIS) is adequate for their measurement systems; and

- $\quad$ Nontrivial complaints from outside the laboratory about performance of the lab.

The technical supervisor and laboratory director assess the results of the audit and, in consultation with Beckman Coulter ${ }^{\circledR}$, implement corrective actions that are warranted.

\subsubsection{Quality Audit: Quality Control}

Quality control addresses the analytic phase of measurement. The purpose of the internal ("bench") quality audit is to ensure that the QC analyses were performed and appropriate actions were taken when out-of-control conditions occurred. The quality audit of quality control shall be conducted at least yearly by the technical supervisor and laboratory director and shall consist of:

- A review of a random 10 percent sample of 5C Cell and Latron control results. The review evaluates whether appropriate corrective action was performed and documented for out-of-control conditions.

- An analysis of the proficiency testing results. The analysis and review will assess PT performance and, if unsatisfactory, whether the deficiencies have been adequately addressed. The technical supervisor and laboratory director compare results to determine the correlation coefficient. This correlation must be greater than 0.95 or the laboratory director may give written approval that the methods are sufficiently similar for the intended use of the data.

- The review of documents that demonstrate that at least once every 6 to 8 weeks the S-Cal calibrator was run on three instruments.

The technical supervisor and laboratory director assess the results of the audit and, in consultation with Beckman Coulter ${ }^{\circledR}$, implement corrective actions that are warranted. 


\subsubsection{Quality Audit: Personnel}

Well-trained and competent personnel are essential components of good laboratory performance. The quality audit of personnel is conducted at least yearly by the technical supervisor and laboratory director and shall consist of:

- A review of the 6C Cell and Latron control ("bench") results or IQAP QC results for 10 percent of the total control runs covering a period of not less than 1 year. The internal ("bench") QC reflects the performance of persons performing the method. If out-of-control conditions occur with a frequency more than twice the expected statistical probability (e.g., $95 \%$ control limits would normally be exceeded by $5 \%$ of the analytical runs), the technical supervisor and laboratory director will assess whether the unacceptable performance is due to the performance of the person in question or other factors (e.g., failing equipment). If the personnel performance is the cause, the technical supervisor and laboratory director determine and implement appropriate remedial action (e.g., training).

- A review of all remedial action since the last audit to assess the effectiveness of the action. Acceptable performance of the method by the person involved for 1 month after remedial action is acceptable evidence of the effectiveness of remedial action.

- The technical supervisor and laboratory director audit the documentation of personnel training and evaluation to check whether the analyst has been appropriately evaluated in the last year (or 6 months if in first year performing a new method). The evaluation criteria are described in the Personnel Training and Evaluation section of the LPM (Chapter 10, Section 10.16).

The technical supervisor maintains documentation of the results of the audit.

\subsubsection{Quality Assurance Review with Staff}

The technical supervisor and laboratory director prepare a report of the results of quality audits within 3 weeks of the completion of the audit. The technical supervisor and laboratory director review pertinent information with appropriate staff to ensure that effective remedial actions are taken. The technical supervisor and laboratory director also notify staff members who have been involved in the audit if performance was acceptable and that no remedial action is needed. At least once every 6 months, the technical supervisor and laboratory director review the results of quality audits. 


\subsection{Personnel Training and Evaluation}

The technical supervisor assures that persons performing laboratory tests receive appropriate training. The performance of persons performing laboratory tests is evaluated every 6 months for the first year testing is performed and annually thereafter. The evaluation shall consist of:

- Direct observation of test performance including survey participant preparation (if applicable), specimen handling, specimen processing, and testing;

- Direct observation of the recording and reporting of test results;

- Review of QC results, IQAP, PT, and linearity results and preventive maintenance records;

- Direct observation of performance of instrument maintenance and function checks;

- Assessment of testing performance using blind QC materials; and

- Assessment of problem-solving skills.

The technical supervisor maintains documentation of the evaluations for at least 7 years. If remedial action is warranted, the technical supervisor shall discuss the results of a personnel evaluation with the person evaluated and implement appropriate action (e.g., training). 


\subsection{Abbreviations}

NCHS - National Center for Health Statistics

CDC - Centers for Disease Control and Prevention

NHANES - National Health and Nutrition Examination Survey

MEC - mobile examination center

LPM - NHANES Laboratory Procedures Manual

U.S. - United States

CBC - complete blood count

ERB - ethics review board

QC - quality control

QA - quality assurance

IQAP- Interlaboratory Quality Assurance Program

PT - proficiency testing

CLIA - Clinical Laboratory Improvement Amendments of 1988

HCFA - Health Care Financing Administration

ISIS - Integrated Survey and Information System

PC - personal computer

LAN - local area network

SP - survey participant 


\section{OPERATIONAL ISSUES}

\subsection{Start of Stand Activities}

The Westat warehouse manager sends supplies for all stands, schedules shipments so that they arrive on or before setup day, and ships supplies requiring refrigeration so that they arrive the day before setup. Store laboratory supplies in the laboratory cabinets or in the MEC trailer compartments.

The advance arrangement staff may assist in completing some setup activities. On setup day, the phlebotomists and medical technologists work together to complete all activities. These tasks include assisting in unpacking the warehouse pallets and boxes, sorting the laboratory supplies by storage location, completing an inventory of all existing and new supplies, organizing and storing all inventoried supplies in MEC cabinets or trailer compartments, and setting up each work area by stocking the area with appropriate supplies. Medical technologists and phlebotomists perform maintenance on and test the functionality of all instruments and equipment. Notify the MEC manager if additional supplies are required and document all instrument and equipment malfunctions in the automated Unusual Field Occurrence utility. Record all lot numbers and expiration dates for consumables (phlebotomy and lab) in the Supply Use Control Log, which is located in the respective QC module.

Mark and store miscellaneous hardware and original packing boxed in a safe location in the laboratory until the end of stand. Hardware includes wood blocks with Velcro, refrigerator and freezer bottle caps, temperature-recording pen protectors, restraining straps, wood boards, and carpeted counter supports and straps for the Beckman Coulter ${ }^{\circledR}$ DxH 800 instrument and printer.

\section{Phlebotomy Room}

- The Phlebotomists' responsibilities include the following tasks:

- Inventory consumables and nonconsumables.

- $\quad$ Check the sink for hot and cold running water and proper drainage.

- $\quad$ Remove the wood block from the refrigerator door. 
- $\quad$ Unlock all cabinets.

- $\quad$ Release the phlebotomy chair restraint.

- Unlock all four casters.

- Pull up on the black lever on the lock and loosen just enough to separate the S bolts.

- $\quad$ Release the phlebotomy cart restraint.

- $\quad$ Turn on the refrigerator in phlebotomy, OGTT, and spare room.

- Verify the refrigerator's operation.

- $\quad$ Check the function of the telephone intercom.

- $\quad$ Clean the refrigerators.

- $\quad$ Install the wall thermometer.

- $\quad$ Clean all work surfaces and storage areas.

- $\quad$ Stock and organize supplies.

- $\quad$ Record all lot numbers and expiration dates in the automated Supply Use Control Log.

- $\quad$ Check the computer operations.

- $\quad$ Store the chair and cart restraint and the wood block in the bottom right drawer.

- Install the temperature monitoring probe into one of the available USB ports on the phlebotomy computers.

\section{Laboratory}

- Medical technologists' responsibilities include the following tasks:

- Inventory consumables and nonconsumables.

- $\quad$ Check the sink and eyewash for cold running water and proper drainage.

- $\quad$ Check telephone operation.

- $\quad$ Check the wall thermometers for damage.

- $\quad$ Remove the wood door blocks from the refrigerators and freezers and store. 
- $\quad$ Replace the refrigerator temperature probe solid bottle caps with the caps with holes and save solid caps for reuse at the end of the stand.

- $\quad$ Place the refrigerator temperature probes back into the bottles.

- $\quad$ Plug in the refrigerators and freezers.

- $\quad$ Verify freezer and refrigerator operation.

- $\quad$ Check light, blowers, and magnehelic pressure gauge reading in the Laminar Flow Biological Safety Cabinet (LFBSC).

- $\quad$ Release the Beckman Coulter ${ }^{\circledR}$ restraining straps until the end of the stand.

- $\quad$ Remove the restraining strap on the Beckman Coulter ${ }^{\circledR}$ printer and store the straps until the end of the stand.

- Contact Beckman Coulter ${ }^{\circledR}$ at 1-800-526-7694 to schedule instrument verification by a service technician. Provide the current address and instrument system ID.

- $\quad$ Perform all Beckman Coulter ${ }^{\circledR}$ beginning of stand operations as described in Chapter 6, This includes installing and priming all new reagents, performing a start-up procedure, and reviewing and verifying the system setup.

- Calibrate the Beckman Coulter ${ }^{\circledR D x H}$ 800. Complete the calibration before performing routine $\mathrm{CBCs}$ on SPs.

- Unpack the Mettler Toledo scale and store the carrying case in an easily accessible area. Perform the QC on the Toledo Scale and complete the QC before performing routine urine flow rates on SPs

- $\quad$ Refrigerate the serum and urine pregnancy controls, and Beckman Coulter ${ }^{\circledR}$ Latron and 6C Cell controls as soon as they arrive.

- $\quad$ Clean and disinfect all work surfaces and storage areas.

- $\quad$ Stock and organize supplies.

- Clean the interior and exterior of refrigerators, freezers, centrifuges, and LFBSC.

- $\quad$ Perform centrifuge maintenance.

- $\quad$ Open and label all new containers using the laboratory application.

- $\quad$ Mentally step through labeling, blood processing, urine processing, pregnancy testing, complete blood count, and specimen storage to make sure all the 
necessary equipment is functioning and all supplies are available in the phlebotomy/laboratory area.

- $\quad$ Document all readings and activities in the Laboratory QC module.

- $\quad$ Call FedEx to check availability (1-800-463-3339).

- NCHS biomedical engineer (annual) responsibilities include the following tasks:

- $\quad$ Determine the rpm of the centrifuges using a strobe or tachometer.

- $\quad$ Perform a timer check.

- $\quad$ Verify that both centrifuges are operating at $4-8^{\circ} \mathrm{C}$.

- $\quad$ Enter the results in the Laboratory QC module.

\subsection{End of Stand Activities}

Turn off the centrifuges. Reboot all computer terminals.

At the end of a stand, shut down all equipment and inventory all supplies. On teardown day, the phlebotomist and medical technologists work together to complete all activities. Carefully wrap all breakable supplies in preparation for the move. Lock all cabinets and drawers. Safely discard all biohazardous waste. Repack the Metler Toledo scale in the blue carrying case.

\section{Packing Phlebotomy Room}

- Phlebotomists' responsibilities include the following tasks:

- Inventory consumables and nonconsumables.

- $\quad$ Turn off and clean the refrigerators in the phlebotomy and OGTT rooms.

- Place wood blocks with Velcro strips on the refrigerator doors to prevent the doors from closing or moving. Be careful not to damage the rubber gasket on the door.

- $\quad$ Pack all juice and crackers in labeled plastic boxes and store them inside the MEC.

- $\quad$ Remove all loose supplies (clock, telephone handset, etc.) and equipment from the countertops; pack loose supplies in boxes and label "Phlebotomy Room." If possible, place the boxes in the cupboards above the counter, under the bench, 
or in the corners on the floor in phlebotomy. If these areas are full, place the boxes in the designated compartments under the trailers. Do not store Vacutainer ${ }^{\circledR}$ tubes in these trailer compartments.

- $\quad$ Bolt the room doors in the open position.

- $\quad$ Close and lock all cabinets.

- $\quad$ Lock all needles in the cabinets.

- $\quad$ Restrain the phlebotomy chair.

- Attach the $\mathrm{S}$ bolt on the end of the orange belt to each of the two wall brackets (under the drop-down chair).

- Attach the center S bolts to each other in the center of the orange belt.

- Tighten the belt by pulling on the free end of the belt.

- $\quad$ Restrain the phlebotomy cart in the staff lounge on the wall between the ISIS rack and the outside entry door to the staff lounge.

\section{Packing the Laboratory}

- Medical technologists' responsibilities include the following tasks:

- $\quad$ Inventory consumables and nonconsumables.

- $\quad$ Perform the Beckman Coulter ${ }^{\circledR}$ end of stand procedures as described in Chapter 6.

- Review, print, and clear the error file.

- Download the IQAP files.

- Print Latron and 6C Cell control cum statistics (files) and graphs.

- Perform daily shut down.

- Bleach the apertures and flow cell.

- Bleach, rinse, and drain reagent lines.

- Turn off the power.

- Rinse exterior of the BSV.

- Wash exterior of the instrument. 
- Dispose of all opened reagents.

- Check the expiration dates on the Beckman Coulter ${ }^{\circledR}$ Latron, Beckman Coulter ${ }^{\circledR}$ 6C Cell control, pregnancy test kit, and pregnancy controls. If unopened and they will not expire before the beginning of the next stand, pack them in a shipper with refrigerant packs, print a label using FedEx Ship, and ship back to the warehouse.

- $\quad$ Unplug the refrigerators and freezers.

- $\quad$ Clean refrigerators and freezers.

- $\quad$ Disinfect and clean the pipettes.

- $\quad$ Replace the refrigerator temperature probe bottle caps (with holes), with the bottle caps without holes, and save the caps with holes for reuse at the start of the next stand.

- Place wood blocks with Velcro strips on each refrigerator and freezer door to prevent the door from closing or moving. Be careful not to damage the rubber gasket on the door.

- $\quad$ Turn off the Mettler Toledo scale. Disconnect the scale from the tan serial cable and black AC adapter power cord. Tape the serial cable and power cord to the countertop. Repack the Mettler Toledo scale in the blue carrying case making sure to remove and store the weigh pan in the lid of the carrying case and cover the scale cone with the plastic cone protector.

- $\quad$ Unplug the LFBSC.

- $\quad$ Close and lock centrifuge lids and turn off centrifuges.

- $\quad$ Pack all tube rockers, Zebra printer, and TB incubator securely in boxes or in drawers.

- $\quad$ Remove all small supplies from the counters and LFBSC and pack in boxes or cabinets.

- $\quad$ Close all cupboards and lock.

- $\quad$ Remove the fire extinguisher from the wall and lay inside the laboratory sink.

- $\quad$ Clean all counters, the LFBSC, and equipment with 1:100 bleach solution.

- $\quad$ Place chairs upside down or lay them down on the floor.

- $\quad$ Attach strap around the bottom front of the instrument. (Do not apply too much pressure.) 
- $\quad$ Secure the Beckman Coulter ${ }^{\circledR}$ printer by strapping it to the printer shelf.

\section{Sending Laboratory Files and Supplies}

At the end of each stand, use FedEx to create a label to send the following hard-copy documentation to the technical supervisor at the home office:

- Beckman Coulter ${ }^{\circledR}$ Latron and 6C Cell Control printouts

- Beckman Coulter ${ }^{\circledR}$ Daily checks printouts

- $\quad$ Beckman Coulter ${ }^{\circledR}$ Calibration, repeatability and carryover printouts

- Beckman Coulter ${ }^{\circledR}$ Latex CBC/Diff cumulative statistic and graph printouts

- Beckman Coulter ${ }^{\circledR}$ Error file printout

- Beckman Coulter ${ }^{\circledR}$ Linearity printouts

- Proficiency testing copies (hematology, urine and serum hCG)

At the end of each stand use FedEx to create a label to send the following supplies to the warehouse supervisor:

- Unopened pregnancy test kits

- Unopened pregnancy urine controls - refrigerated

- Pregnancy serum controls - refrigerated

- Unopened Coulter ${ }^{\circledR} 6 \mathrm{C}$ Cell and Latron controls - refrigerated

Pack supplies using procedures described in Chapter 8.

\section{I3.3 Inventory Procedures and Supplies}

At the start and end of each stand, take a complete inventory. The warehouse uses this inventory to stock the MEC for the next stand.

The equipment and supplies associated with the MEC laboratory are listed in Exhibit 13-1, Equipment and supplies. 
Exhibit 13-1. Equipment and supplies

\begin{tabular}{ll}
\hline -30 Revco freezer ULT 430A18 & Laminar flow biological safety cabinet \\
Two Beckman Coulter Allegra centrifuges & One Jewett refrigerator (phlebotomy) \\
Two refrigerators (OGTT and right side of trailer 3) & Mettler Toledo scale \\
Two Thermolyne Vari-Mix M48700 (lab) & One Thermolyne Speci-Mix (phlebotomy) \\
Two Maxi-Mixer (lab) & One Thermolyne Speci-Mix (lab) \\
Two solar wall thermometers (lab) & One solar wall thermometer (phlebotomy) \\
Two Jewett refrigerators UC5B & Thermometer (centrifuge calibration) \\
Five Jewett temperature monitoring units 8ER-1B & \\
\hline
\end{tabular}

Store supplies in the trailer compartments.

\subsubsection{General Inventory Procedures}

- Since item units can change, verify the "unit of measure" each time you record a count. Examples of units are pack, each, bag, box, case, roll, tray, bottle, pair, and pouch.

- Do not redefine or reiterate the Unit of Measure.

- "PAR Level" represents the number of units the component is to begin a stand with. Note that PAR Levels are set and adjusted by home component staff only. Any/all recommendations to adjust an item's PAR level should be directed to the home component staff.

- Do not write any notes, comments, etc. on the Start-of-Stand Inventory Count Sheets or the End-of-Stand Inventory Count Sheets. Comments or concerns should be written on a separate sheet of paper and attached to the inventory sheet.

- Rotate items by first using items you have in the rooms, then items in the belly compartment. Place items you just received in the back of the stock, unless you need them immediately. Only restock your rooms with items that were just shipped to the stand when you are out of that item in your room. Many items such as gloves, alcohol prep pads, Sani-cloth wipes, blood tubes, etc. deteriorate over time. Always use inventory with the closest expiration date first.

- Multiple inventory items onboard the MEC contain lot numbers and expiration dates. When performing physical inventory counts, pay close attention to these items and mark inventory sheets accordingly for each lot number/expiration date per item. 
- Do not purchase items listed on the inventory sheets. Every item listed on the inventory sheet is supplied by the NHANES Warehouse. Purchasing items locally creates chaos with the Inventory Management System.

- Do not borrow items from other components. Any additional inventory needed by a component should be requested from the NHANES Warehouse. Inventory usage is tracked by component. The "Counted By" tech ID is entered into the database to track inconsistencies and trends. Everyone is accountable for his or her component counts.

\subsubsection{End of Stand Inventory Procedures}

The End-of Stand Inventory Count Sheet (EOSCS) includes the stand number, Site ID (MEC number), a "Counted By" line, and Exam Supply columns for item number, general item description, Unit designation, PAR level, and a count box. The MEC manager prints these EOSCSs for every component. The MEC staff are responsible for accurately counting (inventorying) exam supplies and entering this number into the count box. The MEC staff give the completed sheets back to the MEC manager who sends them to the warehouse manager. The warehouse manager enters the item counts into the Inventory Management System.

- Print only one name in the "Counted By" field. This is the person responsible for taking and verifying the inventory count.

- Please write legibly for all counts.

- Place a number in each "count" box. Do not leave any count box empty. If there is no stock remaining for a specific item, place a "zero" in the count box.

- Do not count partial units; record only whole numbers in the count box. Do not write entries such as: $1 / 2$, partial, some, many, a few, couple, .5 , or multiple.

- If the PAR for an item is more than one (1) and the "unit of measure" is open, do not count that container. Only count the remaining number of items that have not been opened.

- If the PAR for an item is only one (1) unit and it is more than $1 / 2$ empty, place a zero (0) in the count box. A full unit will be supplied to the component at the opening of the next site.

- All active lot numbers and expiration dates are listed on the inventory count sheets if they are applicable for that item. Record the number of supplies remaining for each lot number/expiration date in the count box. If there is no inventory remaining for a listed lot number/expiration date, then place a "zero" in the count box. 
- Any item that has expired during the MEC site or will expire before the MEC reopens at its next location should be removed from the component. If an item is removed, a "zero" should be entered in the count box on the End-of-Stand Inventory Count Sheet for that item. A Delete/Expired Inventory Count Sheet must be filled out, per component, showing the quantity for each item removed from the component. This additional inventory sheet must be attached to the End-of-Stand Inventory Count Sheet for that component when turned into the MEC manager.

\subsubsection{Mid-Stand Inventory Procedures}

Mid-Stand Inventory is performed by each component on the MEC. The Mid-Stand Inventory is performed approximately $2-2 \frac{1}{2}$ weeks into an operating site. This inventory provides the opportunity for MEC staff to request additional supplies from the warehouse that will be needed to complete the current MEC site. The Mid-Stand Inventory is conducted via UFO entry (per component).

NOTE: The Mid-Stand Inventory is only to provide additional supplies needed to complete the current site; it is not designed to bring all supplies up to full par level.

- Each component is to review all remaining inventory to determine if additional supplies are needed to complete the current MEC site.

- A "UFO" will be entered (sighted) by one MEC staff member per component.

- MEC staff will enter a "UFO" by component name and then use "Inventory/Supplies" for the type of UFO entry.

- In the description field, the MEC staff member will request the supply(s) needed for the component.

- Entries are to be made as: item description then quantity requested (per item).

- If no additional supplies are needed for the component, then the entry "Nothing Needed" is to be written in the description field.

- Do not request items in the UFO that are not currently on the component inventory list.

- Do not request changes to set par levels in mid-stand UFOs.

- Do not enter equipment issues in a UFO listed as "Inventory/Supplies."

- All completed UFO entries will be reviewed by the MEC manager for that MEC before the warehouse will pull and ship supplies requested. 
- Once the supplies have been pulled by the warehouse and packed for shipping to the $\mathrm{MEC}$, the warehouse manager will finalize the UFO.

- Mid-Stand deliveries are arranged between the MEC manager and the warehouse manager.

\subsubsection{Start of Stand Inventory Procedures}

The Start-of-Stand Pull Sheets (SOSPS) include the stand number, Site ID (MEC number), and Exam Supply columns for part number, general item description, Previous EOS Count number, PAR level, Unit designation, and a Ship to Stand box. The warehouse manager uses this report to bring each item back to par level. For example, if the Previous EOS Count (captured by the IMS) is four and the Par level is five, the Ship to Stand count box will contain the number one. The warehouse manager prints and packs one SOSPS for each component. The MEC staff are responsible for accurately reconciling the supplies and numbers in the SOSPS. The MEC staff give the completed sheets back to the MEC manager who returns them to the warehouse manager. The warehouse manager reviews each sheet and sends additional supplies to the field if necessary.

- Reconcile the inventory for each item.

- Verify the Previous EOS Count for each item.

- Verify that the start of stand shipment contains the exact number of each item listed in the Ship to Stand box.

- Previous EOS Count added to the Ship to Stand Count Box should equal PAR Level.

- If the total inventory equals the set PAR level, place a checkmark on the right side of the Ship to Stand Count Box (per item).

- If there is a discrepancy, write the physical count on hand to the right of the Ship to Stand Count box (per item).

- If the quantity for an item is higher than the set PAR level, the excess should be removed from the component (leaving PAR) and returned to the warehouse. If an item(s) is being returned to the warehouse, have the MEC manager print a "Transfer Inventory to Warehouse Count Sheet" for the component. Print your name on the inventory sheet. Mark only the quantities for item(s) that are being returned. Give the excess inventory along with the "Transfer Inventory Sheet" to the MEC manager to ship supplies back to the warehouse. 


\subsubsection{Consumables vs. Nonconsumables}

Inventory items are divided into two categories - consumable and nonconsumable. Inventory both types of items during each inventory. The definition for a consumable item is anything that is typically consumed during an examination. A nonconsumable item is one that can be used more than once (example: stopwatches, test tube racks, safety glasses, storage boxes, etc.). There are times when nonconsumable items will need to be replaced. These instances would include items that are used in an emergency, when the item expires, or when the item breaks (example: physician medical kit supplies, stopwatches, thermometers, CPR masks, etc.).

\subsubsection{Shipping Excess Inventory Back to the Warehouse}

When shipping excess inventory back to the warehouse, please use the "Transfer Inventory to Warehouse Manifest," which is found on the Intraweb and can be printed by the MEC manager. This form looks similar to the "End of Stand Count Sheets." Print your name in the "Count By" field and mark only the items (with quantity being returned) in the count boxes that are being shipped back to the warehouse. Give the items to be returned along with the "Transfer Inventory to Warehouse Inventory Sheet" to the MEC manager to ship back to the warehouse. This information is entered into the Inventory Management System by the warehouse manager and is used to adjust the stand inventory and usage information as well as increase the warehouse inventory counts.

\subsubsection{Tracking of Expired and Broken Inventory}

Complete the "Delete Expired/Broken Inventory Report" whenever inventory has expired and must be destroyed or has broken and is no longer usable. Print your name in the "Count By" field and mark only the item(s) in the count box that have expired or broken, and which are being removed from the MEC. This report is found on the Intraweb and should be completed and forwarded to the warehouse manager so that the expired or broken inventory can be removed from the stand inventory. Unless the item is a tool (piece of equipment), under most circumstances the item can be disposed of there in the field and there is no need to return the item to the warehouse. Check with the MEC manager or call the warehouse manager to verify the need to ship the expired/broken item back to the warehouse. 
The chief medical technologist and the phlebotomist are responsible for notifying the MEC manager if additional supplies are needed during the stand. However, each technologist and phlebotomist is responsible for identifying any potential shortage of supplies at each workstation. Contact the warehouse manager directly at 301-330-7092.

\subsection{Dry Run}

After setting up the phlebotomy and laboratory rooms at each new stand, the staff conducts a "dry run" session using volunteers to verify data and specimen collection and processing procedures. One purpose of the dry run session is to help identify any problem areas, particularly with equipment, and to resolve the problems before the stand begins. Another purpose is to provide the contract laboratories with blind split samples for quality control determinations. Two distinct IDs are assigned to each volunteerthe original SP ID and a second unique Sample ID. Document the results of the dry run session in a manner identical to an actual session except enter results twice, first using the SP ID, and second using the Sample ID.

\section{Procedures}

- Collect the amount of blood specified in the Dry Run Venipuncture Protocol. This amount is less than the blood required from a routine examinee.

- Prepare split samples for each volunteer.

- $\quad$ Collect 4-mL of urine and process as specified in Dry Run Urine Processing Protocol

- $\quad$ Process the blood as directed in the Dry Run Blood Processing Protocols

- $\quad$ Store these split samples and ship them to contract laboratories for analysis with the first routine shipment.

\subsubsection{Phlebotomy Protocol and Procedures}

The dry run venipuncture protocol (Exhibit 13-2) is dependent on the last digit in the SP ID. If the last digit is an odd number, the volunteer's protocol includes two 4-mL EDTA, four 2-mL gray, two 
6-mL royal blue and two $15-\mathrm{mL}$ red top tubes. If the last digit is an even number, then the volunteer's protocol includes two 4-mL EDTA, four 2-mL gray, and two $15-\mathrm{mL}$ red top tubes. Since both even and odd dry run volunteers are eligible for the oral glucose tolerance test, their profiles include two additional 2-mL gray tubes.

On dry run day the phlebotomist refers to the following instructions to conduct the phlebotomy procedures:

- Access the phlebotomy application, open the exam, and wand the dry run volunteer into the component.

Exhibit 13-2. Dry run venipuncture protocol

\begin{tabular}{|c|c|c|}
\hline Tube type (in priority order) & $\begin{array}{c}\text { Last digit in } \\
\text { SP ID = Odd }\end{array}$ & $\begin{array}{c}\text { Last digit in } \\
\text { SP ID = Even }\end{array}$ \\
\hline 4-mL EDTA & 2 & 2 \\
\hline 2-mL Gray & 4 & 4 \\
\hline 15-mL Red & 2 & 2 \\
\hline 6 mL Royal Blue & 2 & \\
\hline
\end{tabular}

- Conduct the phlebotomy interview and administer the fasting questionnaire as described in Chapter 3.

- Perform the venipuncture using procedures described in Chapter 3.

The last digit in the SP ID determines the dry run volunteer's venipuncture protocol. The correct venipuncture automatically displays. Note that there are two IDs - an SP ID (110355), and a Sample ID (616625). Both the SP ID and the Sample ID display under the "Obtained all" checkbox. 


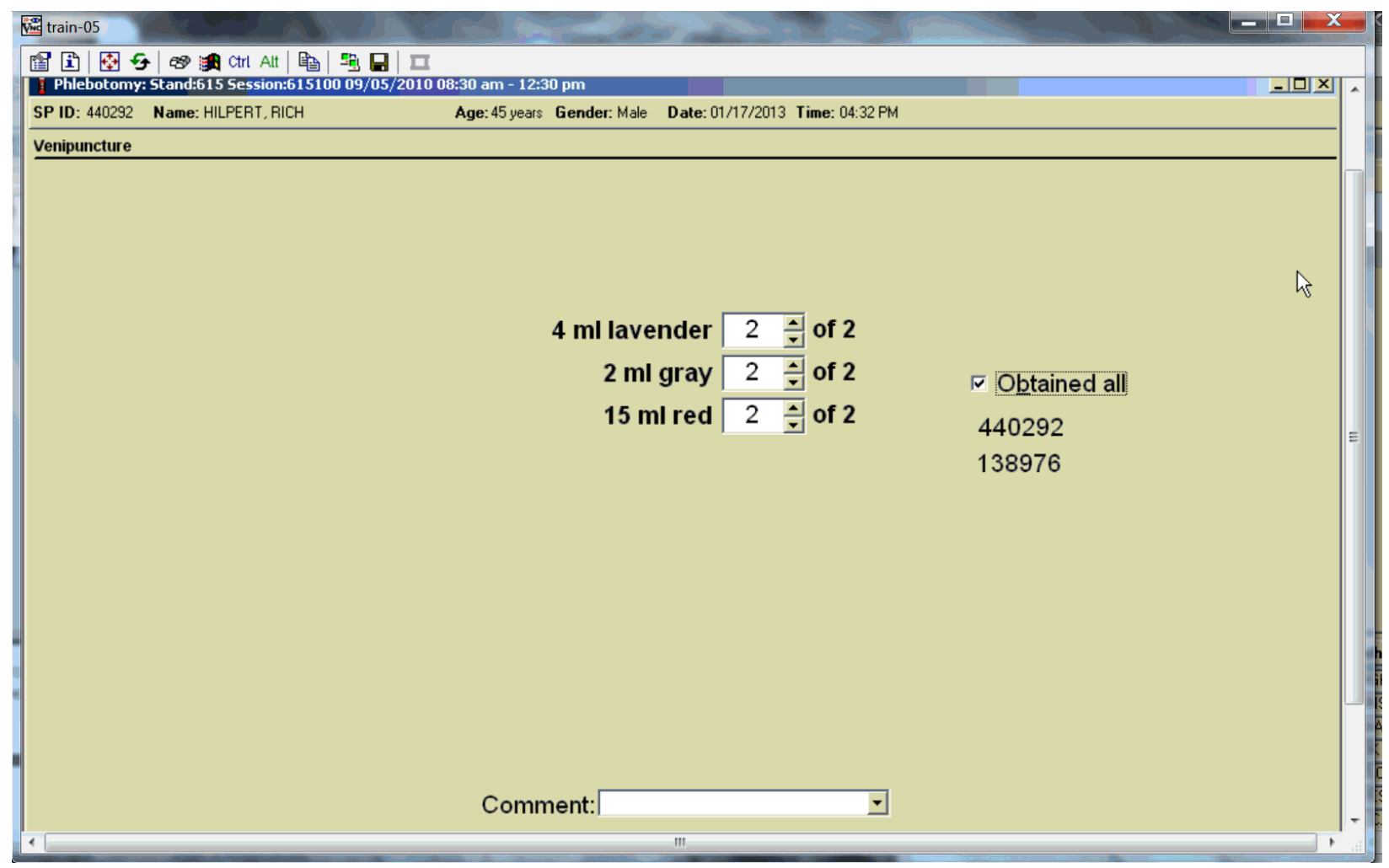

Enter the venipuncture results using procedures described in Chapter 3. Enter any reasons for a tube not being drawn according to protocol, and any comments about the venipuncture. Refer to Chapter 3 for specific instructions.

Label the Vacutainer ${ }^{\circledR}$ tubes for each volunteer; label half of the tubes with the SP ID and the other half with the Sample ID. If only one tube is drawn, discard the tube. If less than two of the 15$\mathrm{mL}$ red tubes are successfully drawn, label the first tube with the original SP ID and the second one with the Sample ID. If an odd number of tubes are drawn, discard the odd tube.

For dry run volunteers with the SP ID and Sample ID ending in an odd number:

- Place one label with the original SP ID on one of the two 4-mL EDTA tubes and place the other label with the Sample ID on the other 4-mL EDTA tube.

- Place the label with the original SP ID on one tube of the pair of 2-mL gray tubes and place the label with the Sample ID on the other 2-mL gray tube.

- Place the label with the original SP ID on one of the two $15-\mathrm{mL}$ red tubes and place the label with the Sample ID on the other $15-\mathrm{mL}$ red tube. 
- Place one label with the original SP ID on one of the two 6-mL royal blue tubes and place the other label with the Sample ID on the other 6-mL royal blue tube.

For dry run volunteers with the SP ID and Sample ID ending in an even number:

- Place two of the labels with the original SP ID on one pair of 3-mL EDTA tubes and place the other two labels with the Sample ID on the other pair of 3-mL EDTA tubes.

- Place the label with the original SP ID on one tube of the pair of 2-mL gray tubes and place the label with the Sample ID on the other 2-mL gray tube.

- Place the labels with the original SP ID on two of the four $15-\mathrm{mL}$ red tubes and place the labels with the Sample ID on the other two 15-mL red tubes.

\subsubsection{Urine Processing and Pregnancy Testing}

Access the laboratory application and logon using the MEC Logon window. 


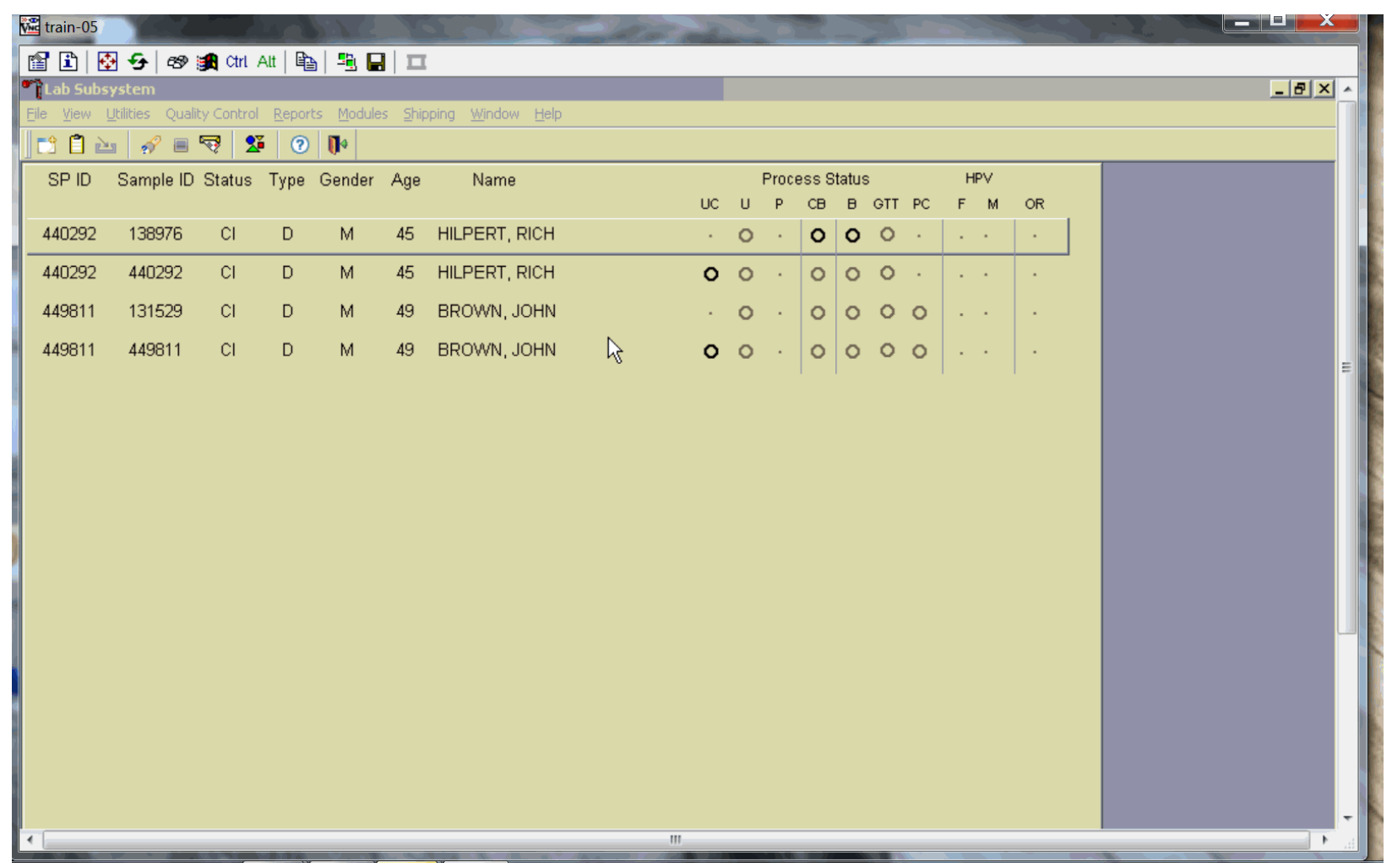

Two distinct IDs are assigned to each volunteer - the original SP ID and a second unique Sample ID.

Place urine and pregnancy labels at workstation 3. Each volunteer has two unique blood processing racks - one with labels containing the SP ID and the second with labels containing the Sample ID. Assemble and label the blood processing racks for each dry run volunteer using procedures described in Chapter 9.

Review the laboratory heads-up display. Use the heads-up display to view the modules for which the dry run volunteer is eligible and the current process status. 


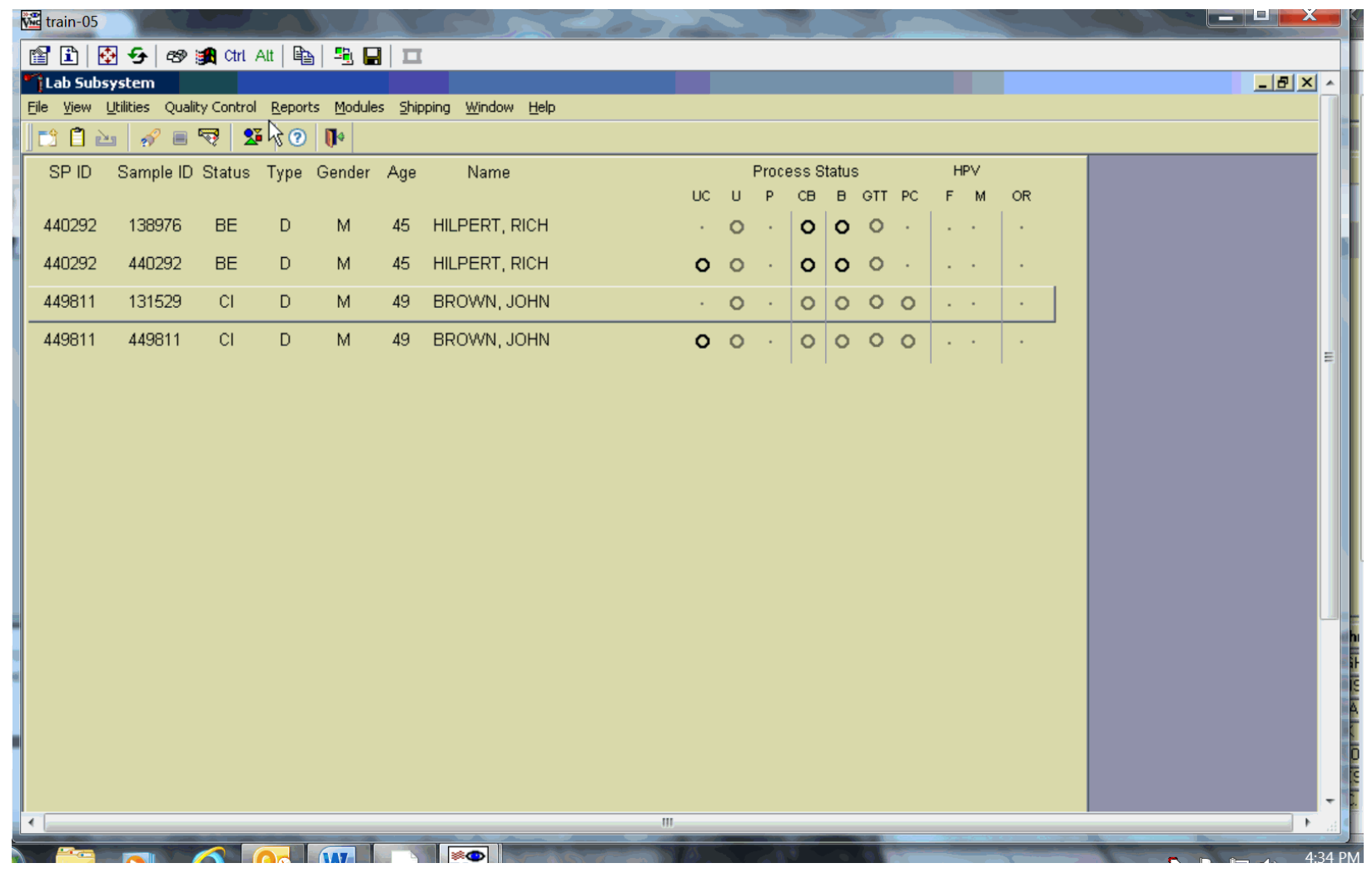

The heads-up display lists each dry run volunteer twice. Note the difference between the SP ID and the Sample ID and the types of tests for which the dry run volunteer is eligible. Dry run volunteers with an SP ID and Sample ID ending in either an odd or even digit are eligible for urine collection and processing, osmolality, CBC, GTT, blood processing, and the pregnancy test (if female.) The urine and blood protocols constitute a limited subset of the primary SP's test protocol. Each test has the same age and gender restrictions as a primary SP. Notice that one urine collection result and one pregnancy test are required for each dry run volunteer when the SP ID and Sample ID end in an odd or even digit.

Collect at least 4-5-mL urine from all dry run volunteers. The MEC coordinator instructs each volunteer to void as soon as they feel they are able to provide a urine specimen. The coordinator or local assistant transports the initial specimen to the laboratory. All dry run volunteers are eligible for at least one urine test. 
Place the urine collection cup on the scale and scan in the urine collection specimen barcode as described in Chapter 4, Section 4.4.

Document the urine collection in the laboratory application.

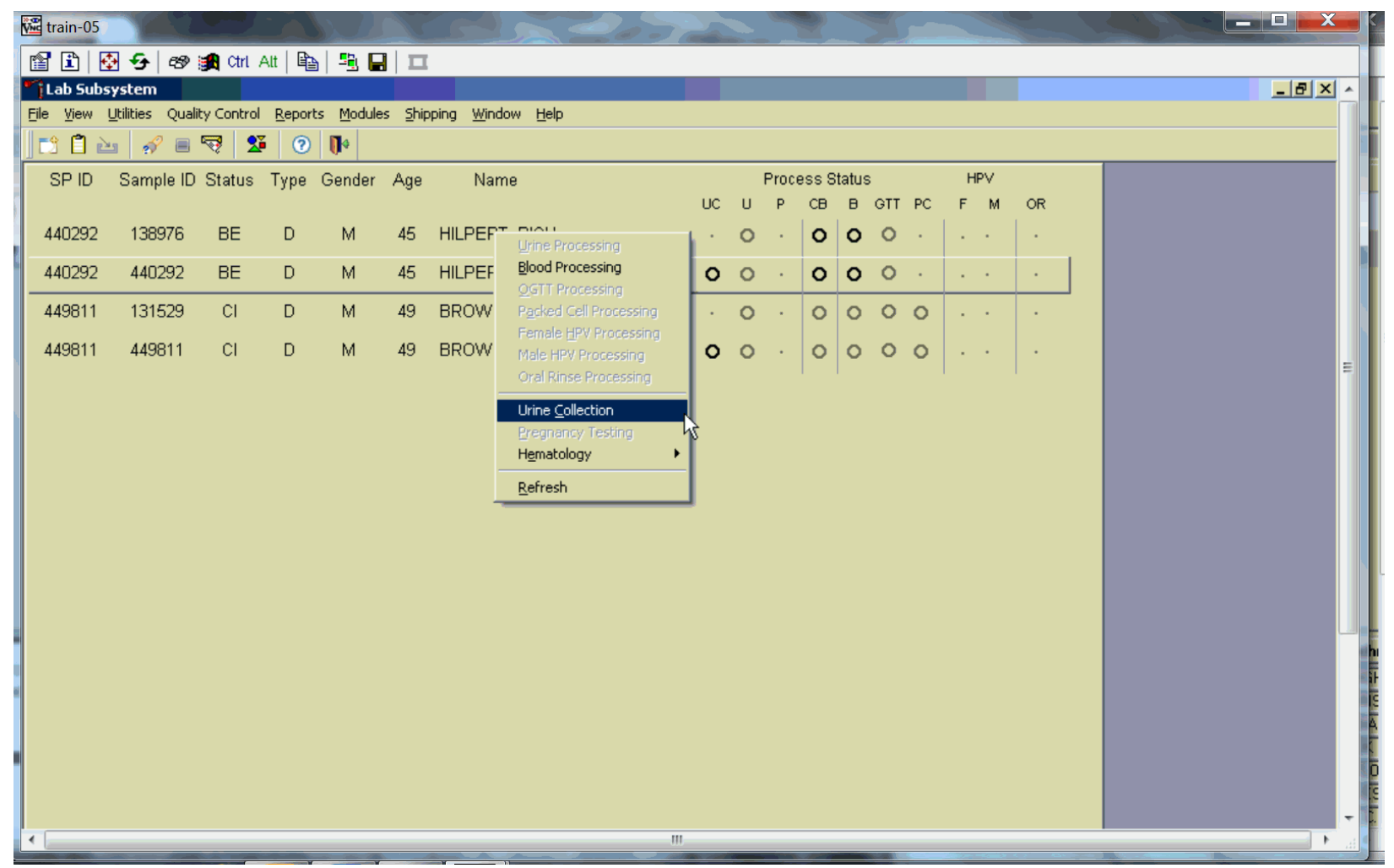

To select the correct dry run volunteer's record, locate the record in the heads-up display that contains the urine collection circle. To access the urine collection module, use the up and down keys to move up and down the list until the correct record is highlighted or drag the mouse arrow to the correct volunteer and right click. Continue by dragging the mouse arrow to \{Urine Collection\}, then left click or type $[\mathrm{C} / \mathrm{c}]$.

The urine collection window displays. 


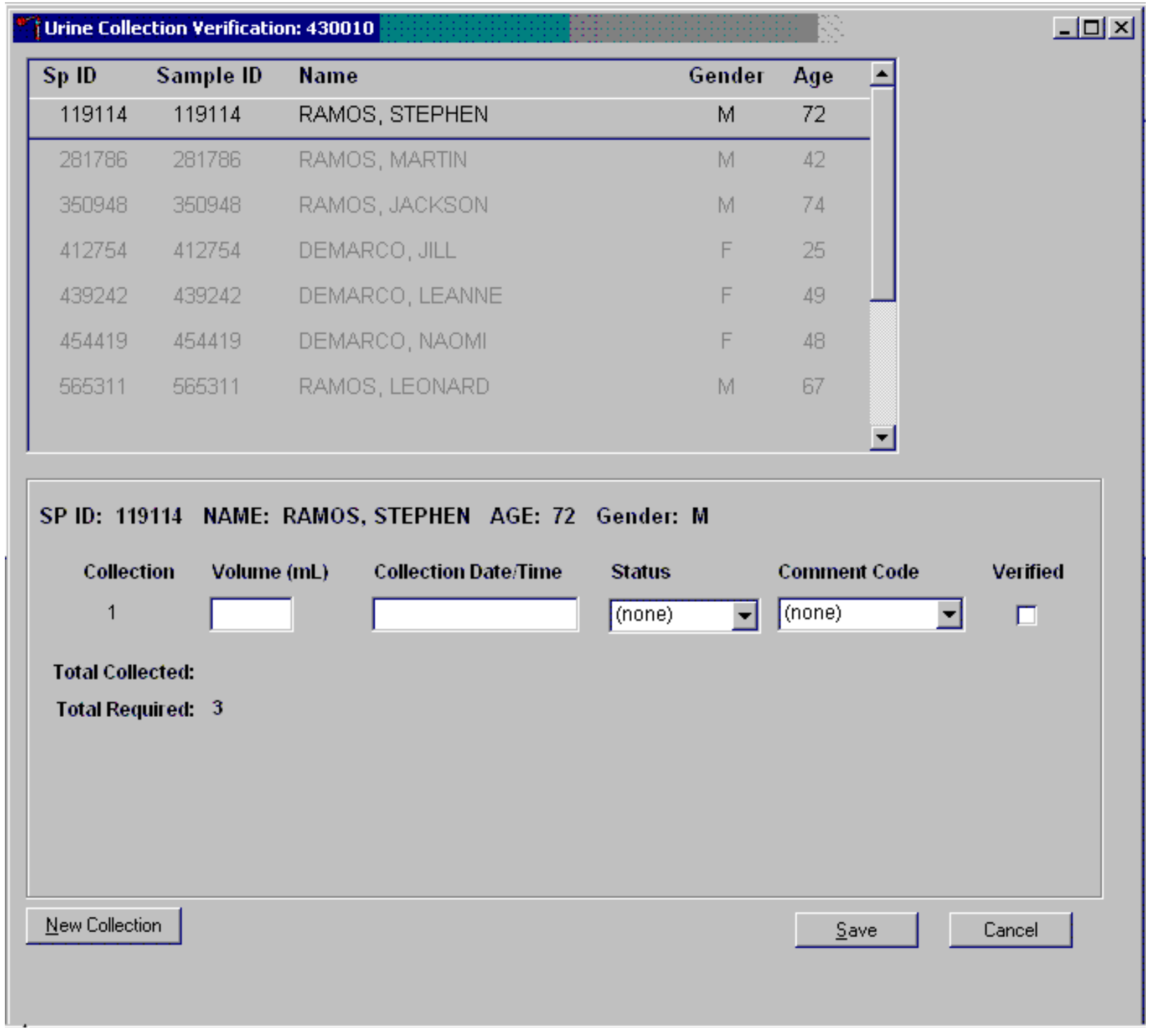

The top of the urine collection window contains columns for the SP ID, Sample ID, Person Name (last, first), Gender, and Age. The row for checked-in SPs is indicated in black, while SPs who have not checked into the MEC session are gray. Only dry run volunteers who have checked into the MEC session are available for processing. SPs who have a urine collection ready for verification display in blue. Use the scroll bar to view all SPs scheduled into the session. 
Verify the sufficiency of the quantity of urine received in the laboratory. Use the measuring tool to verify the amount of urine reported from the scale.

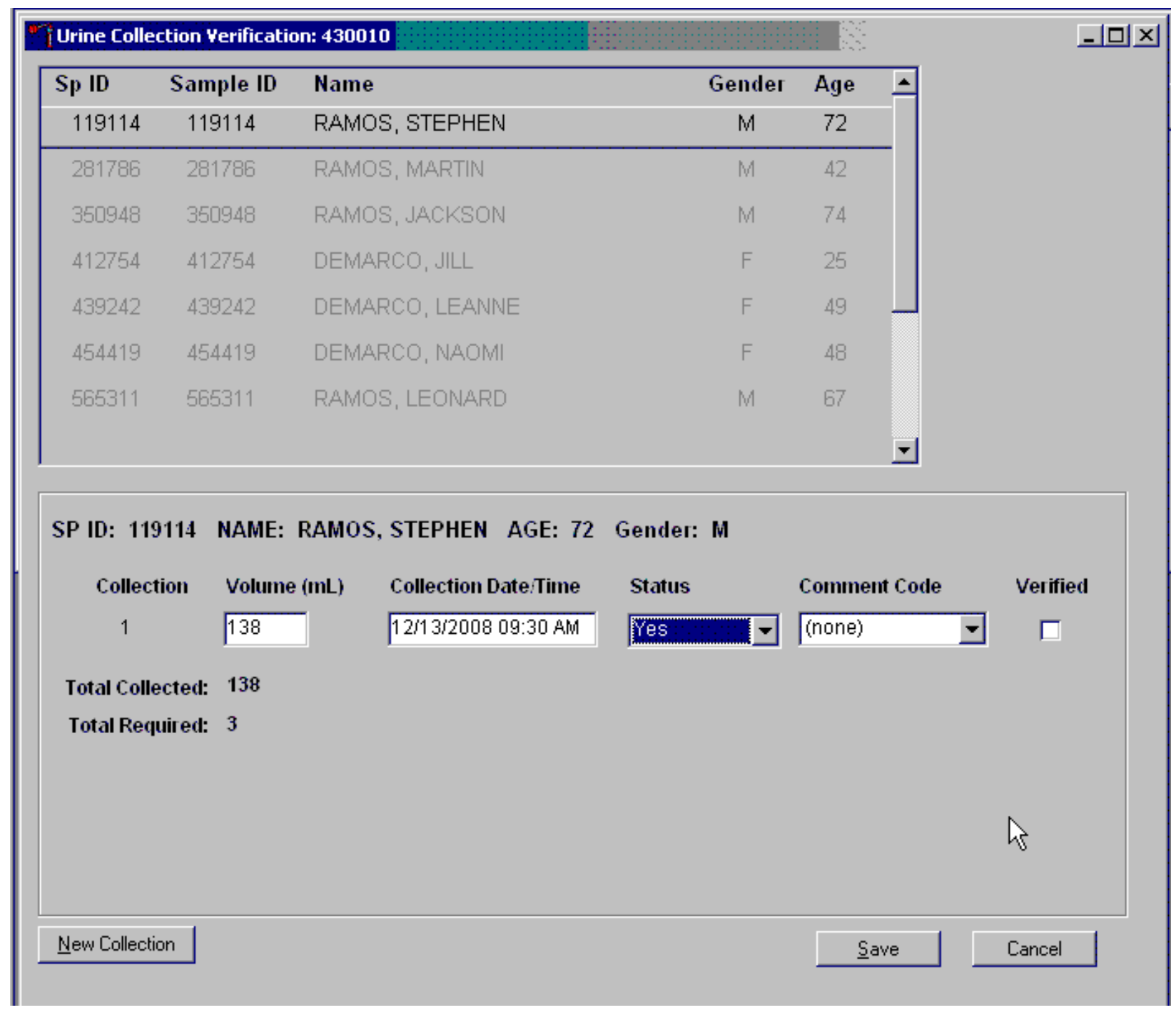

If the SP has submitted a urine collection that has been weighed and scanned into the database the volume, collection status, and collection date and time will automatically display in the lower portion of the Urine Collection screen. 
If the collection did not meet the minimum protocol requirement, the application will automatically insert "QNS" in the Status result text field and "quantity not sufficient" in the Comment Code text box.

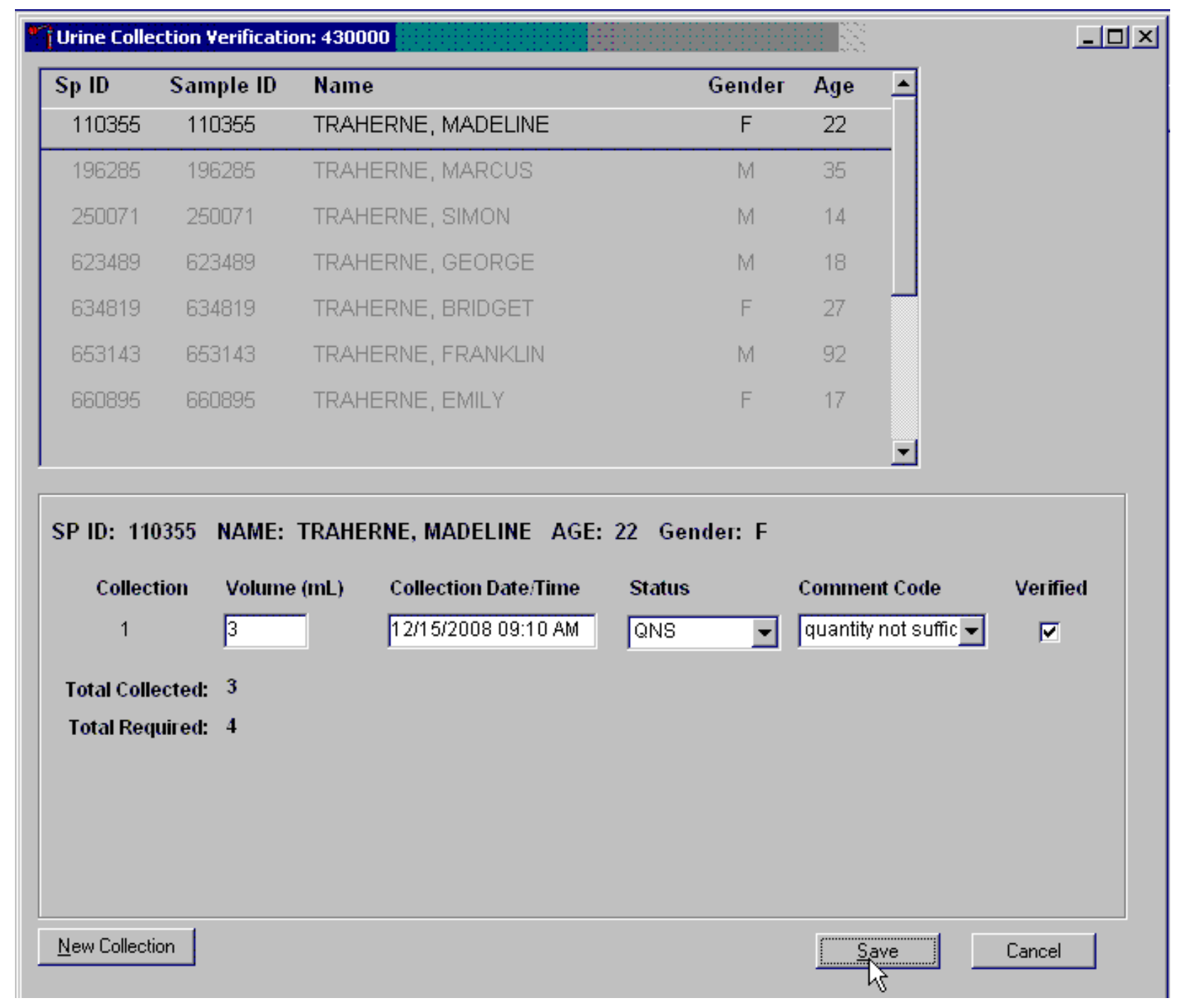

A QNS status immediately prompts and notifies the coordinator to ask the SP for an additional urine sample. 
A second instance of urine collection for the first QNS sample or third instance of urine collection for the second QNS sample will appear below the current sample.

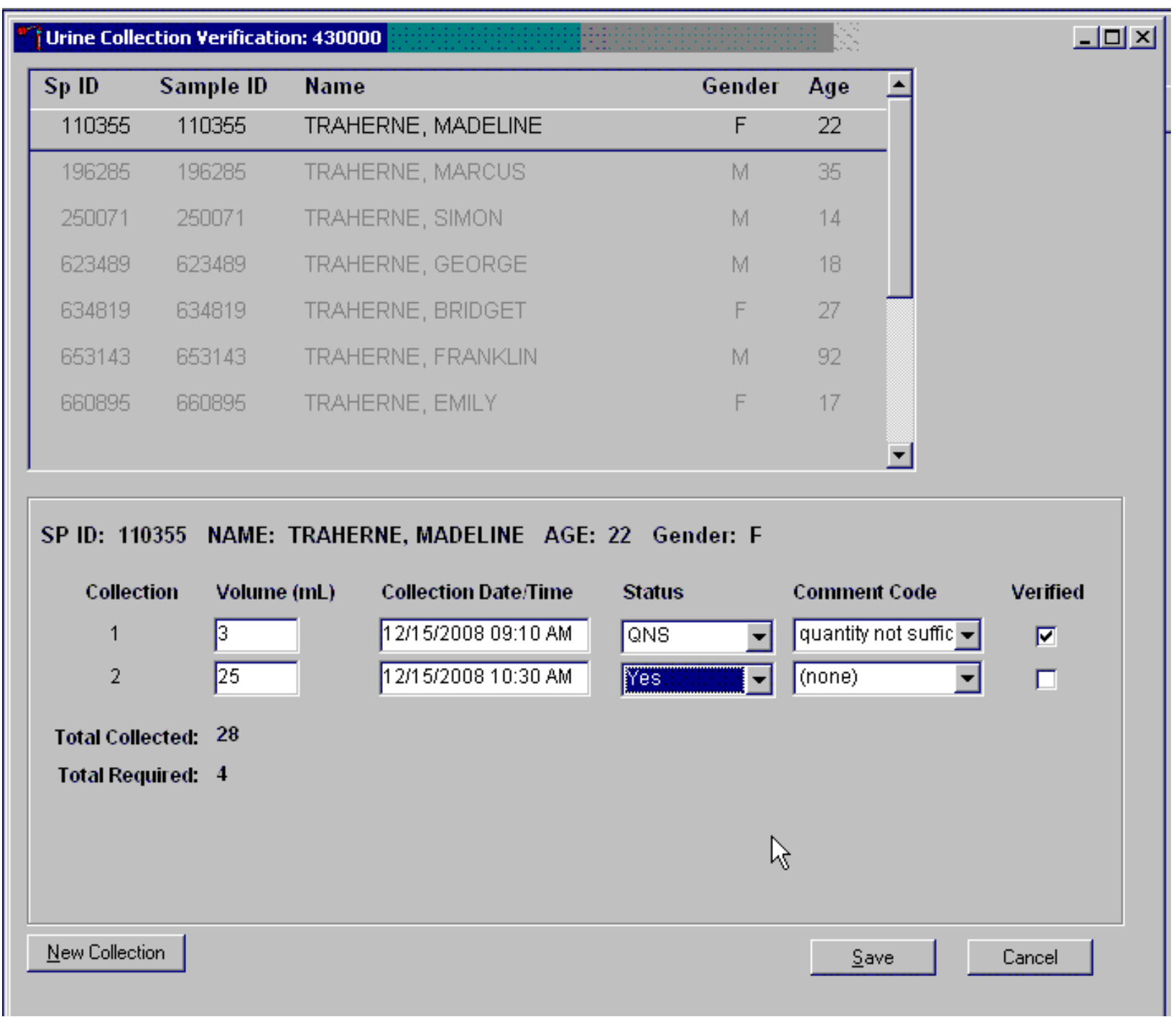

If the quantity of urine collection does meet the requirement of the urine protocol, the application will automatically select a status of "Yes" and no additional collections will appear. 
Evaluate the quantity of urine received in the laboratory. Compare the number displayed in the Total Collected column and the actual volume of urine in the cup.

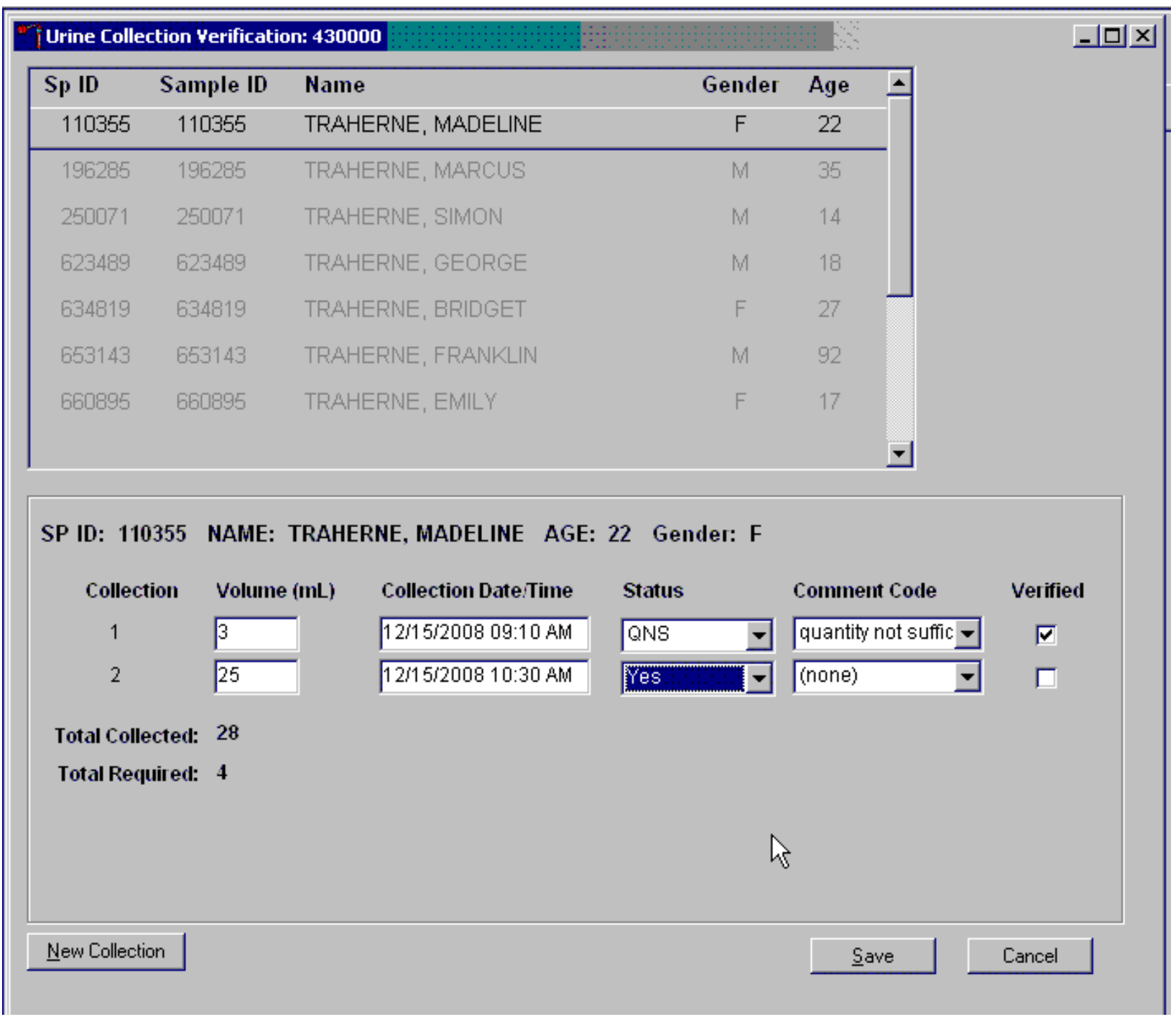

Adjust the Volume (mL) if necessary by using the mouse to direct the mouse arrow to the text box under the Volume ( $\mathrm{mL}$ ) column. Right click on the text box to enable the cursor in the box. Make changes using the number pad on the keyboard. Once any necessary adjustments have been made, use the mouse to direct the mouse arrow to the box under the Collection Date/Time column or press the Tab key. 
A prompt will pop up asking if you would like to subtract the weight of the cup. If the volume of the sample was transmitted from the scale and you have made a manual adjustment to that reading, select "No."

Do not enter a result until urine arrives in the laboratory. At the end of each session, review the results for each dry run volunteer. Enter "No" in the result field for all volunteers who have not produced a urine sample. Record a result other than "(none)" for all records. Record a comment for all insufficient urine collections.

The laboratory application will automatically assign the comment code "quantity not sufficient" for all urine collections that are calculated as QNS by the scale application. The laboratory technician may also assign this comment code to all samples that are changed to QNS during the verification process. 


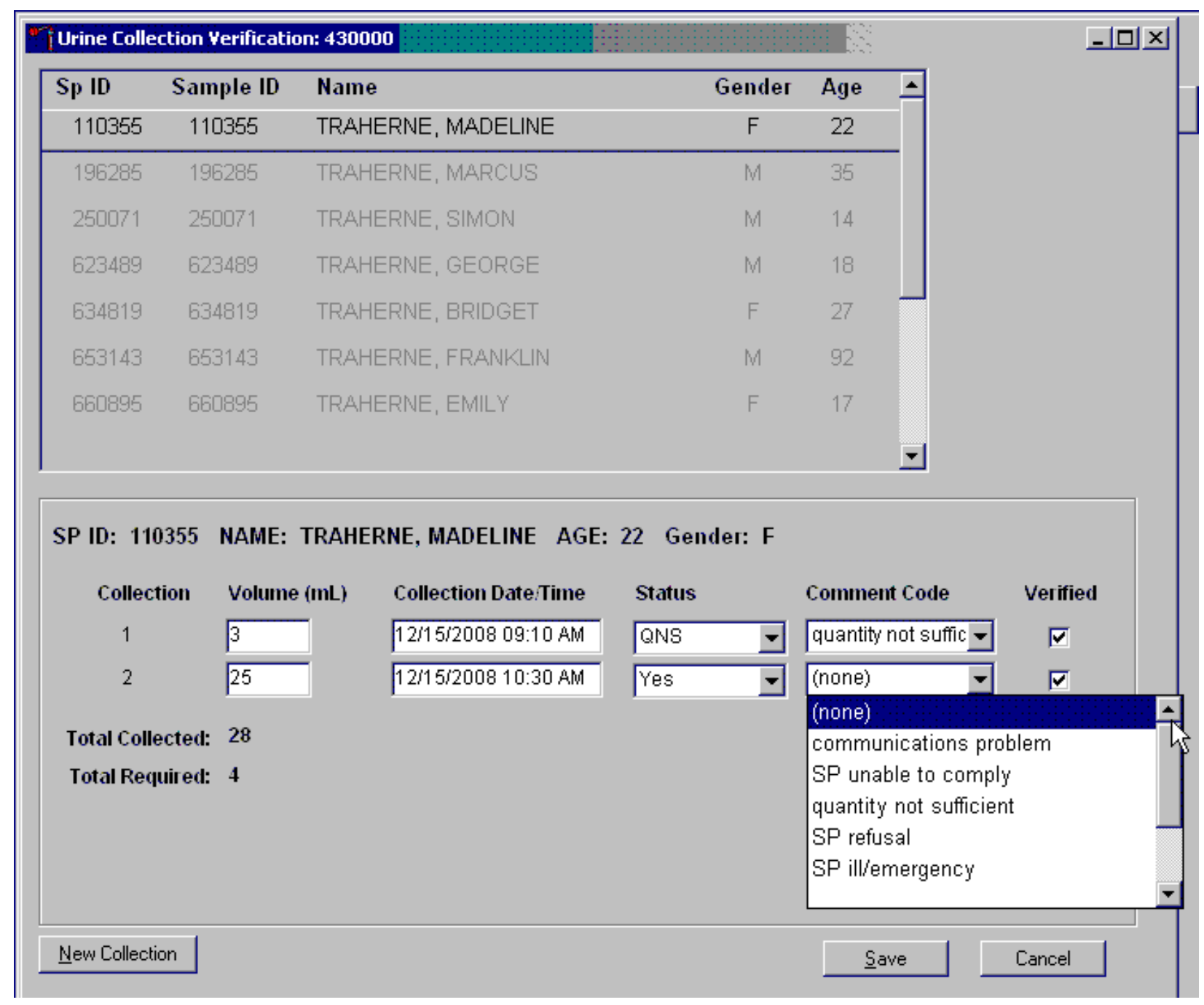

To record a comment, use the mouse to direct the mouse arrow to the drop-down list, click to display the comment codes, and select the most appropriate choice. Alternatively, use the up and down keyboard arrows to scroll through the choices or type the first letter of the desired comment code and when the correct choice is highlighted, left click. 
Verify the results of the urine collection.

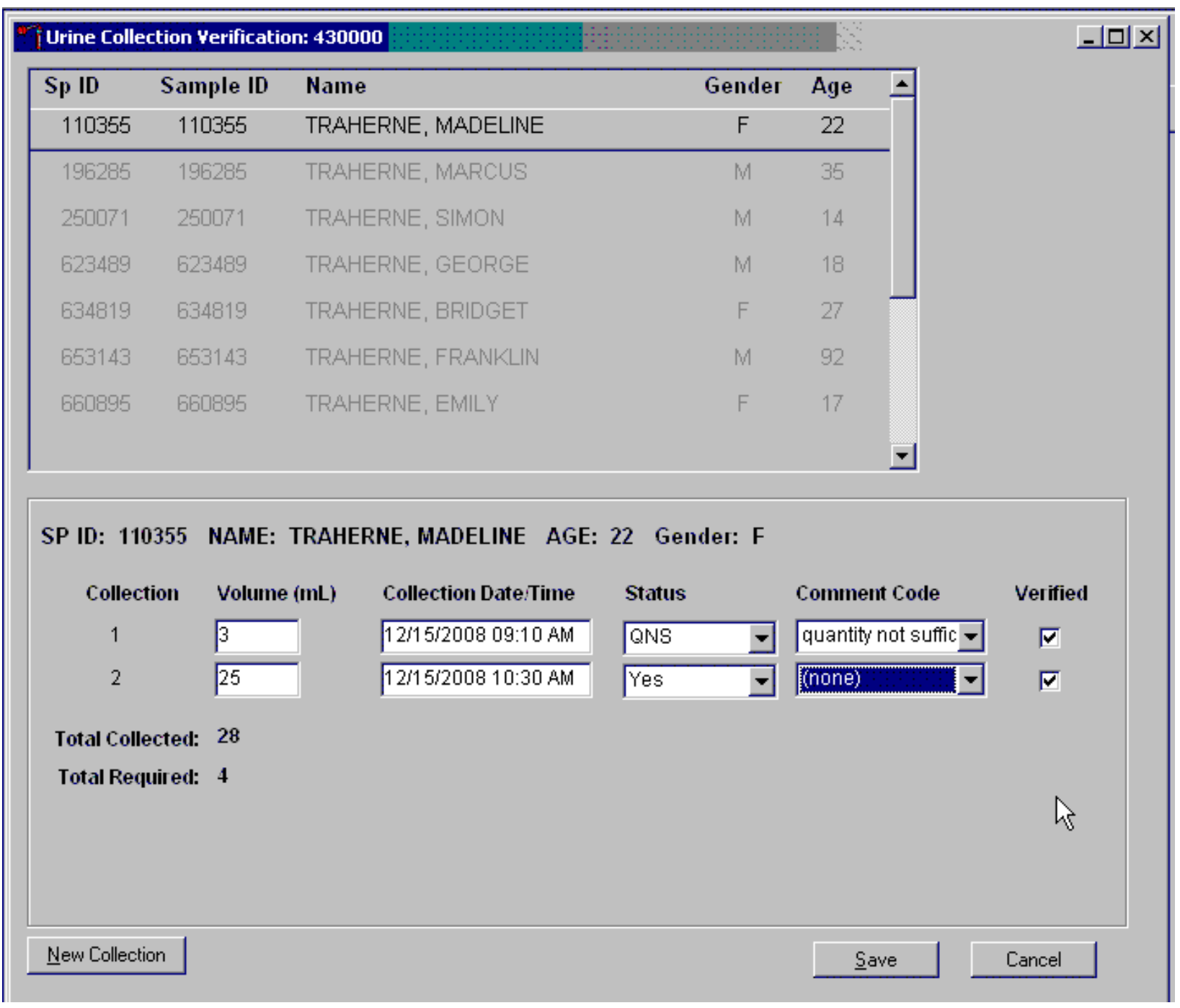

To verify, use the mouse to direct the mouse arrow to the "Verify" checkbox. Left click on the checkbox and a checkmark will appear in the box. Verifying the results will save the results to the database.

To save a result to the database without exiting the module or removing the window, use the mouse to direct the mouse arrow to the "Verify" checkbox. Left click on the checkbox. Alternately, use the mouse to direct the arrow to the "Save" button and left click or press [Enter] when it is highlighted. To save a result to the database and exit the module and to remove the window, use the mouse to direct the mouse arrow to the "OK" button and left click. To exit the module and remove the window without 
saving any information to the database, use the mouse to direct the mouse arrow to the "Cancel" button and left click. To exit the module or close the window after saving results to the database and without recording any further actions, use the mouse to direct the mouse arrow to the $\mathrm{X}$ Close Window button and left click.

\subsubsection{Urine Processing}

Use the urine processing labels printed in advance to label the SP's urine vessels. The urine processing protocol is displayed in Exhibit 13-3. Dry run volunteers are eligible for at least one vessel 45. There are two sets of labels for each dry run volunteer. Label one set of vessels with the first set of labels (SP ID) and the second set of vessels with the second set of labels (Sample ID).

Exhibit 13-3. Dry run urine processing protocol

\begin{tabular}{|c|c|c|c|}
\hline ID & Assay & Sample size $\mathrm{mL}$ & Vessel \\
\hline 45 & Alb/Creat & 3 & 5-mL Cryovial \\
\hline
\end{tabular}

\section{Procedures for Dry Run Volunteers}

- Label one 5-mL cryovial for the first test - \#45. Label one tube with the volunteer's original SP ID. Label the other tube with the volunteer's Sample ID.

- Aliquot 3-mL urine into the vessels using a large plastic transfer pipette.

- Enter the results of urine processing for the original SP ID and again for the Sample ID. 
After filling the urine specimen vessels for each dry run volunteer, use the Urine Processing module to enter the urine processing results.

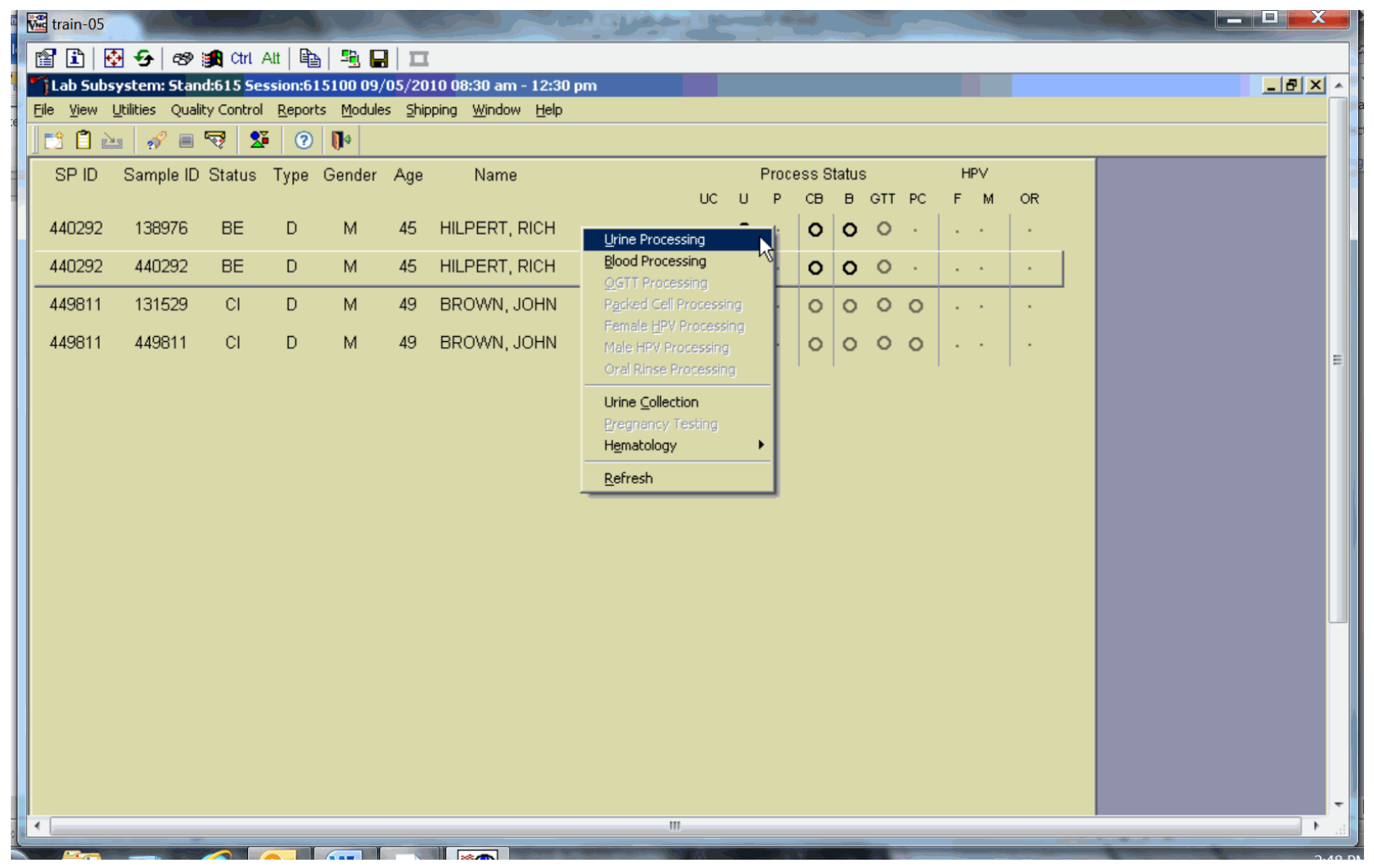

To access the Urine Processing module, use the mouse to direct the mouse arrow to the correct volunteer, right click, drag the mouse arrow to \{Urine Processing\}, and right click or right click and type $[\mathrm{U} / \mathrm{u}]$. Alternatively to access the Urine Processing module, use the up and down keys to move up and down the list until the correct volunteer is highlighted, right click, drag the mouse arrow to \{Urine Processing $\}$, and right click or right click and type $[\mathrm{U} / \mathrm{u}]$. 
The volunteer's urine processing screen displays.

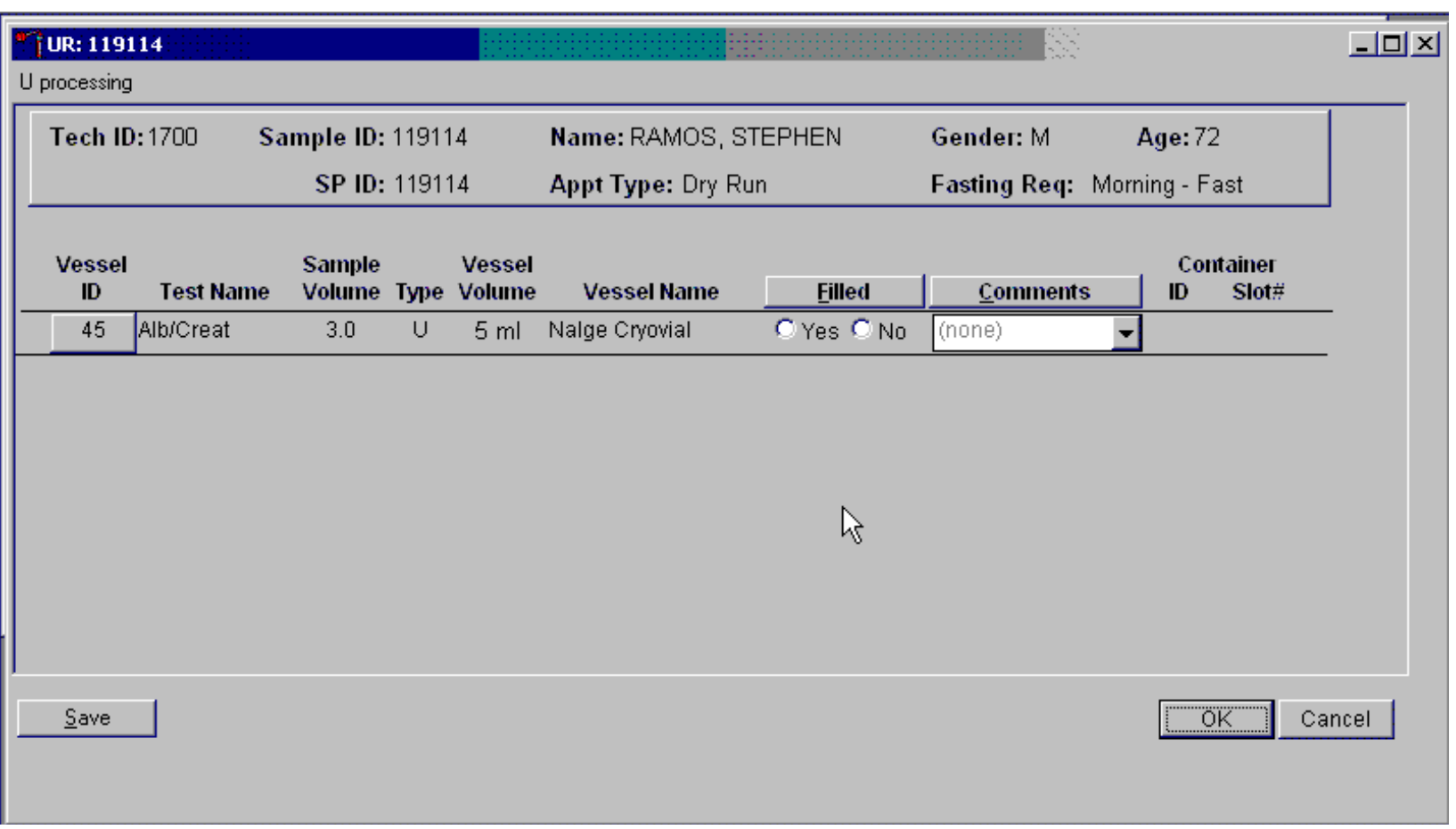

To mark an individual urine vessel as collected or Filled - "Yes," use the mouse to direct the mouse arrow to the "Yes" radio button and left click. As each vessel is marked as Filled - "Yes," it is automatically assigned to a slot in an existing (open) container. To mark an individual urine vessel as not collected or Filled - "No," use the mouse to direct the mouse arrow to the "No" radio button and left click. As each vessel is marked as Filled - "No," the comment, "quantity not sufficient" is automatically entered in the Comments column. To collectively mark all vessels as Filled - "Yes," use the mouse to direct the mouse arrow to the "Filled" button above the radio buttons, left click, and drag the arrow to \{Fill All\} and left click or type [Shift] [F/f]. To mark all vessels as Filled - "No," use the mouse to direct the mouse arrow to the "Filled" button above the radio buttons, left click, drag the mouse arrow to (Not Filled All $\}$, and left click or type [Shift] [F/f], [N/n].

Use the quality comment code to indicate if blood is present. Store each filled vessel in the assigned slot in the assigned container. To record this action or to save this data to the database, use the mouse to direct the mouse arrow to the "Save" button, and left click. To record this action or to save the data to the database and to exit the module, use the mouse to direct the mouse arrow to the "OK" button 
and left click. To close the window without saving any data in the database, use the mouse to direct the mouse arrow to the "Cancel" button, and left click.

Access the Sample ID record on the heads-up display and record the processing results.

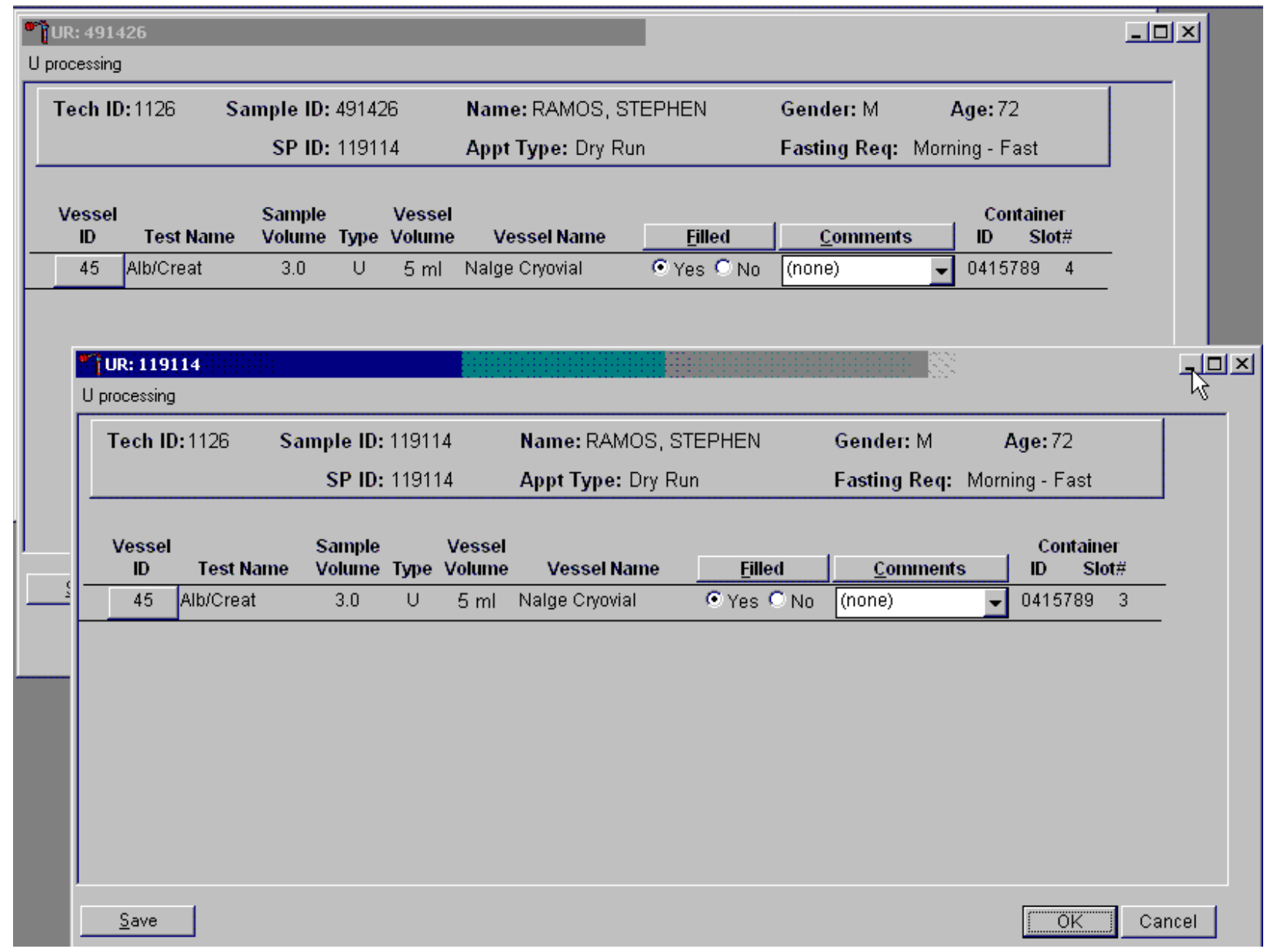

Remember to process vessels in pairs and, if the quantity of urine is insufficient to fill both vessels, do not record either vessel as filled.

Discard any remaining urine specimen at the end of each session. Pour urine down the drain in the laboratory sink. Flush the sink with water. Discard urine cup in the biohazard trash.

The urine-processing technologist performs the pregnancy test on female dry run volunteers aged 8-59 years and documents the results following the procedures in Chapter 5. 


\subsubsection{Hematology}

The technologist at workstation 3 runs two CBCs, in duplicate, for each dry run volunteer after the blood processing technologist processes whole blood from the 4-mL EDTA tubes according to the whole blood processing protocol (see Exhibit 13-4). Run the first CBC in duplicate on the first 4-mL EDTA tube with the original SP ID on the tube. Evaluate results, and save the data using procedures described in Chapter 7.

Run the second CBC in duplicate on the second 4-mL EDTA tube using the Sample ID. Evaluate results, and save the data using procedures described in Chapter 7.

\subsubsection{Blood Processing and Storage}

Print blood-processing labels using procedures described in Chapter 8. There are two sets of labels for each dry run volunteer; one set of labels contains the SP ID and the second set of labels contains the Sample ID. Prepare two blood processing racks (one with the SP ID labels and one with the Sample ID labels) using the blood-processing template for ages $12+$.

Exhibit 13-4. Dry run processing protocol - whole blood

\begin{tabular}{c|l|c|c|c|c|c}
\hline ID & \multicolumn{1}{|c|}{ Test name } & Groups & $\begin{array}{c}\text { Sample } \\
(\mathrm{mL})\end{array}$ & Sample type & Collection type & Vessel type \\
\hline 1 & Lead/Cad/HG/Se/Mn & Both & 0.4 & Whole Blood & 4-mL EDTA & 2-mL \\
\hline 3 & RBC Folate & Even & 0.1 & Whole Blood & 4-mL EDTA & 2-mL \\
\hline 4 & Glycohem & Odd & 0.4 & Whole Blood & 4-mL EDTA & 2-mL \\
\hline 5 & Mercury & Even & 0.5 & Whole Blood & 4-mL EDTA & 2-mL \\
\hline
\end{tabular}

Treat each set of venipuncture tubes and prelabeled blood processing racks as two independent and separate dry run volunteers. Process the complete first set of venipuncture tubes and blood processing vessels on the dry run volunteer using the original SP ID, then process the second set of venipuncture tubes and blood processing vessels using the Sample ID. 
For volunteers whose SP ID and Sample ID ends in an odd digit, process one of the 4-mL EDTA tubes for Lead/Cad/HG/Se/Mn, RBC Foll, and Glycohem. For volunteers whose SP ID and Sample ID ends in an even digit, process one of the 4-mL EDTA tubes for Lead/Cad/HG/Se/Mn, RBC Folate, and Mercury. Use processing procedures described in Chapter 7. Match the EDTA tube with the SP ID to the corresponding blood processing rack. Match the second EDTA tube with the Sample ID to the corresponding blood processing rack.

When the SP ID ends in an even or an odd digit, process each of the 2-mL gray top tubes for Glucose using the same procedures described in Chapter 7. When the SP ID ends in an even or an odd digit, process each of the 2-mL gray top tubes for OGTT using the same procedures described in the Oral Glucose Tolerance test in Chapter 11.

Process pooled serum for the dry run volunteers using the Dry run blood processing protocol - serum (Exhibit 13-5).

Exhibit 13-5. Dry run blood processing protocol - serum

\begin{tabular}{|l|l|l|c|l|l|c|}
\hline \multicolumn{1}{|c|}{ ID } & \multicolumn{1}{|c|}{ Test name } & Groups & $\begin{array}{c}\text { Sample } \\
(\mathrm{mL})\end{array}$ & $\begin{array}{c}\text { Sample } \\
\text { type }\end{array}$ & \multicolumn{1}{c|}{ Collection type } & Vessel type \\
\hline 136 & Se/Cu/Zn & Odd & 1.0 & Serum & 6 mL Royal Blue & $2 \mathrm{~mL}$ \\
\hline 110 & Folate1 & Odd & 0.7 & Serum & $15-\mathrm{mL}$ Red & $2-\mathrm{mL}$ \\
\hline 70 & Vitamin D & Odd & 0.7 & Serum & Serum & $2-\mathrm{mL}$ \\
\hline 122 & HAV & Even & 1.0 & Serum & Serum & $2-\mathrm{mL}$ \\
\hline 17 & Cotinine & Even & 1.8 & Serum & $15-\mathrm{mL}$ Red & $2-\mathrm{mL}$ \\
\hline 21 & Lipids & Both & 1.0 & Serum & $15-\mathrm{mL}$ Red & $2-\mathrm{mL}$ \\
\hline 18 & Biochem & Both & 0.8 & Serum & $15-\mathrm{mL}$ Red & $2-\mathrm{mL}$ \\
\hline 23 & Insulin & Odd & 1.0 & Serum & $15-\mathrm{mL}$ Red & $2-\mathrm{mL}$ \\
\hline 12 & Vit B12/MMA & Even & 0.7 & Serum & $15-\mathrm{mL}$ Red & $2-\mathrm{mL}$ \\
\hline 127 & IgA-TTG/EMA & Both & 0.5 & Serum & $15-\mathrm{mL}$ Red & $2-\mathrm{mL}$ \\
\hline 137 & $\begin{array}{l}\text { Testosterone/Estr } \\
\text { adiol/SHBG }\end{array}$ & Odd & 0.5 & Serum & $15 \mathrm{~mL}$ Red & $2-\mathrm{mL}$ \\
& & & & & \\
\hline
\end{tabular}

- Fill each vessel as described in the blood processing protocol.

- Process the complete first set of venipuncture tubes and blood processing vessels on the dry run volunteer using the original SP ID. Continue to use the labeled vessels in the prepared rack.

- Process the second set of venipuncture tubes and blood processing vessels using the Sample ID. Continue to use the labeled vessels in the prepared rack. 
- Use the same techniques for spinning and separating the blood as described in Chapter 7.

- Check the vessel labels before filling the vessel making sure that each vessel has the correct SP ID or Sample ID.

Match the two processing racks to each other. Record the blood processing results.

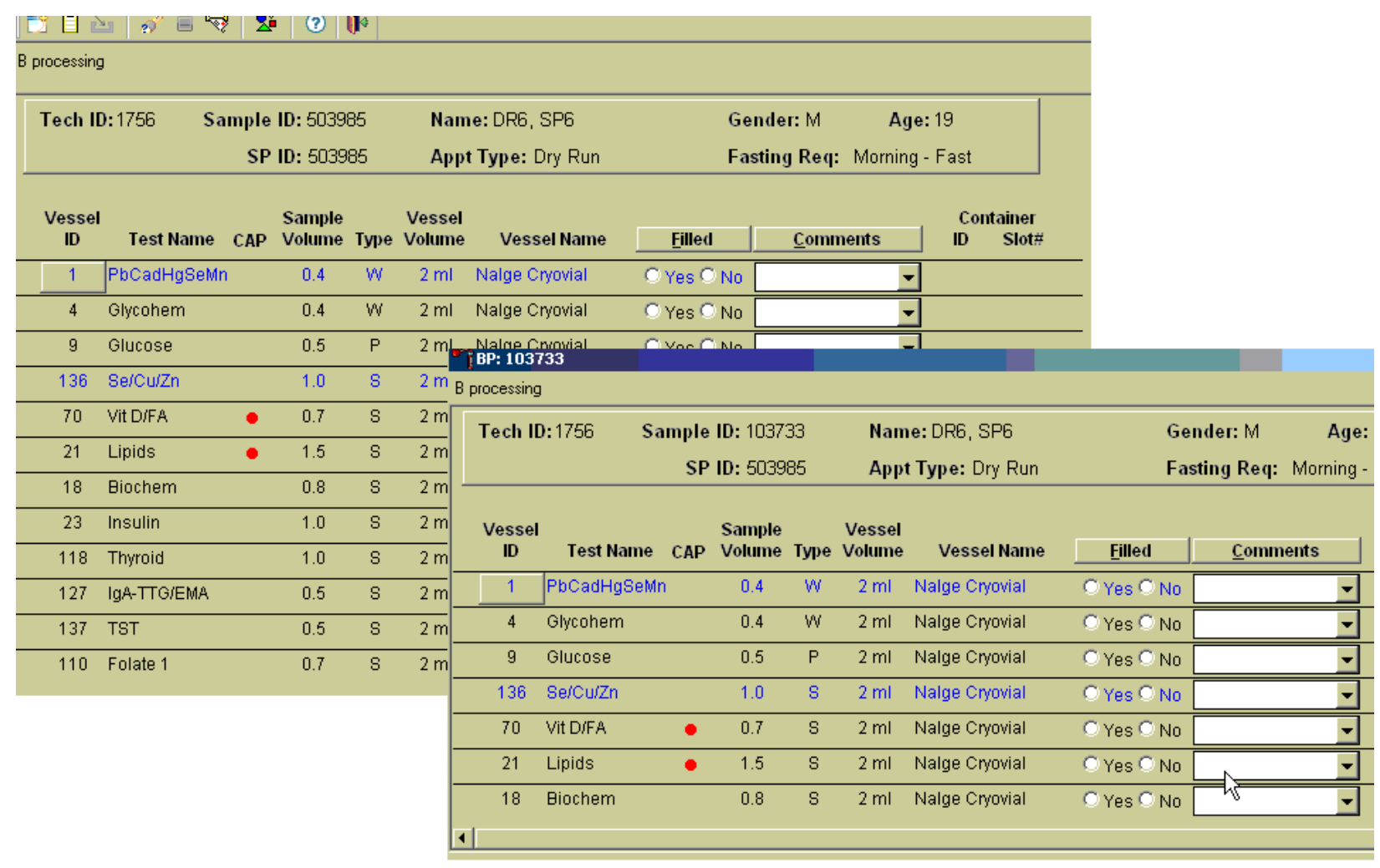

Enter results for those vessels where both vessels are filled for the whole blood, plasma, and serum protocol. Enter the results of blood processing using the correct Sample ID and SP ID numbers and procedures described in Chapter 8, Section 8.8. Store the blood vessels in a manner identical to blood vessels processed during a regular session.

\subsubsection{Shipping Dry Run Specimens}

Ship the split sample blood and urine vessels with samples from the regular sessions following procedures described in Chapter 8 . 


\subsection{Centrifuges - Equipment Maintenance}

Technologists are responsible for inspecting the centrifuges at the beginning of each stand, and inspecting and cleaning the centrifuges at the end of each stand and whenever necessary. Clean the interior of the chamber with a mild soap, as time permits. Rinse thoroughly and dry completely.

- Inspect the rotor yolk and buckets.

- Inspect for rough spots or pitting, white powder deposits or heavy discoloration. If any of these signs are evident, do not use the rotor. Use the second centrifuge and notify the NCHS engineer. Check the condition of the Oring in the tie-down nut; replace it if it is worn or damaged.

- Clean the rotor.

- Use mild detergent, diluted 10 to 1 with water and a soft brush to clean the rotor yolk and tie-down nut. Thoroughly rinse the cleaned rotor and components with water and air-dry upside down. When the rotor is dry, lubricate the bucket sockets and pins by saturating a lintless tissue with TriFlow oil and wipe it on each bucket socket and pin. Also, put a drop of TriFlow oil between the pin and rotor yolk. Before reinstalling the rotor, lightly lubricate the drive hole of the rotor with Anti-Seize to prevent the rotor from sticking, as follows:

1. Apply the lubricant onto a swab.

2. Draw the coated swab through a paper towel to remove excess lubricant.

3 Lightly coat the inside of the drive hole with the lubricant remaining on the swab.

- Clean the buckets.

- $\quad$ Remove the O-ring.

- Clean the bucket and O-ring with a mild detergent diluted 10 to 1 with water, then rinse thoroughly, and dry completely.

- $\quad$ Coat the O-ring with a light film of silicone vacuum grease. Place the O-ring on the ledge inside the bucket.

- Once a week and after every cleaning, lubricate contact areas between the buckets and the pins on the rotor. Saturate a lintless tissue with Tri-Flow oil and wipe it on each bucket socket and pin. Also, put a drop of Tri-Flow oil between the pin and rotor yolk. 
- Inspect and clean Aerosolve canisters.

- $\quad$ Inspect the O-ring for nicks, abrasions, or other damage. Coat the O-ring with a light film of silicone vacuum grease before installing. Place the O-ring in the groove.

- Inspect the canister and lid threads and bottom of canister for heavy crazing or cracks.

- Inspect the tube racks for heavy scratches or cracks and inspect the rubber cushions for damage or for embedded glass from broken tubes.

- $\quad$ Clean the canisters, lids, and racks with a mild detergent, diluted 10 to 1 with water, and a soft brush. Thoroughly rinse the cleaned components with water and air-dry upside down. Coat the O-ring with a light film of silicone vacuum grease before reinstalling it into the groove.

The NCHS biomedical engineer certifies both centrifuges annually. The NCHS engineer determines the rpm of the instruments using a strobe light or a tachometer. The engineer records multiple readings for each centrifuge. The Beckman GS-6R centrifuge should exceed 3,750 rpm to ensure adequate centrifugal force development. The engineer checks each centrifuge timer against a stopwatch and records the values in the Laboratory QC module - NCHS QC.

Document beginning and end of stand cleaning procedures in the Laboratory QC module.

\subsection{Refrigerators and Freezers - Equipment Maintenance}

The freezers require proper ventilation in order to operate efficiently, and the air around the freezers must be free to circulate. Vents in the counters allow the heat from the freezers to rise and dissipate. Keep the vents open and do not obstruct the airflow. Do not store anything between the freezers or between the counter supports and the freezer. Do not store anything on the top grill.

Quality control procedures for the refrigerators and freezers include cleaning them at the beginning and end of the stand and whenever necessary. Continuously monitor the temperatures of refrigerators and freezers on a daily basis and record the results in the Laboratory QC module. Temperatures are automatically captured, graphed, and available in real time. 


\subsection{Use of the Temperature Recording Instrument}

The TempGuard system is a multiple device monitor and alarm system that is equipped with an integrated autodialer and data logging capabilities. The TempGuard system automatically monitors the interior temperature of the refrigerators, freezers, and room temperature.

The system can monitor up to eight temperature sensors and each sensor must have a probe submerged into a bottle of resin. The configuration in the MEC, is as follows:

\begin{tabular}{|c|c|}
\hline TempGuard Port & Refrigerator/Freezer Name \\
\hline 1 & Refrigerator \# 1 \\
\hline 2 & Refrigerator \# 2 \\
\hline 3 & Freezer \# 3 \\
\hline 4 & Freezer \# 4 \\
\hline 5 & Freezer \# 5 \\
\hline 6 & Freezer \# 6 \\
\hline 7 & Room Temp \\
\hline
\end{tabular}

The TempGuard system has been programmed with the following: upper and lower limits for all units including the room temperature and a 60-second delay for the allowable time the freezer door can be left open before the system will alarm. The system has the capability to send out calls and emails to alert staff to temperature issues.

\section{Checking TempGuard Sensors}

All temperature sensors connected will have their temperature readings displayed next to the sensor number on the display

\section{Disabling Alarm}

1. Push the black button on the left side of the TempGuard to see any alarm conditions.

2. Hold the button for 5 seconds to confirm the alarm condition.

3. Alarm conditions must be confirmed individually.

4. The alarm relay will de-energize, and the TempGuard will stop making callouts for the current alarm condition. 


\section{Start of Stand Set-Up (performed by the FES)}

Programming the Autodialer Functions:

(The MEC manager phone \# needs to be updated at each stand.)

1. Enter the "Full Access" PIN (Enter 0000).

2. From the Program Menu, Select $\mathbf{1}$ to set telephone numbers.

3. You will hear "Select contact."

4. Select 1 for the first contact number (FES phone \#).

- $\quad$ Enter your phone number, using this format " 1 + area code +7 digit \#"

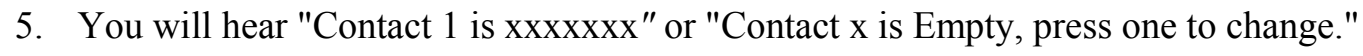

6. Press 1 to make a change or enter a telephone number.

7. You will hear "Enter number then press pound."

8. Enter the number, followed by the \# key.

- $\quad$ Entering the \# key will erase the currently programmed contact telephone number.

9. You will hear the telephone number you just entered.

10. You will be prompted to select another contact to program.

11. Select 2 for the second contact number (MEC managers \#).

- For Dan, enter “1-301-526-8190”

- $\quad$ For David, enter “1-301-529-3502”

12. Press 0 to return to the Program Menu.

The acceptable temperature range for refrigerators used for storage of CLIA-regulated specimens and reagents is $2-8^{\circ} \mathrm{C}$ and is checked and recorded daily.

Freezer temperatures are monitored daily by visual inspection of either a thermometer or graphs and recorded. All freezers are monitored automatically by built-in visual systems (with thermographic or digital reading), which is determined by the probe in the antifreeze solution. The range of allowable temperature for the -30 Revco model is -26 to $-34^{\circ} \mathrm{C}$. If the range is exceeded, the technologist contacts the MEC manager. 


\subsection{Use of the Class II Type A Biological Safety Cabinet}

The Labgard Model NU-425 Class II, Type A2, Laminar Flow Biological Safety Cabinet (LFBSC), with proper technique, provides an effective partial containment system for safe manipulation of moderate- and high-risk microorganisms (see Exhibit 13-6). The vertical laminar airflow barrier prevents contaminated air from entering or escaping from the open-front work chamber, protecting the cabinet contents and the technologist. A high-efficiency filter system decontaminates both the incoming air and the exhaust.

\section{Installation, Certification, and Maintenance}

The NCHS biomedical engineer arranges for a certified engineer to perform the required annual certification that includes replacement of High Efficiency Particulate Air (HEPA) filters as necessary.

The basic concept of the LFBSC is to take air through a blower system, pressurize a plenum, and force air through a filter. Built-in supply and exhaust blowers come with separate HEPA filters. The recirculating airflow pattern directs a portion of the HEPA filtered air straight down over the central work area driving the airborne contaminants into the surrounding grills, allowing a pollution-free environment for particular specifications. If a filter is defective, the filtration system no longer filters and becomes a vacuum cleaner, taking in gross contamination and exploding it through the defective filter onto and around the critical work area. The filter of the LFBSC is a permanent part of the disposable plenum. A magnehelic gauge is calibrated in "inches of water gauge" pressure (w.g.). The gauge indicates when the supply HEPA filter is clogged. As the filter loads with particulate matter, the amount of pressure registered on the gauge will increase. The initial pressure reading is approximately $0.4 \mathrm{w} . \mathrm{g} . \pm 0.05 \mathrm{w} . \mathrm{g}$. depending on the altitude from sea level.

Clean the LFBSC at the beginning and end of each stand. Lift the front glass and wash both the interior and exterior glass. Remove all contents from the interior of the cabinet. Remove and wash the front grill. Lift the cover to the table and wash the base underneath. Put table cover and grill back into cabinet. Lock front glass into place. Document these activities in the Laboratory QC module. Take and record the pressure reading in the Laboratory QC module at the beginning of stand and at the start of each session.

Document all unscheduled maintenance for the LFBSC in the automated Unusual Field Occurrence utility. 
Exhibit 13-6. Operating the laminar flow biological safety cabinet

\section{Operating the NU-425, Laminar Flow Biological Safety Cabinet ${ }^{*}$}

\section{Operator Controls \& Indicators}

The following is a description of the controls and indicators found on both the front panel (see Drawing BCD-05322) and cabinet.

\subsubsection{Circuit Breaker-Blower (Top of Control Center)}

The motor/blower is protected with a circuit breaker. The circuit breaker in conjunction with the motor's thermal protector is designed to open under locked rotor or half-wave power conditions. Should the circuit breaker open (pop-out button will appear) merely depress to reset. If the circuit breaker continually opens, a failure has occurred in the motor or solid-state speed controller. Consult a qualified repair technician or NuAire, Inc. for replacement.

\subsubsection{Circuit Breaker-Outlets (Top of Control Center)}

The duplex outlet located in the sidewall of the work area is protected with a 3 amp circuit breaker. The circuit breaker may trip at 110 percent of load rating but will trip at 145 percent of load rating in less than 2 seconds. Should the circuit breaker open, (pop-out button will appear), unplug the appliance plugged into the outlet and merely depress the pop-out button to reset.

\subsubsection{Fluorescent/U.V. Light Switch}

This switch provides on/off control for the fluorescent light and/or the ultraviolet (U.V.) light if present (optional). With the U.V. light option, the switch provides for on-center off-on operation so that both the fluorescent light and the U.V. light cannot be energized at the same time. The UV light is also interlocked, so it will only operate if the sliding window is closed. Proper care should be exercised when the U.V. light is on.

* Adapted from Operation and Maintenance Manual by NuAire, Inc., 2005. 


\subsubsection{Outlet Switch}

This switch provides on/off control for the 115 VAC power available in the outlet(s) within the cabinet workzone.

\subsubsection{Blower Switch}

The blower switch applies power to the internal motor/blower when in the ON position. The blower switch also has a second set of poles, which are available for use as a contact closure for an exhaust system. In addition, power can be applied to the accessory outlet located on the upper left corner of the cabinet, behind the front window assembly.

The purpose of the outlet is to provide "switched" power for the following (see also 8.1.11):

- Exhaust transition with integral blower

- To power a low power appliance, such as the low airflow alarm.

The rating of the outlet is $115 \mathrm{VAC}$ at 2 amp maximum.

\subsubsection{Indicator Light}

An indicator light is located above the Blower on/off switch and indicates when power is applied to the blower. 


\subsubsection{Audible Alarm Enable}

As the sliding window is raised above its normal working height, a micro switch located in the glide channel activates both an audible and visual alarm. This switch disables the audible alarm but will not disable the red indicator visual alarm.

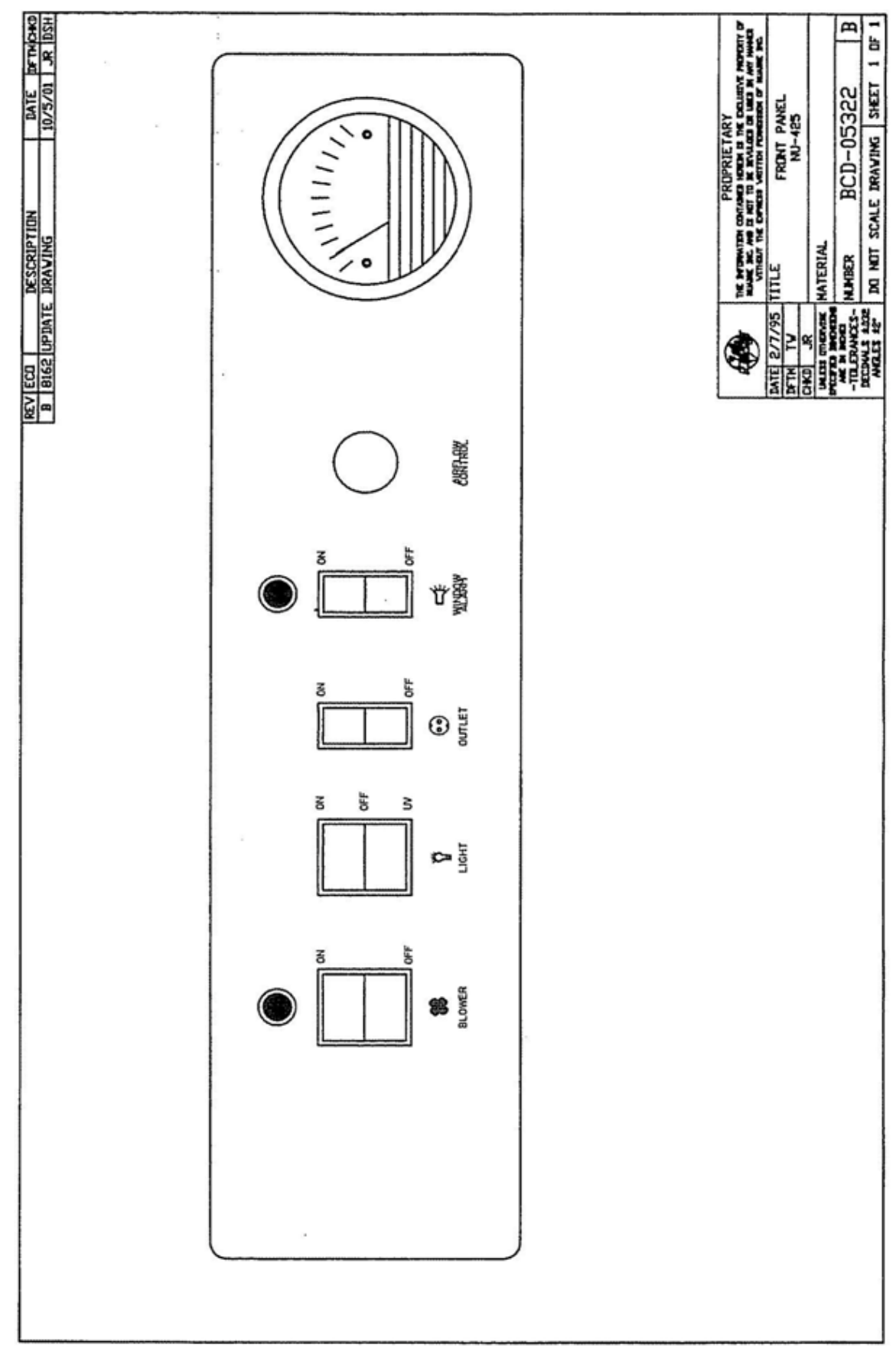




\subsubsection{Airflow Control}

The operating airflows within the cabinet (i.e., 70 LFPM (.35 m/s) downflow and 105 LFPM $(.53 \mathrm{~m} / \mathrm{s})$ air inflow barrier) are controlled by a potentiometer and an exhaust damper. The potentiometer controls the operating voltage applied to the motor/blower. The potentiometer is adjustable over 270 degrees with a slotted screwdriver, which varies the applied voltage from 70 to 115 VAC. THIS ADJUSTMENT SHOULD ONLY BE MADE BY A QUALIFIED TECHNICIAN EMPLOYING THE PROPER INSTRUMENTS IN ORDER TO INSURE AIRFLOWS PER NSF STD. NO. 49.

\subsubsection{Magnehelic Gauge}

The unit is equipped with a magnehelic gauge. The magnehelic gauge displays the static pressure within the pressure plenum supplying the downflow and exhaust filters. The gauge is calibrated in "inches of water gauge" pressure. As the HEPA filters load with particulate matter, the amount of static pressure will increase, giving an indication of the "health" of the cabinet. The initial pressure reading will be approximately 0.5 " w.g. +0.1 " w.g. depending on altitude from sea level. At each 0.1 " w.g. increment increase, the cabinet airflow should be checked by a qualified technician, unless certified on a yearly (or sooner) basis.

\subsubsection{Sliding Window Operation}

The cabinet has a full counter balanced and removable sliding tempered glass window with two operational features. As the window is raised above its specified operating height, an audible alarm alerts the operator of possible compromised personnel protection. At the same time, an audible and visual alarm is activated. The audible alarm may be switched off while the visual alarm remains until lowered to the safe operating position. When the window is lowered below 2 inches $(50 \mathrm{~mm})$, the motor/blower automatically shuts down, to prevent stress on the motor/blower in the form of overheating. The maximum window height is $191 / 2$ inches $(495 \mathrm{~mm})$.

\subsubsection{Convenience/Accessory Outlet}

A convenience/accessory outlet is located in the upper left hand corner in back of the window assembly. The outlet is normally wired "hot" through the motor/blower circuit breaker. If an 
accessory is purchased, the outlet is defined as an accessory outlet supplying $115 \mathrm{VAC}$ at 2.0 Amp maximum, and is wired to the blower switch (see electrical schematic).

\section{$1.2 \quad$ Operating Guidelines}

The intent herein is to present general operational guidelines that will aid in the use of the Laminar Flow Biological Safety Cabinet (LFBSC) to control airborne contaminants of low to moderate risk as stated in Technical Report No. FPS 56500000001 prepared by Dow Chemical U.S.A. for the National Cancer Institute, May 1, 1972.

Procedure protocols defined in terms of the barrier or control concepts unique to LFBSC must be developed in order to obtain a maximum potential for safety and protection. The pre-planning necessary to develop these protocols is based on several fundamental considerations, each of which will contribute to optimum benefits from the equipment:

- Know your "safe working area"

- Minimize disruption of "air curtain"

- Minimize room activity

- Utilize unidirectional airflow

- Employ aseptic techniques

\subsubsection{Know Your "Safe Working Area"}

The LFBSC safe working area is basically the worktray or depressed area. All work should be performed on or above the worktray. The area on or above the front grill is a non-safe working area. 


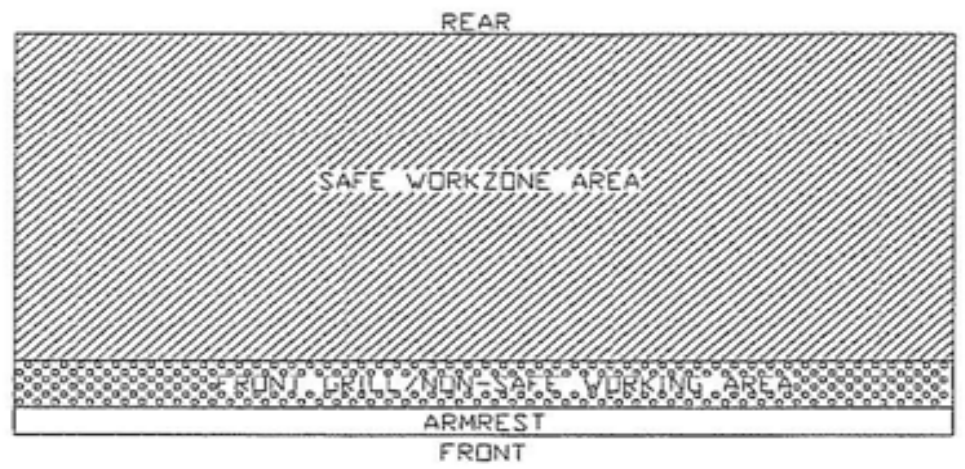

\subsubsection{Minimize Penetration of "Air Curtain"}

The minimum number of items necessary should be placed into the cabinet to prevent overloading, but the work should also be planned to minimize the number of times an operator's hands and arms must enter and leave the air curtain at the open face. The ideal situation is to have everything needed for the complete procedure placed in the hood before starting, so that nothing need pass in or out through the air barrier at the face until the procedure is completed. This is especially important in working with moderate risk agents.

Unnecessary raising of the hands inside the cabinet above the level of the work opening should be avoided. This presents an inclined plane from hands to elbows along which the downflow of air may run to, and possibly out, the open face.

NOTE: When working with agents of lower risk, it is not as important for all materials to be placed in the cabinet before starting, or for the procedure to be completely finished before materials are removed. Also, the time period for a unit may be continued over a more extended period during which entries and withdrawals from the cabinet may be made.

\subsubsection{Minimize Room Activity}

Activity in the room itself should be held to a minimum. Unnecessary activity may create disruptive air currents as well as interfere with the work of the operator. A person walking past the front 
of a cabinet can cause draft velocities up to $175 \mathrm{fpm}(.89 \mathrm{~m} / \mathrm{s})$, which are sufficient to disrupt the air balance of the laminar flow unit.

\subsubsection{Utilize Unidirectional Air Flow}

The operator must keep two important facts in mind: ( I) The air, as supplied to the work area through filters from the top, is contaminant free and (2) Airborne contamination generated in the work area is controlled by the unidirectional flow of parallel air streams in a top-to-bottom direction.

A solid object placed in a laminar air stream will disrupt the parallel flow and consequently, the capability of controlling lateral movement of airborne particulates. A cone of turbulence extends below the object and laminarity of the air stream is not regained until a point is reached downstream, approximately equal to three to six times the diameter of the object. Within the parameters of this cone, particles may be carried laterally by multidirectional eddy currents.

Transfer of viable materials and manipulations which may generate aerosols should not be performed above sterile or uninoculated materials. Items should be localized on the work surface in "clean" and "dirty" groups.

\subsubsection{Employ Aseptic Technique}

The operator must not assume an attitude of "let the cabinet do it" when performing procedures within a LFBSC. Properly balanced and properly used cabinets will do an excellent job of controlling airborne contamination and containing viable agents, but the cabinet will not eliminate contact transmission of contamination. Normal laboratory contamination control procedures and basic aseptic techniques are necessary to obtain maximum benefit from the cabinet. For example, open bottle, tube or flask mounts should be kept as parallel as possible to the downflow to minimize capture of chance particulates. This precaution is merely an extension of good aseptic technique as practiced on open bench

tops. The good laboratory practices designed to minimize creation and/or release of aerosols to the environment should not be discontinued. 
Items of equipment in direct contact with the etiologic agent must remain in the cabinet until enclosed or until surface-decontaminated. Trays of discard pipettes must be covered before removal from the cabinet (aluminum foil may substitute for fabricated covers).

If an accident occurs which spills or splatters suspensions of etiologic agent around the work area, all surfaces and items in the cabinet must be surface-decontaminated before being removed.

\subsection{Operating Sequence}

\subsubsection{Start Up}

Turn on cabinet blower and lights, check air intake and exhaust portals of the cabinet to make sure they are unobstructed.

Good procedure includes the decontamination or wipedown of cabinet surfaces with chemical disinfectant before work commences. This practice eliminates the need for UV lights, whose primary utility in this application is inactivation of surface contamination since the filters effectively remove all airborne contaminants. UV lights, therefore, are not recommended in the LFBSC.

Allow blowers to operate for a minimum of 15 minutes before aseptic manipulations are begun in the cabinet, If the filtered air exhausted from the unit is discharged into the room, as in some installations, an additional advantage is obtained from purification (filtration) of the room air circulated through the equipment. Because of this characteristic contributing to the quality of the laboratory environment, some owners of LFBSC leave them in operation beyond the time of actual use.

\subsubsection{Wipedown}

The interior surfaces of the workspace should next be disinfected (see cleaning procedures) by wiping them thoroughly with $70 \%$ alcohol or similar noncorrosive antimicrobial agents. USE OF CHLORINATED OR HALOGEN MATERIALS IN THE CABINET MAY DAMAGE STAINLESS STEEL. 


\subsubsection{Materials \& Equipment}

The apparatus and materials should next be placed into the cabinet. Care must be exercised that no items be placed over the front intake grills. Materials should be arranged so that clean, dirty (used), and virus materials are well separated. Passage of contaminated materials over uninoculated cultures or clean glassware should be avoided and transfer of viable materials should be performed as deeply into the cabinet (away from open face) as possible.

\subsubsection{Air Purge}

Additional purging of the workspace without user activity should be allowed for 2-3 minutes after materials and apparatus have been placed in it. This will rid the area of all "loose" contamination that may have been introduced with the items.

\subsubsection{Perform Work}

The work can now be performed. The technician performing the work is encouraged to wear a long-sleeved gown with knit cuffs and rubber gloves. This will minimize the shedding of skin flora into the work area and concurrently protect the hands and arms from viable agent contamination. At a minimum, the hands and arms should be washed well with germicidal soap before and after work in the cabinet. For the preparation of antineoplastic drugs, the following procedures summarize those contained in OSHA Instruction PUB 8-1.1, "Work Practice Guidelines for Personnel Dealing with Cytotoxic (Antineoplastic) Drugs." The above document should be thoroughly studied/reviewed prior to drug preparation in the cabinet.

- A sterile plastic-backed absorbent drape should be placed on the work surface during mixing procedures. The drape should be exchanged whenever significant spillage occurs, or at the end of each production sequence.

- Vials should be vented with a filter needle to eliminate internal pressure or vacuum.

- Before opening ampoules, care should be taken to insure that no liquid remains in the tip of the ampoule. A sterile gauze sponge should be wrapped around the neck of the ampoule while opening.

- Final drug measurement should be performed prior to removing the needle from the stopper of the vial. 
- A non-splash collection vessel should be available in the biological safety cabinet to discard excess drug solutions.

\subsubsection{Terminal Purging \& Wipedown}

Following completion of work, allow the cabinet to run for a 2-to 3- minute period without personnel activity to purge the unit. The decontamination of the interior surfaces should be repeated after removal of all materials, cultures, apparatus, etc. A careful check of grills and diffuser grids should be made for spilled or splashed nutrients which may support fungus growth and resulting spore liberation that contaminates the protected work environment.

\subsubsection{Paper Catch/Prefilter}

A permanent paper catch is installed behind the rear divider panel of the work zone. This area forms the return air path to the motor/blower, and if the airflow is blocked, it could seriously affect the performance of the cabinet. Therefore, THE PAPER CATCH SHOULD BE CHECKED AND CLEANED NO LESS THAN ON A WEEKLY BASIS; on a DAILY basis if procedures dictate the use of paper products. Any paper removed must be properly disposed of as Contaminated Hazardous Waste. The above procedures also apply to all units configured with a prefilter.

\subsubsection{Shut Down}

Turn off blowers and lights. Do not use cabinet as a depository for excess lab equipment during periods of non-operation. 


\section{$1.4 \quad$ Ergonomics}

Ergonomics, the study or accommodation of work practices is extremely important for proper cabinet usage and user health and safety. An evaluation of normal work practices should be performed with each user when working in a cabinet. Evaluation criteria should be at a minimum:

- Proper user posture

- Effective workzone layout for work practice

- Vision or sightlines

For each of the above evaluation criterion, several aids may be supplied to accommodate the user.

- Ergonomic chair - Be sure feet are resting on the floor, chair foot support or foot rest. Also be sure back is fully supported with proper chair adjustments.

- Forearm/armrest support - The cabinet is provided with a forearm support on the work access opening. Periodic mini-breaks during work practice should be taken resting the forearm to avoid stress and fatigue.

- Effective workzone layout - Always prepare your work procedure to minimize reach to avoid neck and shoulder stress and fatigue. Rotating tables are optional to maximum workzone and minimize reach.

- Vision and sightline - Always prepare your work procedure to eliminate glare and bright reflections on the window. Keep your window clean and sightlines clear to your effect workzone.

\subsection{Cleaning Procedures}

Cleaning the cabinet is an important function in terms of both containment and sterility. Use the following procedure to effectively clean or surface disinfect the cabinet workzone surfaces.

- Raise the sliding window to a full-open position, if desired.

- Press the audible alarm silence or cleaning key on the front control panel to silence the audible alarm during the cleaning process. 
- Apply appropriate disinfecting solution to cabinet surfaces. Most surface disinfectants require a specific contact time, depending upon the microbiological agents used within the cabinet. Consult appropriate disinfectant documentation for proper application and safety precautions.

- NOTE: Disinfectants that use chlorides and halogens will cause damage to the stainless steel surfaces if left on for long periods of time.

- After the specified contact time, wipe up excess disinfectant. 
Two thermometers monitor ambient temperature in the laboratory. Quality control procedures for ambient air temperatures involve checking these thermometers for damage, monitoring the temperature during each session, and documenting any unusual occurrences. Check the two wall thermometers for damage at the beginning of each stand. Notify the MEC manager of any damage.

The allowable ambient temperature range for the laboratory is $17^{\circ}$ to $25^{\circ} \mathrm{C}\left(63^{\circ}\right.$ to $\left.77^{\circ} \mathrm{F}\right)$.

NOTE: If the room temperature is out of allowable range since the last reading, document this in the automated Unusual Field Occurrence utility and inform the MEC manager immediately.

\subsection{Mettler Toledo Scale}

The Mettler Toledo scale is a very sensitive precision weighing instrument with a weighing limit of 600 grams. Handle the scale with care at all times. Do not weigh anything on the scale other than urine cups and specimens. The weigh pan will get dirty during use. Clean the weigh pan as needed by removing the metal plate from the scale and cleaning with Windex. Replace the weigh pan when clean.

Always use the clear acrylic scale cover to protect the scale from damage while not in use. Use the scale cover to protect from drafts when weighing samples.

\section{Scale Set Up}

Open the blue carrying case. Remove scale from case and place on the counter next to the barcode scanner. Verify that the scale is free of dust and debris. If needed, use the brush in Care Pac M to brush away dust and debris. 
Locate the tan serial cable taped to the counter. The serial cable has a broad end with a trapezoid input with a screw on either side. It looks like this:

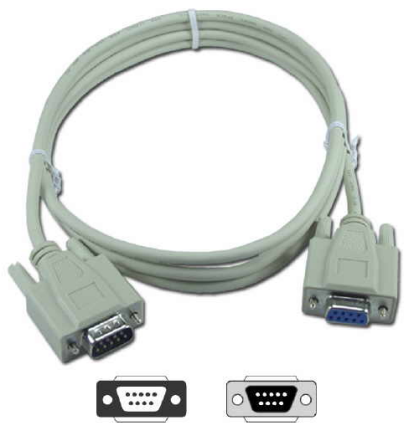

Insert the male end of the cable into the back of the scale. Use the screws to firmly fasten the cable to the back of the scale.

Locate the black AC adapter power cord taped to the counter. Place the round end in the round hole labeled "Power" on the back side of the scale. The LCD display on the front of the scale should say "OFF." Remove the small, round, plastic cone protector from the top of the scale. Be sure to place the cone protector back into the blue carrying case so that it does not get lost.

Remove the weigh pan from the case lid. Gently place the pan on top of the scale, being sure to align the notch on the bottom of the pan with the bump on the right side of the cone. The pan should fit snugly and not wobble around when properly seated.

Never move or turn over the scale without first placing the cone protector on the scale's cone.

Balance the scale using the liquid level in the back right corner of the scale. Adjust the feet of the scale by screwing them in or out until the bubble in the liquid level stays within the center circle. 


\section{Scale Teardown}

Place the carrying case on the counter near the scale. Turn off the scale by pressing and holding the On/Off button. Hold until "OFF" appears on the LCD display. Gently remove weigh pan from scale. Slide weigh pan back into slot in lid of carrying case. Remove the protective cone cover from the carrying case and place over the cone.

Turn the scale around on the counter so the back of the scale is visible. Unplug the AC adapter power cord from the back of the scale. Tape the end of the power cord to the counter so it is easily found at the next setup.

Unplug the serial cable from the back of the scale by unscrewing the two screws and gently pulling the end of the serial cable away from the back of the scale. Tape the cord to the counter so it is easily found at the next setup.

Place the scale in the protective carrying case. Close the lid and snap black locks tightly closed so that the lid and bottom of the case do not come apart. Place the carrying case back into the box for transport to the next stand.

Place the Care Pac M back into the box for transport. Make sure one Styrofoam bumper is on the bottom of the box and set the Care Pac M down into the bumper. Fit the second bumper on top of the Care Pac M and close the box. If Care Pac M has been packed properly, the box should close easily and completely. 


\subsection{Laboratory Quality Control Module}

Document the following items in the Laboratory QC module based on the frequency described in the following table.

\begin{tabular}{|c|c|c|}
\hline Frequency & Item & Acceptable result \\
\hline \multicolumn{3}{|c|}{ Start of Stand } \\
\hline & Freezer \#3 cleaned & Yes \\
\hline & Freezer \#4 cleaned & Yes \\
\hline & Freezer \#5 cleaned & Yes \\
\hline & Freezer \#6 cleaned & Yes \\
\hline & Refrigerator \#1 cleaned & Yes \\
\hline & Refrigerator \#2 cleaned & Yes \\
\hline & Centrifuge \#1 cleaned & Yes \\
\hline & Centrifuge $\# 2$ cleaned & Yes \\
\hline & LFBSCcleaned & Yes \\
\hline & Magnahelic gauge reading & $0.050-0.550$ \\
\hline & Class 6 one hundred gram weight $+/-1.0 \mathrm{~g}$ & $99-101$ \\
\hline & Two Class 6 three hundred gram weight $+/-6.0 \mathrm{~g}$ & $594-606$ \\
\hline \multicolumn{3}{|c|}{ Daily } \\
\hline & Centrifuge \#1 room temperature reading & 17 to $25^{\circ} \mathrm{C}$ \\
\hline & Centrifuge \#2 room temperature reading & 17 to $25^{\circ} \mathrm{C}$ \\
\hline & Magnahelic gauge readings & $0.050-0.550$ \\
\hline & Workstation 2 room temperature reading & 17 to $25^{\circ} \mathrm{C}$ \\
\hline & Workstation 3 room temperature reading & 17 to $25^{\circ} \mathrm{C}$ \\
\hline & Class F2 twenty gram weight $+/-0.2 \mathrm{~g}$ & $19.8-20.2$ \\
\hline & Class F2 five hundred gram weight $+/-5.0 \mathrm{~g}$ & $495-505$ \\
\hline & 1\% Ascorbic Acid & Yes \\
\hline & 7\% Ascorbic Acid & Yes \\
\hline
\end{tabular}




\begin{tabular}{|l|l|c|}
\hline \hline \multicolumn{1}{|c|}{ Item Weekly } & Acceptable result \\
\hline \hline \multicolumn{1}{|c|}{ Erequency } & \multicolumn{1}{|c|}{} \\
\hline & HMX Latron and 6C Cell Control QC data transmitted? & Yes \\
\hline & Flush eyewash for 5 minutes & Yes \\
\hline & Freezer \#3 cleaned & Yes \\
\hline & Freezer \#4 cleaned & Yes \\
\hline & Freezer \#5 cleaned & Yes \\
\hline & Freezer \#6 cleaned & Yes \\
\hline & Refrigerator \#1 cleaned & Yes \\
\hline & Refrigerator \#2 cleaned & Yes \\
\hline & Centrifuge \#1 cleaned & Yes \\
\hline & Centrifuge \#2 cleaned & Yes \\
\hline & LFBSC cleaned & Yes \\
\hline & Coulter printouts sent to home office & Yes \\
\hline
\end{tabular}


Document all instrument quality control activities in the Laboratory QC module.

Access the Laboratory QC module.

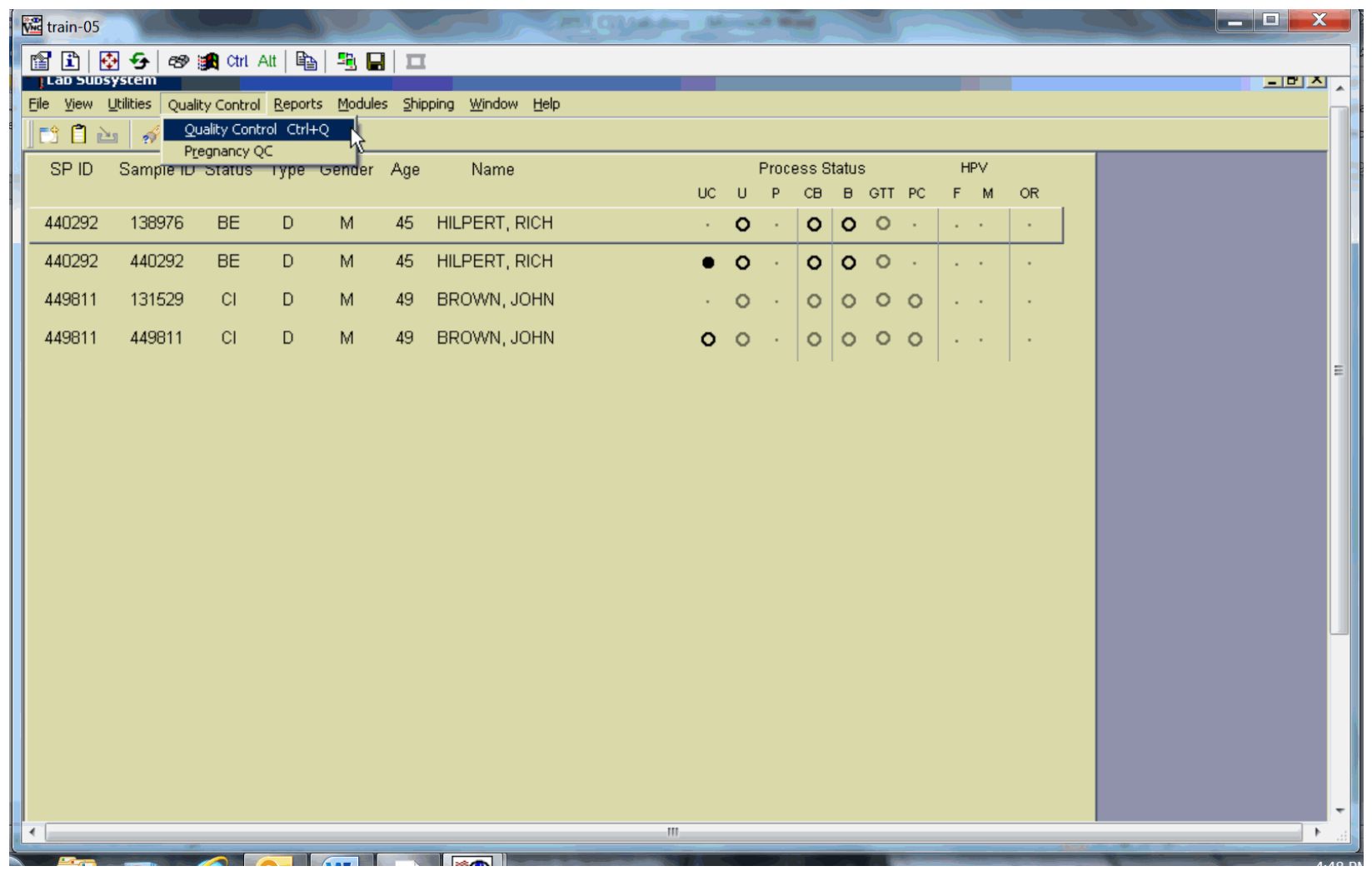


Enter quality control activities and readings.

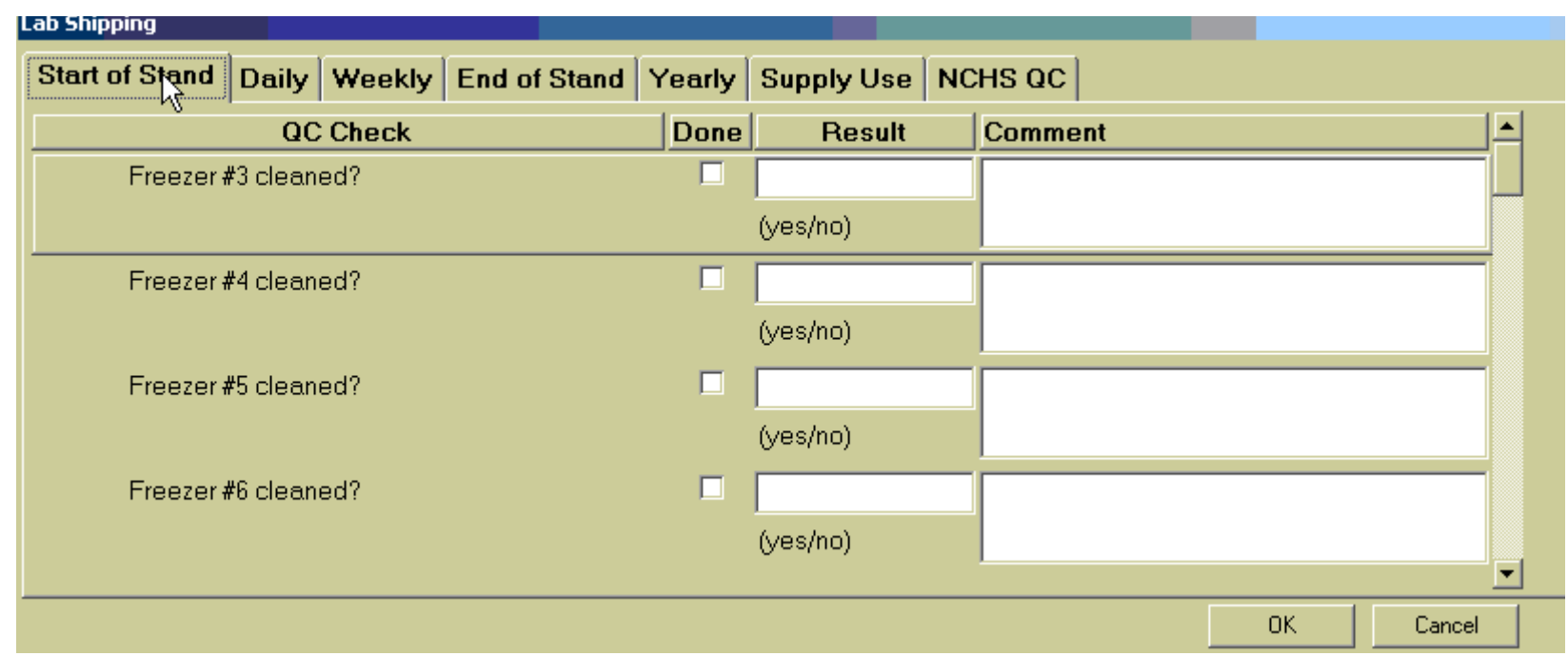

Select the correct activity (Start of Stand, Daily, Weekly, End of Stand, Supply Use, or NCHS QC) by using the mouse to direct the mouse arrow to the correct heading on the top of the file, and left click. At each stand, perform and record weekly activities on the same weekday as set up. 
Mark activities as done and enter the result.

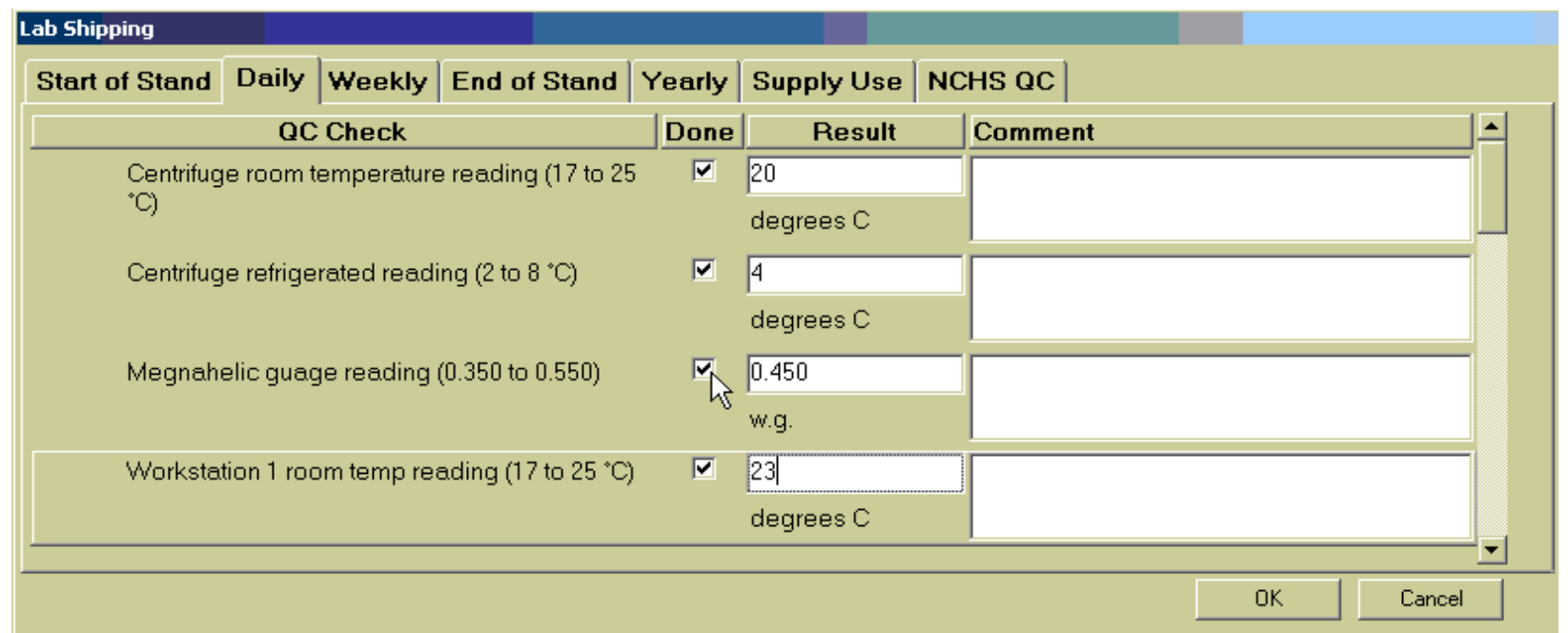

To record an activity as complete or done, use the mouse to direct the mouse arrow to the checkbox in the Done column, and left click to insert a checkmark. Select [Tab] or [Enter] to move to the Result text box.

Enter the response or reading in the Result text box.

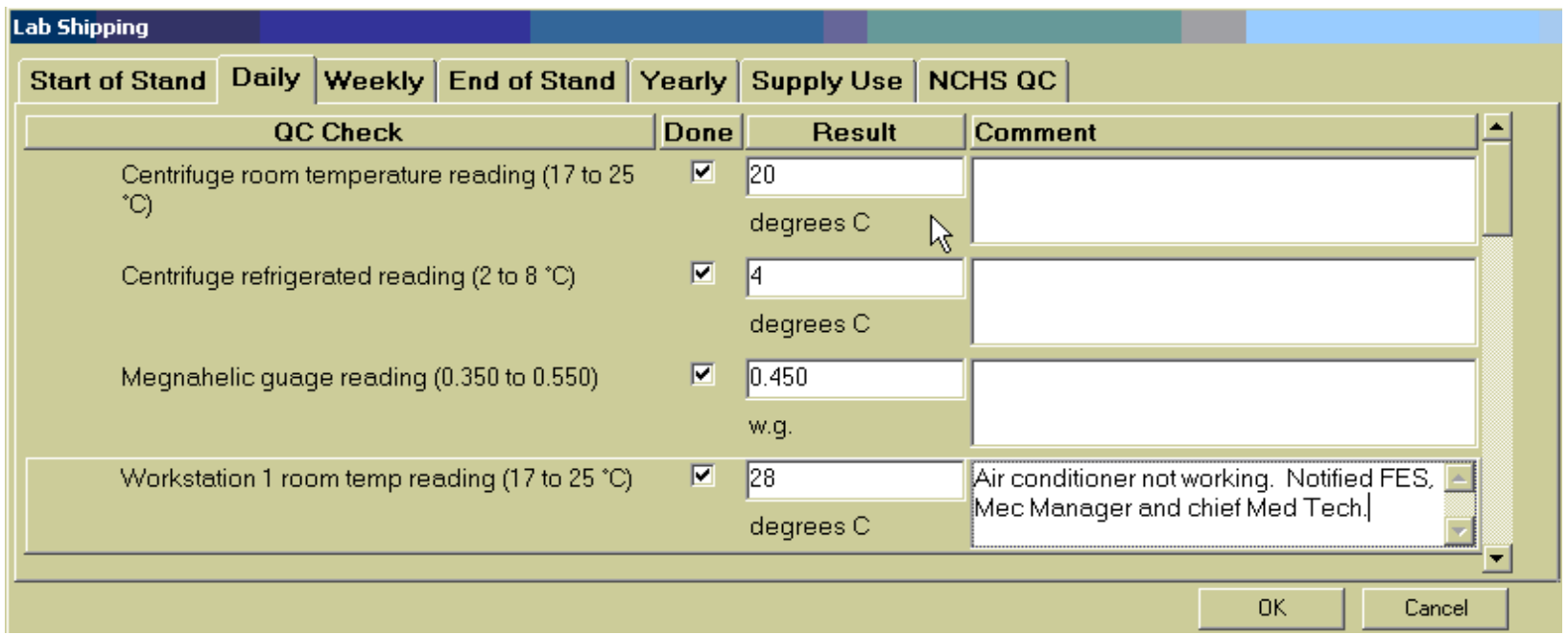

Enter "Yes" or "No" for text responses, whole numbers for rpm measurements, minutes, and seconds for timer readings, three decimals for w.g., and whole negative and positive numbers for thermometer readings in the Result text box. Select [Tab] or [Enter] to move to the Comment text box. Type any actions associated with an unexpected result in the Comment text box. Select [Tab] to move to the next line. 
Enter the lot numbers and expiration dates in the Supply Use activity. This activity lists every inventory item associated with the component. Record the pregnancy test kit and the serum and urine pregnancy control lot numbers and expiration dates even though these are captured in the Pregnancy QC module.

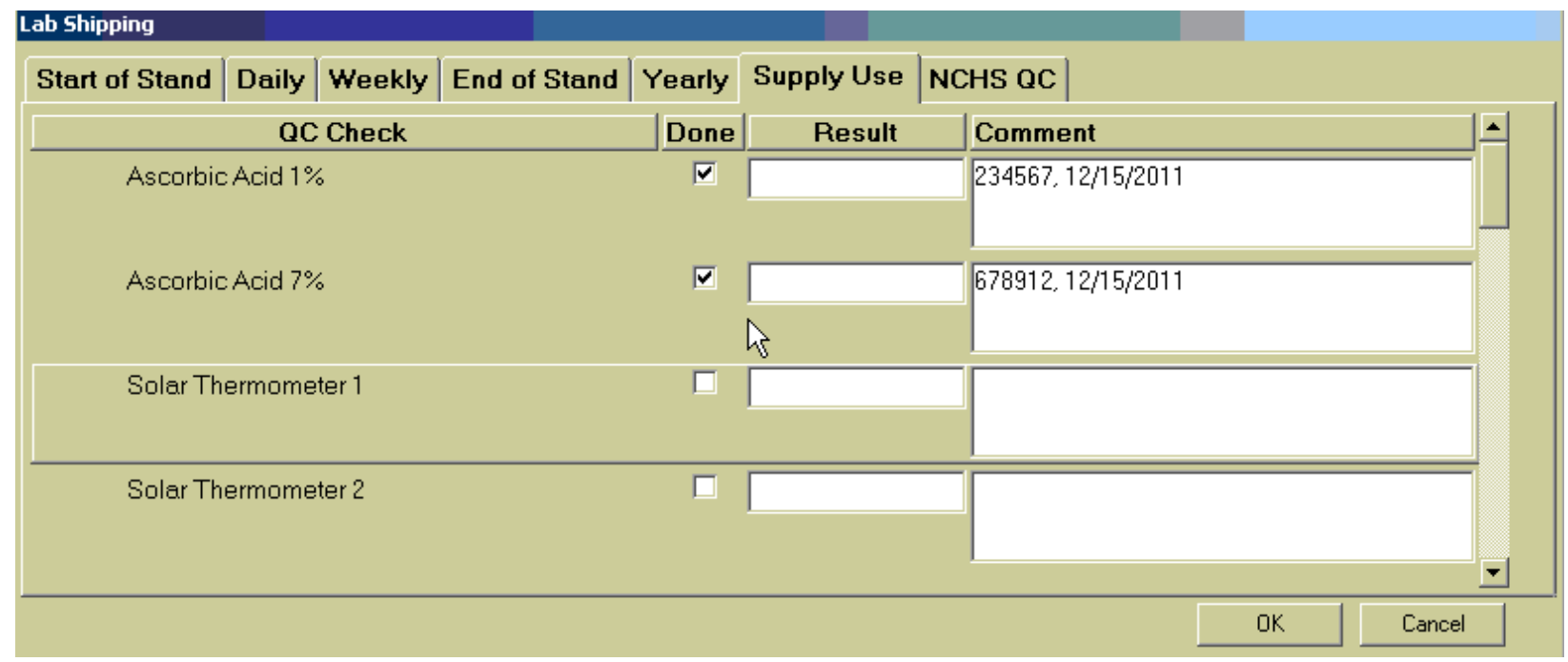

Mark each checkbox and record the lot and expiration date of each item in the Comments text box at the start of the stand and whenever a new lot is put into use. To record an activity as complete or done, use the mouse to direct the mouse arrow to the checkbox in the Done column, and left click to insert a checkmark. Select [Tab] or [Enter] to move to the Result text box. Do not enter the response or reading in the Result text box. Select [Tab] or [Enter] to move to the Comment text box.

- For the ascorbic acid, record the lot number and record the date the reagent was reconstituted using the MM/DD/YYYY format.

- For the timers and thermometers, record the calibration date (14/06), add a comma, and record the expiration date using the MM/DD/YYYY format.

- For items with lot numbers, record the lot number, add a comma, and record the expiration date using the MM/DD/YYYY format.

There is no frequency requirement for this activity within the QC application. 
Update the information each time a new lot is put into use.

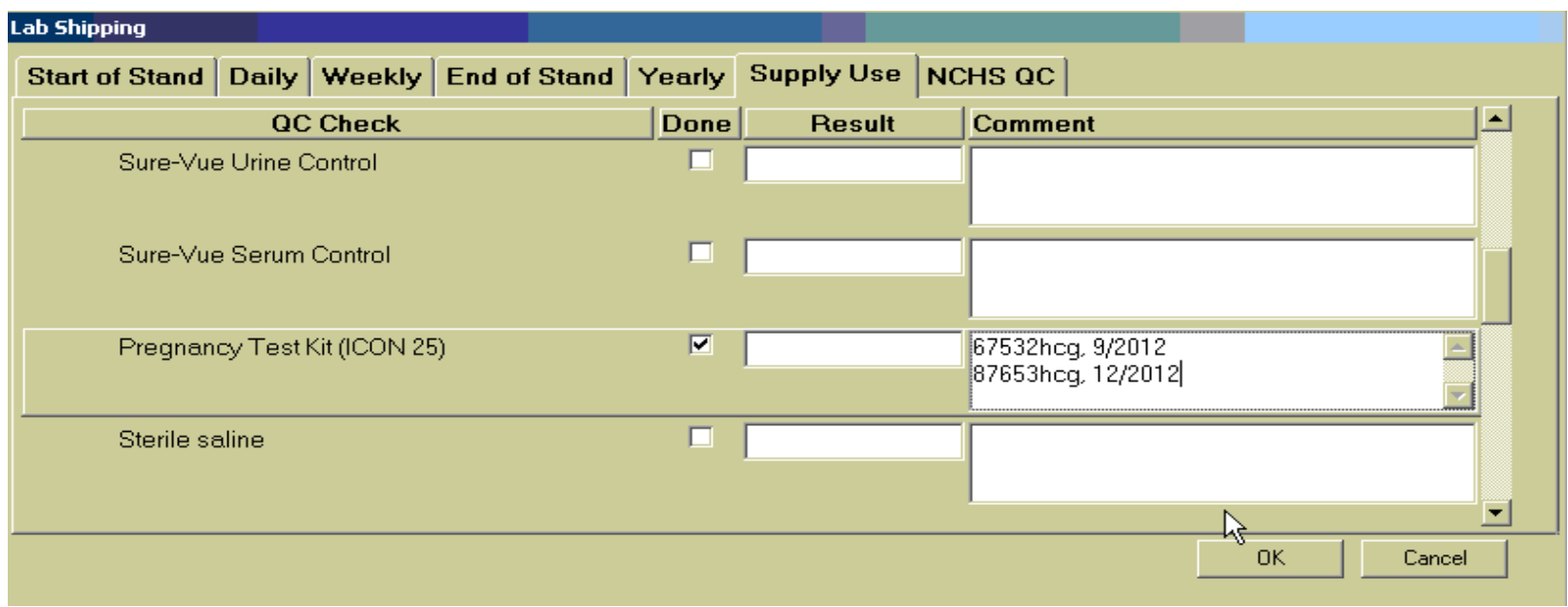

To save the results to the database, use the mouse to direct the mouse arrow to the "OK" button, and left click; to exit the QC module without saving the results, use the mouse to direct the mouse arrow to the "Cancel" button, and left click. Once results are saved to the database, they cannot be changed, except for the information contained in the Supply Use Comments text box.

A Warning Message text box displays if required QC is incomplete.

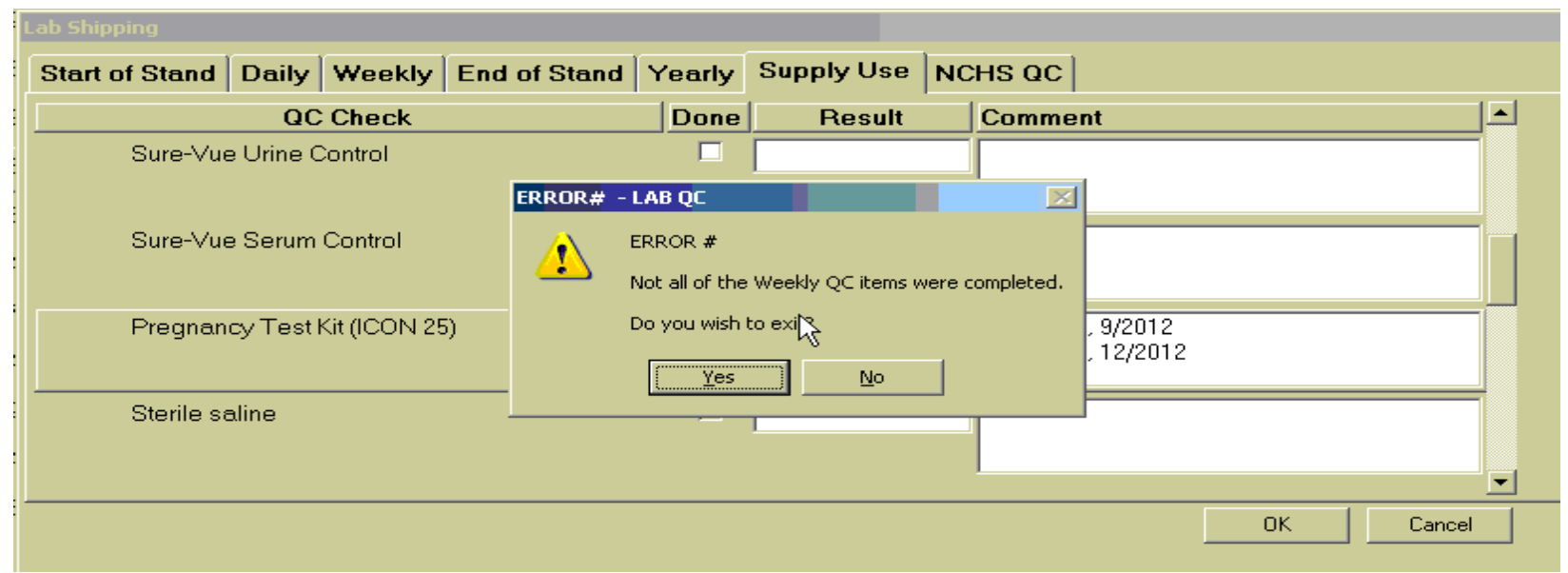

A Warning Message text box displays when an attempt is made to access a laboratory module if the required QC activities have not been performed or if some activities are incomplete. Respond to the Message text box. To exit the QC module, use the mouse to direct the mouse arrow to the "Yes" button, and left click or type [Y/y] or select [Enter]. To remain in the QC module, use the mouse to direct the mouse arrow to the "No" button, and left click or type [N/n]. Continue to perform the required QC activities and record the results. 
A red checkmark on the top of the file header indicates that all QC activities associated with that requirement were successfully recorded and saved to the database.

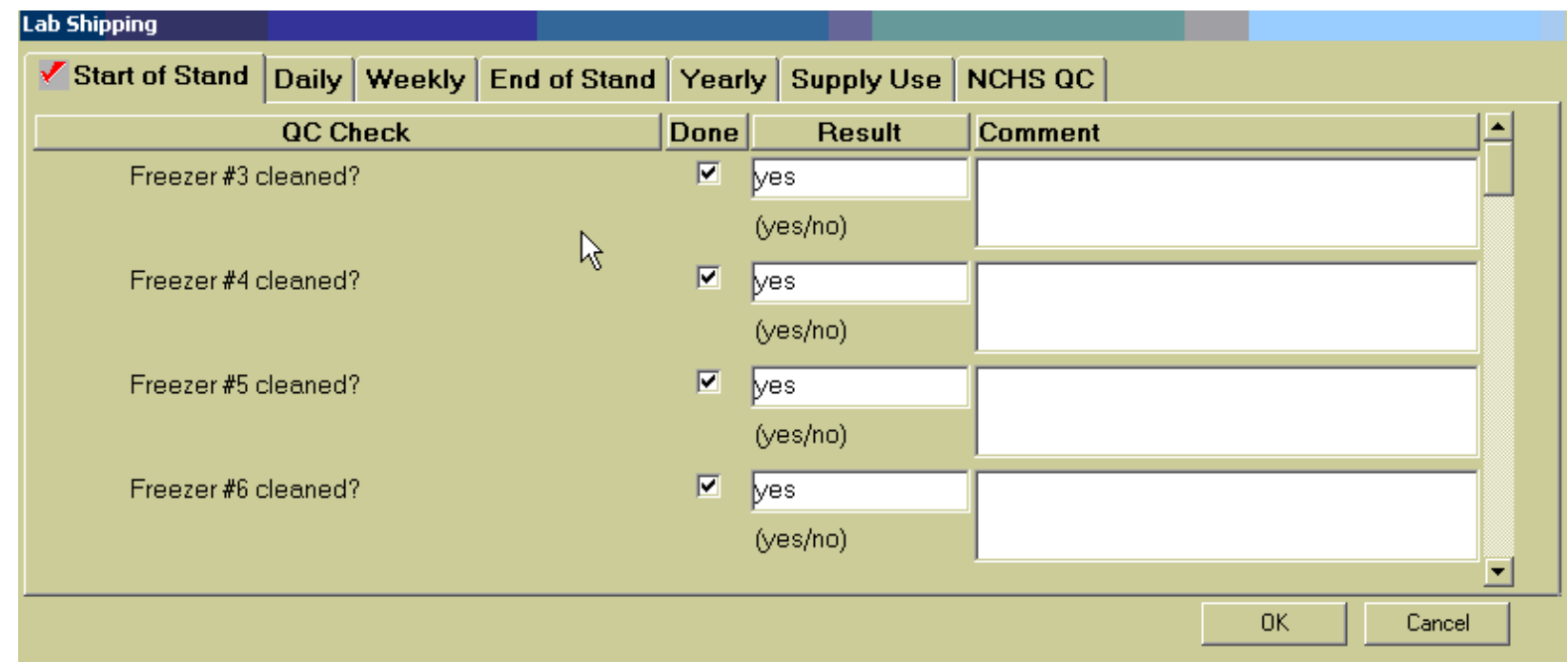

\subsection{Unusual Field Occurrence (UFO)}

Document any unusual occurrence using the Unusual Field Occurrence utility. The hard-copy Unusual Occurrence Log (see Exhibit 13-7) is available as a backup. Record the date, Tech ID number, an explanation of the incident, and the steps to resolve the incident. Be sure to record the model and the serial number of the instrument. Refer to the UFO User Guide for complete instructions. 
Exhibit 13-7. Hard-copy Unusual Occurrence Log

\section{NHANES \\ Unusual Occurrence Log}

\section{Stand Number}

Beginning Stand Date

Technologist Name and ID

Date

Time

Comments or Issue

Action Taken

Resolution

Reviewed by

Date reviewed 
Enter information into the appropriate line(s):

- Stand Number,

- Beginning Stand Date,

- Technologist Name and ID,

- Date,

- Time,

- Comments or Issue,

- Action Taken, and

- Resolution.

NOTE: Do not enter any text in the Reviewed by or Date reviewed lines.

Print these results and file in the QC notebook. Send to the home office at the end of each stand. The data manager can answer any questions about accessing, saving, and printing this document.

\section{Hard-Copy Supply Use Control Log}

Document the lot number and expiration dates for all Vacutainer ${ }^{\circledR}$ tubes, needles, and reagents in the Supply Use Control activity of the Quality Control module. This hard-copy Supply Use Control Log (Exhibit 13-8) is available as a backup.

Enter information into the appropriate column(s):

- Reagent or Supply Name,

- Beginning Stand Date,

- Lot Number,

- Date in Use,

- Expiration Date, and

- Technologist ID. 
Exhibit 13-8. Hard-copy Supply Use Control Log

\section{NHANES}

Supply Use Control Log

\section{Stand Number}

Beginning Stand Date

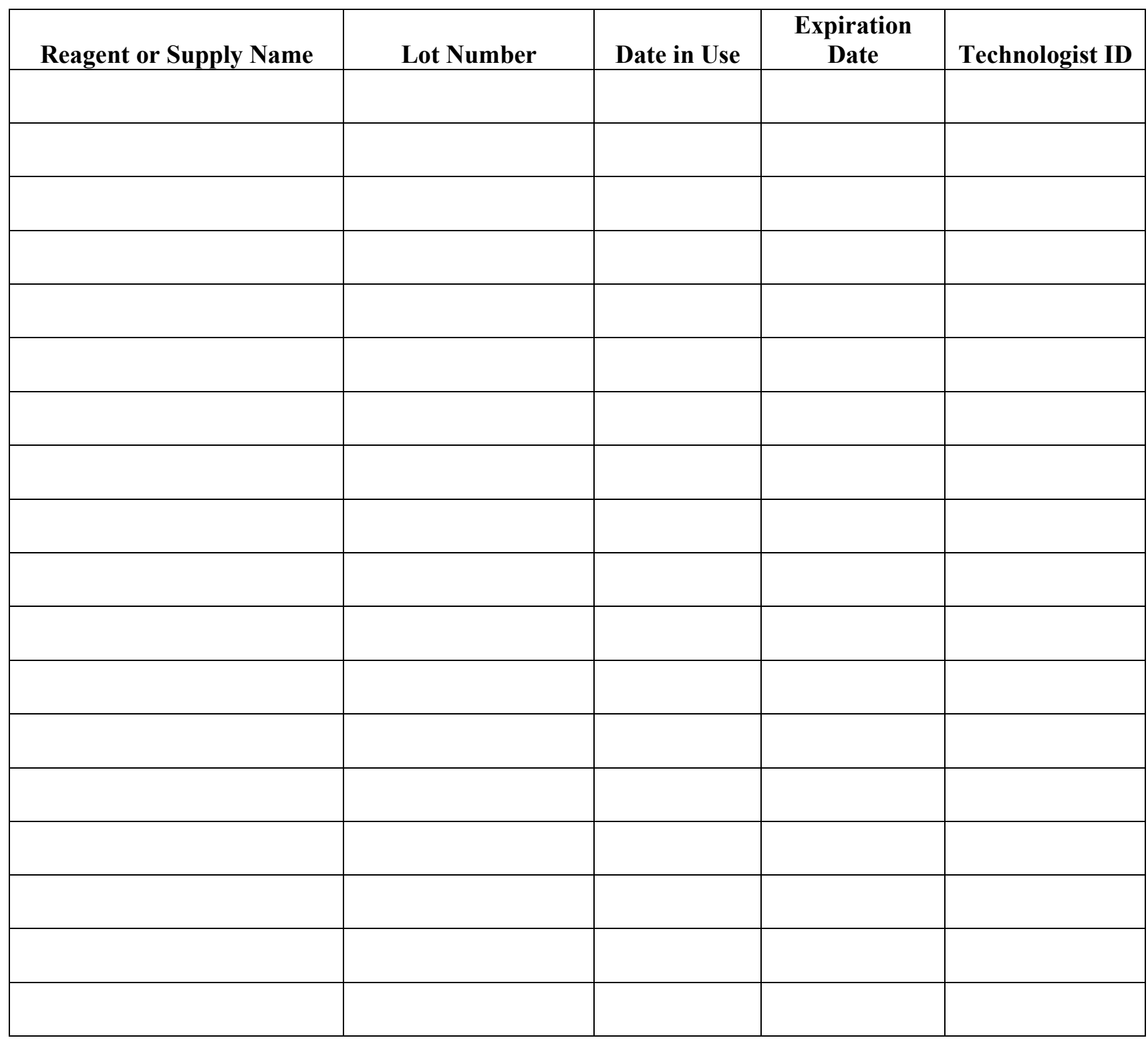


Print these results and file in the QC notebook. Send to the home office at the end of each stand. The data manager can answer any questions about accessing, saving, and printing this document.

\subsection{Eppendorf Pipette Care, Sterilization, Maintenance, and Calibration}

The MEC laboratory has four Eppendorf Research pro electronic pipettes: one 5-100 $\mu \mathrm{L}$, two $50-1000 \mu \mathrm{L}$, and one $100-5000 \mu \mathrm{L}$. The lab also uses $100 \mu \mathrm{L}$ and $1000 \mu \mathrm{L}$ Biopur pipette tips and $5-\mathrm{mL}$ bulk pipette tips. Charge the pipettes in the 4 -stand charger.

- Do not clean the display or any of the labeling using acetone or aggressive solutions.

- Do not allow any liquid to enter the device.

- Only authorized service personnel may carry out repairs.

- Use original spare parts and accessories (battery, pipette tips) only.

\section{Care}

Wipe the outside of the pipette with a moist cloth. The use of water with wetting agent is also permitted.

WARNING: DO NOT ALLOW ANY LIQUID TO ENTER THE PIPETTE. 
Rinse (with distilled water) and dry only the lower part of the pipette. The pipette may be wiped clean with 60 percent isopropanol.

Disassemble the lower half of the Research pro if the pipette is very contaminated or if very aggressive chemicals are dispensed. Rinse the individual parts in distilled water and then dry the parts. Lightly lubricate the piston using silicone grease.

After opening the pipette, check the position of the piston and execute a motor reset. Trigger the motor reset by holding down the Reset key until $\mathbf{R}$ appears in the display.

\section{Sterilization}

Disassemble only the lower half of the pipette. The individual parts of the lower part must be disassembled further into separate pieces. Review the diagrams on page 27 of the Research Pro Instruction Manual before attempting to disassemble any pipette.

To loosen the lower part of the pipette (for all pipettes except the 5,000 $\mu \mathrm{L}$ ):

- Hold down the ejector and pull off the ejector sleeve. Force may be required.

- Unscrew the lower part using the key. During disassembly, do not damage the piston.

- Unscrew the piston. If necessary, first loosen the piston at the upper end using flatnose pliers. The piston is moved to the lowest position.

To loosen the lower part of the $5,000 \mu \mathrm{L}$ pipette:

- Hold down the ejector and pull off the ejector sleeve. Force may be required.

- Unscrew the cylinder. The piston is held in place magnetically. During reassemble, do not damage the piston.

- Pull the piston out of the upper part.

Assemble in reverse order. Engage the magnetic coupling of the 5,000 $\mu \mathrm{L}$ piston as shown in the diagram on page 27 of the Instruction Manual. Check that the dispensing function of the pipette is working correctly. 


\section{Maintenance and Calibration}

Apart from general care and occasional discharging (when the battery symbol starts flashing) and recharging of the pipette, no special maintenance is necessary if the pipette is used correctly. The lower part of the pipette may be replaced. Part B: "Ordering information/service parts," describes the process for replacing the seals in the lower part.

The pipettes are calibrated at least once per year, when the staff are on their break in June/July or December. When instructed, send the pipettes to the warehouse. The warehouse manager will contact the service department at Brinkmann Instruments at 800-645-3050, ext. 404 to schedule the calibration; send the pipettes to Brinkmann, and then back to the field.

A complete list of troubleshooting information is included on pages 28 and 29 of the Instruction Manual.

\subsection{Power Loss and Laboratory Operations}

Three scenarios may occur if power is lost to the MEC. Scenario 1 occurs when power is lost during an exam session and there is no backup generator. Scenario 2 occurs when power is lost when the MEC is not conducting a session. Scenario 3 occurs when power is lost but there is a backup generator available. Procedures to address each scenario are described in detail.

\subsubsection{Scenario 1 - Power Loss during Exam Session with No Backup Generator}

If a power loss occurs during a MEC session, action must be taken to either continue processing the blood or preserve the integrity of the processed specimens. The phlebotomist should not draw blood during a power loss because of safety concerns due to insufficient light. 


\section{Centrifuges}

- Overview - There are two centrifuges in each MEC: one centrifuge is operated at ambient temperature and the second is operated at refrigerated $\left(2-6^{\circ} \mathrm{C}\right)$ temperature. All blood tubes can be spun in the refrigerated centrifuge.

While power is generally required to open or unlock the centrifuge door, an emergency access procedure will allow the technologists to open the door to retrieve the blood tubes if a power failure occurs.

- Consequences - All blood tubes can remain in the locked centrifuge for up to 4 hours.

- Actions

1. It is not possible to routinely spin all tubes in one centrifuge; if a power loss is anticipated, spin all tubes in the refrigerated centrifuge.

2. Use the emergency access procedure to retrieve the blood tubes from the centrifuge. Aliquot the serum and plasma into the vessels if the serum or plasma has sufficiently separated from the cells.

- $\quad$ Move the secondary latch lever to UNLOCK position.

- $\quad$ Insert a 5-mm (3/16 in.) or smaller screwdriver into the override key slot in the front panel (see Figure 4-1 on page 4-4 of the Instruction Manual).

- Turn the screwdriver clockwise until you hear a "click" or until the door pops open.

\section{Laminar Flow Hood}

- Overview - The technologists require the laminar flow hood to process the blood for two reasons: (1) the hood provides an uncontaminated or clean environment for processing environmental blood specimens; (2) the hood is required to provide a fullface shield in case of an accidental blood splash. In addition, the outlet in the hood provides power to the electronic pipettes. The electronic pipette is required to perform an accurate dilution for vessel 3 (RBC Folate).

- Consequences - The environmental specimens may become contaminated. The pipette will lose battery power after $\sim 60$ minutes. 


\section{- Actions}

1. Complete an Unusual Occurrence Log to document the SP IDs of the samples processed without a fully functioning hood. This information is subsequently recorded in an annual summary for NCHS.

2. Attach the comment "equipment failure" to vessels 1, 5, and 29.

3. Use the backup full-face shields when processing blood.

4. Process vessel 3 immediately so specimens are not lost due to battery failure.

\section{Freezers and Refrigerators}

- Overview - There are four freezers and two refrigerators in each MEC lab. The refrigerators and freezers are located in a stacked configuration across from the Coulter. Three freezers are stacked on top of the rack and two refrigerators with the remaining freezer are located on the bottom. Refrigerators and freezers are numbered according to which port they are in on the TempGuard system. Freezers are numbered 3-6 and the refrigerators are numbered 1 and 2. Port 7 is for room temperature monitoring. Refrigerant gel packs are distributed and stored in the freezers. Most specimens are stored in various plastic vials and the vials are placed in cardboard boxes with grids; the cardboard boxes are then stored in the freezers and refrigerators. In addition, some blood tubes (vessels 54 and 55), swabs (vessels 73 and 76), the VOC water (vessel 56), and the Coulter and pregnancy controls are stored in the refrigerator.

- Consequences - Specimen integrity is affected if frozen specimens thaw or if refrigerated specimens reach ambient temperature. Some analytes are more sensitive than other analytes. A full freezer takes approximately 3 hours to rise from $-20^{\circ} \mathrm{C}$ to $10^{\circ} \mathrm{C}$; a $1 / 2$ filled freezer takes 2 hours and 25 minutes to rise to $-10^{\circ} \mathrm{C}$; and a $1 / 4$ filled freezer takes 1 hour and 45 minutes to rise to $-10^{\circ} \mathrm{C}$. Once the temperature reaches $-10^{\circ} \mathrm{C}$ the staff has approximately 30 minutes before the specimens thaw.

\section{- Actions}

1. Do not open the freezer or refrigerator doors, if possible.

2. Refrigerators - After 30 minutes or when the TempGuard reading reaches $7^{\circ} \mathrm{C}$, add 14 frozen gel packs (stored in the bottom of each freezer) to the center of each refrigerator. Alternatively, add 25 pounds of ice cubes or blocks to each refrigerator. Do not add dry ice to the refrigerators; this will cause the refrigerator to explode.

3. Freezers - Contact the local dry ice company and order 200 pounds of dry ice. Obtain four large ice chests or coolers; each cooler should hold the boxes from 
one freezer and 50 pounds of dry ice. Within 2 hours or when the temperature recording chart reading reaches $-10^{\circ} \mathrm{C}$, move the containers (boxes) to the ice chests and add 50 pounds of dry ice to each ice chest. Alternatively, move the containers to Styrofoam shippers and distribute the dry ice across the shippers. Do not add dry ice to the freezers; this will cause the freezer to explode.

\section{Coulter DxH 800}

- Overview - The Coulter consists of the DxH 800, the monitor, hard drive, and printer. The DxH will not function without the hard drive and monitor. The blood components for the CBC (complete blood count) are stable for a maximum of 24 hours when stored at room temperature.

- Consequences - None.

- Actions - None.

NOTE: The data manager should disable the Sensaphone system before a scheduled power loss.

\subsubsection{Scenario 2 - Power Loss when the MEC is Not Conducting Exams}

If the power loss occurs when the MEC is not conducting a session, the freezers and refrigerators require action to protect the integrity of the specimens. The lab staff can continue to ship if all the ISIS processes are complete. If the ISIS processes are not complete, the lab staff can still pack the shippers and complete the ISIS process once power is restored.

\section{Freezers and Refrigerators}

- Overview - All three MECs now have the Temperature Guard system installed. This system monitors the room temperature and the internal temperature of the refrigerators and freezers in the MEC laboratory. When the system detects a significant increase in the temperatures, it begins calling designated staff. The MEC managers and data managers have been identified as those individuals responsible for responding to the call by typing in the response code. The temperature increase could be confined to one freezer or refrigerator, to multiple units, or all units. The temperature increase could be due to any number of circumstances such as a loss of power because a circuit breaker tripped, or due to a compressor malfunction or failure. Whoever enters the code must act to prevent loss of biological specimens. The Temperature Guard 
temperature trigger was established to allow 30 minutes of travel time. After 30 minutes, frozen specimens may begin to thaw.

- Consequences - Same as scenario \#1.

\section{- Actions}

1. Examine each recording unit chart to verify which unit(s) is/are affected. The red pen line on the affected freezer or refrigerator chart should show a distinct movement toward the center of the graph.

2. Refrigerators - If one refrigerator has been affected, move all the specimens to the other refrigerator. If both refrigerators have been affected, keep the specimens cold by adding 14 frozen gel packs (stored in the bottom of each freezer) to the center of each refrigerator. Alternatively, add 25 pounds of ice cubes or blocks to each refrigerator. Do not add dry ice to the refrigerators; this will cause the refrigerator to explode.

3. Freezers - If only one freezer has been affected, move all the specimens from the affected unit to an unaffected unit. If more than one freezer has been affected and there is sufficient space in the unaffected freezers to accommodate the specimens, move the specimens to the other unaffected freezers. If there is insufficient space to accommodate the frozen specimens in the unaffected units, or if all four freezers are affected, try to reach the chief medical technologist or any of the staff medical technologists to assist. Contact the local dry ice company and order 200 pounds of dry ice. Obtain four large ice chests or coolers; each cooler should hold the boxes from one freezer and 50 pounds of dry ice. Within 2 hours or when the TempGuard reading reaches $-10^{\circ} \mathrm{C}$, move the containers (boxes) to the ice chests and add 50 pounds of dry ice to each ice chest. Alternatively, move the containers to Styrofoam shippers and distribute the dry ice across the shippers. Do not add dry ice to the freezers; this will cause the freezer to explode.

4. Leave a note on the unit indicating the new location of the specimens.

5. Turn off the affected unit, by flipping the corresponding wall switch to the left of the units. 


\subsubsection{Scenario 3 - Power Loss with a Backup Generator}

If the power loss occurs when the MEC is conducting a session and there is a backup generator available, continue to monitor the temperature of the freezers and refrigerators and assess the situation with the MEC manager. If the temperature approached the established thresholds:

- Retrieve the generator from the trailer belly compartment,

- Retrieve the power strip,

- Plug the freezers into the power strip,

- Start the generator, and

- If both refrigerators have been affected, keep the specimens cold by adding 14 frozen gel packs (stored in the bottom of each freezer) to the center of each refrigerator. Alternatively, add 25 pounds of ice cubes or blocks to each refrigerator.

If a power loss occurs and the MEC is not conducting a session, the freezers and refrigerators require action to protect the integrity of the specimens. The TempGuard system alerts the MEC and data managers that the temperature of the freezers and/or refrigerators has exceeded established thresholds. The MEC or data managers must respond to the call, travel to the MEC immediately, and do the following:

- Retrieve the generator from the trailer belly compartment,

- Retrieve the power strip,

- Plug the freezers into the power strip,

- Start the generator, and

- If both refrigerators have been affected, keep the specimens cold by adding 14 frozen gel packs (stored in the bottom of each freezer) to the center of each refrigerator. Alternatively, add 25 pounds of ice cubes or blocks to each refrigerator. 
Appendix A

\section{International Air Transport Association (IATA) Dangerous Goods Regulations \\ January 2013}




\section{APPENDIX A \\ INTERNATIONAL AIR TRANSPORT ASSOCIATION (IATA) DANGEROUS GOODS REGULATIONS}

\section{A.1 General Provisions}

Dangerous goods can be transported safely by air transport provided certain principles are strictly followed. The International Air Transport Association (IATA) publishes dangerous goods regulations. NHANES shipments fall under these regulations because diagnostic specimens (UN 3373) are packed in dry ice, which is classified as dangerous goods, and shipped via Federal Express, which uses air transport.

Dangerous goods are articles or substances that are capable of posing a significant risk to health, safety, or to property, when transported by air and which are classified as to the type of hazard. The IATA Dangerous Goods Regulations are applicable to all shippers and agents that offer consignments of dangerous goods to airlines. A shipper must comply fully with these regulations when offering a consignment of dangerous goods to IATA member airlines, and to airlines participating in IATA interline agreements for cargo. Before offering any package for air transport, the shipper must comply with the following specific responsibilities (1.3.2).*

- A shipper must provide such information to his employees as will enable them to carry out their responsibilities with regard to the transport of dangerous goods by air.

- The shipper must ensure that the articles or substances are not prohibited for transport by air.

- The articles or substances must be properly identified, classified, packed, marked, labeled, and documented in accordance with IATA regulations.

- Before a consignment of dangerous goods is offered for air transport, all relevant persons involved in its preparation must have received training to enable them to carry out their responsibilities.

The successful application of regulations concerning the transport of dangerous goods and the achievement of their objectives are greatly dependent on the appreciation of all individuals concerning

\footnotetext{
* Notations in parentheses throughout this appendix represent the appropriate section in the IATA Dangerous Goods Regulations.
} 
the tasks involved and on a detailed understanding of the regulations. Initial and recurrent training programs achieve this for all persons concerned in the transport of dangerous goods. Training must be provided or verified upon the employment of a person in a position involving the transport of dangerous goods by air (1.5.0.2). Recurrent training must take place within 24 months of previous training to ensure knowledge is current. There is a provision for a 3-month window that allows for recurrent training conducted within the final 3 months of the 24-month period to be considered to have been completed on the expiry date of the 24-month period (1.5.0.3). A test must be undertaken following dangerous goods training to verify understanding of the regulation. A certificate must be issued confirming successful completion of the test (1.5.0.4).

Training curricula must include (1.5.2.2):

- General familiarization aimed at providing familiarity with IATA general provisions;

- Function-specific training in the requirements applicable to the function for which that person is responsible; and

- Safety training to cover the hazards presented by dangerous goods, safe handling, and emergency response procedures.

A record of training must be maintained, which must include the following (1.5.5):

- The individual's name;

- The most recent training completion date;

- A description, copy, or reference to training materials used to meet the training requirement;

- The name and address of the organization providing the training; and

- Evidence which shows that a test has been completed satisfactorily.

\section{Training Curricula (table 1.5A)}

Aspects of transport of dangerous goods by air with which packers should be familiar, as a minimum include:

- General philosophy;

- Limitations; 
- General requirements for shippers;

- Classifications;

- List of dangerous goods;

- General packing requirements;

- Packing instructions;

- Labeling and marking;

- Shipper's Declaration and other relevant documentation;

- Recognition of Undeclared Dangerous Goods;

- Provisions for passengers and crew; and

- Emergency procedures.

\section{A.2 Limitations}

Some dangerous goods are too dangerous to be carried by aircraft, others may be carried on cargo aircraft only, and some are acceptable on both cargo and passenger aircraft. A number of limitations are placed on dangerous goods which are permitted to be transported by air. These limitations are established by these Regulations (2.0).

The following goods are permitted on aircraft as checked baggage only and with the approval of the operator(s) (2.3.2).

- Dry Ice - Carbon Dioxide, solid (dry ice) in quantities not exceeding $2.5 \mathrm{~kg}$ (5 lbs.) per person when used to pack perishables not subject to these Regulations, provided the checked baggage (package) permits release of carbon dioxide gas (2.3.4.6).

\section{A.3 Classifications}

Dangerous goods are defined as those goods which meet the criteria of one or more of the nine UN hazard classes and, where applicable, to one of the three UN packing groups according to provisions of IATA regulations. The nine classes relate to the type of hazard, whereas the packing groups 
relate to the degree of danger within the class (3.0.1). The nine classes are: Class 1 Explosives; Class 2 Flammable Gases; Class 3 Flammable Liquids; Class 4 Flammable Solids, Substances Liable to Spontaneous Combustion, Substances Which, in Contact with Water, Emit Flammable Gases; Class 5 Oxidizing Substances and Organic Peroxide; Class 6 Toxic and Infectious Substances; Class 7 Radioactive Material; Class 8 Corrosives; and Class 9 Miscellaneous Dangerous Goods, including dry ice and non-pathogenic genetically modified organisms.

Dangerous goods are assigned to the relevant packing group according to the degree of hazard that they present (3.0.3). The three packing groups are: Packing Group 1 - high danger; Packing Group 2 - medium danger; and Packing Group 3 - low danger.

\section{A.3.1 Division 6.2 - Infectious Substances (3.6.2)}

Division 6.2 defines infectious substances as substances which are known or are reasonably expected to contain pathogens. Pathogens are defined as microorganisms (including bacteria, viruses, rickettsiae, parasites, fungi) and other agents such as prions, which can cause disease in humans or animals. (3.6.2.1).

Infectious substances must be classified in Division 6.2 and assigned to UN 2814, UN 2900, or UN 3373, as appropriate (3.6.2.2.1). Infectious substances are divided into two categories, Category A and Category B. Category A is an infectious substance which is transported in a form that, when exposure to it occurs, is capable of causing permanent disability, or life-threatening or fatal disease in otherwise healthy humans or animals. The proper shipping name for UN 2814 is Infectious substance, affecting humans. The proper shipping name for UN 2900 is Infectious substance, affecting animals. Category B is an infectious substance which does not meet the criteria for inclusion in Category A (3.6.2.2.2.1). Infectious substances in Category B must be assigned to UN3373. The proper name of UN 3373 is Biological substance Category B (3.6.2.2.2.2).

Patient specimens for which there is minimal likelihood that pathogens are present are not subject to these Regulations if the specimen is packed in packaging that will prevent any leakage and that is marked with the words "Exempt human specimen" or "Exempt animal specimen" (3.6.2.2.3.6). The packaging must consist of three components: a leakproof primary receptacle(s), a leakproof secondary receptacle, and an outer packaging of adequate strength for its mass and intended use, and with at least 
one surface having minimum dimensions of $100 \mathrm{~mm} \times 100 \mathrm{~mm}$. For liquids, absorbent material in sufficient quantity to absorb the entire contents must be placed between the primary receptacle(s) and the secondary packaging so that, during transport, any release or leak of a liquid substance will not reach the outer packaging and will not compromise the integrity of the cushioning material. When multiple fragile primary receptacles are placed in a single secondary packaging, they must be either individually wrapped or separated to prevent contact between them (3.6.2.2.3.6).

\section{A.3.2 Class 9- Miscellaneous Dangerous Goods}

Class 9 defines miscellaneous dangerous goods as substances and articles, which, during air transport, present a danger not covered by other classes (3.9.1.1). Included in this class is solid carbon dioxide, or dry ice. Dry ice is regulated by packing instruction 954 .

\section{A.3.3 List of Dangerous Goods}

UN 3373 Biological substance Category B specimens must comply with packing instruction $650(\operatorname{Pg} 463)$.

Dry ice is classified as UN number 1845 and is classified as Class 9 dangerous goods. Shipping packaging must be labeled with a Miscellaneous Class 9 label. A maximum net quantity per package is $200 \mathrm{~kg}$ or $441 \mathrm{lb}$. The relevant packing instruction is 954 . A special provision directs that packing tests are not considered necessary and when dry ice is used as a refrigerant for other dangerous goods loaded in a unit load device or other type of pallet, the quantity limits per package of $200 \mathrm{~kg}$ do not apply.

\section{A.4 General Packing Provisions}

The shipper is responsible for all aspects of the packing of dangerous goods in compliance with these Regulations (5.0.1.1). 
Dry ice (solid carbon dioxide) is produced by expanding liquid carbon dioxide to vapor and "snow" in presses that compact the product into blocks. Dry ice is used primarily for cooling and can cause severe burns (its temperature is about $-79^{\circ} \mathrm{C}$ ) to skin in case of direct contact. When dry ice (solid carbon dioxide) converts (sublimates) directly to gaseous carbon dioxide, it takes heat from its surroundings. The resulting gas is heavier than air and can cause suffocation in confined areas as it displaces air. Packages containing dry ice must be designed and constructed to prevent buildup of pressure due to release of carbon dioxide gas.

Dangerous goods must be packed in good quality packaging, which must be strong enough to withstand the shocks and loadings normally encountered in transport. Packages must be constructed and closed as to prevent any loss of contents when prepared for transport, which might be caused under normal conditions of transport, by vibration, or by changes in temperature, humidity, or pressure (resulting from altitude, for example) (5.0.2.4.1).

New, remanufactured, reused, or reconditioned packaging must be manufactured and tested under a program of quality assurance to ensure that such packaging meets requirements (5.0.2.5).

Packaging for which retention of liquid is a basic function, must be capable of withstanding, without leakage, an internal pressure that produces a pressure differential of not less than $95 \mathrm{kPa}$ for liquids in packing group III, Class 3, or Division 6.1 (5.0.2.9).

Packaging used for solids, which may become liquid at temperatures likely to be encountered during air transport, must also be capable of containing that substance in the liquid state (5.0.2.10).

Inner packaging - cushioning material: Inner packaging must be packed, secured, or cushioned in an outer packaging in such a way that, under normal conditions of transport, they cannot break, be punctured, or leak their contents into outer packaging and so as to control their movement (5.0.2.12.1). 


\section{A.4.1 Packing Instruction 650 - UN 3373}

Packaging must be of good quality materials, strong enough to withstand the shocks and loading normally encountered during transport, including trans-shipment between transport units and warehouses as well as removal from a pallet or overpack for subsequent manual or mechanical handling. Packaging must be constructed and closed to prevent any loss of contents when prepared for transport, which might be caused under normal conditions of transport, by vibration, or by changes in temperature, humidity, or pressure.

The packaging must consist of three components:

1. A primary receptacle(s);

2. A secondary packaging; and

3. A rigid outer packaging.

Primary receptacles must be packed in secondary packaging in such a way that, under normal conditions of transport, they cannot break, be punctured, or leak their contents into secondary packaging. Secondary packaging must be secured in outer packaging with suitable cushioning material. Any leakage of the contents must not substantially impair the protection properties of the cushioning material or of the outer packaging.

Packages must be prepared as follows:

\section{0(a) For Liquids}

- The primary receptacle(s) must be leakproof and must not contain more than $1 \mathrm{~L}$.

- The secondary packaging must be leakproof.

- If multiple fragile primary receptacles are placed in a single secondary packaging, they must be individually wrapped or separated to prevent contact between them.

- Absorbent material must be placed between the primary receptacle and the secondary packaging. The absorbent material, such as cotton wool, must be in sufficient quantity to absorb the entire contents of the primary receptacle (so that any release of the liquid substance will not compromise the integrity of the cushioning material) or of the outer packaging. 
- The primary receptacle or the secondary packaging must be capable of withstanding, without leakage, an internal pressure producing a pressure differential of not less than $95 \mathrm{kPa}$ in the range of $-40^{\circ} \mathrm{C}$ to $+55^{\circ} \mathrm{C}\left(-40^{\circ} \mathrm{F}\right.$ to $\left.130^{\circ} \mathrm{F}\right)$.

- The outer packaging must not contain more than $4 \mathrm{~L}$.

\section{0(b) for Solids}

- The primary receptacles must be siftproof and must not exceed the outer packaging weight limit.

- The secondary packaging must be siftproof.

- If multiple fragile primary receptacles are placed in a single secondary packaging, they must be individually wrapped or separated to prevent contact between them.

- The outer packaging must not contain more than $4 \mathrm{~kg}$. This includes ice, dry ice, or liquid nitrogen when used to keep specimens cold.

- If there is any doubt as to whether or not residual liquid may be present in the primary receptacle during transport then a packaging suitable for liquids, including absorbent material, must be used.

\section{Package Testing}

- Each completed package must be capable of successfully passing the following drop tests described in 6.5.1.1 except that the height of the drop must not be less than $1.2 \mathrm{~m}$. Following the appropriate drop sequence, there must be no leakage from the primary receptacle(s) which must remain protected by absorbent material, when required, in the secondary packaging.

\section{For Liquids and Solids}

- An itemized list of contents must be enclosed between the secondary packaging and the outer packaging.

- Packages must have one side with dimensions of not less than $100 \mathrm{~mm}$ x $100 \mathrm{~mm}$ (4 in. $x 4$ in.)

- For transport, the mark illustrated below must be displayed on the external surface of the outer packaging on a background of contrasting color and must be clearly visible and legible. The mark must be in the form of a square set at an angle of $45^{\circ}$ (diamondshaped) with each side having a length of at least $50 \mathrm{~mm}(2 \mathrm{in}$.), the width of the line must be at least $2 \mathrm{~mm}$ and the letters and numbers must be at least $6 \mathrm{~mm}$ high. 
- The proper shipping name "Biological Substance, Category B" letters at least $6 \mathrm{~mm}$ high must be marked on the outer package adjacent to the diamond-shaped mark.

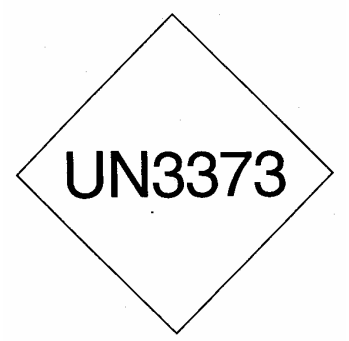

Unless all package markings are clearly visible, the following conditions apply when packages are placed in an overpack:

- The overpack must be marked with the work "Overpack;" and

- The package markings must be reproduced on the outside of the overpack.

Specific Requirements - refrigerated or frozen specimens: ice, dry ice, and liquid nitrogen

- When dry ice or liquid nitrogen is used to keep specimens cold, all applicable requirements of these Regulations must be met. When used, ice or dry ice must be placed outside the secondary packaging or in the outer packaging or an overpack. Interior supports must be provided to secure the secondary packagings in the original position after the ice or dry ice (solid carbon dioxide) has been dissipated. If ice is used, the packaging or overpack must be leakproof. If dry ice (solid carbon dioxide) is used, the outside packaging or overpack must be designed and constructed to permit the release of carbon dioxide gas to prevent a build-up of pressure that could rupture the packaging.

- The primary receptacle and the secondary packaging must maintain their integrity at the temperature of the refrigerant used as well as the temperatures and the pressures, which could result if refrigeration were to be lost.

- Infectious substances assigned to UN 3373 which are packed and marked in accordance with this packing instruction are not subject to any other requirement of these regulations except for the following:

(a) The name, address, and telephone number of the shipper and consignee must be provided on each package;

(b) The name and telephone number of a person responsible must be provided on the air waybill or on the package;

(c) The classification must be in accordance to 3.6.2 (see Section A.3.1); 
(d) The incident reporting requirements in 9.6.1 must be met; and

(e) The inspection for damage or leakage requirements in 9.4.1 and 9.4.2.

9.6.1 - An operator must report dangerous goods accidents or incidents to the appropriate authorities of the State of the operator and the State in which the accident or incident occurred, in accordance with the reporting requirements of those appropriate authorities.

9.4.1 - Packages and overpacks containing dangerous goods must be inspected for signs of damage or leakage upon unloading from the aircraft or unit load device. If evidence of damage or leakage is found, the position where the dangerous goods or unit load was stowed on the aircraft must be inspected for damage or contamination and any hazardous contamination removed. The special responsibilities of operators regarding infectious substances are detailed in 9.4.2. (9.4.1)

9.4.2 - If any person responsible for the carriage of packages containing infectious substances becomes aware of damage to or leakage from such a package, that person must:

- $\quad$ Avoid handling the package or keep handling to a minimum;

- $\quad$ Inspect adjacent packages for contamination and put aside any that may be contaminated;

- Inform the appropriate public health authority, and provide information on any other countries of transit where persons may have been exposed to danger; and

- $\quad$ Notify the shipper and/or consignee.

- If an air waybill is used, the "Nature and Quantity of Goods" box should show the text "BIOLOGICAL SUBSTANCE, CATEGORY B."

- A Shipper's Declaration for Dangerous Goods is not required.

- Clear instructions on filing and closing such packages must be provided by packaging manufacturers and subsequent distributors to the shipper or to the person who prepares the package to enable the package to be correctly prepared for transport.

\section{A.4.2 Packing Instruction 904 - Dry Ice (Pg 499)}

This instruction applies to UN 1845 on passenger and cargo aircraft. The General Packaging Requirements of 5.0.2 must be met. 
Dry ice (solid carbon dioxide), when offered for transport by air, must be in packaging designed and constructed to permit the release of carbon dioxide gas and to prevent a build-up of pressure that could rupture the packaging.

The net weight of dry ice must be marked on the outside of the package.

Arrangements between the shipper and operator(s) must be made for each shipment to ensure that ventilation and safety procedures are followed.

When a Shipper's Declaration is not required, the following information as required by 8.2.3 for dry ice (solid carbon dioxide) must be contained in the "Nature and Quantity of Dangerous Goods" box on the air waybill:

- UN 1845;

- Proper shipping name (Dry Ice or Carbon Dioxide, solid);

- 9 (the word "Class" may be included prior to the number " 9 ");

- The number of packages; and

- The net weight of dry ice in each package.

The net weight of the dry ice must be marked on the outside of the package.

\section{A.5 Packing Specifications and Performance Tests}

Performance tests are designed to ensure that there will be no loss of contents under normal transport conditions. The severity of the tests on a packaging is dependent on the intended contents, taking account of the degree of danger; that is, packing group, relative density (specific gravity), and vapor pressure (for liquids) (6.3.0.2).

The design of each packaging type must be tested as provided in accordance with procedures established by IATA regulation (6.3.1). Tests must be successfully performed on each packaging type before such packaging is used (6.3.1.1.1). 


\section{A.6 Marking and Labeling}

All markings must be so placed on packages or overpacks that they are not covered or obscured by any part of or attachment to the packaging or any other label or marking (7.1.3.1).

All markings must be:

- Durable and printed or otherwise marked on, or affixed to, the external surface of the package or overpack;

- Readily visible and legible;

- Able to withstand open weather exposure without a substantial reduction in effectiveness; and

- Displayed on a background of contrasting color (7.1.3.2).

Unless all markings representative of all dangerous goods in the overpack are clearly visible, the overpack must be marked with the word "Overpack" (7.1.4).

Each package must be marked durably and legible on the outside with each of the following:

- The proper shipping name of the contents and the corresponding UN numbers (7.1.5.1a) (BIOLOGICAL SUBSTANCE, CATEGORY B is UN 3373 and dry ice is UN 1845);

- The full name and address of the shipper and the consignee (7.1.5.1b);

- The NET Quantity of dry ice contained in each package or overpack must be shown. The quantity must be marked on each package or overpack adjacent to the UN number and Proper Shipping Name (7.1.5.1e

- The name and telephone number of a responsible person (7.1.5.1f); and

- For packages containing UN 3373: - "BIOLOGICAL SUBSTANCE, CATEGORY B." (7.1.5.1h).

Empty Packaging

- Other than Class 7, packaging which previously contained dangerous goods must be marked as required for those dangerous goods unless steps such as cleaning, purging of vapors, or refilling with a nondangerous substance are taken to nullify any hazard (7.1.5.6). 
There are two types of labels: hazard labels (in the shape of a square set at $45^{\circ}$ ) which are required for most dangerous goods in all classes and handling labels (in various rectangular shapes), which are required, either alone or in addition to hazard labels for some dangerous goods (7.2.2.2).

A primary hazard label is specified for each listed article and substance. A secondary hazard label or labels must also be applied for each article and substance having subsidiary risk (7.2.3.1). For Class 9 materials, the package must bear the class 9, "Miscellaneous Dangerous Goods" label as required for all dry ice shipments (7.2.3.10).

All labels must be securely affixed or printed on the packaging so that they are readily visible and legible and not obscured by any part of the packaging or by any other label (7.2.6.1a). Each label must be affixed on a background of contrasting color or must have a dotted or solid line outer border (7.2.6.1b). The label must not be folded or affixed in such a manner that different parts of the label appear on different faces of the package (7.2.6.1c). The package must be of such a size that there is adequate space to affix all required labels (7.2.6.1e).

Before empty packaging which had previously contained infectious substances is referred to the shipper, or sent elsewhere, it must be thoroughly disinfected or sanitized and any label or marking indicating that it had contained an infectious substance must be removed or obliterated. (7.2.3.12.2)

Labels must be located on the same surface of the package near the proper shipping name marking if the package dimensions are adequate (7.2.6.2.1).

\section{A.7 Documentation}

Except as otherwise specified, a "Shipper's Declaration for Dangerous Goods" form and an “Air Waybill" must be completed for each consignment of dangerous goods (8.0.1). A "Shipper's Declaration for Dangerous Goods" is not required for shipments containing diagnostic specimens (Pg 463) or dry ice (Pg 499). 
If a Shipper's Declaration is not required for dangerous goods, the Nature and Quantity of Goods" box of the Air Waybill must show in sequence (8.2.3):

- UN or ID number;

- Proper Shipping Name;

- Class or Division number;

- Number of packages; and

- Net quantity per package.

For UN 3373, it is only necessary to show the text "BIOLOGICAL SUBSTANCE; CATEGORY B" and "UN 3373." For dry ice, it is not necessary to show the packing group and packing instruction number on the air waybill.

\section{A.8 References}

1. IATA Dangerous Goods Regulations, 54th Edition, effective January 1, 2013, ISBN 978-92-9233-006-4

2. FedEx Service Guide Vol. 1, effective June 1, 2000. 


\section{A.9 IATA Regulations Examination}

Name

Date

1. IATA is the acronym for:

2. Dangerous goods are articles or substances that are capable of posing a significant risk to health, safety or to property, when transported by air and which are classified as to the type of hazard. (T or F)

3. What are the four responsibilities the shipper must comply with before offering any package for air transport?

4. List the three training requirements:

5. How many UN hazard classes are there?

6. What are the two categories of infectious substances?

7. What is the proper shipping name of UN 3373 ?

8. To what UN hazard class does dry ice belong? 
9. What is the dry ice UN number?

10. Solid carbon dioxide or dry ice is produced by expanding liquid carbon dioxide to vapor and "snow" in presses that compact the product into blocks. Dry ice is used primarily for cooling and can cause severe burns (its temperature is about $-79^{\circ} \mathrm{C}$ ) to skin in case of direct contact. When dry ice (solid carbon dioxide) converts (sublimates) directly to gaseous carbon dioxide, it takes heat from its surroundings. The resulting gas is heavier than air and can cause suffocation in confined areas as it displaces air. Packages containing dry ice must be designed and constructed to prevent buildup of pressure due to release of carbon dioxide gas.

(T or F)

11. Define "Exempt human specimen."

12. What packing instruction regulates UN3373?

13. List the three packaging components in packing instruction 650 :

14. Properly prepared shipments containing liquids must meet the following 6 conditions: 
15. An itemized list of contents must be placed between the secondary packaging and the outer packaging. ( $\mathrm{T}$ or $\mathrm{F})$

16. The proper shipping name "Biological Substance, Category B" with letters at least $6 \mathrm{~mm}$ high must be marked on the outer package adjacent to the diamond-shaped mark. (T or F)

17. When used, ice or dry ice must be placed outside the secondary packaging or in the outer packaging or overpack. Interior supports must be provided to secure secondary packagings in the original position after all the ice or dry ice has dissipated. (T or F)

18. The weight of dry ice is required on the outside of the packaging.

(T or F)

19. The performance tests are designed to ensure that there will be under normal transport conditions.

20. All markings must be:

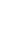

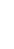

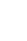

21. For Class 9 goods (dry ice), the package is required to bear the (T or F)

label.

22. All labels must be securely affixed or printed on the packaging so that they are readily visible and legible and not obscured by any part of the packaging or by any other label. (T or F)

23. An "air waybill" must be completed for each consignment of dangerous goods (8.0.1). (T or F) 


\section{A.10 IATA Regulations Examination (Answer Key)}

Name

Date

1. IATA is the acronym for the International Air Transport Association

2. Dangerous goods are articles or substances that are capable of posing a significant risk to health, safety or to property, when transported by air and which are classified as to the type of hazard. (T or F) True

3. What are the four responsibilities the shipper must comply with before offering any package for air transport?

- Information to employees as will enable them to carry out their responsibilities.

- Ensure that articles or substances are not prohibited for transport by air.

- $\quad$ Articles must be properly identified, classified, packed, marked, labeled, and documented.

- All persons involved in preparing consignment must have received training.

4. List the three training requirements:

- General familiarization aimed at providing familiarity with IATA general provisions;

- Function specific training in the requirements applicable to the function for which that person is responsible; and

- Safety training to cover the hazards presented by dangerous goods, safe handling, and emergency response procedures.

5. How many UN hazard classes are there? 9 (nine)

6. What are the two categories of infectious substances?

- $\quad$ ategory A

- Category B

7. What is the proper shipping name of UN 3373? Biological Substance Category B

8. To what UN hazard class does dry ice belong? Class 9 
9. What is the dry ice UN number? UN 1845

10. Solid carbon dioxide or dry ice is produced by expanding liquid carbon dioxide to vapor and "snow" in presses that compact the product into blocks. Dry ice is used primarily for cooling and can cause severe burns (its temperature is about $-79^{\circ} \mathrm{C}$ ) to skin in case of direct contact. When dry ice (solid carbon dioxide) converts (sublimates) directly to gaseous carbon dioxide, it takes heat from its surroundings. The resulting gas is heavier than air and can cause suffocation in confined areas as it displaces air. Packages containing dry ice must be designed and constructed to prevent buildup of pressure due to release of carbon dioxide gas.

(T or F) True

11. Define "Exempt human specimen."

Patient specimens for which there is minimal likelihood that pathogens are present are not subject to these Regulations if the specimen is packed in packaging that will prevent any leakage and that is marked with the words "Exempt human specimen."

12. What packing instruction regulates UN3373? Packing instruction 650

13. List the three packaging components in packing instruction 650 .

- $\quad$ Primary receptacle

- A secondary packaging

- $\quad$ A rigid outer packaging

14. Properly prepared shipments containing liquids must meet the following 6 conditions:

- The primary receptacle must be leakproof.

- The secondary packaging must be leakproof.

- Fragile receptacles must be individually wrapped or separated to prevent contact.

- Absorbent material must be placed between primary receptacle and secondary packaging.

- $\quad$ Primary receptacle or secondary packaging must be capable of withstanding an internal pressure producing a pressure differential of not less than $95 \mathrm{kPa}$ in the range of $-40^{\circ} \mathrm{C}$ to $+55^{\circ} \mathrm{C}$.

- The outer package must not contain more than $4 \mathrm{~L}$. 
15. An itemized list of contents must be placed between the secondary packaging and the outer packaging. (T or F) True

16. The proper shipping name "Biological Substance, Category B" with letters at least $6 \mathrm{~mm}$ high must be marked on the outer package adjacent to the diamond-shaped mark. (T or F) True

17. When used, ice or dry ice must be placed outside the secondary packaging or in the outer packaging or overpack. Interior supports must be provided to secure secondary packagings in the original position after all the ice or dry ice has dissipated. (T or F) True

18. The weight of dry ice is required on the outside of the packaging.

(T or F) True

19. The performance tests are designed to ensure that there will be no loss of contents under normal transport conditions.

20. All markings must be:

- Durable and printed or otherwise marked on, or affixed to, the external surface of the package or overpack;

- Readily visible and legible;

- Able to withstand open weather exposure without a substantial reduction in effectiveness; and

- Displayed on a background of contrasting color.

21. For Class 9 goods (dry ice), the package is required to bear the Class 9 "Miscellaneous" dangerous goods label. ( $\mathrm{T}$ or F) True

22. All labels must be securely affixed or printed on the packaging so that they are readily visible and legible and not obscured by any part of the packaging or by any other label. (T or F) True

23. An "air waybill" must be completed for each consignment of dangerous goods (8.0.1). (T or F) True 


\section{WESTAT \\ Certificate of Test Completion}

International Air Transport Association (IATA) Dangerous Goods Regulations

Name

Date of Successful Exam

Examiner 


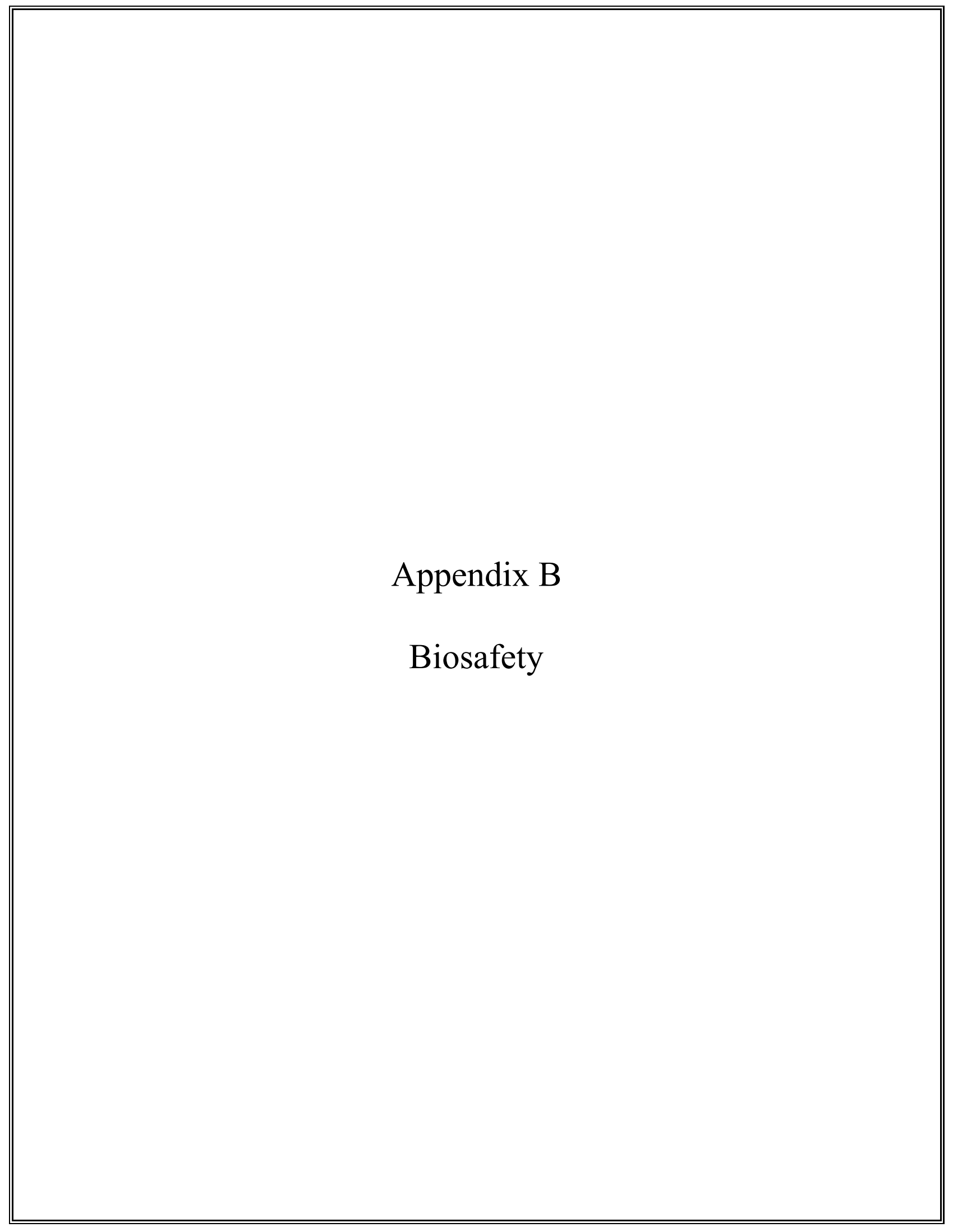




\section{APPENDIX B. BIOSAFETY}

\section{B. POLICY FOR MANAGEMENT OF EXPOSURE TO BLOODBORNE PATHOGENS}

\section{Exposure Control Plan for NHANES Training and Examinations}

\section{B.1 Introduction}

The policy presented in this section is intended to serve as the written policy for the NHANES mobile examination center staff training and main study period. It is based on the OSHA Regulations for exposure to bloodborne pathogens as published in the Federal Register (29CFR 1910.1030), the Updated U.S. Public Health Service Guidelines for the Management of Occupational Exposure to HBV, HCV, and HIV and Recommendations for Postexposure Prophylaxis (MMWR, June 29, 2001), the CDC recommendations, Immunization of Health-Care Workers: Recommendations of the Advisory Committee on Immunization Practices (ACIP) and the Hospital Infection Control Practices Advisory Committee (HICPAC) (December 1997) and the revised Occupational Exposure to Bloodborne Pathogens: Needlestick and Other Sharps injuries; Final Rule.

This manual describes the procedures required to treat and document blood exposure injuries to MEC staff. Universal precautions are the foundation of standard operating procedures and emergency procedures throughout the exam center. MEC staff, the home office staff, and NCHS monitor compliance with these precautions and the pertinent OSHA regulations.

OSHA's rule applies to all persons occupationally exposed to blood or other potentially infectious materials. Blood means human blood, blood products, or blood components. On NHANES, the only body fluids that are collected are blood, vaginal swabs, and urine. Urine is not considered infectious unless the specimens are visibly contaminated with blood or are known to contain an infectious agent.

The Occupational Safety and Health Administration revised the Bloodborne Pathogens standard in conformance with the requirements of the Needlestick Safety and Prevention Act. This Act directs OSHA to: (1) revise the Bloodborne Pathogens standard to include new examples in the definition of engineering controls along with two new definitions; (2) require that Exposure Control Plans reflect how employers implement new developments in control technology; (3) require employers to solicit 
input from employees responsible for direct patient care in the identification, evaluation, and selection of engineering and work practice controls; and (4) require certain employers to establish and maintain a log of percutaneous injuries from contaminated sharps.

\section{B.2 Exposure Determination}

\section{B.2.1 MEC Employees by Group}

Exposure determination is based on the definition of occupational exposure without regard to personal protective clothing and equipment. Job classifications within the work environment are reviewed for exposure and two groups of exposures are identified: Category I job classifications in which all the employees have occupational exposure, and Category II classifications in which some of the employees have occupational exposure. Where all employees have occupational exposure, it is not necessary to list specific work tasks. Where only some employees have occupational exposure, procedures causing occupational exposure must be listed.

Occupational exposure for NHANES staff is categorized as follows:

\section{Category I Exposures}

- Phlebotomist

- Medical technologist

- Dentists

\section{Category II Exposures}

- Physician

- MEC manager

The physician may risk exposure in the event of an emergency in the MEC. The physician is expected to respond to any emergency and perform appropriate procedures, but this does not include the initiation of IV fluids. The MEC manager is responsible for insuring that biohazardous waste is removed and thus may occasionally handle bagged biohazardous waste. The MEC manager is also expected to respond to emergencies and thus may risk exposure if the emergency involves a laceration. 


\section{B.3 Methods of Compliance}

\section{B.3.1 Control for Category I and Category II Exposures}

Risks posed by bloodborne pathogens may be reduced through vaccination, engineering controls, employee work practices, personal protective equipment, and housekeeping procedures. Bloodborne pathogens means pathogenic microorganisms that are present in human blood and can cause disease in humans. These pathogens include, but are not limited to, hepatitis B virus (HBV), hepatitis C virus (HCV), and human immunodeficiency virus (HIV).

Because some infectious diseases can be communicable through contact with SPs and blood, all Category I and Category II staff members are advised to:

- Obtain the hepatitis B vaccine if recommended by Westat for their category of exposure; and

- Use universal precautions (see Section B.3.2) when there is a potential for exposure to blood or body fluids.

\section{B.3.2 General - Universal Precautions}

Universal Precautions is an approach to infection control. According to the concept of Universal Precautions, all human blood and certain human body fluids are treated as if known to be infectious for HIV, HBV, and other bloodborne pathogens. Universal precautions are observed to prevent contact with blood or other potentially infectious materials. Other Potentially Infectious Materials means: (1) the following human body fluids: semen, vaginal secretions, cerebrospinal fluid, synovial fluid, pleural fluid, pericardial fluid, peritoneal fluid, amniotic fluid, saliva in dental procedures, any body fluid that is visibly contaminated with blood, and all body fluids in situations where it is difficult or impossible to differentiate between body fluids; (2) any unfixed tissue or organ (other than intact skin) from a human (living or dead); and (3) HIV-containing cell or tissue cultures, organ cultures, and HIV- or HBVcontaining culture medium or other solutions; and blood, organs, or other tissues from experimental animals infected with HIV or HBV. Universal precautions are applied to procedures involving all sample persons. 


\section{B.3.3 Engineering and Work Practice Controls}

Engineering and work practice controls are the primary methods used to prevent occupational transmission of HBV, HIV, and other bloodborne pathogens. Sharps disposal containers, self-sheathing needles, and biological safety cabinets are examples of engineering controls used in the NHANES.

Biological safety cabinets are similar in appearance to laboratory fume hoods and protect employees from exposure to airborne biohazards by providing an enclosed, mechanically ventilated space for them to work. A laminar flow biological safety cabinet is located in the laboratory in every MEC.

Sharps containers are liquid-tight, puncture-resistant vessels used to minimize the hazards posed by discarded needles, broken glass, scalpels, and other contaminated sharp objects. Light plastic bottles such as those that hold consumer products are unacceptable because the walls are easily penetrated by sharps. Metal, heavy-gauge plastic or laminated multi-ply cardboard containers are a much better choice.

Engineering controls isolate or remove the bloodborne pathogen hazard from the workplace. Work Practice Controls are controls that reduce the likelihood of exposure by altering the manner in which a task is performed (e.g., prohibiting recapping of needles by a two-handed technique). These include procedures for processing and handling blood and blood products, waste disposal, and personal hygiene.

\section{Category I}

Specific procedures for preventing exposures in Category I staff are as follows:

- All materials intended for reuse are inspected and decontaminated on a regular basis and are cleaned and decontaminated immediately upon visible contamination.

- Sinks that can be used for hand washing are provided in the bathrooms in trailers 1 and 4 and in the hallways in trailers 1 and 2, in the laboratory, phlebotomy room, and staff lounge in trailer 3 , and in the dental exam room in trailer 4 . The laboratory and dental sinks include an electronic eye on/off mechanism. 
- All MEC exam staff are instructed to wash hands with soap and water after removing gloves or upon accidental skin contact with blood or body fluids, and between each SP exam.

- Employees are instructed to wash hands and any other skin with soap and water, or flush mucous membranes with water immediately or as soon as feasible following contact of such body areas with blood or other potentially infectious materials.

- Sharps should be handled with extreme care and discarded into the needle disposal containers provided in the MEC. Staff members are instructed that contaminated needles and other contaminated sharps shall not be bent, recapped, or removed. Shearing or breaking of contaminated needles is prohibited. They are required to use the shielding mechanism. Contaminated sharps means any contaminated object that can penetrate the skin including, but not limited to, needles, scalpels, broken glass, broken capillary tubes, and exposed ends of dental wires. Use of the safety shielding mechanism is mandatory.

- Discarded needles and other waste sharps must be placed in an "approved" sharps container. Care must also be taken when mounting a sharps container on the walls and cabinets to make sure the container is accessible to everyone. Immediately or as soon as possible after use, contaminated reusable sharps are placed in puncture resistant and leakproof containers until properly reprocessed. These containers are identified as containing potentially infectious materials and are not allowed to overfill.

- Employees are instructed not to eat, drink, smoke, handle contact lenses, or apply cosmetics or lip balm in the exam rooms.

- No food or drinks are kept in refrigerators, freezers, shelves, cabinets, or work surfaces in the examination rooms. An employee lounge with a refrigerator is provided for personal food storage.

- Procedures involving blood or other potentially infectious materials are performed in such a manner as to minimize splashing, spraying, spattering, or aerosolizing. For example, the splattering of blood or serum that occurs when rubber stoppers are pulled out of specimen tubes is minimized by covering the stopper with gauze before removing it and performing this procedure under the biosafety hood.

- Mouth pipetting/suctioning of blood or other potentially infectious materials is prohibited.

- Equipment should be checked and decontaminated before servicing and shipping. Decontamination means the use of physical or chemical means to remove, inactivate, or destroy bloodborne pathogens on a surface or item to the point where it is no longer capable of transmitting infectious particles and the surface or item is rendered safe for handling, use, or disposal. 
- Work surfaces and or equipment contaminated with blood or body fluids are washed with detergent and disinfected with a 1:10 or 1:100 dilution of bleach at the end of every examination session, as described in the examiner manuals.

- Protective coverings used to cover equipment and environmental surfaces are removed and replaced as soon as feasible when overtly contaminated with blood or body fluids during the exam session.

- Broken glassware is never picked up by hand, but swept up with a brush and dustpan. To minimize aerosol formation, broken glass is not dropped into a disposal container but gently placed in the container.

\section{Category II}

Although the MEC is not set up to treat or manage medical problems, medical emergencies may occur. If the staff must administer CPR, it is recommended that all staff observe the following procedures to prevent exposures:

- Wear gloves and a lab coat when performing emergency procedures. Disposable impervious lab jackets are available in the lab if needed.

- Use the disposable resuscitation masks found in designated areas.

- Follow the instructions of the MEC physician, who will direct the emergency response.

- Decontaminate and/or dispose of all emergency medical supplies.

- Change any protective equipment contaminated with blood or body fluids.

- Wash hands after removing gloves and before working with the next SP.

\section{B.3.4 Personal Protective Equipment}

Personal Protective Equipment is used when occupational exposure remains after instituting engineering and work practice controls. Personal protective equipment is specialized clothing or equipment worn by an employee for protection against a hazard. General work clothes (e.g., uniforms, pants, shirts, or blouses) not intended to function as protection against a hazard are not considered personal protective equipment. 
The selection of protective equipment depends on the nature of the potential exposure but generally includes gloves, lab coats, and gowns. If there is a chance for potentially infectious material to splash into the eyes or mouth, staff members are instructed to wear face protection in the form of a mask and/or safety glasses with side shields. The employer provides all protective equipment in appropriate sizes and sufficient quantities for staff use. Hypoallergenic gloves or other alternatives are available to employees with allergic sensitivity to gloves.

Staff members other than those in Categories I or II are not expected to perform work procedures that carry risk for exposure to blood or body fluids but the following precautions are communicated to the staff in training and monitored:

- Staff should not perform any procedure or task for which they have not been trained.

- Gloves, disposable impervious lab jackets, and/or safety glasses are worn when performing venipuncture, and laboratory procedures. Personal protective equipment is provided to the staff. Disposable impervious lab jackets are provided to the medical technologists, phlebotomists, and health technologists performing the oral health examination. Reusable cloth lab coats are provided to the physician and MEC manager and are laundered by the employer if contaminated with blood or body fluids. Safety glasses are optional for the phlebotomist and are available onsite. Disposable, powder-free hypoallergenic nonlatex powder-free gloves are provided to the medical technologists, phlebotomist, and dental examiners. Wear appropriate gloves if contact with blood, or other potentially infective materials is anticipated. Never wash or decontaminate disposable gloves for reuse.

- Gloves are changed after each sample person exam or when visibly soiled with blood and/or body fluids. Disposable impervious lab jackets worn by the laboratory and phlebotomy staff are discarded when soiled and at the end of each workweek. Disposable impervious lab jackets worn by the health technologists during the oral health examination are changed when visibly soiled with blood and body fluids. All disposable impervious lab jackets are removed before leaving the work area and after a garment becomes contaminated. Place used protective equipment in appropriately designated areas or containers when being stored or discarded.

\section{B.3.5 Housekeeping}

Under the OSHA standard, all areas must be kept clean and sanitary. A cleaning schedule that includes appropriate methods of decontamination procedures has been developed for every component and is included in the component-specific procedure manuals. These procedures are included in component training programs and are part of daily component procedures. Cleaning procedures are 
based on the type of surfaces, the type of contamination present, the tasks or procedures, and their location in the facility. Housekeeping staff also receive a written set of instructions for cleaning designated areas of the MEC.

The following housekeeping procedures must be observed and will be monitored by project staff:

- Clean and decontaminate all equipment and environmental and work surfaces contaminated with blood or other potentially infectious materials.

- Decontaminate work surfaces with an appropriate disinfectant after completion of procedures, immediately when overtly contaminated, after any spill of blood or other potentially infectious material, and at the end of the work shift when surfaces have become contaminated since the last cleaning.

- Remove and replace protective coverings such as plastic wrap.

- Inspect and decontaminate, on a regular basis, reusable receptacles such as bins, pails, and cans that have the likelihood for becoming contaminated. When contamination is visible, clean and decontaminate receptacles immediately, or as soon as possible.

- Place other regulated waste in closable and labeled or color-coded containers. When storing, handling, transporting, or shipping, place other regulated waste in containers that prevent leakage.

- When discarding contaminated sharps, place them in containers that are closable, puncture-resistant, appropriately labeled or color-coded, and leakproof on the sides and bottom.

- Ensure that sharps containers are easily accessible to personnel and located as close as feasible to the use area. Keep sharps containers upright throughout use, replace routinely, close when moved, and do not allow to overfill.

- Never manually open, empty, or clean contaminated sharps disposable containers.

- Discard regulated waste into appropriate containers, labeled as biohazardous waste, and seal to prevent leakage during handling, storage, and transport. Regulated Waste means liquid or semiliquid blood or other potentially infectious materials; contaminated items that would release blood or other potentially infectious materials in a liquid or semiliquid state if compressed; items that are caked with dried blood or other potentially infectious materials and are capable of releasing these materials during handling; contaminated sharps; and pathological and microbiological wastes containing blood or other potentially infectious materials. A hazardous waste company is responsible for the disposal of biohazardous waste from the MEC. 
Specific methods and regular schedules for cleaning bench tops, equipment, and other environmental surfaces contaminated with potentially infectious materials are documented in the Infection Control Manual and in the respective component procedure manuals. The time required for cleanup may be reduced if surfaces are covered before work with an impermeable, disposable covering such as plastic sheeting or absorbent bench pads.

Good housekeeping procedures are not limited to areas in which infectious materials are handled. Prudent safety practice dictates that halls and passageways are kept clear of obstructions such as carts, obsolete equipment, and shipping containers. Fire doors, other than those interconnected to smokedetection systems, are kept closed. Stairs are not obstructed and exits are clearly marked.

Contaminated laundry is handled as little as possible and placed in labeled bags at locations where it is used. Leakproof bags are used to transport wet laundry. In addition, all employees who work with contaminated laundry wear gloves.

\section{B.4 Hepatitis B Vaccine}

The hepatitis B vaccine is made available to Category I and II MEC staff at no cost and at a reasonable time and place within 10 working days of initial assignment, and to any MEC staff following an exposure incident. Employees who refuse the hepatitis vaccine must sign the Declination Form, which is kept with the employee's medical records. Employees who have previously received the vaccine should provide evidence of antibody testing or completion of the vaccine series.

If an exposure incident occurs, the MEC manager and MEC physician must be notified immediately. The MEC physician must complete a Medical Incident Form, and the employee must receive an immediate medical evaluation to assess his or her level of vaccine protection and level of exposure. The MEC physician must also recommend an appropriate treatment regimen per the Updated U.S. Public Health Service Guidelines for the Management of Occupational Exposure to HBV, HCV, and HIV and Recommendations for Postexposure Prophylaxis (MMWR, June 29, 2001). 


\section{B.4.1 Procedures for Evaluating Exposure Incidents}

The OSHA standard requires that following a report of an exposure incident, the employer shall make immediately available to the exposed employee a confidential medical evaluation and followup. Exposure Incident means a specific eye, mouth, other mucous membrane, nonintact skin, or parenteral contact with blood or other potentially infectious materials that results from the performance of an employee's duties. The exposure report must include at least the following elements:

1. Date and time of exposure;

2. Documentation of the route(s) of exposure, and the circumstances under which the exposure incident occurred;

3. Identification and documentation of the source individual, unless the employer can establish that identification is infeasible or prohibited by state or local law;

a. The source individual's blood shall be tested as soon as feasible and after consent is obtained in order to determine HBV, HCV, and HIV infectivity. If consent is not obtained, the employer shall establish that legally required consent cannot be obtained. When the source individual's consent is not required by law, the source individual's blood, if available, shall be tested and the results documented.

b. Results of the source individual's testing shall be made available to the exposed employee's physician, and the employee shall be informed of applicable laws and regulations concerning disclosure of the identity and infectious status of the source individual.

4. Collection and testing of blood for HBV, HCV, and HIV serological status;

a. The exposed employee's blood shall be collected as soon as feasible and tested after consent is obtained.

b. If the employee consents to baseline blood collection, but does not give consent at that time for HIV serologic testing, the sample shall be preserved for at least 90 days. If, within 90 days of the exposure incident, the employee elects to have the baseline sample tested, such testing shall be done as soon as feasible.

5. Postexposure prophylaxis, when medically indicated, as recommended by the U.S. Public Health Service;

6. Counseling; and

7. Evaluation of reported illnesses. 
It is important that the employee have access to treatment for possible $\mathrm{HBV}, \mathrm{HCV}$, or HIV exposure as soon as possible after the incident because HBIG (Hepatitis B immunoglobulin), hepatitis B vaccine, and HIV PEP (postexposure prophylaxis) are most likely to be effective if administered as soon after the exposure as possible. During training, the training supervisor, the Director of MEC Operations, Catherine Novak, will oversee the response to a needlestick or other exposure. During the examination period, the MEC physician is responsible for initiating the response, referring the employee to the nearest emergency department, notifying home office staff, and documenting the incident.

At a minimum, the evaluation and followup must include the following elements:

- Treatment of Exposure Site

- Wounds and skin sites that have been in contact with blood or body fluids should be washed with soap and water; mucous membranes should be flushed with water. No evidence exists that using antiseptics for wound care or expressing fluid by squeezing the wound further reduces the risk of bloodborne pathogen transmission; however, the use of antiseptics is not contraindicated. The application of caustic agents (e.g., bleach) or the injection of antiseptics or disinfectants into the wound is not recommended.

- Exposure Report

- Document the routes of exposure and how the exposure occurred on a Medical Incident Form and OSHA's Forms 300 and 301 (See Section 5.6 in Chapter 5 of the Infection Control Procedures Manual). The Medical Incident Form can be accessed from any exam room by clicking on the ambulance icon on the toolbar.

- Evaluation of the Employee and the Exposure

- The employee should proceed immediately to the closest emergency department where he or she can be evaluated and counseled. Hospitals are available during all working hours, including nights and weekends. HBIG, hepatitis B vaccine, and antiretroviral agents for HIV PEP should be available for timely administration (i.e., either by providing access on site or by creating linkages with other facilities or providers to make them available off site). The evaluating emergency physician is responsible for providing postexposure management and counseling. He or she is familiar with evaluation and treatment protocols and whether the hospital has access to HBIG, hepatitis B vaccine, and antiretroviral drugs for HIV PEP.

- $\quad$ The exposure will be evaluated by the hospital physician for the potential to transmit HBV, HCV, and HIV based on the type of body substance involved and the route and severity of the exposure. Blood, fluid containing visible 
blood or other potentially infectious fluid, can be infectious for bloodborne viruses. Exposures to these fluids through a percutaneous injury (i.e., needlestick or other penetrating sharps-related event) or through contact with a mucous membrane are situations that pose a risk for bloodborne virus transmission and require further evaluation. For HCV and HIV, exposure to a blood-filled hollow needle or visibly bloody device suggests a higher risk exposure than exposure to a needle that was most likely used for giving an injection.

The physician evaluating the employee must be provided with a copy of the OSHA bloodborne pathogen regulations, a description of the employee's duties relevant to the exposure incident, and documentation of the route and circumstances of exposure. Within 15 days after the evaluation of the exposed employee, the employer must obtain a copy of the report from the health care professional responsible for the postexposure evaluation and ensure that the employee receives a copy of the written opinion. The evaluating physician must provide a written opinion regarding vaccination status and postexposure evaluation and followup. The written opinion to the employer is limited to whether the vaccine is necessary, documentation of administration, and whether the employee was informed of the results of the evaluation, including any medical conditions resulting from exposure which require further evaluation or treatment. However, all diagnoses not related to the exposure incident are protected by patient/physician confidentiality and should not be included in the written report to the employer.

For skin exposure, followup is indicated only if it involves exposure to a body fluid previously listed and evidence exists of compromised skin integrity (e.g., dermatitis, abrasion, or open wound).

- Evaluation of the Exposure Source

- The person whose blood or body fluid is the source of an occupational exposure should be evaluated for HBV, HCV, and HIV infection.

- The HBV, HCV, and/or HIV infection status of the source is unknown. The source person should be informed of the incident and asked to undergo testing at the emergency department or to consent to have their blood tested by an independent reference laboratory. It will be explained to the SP that a blood test is needed to determine treatment options for the exposed person. Offer to use serum that has already been collected for testing so that a second venipuncture will not be necessary. The SP may also choose to go to his or her physician to obtain the test at NHANES project expense. The SP will be asked to consent to release the results of the tests to the exposed staff person by sending the results to the staff person's physician. The SP should sign the consent form (See Exhibit 5-3 in Chapter 5 of the Infection Control Procedures Manual.) and a copy of the consent should be provided to the SP. If the SP refuses to be tested, the MEC physician or other supervisor named above should document the refusal in writing. 
- $\quad$ Procedures should be followed for testing source persons, including obtaining informed consent, in accordance with applicable state and local laws. Any persons determined to be infected with $\mathrm{HBV}, \mathrm{HCV}$, or HIV should be referred for appropriate counseling and treatment. Confidentiality of the source person should be maintained at all times.

- $\quad$ Testing to determine the HBV, HCV, and HIV infection status of an exposure source should be performed as soon as possible. An FDA-approved rapid HIVantibody test kit should be considered for use in this situation, particularly if testing by EIA cannot be completed within $24-48$ hours. Repeatedly reactive results by EIA or rapid HIV-antibody tests are considered highly suggestive of infection, whereas a negative result is an excellent indicator of the absence of HIV antibody. Confirmation of a reactive result by Western blot or immunofluorescent antibody is not necessary to make initial decisions about postexposure management but should be done to complete the testing process and before informing the source person. Repeatedly reactive results by EIA for anti-HCV should be confirmed by a supplemental test (i.e., recombinant immunoblot assay [RIBA ${ }^{\mathrm{TM}}$ ] or HCV PCR). Direct virus assays (e.g., HIV p24 antigen EIA or tests for HIV RNA or HCV RNA) for routine HIV or HCV screening of source persons are not recommended.

- If the exposure source cannot be tested or refuses to provide consent, information about where and under what circumstances the exposure occurred should be assessed epidemiologically for the likelihood of transmission of $\mathrm{HBV}, \mathrm{HCV}$, or HIV. Certain situations as well as the type of exposure might suggest an increased or decreased risk; an important consideration is the prevalence of $\mathrm{HBV}, \mathrm{HCV}$, or HIV in the population group (i.e., institution or community) from which the contaminated source material is derived.

- Testing of needles or other sharp instruments implicated in an exposure, regardless of whether the source is known or unknown, is not recommended. The reliability and interpretation of findings in such circumstances are unknown, and testing might be hazardous to persons handling the sharp instrument.

- The exposed employee's physician is provided with the source individual's test result and information about the applicable disclosure laws and regulations concerning the source identity and infectious status. (This will be accomplished through the evaluating physician and not through the employer.)

A copy of all documentation related to the incident, including the Medical Incident Form and OSHA's Forms 300 and 301, should be sent to the Director of Mobile Examination Center Operations, Catherine Novak, as soon as possible. 
A more detailed description of these recommendations is in the Updated U.S. Public Health Service Guidelines for the Management of Occupational Exposure to $H B V, H C V$, and $H I V$ and Recommendations for Postexposure Prophylaxis (MMWR, June 29, 2001). Copies of these guidelines are available in Appendix $\mathrm{C}$ of the Infection Control Manual.

\section{B.5 Labeling}

The OSHA standard requires that fluorescent orange and orange-red warning labels be attached to containers of regulated waste, to refrigerators and freezers containing blood and other potentially infectious materials, and to other containers used to store, transport, or ship blood or other potentially infectious materials. These labels are not necessary when (1) using red bags or red containers; (2) labels designate the contents of containers of blood, blood components, or blood products; and (3) individual containers of blood or other potentially infectious materials are placed in a labeled container during storage, transport, or disposal. The warning label must be fluorescent orange or orange-red, contain the biohazard symbol and the word BIOHAZARD, in a contrasting color, and be attached to each object by string, wire, adhesive, or other method to prevent loss or unintentional removal of the label.

In the MEC, labels are affixed to containers of infectious waste, centrifuges, refrigerators and freezers containing blood or body fluids, and containers used to store, transport, or ship blood or body fluids.

\section{B.6 Communication of Hazards to Employees - Training}

All MEC employees, including those designated as Category I and II, will receive information and training during their working hours regarding the OSHA regulation and their exposure category at the time of initial assignment and at no cost to the employee. The training program will be provided at least once a year thereafter. Additional training will be provided as necessary when existing tasks are modified or tasks are added that affect the employee's exposure risk. Persons conducting the training will be knowledgeable about the subject matter, and the information will be appropriate in context and vocabulary to the education level, literacy, and language of the audience. All training programs for MEC staff will include instruction in the OSHA regulations, general infection control 
practices, and specific infection control procedures relevant to each examination component. The training must include the following elements:

- How to obtain a copy of the regulatory text and an explanation of its contents;

- Information on the epidemiology and symptoms of bloodborne diseases;

- Ways in which bloodborne pathogens are transmitted;

- Explanation of the exposure control plan and how to obtain a copy;

- Information on how to recognize tasks that might result in occupational exposure (categories of exposure);

- Explanation of the use and limitations of work practice and engineering controls, and personal protective equipment;

- Information on the types, selection, proper use, location, removal, handling, decontamination, and disposal of personal protective equipment;

- Information on hepatitis B vaccination such as safety, benefits, efficacy, methods of administration, and availability;

- Information on whom to contact and what to do in an emergency;

- Information on how to report an exposure incident and on postexposure evaluation and followup;

- Information on warning labels, and signs, where applicable, and color coding;

- Information on other infectious disease concerns in the MEC; and

- Question and answer session on any aspect of the training.

\section{B.7 Recordkeeping}

The exposure control plan is reviewed and updated at least annually or whenever new tasks and procedures affect occupational exposure. A copy of the Exposure Plan is kept in every copy of the Infection Control Manual. Copies of this manual are kept in the staff lounge, in the physician exam room, and in each field office. The plan must be made available to the Assistant Secretary for OSHA and to the Director of the National Institute for Occupational Safety and Health for examination and copying if required. 
Employers also must preserve and maintain for each employee an accurate record of occupational exposure. Under the OSHA bloodborne pathogens standard, medical records must include the following information:

- Employee's name and Social Security number;

- Employee's hepatitis B vaccination status including vaccination dates and any medical records relating to the employee's ability to receive vaccinations;

- Results of examinations, medical testing, and postexposure evaluation and followup procedures;

- Health care professional's written opinion; and

- A copy of the information provided to the health care professional.

Medical records must be kept confidential and maintained for at least the duration of employment plus 30 years.

Training records must also be maintained and kept accurate by the employer for 3 years and must include the following:

- Training dates;

- Content or a summary of the training;

- Names and qualifications of trainer(s); and

- Names and job titles of trainees.

Upon request, both medical and training records must be available to the Director of the National Institute for Occupational Safety and Health and to the Assistant Secretary of Labor for Occupational Safety and Health. Training records must be available to employees or employee representatives upon request. The employee or anyone having the employee's written consent can obtain an employee's medical records. In addition, if the employer ceases to do business, medical and training records must be transferred to the successor employer. If there is no successor employer, the employer must notify the Director, NIOSH, U.S. Department of Health and Human Services, for specific directions regarding disposition of the records at least 3 months before intended disposal. 


\section{B.8 Schedule and Methods for Implementing Sections of the Standard Covering the Methods of Compliance}

Procedures for compliance with OSHA bloodborne pathogen recommendations are included in initial NHANES training programs and incorporated into all specific component procedures and operations procedures in the MEC. All procedures should be operational at the time of training and during the main study.

\section{B.9 Sharps Injury Log}

29 CFR Part 1904 - Recording and Reporting Occupational Injuries and Illnesses requires establishment and maintenance of a sharps injury log. All work-related needlestick injuries and cuts from sharp objects that are contaminated with another person's blood or other potentially infectious material must be entered on the OSHA's Forms 300 and 301 as an injury. The OSHA's Forms 300 and 301 serve as the sharps injury log. Do not enter the employee's name to maintain confidentiality. The sharps injury log must contain, at a minimum:

1. The type and brand of device involved in the incident;

2. The department or work area where the exposure incident occurred; and

3. An explanation of how the incident occurred.

The sharps injury log is maintained for the period required by 29 CFR 1904.03; the OSHA's Forms 300 and 301 are maintained for five (5) years following the end of the calendar year that these records cover.

\section{B.10 Needlestick and other Sharps Injuries}

In January 2001, the Occupational Safety and Health Administration revised the Bloodborne Pathogens (BBP) standard in conformance with the requirements of the Needlestick Safety and Prevention Act. The revisions to OSHA's BBP standard required under the Needlestick Safety and Prevention Act can be broadly categorized into four areas: (1) modification of definitions relating to 
engineering controls; (2) revision and updating of the Exposure Control Plan; (3) solicitation of employee input; and (4) recordkeeping.

The standard has been revised to add new requirements to the annual review and update of the Exposure Control Plan. Consideration and implementation of safer medical devices must be documented in the Exposure Control Plan by describing the safer devices identified as candidates for adoption, the method or methods used to evaluate devices and the results of evaluations, and justification for selection decisions. The revised standard requires that solicitation of input from employees be documented in the Exposure Control Plan. This information must be updated at least annually. The recordkeeping requirements of the standard have been amended by adding a paragraph to require that employers maintain a sharps injury log to serve as a tool for identifying high risk areas and evaluating devices.

In November 1999, NIOSH published ALERT, Preventing Needlestick Injuries in Health Care Settings. A comprehensive plan and program to prevent transmission of bloodborne pathogens and to meet (and exceed, whenever possible) regulations was implemented to identify and evaluate various new safer needle technologies and devices. The intent was to replace the existing nonshielded blood collection needles.

Annual formal evaluations have been conducted since 2000. A notebook detailing each evaluation is kept at the Westat home office and is available for review and inspection. 


\section{ATTACHMENT A \\ Safety Blood Collection Set \\ Evaluation Form - Stands 245 and 246 \\ Page 1}

\section{Your Name \\ Today's Date \\ Product Name \\ Product Needle Size}

\section{Key Performance Issues}

1. The product is satisfactory for standard phlebotomy purposes

2. The product is equally satisfactory for different or diverse patient populations (adults, children, heavy, thin, etc.)

Comment:

3. What percentage of typical clinical procedures does this product address?

4. The product is satisfactory for SPs with fragile veins

5. The product is simple to operate

6. The use of this product requires no training

7. The safety feature activated with a one-handed technique

8. The safety feature worked reliably

9. Both hands remain behind the needle during engagement of safety feature

10. The safety feature did not interfere with the blood draw

11. This product allowed me to see what I needed to see during the blood draw

12. The safety feature could not be bypassed

13. The use of this product did not create any extra risk of sprays, blood leakage, and/or drips

14. The product could be disposed of into the sharps container that is available for my use

\section{Feedback}

15. About how many times did you use the blood collection set before you were comfortable using it? (check one)
1 time
5 times
10 times
15 times
20 times
I never felt comfortable

16. Did you have any problems with this device?

Yes

No If yes, please briefly explain below. 
17. Which device would you rather use: (Please check one)

$\square 1$. The product we normally use

$\square$ 2. This test one $\square$ 3. Other, please indicate

Comments: 


\section{ATTACHMENT B \\ Sharps Disposal \\ Evaluation Form}

Date:

Position:

Manufacturer:

Name:

Stand:

Capacity:

Indicate the degree to which you agree or disagree with each statement by circling the most appropriate number for each statement.

Not applicable (N/A) may be used if the question does not apply to this product.

\section{Please Rate:}

agree disagree

1. The container is puncture resistant

2. The container does not leak

3. The container is stable

4. The container is accessible from any direction

5. The container is located where the needles are used

6. The container allows single handed use

7. It is possible to drop sharps or tubes into the container vertically

8. No force is required to put sharps or tubes into the container

9. Needles/tubing do not get caught on the opening or interior shape

10. The container opens and closes easily

11. The container has a solid tight-fitting cover

12. The box accommodates a range of sharp sizes or tubes

13. The size of the container is appropriate for its use

14. No one (including a child) can access the contents

15. There is a permanent lock for final disposal that is not reversible

16. The user can determine the fill level visually

17. The container is appropriately labeled

18. The box is not threatening to participants

19. Use of this container does not compromise infection control practices $\begin{array}{llllll}1 & 2 & 3 & 4 & 5 & \mathrm{~N} / \mathrm{A}\end{array}$

$\begin{array}{llllll}1 & 2 & 3 & 4 & 5 & \mathrm{~N} / \mathrm{A}\end{array}$

$\begin{array}{lllllll}1 & 2 & 3 & 4 & 5 & \mathrm{~N} / \mathrm{A}\end{array}$

$\begin{array}{llllll}1 & 2 & 3 & 4 & 5 & \mathrm{~N} / \mathrm{A}\end{array}$

$\begin{array}{llllll}1 & 2 & 3 & 4 & 5 & \mathrm{~N} / \mathrm{A}\end{array}$

$\begin{array}{llllll}1 & 2 & 3 & 4 & 5 & \mathrm{~N} / \mathrm{A}\end{array}$

$\begin{array}{lllll}1 & 2 & 3 & 4 & 5\end{array} \mathrm{~N} / \mathrm{A}$

$\begin{array}{lllll}1 & 2 & 3 & 4 & 5\end{array} \mathrm{~N} / \mathrm{A}$

$\begin{array}{lllll}1 & 2 & 3 & 4 & 5\end{array} \mathrm{~N} / \mathrm{A}$

$\begin{array}{lllll}1 & 2 & 3 & 4 & 5\end{array} \mathrm{~N} / \mathrm{A}$

$\begin{array}{lllll}1 & 2 & 3 & 4 & 5\end{array} \mathrm{~N} / \mathrm{A}$

$\begin{array}{lllll}1 & 2 & 3 & 4 & 5\end{array} \mathrm{~N} / \mathrm{A}$

$\begin{array}{lllll}1 & 2 & 3 & 4 & 5\end{array} \mathrm{~N} / \mathrm{A}$

$\begin{array}{lllll}1 & 2 & 3 & 4 & 5\end{array} \mathrm{~N} / \mathrm{A}$

$\begin{array}{lllll}1 & 2 & 3 & 4 & 5\end{array} \mathrm{~N} / \mathrm{A}$

$\begin{array}{lllll}1 & 2 & 3 & 4 & 5\end{array} \mathrm{~N} / \mathrm{A}$

$\begin{array}{lllll}1 & 2 & 3 & 4 & 5\end{array} \mathrm{~N} / \mathrm{A}$

$\begin{array}{llllll}1 & 2 & 3 & 4 & 5 & \mathrm{~N} / \mathrm{A}\end{array}$

$\begin{array}{lllll}1 & 2 & 3 & 4 & 5\end{array}$

Did this sharps container interfere with your ability to complete the venipuncture or blood processing protocol?

Yes or No

Are there other questions that you feel should be asked regarding the safety/utility of this product? 


\section{B.11 Laboratory and Phlebotomy Infection Control}

This section contains a summary of all procedures used for infection control in the MEC laboratory and phlebotomy components. For detailed information regarding these procedures, refer to the component's manual of operation.

\section{B.11.1 Glossary}

Decontamination: The use of physical or chemical means to remove, inactivate, or destroy bloodborne pathogens on a surface or item to the point where they are no longer capable of transmitting infectious particles and the surface or item is rendered safe for handling, use, or disposal.

Detergent: $\quad$ A cleansing agent used to facilitate the removal of gross debris from an object.

Disinfect: Inactivation of virtually all recognized pathogenic microorganisms but not necessarily all microbial forms (e.g., bacterial endospores) on inanimate objects.

Engineering Controls (e.g., sharps disposal containers, self-sheathing needles, safer Controls: medical devices, such as sharps with engineered sharps injury protections and needleless systems) that isolate or remove the bloodborne pathogens hazard from the workplace.

Mucous membrane Exposure that occurs from droplet contamination (splashes, spatters) exposure: of the eyes, nose, or mouth.

Parenteral Exposure that occurs from piercing mucous membranes or the skin exposure: barrier through such events as needlesticks, human bites, cuts, and abrasions.

Personal Protective Specialized clothing or equipment worn by an employee for protection Equipment: against a hazard. General work clothes (e.g., uniforms, pants, shirts or blouses) not intended to function as protection against a hazard are not considered personal protective equipment. 
Regulated Waste: $\quad$ Liquid or semiliquid blood or other potentially infectious materials; contaminated items that would release blood or other potentially infectious materials in a liquid or semiliquid state if compressed; items that are caked with dried blood or other potentially infectious materials and are capable of releasing these materials during handling; contaminated sharps; and pathological and microbiological wastes containing blood or other potentially infectious materials.

Sharps with Non-needle sharps or a needle device used for withdrawing body engineered sharps fluids, accessing a vein or artery, or administering medications or injury protections: other fluids, with a built-in safety feature or mechanism that effectively reduces the risk of an exposure incident.

Sterilize: $\quad$ The use of a physical or chemical procedure to destroy all microbial life including highly resistant bacterial endospores.

Universal An approach to infection control. According to the concept of precautions: $\quad$ Universal Precautions, all human blood and certain human body fluids are treated as if known to be infectious for HIV, HBV, and other bloodborne pathogens.

Work Practice Controls that reduce the likelihood of exposure by altering the manner Controls: $\quad$ in which a task is performed (e.g., prohibiting recapping of needles by a two-handed technique).

In order to comply with Federal regulations, minimize the spread of infectious diseases, and provide a safe working environment, follow defined safety procedures at all times.

\section{B.11.2 Laboratory Access}

- Unauthorized people should not enter the MEC laboratory.

- All authorized persons must wear gloves when working with equipment, and when handling biological specimens such as blood, blood products, or urine in the laboratory. Examples are lab staff, biomedical engineers, Coulter technical representatives, NCHS or observers, coordinator, assistant coordinator, health techs, and MEC manager. People who visit the lab to observe should not touch countertops, equipment, or supplies if they are not wearing gloves. 


\section{B.11.3 Laboratory/Phlebotomy - Universal Precautions}

A complete description of Universal Precautions is described in Section B.3.2. Laboratory staff, including the phlebotomist, observe Universal Precautions at all times.

\section{B.11.4 Laboratory/Phlebotomy - Specific Engineering and Work Practice Controls}

Engineering and work practice controls are described in Section B.3.3. Engineering controls eliminate hazards at their source. The biological safety cabinet and waste containers for sharps are examples of engineering controls used in the NHANES. A laminar flow biological safety cabinet is located in the laboratory in every MEC.

All health care workers take precautions to prevent injuries caused by needles and other sharp instruments or devices during procedures, when cleaning used instruments, during disposal of used needles, and when handling sharp instruments after procedures. It is mandatory and essential to use the shielded mechanism of the needle.

Specific measures of protection for the laboratory staff (including the phlebotomist) are described below.

Hang outside street clothing in a designated separate clean area.

\section{Work Practice Controls}

- Do not eat, drink, smoke, handle contact lenses, or apply cosmetics or lip balm in the MEC laboratory or in the examination rooms.

- Do not keep food or drinks in refrigerators, freezers, shelves, cabinets, or work surfaces in the examination rooms. An employee lounge with a refrigerator is provided for personal food storage.

- Do not mouth pipette.

- Procedures involving blood or other potentially infectious materials are performed in such a manner as to minimize splashing, spraying, or aerosolizing. For example, the splattering of blood or serum that occurs when rubber stoppers are pulled out of specimen tubes is minimized by covering the stopper with gauze before removing it and performing this procedure under the biosafety hood. 
- Perform all blood processing tasks in the LSBSC.

- Use Aerosolve containers when centrifuging blood tubes.

- Used needles must never be sheared, bent, clipped, broken, or recapped by hand. Discarded needles and other waste sharps must be placed in an "approved" sharps container. Use either a one-handed "scoop" technique or a mechanical device designed for holding the needle sheath.

- Use of the safety shielding mechanism on the blood collection needle is mandatory.

- Never point (direct) or move the unsheathed tip of a needle toward anyone.

- Always be alert and extremely cautious when handling unsheathed needles.

- Immediately after use, dispose of sharps in sharps containers.

- When discarding contaminated sharps, place them in containers that are closable, puncture-resistant, appropriately labeled or color-coded, and leakproof on the sides and bottom.

- Ensure that sharps containers are easily accessible to personnel and located as close as feasible to the use area.

- Keep sharps containers upright throughout use, replace routinely, close when moved, and do not overfill. Do not overstuff the biohazard waste containers by poking the trash down with another object. Shaking the container back and forth is acceptable.

- Never manually open, empty, or clean contaminated sharps disposable containers.

- Never discard needles directly into a container used for ordinary ("noninfectious") waste.

\section{B.11.5 Laboratory/Phlebotomy - Personal Protective Equipment}

A complete description of personal protective equipment is contained in Section B.3.4.

- Wear impervious lab jackets when performing venipuncture and laboratory procedures. Disposable impervious lab jackets are provided to the medical technologists and phlebotomists.

- Remove protective equipment before leaving the work area and after a garment becomes contaminated. 
- Place used protective equipment in appropriately designated areas inside the laboratory and place in designated biohazardous containers when being discarded.

- Discard lab jackets when soiled and at the end of each workweek.

- If the LFBSC is nonfunctional, then wear safety glasses when processing blood. Safety glasses are optional for the phlebotomist but are available onsite.

- Wear appropriate face and eye protection such as a mask with glasses with solid side shields or a chin-length face shield when splashes, sprays, spatters, or droplets of blood or other potentially infectious materials pose a hazard to the eye, nose, or mouth.

- Wear gloves when performing venipuncture, processing blood specimens, processing urine, processing vaginal swabs, and performing hematology testing. Disposable, powder-free hypoallergenic nonlatex powder-free gloves are provided to the medical technologists and phlebotomist.

- Change gloves after each sample person's exam or when visibly soiled with blood and/or body fluids.

- Never wash or decontaminate disposable gloves for reuse.

- Discard utility gloves at the first sign of deterioration.

- Remove gloves before leaving the laboratory or phlebotomy room.

Personal Hygiene - Infection Control

- Wash hands with soap and water after removing gloves, or upon accidental skin contact with blood or body fluids, and before working with the next SP.

- Hand washing sinks are located in the bathrooms in trailers 1 and 4, in the hallways in trailers 1 and 2, in the laboratory, phlebotomy room, and staff lounge in trailer 3 , and in the dental exam room in trailer 4. The laboratory and dental sinks include an electronic eye on/off mechanism.

- If using liquid soap for hand washing, replace with fresh product when empty. Do not add liquids to a partially full dispenser.

\section{B.11.6 Laboratory/Phlebotomy - Waste Management}

A complete description of the waste management procedures is described in Section B.3.5. 
- Discard hazardous waste into appropriate containers. Biohazardous waste containers are labeled and sealed to prevent leakage during handling storage and transport. A hazardous waste company is responsible for the disposal of biohazardous waste from the MEC. Do not overstuff the biohazard waste containers by poking the trash down with another object. Shaking the container back and forth is acceptable.

- Dispose of all potentially infectious materials in a biohazard waste container.

- When discarding glass blood tubes, place them in sharps containers that are closable, puncture-resistant, appropriately labeled or color coded, and leakproof on the sides and bottom.

- Place solid waste from the laboratory in biohazard bags and transport to an area designated to hold these bags. A single bag is adequate if the bag is sturdy (not easily penetrated) and if the waste can be put in the bag without contaminating the outside of the bag; otherwise, double bagging is indicated.

- To minimize the potential risk for accidental transmission of disease or injury, store infectious waste waiting terminal processing in a pre-incineration storage area in the MEC accessible only to personnel involved in the disposal process.

- Place regulated waste in closable and labeled or color-coded containers. When storing, handling, transporting, or shipping, place other regulated waste in containers that prevent leakage.

- Transport closed sharps containers and biohazard bags destined for incineration or steam sterilization to the designated pre-incineration storage.

- Discard all regulated waste according to Federal, state, and local regulations.

- Refer to "infectious waste" in the glossary for any questions about identifying wastes for which special precautions appear prudent.

- Liquid Wastes

- $\quad$ Pour urine down the sink connected to the sewer before discarding the cup in biohazard container.

- Pour Coulter waste down the sink connected to a sewer. Add $13 / 4$ cups $(3.75-\mathrm{mL})$ undiluted bleach to the 10 -gallon waste container and reattach to the instrument. 


\section{B.11.7 Laboratory/Phlebotomy - Labeling}

In the MEC, labels are affixed to containers of infectious waste, centrifuges, refrigerators, and freezers containing blood or body fluids and containers used to store, transport, or ship blood or body fluids.

A complete description of the OSHA label requirements is provided in Section B.5.

\section{B.11.8 Laboratory/Phlebotomy - Housekeeping}

A complete description of OSHA's housekeeping requirements is provided in Section B.3.5.

All housekeeping procedures must be observed and will be monitored by project staff:

- Wash work surfaces and or equipment contaminated with blood or body fluids with detergent and disinfect with a 1:10 dilution of bleach.

- At the end of every examination session, disinfect work surfaces with a 1:10 dilution of bleach.

- Check and decontaminate equipment before servicing and shipping.

- Remove protective coverings used to cover equipment and environmental surface and replace as soon as feasible when overtly contaminated with blood or body fluids during the exam session.

- Inspect and decontaminate all materials intended for reuse on a regular basis and clean and decontaminate immediately upon visible contamination.

- Inspect and decontaminate, on a regular basis, reusable receptacles such as bins, pails, and cans that have the likelihood to become contaminated. When contamination is visible, clean and decontaminate receptacles immediately, or as soon as possible.

- Place other regulated waste in closable and labeled or color-coded containers. When storing, handling, transporting, or shipping, place other regulated waste in containers that prevent leakage.

- Clean and decontaminate all equipment and environmental and work surfaces contaminated with blood or other potentially infectious materials. 
- Decontaminate work surfaces with an appropriate disinfectant after completion of procedures, immediately when overtly contaminated, after any spill of blood or other potentially infectious material, and at the end of the work shift when surfaces have become contaminated since the last cleaning.

- Remove and replace protective coverings such as plastic wrap and aluminum foil when contaminated.

- Clean and disinfect equipment and working surfaces, including keyboard covers, with a 1:10 bleach solution (5-mL bleach to $50-\mathrm{mL}$ tap water) or 1:100 bleach solution (1-mL bleach to $100-\mathrm{mL}$ water) after completion of procedures, when surfaces are overtly contaminated, immediately after any spill of blood or other potentially infectious materials, and at the end of the work shift.

- Remove and replace nonabsorbent paper used to cover equipment and environmental surfaces at the end of the work shift or when they become overtly contaminated.

- Check equipment routinely and decontaminate as necessary before servicing or shipping.

- Decontaminate reusable items contaminated with blood or other potentially infectious materials before washing and/or reprocessing. Routinely check equipment and decontaminate it before servicing and shipping.

- Clean and decontaminate all equipment and work surfaces contaminated with blood or other potentially infectious materials.

- On a regular basis inspect and decontaminate reusable receptacles such as bins, pails, and cans that have a likelihood to become contaminated. When contamination is visible, clean and decontaminate receptacles immediately, or as soon as possible.

\section{B.11.9 Laboratory/Phlebotomy - Cleaning a Blood Spill}

If a blood spill occurs, wear gloves and, if needed, a gown and facial protection during the cleanup procedure.

If the spill contains broken glass, use dustpan and broom to collect glass pieces. Dispose of broken glass and contaminated paper towels in sharps containers that are closable, puncture-resistant, appropriately labeled or color coded, and leakproof on the sides and bottom. Disinfect the dustpan and broom with bleach solution. Always use mechanical means such as forceps or a brush and dust pan to pick up contaminated broken glass; never pick up broken glass with hands even if wearing gloves. To 
minimize aerosol formation, do not drop broken glass into a disposal container but gently place it in the container.

To clean a blood spill, perform the following procedure:

- Wear gloves;

- Cover spill with disposable paper towels;

- Soak paper towels with 10 percent bleach solution;

- Let solution soak for at least 30 seconds;

- Dispose of blood/bleach soaked paper towels in biohazard container;

- Allow area to dry and then remove gloves; and

- Wash hands.

\section{B.12 References}

The following references were used to prepare the Exposure Plan:

1. Occupational Exposure to Bloodborne Pathogens, U.S. Department of Labor, Occupational Safety and Health Administration. OSHA 3127, 1996 (Revised). Title 29 Code of Federal Regulations, Part 1910.1030. [56 FR 64004, Dec. 06, 1991, as amended at 57 FR 12717, April 13, 1992; 57 FR 29206, July 1, 1992; 61 FR 5507, Feb. 13, 1996; 66 FR 5325 Jan., 18, 2001]

2. Immunization of Health-Care Workers, USPHS, CDC,, MMWR 1997;46(No. RR-18).

3. Updated U.S. Public Health Service Guidelines for the Management of Occupational Exposure to $\mathrm{HBV}, \mathrm{HCV}$, and $\mathrm{HIV}$ and Recommendations for Postexposure Prophylaxis (MMWR, June 29, 2001)

4. Occupational Exposure to Bloodborne Pathogens, Needlestick and other Sharps injuries: Final Rule, U.S. Department of Labor, Occupational Safety and Health Administration. OSHA, 2000 (Revised). 
5. Title 29 Code of Federal Regulations, Part 1904.

6. CDC. Guideline for Hand Hygiene in Health-Care Settings Recommendations of the Healthcare Infection Control Practices Advisory Committee and the HICPAC/SHEA/APIC/IDSA Hand Hygiene Task Force. MMWR 2002:51(RR-16).

Copies of these materials are included in the Appendixes of the Infection Control Manual. 
Appendix C

Laboratory Protocol 
Table C-1. Phlebotomy protocol

Venipuncture Protocol (January 2013)

\begin{tabular}{|c|c|c|c|c|c|c|c|c|c|c|c|c|c|}
\hline Tube Type & $\begin{array}{c}\text { Primary } \\
1-2 \\
\end{array}$ & $\begin{array}{c}\text { Primary } \\
3-5 \\
\end{array}$ & $\begin{array}{c}\text { Primary } \\
6-11 \\
\end{array}$ & $\begin{array}{c}\text { Primary } \\
12-19 \\
\end{array}$ & $\begin{array}{c}\text { Primary } \\
20+ \\
\end{array}$ & $\begin{array}{l}\text { Dry Run } \\
\text { Odd 12+ } \\
\end{array}$ & $\begin{array}{c}\text { Dry Run } \\
\text { Even } \\
12+ \\
\end{array}$ & $\begin{array}{c}\text { VIP } \\
\text { Guest } \\
1-2 \\
\end{array}$ & $\begin{array}{c}\text { VIP } \\
\text { Guest } \\
3-5 \\
\end{array}$ & $\begin{array}{c}\text { VIP } \\
\text { Guest } \\
6-11 \\
\end{array}$ & $\begin{array}{c}\text { VIP } \\
\text { Guest } \\
12+ \\
\end{array}$ & $\begin{array}{c}\text { Guests } \\
1-11 \\
\end{array}$ & $\begin{array}{c}\text { Guests } \\
12+ \\
\end{array}$ \\
\hline 3-mL Lavender & 1 & 2 & & & & & & 1 & 1 & 1 & 1 & 1 & 1 \\
\hline 4-mL Lavender & & & 2 & 2 & 2 & 2 & 2 & & & & & & \\
\hline 2-mL Gray & & & & 1 & 1 & 2 & 2 & & & & 1 & & \\
\hline 6 mL Royal Blue ${ }^{3}$ & & & 1 & 1 & 1 & 2 & & & & & & & \\
\hline 3-mL Red & 2 & 1 & & & & & & 1 & 1 & & & & 1 \\
\hline 7-mL Red & & 1 & 1 & 1 & & & & & & 1 & & & \\
\hline 15-mL Red & & & & 2 & 3 & 2 & 2 & & & & & & \\
\hline 10-mL Red & & & 1 & & & & & & & & 1 & & \\
\hline $15-\mathrm{mL} \mathrm{Red}^{\circ}$ & & & & 3 & 3 & & & & & & & & \\
\hline 7-mL Gray ${ }^{1}$ & & & & 1 & 1 & & & & & & & & \\
\hline 2-mL Gray ${ }^{2}$ & & & & 1 & 1 & 2 & 2 & & & & & & \\
\hline Milliliters & 9 & 16 & 31 & 107 & 115 & 58 & 46 & 6 & 6 & 10 & 15 & 3 & 6 \\
\hline Ounces & 0.3 & 0.5 & 1.0 & 3.6 & 3.9 & 2.0 & 1.6 & 0.2 & 0.2 & 0.3 & 0.5 & 0.1 & 0.2 \\
\hline Tablespoons & 0.6 & 1.0 & 2.0 & 7.2 & 7.8 & 4.0 & 3.2 & 0.4 & 0.4 & 0.7 & 1.0 & 0.2 & 0.4 \\
\hline
\end{tabular}

${ }^{\circ}$ SPs selected for inclusion in the Persistent Pesticides, BFR subsamples, and Perfluoronated samples

${ }^{1}$ SPs selected for inclusion in the VOC subsample

${ }^{2}$ Glucose tolerance test

${ }^{3}$ Selenium/Copper/Zinc 
Table C-2. 2013 Lab protocol

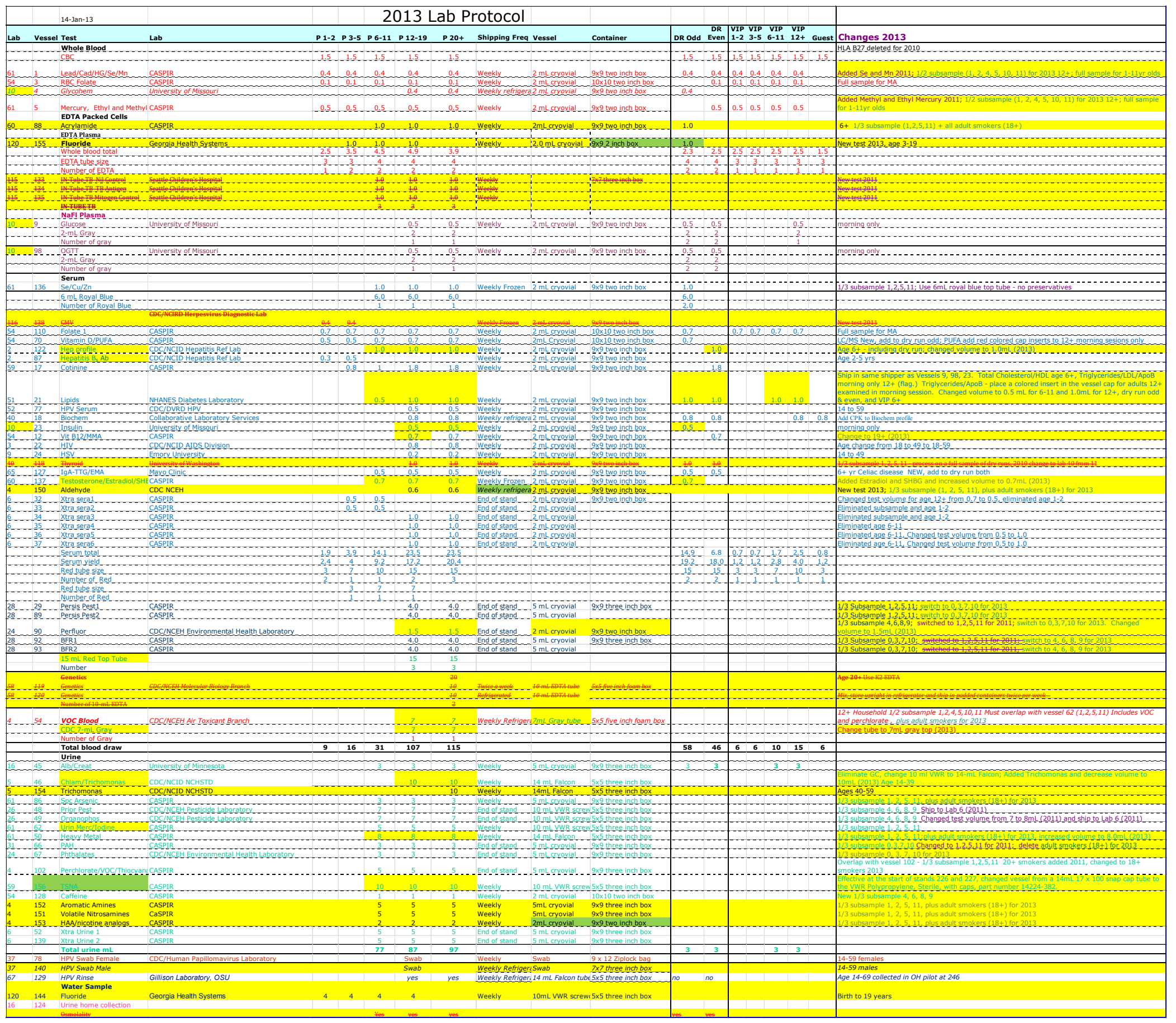


Appendix D

Laboratory and Phlebotomy Inventory 


\section{LABORATORY INVENTORY}

CONSUMABLE ITEMS

$\underline{\text { Part number }} \quad \underline{\text { Description }} \quad \underline{\text { Par level Unit distribution }}$

EXAM SUPPLIES

56616-032

ABS $3 \times 3$

$\mathrm{SO}-\mathrm{AA}-1 \%$

$\mathrm{SO}-\mathrm{AA}-7 \%$

58-104

RD 06173

24-54

$24-400-102$

$14-666-301$

$108 \mathrm{M}$

14-830-132

SO-Bleach

1441266

23-751610

19-037-189

761282

747112

747113

GOJ-9639-12CT

34155

05-403-11

05-403-09

$05-403-62$

721032

06-66997

1371136

86.1171

15-235-61
Absorbent Bench Pad - blue chuck

Absorbent Sheets (3" x 3")

Ascorbic Acid - 1\%

Ascorbic Acid - 7\%

Alcohol Wipes

Aluminum Foil - 50 Sq. Feet Roll

Applicator Sticks, cotton tipped

Applicator Sticks, wooden

Bench Kote Paper - 46" x 57" Paper for Lab Counter

Bio-Hazard Bags (25" x 35")

Bio-Hazard Container (8 Gallon)

Bleach - Regular - 5 - 5.25 Hydrochloride, Fragra

Bleach Right Disinfecting Spray - 32 Ounce

Spray Bottle

Deionized Water - 1 Gallon Plastic Bottle

Glove Liners - Full Finger

Gloves, Sterling Nitrile Gray(Lg.)

Gloves, Sterling Nitrile Gray (Med.)

Gloves, Sterling Nitrile Gray (Sm.)

Handsoap-Waterless (Purell)

Kimwipes (4 1/2 x $81 / 2)$

Pipette Tips $1000 \mathrm{uL}$ - 5 Racks of 96 tips each/per case

Pipette Tips 200uL

Pipette Tips 5mL (Bulk Pack) - 500 Pcs. per case

Saline Solution 09\% - 1L

Towlettes, Sani-Cloth (Cleaner)

Transfer Pipette (15mL ,8.0 Bulb Clear) - 250 per box

Transfer Pipette (3.5 clear, plastic)

Wipes, Terri (23" x 11")
Each

Pack

Each

Vial

Box

Roll

Bag

Box

Case

Box

Each

Gallon

Bottle

Gallon

Pack

Box

Box

Box

Bottle

Box

Box

Box

Case

Bottle

Bottle

Box

Box

Box 
OFFICE SUPPLIES

2251

107708

AVE 5165

AVE 5160

31155

T650A11A

HEWQ2610A

SAN 30001

AVE 08888

95.954

MMM 670-5AU

FED1104S

SPR-33-1LB

SWI 35108
3-Hole Copier Paper - 500 sheets per pack

Dymo Costar Label (1"x2 1/8") - 500 labels per roll

Label - Full Sheet 8.5" x 11" - Lab Label

Labels, Mailing Label 1" x 2 5/8"

Labels, Zebra (1" x 2.625", clear) - Black Bar Added

Laser Printer Toner - Lexmark - Black

Laser Printer Toner Cartridge-HP2300DN

Magic Markers - Sharpie - Fine Point

Magic Markers, Black

Magic Markers, Felt Tip Fine Line (Waterproof)

Post-It Page Markers - 100 pcs per pad/5 colors

Printer Ribbon, Zebra (Thermal Transfer)

Rubber Bands, \#33

Staples, Standard
Pack

Box

Box

Box

Roll

Each

Each

Each

Each

Each

Pack

Roll

Box

Box

\section{HEMATOLOGY SUPPLIES}

628027
628018
628023
628020
628017
628024
628026

SPECIALIZED STICKERS \& LABELS

3WRY4

3WRY5
DxH6C Cell Control - REFRIGERATED

DxH Cell Lyse - 1L Bottle

DxH Cleaner - 10 L Box

DxH Diff Pak

DxH Diluent - 10L Box

DxHLatron - REFRIGERATED

DxH S-Cal - REFRIGERATED
Pack

Bottle

Box

Box

Box

Pack

Pack

\section{PREGNANCY TEST SUPPLIES}

22247114

SA087712

SA087413
FedEx Class 9 Label - Dry Ice Label

Sticker, "Keep Frozen " - 500/roll

Sticker, "Refrigerate Do Not Freeze" - 500/roll
$1 \quad$ Roll
Roll

Roll
Pregnancy Test Kit (ICON 25) - Non-Refrigerated Sure-Vue hCG Serum Control Set - Refrigerated Sure-Vue hCG Urine Control Set - Refrigerated
Box

Pack

Pack 
JACKETS, GOWNS \& SLIPPERS

$\begin{array}{llll}\text { ML0795 } & \text { Lab Jackets, Disp. (Blue, 2XL) } & 15 & \text { Each } \\ 10078 & \text { Lab Jackets, Disp. (Blue, Lg.) - Kimberly Clark } & 10 & \text { Each } \\ 10067 & \text { Lab Jackets, Disp. (Blue, Med.) - Kimberly Clark } & 20 & \text { Each } \\ 10087 & \text { Lab Jackets, Disp. (Blue, XL) - - Kimberly Clark } & 10 & \text { Each }\end{array}$

\section{TUBES, VIALS, FILTERS}

\begin{tabular}{|c|c|c|c|}
\hline 62.559 .001 & $\begin{array}{l}\text { Conical Tube }(50 \mathrm{~mL} \text {, red top) - } 25 \text { Pieces Per Bag/300 } \\
\text { per case }\end{array}$ & 16 & Bag \\
\hline $15-350-49$ & Cryogenic Vial Color Coders - Red Only & 5 & Pack \\
\hline $\mathrm{SO}-10 \mathrm{~mL}$ & $\begin{array}{l}\text { CryoPro 10mL Vial - WITH ADDITIVE } \\
\text { (urine/mercury) }\end{array}$ & 150 & Each \\
\hline 62.551 .201 & CryoPro Cryogenic 10mL Vial - Screw On Cap & 7 & Case \\
\hline $14-959-11 B$ & Falcon Culture Test Tube (14 mL) & 25 & Bag \\
\hline $679800-5840$ & Nalgene Cryovial (2.0 mL, Non-Sterile) & 8 & Case \\
\hline 03-337-7D & Nalgene Cryovial (2.0 mL, Sterile) & 2 & Case \\
\hline $03-337-7 \mathrm{H}$ & Nalgene Cryovial (5.0 mL, Sterile) & 10 & Case \\
\hline $02-681-50$ & Serum Filter Tube (13mm x 4") & 4 & Pack \\
\hline $02-681-52$ & Serum Filter Tube (16mm x 6") & 20 & Pack \\
\hline
\end{tabular}

\section{SHIPPING SUPPLIES}

$\begin{array}{llcl}\text { 1A-55968 } & \text { Bag, Ziplock (9" x 12" Clear) - Bitran Replacement Bag } & 1 & \text { Case } \\ \text { CJ-3-D-25 } & \text { Box, Cardboard 3" with 5X5 grid, white } & 60 & \text { Each } \\ \text { RB-2-100 } & \text { Box, Cardboard 2" with 10 x 10 grid } & 30 & \text { Each } \\ \text { BX-2-D-81 } & \text { Box, Cardboard 2" with 9 x 9 grid } & 140 & \text { Each } \\ \text { CJ-3-D-49-2.25 } & \text { Box, Cardboard 3" with 7 x 7 grid } & 25 & \text { Each } \\ \text { CJ-3-D-81 } & \text { Box, Cardboard 3" with 9 x 9 grid, white } & 40 & \text { Each } \\ \text { 95.064.925 } & \text { Box, Cardboard 5.25" x 5.25" with foam insert } & 30 & \text { Each } \\ \text { STP 710 } & \text { Combination Bag and Envelope System - 50 Units } & & \\ & \text { per case } & 2 & \text { Case } \\ \text { STP 730 } & \text { Combination Bag and Envelope System - 50 Units } & & \\ & \text { per case } & 4 & \text { Case } \\ \text { STP 740 } & \text { Combination Bag and Envelope System - 50 Units } & & \\ & \text { per case } & 1 & \text { Case } \\ \text { SEL 10190 } & \text { Envelopes, Large Padded - 10.5" x 16" } & 10 & \text { Each } \\ & \text { Fedex Airbill Pouches } & 2 & \text { Pack } \\ & \text { Fedex Blank Airbills } & 1 & \text { Pack } \\ & \text { Fedex Large Box } & 1 & \text { Each } \\ & \text { Fedex Preprinted Airbill - Warehouse - Stand } & \\ \text { 240-20 } & \text { Specific/Throw Away At } & 200 & \text { Each } \\ \text { 03-528-18 } & \text { News-Pak (newsprint on roll) } & 1 & \text { Roll } \\ 03-530-71 & \text { Shipper (Insulated, Styrofoam, Medium) - 16 x 16 x 16 } & 48 & \text { Each } \\ \text { MMM 3710-2-TT } & \text { Shipper (Insulated, Styrofoam, Small) - 14 x 14 x 10 } & 40 & \text { Each } \\ \text { 03-528C } & \text { Tape, 2" Packing } & 10 & \text { Roll } \\ & \text { U-Tek Large Refrigerant Packs (24 oz.) } & 14 & \text { Case }\end{array}$


Part number Description

$\underline{\text { Par level Unit distribution }}$

COMPONENT TOOLS

$6 \mathrm{Y} 762$

Valve Grease - Dow Corning Lubricant \#111

Tube

MEC SUPPLIES

PC22160

Tissues - Facial

2

Box

\section{NON-CONSUMABLE SUPPLIES}

\section{OFFICE SUPPLIES}

OIC $83100 \quad$ Clipboard

BOS B440BK Stapler, Standard Size

$2 \quad$ Each

SPARE PARTS \& TOOLS

$\begin{array}{llll}11779 & \text { Cordless Screwdriver w/Socket - NEW - Craftsman } & 1 & \text { Each } \\ & \text { 4.8 Volt Unit } & & \text { Each } \\ 22-46-139-7 & \text { Eppendorf Pipettor 100-5000uL } & 2 & \text { Each } \\ 22-46-138-9 & \text { Eppendorf Pipettor 50-1000uL } & 1 & \text { Each } \\ 22-46-136-2 & \text { Eppendorf Pipettor 5-100uL } & 2 & \text { Each } \\ 8395 & \text { Glass Fuse - 1 amp/250 volts - Centrifuge } & & \\ & \text { Hammer (16 oz. Claw) - Used for breaking } & & \text { Each } \\ 99833 & \text { down dry ice } & 2 & \text { Each } \\ \text { NC0090150 } & \text { Pipettor Battery - Spare/Backup Battery } & 1 & \text { Each } \\ 22461559 & \text { Pipettor Charging Stand } & 1 & \text { Each } \\ 40822 & \text { Screwdriver (4 in 1 multi-tip screwdriver) } & 1 & \text { Each } \\ 66-039-6 & \text { Screwdriver Set - 6 piece precision-Mfg. Name: Stanley } & 1\end{array}$

\section{SHIPPING SUPPLIES}
14-241B
$8 \mathrm{~A}-18116$
138465
138465
$1 \mathrm{E}-24039$
$\mathrm{H}-150$

\author{
Aluminum Ice Scoop \\ Full Face Shield \\ Gloves, Cryo Insulated, Large \\ Gloves, Cryo Insulated, Medium \\ Leather Utility Gloves \\ Tape Gun, 2"
}

Each
Each
Pair
Pair
Pair
Each 


\section{COMPONENT TOOLS}

\begin{tabular}{|c|c|c|c|}
\hline BPT - Custom & $\begin{array}{l}\text { Blood Processing Template - All Ages 1-2, 3-5, 6-11, } \\
12+\end{array}$ & 100 & Each \\
\hline $03-572-5$ & Bottle Brush - 11" & 2 & Each \\
\hline \multirow[t]{2}{*}{$21-102-52$} & Cube Rack (Clear) for Absorbic Acid - 4 Way & & \\
\hline & Flipper Rack & 2 & Each \\
\hline $15-059-147$ & $\begin{array}{l}\text { Exact -Temp Refrigerator Thermometer - Red Liquid } \\
\text { Therm. with Bottle }\end{array}$ & 1 & Each \\
\hline $15-059-145$ & $\begin{array}{l}\text { Exact-Temp Freezer Thermometer (Ultra-Low, -90C) } \\
\text { - Red Liquid Therm. with Bottle }\end{array}$ & 2 & Each \\
\hline OA-9188 & Flashlight - Black & 1 & Each \\
\hline ML4042 & Footstool - Metal & 2 & Each \\
\hline 03-007-38 & Graduated Cylinder $10 \mathrm{~mL}$ & 1 & Each \\
\hline $08-572-7 \mathrm{C}$ & Graduated Cylinder 50mL & 1 & Each \\
\hline 7BC-12097-2 & LAB- Wash Bottle- DI Water - Clear Squeeze Bottle & 1 & Each \\
\hline $13-812-26$ & Hemostat Forceps - Metal 6 1/4" Forceps & & \\
\hline $60985-444$ & Multicolor 4-Way Flip Racks - Set of 4 Racks & 1 & Each \\
\hline 14-809-101 & Nalge 13mm Half Rack - Unwire Plastic & 3 & Each \\
\hline 14-809-134 & Nalge 16mm Full Rack - Unwire Plastic & 4 & Each \\
\hline 14-809-117 & Nalge 16mm Half Rack - Unwire Plastic & 5 & Each \\
\hline $03-337-7 \mathrm{E}$ & Nalgene Cryovial Rack - Plastic, 50 Well Tray & 100 & Each \\
\hline $9 A-33810$ & Protective Eyewear & 3 & Each \\
\hline WX-167 & Public Alert Radio - Weather Radio & 1 & Each \\
\hline $03-576$ & Test Tube Brush - 9" & 2 & Each \\
\hline $15-078 \mathrm{~J}$ & Thermometer, Calibration - For Centerfuge & 1 & Each \\
\hline 15-077-19 & Thermometer, Solar & 2 & Each \\
\hline $06-662-51$ & Timer & 1 & Each \\
\hline $06-662-3$ & Timer, Triple Display & 2 & Each \\
\hline 7BC-12097-2 & Wash Bottle - Deionized Water Clear Squeeze Bottle & 2 & Each \\
\hline 7BC-12097-2 & Wash Bottle - Saline - Clear Squeeze Bottle & 1 & Each \\
\hline
\end{tabular}




\section{PHLEBOTOMY INVENTORY}

\section{CONSUMABLE ITEMS}

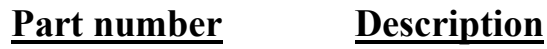

$\underline{\text { Par level Unit distribution }}$

EXAM SUPPLIES

$7000 \mathrm{HP}$

58-104

$17-98797 \mathrm{E}$

AB01

29501

ML8532

367281

367283

19-003-428D

9022

GOJ-9639 12CT

209269

Sticker

713267

519018

18-917

934-0852

4437

$02-6576$

06-66997

$1530-0$

$1527-0$

DPC-050

115000
Air Sickness Bags

Alcohol Wipes

Ammonia Ampules

Bandaids - Cartoon Characters

Bandaids (3/4" x 3" Flex Adhesive) - Coverlet

Bio-Hazard Sharps Container - 8 Quart Container

Butterfly Needle $21 \mathrm{~g}$ Safety - Lok - 50 pcs. box $/ 4$

boxes per case

Butterfly Needle 23g Safety - Lok - 50 pcs. box/4 boxes

per case

Co-Flex NL Cohesive Bandage 1Inch - Multi -

Colored Rolls

Gauze Pads (2 x 2)

Handsoap-Waterless (Purrell) -

Heel Warmers (Infant)

Kids Cartoon Stickers

NIPRO 19G X 3/4”Butterfly Needle - 50 pieces/box

NIPRO Luer Adapter - 100 per box

Pillow Cover - for 17" x 23" pillows

Pillow, (disposable, $17 \times 23$ )

Squeeze Ball (for drawing blood) - Foam 3" Ball

Tourniquets, Stretch (Blue, Vcutnr.)

Towlettes, Sani-Cloth (Cleaner)

Transpore Paper (1/2 " x 10 yds)

Transpore Tape (1/2 " x 10 yds)

Venolite Disposable Covers - Clear Plastic

White Ice Wings - Disposable Ice Cubes
Each

Box

Box

Box

Box

Each

Box

Box

Case

Pack

Bottle

Each

Roll

Box

Box

Each

Each

Each

Box

Bottle

Roll

Roll

Pack

Pack 
OFFICE SUPPLIES

DUR PC1500

DUR PC2400

107708

37001

FORMS
Batteries, AA

Batteries, AAA

Dymo Costar Label (1"x2 1/8") - 500 labels per roll

Magic Marker - Ultra Fine Sharpie
$6 \quad$ Each

8 Each

8 Box

$4 \quad$ Each

List of Reported Tests- English (Handout) - Counted Individually

List of Reported Tests Spanish (Handout) - Counted Individually
10

10

Each

Each

VOC SUPPLIES

VOC $7 \mathrm{~mL}$

BD Vacutainer 7mL Gray - VOC Blood Draw Tube

175

Each

\section{FASTING SNACK SUPPLIES}

30802188
PC9904
188670
$401526 \mathrm{FB}$
$401025 \mathrm{FB}$
$401223 \mathrm{FB}$

Animal Crackers

Juice, Apple - Motts

Juice, Orange - Minute Maid

Plastic Drinking Straw - Individually Wrapped

Plastic Medicine Cup - 1 Ounce

Trutol Glucose Bevarage - Fruit Punch - 24 per case

Trutol Glucose Bevarage - Lemon / Lime - 24 per case

$\begin{array}{cc}288 & \text { Each } \\ 200 & \text { Each } \\ 150 & \text { Each } \\ 1 & \text { Box } \\ 1 & \text { Sleeve } \\ 48 & \text { Each } \\ 30 & \text { Each } \\ 48 & \text { Each }\end{array}$


TUBES, VIALS, FILTERS

367921

367856

366668

367861

368380

366431

366430

22-289-953

8881301819
BD Hemogard 2 mL Gray Top

BD Hemogard 3mL EDTA Lavender Top

BD Hemogard 3mL Red Top

BD Hemogard 4 mL EDTA Purple Top

BD Hemogard $6 \mathrm{~mL}$ Royal Blue

BD Hemogard $7 \mathrm{~mL}$ Red Top

BD Vacutainer $10 \mathrm{~mL}$ Red Top

BD Vacutainer Tube Holder - 250 pcs. bag/4 bags per case

KS Vacutainer 15mL Red Top
Tray

Tray

Tray

Tray

Tray

Tray

Tray

$500 \quad$ Each

10 Tray

\section{EMERGENCY SUPPLIES}

8BZ-153919

Emergency Blanket - Disposable - Single Use

Each

COMPONENT TOOLS

415S0023BY

NOTRAX, Floor Mat - Shielded Sof-Tred, 2x3

1

Each

MEC SUPPLIES

PC22160

Tissues - Facial

2

Box

\section{NON-CONSUMABLE SUPPLIES}

\section{EMERGENCY SUPPLIES}

CPR Mask - Adult

Each

\section{COMPONENT TOOLS}

ML10043

111000

ML4042

14-809-130

14-809-101

14-809-117

232-237

SECA

08-952

15-077-19

VeinLite EMS
Bio-Hazard Trashcan - 7 Gallon Red Can

Buzzy Bee - Striped Vibrating Bee

Footstool - Bariatric- Metal Reinforced One Step Stool

Nalge 13mm Full Rack - Unwire Plastic

Nalge 13mm Half Rack - Unwire Plastic

Nalge 16mm Half Rack - Unwire Plastic

Plastic Supply Basket, black

Scale - Portable

Scissors - Lister bandage (5 1/2 " Stainless Steel)

Thermometer, Solar

Vein Lite EMS - Hand Held Unit
Each

Each

Each

Each

Each

Each

Each

Each

Each

Each

Each 